HYDROLOGIC DATA FOR PALEOZOIC ROCKS IN THE UPPER COLORADO RIVER BASIN, COLORADO, UTAH, WYOMING, AND ARIZONA

REGIONAL AQUIFER-SYSTEM ANALYSIS PROGRAM

By A.L. Geldon

U.S. GEOLOGICAL SURVEY

Open-File Report 89-59 


\section{DEPARTMENT OF THE INTERIOR}

DONALD PAUL HODEL, Secretary

U.S . GEOLOGICAL SURVEY

Dallas L. Peck, Director

For additional information write to:

District Chief U.S. Geological Survey Box 25046, Mail Stop 415

Federal Center Denver, CO 80225-0046
Copies of this report can be purchased from:

U.S. Geological Survey Books and Open-File Reports Section Federal Center

Box 25425

Denver, CO 80225-0425

[Telephone: (303) 236-7476] 
FIGURE

Figure 1. Map showing location of the Upper Colorado River Basin

Page and areal coverage by the principal survey meridians within the basin--

\section{TABLES}

Table 1. Statigraphic codes for geologic units named in the report----- 3

2. Equations relating porosity to intergranular permeability for Paleozoic hydrogeologic units-_. 8

3. Artesian yields from wells and springs-_- 15

4. Laboratory-determined porosity and permeability-_- 33

5. In-situ permeability and hydraulic conductivity-_- 195

\section{CONVERSION FACTORS}

This report uses inch-pound units as the primary system for numerical data. Inch-pound units can be converted to metric units with the following multiplication factors:

\section{Multiply}

barrel (bb1)

foot $(f t)$

foot squared per day $\left(\mathrm{ft}^{2} / \mathrm{d}\right)$

foot per day $(\mathrm{ft} / \mathrm{d})$

gallon (gal)

gallon per minute (gal/min)

inch (in.)

mile (mi)

millidarcy $(\mathrm{mD})$

pound per square inch $\left(1 \mathrm{~b} / \mathrm{in}^{2}\right)$

square mile $\left(\mathrm{mi}^{2}\right)$
$B y$

158.9

0.3048

0.09290

0.3048

3.7854

0.06308

25.4

1.6093

$9.87 \times 10^{-12}$

6.895

2.59
To obtain

liter

meter

meter squared per day

meter per day

liter

liter per second

millimeter

kilometer

square centimeter

kilopascal

square kilometer

Degree Fahrenheit $\left({ }^{\circ} \mathrm{F}\right)$

${ }^{\circ} \mathrm{C}=5 / 9\left({ }^{\circ} \mathrm{F}-32\right)$

degree Celsius $\left({ }^{\circ} \mathrm{C}\right)$ 


\title{
HYDROLOGIC DATA FOR PALEOZOIC ROCKS IN THE \\ UPPER COLORADO RIVER BASIN, COLORADO, UTAH, WYOMING, AND ARIZONA
}

By A.L. Geldon

\begin{abstract}
This report contains data that were used to interpret the hydrology of Paleozoic rocks in the Upper Colorado River Basin under the U.S. Geological Survey's Regional Aquifer-System Analysis program. The study area includes the drainages of the Green and Colorado Rivers from their headwaters to Lees Ferry, Arizona. Hydrologic data presented in this report include artesian yields from wells and springs and values of porosity, intrinsic permeability, and hydraulic conductivity determined by laboratory analyses and aquifer tests.
\end{abstract}

\section{INTRODUCTION}

In anticipation of increasing ground-water use in the Upper Colorado River Basin, the U.S. Geological Survey undertook a systematic appraisal of the area's ground-water resources from 1981 to 1986. This study is known as the Upper Colorado River Basin Regional Aquifer-System Analysis (RASA). The plan of investigation for this study is discussed by Taylor and others (1983). This report presents hydrologic data compiled from diverse published and unpublished sources that were used in the investigation of Paleozoic aquifers and confining layers.

\section{LOCATION}

The Upper Colorado River Basin (fig. 1) extends from the headwaters of the Colorado and Green Rivers to Lees Ferry, Arizona, and covers parts of five States--Colorado, Utah, Wyoming, Arizona, and New Mexico. Excluding the San Juan Basin (which is being studied separately), the Upper Colorado River Basin has a surface area of approximately $100,000 \mathrm{mi}^{2}$.

\section{GEOLOGIC SETTING}

The Upper Colorado River Basin includes parts of four physiographic provinces--the Middle Rocky Mountains, Southern Rocky Mountains, Wyoming Basin, and Colorado Plateaus (Taylor and others, 1983, p. 5). Within this region, geologic formations representing all Paleozoic systems except the Silurian are present (Taylor and others, 1986; Lindner-Lunsford and others, 1985). Cambrian to Mississippian formations predominantly consist of limestone, dolomite, quartz sandstone, and quartzite. Pennsylvanian and Permian formations predominantly consist of arkosic and quartz sandstone 


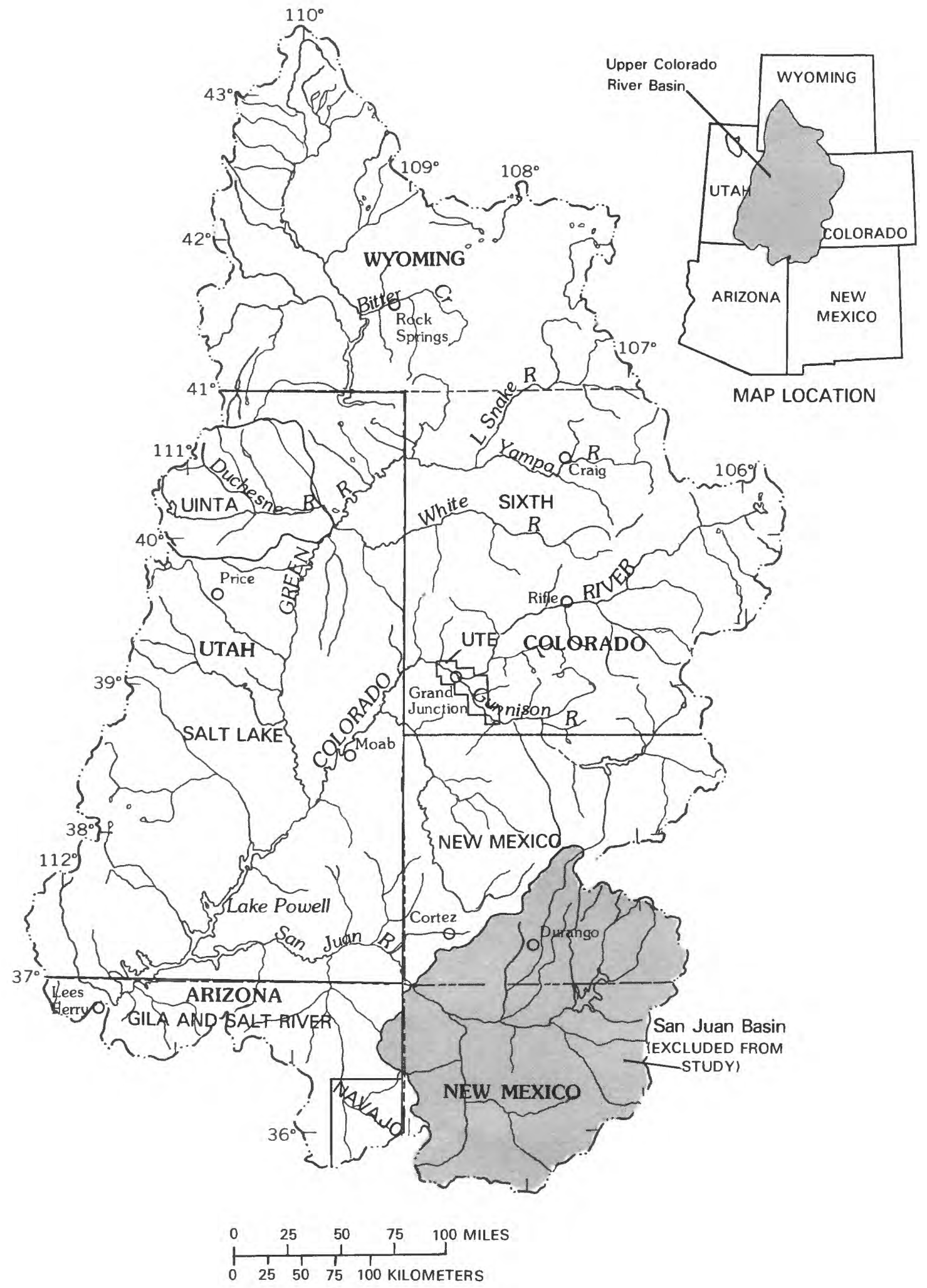

Figure 1.--Location of Upper Colorado River Basin 
and shale; thick carbonate or evaporite sequences are present locally. The Paleozoic rocks range in thickness from 0 to $18,000 \mathrm{ft}$ and comprise 11 hydrogeologic units--2 regional aquifers, 4 local aquifers, and 5 confining layers (table 1). They are buried by as much as $27,000 \mathrm{ft}$ of Mesozoic and Tertiary rocks in deep structural basins but are at or near land surface or missing in the center of most uplifted areas (Geldon, in press).

Table 1.--Stratigraphic codes for geologic units named in the report

[The codes are derived from the GEONAMES data base of the U.S. Geological Survey and are listed by hydrogeologic unit. The data base is an annotated index lexicon of formal geologic nomeclature of the United States]

\begin{tabular}{ll}
\hline Code Geologic unit $\quad$ Approximate age \\
\hline
\end{tabular}

Park City-State Bridge confining unit

317 GSEG

317SBDG

$317 \mathrm{KIBB}$

317PRKC

317PSPR

317TRWP

Goose Egg Formation------------- Permian and Triassic

State Bridge Formation------ Permian and Triassic

Kaibab Formation---_-_-_-_------ Permian

Park City Formation--------- Permian

Phosphoria Formation--..-.-.-- Permian

Toroweap Formation----.--- Permian

Weber-De Chelly aquifer

317DCLL

317WTRM

321WEBR

321TSLP

321WLLS

310PMPV

317RICO

317CDRM

317CTLR

317ELPC

317HLGT

3170GRK

321HKTL

324HRMS

324MNRN

324MRGN

324MRON

324AMSD

\begin{abstract}
De Chelly Sandstone---_.
White Rim Sandstone---

Weber Sandstone---

Tensleep Sandstone---_-.--

Wells Formation--
\end{abstract}

Permian

Permian

Pennsylvanian and Permian

Pennsylvanian and Permian

Pennsylvanian and Permian

\section{Cutler-Maroon aquifer}

Pennsylvanian and Permian rocks---- Pennsylvanian and Permian

Rico Formation----- Pennsylvanian and Permian

Cedar Mesa Sandstone--.-.--.---- Permian

Cutler Formation (Group)-..----- Pennsylvanian and Permian

Elephant Canyon Formation-------- Permian

Halgaito Shale (Formation)-------- Permian

Organ Rock Shale------_-------- Permian

Honaker Trail Formation (Upper

Unit Hermosa Group)--_-.------ Pennsylvanian

Hermosa Formation---..- Pennsylvanian

Minturn Formation---.- Pennsylvanian

Morgan Formation----------- Pennsylvanian

Maroon Formation------------ Pennsylvanian and Permian

Amsden Formation---- Mississippian and Pennsylvanian

Paradox-Eagle Valley confining unit

324RDVL Round Valley Limestone---.---.--- Pennsylvanian

324EGLV Eagle Valley Evaporite (Formation)-- Pennsylvanian

324PRDX Paradox Member of Hermosa Formation- Pennsylvanian 
Table 1.--Stratigraphic codes for geologic units named in the report--Continued

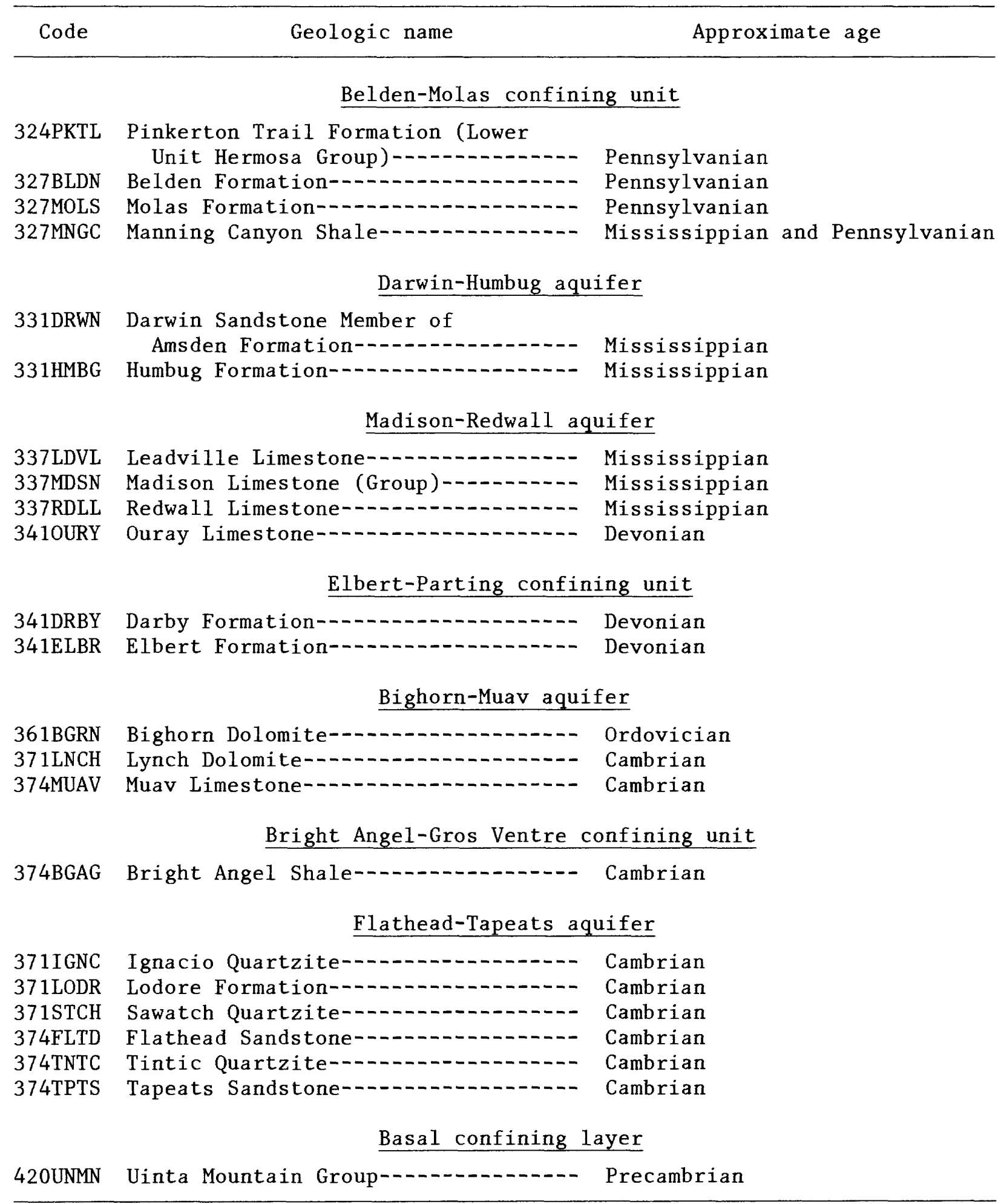


Wells and springs are numbered in this report according to the U.S. Bureau of Land Management system. The first one or two letters in the siteidentification number represent the principal survey meridian. Principal survey meridians to which the study area is referenced (fig. 1) and the symbols adopted for them in this report are:

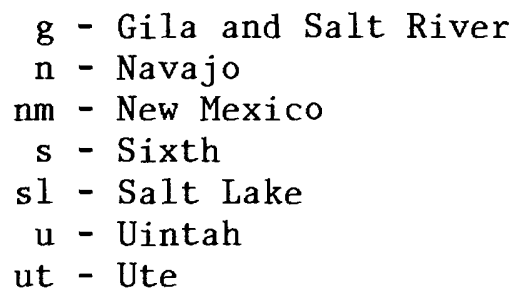

Letters and numbers following the symbol for the principal survey meridian in the site identification number refer, in order, to quadrant, township, range, section, quarter section, quarter-quarter section, quarter-quarterquarter section, and number of well or spring within the smallest physical boundary (multiple ground-water sites within the smallest physical boundary are numbered consecutively). Quadrants and divisions of sections are labeled from a to $d$ in a counter-clockwise direction starting with the northeast quadrant or section division. Dashes are used to separate quadrant, township, range, and section designations. As an example, a well numbered sc06-89-09 bda1 is the first well in the northeast quarter of the southeast quarter of the northwest quarter of Section 9, Township 6 South, Range 89 West, in the southwest quadrant of the Sixth principal survey meridian.

\section{HYDROLOGIC DATA}

Artesian yields from wells and springs, porosity, laboratory-determined (integranular) and field-determined (in-situ) permeability, and hydraulicconductivity data are presented in tables 3, 4, and 5 (following the References section). Stratigraphic codes used in these tables are explained in table 1. Sources for the data are listed in the tables.

Data presented in this report were obtained by a variety of methods. The 779 yield values reported in table 3 are spring flows and artesian flows into or from wells. The well flows include flows measured at the surface and flows from specific horizons recorded during drilling or aquifer tests. Much of the yield data were calculated from the volume of fluid recovered during drillstem tests, using equation 1:

$$
q=\frac{0.041 L d^{2}}{t}
$$

where $q=$ discharge, in gallons per minute;

$L=$ length of fluid-filled drill pipe, in feet;

$d=$ diameter of drill pipe (unless known, it is assumed to be 3.83 in., based on information in Bredehoeft, 1965); and

$t=$ the flow period, in minutes. 
In order to use a drill-stem test for hydrological interpretation, it was decided arbitrarily that the recovered fluid had to be at least 75 percent water or some form of water, such as muddy water, salty water, oil-cut water, or gas-cut water. If more than one flowing interval was encountered in a formation in the same borehole or in closely spaced boreholes within the same section, generally only the largest yield was recorded. At sites where discharges from springs or wells were measured on more than one occasion, only the maximum discharge was recorded in table 3.

Values of laboratory-determined porosity and integranular permeability are listed in table 4. For each hydrologic property, there are 7,659 values determined from plugs of core taken from petroleum industry exploration boreholes (Petroleum Information Corp., unpublished data) and 6 values determined from outcrop samples (compiled from Hood, 1976; Hood and Patterson, 1984, and U.S. Geological Survey files). Although generally unstated, the specific method of analysis presumably followed conventional procedures described by Freeze and Cherry, 1979, p. 335-337). In the standard method of analyzing porosity, samples are saturated, weighed, oven-dried, and reweighed. Porosity is calculated from the weight of the water lost in drying. Intergranular permeability routinely is measured with a permeameter, a device in which water or air under constant or falling head is passed through a sample.

Values of in-situ permeability and hydraulic conductivity are listed in table 5. Most of the in-situ permeability values (406) were obtained from dril1-stem tests, either by the method of Horner (1951) or by less precise methods described by Geldon (in press) and Earlougher (1977). In the Horner (1951) method, as described by Bredehoeft (1965), pressure in the well bore during a shut-in period is plotted versus time, and permeability is calculated from the straight-line portion of the plot by the equation:

$$
k=\frac{162.6 q v \log [(t+\Delta t) / \Delta t]}{h\left(\rho_{0}-\rho_{w}\right)} ;
$$

where $k=$ in-situ permeability, in millidarcies;

$q=$ discharge rate, in barrels per day;

$p_{w}=$ well-bore pressure, in pounds per square inch;

$p_{0}=$ undisturbed formation pressure, in pounds per square inch;

$h=$ thickness of the tested interval, in feet;

$v=$ viscosity, in centipoise;

$t=$ flow period, in minutes; and

$\Delta t=$ shut-in period, in minutes.

The Geldon (in press) and Earlougher (1977) methods use variations of the Horner (1951) equation to estimate permeability from summary drill-stem test data, where detailed records of pressure versus time are not available. Respectively, the equations used are:

$$
k=\frac{162.6 \mathrm{vq} \log \left[\left(\Delta t_{1} / \Delta t_{2}\right) \times\left(t_{2}+\Delta t_{2}\right) /\left(t_{1}+\Delta t_{1}\right)\right]}{h\left(p_{1}-p_{2}\right)}
$$

and 


$$
k=\frac{162.6 q v \beta \log [(t+\Delta t) / \Delta t]}{h(p i-p f)}
$$

where $k=$ in-situ permeability, in millidarcies;

$\mathrm{v}=$ viscosity, in centipoise;

$q=$ discharge rate, in barrels per day;

$h=$ thickness of test interval, in feet;

$t=$ flow period, in minutes;

$t_{1}=$ initial flow period, in minutes;

$t_{2}=$ final flow period, in minutes;

$\Delta t=$ shut-in period, in minutes;

$\Delta t_{1}=$ initial shut-in period, in minutes;

$\Delta t_{2}=$ final shut-in period, in minutes;

$p_{1}=$ reported pressure during initial shut-in period, in pounds per square inch;

$p_{2}=$ reported pressure during final shut-in period, in pounds per square inch;

$p i=$ reported pressure before flow period, in pounds per square inch;

$p f=$ reported pressure after flow period, in pounds per square inch; and

$\beta=$ a formation constant between 0.99 and 1.06 (unknown but assigned a value of 1 because errors resulting from a wrong value of $\beta$, within its narrow range, were felt to be less significant in calculating permeability than errors resulting from inaccurate estimates of viscosity or the imprecision of the method).

Additional (182) values of in-situ permeability listed in table 5 were estimated from porosity and intergranular permeability by empirical equations developed during the RASA study. Equations relating intergranular permeability to porosity by rock type and hydrostratigraphic unit are given in table 2 . Although these equations were derived entirely from laboratory data, they were applied also to geophysically determined values of porosity in estimating permeability. Geophysically-determined values of porosity were furnished by G.A. Wetherbee and W.P. Van Liew (U.S. Geological Survey, written commun., 1985) or obtained from a report by Fox and others (1975). Wetherbee and Van Liew determined porosity using a combination of sonic, bulk-density, and neutron logs calibrated against lithologic logs and measured porosity. Fox and others determined porosity from sonic logs calibrated against a curve of interval transit time versus measured porosity. Procedures used in these porosity determinations are described by Keys and MacCary (1981). Equations relating intergranular to in-situ permeability (based on data from 22 paired boreholes) varied according to whether the interval was sandstone or carbonate rock. These equations are:

and

$$
k_{d}=1.4 \times k_{s}^{0.65}
$$

$$
k_{d}=3.5 \times k_{c}^{0.80}
$$

where $k_{d}$ = equivalent in-situ permeability, in millidarcies;

$k_{s}=$ geometric mean of intergranular sandstone permeability values in a borehole or outcrop area, in millidarcies; and

$k_{C}=$ arithmetic mean of intergranular carbonate permeability values in a borehole or outcrop area, in millidarcies. 
Table 2.--Equations relating porosity to intergranular permeability for Paleozoic hydrogeologic units

\begin{tabular}{|c|c|c|c|c|}
\hline $\begin{array}{l}\text { Hydrogeologic } \\
\text { unit }\end{array}$ & Rock type & Equation & $\begin{array}{l}\text { Correlation } \\
\text { coefficient } \\
\qquad\left(R^{2}\right)\end{array}$ & $\begin{array}{c}\text { Number } \\
\text { of } \\
\text { observations }\end{array}$ \\
\hline $\begin{array}{l}\text { Park City-State Bridge } \\
\text { confining unit }\end{array}$ & $\begin{array}{l}\text { Sandstone } \\
\text { Dolomite } \\
\text { Limestone }\end{array}$ & $\begin{array}{l}k=0.022 \mathrm{e}^{0.41 \theta} \\
k=0.0091 \mathrm{e}^{0.46 \theta} \\
\text { Insignificant }\end{array}$ & $\begin{array}{r}0.67 \\
.83 \\
<.60\end{array}$ & $\begin{array}{r}30 \\
521 \\
76\end{array}$ \\
\hline $\begin{array}{l}\text { Weber-De Chelly } \\
\text { aquifer }\end{array}$ & Sandstone & $k=0.017 \mathrm{e}^{0.46 \theta}$ & .87 & 2,636 \\
\hline Cutler-Maroon aquifer & $\begin{array}{l}\text { Sands tone } \\
\text { Limestone }\end{array}$ & $\begin{array}{l}k=0.017 \mathrm{e}^{0.35 \theta} \\
\text { Insignificant }\end{array}$ & $\begin{array}{r}.66 \\
<.60\end{array}$ & $\begin{array}{l}292 \\
195\end{array}$ \\
\hline $\begin{array}{l}\text { Paradox-Eagle Valley } \\
\text { confining unit }\end{array}$ & $\begin{array}{l}\text { Sandstone } \\
\text { Dolomite } \\
\text { Limestone } \\
\text { Shale } \\
\text { Anhydrite }\end{array}$ & $\begin{array}{l}k=0.035 \mathrm{e}^{0.25 \theta} \\
\text { Insignificant } \\
k=0.019 \mathrm{e}^{0.37 \theta} \\
\text { Insignificant } \\
\text { Insignificant }\end{array}$ & $\begin{array}{r}.61 \\
<.60 \\
.62 \\
<.60 \\
<.60\end{array}$ & $\begin{array}{r}37 \\
722 \\
1,649 \\
41 \\
15\end{array}$ \\
\hline $\begin{array}{l}\text { Belden-Molas } \\
\text { confining unit }\end{array}$ & $\begin{array}{l}\text { Dolomite } \\
\text { Limestone }\end{array}$ & $\begin{array}{l}k=0.017 \mathrm{e}^{1.2 \theta} \\
\text { Insignificant }\end{array}$ & $\begin{array}{r}.95 \\
<.60\end{array}$ & $\begin{array}{r}7 \\
10\end{array}$ \\
\hline Darwin-Humbug aquifer & $\begin{array}{l}\text { Sandstone } \\
\text { Dolomite }\end{array}$ & $\begin{array}{l}\text { Insignificant } \\
\text { Insignificant }\end{array}$ & $\begin{array}{l}<.60 \\
<.60\end{array}$ & $\begin{array}{r}19 \\
9\end{array}$ \\
\hline $\begin{array}{l}\text { Madison-Redwall } \\
\text { aquifer }\end{array}$ & $\begin{array}{l}\text { Dolomite } \\
\text { Limestone }\end{array}$ & $\begin{array}{l}\text { Insignificant } \\
\text { Insignificant }\end{array}$ & $\begin{array}{l}<.60 \\
<.60\end{array}$ & $\begin{array}{l}435 \\
513\end{array}$ \\
\hline $\begin{array}{l}\text { Elbert-Parting } \\
\text { confining unit }\end{array}$ & $\begin{array}{l}\text { Sandstone } \\
\text { Dolomite }\end{array}$ & $\begin{array}{l}k=0.010 \mathrm{e}^{0.69 \theta} \\
\text { Insignificant }\end{array}$ & $\begin{array}{r}.77 \\
<.60\end{array}$ & $\begin{array}{r}125 \\
89\end{array}$ \\
\hline Bighorn-Muav aquifer & $\begin{array}{l}\text { Sandstone } \\
\text { Dolomite }\end{array}$ & $\begin{array}{l}k=0.001 \mathrm{e}^{0.94 \theta} \\
k=0.0032 \mathrm{e}^{0.74 \theta}\end{array}$ & $\begin{array}{l}.88 \\
.81\end{array}$ & $\begin{array}{r}9 \\
45\end{array}$ \\
\hline
\end{tabular}

${ }^{1} \mathrm{~A}$ relation between porosity and intergranular permeability was considered significant and useful for estimating hydrologic properties if the correlation coefficient for the analysis was greater than 0.60 . Equations could have been developed from regression analyses with correlation coefficients of less than 0.60 , but the credibility of calculated values would have been questionable. 
Hydraulic-conductivity values reported in table 5 were determined either by conversion from in-situ permeability, by analysis of pressure-injection tests, or by conversion from transmissivity values determined by constant-rate, airlift, or step-drawdown pumping tests, flowing-well tests, slug tests, or specific-capacity (pumping and bailing) tests. Conversion from in-situ permeability was based on the equation (Freeze and Cherry, 1979, p. 27):

$$
K=\frac{k p g}{\mu}
$$

where $K=$ hydraulic conductivity of water at $60^{\circ} \mathrm{F}$;

$k=$ in-situ permeability;

$p=$ density of water at $60^{\circ} \mathrm{F}$;

$g=$ acceleration due to gravity at $60^{\circ} \mathrm{F}$;

$\mu=$ viscosity of water at $60^{\circ} \mathrm{F}$;

Results of 84 pressure-injection tests done in 13 wells at 6 damsites were made available by the U.S. Bureau of Reclamation. The methods and analytical procedures for this kind of test are described by the U.S. Water and Power Resources Service (1981, p. 249-266). The relevant equation is:

$$
K=\frac{30.6 \& \operatorname{Ln}(L / r)}{L H}
$$

where $K=$ hydraulic conductivity, in feet per day;

$Q=$ discharge, in gallons per minute;

$L=$ test interval, in feet;

$r=$ well radius, in feet; and

$H=$ head, in feet.

Results of 4 pressure-injection tests of the Leadville (Redwall) Limestone and 15 pumping tests--2 in the Weber Sandstone, 6 in the De Chelly Sandstone, 6 in the Paradox Member of the Hermosa Formation, and 1 in the Cutler Group and upper member of the Hermosa Formation, were compiled from reports by Cooley and others (1969), Hood (1976), Sumsion (1976), Wollitz and others (1982), and Thackston and others (1984). The pressure-injection tests of the Leadville Limestone were analyzed by equation 2 and by the Cooper-Jacob (1946) modification of the Theis (1935) nonequilibrium solution. The relevant equation in the Cooper-Jacob (1946) solution is:

$$
T=\frac{35.2 Q}{\Delta s_{d}}
$$

where $T=$ transmissivity, in feet squared per day;

$Q=$ discharge, in gallons per minute; and

$\Delta s_{d}=$ change in drawdown, residual drawdown, or recovery, in feet, over one log cycle of time.

The four injection tests yielded similar values of transmissivity, and a median value is reported in table 5. The pumping tests were analyzed by either equation 9, the Theis (1935) recovery formula (a variation of equation 9 in which residual drawdown is substituted for drawdown), or the Theis (1935) nonequilibrium solution. The relevant equation in the latter solution is: 


$$
T=\frac{Q}{4 \pi s} W(u)
$$

where $T=$ transmissivity, in feet squared per day;

$Q=$ discharge, in cubic feet per day;

$s=$ drawdown, in feet at the type-curve match point; and

$W(u)=$ the well function of a non leaky aquifer, at the type curve match point.

Transmissivity values determined from the above-cited injection and pumping tests were converted to hydraulic conductivity values by dividing by the test interval thickness.

Thirty-nine pumping and bailing-test results in the area were obtained from reports and files of State and Federal agencies that are cited in table 5. These tests were not analyzable by conventional methods because head drawdown versus time data were not available. Estimates of transmissivity from specific capacity, however, could be made using equations given by Lohman (1979, p. 52) and Driscoll (1986, p. 1021). These equations are:

$$
T=\frac{2.3 \& \log \frac{2.25 T t}{r_{w}{ }^{2} S}}{4 \pi S_{w}} ;
$$

and

$$
T=\frac{2000 Q}{S w}
$$

where $T=$ transmissivity, in feet squared per day (equation 11) or gallons per day per foot (equation 12);

$Q=$ discharge in cubic feet per day (equation 11) or gallons per minute (equation 12);

$S_{w}=$ drawdown, in feet;

$t=$ pumping time, in days;

$r_{w}=$ radius of well, in feet; and

$S=$ storage coefficient, dimensionless.

If unknown, the storage coefficient was estimated by multiplying the thickness of the tested interval by $1 \times 10^{-6}$, as suggested by Lohman (1979, p. 53). To determine hydraulic conductivity, the calculated value of transmissivity, in feet squared per day, was divided by the test interval thickness.

A flowing-well test of the Leadville Limestone at Glenwood Springs, Colo. (Geldon, in press), was made during this study. Changes in head in the production well were observed directly by means of a standpipe attached to the well. Discharge was measured by the orifice-plate method (U.S. Water and Power Resources Service, 1981, p. 233-242). Four observation wells and three springs also were monitored. A recording barometer was set up to adjust recorded water levels for changes in atmospheric pressure. Test data were analyzed by the solutions of Jacob and Lohman (1952) and Hantush (1960) and by the Theis recovery formula. The equations for the Jacob-Lohman (1952) and Hantush (1960) solutions are: 


$$
T=\frac{0.183 \times 1,440}{\Delta(s / 2)_{d}}
$$

and

$$
T=\frac{192.5 Q H(u, \beta)}{4 \pi s}
$$

where $T=$ transmissivity, in feet squared per day;

$Q=$ discharge, in gallons per minute;

$s=$ drawdown or recovery at the type-curve match point, in feet;

$\Delta(s / Q)=$ change in specific discharge over 1 log cycle of time; and

$H(u, \beta)=$ the well function of a leaky aquifer with storage in the confining beds, at the type-curve match point.

Three slug-injection tests done in salt beds of the Paradox Member of the Hermosa Formation and an "airlift" test of the Cutler Group provided additional transmissivity data. The slug tests were analyzed by a method of type-curve matching described by Cooper and others (1967) and by Lohman (1979, p. 27-30). The "airlift" test result was reported by Woodward-Clyde Consultants (1982); the test was described by Thackston and others (1984). The latter report indicates that the airlift test involved displacement of water from a borehole by injection of compressed air and monitoring the recovery of formation pressure. Presumably, the pressure recovery data were analyzed using equation 9. Hydraulic conductivity was determined in both the slug and airlift tests by dividing transmissivity by test interval thickness.

The data presented in the following tables (3, 4, and 5) are reported without interpretation. Some interpretive reports for the Upper Colorado River Basin have been published prior to this data report, and others are planned. The data are given to support the interpretive reports and to provide the most comprehensive hydrologic-data base for Paleozoic rocks in the Upper Colorado River Basin assembled to date (1987). The data should be useful as a starting point for hydrologic studies in the basin. However, the data are distributed very unevenly and vary in quality from marginal to very good. Thus, it is recommended that additional data be collected wherever studies of local or sub-regional scale are planned.

\section{REFERENCES}

Barrett, J.K., and Pearl, R.H., 1977, An appraisal of Colorado's geothermal resources: Denver, Colorado Geological Survey Final Technical Report, $290 \mathrm{p}$.

Berry, D.W., 1960, Geology and ground-water resources of the Rawlins area, Carbon County, Wyoming: U.S. Geological Survey Water-Supply Paper 1458, $74 \mathrm{p}$.

Bredehoeft, J.D., 1965, The drill-stem test, the petroleum industry's deepwell pumping test: Groundwater, v. 3, no. 3, p. 31-36.

Cooley, M.E., 1976, Spring flow from pre-Pennsylvanian rocks in the southwestern part of the Navajo Indian Reservation, Arizona: U.S. Geological Survey Professional Paper 521-F, 15 p. 
Cooley, M.E., Harshbarger, J.W., Akers, J.P., and Hardt, W.F., 1969, Regional hydrology of the Navajo and Hopi Indian Reservations, Arizona, New Mexico, and Utah: U.S. Geological Survey Professional Paper 521-A, 61 p.

Cooper, H.H., and Jacob, C.E., 1946, A generalized graphical method for evaluating formation constants and summarizing well-field history: American Geophysical Union Transactions, v. 27, no. 4, p. 526-534.

Cooper, H.H., Jr., Bredehoeft, J.D., and Papadopolos, S.S., 1967, Response of a finite-diameter well to an instantaneous charge of water: Water Resources Research, v. 3, no. 1, p. 263-269.

Davis, G.E., Hardt, W.F., Thompson, L.K., and Cooley, M.E., 1963, Geohydrologic data in the Navajo and Hopi Indian Reservations, Arizona, New Mexico, and Utah, Part I--Records of ground-water supplies: Tucson, Arizona State Land Department Water Resources Report 12-A, 159 p.

Driscoll, F.G., 1986, Groundwater and wells, second edition: St. Paul, Minn., Johnson Division, UOP, $1089 \mathrm{p}$.

Earlougher, R.C., Jr., 1977, Advances in well-test analysis: Dallas, Tex., American Institute of Mining, Metallurgical, and Petroleum Engineers, Monograph 5, $264 \mathrm{p}$.

Feltis, R.D., 1966, Water from bedrock in the Colorado Plateau of Utah: Salt Lake City, Utah Department of Natural Resources Technical Publication 15, $82 \mathrm{p}$.

Fox, J.E., Lambert, P.W., Mast, R.F., Nuss, N.W., and Rein, R.D., 1975, Porosity variation in the Tensleep and its equivalent, the Weber Sandstone, western Wyoming--A $\log$ and petrographic analysis, in Balyard, D.W., ed., Deep drilling frontiers of the central Rocky Mountains: Denver, Colo., Rocky Mountain Association of Geologists, p. 185-216.

Freeze, R.A., and Cherry, J.A., 1979, Groundwater: Englewood Cliffs, N.J., Prentice-Ha11, Inc., $604 \mathrm{p}$.

Geldon, A.L., in press, Hydrogeology of the Leadville Limestone and other Paleozoic rocks in northwestern Colorado, with results of aquifer tests at Glenwood Springs: U.S. Geological Survey Water-Resources Investigations Report 87-4195.

Hampton, E.R., 1974, Preliminary evaluation of ground water in the prePennsylvanian carbonate rocks, McCoy area, Colorado: U.S. Geological Survey open-file report, $11 \mathrm{p}$.

Hand, F.E., Jr., 1979, Groundwater resources in the northern part of the Glen Canyon National Recreation Area and adjacent lands west of the Colorado and Green Rivers, Utah: Laramie, Wyoming Water Resources Research Institute, University of Wyoming, $44 \mathrm{p}$.

Hantush, M.S., 1960, Modification of the theory of leaky aquifers: Journal of Geophysical Research, v. 65, no. 11, p. 3713-3725.

Harshbarger, J.W., and Repenning, C.A., 1954, Water resources of the Chuska Mountains area, Navajo Indian Reservation, Arizona and New Mexico: U.S. Geological Survey Circular 308, 16 p.

Hood, J.W., 1976, Characteristics of aquifers in the northern Uinta Basin, Utah and Colorado: Salt Lake City, Utah Department of Natural Resources Technical Publication 53, 71 p.

Hood, J.W., and Danielson, T.W., 1981, Bedrock aquifers in the lower Dirty Devil River basin area, Utah, with special emphasis on the Navajo Sandstone: Salt Lake City, Utah Department of Natural Resources Technical Publication $68,143 \mathrm{p}$.

Hood, J.W., Mundorff, J.C., and Price, Don, 1976, Selected hydrologic data, Uinta Basin area, Utah and Colorado: U.S. Geological Survey Utah BasicData Release 26, 321 p. 
Hood, J.W., and Patterson, D.J., 1984, Bedrock aquifers in the northern San Rafael Swell area, Utah, with special emphasis on the Navajo Sandstone: Salt Lake City, Utah Department of Natural Resources Technical Publication $78,128 \mathrm{p}$.

Horner, D.R., 1951, Pressure buildup in wells, in Proceedings, Third World Petroleum Congress, Part II: Leiden, E.J. Brill, p. 503-521.

Huntoon, P.W., 1977, The hydrologic feasibility of developing ground-water supplies in the northern part of Canyonlands National Park and Bridges National Monument, Utah: Laramie, Wyoming Water Resources Research Institute, University of Wyoming, $24 \mathrm{p}$.

Iorns, W.V., Hembree, C.H., Phoenix, D.A., and Oakland, G.L., 1964, Water resources of the Upper Colorado River Basin--Basic data: U.S. Geological Survey Professional Paper 442, 1036 p.

Jacob, C.E., and Lohman, S.W., 1952, Nonsteady flow to a well of constant drawdown in an extensive aquifer: Washington, D.C., American Geophysical Union Transactions, v. 33, no. 3, p. 559-569.

Jobin, D.A., 1962, Relation of the transmissive character of the sedimentary rocks of the Colorado Plateau to the distribution of uranium deposits: U.S. Geological Survey Bulletin 1124, 151 p.

Keys, W.S., and MacCary, L.M., 1981, Application of borehole geophysics to water-resources investigations: U.S. Geological Survey Techniques of Water-Resources Investigations Report, bk. 2, chap. E1, 126 p.

Linder-Lunsford, J.B., Kimba11, B.A., Chafin, D.T., and Bryant, C.G., 1985, Hydrogeology of aquifers of Paleozoic age, Upper Colorado River Basin, excluding the San Juan Basin, in Colorado, Utah, Wyoming, and Arizona: U.S. Geological Survey Hydrologic Atlas HA-702, Scale 1:2,500,000, 2 sheets.

Lines, G.C., and Glass, W.R., 1975, Water resources of the thrust belt of western Wyoming: U.S. Geological Survey Hydrologic Investigations Atlas HA-539, scale $1: 25,000,3$ sheets.

Lohman, S.W., 1979, Ground-water hydraulics: U.S. Geological Survey Professional Paper 708, 70 p.

McGavock, E.H., Edmonds, N.J., Gillespie, E.L., and Halpenny, P.C., 1966, Geohydrologic data in the Navajo and Hopi Indian Reservations, Arizona, New Mexico, and Utah, Part IA--Supplemental records of ground-water supplies: Tucson, Arizona State Land Department Water Resources Report 12-E, 55 p.

Richter, H.R., Jr., 1980, Ground-water resources in the part of Canyonlands National Park east of the Colorado River and contiguous Bureau of Land Management lands: Laramie, University of Wyoming, unpublished Master of Science thesis, $80 \mathrm{p}$.

Ritzma, H.R., and Doelling, H.H., 1969, Mineral resources, San Juan County, Utah, and adjacent areas, Part I: Petroleum, potash, ground water, and miscellaneous minerals: Salt Lake City, Utah Geological and Mineralogical Survey Special Studies 24, 125 p.

Rouse, J.V., 1967, Mineral springs and other natural point sources of saline pollution in the Colorado River Basin: Phoenix, Ariz., U.S. Department of Interior Federal Water Pollution Control Administration, Southwest Region, Colorado River Basin Water Quality Control Project Field Station, $23 \mathrm{p}$.

Rush, F.E., Hart, I.M., Whitfield, M.S., Giles, T.F., and D'Epagnier, T.G., 1980, Results of hydraulic tests in wells DOE-1, 2, and 3, Salt Valley, Grand County, Utah: U.S. Geological Survey Open-File Report 80-205, $33 \mathrm{p}$. 
Sumsion, C.T., 1971, Geology and water resources of the Spanish Valley area, Grand and San Juan Counties, Utah: Salt Lake City, Utah Department of Natural Resources Technical Publication 32, 45 p. 1976, Water resources of Dinosaur National Monument, Colorado and Utah: U.S. Geological Survey Open-File Report 76-580, 68 p.

Sumsion, C.T., and Bolke, E.L., 1972, Water resources of part of Canyonlands National Park, southeastern Utah: U.S. Geological Survey Open-File Report 72-363, 75 p.

Taylor, 0.J., Hood, J.W., and Zimmerman, E.A., 1983, Plan of study for the regional aquifer systems analysis of the Upper Colorado River Basin in Colorado, Utah, Wyoming, and Arizona: U.S. Geological Survey WaterResources Investigations Report 83-4184, $23 \mathrm{p}$. , 1986, Hydrogeologic framework of the Upper Colorado River Basin excluding the San Juan River Basin, Colorado, Utah, Wyoming, and Arizona: U.S. Geological Survey Hydrologic Investigations Atlas HA-687, Scale $1: 2,500,000$.

Teller, R.W., and Chafin, D.T., 1986, Selected drill-stem test data for the Upper Colorado River Basin: U.S. Geological Survey Water-Resources Investigations Report 84-4146, $112 \mathrm{p}$.

Teller, R.W., and Welder, F.A., 1983, Ground-water potential of the Leadville Limestone on the White River Uplift in Garfield and Rio Blanco Counties, Colorado: U.S. Geological Survey Water-Resources Investigations Report 83-4036, $24 \mathrm{p}$.

Thackston, J.W., Preslo, L.M., Huexter, D.E., and Donnelly, Nancy, 1984, Results of hydraulic test at Gibson Dome No. 1., Elk Ridge, No. 1., and E.J. Kubat boreholes, Paradox basin, Utah: Columbus, Ohio, Battelle Memorial Institute, Office of Nuclear Waste Isolation Report ONWI-491, $93 \mathrm{p}$.

Theis, C.V., 1935, The relation between the lowering of the piezometric surface and the rate and duration of discharge of a well using ground-water storage: American Geophysical Union Transactions, v. 16, p. 519-524.

U.S. Water and Power Resources Service (Bureau of Reclamation), 1981, Ground water manual: $480 \mathrm{p}$.

Weir, J.E., Jr., Maxfield, E.B., and Hart, I.M., 1983, Reconnaissance of the geohydrology of the Moab-Monticello area, western Paradox Basin, Grand and San Juan Counties, Utah: U.S. Geological Survey Water-Resources Investigations Report 83-4098, $59 \mathrm{p}$.

Whitcomb, H.A., and Lowry, M.E., 1968, Ground-water resources and geology of the Wind River Basin area, central Wyoming: U.S. Geological Survey Hydrologic Investigations Atlas HA-270, 13 p., scale 1:250,000, 3 sheets. Wollitz, L.E., Thordarson, William, Whitfield, M.S., Jr., and Weir, J.E., Jr., 1982, Results of hydraulic tests in U.S. Department of Energy's wells DOE-4, 5, 6, 7, 8, and 9, Salt Valley, Grand County, Utah: U.S. Geological Survey Open-File Report 82-346, $71 \mathrm{p}$.

Woodward-C1yde Consu1tants, 1982, Geologica1 characterization report for the Paradox study region, Utah study areas: Columbus, Ohio, Battelle Memorial Institute, Office of Nuclear Waste Isolation Report ONWI-290, v. I-VI. 
Table 3.--Artesian yields from wells and springs

1. Formation codes are defined in table 1 .

2. Depths to the top and bottom of tested intervals were measured from the land surface, top of casing, drilling platform, or kelly bushing. The measuring point was unknown or was not recorded in compiling the data in this table. An error of no more than 20 feet is introduced by assuming that the measuring point was the land surface. The number 99,999 in the depth columns indicates that the depth is unknown. Zeroes in both depth columns indicate that the site is a spring.

3. All yields reported are artesian. They are either spring flows, flows from well heads, or flows from intervals into a well during construction, drill-stem testing, or other aquifer testing. If yields varied at a site during multiple visits, generally the largest value is reported. Most sites have only one recorded measurement. Measurements in this table span a period of approximately 41 years, from about 1945 to 1986. Dates of measurement were not recorded in compiling the data in this table.

4. Some of the data in this table may be duplicates. This situation results because the data have been compiled from numerous reports and unpublished files. The same well or spring can be given different sitelocation numbers by different investigators. Alternatively, discharge from a well or spring can be attributed to different formations at the same site. As a further complication, the same well or spring can be given different location numbers, and the flow can be attributed to different formations by different investigators. Every attempt has been made to avoid duplicate listings in this table. Nevertheless, some questions were impossible to resolve, and some duplication could not be avoided. 
Table 3.--Artesian yields from wells and springs

\begin{tabular}{|c|c|c|c|c|}
\hline Site & $\begin{array}{l}\text { Depth } \\
\text { to top } \\
\text { (feet) }\end{array}$ & $\begin{array}{l}\text { Depth to } \\
\text { bottom } \\
\text { (feet) }\end{array}$ & $\begin{array}{l}\text { Yield } \\
\text { (gallons per } \\
\text { minute) }\end{array}$ & Remarks \\
\hline
\end{tabular}

ga41-22-10

slc35-02-27aa

s 1 c $35-02-35 b d$

s lc 36-04-10bbd

s 1 d 29-10-08cc

sld29-18-20ccd

sld30-12-19bb

s $1 \mathrm{~d} 30-18-06 \mathrm{dbc}$

sld30-18-15cca

s ld30-19-14a db

s ld30-19-23dad

s ld 30-19-25cdc

sld30-19-27cdd

s ld30-20-20cdd

sld30.5-19-34cac

s 1d31-19-03bad

s 1d31-19-04adc

sld31-20-04abb

sld31-20-06ada

s 1 d $31-22-22 \mathrm{cb}$

sld32-18-27bca

sld32-18-29dbd

sld32-18-32da

sld $32-18-33 \mathrm{ccc}$

sld32-18-36cd

s 1d32-19-32dbc

sld33-18-24acc

sld36-16-34

s ld37-02-07bbd

s 1d37-03-30dd

s $1 d 37-18-35 d$

s ld38-18-15

sld40-15-29

s ld41-17-33

s ld42-15-13

sld42-16-19

sld42-16-30d

s 1d42-17-04

nmb37-18-20bdb

nmb38-15-21bcb

nmb40-20-12 cd

nmb42-09-34adb

nmb42-09-35daa

nmb42-18-14ad

nmb42-18-14ad

nmb42-18-14ad

nmb42-19-09aa

nmb43-09-36dcb

\section{$317 \mathrm{CDRM}$}

\begin{tabular}{|c|c|c|}
\hline 0 & 0 & 25.00 \\
\hline 8,630 & 8,702 & 0.15 \\
\hline 9,158 & 9,191 & 13.00 \\
\hline 11,122 & 11,221 & 7.90 \\
\hline 6,035 & 6,060 & 2.30 \\
\hline 0 & 0 & 0.10 \\
\hline 3,574 & 3,615 & 4.30 \\
\hline 0 & 0 & 2.00 \\
\hline 0 & 0 & 1.80 \\
\hline 0 & 0 & 13.00 \\
\hline 0 & 0 & 1.30 \\
\hline 0 & 0 & 10.00 \\
\hline 0 & 0 & 0.10 \\
\hline 0 & 0 & 0.20 \\
\hline 0 & 0 & 0.20 \\
\hline 0 & 0 & 3.00 \\
\hline 0 & 0 & 0.30 \\
\hline 0 & 0 & 0.20 \\
\hline 0 & 0 & 2.50 \\
\hline 2,300 & 2,404 & 25.00 \\
\hline 0 & 0 & 0.80 \\
\hline 0 & 0 & 125.00 \\
\hline 0 & 0 & 1.00 \\
\hline 0 & 0 & 1.10 \\
\hline 0 & 0 & 11.00 \\
\hline 0 & 0 & 0.40 \\
\hline 0 & 0 & 2.50 \\
\hline 0 & 0 & 5.00 \\
\hline 7,850 & 7,964 & 23.00 \\
\hline 8,172 & 8,201 & 2.00 \\
\hline 0 & 0 & 10.00 \\
\hline 0 & 0 & 0.20 \\
\hline 0 & 0 & 5.00 \\
\hline 0 & 0 & 0.20 \\
\hline 0 & 0 & 2.00 \\
\hline 0 & 0 & 0.20 \\
\hline 0 & 0 & 0.20 \\
\hline 0 & 0 & 1.00 \\
\hline
\end{tabular}

Davis and others (1963)

Petroleum Information Corp. (unpublished)

Petroleum Information Corp. (unpublished)

Petroleum Information Corp. (unpublished)

Petroleum Information Corp. (unpublished)

Horse Canyon Spring (Hand, 1979)

Petroleum Information Corp. (unpublished)

Pictograph Spring (Hand, 1979)

Water Canyon Spring (Hand, 1979)

Lower Little Spring (Richter, 1980)

Little Spring (Richter,1980)

Squaw Spring (Richter,1980)

Soda Spring (Sumsion and Bolke, 1972)

Cave Spring (Sumsion and Bolke, 1972)

Hannover Spring (Richter, 1980)

Echo Spring (Richter, 1980)

Dorius Spring (Richter, 1980)

Paul Bunyun Spring (Richter, 1980)

Peekaboo Spring (Sumsion and Bolke, 1972)

Richter (1980)

Little Home Water Spring (Richter, 1980)

Big Spring (Richter, 1980)

Homewater Spring (Weir and others, 1983)

South Spring (Richter, 1980)

Calf Wash Spring (Weir and others, 1983)

Iron Spring (Richter, 1980)

Sweet Alice Spring (Weir and others, 1983)

Fry Spring (Ritzma and Doelling, 1969)

Petroleum Information Corp. (unpublished)

Petroleum Information Corp. (unpublished)

Kane Gulch Spring (Iorns and others, 1964)

Todie Spring (Ritzma and Doelling, 1969)

Iorns and others (1964)

Ritzma and Doelling (1969)

Iorns and others (1964)

Spring 8A-293 (Ritzma and Doelling, 1969)

Cedar Spring (Ritzma and Doelling, 1969)

Spring 8A-191 (Ritzma and Doelling, 1969)

\section{CTLR}

$\begin{array}{rrr}4,107 & 4,154 & 11.00 \\ 3,628 & 3,655 & 1.80 \\ 4,159 & 4,177 & 5.80 \\ 0 & 0 & 20.00 \\ 0 & 0 & 20.00 \\ & & \\ 2,529 & 2,552 & 6.90 \\ 2,971 & 3,062 & 5.10 \\ 3,545 & 3,616 & 5.70 \\ 4,243 & 4,261 & 2.90 \\ 0 & 0 & 0.30\end{array}$

Petroleum Information Corp. (unpublished) Petroleum Information Corp. (unpublished) Petroleum Information Corp. (unpublished) U.S. Geological Survey (unpublished)

U.S. Geological Survey (unpublished)

Petroleum Information Corp. (unpublished) Petroleum Information Corp. (unpublished) Petroleum Information Corp. (unpublished) Petroleum Information Corp. (unpublished) Telluride Spring (U.S. Geological Survey, unpublished) 
Table 3.--Artesian yields from wells and springs--Continued

\begin{tabular}{llcc}
\hline Site & $\begin{array}{l}\text { Depth } \\
\text { to top } \\
\text { (feet) }\end{array}$ & $\begin{array}{c}\text { Depth to } \\
\text { bottom } \\
\text { (feet) }\end{array}$ & $\begin{array}{c}\text { Yield } \\
\text { (gallons per } \\
\text { minute) }\end{array}$ \\
\hline
\end{tabular}

nmb43-10-17baa nmb43-11-11 a a nmb44-11-34ddd nmb44-13-09ad nmb44-14-16bca

nmb44-15-01adb s1d19-13-12ddd s1d21-14-05adc s 1d22-16-25bbb s 1d23-16-03bca

s 1d24-13-03dbb s1d24-24-21cca s 1d25-14-21bdd s1d25-23-08baa s1d26-22-20acd

s1d27-20-09a a s1d27-21-20cbb sld28-12-32da s1d28-20-16dbd s1d28-20-21dcc

s1d28-20-26bda s1d28-20-27aab s1d30-24-12dab sld30-25-10ca sld30-25-24ba

s 1d31-12-04da s1d33-16-19a sld35-26-20ad s 1d35-26-31dd s ld43-19-20

ga 40-22-18 nb0 1-07-08 nb01-08-06 nb01-08-13 nb02-09-04

nb02-09-23 nb03-09-02 nb04-09-36 nb07-09-29 nb07-10-11

nmb23-19-07d s 1d40-10-10c sld41-24-19ac s 1d42-13-09 s $1 \mathrm{~d} 42-24-35 \mathrm{cb}$

\section{CTLR--Continued}

$\begin{array}{rrr}0 & 0 & 6.00 \\ 2,647 & 2,708 & 6.70 \\ 0 & 0 & 3.50 \\ 3,962 & 4,086 & 20.00 \\ 5,715 & 5,743 & 8.00 \\ 3,275 & 3,310 & 8.10 \\ 3,671 & 3,702 & 2.80 \\ 4,295 & 4,334 & 30.00 \\ 2,585 & 2,605 & 70.00 \\ 2,990 & 3,000 & 280.00 \\ & & \\ 2,160 & 2,583 & 175.00 \\ 0 & 0 & 0.21 \\ 2,158 & 2,765 & 70.00 \\ 0 & 285 & 200.00 \\ 0 & 133 & 20.00 \\ & & \\ 530 & 535 & 60.00 \\ 0 & 0 & 0.10 \\ 2,330 & 2,438 & 9.90 \\ 0 & 0 & 0.20 \\ 0 & 0 & 1.00 \\ & & \\ 0 & 0 & 0.20 \\ 0 & 0 & 2.60 \\ 104 & 670 & 1.80 \\ 3,214 & 3,239 & 12.00 \\ 4,548 & 4,650 & 18.00 \\ 3,969 & 3,979 & 2.10 \\ 500 & 1,200 & 360.00 \\ 4,369 & 4,452 & 9.00 \\ 4,648 & 4,686 & 4.80 \\ 0 & 0 & 4.00 \\ & & \end{array}$

6.00 U.S. Geological Survey (unpublished)

6.70 Petroleum Information Corp. (unpublished)

Placerville Spring (U.S. Geological Survey, unpublished)

Petroleum Information Corp. (unpublished)

Petroleum Information Corp. (unpublished)

Petroleum Information Corp. (unpublished)

Petroleum Information Corp. (unpublished)

Petroleum Information Corp. (unpublished)

Petroleum Information Corp. (unpublished)

Petroleum Information Corp. (unpublished)

Petroleum Information Corp. (unpublished) Stinking Spring (Weir and others, 1983) Petroleum Information Corp. (unpublished) Weir and others (1983)

Sumsion (1971)

Richter (1980)

Dripping Spring (Richter, 1980)

Petroleum Information Corp. (unpublished)

My Spring (Richter, 1980)

Eric Spring (Richter, 1980)

Lockhart Canyon Spring (Richter, 1980)

Lockhart Spring (Weir and others, 1983)

U.S. Geological Survey (unpublished)

Petroleum Information Corp. (unpublished)

Petroleum Information Corp. (unpublished)

Petroleum Information Corp. (unpublished) Richter (1980)

Petroleum Information Corp. (unpublished)

Petroleum Information Corp. (unpublished)

Iorns and others (1964)

\section{DCLL}

0.20

2.00

1.00

0.50

30.00

55.00

2.50

2.50

2.50

3.50

80.00

150.00

8.00

5.00

27.00
Davis and others (1963); McGavock and others (1966)

Davis and others (1963); McGavock and others (1966)

Davis and others (1963); McGavock and others (1966)

Davis and others (1963); McGavock and others (1966)

Davis and others (1963); McGavock and others (1966)

Davis and others (1963); McGavock and OTHERS (1966)

Davis and others (1963); McGavock and others (1966)

Davis and others (1963); McGavock and others (1966)

Davis and others (1963); McGavock and others (1966)

Toadlena Springs (Harshbarger and Repenning, 1954)

Spring GJ-200 (Ritzma and Doelling, 1969)

Petroleum Information Corp. (unpublished)

Spring 8GS-12-2 (Ritzma and Doelling, 1969)

Petroleum Information Corp. (unpublished)
Davis and others (1963); McGavock and others (1966) 
Table 3.--Artesian yields from wells and springs--Continued

\begin{tabular}{cccc}
\hline Site & $\begin{array}{l}\text { Depth } \\
\text { to top } \\
\text { (feet) }\end{array}$ & $\begin{array}{c}\text { Depth to } \\
\text { bottom } \\
\text { (feet) }\end{array}$ & $\begin{array}{c}\text { Yield } \\
\text { (gallons per } \\
\text { minute) }\end{array}$ \\
\hline
\end{tabular}

s $1 d 43-14-11$

s1d43-14-13

sld43-19-29

sld43-21-09bbd

sld43-21-10cca

s1d43-22-15cc

s1d43-22-24dda

sld43-23-32ac

sld43-23-33bb

sb $13-88-36 \mathrm{ca}$

sb15-91-11bac

sb 19-88-10cab

sb19-89-03bbc

sid41-19-10

sld42-17-15

s 1c35-02-22dba

s1d20-07-02cca

s1d25-12-24cc

s1d28-08-33cdd

s $1 \mathrm{~d} 29-10-08 \mathrm{cc}$

s1d29-12-30ab

s1d36-01-12bc

s1d36-06-18ccc

s $1 \mathrm{~d} 37-02-08 \mathrm{bbb}$

s1d37-05-17dda

s1d38-03-08ad

s 1d40-02-19a ac

s1d34-14-09

s1d34-14-19

sld43-16-23

sb06-99-29bdb sb08-99-16bcd sb14-103-10ad sb16-104-16ddb sb19-102-35aa

sb30-96-07b

sla03-22-34ba

sld02-21-33dcd

sld02-22-24ccd

s1d04-21-36cd

\section{DCLL--Continued}

0.10

0.20

4.00

19.00

15.00

9.60

19.00

12.00

11.00
Spring 8A-213 (Ritzma and Doelling, 1969) Spring 8K-550 (Ritzma and Doelling, 1969) Spring 8A-260 (Ritzma and Doelling, 1969) Petroleum Information Corp. (unpublished) Petroleum Information Corp. (unpublished)

Petroleum Information Corp. (unpublished) Petroleum Information Corp. (unpublished) Petroleum Information Corp. (unpublished) Petroleum Information Corp. (unpublished)

\section{GSEG}

$\begin{array}{rrr}7,546 & 7,641 & 25.00 \\ 10,088 & 10,100 & 0.39 \\ 6,095 & 6,140 & 33.00 \\ 10,521 & 10,718 & 14.00\end{array}$

Petroleum Information Corp. (unpublished) Petroleum Information Corp. (unpublished) Petroleum Information Corp. (unpublished) Petroleum Information Corp. (unpublished)

\section{HLGT}

1.00

2.00

Iorns and others (1964)

Iorns and others (1964)

\section{KIBB}

10,050

7,244

2,305

3,595

4,840

2,663

6,193

2,248

6,921

3,330

7,298

8,115

10,085

7,294

2,391

3,700

4,868

2,704

6,271

2,300

6,967

3,402

7,330

8,142

12.00

5.60

3.20

23.00

1.30

3.80

41.00

4.20

4. 70

8.00

1.80

6.20

\section{GRK}

50.00

30.00

0.10

Hite Store Spring (U.S. Geological Survey, unpublished) Ritzma and Doelling (1969)

Spring 8A-229 (Ritzma and Doelling, 1969)

\section{PRKC}

3.00

20.00

44.00

18.00

3.80

5,459

8,378

9,042

5,489

8,481

99,999

99,999

2,972

3,021

700.00

21.00

20.00

400.00

8.10
Disappointment Spring (Sumsion, 1976)

U.S. Geological Survey (unpublished)

Petroleum Information Corp. (unpublished)

Petroleum Information Corp. (unpublished)

Petroleum Information Corp. (unpublished)

Whitcomb and Lowry (1968)

Petroleum Information Corp. (unpublished)

Squaw Spring (Hood and others, 1976)

Hood and others (1976)

Petroleum Information Corp. (unpublished) 
Table 3.--Artesian yields from wells and springs--Continued

\begin{tabular}{llcc} 
Site & $\begin{array}{l}\text { Depth } \\
\text { to top } \\
\text { (feet) }\end{array}$ & $\begin{array}{c}\text { Depth to } \\
\text { bottom } \\
\text { (feet) }\end{array}$ & $\begin{array}{c}\text { Yield } \\
\text { (gallons per } \\
\text { minute) }\end{array}$ \\
\hline
\end{tabular}

sld04-22-35dbc sld04-23-23dda s1d05-22-26bca sld06-24-05cdc sld18-14-30cc

ub02-01-20cbd ub02-03-22dd

$\begin{array}{rrr}4,745 & 4,779 & 19.00 \\ 0 & 0 & 4.00 \\ 4,372 & 4,393 & 14.00 \\ 1,181 & 1,210 & 10.00 \\ 3,605 & 3,673 & 7.80 \\ & & \\ 6,211 & 6,235 & 92.00 \\ 4,555 & 4,620 & 57.00\end{array}$

\section{PRKC--Cont inued}

9.00 Petroleum Information Corp. (unpublished)

4.00 Red Wash Spring (Hood and others, 1976)

4.00 Petroleum Information Corp. (unpublished)

Petroleum Information Corp. (unpublished)

Petroleum Information Corp. (unpublished)

2.00 Petroleum Information Corp. (unpublished)

Petroleum Information Corp. (unpublished)

\section{PSPR}

$\begin{array}{rrr}18,447 & 18,521 & 47.00 \\ 4,510 & 4,529 & 4.50 \\ 2,816 & 2,949 & 21.00 \\ 7,232 & 7,262 & 15.00 \\ 6,776 & 6,812 & 29.00 \\ & & \\ 11,800 & 11,860 & 0.77 \\ 6,353 & 6,420 & 15.00 \\ 2,285 & 2,355 & 2.00 \\ 0 & 0 & 200.00 \\ 99,999 & 99,999 & 300.00 \\ & & \\ 6,604 & 6,877 & 12.00 \\ 12,653 & 12,718 & 3.30 \\ 14,268 & 14,374 & 19.00 \\ 0 & 0 & 628.00 \\ 0 & 0 & 2,870.00\end{array}$

Petroleum Information Corp. (unpub1ished) Petroleum Information Corp. (unpublished) Petroleum Information Corp. (unpublished) Petroleum Information Corp. (unpublished) Petroleum Information Corp. (unpublished)

Petroleum Information Corp. (unpublished) Petroleum Information Corp. (unpublished) Petroleum Information Corp. (unpublished) Lines and Glass (1975)

Lines and Glass (1975)

Petroleum Information Corp. (unpublished) Petroleum Information Corp. (unpub1ished) Petroleum Information Corp. (unpublished) Kendall Cold Spring (Rouse, 1967)

Kendall Warm Spring (Rouse, 1967)

\section{RIC0}

$\begin{array}{rrr}2,790 & 2,840 & 6.00 \\ 1,047 & 1,500 & 100.00 \\ 0 & 0 & 1.20 \\ 0 & 0 & 2.00 \\ 0 & 0 & 5.20 \\ & & \\ 0 & 0 & 13.00 \\ 0 & 0 & 4.30 \\ 0 & 0 & 2.00 \\ 0 & 0 & 3.30 \\ 0 & 0 & 20.00 \\ & & \\ 500 & 800 & 2.00 \\ 500 & 800 & 15.00 \\ 400 & 918 & 25.00 \\ 1,030 & 1,420 & 25.00 \\ 0 & 0 & 5.00 \\ 0 & 0 & 5.00\end{array}$

Richter (1980)

Richter (1980)

Loop Trail Spring (Richter, 1980)

Elephant Spring (Sumsion and Bolke, 1972)

Lower Big Spring (Richter, 1980)

Lower Jump Spring (Richter, 1980)

Big Springs (Richter, 1980)

Upper Jump Spring (Richter, 1980)

North Home Spring (Richter, 1980)

Lime Creek Spring (Ritzma and Doelling, 1969)

Richter (1980)

Richter (1980)

Richter (1980)

Richter (1980)

Johns Canyon Spring (Iorns and others, 1964)

Goodrich Sulphur Spring (Iorns and others, 1964)

\section{TRWP}

sld35-03-29aa s1d36-03-15 cb s1d42-07-16ac

$\begin{array}{lll}4,985 & 5,015 & 5.70 \\ 5,297 & 5,320 & 9.00 \\ 4,590 & 4,604 & 1.70\end{array}$

Petroleum Information Corp. (unpublished)
Petroleum Information Corp. (unpublished)
Petroleum Information Corp. (unpublished) 
Table 3.--Artesian yields from wells and springs--Continued

\begin{tabular}{llcc}
\hline Site & $\begin{array}{l}\text { Depth } \\
\text { to top } \\
\text { (feet) }\end{array}$ & $\begin{array}{c}\text { Depth to } \\
\text { bottom } \\
\text { (feet) }\end{array}$ & $\begin{array}{c}\text { Yield } \\
\text { (gallons per } \\
\text { minute) }\end{array}$ \\
\hline
\end{tabular}

\begin{tabular}{|c|c|c|c|}
\hline $\mathrm{s} 1 \mathrm{c} 32-02-23 \mathrm{dbd}$ & 9,222 & 9,288 & 30.00 \\
\hline $\mathrm{s} 1 \mathrm{~d} 18-14-30 \mathrm{cc}$ & 3,717 & 3,868 & 3.60 \\
\hline $\mathrm{s} 1 \mathrm{~d} 22-17-32 \mathrm{aa}$ & 2,333 & 2,388 & 31.00 \\
\hline sld $23-16-15 d c a$ & 2,530 & 2,570 & 3.30 \\
\hline $\operatorname{s} 1 \mathrm{~d} 23-19-31 \mathrm{ccc}$ & 2,386 & 2,415 & 13.00 \\
\hline $\operatorname{s} 1 \mathrm{~d} 25-12-24 \mathrm{cc}$ & 2,395 & 2,422 & 4.70 \\
\hline $\mathrm{s} 1 \mathrm{~d} 25-13-14 \mathrm{cc}$ & 2,174 & 2,224 & 14.00 \\
\hline s1d26-07-18bdb & 3,348 & 3,366 & 32.00 \\
\hline sld $26-07-19 b b$ & 3,706 & 3,921 & 11.00 \\
\hline $\mathrm{s} 1 \mathrm{~d} 26-16-31 \mathrm{cc}$ & 2,280 & 2,550 & 137.00 \\
\hline $\mathrm{s} 1 \mathrm{~d} 27-14-17 \mathrm{cc}$ & 2,730 & 2,825 & 9.60 \\
\hline $\operatorname{sld} 27-15-32 \mathrm{ad}$ & 2,330 & 2,366 & 3.30 \\
\hline $\mathrm{s} 1 \mathrm{~d} 27-17.5-01 \mathrm{ddc}$ & 373 & 500 & 45.00 \\
\hline $\mathrm{s} 1 \mathrm{~d} 27-17.5-13 \mathrm{cba}$ & 0 & 0 & 0.10 \\
\hline $\mathrm{s} 1 \mathrm{~d} 28-12-32 \mathrm{da}$ & 2,443 & 2,474 & 35.00 \\
\hline $\mathrm{s} 1 \mathrm{~d} 30-12-19 \mathrm{bb}$ & 2,900 & 2,933 & 8.50 \\
\hline s1d31-12-04da & 2,251 & 2,261 & 3.90 \\
\hline s1d31-12-05dac & 2,625 & 2,733 & 4.80 \\
\hline $\mathrm{s} 1 \mathrm{~d} 36-10-20 \mathrm{dc}$ & 3,799 & 3,869 & 19.00 \\
\hline $\operatorname{sld} 36-11-19 a d c$ & 2,900 & 3,032 & 38.00 \\
\hline sld38-02-31bad & 4,325 & 4,362 & 5.10 \\
\hline s $1 d 41-07-19 b b d$ & 3,180 & 3,225 & 4.80 \\
\hline $\mathrm{nmb} 26-18-18 \mathrm{db}$ & 6,405 & 6,450 & 6.60 \\
\hline $\mathrm{nmb} 33.5-20-16 \mathrm{bc}$ & 5,890 & 5,917 & 13.00 \\
\hline $\mathrm{nmb} 37-19-15 \mathrm{acd}$ & 4,798 & 4,828 & 18.00 \\
\hline nmb38-19-29da & 5,041 & 5,111 & 0.45 \\
\hline$n m b 39-14-19 c b c$ & 5,247 & 5,278 & 3.90 \\
\hline nmb40-16-26cd & 5,708 & 5,758 & 2.40 \\
\hline nmb40-20-12cd & 5,200 & 5,358 & 0.51 \\
\hline $\mathrm{nmb} 41-14-12 \mathrm{aaa}$ & 5,886 & 5,936 & 0.86 \\
\hline $\mathrm{nmb} 44-08-31$ & 0 & 0 & 10.00 \\
\hline $\mathrm{nmb} 44-14-16 \mathrm{bca}$ & 5,715 & 5,743 & 7.70 \\
\hline nmb44-17-14abd & 9,148 & 9,285 & 37.00 \\
\hline nmb44-18-21dda & 6,409 & 6,450 & 1.10 \\
\hline$n m b 44-19-28 c d$ & 4,234 & 4,307 & 12.00 \\
\hline nmb46-14-14ab & 7,361 & 7,545 & 1.30 \\
\hline $\mathrm{nmb} 46-19-30 \mathrm{bb}$ & 8,317 & 8,446 & 41.00 \\
\hline $\mathrm{s} 1 \mathrm{~d} 22-16-14 \mathrm{ba}$ & 4,764 & 4,846 & 8.10 \\
\hline s $1 d 24-15-05 c a a$ & 3,618 & 3,712 & 18.00 \\
\hline s 1 d $24-19-22 d a c$ & 3,248 & 3,316 & 12.00 \\
\hline $\operatorname{s} 1 \mathrm{~d} 25-14-22 \mathrm{~cd}$ & 3,975 & 4,070 & 7.20 \\
\hline $\mathrm{s} 1 \mathrm{~d} 26-07-18 \mathrm{bdb}$ & 4,573 & 4,695 & 76.00 \\
\hline $\mathrm{s} 1 \mathrm{~d} 27-19-27 \mathrm{da}$ & 3,190 & 4,740 & 21.00 \\
\hline $\mathrm{s} 1 \mathrm{~d} 28-20-36 \mathrm{ca}$ & 1,500 & 2,850 & 200.00 \\
\hline $\operatorname{s} 1 d 28-22-34 a a$ & 4,454 & 4,534 & 5.50 \\
\hline $\mathrm{s} 1 \mathrm{~d} 29-20-23 \mathrm{cb}$ & 1,800 & 2,400 & 20.00 \\
\hline $\mathrm{s} 1 \mathrm{~d} 30-13-34 \mathrm{ab}$ & 4,020 & 4,071 & 5.30 \\
\hline
\end{tabular}

\section{WTRM}

Petroleum Information Corp. (unpublished) Petroleum Information Corp. (unpublished) Petroleum Information Corp. (unpublished) Feltis (1966)

Petroleum Information Corp. (unpublished)

Petroleum Information Corp. (unpublished) Petroleum Information Corp. (unpublished) Petroleum Information Corp. (unpublished) Petroleum Information Corp. (unpublished) Richter (1980)

Richter (1980)

Petroleum Information Corp. (unpublished) Sumsion and Bolke (1972)

Hardscrabble Spring (Sumsion and Bolke, 1972)

Petroleum Information Corp. (unpublished)

Petroleum Information Corp. (unpublished)

Petroleum Information Corp. (unpublished) Petroleum Information Corp. (unpublished) Petroleum Information Corp. (unpublished) Petroleum Information Corp. (unpublished) Petroleum Information Corp. (unpublished) Petroleum Information Corp. (unpublished)

\section{HKTL}

Petroleum Information Corp. (unpublished) Petroleum Information Corp. (unpublished) Petroleum Information Corp. (unpublished) Petroleum Information Corp. (unpublished) Petroleum Information Corp. (unpublished)

Petroleum Information Corp. (unpublished) Petroleum Information Corp. (unpublished) Petroleum Information Corp. (unpublished) Pavillion Hot Spring (Iorns and others, 1964) Petroleum Information Corp. (unpublished)

Petroleum Information Corp. (unpublished) Petroleum Information Corp. (unpublished) Petroleum Information Corp. (unpublished) Petroleum Information Corp. (unpublished) Petroleum Information Corp. (unpublished)

Petroleum Information Corp. (unpublished) Petroleum Information Corp. (unpublished) Petroleum Information Corp. (unpublished) Petroleum Information Corp. (unpublished) Petroleum Information Corp. (unpublished)

Richter (1980)

Richter (1980)

Petroleum Information Corp. (unpublished) Richter (1980)

Petroleum Information Corp. (unpublished) 
Table 3.--Artesian yields from wells and springs--Continued

\begin{tabular}{llcc} 
Site & $\begin{array}{l}\text { Depth } \\
\text { to top } \\
\text { (feet) }\end{array}$ & $\begin{array}{c}\text { Depth to } \\
\text { bottom } \\
\text { (feet) }\end{array}$ & $\begin{array}{c}\text { Yield } \\
\text { (gallons per } \\
\text { minute) }\end{array}$ \\
\hline
\end{tabular}

\begin{abstract}
s1d $32-15-33 a b$
sld33-16-19ba

sld34-26-29cca

sld36-26-16 cac

s1d38-21-06bb

sld40-12-27bb

sld $40-26-16 \mathrm{dc}$

sld41-26-34ad
\end{abstract}

$\mathrm{sb} 13-88-36 \mathrm{ca}$

sb15-103-08bc

sb15-105-11dac

sb15-91-11bac

sb16-101-11ddc

sb16-104-21aca

sb16-90-31 abd

sb16-92-12db

sb $17-102-17 \mathrm{~cd}$

sb18-101-32adb

sb18-103-18dad

sb18-90-11bab

sb19-102-35a a

sb19-104-11ac

sb19-104-11ac

sb19-87-29da

sb19-88-02db

sb19-88-10cb

sb19-89-03bbc

sb19-89-30bdd

sb20-104-36ccc

sb20-116-23ab

sb20-84-28cc

sb20-87-30ddc

sb20-88-36ccc

sb22-115-35cdc

sb22-86-34dcc

sb22-88-28bdd

sb23-115-26cab

sb23-86-16cc

sb23-88-20aa

sb24-87-06ddb

sb24-88-06bbb

sb24-88-32ba

sb25-86-34da

sb25-87-11bad

sb25-88-03cc

$\mathrm{sb} 25-89-14 \mathrm{dc}$

sb26-86-07dcc

sb26-87-30dd

$\begin{array}{rrr}2,760 & 2,880 & 6.60 \\ 930 & 99,999 & 450.00 \\ 4,509 & 4,519 & 0.66 \\ 4,995 & 5,074 & 4.40 \\ 2,158 & 2,218 & 10.00 \\ & & \\ 4,342 & 4,444 & 5.60 \\ 5,590 & 5,650 & 2.50 \\ 5,968 & 5,983 & 12.00\end{array}$

7,644

6,670

11,435

10,280

6,665

7,672

6,720

11,610

10,307

6,691

6,065

10,082

11,137

7,810

6,172

10,191

11,180

7,850

10,048

10,066

5,485

10,265

8,587

6,217

6,632

5,499

10,330

8,627

6,247

6,705

5,216

6,128

6,167

10,521

10,486

5,287

6,176

6,220

10,718

10,510

6,340

5,758

9,485

8,176

6,347

6,370

5,990

9,638

8,208

6,390

5,585

4,809

2,160

6,743

9,133

5,590

4,840

2,217

6,791

9,235

0
4,889
5,128

5,128

3,857

7,070

6,360

4,805

5,260

4,311

4,414

61.00

56.00

24.00

8.60

3.20

40.00

9.20

1.40

37.00

2.50

11.00

27.00

20.00

0.13

8.60

35.00

20.00

65.00

16.00

32.00

20.00

27.00

43.00

3.20

1.50

10.00

1.00

16.00

12.00

30.00

200.00

5.30

171.00

6.20

37.00

16.00

24.00

18.00

10.00

7.80

\section{HKTL}

Petroleum Information Corp. (unpublished) Richter (1980)

Petroleum Information Corp. (unpublished)

Petroleum Information Corp. (unpublished)

Petroleum Information Corp. (unpublished)

Petroleum Information Corp. (unpublished) Petroleum Information Corp. (unpublished)

Petroleum Information Corp. (unpublished)

\section{TSLP}

Petroleum Information Corp. (unpublished) Petroleum Information Corp. (unpublished) Petroleum Information Corp. (unpublished) Petroleum Information Corp. (unpublished) Petroleum Information Corp. (unpublished)

Petroleum Information Corp. (unpublished) Petroleum Information Corp. (unpublished) Petroleum Information Corp. (unpublished) Petroleum Information Corp. (unpublished) Petroleum Information Corp. (unpublished)

Petroleum Information Corp. (unpublished) Petroleum Information Corp. (unpublished) Petroleum Information Corp. (unpublished) Petroleum Information Corp. (unpublished) Petroleum Information Corp. (unpublished)

Petroleum Information Corp. (unpublished) Petroleum Information Corp. (unpublished) Petroleum Information Corp. (unpublished) Petroleum Information Corp. (unpublished) Petroleum Information Corp. (unpublished)

Petroleum Information Corp. (unpublished) Petroleum Information Corp. (unpublished) Petroleum Information Corp. (unpublished) Petroleum Information Corp. (unpublished) Petroleum Information Corp. (unpublished)

Petroleum Information Corp. (unpublished) Petroleum Information Corp. (unpublished) Petroleum Information Corp. (unpublished) Petroleum Information Corp. (unpublished) Petroleum Information Corp. (unpublished)

01son Spring (Berry, 1960)

Petroleum Information Corp. (unpublished) Petroleum Information Corp. (unpublished) Petroleum Information Corp. (unpublished) Petroleum Information Corp. (unpublished)

Petroleum Information Corp. (unpublished) Petroleum Information Corp. (unpublished) Petroleum Information Corp. (unpublished) Petroleum Information Corp. (unpublished) Petroleum Information Corp. (unpublished) 
Table 3.--Artesian yields from wells and springs--Continued

\begin{tabular}{llccc}
\hline Site & $\begin{array}{l}\text { Depth } \\
\text { to top } \\
\text { (feet) }\end{array}$ & $\begin{array}{c}\text { Depth to } \\
\text { bottom } \\
\text { (feet) }\end{array}$ & $\begin{array}{c}\text { Yield } \\
\text { (gallons per } \\
\text { minute) }\end{array}$ & Remarks \\
\hline
\end{tabular}

\begin{abstract}
$\operatorname{sb} 26-87-32 b b$ sb26-89-06ca sb26-90-14bab sb26-95-06dad sb28-113-19bbd

$\mathrm{sb} 33-100-18 \mathrm{ac}$ sb33-100-25ca sb33-100-32ad sb33-101-13ac sb33-99-35ad
\end{abstract}

sb01-93-06cb sb02-101-31bdc sb02-102-17ba sb02-102-21bc sb02-103-05dd

sb02-103-15cb sb02-103-36dd sb02-92-06cca sb02-94-04cd

sb03-100-12ba

sb03-102-05adb sb03-103-12bb sb03-103-16ba sb03-90-23dd

sb03-90-26baa

sb03-97-29dba sb04-104-36bcd sb04-92-22dc sb05-91-33dd sb05-94-17cb

sb05-95-02dda sb05-96-14ab sb06-102-18add sb06-102-22baa sb06-103-07bbb

sb06-94-10cc sb07-103-32adb sb07-87-13 sb07-95-32bb

sb10-101-25da

sb12-104-17cac sb14-101-18bbd sb15-112-29ad sc02-104-12bca sla03-22-34ba

sla03-25-28bb sld02-22-13ccd sld02-22-31adc sld02-22-32bcb sld03-08-33bda

$\begin{array}{rrr}4,460 & 4,513 & 6.10 \\ 6,418 & 6,472 & 7.70 \\ 6,200 & 6,250 & 62.00 \\ 7,791 & 7,859 & 65.00 \\ 12,929 & 12,997 & 1.00 \\ & & \\ 99,999 & 99,999 & 100.00 \\ 99,999 & 99,999 & 539.00 \\ 99,999 & 99,999 & 16.00 \\ 99,999 & 99,999 & 75.00 \\ 99,999 & 99,999 & 500.00\end{array}$

321TSLP--Continued

6.10 Petroleum Information Corp. (unpublished)

7.70 Petroleum Information Corp. (unpublished)

Petroleum Information Corp. (unpublished)

Petroleum Information Corp. (unpublished)

Petroleum Information Corp. (unpublished)

Whitcomb and Lowry (1968)

Whitcomb and Lowry (1968)

Whit comb and Lowry (1968)

Whitcomb and Lowry (1968)

Whitcomb and Lowry (1968)

\section{$321 \mathrm{WEBR}$}

$\begin{array}{rrr}5,440 & 5,490 & 12.00 \\ 6,310 & 6,377 & 0.17 \\ 6,686 & 6,709 & 4.80 \\ 6,578 & 6,605 & 1.50 \\ 7,455 & 7,480 & 15.00 \\ & & \\ 6,794 & 6,816 & 8.00 \\ 6,250 & 6,274 & 14.00 \\ 9,261 & 9,325 & 20.00 \\ 10,614 & 10,675 & 11.00 \\ 3,323 & 3,425 & 57.00 \\ & & \\ 99,999 & 750 & 500.00 \\ 9,520 & 9,763 & 31.00 \\ 8,735 & 8,870 & 61.00 \\ 6,145 & 6,183 & 14.00 \\ 6,070 & 6,194 & 2.40 \\ & & \\ 11,940 & 12,045 & 1.90 \\ 1,340 & 1,410 & 18.00 \\ 4,622 & 4,652 & 1.80 \\ 6,081 & 6,097 & 3.60 \\ 3,007 & 3,022 & 77.00 \\ & & \\ 2,525 & 2,582 & 11.00 \\ 6,548 & 6,600 & 11.00 \\ 0 & 0 & 15.00 \\ 0 & 0 & 2.00 \\ 0 & 0 & 270.00 \\ & & \\ 3,950 & 4,076 & 0.33 \\ 165 & 300 & 35.00 \\ 5,535 & 5,607 & 9.00 \\ 2,070 & 2,107 & 17.00 \\ 1,855 & 1,907 & 16.00 \\ 14,214 & 14,295 & 20.00 \\ 12,771 & 12,800 & 4.60 \\ 17,210 & 18,140 & 1.40 \\ 10,713 & 10,742 & 8.70 \\ 3,203 & 3,277 & 23.00 \\ 11,675 & 11,885 & 4.80 \\ 0 & 0 & 500.00 \\ 0 & 0 & 1,350.00 \\ 30 & 368 & 40.00 \\ 0 & 0 & 230.00\end{array}$

Petroleum Information Corp. (unpublished) Petroleum Information Corp. (unpublished) Petroleum Information Corp. (unpublished) Petroleum Information Corp. (unpublished) Petroleum Information Corp. (unpublished)

Petroleum Information Corp. (unpublished) Petroleum Information Corp. (unpublished) Petroleum Information Corp. (unpublished) Petroleum Information Corp. (unpublished) Petroleum Information Corp. (unpublished)

Hood and others (1976)

Petroleum Information Corp. (unpublished) Petroleum Information Corp. (unpublished) Petroleum Information Corp. (unpublished) Petroleum Information Corp. (unpublished)

Petroleum Information Corp. (unpublished) Petroleum Information Corp. (unpublished) Petroleum Information Corp. (unpublished) Petroleum Information Corp. (unpublished) Petroleum Information Corp. (unpublished)

Petroleum Information Corp. (unpublished) Petroleum Information Corp. (unpublished) Castle Park Spring (Sumsion, 1976)

Bull Park Spring (Sumsion, 1976)

Pool Creek Spring (Sumsion, 1976)

Petroleum Information Corp. (unpublished) Sumsion (1976)

Petroleum Information Corp. (unpublished)

Petroleum Information Corp. (unpublished)

Petroleum Information Corp. (unpublished)

Petroleum Information Corp. (unpublished)

Petroleum Information Corp. (unpublished)

Petroleum Information Corp. (unpublished)

Petroleum Information Corp. (unpublished)

Petroleum Information Corp. (unpublished)

Petroleum Information Corp. (unpublished) Stauffer Chemical Spring (Hood and others, 1976)

Ratliff Spring (Hood and others, 1976)

Hood and others (1976)

Hood and others (1976) 
Table 3.--Artesian yields from wells and springs--Continued

\begin{tabular}{|c|c|c|c|c|}
\hline Site & $\begin{array}{l}\text { Depth } \\
\text { to top } \\
\text { (feet) }\end{array}$ & $\begin{array}{l}\text { Depth to } \\
\text { bottom } \\
\text { (feet) }\end{array}$ & $\begin{array}{c}\text { Yield } \\
\text { (gallons per } \\
\text { minute) }\end{array}$ & Remarks \\
\hline \multicolumn{5}{|c|}{ 321WEBR--Continued } \\
\hline $\begin{array}{l}\text { sld03-21-20baa } \\
\text { sld03-21-28dba } \\
\text { sld03-21-30ddc } \\
\text { sld03-22-30ddd } \\
\text { sld03-25-11dcc }\end{array}$ & $\begin{array}{r}148 \\
1,318 \\
214 \\
5,484 \\
0\end{array}$ & $\begin{array}{r}210 \\
2,550 \\
2715 \\
5,517 \\
0\end{array}$ & $\begin{array}{r}10.00 \\
230.00 \\
250.00 \\
41.00 \\
100.00\end{array}$ & $\begin{array}{l}\text { Hood and others (1976) } \\
\text { Hood and others (1976) } \\
\text { Hood and others (1976) } \\
\text { Petroleum Information Corp. (unpublished) } \\
\text { Big Draw Spring (Sumsion, 1976) }\end{array}$ \\
\hline $\begin{array}{l}\text { sld03-25-12cca } \\
\text { sld03-25-22dda } \\
\text { sld04-20-04ddc } \\
\text { sld04-23-25b } \\
\text { sld04-24-35dad }\end{array}$ & $\begin{array}{r}0 \\
0 \\
4,868 \\
0 \\
0\end{array}$ & $\begin{array}{r}0 \\
0 \\
4,983 \\
0 \\
0\end{array}$ & $\begin{array}{r}10.00 \\
100.00 \\
11.00 \\
3.00 \\
23.00\end{array}$ & $\begin{array}{l}\text { Ely Creek Spring (Sumsion, 1976) } \\
\text { Sage Creek Spring (Sumsion, 1976) } \\
\text { Petroleum Information Corp. (unpublished) } \\
\text { Red Wash Spring (Feltis, 1966) } \\
\text { Morris Ranch Spring (Sumsion, 1976) }\end{array}$ \\
\hline $\begin{array}{l}\text { sld04-24-35dad } \\
\text { sld04-24-36bdc } \\
\text { sld04-25-31cca } \\
\text { sld05-22-06ca } \\
\text { sld05-22-26cc }\end{array}$ & $\begin{array}{r}30 \\
0 \\
0 \\
7,035 \\
4,781\end{array}$ & $\begin{array}{r}300 \\
0 \\
0 \\
7,085 \\
4,820\end{array}$ & $\begin{array}{r}14.00 \\
56.00 \\
117.00 \\
3.70 \\
34.00\end{array}$ & $\begin{array}{l}\text { Hood and others (1976) } \\
\text { Hog Canyon Spring (Sumsion, 1976) } \\
\text { Cub Creek Spring (Hood and others, 1976) } \\
\text { Petroleum Information Corp. (unpublished) } \\
\text { Petroleum Information Corp. (unpublished) }\end{array}$ \\
\hline $\begin{array}{l}\text { sld05-23-20ccc } \\
\text { sld05-24-11dac } \\
\text { sld05-24-32 } \\
\text { sld06-23-01bad } \\
\text { sld06-23-05dad }\end{array}$ & $\begin{array}{r}3,752 \\
0 \\
0 \\
2,077 \\
5,805\end{array}$ & $\begin{array}{r}3,766 \\
0 \\
0 \\
2,650 \\
5,929\end{array}$ & $\begin{array}{r}56.00 \\
50.00 \\
10.00 \\
200.00 \\
30.00\end{array}$ & $\begin{array}{l}\text { Petroleum Information Corp. (unpublished) } \\
\text { South Fork Springs (Hood and others, 1976) } \\
\text { Morris Ranch Spring (Feltis, 1966) } \\
\text { Hood and others (1976) } \\
\text { Petroleum Information Corp. (unpublished) }\end{array}$ \\
\hline $\begin{array}{l}\text { sld06-24-05 } \\
\text { sld06-25-05da } \\
\text { sld15-13-11dd } \\
\text { sld16-12-27ab } \\
\text { ua02-02-32bbb } \\
\text { ub01-08-30ddb }\end{array}$ & $\begin{array}{r}0 \\
3,264 \\
5,734 \\
4,442 \\
4,412 \\
0\end{array}$ & $\begin{array}{r}0 \\
3,300 \\
5,765 \\
99,999 \\
4,447 \\
0\end{array}$ & $\begin{array}{r}10.00 \\
19.00 \\
13.00 \\
2.00 \\
89.00 \\
200.00\end{array}$ & $\begin{array}{l}\text { Morris Ranch Spring (Feltis, 1966) } \\
\text { Petroleum Information Corp. (unpublished) } \\
\text { Petroleum Information Corp. (unpublished) } \\
\text { Feltis (1966) } \\
\text { Petroleum Information Corp. (unpublished) } \\
\text { Warm Spring (Hood and others, 1976) }\end{array}$ \\
\hline \multicolumn{5}{|c|}{ 321WLLS } \\
\hline $\begin{array}{l}\text { sb13-118-19cc } \\
\text { sb14-113-16dad } \\
\text { sb15-117-31ccc } \\
\text { sb16-117-03ddd } \\
\text { sb16-117-32cca }\end{array}$ & $\begin{array}{r}7,670 \\
17,805 \\
4,746 \\
5,035 \\
4,967\end{array}$ & $\begin{array}{r}7,720 \\
17,874 \\
4,781 \\
5,557 \\
5,066\end{array}$ & $\begin{array}{r}59.00 \\
2.30 \\
0.92 \\
2.10 \\
75.00\end{array}$ & $\begin{array}{l}\text { Petroleum Information Corp. (unpublished) } \\
\text { Petroleum Information Corp. (unpublished) } \\
\text { Petroleum Information Corp. (unpublished) } \\
\text { Petroleum Information Corp. (unpublished) } \\
\text { Petroleum Information Corp. (unpublished) }\end{array}$ \\
\hline $\begin{array}{l}\text { sb21-119-28acd } \\
\text { sb26-118-13bad } \\
\text { sb29-114-19bda } \\
\text { sb29-116-07bb } \\
\text { sb29-117-01ad }\end{array}$ & $\begin{array}{r}7,549 \\
0 \\
14,522 \\
0 \\
0\end{array}$ & $\begin{array}{r}7,679 \\
0 \\
14,669 \\
0 \\
0\end{array}$ & $\begin{array}{r}5.60 \\
1,600.00 \\
32.00 \\
35.00 \\
200.00\end{array}$ & $\begin{array}{l}\text { Petroleum Information Corp. (unpublished) } \\
\text { Lines and Glass (1975) } \\
\text { Petroleum Information Corp. (unpublished) } \\
\text { Lines and Glass (1975) } \\
\text { Lines and Glass (1975) }\end{array}$ \\
\hline $\begin{array}{l}\text { sb30-117-35c } \\
\text { sb33-116-11c } \\
\text { sb33-116-12b } \\
\text { sb34-116-10c } \\
\text { sb37-115-31cb }\end{array}$ & $\begin{array}{l}0 \\
0 \\
0 \\
0 \\
0\end{array}$ & $\begin{array}{l}0 \\
0 \\
0 \\
0 \\
0\end{array}$ & $\begin{array}{r}2,200.00 \\
30.00 \\
1,500.00 \\
25.00 \\
175.00\end{array}$ & $\begin{array}{l}\text { Lines and Glass (1975) } \\
\text { Lines and Glass (1975) } \\
\text { Lines and Glass (1975) } \\
\text { Lines and Glass (1975) } \\
\text { Lines and Glass (1975) }\end{array}$ \\
\hline $\begin{array}{l}\text { sb37-116-25a } \\
\text { sb37-118-12b }\end{array}$ & $\begin{array}{l}0 \\
0\end{array}$ & $\begin{array}{l}0 \\
0\end{array}$ & $\begin{array}{r}50.00 \\
200.00\end{array}$ & $\begin{array}{l}\text { Lines and Glass (1975) } \\
\text { Lines and Glass (1975) }\end{array}$ \\
\hline
\end{tabular}


Table 3.--Artesian yields from wells and springs--Continued

\begin{tabular}{cccc}
\hline Site & $\begin{array}{l}\text { Depth } \\
\text { to top } \\
\text { (feet) }\end{array}$ & $\begin{array}{c}\text { Depth to } \\
\text { bottom } \\
\text { (feet) }\end{array}$ & $\begin{array}{c}\text { Yield } \\
\text { (gallons per } \\
\text { minute) }\end{array}$ \\
\hline
\end{tabular}

\begin{tabular}{|c|c|c|c|}
\hline $\begin{array}{l}\text { sb16-104-16ddb } \\
\text { sb19-104-11ac }\end{array}$ & $\begin{array}{l}6,150 \\
6,961\end{array}$ & $\begin{array}{l}6,173 \\
7,071\end{array}$ & $\begin{array}{r}12.00 \\
5.50\end{array}$ \\
\hline $\begin{array}{l}\text { sb01-93-08dd } \\
\text { sb01-93-17ac } \\
\text { sc05-86-05 } \\
\text { sc08-84-16aa }\end{array}$ & $\begin{array}{r}6,081 \\
5,234 \\
0 \\
0\end{array}$ & $\begin{array}{r}6,131 \\
5,386 \\
0 \\
0\end{array}$ & $\begin{array}{r}49.00 \\
42.00 \\
450.00 \\
150.00\end{array}$ \\
\hline $\begin{array}{l}\text { sb02-92-15cc } \\
\text { sc02-83-21ab }\end{array}$ & $\begin{array}{r}2,302 \\
220\end{array}$ & $\begin{array}{r}2,350 \\
300\end{array}$ & $\begin{array}{r}5.20 \\
15.00\end{array}$ \\
\hline $\begin{array}{l}\text { sb04-101-19ab } \\
\text { sb07-103-20dbc } \\
\text { sb07-103-25bcc } \\
\text { sb07-104-13bca } \\
\text { sc02-104-12bca }\end{array}$ & $\begin{array}{r}1,790 \\
0 \\
0 \\
0 \\
10,713\end{array}$ & $\begin{array}{r}1,820 \\
0 \\
0 \\
0 \\
10,742\end{array}$ & $\begin{array}{r}1.40 \\
580.00 \\
20.00 \\
100.00 \\
8.70\end{array}$ \\
\hline $\begin{array}{l}\text { sld03-25-01bda } \\
\text { sld05-22-01cc }\end{array}$ & $\begin{array}{r}0 \\
7,148\end{array}$ & $\begin{array}{r}0 \\
7,232\end{array}$ & $\begin{array}{r}200.00 \\
6.60\end{array}$ \\
\hline $\begin{array}{l}\text { sb01-90-22bc } \\
\text { sc02-83-04 } \\
\text { sc05-91-13da } \\
\text { sc07-87-14ca } \\
\text { sc08-86-17dd }\end{array}$ & $\begin{array}{r}80 \\
0 \\
156 \\
200 \\
145\end{array}$ & $\begin{array}{r}119 \\
0 \\
207 \\
235 \\
195\end{array}$ & $\begin{array}{r}15.00 \\
15.00 \\
2.00 \\
15.00 \\
15.00\end{array}$ \\
\hline $\begin{array}{l}\text { sc08-88-27dd } \\
\text { sc10-88-04 } \\
\text { sc } 10-88-04 \text { ba } \\
\text { sc11-87-35 } \\
\text { sc12-85-16bac }\end{array}$ & $\begin{array}{r}160 \\
0 \\
0 \\
0 \\
0\end{array}$ & $\begin{array}{r}180 \\
0 \\
0 \\
0 \\
0\end{array}$ & $\begin{array}{r}20.00 \\
50.00 \\
10.00 \\
898.00 \\
75.00\end{array}$ \\
\hline
\end{tabular}

324AMSD

Petroleum Information Corp. (unpublished)

\section{EGLV}

Petroleum Information Corp. (unpublished)

Petroleum Information Corp. (unpublished)

Big Spring (Iorns and others, 1964)

Big Sulphur Spring (Iorns and others, 1964)

\section{MNRN}

Petroleum Information Corp. (unpublished)

Colorado Division of Water Resources (unpublished)

\section{MRGN}

Petroleum Information Corp. (unpublished)
Mitten Park Fault Spring (Sumsion, 1976)
Warm Springs (Sumsion, 1976)
Burnt Springs (Sumsion, 1976)
Petroleum Information Corp. (unpublished)

North Jones Hole Spring (Hood and others, 1976)

Petroleum Information Corp. (unpublished)

\section{MRON}

Colorado Division of Water Resources (unpublished) Iorns and others (1964)

Colorado Division of Water Resources (unpublished)

Colorado Division of Water Resources (unpublished)

Colorado Division of Water Resources (unpublished)

Colorado Division of Water Resources (unpublished)

Cold Iron Spring (Iorns and others, 1964)

Penny Hot Spring (Barrett and Pear1, 1977)

Arsenic Spring (Rouse, 1967)

Conundrum Hot Springs (Barrett and Pear1, 1977)

\section{PKTL}

ga $41-30-10 \mathrm{cb}$ nmb33-13-15ac s $1 \mathrm{c} 35-02-22 \mathrm{dba}$ s $1 d 26-13-25 \mathrm{cb}$ s1d26-13-25cb

sld27-14-05bbd s1d $29-10-08 \mathrm{cc}$ s1d30-13-04dc sld42-21-02aa sld43-23-25cd

ga40-25-06ac ga41-26-31cc ga 41-26-33cc ga41-28-11 ca ga $41-28-22 b c$

$\begin{array}{rrr}5,550 & 5,620 & 4.60 \\ 9,240 & 9,422 & 30.00 \\ 10,050 & 10,085 & 11.00 \\ 5,147 & 5,220 & 4.90 \\ 5,147 & 5,220 & 10.00 \\ & & \\ 5,141 & 5,261 & 1.30 \\ 7,370 & 7,470 & 16.00 \\ 5,540 & 5,610 & 15.00 \\ 5,730 & 5,782 & 7.10 \\ 5,380 & 5,498 & 9.40\end{array}$

Petroleum Information Corp. (unpublished) Petroleum Information Corp. (unpublished) Petroleum Information Corp. (unpublished) Petroleum Information Corp. (unpublished) Petroleum Information Corp. (unpublished)

Petroleum Information Corp. (unpublished) Petroleum Information Corp. (unpublished) Petroleum Information Corp. (unpublished) Petroleum Information Corp. (unpublished) Petroleum Information Corp. (unpublished)

\section{PRDX}

Petroleum Information Corp. (unpublished) Petrcleum Information Corp. (unpublished) Petroleum Information Corp. (unpublished) Petroleum Information Corp. (unpublished) Petroleum Information Corp. (unpublished) 
Table 3.--Artesian yields from wells and springs--Continued

\begin{tabular}{llcc} 
Site & $\begin{array}{l}\text { Depth } \\
\text { to top } \\
\text { (feet) }\end{array}$ & $\begin{array}{c}\text { Depth to } \\
\text { bottom } \\
\text { (feet) }\end{array}$ & $\begin{array}{c}\text { Yield } \\
\text { (gallons per } \\
\text { minute) }\end{array}$ \\
\hline
\end{tabular}

\begin{tabular}{|c|c|c|c|}
\hline $\begin{array}{l}\text { ga41-29-22aa } \\
\text { ga41-31-07cc } \\
\text { nmb26-18-18db } \\
\text { nmb26-18-28dd } \\
\text { nmb31-12-31d }\end{array}$ & $\begin{array}{l}5,240 \\
5,460 \\
6,405 \\
6,302 \\
9,485\end{array}$ & $\begin{array}{l}5,427 \\
5,517 \\
6,450 \\
6,356 \\
9,535\end{array}$ & $\begin{array}{r}40.00 \\
20.00 \\
5.90 \\
61.00 \\
5.00\end{array}$ \\
\hline $\begin{array}{l}\text { nmb32-15-21cd } \\
\text { nmb33-14-36acd } \\
\text { nmb33-20-16ac } \\
\text { nmb33.5-20-25cb } \\
\text { nmb33.5-20-33ab }\end{array}$ & $\begin{array}{l}8,195 \\
9,498 \\
5,888 \\
5,850 \\
5,860\end{array}$ & $\begin{array}{l}8,300 \\
9,633 \\
5,950 \\
5,902 \\
5,893\end{array}$ & $\begin{array}{r}3.30 \\
10.00 \\
64.00 \\
15.00 \\
33.00\end{array}$ \\
\hline $\begin{array}{l}n m b 34-13-01 c a \\
n m b 35-12-17 a b \\
n m b 35-13-14 c d \\
n m b 36-09-10 b c b \\
n m b 36-09-16 b\end{array}$ & $\begin{array}{r}8,488 \\
7,125 \\
7,412 \\
0 \\
0\end{array}$ & $\begin{array}{r}8,719 \\
7,216 \\
7,454 \\
0 \\
0\end{array}$ & $\begin{array}{l}23.00 \\
56.00 \\
34.00 \\
24.00 \\
15.00\end{array}$ \\
\hline $\begin{array}{l}\mathrm{nmb} 36-19-02 \mathrm{ca} \\
\mathrm{nmb} 37-13-31 \mathrm{ab} \\
\mathrm{nmb} 37-19-06 \mathrm{bac} \\
\mathrm{nmb} 37-20-24 \mathrm{dd} \\
\mathrm{nmb} 38-19-29 \mathrm{da}\end{array}$ & $\begin{array}{l}5,152 \\
5,336 \\
6,114 \\
6,061 \\
5,364\end{array}$ & $\begin{array}{l}5,224 \\
5,352 \\
6,146 \\
6,077 \\
5,722\end{array}$ & $\begin{array}{r}0.21 \\
30.00 \\
38.00 \\
6.00 \\
5.40\end{array}$ \\
\hline $\begin{array}{l}n m b 39-20-10 d c \\
n m b 39-20-12 b a \\
n m b 43-15-12 b b \\
n m b 44-14-16 b c a \\
n m b 44-18-21 d d a\end{array}$ & $\begin{array}{r}6,305 \\
6,327 \\
11,006 \\
6,600 \\
7,035\end{array}$ & $\begin{array}{r}6,332 \\
6,361 \\
11,103 \\
6,655 \\
7,163\end{array}$ & $\begin{array}{r}5.00 \\
4.50 \\
36.00 \\
1.90 \\
4.30\end{array}$ \\
\hline $\begin{array}{l}\text { nmb45-18-26ccb } \\
\text { s } 1 \mathrm{~d} 22-16-02 \mathrm{bbd} \\
\text { s1d23-14-11aa } \\
\text { s1d24-24-21c } \\
\text { s } 1 \mathrm{~d} 24-24-22\end{array}$ & $\begin{array}{r}9,605 \\
5,792 \\
5,534 \\
0 \\
0\end{array}$ & $\begin{array}{r}9,700 \\
5,896 \\
5,624 \\
0 \\
0\end{array}$ & $\begin{array}{r}11.00 \\
146.00 \\
103.00 \\
25.00 \\
55.00\end{array}$ \\
\hline $\begin{array}{l}\text { sld25-12-34cc } \\
\text { sld25-15-22ac } \\
\text { sld26-19-14ac } \\
\text { sld26-19-23aa } \\
\text { sld26-20-31bbb }\end{array}$ & $\begin{array}{l}4,785 \\
4,790 \\
6,266 \\
4,432 \\
6,036\end{array}$ & $\begin{array}{l}4,788 \\
4,935 \\
6,373 \\
4,445 \\
6,052\end{array}$ & $\begin{array}{r}22.00 \\
11.00 \\
5.40 \\
5.10 \\
21.00\end{array}$ \\
\hline $\begin{array}{l}\text { sld29-24-28ac } \\
\text { sld30-12-24adc } \\
\text { sld30-24-24ba } \\
\text { sld31-12-04acc } \\
\text { sld32-17-08dc }\end{array}$ & $\begin{array}{r}3,644 \\
4,900 \\
3,600 \\
4,922 \\
0\end{array}$ & $\begin{array}{r}3,705 \\
4,936 \\
3,710 \\
4,972 \\
0\end{array}$ & $\begin{array}{r}19.00 \\
2.50 \\
4.80 \\
21.00 \\
90.00\end{array}$ \\
\hline $\begin{array}{l}\text { sld32-21-02baa } \\
\text { s1d36-21-22bd } \\
\text { sld36-25-13adb } \\
\text { sld37-14-03cad } \\
\text { sld37-19-23cc }\end{array}$ & $\begin{array}{l}3,004 \\
5,555 \\
5,817 \\
3,145 \\
2,369\end{array}$ & $\begin{array}{l}3,134 \\
5,594 \\
5,890 \\
3,228 \\
2,396\end{array}$ & $\begin{array}{r}2.70 \\
6.30 \\
20.00 \\
9.60 \\
1.90\end{array}$ \\
\hline $\begin{array}{l}\text { sld38-22-34ad } \\
\text { sld38-25-09bab } \\
\text { sld38-26-16baa } \\
\text { sld39-13-24dd } \\
\text { sld39-21-13da }\end{array}$ & $\begin{array}{l}6,247 \\
5,513 \\
5,144 \\
5,011 \\
5,649\end{array}$ & $\begin{array}{l}6,309 \\
5,529 \\
5,265 \\
5,231 \\
5,710\end{array}$ & $\begin{array}{r}6.50 \\
3.80 \\
17.00 \\
18.00 \\
21.00\end{array}$ \\
\hline
\end{tabular}

\section{PRDX}

40.00 Petroleum Information Corp. (unpublished)

Petroleum Information Corp. (unpublished) Petroleum Information Corp. (unpublished) Petroleum Information Corp. (unpublished) Petroleum Information Corp. (unpublished)

Petroleum Information Corp. (unpublished) Petroleum Information Corp. (unpublished) Petroleum Information Corp. (unpublished) Petroleum Information Corp. (unpublished) Petroleum Information Corp. (unpublished)

Petroleum Information Corp. (unpublished) Petroleum Information Corp. (unpublished) Petroleum Information Corp. (unpublished) Tripp Hot Spring (Barrett and Pearl, 1977) Trimble Hot Spring (Torns and others, 1964)

Petroleum Information Corp. (unpublished) Petroleum Information Corp. (unpublished) Petroleum Information Corp. (unpublished) Petroleum Information Corp. (unpublished) Petroleum Information Corp. (unpublished)

Petroleum Information Corp. (unpublished) Petroleum Information Corp. (unpublished) Petroleum Information Corp. (unpublished) Petroleum Information Corp. (unpublished) Petroleum Information Corp. (unpublished)

Petroleum Information Corp. (unpublished) Petroleum Information Corp. (unpublished) Petroleum Information Corp. (unpublished) Stinking Spring (Ritzma and Doelling, 1969) Onion Creek Spring (Rouse, 1967)

Petroleum Information Corp. (unpublished) Petroleum Information Corp. (unpublished) Petroleum Information Corp. (unpublished) Petroleum Information Corp. (unpublished) Petroleum Information Corp. (unpublished)

Petroleum Information Corp. (unpublished) Petroleum Information Corp. (unpublished) Petroleum Information Corp. (unpublished) Petroleum Information Corp. (unpublished) U.S. Geological Survey (unpublished)

Petroleum Information Corp. (unpublished) Petroleum Information Corp. (unpublished) Petroleum Information Corp. (unpublished) Petroleum Information Corp. (unpublished) Petroleum Information Corp. (unpublished)

Petroleum Information Corp. (unpublished) Petroleum Information Corp. (unpublished) Petroleum Information Corp. (unpublished) Petroleum Information Corp. (unpublished) Petroleum Information Corp. (unpublished) 
Table 3.--Artesian yields from wells and springs--Continued

\begin{tabular}{|c|c|c|c|c|}
\hline Site & $\begin{array}{l}\text { Depth } \\
\text { to top } \\
\text { (feet) }\end{array}$ & $\begin{array}{l}\text { Depth to } \\
\text { bottom } \\
\text { (feet) }\end{array}$ & $\begin{array}{l}\text { Yield } \\
\text { (gallons per } \\
\text { minute) }\end{array}$ & Remarks \\
\hline \multicolumn{5}{|c|}{ 324PRDX--Cont inued } \\
\hline $\begin{array}{l}\text { s } 1 d 39-21-15 c d \\
\text { sld39-22-29cb } \\
\text { s } 1 d 39-24-31 c c \\
\text { s } 1 d 40-22-24 c c \\
\text { s } 1 d 40-23-20 d b b\end{array}$ & $\begin{array}{l}5,650 \\
5,782 \\
6,086 \\
5,568 \\
5,523\end{array}$ & $\begin{array}{l}5,751 \\
5,819 \\
6,162 \\
5,615 \\
5,600\end{array}$ & $\begin{array}{r}2.30 \\
21.00 \\
14.00 \\
2.40 \\
9.50\end{array}$ & $\begin{array}{l}\text { Petroleum Information Corp. (unpublished) } \\
\text { Petroleum Information Corp. (unpublished) } \\
\text { Petroleum Information Corp. (unpublished) } \\
\text { Petroleum Information Corp. (unpublished) } \\
\text { Petroleum Information Corp. (unpublished) }\end{array}$ \\
\hline $\begin{array}{l}\text { s } 1 d 40-25-14 c c d \\
\text { s } 1 d 40-26-32 a a \\
\text { s } 1 d 41-23-35 d d \\
\text { s } 1 d 41-24-32 b c a \\
\text { s } 1 d 41-25-26 c c\end{array}$ & $\begin{array}{l}5,788 \\
5,465 \\
5,315 \\
5,873 \\
5,364\end{array}$ & $\begin{array}{l}5,843 \\
5,552 \\
5,330 \\
5,935 \\
5,394\end{array}$ & $\begin{array}{l}21.00 \\
13.00 \\
20.00 \\
21.00 \\
22.00\end{array}$ & $\begin{array}{l}\text { Petroleum Information Corp. (unpublished) } \\
\text { Petroleum Information Corp. (unpublished) } \\
\text { Petroleum Information Corp. (unpublished) } \\
\text { Petroleum Information Corp. (unpublished) } \\
\text { Petroleum Information Corp. (unpublished) }\end{array}$ \\
\hline \multicolumn{5}{|c|}{$324 \mathrm{RDVL}$} \\
\hline $\begin{array}{l}\text { sb14-113-01adc } \\
\text { sb15-112-29ca }\end{array}$ & $\begin{array}{l}18,440 \\
18,140\end{array}$ & $\begin{array}{l}18,605 \\
18,480\end{array}$ & $\begin{array}{l}1.60 \\
0.83\end{array}$ & $\begin{array}{l}\text { Petroleum Information Corp. (unpublished) } \\
\text { Petroleum Information Corp. (unpublished) }\end{array}$ \\
\hline \multicolumn{5}{|c|}{ 327BLDN } \\
\hline $\begin{array}{l}\text { sb04-91-10bbb } \\
\text { s c08-84-14baa }\end{array}$ & $\begin{array}{c}9,480 \\
242\end{array}$ & $\begin{array}{r}9,550 \\
297\end{array}$ & $\begin{array}{r}4.00 \\
100.00\end{array}$ & $\begin{array}{l}\text { Petroleum Information Corp. (unpublished) } \\
\text { U.S. Bureau of Reclamation (unpublished) }\end{array}$ \\
\hline \multicolumn{5}{|c|}{$327 \mathrm{MNGC}$} \\
\hline \multicolumn{5}{|c|}{ 331DRWN } \\
\hline $\mathrm{sb} 26-90-14 \mathrm{bab}$ & \multicolumn{4}{|r|}{$\underline{331 H M B G}$} \\
\hline $\begin{array}{l}\text { sb08-99-17 } \\
\text { sb19-112-22cc } \\
\text { s1d04-20-04ddc } \\
\text { ub02-07-36aba }\end{array}$ & $\begin{array}{r}5,085 \\
17,424 \\
6,661 \\
0\end{array}$ & $\begin{array}{r}5,150 \\
17,498 \\
6,730 \\
0\end{array}$ & $\begin{array}{r}34.00 \\
8.90 \\
7.20 \\
900.00\end{array}$ & $\begin{array}{l}\text { U.S. Geological Survey (unpublished) } \\
\text { Petroleum Information Corp. (unpublished) } \\
\text { Petroleum Information Corp. (unpublished) } \\
\text { Hood and others (1976) }\end{array}$ \\
\hline \multicolumn{5}{|c|}{ 337LDVL } \\
\hline $\begin{array}{l}\text { ga40-30-05cc } \\
\text { nmb27-18-34cc } \\
\text { nmb32-15-21cd } \\
\text { nmb34-12-27cad } \\
\text { nmb34-12-28cd }\end{array}$ & $\begin{array}{l}6,059 \\
6,812 \\
9,364 \\
9,831 \\
9,972\end{array}$ & $\begin{array}{r}6,120 \\
6,828 \\
9,494 \\
9,885 \\
10,026\end{array}$ & $\begin{array}{r}27.00 \\
9.50 \\
7.30 \\
26.00 \\
15.00\end{array}$ & $\begin{array}{l}\text { Petroleum Information Corp. (unpublished) } \\
\text { Petroleum Information Corp. (unpublished) } \\
\text { Petroleum Information Corp. (unpublished) } \\
\text { Petroleum Information Corp. (unpublished) } \\
\text { Petroleum Information Corp. (unpublished) }\end{array}$ \\
\hline $\begin{array}{l}\mathrm{nmb} 37-09-25 \mathrm{ab} \\
\mathrm{nmb} 37-15-02 \mathrm{cc} \\
\mathrm{nmb} 37-17-27 \mathrm{bb} \\
\mathrm{nmb} 39-13-02 \mathrm{bb} \\
\mathrm{nmb} 40-17-13 \mathrm{a} a\end{array}$ & $\begin{array}{r}0 \\
7,840 \\
7,715 \\
6,590 \\
9,140\end{array}$ & $\begin{array}{r}0 \\
8,047 \\
7,740 \\
6,698 \\
9,207\end{array}$ & $\begin{array}{r}54.00 \\
79.00 \\
0.60 \\
9.60 \\
0.68\end{array}$ & $\begin{array}{l}\text { Mound Warm Spring (Barrett and Pearl, 1977) } \\
\text { Petroleum Information Corp. (unpublished) } \\
\text { Petroleum Information Corp. (unpublished) } \\
\text { Petroleum Information Corp. (unpublished) } \\
\text { Petroleum Information Corp. (unpublished) }\end{array}$ \\
\hline
\end{tabular}


Table 3.--Artesian yields from wells and springs--Continued

\begin{tabular}{|c|c|c|c|c|}
\hline Site & $\begin{array}{l}\text { Depth } \\
\text { to top } \\
\text { (feet) }\end{array}$ & $\begin{array}{l}\text { Depth to } \\
\text { bottom } \\
\text { (feet) }\end{array}$ & $\begin{array}{c}\text { Yield } \\
\text { (gallons per } \\
\text { minute) }\end{array}$ & Remarks \\
\hline \multicolumn{5}{|c|}{ 337LDVL--Continued } \\
\hline $\begin{array}{l}\text { nmb41-17-24aa } \\
\text { nmb41-18-17dd } \\
\text { nmb42-18-34bdc } \\
\text { nmb43-17-07 } \\
\text { nmb44-07-31cbc }\end{array}$ & $\begin{array}{r}10,163 \\
9,104 \\
10,044 \\
9,067 \\
0\end{array}$ & $\begin{array}{r}10,465 \\
9,265 \\
10,079 \\
9,272 \\
0\end{array}$ & $\begin{array}{r}15.00 \\
34.00 \\
0.86 \\
73.00 \\
200.00\end{array}$ & $\begin{array}{l}\text { Petroleum Information Corp. (unpublished) } \\
\text { Petroleum Information Corp. (unpublished) } \\
\text { Petroleum Information Corp. (unpublished) } \\
\text { Petroleum Information Corp. (unpublished) } \\
\text { Pool Hot Spring (Barrett and Pearl, 1977) }\end{array}$ \\
\hline $\begin{array}{l}\text { nmb44-17-36ad } \\
\text { nmb44-19-16bcc } \\
\text { nmb45-19-31ac } \\
\text { nmb46-14-14ab } \\
\text { nmb47-15-10bd }\end{array}$ & $\begin{array}{r}9,264 \\
9,150 \\
10,157 \\
9,541 \\
6,664\end{array}$ & $\begin{array}{r}9,431 \\
9,203 \\
10,335 \\
9,873 \\
6,700\end{array}$ & $\begin{array}{r}4.50 \\
8.90 \\
68.00 \\
5.20 \\
58.00\end{array}$ & $\begin{array}{l}\text { Petroleum Information Corp. (unpublished) } \\
\text { Petroleum Information Corp. (unpublished) } \\
\text { Petroleum Information Corp. (unpublished) } \\
\text { Petroleum Information Corp. (unpublished) } \\
\text { Petroleum Information Corp. (unpublished) }\end{array}$ \\
\hline $\begin{array}{l}\mathrm{sc0} 1-91-32 \mathrm{bcc} \\
\mathrm{sc0} 1-92-36 \mathrm{daa} \\
\mathrm{sc0}-84-02 \mathrm{bc} \\
\mathrm{sc0}-84-03 \mathrm{ad} \\
\mathrm{sc0}-90-19 \mathrm{bdd}\end{array}$ & $\begin{array}{r}0 \\
0 \\
140 \\
149 \\
0\end{array}$ & $\begin{array}{r}0 \\
0 \\
757 \\
980 \\
0\end{array}$ & $\begin{array}{r}0.97 \\
1.50 \\
3,200.00 \\
7,500.00 \\
370.00\end{array}$ & $\begin{array}{l}\text { Teller and Welder (1983) } \\
\text { Teller and Welder (1983) } \\
\text { Hampton (1974) } \\
\text { Colorado Division of Water Resources (unpublished) } \\
\text { Teller and Welder (1983) }\end{array}$ \\
\hline $\begin{array}{l}\text { sc02-91-06acb } \\
\text { sc03-90-07aba } \\
\text { sc03-91-10cad } \\
\text { sc03-92-23baa } \\
\text { sc03-92-33bbc }\end{array}$ & $\begin{array}{l}0 \\
0 \\
0 \\
0 \\
0\end{array}$ & $\begin{array}{l}0 \\
0 \\
0 \\
0 \\
0\end{array}$ & $\begin{array}{r}34.00 \\
102.00 \\
266.00 \\
400.00 \\
23.00\end{array}$ & $\begin{array}{l}\text { Teller and Welder }(1983) \\
\text { Teller and Welder }(1983) \\
\text { Teller and Welder }(1983) \\
\text { Teller and Welder }(1983) \\
\text { Teller and Welder }(1983)\end{array}$ \\
\hline $\begin{array}{l}\text { sc14-83-19cbc } \\
\text { sld22-20-17cb } \\
\text { sld24-20-11bc } \\
\text { sld29.5-23-36dac } \\
\text { sld34-25-23ad }\end{array}$ & $\begin{array}{r}0 \\
14,850 \\
11,795 \\
9,310 \\
8,145\end{array}$ & $\begin{array}{r}0 \\
14,994 \\
11,913 \\
9,540 \\
8,205\end{array}$ & $\begin{array}{r}300.00 \\
13.00 \\
22.00 \\
12.00 \\
12.00\end{array}$ & $\begin{array}{l}\text { White Water Spring (U.S. Geological Survey, unpublished) } \\
\text { Petroleum Information Corp. (unpublished) } \\
\text { Petroleum Information Corp. (unpublished) } \\
\text { Petroleum Information Corp. (unpublished) } \\
\text { Petroleum Information Corp. (unpublished) }\end{array}$ \\
\hline $\begin{array}{l}\text { sld } 35-25-09 \text { ad } \\
\text { sld35-26-20ad } \\
\text { sld } 39-24-04 \text { aaa } \\
\text { sld40-25-21dc } \\
\text { sld40-26-19ad }\end{array}$ & $\begin{array}{l}7,034 \\
7,588 \\
7,830 \\
7,515 \\
7,345\end{array}$ & $\begin{array}{l}7,150 \\
7,653 \\
7,881 \\
7,637 \\
7,410\end{array}$ & $\begin{array}{r}6.10 \\
0.52 \\
53.00 \\
58.00 \\
28.00\end{array}$ & $\begin{array}{l}\text { Petroleum Information Corp. (unpublished) } \\
\text { Petroleum Information Corp. (unpublished) } \\
\text { Petroleum Information Corp. (unpublished) } \\
\text { Petroleum Information Corp. (unpublished) } \\
\text { Petroleum Information Corp. (unpublished) }\end{array}$ \\
\hline \multicolumn{5}{|c|}{ 337MDSN } \\
\hline $\begin{array}{l}\text { sb06-96-35ccd } \\
\text { sb07-103-20cad } \\
\text { sb08-99-16bcd } \\
\text { sb12-101-24cc } \\
\text { sb15-112-29ac }\end{array}$ & $\begin{array}{r}3,731 \\
0 \\
5,871 \\
16,600 \\
18,552\end{array}$ & $\begin{array}{r}3,817 \\
0 \\
5,877 \\
16,760 \\
18,741\end{array}$ & $\begin{array}{r}47.00 \\
450.00 \\
46.00 \\
20.00 \\
43.00\end{array}$ & $\begin{array}{l}\text { Petroleum Information Corp. (unpublished) } \\
\text { Mitten Park Fault Spring (Hood and others, 1976) } \\
\text { U.S. Geological Survey (unpublished) } \\
\text { Petroleum Information Corp. (unpublished) } \\
\text { Petroleum Information Corp. (unpublished) }\end{array}$ \\
\hline
\end{tabular}


Table 3.--Artesian yields from wells and springs--Continued

$\begin{array}{cccc}\text { Site } & \begin{array}{l}\text { Depth } \\ \text { to top } \\ \text { (feet) }\end{array} & \begin{array}{c}\text { Depth to } \\ \text { bottom } \\ \text { (feet) }\end{array} & \begin{array}{c}\text { Yield } \\ \text { (gallons per } \\ \text { minute) }\end{array}\end{array}$ Remarks

\begin{abstract}
sb16-101-11ba
sb16-104-16ddb

sb16-112-08ad

sb16-112-28dd

sb16-117-32cca

sb18-98-21db

sb19-104-11ac

sb19-112-22cc

sb19-87-29da

sb20-84-28cc

sb21-117-14cc

sb22-105-11bcc

sb25-88-31ac

sb26-114-01bac

sb26-114-01dcc

sb26-88-34a

sb26-89-24dc

sb26-90-14bab

sb28-113-19bbd

sb30-117-24a

sb33-118-13acc

sb37-118-34dcd sb39-116-32daa sc05-103-25cc

sla 02-19-16bcb

sla 03-24-22bdb s1d04-24-16cdd ub0 1-08-17cbb ub02-02-05dbb ub02-07-25cab ub03-02-34
\end{abstract}

ga32-06-01c ga32-07-31b ga $36-05-27 b$ ga 36-28-06bb ga38-24-29dba

ga39-25-28bd ga 40-25-06aa ga40-25-11dac ga 40-27-06bb ga40-28-01cc

ga 40-29-15cca ga40-29-16bdd ga 40-29-18da ga41-28-04da ga41-28-09ac

ga41-29-06dd ga $41-30-10 \mathrm{cb}$ ga41-30-16ccd ga 41-30-23cd nmb32-20-17dd

$\begin{array}{rr}15,013 & 15,035 \\ 7,074 & 7,130 \\ 18,313 & 18,431 \\ 18,240 & 18,288 \\ 5,778 & 5,880 \\ & \\ 18,652 & 19,171 \\ 7,507 & 7,670 \\ 18,004 & 18,128 \\ 5,711 & 5,811 \\ 10,060 & 10,194 \\ & \\ 3,228 & 3,285 \\ 17,998 & 18,150 \\ 5,935 & 6,015 \\ 0 & 0 \\ 0 & 0 \\ & \\ 5,204 & 5,259 \\ 6,860 & 6,905 \\ 6,717 & 6,806 \\ 13,718 & 14,033 \\ 0 & 0 \\ & \\ 0 & 0 \\ 0 & 0 \\ 0 & 0 \\ 8,553 & 8,600 \\ 0 & 0 \\ 10,764 & 10,912 \\ 0 & 0 \\ 0 & 0 \\ 0 & 0 \\ 0 & 0 \\ 0 & 0\end{array}$

0

3,773

5,555

5,020

5,925

5,736

5,925

5,592

5,695

5,710

6,226

5,689

6,068

5,738

6,256

5,923

6,068

6,589
337MDSN--Cont inued

$\begin{array}{ll}27.00 & \text { Petroleum Information Corp. (unpublished) } \\ 23.00 & \text { Petroleum Information Corp. (unpublished) }\end{array}$

23.00

5.30

4.20

39.00

27.00

2.80

31.00

37.00

25.00

1.40

37.00

38.00

$5,500.00$

15.00

102.00

5.70

36.00

2. 10

100.00

150.00

15.00

350.00

2.00

$2,900.00$

40.00

$2,700.00$

$6,940.00$

$4,500.00$

678.00

$5,164.00$

100.00

$44,550.00$

$4,500.00$

7. 20

7.10

9.30

24.00

10.00

7.80

16.00

12.00

15.00

10.00

18.00

27.00

13.00

12.00

15.00

27.00

12.00

Petroleum Information Corp. (unpublished)

Petroleum Information Corp. (unpublished)

Petroleum Information Corp. (unpublished)

Petroleum Information Corp. (unpublished)

Petroleum Information Corp. (unpublished)

Petroleum Information Corp. (unpublished)

Petroleum Information Corp. (unpublished)

Petroleum Information Corp. (unpublished)

Petroleum Information Corp. (unpublished)

Petroleum Information Corp. (unpublished)

Petroleum Information Corp. (unpublished)

Hogsback Spring (Lines and Glass, 1975)

Lines and Glass (1975)

Petroleum Information Corp. (unpublished)

Petroleum Information Corp. (unpublished)

Petroleum Information Corp. (unpublished)

Petroleum Information Corp. (unpublished)

Lines and Glass (1975)

Lines and Glass (1975)

Lines and Glass (1975)

Lines and Glass (1975)

Petroleum Information Corp. (unpublished)

Petroleum Information Corp. (unpublished)

Big Spring (Hood and others, 1976)

Indian Big Spring (Hood and others, 1976)

Hood and others (1976)

U.S. Geological Survey (unpublished)

\section{RDLL}

Cooley (1976)

Blue Spring (Cooley, 1976)

Vasey's Paradise Spring (Cooley, 1976)

Petroleum Information Corp. (unpublished)

Petroleum Information Corp. (unpublished)

Petroleum Information Corp. (unpublished)

Petroleum Information Corp. (unpublished)

Petroleum Information Corp. (unpublished)

Petroleum Information Corp. (unpublished)

Petroleum Information Corp. (unpublished)

Petroleum Information Corp. (unpublished)

Petroleum Information Corp. (unpublished)

Petroleum Information Corp. (unpublished)

Petroleum Information Corp. (unpublished)

Petroleum Information Corp. (unpublished)

Petroleum Information Corp. (unpublished)

Petroleum Information Corp. (unpublished)

Petroleum Information Corp. (unpublished)

Petroleum Information Corp. (unpublished)

Petroleum Information Corp. (unpublished)
Sheep Creek Spring (U.S. Geological Survey, unpublished)

Split Mountain Warm Spring (Hood and others, 1976) 
Table 3.--Artesian yields from wells and springs--Continued

\begin{tabular}{cccc} 
Site & $\begin{array}{l}\text { Depth } \\
\text { to top } \\
\text { (feet) }\end{array}$ & $\begin{array}{c}\text { Depth to } \\
\text { bottom } \\
\text { (feet) }\end{array}$ & $\begin{array}{c}\text { Yield } \\
\text { (gallons per } \\
\text { minute) }\end{array}$ \\
\hline
\end{tabular}

\begin{tabular}{|c|c|c|c|}
\hline $\begin{array}{l}\text { nmb46-19-06dc } \\
\text { sb03-103-03cb } \\
\text { s } 1 \text { c32-02-23dbd } \\
\text { s } 1 c 35-02-22 d b a \\
\text { s } 1 d 09-20-22 c c b\end{array}$ & $\begin{array}{r}11,182 \\
4,384 \\
11,065 \\
10,186 \\
19,326\end{array}$ & $\begin{array}{r}11,261 \\
4,563 \\
11,130 \\
10,310 \\
20,052\end{array}$ & $\begin{array}{r}20.00 \\
28.00 \\
51.00 \\
55.00 \\
7.20\end{array}$ \\
\hline $\begin{array}{l}\text { sld15-12-08cc } \\
\text { sld15-13-17db } \\
\text { s } 1 d 16-12-27 a b \\
\text { sld17-14-29ac } \\
\text { sld17-14-29ac }\end{array}$ & $\begin{array}{l}8,323 \\
7,990 \\
6,998 \\
8,340 \\
8,442\end{array}$ & $\begin{array}{l}9,174 \\
8,080 \\
7,132 \\
8,507 \\
8,508\end{array}$ & $\begin{array}{r}34.00 \\
24.00 \\
8.40 \\
4.10 \\
47.00\end{array}$ \\
\hline $\begin{array}{l}\text { sld18-12-12ad } \\
\text { sld } 18-14-08 c c \\
\text { sld } 18-14-30 c c \\
\text { s } 1 d 19-12-09 c c \\
\text { s } 1 d 19-13-12 d d\end{array}$ & $\begin{array}{l}5,050 \\
7,640 \\
6,963 \\
4,803 \\
6,518\end{array}$ & $\begin{array}{l}5,150 \\
7,920 \\
7,083 \\
4,853 \\
6,608\end{array}$ & $\begin{array}{r}40.00 \\
33.00 \\
8.40 \\
6.30 \\
26.00\end{array}$ \\
\hline $\begin{array}{l}\text { sld21-15-24cc } \\
\text { s } 1 d 22-13-24 a \text { a } \\
\text { sld22-13-24aa } \\
\text { sld22-15-09ac } \\
\text { sld22-15-26ca }\end{array}$ & $\begin{array}{l}9,705 \\
6,443 \\
6,645 \\
8,704 \\
8,383\end{array}$ & $\begin{array}{l}9,753 \\
6,749 \\
6,755 \\
8,747 \\
8,490\end{array}$ & $\begin{array}{r}57.00 \\
161.00 \\
32.00 \\
78.00 \\
47.00\end{array}$ \\
\hline $\begin{array}{l}\text { s } 1 d 22-16-25 b b \\
\text { s } 1 d 22-17-34 b d \\
\text { s } 1 d 23-15-21 b a \\
\text { s } 1 d 23-16-03 b c \\
\text { s } 1 d 23-17-16 d a\end{array}$ & $\begin{array}{r}9,225 \\
10,053 \\
7,500 \\
8,530 \\
8,858\end{array}$ & $\begin{array}{r}9,280 \\
10,173 \\
7,702 \\
8,715 \\
8,887\end{array}$ & $\begin{array}{l}34.00 \\
32.00 \\
11.00 \\
82.00 \\
26.00\end{array}$ \\
\hline $\begin{array}{l}\text { sld23-17-21dda } \\
\text { sld23-18-21bbb } \\
\text { sld24-14-10aa } \\
\text { sld24-14-21daa } \\
\text { sld24-16-19ac }\end{array}$ & $\begin{array}{r}8,465 \\
10,240 \\
6,120 \\
7,200 \\
7,968\end{array}$ & $\begin{array}{r}8,525 \\
10,333 \\
6,353 \\
7,350 \\
8,067\end{array}$ & $\begin{array}{r}5.80 \\
41.00 \\
21.00 \\
17.00 \\
46.00\end{array}$ \\
\hline $\begin{array}{l}\text { s } 1 d 24-17-26 c c a \\
\text { s } 1 d 25-12-34 c c \\
\text { s } 1 d 25-12-34 c c \\
\text { s } 1 d 25-15-32 c a \\
\text { s } 1 d 25-16-29 c d\end{array}$ & $\begin{array}{l}8,525 \\
5,595 \\
5,789 \\
5,631 \\
6,486\end{array}$ & $\begin{array}{l}8,615 \\
5,720 \\
6,008 \\
5,843 \\
6,594\end{array}$ & $\begin{array}{l}49.00 \\
14.00 \\
58.00 \\
31.00 \\
55.00\end{array}$ \\
\hline $\begin{array}{l}\text { s } 1 d 25-17-20 d a \\
\text { s1d25-18-35cac } \\
\text { s } 1 d 25-19-27 d b \\
\text { s } 1 d 26-13-35 a c \\
\text { sld26-14-26dd }\end{array}$ & $\begin{array}{l}6,361 \\
7,990 \\
7,602 \\
5,940 \\
6,500\end{array}$ & $\begin{array}{l}6,386 \\
8,087 \\
7,630 \\
6,040 \\
6,700\end{array}$ & $\begin{array}{l}51.00 \\
19.00 \\
32.00 \\
25.00 \\
25.00\end{array}$ \\
\hline $\begin{array}{l}\text { s1d26-14-30cc } \\
\text { s1d26-17-05cc } \\
\text { s1d26-18-07dad } \\
\text { s } 1 d 26-19-14 d c \\
\text { s } 1 d 26-20-31 b b b\end{array}$ & $\begin{array}{l}5,860 \\
6,410 \\
6,978 \\
7,486 \\
7,543\end{array}$ & $\begin{array}{l}6,007 \\
6,466 \\
7,086 \\
7,534 \\
7,583\end{array}$ & $\begin{array}{r}18.00 \\
23.00 \\
60.00 \\
1.50 \\
42.00\end{array}$ \\
\hline $\begin{array}{l}\text { s1d26-22-01acc } \\
\text { s } 1 d 27-12-09 a a c \\
\text { s } 1 d 27-13-36 c d \\
\text { s } 1 d 27-15-35 b c \\
\text { s } 1 d 27-16-33 a c\end{array}$ & $\begin{array}{r}10,807 \\
6,210 \\
6,712 \\
5,170 \\
5,894\end{array}$ & $\begin{array}{r}10,944 \\
6,430 \\
6,820 \\
5,410 \\
5,994\end{array}$ & $\begin{array}{l}12.00 \\
49.00 \\
56.00 \\
15.00 \\
64.00\end{array}$ \\
\hline $\begin{array}{l}\text { s1d27-18-26ba } \\
\text { s } 1 \mathrm{~d} 27-19-27 \mathrm{cad} \\
\text { s } 1 \mathrm{~d} 27-20-06 \mathrm{ca} \\
\text { s } 1 \mathrm{~d} 27-21-03 \mathrm{~cd} \\
\text { s } 1 \mathrm{~d} 27-21-34 \mathrm{cab}\end{array}$ & $\begin{array}{l}6,260 \\
7,645 \\
6,230 \\
6,150 \\
7,908\end{array}$ & $\begin{array}{l}6,400 \\
7,735 \\
6,286 \\
6,274 \\
7,940\end{array}$ & $\begin{array}{r}8.50 \\
48.00 \\
79.00 \\
25.00 \\
17.00\end{array}$ \\
\hline
\end{tabular}

\section{RDLL--Continued}

20.00 Petroleum Information Corp. (unpublished)

28.00 Petroleum Information Corp. (unpublished)

51.00 Petroleum Information Corp. (unpublished)

55.00 Petroleum Information Corp. (unpublished)

7.20 Petroleum Information Corp. (unpublished)

34.00 Petroleum Information Corp. (unpublished)

Petroleum Information Corp. (unpublished)

Petroleum Information Corp. (unpublished) Petroleum Information Corp. (unpublished) Petroleum Information Corp. (unpublished)

Petroleum Information Corp. (unpublished) Petroleum Information Corp. (unpublished) Petroleum Information Corp. (unpublished) Petroleum Information Corp. (unpublished) Petroleum Information Corp. (unpublished)

Petroleum Information Corp. (unpublished) Petroleum Information Corp. (unpublished) Petroleum Information Corp. (unpublished) Petroleum Information Corp. (unpublished) Petroleum Information Corp. (unpublished)

Petroleum Information Corp. (unpublished) Petroleum Information Corp. (unpublished) Petroleum Information Corp. (unpublished) Petroleum Information Corp. (unpublished) Petroleum Information Corp. (unpublished)

Petroleum Information Corp. (unpublished) Petroleum Information Corp. (unpublished) Petroleum Information Corp. (unpublished) Petroleum Information Corp. (unpublished) Petroleum Information Corp. (unpublished)

Petroleum Information Corp. (unpublished) Petroleum Information Corp. (unpublished) Petroleum Information Corp. (unpublished) Petroleum Information Corp. (unpublished) Petroleum Information Corp. (unpublished)

Petroleum Information Corp. (unpublished) Petroleum Information Corp. (unpublished) Petroleum Information Corp. (unpublished) Petroleum Information Corp. (unpublished) Petroleum Information Corp. (unpublished)

Petroleum Information Corp. (unpublished) Petroleum Information Corp. (unpublished) Petroleum Information Corp. (unpublished) Petroleum Information Corp. (unpublished) Petroleum Information Corp. (unpublished)

Petroleum Information Corp. (unpublished) Petroleum Information Corp. (unpublished) Petroleum Information Corp. (unpublished) Petroleum Information Corp. (unpublished) Petroleum Information Corp. (unpublished)

Petroleum Information Corp. (unpublished) Petroleum Information Corp. (unpublished) Petroleum Information Corp. (unpublished) Petroleum Information Corp. (unpublished) Petroleum Information Corp. (unpublished) 
Table 3.--Artesian yields from wells and springs--Continued

\begin{tabular}{|c|c|c|c|c|}
\hline Site & $\begin{array}{l}\text { Depth } \\
\text { to top } \\
\text { (feet) }\end{array}$ & $\begin{array}{l}\text { Depth to } \\
\text { bottom } \\
\text { (feet) }\end{array}$ & $\begin{array}{l}\text { Yield } \\
\text { (gallons per } \\
\text { minute) }\end{array}$ & Remarks \\
\hline
\end{tabular}

\begin{tabular}{|c|c|c|c|c|c|c|c|}
\hline $\begin{array}{l}\text { s } 1 d 27-22-17 d d b \\
\text { s } 1 d 27-22-32 a a \\
\text { sld } 28-05-13 d b \\
\text { s } 1 d 28-08-29 c d \\
\text { s } 1 d 28-11-05 c d\end{array}$ & $\begin{array}{l}7,025 \\
7,210 \\
4,934 \\
6,556 \\
7,100\end{array}$ & $\begin{array}{l}7,083 \\
7,248 \\
5,059 \\
6,618 \\
7,301\end{array}$ & $\begin{array}{l}24.00 \\
42.00 \\
28.00 \\
20.00 \\
26.00\end{array}$ & $\begin{array}{l}\text { Petroleum } \\
\text { Petroleum } \\
\text { Petroleum } \\
\text { Petroleum } \\
\text { Petroleum }\end{array}$ & $\begin{array}{l}\text { Information } \\
\text { Information } \\
\text { Information } \\
\text { Information } \\
\text { Information }\end{array}$ & $\begin{array}{l}\text { Corp. } \\
\text { Corp. } \\
\text { Corp. } \\
\text { Corp. } \\
\text { Corp. }\end{array}$ & $\begin{array}{l}\text { (unpublished) } \\
\text { (unpublished) } \\
\text { (unpublished) } \\
\text { (unpublished) } \\
\text { (unpublished) }\end{array}$ \\
\hline $\begin{array}{l}\text { s } 1 d 28-14-14 \mathrm{cc} \\
\text { s } 1 \mathrm{~d} 28-18-12 \mathrm{ba} \\
\text { s } 1 \mathrm{~d} 28-20-23 \mathrm{dd} \\
\text { s } 1 \mathrm{~d} 28-21-22 \mathrm{ca} \\
\text { s } 1 \mathrm{~d} 28-21-31 \mathrm{bd}\end{array}$ & $\begin{array}{l}6,798 \\
5,652 \\
4,680 \\
7,726 \\
3,589\end{array}$ & $\begin{array}{l}6,974 \\
5,825 \\
4,712 \\
7,786 \\
3,714\end{array}$ & $\begin{array}{r}18.00 \\
20.00 \\
23.00 \\
51.00 \\
4.40\end{array}$ & $\begin{array}{l}\text { Petroleum } \\
\text { Petroleum } \\
\text { Petroleum } \\
\text { Petroleum } \\
\text { Petroleum }\end{array}$ & $\begin{array}{l}\text { Information } \\
\text { Information } \\
\text { Information } \\
\text { Information } \\
\text { Information }\end{array}$ & $\begin{array}{l}\text { Corp. } \\
\text { Corp. } \\
\text { Corp. } \\
\text { Corp. } \\
\text { Corp. }\end{array}$ & $\begin{array}{l}\text { (unpublished) } \\
\text { (unpublished) } \\
\text { (unpublished) } \\
\text { (unpublished) } \\
\text { (unpublished) }\end{array}$ \\
\hline $\begin{array}{l}\text { sld28-22-34aa } \\
\text { sld 28-23-17cd } \\
\text { sld } 28-25-28 \mathrm{ca} \\
\text { s } 1 d 29-10-08 \mathrm{cc} \\
\text { sld29-13-01aab }\end{array}$ & $\begin{array}{r}8,125 \\
8,370 \\
12,475 \\
7,920 \\
6,230\end{array}$ & $\begin{array}{r}8,227 \\
8,450 \\
12,572 \\
7,953 \\
6,404\end{array}$ & $\begin{array}{l}15.00 \\
57.00 \\
16.00 \\
23.00 \\
48.00\end{array}$ & $\begin{array}{l}\text { Petroleum } \\
\text { Petroleum } \\
\text { Petroleum } \\
\text { Petroleum } \\
\text { Petroleum }\end{array}$ & $\begin{array}{l}\text { Information } \\
\text { Information } \\
\text { Information } \\
\text { Information } \\
\text { Information }\end{array}$ & $\begin{array}{l}\text { Corp. } \\
\text { Corp. } \\
\text { Corp. } \\
\text { Corp. } \\
\text { Corp. }\end{array}$ & $\begin{array}{l}\text { (unpublished) } \\
\text { (unpublished) } \\
\text { (unpublished) } \\
\text { (unpublished) } \\
\text { (unpublished) }\end{array}$ \\
\hline $\begin{array}{l}\text { sld } 29-15-20 \mathrm{ad} \\
\text { sld29-16-08dc } \\
\text { sld29-20-04cba } \\
\text { sld29-21-18cb } \\
\text { sld29-24-28ac }\end{array}$ & $\begin{array}{l}6,685 \\
6,634 \\
4,334 \\
6,420 \\
9,876\end{array}$ & $\begin{array}{l}6,846 \\
6,675 \\
4,344 \\
6,540 \\
9,951\end{array}$ & $\begin{array}{r}7.90 \\
41.00 \\
9.30 \\
53.00 \\
68.00\end{array}$ & $\begin{array}{l}\text { Petroleum } \\
\text { Petroleum } \\
\text { Petroleum } \\
\text { Petroleum } \\
\text { Petroleum }\end{array}$ & $\begin{array}{l}\text { Information } \\
\text { Information } \\
\text { Information } \\
\text { Information } \\
\text { Information }\end{array}$ & $\begin{array}{l}\text { Corp. } \\
\text { Corp. } \\
\text { Corp. } \\
\text { Corp. } \\
\text { Corp. }\end{array}$ & $\begin{array}{l}\text { (unpublished) } \\
\text { (unpublished) } \\
\text { (unpublished) } \\
\text { (unpublished) } \\
\text { (unpublished) }\end{array}$ \\
\hline $\begin{array}{l}\text { sld29-26-05dd } \\
\text { sid29.5-20-35ada } \\
\text { sld30-12-24adc } \\
\text { sld30-13-04dc } \\
\text { sld30-16-27bd }\end{array}$ & $\begin{array}{r}11,406 \\
5,955 \\
6,088 \\
6,043 \\
5,764\end{array}$ & $\begin{array}{r}11,557 \\
5,974 \\
6,110 \\
6,129 \\
5,916\end{array}$ & $\begin{array}{r}5.90 \\
33.00 \\
20.00 \\
31.00 \\
16.00\end{array}$ & $\begin{array}{l}\text { Petroleum } \\
\text { Petroleum } \\
\text { Petroleum } \\
\text { Petroleum } \\
\text { Petroleum }\end{array}$ & $\begin{array}{l}\text { Information } \\
\text { Information } \\
\text { Information } \\
\text { Information } \\
\text { Information }\end{array}$ & $\begin{array}{l}\text { Corp. } \\
\text { Corp. } \\
\text { Corp. } \\
\text { Corp. } \\
\text { Corp. }\end{array}$ & $\begin{array}{l}\text { (unpublished) } \\
\text { (unpublished) } \\
\text { (unpublished) } \\
\text { (unpublished) } \\
\text { (unpublished) }\end{array}$ \\
\hline $\begin{array}{l}\text { s } 1 d 30-20-19 d c \\
\text { s } 1 d 30-23-13 d a \\
\text { s } 1 d 30-24-30 d b \\
\text { s } 1 d 30-25-24 b a \\
\text { s } 1 d 30-25-28 b c\end{array}$ & $\begin{array}{r}4,428 \\
8,545 \\
8,177 \\
10,080 \\
9,680\end{array}$ & $\begin{array}{r}4,528 \\
8,650 \\
8,289 \\
10,244 \\
9,712\end{array}$ & $\begin{array}{r}5.60 \\
42.00 \\
6.40 \\
23.00 \\
44.00\end{array}$ & $\begin{array}{l}\text { Petroleum } \\
\text { Petroleum } \\
\text { Petroleum } \\
\text { Petroleum } \\
\text { Petroleum }\end{array}$ & $\begin{array}{l}\text { Information } \\
\text { Information } \\
\text { Information } \\
\text { Information } \\
\text { Information }\end{array}$ & $\begin{array}{l}\text { Corp. } \\
\text { Corp. } \\
\text { Corp. } \\
\text { Corp. } \\
\text { Corp. }\end{array}$ & $\begin{array}{l}\text { (unpublished) } \\
\text { (unpublished) } \\
\text { (unpublished) } \\
\text { (unpublished) } \\
\text { (unpublished) }\end{array}$ \\
\hline $\begin{array}{l}\text { s } 1 d 30-26-22 \mathrm{cb} \\
\text { s } 1 \mathrm{~d} 31-07-36 \mathrm{da} \\
\text { s } 1 \mathrm{~d} 31-12-24 \mathrm{da} \\
\text { s } 1 \mathrm{~d} 31-22-22 \mathrm{cbb} \\
\text { s } 1 \mathrm{~d} 31-23-26 \mathrm{bd}\end{array}$ & $\begin{array}{r}10,722 \\
6,518 \\
6,070 \\
7,480 \\
8,361\end{array}$ & $\begin{array}{r}10,854 \\
6,648 \\
6,150 \\
7,564 \\
8,441\end{array}$ & $\begin{array}{r}77.00 \\
36.00 \\
18.00 \\
132.00 \\
11.00\end{array}$ & $\begin{array}{l}\text { Petroleum } \\
\text { Petroleum } \\
\text { Petroleum } \\
\text { Petroleum } \\
\text { Petroleum }\end{array}$ & $\begin{array}{l}\text { Information } \\
\text { Information } \\
\text { Information } \\
\text { Information } \\
\text { Information }\end{array}$ & $\begin{array}{l}\text { Corp. } \\
\text { Corp. } \\
\text { Corp. } \\
\text { Corp. } \\
\text { Corp. }\end{array}$ & $\begin{array}{l}\text { (unpublished) } \\
\text { (unpublished) } \\
\text { (unpublished) } \\
\text { (unpublished) } \\
\text { (unpublished) }\end{array}$ \\
\hline $\begin{array}{l}\text { s } 1 d 31-25-21 c c \\
\text { s } 1 d 35-05-18 c a \\
\text { s } 1 d 35-20-18 d c \\
\text { sId36-01-12bc } \\
\text { sId37-15-33ba }\end{array}$ & $\begin{array}{l}9,700 \\
4,372 \\
4,247 \\
8,848 \\
4,184\end{array}$ & $\begin{array}{l}9,865 \\
4,400 \\
4,347 \\
8,971 \\
4,310\end{array}$ & $\begin{array}{r}20.00 \\
24.00 \\
6.10 \\
50.00 \\
29.00\end{array}$ & $\begin{array}{l}\text { Petroleum } \\
\text { Petroleum } \\
\text { Petroleum } \\
\text { Petroleum } \\
\text { Petroleum }\end{array}$ & $\begin{array}{l}\text { Information } \\
\text { Information } \\
\text { Information } \\
\text { Information } \\
\text { Information }\end{array}$ & $\begin{array}{l}\text { Corp. } \\
\text { Corp. } \\
\text { Corp. } \\
\text { Corp. } \\
\text { Corp. }\end{array}$ & $\begin{array}{l}\text { (unpublished) } \\
\text { (unpublished) } \\
\text { (unpublished) } \\
\text { (unpublished) } \\
\text { (unpublished) }\end{array}$ \\
\hline $\begin{array}{l}\text { s } 1 d 37-20-32 \\
\text { s } 1 d 37-21-29 b a c \\
\text { s } 1 d 37-22-17 c d d \\
\text { s } 1 \text { d } 37-24-20 a b \\
\text { s } 1 d 38-20-26 b c a\end{array}$ & $\begin{array}{l}4,350 \\
5,903 \\
7,712 \\
8,210 \\
2,452\end{array}$ & $\begin{array}{l}4,400 \\
5,995 \\
7,728 \\
8,259 \\
2,464\end{array}$ & $\begin{array}{r}8.50 \\
14.00 \\
2.60 \\
6.70 \\
2.30\end{array}$ & $\begin{array}{l}\text { Petroleum } \\
\text { Petroleum } \\
\text { Petroleum } \\
\text { Petroleum } \\
\text { Petroleum }\end{array}$ & $\begin{array}{l}\text { Information } \\
\text { Information } \\
\text { Information } \\
\text { Information } \\
\text { Information }\end{array}$ & $\begin{array}{l}\text { Corp. } \\
\text { Corp. } \\
\text { Corp. } \\
\text { Corp. } \\
\text { Corp. }\end{array}$ & $\begin{array}{l}\text { (unpublished) } \\
\text { (unpublished) } \\
\text { (unpublished) } \\
\text { (unpublished) } \\
\text { (unpublished) }\end{array}$ \\
\hline $\begin{array}{l}\text { sld39-22-29cb } \\
\text { s } 1 d 39-23-32 a b b \\
\text { sld40-07-02bd } \\
\text { sld40-10-03cd } \\
\text { sld40-21-33da }\end{array}$ & $\begin{array}{l}7,571 \\
7,597 \\
6,142 \\
4,412 \\
6,065\end{array}$ & $\begin{array}{l}7,614 \\
7,681 \\
7,155 \\
4,466 \\
6,104\end{array}$ & $\begin{array}{r}12.00 \\
22.00 \\
14.00 \\
21.00 \\
3.30\end{array}$ & $\begin{array}{l}\text { Petroleum } \\
\text { Petroleum } \\
\text { Petroleum } \\
\text { Petroleum } \\
\text { Petroleum }\end{array}$ & $\begin{array}{l}\text { Information } \\
\text { Information } \\
\text { Information } \\
\text { Information } \\
\text { Information }\end{array}$ & $\begin{array}{l}\text { Corp. } \\
\text { Corp. } \\
\text { Corp. } \\
\text { Corp. } \\
\text { Corp. }\end{array}$ & $\begin{array}{l}\text { (unpublished) } \\
\text { (unpublished) } \\
\text { (unpublished) } \\
\text { (unpublished) } \\
\text { (unpublished) }\end{array}$ \\
\hline $\begin{array}{l}\text { s1d40-25-19caa } \\
\text { sld41-18-01aa } \\
\text { s } 1 d 41-20-36 \mathrm{bb} \\
\text { sld41-21-35dc } \\
\text { s1d41-23-35dd }\end{array}$ & $\begin{array}{l}7,347 \\
2,300 \\
6,330 \\
6,265 \\
6,400\end{array}$ & $\begin{array}{l}7,378 \\
2,380 \\
6,405 \\
6,345 \\
6,506\end{array}$ & $\begin{array}{r}13.00 \\
8.60 \\
6.80 \\
11.00 \\
21.00\end{array}$ & $\begin{array}{l}\text { Petroleum } \\
\text { Petroleum } \\
\text { Petroleum } \\
\text { Petroleum } \\
\text { Petroleum }\end{array}$ & $\begin{array}{l}\text { Information } \\
\text { Information } \\
\text { Information } \\
\text { Information } \\
\text { Information }\end{array}$ & $\begin{array}{l}\text { Corp. } \\
\text { Corp. } \\
\text { Corp. } \\
\text { Corp. } \\
\text { Corp. }\end{array}$ & $\begin{array}{l}\text { (unpublished) } \\
\text { (unpublished) } \\
\text { (unpublished) } \\
\text { (unpublished) } \\
\text { (unpublished) }\end{array}$ \\
\hline
\end{tabular}

337RDLL--Continued 
Table 3.--Artesian yields from wells and springs--Continued

\begin{tabular}{|c|c|c|c|c|}
\hline & $\begin{array}{l}\text { Depth } \\
\text { to top } \\
\text { (feet) }\end{array}$ & $\begin{array}{l}\text { Depth to } \\
\text { bottom } \\
\text { (feet) }\end{array}$ & $\begin{array}{l}\text { Yield } \\
\text { (gallons per } \\
\text { minute) }\end{array}$ & Remarks \\
\hline
\end{tabular}

$\begin{array}{lllr}\text { s } 1 d 42-23-02 c b & 5,855 & 5,890 & 20.00 \\ \text { s } 1 d 42-24-01 \mathrm{aa} & 6,698 & 6,750 & 10.00 \\ \text { sld43-02-19bc } & 6,390 & 6,505 & 17.00 \\ \text { sld43-21-19ba } & 6,050 & 6,130 & 25.00 \\ \text { s } 1 d 43-24-06 \mathrm{bb} & 6,805 & 6,925 & 34.00 \\ & & & \\ \text { s } 1 d 43-24-13 \mathrm{aa} & 6,490 & 6,530 & 4.00 \\ \text { s } 1 d 43-25-33 \mathrm{ba} & 6,680 & 6,800 & 8.30 \\ \text { s } 1 d 43-26-31 \mathrm{bb} & 6,775 & 6,947 & 14.00\end{array}$

\section{RDLL--Continued}

20.00

10.00

17.00

5.00

4.00

14.00 sb33-117-24b

sb38-115-03bca

sb38-115-03bcd

ga $35-28-25 d d d$

ga $37-29-35 b b$

ga38-27-20dd

ga39-24-07dd

ga40-24-08ca

ga $40-28-18 b b$

ga41-25-20aa

ga41-28-09ac

ga41-29-06dd

ga41-29-06dd

nb06-06-20bba

nb06-07-32aa

nb06-10-14bc

nb07-09-14dd

nmb26-18-28ad

nmb 27-17-20ba

nmb40-18-13db

nmb44-18-16bca

s ld25-14-22cd

sld27-15-32ad

s1d28-18-12ba

s 1 d 28-20-23dd

sld28-21-31bd

s 1 d $30-24-30 d b$

s1d31-25-21cc

sld36-10-18-aa

s 1d38-20-26bca

sld39-24-04aa a

s 1d41-23-35dd

sld43-22-21abd

nmb45-08-15a a

nmb45-12-02bd

sld39-23-32cd

sld42-22-33ac

sld43-22-22ba

$\begin{array}{rrr}2,748 & 2,810 & 11.00 \\ 3,415 & 3,483 & 4.80 \\ 5,590 & 5,690 & 7.30 \\ 6,040 & 6,133 & 9.60 \\ 6,322 & 6,349 & 3.10 \\ & & \\ 6,735 & 6,900 & 13.00 \\ 6,500 & 6,535 & 7.20 \\ 6,500 & 6,520 & 30.00 \\ 6,014 & 6,080 & 12.00 \\ 6,136 & 6,246 & 4.60 \\ & & \\ 2,644 & 2,674 & 18.00 \\ 2,441 & 2,516 & 3.50 \\ 2,813 & 2,959 & 7.20 \\ 2,434 & 2,494 & 5.60 \\ 6,795 & 6,873 & 12.00 \\ & & \\ 7,750 & 7,850 & 1.50 \\ 8,806 & 8,901 & 8.50 \\ 8,401 & 8,570 & 3.70 \\ 5,340 & 5,481 & 44.00 \\ 6,510 & 6,613 & 42.00 \\ & & \\ 6,391 & 6,509 & 8.00 \\ 5,450 & 5,613 & 18.00 \\ 4,150 & 4,193 & 12.00 \\ 8,789 & 8,957 & 4.50 \\ 10,280 & 10,450 & 18.00 \\ & & \\ 8,279 & 8,314 & 58.00 \\ 3,020 & 3,029 & 0.05 \\ 8,295 & 8,406 & 10.00 \\ 6,889 & 7,020 & 8.00 \\ 5,877 & 5,896 & 5.60\end{array}$

40.00

900.00

$1,100.00$

tines and Glass (1975)

nes and Glass (1975)

Lines and Glass (1975)

341ELBR

Petroleum Information Corp. (unpublished) Petroleum Information Corp. (unpublished) Petroleum Information Corp. (unpublished) Petroleum Information Corp. (unpublished) Petroleum Information Corp. (unpublished)

Petroleum Information Corp. (unpublished) Petroleum Information Corp. (unpublished) Petroleum Information Corp. (unpublished) Petroleum Information Corp. (unpublished) Petroleum Information Corp. (unpublished)

Petroleum Information Corp. (unpublished) Petroleum Information Corp. (unpublished) Petroleum Information Corp. (unpublished) Petroleum Information Corp. (unpublished) Petroleum Information Corp. (unpublished)

Petroleum Information Corp. (unpublished) Petroleum Information Corp. (unpublished) Petroleum Information Corp. (unpublished) Petroleum Information Corp. (unpublished) Petroleum Information Corp. (unpublished)

Petroleum Information Corp. (unpublished) Petroleum Information Corp. (unpublished) Petroleum Information Corp. (unpublished) Petroleum Information Corp. (unpublished) Petroleum Information Corp. (unpublished)

Petroleum Information Corp. (unpublished) Petroleum Information Corp. (unpublished) Petroleum Information Corp. (unpublished) Petroleum Information Corp. (unpublished) Petroleum Information Corp. (unpublished)

\section{URY}

4,345

3,140

4,356

12.00

21.00

6.20

$\begin{array}{rrr}5,807 & 5,930 & 5.00 \\ 5,855 & 5,978 & 32.00\end{array}$
Petroleum Information Corp. (unpublished) Petroleum Information Corp. (unpublished) Petroleum Information Corp. (unpublished) Petroleum Information Corp. (unpublished) Petroleum Information Corp. (unpublished) 
Table 3.--Artesian yields from wells and springs--Continued

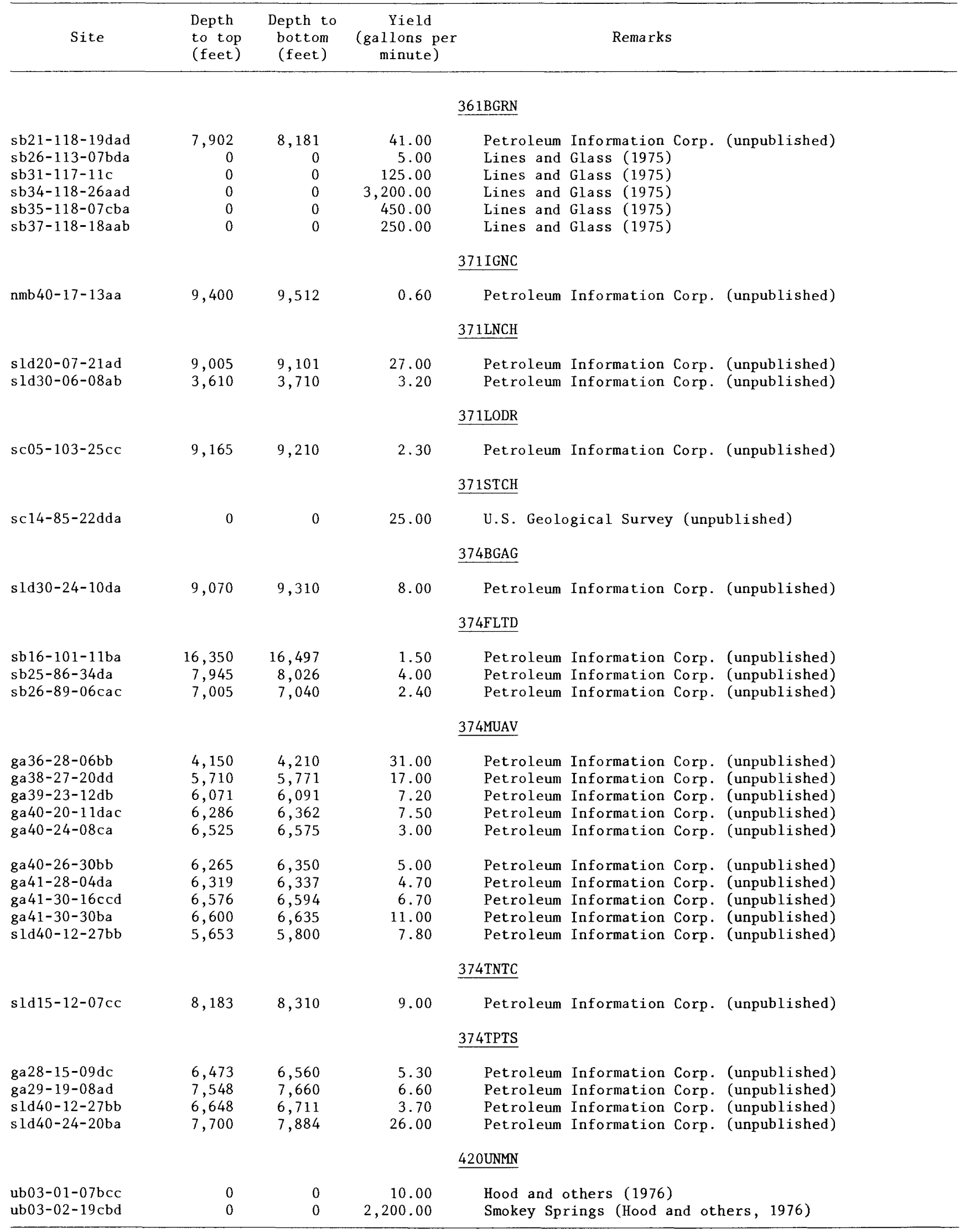


Table 4.--Laboratory-determined porosity and permeability

1. Depths to the top and bottom of intervals are measured from the land surface, drilling platform, or kelly bushing. The measuring point was unspecified or was not recorded in compiling the information in this table. An error of no more than 20 feet is introduced by assuming the measuring point was the land surface. Depths of less than 5 feet in either depth column at 6 sites indicate an outcrop sample. All other values in this table were determined for core samples.

2. Outcrop-sample data were compiled from reports by Hood (1976) and Hood and Patterson (1984) and from unpublished files of the U.S. Geological Survey. Core-sample data were compiled from unpublished files of Petroleum Information Corp. (Denver, Colo.).

3. Formation codes are defined in table 1 .

4. For each site, porosity and permeability values are averaged by formation ("AVERAGE") and by site ("SITE AVERAGE").

5. Porosity and permeability values listed in this table were determined by laboratory analyses. Thus, the permeability values represent intergranular permeability only. They generally are different from values of permeability determined by drill-stem tests, which take into account large secondary openings in addition to pore openings. Thus, the values of permeability in this table do not agree with in-situ values of permeability listed in table 5 for the same sites.

6. Values are reported to three significant figures (trailing zeros are not significant). Values of permeability listed as 0.000000 indicate a permeability of less than the lower limit of detection, which in different analyses was either 0.01 or, less commonly, 0.1 millidarcies.

7. Lithologic descriptions in this table are taken from geologic logs and outcrop descriptions. They generally should be considered nonquantitative.

8. To comply with format restrictions, the term $w /$ is used in places to mean "with." 
Table 4.--Laboratory-determined porosity and permeability

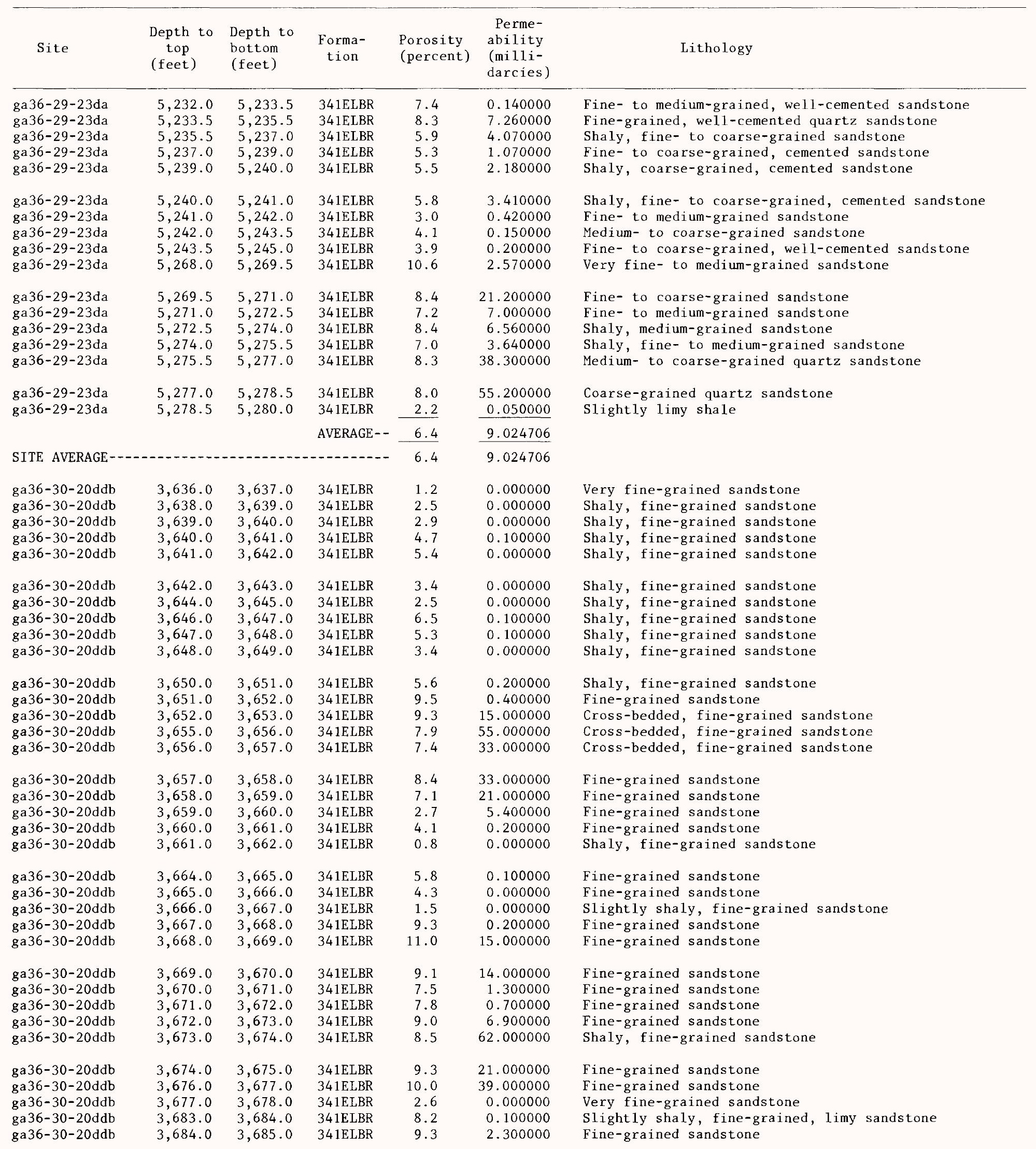


Table 4.--Laboratory-determined porosity and permeability--Continued

\begin{tabular}{|c|c|c|c|c|c|c|}
\hline Site & $\begin{array}{l}\text { Depth to } \\
\text { top } \\
\text { (feet) }\end{array}$ & $\begin{array}{l}\text { Depth to } \\
\text { bottom } \\
\text { (feet) }\end{array}$ & $\begin{array}{c}\text { Forma- } \\
\text { tion }\end{array}$ & $\begin{array}{l}\text { Porosity } \\
\text { (percent) }\end{array}$ & $\begin{array}{l}\text { Perme- } \\
\text { ability } \\
\text { (milli- } \\
\text { darcies) }\end{array}$ & Lithology \\
\hline ga $36-30-20 d d b$ & $3,685.0$ & $3,686.0$ & $341 E L B R$ & 10.5 & 8.600000 & Fine-grained sandstone \\
\hline ga $36-30-20 d d b$ & $3,686.0$ & $3,687.0$ & $341 \mathrm{ELBR}$ & 6.1 & 4.800000 & Fine-grained sandstone \\
\hline ga $36-30-20 d d b$ & $3,687.0$ & $3,688.0$ & $341 \mathrm{ELBR}$ & 8.6 & 1.500000 & Fine-grained sandstone \\
\hline ga $36-30-20 d d b$ & $3,688.0$ & $3,689.0$ & 341ELBR & 8.7 & 36.000000 & Fine-grained sandstone \\
\hline ga $36-30-20 d d b$ & $3,689.0$ & $3,690.0$ & 341ELBR & 8.1 & 17.000000 & Slightly shaly, fine-grained sandstone \\
\hline ga $36-30-20 d d b$ & $3,690.0$ & $3,691.0$ & $341 E L B R$ & 6.1 & 1.000000 & Fine-grained sandstone \\
\hline ga $36-30-20 d d b$ & $3,691.0$ & $3,692.0$ & $341 E L B R$ & 3.1 & 0.000000 & Very fine-grained sandstone \\
\hline ga $36-30-20 d d b$ & $3,692.0$ & $3,693.0$ & 341 ELBR & 3.4 & 0.000000 & Very fine-grained sandstone \\
\hline ga $36-30-20 d d b$ & $3,693.0$ & $3,694.0$ & $341 \mathrm{ELBR}$ & 5.1 & 0.100000 & Fine-grained sandstone \\
\hline ga $36-30-20 d d b$ & $3,696.0$ & $3,697.0$ & $341 \mathrm{ELBR}$ & 6.1 & 0.200000 & Fine-grained dolomitic sandstone \\
\hline \multirow[t]{2}{*}{ ga $36-30-20 d d b$} & $3,697.0$ & $3,698.0$ & 341ELBR & 6.4 & 0.100000 & Fine-grained dolomitic sandstone \\
\hline & & & AVERAGE - - & 6.3 & 10.763265 & \\
\hline ga $36-30-20 d d b$ & $3,727.5$ & $3,728.0$ & 374MUAV & 2.0 & 0.000000 & Slightly shaly, fine-grained dolomite \\
\hline ga $36-30-20 \mathrm{ddb}$ & $3,728.0$ & $3,729.0$ & 374MUAV & 8.1 & 1.300000 & Slightly shaly, fine-grained dolomite \\
\hline ga $36-30-20 \mathrm{ddb}$ & $3,729.0$ & $3,730.5$ & 374MUAV & 1.1 & 0.000000 & Fractured, slightly shaly, fine-grained dolomite \\
\hline ga $36-30-20 \mathrm{ddb}$ & $3,777.0$ & $3,778.0$ & 374MUAV & 0.8 & 0.000000 & Fractured, slightly shaly, fine-grained dolomite \\
\hline ga $36-30-20 d d b$ & $3,781.0$ & $3,782.0$ & 374MUAV & 2.2 & 0.000000 & Fractured, slightly shaly, fine-grained dolomite \\
\hline ga $36-30-20 d d b$ & $3,782.0$ & $3,783.0$ & 374MUAV & 1.3 & 0.000000 & Fractured, slightly shaly, fine-grained dolomite \\
\hline ga $36-30-20 d d b$ & $3,783.0$ & $3,784.0$ & 374MUAV & 1.4 & 0.000000 & Fractured, slightly shaly, fine-grained dolomite \\
\hline ga $36-30-20 d d b$ & $3,785.0$ & $3,786.0$ & 374MUAV & 1.3 & 0.000000 & Fractured, shaly,fine-grained dolomite \\
\hline ga $36-30-20 d d b$ & $3,806.0$ & $3,807.0$ & 374MUAV & 4.0 & 0.100000 & Shaly, carbonaceous, fine-grained dolomite \\
\hline$g a 36-30-20 d d b$ & $3,807.0$ & $3,808.0$ & 374MUAV & 2.0 & 0.000000 & Slightly shaly, fine-grained dolomite \\
\hline ga $36-30-20 d d b$ & $3,808.0$ & $3,809.0$ & 374MUAV & 2.4 & 0.000000 & Slightly shaly, fine-grained dolomite \\
\hline ga36-30-20ddb & $3,809.0$ & $3,810.0$ & 374MUAV & 3.4 & 0.000000 & Slightly shaly, fine-grained dolomite \\
\hline ga36-30-20ddb & $3,810.0$ & $3,811.0$ & 374MUAV & 7.0 & 0.100000 & Shaly, medium-grained dolomite \\
\hline ga $36-30-20 d d b$ & $3,811.0$ & $3,812.0$ & 374MUAV & 11.7 & 6.300000 & Shaly, medium-grained dolomite \\
\hline ga $36-30-20 d d b$ & $3,812.0$ & $3,813.0$ & 374MUAV & 12.6 & 157.000000 & Shaly, slightly vuggy, medium-grained dolomite \\
\hline \multirow[t]{2}{*}{ ga $36-30-20 d d b$} & $3,813.0$ & $3,814.0$ & 374MUAV & 10.6 & 1.000000 & Shaly, medium-grained dolomite \\
\hline & & & AVERAGE-- & 4.5 & 10.362500 & \\
\hline \multicolumn{4}{|c|}{ SITE AVERAGE- } & 5.9 & 10.664615 & \\
\hline ga36-30-30ada & $3,068.0$ & $3,069.0$ & $324 \mathrm{HRMS}$ & 9.5 & 0.000000 & Baked, shaly, very fine-grained limestone \\
\hline ga $36-30-30$ ada & $3,069.0$ & $3,070.0$ & $324 \mathrm{HRMS}$ & 4.2 & 0.010000 & Shaly, very fine-grained limestone \\
\hline ga $36-30-30$ ada & $3,070.0$ & $3,071.0$ & $324 \mathrm{HRMS}$ & 1.4 & 0.000000 & Shaly, very fine-grained limestone \\
\hline ga $36-30-30 a d a$ & $3,071.0$ & $3,072.0$ & 324 HRMS & 1.4 & 0.000000 & Shaly, very fine-grained limestone \\
\hline ga $36-30-30$ ada & $3,072.0$ & $3,073.0$ & 324HRMS & 1.7 & 0.000000 & Very fine-grained limestone \\
\hline ga $36-30-30 a d a$ & $3,073.0$ & $3,074.0$ & 324HRMS & 1.1 & 0.000000 & Very fine-grained limestone \\
\hline ga $36-30-30$ ada & $3,074.0$ & $3,075.0$ & $324 \mathrm{HRMS}$ & 1.0 & 0.010000 & Very fine-grained limestone \\
\hline ga $36-30-30$ ada & $3,075.0$ & $3,076.0$ & $324 \mathrm{HRMS}$ & 1.6 & 0.050000 & Very fine-grained limestone \\
\hline ga $36-30-30$ ada & $3,076.0$ & $3,077.0$ & 324HRMS & 2.2 & 0.000000 & Very fine-grained limestone \\
\hline ga $36-30-30$ ada & $3,077.0$ & $3,078.0$ & 324HRMS & 2.3 & 0.000000 & Very fine-grained limestone \\
\hline \multirow{2}{*}{\multicolumn{3}{|c|}{ SITE AVERAGE--. }} & AVERAGE-- & 2.6 & 0.007000 & \\
\hline & & & $-\cdots-n-\cdots$ & 2.6 & 0.007000 & \\
\hline ga $37-28-32 a b c$ & $3,655.0$ & $3,656.0$ & $324 \mathrm{PRDX}$ & 1.6 & 0.300000 & Shaly, fine-grained limestone \\
\hline ga $37-28-32 a b c$ & $3,656.0$ & $3,657.0$ & $324 \mathrm{PRDX}$ & 4.3 & 0.000000 & Fine-grained limestone \\
\hline ga $37-28-32 a b c$ & $3,657.0$ & $3,658.0$ & 324PRDX & 4.2 & 0.000000 & Fine-grained limestone \\
\hline ga $37-28-32 a b c$ & $3,659.0$ & $3,660.0$ & $324 \mathrm{PRDX}$ & 1.6 & 11.000000 & Shaly, fine-grained limestone \\
\hline ga $37-28-32 a b c$ & $3,661.0$ & $3,662.0$ & 324PRDX & 3.1 & 0.000000 & Shaly, fine-grained limestone \\
\hline
\end{tabular}


Table 4.--Laboratory-determined porosity and permeability--Continued

\begin{tabular}{|c|c|c|c|c|c|c|}
\hline Site & $\begin{array}{l}\text { Depth to } \\
\text { top } \\
\text { (feet) }\end{array}$ & $\begin{array}{l}\text { Depth to } \\
\text { bottom } \\
\text { (feet) }\end{array}$ & $\begin{array}{c}\text { Forma- } \\
\text { tion }\end{array}$ & $\begin{array}{l}\text { Porosity } \\
\text { (percent) }\end{array}$ & $\begin{array}{l}\text { Perme- } \\
\text { ability } \\
\text { (milli- } \\
\text { darcies) }\end{array}$ & Lithology \\
\hline ga $37-28-32 a b c$ & $3,663.0$ & $3,664.0$ & 324PRDX & 1.2 & 1.600000 & Shaly, fine-grained limestone \\
\hline ga $37-28-32 a b c$ & $3,665.0$ & $3,666.0$ & $324 \mathrm{PRDX}$ & 1.4 & 0.000000 & Shaly, fine-grained limestone \\
\hline ga $37-28-32 a b c$ & $3,667.0$ & $3,668.0$ & 324PRDX & 8.4 & 1.400000 & Vuggy, fine-grained limestone \\
\hline ga $37-28-32 a b c$ & $3,668.0$ & $3,669.0$ & 324PRDX & 7.6 & 19.000000 & Vuggy, fine-grained limestone \\
\hline ga $37-28-32 a b c$ & $3,669.0$ & $3,670.0$ & $324 \mathrm{PRDX}$ & 7.2 & 54.000000 & Vuggy, fine-grained limestone \\
\hline \multirow[t]{2}{*}{ ga $37-28-32 a b c$} & \multirow[t]{2}{*}{$3,670.0$} & \multirow[t]{2}{*}{$3,671.0$} & $324 \mathrm{PRDX}$ & $\underline{10.9}$ & 3.200000 & \multirow[t]{3}{*}{ Vuggy, fine-grained limestone } \\
\hline & & & AVERAGE-- & 4.7 & 8.227273 & \\
\hline \multicolumn{4}{|c|}{ SITE AVERAGE-1. } & 4.7 & 8.227273 & \\
\hline$g a 37-29-22 b b b$ & $3,559.0$ & $3,560.0$ & 324PRDX & 10.1 & 5.100000 & \multirow{5}{*}{$\begin{array}{l}\text { Shaly, very fine-grained limestone } \\
\text { Very fine-grained limestone } \\
\text { Shaly, very fine-grained limestone } \\
\text { Shaly, very fine-grained limestone } \\
\text { Very fine-grained dolomitic limestone }\end{array}$} \\
\hline ga $37-29-22 b b b$ & $3,560.0$ & $3,561.0$ & 324PRDX & 12.3 & 7.600000 & \\
\hline ga $37-29-22 b b b$ & $3,561.0$ & $3,562.0$ & 324PRDX & 6.9 & 1.400000 & \\
\hline ga37-29-22bbb & $3,562.0$ & $3,563.0$ & 324PRDX & 15.8 & 1.000000 & \\
\hline ga $37-29-22 b b b$ & $3,563.0$ & $3,564.0$ & 324PRDX & 13.9 & 0.200000 & \\
\hline ga37-29-22bbb & $3,564.0$ & $3,565.0$ & 324PRDX & 27.3 & 2.000000 & Very fine-grained dolomitic limestone \\
\hline ga37-29-22bbb & $3,565.0$ & $3,566.0$ & $324 \mathrm{PRDX}$ & 13.7 & 9.600000 & Very fine-grained, slightly limy dolomite \\
\hline ga $37-29-22 b b b$ & $3,566.0$ & $3,567.0$ & 324PRDX & 7.8 & 1.000000 & Very fine-grained, slightly limy dolomite \\
\hline ga $37-29-22 b b b$ & $3,567.0$ & $3,568.0$ & 324PRDX & 4.8 & 0.500000 & Very fine-grained dolomitic limestone \\
\hline ga $37-29-22 b b b$ & $3,568.0$ & $3,569.0$ & 324PRDX & 5.8 & 0.600000 & Very fine-grained dolomitic limestone \\
\hline ga $37-29-22 b b b$ & $3,569.0$ & $3,570.0$ & 324PRDX & 7.0 & 0.300000 & \multirow{4}{*}{$\begin{array}{l}\text { Very fine-grained dolomitic limestone } \\
\text { Very fine-grained dolomitic limestone }\end{array}$} \\
\hline \multirow[t]{2}{*}{ ga $37-29-22 b b b$} & $3,570.0$ & $3,571.0$ & 324PRDX & 6.4 & 0.000000 & \\
\hline & & & AVERAGE-- & 11.0 & 2.441667 & \\
\hline \multicolumn{3}{|l|}{ SITE AVERAGE-- } & -------- & $\overline{11.0}$ & 2.441667 & \\
\hline ga $37-29-35 b b c$ & $3,442.0$ & $3,443.0$ & 341ELBR & 9.8 & 18.000000 & \multirow{5}{*}{$\begin{array}{l}\text { Fine-grained dolomitic sandstone } \\
\text { Fine-grained dolomitic sandstone } \\
\text { Fine-grained dolomitic sandstone } \\
\text { Fine-grained dolomitic sandstone } \\
\text { Fine dolomitic sandstone }\end{array}$} \\
\hline ga37 & $3,443.0$ & $3,444.0$ & 341ELBR & 9.2 & 12.000000 & \\
\hline ga $37-29-35 b b c$ & $3,444.0$ & $3,445.0$ & $341 E L B R$ & 10.1 & 21.000000 & \\
\hline ga $37-29-35 b b c$ & $3,445.0$ & $3,446.0$ & $341 \mathrm{ELBR}$ & 9.6 & 22.000000 & \\
\hline ga $37-29-35 b b c$ & $3,446.0$ & $3,447.0$ & 341ELBR & 7.8 & 3.000000 & \\
\hline ga $37-29-35 b b c$ & $3,447.0$ & $3,448.0$ & 341ELBR & 10.4 & 10.000000 & \multirow{5}{*}{$\begin{array}{l}\text { Fine-grained dolomitic sandstone } \\
\text { Fine-grained dolomitic sandstone } \\
\text { Fine-grained dolomitic sandstone } \\
\text { Fine-grained dolomitic sandstone } \\
\text { Fine-grained dolomitic sandstone }\end{array}$} \\
\hline ga $37-29-35 b b c$ & $3,448.0$ & $3,449.0$ & $341 E L B R$ & 8.3 & 18.000000 & \\
\hline ga $37-29-35 b b c$ & $3,449.0$ & $3,450.0$ & 341ELBR & 11.0 & 24.000000 & \\
\hline ga $37-29-35 b b c$ & $3,450.0$ & $3,451.0$ & $341 \mathrm{ELBR}$ & 6.5 & 1.500000 & \\
\hline ga $37-29-35 b b c$ & $3,451.0$ & $3,452.0$ & 341 ELBR & 8.1 & 11.000000 & \\
\hline ga $37-29-35 b b c$ & $3,452.0$ & $3,453.0$ & 341ELBR & 7.8 & 1.900000 & \multirow{5}{*}{$\begin{array}{l}\text { Fine-grained dolomitic sandstone } \\
\text { Fine-grained glauconitic sandstone } \\
\text { Fine-grained glauconitic sandstone } \\
\text { Fine-grained glauconitic sandstone } \\
\text { Fine-grained glauconitic sandstone }\end{array}$} \\
\hline ga $37-29-35 b b c$ & $3,456.0$ & $3,457.0$ & 341ELBR & 5.2 & 0.010000 & \\
\hline ga $37-29-35 b b c$ & $3,457.0$ & $3,458.0$ & 341ELBR & 5.7 & 0.010000 & \\
\hline ga $37-29-35 b b c$ & $3,458.0$ & $3,459.0$ & 341ELBR & 5.0 & 0.010000 & \\
\hline ga $37-29-35$ bbc & $3,459.0$ & $3,460.0$ & 341ELBR & 3.3 & 0.020000 & \\
\hline \multirow[t]{2}{*}{ ga $37-29-35 b b c$} & $3,465.0$ & $3,466.0$ & $341 \mathrm{ELBR}$ & 1.0 & 0.010000 & \multirow[t]{2}{*}{ Shaly, fine-grained sandstone } \\
\hline & & & AVERAGE-- & 7.4 & 8.903750 & \\
\hline ga $37-29-35 b b c$ & $3,580.0$ & $3,581.0$ & 374MUAV & 2.3 & 0.300000 & Vuggy, fine-grained dolomite \\
\hline ga $37-29-35 b b c$ & $3,586.0$ & $3,587.0$ & 374MUAV & 3.2 & 0.300000 & Shaly, carbonaceous dolomite \\
\hline ga $37-29-35 b b c$ & $3,587.0$ & $3,588.0$ & 374MUAV & 3.5 & 1.300000 & Vuggy, fine-grained dolomite \\
\hline ga $37-29-35 b b c$ & $3,588.0$ & $3,589.0$ & 374MUAV & 4.1 & 2.000000 & Vuggy, fine-grained dolomite \\
\hline ga $37-29-35 b b c$ & $3,589.0$ & $3,590.0$ & 374MUAV & 3.0 & 1.600000 & Vuggy, fine-grained dolomite \\
\hline ga $37-29-35 b b c$ & $3,590.0$ & $3,591.0$ & 374MUAV & 2.9 & 1.800000 & Vuggy, cherty, fine-grained dolomite \\
\hline ga $37-29-35 b b c$ & $3,592.0$ & $3,593.0$ & 374MUAV & 1.2 & 0.000000 & Shaly, carbonaceous, fine-grained dolomite \\
\hline ga $37-29-35 b b c$ & $3,593.0$ & $3,594.0$ & 374MUAV & 1.3 & 0.000000 & Shaly, carbonaceous, fine-grained dolomite \\
\hline ga $37-29-35 b b c$ & $3,594.0$ & $3,595.0$ & 374MUAV & 1.0 & 0.000000 & Shaly, carbonaceous, fine-grained dolomite \\
\hline ga $37-29-35 b b c$ & $3,595.0$ & $3,596.0$ & 374MUAV & 1.0 & 0.000000 & Carbonaceous, fine-grained dolomite \\
\hline
\end{tabular}


Table 4.--Laboratory-determined porosity and permeability--Continued

\begin{tabular}{|c|c|c|c|c|c|c|}
\hline Site & $\begin{array}{l}\text { Depth to } \\
\text { top } \\
\text { (feet) }\end{array}$ & $\begin{array}{l}\text { Depth to } \\
\text { bottom } \\
\text { (feet) }\end{array}$ & $\begin{array}{c}\text { Forma- } \\
\text { tion }\end{array}$ & $\begin{array}{l}\text { Porosity } \\
\text { (percent) }\end{array}$ & $\begin{array}{l}\text { Perme- } \\
\text { ability } \\
\text { (milli- } \\
\text { darcies) }\end{array}$ & Lithology \\
\hline $\begin{array}{l}\text { ga } 37-29-35 b b c \\
\text { ga } 37-29-35 b b c \\
\text { ga } 37-29-35 b b c \\
\text { ga } 37-29-35 b b c \\
\text { ga } 37-29-35 b b c\end{array}$ & $\begin{array}{l}3,596.0 \\
3,597.0 \\
3,598.0 \\
3,599.0 \\
3,600.0\end{array}$ & $\begin{array}{l}3,597.0 \\
3,598.0 \\
3,599.0 \\
3,600.0 \\
3,601.0\end{array}$ & $\begin{array}{l}\text { 374MUAV } \\
374 M U A V \\
374 M U A V \\
374 M U A V \\
374 M U A V\end{array}$ & $\begin{array}{l}1.7 \\
7.0 \\
6.8 \\
1.4 \\
8.2\end{array}$ & $\begin{array}{l}0.010000 \\
1.800000 \\
0.200000 \\
0.000000 \\
1.500000\end{array}$ & $\begin{array}{l}\text { Vuggy, fine-grained dolomite } \\
\text { Vuggy, fine-grained dolomite } \\
\text { Vuggy, fine-grained dolomite } \\
\text { Fine-grained dolomite } \\
\text { Fine-grained quartz sandstone }\end{array}$ \\
\hline $\begin{array}{l}\text { ga } 37-29-35 b b c \\
\text { ga } 37-29-35 b b c \\
\text { ga } 37-29-35 b b c \\
\text { ga } 37-29-35 b b c \\
\text { ga } 37-29-35 b b c\end{array}$ & $\begin{array}{l}3,601.0 \\
3,602.0 \\
3,603.0 \\
3,604.0 \\
3,606.0\end{array}$ & $\begin{array}{l}3,602.0 \\
3,603.0 \\
3,604.0 \\
3,605.0 \\
3,607.0\end{array}$ & $\begin{array}{l}\text { 374MUAV } \\
374 \mathrm{MUAV} \\
374 \mathrm{MUAV} \\
374 \mathrm{MUAV} \\
\text { 374MUAV }\end{array}$ & $\begin{array}{l}7.7 \\
7.2 \\
8.2 \\
4.9 \\
4.2\end{array}$ & $\begin{array}{l}0.860000 \\
5.500000 \\
2.500000 \\
0.010000 \\
0.020000\end{array}$ & $\begin{array}{l}\text { Fine-grained quartz sandstone } \\
\text { Fine-grained quartz sandstone } \\
\text { Fine-grained quartz sandstone } \\
\text { Slightly shaly, medium-grained sandstone } \\
\text { Medium-grained, slightly arkosic sandstone }\end{array}$ \\
\hline $\begin{array}{l}\text { ga } 37-29-35 b b c \\
\text { ga } 37-29-35 b b c \\
\text { ga37-29-35bbc } \\
\text { ga } 37-29-35 b b c\end{array}$ & $\begin{array}{l}3,608.0 \\
3,610.0 \\
3,612.0 \\
3,619.0\end{array}$ & $\begin{array}{l}3,609.0 \\
3,611.0 \\
3,613.0 \\
3,620.0\end{array}$ & $\begin{array}{l}\text { 374MUAV } \\
\text { 374MUAV } \\
\text { 374MUAV } \\
\text { 374MUAV }\end{array}$ & $\begin{array}{l}4.1 \\
2.9 \\
2.7 \\
2.5\end{array}$ & $\begin{array}{l}0.110000 \\
0.060000 \\
0.010000 \\
0.010000\end{array}$ & $\begin{array}{l}\text { Medium-grained arkosic sandstone } \\
\text { Shaly, gravelly sandstone } \\
\text { Shaly, medium-grained sandstone } \\
\text { Gravelly sandstone }\end{array}$ \\
\hline SITE AVERAGE-- & $8-0-2-0$ & 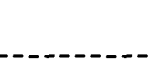 & AVERAGE-- & $\frac{3.9}{5.3}$ & $\frac{0.828750}{4.058750}$ & \\
\hline $\begin{array}{l}\text { ga } 40-28-11 \text { a a } \\
\text { ga } 40-28-11 \text { a a a } \\
\text { ga40-28-11aaa } \\
\text { ga40-28-11aaa } \\
\text { ga40-28-11aaa }\end{array}$ & $\begin{array}{l}5,566.0 \\
5,568.5 \\
5,570.2 \\
5,572.4 \\
5,573.9\end{array}$ & $\begin{array}{l}5,568 \cdot 5 \\
5,570 \cdot 2 \\
5,572 \cdot 4 \\
5,573.9 \\
5,575.4\end{array}$ & $\begin{array}{l}\text { 337RDLL } \\
\text { 337RDLL } \\
\text { 337RDLL } \\
\text { 337RDLL } \\
\text { 337RDLL }\end{array}$ & $\begin{array}{l}3.4 \\
2.1 \\
3.9 \\
4.5 \\
6.7\end{array}$ & $\begin{array}{l}0.040000 \\
0.100000 \\
0.200000 \\
0.100000 \\
0.300000\end{array}$ & $\begin{array}{l}\text { Dolomite } \\
\text { Dolomite } \\
\text { Fractured, dolomitic limestone } \\
\text { Fractured, dolomitic limestone } \\
\text { Fractured, dolomitic limestone }\end{array}$ \\
\hline $\begin{array}{l}\text { ga } 40-28-11 \text { aa } \\
\text { ga } 40-28-11 \text { a aa } \\
\text { ga40-28-11a a a } \\
\text { ga } 40-28-11 \text { a a a } \\
\text { ga } 40-28-11 \text { aa }\end{array}$ & $\begin{array}{l}5,575 \cdot 4 \\
5,576.5 \\
5,577.7 \\
5,579.4 \\
5,581.0\end{array}$ & $\begin{array}{l}5,576.5 \\
5,577.7 \\
5,579.4 \\
5,581.3 \\
5,582.0\end{array}$ & $\begin{array}{l}\text { 337RDLL } \\
\text { 337RDLL } \\
\text { 337RDLL } \\
\text { 337RDLL } \\
\text { 337RDLL }\end{array}$ & $\begin{array}{l}5.1 \\
3.0 \\
3.7 \\
4.7 \\
9.8\end{array}$ & $\begin{array}{l}0.200000 \\
0.100000 \\
3.900000 \\
0.900000 \\
0.010000\end{array}$ & $\begin{array}{l}\text { Limestone } \\
\text { Limestone } \\
\text { Limestone } \\
\text { Limestone } \\
\text { Limestone }\end{array}$ \\
\hline $\begin{array}{l}\text { ga } 40-28-11 \text { a a } \\
\text { ga40-28-11aaa } \\
\text { ga40-28-11 a aa } \\
\text { ga40-28-11a aa } \\
\text { ga40-28-11aaa }\end{array}$ & $\begin{array}{l}5,582.0 \\
5,583.0 \\
5,584.0 \\
5,585.0 \\
5,586.0\end{array}$ & $\begin{array}{l}5,583.0 \\
5,584.0 \\
5,585.0 \\
5,586.0 \\
5,587.0\end{array}$ & $\begin{array}{l}\text { 337RDLL } \\
\text { 337RDLL } \\
\text { 337RDLL } \\
\text { 337RDLL } \\
\text { 337RDLL }\end{array}$ & $\begin{array}{r}13.1 \\
7.1 \\
7.5 \\
4.1 \\
12.3\end{array}$ & $\begin{array}{l}0.060000 \\
0.030000 \\
0.040000 \\
0.000000 \\
0.900000\end{array}$ & $\begin{array}{l}\text { Limes tone } \\
\text { Limes tone } \\
\text { Limestone } \\
\text { Limestone } \\
\text { Limestone }\end{array}$ \\
\hline $\begin{array}{l}\text { ga40-28-11 a aa } \\
\text { ga 40-28-11 a a a } \\
\text { ga40-28-11a a a } \\
\text { ga 40-28-11 aaa } \\
\text { ga40-28-11aaa }\end{array}$ & $\begin{array}{l}5,587.0 \\
5,588.0 \\
5,592.8 \\
5,597.9 \\
5,601.0\end{array}$ & $\begin{array}{l}5,588.0 \\
5,589.0 \\
5,594.1 \\
5,599.0 \\
5,602.5\end{array}$ & $\begin{array}{l}\text { 337RDLL } \\
\text { 337RDLL } \\
\text { 337RDLL } \\
\text { 337RDLL } \\
\text { 337RDLL }\end{array}$ & $\begin{array}{l}6.2 \\
8.7 \\
5.6 \\
6.3 \\
4.7\end{array}$ & $\begin{array}{l}0.170000 \\
0.030000 \\
0.000000 \\
0.000000 \\
0.300000\end{array}$ & $\begin{array}{l}\text { Limestone } \\
\text { Limestone } \\
\text { Fractured limestone } \\
\text { Fractured limestone } \\
\text { Fractured limestone }\end{array}$ \\
\hline $\begin{array}{l}\text { ga 40-28-11 aaa } \\
\text { ga40-28-11 a a a } \\
\text { ga40-28-11 a a a } \\
\text { ga40-28-11 a aa } \\
\text { ga40-28-11aaa }\end{array}$ & $\begin{array}{l}5,602 \cdot 5 \\
5,604.1 \\
5,606.0 \\
5,607.8 \\
5,609.5\end{array}$ & $\begin{array}{l}5,604.1 \\
5,606.0 \\
5,607.8 \\
5,609.5 \\
5,611.1\end{array}$ & $\begin{array}{l}\text { 337RDLL } \\
\text { 337RDLL } \\
\text { 337RDLL } \\
\text { 337RDLL } \\
\text { 337RDLL }\end{array}$ & $\begin{array}{l}5.8 \\
7.5 \\
6.4 \\
5.1 \\
4.9\end{array}$ & $\begin{array}{l}0.400000 \\
0.300000 \\
0.100000 \\
0.100000 \\
0.000000\end{array}$ & $\begin{array}{l}\text { Fractured limestone } \\
\text { Fractured limestone } \\
\text { Fractured limestone } \\
\text { Fractured limestone } \\
\text { Fractured limestone }\end{array}$ \\
\hline $\begin{array}{l}\text { ga 40-28-11aaa } \\
\text { ga40-28-11aaa } \\
\text { ga40-28-11aaa } \\
\text { ga40-28-11aaa } \\
\text { ga40-28-11aaa }\end{array}$ & $\begin{array}{l}5,611.1 \\
5,613.1 \\
5,618.0 \\
5,620.0 \\
5,622.5\end{array}$ & $\begin{array}{l}5,613 \cdot 1 \\
5,614.9 \\
5,620.0 \\
5,622 \cdot 5 \\
5,624.3\end{array}$ & $\begin{array}{l}\text { 337RDLL } \\
\text { 337RDLL } \\
\text { 337RDLL } \\
\text { 337RDLL } \\
\text { 337RDLL }\end{array}$ & $\begin{array}{l}5.6 \\
8.7 \\
5.6 \\
3.3 \\
2.6\end{array}$ & $\begin{array}{l}0.100000 \\
0.100000 \\
2.900000 \\
7.600000 \\
0.200000\end{array}$ & $\begin{array}{l}\text { Fractured limestone } \\
\text { Fractured limestone } \\
\text { Fractured limestone } \\
\text { Fractured limestone } \\
\text { Fractured limestone }\end{array}$ \\
\hline $\begin{array}{l}\text { ga } 40-28-11 \text { aaa } \\
\text { ga } 40-28-11 \text { aaa } \\
\text { ga } 40-28-11 \text { aaa } \\
\text { ga40-28-11aaa } \\
\text { ga 40-28-11aaa }\end{array}$ & $\begin{array}{l}5,624 \cdot 3 \\
5,626.2 \\
5,628 \cdot 0 \\
5,629.8 \\
5,631 \cdot 5\end{array}$ & $\begin{array}{l}5,626.2 \\
5,628.0 \\
5,629.8 \\
5,631.5 \\
5,633.1\end{array}$ & $\begin{array}{l}\text { 337RDLL } \\
\text { 337RDLL } \\
\text { 337RDLL } \\
\text { 337RDLL } \\
\text { 337RDLL }\end{array}$ & $\begin{array}{l}4.1 \\
6.2 \\
1.5 \\
5.1 \\
4.5\end{array}$ & $\begin{array}{r}0.000000 \\
0.010000 \\
0.100000 \\
12.000000 \\
0.010000\end{array}$ & $\begin{array}{l}\text { Fractured limestone } \\
\text { Fractured limestone } \\
\text { Fractured limestone } \\
\text { Fractured limestone } \\
\text { Fractured limestone }\end{array}$ \\
\hline
\end{tabular}


Table 4.--Laboratory-determined porosity and permeability--Continued

\begin{tabular}{|c|c|c|c|c|c|c|}
\hline Site & $\begin{array}{l}\text { Depth to } \\
\text { top } \\
\text { (feet) }\end{array}$ & $\begin{array}{l}\text { Depth to } \\
\text { bottom } \\
\text { (feet) }\end{array}$ & $\begin{array}{l}\text { Forma- } \\
\text { tion }\end{array}$ & $\begin{array}{l}\text { Porosity } \\
\text { (percent) }\end{array}$ & $\begin{array}{l}\text { Perme- } \\
\text { ability } \\
\text { (milli- } \\
\text { darcies) }\end{array}$ & Lithology \\
\hline $\begin{array}{l}\text { ga } 40-28-11 \text { aa } \\
\text { ga } 40-28-11 \text { aaa } \\
\text { ga40-28-11aaa }\end{array}$ & $\begin{array}{l}5,633.1 \\
5,634.7 \\
5,637.0\end{array}$ & $\begin{array}{l}5,634.7 \\
5,637.0 \\
5,638.3\end{array}$ & $\begin{array}{l}\text { 337RDLL } \\
\text { 337RDLL } \\
\text { 337RDLL }\end{array}$ & $\begin{array}{l}3.4 \\
5.5 \\
4.8 \\
\end{array}$ & $\begin{array}{l}0.100000 \\
0.100000 \\
0.000000 \\
\end{array}$ & $\begin{array}{l}\text { Fractured limestone } \\
\text { Fractured limestone } \\
\text { Fractured limestone }\end{array}$ \\
\hline SITE AVERAGE-- & -1 & . & AVERAGE-- & $\frac{5.6}{5.6}$ & $\frac{0.828947}{0.828947}$ & \\
\hline $\begin{array}{l}\text { ga } 40-29-15 \text { ca } \\
\text { ga40-29-15 caa } \\
\text { ga40-29-15 caa } \\
\text { ga40-29-15 ca } \\
\text { ga40-29-15 caa }\end{array}$ & $\begin{array}{l}5,229.0 \\
5,230.0 \\
5,231.0 \\
5,249.0 \\
5,250.0\end{array}$ & $\begin{array}{l}5,230.0 \\
5,231.0 \\
5,232.0 \\
5,250.0 \\
5,251.0\end{array}$ & $\begin{array}{l}\text { 324PKTL } \\
324 \mathrm{PKTL} \\
324 \mathrm{PKTL} \\
324 \mathrm{PKTL} \\
324 \mathrm{PKTL}\end{array}$ & $\begin{array}{l}2.3 \\
2.0 \\
1.4 \\
2.1 \\
2.4\end{array}$ & $\begin{array}{l}3.000000 \\
0.400000 \\
0.000000 \\
0.000000 \\
0.000000\end{array}$ & $\begin{array}{l}\text { Limestone } \\
\text { Limestone } \\
\text { Limestone } \\
\text { Limestone } \\
\text { Limestone }\end{array}$ \\
\hline $\begin{array}{l}\text { ga } 40-29-15 \text { ca } \\
\text { ga40-29-15caa }\end{array}$ & $\begin{array}{l}5,251.0 \\
5,252.0\end{array}$ & $\begin{array}{l}5,252.0 \\
5,253.0\end{array}$ & $\begin{array}{l}\text { 324PKTL } \\
\text { 324PKTL }\end{array}$ & $\begin{array}{l}2.1 \\
1.8 \\
\end{array}$ & $\begin{array}{l}0.000000 \\
0.000000 \\
\end{array}$ & $\begin{array}{l}\text { Limestone } \\
\text { Limestone }\end{array}$ \\
\hline & & & AVERAGE-- & 2.0 & 0.485714 & \\
\hline $\begin{array}{l}\text { ga40-29-15caa } \\
\text { ga40-29-15caa } \\
\text { ga40-29-15caa }\end{array}$ & $\begin{array}{l}5,725.0 \\
5,726.0 \\
5,727.0\end{array}$ & $\begin{array}{l}5,726.0 \\
5,727.0 \\
5,728.0\end{array}$ & $\begin{array}{l}\text { 337RDLL } \\
\text { 337RDLL } \\
\text { 337RDLL }\end{array}$ & $\begin{array}{l}13.5 \\
13.7 \\
12.8 \\
\end{array}$ & $\begin{array}{l}1.300000 \\
6.000000 \\
4.400000 \\
\end{array}$ & $\begin{array}{l}\text { Dolomite } \\
\text { Dolomite } \\
\text { Dolomite }\end{array}$ \\
\hline SITE AVERAGE - & & 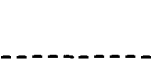 & AVERAGE -- & $\frac{13.3}{5.4}$ & $\frac{3.900000}{1.510000}$ & \\
\hline $\begin{array}{l}\text { ga } 41-28-03 c a b \\
\text { ga } 41-28-03 c a b \\
\text { ga } 41-28-03 c a b \\
\text { ga } 41-28-03 c a b \\
\text { ga41-28-03cab }\end{array}$ & $\begin{array}{l}4,626.0 \\
4,627.0 \\
4,628.0 \\
4,629.0 \\
4,630.0\end{array}$ & $\begin{array}{l}4,627.0 \\
4,628.0 \\
4,629.0 \\
4,630.0 \\
4,631.0\end{array}$ & $\begin{array}{l}\text { 324PRDX } \\
324 \mathrm{PRDX} \\
324 \mathrm{PRDX} \\
324 \mathrm{PRDX} \\
324 \mathrm{PRDX}\end{array}$ & $\begin{array}{l}0.5 \\
0.4 \\
0.5 \\
0.7 \\
0.6\end{array}$ & $\begin{array}{l}0.000000 \\
0.000000 \\
0.000000 \\
0.000000 \\
0.010000\end{array}$ & $\begin{array}{l}\text { Fine-grained to sucrose limestone } \\
\text { Fine-grained to sucrose limestone } \\
\text { Fine-grained to sucrose limestone } \\
\text { Fine-grained to sucrose limestone } \\
\text { Fine-grained to sucrose limestone }\end{array}$ \\
\hline $\begin{array}{l}\text { ga41-28-03cab } \\
\text { ga41-28-03cab } \\
\text { ga41-28-03cab } \\
\text { ga41-28-03cab } \\
\text { ga41-28-03cab }\end{array}$ & $\begin{array}{l}4,631.0 \\
4,632.0 \\
4,633.0 \\
4,634.0 \\
4,635.0\end{array}$ & $\begin{array}{l}4,632.0 \\
4,633.0 \\
4,634.0 \\
4,635.0 \\
4,636.0\end{array}$ & $\begin{array}{l}324 \mathrm{PRDX} \\
324 \mathrm{PRDX} \\
324 \mathrm{PRDX} \\
324 \mathrm{PRDX} \\
324 \mathrm{PRDX}\end{array}$ & $\begin{array}{l}0.8 \\
1.7 \\
1.7 \\
1.6 \\
1.0\end{array}$ & $\begin{array}{l}0.040000 \\
0.000000 \\
0.000000 \\
0.000000 \\
0.000000\end{array}$ & $\begin{array}{l}\text { Fine-grained to sucrose limestone } \\
\text { Fine-grained to sucrose limestone } \\
\text { Fine-grained to sucrose limestone } \\
\text { Fine-grained to sucrose limestone } \\
\text { Fine-grained to sucrose limestone }\end{array}$ \\
\hline $\begin{array}{l}\text { ga } 41-28-03 c a b \\
\text { ga } 41-28-03 c a b \\
\text { ga41-28-03cab } \\
\text { ga41-28-03cab } \\
\text { ga41-28-03cab }\end{array}$ & $\begin{array}{l}4,636.0 \\
4,637.0 \\
4,638.0 \\
4,639.0 \\
4,640.0\end{array}$ & $\begin{array}{l}4,637 \cdot 0 \\
4,638 \cdot 0 \\
4,639 \cdot 0 \\
4,640.0 \\
4,641.0\end{array}$ & $\begin{array}{l}\text { 324PRDX } \\
324 \mathrm{PRDX} \\
324 \mathrm{PRDX} \\
324 \mathrm{PRDX} \\
324 \mathrm{PRDX}\end{array}$ & $\begin{array}{l}1.0 \\
1.5 \\
1.0 \\
1.1 \\
0.3\end{array}$ & $\begin{array}{l}0.000000 \\
0.000000 \\
0.000000 \\
0.000000 \\
0.000000\end{array}$ & $\begin{array}{l}\text { Fine-grained to sucrose limestone } \\
\text { Fine-grained to sucrose limestone } \\
\text { Sucrose limestone } \\
\text { Sucrose limestone } \\
\text { Sucrose limestone }\end{array}$ \\
\hline $\begin{array}{l}\text { ga } 41-28-03 c a b \\
\text { ga } 41-28-03 c a b \\
\text { ga } 41-28-03 c a b \\
\text { ga } 41-28-03 c a b \\
\text { ga41-28-03cab }\end{array}$ & $\begin{array}{l}4,641.0 \\
4,642.0 \\
4,643.0 \\
4,644.0 \\
4,645.0\end{array}$ & $\begin{array}{l}4,642.0 \\
4,643.0 \\
4,644.0 \\
4,645.0 \\
4,646.0\end{array}$ & $\begin{array}{l}\text { 324PRDX } \\
324 \mathrm{PRDX} \\
324 \mathrm{PRDX} \\
324 \mathrm{PRDX} \\
324 \mathrm{PRDX}\end{array}$ & $\begin{array}{l}0.4 \\
0.5 \\
0.4 \\
0.4 \\
3.0\end{array}$ & $\begin{array}{l}0.000000 \\
0.000000 \\
0.000000 \\
0.000000 \\
0.000000\end{array}$ & $\begin{array}{l}\text { Sucrose limestone } \\
\text { Sucrose limestone } \\
\text { Sucrose limestone } \\
\text { Sucrose limestone } \\
\text { Sucrose, carbonaceous limestone }\end{array}$ \\
\hline $\begin{array}{l}\text { ga } 41-28-03 c a b \\
\text { ga41-28-03cab } \\
\text { ga } 41-28-03 c a b \\
\text { ga } 41-28-03 c a b \\
\text { ga41-28-03cab }\end{array}$ & $\begin{array}{l}4,646.0 \\
4,647.0 \\
4,648.0 \\
4,649.0 \\
4,650.0\end{array}$ & $\begin{array}{l}4,647.0 \\
4,648.0 \\
4,649.0 \\
4,650.0 \\
4,651.0\end{array}$ & $\begin{array}{l}324 \mathrm{PRDX} \\
324 \mathrm{PRDX} \\
324 \mathrm{PRDX} \\
324 \mathrm{PRDX} \\
324 \mathrm{PRDX}\end{array}$ & $\begin{array}{l}0.5 \\
0.4 \\
1.3 \\
0.5 \\
1.1\end{array}$ & $\begin{array}{l}0.000000 \\
0.000000 \\
0.000000 \\
0.000000 \\
0.000000\end{array}$ & $\begin{array}{l}\text { Sucrose limestone } \\
\text { Sucrose limestone } \\
\text { Sucrose limestone } \\
\text { Sucrose limestone } \\
\text { Sucrose limestone }\end{array}$ \\
\hline $\begin{array}{l}\text { ga } 41-28-03 c a b \\
\text { ga } 41-28-03 c a b \\
\text { ga } 41-28-03 c a b \\
\text { ga } 41-28-03 c a b \\
\text { ga41-28-03cab }\end{array}$ & $\begin{array}{l}4,651.0 \\
4,652.0 \\
4,653.0 \\
4,654.0 \\
4,655.0\end{array}$ & $\begin{array}{l}4,652 \cdot 0 \\
4,653.0 \\
4,654.0 \\
4,655.0 \\
4,656.0\end{array}$ & $\begin{array}{l}\text { 324PRDX } \\
324 \mathrm{PRDX} \\
324 \mathrm{PRDX} \\
324 \mathrm{PRDX} \\
324 \mathrm{PRDX}\end{array}$ & $\begin{array}{l}1.9 \\
2.3 \\
2.4 \\
1.6 \\
2.1\end{array}$ & $\begin{array}{l}0.000000 \\
0.000000 \\
0.000000 \\
0.000000 \\
0.000000\end{array}$ & $\begin{array}{l}\text { Sucrose limestone } \\
\text { Sucrose limestone } \\
\text { Sucrose limestone } \\
\text { Sucrose limestone } \\
\text { Sucrose limestone }\end{array}$ \\
\hline
\end{tabular}


Table 4.--Laboratory-determined porosity and permeability--Continued

\begin{tabular}{|c|c|c|c|c|c|c|}
\hline Site & $\begin{array}{l}\text { Depth to } \\
\text { top } \\
\text { (feet) }\end{array}$ & $\begin{array}{l}\text { Depth to } \\
\text { bottom } \\
\text { (feet) }\end{array}$ & $\begin{array}{c}\text { Forma- } \\
\text { tion }\end{array}$ & $\begin{array}{l}\text { Porosity } \\
\text { (percent) }\end{array}$ & $\begin{array}{c}\text { Perme- } \\
\text { ability } \\
\text { (milli- } \\
\text { darcies) }\end{array}$ & Lithology \\
\hline$g a 41-28-03 c a b$ & $4,656.0$ & $4,657.0$ & 324PRDX & 2.0 & 0.000000 & Sucrose limestone \\
\hline ga 41-28-03cab & $4,657.0$ & $4,658.0$ & 324PRDX & 0.9 & 0.000000 & Sucrose limestone \\
\hline ga41-28-03cab & $4,658.0$ & $4,659.0$ & 324PRDX & 1.0 & 0.000000 & Sucrose limestone \\
\hline ga41-28-03cab & $4,659.0$ & $4,660.0$ & 324PRDX & 0.4 & 0.000000 & Sucrose limestone \\
\hline$g a 41-28-03 c a b$ & $4,661.0$ & $4,662.0$ & $324 \mathrm{PRDX}$ & 4.9 & 0.000000 & Sucrose, carbonaceous dolomite \\
\hline ga41-28-03cab & $4,662.0$ & $4,663.0$ & 324PRDX & 0.4 & 0.000000 & Sucrose limestone \\
\hline ga $41-28-03 c a b$ & $4,663.0$ & $4,664.0$ & 324PRDX & 0.4 & 0.000000 & Sucrose limestone \\
\hline $\mathrm{ga} 41-28-03 \mathrm{cab}$ & $4,664.0$ & $4,665.0$ & 324PRDX & 0.5 & 0.000000 & Sucrose limestone \\
\hline ga41-28-03cab & $4,665.0$ & $4,666.0$ & $324 \mathrm{PRDX}$ & 0.4 & 0.000000 & Sucrose limestone \\
\hline ga41-28-03cab & $4,671.0$ & $4,672.0$ & 324PRDX & 0.6 & 0.000000 & Sucrose limestone \\
\hline ga41-28-03cab & $4,672.0$ & $4,673.0$ & 324PRDX & 0.5 & 0.000000 & Sucrose, dolomitic limestone \\
\hline ga $41-28-03 c a b$ & $4,775.0$ & $4,776.0$ & 324PRDX & 15.0 & 43.000000 & Vuggy, sucrose dolomite \\
\hline ga $41-28-03 c a b$ & $4,776.0$ & $4,777.0$ & 324PRDX & 17.3 & 115.000000 & Vuggy, sucrose dolomite \\
\hline ga $41-28-03 c a b$ & $4,777.0$ & $4,778.0$ & 324PRDX & 19.0 & 34.000000 & Vuggy, sucrose dolomite \\
\hline ga41-28-03cab & $4,778.0$ & $4,779.0$ & $324 \mathrm{PRDX}$ & 20.7 & 58.000000 & Vuggy, sucrose dolomite \\
\hline ga41-28-03cab & $4,779.0$ & $4,780.0$ & 324PRDX & 17.7 & 8.500000 & Vuggy, sucrose dolomite \\
\hline ga $41-28-03 c a b$ & $4,780.0$ & $4,781.0$ & 324PRDX & 15.3 & 10.000000 & Vuggy, sucrose dolomite \\
\hline ga41-28-03cab & $4,781.0$ & $4,782.0$ & 324PRDX & 13.6 & 1.200000 & Vuggy, sucrose dolomite \\
\hline ga $41-28-03 \mathrm{cab}$ & $4,782.0$ & $4,783.0$ & 324PRDX & 12.4 & 0.100000 & Sucrose dolomite \\
\hline ga41-28-03cab & $5,100.0$ & $5,101.0$ & 324PRDX & 0.5 & 0.000000 & Sucrose limestone \\
\hline $\mathrm{ga4} 1-28-03 \mathrm{cab}$ & $5,101.0$ & $5,102.0$ & 324PRDX & 0.7 & 0.000000 & Sucrose limestone \\
\hline$g a 41-28-03 c a b$ & $5,102.0$ & $5,103.0$ & 324PRDX & 0.4 & 0.000000 & Sucrose limestone \\
\hline $\mathrm{ga} 41-28-03 \mathrm{cab}$ & $5,103.0$ & $5,104.0$ & 324PRDX & 0.6 & 0.000000 & Sucrose limestone \\
\hline $\mathrm{ga} 41-28-03 \mathrm{cab}$ & $5,104.0$ & $5,105.0$ & 324PRDX & 0.4 & 0.000000 & Sucrose limestone \\
\hline ga $41-28-03 \mathrm{cab}$ & $5,105.0$ & $5,106.0$ & $324 \mathrm{PRDX}$ & 0.5 & 0.000000 & Sucrose limestone \\
\hline $\mathrm{ga4} 1-28-03 \mathrm{cab}$ & $5,106.0$ & $5,107.0$ & $324 \mathrm{PRDX}$ & 0.4 & 0.000000 & Sucrose limestone \\
\hline ga 41-28-03cab & $5,107.0$ & $5,108.0$ & $324 \mathrm{PRDX}$ & 1.2 & 0.000000 & Sucrose limestone \\
\hline ga41-28-03cab & $5,108.0$ & $5,109.0$ & $324 \mathrm{PRDX}$ & 0.5 & 0.000000 & Sucrose, limy dolomite \\
\hline ga41-28-03cab & $5,109.0$ & $5,110.0$ & 324PRDX & 1.0 & 0.000000 & Sucrose dolomite \\
\hline ga41-28-03cab & $5,110.0$ & $5,111.0$ & 324 PRDX & 0.9 & 0.000000 & Sucrose dolomite \\
\hline ga $41-28-03 \mathrm{cab}$ & $5,111.0$ & $5,112.0$ & 324PRDX & 1.8 & 0.000000 & Sucrose, dolomitic limestone \\
\hline$g a 41-28-03 c a b$ & $5,112.0$ & $5,113.0$ & 324 PRDX & 4.3 & 0.000000 & Sucrose, dolomitic limestone \\
\hline ga41-28-03cab & $5,113.0$ & $5,114.0$ & 324PRDX & 3.4 & 0.000000 & Sucrose dolomite \\
\hline ga $41-28-03 \mathrm{cab}$ & $5,114.0$ & $5,115.0$ & 324PRDX & 2.8 & 0.000000 & Sucrose, dolomitic limestone \\
\hline ga41-28-03cab & $5,115.0$ & $5,116.0$ & 324PRDX & 2.8 & 0.000000 & Shaly, fine-grained dolomite \\
\hline SITE AVERAGE-- & -------1 & 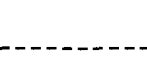 & AVERAGE-- & $\frac{3.1}{3.1}$ & $\frac{3.457342}{3.457342}$ & \\
\hline
\end{tabular}


Table 4.--Laboratory-determined porosity and permeability--Continued

\begin{tabular}{|c|c|c|c|c|c|c|}
\hline Site & $\begin{array}{l}\text { Depth to } \\
\text { top } \\
\text { (feet) }\end{array}$ & $\begin{array}{l}\text { Depth to } \\
\text { bottom } \\
\text { (feet) }\end{array}$ & $\begin{array}{c}\text { Forma- } \\
\text { tion }\end{array}$ & $\begin{array}{l}\text { Porosity } \\
\text { (percent) }\end{array}$ & $\begin{array}{l}\text { Perme- } \\
\text { ability } \\
\text { (milli- } \\
\text { darcies) }\end{array}$ & Lithology \\
\hline ga41-28-04dab & $4,608.0$ & $4,609.0$ & 324PRDX & 1.1 & 0.040000 & Crystalline limestone \\
\hline ga41-28-04dab & $4,609.0$ & $4,610.0$ & $324 \mathrm{PRDX}$ & 4.7 & 0.130000 & Crystalline limestone \\
\hline ga $41-28-04 d a b$ & $4,610.0$ & $4,611.0$ & $324 \mathrm{PRDX}$ & 0.4 & 0.000000 & Fractured, crystalline limestone \\
\hline ga41-28-04dab & $4,611.0$ & $4,612.0$ & 324PRDX & 3.7 & 0.000000 & Fractured, crystalline limestone \\
\hline ga $41-28-04 d a b$ & $4,612.0$ & $4,613.0$ & $324 \mathrm{PRDX}$ & 3.5 & 0.000000 & Crystalline limestone \\
\hline ga41-28-04dab & $4,613.0$ & $4,614.0$ & 324PRDX & 12.2 & 0.130000 & Crystalline limestone \\
\hline ga41-28-04dab & $4,614.0$ & $4,615.0$ & 324PRDX & 0.7 & 0.000000 & Crystalline limestone \\
\hline ga41-28-04dab & $4,615.0$ & $4,616.0$ & $324 \mathrm{PRDX}$ & 0.7 & 0.000000 & Crystalline limestone \\
\hline ga41-28-04dab & $4,616.0$ & $4,617.0$ & $324 \mathrm{PRDX}$ & 0.4 & 0.000000 & Crystalline limestone \\
\hline ga41-28-04dab & $4,617.0$ & $4,618.0$ & 324PRDX & 1.1 & 0.000000 & Crystalline limestone \\
\hline ga41-28-04dab & $4,618.0$ & $4,619.0$ & 324PRDX & 1.5 & 0.000000 & Crystalline limestone \\
\hline ga41-28-04dab & $4,619.0$ & $4,620.0$ & $324 \mathrm{PRDX}$ & 1.8 & 0.000000 & Crystalline limestone \\
\hline ga41-28-04dab & $4,620.0$ & $4,621.0$ & 324PRDX & 0.7 & 0.000000 & Crystalline limestone \\
\hline ga41-28-04dab & $4,621.0$ & $4,622.0$ & $324 \mathrm{PRDX}$ & 3.9 & 0.640000 & Crystalline limestone \\
\hline ga41-28-04dab & $4,622.0$ & $4,623.0$ & $324 \mathrm{PRDX}$ & 1.1 & 0.000000 & Crystalline limestone \\
\hline$g a 41-28-04 d a b$ & $4,629.0$ & $4,630.0$ & 324PRDX & 1.0 & 0.000000 & Crystalline limestone \\
\hline ga41-28-04dab & $4,630.0$ & $4,631.0$ & 324PRDX & 6.9 & 0.380000 & Sandy to crystalline limestone \\
\hline ga41-28-04dab & $4,631.0$ & $4,632.0$ & $324 \mathrm{PRDX}$ & 8.5 & 0.940000 & Sandy to crystalline limestone \\
\hline ga41-28-04dab & $4,632.0$ & $4,633.0$ & 324PRDX & 8.0 & 0.130000 & Sandy to crystalline limestone \\
\hline ga $41-28-04 d a b$ & $4,633.0$ & $4,634.0$ & 324PRDX & 17.7 & 3.180000 & Vuggy limestone \\
\hline ga41-28-04dab & $4,634.0$ & $4,635.0$ & 324PRDX & 20.2 & 3.950000 & Vuggy limestone \\
\hline ga41-28-04dab & $4,635.0$ & $4,636.0$ & $324 \mathrm{PRDX}$ & 22.3 & 0.810000 & Vuggy limestone \\
\hline ga41-28-04dab & $4,636.0$ & $4,637.0$ & $324 \mathrm{PRDX}$ & 6.6 & 0.000000 & Very fractured, sandy limestone \\
\hline ga4 $1-28-04 d a b$ & $4,637.0$ & $4,638.0$ & $324 \mathrm{PRDX}$ & 8.0 & 0.000000 & Very fractured, sandy limestone \\
\hline ga41-28-04dab & $4,638.0$ & $4,639.0$ & $324 \mathrm{PRDX}$ & 8.7 & 0.000000 & Very fractured, sandy limestone \\
\hline ga41-28-04dab & $4,640.0$ & $4,641.0$ & 324PRDX & 5.6 & 0.000000 & Crystalline limestone \\
\hline ga $41-28-04 d a b$ & $4,641.0$ & $4,642.0$ & 324PRDX & 3.8 & 0.000000 & Crystalline limestone \\
\hline ga41-28-04dab & $4,642.0$ & $4,643.0$ & $324 \mathrm{PRDX}$ & 2.3 & 0.000000 & Crystalline limestone \\
\hline ga4 $1-28-04 d a b$ & $4,643.0$ & $4,644.0$ & $324 \mathrm{PRDX}$ & 2.5 & 0.380000 & Crystalline limestone \\
\hline ga $41-28-04 d a b$ & $4,644.0$ & $4,645.0$ & $324 \mathrm{PRDX}$ & 3.6 & 0.000000 & Crystalline limestone \\
\hline ga41-28-04dab & $4,645.0$ & $4,646.0$ & $324 \mathrm{PRDX}$ & 0.4 & 0.000000 & Crystalline limestone \\
\hline ga41-28-04dab & $4,646.0$ & $4,647.0$ & 324PRDX & 0.7 & 0.000000 & Crystalline limestone \\
\hline ga $41-28-04 d a b$ & $4,647.0$ & $4,648.0$ & 324PRDX & 0.5 & 0.000000 & Crystalline limestone \\
\hline ga41-28-04dab & $4,648.0$ & $4,649.0$ & 324PRDX & 0.8 & 0.000000 & Crystalline limestone \\
\hline ga $41-28-04 d a b$ & $4,649.0$ & $4,650.0$ & $324 \mathrm{PRDX}$ & 0.5 & 0.000000 & Crystalline limestone \\
\hline ga $41-28-04 \mathrm{dab}$ & $4,650.0$ & $4,651.0$ & $324 \mathrm{PRDX}$ & 0.4 & 0.000000 & Crystalline limestone \\
\hline ga41-28-04dab & $4,651.0$ & $4,652.0$ & 324PRDX & 0.6 & 0.000000 & Crystalline limestone \\
\hline ga41-28-04dab & $4,652.0$ & $4,653.0$ & $324 \mathrm{PRDX}$ & 1.1 & 0.000000 & Crystalline limestone \\
\hline ga41-28-04dab & $4,653.0$ & $4,654.0$ & $324 \mathrm{PRDX}$ & 0.4 & 0.000000 & Crystalline limestone \\
\hline ga41-28-04dab & $4,654.0$ & $4,655.0$ & 324PRDX & 1.1 & 0.000000 & Crystalline limestone \\
\hline $\mathrm{ga41-28-04dab}$ & $4,655.0$ & $4,656.0$ & $324 \mathrm{PRDX}$ & 0.5 & 0.000000 & Crystalline limestone \\
\hline ga41-28-04dab & $4,656.0$ & $4,657.0$ & $324 \mathrm{PRDX}$ & 0.5 & 0.000000 & Crystalline limestone \\
\hline ga41-28-04dab & $4,657.0$ & $4,658.0$ & $324 \mathrm{PRDX}$ & 0.5 & 0.000000 & Crystalline 1imestone \\
\hline ga41-28-04dab & $4,658.0$ & $5,659.0$ & 324PRDX & 0.6 & 0.000000 & Crystalline limestone \\
\hline ga41-28-04dab & $4,659.0$ & $4,660.0$ & $324 \mathrm{PRDX}$ & 1.1 & 0.000000 & Crystalline limestone \\
\hline ga41-28-04dab & $4,660.0$ & $4,661.0$ & $324 \mathrm{PRDX}$ & 0.7 & 0.000000 & Crystalline 1imestone \\
\hline ga41-28-04dab & $4,661.0$ & $4,662.0$ & $324 \mathrm{PRDX}$ & 2.1 & 0.000000 & Crystalline limestone \\
\hline ga $41-28-04 d a b$ & $4,662.0$ & $4,663.0$ & $324 \mathrm{PRDX}$ & 0.3 & 0.000000 & Crystalline limestone \\
\hline ga41-28-04dab & $4,663.0$ & $4,664.0$ & $324 \mathrm{PRDX}$ & 0.4 & 0.000000 & Crystalline 1 imestone \\
\hline ga $41-28-04 d a b$ & $4,664.0$ & $4,665.0$ & $324 \mathrm{PRDX}$ & 0.7 & 0.000000 & Crystalline limestone \\
\hline
\end{tabular}


Table 4.--Laboratory-determined porosity and permeability--Continued

\begin{tabular}{|c|c|c|c|c|c|c|}
\hline Site & $\begin{array}{l}\text { Depth to } \\
\text { top } \\
\text { (feet) }\end{array}$ & $\begin{array}{l}\text { Depth to } \\
\text { bottom } \\
\text { (feet) }\end{array}$ & $\begin{array}{c}\text { Forma- } \\
\text { tion }\end{array}$ & $\begin{array}{l}\text { Porosity } \\
\text { (percent) }\end{array}$ & $\begin{array}{l}\text { Perme- } \\
\text { ability } \\
\text { (milli- } \\
\text { darcies) }\end{array}$ & Lithology \\
\hline ga $41-28-04 d a b$ & $4,665.0$ & $4,666.0$ & 324PRDX & 1.2 & 0.000000 & Crystalline limestone \\
\hline ga41-28-04dab & $4,666.0$ & $4,667.0$ & $324 \mathrm{PRDX}$ & 1.3 & 0.000000 & Crystalline limestone \\
\hline ga41-28-04dab & $4,667.0$ & $4,668.0$ & $324 \mathrm{PRDX}$ & 1.1 & 0.000000 & Crystalline limestone \\
\hline ga $41-28-04 d a b$ & $4,668.0$ & $4,669.0$ & $324 \mathrm{PRDX}$ & 0.7 & 0.000000 & Crystalline limestone \\
\hline ga41-28-04dab & $4,669.0$ & $4,670.0$ & $324 \mathrm{PRDX}$ & 0.9 & 0.000000 & Crystalline limestone \\
\hline ga 41-28-04dab & $4,670.0$ & $4,671.0$ & 324PRDX & 1.0 & 0.000000 & Crystalline limestone \\
\hline ga $41-28-04 \mathrm{dab}$ & $4,671.0$ & $4,672.0$ & $324 \mathrm{PRDX}$ & 0.4 & 0.000000 & Crystalline limestone \\
\hline ga $41-28-04 \mathrm{dab}$ & $4,672.0$ & $4,673.0$ & $324 \mathrm{PRDX}$ & 0.3 & 0.000000 & Crystalline limestone \\
\hline $9 a 41-28-04 d a b$ & $4,673.0$ & $4,674.0$ & $324 \mathrm{PRDX}$ & 0.5 & 0.000000 & Crystalline limestone \\
\hline ga41-28-04dab & $4,674.0$ & $4,675.0$ & 324PRDX & 0.6 & 0.000000 & Crystalline limestone \\
\hline ga41-28-04dab & $4,675.0$ & $4,676.0$ & $324 \mathrm{PRDX}$ & 0.4 & 0.000000 & Crystalline limestone \\
\hline ga $41-28-04 d a b$ & $4,676.0$ & $4,677.0$ & $324 \mathrm{PRDX}$ & 0.3 & 0.000000 & Crystalline limestone \\
\hline ga41-28-04dab & $4,677.0$ & $4,678.0$ & 324PRDX & 0.1 & 0.000000 & Crystalline limestone \\
\hline ga41-28-04dab & $4,678.0$ & $4,679.0$ & $324 \mathrm{PRDX}$ & 1.8 & 0.000000 & Crystalline limestone \\
\hline ga41-28-04dab & $4,679.0$ & $4,680.0$ & $324 \mathrm{PRDX}$ & 1.0 & 0.000000 & Crystalline limestone \\
\hline ga41-28-04dab & $4,680.0$ & $4,681.0$ & $324 \mathrm{PRDX}$ & 0.4 & 0.000000 & Crystalline limestone \\
\hline ga $41-28-04 d a b$ & $4,681.0$ & $4,682.0$ & $324 \mathrm{PRDX}$ & 0.3 & 0.000000 & Limy shale \\
\hline ga41-28-04dab & $4,682.0$ & $4,683.0$ & $324 \mathrm{PRDX}$ & 0.4 & 0.000000 & Limy shale \\
\hline ga41-28-04dab & $4,683.0$ & $4,684.0$ & 324PRDX & 1.2 & 0.000000 & Crystalline limestone \\
\hline ga41-28-04dab & $4,684.0$ & $4,685.0$ & 324PRDX & 1.5 & 0.000000 & Crystalline limestone \\
\hline ga41-28-04dab & $4,685.0$ & $4,686.0$ & $324 \mathrm{PRDX}$ & 1.4 & 0.000000 & Crystalline limestone \\
\hline ga $41-28-04 d a b$ & $4,686.0$ & $4,687.0$ & $324 \mathrm{PRDX}$ & 2.4 & 0.000000 & Crystalline limestone \\
\hline ga41-28-04dab & $4,687.0$ & $4,688.0$ & $324 \mathrm{PRDX}$ & 1.0 & 3.180000 & Crystalline limestone \\
\hline ga $41-28-04 d a b$ & $4,688.0$ & $4,689.0$ & $324 \mathrm{PRDX}$ & 0.7 & 0.810000 & Vuggy limestone \\
\hline ga41-28-04dab & $4,689.0$ & $4,690.0$ & 324PRDX & 3.8 & 0.000000 & Vuggy limestone \\
\hline ga41-28-04dab & $4,690.0$ & $4,691.0$ & $324 \mathrm{PRDX}$ & 8.5 & 0.130000 & Vuggy limestone \\
\hline ga $41-28-04 \mathrm{dab}$ & $4,691.0$ & $4,692.0$ & $324 \mathrm{PRDX}$ & 3.6 & 0.000000 & Crystalline limestone \\
\hline ga41-28-04dab & $4,692.0$ & $4,693.0$ & 324PRDX & 0.7 & 0.000000 & Crystalline limestone \\
\hline ga $41-28-04 \mathrm{dab}$ & $4,693.0$ & $4,694.0$ & 324PRDX & 0.9 & 0.000000 & Crystalline limestone \\
\hline ga $41-28-04 d a b$ & $4,694.0$ & $4,695.0$ & $324 \mathrm{PRDX}$ & 0.7 & 0.000000 & Crystalline limestone \\
\hline ga41-28-04dab & $4,695.0$ & $4,696.0$ & $324 \mathrm{PRDX}$ & 0.4 & 0.000000 & Crystalline limestone \\
\hline ga41-28-04dab & $4,696.0$ & $4,697.0$ & 324PRDX & 0.8 & 0.000000 & Crystalline limestone \\
\hline ga $41-28-04 d a b$ & $4,697.0$ & $4,698.0$ & 324PRDX & 1.0 & 0.000000 & Crystalline limestone \\
\hline $\mathrm{ga} 41-28-04 \mathrm{dab}$ & $4,698.0$ & $4,699.0$ & $324 \mathrm{PRDX}$ & 2.0 & 0.000000 & Crystalline limestone \\
\hline ga $41-28-04 \mathrm{dab}$ & $4,699.0$ & $4,700.0$ & 324PRDX & 2.3 & 0.000000 & Crystalline limestone \\
\hline ga41-28-04dab & $4,700.0$ & $4,701.0$ & 324PRDX & 0.9 & 0.000000 & Crystalline limestone \\
\hline ga $41-28-04 \mathrm{dab}$ & $4,701.0$ & $4,702.0$ & 324PRDX & 0.2 & 0.000000 & Fractured, crystalline limestone \\
\hline ga41-28-04dab & $4,702.0$ & $4,703.0$ & 324PRDX & 1.2 & 0.000000 & Fractured, crystalline limestone \\
\hline ga41-28-04dab & $4,703.0$ & $4,704.0$ & $324 \mathrm{PRDX}$ & 0.9 & 0.000000 & Fractured, crystalline limestone \\
\hline ga $41-28-04 d a b$ & $4,704.0$ & $4,705.0$ & $324 \mathrm{PRDX}$ & 0.6 & 0.380000 & Fractured, crystalline limestone \\
\hline ga41-28-04dab & $4,705.0$ & $4,706.0$ & $324 \mathrm{PRDX}$ & 0.8 & 0.000000 & Crystalline limestone \\
\hline ga $41-28-04 \mathrm{dab}$ & $4,706.0$ & $4,707.0$ & 324PRDX & 0.5 & 0.000000 & Fossiliferous, crystalline limestone \\
\hline ga $41-28-04 \mathrm{dab}$ & $4,707.0$ & $4,708.0$ & $324 \mathrm{PRDX}$ & 0.7 & 0.130000 & Fossiliferous, crystalline limestone \\
\hline ga $41-28-04 \mathrm{dab}$ & $4,708.0$ & $4,709.0$ & 324PRDX & 0.3 & 0.000000 & Fossiliferous, crystalline limestone \\
\hline ga41-28-04dab & $4,709.0$ & $4,710.0$ & $324 \mathrm{PRDX}$ & 0.4 & 0.000000 & Fossiliferous, crystalline limestone \\
\hline ga41-28-04dab & $4,710.0$ & $4,711.0$ & 324PRDX & 0.5 & 0.000000 & Fossiliferous, crystalline limestone \\
\hline ga41-28-04dab & $4,711.0$ & $4,712.0$ & 324PRDX & 0.4 & 0.000000 & Fossiliferous, crystalline limestone \\
\hline ga $41-28-04 \mathrm{dab}$ & $4,712.0$ & $4,713.0$ & $324 \mathrm{PRDX}$ & 0.4 & 0.000000 & Fossiliferous, crystalline limestone \\
\hline ga41-28-04dab & $4,713.0$ & $4,714.0$ & 324PRDX & 0.6 & 0.000000 & Fossiliferous, crystalline limestone \\
\hline ga $41-28-04 d a b$ & $4,714.0$ & $4,715.0$ & 324PRDX & 0.8 & 0.000000 & Fossiliferous, crystalline limestone \\
\hline
\end{tabular}


Table 4.--Laboratory-determined porosity and permeability--Continued

\begin{tabular}{|c|c|c|c|c|c|c|}
\hline Site & $\begin{array}{l}\text { Depth to } \\
\text { top } \\
\text { (feet) }\end{array}$ & $\begin{array}{l}\text { Depth to } \\
\text { bottom } \\
\text { (feet) }\end{array}$ & $\begin{array}{c}\text { Forma- } \\
\text { tion }\end{array}$ & $\begin{array}{l}\text { Porosity } \\
\text { (percent) }\end{array}$ & $\begin{array}{l}\text { Perme- } \\
\text { ability } \\
\text { (milli- } \\
\text { darcies) }\end{array}$ & Lithology \\
\hline ga41-28-04dab & $4,715.0$ & $4,716.0$ & 324PRDX & 0.9 & 0.000000 & Fossiliferous, crystalline limestone \\
\hline ga41-28-04dab & $4,716.0$ & $4,717.0$ & $324 \mathrm{PRDX}$ & 0.2 & 0.000000 & Fossiliferous, crystalline limestone \\
\hline ga41-28-04dab & $4,717.0$ & $4,718.0$ & 324PRDX & 0.5 & 0.000000 & Crystalline limestone \\
\hline ga41-28-04dab & $4,718.0$ & $4,719.0$ & 324PRDX & 0.8 & 0.000000 & Crystalline limestone \\
\hline ga41-28-04dab & $4,719.0$ & $4,720.0$ & $324 \mathrm{PRDX}$ & 0.8 & 0.000000 & Fossiliferous, crystalline limestone \\
\hline ga41-28-04dab & $4,720.0$ & $4,721.0$ & 324PRDX & 0.5 & 0.000000 & Fossiliferous, crystalline limestone \\
\hline ga41-28-04dab & $4,721.0$ & $4,722.0$ & $324 \mathrm{PRDX}$ & 1.5 & 0.640000 & Fossiliferous, crystalline limestone \\
\hline ga $41-28-04 d a b$ & $4,722.0$ & $4,723.0$ & $324 \mathrm{PRDX}$ & 1.9 & 0.000000 & Fossiliferous, crystalline limestone \\
\hline ga41-28-04dab & $4,723.0$ & $4,724.0$ & 324PRDX & 1.3 & 0.000000 & Crystalline limestone \\
\hline ga $41-28-04 \mathrm{dab}$ & $4,724.0$ & $4,725.0$ & $324 \mathrm{PRDX}$ & 1.2 & 0.000000 & Crystalline limestone \\
\hline ga41-28-04dab & $4,725.0$ & $4,726.0$ & $324 \mathrm{PRDX}$ & 1.7 & 0.000000 & Crystalline limestone \\
\hline ga $41-28-04 \mathrm{dab}$ & $4,726.0$ & $4,727.0$ & $324 \mathrm{PRDX}$ & 1.2 & 0.000000 & Fossiliferous, crystalline limestone \\
\hline ga41-28-04dab & $4,727.0$ & $4,728.0$ & 324PRDX & 1.1 & 0.000000 & Fossiliferous, crystalline limestone \\
\hline ga41-28-04dab & $4,782.0$ & $4,783.0$ & $324 \mathrm{PRDX}$ & 3.5 & 0.040000 & Crystalline limestone \\
\hline ga $41-28-04 d a b$ & $4,783.0$ & $4,784.0$ & 324PRDX & 4.3 & 0.850000 & Crystalline limestone \\
\hline ga41-28-04dab & $4,784.0$ & $4,785.0$ & $324 \mathrm{PRDX}$ & 2.7 & 0.000000 & Crystalline limestone \\
\hline ga41-28-04dab & $4,785.0$ & $4,786.0$ & 324PRDX & 1.3 & 0.000000 & Crystalline limestone \\
\hline ga41-28-04dab & $4,786.0$ & $4,787.0$ & 324PRDX & 3.3 & 0.000000 & Crystalline limestone \\
\hline ga $41-28-04 \mathrm{dab}$ & $4,787.0$ & $4,788.0$ & $324 \mathrm{PRDX}$ & 6.2 & 0.000000 & Crystalline limestone \\
\hline ga $41-28-04 d a b$ & $4,788.0$ & $4,789.0$ & 324PRDX & 5.7 & 0.000000 & Crystalline limestone \\
\hline ga41-28-04dab & $4,789.0$ & $4,790.0$ & $324 \mathrm{PRDX}$ & 4.3 & 0.000000 & Crystalline limestone \\
\hline ga 41-28-04dab & $4,790.0$ & $4,791.0$ & 324PRDX & 14.4 & 1.630000 & Sucrose limestone \\
\hline ga $41-28-04 d a b$ & $4,791.0$ & $4,792.0$ & $324 \mathrm{PRDX}$ & 10.0 & 1.200000 & Sucrose limestone \\
\hline ga41-28-04dab & $4,792.0$ & $4,793.0$ & $324 \mathrm{PRDX}$ & 12.3 & 0.930000 & Sucrose limestone \\
\hline ga41-28-04dab & $4,793.0$ & $4,794.0$ & $324 \mathrm{PRDX}$ & 12.0 & 0.040000 & Cherty, sucrose limestone \\
\hline ga $41-28-04 d a b$ & $4,794.0$ & $4,795.0$ & $324 \mathrm{PRDX}$ & 16.3 & 1.630000 & Cherty, sucrose limestone \\
\hline ga4 $1-28-04 \mathrm{dab}$ & $4,795.0$ & $4,796.0$ & 324 PRDX & 1.4 & 0.810000 & Cherty, sucrose limestone \\
\hline ga41-28-04dab & $4,796.0$ & $4,797.0$ & 324PRDX & 7.9 & 1.460000 & Cherty, sucrose limestone \\
\hline ga41-28-04dab & $4,799.0$ & $4,800.0$ & 324PRDX & 2.9 & 0.000000 & Sucrose limestone \\
\hline ga $41-28-04 d a b$ & $4,800.0$ & $4,801.0$ & $324 \mathrm{PRDX}$ & 7.3 & 0.000000 & Fractured, crystalline dolomite \\
\hline ga $41-28-04 d a b$ & $4,801.0$ & $4,802.0$ & $324 \mathrm{PRDX}$ & 10.8 & 0.000000 & Fractured, crystalline dolomite \\
\hline ga41-28-04dab & $4,802.0$ & $4,803.0$ & $324 \mathrm{PRDX}$ & 11.0 & 0.000000 & Fractured, crystalline dolomite \\
\hline ga $41-28-04 d a b$ & $4,803.0$ & $4,804.0$ & 324PRDX & 10.0 & 8.540000 & Cherty dolomite \\
\hline ga41-28-04dab & $4,804.0$ & $4,805.0$ & $324 \mathrm{PRDX}$ & 24.6 & 183.000000 & Cherty dolomite \\
\hline ga41-28-04dab & $4,805.0$ & $4,806.0$ & 324PRDX & 16.0 & 48.000000 & Cherty dolomite \\
\hline ga41-28-04dab & $4,806.0$ & $4,807.0$ & $324 \mathrm{PRDX}$ & 12.6 & 209.000000 & Cherty dolomite \\
\hline ga41-28-04dab & $4,807.0$ & $4,808.0$ & $324 \mathrm{PRDX}$ & 15.8 & 209.000000 & Cherty dolomite \\
\hline ga $41-28-04 \mathrm{dab}$ & $4,808.0$ & $4,809.0$ & $324 \mathrm{PRDX}$ & 16.5 & 348.000000 & Cherty dolomite \\
\hline ga41-28-04dab & $4,809.0$ & $4,810.0$ & 324PRDX & 16.4 & 93.000000 & Cherty dolomite \\
\hline ga41-28-04dab & $4,810.0$ & $4,811.0$ & 324PRDX & 17.5 & 81.000000 & Cherty dolomite \\
\hline ga $41-28-04 d a b$ & $4,811.0$ & $4,812.0$ & 324PRDX & 19.7 & 55.000000 & Cherty dolomite \\
\hline ga $41-28-04 \mathrm{dab}$ & $4,812.0$ & $4,813.0$ & $324 \mathrm{PRDX}$ & 16.1 & 699.000000 & Cherty dolomite \\
\hline ga $41-28-04 d a b$ & $4,813.0$ & $4,814.0$ & $324 \mathrm{PRDX}$ & 13.4 & 0.000000 & Cherty dolomite \\
\hline ga41-28-04dab & $4,814.0$ & $4,815.0$ & 324PRDX & 11.5 & 0.000000 & Cherty dolomite \\
\hline ga $41-28-04$ dab & $4,815.0$ & $4,816.0$ & $324 \mathrm{PRDX}$ & 10.7 & 0.810000 & Cherty dolomite \\
\hline ga41-28-04dab & $4,816.0$ & $4,817.0$ & $324 \mathrm{PRDX}$ & 15.1 & 0.300000 & Cherty dolomite \\
\hline ga41-28-04dab & $4,817.0$ & $4,818.0$ & $324 \mathrm{PRDX}$ & 11.9 & 0.380000 & Cherty, crystalline dolomite \\
\hline ga $41-28-04 d a b$ & $4,818.0$ & $4,819.0$ & 324PRDX & 9.5 & 0.300000 & Cherty, crystalline dolomite \\
\hline ga $41-28-04 \mathrm{dab}$ & $4,819.0$ & $4,820.0$ & $324 \mathrm{PRDX}$ & 2.5 & 0.130000 & Cherty, crystalline dolomite \\
\hline ga41-28-04dab & $4,820.0$ & $4,821.0$ & $324 \mathrm{PRDX}$ & 9.6 & 0.210000 & Shaly dolomite \\
\hline
\end{tabular}


Table 4.--Laboratory-determined porosity and permeability--Continued

\begin{tabular}{|c|c|c|c|c|c|c|}
\hline Site & $\begin{array}{l}\text { Depth to } \\
\text { top } \\
\text { (feet) }\end{array}$ & $\begin{array}{l}\text { Depth to } \\
\text { bottom } \\
\text { (feet) }\end{array}$ & $\begin{array}{c}\text { Forma- } \\
\text { tion }\end{array}$ & $\begin{array}{l}\text { Porosity } \\
\text { (percent) }\end{array}$ & $\begin{array}{l}\text { Perme- } \\
\text { ability } \\
\text { (milli- } \\
\text { darcies) }\end{array}$ & Lithology \\
\hline $\begin{array}{l}\text { ga } 41-28-04 \mathrm{dab} \\
\text { ga } 41-28-04 \mathrm{dab} \\
\text { ga } 41-28-04 \mathrm{dab} \\
\text { ga } 41-28-04 \mathrm{dab} \\
\text { ga41-28-04dab }\end{array}$ & $\begin{array}{l}4,821.0 \\
4,822.0 \\
4,823.0 \\
4,824.0 \\
4,825.0\end{array}$ & $\begin{array}{l}4,822.0 \\
4,823.0 \\
4,824.0 \\
4,825.0 \\
4,826.0\end{array}$ & $\begin{array}{l}\text { 324PRDX } \\
324 \mathrm{PRDX} \\
324 \mathrm{PRDX} \\
324 \mathrm{PRDX} \\
324 \mathrm{PRDX}\end{array}$ & $\begin{array}{r}10.1 \\
8.5 \\
12.5 \\
4.5 \\
2.0\end{array}$ & $\begin{array}{l}0.000000 \\
0.130000 \\
0.210000 \\
0.210000 \\
0.040000\end{array}$ & $\begin{array}{l}\text { Shaly dolomite } \\
\text { Cherty dolomite } \\
\text { Cherty dolomite } \\
\text { Cherty dolomite } \\
\text { Cherty dolomite }\end{array}$ \\
\hline $\begin{array}{l}\text { ga } 41-28-04 \mathrm{dab} \\
\text { ga } 41-28-04 \mathrm{dab} \\
\text { ga } 41-28-04 \mathrm{dab} \\
\text { ga } 41-28-04 \mathrm{dab} \\
\text { ga4 } 1-28-04 \mathrm{dab}\end{array}$ & $\begin{array}{l}4,877.0 \\
4,878.0 \\
4,879.0 \\
4,880.0 \\
4,881.0\end{array}$ & $\begin{array}{l}4,878.0 \\
4,879.0 \\
4,880.0 \\
4,881.0 \\
4,882.0\end{array}$ & $\begin{array}{l}324 \mathrm{PRDX} \\
324 \mathrm{PRDX} \\
324 \mathrm{PRDX} \\
324 \mathrm{PRDX} \\
324 \mathrm{PRDX}\end{array}$ & $\begin{array}{l}0.8 \\
2.3 \\
4.1 \\
6.6 \\
7.7\end{array}$ & $\begin{array}{l}0.040000 \\
0.040000 \\
0.300000 \\
0.810000 \\
0.640000\end{array}$ & $\begin{array}{l}\text { Fractured limestone } \\
\text { Fractured limestone } \\
\text { Fractured limestone } \\
\text { Fractured limestone } \\
\text { Fractured limestone }\end{array}$ \\
\hline $\begin{array}{l}\text { ga } 41-28-04 \mathrm{dab} \\
\text { ga } 41-28-04 \mathrm{dab} \\
\text { ga } 41-28-04 \mathrm{dab} \\
\text { ga } 41-28-04 \mathrm{dab} \\
\text { ga } 41-28-04 \mathrm{dab}\end{array}$ & $\begin{array}{l}4,882.0 \\
4,883.0 \\
4,884.0 \\
4,885.0 \\
4,886.0\end{array}$ & $\begin{array}{l}4,883.0 \\
4,884.0 \\
4,885.0 \\
4,886.0 \\
4,887.0\end{array}$ & $\begin{array}{l}324 \mathrm{PRDX} \\
324 \mathrm{PRDX} \\
324 \mathrm{PRDX} \\
324 \mathrm{PRDX} \\
324 \mathrm{PRDX}\end{array}$ & $\begin{array}{l}5.4 \\
6.4 \\
7.5 \\
2.7 \\
3.0\end{array}$ & $\begin{array}{l}0.300000 \\
4.750000 \\
1.840000 \\
1.200000 \\
0.640000\end{array}$ & $\begin{array}{l}\text { Fractured limestone } \\
\text { Fractured limestone } \\
\text { Crystalline limestone } \\
\text { Crystalline limestone } \\
\text { Crystalline limestone }\end{array}$ \\
\hline $\begin{array}{l}\text { ga } 41-28-04 \mathrm{dab} \\
\text { ga } 41-28-04 \mathrm{dab} \\
\text { ga } 41-28-04 \mathrm{dab} \\
\text { ga } 41-28-04 \mathrm{dab} \\
\text { ga41-28-04dab }\end{array}$ & $\begin{array}{l}4,887.0 \\
4,888.0 \\
4,889.0 \\
4,890.0 \\
4,891.0\end{array}$ & $\begin{array}{l}4,888.0 \\
4,889.0 \\
4,890.0 \\
4,891.0 \\
4,892.0\end{array}$ & $\begin{array}{l}324 \mathrm{PRDX} \\
324 \mathrm{PRDX} \\
324 \mathrm{PRDX} \\
324 \mathrm{PRDX} \\
324 \mathrm{PRDX}\end{array}$ & $\begin{array}{l}0.7 \\
1.6 \\
1.5 \\
1.1 \\
2.1\end{array}$ & $\begin{array}{l}4.750000 \\
0.470000 \\
0.380000 \\
0.040000 \\
0.000000\end{array}$ & $\begin{array}{l}\text { Crystalline limestone } \\
\text { Crystalline limestone } \\
\text { Crystalline limestone } \\
\text { Vuggy limestone } \\
\text { Vuggy limestone }\end{array}$ \\
\hline $\begin{array}{l}\text { ga } 41-28-04 \mathrm{dab} \\
\text { ga } 41-28-04 \mathrm{dab} \\
\text { ga } 41-28-04 \mathrm{dab} \\
\text { ga } 41-28-04 \mathrm{dab} \\
\text { ga41-28-04dab }\end{array}$ & $\begin{array}{l}4,892.0 \\
4,893.0 \\
4,894.0 \\
4,895.0 \\
4,896.0\end{array}$ & $\begin{array}{l}4,893.0 \\
4,894.0 \\
4,895.0 \\
4,896.0 \\
4,897.0\end{array}$ & $\begin{array}{l}\text { 324PRDX } \\
324 \mathrm{PRDX} \\
324 \mathrm{PRDX} \\
324 \mathrm{PRDX} \\
\text { 324PRDX }\end{array}$ & $\begin{array}{l}2.4 \\
1.1 \\
7.5 \\
5.7 \\
3.7\end{array}$ & $\begin{array}{l}0.130000 \\
0.000000 \\
0.000000 \\
0.040000 \\
0.000000\end{array}$ & $\begin{array}{l}\text { Vuggy limestone } \\
\text { Vuggy limestone } \\
\text { Shaly dolomite } \\
\text { Shaly dolomite } \\
\text { Shaly dolomite }\end{array}$ \\
\hline $\begin{array}{l}\text { ga } 41-28-04 d a b \\
\text { ga } 41-28-04 d a b \\
\text { ga41-28-04dab }\end{array}$ & $\begin{array}{l}4,897.0 \\
4,898.0 \\
4,899.0\end{array}$ & $\begin{array}{l}4,898.0 \\
4,899.0 \\
4,900.0\end{array}$ & $\begin{array}{l}\text { 324PRDX } \\
\text { 324PRDX } \\
\text { 324PRDX } \\
\text { AVERAGE-- }\end{array}$ & $\begin{array}{l}9.9 \\
9.7 \\
9.4 \\
4.3\end{array}$ & $\begin{array}{r}0.000000 \\
0.000000 \\
0.380000 \\
11.110000\end{array}$ & $\begin{array}{l}\text { Shaly dolomite } \\
\text { Shaly dolomite } \\
\text { Shaly dolomite }\end{array}$ \\
\hline $\begin{array}{l}\text { ga } 41-28-04 \mathrm{dab} \\
\text { ga } 41-28-04 \mathrm{dab} \\
\text { ga } 41-28-04 \mathrm{dab} \\
\text { ga } 41-28-04 \mathrm{dab} \\
\text { ga } 41-28-04 \mathrm{dab}\end{array}$ & $\begin{array}{l}5,516.0 \\
5,517.0 \\
5,518.0 \\
5,519.0 \\
5,520.0\end{array}$ & $\begin{array}{l}5,517.0 \\
5,518.0 \\
5,519.0 \\
5,520.0 \\
5,521.0\end{array}$ & $\begin{array}{l}\text { 337RDLL } \\
\text { 337RDLL } \\
\text { 337RDLL } \\
\text { 337RDLL } \\
\text { 337RDLL }\end{array}$ & $\begin{array}{l}3.2 \\
3.1 \\
2.6 \\
7.6 \\
8.2\end{array}$ & $\begin{array}{l}0.000000 \\
0.000000 \\
0.000000 \\
0.000000 \\
0.000000\end{array}$ & $\begin{array}{l}\text { Crystalline limestone } \\
\text { Crystalline limestone } \\
\text { Crystalline limestone } \\
\text { Crystalline limestone } \\
\text { Crystalline limestone }\end{array}$ \\
\hline $\begin{array}{l}\text { ga } 41-28-04 \mathrm{dab} \\
\text { ga } 41-28-04 \mathrm{dab} \\
\text { ga } 41-28-04 \mathrm{dab} \\
\text { ga } 41-28-04 \mathrm{dab} \\
\text { ga } 41-28-04 \mathrm{dab}\end{array}$ & $\begin{array}{l}5,521.0 \\
5,606.0 \\
5,607.0 \\
5,608.0 \\
5,609.0\end{array}$ & $\begin{array}{l}5,522.0 \\
5,607.0 \\
5,608.0 \\
5,609.0 \\
5,610.0\end{array}$ & $\begin{array}{l}\text { 337RDLL } \\
\text { 337RDLL } \\
\text { 337RDLL } \\
\text { 337RDLL } \\
\text { 337RDLL }\end{array}$ & $\begin{array}{l}4.6 \\
1.0 \\
2.8 \\
2.0 \\
2.1\end{array}$ & $\begin{array}{l}0.000000 \\
0.040000 \\
0.000000 \\
0.000000 \\
0.000000\end{array}$ & $\begin{array}{l}\text { Crystalline limestone } \\
\text { Crystalline limestone } \\
\text { Crystalline limestone } \\
\text { Crystalline limestone } \\
\text { Crystalline limestone }\end{array}$ \\
\hline $\begin{array}{l}\text { ga } 41-28-04 \mathrm{dab} \\
\text { ga } 41-28-04 \mathrm{dab} \\
\text { ga } 41-28-04 \mathrm{dab} \\
\text { ga } 41-28-04 \mathrm{dab} \\
\text { ga } 41-28-04 \mathrm{dab}\end{array}$ & $\begin{array}{l}5,610.0 \\
5,611.0 \\
5,612.0 \\
5,613.0 \\
5,614.0\end{array}$ & $\begin{array}{l}5,611.0 \\
5,612.0 \\
5,613.0 \\
5,614.0 \\
5,615.0\end{array}$ & $\begin{array}{l}\text { 337RDLL } \\
\text { 337RDLL } \\
\text { 337RDLL } \\
\text { 337RDLL } \\
\text { 337RDLL }\end{array}$ & $\begin{array}{l}2.0 \\
1.9 \\
3.5 \\
2.0 \\
3.1\end{array}$ & $\begin{array}{l}0.000000 \\
0.000000 \\
0.040000 \\
0.130000 \\
0.000000\end{array}$ & $\begin{array}{l}\text { Crystalline limestone } \\
\text { Crystalline limestone } \\
\text { Crystalline limestone } \\
\text { Crystalline limestone } \\
\text { Crystalline limestone }\end{array}$ \\
\hline $\begin{array}{l}\text { ga } 41-28-04 \mathrm{dab} \\
\text { ga } 41-28-04 \mathrm{dab} \\
\text { ga } 41-28-04 \mathrm{dab} \\
\text { ga } 41-28-04 \mathrm{dab} \\
\text { ga } 41-28-04 \mathrm{dab}\end{array}$ & $\begin{array}{l}5,615.0 \\
5,622.0 \\
5,623.0 \\
5,624.0 \\
5,625.0\end{array}$ & $\begin{array}{l}5,616.0 \\
5,623.0 \\
5,624.0 \\
5,625.0 \\
5,626.0\end{array}$ & $\begin{array}{l}\text { 337RDLL } \\
\text { 337RDLL } \\
\text { 337RDLL } \\
\text { 337RDLL } \\
\text { 337RDLL }\end{array}$ & $\begin{array}{l}2.0 \\
3.1 \\
1.0 \\
1.2 \\
1.0\end{array}$ & $\begin{array}{l}0.000000 \\
0.000000 \\
0.000000 \\
0.040000 \\
0.000000\end{array}$ & $\begin{array}{l}\text { Crystalline limestone } \\
\text { Crystalline dolomite } \\
\text { Crystalline limestone } \\
\text { Fractured, crystalline limestone } \\
\text { Shaly, crystalline limestone }\end{array}$ \\
\hline
\end{tabular}


Table 4.--Laboratory-determined porosity and permeability--Continued

\begin{tabular}{|c|c|c|c|c|c|c|}
\hline Site & $\begin{array}{l}\text { Depth to } \\
\text { top } \\
\text { (feet) }\end{array}$ & $\begin{array}{l}\text { Depth to } \\
\text { bottom } \\
\text { (feet) }\end{array}$ & $\begin{array}{c}\text { Forma- } \\
\text { tion }\end{array}$ & $\begin{array}{l}\text { Porosity } \\
\text { (percent) }\end{array}$ & $\begin{array}{l}\text { Perme- } \\
\text { ability } \\
\text { (milli- } \\
\text { darcies) }\end{array}$ & Lithology \\
\hline ga41-28-04dab & $5,626.0$ & $5,627.0$ & 337RDLL & 1.2 & 0.000000 & Crystalline limestone \\
\hline ga $41-28-04 \mathrm{dab}$ & $5,627.0$ & $5,628.0$ & 337RDLL & 1.3 & 0.000000 & Crystalline limestone \\
\hline ga41-28-04dab & $5,628.0$ & $5,629.0$ & 337RDLL & 1.5 & 0.000000 & Limy shale \\
\hline ga $41-28-04 \mathrm{dab}$ & $5,702.0$ & $5,703.0$ & 337RDLL & 1.4 & 0.000000 & Crystalline limestone \\
\hline ga41-28-04dab & $5,703.0$ & $5,704.0$ & 337RDLL & 15.3 & 4.400000 & Vuggy, sucrose dolomite \\
\hline ga41-28-04dab & $5,704.0$ & $5,705.0$ & 337RDLL & 12.2 & 0.930000 & Vuggy, sucrose dolomite \\
\hline ga41-28-04dab & $5,705.0$ & $5,706.0$ & 337 RDLL & 7.3 & 1.110000 & Vuggy, sucrose dolomite \\
\hline ga $41-28-04 d a b$ & $5,706.0$ & $5,707.0$ & 337RDLL & 2.0 & 0.000000 & Vuggy, cherty, sucrose dolomite \\
\hline ga4l-28-04dab & $5,707.0$ & $5,708.0$ & 337RDLL & 11.2 & 3.310000 & Vuggy, cherty, sucrose dolomite \\
\hline ga41-28-04dab & $5,708.0$ & $5,709.0$ & 337RDLL & 15.4 & 1.630000 & Vuggy, cherty, sucrose dolomite \\
\hline ga41-28-04dab & $5,709.0$ & $5,710.0$ & 337RDLL & 12.8 & 3.780000 & Vuggy, cherty, sucrose dolomite \\
\hline ga41-28-04dab & $5,710.0$ & $5,711.0$ & 337RDLL & 7.5 & 2.400000 & Vuggy, cherty, sucrose dolomite \\
\hline ga $41-28-04 d a b$ & $5,711.0$ & $5,712.0$ & 337RDLL & 10.2 & 0.380000 & Vuggy, cherty, sucrose dolomite \\
\hline ga41-28-04dab & $5,712.0$ & $5,713.0$ & 337RDLL & 3.5 & 4.320000 & Vuggy, cherty, sucrose dolomite \\
\hline ga41-28-04dab & $5,713.0$ & $5,714.0$ & 337RDLL & 6.8 & 1.630000 & Vuggy, cherty, sucrose dolomite \\
\hline ga41-28-04dab & $5,714.0$ & $5,715.0$ & 337RDLL & 11.9 & 2.230000 & Vuggy, cherty, sucrose dolomite \\
\hline ga41-28-04dab & $5,715.0$ & $5,716.0$ & 337RDLL & 14.4 & 7.440000 & Vuggy, cherty, sucrose dolomite \\
\hline ga41-28-04dab & $5,716.0$ & $5,717.0$ & 337RDLL & 8.2 & 2.400000 & Vuggy, cherty, sucrose dolomite \\
\hline ga41-28-04dab & $5,717.0$ & $5,718.0$ & 337RDLL & 7.8 & 1.460000 & Vuggy, cherty, sucrose dolomite \\
\hline ga41-28-04dab & $5,718.0$ & $5,719.0$ & 337RDLL & 11.2 & 2.870000 & Vuggy, cherty, sucrose dolomite \\
\hline \multirow[t]{2}{*}{ ga41-28-04dab } & \multirow[t]{2}{*}{$5,719.0$} & \multirow[t]{2}{*}{$5,720.0$} & 337RDLL & 7.8 & 13.300000 & \multirow[t]{2}{*}{ Vuggy, cherty, sucrose dolomite } \\
\hline & & & AVERAGE-- & 5.6 & 1.313171 & \\
\hline$g a 41-28-04 d a b$ & $5,974.0$ & $5,975.0$ & 341ELBR & 11.5 & 51.000000 & Fine-grained sandstone \\
\hline ga41-28-04dab & $5,975.0$ & $5,976.0$ & 341ELBR & 12.2 & 56.000000 & Fine-grained sandstone \\
\hline ga41-28-04dab & $5,976.0$ & $5,977.0$ & $341 E L B R$ & 11.3 & 179.000000 & Fine-grained sandstone \\
\hline ga41-28-04dab & $5,977.0$ & $5,978.0$ & $341 \mathrm{ELBR}$ & 8.2 & 20.000000 & Fine-grained sandstone \\
\hline ga41-28-04dab & $5,978.0$ & $5,979.0$ & $341 \mathrm{ELBR}$ & 7.2 & 6.700000 & Fine-grained sandstone \\
\hline ga41-28-04dab & $5,979.0$ & $5,980.0$ & $341 E L B R$ & 5.8 & 5.000000 & Fine-grained sandstone \\
\hline ga41-28-04dab & $5,980.0$ & $5,981.0$ & $341 E L B R$ & 3.5 & 0.810000 & Fine-grained sandstone \\
\hline ga41-28-04dab & $5,981.0$ & $5,982.0$ & 341ELBR & 3.9 & 0.380000 & Fine-grained sandstone \\
\hline ga41-28-04dab & $5,982.0$ & $5,983.0$ & $341 E L B R$ & 4.2 & 0.300000 & Fine-grained sandstone \\
\hline ga41-28-04dab & $5,983.0$ & $5,984.0$ & $341 \mathrm{ELBR}$ & 2.4 & 0.300000 & Fine-grained sandstone \\
\hline $\mathrm{ga} 41-28-04 \mathrm{dab}$ & $5,984.0$ & $5,985.0$ & 341ELBR & 5.5 & 2.320000 & Fine-grained sandstone \\
\hline ga41-28-04dab & $5,985.0$ & $5,986.0$ & $341 \mathrm{ELBR}$ & 3.3 & 0.000000 & Fine-grained sandstone \\
\hline ga 41-28-04dab & $5,986.0$ & $5,987.0$ & 341ELBR & 1.1 & 0.000000 & Shale \\
\hline \multirow[t]{2}{*}{ ga $41-28-04 \mathrm{dab}$} & $5,987.0$ & $5,988.0$ & $341 \mathrm{ELBR}$ & 0.8 & 0.040000 & Fine-grained sandstone \\
\hline & & & AVERAGE-- & 5.8 & 22.989286 & \\
\hline ga41-28-04dab & $6,322.0$ & $6,323.0$ & $374 \mathrm{MUAV}$ & 1.1 & 0.000000 & Dolomite \\
\hline ga4 $1-28-04 \mathrm{dab}$ & $6,323.0$ & $6,324.0$ & $374 \mathrm{MUAV}$ & 0.5 & 0.000000 & Dolomite \\
\hline ga $41-28-04 d a b$ & $6,324.0$ & $6,325.0$ & $374 \mathrm{MUAV}$ & 0.3 & 0.000000 & Dolomite \\
\hline ga 41-28-04dab & $6,325.0$ & $6,326.0$ & 374MUAV & 0.8 & 0.000000 & Dolomite \\
\hline ga41-28-04dab & $6,326.0$ & $6,327.0$ & $374 \mathrm{MUAV}$ & 0.5 & 0.000000 & Dolomite \\
\hline ga41-28-04dab & $6,327.0$ & $6,328.0$ & $374 M U A V$ & 0.3 & 0.000000 & Dolomite \\
\hline ga41-28-04dab & $6,328.0$ & $6,329.0$ & 374MUAV & 1.5 & 0.000000 & Dolomite \\
\hline ga41-28-04dab & $6,329.0$ & $6,330.0$ & $374 \mathrm{MUAV}$ & 0.8 & 0.000000 & Dolomite \\
\hline ga41-28-04dab & $6,330.0$ & $6,331.0$ & $374 \mathrm{MUAV}$ & 0.6 & 0.000000 & Dolomite \\
\hline ga $41-28-04 d a b$ & $6,331.0$ & $6,332.0$ & $374 \mathrm{MUAV}$ & 4.2 & 0.040000 & Dolomite \\
\hline
\end{tabular}


Table 4.--Laboratory-determined porosity and permeability--Continued

\begin{tabular}{|c|c|c|c|c|c|c|}
\hline Site & $\begin{array}{l}\text { Depth to } \\
\text { top } \\
\text { (feet) }\end{array}$ & $\begin{array}{l}\text { Depth to } \\
\text { bottom } \\
\text { (feet) }\end{array}$ & $\begin{array}{c}\text { Forma- } \\
\text { tion }\end{array}$ & $\begin{array}{l}\text { Porosity } \\
\text { (percent) }\end{array}$ & $\begin{array}{l}\text { Perme- } \\
\text { ability } \\
\text { (milli- } \\
\text { darcies) }\end{array}$ & Lithology \\
\hline ga $41-28-04 d a b$ & $6,332.0$ & $6,333.0$ & 374MUAV & 2.7 & 0.000000 & Dolomite \\
\hline ga41-28-04dab & $6,333.0$ & $6,334.0$ & 374MUAV & 2.3 & 0.000000 & Dolomite \\
\hline ga $41-28-04 d a b$ & $6,334.0$ & $6,335.0$ & 374MUAV & 4.9 & 0.000000 & Dolomite \\
\hline ga $41-28-04 d a b$ & $6,335.0$ & $6,336.0$ & 374MUAV & 0.9 & 0.000000 & Dolomite \\
\hline ga $41-28-04 \mathrm{dab}$ & $6,336.0$ & $6,337.0$ & 374MUAV & 1.1 & 0.000000 & Dolomite \\
\hline ga $41-28-04 d a b$ & $6,337.0$ & $6,338.0$ & 374MUAV & 0.9 & 0.000000 & Dolomite \\
\hline & & & AVERAGE -- & 1.5 & 0.002500 & \\
\hline SITE AVERAGE-- & & & 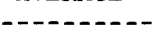 & 4.4 & 9.451044 & \\
\hline ga 41-30-21cab & $4,947.0$ & $4,948.0$ & 324PRDX & 5.0 & 0.200000 & Fine-grained limestone \\
\hline ga $41-30-21 \mathrm{cab}$ & $4,948.0$ & $4,949.0$ & $324 \mathrm{PRDX}$ & 3.1 & 0.230000 & Fine-grained limestone \\
\hline ga $41-30-21 c a b$ & $4,949.0$ & $4,950.0$ & 324PRDX & 1.7 & 0.010000 & Fine-grained limestone \\
\hline ga $41-30-21 \mathrm{cab}$ & $4,950.0$ & $4,951.0$ & 324PRDX & 1.0 & 0.000000 & Fine-grained limestone \\
\hline ga $41-30-21 c a b$ & $4,951.0$ & $4,952.0$ & 324PRDX & 0.7 & 0.000000 & Fine-grained limestone \\
\hline $\mathrm{ga} 41-30-21 \mathrm{cab}$ & $4,952.0$ & $4,953.0$ & 324PRDX & 0.7 & 0.000000 & Fine-grained limestone \\
\hline ga41-30-21 cab & $4,953.0$ & $4,954.0$ & 324PRDX & 6.8 & 0.240000 & Fine-grained limestone \\
\hline ga41-30-21cab & $4,954.0$ & $4,955.0$ & $324 \mathrm{PRDX}$ & 11.6 & 0.170000 & Vuggy limestone \\
\hline $\mathrm{ga} 41-30-21 \mathrm{cab}$ & $4,955.0$ & $4,956.0$ & $324 \mathrm{PRDX}$ & 6.6 & 0.070000 & Vuggy limestone \\
\hline $\mathrm{ga} 41-30-21 \mathrm{cab}$ & $4,956.0$ & $4,957.0$ & $324 \mathrm{PRDX}$ & 2.3 & 0.000000 & Vuggy limestone \\
\hline $\mathrm{ga} 41-30-21 \mathrm{cab}$ & $4,957.0$ & $4,958.0$ & 324PRDX & 4.0 & 0.080000 & Vuggy limestone \\
\hline ga $41-30-21 \mathrm{cab}$ & $4,958.0$ & $4,959.0$ & 324PRDX & 14.4 & 2.600000 & Vuggy limestone \\
\hline ga $41-30-21 c a b$ & $4,959.0$ & $4,960.0$ & $324 \mathrm{PRDX}$ & 26.4 & 0.250000 & Vuggy limestone \\
\hline ga41-30-21cab & $4,960.0$ & $4,961.0$ & $324 \mathrm{PRDX}$ & 20.8 & 0.380000 & Vuggy limestone \\
\hline ga $41-30-21 c a b$ & $4,961.0$ & $4,962.0$ & 324PRDX & 28.5 & 0.680000 & Vuggy limestone \\
\hline$g a 41-30-21 c a b$ & $4,962.0$ & $4,963.0$ & 324PRDX & 11.1 & 0.070000 & Vuggy limestone \\
\hline$g a 41-30-21 c a b$ & $4,963.0$ & $4,964.0$ & 324PRDX & 15.1 & 0.270000 & Vuggy limestone \\
\hline ga41-30-21cab & $4,964.0$ & $4,965.0$ & $324 \mathrm{PRDX}$ & 15.5 & 0.060000 & Vuggy limestone \\
\hline ga $41-30-21 c a b$ & $4,965.0$ & $4,966.0$ & 324PRDX & 13.9 & 0.100000 & Vuggy limestone \\
\hline$g a 41-30-21 c a b$ & $4,966.0$ & $4,967.0$ & 324PRDX & 6.1 & 0.250000 & Vuggy limestone \\
\hline $\mathrm{ga} 41-30-21 \mathrm{cab}$ & $5,022.0$ & $5,023.0$ & $324 \mathrm{PRDX}$ & 3.9 & 0.000000 & Fine-grained limestone \\
\hline ga $41-30-21 c a b$ & $5,023.0$ & $5,024.0$ & 324PRDX & 13.5 & 14.000000 & Fine-grained limestone \\
\hline ga $41-30-21 \mathrm{cab}$ & $5,024.0$ & $5,025.0$ & 324PRDX & 6.1 & 0.190000 & Fine-grained limestone \\
\hline ga $41-30-21 c a b$ & $5,025.0$ & $5,026.0$ & 324PRDX & 7.8 & 0.020000 & Fine-grained limestone \\
\hline ga $41-30-21 c a b$ & $5,026.0$ & $5,027.0$ & $324 \mathrm{PRDX}$ & 2.9 & 0.000000 & Fine-grained limestone \\
\hline$g a 41-30-21 c a b$ & $5,027.0$ & $5,028.0$ & 324PRDX & 1.0 & 0.000000 & Fine-grained limestone \\
\hline ga41-30-21cab & $5,028.0$ & $5,029.0$ & 324PRDX & 3.0 & 0.000000 & Fine-grained limestone \\
\hline ga $41-30-21 c a b$ & $5,029.0$ & $5,030.0$ & 324PRDX & 6.3 & 0.800000 & Fossiliferous limestone \\
\hline$g a 41-30-21 c a b$ & $5,030.0$ & $5,031.0$ & 324PRDX & 3.8 & 19.000000 & Fossiliferous limestone \\
\hline$g a 41-30-21 c a b$ & $5,031.0$ & $5,032.0$ & $324 \mathrm{PRDX}$ & 4.4 & 0.170000 & Fossiliferous limestone \\
\hline$g a 41-30-21 c a b$ & $5,032.0$ & $5,033.0$ & 324PRDX & 5.2 & 0.050000 & Fossiliferous limestone \\
\hline ga $41-30-21 c a b$ & $5,033.0$ & $5,034.0$ & 324PRDX & 4.5 & 0.000000 & Fossiliferous limestone \\
\hline ga $41-30-21 c a b$ & $5,034.0$ & $5,035.0$ & $324 \mathrm{PRDX}$ & 7.5 & 1.200000 & Fossiliferous limestone \\
\hline$g a 41-30-21 c a b$ & $5,035.0$ & $5,036.0$ & 324PRDX & 7.3 & 7.100000 & Fossiliferous limestone \\
\hline $\mathrm{ga} 41-30-21 \mathrm{cab}$ & $5,036.0$ & $5,037.0$ & 324PRDX & 5.9 & 0.360000 & Fossiliferous limestone \\
\hline$g a 41-30-21 c a b$ & $5,037.0$ & $5,038.0$ & 324PRDX & 1.9 & 0.000000 & Fine-grained limestone \\
\hline$g a 41-30-21 c a b$ & $5,074.0$ & $5,075.0$ & $324 \mathrm{PRDX}$ & 1.8 & 0.020000 & Fine-grained limestone \\
\hline ga $41-30-21 \mathrm{cab}$ & $5,075.0$ & $5,076.0$ & $324 \mathrm{PRDX}$ & 4.1 & 0.480000 & Fine-grained limestone \\
\hline ga $41-30-21 c a b$ & $5,076.0$ & $5,077.0$ & $324 \mathrm{PRDX}$ & 5.1 & 0.070000 & Fine-grained limestone \\
\hline ga41-30-21cab & $5,077.0$ & $5,078.0$ & $324 \mathrm{PRDX}$ & 7.2 & 0.250000 & Fine-grained limestone \\
\hline
\end{tabular}


Table 4.--Laboratory-determined porosity and permeability--Continued

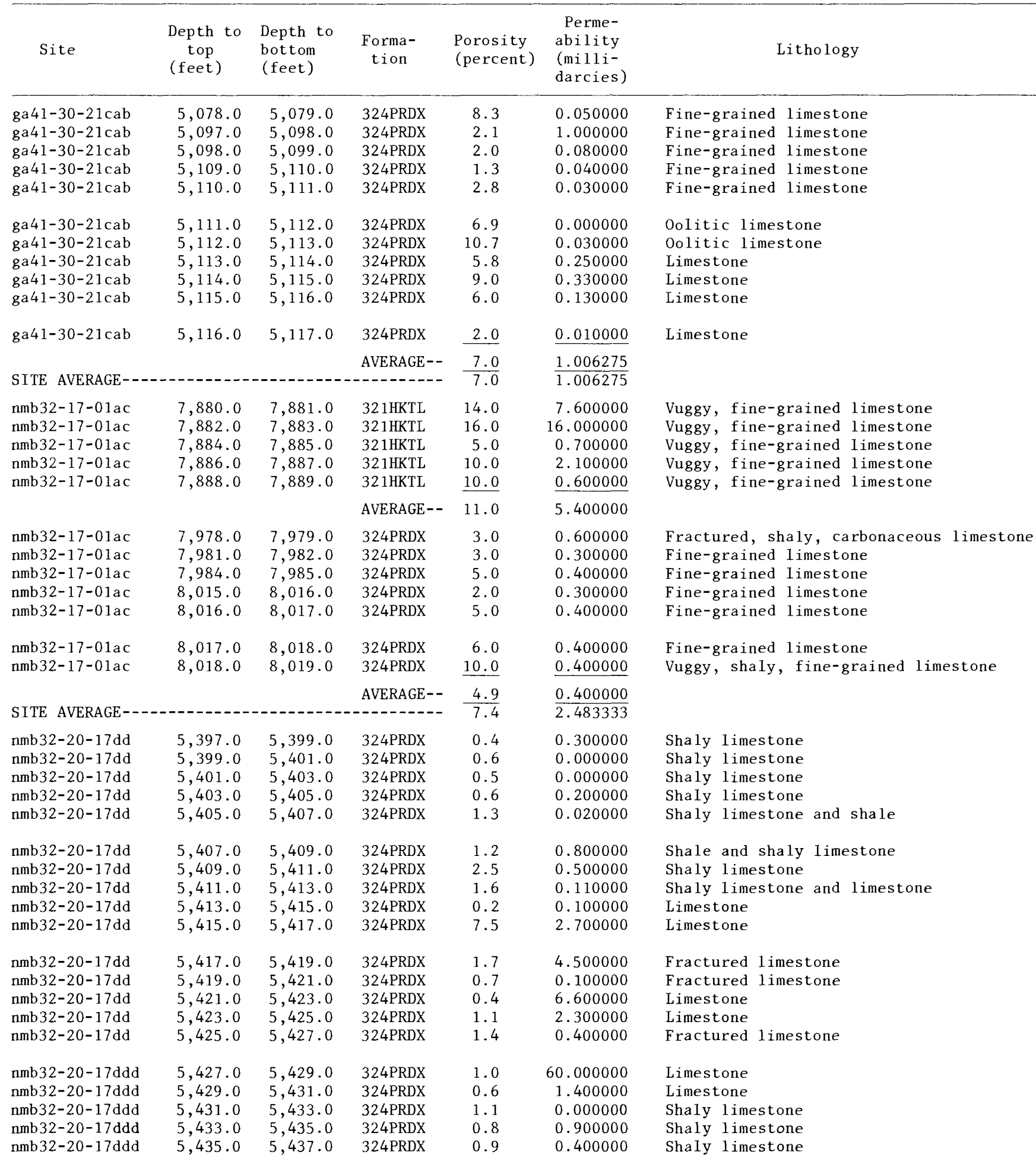


Table 4.--Laboratory-determined porosity and permeability--Continued

\begin{tabular}{|c|c|c|c|c|c|c|}
\hline Site & $\begin{array}{l}\text { Depth to } \\
\text { top } \\
\text { (feet) }\end{array}$ & $\begin{array}{l}\text { Depth to } \\
\text { bottom } \\
\text { (feet) }\end{array}$ & $\begin{array}{c}\text { Forma- } \\
\text { tion }\end{array}$ & $\begin{array}{l}\text { Porosity } \\
\text { (percent) }\end{array}$ & $\begin{array}{l}\text { Perme- } \\
\text { ability } \\
\text { (milli- } \\
\text { darcies) }\end{array}$ & Lithology \\
\hline $\mathrm{nmb} 32-20-17 \mathrm{ddd}$ & $5,437.0$ & $5,439.0$ & 324PRDX & 0.3 & 1.800000 & Shaly limestone \\
\hline $\mathrm{nmb} 32-20-17 \mathrm{ddd}$ & $5,439.0$ & $5,441.0$ & $324 \mathrm{PRDX}$ & 0.6 & 3.500000 & Fractured limestone \\
\hline nmb32-20-17ddd & $5,441.0$ & $5,442.0$ & 324PRDX & 0.6 & 0.300000 & Fractured limestone \\
\hline nmb32-20-17ddd & $5,511.0$ & $5,513.0$ & $324 \mathrm{PRDX}$ & 1.9 & 26.000000 & Limestone \\
\hline nmb32-20-17ddd & $5,513.0$ & $5,515.0$ & $324 \mathrm{PRDX}$ & 1.2 & 48.000000 & Limestone \\
\hline $\mathrm{nmb} 32-20-17 \mathrm{ddd}$ & $5,515.0$ & $5,517.0$ & $324 \mathrm{PRDX}$ & 1.3 & 3.000000 & Limestone \\
\hline$n m b 32-20-17 d d d$ & $5,517.0$ & $5,519.0$ & $324 \mathrm{PRDX}$ & 0.6 & 0.000000 & Limes tone \\
\hline nmb32-20-17ddd & $5,519.0$ & $5,521.0$ & $324 \mathrm{PRDX}$ & 0.9 & 0.000000 & Limestone \\
\hline nmb32-20-17ddd & $5,521.0$ & $5,523.0$ & $324 \mathrm{PRDX}$ & 1.5 & 0.500000 & Limestone \\
\hline nmb32-20-17ddd & $5,523.0$ & $5,525.0$ & $324 \mathrm{PRDX}$ & 2.2 & 0.900000 & Limestone \\
\hline$n m b 32-20-17 d d d$ & $5,525.0$ & $5,527.0$ & 324PRDX & 1.1 & 0.000000 & Limestone \\
\hline $\mathrm{nmb} 32-20-17 \mathrm{ddd}$ & $5,586.0$ & $5,588.0$ & $324 \mathrm{PRDX}$ & 3.2 & 1.000000 & Dolomite and shaly dolomite \\
\hline nmb32-20-17ddd & $5,588.0$ & $5,590.0$ & $324 \mathrm{PRDX}$ & 2.3 & 0.000000 & Anhydrite and dolomite \\
\hline nmb32-20-17ddd & $5,590.0$ & $5,592.0$ & $324 \mathrm{PRDX}$ & 9.8 & 0.600000 & Dolomite and anhydrite \\
\hline nmb32-20-17ddd & $5,592.0$ & $5,594.0$ & 324PRDX & 8.9 & 0.400000 & Dolomite and anhydrite \\
\hline nmb32-20-17ddd & $5,838.0$ & $5,840.0$ & $324 \mathrm{PRDX}$ & 1.6 & 3.100000 & Shaly dolomite \\
\hline nmb32-20-17ddd & $5,840.0$ & $5,842.0$ & $324 \mathrm{PRDX}$ & 3.4 & 0.300000 & Shaly dolomite \\
\hline$n m b 32-20-17 d d d$ & $5,842.0$ & $5,844.0$ & $324 \mathrm{PRDX}$ & 5.5 & 0.200000 & Shaly dolomite \\
\hline nmb32-20-17ddd & $5,844.0$ & $5,846.0$ & $324 \mathrm{PRDX}$ & 5.0 & 0.000000 & Shaly dolomite \\
\hline nmb32-20-17ddd & $5,846.0$ & $5,848.0$ & 324PRDX & 2.0 & 0.100000 & Shaly dolomite \\
\hline nmb32-20-17ddd & $5,848.0$ & $5,850.0$ & 324PRDX & 5.1 & 0.100000 & Shaly dolomite \\
\hline nmb32-20-17ddd & $5,850.0$ & $5,852.0$ & $324 \mathrm{PRDX}$ & 1.9 & 0.900000 & Shaly dolomite \\
\hline nmb32-20-17ddd & $5,852.0$ & $5,854.0$ & 324PRDX & 3.2 & 1.100000 & Shaly dolomite \\
\hline nmb32-20-17ddd & $5,861.0$ & $5,863.0$ & 324 PRDX & 1.4 & 0.200000 & Dolomite \\
\hline nmb32-20-17ddd & $5,863.0$ & $5,865.0$ & 324PRDX & 3.1 & 0.000000 & Dolomite \\
\hline $\mathrm{nmb} 32-20-17 \mathrm{ddd}$ & $5,865.0$ & $5,867.0$ & 324PRDX & 2.6 & 6.300000 & Dolomite \\
\hline $\mathrm{nmb} 32-20-17 \mathrm{ddd}$ & $5,867.0$ & $5,869.0$ & $324 \mathrm{PRDX}$ & 2.8 & 5.800000 & Dolomite \\
\hline nmb32-20-17ddd & $5,932.0$ & $5,933.5$ & $324 \mathrm{PRDX}$ & 2.7 & 20.000000 & Dolomite \\
\hline nmb32-20-17ddd & $5,933.5$ & $5,935.0$ & $324 \mathrm{PRDX}$ & 0.6 & 0.200000 & Dolomite \\
\hline nmb $32-20-17 d d d$ & $5,935.0$ & $5,936.5$ & 324PRDX & 0.7 & 0.200000 & Dolomite \\
\hline $\mathrm{nmb} 32-20-17 \mathrm{ddd}$ & $5,936.5$ & $5,938.0$ & 324PRDX & 0.9 & 37.000000 & Dolomite \\
\hline nmb $32-20-17 d d d$ & $5,938.0$ & $5,939.5$ & $324 \mathrm{PRDX}$ & 1.3 & 0.200000 & Dolomite \\
\hline$n m b 32-20-17 d d d$ & $5,939.5$ & $5,941.0$ & $324 \mathrm{PRDX}$ & 4.6 & 0.300000 & Dolomite \\
\hline nmb32-20-17ddd & $5,941.0$ & $5,942.5$ & $324 \mathrm{PRDX}$ & 1.5 & 0.100000 & Dolomite \\
\hline $\mathrm{nmb} 32-20-17 \mathrm{ddd}$ & $5,942.5$ & $5,944.0$ & 324PRDX & 0.7 & 0.000000 & Dolomite \\
\hline $\mathrm{nmb} 32-20-17 \mathrm{ddd}$ & $5,944.0$ & $5,945.5$ & 324PRDX & 0.9 & 0.000000 & Dolomite \\
\hline $\mathrm{nmb} 32-20-17 \mathrm{ddd}$ & $5,945.5$ & $5,947.0$ & $324 \mathrm{PRDX}$ & 1.3 & 0.800000 & Dolomite \\
\hline $\mathrm{nmb} 32-20-17 \mathrm{ddd}$ & $5,947.0$ & $5,948.5$ & 324PRDX & 0.5 & 15.000000 & Dolomite \\
\hline $\mathrm{nmb} 32-20-17 \mathrm{ddd}$ & $5,948.5$ & $5,950.0$ & 324PRDX & 2.4 & 3.100000 & Dolomite \\
\hline $\mathrm{nmb} 32-20-17 \mathrm{ddd}$ & $5,950.0$ & $5,951.5$ & 324PRDX & 0.8 & 0.200000 & Dolomite \\
\hline $\mathrm{nmb} 32-20-17 \mathrm{ddd}$ & $5,951.5$ & $5,953.0$ & 324PRDX & 0.4 & 2.800000 & Dolomite \\
\hline $\mathrm{nmb} 32-20-17 \mathrm{ddd}$ & $5,953.0$ & $5,954.5$ & $324 \mathrm{PRDX}$ & 1.9 & 0.000000 & Dolomite \\
\hline $\mathrm{nmb} 32-20-17 \mathrm{ddd}$ & $5,954.5$ & $5,956.0$ & $324 \mathrm{PRDX}$ & 0.4 & 0.000000 & Dolomite \\
\hline $\mathrm{nmb} 32-20-17 \mathrm{ddd}$ & $5,956.0$ & $5,957.5$ & $324 \mathrm{PRDX}$ & 0.3 & 0.000000 & Dolomite \\
\hline nmb32-20-17ddd & $5,957.5$ & $5,959.0$ & 324PRDX & 0.4 & 0.000000 & Dolomite \\
\hline$n m b 32-20-17 d d d$ & $5,959.0$ & $5,960.5$ & $324 \mathrm{PRDX}$ & 0.6 & 0.000000 & Dolomite \\
\hline $\mathrm{nmb} 32-20-17 \mathrm{ddd}$ & $5,960.5$ & $5,962.0$ & $324 \mathrm{PRDX}$ & 3.1 & 0.100000 & Dolomite \\
\hline $\mathrm{nmb} 32-20-17 \mathrm{ddd}$ & $5,962.0$ & $5,963.5$ & 324 PRDX & 1.6 & 0.300000 & Dolomite \\
\hline $\mathrm{nmb} 32-20-17 \mathrm{ddd}$ & $5,963.5$ & $5,965.0$ & $324 \mathrm{PRDX}$ & 1.6 & 0.100000 & Dolomite \\
\hline $\mathrm{nmb} 32-20-17 \mathrm{ddd}$ & $5,965.0$ & $5,966.5$ & 324PRDX & 1.2 & 0.300000 & Dolomite \\
\hline
\end{tabular}


Table 4.--Laboratory-determined porosity and permeability--Continued

\begin{tabular}{|c|c|c|c|c|c|c|c|}
\hline Site & $\begin{array}{l}\text { Depth to } \\
\text { top } \\
\text { (feet) }\end{array}$ & $\begin{array}{l}\text { Depth to } \\
\text { bottom } \\
\text { (feet) }\end{array}$ & $\begin{array}{c}\text { Forma- } \\
\text { tion }\end{array}$ & $\begin{array}{l}\text { Porosity } \\
\text { (percent) }\end{array}$ & $\begin{array}{l}\text { Perme- } \\
\text { ability } \\
\text { (milli- } \\
\text { darcies) }\end{array}$ & & Lithology \\
\hline nmb32-20-17ddd & $6,540.0$ & $6,541.5$ & 337RDLL & 1.1 & 3.800000 & Limestone & \\
\hline nmb32-20-17ddd & $6,541.5$ & $6,543.0$ & $337 \mathrm{RDLL}$ & 0.8 & 1.100000 & Limes tone & \\
\hline nmb $32-20-17 \mathrm{ddd}$ & $6,543.0$ & $6,544.5$ & 337RDLL & 0.9 & 0.300000 & Limestone & \\
\hline nmb32-20-17ddd & $6,544.5$ & $6,546.0$ & $337 \mathrm{RDLL}$ & 1.0 & 39.000000 & Limes tone & \\
\hline nmb32-20-17ddd & $6,546.0$ & $6,547.5$ & 337RDLL & 1.3 & 0.100000 & Limestone & \\
\hline $\mathrm{nmb} 32-20-17 \mathrm{ddd}$ & $6,547.5$ & $6,549.0$ & 337RDLL & 0.9 & 0.100000 & Limestone & \\
\hline nmb32-20-17ddd & $6,549.0$ & $6,550.5$ & $337 \mathrm{RDLL}$ & 1.8 & 0.200000 & Limestone & \\
\hline nmb32-20-17ddd & $6,550.5$ & $6,552.0$ & 337RDLL & 1.6 & 0.000000 & Limestone & \\
\hline nmb32-20-17ddd & $6,552.0$ & $6,553.5$ & 337RDLL & 1.2 & 0.000000 & Limestone & \\
\hline nmb32-20-17ddd & $6,553.5$ & $6,555.0$ & 337RDLL & 0.8 & 0.200000 & Limes tone & \\
\hline nmb32-20-17ddd & $6,555.0$ & $6,556.5$ & 337RDLL & 0.7 & 0.000000 & Limestone & \\
\hline nmb32-20-17ddd & $6,556.5$ & $6,558.0$ & 337RDLL & 0.9 & 0.000000 & Limestone & \\
\hline nmb32-20-17ddd & $6,558.0$ & $6,559.5$ & 337 RDLL & 1.4 & 0.600000 & Limestone & \\
\hline nmb32-20-17ddd & $6,559.5$ & $6,561.0$ & $337 \mathrm{RDLL}$ & 1.9 & 0.300000 & Limestone & \\
\hline nmb32-20-17ddd & $6,561.0$ & $6,562.5$ & 337RDLL & 2.0 & 0.000000 & Limestone & \\
\hline nmb32-20-17ddd & $6,562.5$ & $6,564.0$ & 337RDLL & 2.0 & 0.200000 & Limestone & \\
\hline nmb32-20-17ddd & $6,564.0$ & $6,565.5$ & 337 RDLL & 1.9 & 0.200000 & Limestone & \\
\hline nmb32-20-17ddd & $6,565.5$ & $6,567.0$ & $337 \mathrm{RDLL}$ & 1.3 & 0.000000 & Limestone & \\
\hline nmb32-20-17ddd & $6,567.0$ & $6,568.5$ & 337RDLL & 1.9 & 0.000000 & Limestone & \\
\hline nmb32-20-17ddd & $6,568.5$ & $6,570.0$ & 337RDLL & 1.6 & 0.200000 & Limestone & \\
\hline nmb32-20-17ddd & $6,570.0$ & $6,571.5$ & 337RDLL & 1.4 & 2.100000 & Limestone & \\
\hline nmb32-20-17ddd & $6,571.5$ & $6,573.0$ & 337 RDLL & 1.0 & 0.200000 & Limes tone & \\
\hline nmb32-20-17ddd & $6,573.0$ & $6,574.5$ & 337 RDLL & 1.4 & 0.300000 & Limestone & \\
\hline nmb32-20-17ddd & $6,574.5$ & $6,576.0$ & 337RDLL & 1.3 & 0.000000 & Limestone & \\
\hline nmb32-20-17ddd & $6,576.0$ & $6,577.5$ & 337RDLL & 0.6 & 0.300000 & Limestone & \\
\hline nmb32-20-17ddd & $6,577.5$ & $6,579.0$ & 337RDLL & 1.1 & 0.200000 & Limestone & \\
\hline nmb32-20-17ddd & $6,579.0$ & $6,580.5$ & 337RDLL & 0.6 & 3.800000 & Limestone & \\
\hline nmb $32-20-17 \mathrm{ddd}$ & $6,580.5$ & $6,582.0$ & 337RDLL & 1.2 & 1.300000 & Limestone & \\
\hline nmb $32-20-17 d d d$ & $6,582.0$ & $6,583.5$ & 337RDLL & 1.6 & 6.000000 & Limestone & \\
\hline nmb32-20-17ddd & $6,583.5$ & $6,585.0$ & 337RDLL & 1.2 & 1.000000 & Limestone & \\
\hline nmb32-20-17ddd & $6,585.0$ & $6,586.5$ & 337RDLL & 1.1 & 0.200000 & Limestone & \\
\hline nmb32-20-17ddd & $6,586.5$ & $6,588.0$ & 337RDLL & 1.2 & 0.000000 & Limestone & \\
\hline nmb32-20-17ddd & $6,588.0$ & $6,590.0$ & 337RDLL & 1.1 & 0.300000 & Limestone & \\
\hline nmb32-20-17ddd & $6,590.0$ & $6,592.0$ & 337 RDLL & 0.8 & 0.100000 & Limestone & and dolomite \\
\hline nmb32-20-17ddd & $6,592.0$ & $6,594.0$ & 337RDLL & 1.0 & 0.600000 & Limestone & and dolomite \\
\hline nmb32-20-17ddd & $6,594.0$ & $6,596.0$ & 337RDLL & 1.6 & 0.100000 & Limestone & and dolomite \\
\hline nmb32-20-17ddd & $6,596.0$ & $6,598.0$ & 337RDLL & 2.1 & 1.300000 & Limestone & and dolomite \\
\hline nmb32-20-17ddd & $6,598.0$ & $6,600.0$ & 337RDLL & 2.6 & 0.200000 & Limestone & and dolomite \\
\hline nmb32-20-17ddd & $6,600.0$ & $6,602.0$ & 337 RDLL & 2.1 & 0.100000 & Limes tone & and dolomite \\
\hline nmb32-20-17ddd & $6,602.0$ & $6,604.0$ & 337RDLL & 1.5 & 0.500000 & Limestone & and dolomite \\
\hline nmb32-20-17ddd & $6,604.0$ & $6,606.0$ & 337RDLL & 1.2 & 0.400000 & Limestone & and dolomite \\
\hline nmb32-20-17ddd & $6,606.0$ & $6,608.0$ & 337RDLL & 1.3 & 1.900000 & Limestone & and dolomite \\
\hline nmb32-20-17ddd & $6,608.0$ & $6,610.0$ & 337RDLL & 1.6 & 0.700000 & Limestone & and dolomite \\
\hline nmb32-20-17ddd & $6,610.0$ & $6,612.0$ & 337 RDLL & 1.2 & 1.100000 & Limestone & and dolomite \\
\hline nmb32-20-17ddd & $6,612.0$ & $6,614.0$ & 337RDLL & 1.8 & 0.100000 & Limestone & and dolomite \\
\hline nmb32-20-17ddd & $6,614.0$ & $6,616.0$ & 337 RDLL & 3.1 & 0.200000 & Dolomite & \\
\hline nmb $32-20-17 d d d$ & $6,616.0$ & $6,618.0$ & 337 RDLL & 6.3 & 14.000000 & Dolomite & \\
\hline nmb32-20-17ddd & $6,618.0$ & $6,620.0$ & 337RDLL & 10.0 & 0.200000 & Dolomite & \\
\hline nmb32-20-17ddd & $6,620.0$ & $6,622.0$ & 337RDLL & 1.7 & 0.100000 & Dolomite & \\
\hline nmb32-20-17ddd & $6,622.0$ & $6,624.0$ & 337RDLL & 2.3 & 48.000000 & Dolomite & \\
\hline
\end{tabular}


Table 4.--Laboratory-determined porosity and permeability--Continued

\begin{tabular}{|c|c|c|c|c|c|c|}
\hline Site & $\begin{array}{l}\text { Depth to } \\
\text { top } \\
\text { (feet) }\end{array}$ & $\begin{array}{l}\text { Depth to } \\
\text { bottom } \\
\text { (feet) }\end{array}$ & $\begin{array}{l}\text { Forma- } \\
\text { tion }\end{array}$ & $\begin{array}{l}\text { Porosity } \\
\text { (percent) }\end{array}$ & $\begin{array}{l}\text { Perme- } \\
\text { ability } \\
\text { (milli- } \\
\text { darcies) }\end{array}$ & Lithology \\
\hline $\begin{array}{l}\text { nmb32-20-17ddd } \\
\text { nmb32-20-17ddd } \\
\text { nmb32-20-17ddd } \\
\text { nmb32-20-17ddd } \\
\text { nmb32-20-17ddd }\end{array}$ & $\begin{array}{l}6,624.0 \\
6,626.0 \\
6,628.0 \\
6,630.0 \\
6,632.0\end{array}$ & $\begin{array}{l}6,626.0 \\
6,628.0 \\
6,630.0 \\
6,632.0 \\
6,634.0\end{array}$ & $\begin{array}{l}\text { 337RDLL } \\
\text { 337RDLL } \\
\text { 337RDLL } \\
\text { 337RDLL } \\
\text { 337RDLL }\end{array}$ & $\begin{array}{l}3.6 \\
5.2 \\
7.1 \\
8.4 \\
6.7\end{array}$ & $\begin{array}{r}0.200000 \\
0.900000 \\
0.200000 \\
1.400000 \\
19.000000\end{array}$ & $\begin{array}{l}\text { Dolomite } \\
\text { Dolomite } \\
\text { Dolomite } \\
\text { Dolomite } \\
\text { Dolomite }\end{array}$ \\
\hline $\begin{array}{l}\text { nmb32-20-17ddd } \\
\text { nmb32-20-17ddd } \\
\text { nmb32-20-17ddd } \\
\text { nmb32-20-17ddd } \\
\text { nmb32-20-17ddd }\end{array}$ & $\begin{array}{l}6,634.0 \\
6,636.0 \\
6,638.0 \\
6,640.0 \\
6,642.0\end{array}$ & $\begin{array}{l}6,636.0 \\
6,638.0 \\
6,640.0 \\
6,642.0 \\
6,644.0\end{array}$ & $\begin{array}{l}\text { 337RDLL } \\
\text { 337RDLL } \\
\text { 337RDLL } \\
\text { 337RDLL } \\
\text { 337RDLL }\end{array}$ & $\begin{array}{r}2.4 \\
3.9 \\
8.6 \\
10.4 \\
0.8\end{array}$ & $\begin{array}{l}0.400000 \\
0.100000 \\
0.500000 \\
4.700000 \\
4.900000\end{array}$ & $\begin{array}{l}\text { Dolomite } \\
\text { Dolomite } \\
\text { Dolomite } \\
\text { Dolomite } \\
\text { Dolomite }\end{array}$ \\
\hline $\begin{array}{l}\text { nmb32-20-17ddd } \\
\text { nmb32-20-17ddd } \\
\text { nmb32-20-17ddd } \\
\text { nmb32-20-17ddd } \\
\text { nmb32-20-17ddd }\end{array}$ & $\begin{array}{l}6,644.0 \\
6,646.0 \\
6,648.0 \\
6,650.0 \\
6,652.0\end{array}$ & $\begin{array}{l}6,646.0 \\
6,648.0 \\
6,650.0 \\
6,652.0 \\
6,654.0\end{array}$ & $\begin{array}{l}\text { 337RDLL } \\
\text { 337RDLL } \\
\text { 337RDLL } \\
\text { 337RDLL } \\
\text { 337RDLL }\end{array}$ & $\begin{array}{l}0.9 \\
1.4 \\
2.1 \\
0.6 \\
0.5\end{array}$ & $\begin{array}{r}0.200000 \\
115.000000 \\
0.200000 \\
0.400000 \\
0.300000\end{array}$ & $\begin{array}{l}\text { Dolomite } \\
\text { Dolomite } \\
\text { Dolomite } \\
\text { Dolomite } \\
\text { Dolomite }\end{array}$ \\
\hline $\begin{array}{l}\text { nmb32-20-17ddd } \\
\text { nmb32-20-17ddd } \\
\text { nmb32-20-17ddd } \\
\text { nmb32-20-17ddd } \\
\text { nmb32-20-17ddd }\end{array}$ & $\begin{array}{l}6,654.0 \\
6,656.0 \\
6,658.0 \\
6,660.0 \\
6,662.0\end{array}$ & $\begin{array}{l}6,656.0 \\
6,658.0 \\
6,660.0 \\
6,662.0 \\
6,664.0\end{array}$ & $\begin{array}{l}\text { 337RDLL } \\
\text { 337RDLL } \\
\text { 337RDLL } \\
\text { 337RDLL } \\
\text { 337RDLL }\end{array}$ & $\begin{array}{l}0.4 \\
0.5 \\
0.4 \\
0.6 \\
0.3 \\
\end{array}$ & $\begin{array}{l}0.000000 \\
0.000000 \\
0.200000 \\
0.000000 \\
0.000000 \\
\end{array}$ & $\begin{array}{l}\text { Dolomite } \\
\text { Dolomite } \\
\text { Dolomite } \\
\text { Dolomite } \\
\text { Dolomite }\end{array}$ \\
\hline SITE AVERAGE--- & ------ & $--1--1$ & AVERAGE-- & $\frac{2.1}{2.2}$ & $\frac{4.002857}{6.298011}$ & \\
\hline nmb33-14-08cdc & $8,674.0$ & $8,674.1$ & $\begin{array}{l}\text { 321HKTL } \\
\text { AVERAGE-- }\end{array}$ & $\frac{4.1}{4.1}$ & $\frac{0.000000}{0.000000}$ & Siltstone \\
\hline nmb33-14-08cdc & $9,206.0$ & $9,207.0$ & 324PRDX & 3.1 & 0.000000 & $\begin{array}{l}\text { Very fine-grained sandstone } \\
\text { with salt veins }\end{array}$ \\
\hline nmb33-14-08cdc & $9,208.0$ & $9,209.0$ & $324 \mathrm{PRDX}$ & 2.5 & 0.000000 & $\begin{array}{l}\text { Very fine-grained sandstone } \\
\text { with salt veins }\end{array}$ \\
\hline nmb33-14-08cdc & $9,281.0$ & $9,282.0$ & $324 \mathrm{PRDX}$ & 6.2 & $\underline{0.000000}$ & Crystalline dolomite \\
\hline SITE AVERAGE-- & & & AVERAGE-- & $\frac{3.9}{4.0}$ & $\frac{0.000000}{0.000000}$ & \\
\hline $\begin{array}{l}\text { nmb33-18-22ddd } \\
\text { nmb33-18-22ddd } \\
\text { nmb33-18-22ddd } \\
\text { nmb33-18-22ddd } \\
\text { nmb33-18-22ddd }\end{array}$ & $\begin{array}{l}6,466.0 \\
6,467.0 \\
6,468.0 \\
6,469.0 \\
6,473.0\end{array}$ & $\begin{array}{l}6,467.0 \\
6,468.0 \\
6,469.0 \\
6,470.0 \\
6,474.0\end{array}$ & $\begin{array}{l}324 \mathrm{PRDX} \\
324 \mathrm{PRDX} \\
324 \mathrm{PRDX} \\
324 \mathrm{PRDX} \\
324 \mathrm{PRDX}\end{array}$ & $\begin{array}{l}1.1 \\
0.8 \\
1.2 \\
2.2 \\
1.7\end{array}$ & $\begin{array}{l}0.000000 \\
0.000000 \\
0.000000 \\
0.000000 \\
0.000000\end{array}$ & $\begin{array}{l}\text { Fractured dolomite } \\
\text { Dolomite } \\
\text { Dolomite } \\
\text { Dolomite } \\
\text { Limestone }\end{array}$ \\
\hline $\begin{array}{l}\text { nmb33-18-22ddd } \\
\text { nmb33-18-22ddd } \\
\text { nmb33-18-22ddd } \\
\text { nmb33-18-22ddd } \\
\text { nmb33-18-22ddd }\end{array}$ & $\begin{array}{l}6,474.0 \\
6,480.0 \\
6,481.0 \\
6,483.0 \\
6,484.0\end{array}$ & $\begin{array}{l}6,475.0 \\
6,481.0 \\
6,482.0 \\
6,484.0 \\
6,485.0\end{array}$ & $\begin{array}{l}324 \mathrm{PRDX} \\
324 \mathrm{PRDX} \\
324 \mathrm{PRDX} \\
324 \mathrm{PRDX} \\
324 \mathrm{PRDX}\end{array}$ & $\begin{array}{l}1.7 \\
4.3 \\
3.5 \\
3.6 \\
4.0\end{array}$ & $\begin{array}{l}0.000000 \\
0.000000 \\
0.010000 \\
0.000000 \\
0.000000\end{array}$ & $\begin{array}{l}\text { Limestone } \\
\text { Dolomite } \\
\text { Dolomite } \\
\text { Dolomite and anhydrite } \\
\text { Dolomite }\end{array}$ \\
\hline $\begin{array}{l}\text { nmb33-18-22ddd } \\
\text { SITE AVERAGE--- }\end{array}$ & $6,487.0$ & $6,488.0$ & $\begin{array}{l}\text { 324PRDX } \\
\text { AVERAGE-- }\end{array}$ & $\frac{2.1}{\frac{2.4}{2.4}}$ & $\frac{0.010000}{\frac{0.001818}{0.001818}}$ & Dolomite \\
\hline $\begin{array}{l}\text { nmb33-20-04ddd } \\
\text { nmb33-20-04ddd } \\
\text { nmb33-20-04ddd } \\
\text { nmb33-20-04ddd } \\
\text { nmb33-20-04ddd }\end{array}$ & $\begin{array}{l}6,002 \cdot 0 \\
6,003 \cdot 0 \\
6,004.0 \\
6,005.0 \\
6,006.0\end{array}$ & $\begin{array}{l}6,003 \cdot 0 \\
6,004 \cdot 0 \\
6,005 \cdot 0 \\
6,006.0 \\
6,007.0\end{array}$ & $\begin{array}{l}324 \mathrm{PRDX} \\
324 \mathrm{PRDX} \\
324 \mathrm{PRDX} \\
324 \mathrm{PRDX} \\
324 \mathrm{PRDX}\end{array}$ & $\begin{array}{r}10.8 \\
8.7 \\
8.5 \\
6.1 \\
8.1\end{array}$ & $\begin{array}{r}39.000000 \\
22.000000 \\
4.800000 \\
67.000000 \\
0.080000\end{array}$ & $\begin{array}{l}\text { Vuggy, medium- to coarsely crystalline limestone } \\
\text { Vuggy, medium- to coarsely crystalline limestone } \\
\text { Vuggy, medium- to coarsely crystalline limestone } \\
\text { Vuggy, medium- to coarsely crystalline limestone } \\
\text { Medium- to coarsely crystalline limestone }\end{array}$ \\
\hline
\end{tabular}


Table 4.--Laboratory-determined porosity and permeability--Continued

\begin{tabular}{|c|c|c|c|c|c|c|}
\hline Site & $\begin{array}{l}\text { Depth to } \\
\text { top } \\
\text { (feet) }\end{array}$ & $\begin{array}{l}\text { Depth to } \\
\text { bottom } \\
\text { (feet) }\end{array}$ & $\begin{array}{c}\text { Forma- } \\
\text { tion }\end{array}$ & $\begin{array}{l}\text { Porosity } \\
\text { (percent) }\end{array}$ & $\begin{array}{l}\text { Perme- } \\
\text { ability } \\
\text { (milli- } \\
\text { darcies) }\end{array}$ & Lithology \\
\hline $\mathrm{nmb} 33-20-04 \mathrm{ddd}$ & $6,011.0$ & $6,012.0$ & $324 \mathrm{PRDX}$ & 5.8 & 0.710000 & Vuggy, anhydritic, medium-crystalline limestone \\
\hline nmb33-20-04ddd & $6,012.0$ & $6,013.0$ & $324 \mathrm{PRDX}$ & 14.0 & 10.000000 & Vuggy, coarsely crystalline limestone \\
\hline nmb33-20-04ddd & $6,013.0$ & $6,014.0$ & $324 \mathrm{PRDX}$ & 13.0 & 2.900000 & Medium-crystalline limestone \\
\hline $\mathrm{nmb} 33-20-04 \mathrm{ddd}$ & $6,014.0$ & $6,015.0$ & $324 \mathrm{PRDX}$ & 14.7 & 7.900000 & Medium-crystalline limestone \\
\hline nmb33-20-04ddd & $6,015.0$ & $6,016.0$ & 324PRDX & 9.8 & 12.000000 & Anhydritic, medium-crystalline limestone \\
\hline nmb33-20-04ddd & $6,016.0$ & $6,017.0$ & 324PRDX & 11.0 & 0.020000 & Vuggy, anhydritic, fine-grained limestone \\
\hline nmb 33-20-04ddd & $6,017.0$ & $6,018.0$ & $324 \mathrm{PRDX}$ & 2.7 & 0.000000 & Fine-grained limestone \\
\hline & & & AVERAGE - - & 9.4 & 13.867500 & \\
\hline SITE AVERAGE-- & - & - & $\cdots-\cdots-1$ & 9.4 & $\overline{13.867500}$ & \\
\hline$n m b 33.5-12-29 \mathrm{dbd}$ & $7,657.0$ & $7,658.0$ & $321 \mathrm{HKTL}$ & 0.5 & 0.060000 & Limestone \\
\hline$n m b 33.5-12-29 \mathrm{dbd}$ & $7,658.0$ & $7,659.0$ & 321HKTL & 3.0 & 0.050000 & Limestone \\
\hline$n m b 33.5-12-29 \mathrm{dbd}$ & $7,659.0$ & $7,660.0$ & $321 \mathrm{HKTL}$ & 0.6 & 0.040000 & Limestone \\
\hline $\mathrm{nmb} 33.5-12-29 \mathrm{dbd}$ & $7,660.0$ & $7,661.0$ & $321 \mathrm{HKTL}$ & 1.6 & 0.020000 & Limestone \\
\hline $\mathrm{nmb} 33.5-12-29 \mathrm{dbd}$ & $7,661.0$ & $7,662.0$ & $321 \mathrm{HKTL}$ & 1.1 & 0.040000 & Limestone \\
\hline $\mathrm{nmb} 33.5-12-29 \mathrm{dbd}$ & $7,662.0$ & $7,663.0$ & 321HKTL & 1.8 & 0.050000 & Limestone \\
\hline $\mathrm{nmb} 33.5-12-29 \mathrm{dbd}$ & $7,663.0$ & $7,664.0$ & $321 \mathrm{HKTL}$ & 1.1 & 0.020000 & Limestone \\
\hline $\mathrm{nmb} 33.5-12-29 \mathrm{dbd}$ & $7,664.0$ & $7,665.0$ & $321 \mathrm{HKTL}$ & 0.8 & 0.040000 & Limestone \\
\hline$n m b 33.5-12-29 d b d$ & $7,665.0$ & $7,666.0$ & 321HKTL & 2.0 & 0.040000 & Limestone \\
\hline nmb33.5-12-29dbd & $7,666.0$ & $7,667.0$ & 321HKTL & 0.9 & 0.060000 & Limestone \\
\hline nmb33.5-12-29dbd & $7,667.0$ & $7,668.0$ & $321 \mathrm{HKTL}$ & 1.5 & 0.040000 & Limestone \\
\hline nmb33.5-12-29dbd & $7,668.0$ & $7,669.0$ & $321 \mathrm{HKTL}$ & 1.8 & 0.030000 & Limestone \\
\hline nmb33.5-12-29dbd & $7,669.0$ & $7,670.0$ & $321 \mathrm{HKTL}$ & 0.7 & 0.050000 & Limestone \\
\hline nmb33.5-12-29dbd & $7,670.0$ & $7,671.0$ & $321 \mathrm{HKTL}$ & 1.2 & 0.040000 & Limestone \\
\hline$n m b 33.5-12-29 d b d$ & $7,671.0$ & $7,672.0$ & 321HKTL & 0.8 & 0.060000 & Limestone and shale \\
\hline$n m b 33.5-12-29 d b d$ & $7,672.0$ & $7,673.0$ & $321 \mathrm{HKTL}$ & 0.8 & 0.020000 & Shale \\
\hline nmb33.5-12-29dbd & $7,676.0$ & $7,677.0$ & $321 \mathrm{HKTL}$ & 2.7 & 0.020000 & Sandstone \\
\hline$n m b 33.5-12-29 d b d$ & $7,677.0$ & $7,678.0$ & $321 \mathrm{HKTL}$ & 3.8 & 0.080000 & Sandstone \\
\hline nmb33.5-12-29dbd & $7,678.0$ & $7,679.0$ & $321 \mathrm{HKTL}$ & 3.7 & 0.020000 & Sandstone \\
\hline nmb33.5-12-29dbd & $7,679.0$ & $7,680.0$ & $321 \mathrm{HKTL}$ & 5.3 & 0.480000 & Sandstone \\
\hline$n m b 33.5-12-29 d b d$ & $7,680.0$ & $7,681.0$ & $321 \mathrm{HKTL}$ & 12.5 & 1.800000 & Sands tone \\
\hline nmb33.5-12-29dbd & $7,681.0$ & $7,682.0$ & $321 \mathrm{HKTL}$ & 13.7 & 1.400000 & Sandstone \\
\hline nmb33.5-12-29dbd & $7,682.0$ & $7,683.0$ & $321 \mathrm{HKTL}$ & 16.5 & 0.800000 & Sandstone \\
\hline $\mathrm{nmb} 33.5-12-29 \mathrm{dbd}$ & $7,683.0$ & $7,684.0$ & $321 \mathrm{HKTL}$ & 18.3 & 1.100000 & Sandstone \\
\hline nmb33.5-12-29dbd & $7,684.0$ & $7,685.0$ & $321 \mathrm{HKTL}$ & 19.1 & 7.200000 & Sandstone \\
\hline$n m b 33.5-12-29 \mathrm{dbd}$ & $7,689.0$ & $7,690.0$ & 321HKTL & 16.0 & 8.700000 & Sandstone \\
\hline nmb33.5-12-29dbd & $7,690.0$ & $7,691.0$ & $321 \mathrm{HKTL}$ & 13.1 & 0.800000 & Sandstone \\
\hline$n m b 33.5-12-29 d b d$ & $7,691.0$ & $7,692.0$ & $321 \mathrm{HKTL}$ & 16.4 & 5.500000 & Sands tone \\
\hline $\mathrm{nmb} 33.5-12-29 \mathrm{dbd}$ & $7,692.0$ & $7,693.0$ & $321 \mathrm{HKTL}$ & 17.7 & 10.000000 & Sandstone \\
\hline$n m b 33.5-12-29 d b d$ & $7,693.0$ & $7,694.0$ & $321 \mathrm{HKTL}$ & 17.6 & 2.800000 & Sandstone \\
\hline$n m b 33.5-12-29 d b d$ & $7,694.0$ & $7,695.0$ & $321 \mathrm{HKTL}$ & 18.6 & 6.100000 & Sandstone \\
\hline $\mathrm{nmb} 33.5-12-29 \mathrm{dbd}$ & $7,695.0$ & $7,696.0$ & 321 HKTL & 17.3 & 3.000000 & Sandstone \\
\hline $\mathrm{nmb} 33.5-12-29 \mathrm{dbd}$ & $7,696.0$ & $7,697.0$ & $321 \mathrm{HKTL}$ & 17.5 & 15.000000 & Sandstone \\
\hline$n m b 33.5-12-29 d b d$ & $7,697.0$ & $7,698.0$ & $321 \mathrm{HKTL}$ & 20.0 & 25.000000 & Sandstone \\
\hline nmb33.5-12-29dbd & $7,698.0$ & $7,699.0$ & $321 \mathrm{HKTL}$ & 18.4 & 10.000000 & Sandstone \\
\hline$n m b 33.5-12-29 d b d$ & $7,699.0$ & $7,700.0$ & $321 \mathrm{HKTL}$ & 18.5 & 13.000000 & Sandstone \\
\hline $\mathrm{nmb} 33.5-12-29 \mathrm{dbd}$ & $7,700.0$ & $7,701.0$ & $321 \mathrm{HKTL}$ & 13.9 & 11.000000 & Sands tone \\
\hline nmb33.5-12-29dbd & $7,701.0$ & $7,702.0$ & $321 \mathrm{HKTL}$ & 12.3 & 7.200000 & Sands tone \\
\hline nmb33.5-12-29dbd & $7,702.0$ & $7,703.0$ & $321 \mathrm{HKTL}$ & 7.0 & 0.520000 & Sands tone \\
\hline$n m b 33.5-12-29 d b d$ & $7,703.0$ & $7,704.0$ & 321HKTL & 2.3 & 0.030000 & Sandstone \\
\hline
\end{tabular}


Table 4.--Laboratory-determined porosity and permeability--Continued

\begin{tabular}{|c|c|c|c|c|c|c|}
\hline Site & $\begin{array}{l}\text { Depth to } \\
\text { top } \\
\text { (feet) }\end{array}$ & $\begin{array}{l}\text { Depth to } \\
\text { bottom } \\
\text { (feet) }\end{array}$ & $\begin{array}{c}\text { Forma- } \\
\text { tion }\end{array}$ & $\begin{array}{l}\text { Porosity } \\
\text { (percent) }\end{array}$ & $\begin{array}{l}\text { Perme- } \\
\text { ability } \\
\text { (milli- } \\
\text { darcies) }\end{array}$ & Lithology \\
\hline $\begin{array}{l}\text { nmb33.5-12-29dbd } \\
\text { nmb33.5-12-29dbd } \\
\text { nmb33.5-12-29dbd } \\
\text { nmb33.5-12-29dbd } \\
\text { nmb33.5-12-29dbd }\end{array}$ & $\begin{array}{l}7,704.0 \\
7,705.0 \\
7,706.0 \\
7,707.0 \\
7,708.0\end{array}$ & $\begin{array}{l}7,705.0 \\
7,706.0 \\
7,707.0 \\
7,708.0 \\
7,709.0\end{array}$ & $\begin{array}{l}321 \mathrm{HKTL} \\
321 \mathrm{HKTL} \\
321 \mathrm{HKTL} \\
321 \mathrm{HKTL} \\
321 \mathrm{HKTL}\end{array}$ & $\begin{array}{r}7.2 \\
11.9 \\
15.6 \\
16.3 \\
11.6\end{array}$ & $\begin{array}{l}0.080000 \\
0.170000 \\
0.190000 \\
0.330000 \\
0.340000\end{array}$ & $\begin{array}{l}\text { Sandstone } \\
\text { Sandstone } \\
\text { Sandstone } \\
\text { Sandstone } \\
\text { Sandstone }\end{array}$ \\
\hline $\begin{array}{l}\text { nmb33.5-12-29dbd } \\
\text { nmb33.5-12-29dbd } \\
\text { nmb33.5-12-29dbd } \\
\text { nmb33.5-12-29dbd }\end{array}$ & $\begin{array}{l}7,709.0 \\
7,710.0 \\
7,711.0 \\
7,712.0\end{array}$ & $\begin{array}{l}7,710.0 \\
7,711.0 \\
7,712.0 \\
7,713.0\end{array}$ & $\begin{array}{l}321 \mathrm{HKTL} \\
321 \mathrm{HKTL} \\
321 \mathrm{HKTL} \\
321 \mathrm{HKTL}\end{array}$ & $\begin{array}{r}15.6 \\
13.2 \\
14.2 \\
5.2 \\
\end{array}$ & $\begin{array}{l}0.280000 \\
0.560000 \\
0.750000 \\
0.070000 \\
\end{array}$ & $\begin{array}{l}\text { Sandstone } \\
\text { Sandstone } \\
\text { Sandstone } \\
\text { Sandstone }\end{array}$ \\
\hline & & & AVERAGE-- & 9.2 & 2.754694 & \\
\hline $\begin{array}{l}\text { nmb33.5-12-29dbd } \\
\text { nmb33.5-12-29dbd } \\
\text { nmb33.5-12-29dbd } \\
\text { nmb33.5-12-29dbd } \\
\text { nmb33.5-12-29dbd }\end{array}$ & $\begin{array}{l}9,400.0 \\
9,401.0 \\
9,402.0 \\
9,403.0 \\
9,404.0\end{array}$ & $\begin{array}{l}9,401.0 \\
9,402.0 \\
9,403.0 \\
9,404.0 \\
9,405.0\end{array}$ & $\begin{array}{l}\text { 324PKTL } \\
324 \mathrm{PKTL} \\
324 \mathrm{PKTL} \\
324 \mathrm{PKTL} \\
324 \mathrm{PKTL}\end{array}$ & $\begin{array}{l}0.3 \\
0.5 \\
0.3 \\
0.3 \\
0.5\end{array}$ & $\begin{array}{l}0.000000 \\
0.000000 \\
0.000000 \\
0.000000 \\
0.000000\end{array}$ & $\begin{array}{l}\text { Limestone } \\
\text { Limestone } \\
\text { Limestone } \\
\text { Dolomite } \\
\text { Dolomite }\end{array}$ \\
\hline $\begin{array}{l}\text { nmb33.5-12-29dbd } \\
\text { nmb33.5-12-29dbd } \\
\text { nmb33.5-12-29dbd } \\
\text { nmb33.5-12-29dbd } \\
\text { nmb33.5-12-29dbd }\end{array}$ & $\begin{array}{l}9,416.5 \\
9,418.4 \\
9,420.2 \\
9,422.0 \\
9,423.5\end{array}$ & $\begin{array}{l}9,418.4 \\
9,420.2 \\
9,422.0 \\
9,423.5 \\
9,425.0\end{array}$ & $\begin{array}{l}\text { 324PKTL } \\
324 \mathrm{PKTL} \\
324 \mathrm{PKTL} \\
324 \mathrm{PKTL} \\
324 \mathrm{PKTL}\end{array}$ & $\begin{array}{l}3.0 \\
6.5 \\
7.9 \\
6.7 \\
4.6 \\
\end{array}$ & $\begin{array}{r}0.100000 \\
2.900000 \\
45.000000 \\
18.000000 \\
17.000000 \\
\end{array}$ & $\begin{array}{l}\text { Dolomite } \\
\text { Dolomite } \\
\text { Dolomite } \\
\text { Dolomite } \\
\text { Dolomite }\end{array}$ \\
\hline SITE AVERAGE & - & 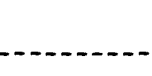 & AVERAGE-- & $\frac{3.1}{8.2}$ & $\frac{8.300000}{3.694576}$ & \\
\hline $\begin{array}{l}\text { nmb33.5-20-03aba } \\
\text { nmb33.5-20-03aba } \\
\text { nmb33.5-20-03aba } \\
\text { nmb33.5-20-03aba } \\
\text { nmb33.5 -20-03aba }\end{array}$ & $\begin{array}{l}5,901.0 \\
5,902.0 \\
5,903.0 \\
5,904.0 \\
5,905.0\end{array}$ & $\begin{array}{l}5,902.0 \\
5,903.0 \\
5,904.0 \\
5,905.0 \\
5,906.0\end{array}$ & $\begin{array}{l}324 \mathrm{PRDX} \\
324 \mathrm{PRDX} \\
324 \mathrm{PRDX} \\
324 \mathrm{PRDX} \\
324 \mathrm{PRDX}\end{array}$ & $\begin{array}{l}4.6 \\
3.0 \\
7.5 \\
8.8 \\
5.7\end{array}$ & $\begin{array}{l}0.000000 \\
0.040000 \\
0.000000 \\
0.000000 \\
0.000000\end{array}$ & $\begin{array}{l}\text { Dolomite } \\
\text { Dolomite } \\
\text { Dolomite } \\
\text { Dolomite } \\
\text { Dolomite }\end{array}$ \\
\hline $\begin{array}{l}\text { nmb33.5-20-03aba } \\
\text { nmb33.5-20-03aba } \\
\text { nmb33.5-20-03aba } \\
\text { nmb33.5-20-03aba } \\
\text { nmb33.5-20-03aba }\end{array}$ & $\begin{array}{l}5,906.0 \\
5,907.0 \\
5,908.0 \\
5,909.0 \\
5,910.0\end{array}$ & $\begin{array}{l}5,907.0 \\
5,908.0 \\
5,909.0 \\
5,910.0 \\
5,911.0\end{array}$ & $\begin{array}{l}\text { 324PRDX } \\
324 \mathrm{PRDX} \\
\text { 324PRDX } \\
324 \mathrm{PRDX} \\
324 \mathrm{PRDX}\end{array}$ & $\begin{array}{r}4.9 \\
9.0 \\
10.1 \\
11.1 \\
10.3\end{array}$ & $\begin{array}{l}0.000000 \\
0.000000 \\
0.000000 \\
0.000000 \\
0.000000\end{array}$ & $\begin{array}{l}\text { Dolomite } \\
\text { Dolomite } \\
\text { Dolomite } \\
\text { Dolomite } \\
\text { Dolomite }\end{array}$ \\
\hline $\begin{array}{l}\mathrm{nmb} 33.5-20-03 a \mathrm{aba} \\
\mathrm{nmb33} .5-20-03 a b a \\
\mathrm{nmb} 33.5-20-03 a b a \\
\text { nmb33.5-20-03aba } \\
\text { nmb33.5-20-03aba }\end{array}$ & $\begin{array}{l}5,911.0 \\
5,912.0 \\
5,913.0 \\
5,916.0 \\
5,917.0\end{array}$ & $\begin{array}{l}5,912.0 \\
5,913.0 \\
5,914.0 \\
5,917.0 \\
5,918.0\end{array}$ & $\begin{array}{l}\text { 324PRDX } \\
324 \mathrm{PRDX} \\
324 \mathrm{PRDX} \\
324 \mathrm{PRDX} \\
\text { 324PRDX }\end{array}$ & $\begin{array}{l}9.6 \\
2.5 \\
9.1 \\
1.2 \\
1.2\end{array}$ & $\begin{array}{l}0.000000 \\
0.000000 \\
0.000000 \\
0.000000 \\
0.000000\end{array}$ & $\begin{array}{l}\text { Dolomite } \\
\text { Dolomite } \\
\text { Dolomite } \\
\text { Dolomite } \\
\text { Dolomite }\end{array}$ \\
\hline $\begin{array}{l}\text { nmb33.5-20-03aba } \\
\text { nmb33.5-20-03aba } \\
\text { nmb33.5-20-03aba } \\
\text { nmb33.5-20-03aba } \\
\text { nmb33.5-20-03aba }\end{array}$ & $\begin{array}{l}5,918.0 \\
5,919.0 \\
5,920.0 \\
5,921.0 \\
5,922.0\end{array}$ & $\begin{array}{l}5,919.0 \\
5,920.0 \\
5,921.0 \\
5,922.0 \\
5,923.0\end{array}$ & $\begin{array}{l}\text { 324PRDX } \\
324 \mathrm{PRDX} \\
324 \mathrm{PRDX} \\
324 \mathrm{PRDX} \\
\text { 324PRDX }\end{array}$ & $\begin{array}{l}1.5 \\
0.8 \\
1.0 \\
1.1 \\
1.2\end{array}$ & $\begin{array}{l}0.000000 \\
0.010000 \\
0.000000 \\
0.000000 \\
0.000000\end{array}$ & $\begin{array}{l}\text { Dolomite } \\
\text { Dolomite and limestone } \\
\text { Limestone } \\
\text { Limestone } \\
\text { Limestone }\end{array}$ \\
\hline $\begin{array}{l}\text { nmb33.5-20-03aba } \\
\text { nmb33.5-20-03aba } \\
\text { nmb33.5-20-03aba } \\
\text { nmb33.5-20-03aba } \\
\text { nmb33.5-20-03aba }\end{array}$ & $\begin{array}{l}5,923.0 \\
5,924.0 \\
5,925.0 \\
5,926.5 \\
5,928.0\end{array}$ & $\begin{array}{l}5,924.0 \\
5,925.0 \\
5,926.5 \\
5,928.0 \\
5,929.0\end{array}$ & $\begin{array}{l}324 \mathrm{PRDX} \\
324 \mathrm{PRDX} \\
324 \mathrm{PRDX} \\
324 \mathrm{PRDX} \\
\text { 324PRDX }\end{array}$ & $\begin{array}{l}1.1 \\
1.0 \\
6.6 \\
5.5 \\
1.4\end{array}$ & $\begin{array}{l}0.010000 \\
0.000000 \\
0.000000 \\
0.100000 \\
0.000000\end{array}$ & $\begin{array}{l}\text { Fractured limestone } \\
\text { Fractured limestone } \\
\text { Limestone } \\
\text { Dolomite } \\
\text { Fractured limestone }\end{array}$ \\
\hline $\begin{array}{l}\text { nmb33.5-20-03aba } \\
\text { nmb33.5-20-03aba } \\
\text { nmb33.5-20-03aba } \\
\text { nmb33.5-20-03aba } \\
\text { nmb33.5-20-03aba }\end{array}$ & $\begin{array}{l}5,929.0 \\
5,930.0 \\
5,931.0 \\
5,932.0 \\
5,933.0\end{array}$ & $\begin{array}{l}5,930.0 \\
5,931.0 \\
5,932.0 \\
5,933.0 \\
5,934.0\end{array}$ & $\begin{array}{l}\text { 324PRDX } \\
324 \mathrm{PRDX} \\
324 \mathrm{PRDX} \\
\text { 324PRDX } \\
\text { 324PRDX }\end{array}$ & $\begin{array}{l}3.0 \\
6.4 \\
7.7 \\
7.8 \\
9.9\end{array}$ & $\begin{array}{l}0.000000 \\
0.000000 \\
0.010000 \\
0.010000 \\
0.000000\end{array}$ & $\begin{array}{l}\text { Fractured limestone } \\
\text { Limestone } \\
\text { Limestone } \\
\text { Limestone } \\
\text { Limestone }\end{array}$ \\
\hline
\end{tabular}


Table 4.--Laboratory-determined porosity and permeability--Continued

\begin{tabular}{|c|c|c|c|c|c|c|}
\hline Site & $\begin{array}{l}\text { Depth to } \\
\text { top } \\
\text { (feet) }\end{array}$ & $\begin{array}{l}\text { Depth to } \\
\text { bottom } \\
\text { (feet) }\end{array}$ & $\begin{array}{c}\text { Forma- } \\
\text { tion }\end{array}$ & $\begin{array}{l}\text { Porosity } \\
\text { (percent) }\end{array}$ & $\begin{array}{l}\text { Perme- } \\
\text { ability } \\
\text { (milli- } \\
\text { darcies) }\end{array}$ & Lithology \\
\hline nmb33.5-20-03aba & $5,934.0$ & $5,935.0$ & 324PRDX & 7.1 & 0.010000 & Limestone \\
\hline nmb33.5-20-03aba & $5,935.0$ & $5,936.0$ & $324 \mathrm{PRDX}$ & 3.7 & 0.000000 & Limestone \\
\hline nmb33.5-20-03aba & $5,936.0$ & $5,937.0$ & $324 \mathrm{PRDX}$ & 1.3 & 0.010000 & Limestone \\
\hline nmb33.5-20-03aba & $5,937.0$ & $5,939.0$ & 324PRDX & 1.5 & 0.000000 & Fractured dolomite \\
\hline nmb33.5-20-03aba & $5,939.0$ & $5,940.7$ & 324PRDX & 5.3 & 0.000000 & Fractured dolomite \\
\hline nmb33.5-20-03aba & $5,940.7$ & $5,942.2$ & 324PRDX & 8.8 & 0.000000 & Fractured dolomite \\
\hline nmb33.5-20-03aba & $5,942.2$ & $5,944.2$ & $324 \mathrm{PRDX}$ & 7.2 & 0.000000 & Fractured dolomite \\
\hline nmb33.5-20-03aba & $5,944.2$ & $5,946.0$ & 324PRDX & 3.6 & 0.000000 & Dolomite \\
\hline nmb33.5-20-03aba & $5,946.0$ & $5,947.0$ & $324 \mathrm{PRDX}$ & 1.3 & 0.000000 & Limestone \\
\hline nmb33.5-20-03aba & $5,947.0$ & $5,948.0$ & $324 \mathrm{PRDX}$ & 2.4 & 0.060000 & Limestone \\
\hline $\mathrm{nmb} 33.5-20-03 \mathrm{aba}$ & $5,948.0$ & $5,949.0$ & 324PRDX & 1.8 & 0.000000 & Limestone \\
\hline nmb33.5-20-03aba & $5,949.0$ & $5,950.0$ & 324PRDX & 1.9 & 0.000000 & Limestone \\
\hline & & & AVERAGE -- & 4.8 & 0.006190 & \\
\hline SITE AVERAGE---- & - & - & ------- & 4.8 & 0.006190 & \\
\hline nmb33.5-20-16bca & $5,717.0$ & $5,718.0$ & 324PRDX & 1.6 & 0.000000 & Shale \\
\hline nmb33.5-20-16bca & $5,719.0$ & $5,720.0$ & $324 \mathrm{PRDX}$ & 1.8 & 0.000000 & Shale \\
\hline nmb33.5-20-16bca & $5,721.0$ & $5,722.0$ & $324 \mathrm{PRDX}$ & 1.9 & 0.000000 & Shale \\
\hline nmb 33.5-20-16bca & $5,723.0$ & $5,724.0$ & $324 \mathrm{PRDX}$ & 4.0 & 0.000000 & Shale \\
\hline nmb $33.5-20-16$ bca & $5,725.0$ & $5,726.0$ & 324PRDX & 2.3 & 0.000000 & Shale \\
\hline nmb33.5-20-16bca & $5,727.0$ & $5,728.0$ & 324PRDX & 3.3 & 0.000000 & Finely crystalline limestone \\
\hline nmb33.5-20-16bca & $5,729.0$ & $5,730.0$ & $324 \mathrm{PRDX}$ & 2.0 & 0.000000 & Finely crystalline limestone \\
\hline nmb33.5-20-16bca & $5,731.0$ & $5,732.0$ & 324PRDX & 2.7 & 0.000000 & Finely crystalline limestone \\
\hline nmb33.5-20-16bca & $5,733.0$ & $5,734.0$ & $324 \mathrm{PRDX}$ & 1.1 & 0.000000 & Finely crystalline limestone \\
\hline nmb33.5-20-16bca & $5,735.0$ & $5,736.0$ & $324 \mathrm{PRDX}$ & 1.7 & 0.000000 & Finely crystalline limestone \\
\hline nmb33.5-20-16bca & $5,739.0$ & $5,740.0$ & 324PRDX & 2.7 & 0.000000 & Finely crystalline limestone \\
\hline nmb33.5-20-16bca & $5,741.0$ & $5,742.0$ & 324PRDX & 3.3 & 0.000000 & Finely crystalline limestone \\
\hline $\mathrm{nmb} 33.5-20-16 \mathrm{bca}$ & $5,743.0$ & $5,744.0$ & $324 \mathrm{PRDX}$ & 2.9 & 0.000000 & Finely crystalline limestone \\
\hline nmb33.5-20-16bca & $5,745.0$ & $5,746.0$ & 324PRDX & 5.7 & 0.000000 & Finely crystalline limestone \\
\hline nmb33.5-20-16bca & $5,747.0$ & $5,748.0$ & $324 \mathrm{PRDX}$ & 4.7 & 0.000000 & Finely crystalline limestone \\
\hline $\mathrm{nmb} 33.5-20-16 \mathrm{bca}$ & $5,749.0$ & $5,750.0$ & $324 \mathrm{PRDX}$ & 3.6 & 0.000000 & Finely crystalline limestone \\
\hline nmb33.5-20-16bca & $5,751.0$ & $5,752.0$ & $324 \mathrm{PRDX}$ & 3.2 & 0.000000 & Finely crystalline limestone \\
\hline nmb33.5-20-16bca & $5,753.0$ & $5,754.0$ & $324 \mathrm{PRDX}$ & 2.5 & 0.000000 & Finely crystalline limestone \\
\hline nmb33.5-20-16bca & $5,755.0$ & $5,756.0$ & $324 \mathrm{PRDX}$ & 2.0 & 0.000000 & Finely crystalline limestone \\
\hline nmb33.5-20-16bca & $5,757.0$ & $5,758.0$ & 324PRDX & 4.3 & 0.000000 & Finely crystalline limestone \\
\hline nmb33.5-20-16bca & $5,759.0$ & $5,760.0$ & $324 \mathrm{PRDX}$ & 1.8 & 0.000000 & Finely crystalline limestone \\
\hline nmb33.5-20-16bca & $5,814.0$ & $5,815.0$ & 324PRDX & 1.9 & 0.000000 & Shale and limestone \\
\hline nmb33.5-20-16bca & $5,816.0$ & $5,817.0$ & 324PRDX & 8.6 & 0.000000 & Shale and limestone \\
\hline nmb33.5-20-16bca & $5,817.0$ & $5,818.0$ & $324 \mathrm{PRDX}$ & 13.1 & 0.000000 & Shale and limestone \\
\hline nmb33.5-20-16bca & $5,819.0$ & $5,820.0$ & $324 \mathrm{PRDX}$ & 3.3 & 0.000000 & Shale and limestone \\
\hline nmb33.5-20-16bca & $5,821.0$ & $5,822.0$ & 324PRDX & 3.3 & 0.000000 & Fine-grained dolomite \\
\hline nmb33.5-20-16bca & $5,823.0$ & $5,824.9$ & $324 \mathrm{PRDX}$ & 3.3 & 0.000000 & Sucrose dolomite \\
\hline $\mathrm{nmb} 33.5-20-16 \mathrm{bca}$ & $5,824.9$ & $5,826.8$ & $324 \mathrm{PRDX}$ & 6.3 & 0.000000 & Sucrose dolomite \\
\hline nmb33.5-20-16bca & $5,826.8$ & $5,828.4$ & 324PRDX & 8.0 & 0.300000 & Vuggy, finely to coarsely crystalline limestone \\
\hline nmb33.5-20-16bca & $5,828.4$ & $5,830.0$ & $324 \mathrm{PRDX}$ & 2.9 & 0.200000 & Vuggy, finely to coarsely crystalline limestone \\
\hline nmb33.5-20-16bca & $5,830.0$ & $5,831.1$ & 324PRDX & 4.3 & 0.000000 & Vuggy, finely to coarsely crystalline limestone \\
\hline nmb33.5-20-16bca & $5,831.1$ & $5,832.6$ & $324 \mathrm{PRDX}$ & 2.4 & 0.000000 & Vuggy, finely to coarsely crystalline limestone \\
\hline nmb33.5-20-16bca & $5,832.6$ & $5,834.5$ & $324 \mathrm{PRDX}$ & 5.9 & 0.000000 & Vuggy, finely to coarsely crystalline limestone \\
\hline nmb33.5-20-16bca & $5,834.5$ & $5,836.0$ & $324 \mathrm{PRDX}$ & 5.1 & 0.300000 & Vuggy, finely to coarsely crystalline limestone \\
\hline nmb33.5-20-16bca & $5,836.0$ & $5,837.3$ & 324PRDX & 1.6 & 13.000000 & Fine-grained limestone \\
\hline
\end{tabular}


Table 4.--Laboratory-determined porosity and permeability--Continued

\begin{tabular}{|c|c|c|c|c|c|c|}
\hline Site & $\begin{array}{l}\text { Depth to } \\
\text { top } \\
\text { (feet) }\end{array}$ & $\begin{array}{l}\text { Depth to } \\
\text { bottom } \\
\text { (feet) }\end{array}$ & $\begin{array}{c}\text { Forma- } \\
\text { tion }\end{array}$ & $\begin{array}{l}\text { Porosity } \\
\text { (percent) }\end{array}$ & $\begin{array}{c}\text { Perme- } \\
\text { ability } \\
\text { (milli- } \\
\text { darcies) }\end{array}$ & Lithology \\
\hline $\mathrm{nmb} 33.5-20-16 \mathrm{bca}$ & $5,837.3$ & $5,839.0$ & 324PRDX & 3.3 & 0.000000 & Sucrose, dolomitic limestone \\
\hline nmb33.5-20-16bca & $5,839.0$ & $5,840.7$ & 324PRDX & 5.2 & 0.100000 & Sucrose, dolomitic limestone \\
\hline $\mathrm{nmb} 33.5-20-16 \mathrm{bca}$ & $5,840.7$ & $5,842.2$ & $324 \mathrm{PRDX}$ & 9.7 & 0.000000 & Sucrose, dolomitic limestone \\
\hline $\mathrm{nmb} 33.5-20-16 \mathrm{bca}$ & $5,842.2$ & $5,843.0$ & 324PRDX & 17.0 & 0.000000 & Sucrose, dolomitic limestone \\
\hline nmb33.5-20-16bca & $5,845.0$ & $5,846.0$ & 324PRDX & 1.6 & 0.300000 & Fine-grained limestone \\
\hline $\mathrm{nmb} 33.5-20-16 \mathrm{bca}$ & $5,846.0$ & $5,847.0$ & $324 \mathrm{PRDX}$ & 0.9 & 4.000000 & Fine-grained limestone \\
\hline $\mathrm{nmb} 33.5-20-16 \mathrm{bca}$ & $5,847.0$ & $5,848.0$ & $324 \mathrm{PRDX}$ & 6.6 & 0.500000 & Vuggy limestone \\
\hline $\mathrm{nmb} 33.5-20-16 \mathrm{bca}$ & $5,849.0$ & $5,850.0$ & $324 \mathrm{PRDX}$ & 6.8 & 0.300000 & Vuggy limestone \\
\hline $\mathrm{nmb} 33.5-20-16 \mathrm{bca}$ & $5,851.0$ & $5,852.0$ & 324PRDX & 4.5 & 0.200000 & Vuggy limestone \\
\hline nmb33.5-20-16bca & $5,852.0$ & $5,853.0$ & $324 \mathrm{PRDX}$ & 3.1 & 4.900000 & Vuggy limestone \\
\hline $\mathrm{nmb} 33.5-20-16 \mathrm{bca}$ & $5,853.0$ & $5,854.0$ & $324 \mathrm{PRDX}$ & 3.6 & 0.700000 & Vuggy limestone \\
\hline$n m b 33 \cdot 5-20-16 \mathrm{bca}$ & $5,854.0$ & $5,855.0$ & $324 \mathrm{PRDX}$ & 3.0 & 0.400000 & Vuggy limestone \\
\hline nmb33.5-20-16bca & $5,855.0$ & $5,856.0$ & 324PRDX & 1.2 & 0.000000 & Vuggy limestone \\
\hline $\mathrm{nmb} 33.5-20-16 \mathrm{bca}$ & $5,857.0$ & $5,858.0$ & $324 \mathrm{PRDX}$ & 4.1 & 0.000000 & Fine-grained dolomite \\
\hline $\mathrm{nmb} 33.5-20-16 \mathrm{bca}$ & $5,859.0$ & $5,860.0$ & $324 \mathrm{PRDX}$ & 4.4 & 0.000000 & Fine-grained dolomite \\
\hline $\mathrm{nmb} 33.5-20-16 \mathrm{bca}$ & $5,861.0$ & $5,862.0$ & $324 \mathrm{PRDX}$ & 1.4 & 0.000000 & Fine-grained dolomite \\
\hline $\mathrm{nmb} 33.5-20-16 \mathrm{bca}$ & $5,863.0$ & $5,864.0$ & 324PRDX & 0.5 & 0.000000 & Fine-grained limestone \\
\hline $\mathrm{nmb} 33.5-20-16 \mathrm{bca}$ & $5,865.0$ & $5,866.0$ & 324PRDX & 1.9 & 0.000000 & Fine-grained limestone \\
\hline nmb33.5-20-16bca & $5,867.0$ & $5,868.0$ & $324 \mathrm{PRDX}$ & 1.2 & 0.000000 & Fine-grained limestone \\
\hline$n m b 33.5-20-16 b c a$ & $5,869.0$ & $5,870.0$ & $324 \mathrm{PRDX}$ & 3.7 & 1.300000 & Fine-grained limestone \\
\hline $\mathrm{nmb} 33.5-20-16 \mathrm{bca}$ & $5,871.0$ & $5,872.0$ & 324PRDX & 4.3 & 0.000000 & Sucrose dolomite \\
\hline $\mathrm{nmb} 33 \cdot 5-20-16 \mathrm{bca}$ & $5,873.0$ & $5,874.0$ & 324PRDX & 4.2 & 0.000000 & Sucrose dolomite \\
\hline $\mathrm{nmb} 33.5-20-16 \mathrm{bca}$ & $5,875.0$ & $5,876.0$ & $324 \mathrm{PRDX}$ & 4.1 & 0.000000 & Sucrose dolomite \\
\hline $\mathrm{nmb} 33.5-20-16 \mathrm{bca}$ & $5,877.0$ & $5,878.0$ & $324 \mathrm{PRDX}$ & 3.9 & 0.000000 & Finely crystalline limestone \\
\hline$n m b 33.5-20-16 b c a$ & $5,879.0$ & $5,880.0$ & $324 \mathrm{PRDX}$ & 1.9 & 0.000000 & Finely crystalline limestone \\
\hline $\mathrm{nmb} 33.5-20-16 \mathrm{bca}$ & $5,881.0$ & $5,882.0$ & 324PRDX & 2.5 & 0.000000 & Finely crystalline limestone \\
\hline $\mathrm{nmb} 33.5-20-16 \mathrm{bca}$ & $5,883.0$ & $5,884.0$ & 324PRDX & 1.6 & 0.000000 & Finely crystalline limestone \\
\hline nmb33.5-20-16bca & $5,885.0$ & $5,886.0$ & $324 \mathrm{PRDX}$ & 2.4 & 0.000000 & Finely crystalline limestone \\
\hline$n m b 33.5-20-16 b c a$ & $5,887.0$ & $5,888.0$ & $324 \mathrm{PRDX}$ & 2.2 & 0.000000 & Finely crystalline limestone \\
\hline $\mathrm{nmb} 33.5-20-16 \mathrm{bca}$ & $5,889.0$ & $5,890.0$ & $324 \mathrm{PRDX}$ & 1.9 & 0.000000 & Finely crystalline limestone \\
\hline $\mathrm{nmb} 33.5-20-16 \mathrm{bca}$ & $5,891.0$ & $5,892.0$ & 324PRDX & 1.9 & 0.000000 & Finely crystalline limestone \\
\hline $\mathrm{nmb} 33.5-20-16 \mathrm{bca}$ & $5,892.0$ & $5,893.0$ & 324PRDX & 3.5 & 0.400000 & Vuggy limestone \\
\hline $\mathrm{nmb} 33.5-20-16 \mathrm{bca}$ & $5,894.0$ & $5,895.0$ & 324PRDX & 8.4 & 2.800000 & Vuggy limestone \\
\hline $\mathrm{nmb} 33.5-20-16 \mathrm{bca}$ & $5,896.0$ & $5,897.0$ & $324 \mathrm{PRDX}$ & 0.8 & 1.400000 & Vuggy limestone \\
\hline $\mathrm{nmb} 33.5-20-16 \mathrm{bca}$ & $5,898.0$ & $5,899.0$ & $324 \mathrm{PRDX}$ & 0.7 & 3.300000 & Vuggy limestone \\
\hline$n m b 33.5-20-16 b c a$ & $5,900.0$ & $5,901.0$ & $324 \mathrm{PRDX}$ & 0.3 & 0.400000 & Vuggy limestone \\
\hline $\mathrm{nmb} 33.5-20-16 \mathrm{bca}$ & $5,901.0$ & $5,902.0$ & $324 \mathrm{PRDX}$ & 6.1 & 213.000000 & Vuggy limestone \\
\hline $\mathrm{nmb} 33.5-20-16 \mathrm{bca}$ & $5,903.0$ & $5,904.0$ & 324PRDX & 6.9 & 4.600000 & Vuggy limestone \\
\hline nmb33.5-20-16bca & $5,905.0$ & $5,906.0$ & $324 \mathrm{PRDX}$ & 6.8 & 2.700000 & Vuggy limestone \\
\hline$n m b 33.5-20-16 b c a$ & $5,907.0$ & $5,908.0$ & 324PRDX & 5.7 & 19.000000 & Vuggy limestone \\
\hline $\mathrm{nmb} 33.5-20-16 \mathrm{bca}$ & $5,909.0$ & $5,910.0$ & 324PRDX & 10.5 & 14.000000 & Vuggy limestone \\
\hline $\mathrm{nmb} 33.5-20-16 \mathrm{bca}$ & $5,911.0$ & $5,912.0$ & 324PRDX & 7.8 & 2.500000 & Vuggy limestone \\
\hline $\mathrm{nmb} 33.5-20-16 \mathrm{bca}$ & $5,912.0$ & $5,913.0$ & $324 \mathrm{PRDX}$ & 12.4 & 9.100000 & Vuggy limestone \\
\hline $\mathrm{nmb} 33.5-20-16 \mathrm{bca}$ & $5,913.0$ & $5,914.0$ & 324PRDX & 9.3 & 4.300000 & Vuggy limestone \\
\hline $\mathrm{nmb} 33.5-20-16 \mathrm{bca}$ & $5,914.0$ & $5,915.0$ & 324PRDX & 8.1 & 3.900000 & Vuggy limestone \\
\hline $\mathrm{nmb} 33.5-20-16 \mathrm{bca}$ & $5,915.0$ & $5,916.0$ & 324PRDX & 4.7 & 0.500000 & Vuggy limestone \\
\hline $\mathrm{nmb} 33.5-20-16 \mathrm{bca}$ & $5,916.0$ & $5,917.0$ & 324PRDX & 9.3 & 2.700000 & Vuggy limestone \\
\hline$n m b 33.5-20-16 b c a$ & $5,917.0$ & $5,918.0$ & 324PRDX & 28.8 & 107.000000 & Sucrose dolomite \\
\hline nmb33.5-20-16bca & $5,918.0$ & $5,919.0$ & 324PRDX & 27.6 & 65.000000 & Sucrose dolomite \\
\hline $\mathrm{nmb} 33.5-20-16 \mathrm{bca}$ & $5,919.0$ & $5,920.0$ & 324PRDX & 22.5 & 11.000000 & Sucrose dolomite \\
\hline
\end{tabular}


Table 4.--Laboratory-determined porosity and permeability--Continued

\begin{tabular}{|c|c|c|c|c|c|c|}
\hline Site & $\begin{array}{l}\text { Depth to } \\
\text { top } \\
\text { (feet) }\end{array}$ & $\begin{array}{l}\text { Depth to } \\
\text { bottom } \\
\text { (feet) }\end{array}$ & $\begin{array}{c}\text { Forma- } \\
\text { tion }\end{array}$ & $\begin{array}{l}\text { Porosity } \\
\text { (percent) }\end{array}$ & $\begin{array}{l}\text { Perme- } \\
\text { ability } \\
\text { (milli- } \\
\text { darcies) }\end{array}$ & Lithology \\
\hline $\mathrm{nmb} 33.5-20-16 \mathrm{bca}$ & $5,921.0$ & $5,922.0$ & 324PRDX & 17.5 & 2.300000 & Sucrose dolomite \\
\hline nmb33.5-20-16bca & $5,923.0$ & $5,924.0$ & 324PRDX & 11.8 & 0.700000 & Sucrose dolomite \\
\hline nmb33.5-20-16bca & $5,925.0$ & $5,926.0$ & $324 \mathrm{PRDX}$ & 24.4 & 12.000000 & Sucrose dolomite \\
\hline $\mathrm{nmb} 33.5-20-16 \mathrm{bca}$ & $5,927.0$ & $5,928.0$ & $324 \mathrm{PRDX}$ & 25.6 & 19.000000 & Sucrose dolomite \\
\hline nmb33.5-20-16bca & $5,929.0$ & $5,930.0$ & 324PRDX & 31.0 & 51.000000 & Sucrose dolomite \\
\hline nmb33.5-20-16bca & $5,931.0$ & $5,932.0$ & $324 \mathrm{PRDX}$ & 26.0 & 30.000000 & Sucrose dolomite \\
\hline nmb33.5-20-16bca & $5,933.0$ & $5,934.0$ & 324PRDX & 19.2 & 3.800000 & Sucrose dolomite \\
\hline $\mathrm{nmb} 33.5-20-16 \mathrm{bca}$ & $5,935.0$ & $5,936.0$ & 324PRDX & 16.8 & 6.900000 & Chalky limestone \\
\hline nmb33.5-20-16bca & $5,937.0$ & $5,938.0$ & $324 \mathrm{PRDX}$ & 12.0 & 2.600000 & Chalky limestone \\
\hline nmb33.5-20-16bca & $5,939.0$ & $5,940.0$ & 324PRDX & 16.8 & 3.400000 & Chalky limestone \\
\hline nmb33.5-20-16bca & $5,941.0$ & $5,942.0$ & 324PRDX & 3.9 & 0.000000 & Chalky limestone \\
\hline nmb33.5-20-16bca & $5,997.0$ & $5,998.0$ & $324 \mathrm{PRDX}$ & 3.0 & 0.000000 & Shaly, fine-grained dolomite \\
\hline $\mathrm{nmb} 33.5-20-16 \mathrm{bca}$ & $5,999.0$ & $6,000.0$ & $324 \mathrm{PRDX}$ & 1.9 & 0.000000 & Shaly, fine-grained dolomite \\
\hline nmb33.5-20-16bca & $6,001.0$ & $6,002.0$ & $324 \mathrm{PRDX}$ & 2.7 & 0.000000 & Shaly, fine-grained dolomite \\
\hline $\mathrm{nmb} 33.5-20-16 \mathrm{bca}$ & $6,003.0$ & $6,004.0$ & $324 \mathrm{PRDX}$ & 2.4 & 0.000000 & Limy shale \\
\hline nmb33.5-20-16bca & $6,005.0$ & $6,006.0$ & 324PRDX & 3.6 & 0.000000 & Limy shale \\
\hline nmb33.5-20-16bca & $6,007.0$ & $6,008.0$ & 324PRDX & 3.0 & 0.000000 & Limy shale \\
\hline nmb33.5-20-16bca & $6,023.0$ & $6,024.0$ & $324 \mathrm{PRDX}$ & 3.8 & 0.000000 & Shaly, fine-grained limestone \\
\hline nmb33.5-20-16bca & $6,025.0$ & $6,026.0$ & $324 \mathrm{PRDX}$ & 4.0 & 0.000000 & Shaly, fine-grained limestone \\
\hline nmb33.5-20-16bca & $6,027.0$ & $6,028.0$ & $324 \mathrm{PRDX}$ & 2.8 & 0.000000 & Shaly, fine-grained limestone \\
\hline nmb33.5-20-16bca & $6,029.0$ & $6,030.0$ & 324PRDX & 3.3 & 0.000000 & Shaly, fine-grained limestone \\
\hline nmb33.5-20-16bca & $6,031.0$ & $6,032.0$ & $324 \mathrm{PRDX}$ & 4.0 & 0.000000 & Limy shale \\
\hline nmb33.5-20-16bca & $6,033.0$ & $6,034.0$ & 324PRDX & 3.6 & 0.000000 & Limy shale \\
\hline $\mathrm{nmb} 33.5-20-16 \mathrm{bca}$ & $6,035.0$ & $6,036.0$ & 324PRDX & 2.8 & 0.000000 & Limy shale \\
\hline nmb33.5-20-16bca & $6,037.0$ & $6,038.0$ & $324 \mathrm{PRDX}$ & 2.3 & 0.000000 & Dolomite and limestone \\
\hline nmb33.5-20-16bca & $6,039.0$ & $6,040.0$ & $324 \mathrm{PRDX}$ & 3.3 & 00 & Dolomite and limestone \\
\hline nmb33.5-20-16bca & $6,041.0$ & $6,042.0$ & 324PRDX & 3.5 & 0.000000 & Dolomite and limestone \\
\hline $\mathrm{nmb} 33.5-20-16 \mathrm{bca}$ & $6,044.0$ & $6,045.0$ & $324 \mathrm{PRDX}$ & 1.6 & 0.000000 & Dolomite and limestone \\
\hline nmb33.5-20-16bca & $6,046.0$ & $6,047.0$ & $324 \mathrm{PRDX}$ & 1.8 & 0.000000 & Dolomite and limestone \\
\hline nmb33.5-20-16bca & $6,048.0$ & $6,049.0$ & $324 \mathrm{PRDX}$ & 3.5 & 0.000000 & Dolomite and limestone \\
\hline $\mathrm{nmb} 33.5-20-16 \mathrm{bca}$ & $6,050.0$ & $6,051.0$ & $324 \mathrm{PRDX}$ & 3.7 & 0.000000 & Dolomite and limestone \\
\hline nmb33.5-20-16bca & $6,058.0$ & $6,059.0$ & 324PRDX & 4.8 & 0.000000 & Chalky dolomite \\
\hline nmb33.5-20-16bca & $6,060.0$ & $6,061.0$ & $324 \mathrm{PRDX}$ & 4.9 & 0.000000 & Chalky dolomite \\
\hline nmb33.5-20-16bca & $6,062.0$ & $6,063.0$ & $324 \mathrm{PRDX}$ & 4.2 & 0.000000 & Chalky dolomite \\
\hline nmb33.5-20-16bca & $6,064.0$ & $6,065.0$ & $324 \mathrm{PRDX}$ & 4.1 & 0.000000 & Shaly, fine-grained limestone \\
\hline nmb33.5-20-16bca & $6,066.0$ & $6,067.0$ & 324PRDX & 2.6 & 0.000000 & Shaly, fine-grained limestone \\
\hline nmb33.5-20-16bca & $6,068.0$ & $6,069.0$ & 324PRDX & 6.2 & 0.000000 & Shaly, fine-grained limestone \\
\hline nmb33.5-20-16bca & $6,071.0$ & $6,072.0$ & $324 \mathrm{PRDX}$ & 3.0 & 0.000000 & Shaly, fine-grained limestone \\
\hline nmb33.5-20-16bca & $6,073.0$ & $6,074.0$ & 324PRDX & 2.3 & 0.000000 & Shaly, fine-grained limestone \\
\hline nmb33.5-20-16bca & $6,075.0$ & $6,076.0$ & $324 \mathrm{PRDX}$ & 4.9 & 0.000000 & Shaly, fine-grained limestone \\
\hline nmb33.5-20-16bca & $6,077.0$ & 6 & $324 \mathrm{PRDX}$ & 3.5 & 0.000000 & Shaly, fine-grained limestone \\
\hline nmb33.5-20-16bca & $6,079.0$ & $6,080.0$ & $324 \mathrm{PRDX}$ & 3.0 & 0.000000 & Shaly, fine-grained limestone \\
\hline nmb33.5-20-16bca & $6,081.0$ & $6,082.0$ & 324PRDX & 2.2 & 0.000000 & Shaly, fine-grained limestone \\
\hline nmb33.5-20-16bca & $6,083.0$ & $6,084.0$ & $324 \mathrm{PRDX}$ & 2.8 & 0.000000 & Shaly, fine-grained limestone \\
\hline nmb 33.5-20-16bca & $6,091.0$ & $6,092.0$ & $324 \mathrm{PRDX}$ & 1.8 & 0.000000 & Shaly, fine-grained limestone \\
\hline nmb33.5-20-16bca & $6,093.0$ & $6,094.0$ & 324PRDX & 1.5 & 0.000000 & Shaly, fine-grained limestone \\
\hline nmb33.5-20-16bca & $6,095.0$ & $6,096.0$ & 324PRDX & 2.4 & 0.000000 & Shaly, fine-grained limestone \\
\hline nmb33.5-20-16bca & $6,097.0$ & $6,098.0$ & 324PRDX & 2.2 & 0.000000 & Shaly, fine-grained limestone \\
\hline nmb33.5-20-16bca & $6,100.0$ & $6,101.0$ & $324 \mathrm{PRDX}$ & 1.4 & 0.000000 & Shaly, fine-grained limestone \\
\hline nmb33.5-20-16bca & $6,108.0$ & $6,109.0$ & $324 \mathrm{PRDX}$ & 5.2 & 0.000000 & Shaly, fine-grained dolomite \\
\hline
\end{tabular}


Table 4.--Laboratory-determined porosity and permeability--Continued

\begin{tabular}{|c|c|c|c|c|c|c|}
\hline Site & $\begin{array}{l}\text { Depth to } \\
\text { top } \\
\text { (feet) }\end{array}$ & $\begin{array}{l}\text { Depth to } \\
\text { bottom } \\
\text { (feet) }\end{array}$ & $\begin{array}{l}\text { Forma- } \\
\text { tion }\end{array}$ & $\begin{array}{l}\text { Porosity } \\
\text { (percent) }\end{array}$ & $\begin{array}{l}\text { Perme- } \\
\text { ability } \\
\text { (milli- } \\
\text { darcies) }\end{array}$ & Lithology \\
\hline $\mathrm{nmb} 33.5-20-16 \mathrm{bca}$ & $6,112.0$ & $6,113.0$ & $324 \mathrm{PRDX}$ & 5.3 & 1.000000 & Shaly, fine-grained dolomite \\
\hline SITE AVERAGE---- & $----n--$ & ------ & AVERAGE-- & $\frac{5.6}{5.6}$ & $\frac{4.575182}{4.575182}$ & \\
\hline $\begin{array}{l}\text { nmb33.5-20-21dac } \\
\text { nmb33.5-20-21dac } \\
\text { nmb33.5-20-21dac } \\
\text { nmb33.5-20-21dac } \\
\text { nmb33.5-20-21dac }\end{array}$ & $\begin{array}{l}5,691.0 \\
5,697.0 \\
5,700.0 \\
5,701.6 \\
5,716.0\end{array}$ & $\begin{array}{l}5,693.0 \\
5,698.0 \\
5,701.6 \\
5,703.0 \\
5,718.0\end{array}$ & $\begin{array}{l}324 \mathrm{PRDX} \\
324 \mathrm{PRDX} \\
324 \mathrm{PRDX} \\
324 \mathrm{PRDX} \\
324 \mathrm{PRDX}\end{array}$ & $\begin{array}{r}5.4 \\
14.5 \\
1.8 \\
3.2 \\
4.1\end{array}$ & $\begin{array}{l}0.100000 \\
0.200000 \\
0.700000 \\
0.800000 \\
0.000000\end{array}$ & $\begin{array}{l}\text { Limes tone } \\
\text { Limes tone } \\
\text { Limestone } \\
\text { Limes tone } \\
\text { Limestone }\end{array}$ \\
\hline $\begin{array}{l}\text { nmb33.5-20-21dac } \\
\text { nmb33.5-20-21dac } \\
\text { nmb33.5-20-21dac } \\
\text { nmb33.5-20-21dac } \\
\text { nmb33.5-20-21dac }\end{array}$ & $\begin{array}{l}5,721.0 \\
5,722.6 \\
5,724.4 \\
5,726.0 \\
5,727.5\end{array}$ & $\begin{array}{l}5,722.6 \\
5,724.4 \\
5,726.0 \\
5,727.5 \\
5,729.5\end{array}$ & $\begin{array}{l}\text { 324PRDX } \\
324 \mathrm{PRDX} \\
324 \mathrm{PRDX} \\
324 \mathrm{PRDX} \\
324 \mathrm{PRDX}\end{array}$ & $\begin{array}{r}7.3 \\
10.0 \\
8.6 \\
7.1 \\
7.5\end{array}$ & $\begin{array}{r}1.700000 \\
3.800000 \\
8.100000 \\
60.000000 \\
13.000000\end{array}$ & $\begin{array}{l}\text { Dolomite } \\
\text { Dolomite } \\
\text { Dolomite } \\
\text { Dolomite } \\
\text { Dolomite }\end{array}$ \\
\hline $\begin{array}{l}\mathrm{nmb} 33.5-20-21 \mathrm{dac} \\
\mathrm{nmb} 33.5-20-21 \mathrm{dac} \\
\mathrm{nmb} 33.5-20-21 \mathrm{dac} \\
\mathrm{nmb} 33.5-20-21 \mathrm{dac} \\
\mathrm{nmb} 33.5-20-21 \mathrm{dac}\end{array}$ & $\begin{array}{l}5,729.5 \\
5,731.2 \\
5,738.0 \\
5,739.5 \\
5,742.0\end{array}$ & $\begin{array}{l}5,731.2 \\
5,733.0 \\
5,739.5 \\
5,742.0 \\
5,743.5\end{array}$ & $\begin{array}{l}324 \mathrm{PRDX} \\
324 \mathrm{PRDX} \\
324 \mathrm{PRDX} \\
324 \mathrm{PRDX} \\
324 \mathrm{PRDX}\end{array}$ & $\begin{array}{r}6.6 \\
7.7 \\
10.6 \\
14.0 \\
13.3\end{array}$ & $\begin{array}{r}0.300000 \\
0.000000 \\
5.900000 \\
19.000000 \\
31.000000\end{array}$ & $\begin{array}{l}\text { Dolomite } \\
\text { Dolomite } \\
\text { Shale } \\
\text { Limestone and dolomite } \\
\text { Dolomite }\end{array}$ \\
\hline $\begin{array}{l}\mathrm{nmb} 33 \cdot 5-20-21 \mathrm{dac} \\
\mathrm{nmb} 33 \cdot 5-20-21 \mathrm{dac} \\
\mathrm{nmb} 33 \cdot 5-20-21 \mathrm{dac} \\
\mathrm{nmb} 33.5-20-21 \mathrm{dac} \\
\mathrm{nmb} 33.5-20-21 \mathrm{dac}\end{array}$ & $\begin{array}{l}5,743.5 \\
5,745.2 \\
5,747.0 \\
5,748.7 \\
5,750.3\end{array}$ & $\begin{array}{l}5,745.2 \\
5,747.0 \\
5,748.7 \\
5,750.3 \\
5,752.0\end{array}$ & $\begin{array}{l}\text { 324PRDX } \\
324 \mathrm{PRDX} \\
324 \mathrm{PRDX} \\
324 \mathrm{PRDX} \\
324 \mathrm{PRDX}\end{array}$ & $\begin{array}{r}15.8 \\
14.7 \\
10.2 \\
8.2 \\
11.0\end{array}$ & $\begin{array}{r}43.000000 \\
14.000000 \\
3.000000 \\
0.000000 \\
0.000000\end{array}$ & $\begin{array}{l}\text { Dolomite } \\
\text { Dolomite } \\
\text { Dolomite } \\
\text { Dolomite } \\
\text { Dolomite }\end{array}$ \\
\hline $\begin{array}{l}\text { nmb33.5-20-21dac } \\
\text { nmb33.5-20-21dac } \\
\text { nmb33.5-20-21dac } \\
\text { nmb33.5-20-21dac } \\
\text { nmb33.5-20-21dac }\end{array}$ & $\begin{array}{l}5,752.0 \\
5,762.0 \\
5,763.8 \\
5,765.6 \\
5,767.2\end{array}$ & $\begin{array}{l}5,753.0 \\
5,763.8 \\
5,765.6 \\
5,767.2 \\
5,768.9\end{array}$ & $\begin{array}{l}324 \mathrm{PRDX} \\
324 \mathrm{PRDX} \\
324 \mathrm{PRDX} \\
324 \mathrm{PRDX} \\
324 \mathrm{PRDX}\end{array}$ & $\begin{array}{l}8.4 \\
2.9 \\
4.8 \\
5.2 \\
5.2\end{array}$ & $\begin{array}{l}0.000000 \\
0.100000 \\
0.100000 \\
0.900000 \\
1.400000\end{array}$ & $\begin{array}{l}\text { Dolomite } \\
\text { Shale } \\
\text { Dolomite } \\
\text { Dolomite } \\
\text { Dolomite }\end{array}$ \\
\hline $\begin{array}{l}\text { nmb33.5-20-21dac } \\
\text { nmb33.5-20-21dac } \\
\text { nmb33.5-20-21dac } \\
\text { nmb33.5-20-21dac } \\
\text { nmb33.5-20-21dac }\end{array}$ & $\begin{array}{l}5,768.9 \\
5,770.6 \\
5,772.3 \\
5,773.9 \\
5,775.7\end{array}$ & $\begin{array}{l}5,770.6 \\
5,772.3 \\
5,773.9 \\
5,775.7 \\
5,777.5\end{array}$ & $\begin{array}{l}324 \mathrm{PRDX} \\
324 \mathrm{PRDX} \\
324 \mathrm{PRDX} \\
324 \mathrm{PRDX} \\
324 \mathrm{PRDX}\end{array}$ & $\begin{array}{l}8.3 \\
9.8 \\
9.0 \\
9.2 \\
4.8\end{array}$ & $\begin{array}{r}0.800000 \\
34.000000 \\
6.500000 \\
23.000000 \\
1.400000\end{array}$ & $\begin{array}{l}\text { Dolomite and limestone } \\
\text { Limestone } \\
\text { Limestone } \\
\text { Limestone } \\
\text { Limestone }\end{array}$ \\
\hline $\begin{array}{l}\text { nmb33.5-20-21dac } \\
\text { nmb33.5-20-21dac } \\
\text { nmb33.5-20-21dac } \\
\text { nmb33.5-20-21dac } \\
\text { nmb33.5-20-21dac }\end{array}$ & $\begin{array}{l}5,777.5 \\
5,779.0 \\
5,780.6 \\
5,782.4 \\
5,784.0\end{array}$ & $\begin{array}{l}5,779.0 \\
5,780.6 \\
5,782.4 \\
5,784.0 \\
5,785.8\end{array}$ & $\begin{array}{l}\text { 324PRDX } \\
324 \mathrm{PRDX} \\
324 \mathrm{PRDX} \\
324 \mathrm{PRDX} \\
324 \mathrm{PRDX}\end{array}$ & $\begin{array}{l}13.5 \\
12.3 \\
10.9 \\
10.9 \\
12.7\end{array}$ & $\begin{array}{l}17.000000 \\
68.000000 \\
91.000000 \\
91.000000 \\
48.000000\end{array}$ & $\begin{array}{l}\text { Limestone } \\
\text { Limestone } \\
\text { Limestone } \\
\text { Limestone } \\
\text { Limestone }\end{array}$ \\
\hline $\begin{array}{l}\text { nmb33.5-20-21dac } \\
\text { nmb33.5-20-21dac } \\
\text { nmb33.5-20-21dac } \\
\text { nmb33.5-20-21dac } \\
\text { nmb33.5-20-21dac }\end{array}$ & $\begin{array}{l}5,785.8 \\
5,787.4 \\
5,789.9 \\
5,790.8 \\
5,792.8\end{array}$ & $\begin{array}{l}5,787.4 \\
5,789.9 \\
5,790.8 \\
5,792.8 \\
5,793.6\end{array}$ & $\begin{array}{l}324 \mathrm{PRDX} \\
324 \mathrm{PRDX} \\
324 \mathrm{PRDX} \\
324 \mathrm{PRDX} \\
324 \mathrm{PRDX}\end{array}$ & $\begin{array}{r}12.6 \\
6.9 \\
2.9 \\
3.1 \\
6.0\end{array}$ & $\begin{array}{r}117.000000 \\
3.500000 \\
12.000000 \\
0.700000 \\
0.100000\end{array}$ & $\begin{array}{l}\text { Limestone } \\
\text { Limestone and dolomite } \\
\text { Limestone and dolomite } \\
\text { Limestone } \\
\text { Limestone }\end{array}$ \\
\hline $\begin{array}{l}\text { nmb33.5-20-21dac } \\
\text { nmb33.5-20-21dac } \\
\text { nmb33.5-20-21dac } \\
\text { nmb33.5-20-21dac }\end{array}$ & $\begin{array}{l}5,793.6 \\
5,795.2 \\
5,797.0 \\
5,798.7\end{array}$ & $\begin{array}{l}5,795.2 \\
5,797.0 \\
5,798.7 \\
5,800.5\end{array}$ & $\begin{array}{l}\text { 324PRDX } \\
324 \mathrm{PRDX} \\
324 \mathrm{PRDX} \\
324 \mathrm{PRDX}\end{array}$ & $\begin{array}{r}12.0 \\
6.4 \\
10.3 \\
2.0 \\
\end{array}$ & $\begin{array}{l}0.300000 \\
0.000000 \\
0.000000 \\
0.000000 \\
\end{array}$ & $\begin{array}{l}\text { Limestone } \\
\text { Limestone } \\
\text { Limestone } \\
\text { Limestone }\end{array}$ \\
\hline SITE AVERAGE----. & ------1 & 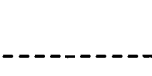 & AVERAGE -- & $\frac{8.4}{8.4}$ & $\frac{16.395455}{16.395455}$ & \\
\hline
\end{tabular}


Table 4.--Laboratory-determined porosity and permeability--Continued

\begin{tabular}{|c|c|c|c|c|c|c|}
\hline Site & $\begin{array}{l}\text { Depth to } \\
\text { top } \\
\text { (feet) }\end{array}$ & $\begin{array}{l}\text { Depth to } \\
\text { bottom } \\
\text { (feet) }\end{array}$ & $\begin{array}{c}\text { Forma- } \\
\text { tion }\end{array}$ & $\begin{array}{l}\text { Porosity } \\
\text { (percent) }\end{array}$ & $\begin{array}{l}\text { Perme- } \\
\text { ability } \\
\text { (milli- } \\
\text { darcies) }\end{array}$ & Lithology \\
\hline $\mathrm{nmb} 33.5-20-22 \mathrm{bcb}$ & $5,641.0$ & $5,642.0$ & 324PRDX & 2.0 & 0.000000 & Limestone \\
\hline$n m b 33.5-20-22 b c b$ & $5,642.0$ & $5,643.0$ & 324PRDX & 1.0 & 0.000000 & Limestone \\
\hline nmb33.5-20-22bcb & $5,643.0$ & $5,644.0$ & $324 \mathrm{PRDX}$ & 3.4 & 0.000000 & Limestone \\
\hline $\mathrm{nmb} 33.5-20-22 \mathrm{bcb}$ & $5,644.0$ & $5,645.0$ & 324PRDX & 2.3 & 0.000000 & Limestone \\
\hline $\mathrm{nmb} 33.5-20-22 \mathrm{bcb}$ & $5,645.0$ & $5,646.0$ & $324 \mathrm{PRDX}$ & 4.1 & 0.010000 & Limestone \\
\hline $\mathrm{nmb} 33.5-20-22 \mathrm{bcb}$ & $5,646.0$ & $5,647.0$ & 324PRDX & 2.8 & 0.000000 & Limestone \\
\hline $\mathrm{nmb} 33.5-20-22 \mathrm{bcb}$ & $5,647.0$ & $5,648.0$ & 324PRDX & 2.2 & 0.000000 & Limestone \\
\hline$n m b 33.5-20-22 b c b$ & $5,648.0$ & $5,649.0$ & 324PRDX & 2.7 & 0.000000 & Limestone \\
\hline $\mathrm{nmb} 33.5-20-22 \mathrm{bcb}$ & $5,649.0$ & $5,650.0$ & 324PRDX & 3.5 & 0.000000 & Limestone \\
\hline nmb33.5-20-22bcb & $5,650.0$ & $5,651.0$ & $324 \mathrm{PRDX}$ & 2.2 & 0.000000 & Limestone \\
\hline $\mathrm{nmb} 33.5-20-22 \mathrm{bcb}$ & $5,651.0$ & $5,652.0$ & $324 \mathrm{PRDX}$ & 3.9 & 0.000000 & Limestone \\
\hline $\mathrm{nmb} 33.5-20-22 \mathrm{bcb}$ & $5,652.0$ & $5,653.0$ & 324PRDX & 2.3 & 0.000000 & Limestone \\
\hline nmb33.5-20-22bcb & $5,653.0$ & $5,654.0$ & $324 \mathrm{PRDX}$ & 1.2 & 0.000000 & Limestone \\
\hline $\mathrm{nmb} 33.5-20-22 \mathrm{bcb}$ & $5,654.0$ & $5,655.0$ & $324 \mathrm{PRDX}$ & 2.1 & 0.000000 & Limestone \\
\hline nmb33.5-20-22bcb & $5,655.0$ & $5,656.0$ & $324 \mathrm{PRDX}$ & 2.0 & 0.000000 & Limestone \\
\hline $\mathrm{nmb} 33.5-20-22 \mathrm{bcb}$ & $5,656.0$ & $5,657.0$ & $324 \mathrm{PRDX}$ & 1.4 & 0.000000 & Limestone \\
\hline nmb33.5-20-22bcb & $5,690.0$ & $5,691.0$ & $324 \mathrm{PRDX}$ & 0.4 & 0.000000 & Anhydrite \\
\hline nmb33.5-20-22bcb & $5,692.0$ & $5,693.0$ & $324 \mathrm{PRDX}$ & 0.4 & 0.000000 & Anhydrite \\
\hline $\mathrm{nmb} 33.5-20-22 \mathrm{bcb}$ & $5,694.0$ & $5,695.0$ & 324PRDX & 1.4 & 0.000000 & Dolomite \\
\hline nmb33.5-20-22bcb & $5,695.0$ & $5,696.0$ & $324 \mathrm{PRDX}$ & 3.5 & 0.000000 & Dolomite \\
\hline nmb33.5-20-22bcb & $5,696.0$ & $5,697.0$ & $324 \mathrm{PRDX}$ & 4.2 & 0.000000 & Dolomite \\
\hline $\mathrm{nmb} 33.5-20-22 \mathrm{bcb}$ & $5,697.0$ & $5,698.0$ & 324PRDX & 0.8 & 0.000000 & Limestone \\
\hline $\mathrm{nmb} 33.5-20-22 \mathrm{bcb}$ & $5,698.0$ & $5,699.0$ & $324 \mathrm{PRDX}$ & 1.2 & 0.000000 & Limestone \\
\hline nmb33.5-20-22bcb & $5,699.0$ & $5,700.0$ & $324 \mathrm{PRDX}$ & 1.7 & 0.000000 & Limestone \\
\hline $\mathrm{nmb} 33.5-20-22 \mathrm{bcb}$ & $5,700.0$ & $5,701.0$ & $324 \mathrm{PRDX}$ & 0.7 & 0.000000 & Shaly limestone \\
\hline nmb33.5-20-22bcb & $5,701.0$ & $5,702.0$ & 324PRDX & 1.2 & 0.000000 & Shaly limestone \\
\hline $\mathrm{nmb} 33.5-20-22 \mathrm{bcb}$ & $5,702.0$ & $5,703.0$ & $324 \mathrm{PRDX}$ & 0.9 & 0.000000 & Shaly limestone \\
\hline nmb33.5-20-22bcb & $5,703.0$ & $5,704.0$ & 324PRDX & 0.5 & 0.000000 & Shaly limestone \\
\hline $\mathrm{nmb} 33.5-20-22 \mathrm{bcb}$ & $5,704.0$ & $5,705.0$ & $324 \mathrm{PRDX}$ & 1.0 & 0.000000 & Shaly limestone \\
\hline $\mathrm{nmb} 33.5-20-22 \mathrm{bcb}$ & $5,705.0$ & $5,706.0$ & $324 \mathrm{PRDX}$ & 1.2 & 0.000000 & Shaly limestone \\
\hline $\mathrm{nmb} 33.5-20-22 \mathrm{bcb}$ & $5,706.0$ & $5,707.0$ & $324 \mathrm{PRDX}$ & 1.8 & 0.000000 & Limestone and dolomite \\
\hline $\mathrm{nmb} 33.5-20-22 \mathrm{bcb}$ & $5,707.0$ & $5,708.0$ & 324PRDX & 1.2 & 0.000000 & Limestone and dolomite \\
\hline $\mathrm{nmb} 33.5-20-22 \mathrm{bcb}$ & $5,708.0$ & $5,709.0$ & 324PRDX & 1.4 & 0.000000 & Limestone and dolomite \\
\hline $\mathrm{nmb} 33.5-20-22 \mathrm{bcb}$ & $5,709.0$ & $5,710.0$ & $324 \mathrm{PRDX}$ & 2.9 & 0.000000 & Limestone and dolomite \\
\hline $\mathrm{nmb} 33.5-20-22 \mathrm{bcb}$ & $5,710.0$ & $5,711.0$ & $324 \mathrm{PRDX}$ & 0.3 & 0.000000 & Limestone and dolomite \\
\hline $\mathrm{nmb} 33.5-20-22 \mathrm{bcb}$ & $5,711.0$ & $5,712.0$ & $324 \mathrm{PRDX}$ & 0.3 & 0.000000 & Limestone and dolomite \\
\hline $\mathrm{nmb} 33.5-20-22 \mathrm{bcb}$ & $5,712.0$ & $5,713.0$ & $324 \mathrm{PRDX}$ & 1.5 & 0.000000 & Limestone and dolomite \\
\hline $\mathrm{nmb} 33.5-20-22 \mathrm{bcb}$ & $5,713.0$ & $5,714.0$ & 324PRDX & 2.3 & 0.000000 & Limestone and dolomite \\
\hline $\mathrm{nmb} 33.5-20-22 \mathrm{bcb}$ & $5,714.0$ & $5,715.0$ & 324PRDX & 8.3 & 0.010000 & Limestone and dolomite \\
\hline $\mathrm{nmb} 33.5-20-22 \mathrm{bcb}$ & $5,715.0$ & $5,716.0$ & 324PRDX & 1.8 & 0.000000 & Limestone and dolomite \\
\hline nmb33.5-20-22bcb & $5,716.0$ & $5,717.0$ & $324 \mathrm{PRDX}$ & 1.8 & 0.020000 & Shaly limestone and shaly dolomite \\
\hline $\mathrm{nmb} 33.5-20-22 \mathrm{bcb}$ & $5,717.0$ & $5,718.0$ & $324 \mathrm{PRDX}$ & 0.8 & 0.260000 & Shaly limestone and shaly dolomite \\
\hline $\mathrm{nmb} 33.5-20-22 \mathrm{bcb}$ & $5,718.0$ & $5,719.8$ & 324PRDX & 4.8 & 45.000000 & Limestone and dolomite \\
\hline nmb33.5-20-22bcb & $5,719.8$ & $5,721.7$ & 324PRDX & 7.1 & 0.800000 & Limestone \\
\hline $\mathrm{nmb} 33.5-20-22 \mathrm{bcb}$ & $5,721.7$ & $5,723.5$ & $324 \mathrm{PRDX}$ & 7.3 & 4.800000 & Limestone \\
\hline $\mathrm{nmb} 33.5-20-22 \mathrm{bcb}$ & $5,723.5$ & $5,725.4$ & $324 \mathrm{PRDX}$ & 8.7 & 14.000000 & Limestone \\
\hline nmb33.5-20-22bcb & $5,725.4$ & $5,726.6$ & 324PRDX & 7.2 & 1.800000 & Limestone \\
\hline $\mathrm{nmb} 33.5-20-22 \mathrm{bcb}$ & $5,726.6$ & $5,728.0$ & 324PRDX & 7.8 & 4.300000 & Limestone \\
\hline $\mathrm{nmb} 33.5-20-22 \mathrm{bcb}$ & $5,728.0$ & $5,729.0$ & 324PRDX & 5.6 & 0.000000 & Sandy limestone \\
\hline $\mathrm{nmb} 33.5-20-22 \mathrm{bcb}$ & $5,729.0$ & $5,730.0$ & 324PRDX & 8.5 & 0.010000 & Sandy limestone \\
\hline $\mathrm{nmb} 33.5-20-22 \mathrm{bcb}$ & $5,730.0$ & $5,731.0$ & $324 \mathrm{PRDX}$ & 4.5 & 0.020000 & Sandy limestone \\
\hline
\end{tabular}


Table 4.--Laboratory-determined porosity and permeability--Continued

\begin{tabular}{|c|c|c|c|c|c|c|}
\hline Site & $\begin{array}{l}\text { Depth to } \\
\text { top } \\
\text { (feet) }\end{array}$ & $\begin{array}{l}\text { Depth to } \\
\text { bottom } \\
\text { (feet) }\end{array}$ & $\begin{array}{c}\text { Forma- } \\
\text { tion }\end{array}$ & $\begin{array}{l}\text { Porosity } \\
\text { (percent) }\end{array}$ & $\begin{array}{l}\text { Perme- } \\
\text { ability } \\
\text { (milli- } \\
\text { darcies) }\end{array}$ & Lithology \\
\hline $\mathrm{nmb} 33.5-20-22 \mathrm{bcb}$ & $5,731.0$ & $5,732.0$ & $324 \mathrm{PRDX}$ & 5.7 & 0.000000 & Limestone \\
\hline $\mathrm{nmb} 33.5-20-22 \mathrm{bcb}$ & $5,732.0$ & $5,733.0$ & $324 \mathrm{PRDX}$ & 2.8 & 0.000000 & Dolomite \\
\hline $\mathrm{nmb} 33.5-20-22 \mathrm{bcb}$ & $5,733.0$ & $5,734.0$ & 324PRDX & 5.8 & 0.000000 & Dolomite \\
\hline $\mathrm{nmb} 33.5-20-22 \mathrm{bcb}$ & $5,734.0$ & $5,735.0$ & 324PRDX & 5.2 & 0.000000 & Dolomite \\
\hline nmb33.5-20-22bcb & $5,735.0$ & $5,736.0$ & $324 \mathrm{PRDX}$ & 2.8 & 0.040000 & Dolomite \\
\hline$n m b 33.5-20-22 b c b$ & $5,736.0$ & $5,737.0$ & $324 \mathrm{PRDX}$ & 1.6 & 0.000000 & Dolomite \\
\hline nmb33.5-20-22bcb & $5,737.0$ & $5,738.0$ & 324PRDX & 2.3 & 0.000000 & Dolomite \\
\hline nmb33.5-20-22bcb & $5,738.0$ & $5,739.0$ & 324PRDX & 1.5 & 0.000000 & Dolomite \\
\hline nmb33.5-20-22bcb & $5,739.0$ & $5,740.0$ & 324PRDX & 0.7 & 0.000000 & Dolomite \\
\hline nmb33.5-20-22bcb & $5,740.0$ & $5,741.0$ & $324 \mathrm{PRDX}$ & 2.3 & 0.000000 & Shaly dolomite \\
\hline nmb33.5-20-22bcb & $5,741.0$ & $5,742.0$ & 324PRDX & 2.3 & 0.000000 & Shaly dolomite \\
\hline nmb33.5-20-22bcb & $5,742.0$ & $5,743.0$ & $324 \mathrm{PRDX}$ & 0.7 & 0.000000 & Dolomite \\
\hline$n m b 33.5-20-22 b c b$ & $5,743.0$ & $5,744.0$ & 324PRDX & 2.6 & 0.000000 & Dolomite \\
\hline nmb33.5-20-22bcb & $5,744.0$ & $5,745.0$ & 324PRDX & 0.9 & 0.000000 & Dolomite \\
\hline nmb33.5-20-22bcb & $5,745.0$ & $5,746.0$ & 324PRDX & 1.2 & 0.000000 & Dolomite \\
\hline$n m b 33.5-20-22 b c b$ & $5,746.0$ & $5,747.0$ & $324 \mathrm{PRDX}$ & 0.7 & 0.000000 & Dolomite \\
\hline nmb33.5-20-22bcb & $5,747.0$ & $5,748.0$ & $324 \mathrm{PRDX}$ & 0.5 & 0.000000 & Dolomite \\
\hline nmb33.5-20-22bcb & $5,748.0$ & $5,749.0$ & $324 \mathrm{PRDX}$ & 0.3 & 0.010000 & Dolomite \\
\hline nmb33.5-20-22bcb & $5,749.0$ & $5,750.0$ & $324 \mathrm{PRDX}$ & 2.1 & 0.010000 & Dolomite \\
\hline nmb33.5-20 -22bcb & $5,750.0$ & $5,751.0$ & $324 \mathrm{PRDX}$ & 1.6 & 0.000000 & Dolomite \\
\hline nmb33.5-20-22bcb & $5,751.0$ & $5,752.0$ & $324 \mathrm{PRDX}$ & 1.8 & 0.000000 & Dolomite \\
\hline nmb33.5-20-22bcb & $5,754.0$ & $5,755.0$ & 324PRDX & 1.4 & 0.000000 & Limestone \\
\hline nmb33.5-20-22bcb & $5,755.0$ & $5,756.0$ & $324 \mathrm{PRDX}$ & 1.4 & 0.000000 & Limestone \\
\hline nmb33.5-20-22bcb & $5,756.0$ & $5,757.0$ & $324 \mathrm{PRDX}$ & 1.0 & 0.000000 & Limestone \\
\hline nmb33.5-20-22bcb & $5,757.0$ & $5,758.0$ & 324PRDX & 0.9 & 0.000000 & Limestone \\
\hline nmb33.5-20-22bcb & $5,758.0$ & $5,759.0$ & 324PRDX & 0.9 & 0.000000 & Limestone \\
\hline nmb33.5-20-22bcb & $5,759.0$ & $5,760.0$ & $324 \mathrm{PRDX}$ & 0.5 & 0.000000 & Limestone \\
\hline nmb33.5-20-22bcb & $5,760.0$ & $5,761.0$ & 324PRDX & 0.3 & 0.000000 & Limestone \\
\hline nmb33.5-20-22bcb & $5,761.0$ & $5,762.0$ & $324 \mathrm{PRDX}$ & 0.3 & 0.000000 & Limestone \\
\hline nmb33.5-20-22bcb & $5,762.0$ & $5,763.0$ & $324 \mathrm{PRDX}$ & 0.6 & 0.000000 & Limestone \\
\hline nmb33.5-20-22bcb & $5,763.0$ & $5,764.0$ & $324 \mathrm{PRDX}$ & 0.9 & 0.000000 & Limestone \\
\hline nmb33.5-20-22bcb & $5,764.0$ & $5,765.0$ & 324PRDX & 0.6 & 0.000000 & Limestone \\
\hline $\mathrm{nmb} 33.5-20-22 \mathrm{bcb}$ & $5,765.0$ & $5,766.0$ & 324PRDX & 0.8 & 0.000000 & Limestone \\
\hline nmb33.5-20-22bcb & $5,766.0$ & $5,767.0$ & $324 \mathrm{PRDX}$ & 1.0 & 0.000000 & Limestone \\
\hline nmb33.5-20-22bcb & $5,767.0$ & $5,768.0$ & 324PRDX & 1.0 & 0.000000 & Limestone \\
\hline$n m b 33.5-20-22 b c b$ & $5,768.0$ & $5,769.0$ & $324 \mathrm{PRDX}$ & 2.4 & 0.000000 & Limestone \\
\hline nmb33.5-20-22bcb & $5,769.0$ & $5,770.0$ & 324PRDX & 1.1 & 0.000000 & Limestone \\
\hline nmb33.5-20-22bcb & $5,770.0$ & $5,771.0$ & $324 \mathrm{PRDX}$ & 0.8 & 0.000000 & Limestone \\
\hline nmb33.5-20-22bcb & $5,771.0$ & $5,772.0$ & $324 \mathrm{PRDX}$ & 0.7 & 0.000000 & Limestone \\
\hline nmb33.5-20-22bcb & $5,772.0$ & $5,773.0$ & $324 \mathrm{PRDX}$ & 1.0 & 0.000000 & Limestone \\
\hline nmb33.5-20-22bcb & $5,773.0$ & $5,774.0$ & 324PRDX & 0.7 & 0.010000 & Limestone \\
\hline nmb33.5-20-22bcb & $5,774.0$ & $5,775.0$ & $324 \mathrm{PRDX}$ & 1.9 & 0.000000 & Limestone \\
\hline nmb33.5-20-22bcb & $5,775.0$ & $5,776.0$ & 324PRDX & 1.5 & 0.000000 & Limestone \\
\hline nmb33.5-20-22bcb & $5,776.0$ & $5,777.0$ & 324PRDX & 1.5 & 0.000000 & Limestone \\
\hline nmb33.5-20-22bcb & $5,777.0$ & $5,778.0$ & $324 \mathrm{PRDX}$ & 1.5 & 0.000000 & Limestone \\
\hline $\mathrm{nmb} 33.5-20-22 \mathrm{bcb}$ & $5,778.0$ & $5,779.0$ & $324 \mathrm{PRDX}$ & 2.9 & 0.000000 & Limestone \\
\hline nmb33.5-20-22bcb & $5,779.0$ & $5,780.0$ & $324 \mathrm{PRDX}$ & 2.3 & 0.000000 & Limestone \\
\hline nmb33.5-20-22bcb & $5,780.0$ & $5,781.0$ & 324PRDX & 2.0 & 0.000000 & Limestone \\
\hline nmb33.5-20-22bcb & $5,781.0$ & $5,782.0$ & $324 \mathrm{PRDX}$ & 3.7 & 0.000000 & Limestone \\
\hline nmb33.5-20-22bcb & $5,782.0$ & $5,783.0$ & 324PRDX & 3.2 & 0.000000 & Limestone \\
\hline
\end{tabular}


Table 4.--Laboratory-determined porosity and permeability--Continued

\begin{tabular}{|c|c|c|c|c|c|c|}
\hline Site & $\begin{array}{l}\text { Depth to } \\
\text { top } \\
\text { (feet) }\end{array}$ & $\begin{array}{l}\text { Depth to } \\
\text { bottom } \\
\text { (feet) }\end{array}$ & $\begin{array}{c}\text { Forma- } \\
\text { tion }\end{array}$ & $\begin{array}{l}\text { Porosity } \\
\text { (percent) }\end{array}$ & $\begin{array}{l}\text { Perme- } \\
\text { ability } \\
\text { (milli- } \\
\text { darcies) }\end{array}$ & Lithology \\
\hline \multirow[t]{2}{*}{ nmb33.5-20-22bcb } & $\begin{array}{l}5,783.0 \\
5,784.0\end{array}$ & $\begin{array}{l}5,784.0 \\
5,785.0\end{array}$ & $\begin{array}{l}\text { 324PRDX } \\
\text { 324PRDX }\end{array}$ & $\begin{array}{l}2.8 \\
2.6 \\
\end{array}$ & $\begin{array}{l}0.000000 \\
0.000000 \\
\end{array}$ & $\begin{array}{l}\text { Limestone } \\
\text { Limestone }\end{array}$ \\
\hline & & & AVERAGE -- & 2.3 & 0.690291 & \\
\hline SITE AVERAGE- - & 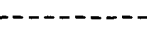 & $-\cdots+-1$ & - - $-\cdots-1$ & 2.3 & 0.690291 & \\
\hline $\begin{array}{l}\text { nmb34-20-03baa } \\
\text { nmb34-20-03baa } \\
\text { nmb34-20-03baa } \\
\text { nmb34-20-03baa } \\
\text { nmb34-20-03baa }\end{array}$ & $\begin{array}{l}5,562.0 \\
5,563.0 \\
5,565.0 \\
5,567.0 \\
5,569.0\end{array}$ & $\begin{array}{l}5,563.0 \\
5,565.0 \\
5,567.0 \\
5,569.0 \\
5,571.0\end{array}$ & $\begin{array}{l}\text { 324PRDX } \\
324 \mathrm{PRDX} \\
324 \mathrm{PRDX} \\
324 \mathrm{PRDX} \\
324 \mathrm{PRDX}\end{array}$ & $\begin{array}{l}3.2 \\
1.2 \\
0.9 \\
3.7 \\
0.5\end{array}$ & $\begin{array}{l}0.300000 \\
0.000000 \\
0.000000 \\
0.000000 \\
0.000000\end{array}$ & $\begin{array}{l}\text { Shale and anhydrite } \\
\text { Shale, limestone, and anhydrite } \\
\text { Limestone } \\
\text { Limestone } \\
\text { Limestone }\end{array}$ \\
\hline $\begin{array}{l}\text { nmb34-20-03baa } \\
\text { nmb34-20-03baa } \\
\text { nmb34-20-03baa } \\
\text { nmb34-20-03baa } \\
\text { nmb34-20-03baa }\end{array}$ & $\begin{array}{l}5,571.0 \\
5,573.0 \\
5,575.0 \\
5,577.0 \\
5,579.0\end{array}$ & $\begin{array}{l}5,573.0 \\
5,575.0 \\
5,577.0 \\
5,579.0 \\
5,581.0\end{array}$ & $\begin{array}{l}324 \mathrm{PRDX} \\
324 \mathrm{PRDX} \\
324 \mathrm{PRDX} \\
324 \mathrm{PRDX} \\
324 \mathrm{PRDX}\end{array}$ & $\begin{array}{l}0.5 \\
0.7 \\
1.0 \\
0.6 \\
6.2\end{array}$ & $\begin{array}{l}0.000000 \\
0.000000 \\
0.000000 \\
0.000000 \\
0.000000\end{array}$ & $\begin{array}{l}\text { Limestone } \\
\text { Limestone } \\
\text { Limestone and limy shale } \\
\text { Limy shale } \\
\text { Dolomite and shaly dolomite }\end{array}$ \\
\hline $\begin{array}{l}\text { nmb34-20-03baa } \\
\text { nmb34-20-03baa } \\
\text { nmb34-20-03baa } \\
\text { nmb34-20-03baa } \\
\text { nmb34-20-03baa }\end{array}$ & $\begin{array}{l}5,581.0 \\
5,583.0 \\
5,585.0 \\
5,587.0 \\
5,589.0\end{array}$ & $\begin{array}{l}5,583.0 \\
5,585.0 \\
5,587.0 \\
5,589.0 \\
5,590.0\end{array}$ & $\begin{array}{l}\text { 324PRDX } \\
324 \mathrm{PRDX} \\
324 \mathrm{PRDX} \\
324 \mathrm{PRDX} \\
324 \mathrm{PRDX}\end{array}$ & $\begin{array}{l}5.8 \\
7.9 \\
6.1 \\
3.3 \\
4.6\end{array}$ & $\begin{array}{l}0.000000 \\
0.000000 \\
0.000000 \\
0.200000 \\
0.200000\end{array}$ & $\begin{array}{l}\text { Shaly dolomite } \\
\text { Shaly dolomite } \\
\text { Shaly dolomite } \\
\text { Shaly dolomite } \\
\text { Shaly dolomite }\end{array}$ \\
\hline $\begin{array}{l}\text { nmb34-20-03baa } \\
\text { nmb34-20-03baa } \\
\text { nmb34-20-03baa } \\
\text { nmb34-20-03baa } \\
\text { nmb34-20-03baa }\end{array}$ & $\begin{array}{l}5,591.0 \\
5,593.0 \\
5,595.0 \\
5,597.0 \\
5,599.0\end{array}$ & $\begin{array}{l}5,592.0 \\
5,594.0 \\
5,596.0 \\
5,598.0 \\
5,600.0\end{array}$ & $\begin{array}{l}324 \mathrm{PRDX} \\
324 \mathrm{PRDX} \\
324 \mathrm{PRDX} \\
324 \mathrm{PRDX} \\
324 \mathrm{PRDX}\end{array}$ & $\begin{array}{r}6.0 \\
5.6 \\
10.6 \\
6.5 \\
7.8\end{array}$ & $\begin{array}{l}0.000000 \\
0.000000 \\
0.000000 \\
0.000000 \\
0.100000\end{array}$ & $\begin{array}{l}\text { Shaly limestone and dolomite } \\
\text { Shaly limestone and dolomite } \\
\text { Shaly limestone and dolomite } \\
\text { Shaly limestone and dolomite } \\
\text { Shaly limestone and dolomite }\end{array}$ \\
\hline $\begin{array}{l}\text { nmb34-20-03baa } \\
\text { nmb34-20-03baa } \\
\text { nmb34-20-03ba a } \\
\text { nmb34-20-03baa } \\
\text { nmb34-20-03baa }\end{array}$ & $\begin{array}{l}5,601.0 \\
5,603.0 \\
5,605.0 \\
5,607.0 \\
5,609.0\end{array}$ & $\begin{array}{l}5,602.0 \\
5,604.0 \\
5,606.0 \\
5,608.0 \\
5,610.0\end{array}$ & $\begin{array}{l}\text { 324PRDX } \\
324 \mathrm{PRDX} \\
324 \mathrm{PRDX} \\
324 \mathrm{PRDX} \\
324 \mathrm{PRDX}\end{array}$ & $\begin{array}{l}0.5 \\
1.6 \\
0.6 \\
0.5 \\
1.1\end{array}$ & $\begin{array}{l}0.000000 \\
0.000000 \\
0.000000 \\
0.000000 \\
0.000000\end{array}$ & $\begin{array}{l}\text { Limy shale and anhydrite } \\
\text { Shaly limestone } \\
\text { Shale and anhydrite } \\
\text { Limy shale } \\
\text { Limy shale }\end{array}$ \\
\hline $\begin{array}{l}\text { nmb34-20-03baa } \\
\text { nmb34-20-03baa } \\
\text { nmb34-20-03baa } \\
\text { nmb34-20-03baa } \\
\text { nmb34-20-03baa }\end{array}$ & $\begin{array}{l}5,611.0 \\
5,613.0 \\
5,615.0 \\
5,617.0 \\
5,619.0\end{array}$ & $\begin{array}{l}5,612.0 \\
5,614.0 \\
5,616.0 \\
5,618.0 \\
5,620.0\end{array}$ & $\begin{array}{l}\text { 324PRDX } \\
324 \mathrm{PRDX} \\
324 \mathrm{PRDX} \\
324 \mathrm{PRDX} \\
324 \mathrm{PRDX}\end{array}$ & $\begin{array}{l}1.7 \\
0.5 \\
1.5 \\
1.3 \\
2.9\end{array}$ & $\begin{array}{l}0.000000 \\
0.000000 \\
0.000000 \\
0.000000 \\
0.000000\end{array}$ & $\begin{array}{l}\text { Limy shale } \\
\text { Limy shale } \\
\text { Limy shale } \\
\text { Shaly limestone } \\
\text { Shaly limestone }\end{array}$ \\
\hline $\begin{array}{l}\text { nmb34-20-03ba a } \\
\text { nmb34-20-03ba a } \\
\text { nmb34-20-03ba a } \\
n m b 34-20-03 \text { ba a } \\
\text { nmb34-20-03ba a }\end{array}$ & $\begin{array}{l}5,621.0 \\
5,623.0 \\
5,625.0 \\
5,627.0 \\
5,629.0\end{array}$ & $\begin{array}{l}5,622.0 \\
5,624.0 \\
5,626.0 \\
5,628.0 \\
5,630.0\end{array}$ & $\begin{array}{l}324 \mathrm{PRDX} \\
324 \mathrm{PRDX} \\
324 \mathrm{PRDX} \\
324 \mathrm{PRDX} \\
324 \mathrm{PRDX}\end{array}$ & $\begin{array}{l}3.5 \\
1.7 \\
2.0 \\
1.3 \\
2.6\end{array}$ & $\begin{array}{l}0.000000 \\
0.000000 \\
0.200000 \\
0.000000 \\
0.100000\end{array}$ & $\begin{array}{l}\text { Shaly limestone } \\
\text { Shaly limestone } \\
\text { Shaly limestone } \\
\text { Shaly limestone } \\
\text { Shaly limestone }\end{array}$ \\
\hline $\begin{array}{l}\text { nmb34-20-03baa } \\
\text { nmb34-20-03baa } \\
\text { nmb34-20-03baa } \\
\text { nmb34-20-03baa } \\
\text { nmb34-20-03baa }\end{array}$ & $\begin{array}{l}5,631.0 \\
5,633.0 \\
5,635.0 \\
5,637.0 \\
5,638.0\end{array}$ & $\begin{array}{l}5,632.0 \\
5,634.0 \\
5,636.0 \\
5,638.0 \\
5,639.5\end{array}$ & $\begin{array}{l}324 \mathrm{PRDX} \\
324 \mathrm{PRDX} \\
324 \mathrm{PRDX} \\
324 \mathrm{PRDX} \\
324 \mathrm{PRDX}\end{array}$ & $\begin{array}{r}3.2 \\
6.3 \\
10.8 \\
18.7 \\
19.0 \\
\end{array}$ & $\begin{array}{l}0.100000 \\
0.000000 \\
0.000000 \\
4.300000 \\
\underline{0.400000} \\
\end{array}$ & $\begin{array}{l}\text { Limestone } \\
\text { Limestone } \\
\text { Limestone } \\
\text { Shaly limestone } \\
\text { Shaly limestone }\end{array}$ \\
\hline SITE AVERAGE-- & & & AVERAGE-- & $\frac{4.1}{4.1}$ & $\frac{0.147500}{0.147500}$ & \\
\hline
\end{tabular}


Table 4.--Laboratory-determined porosity and permeability--Continued

\begin{tabular}{|c|c|c|c|c|c|c|}
\hline Site & $\begin{array}{l}\text { Depth to } \\
\text { top } \\
\text { (feet) }\end{array}$ & $\begin{array}{l}\text { Depth to } \\
\text { bottom } \\
\text { (feet) }\end{array}$ & $\begin{array}{l}\text { Forma- } \\
\text { tion }\end{array}$ & $\begin{array}{l}\text { Porosity } \\
\text { (percent) }\end{array}$ & $\begin{array}{l}\text { Perme- } \\
\text { ability } \\
\text { (milli- } \\
\text { darcies) }\end{array}$ & Lithology \\
\hline nmb35-20-15ccc & $5,602.0$ & $5,603.9$ & $324 \mathrm{PRDX}$ & 5.7 & 6.500000 & Dolomite and limestone \\
\hline $\mathrm{nmb} 35-20-15 \mathrm{ccc}$ & $5,603.9$ & $5,606.0$ & 324PRDX & 4.1 & 0.800000 & Dolomite and limestone \\
\hline nmb35-20-15ccc & $5,606.0$ & $5,608.0$ & $324 \mathrm{PRDX}$ & 5.2 & 3.900000 & Dolomite and limestone \\
\hline$n m b 35-20-15 c c c$ & $5,608.0$ & $5,609.9$ & 324PRDX & 8.4 & 1.100000 & Dolomite and limestone \\
\hline$n m b 35-20-15 c c c$ & $5,609.9$ & $5,611.9$ & $324 \mathrm{PRDX}$ & 10.7 & 7.200000 & Dolomite and limestone \\
\hline$n m b 35-20-15 c c c$ & $5,611.9$ & $5,613.5$ & 324PRDX & 11.4 & 2.400000 & Dolomite and limestone \\
\hline$n m b 35-20-15 c c c$ & $5,613.5$ & $5,615.5$ & $324 \mathrm{PRDX}$ & 11.9 & 1.800000 & Dolomite and limestone \\
\hline $\mathrm{nmb} 35-20-15 \mathrm{ccc}$ & $5,615.5$ & $5,617.3$ & 324PRDX & 12.2 & 2.100000 & Dolomite and limestone \\
\hline$n m b 35-20-15 c c c$ & $5,617.3$ & $5,619.0$ & 324PRDX & 11.9 & 4.500000 & Dolomite and limestone \\
\hline$n m b 35-20-15 c c c$ & $5,619.0$ & $5,620.9$ & 324PRDX & 8.4 & 0.300000 & Dolomite and limestone \\
\hline$n m b 35-20-15 c c c$ & $5,620.9$ & $5,623.0$ & 324PRDX & 2.7 & 2.100000 & Limestone and dolomite \\
\hline$n m b 35-20-15 c c c$ & $5,625.0$ & $5,626.0$ & $324 \mathrm{PRDX}$ & 7.2 & 1.000000 & Dolomite \\
\hline $\mathrm{nmb} 35-20-15 \mathrm{ccc}$ & $5,629.0$ & $5,630.0$ & $324 \mathrm{PRDX}$ & 9.7 & 0.000000 & Dolomite \\
\hline$n m b 35-20-15 c c c$ & $5,631.0$ & $5,632.0$ & 324PRDX & 9.5 & 0.100000 & Dolomite \\
\hline nmb35-20-15ccc & $5,633.0$ & $5,634.0$ & $324 \mathrm{PRDX}$ & 4.5 & 0.000000 & Dolomite \\
\hline $\mathrm{nmb} 35-20-15 \mathrm{ccc}$ & $5,635.0$ & $5,637.0$ & $324 \mathrm{PRDX}$ & 3.9 & 0.100000 & Dolomite \\
\hline $\mathrm{nmb} 35-20-15 \mathrm{ccc}$ & $5,638.0$ & $5,639.0$ & 324PRDX & 2.7 & 0.100000 & Dolomite \\
\hline$n m b 35-20-15 c c c$ & $5,643.0$ & $5,645.0$ & 324PRDX & 4.1 & 0.300000 & Limestone \\
\hline nmb $35-20-15 c c c$ & $5,645.0$ & $5,647.0$ & $324 \mathrm{PRDX}$ & 5.5 & 1.100000 & Limestone \\
\hline$n m b 35-20-15 c c c$ & $5,647.0$ & $5,648.7$ & $324 \mathrm{PRDX}$ & 5.0 & 2.200000 & Limestone \\
\hline $\mathrm{nmb} 35-20-15 \mathrm{ccc}$ & $5,648.7$ & $5,650.3$ & 324PRDX & 5.8 & 3.600000 & Limestone \\
\hline $\mathrm{nmb} 35-20-15 \mathrm{ccc}$ & $5,650.3$ & $5,652.3$ & $324 \mathrm{PRDX}$ & 2.0 & 1.400000 & Limestone \\
\hline$n m b 35-20-15 c c c$ & $5,652.3$ & $5,654.2$ & 324PRDX & 3.1 & 1.800000 & Limestone \\
\hline $\mathrm{nmb} 35-20-15 \mathrm{ccc}$ & $5,654.2$ & $5,656.0$ & $324 \mathrm{PRDX}$ & 4.4 & 4.300000 & Limestone \\
\hline $\mathrm{nmb} 35-20-15 \mathrm{ccc}$ & $5,656.0$ & $5,658.0$ & 324PRDX & 5.2 & 7.400000 & Limestone \\
\hline$n m b 35-20-15 c c c$ & $5,658.0$ & $5,660.0$ & $324 \mathrm{PRDX}$ & 6.5 & 5.700000 & Limestone \\
\hline $\mathrm{nmb} 35-20-15 \mathrm{ccc}$ & $5,660.0$ & $5,662.0$ & $324 \mathrm{PRDX}$ & 5.7 & 3.000000 & Limestone \\
\hline$n m b 35-20-15 c c c$ & $5,662.0$ & $5,663.8$ & 324PRDX & 5.1 & 9.500000 & Limestone \\
\hline $\mathrm{nmb} 35-20-15 \mathrm{ccc}$ & $5,663.8$ & $5,665.5$ & 324PRDX & 5.6 & 21.000000 & Limestone \\
\hline$n m b 35-20-15 c c c$ & $5,665.5$ & $5,667.2$ & $324 \mathrm{PRDX}$ & 5.0 & 4.000000 & Limestone \\
\hline nmb35-20-15ccc & $5,667.2$ & $5,669.3$ & 324PRDX & 5.3 & 6.500000 & Limestone \\
\hline $\mathrm{nmb} 35-20-15 \mathrm{ccc}$ & $5,669 \cdot 3$ & $5,671.3$ & 324PRDX & 5.3 & 0.800000 & Limestone \\
\hline $\mathrm{nmb} 35-20-15 \mathrm{ccc}$ & $5,671.3$ & $5,673.3$ & $324 \mathrm{PRDX}$ & 8.5 & 6.000000 & Limestone \\
\hline$n m b 35-20-15 c c c$ & $5,673.3$ & $5,675.1$ & 324PRDX & 6.2 & 4.000000 & Limestone \\
\hline$n m b 35-20-15 c c c$ & $5,675.1$ & $5,677.0$ & 324PRDX & 5.5 & 0.500000 & Dolomite \\
\hline$n m b 35-20-15 c c c$ & $5,677.0$ & $5,679.0$ & $324 \mathrm{PRDX}$ & 11.3 & 3.600000 & Dolomite \\
\hline $\mathrm{nmb} 35-20-15 \mathrm{ccc}$ & $5,679.0$ & $5,680.4$ & 324PRDX & 8.9 & 0.300000 & Dolomite \\
\hline$n m b 35-20-15 c c c$ & $5,680.4$ & $5,683.0$ & 324PRDX & 7.3 & 0.600000 & Dolomite \\
\hline $\mathrm{nmb} 35-20-15 \mathrm{ccc}$ & $5,683.0$ & $5,685.0$ & 324PRDX & 8.5 & 8.300000 & Dolomite \\
\hline$n m b 35-20-15 c c c$ & $5,685.0$ & $5,687.1$ & 324PRDX & 19.2 & 3.500000 & Dolomite \\
\hline $\mathrm{nmb} 35-20-15 \mathrm{ccc}$ & $5,687.1$ & $5,688.8$ & $324 \mathrm{PRDX}$ & 21.9 & 7.600000 & Dolomite \\
\hline$n m b 35-20-15 c c c$ & $5,688.8$ & $5,690.7$ & 324PRDX & 21.9 & 13.000000 & Dolomite \\
\hline $\mathrm{nmb} 35-20-15 \mathrm{ccc}$ & $5,690.7$ & $5,693.0$ & 324PRDX & 22.2 & 8.500000 & Dolomite \\
\hline$n m b 35-20-15$ ccc & $5,693.0$ & $5,695.1$ & 324PRDX & 20.4 & 5.500000 & Dolomite \\
\hline $\mathrm{nmb} 35-20-15 \mathrm{ccc}$ & $5,695.1$ & $5,697.0$ & 324PRDX & 16.9 & 0.000000 & Dolomite \\
\hline \multirow[t]{3}{*}{ nmb35-20-15ccc } & $5,697.0$ & $5,699.0$ & 324PRDX & 4.5 & 0.000000 & Dolomite \\
\hline & & & AVERAGE-- & 8.4 & 3.652174 & \\
\hline & & & & 8.4 & 3.652174 & \\
\hline
\end{tabular}


Table 4.--Laboratory-determined porosity and permeability--Continued

\begin{tabular}{|c|c|c|c|c|c|c|}
\hline Site & $\begin{array}{l}\text { Depth to } \\
\text { top } \\
\text { (feet) }\end{array}$ & $\begin{array}{l}\text { Depth to } \\
\text { bottom } \\
\text { (feet) }\end{array}$ & $\begin{array}{c}\text { Forma- } \\
\text { tion }\end{array}$ & $\begin{array}{l}\text { Porosity } \\
\text { (percent) }\end{array}$ & $\begin{array}{c}\text { Perme- } \\
\text { ability } \\
\text { (milli- } \\
\text { darcies) }\end{array}$ & Lithology \\
\hline nmb35-20-34ddd & $5,493.0$ & $5,494.0$ & $324 \mathrm{PRDX}$ & 12.6 & 1.100000 & Sucrose dolomite \\
\hline nmb35-20-34ddd & $5,498.0$ & $5,499.0$ & $324 \mathrm{PRDX}$ & 11.6 & 1.300000 & Crystalline to sucrose dolomite \\
\hline nmb35-20-34ddd & $5,507.0$ & $5,508.0$ & 324PRDX & 13.7 & 3.300000 & Anhydritic, sucrose dolomite \\
\hline nmb $35-20-34 d d d$ & $5,512.0$ & $5,513.0$ & $324 \mathrm{PRDX}$ & 12.0 & 4.800000 & Anhydritic, sucrose to crystalline dolomite \\
\hline nmb35-20-34ddd & $5,517.0$ & $5,518.0$ & $324 \mathrm{PRDX}$ & 2.1 & 0.060000 & Vuggy, anhydritic, crystalline limestone \\
\hline nmb35-20-34ddd & $5,518.0$ & $5,519.0$ & 324PRDX & 4.7 & 0.070000 & Vuggy, anhydritic, crystalline limestone \\
\hline nmb $35-20-34 d d d$ & $5,519.0$ & $5,520.0$ & 324PRDX & 2.1 & 1.100000 & Vuggy, anhydritic, crystalline limestone \\
\hline nmb35-20-34ddd & $5,520.0$ & $5,521.0$ & $324 \mathrm{PRDX}$ & 4.1 & 2.400000 & Vuggy, anhydritic, crystalline limestone \\
\hline nmb35-20-34ddd & $5,521.0$ & $5,522.0$ & $324 \mathrm{PRDX}$ & 1.6 & 0.060000 & Vuggy, anhydritic, crystalline limestone \\
\hline nmb35-20-34ddd & $5,522.0$ & $5,523.0$ & 324PRDX & 7.8 & 0.350000 & Vuggy, anhydritic, crystalline limestone \\
\hline nmb35-20-34ddd & $5,523.0$ & $5,524.0$ & $324 \mathrm{PRDX}$ & 4.7 & 0.790000 & Vuggy, anhydritic, crystalline limestone \\
\hline nmb35-20-34ddd & $5,532.0$ & $5,533.0$ & $324 \mathrm{PRDX}$ & 21.7 & 0.390000 & Sucrose to earthy dolomite \\
\hline nmb $35-20-34 d d d$ & $5,534.0$ & $5,535.0$ & $324 \mathrm{PRDX}$ & 10.0 & 0.020000 & Sucrose to earthy dolomite \\
\hline nmb35-20-34ddd & $5,535.0$ & $5,536.0$ & $324 \mathrm{PRDX}$ & 15.0 & 0.200000 & Anhydritic, sucrose to earthy dolomite \\
\hline nmb $35-20-34 d d d$ & $5,536.0$ & $5,537.0$ & $324 \mathrm{PRDX}$ & 17.8 & 0.330000 & Anhydritic, sucrose to earthy dolomite \\
\hline nmb35-20-34ddd & $5,538.0$ & $5,539.0$ & $324 \mathrm{PRDX}$ & 8.8 & 5.200000 & Anhydritic, sucrose to earthy dolomite \\
\hline nmb35-20-34ddd & $5,539.0$ & $5,540.0$ & $324 \mathrm{PRDX}$ & 6.4 & 0.280000 & Anhydritic, sucrose to earthy dolomite \\
\hline nmb35-20-34ddd & $5,540.0$ & $5,541.0$ & 324PRDX & 3.3 & 0.120000 & Vuggy, anhydritic limestone \\
\hline nmb35-20-34ddd & $5,541.0$ & $5,542.0$ & $324 \mathrm{PRDX}$ & 5.5 & 1.900000 & Vuggy, anhydritic limestone \\
\hline nmb35-20-34ddd & $5,542.0$ & $5,543.0$ & $324 \mathrm{PRDX}$ & 3.5 & 0.750000 & Vuggy, anhydritic limestone \\
\hline nmb35-20-34ddd & $5,544.0$ & $5,545.0$ & $324 \mathrm{PRDX}$ & 5.9 & 8.000000 & Vuggy, anhydritic, dolomitic limestone \\
\hline nmb $35-20-34 d d d$ & $5,545.0$ & $5,546.0$ & 324PRDX & 7.8 & 1.300000 & Vuggy, anhydritic, dolomitic limestone \\
\hline nmb $35-20-34 d d d$ & $5,551.0$ & $5,552.0$ & $324 \mathrm{PRDX}$ & 5.2 & 3.300000 & Slightly vuggy, dolomitic limestone \\
\hline nmb35-20-34ddd & $5,552.0$ & $5,553.0$ & $324 \mathrm{PRDX}$ & 9.8 & 4.400000 & Vuggy, sucrose, dolomitic limestone \\
\hline nmb35-20-34ddd & $5,553.0$ & $5,554.0$ & $324 \mathrm{PRDX}$ & 10.3 & 0.630000 & Vuggy, anhydritic, sucrose, dolomitic limestone \\
\hline nmb35-20-34ddd & $5,554.0$ & $5,555.0$ & $324 \mathrm{PRDX}$ & 11.6 & 43.000000 & Vuggy, anhydritic, sucrose, dolomitic limestone \\
\hline nmb35-20-34ddd & $5,555.0$ & $5,556.0$ & $324 \mathrm{PRDX}$ & 16.9 & 35.000000 & Vuggy, sucrose, dolomitic limestone \\
\hline nmb $35-20-34 d d d$ & $5,556.0$ & $5,557.0$ & $324 \mathrm{PRDX}$ & 18.0 & 39.000000 & Vuggy, sucrose dolomite with limestone fragments \\
\hline nmb35-20-34ddd & $5,557.0$ & $5,558.0$ & 324PRDX & 14.2 & 10.000000 & Vuggy, anhydritic, sucrose dolomite \\
\hline $\mathrm{nmb} 35-20-34 \mathrm{ddd}$ & $5,558.0$ & $5,559.0$ & $324 \mathrm{PRDX}$ & 20.8 & 1.100000 & Vuggy, anhydritic, sucrose dolomite \\
\hline nmb $35-20-34 d d d$ & $5,559.0$ & $5,560.0$ & $324 \mathrm{PRDX}$ & 20.7 & 15.000000 & Vuggy, anhydritic, sucrose dolomite \\
\hline nmb35-20-34ddd & $5,560.0$ & $5,561.0$ & $324 \mathrm{PRDX}$ & 17.6 & 53.000000 & Vuggy, anhydritic, sucrose dolomite \\
\hline nmb $35-20-34 d d d$ & $5,561.0$ & $5,562.0$ & $324 \mathrm{PRDX}$ & 13.5 & 123.000000 & Vuggy, anhydritic, sucrose dolomite \\
\hline nmb35-20-34ddd & $5,562.0$ & $5,563.0$ & $324 \mathrm{PRDX}$ & 13.0 & 81.000000 & Vuggy, sucrose, dolomitic limestone \\
\hline nmb35-20-34ddd & $5,563.0$ & $5,564.0$ & $324 \mathrm{PRDX}$ & 16.1 & 84.000000 & Vuggy, sucrose, dolomitic limestone \\
\hline nmb35-20-34ddd & $5,564.0$ & $5,565.0$ & 324PRDX & 12.3 & 39.000000 & Vuggy, sucrose, dolomitic limestone \\
\hline nmb35-20-34ddd & $5,565.0$ & $5,566.0$ & 324PRDX & 15.9 & 4.800000 & Vuggy, sucrose, dolomitic limestone \\
\hline nmb35-20-34ddd & $5,566.0$ & $5,567.0$ & $324 \mathrm{PRDX}$ & 14.5 & 20.000000 & Vuggy, sucrose, dolomitic limestone \\
\hline nmb35-20-34ddd & $5,567.0$ & $5,568.0$ & 324PRDX & 10.2 & 4.800000 & Vuggy, sucrose, dolomitic limestone \\
\hline nmb35-20-34ddd & $5,568.0$ & $5,569.0$ & $324 \mathrm{PRDX}$ & 9.1 & 0.210000 & Vuggy, sucrose, dolomitic limestone \\
\hline nmb35-20-34ddd & $5,569.0$ & $5,570.0$ & $324 \mathrm{PRDX}$ & 22.6 & 15.000000 & Anhydritic, sucrose to earthy dolomite \\
\hline nmb35-20-34ddd & $5,570.0$ & $5,571.0$ & $324 \mathrm{PRDX}$ & 18.5 & 3.300000 & Anhydritic, sucrose to earthy dolomite \\
\hline nmb35-20-34ddd & $5,571.0$ & $5,572.0$ & 324PRDX & 19.1 & 9.200000 & Anhydritic, sucrose to earthy dolomite \\
\hline nmb35-20-34ddd & $5,572.0$ & $5,573.0$ & $324 \mathrm{PRDX}$ & 11.9 & 0.850000 & Anhydritic, sucrose to earthy dolomite \\
\hline nmb35-20-34ddd & $5,573.0$ & $5,574.0$ & $324 \mathrm{PRDX}$ & 5.7 & 0.100000 & Shaly, anhydritic, dolomitic limestone \\
\hline nmb35-20-34ddd & $5,575.0$ & $5,576.0$ & 324PRDX & 9.0 & 0.040000 & Anhydritic, earthy to sucrose dolomite \\
\hline nmb35-20-34ddd & $5,577.0$ & $5,578.0$ & $324 \mathrm{PRDX}$ & 9.6 & 0.010000 & Anhydritic, earthy to sucrose dolomite \\
\hline nmb35-20-34ddd & $5,579.0$ & $5,580.0$ & $324 \mathrm{PRDX}$ & 5.9 & 0.010000 & Earthy, anhydritic dolomite \\
\hline nmb $35-20-34 d d d$ & $5,581.0$ & $5,582.0$ & $324 \mathrm{PRDX}$ & 6.5 & 0.010000 & Shaly dolomite \\
\hline nmb35-20-34ddd & $5,583.0$ & $5,584.0$ & 324PRDX & 10.0 & 0.050000 & Finely crystalline, dolomitic limestone \\
\hline nmb35-20-34ddd & $5,585.0$ & $5,586.0$ & $324 \mathrm{PRDX}$ & 0.8 & 0.000000 & Finely crystalline limestone \\
\hline
\end{tabular}


Table 4.--Laboratory-determined porosity and permeability--Continued

\begin{tabular}{|c|c|c|c|c|c|c|}
\hline Site & $\begin{array}{l}\text { Depth to } \\
\text { top } \\
\text { (feet) }\end{array}$ & $\begin{array}{l}\text { Depth to } \\
\text { bottom } \\
\text { (feet) }\end{array}$ & $\begin{array}{c}\text { Forma- } \\
\text { tion }\end{array}$ & $\begin{array}{l}\text { Porosity } \\
\text { (percent) }\end{array}$ & $\begin{array}{c}\text { Perme- } \\
\text { ability } \\
\text { (milli- } \\
\text { darcies) }\end{array}$ & Lithology \\
\hline nmb35-20-34ddd & $5,587.0$ & $5,588.0$ & $324 \mathrm{PRDX}$ & 0.4 & 0.000000 & Crystalline limestone \\
\hline nmb35-20-34ddd & $5,589.0$ & $5,590.0$ & $324 \mathrm{PRDX}$ & 0.9 & 0.000000 & Crystalline limestone \\
\hline nmb35-20-34ddd & $5,591.0$ & $5,592.0$ & $324 \mathrm{PRDX}$ & 0.8 & 0.000000 & Crystalline limestone \\
\hline nmb $35-20-34 d d d$ & $5,594.0$ & $5,595.0$ & 324PRDX & 0.5 & 0.000000 & Crystalline limestone \\
\hline nmb35-20-34ddd & $5,596.0$ & $5,597.0$ & 324PRDX & 3.9 & 5.400000 & Vuggy limestone \\
\hline nmb35-20-34ddd & $5,597.0$ & $5,598.0$ & 324PRDX & 7.9 & 123.000000 & Vuggy limestone \\
\hline nmb35-20-34ddd & $5,598.0$ & $5,599.0$ & 324PRDX & 11.1 & 489.000000 & Vuggy limestone \\
\hline nmb35-20-34ddd & $5,599.0$ & $5,600.0$ & $324 \mathrm{PRDX}$ & 6.9 & 7.700000 & Vuggy limestone \\
\hline nmb35-20-34ddd & $5,600.0$ & $5,601.0$ & $324 \mathrm{PRDX}$ & 7.6 & 4.200000 & Vuggy limestone \\
\hline nmb35-20-34ddd & $5,601.0$ & $5,602.0$ & 324PRDX & 8.8 & 23.000000 & Vuggy limestone \\
\hline nmb35-20-34ddd & $5,602.0$ & $5,603.0$ & 324PRDX & 4.3 & 1.600000 & Vuggy limestone \\
\hline nmb $35-20-34 d d d$ & $5,603.0$ & $5,604.0$ & 324PRDX & 2.6 & 6.300000 & Slightly vuggy limestone \\
\hline nmb35-20-34ddd & $5,604.0$ & $5,605.0$ & 324PRDX & 1.1 & 0.200000 & Slightly vuggy, limestone conglomerate \\
\hline nmb35-20-34ddd & $5,606.0$ & $5,607.0$ & $324 \mathrm{PRDX}$ & 2.4 & 0.170000 & Slightly vuggy, limestone conglomerate \\
\hline nmb35-20-34ddd & $5,607.0$ & $5,608.0$ & 324PRDX & 4.3 & 0.880000 & Vuggy limestone conglomerate \\
\hline nmb35-20-34ddd & $5,608.0$ & $5,609.0$ & $324 \mathrm{PRDX}$ & 4.2 & 0.220000 & Slightly vuggy, limestone conglomerate \\
\hline nmb35-20-34ddd & $5,610.0$ & $5,611.0$ & 324PRDX & 2.9 & 0.120000 & Slightly vuggy, limestone conglomerate \\
\hline nmb35-20-34ddd & $5,612.0$ & $5,613.0$ & 324PRDX & 3.6 & 0.220000 & Vuggy limestone conglomerate \\
\hline nmb35-20-34ddd & $5,613.0$ & $5,614.0$ & 324PRDX & 2.2 & 114.000000 & Slightly vuggy, limestone conglomerate \\
\hline nmb35-20-34ddd & $5,614.0$ & $5,615.0$ & 324PRDX & 8.4 & 0.000000 & Finely crystalline, dolomitic limestone \\
\hline nmb35-20-34ddd & $5,615.0$ & $5,616.0$ & 324PRDX & 8.7 & 0.540000 & Earthy to finely crystalline limestone \\
\hline nmb35-20-34ddd & $5,616.0$ & $5,617.0$ & 324PRDX & 10.0 & 0.060000 & Earthy to finely crystalline, dolomitic limestone \\
\hline nmb35-20-34ddd & $5,617.0$ & $5,618.0$ & 324PRDX & 13.9 & 1.500000 & Anhydritic, earthy to crystalline, dolomitic limestone \\
\hline nmb35-20-34ddd & $5,618.0$ & $5,619.0$ & $324 \mathrm{PRDX}$ & 17.0 & 2.200000 & Sucrose to earthy dolomite \\
\hline nmb35-20-34ddd & $5,619.0$ & $5,620.0$ & $324 \mathrm{PRDX}$ & 12.4 & 1.000000 & Anhydritic, dolomitic limestone \\
\hline nmb35-20-34ddd & $5,620.0$ & $5,621.0$ & 324PRDX & 11.9 & 1.800000 & Anhydritic, dolomitic limestone \\
\hline nmb35-20-34ddd & $5,621.0$ & $5,622.0$ & 324PRDX & 9.0 & 0.140000 & Earthy dolomitic limestone \\
\hline nmb35-20-34ddd & $5,622.0$ & $5,623.0$ & $324 \mathrm{PRDX}$ & 11.5 & 0.450000 & Earthy dolomitic limestone \\
\hline nmb35-20-34ddd & $5,624.0$ & $5,625.0$ & 324PRDX & 16.0 & 0.190000 & Earthy dolomitic limestone \\
\hline nmb35-20-34ddd & $5,626.0$ & $5,627.0$ & $324 \mathrm{PRDX}$ & 14.2 & 0.680000 & Earthy dolomitic limestone \\
\hline nmb35-20-34ddd & $5,627.0$ & $5,628.0$ & 324PRDX & 19.7 & 4.400000 & Earthy dolomitic limestone \\
\hline nmb35-20-34ddd & $5,628.0$ & $5,629.0$ & 324PRDX & 15.0 & 0.590000 & Anhydritic, earthy, dolomitic limestone \\
\hline nmb35-20-34ddd & $5,629.0$ & $5,630.0$ & 324PRDX & 13.3 & 1.800000 & Anhydritic, earthy, dolomitic limestone \\
\hline nmb35-20-34ddd & $5,630.0$ & $5,631.0$ & $324 \mathrm{PRDX}$ & 12.6 & 0.440000 & Anhydritic, earthy, dolomitic limestone \\
\hline \multirow[t]{2}{*}{ nmb $35-20-34 d d d$} & $5,631.0$ & $5,632.0$ & 324PRDX & 7.6 & 0.080000 & Earthy, dolomitic limestone \\
\hline & & & AVERAGE-- & 9.6 & 16.459419 & \\
\hline SITE AVERAGE-- & -......- & -----1 & 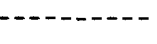 & 9.6 & $\overline{16.459419}$ & \\
\hline nmb36-17-13dcd & $5,558.0$ & $5,559.0$ & 324PRDX & 0.7 & 0.060000 & Finely crystalline, dolomitic limestone \\
\hline nmb36-17-13dcd & $5,560.0$ & $5,561.0$ & 324PRDX & 1.5 & 0.070000 & Finely crystalline, dolomitic limestone \\
\hline $\mathrm{nmb} 36-17-13 \mathrm{dcd}$ & $5,561.0$ & $5,562.0$ & 324PRDX & 2.0 & 0.060000 & Finely crystalline, dolomitic limestone \\
\hline nmb36-17-13dcd & $5,562.0$ & $5,563.0$ & 324PRDX & 2.0 & 0.080000 & Finely crystalline, dolomitic limestone \\
\hline nmb36-17-13dcd & $5,563.0$ & $5,564.0$ & $324 \mathrm{PRDX}$ & 2.1 & 0.090000 & Finely crystalline, dolomitic limestone \\
\hline nmb36-17-13dcd & $5,564.0$ & $5,565.0$ & $324 \mathrm{PRDX}$ & 2.2 & 0.070000 & Vuggy, finely crystalline, dolomitic limestone \\
\hline $\mathrm{nmb} 36-17-13 \mathrm{dcd}$ & $5,565.0$ & $5,566.0$ & $324 \mathrm{PRDX}$ & 9.0 & 8.500000 & Vuggy, finely crystalline, limy dolomite \\
\hline nmb36-17-13dcd & $5,566.0$ & $5,567.0$ & 324PRDX & 15.0 & 54.000000 & Vuggy, finely crystalline, limy dolomite \\
\hline nmb36-17-13dcd & $5,567.0$ & $5,568.0$ & 324PRDX & 21.5 & 151.000000 & Vuggy, finely crystalline, limy dolomite \\
\hline nmb36-17-13dcd & $5,568.0$ & $5,569.0$ & $324 \mathrm{PRDX}$ & 14.7 & 34.000000 & Vuggy, finely crystalline, limy dolomite \\
\hline nmb36-17-13dcd & $5,569.0$ & $5,570.0$ & $324 \mathrm{PRDX}$ & 6.3 & 5.000000 & Vuggy, finely crystalline, limy dolomite \\
\hline nmb36-17-13dcd & $5,570.0$ & $5,571.0$ & 324PRDX & 14.5 & 6.300000 & Vuggy, finely crystalline, limy dolomite \\
\hline nmb36-17-13dcd & $5,571.0$ & $5,572.0$ & 324PRDX & 13.4 & 3.100000 & Vuggy, finely crystalline, limy dolomite \\
\hline nmb $36-17-13 d c d$ & $5,572.0$ & $5,573.0$ & 324PRDX & 15.8 & 0.690000 & Vuggy, finely crystalline, limy dolomite \\
\hline $\mathrm{nmb} 36-17-13 \mathrm{dcd}$ & $5,573.0$ & $5,574.0$ & 324PRDX & 16.6 & 54.000000 & Vuggy, finely crystalline, limy dolomite \\
\hline
\end{tabular}


Table 4.--Laboratory-determined porosity and permeability--Continued

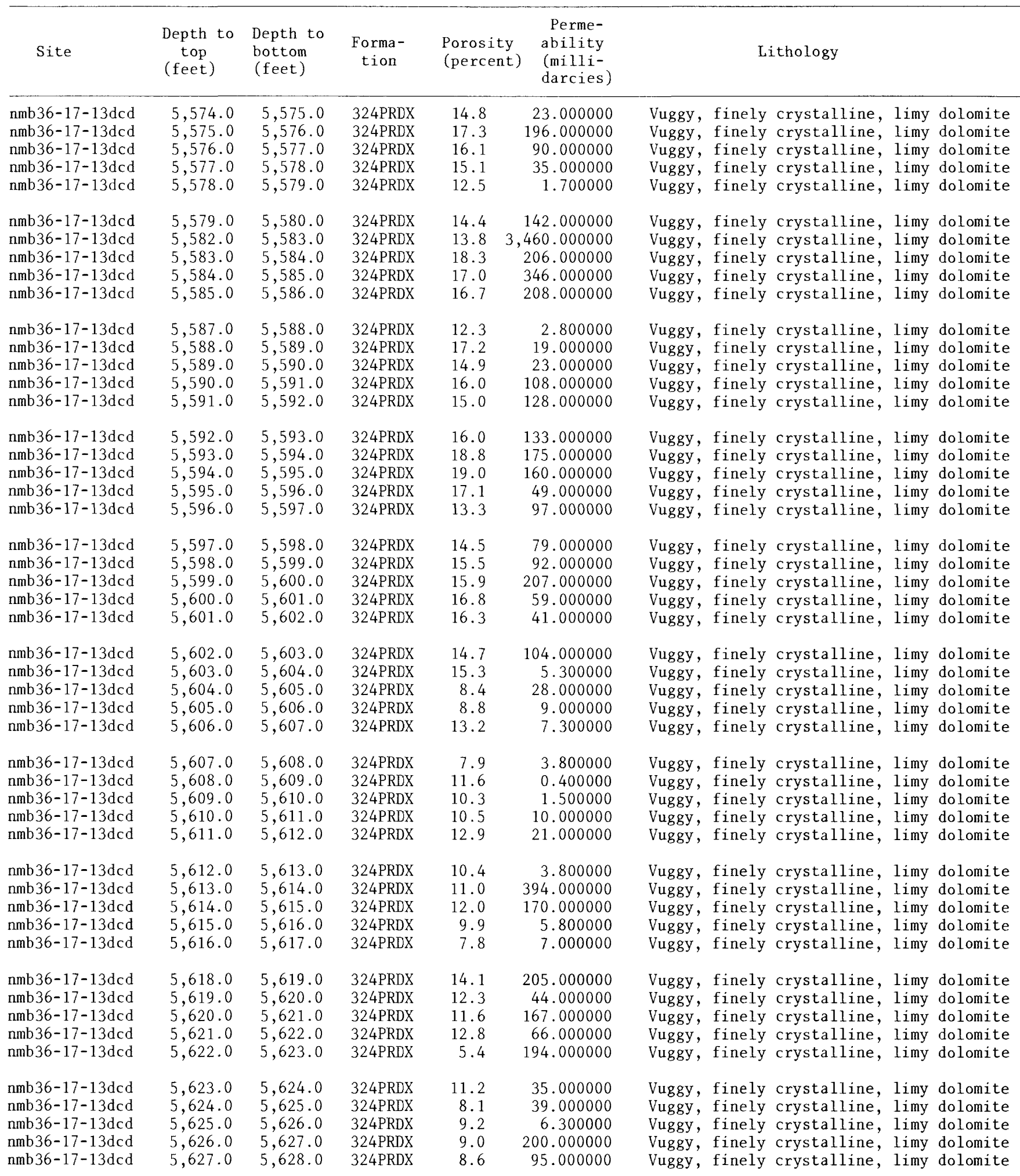


Table 4.--Laboratory-determined porosity and permeability--Continued

\begin{tabular}{|c|c|c|c|c|c|c|}
\hline Site & $\begin{array}{l}\text { Depth to } \\
\text { top } \\
\text { (feet) }\end{array}$ & $\begin{array}{l}\text { Depth to } \\
\text { bottom } \\
\text { (feet) }\end{array}$ & $\begin{array}{c}\text { Forma- } \\
\text { tion }\end{array}$ & $\begin{array}{l}\text { Porosity } \\
\text { (percent) }\end{array}$ & $\begin{array}{c}\text { Perme- } \\
\text { ability } \\
\text { (milli- } \\
\text { darcies) }\end{array}$ & Lithology \\
\hline $\begin{array}{l}\text { nmb36-17-13dcd } \\
\text { nmb36-17-13dcd } \\
\text { nmb36-17-13dcd } \\
\text { nmb36-17-13dcd } \\
\text { nmb36-17-13dcd }\end{array}$ & $\begin{array}{l}5,628 \cdot 0 \\
5,629 \cdot 0 \\
5,630 \cdot 0 \\
5,631.0 \\
5,632.0\end{array}$ & $\begin{array}{l}5,629.0 \\
5,630.0 \\
5,631.0 \\
5,632.0 \\
5,633.0\end{array}$ & $\begin{array}{l}\text { 324PRDX } \\
\text { 324PRDX } \\
\text { 324PRDX } \\
\text { 324PRDX } \\
\text { 324PRDX } \\
\text { AVERAGE-- }\end{array}$ & $\begin{array}{r}5.5 \\
3.6 \\
0.5 \\
0.3 \\
0.5 \\
11.7 \\
\end{array}$ & $\begin{array}{r}0.060000 \\
0.060000 \\
0.060000 \\
0.060000 \\
0.070000 \\
116.500417 \\
\end{array}$ & $\begin{array}{l}\text { Finely crystalline, dolomitic limestone } \\
\text { Finely crystalline, dolomitic limestone } \\
\text { Shaly, finely crystalline, dolomitic limestone } \\
\text { Shaly, finely crystalline, dolomitic limestone } \\
\text { Shaly, finely crystalline, dolomitic limestone }\end{array}$ \\
\hline SITE AVERAGE-- & $-\cdots$ & --- & $-\cdots-1-1$ & 11.7 & 116.500417 & \\
\hline $\begin{array}{l}\text { nmb36-17-23bac } \\
\text { nmb36-17-23bac } \\
\text { nmb36-17-23bac } \\
\text { nmb36-17-23bac } \\
\text { nmb36-17-23bac }\end{array}$ & $\begin{array}{l}2,298.0 \\
2,299.0 \\
2,300.0 \\
2,301.0 \\
2,302.0\end{array}$ & $\begin{array}{l}2,299.0 \\
2,300.0 \\
2,301.0 \\
2,302.0 \\
2,303.0\end{array}$ & $\begin{array}{l}\text { 317CTLR } \\
\text { 317CTLR } \\
\text { 317CTLR } \\
\text { 317CTLR } \\
\text { 317CTLR }\end{array}$ & $\begin{array}{r}6.7 \\
17.8 \\
16.8 \\
12.1 \\
16.1\end{array}$ & $\begin{array}{r}0.050000 \\
232.000000 \\
25.000000 \\
21.000000 \\
33.000000\end{array}$ & $\begin{array}{l}\text { Shale } \\
\text { Medium- to coarse-grained, arkosic sandstone } \\
\text { Medium- to coarse-grained, arkosic sandstone } \\
\text { Medium- to coarse-grained, arkosic sandstone } \\
\text { Gravelly, medium- to coarse-grained sandstone }\end{array}$ \\
\hline $\begin{array}{l}\text { nmb36-17-23bac } \\
\text { nmb36-17-23bac } \\
\text { nmb36-17-23bac } \\
\text { nmb36-17-23bac } \\
\text { nmb36-17-23bac }\end{array}$ & $\begin{array}{l}2,303 \cdot 0 \\
2,304.0 \\
2,305 \cdot 0 \\
2,306.0 \\
2,307.0\end{array}$ & $\begin{array}{l}2,304 \cdot 0 \\
2,305.0 \\
2,306.0 \\
2,307.0 \\
2,308.0\end{array}$ & $\begin{array}{l}317 \text { CTLR } \\
317 \text { CTLR } \\
317 \text { CTLR } \\
\text { 317CTLR } \\
\text { 317CTLR }\end{array}$ & $\begin{array}{l}13.8 \\
15.2 \\
14.9 \\
14.0 \\
13.8\end{array}$ & $\begin{array}{r}11.000000 \\
21.000000 \\
13.000000 \\
11.000000 \\
6.500000\end{array}$ & $\begin{array}{l}\text { Gravelly, medium- to coarse-grained sandstone } \\
\text { Gravelly, medium- to coarse-grained sandstone } \\
\text { Gravelly, medium- to coarse-grained sandstone } \\
\text { Gravelly, medium- to coarse-grained sandstone } \\
\text { Gravelly, fine- to medium-grained sandstone }\end{array}$ \\
\hline $\begin{array}{l}\text { nmb36-17-23bac } \\
\text { nmb36-17-23bac } \\
\text { nmb36-17-23bac } \\
\text { nmb36-17-23bac } \\
\text { nmb36-17-23bac }\end{array}$ & $\begin{array}{l}2,308.0 \\
2,309.0 \\
2,310.0 \\
2,311.0 \\
2,312.0\end{array}$ & $\begin{array}{l}2,309.0 \\
2,310.0 \\
2,311.0 \\
2,312.0 \\
2,313.0\end{array}$ & $\begin{array}{l}\text { 317CTLR } \\
\text { 317CTLR } \\
\text { 317CTLR } \\
\text { 317CTLR } \\
\text { 317CTLR }\end{array}$ & $\begin{array}{l}13.7 \\
13.5 \\
11.8 \\
13.6 \\
13.2\end{array}$ & $\begin{array}{r}11.000000 \\
6.700000 \\
1.800000 \\
4.500000 \\
2.900000\end{array}$ & $\begin{array}{l}\text { Gravelly, medium- to coarse-grained sandstone } \\
\text { Gravelly, medium- to coarse-grained sandstone } \\
\text { Sandstone } \\
\text { Sandstone } \\
\text { Sandstone }\end{array}$ \\
\hline $\begin{array}{l}\text { nmb36-17-23bac } \\
\text { nmb36-17-23bac }\end{array}$ & $\begin{array}{l}2,313.0 \\
2,314.0\end{array}$ & $\begin{array}{l}2,314.0 \\
2,315.0\end{array}$ & $\begin{array}{l}\text { 317CTLR } \\
\text { 317CTLR }\end{array}$ & $\begin{array}{r}12.9 \\
8.5 \\
\end{array}$ & $\begin{array}{l}1.500000 \\
3.100000 \\
\end{array}$ & $\begin{array}{l}\text { Sandstone } \\
\text { Sandstone and shale }\end{array}$ \\
\hline & & & AVERAGE-- & 13.4 & 23.826471 & \\
\hline SITE AVERAGE- - & 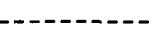 & $\ldots-1 . .-1$ & --------- & 13.4 & 23.826471 & \\
\hline $\begin{array}{l}\text { nmb36-18-06ccc } \\
\text { nmb36-18-06ccc } \\
\text { nmb36-18-06ccc } \\
\text { nmb36-18-06ccc } \\
\text { nmb36-18-06ccc }\end{array}$ & $\begin{array}{l}5,395.0 \\
5,396.0 \\
5,397.0 \\
5,398.0 \\
5,399.0\end{array}$ & $\begin{array}{l}5,396.0 \\
5,397.0 \\
5,398.0 \\
5,399.0 \\
5,400.0\end{array}$ & $\begin{array}{l}\text { 324PRDX } \\
324 \mathrm{PRDX} \\
324 \mathrm{PRDX} \\
324 \mathrm{PRDX} \\
324 \mathrm{PRDX}\end{array}$ & $\begin{array}{r}9.2 \\
14.3 \\
14.8 \\
6.2 \\
8.4\end{array}$ & $\begin{array}{l}0.020000 \\
0.060000 \\
0.900000 \\
0.020000 \\
0.020000\end{array}$ & $\begin{array}{l}\text { Limestone } \\
\text { Limestone } \\
\text { Limestone } \\
\text { Limestone } \\
\text { Limestone }\end{array}$ \\
\hline $\begin{array}{l}\mathrm{nmb} 36-18-06 \mathrm{ccc} \\
\mathrm{nmb} 36-18-06 \mathrm{ccc} \\
\mathrm{nmb} 36-18-06 \mathrm{ccc} \\
\mathrm{nmb36}-18-06 \mathrm{ccc} \\
\mathrm{nmb36}-18-06 \mathrm{ccc}\end{array}$ & $\begin{array}{l}5,400.0 \\
5,401.0 \\
5,402.0 \\
5,403.0 \\
5,404.0\end{array}$ & $\begin{array}{l}5,401.0 \\
5,402.0 \\
5,403.0 \\
5,404.0 \\
5,405.0\end{array}$ & $\begin{array}{l}\text { 324PRDX } \\
\text { 324PRDX } \\
\text { 324PRDX } \\
\text { 324PRDX } \\
\text { 324PRDX }\end{array}$ & $\begin{array}{r}3.3 \\
5.2 \\
9.3 \\
11.2 \\
10.9\end{array}$ & $\begin{array}{l}0.000000 \\
0.030000 \\
0.000000 \\
0.250000 \\
0.190000\end{array}$ & $\begin{array}{l}\text { Limestone } \\
\text { Limestone } \\
\text { Limestone } \\
\text { Limestone } \\
\text { Limestone }\end{array}$ \\
\hline $\begin{array}{l}\mathrm{nmb} 36-18-06 \mathrm{ccc} \\
\mathrm{nmb} 36-18-06 \mathrm{ccc} \\
\mathrm{nmb} 36-18-06 \mathrm{ccc} \\
\mathrm{nmb} 36-18-06 \mathrm{ccc} \\
\mathrm{nmb} 36-18-06 \mathrm{ccc}\end{array}$ & $\begin{array}{l}5,405.0 \\
5,406.0 \\
5,407.0 \\
5,408.0 \\
5,409.0\end{array}$ & $\begin{array}{l}5,406.0 \\
5,407.0 \\
5,408.0 \\
5,409.0 \\
5,410.0\end{array}$ & $\begin{array}{l}\text { 324PRDX } \\
324 \mathrm{PRDX} \\
\text { 324PRDX } \\
324 \mathrm{PRDX} \\
\text { 324PRDX }\end{array}$ & $\begin{array}{r}6.6 \\
7.7 \\
10.1 \\
8.9 \\
9.4\end{array}$ & $\begin{array}{l}0.040000 \\
0.340000 \\
0.120000 \\
1.900000 \\
0.140000\end{array}$ & $\begin{array}{l}\text { Limestone } \\
\text { Limestone } \\
\text { Limestone } \\
\text { Limestone } \\
\text { Limestone }\end{array}$ \\
\hline $\begin{array}{l}\text { nmb36-18-06ccc } \\
\text { nmb36-18-06ccc } \\
\text { nmb36-18-06ccc } \\
\text { nmb36-18-06ccc } \\
\text { nmb36-18-06cc }\end{array}$ & $\begin{array}{l}5,410.0 \\
5,411.0 \\
5,412.0 \\
5,413.0 \\
5,414.0\end{array}$ & $\begin{array}{l}5,411.0 \\
5,412.0 \\
5,413.0 \\
5,414.0 \\
5,415.0\end{array}$ & $\begin{array}{l}\text { 324PRDX } \\
\text { 324PRDX } \\
\text { 324PRDX } \\
\text { 324PRDX } \\
\text { 324PRDX }\end{array}$ & $\begin{array}{l}5.0 \\
5.1 \\
3.0 \\
8.2 \\
4.7\end{array}$ & $\begin{array}{l}0.000000 \\
0.020000 \\
0.000000 \\
0.020000 \\
0.000000\end{array}$ & $\begin{array}{l}\text { Limestone } \\
\text { Limestone } \\
\text { Limestone } \\
\text { Limestone } \\
\text { Limes tone }\end{array}$ \\
\hline
\end{tabular}


Table 4.--Laboratory-determined porosity and permeability--Continued

\begin{tabular}{|c|c|c|c|c|c|c|}
\hline Site & $\begin{array}{l}\text { Depth to } \\
\text { top } \\
\text { (feet) }\end{array}$ & $\begin{array}{l}\text { Depth to } \\
\text { bottom } \\
\text { (feet) }\end{array}$ & $\begin{array}{c}\text { Forma- } \\
\text { tion }\end{array}$ & $\begin{array}{l}\text { Porosity } \\
\text { (percent) }\end{array}$ & $\begin{array}{l}\text { Perme- } \\
\text { ability } \\
\text { (milli- } \\
\text { darcies) }\end{array}$ & Lithology \\
\hline $\begin{array}{l}\text { nmb36-18-06ccc } \\
\text { nmb36-18-06ccc } \\
\text { nmb36-18-06ccc } \\
\text { nmb36-18-06ccc } \\
\text { nmb36-18-06ccc }\end{array}$ & $\begin{array}{l}5,415.0 \\
5,416.0 \\
5,418.0 \\
5,420.0 \\
5,421.0\end{array}$ & $\begin{array}{l}5,416.0 \\
5,417.0 \\
5,419.0 \\
5,421.0 \\
5,422.0\end{array}$ & $\begin{array}{l}324 \mathrm{PRDX} \\
324 \mathrm{PRDX} \\
324 \mathrm{PRDX} \\
324 \mathrm{PRDX} \\
324 \mathrm{PRDX}\end{array}$ & $\begin{array}{l}5.4 \\
7.5 \\
2.5 \\
4.6 \\
4.4\end{array}$ & $\begin{array}{l}0.020000 \\
0.000000 \\
0.000000 \\
0.000000 \\
0.050000\end{array}$ & $\begin{array}{l}\text { Limestone } \\
\text { Limestone } \\
\text { Limestone } \\
\text { Limestone } \\
\text { Limestone }\end{array}$ \\
\hline $\begin{array}{l}\text { nmb36-18-06ccc } \\
\text { nmb36-18-06ccc } \\
\text { nmb36-18-06ccc } \\
\text { nmb36-18-06ccc } \\
\text { nmb36-18-06ccc }\end{array}$ & $\begin{array}{l}5,423.0 \\
5,425.0 \\
5,426.0 \\
5,431.0 \\
5,432.0\end{array}$ & $\begin{array}{l}5,424.0 \\
5,426.0 \\
5,427.0 \\
5,432.0 \\
5,433.0\end{array}$ & $\begin{array}{l}324 \mathrm{PRDX} \\
324 \mathrm{PRDX} \\
324 \mathrm{PRDX} \\
324 \mathrm{PRDX} \\
324 \mathrm{PRDX}\end{array}$ & $\begin{array}{l}3.6 \\
3.7 \\
1.2 \\
4.7 \\
4.7\end{array}$ & $\begin{array}{l}0.000000 \\
0.010000 \\
0.000000 \\
0.010000 \\
0.000000\end{array}$ & $\begin{array}{l}\text { Limestone } \\
\text { Limestone } \\
\text { Shaly limestone } \\
\text { Anhydritic dolomite } \\
\text { Anhydritic dolomite }\end{array}$ \\
\hline $\begin{array}{l}\text { nmb36-18-06ccc } \\
\text { nmb36-18-06ccc } \\
\text { nmb36-18-06ccc } \\
\text { nmb36-18-06ccc } \\
\text { nmb36-18-06ccc }\end{array}$ & $\begin{array}{l}5,433.0 \\
5,434.0 \\
5,443.0 \\
5,445.0 \\
5,448.0\end{array}$ & $\begin{array}{l}5,434.0 \\
5,435.0 \\
5,444.0 \\
5,446.0 \\
5,449.0\end{array}$ & $\begin{array}{l}324 \mathrm{PRDX} \\
324 \mathrm{PRDX} \\
324 \mathrm{PRDX} \\
324 \mathrm{PRDX} \\
324 \mathrm{PRDX}\end{array}$ & $\begin{array}{l}6.2 \\
2.4 \\
1.4 \\
0.7 \\
1.0\end{array}$ & $\begin{array}{l}0.010000 \\
0.000000 \\
0.000000 \\
0.000000 \\
0.020000\end{array}$ & $\begin{array}{l}\text { Anhydritic dolomite } \\
\text { Anhydritic dolomite } \\
\text { Limestone } \\
\text { Limestone } \\
\text { Limestone }\end{array}$ \\
\hline $\begin{array}{l}\text { nmb36-18-06ccc } \\
\text { nmb36-18-06ccc } \\
\text { nmb36-18-06ccc } \\
\text { nmb36-18-06ccc } \\
\text { nmb36-18-06ccc }\end{array}$ & $\begin{array}{l}5,450.0 \\
5,452.0 \\
5,454.0 \\
5,456.0 \\
5,458.0\end{array}$ & $\begin{array}{l}5,451.0 \\
5,453.0 \\
5,455.0 \\
5,457.0 \\
5,459.0\end{array}$ & $\begin{array}{l}324 \mathrm{PRDX} \\
324 \mathrm{PRDX} \\
324 \mathrm{PRDX} \\
324 \mathrm{PRDX} \\
324 \mathrm{PRDX}\end{array}$ & $\begin{array}{l}1.2 \\
1.2 \\
1.7 \\
1.1 \\
0.5\end{array}$ & $\begin{array}{l}0.010000 \\
0.000000 \\
0.000000 \\
0.000000 \\
0.000000\end{array}$ & $\begin{array}{l}\text { Limes tone } \\
\text { Limestone } \\
\text { Limes tone } \\
\text { Limes tone } \\
\text { Limes tone }\end{array}$ \\
\hline $\begin{array}{l}\text { nmb36-18-06ccc } \\
\text { nmb36-18-06ccc } \\
\text { nmb36-18-06ccc } \\
\text { nmb36-18-06ccc } \\
\text { nmb36-18-06ccc }\end{array}$ & $\begin{array}{l}5,460.0 \\
5,462.0 \\
5,464.0 \\
5,466.0 \\
5,467.0\end{array}$ & $\begin{array}{l}5,461.0 \\
5,463.0 \\
5,465.0 \\
5,467.0 \\
5,468.0\end{array}$ & $\begin{array}{l}324 \mathrm{PRDX} \\
324 \mathrm{PRDX} \\
324 \mathrm{PRDX} \\
324 \mathrm{PRDX} \\
324 \mathrm{PRDX}\end{array}$ & $\begin{array}{l}1.4 \\
1.4 \\
1.3 \\
1.2 \\
1.2\end{array}$ & $\begin{array}{l}0.000000 \\
0.000000 \\
0.000000 \\
0.040000 \\
0.000000\end{array}$ & $\begin{array}{l}\text { Limes tone } \\
\text { Limes tone } \\
\text { Limes tone } \\
\text { Limes tone } \\
\text { Limes tone }\end{array}$ \\
\hline $\begin{array}{l}\text { nmb36-18-06ccc } \\
\text { nmb36-18-06ccc } \\
\text { nmb36-18-06ccc } \\
\text { nmb36-18-06ccc } \\
\text { nmb36-18-06ccc }\end{array}$ & $\begin{array}{l}5,584.0 \\
5,585.0 \\
5,586.0 \\
5,587.0 \\
5,588.0\end{array}$ & $\begin{array}{l}5,585.0 \\
5,586.0 \\
5,587.0 \\
5,588.0 \\
5,589.0\end{array}$ & $\begin{array}{l}324 \mathrm{PRDX} \\
324 \mathrm{PRDX} \\
324 \mathrm{PRDX} \\
324 \mathrm{PRDX} \\
324 \mathrm{PRDX}\end{array}$ & $\begin{array}{l}2.5 \\
4.4 \\
3.1 \\
2.9 \\
3.5\end{array}$ & $\begin{array}{l}0.000000 \\
0.000000 \\
0.000000 \\
0.010000 \\
0.000000\end{array}$ & $\begin{array}{l}\text { Dolomite } \\
\text { Dolomite } \\
\text { Dolomite } \\
\text { Dolomite } \\
\text { Dolomite }\end{array}$ \\
\hline $\begin{array}{l}\text { nmb36-18-06ccc } \\
\text { nmb36-18-06ccc } \\
\text { nmb36-18-06ccc } \\
\text { nmb36-18-06ccc } \\
\text { nmb36-18-06ccc }\end{array}$ & $\begin{array}{l}5,589.0 \\
5,590.0 \\
5,608.0 \\
5,643.0 \\
5,644.0\end{array}$ & $\begin{array}{l}5,590.0 \\
5,591.0 \\
5,609.0 \\
5,644.0 \\
5,645.0\end{array}$ & $\begin{array}{l}324 \mathrm{PRDX} \\
324 \mathrm{PRDX} \\
324 \mathrm{PRDX} \\
324 \mathrm{PRDX} \\
324 \mathrm{PRDX}\end{array}$ & $\begin{array}{l}3.4 \\
5.9 \\
1.4 \\
2.8 \\
2.8\end{array}$ & $\begin{array}{l}0.000000 \\
0.000000 \\
0.000000 \\
0.000000 \\
0.000000\end{array}$ & $\begin{array}{l}\text { Dolomite } \\
\text { Dolomite } \\
\text { Shaly dolomite } \\
\text { Dolomite } \\
\text { Dolomite }\end{array}$ \\
\hline $\begin{array}{l}\text { nmb36-18-06ccc } \\
\text { nmb36-18-06ccc } \\
\text { nmb36-18-06ccc } \\
\text { nmb36-18-06ccc } \\
\text { nmb36-18-06ccc }\end{array}$ & $\begin{array}{l}5,668.0 \\
5,669.0 \\
5,670.0 \\
5,671.0 \\
5,672.0\end{array}$ & $\begin{array}{l}5,669.0 \\
5,670.0 \\
5,671.0 \\
5,672.0 \\
5,673.0\end{array}$ & $\begin{array}{l}324 \mathrm{PRDX} \\
324 \mathrm{PRDX} \\
324 \mathrm{PRDX} \\
324 \mathrm{PRDX} \\
324 \mathrm{PRDX}\end{array}$ & $\begin{array}{r}8.7 \\
13.4 \\
15.8 \\
13.1 \\
6.8\end{array}$ & $\begin{array}{l}4.000000 \\
1.100000 \\
0.120000 \\
4.700000 \\
0.560000\end{array}$ & $\begin{array}{l}\text { Dolomite } \\
\text { Dolomite } \\
\text { Dolomite } \\
\text { Dolomite } \\
\text { Dolomite }\end{array}$ \\
\hline $\begin{array}{l}\text { nmb36-18-06ccc } \\
\text { nmb36-18-06ccc } \\
\text { nmb36-18-06ccc } \\
\text { nmb36-18-06ccc } \\
\text { nmb36-18-06ccc }\end{array}$ & $\begin{array}{l}5,673.0 \\
5,674.0 \\
5,675.0 \\
5,676.0 \\
5,677.0\end{array}$ & $\begin{array}{l}5,674.0 \\
5,675.0 \\
5,676.0 \\
5,677.0 \\
5,678.0\end{array}$ & $\begin{array}{l}324 \mathrm{PRDX} \\
324 \mathrm{PRDX} \\
324 \mathrm{PRDX} \\
324 \mathrm{PRDX} \\
324 \mathrm{PRDX}\end{array}$ & $\begin{array}{r}10.1 \\
11.2 \\
9.8 \\
0.9 \\
5.8\end{array}$ & $\begin{array}{r}0.030000 \\
0.600000 \\
19.000000 \\
0.600000 \\
0.030000\end{array}$ & $\begin{array}{l}\text { Limes tone } \\
\text { Limes tone } \\
\text { Limes tone } \\
\text { Limes tone } \\
\text { Limes tone }\end{array}$ \\
\hline $\begin{array}{l}\text { nmb36-18-06ccc } \\
\text { nmb36-18-06ccc } \\
\text { nmb36-18-06ccc } \\
\text { nmb36-18-06ccc } \\
\text { nmb36-18-06ccc }\end{array}$ & $\begin{array}{l}5,678.0 \\
5,679.0 \\
5,680.0 \\
5,681.0 \\
5,682.0\end{array}$ & $\begin{array}{l}5,679.0 \\
5,680.0 \\
5,681.0 \\
5,682.0 \\
5,683.0\end{array}$ & $\begin{array}{l}324 \mathrm{PRDX} \\
324 \mathrm{PRDX} \\
324 \mathrm{PRDX} \\
324 \mathrm{PRDX} \\
324 \mathrm{PRDX}\end{array}$ & $\begin{array}{r}8.6 \\
8.5 \\
4.8 \\
5.1 \\
10.1\end{array}$ & $\begin{array}{l}0.260000 \\
0.750000 \\
1.000000 \\
0.290000 \\
0.800000\end{array}$ & $\begin{array}{l}\text { Limes tone } \\
\text { Limestone } \\
\text { Limestone } \\
\text { Limestone } \\
\text { Limestone }\end{array}$ \\
\hline
\end{tabular}


Table 4.--Laboratory-determined porosity and permeability--Continued

\begin{tabular}{|c|c|c|c|c|c|c|}
\hline Site & $\begin{array}{l}\text { Depth to } \\
\text { top } \\
\text { (feet) }\end{array}$ & $\begin{array}{l}\text { Depth to } \\
\text { bottom } \\
\text { (feet) }\end{array}$ & $\begin{array}{c}\text { Forma- } \\
\text { tion }\end{array}$ & $\begin{array}{l}\text { Porosity } \\
\text { (percent) }\end{array}$ & $\begin{array}{l}\text { Perme- } \\
\text { ability } \\
\text { (milli- } \\
\text { darcies) }\end{array}$ & Lithology \\
\hline $\mathrm{nmb} 36-18-06 \mathrm{ccc}$ & $5,683.0$ & $5,684.0$ & 324PRDX & 9.5 & 0.150000 & Limestone \\
\hline nmb36-18-06ccc & $5,684.0$ & $5,685.0$ & $324 \mathrm{PRDX}$ & 7.2 & 0.020000 & Limestone \\
\hline $\mathrm{nmb} 36-18-06 \mathrm{ccc}$ & $5,685.0$ & $5,686.0$ & 324PRDX & 9.7 & 6.100000 & Limestone \\
\hline nmb36-18-06ccc & $5,686.0$ & $5,687.0$ & 324PRDX & 11.0 & 0.650000 & Limestone \\
\hline nmb36-18-06ccc & $5,687.0$ & $5,688.0$ & 324PRDX & 19.1 & 0.130000 & Limestone \\
\hline$n m b 36-18-06 c c c$ & $5,688.0$ & $5,689.0$ & 324PRDX & 22.3 & 4.800000 & Limestone \\
\hline nmb36-18-06ccc & $5,689.0$ & $5,690.0$ & $324 \mathrm{PRDX}$ & 21.6 & 2.500000 & Limes tone \\
\hline nmb36-18-06ccc & $5,690.0$ & $5,691.0$ & 324PRDX & 21.6 & 9.000000 & Limestone \\
\hline nmb36-18-06ccc & $5,691.0$ & $5,692.0$ & 324PRDX & 21.8 & 61.000000 & Limestone \\
\hline nmb36-18-06ccc & $5,692.0$ & $5,693.0$ & 324PRDX & 24.1 & 25.000000 & Limestone \\
\hline $\mathrm{nmb} 36-18-06 \mathrm{ccc}$ & $5,693.0$ & $5,694.0$ & $324 \mathrm{PRDX}$ & 15.3 & 22.000000 & Limestone \\
\hline nmb $36-18-06 \mathrm{ccc}$ & $5,694.0$ & $5,695.0$ & $324 \mathrm{PRDX}$ & 14.9 & 10.000000 & Limestone \\
\hline $\mathrm{nmb} 36-18-06 \mathrm{ccc}$ & $5,695.0$ & $5,696.0$ & 324PRDX & 16.1 & 10.000000 & Limestone \\
\hline $\mathrm{nmb} 36-18-06 \mathrm{ccc}$ & $5,696.0$ & $5,697.0$ & 324PRDX & 15.4 & 85.000000 & Limes tone \\
\hline $\mathrm{nmb} 36-18-06 \mathrm{ccc}$ & $5,697.0$ & $5,698.0$ & $324 \mathrm{PRDX}$ & 8.5 & 6.500000 & Limestone \\
\hline $\mathrm{nmb} 36-18-06 \mathrm{ccc}$ & $5,698.0$ & $5,699.0$ & 324PRDX & 11.3 & 1.300000 & Limestone \\
\hline nmb36-18-06ccc & $5,699.0$ & $5,700.0$ & 324PRDX & 10.5 & 4.000000 & Limestone \\
\hline nmb36-18-06ccc & $5,700.0$ & $5,701.0$ & $324 \mathrm{PRDX}$ & 4.8 & 0.020000 & Limestone \\
\hline nmb36-18-06ccc & $5,701.0$ & $5,702.0$ & $324 \mathrm{PRDX}$ & 7.3 & 0.230000 & Limestone \\
\hline $\mathrm{nmb} 36-18-06 \mathrm{ccc}$ & $5,702.0$ & $5,703.0$ & 324PRDX & 32.1 & 87.000000 & Limestone \\
\hline nmb36-18-06ccc & $5,703.0$ & $5,704.0$ & $324 \mathrm{PRDX}$ & 26.6 & 82.000000 & Limestone \\
\hline $\mathrm{nmb} 36-18-06 \mathrm{ccc}$ & $5,704.0$ & $5,705.0$ & 324PRDX & 22.5 & 1.000000 & Limestone \\
\hline $\mathrm{nmb} 36-18-06 \mathrm{ccc}$ & $5,705.0$ & $5,706.0$ & 324PRDX & 17.0 & 0.080000 & Limestone \\
\hline $\mathrm{nmb} 36-18-06 \mathrm{ccc}$ & $5,706.0$ & $5,707.0$ & 324PRDX & 17.3 & 0.010000 & Limestone \\
\hline $\mathrm{nmb} 36-18-06 \mathrm{ccc}$ & $5,707.0$ & $5,708.0$ & 324PRDX & 12.3 & 0.090000 & Limestone \\
\hline & & & AVERAGE-- & $\frac{8.3}{8.2}$ & 4.807053 & \\
\hline SITE AVERAGE-- & $-.-1-1$ & ------ & -------- & 8.3 & 4.807053 & \\
\hline nmb37-17-24cca & $7,700.0$ & $7,701.0$ & 337LDVL & 1.3 & 0.000000 & Finely crystalline limestone \\
\hline nmb37-17-24cca & $7,702.0$ & $7,703.0$ & 337LDVL & 0.9 & 0.000000 & Finely crystalline limestone \\
\hline nmb37-17-24cca & $7,704.0$ & $7,705.0$ & 337LDVL & 1.2 & 19.000000 & Finely crystalline limestone \\
\hline nmb37-17-24cca & $7,706.0$ & $7,707.0$ & $337 \mathrm{LDVL}$ & 1.1 & 0.000000 & Finely crystalline limestone \\
\hline $\mathrm{nmb} 37-17-24 \mathrm{cca}$ & $7,708.0$ & $7,709.0$ & 337LDVL & 1.6 & 0.000000 & Finely crystalline limestone \\
\hline $\mathrm{nmb} 37-17-24 \mathrm{cca}$ & $7,710.0$ & $7,711.0$ & 337LDVL & 1.1 & 0.000000 & Finely crystalline limestone \\
\hline $\mathrm{nmb} 37-17-24 \mathrm{cca}$ & $7,712.0$ & $7,713.0$ & $337 \mathrm{LDVL}$ & 1.5 & 4.500000 & Finely crystalline limestone \\
\hline $\mathrm{nmb} 37-17-24 \mathrm{cca}$ & $7,714.0$ & $7,715.0$ & 337LDVL & 1.3 & 1.900000 & Finely crystalline limestone \\
\hline nmb37-17-24cca & $7,716.0$ & $7,717.0$ & 337 LDVL & 1.0 & 0.000000 & Finely crystalline limestone \\
\hline nmb37-17-24cca & $7,718.0$ & $7,719.0$ & 337LDVL & 1.4 & 0.000000 & Anhydritic, finely crystalline limestone \\
\hline nmb37-17-24cca & $7,720.0$ & $7,721.0$ & 337LDVL & 1.7 & 0.000000 & Anhydritic, finely crystalline limestone \\
\hline $\mathrm{nmb} 37-17-24 \mathrm{cca}$ & $7,722.0$ & $7,723.0$ & $337 L D V L$ & 1.3 & 0.000000 & Finely crystalline limestone \\
\hline $\mathrm{nmb} 37-17-24 \mathrm{cca}$ & $7,724.0$ & $7,725.0$ & 337LDVL & 1.5 & 0.000000 & Finely crystalline limestone \\
\hline nmb37-17-24cca & $7,726.0$ & $7,727.0$ & 337LDVL & 0.9 & 0.000000 & Shaly, finely crystalline limestone \\
\hline nmb37-17-24cca & $7,728.0$ & $7,729.0$ & 337LDVL & 1.2 & 0.100000 & Shaly, anhydritic, finely crystalline limestone \\
\hline $\mathrm{nmb} 37-17-24 \mathrm{cca}$ & $7,729.0$ & $7,730.0$ & $337 \mathrm{LDVL}$ & 1.2 & 12.000000 & Shaly, anhydritic, finely crystalline limestone \\
\hline $\mathrm{nmb} 37-17-24 \mathrm{cca}$ & $7,731.0$ & $7,732.0$ & 337LDVL & 1.2 & 0.000000 & Finely crystalline limestone \\
\hline nmb37-17-24cca & $7,733.0$ & $7,734.0$ & $337 \mathrm{LDVL}$ & 1.1 & 0.100000 & Finely crystalline limestone \\
\hline nmb37-17-24cca & $7,735.0$ & $7,736.0$ & 337LDVL & 1.2 & 0.000000 & Finely crystalline limestone \\
\hline nmb37-17-24cca & $7,737.0$ & $7,738.0$ & $337 \mathrm{LDVL}$ & 1.6 & 0.000000 & Finely crystalline limestone \\
\hline $\mathrm{nmb} 37-17-24 \mathrm{cca}$ & $7,739.0$ & $7,740.0$ & $337 \mathrm{LDVL}$ & 1.7 & 0.000000 & Finely crystalline limestone \\
\hline nmb37-17-24cca & $7,741.0$ & $7,742.0$ & 337LDVL & 0.9 & 0.000000 & Finely crystalline limestone \\
\hline $\mathrm{nmb} 37-17-24 \mathrm{cca}$ & $7,743.0$ & $7,744.0$ & $337 \mathrm{LDVL}$ & 1.1 & 0.000000 & Finely crystalline limestone \\
\hline $\mathrm{nmb} 37-17-24 \mathrm{cca}$ & $7,745.0$ & $7,746.0$ & 337LDVL & 1.3 & 2.100000 & Finely crystalline limestone \\
\hline nmb37-17-24cca & $7,747.0$ & $7,748.0$ & 337LDVL & 2.0 & 0.000000 & Finely crystalline limestone \\
\hline
\end{tabular}


Table 4.--Laboratory-determined porosity and permeability--Continued

\begin{tabular}{|c|c|c|c|c|c|c|}
\hline Site & $\begin{array}{c}\text { Depth to } \\
\text { top } \\
\text { (feet) }\end{array}$ & $\begin{array}{l}\text { Depth to } \\
\text { bottom } \\
\text { (feet) }\end{array}$ & $\begin{array}{c}\text { Forma- } \\
\text { tion }\end{array}$ & $\begin{array}{l}\text { Porosity } \\
\text { (percent) }\end{array}$ & $\begin{array}{c}\text { Perme- } \\
\text { ability } \\
\text { (milli- } \\
\text { darcies) }\end{array}$ & Lithology \\
\hline $\mathrm{nmb} 37-17-24 \mathrm{cca}$ & $7,749.0$ & $7,750.0$ & 337LDVL & 1.3 & 0.100000 & Finely crystalline limestone \\
\hline $\mathrm{nmb} 37-17-24 \mathrm{cca}$ & $7,751.0$ & $7,752.0$ & 337LDVL & 1.0 & 0.000000 & Finely crystalline limestone \\
\hline nmb $37-17-24 \mathrm{cca}$ & $7,753.0$ & $7,754.0$ & 337LDVL & 2.1 & 4.700000 & Anhydritic, finely crystalline lim \\
\hline $\mathrm{nmb} 37-17-24 \mathrm{cca}$ & $7,755.0$ & $7,756.0$ & $337 \mathrm{LDVL}$ & 3.9 & 0.000000 & Anhydritic, finely crystalline limes \\
\hline $\mathrm{nmb} 37-17-24 \mathrm{cca}$ & $7,757.0$ & $7,758.0$ & $337 \mathrm{LDVL}$ & 1.5 & 356.000000 & Anhydritic, finely crystalline limes \\
\hline $\mathrm{nmb} 37-17-24 \mathrm{cca}$ & $7,759.0$ & $7,760.0$ & $337 \mathrm{LDVL}$ & 1.6 & 0.000000 & Finely crystalline limestone \\
\hline $\mathrm{nmb} 37-17-24 \mathrm{cca}$ & $7,760.0$ & 7,7 & 337LDVL & 1.7 & 389.0 & Finely crystalline, algal limestone \\
\hline$n m b 37-17-24 c c a$ & $7,762.0$ & $7,763.0$ & 337LDVL & 1.9 & 0.100000 & Finely crystalline, algal limestone \\
\hline $\mathrm{nmb} 37-17-24 \mathrm{cca}$ & $7,764.0$ & $7,765.0$ & 337 LDVL & 1.1 & 0.000000 & Finely crystalline, algal limestone \\
\hline $\mathrm{nmb} 37-17-24 \mathrm{cca}$ & $7,766.0$ & $7,767.0$ & 337LDVL & 1.2 & 0.000000 & Finely crystalline, algal limestone \\
\hline $\mathrm{nmb} 37-17-24 \mathrm{cca}$ & $7,768.0$ & $7,769.0$ & 337LDVL & 1.5 & 106.000000 & Finely crystalline, algal limestone \\
\hline $\mathrm{nmb} 37-17-24 \mathrm{cca}$ & $7,770.0$ & $7,771.0$ & 337LDVL & 1.2 & 904.0 & Finely crystalline limestone \\
\hline nmb37-17-24cca & $7,772.0$ & $7,773.0$ & 337LDVL & 1.1 & 0.000000 & Finely crystalline limestone \\
\hline $\mathrm{nmb} 37-17-24 \mathrm{cca}$ & $7,774.0$ & $7,775.0$ & 337LDVL & 1.1 & 0.0 & Finely crystalline limestone \\
\hline $\mathrm{nmb} 37-17-24 \mathrm{cca}$ & $7,776.0$ & $7,777.0$ & 337LDVL & 1.7 & 0.000000 & Finely crystalline, algal limestone \\
\hline $\mathrm{nmb} 37-17-24 \mathrm{cca}$ & $7,778.0$ & $7,779.0$ & 337 LDVL & 1.5 & 0.000000 & Finely crystalline, algal limestone \\
\hline nmb37-17-24cca & $7,780.0$ & $7,781.0$ & 337LDVL & 1.4 & 0.000000 & Finely crystalline, algal limestone \\
\hline $\mathrm{nmb} 37-17-24 \mathrm{cca}$ & $7,782.0$ & $7,783.0$ & 337LDVL & 1.0 & 0.000000 & Finely crystalline, algal limestone \\
\hline nmb37-17-24cca & $7,784.0$ & $7,785.0$ & 337LDVL & 6.4 & 0.200000 & Finely crystalline dolomite \\
\hline $\mathrm{nmb} 37-17-24 \mathrm{cca}$ & $7,785.0$ & $7,786.0$ & 337LDVL & 10.0 & 5.900000 & Shaly, limy dolomite \\
\hline $\mathrm{nmb} 37-17-24 \mathrm{cca}$ & $7,786.0$ & $7,787.0$ & 337LDVL & 6.3 & 0.6 & Shaly, limy dolomite \\
\hline$n m b 37-17-24 c c a$ & $7,787.0$ & $7,788.0$ & $337 \mathrm{LDVL}$ & 9.6 & 0.2 & Shaly, limy dolomite \\
\hline $\mathrm{nmb} 37-17-24 \mathrm{cca}$ & $7,788.0$ & $7,789.0$ & 337LDVL & 9.7 & 0.100000 & Shaly, limy dolomite \\
\hline $\mathrm{nmb} 37-17-24 \mathrm{cca}$ & $7,789.0$ & $7,790.0$ & 337LDVL & 9.4 & 0.100000 & Finely crystalline dolomite \\
\hline $\mathrm{nmb} 37-17-24 \mathrm{cca}$ & $7,790.0$ & $7,791.0$ & 337LDVL & 7.5 & 3.700000 & Finely crystalline dolomite \\
\hline $\mathrm{nmb} 37-17-24 \mathrm{cca}$ & $7,791.0$ & $7,792.0$ & 337LDVL & 13.2 & 1.100000 & Finely crystalline dolomite \\
\hline $\mathrm{nmb} 37-17-24 \mathrm{cca}$ & $7,792.0$ & $7,793.0$ & 337LDVL & 11.1 & 15.000000 & Finely crystalline dolomite \\
\hline $\mathrm{nmb} 37-17-24 \mathrm{cca}$ & $7,793.0$ & $7,794.0$ & 337LDVL & 7.8 & 2.500000 & Finely crystalline dolomite \\
\hline $\mathrm{nmb} 37-17-24 \mathrm{cca}$ & $7,794.0$ & $7,795.0$ & 337LDVL & 7.0 & 0.000000 & Finely crystalline dolomite \\
\hline $\mathrm{nmb} 37-17-24 \mathrm{cca}$ & $7,796.0$ & $7,797.0$ & 337LDVL & 1.7 & 0.000000 & Finely crystalline, algal limestone \\
\hline nmb37-17-24cca & $7,798.0$ & 799.0 & VL & 1.6 & 86. & Finely crystalline, algal limestone \\
\hline nmb37-17-24cca & $7,800.0$ & $7,801.0$ & 337LDVL & 2.0 & 0.000000 & Finely crystalline, algal limestone \\
\hline $\mathrm{nmb} 37-17-24 \mathrm{cca}$ & $7,801.0$ & $7,802.0$ & 337LDVL & 1.8 & 20.000000 & Finely crystalline, algal limestone \\
\hline $\mathrm{amb} 37-17-24 \mathrm{cca}$ & $7,802.0$ & $7,803.0$ & 337LDVL & 1.5 & 0.000000 & Finely crystalline, algal limestone \\
\hline$n m b 37-17-24 c c a$ & $7,803.0$ & $7,804.0$ & 337LDVL & 1.8 & 134.000000 & Finely crystalline, algal limestone \\
\hline $\mathrm{nmb} 37-17-24 \mathrm{cca}$ & 7,8 & 0 & $\mathrm{VL}$ & 1.7 & 00 & Finely crystalline limestone \\
\hline nmb37-17-24cca & 7,80 & 7,8 & 337LDVL & 1.4 & 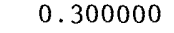 & Finely crystalline limestone \\
\hline $\mathrm{nmb} 37-17-24 \mathrm{cca}$ & $7,806.0$ & $7,807.0$ & 337LDVL & 1.4 & 940.000000 & Finely crystalline limestone \\
\hline $\mathrm{nmb} 37-17-24 \mathrm{cca}$ & $7,807.0$ & $7,808.0$ & 337LDVL & 1.8 & 0.0 & Finely crystalline limestone \\
\hline $\mathrm{nmb} 37-17-24 \mathrm{cca}$ & $7,809.0$ & $7,810.0$ & $337 \mathrm{LDVL}$ & 1.0 & 0.000000 & Finely crystalline limestone \\
\hline $\mathrm{nmb} 37-17-24 \mathrm{cca}$ & $7,810.0$ & 1.0 & $J$ & 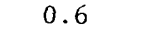 & 280 & Finely crystalline lim \\
\hline $\mathrm{nmb} 37-17-24 \mathrm{cca}$ & $7,811.0$ & $7,812.0$ & 337LDVL & 0.8 & 0.000000 & Finely crystalline limestone \\
\hline $\mathrm{nmb} 37-17-24 \mathrm{cca}$ & $7,812.0$ & $7,813.0$ & 337LDVL & 1.5 & 0.000000 & Finely crystalline limestone \\
\hline$n m b 37-17-24 c c a$ & $7,813.0$ & $7,814.0$ & $337 \mathrm{LDVL}$ & 1.2 & 0.000000 & Finely crystalline limestone \\
\hline $\mathrm{nmb} 37-17-24 \mathrm{cca}$ & $7,814.0$ & $7,815.0$ & 337LDVL & 1.2 & 840.000000 & Finely crystalline limestone \\
\hline $\mathrm{nmb} 37-17-24 \mathrm{cca}$ & $7,816.0$ & $7,817.0$ & 337LDVL & 1.3 & 0.100000 & Finely crystalline limestone \\
\hline $\mathrm{nmb} 37-17-24 \mathrm{cca}$ & $7,818.0$ & $7,819.0$ & 337LDVL & 1.6 & 0.000000 & Finely crystalline limestone \\
\hline nmb37-17-24cca & $7,820.0$ & $7,821.0$ & 337LDVL & 1.3 & 0.000000 & Finely crystalline limestone \\
\hline $\mathrm{nmb} 37-17-24 \mathrm{cca}$ & $7,822.0$ & $7,823.0$ & 337LDVL & 1.5 & 0.700000 & Finely crystalline limestone \\
\hline $\mathrm{nmb} 37-17-24 \mathrm{cca}$ & $7,824.0$ & $7,825.0$ & 337LDVL & 10.6 & 0.300000 & Finely crystalline dolomite \\
\hline
\end{tabular}


Table 4.--Laboratory-determined porosity and permeability--Continued

\begin{tabular}{|c|c|c|c|c|c|c|}
\hline Site & $\begin{array}{l}\text { Depth to } \\
\text { top } \\
\text { (feet) }\end{array}$ & $\begin{array}{l}\text { Depth to } \\
\text { bottom } \\
\text { (feet) }\end{array}$ & $\begin{array}{c}\text { Forma- } \\
\text { tion }\end{array}$ & $\begin{array}{l}\text { Porosity } \\
\text { (percent) }\end{array}$ & $\begin{array}{c}\text { Perme- } \\
\text { ability } \\
\text { (milli- } \\
\text { darcies) }\end{array}$ & Lithology \\
\hline $\begin{array}{l}\text { nmb37-17-24cca } \\
\text { nmb37-17-24cca } \\
\text { nmb37-17-24cca } \\
\text { nmb37-17-24cca } \\
\text { nmb37-17-24cca }\end{array}$ & $\begin{array}{l}7,826.0 \\
7,827.0 \\
7,828.0 \\
7,829.0 \\
7,830.0\end{array}$ & $\begin{array}{l}7,827.0 \\
7,828.0 \\
7,829.0 \\
7,830.0 \\
7,831.0\end{array}$ & $\begin{array}{l}\text { 337LDVL } \\
\text { 337LDVL } \\
\text { 337LDVL } \\
\text { 337LDVL } \\
\text { 337LDVL }\end{array}$ & $\begin{array}{r}10.6 \\
8.1 \\
13.9 \\
12.1 \\
12.9\end{array}$ & $\begin{array}{r}0.300000 \\
14.000000 \\
0.800000 \\
0.800000 \\
5.000000\end{array}$ & $\begin{array}{l}\text { Finely crystalline dolomite } \\
\text { Finely crystalline dolomite } \\
\text { Finely crystalline dolomite } \\
\text { Finely crystalline dolomite } \\
\text { Finely crystalline dolomite }\end{array}$ \\
\hline $\begin{array}{l}\text { nmb37-17-24cca } \\
\text { nmb37-17-24cca } \\
\text { nmb37-17-24cca } \\
\text { nmb37-17-24cca } \\
\text { nmb37-17-24cca }\end{array}$ & $\begin{array}{l}7,832.0 \\
7,834.0 \\
7,836.0 \\
7,838.0 \\
7,840.0\end{array}$ & $\begin{array}{l}7,833.0 \\
7,835.0 \\
7,837.0 \\
7,839.0 \\
7,841.0\end{array}$ & $\begin{array}{l}\text { 337LDVL } \\
\text { 337LDVL } \\
\text { 337LDVL } \\
\text { 337LDVL } \\
\text { 337LDVL }\end{array}$ & $\begin{array}{r}13.0 \\
9.6 \\
11.6 \\
10.4 \\
9.7\end{array}$ & $\begin{array}{l}0.100000 \\
0.300000 \\
1.300000 \\
1.300000 \\
0.100000\end{array}$ & $\begin{array}{l}\text { Finely crystalline dolomite } \\
\text { Finely crystalline dolomite } \\
\text { Finely crystalline dolomite } \\
\text { Finely crystalline dolomite } \\
\text { Finely crystalline dolomite }\end{array}$ \\
\hline $\begin{array}{l}\text { nmb37-17-24cca } \\
\mathrm{nmb} 37-17-24 \mathrm{cca} \\
\mathrm{nmb} 37-17-24 \mathrm{cca} \\
\mathrm{nmb} 37-17-24 \mathrm{cca} \\
\mathrm{nmb} 37-17-24 \mathrm{cca}\end{array}$ & $\begin{array}{l}7,842.0 \\
7,844.0 \\
7,846.0 \\
7,848.0 \\
7,850.0\end{array}$ & $\begin{array}{l}7,843.0 \\
7,845.0 \\
7,847.0 \\
7,849.0 \\
7,851.0\end{array}$ & $\begin{array}{l}\text { 337LDVL } \\
\text { 337LDVL } \\
\text { 337LDVL } \\
\text { 337LDVL } \\
\text { 337LDVL }\end{array}$ & $\begin{array}{r}11.1 \\
8.3 \\
7.9 \\
7.5 \\
5.4\end{array}$ & $\begin{array}{r}0.300000 \\
0.700000 \\
0.200000 \\
0.100000 \\
335.000000\end{array}$ & $\begin{array}{l}\text { Anhydritic, finely crystalline dolomite } \\
\text { Finely crystalline dolomite } \\
\text { Finely crystalline dolomite } \\
\text { Anhydritic, finely crystalline dolomite } \\
\text { Finely crystalline dolomite }\end{array}$ \\
\hline $\begin{array}{l}\text { nmb37-17-24cca } \\
\mathrm{nmb} 37-17-24 \mathrm{cca} \\
\mathrm{nmb} 37-17-24 \mathrm{cca} \\
\mathrm{nmb} 37-17-24 \mathrm{cca} \\
\mathrm{nmb} 37-17-24 \mathrm{cca}\end{array}$ & $\begin{array}{l}7,852.0 \\
7,854.0 \\
7,856.0 \\
7,858.0 \\
7,860.0\end{array}$ & $\begin{array}{l}7,853.0 \\
7,855.0 \\
7,857.0 \\
7,859.0 \\
7,861.0\end{array}$ & $\begin{array}{l}\text { 337LDVL } \\
\text { 337LDVL } \\
\text { 337LDVL } \\
\text { 337LDVL } \\
\text { 337LDVL }\end{array}$ & $\begin{array}{r}10.2 \\
11.6 \\
8.3 \\
10.6 \\
13.1\end{array}$ & $\begin{array}{l}1.200000 \\
1.200000 \\
0.300000 \\
1.200000 \\
2.000000\end{array}$ & $\begin{array}{l}\text { Finely crystalline dolomite } \\
\text { Finely crystalline dolomite } \\
\text { Finely crystalline dolomite } \\
\text { Anhydritic, finely crystalline dolomite } \\
\text { Finely crystalline dolomite }\end{array}$ \\
\hline $\begin{array}{l}\mathrm{nmb} 37-17-24 \mathrm{cca} \\
\mathrm{nmb} 37-17-24 \mathrm{cca} \\
\mathrm{nmb} 37-17-24 \mathrm{cca} \\
\mathrm{nmb} 37-17-24 \mathrm{cca} \\
\mathrm{nmb} 37-17-24 \mathrm{cca}\end{array}$ & $\begin{array}{l}7,862.0 \\
7,864.0 \\
7,866.0 \\
7,868.0 \\
7,870.0\end{array}$ & $\begin{array}{l}7,863.0 \\
7,865.0 \\
7,867.0 \\
7,869.0 \\
7,871.0\end{array}$ & $\begin{array}{l}\text { 337LDVL } \\
\text { 337LDVL } \\
\text { 337LDVL } \\
\text { 337LDVL } \\
\text { 337LDVL }\end{array}$ & $\begin{array}{r}9.1 \\
5.7 \\
6.2 \\
10.8 \\
11.7\end{array}$ & $\begin{array}{l}0.500000 \\
0.000000 \\
0.000000 \\
3.400000 \\
5.500000\end{array}$ & $\begin{array}{l}\text { Finely crystalline dolomite } \\
\text { Finely crystalline dolomite } \\
\text { Finely crystalline dolomite } \\
\text { Anhydritic, finely crystalline dolomite } \\
\text { Anhydritic, finely crystalline dolomite }\end{array}$ \\
\hline $\begin{array}{l}\mathrm{nmb} 37-17-24 c c a \\
\mathrm{nmb} 37-17-24 \mathrm{cca} \\
\mathrm{nmb} 37-17-24 \mathrm{cca} \\
\mathrm{nmb} 37-17-24 \mathrm{cca} \\
\mathrm{nmb} 37-17-24 \mathrm{cca}\end{array}$ & $\begin{array}{l}7,872.0 \\
7,874.0 \\
7,876.0 \\
7,878.0 \\
7,880.0\end{array}$ & $\begin{array}{l}7,873.0 \\
7,875.0 \\
7,877.0 \\
7,879.0 \\
7,881.0\end{array}$ & $\begin{array}{l}\text { 337LDVL } \\
\text { 337LDVL } \\
\text { 337LDVL } \\
\text { 337LDVL } \\
\text { 337LDVL }\end{array}$ & $\begin{array}{l}9.5 \\
6.1 \\
4.9 \\
6.7 \\
5.9\end{array}$ & $\begin{array}{l}0.000000 \\
0.100000 \\
0.700000 \\
0.200000 \\
0.000000\end{array}$ & $\begin{array}{l}\text { Finely crystalline dolomite } \\
\text { Finely crystalline dolomite } \\
\text { Finely crystalline dolomite } \\
\text { Anhydritic, finely crystalline dolomite } \\
\text { Finely crystalline dolomite }\end{array}$ \\
\hline $\begin{array}{l}\text { nmb37-17-24cca } \\
\mathrm{nmb} 37-17-24 \mathrm{cca} \\
\mathrm{nmb} 37-17-24 \mathrm{cca} \\
\mathrm{nmb} 37-17-24 \mathrm{cca} \\
\mathrm{nmb} 37-17-24 \mathrm{cca}\end{array}$ & $\begin{array}{l}7,882.0 \\
7,884.0 \\
7,888.0 \\
7,890.0 \\
7,892.0\end{array}$ & $\begin{array}{l}7,883.0 \\
7,885.0 \\
7,889.0 \\
7,891.0 \\
7,893.0\end{array}$ & $\begin{array}{l}\text { 337LDVL } \\
\text { 337LDVL } \\
\text { 337LDVL } \\
\text { 337LDVL } \\
\text { 337LDVL }\end{array}$ & $\begin{array}{l}4.5 \\
8.3 \\
5.3 \\
1.2 \\
0.8\end{array}$ & $\begin{array}{r}0.700000 \\
24.000000 \\
0.000000 \\
0.000000 \\
0.000000\end{array}$ & $\begin{array}{l}\text { Finely crystalline dolomite } \\
\text { Finely crystalline dolomite } \\
\text { Finely crystalline dolomite } \\
\text { Finely crystalline dolomite } \\
\text { Finely crystalline dolomite }\end{array}$ \\
\hline $\begin{array}{l}\mathrm{nmb} 37-17-24 c \mathrm{ca} \\
\mathrm{nmb} 37-17-24 \mathrm{cca} \\
\mathrm{nmb} 37-17-24 c \mathrm{ca} \\
\mathrm{nmb} 37-17-24 c \mathrm{ca} \\
\mathrm{nmb} 37-17-24 \mathrm{cca}\end{array}$ & $\begin{array}{l}7,894.0 \\
7,896.0 \\
7,898.0 \\
7,902.5 \\
7,903.0\end{array}$ & $\begin{array}{l}7,895.0 \\
7,897.0 \\
7,899.0 \\
7,903.0 \\
7,904.0\end{array}$ & $\begin{array}{l}\text { 337LDVL } \\
\text { 337LDVL } \\
\text { 337LDVL } \\
\text { 337LDVL } \\
\text { 337LDVL }\end{array}$ & $\begin{array}{l}1.5 \\
0.8 \\
8.9 \\
3.8 \\
3.3\end{array}$ & $\begin{array}{l}2.000000 \\
0.400000 \\
0.000000 \\
0.000000 \\
0.700000\end{array}$ & $\begin{array}{l}\text { Anhydritic, finely crystalline dolomite } \\
\text { Anhydritic, finely crystalline dolomite } \\
\text { Finely crystalline dolomite } \\
\text { Finely crystalline dolomite } \\
\text { Finely crystalline dolomite }\end{array}$ \\
\hline $\begin{array}{l}\text { nmb37-17-24cca } \\
\text { nmb37-17-24cca } \\
\mathrm{nmb} 37-17-24 c c a \\
\mathrm{nmb} 37-17-24 c \mathrm{ca} \\
\mathrm{nmb} 37-17-24 c \mathrm{ca}\end{array}$ & $\begin{array}{l}7,904.0 \\
7,905.0 \\
7,906.0 \\
7,907.0 \\
7,908.0\end{array}$ & $\begin{array}{l}7,905.0 \\
7,906.0 \\
7,907.0 \\
7,908.0 \\
7,909.0\end{array}$ & $\begin{array}{l}\text { 337LDVL } \\
\text { 337LDVL } \\
\text { 337LDVL } \\
\text { 337LDVL } \\
\text { 337LDVL }\end{array}$ & $\begin{array}{l}6.9 \\
6.3 \\
7.1 \\
5.2 \\
5.3\end{array}$ & $\begin{array}{r}0.900000 \\
3.900000 \\
184.000000 \\
17.000000 \\
0.000000\end{array}$ & $\begin{array}{l}\text { Finely crystalline dolomite } \\
\text { Finely crystalline dolomite } \\
\text { Finely crystalline dolomite } \\
\text { Finely crystalline dolomite } \\
\text { Finely crystalline dolomite }\end{array}$ \\
\hline $\begin{array}{l}\mathrm{nmb} 37-17-24 c \mathrm{ca} \\
\mathrm{nmb} 37-17-24 c \mathrm{ca} \\
\mathrm{nmb} 37-17-24 c \mathrm{ca} \\
\mathrm{nmb} 37-17-24 c c a \\
\mathrm{nmb} 37-17-24 \mathrm{cca}\end{array}$ & $\begin{array}{l}7,909.0 \\
7,910.0 \\
7,911.0 \\
7,912.0 \\
7,914.0\end{array}$ & $\begin{array}{l}7,910.0 \\
7,911.0 \\
7,912.0 \\
7,913.0 \\
7,915.0\end{array}$ & $\begin{array}{l}\text { 337LDVL } \\
\text { 337LDVL } \\
\text { 337LDVL } \\
\text { 337LDVL } \\
\text { 337LDVL }\end{array}$ & $\begin{array}{l}8.5 \\
8.4 \\
8.1 \\
5.7 \\
9.5\end{array}$ & $\begin{array}{r}16.000000 \\
2.900000 \\
0.300000 \\
0.000000 \\
0.300000\end{array}$ & $\begin{array}{l}\text { Finely crystalline dolomite } \\
\text { Finely crystalline dolomite } \\
\text { Finely crystalline dolomite } \\
\text { Finely crystalline dolomite } \\
\text { Finely crystalline dolomite }\end{array}$ \\
\hline
\end{tabular}


Table 4.--Laboratory-determined porosity and permeability--Continued

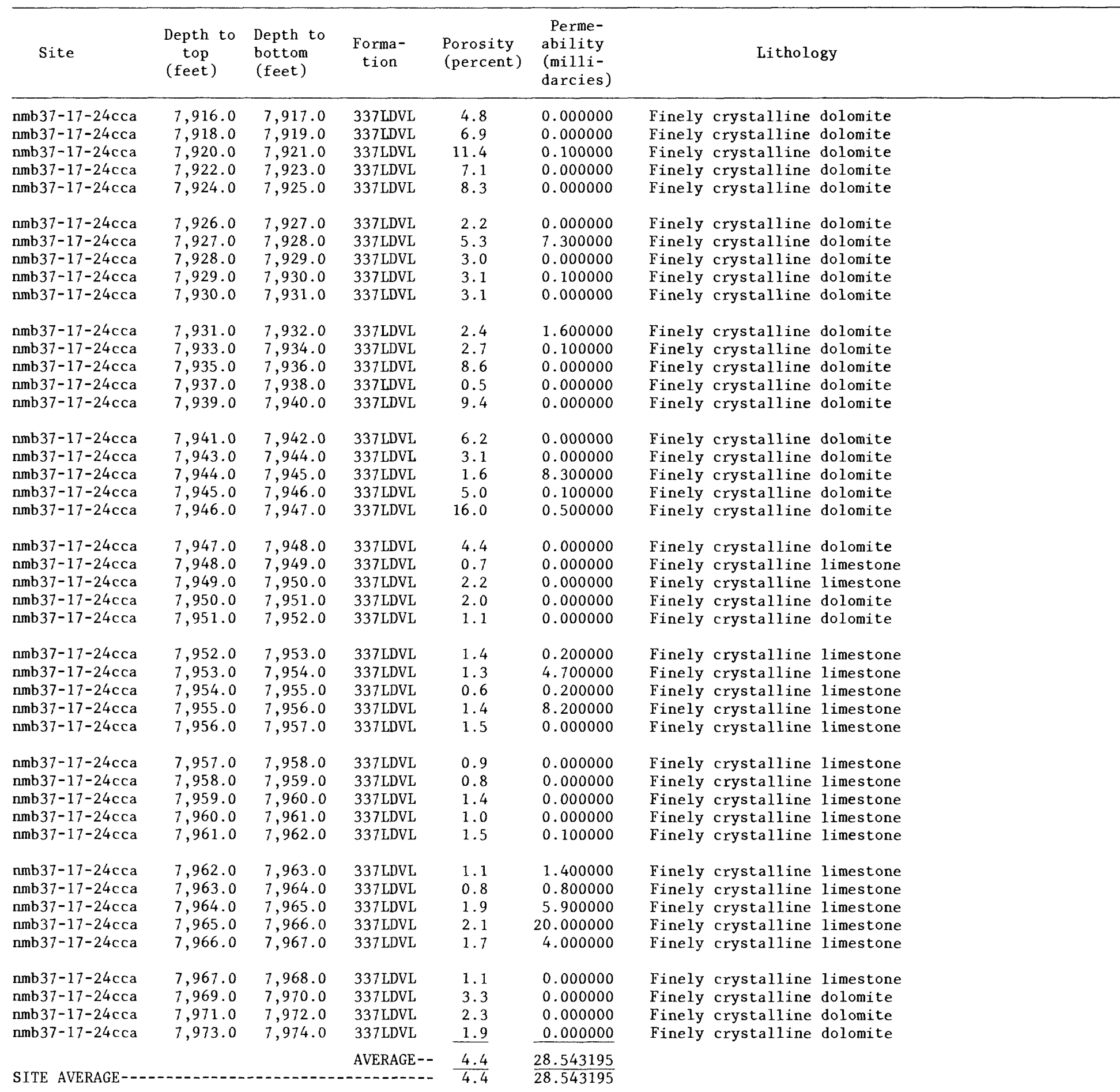


Table 4.--Laboratory-determined porosity and permeability--Continued

\begin{tabular}{|c|c|c|c|c|c|c|}
\hline Site & $\begin{array}{l}\text { Depth to } \\
\text { top } \\
\text { (feet) }\end{array}$ & $\begin{array}{l}\text { Depth to } \\
\text { bottom } \\
\text { (feet) }\end{array}$ & $\begin{array}{c}\text { Forma- } \\
\text { tion }\end{array}$ & $\begin{array}{l}\text { Porosity } \\
\text { (percent) }\end{array}$ & $\begin{array}{l}\text { Perme- } \\
\text { ability } \\
(m i l l i- \\
\text { darcies) }\end{array}$ & Lithology \\
\hline $\begin{array}{l}\text { nmb37-20-11dbc } \\
\text { nmb37-20-11dbc } \\
\text { nmb37-20-11dbc } \\
\text { nmb37-20-11dbc } \\
\text { nmb37-20-11dbc }\end{array}$ & $\begin{array}{l}5,530.0 \\
5,531.8 \\
5,533.2 \\
5,535.1 \\
5,536.0\end{array}$ & $\begin{array}{l}5,531 \cdot 8 \\
5,533.2 \\
5,535.1 \\
5,536.0 \\
5,537.5\end{array}$ & $\begin{array}{l}\text { 324PRDX } \\
\text { 324PRDX } \\
324 \mathrm{PRDX} \\
\text { 324PRDX } \\
\text { 324PRDX }\end{array}$ & $\begin{array}{r}6.7 \\
9.5 \\
10.9 \\
7.6 \\
6.6\end{array}$ & $\begin{array}{r}1.200000 \\
46.000000 \\
16.000000 \\
2.200000 \\
5.200000\end{array}$ & $\begin{array}{l}\text { Limestone } \\
\text { Limestone } \\
\text { Limestone } \\
\text { Limestone } \\
\text { Limestone }\end{array}$ \\
\hline $\begin{array}{l}\text { nmb37-20-11dbc } \\
\text { nmb37-20-11dbc } \\
\text { nmb37-20-11dbc } \\
\text { nmb37-20-11dbc } \\
\text { nmb37-20-11dbc }\end{array}$ & $\begin{array}{l}5,537.5 \\
5,539.2 \\
5,541.2 \\
5,543.0 \\
5,544.9\end{array}$ & $\begin{array}{l}5,539.2 \\
5,541.2 \\
5,543.0 \\
5,544.9 \\
5,546.2\end{array}$ & $\begin{array}{l}\text { 324PRDX } \\
324 \mathrm{PRDX} \\
324 \mathrm{PRDX} \\
\text { 324PRDX } \\
\text { 324PRDX }\end{array}$ & $\begin{array}{r}10.8 \\
8.2 \\
9.8 \\
9.5 \\
6.8\end{array}$ & $\begin{array}{r}37.000000 \\
2.900000 \\
20.000000 \\
48.000000 \\
0.000000\end{array}$ & $\begin{array}{l}\text { Limestone } \\
\text { Limestone } \\
\text { Limestone } \\
\text { Limestone } \\
\text { Limestone }\end{array}$ \\
\hline $\begin{array}{l}\text { nmb37-20-11dbc } \\
\text { SITE AVERAGE- - }\end{array}$ & $5,546.2$ & $5,547.5$ & $\begin{array}{l}\text { 324PRDX } \\
\text { AVERAGE-- }\end{array}$ & $\begin{array}{l}6.4 \\
8.4 \\
8.4\end{array}$ & $\begin{array}{l}0.000000 \\
16.227273 \\
16.227273\end{array}$ & Limestone \\
\hline $\begin{array}{l}\text { nmb37-20-24ddd } \\
\text { nmb37-20-24ddd } \\
\text { nmb37-20-24ddd } \\
\text { nmb37-20-24ddd } \\
\text { nmb37-20-24ddd }\end{array}$ & $\begin{array}{l}5,822.0 \\
5,823.0 \\
5,830.0 \\
5,831.0 \\
5,838.0\end{array}$ & $\begin{array}{l}5,823.0 \\
5,824.0 \\
5,831.0 \\
5,832.0 \\
5,839.0\end{array}$ & $\begin{array}{l}321 \mathrm{HKTL} \\
321 \mathrm{HKTL} \\
321 \mathrm{HKTL} \\
321 \mathrm{HKTL} \\
321 \mathrm{HKTL}\end{array}$ & $\begin{array}{r}4.3 \\
8.4 \\
10.0 \\
6.1 \\
14.0\end{array}$ & $\begin{array}{l}0.080000 \\
0.270000 \\
0.280000 \\
0.080000 \\
0.900000\end{array}$ & $\begin{array}{l}\text { Limestone } \\
\text { Limestone } \\
\text { Limestone } \\
\text { Limestone } \\
\text { Dolomite }\end{array}$ \\
\hline $\begin{array}{l}\text { nmb37-20-24ddd } \\
\text { nmb37-20-24ddd } \\
\text { nmb37-20-24ddd } \\
\text { nmb37-20-24ddd } \\
\text { nmb37-20-24ddd }\end{array}$ & $\begin{array}{l}5,839.0 \\
5,842.0 \\
5,848.0 \\
5,855.0 \\
5,860.0\end{array}$ & $\begin{array}{l}5,840.0 \\
5,843.0 \\
5,849.0 \\
5,856.0 \\
5,861.0\end{array}$ & $\begin{array}{l}321 \mathrm{HKTL} \\
321 \mathrm{HKTL} \\
321 \mathrm{HKTL} \\
321 \mathrm{HKTL} \\
321 \mathrm{HKTL}\end{array}$ & $\begin{array}{r}11.3 \\
8.1 \\
1.8 \\
1.2 \\
2.5\end{array}$ & $\begin{array}{l}0.190000 \\
0.020000 \\
0.020000 \\
0.080000 \\
0.020000\end{array}$ & $\begin{array}{l}\text { Dolomite } \\
\text { Limestone } \\
\text { Limestone } \\
\text { Limestone } \\
\text { Limestone }\end{array}$ \\
\hline \multirow[t]{2}{*}{$\begin{array}{l}\text { nmb37-20-24ddd } \\
\text { nmb37-20-24ddd }\end{array}$} & $\begin{array}{l}5,864.0 \\
5,869.0\end{array}$ & $\begin{array}{l}5,865.0 \\
5,870.0\end{array}$ & $\begin{array}{l}\text { 321HKTL } \\
321 \mathrm{HKTL}\end{array}$ & $\begin{array}{l}1.0 \\
2.0 \\
\end{array}$ & $\begin{array}{l}0.020000 \\
0.010000 \\
\end{array}$ & $\begin{array}{l}\text { Limestone } \\
\text { Limestone }\end{array}$ \\
\hline & & & AVERAGE -- & 5.9 & 0.164167 & \\
\hline $\begin{array}{l}\text { nmb37-20-24ddd } \\
\text { nmb37-20-24ddd } \\
\text { nmb37-20-24ddd } \\
\text { nmb37-20-24ddd } \\
\text { nmb37-20-24ddd }\end{array}$ & $\begin{array}{l}6,065.0 \\
6,066.0 \\
6,067.0 \\
6,068.0 \\
6,069.0\end{array}$ & $\begin{array}{l}6,066.0 \\
6,067.0 \\
6,068.0 \\
6,069.0 \\
6,070.0\end{array}$ & $\begin{array}{l}\text { 324PRDX } \\
324 \mathrm{PRDX} \\
324 \mathrm{PRDX} \\
324 \mathrm{PRDX} \\
\text { 324PRDX }\end{array}$ & $\begin{array}{r}17.5 \\
6.8 \\
5.0 \\
7.0 \\
7.7\end{array}$ & $\begin{array}{l}0.100000 \\
0.040000 \\
0.010000 \\
0.000000 \\
0.000000\end{array}$ & $\begin{array}{l}\text { Limestone } \\
\text { Limestone } \\
\text { Limestone } \\
\text { Limestone } \\
\text { Limes tone }\end{array}$ \\
\hline $\begin{array}{l}\text { nmb37-20-24ddd } \\
\text { nmb37-20-24ddd } \\
\text { nmb37-20-24ddd } \\
\text { nmb37-20-24ddd } \\
\text { nmb37-20-24ddd }\end{array}$ & $\begin{array}{l}6,070.0 \\
6,071.0 \\
6,072.0 \\
6,073.0 \\
6,074.0\end{array}$ & $\begin{array}{l}6,071.0 \\
6,072.0 \\
6,073.0 \\
6,074.0 \\
6,075.0\end{array}$ & $\begin{array}{l}324 \mathrm{PRDX} \\
324 \mathrm{PRDX} \\
324 \mathrm{PRDX} \\
324 \mathrm{PRDX} \\
324 \mathrm{PRDX}\end{array}$ & $\begin{array}{r}3.3 \\
13.6 \\
8.0 \\
7.3 \\
13.1\end{array}$ & $\begin{array}{r}0.290000 \\
13.000000 \\
21.000000 \\
87.000000 \\
331.000000\end{array}$ & $\begin{array}{l}\text { Limes tone } \\
\text { Limes tone } \\
\text { Limes tone } \\
\text { Limes tone } \\
\text { Limes tone }\end{array}$ \\
\hline $\begin{array}{l}\text { nmb37-20-24ddd } \\
\text { nmb37-20-24ddd }\end{array}$ & $\begin{array}{l}6,075.0 \\
6,076.0\end{array}$ & $\begin{array}{l}6,076.0 \\
6,077.0\end{array}$ & $\begin{array}{l}\text { 324PRDX } \\
\text { 324PRDX }\end{array}$ & $\begin{array}{r}6.7 \\
10.0 \\
\end{array}$ & $\begin{array}{l}3.200000 \\
7.400000 \\
\end{array}$ & $\begin{array}{l}\text { Limestone } \\
\text { Limestone }\end{array}$ \\
\hline SITE AVERAGE- - & $x--\infty-\infty-\infty$ & $x--\infty-\infty$ & AVERAGE-- & $\frac{8.8}{7.4}$ & $\frac{38.586667}{19.375417}$ & \\
\hline $\begin{array}{l}\text { nmb38-15-20ccb } \\
\text { nmb38-15-20ccb } \\
\text { nmb38-15-20ccb } \\
\text { nmb38-15-20ccb } \\
\text { nmb38-15-20ccb }\end{array}$ & $\begin{array}{l}2,888.0 \\
2,889.0 \\
2,890.0 \\
2,891.0 \\
2,892.0\end{array}$ & $\begin{array}{l}2,889.0 \\
2,890.0 \\
2,891.0 \\
2,892.0 \\
2,893.0\end{array}$ & $\begin{array}{l}\text { 317CTLR } \\
\text { 317CTLR } \\
\text { 317CTLR } \\
\text { 317CTLR } \\
\text { 317CTLR }\end{array}$ & $\begin{array}{l}10.8 \\
11.0 \\
12.8 \\
13.2 \\
15.1\end{array}$ & $\begin{array}{l}9.000000 \\
3.000000 \\
5.600000 \\
2.500000 \\
4.400000\end{array}$ & $\begin{array}{l}\text { Friable arkosic sandstone } \\
\text { Friable arkosic sandstone } \\
\text { Friable arkosic sandstone } \\
\text { Friable arkosic sandstone } \\
\text { Friable arkosic sandstone }\end{array}$ \\
\hline $\begin{array}{l}\text { nmb38-15-20ccb } \\
\text { nmb38-15-20ccb } \\
\text { nmb38-15-20ccb } \\
\text { nmb38-15-20ccb } \\
\text { nmb38-15-20ccb }\end{array}$ & $\begin{array}{l}2,893.0 \\
2,894.0 \\
2,895.0 \\
2,896.0 \\
2,897.0\end{array}$ & $\begin{array}{l}2,894.0 \\
2,895.0 \\
2,896.0 \\
2,897.0 \\
2,898.0\end{array}$ & $\begin{array}{l}\text { 317CTLR } \\
\text { 317CTLR } \\
\text { 317CTLR } \\
\text { 317CTLR } \\
\text { 317CTLR }\end{array}$ & $\begin{array}{r}12.8 \\
11.6 \\
12.1 \\
5.8 \\
10.3\end{array}$ & $\begin{array}{l}1.900000 \\
1.200000 \\
1.000000 \\
0.000000 \\
0.700000\end{array}$ & $\begin{array}{l}\text { Friable arkosic sandstone } \\
\text { Friable arkosic sandstone } \\
\text { Friable arkosic sandstone } \\
\text { Friable arkosic sandstone } \\
\text { Friable arkosic sandstone }\end{array}$ \\
\hline
\end{tabular}


Table 4.--Laboratory-determined porosity and permeability--Continued

\begin{tabular}{|c|c|c|c|c|c|c|}
\hline Site & $\begin{array}{l}\text { Depth to } \\
\text { top } \\
\text { (feet) }\end{array}$ & $\begin{array}{l}\text { Depth to } \\
\text { bottom } \\
\text { (feet) }\end{array}$ & $\begin{array}{c}\text { Forma- } \\
\text { tion }\end{array}$ & $\begin{array}{l}\text { Porosity } \\
\text { (percent) }\end{array}$ & $\begin{array}{l}\text { Perme- } \\
\text { ability } \\
\text { (milli- } \\
\text { darcies) }\end{array}$ & Lithology \\
\hline $\mathrm{nmb} 38-15-20 \mathrm{ccb}$ & $2,898.0$ & $2,899.0$ & 317CTLR & 9.6 & 0.000000 & Clays tone \\
\hline $\mathrm{nmb} 38-15-20 \mathrm{ccb}$ & $2,899.0$ & $2,900.0$ & 317CTLR & 6.9 & 0.000000 & Clays tone \\
\hline$n m b 38-15-20 c c b$ & $3,205.0$ & $3,206.0$ & 317 CTLR & 7.7 & 1.900000 & Coarse-grained arkosic sandstone \\
\hline $\mathrm{nmb} 38-15-20 \mathrm{ccb}$ & $3,206.0$ & $3,207.0$ & 317 CTLR & 17.3 & 63.000000 & Coarse-grained arkosic sandstone \\
\hline $\mathrm{nmb} 38-15-20 \mathrm{ccb}$ & $3,207.0$ & $3,208.0$ & 317CTLR & 17.3 & 69.000000 & Coarse-grained arkosic sandstone \\
\hline $\mathrm{nmb} 38-15-20 \mathrm{ccb}$ & $3,208.0$ & $3,209.0$ & 317CTLR & 16.6 & 63.000000 & Coarse-grained arkosic sandstone \\
\hline $\mathrm{nmb} 38-15-20 \mathrm{ccb}$ & $3,209.0$ & $3,210.0$ & 317 CTLR & 14.2 & 16.000000 & Conglomeratic arkosic sandstone \\
\hline $\mathrm{nmb} 38-15-20 \mathrm{ccb}$ & $3,210.0$ & $3,211.0$ & 317CTLR & 14.9 & 23.000000 & Conglomeratic arkosic sandstone \\
\hline $\mathrm{nmb} 38-15-20 \mathrm{ccb}$ & $3,211.0$ & $3,212.0$ & 317 CTLR & 13.3 & 4.400000 & Conglomeratic arkosic sandstone \\
\hline$n m b 38-15-20 c c b$ & $3,212.0$ & $3,213.0$ & 317CTLR & 12.0 & 0.000000 & Conglomeratic arkosic sandstone \\
\hline $\mathrm{nmb} 38-15-20 \mathrm{ccb}$ & $3,213.0$ & $3,214.0$ & 317CTLR & 18.9 & 45.000000 & Conglomeratic arkosic sandstone \\
\hline $\mathrm{nmb} 38-15-20 \mathrm{ccb}$ & $3,214.0$ & $3,215.0$ & 317 CTLR & 11.0 & 0.000000 & Conglomeratic arkosic sandstone \\
\hline $\mathrm{nmb} 38-15-20 \mathrm{ccb}$ & $3,215.0$ & $3,216.0$ & 317CTLR & 9.2 & 0.000000 & Conglomeratic arkosic sandstone \\
\hline nmb38-15-20ccb & $3,216.0$ & $3,217.0$ & 317CTLR & 13.7 & 6.100000 & Conglomeratic arkosic sandstone \\
\hline $\mathrm{nmb} 38-15-20 \mathrm{ccb}$ & $3,217.0$ & $3,218.0$ & 317CTLR & 10.5 & 0.000000 & Conglomeratic arkosic sandstone \\
\hline $\mathrm{nmb} 38-15-20 \mathrm{ccb}$ & $3,218.0$ & $3,219.0$ & 317CTLR & 9.7 & 0.600000 & Conglomeratic arkosic sandstone \\
\hline$n m b 38-15-20 \mathrm{ccb}$ & $3,219.0$ & $3,220.0$ & 317CTLR & 14.8 & 15.000000 & Conglomeratic arkosic sandstone \\
\hline nmb38-15-20ccb & $3,220.0$ & $3,221.0$ & 317 CTLR & 12.9 & 0.500000 & Conglomeratic arkosic sandstone \\
\hline \multirow[t]{2}{*}{$\mathrm{nmb} 38-15-20 \mathrm{ccb}$} & $3,221.0$ & $3,222.0$ & 317CTLR & 11.9 & 0.700000 & Conglomeratic arkosic sandstone \\
\hline & & & AVERAGE-- & 12.3 & 11.637931 & \\
\hline $\mathrm{nmb} 38-15-20 \mathrm{ccb}$ & $5,744.0$ & $5,745.0$ & $324 \mathrm{PRDX}$ & 2.7 & 0.000000 & Cherty limestone \\
\hline$n m b 38-15-20 c c b$ & $5,745.0$ & $5,746.0$ & $324 \mathrm{PRDX}$ & 2.5 & 0.000000 & Cherty limestone \\
\hline $\mathrm{nmb} 38-15-20 \mathrm{ccb}$ & $5,746.0$ & $5,747.0$ & $324 \mathrm{PRDX}$ & 3.5 & 0.000000 & Cherty limestone \\
\hline $\mathrm{nmb} 38-15-20 \mathrm{ccb}$ & $5,747.0$ & $5,748.0$ & $324 \mathrm{PRDX}$ & 2.9 & 0.000000 & Cherty limestone \\
\hline$n m b 38-15-20 c c b$ & $5,748.0$ & $5,749.0$ & $324 \mathrm{PRDX}$ & 2.9 & 0.000000 & Cherty limestone \\
\hline $\mathrm{nmb} 38-15-20 \mathrm{ccb}$ & $5,749.0$ & $5,750.0$ & 324PRDX & 3.7 & 0.000000 & Cherty limestone \\
\hline $\mathrm{nmb} 38-15-20 \mathrm{ccb}$ & $5,750.0$ & $5,751.0$ & $324 \mathrm{PRDX}$ & 3.1 & 0.000000 & Cherty limestone \\
\hline $\mathrm{nmb} 38-15-20 \mathrm{ccb}$ & $5,751.0$ & $5,752.0$ & 324PRDX & 3.6 & 0.000000 & Cherty limestone \\
\hline \multirow[t]{2}{*}{$\mathrm{nmb} 38-15-20 \mathrm{ccb}$} & $5,752.0$ & $5,753.0$ & $324 \mathrm{PRDX}$ & 3.0 & 0.000000 & Cherty limestone \\
\hline & & & AVERAGE-- & 3.1 & 0.000000 & \\
\hline $\mathrm{nmb} 38-15-20 \mathrm{ccb}$ & $7,408.0$ & $7,409.0$ & 337LDVL & 1.8 & 0.000000 & Coquina limestone \\
\hline $\mathrm{nmb} 38-15-20 \mathrm{ccb}$ & $7,409.0$ & $7,410.0$ & 337LDVL & 3.0 & 0.000000 & Coquina limestone \\
\hline $\mathrm{nmb} 38-15-20 \mathrm{ccb}$ & $7,410.0$ & $7,411.0$ & 337LDVL & 2.1 & 0.500000 & Coquina limestone \\
\hline $\mathrm{nmb} 38-15-20 \mathrm{ccb}$ & $7,411.0$ & $7,412.0$ & $337 \mathrm{LDVL}$ & 1.7 & 0.400000 & Coquina limestone \\
\hline$n m b 38-15-20 c c b$ & $7,412.0$ & $7,413.0$ & 337LDVL & 1.2 & 0.600000 & Coquina limestone \\
\hline $\mathrm{nmb} 38-15-20 \mathrm{ccb}$ & $7,413.0$ & $7,414.0$ & 337LDVL & 1.7 & 0.000000 & Coquina limestone \\
\hline nmb $38-15-20 \mathrm{ccb}$ & $7,414.0$ & $7,415.0$ & 337LDVL & 1.0 & 0.500000 & Coquina limestone \\
\hline $\mathrm{nmb} 38-15-20 \mathrm{ccb}$ & $7,415.0$ & $7,416.0$ & 337LDVL & 2.4 & 0.000000 & Coquina limestone \\
\hline $\mathrm{nmb} 38-15-20 \mathrm{ccb}$ & $7,416.0$ & $7,417.0$ & 337LDVL & 1.0 & 0.000000 & Coquina limestone \\
\hline$n m b 38-15-20 c c b$ & $7,417.0$ & $7,418.0$ & 337LDVL & 2.3 & 0.000000 & Coquina limestone \\
\hline $\mathrm{nmb} 38-15-20 \mathrm{ccb}$ & $7,418.0$ & $7,419.0$ & 337LDVL & 1.0 & 0.000000 & Coquina limestone \\
\hline$n m b 38-15-20 c c b$ & $7,419.0$ & $7,420.0$ & 337LDVL & 1.2 & 0.000000 & Coquina limestone \\
\hline $\mathrm{nmb} 38-15-20 \mathrm{ccb}$ & $7,420.0$ & $7,421.0$ & 337LDVL & 1.5 & 0.400000 & Coquina limestone \\
\hline $\mathrm{nmb} 38-15-20 \mathrm{ccb}$ & $7,421.0$ & $7,422.0$ & $337 \mathrm{LDVL}$ & 2.5 & 0.000000 & Coquina limestone \\
\hline $\mathrm{nmb} 38-15-20 \mathrm{ccb}$ & $7,422.0$ & $7,423.0$ & $337 \mathrm{LDVL}$ & 1.6 & 0.000000 & Coquina limestone \\
\hline $\mathrm{nmb} 38-15-20 \mathrm{ccb}$ & $7,423.0$ & $7,424.0$ & 337LDVL & 2.5 & 0.000000 & Coquina limestone \\
\hline $\mathrm{nmb} 38-15-20 \mathrm{ccb}$ & $7,424.0$ & $7,425.0$ & 337LDVL & 1.6 & 0.000000 & Coquina limestone \\
\hline $\mathrm{nmb} 38-15-20 \mathrm{ccb}$ & $7,425.0$ & $7,426.0$ & 337LDVL & 1.2 & 0.000000 & Coquina limestone \\
\hline $\mathrm{nmb} 38-15-20 \mathrm{ccb}$ & $7,426.0$ & $7,427.0$ & $337 \mathrm{LDVL}$ & 2.7 & 0.000000 & Coquina limestone \\
\hline $\mathrm{nmb} 38-15-20 \mathrm{ccb}$ & $7,427.0$ & $7,428.0$ & 337LDVL & 1.7 & 1.400000 & Coquina limestone \\
\hline
\end{tabular}


Table 4.--Laboratory-determined porosity and permeability--Continued

\begin{tabular}{|c|c|c|c|c|c|c|}
\hline Site & $\begin{array}{l}\text { Depth to } \\
\text { top } \\
\text { (feet) }\end{array}$ & $\begin{array}{l}\text { Depth to } \\
\text { bottom } \\
\text { (feet) }\end{array}$ & $\begin{array}{c}\text { Forma- } \\
\text { tion }\end{array}$ & $\begin{array}{l}\text { Porosity } \\
\text { (percent) }\end{array}$ & $\begin{array}{l}\text { Perme- } \\
\text { ability } \\
\text { (milli- } \\
\text { darcies) }\end{array}$ & Lithology \\
\hline $\begin{array}{l}n m b 38-15-20 c c b \\
n m b 38-15-20 c c b \\
n m b 38-15-20 c c b \\
n m b 38-15-20 c c b \\
n m b 38-15-20 c c b\end{array}$ & $\begin{array}{l}7,428.0 \\
7,429.0 \\
7,430.0 \\
7,431.0 \\
7,432.0\end{array}$ & $\begin{array}{l}7,429.0 \\
7,430.0 \\
7,431.0 \\
7,432.0 \\
7,433.0\end{array}$ & $\begin{array}{l}\text { 337LDVL } \\
\text { 337LDVL } \\
\text { 337LDVL } \\
\text { 337LDVL } \\
\text { 337LDVL }\end{array}$ & $\begin{array}{l}3.8 \\
3.1 \\
4.0 \\
2.5 \\
2.7\end{array}$ & $\begin{array}{l}1.500000 \\
1.200000 \\
2.500000 \\
2.100000 \\
3.000000\end{array}$ & $\begin{array}{l}\text { Coquina limestone } \\
\text { Coquina limestone } \\
\text { Coquina limestone } \\
\text { Coquina limestone } \\
\text { Coquina limestone }\end{array}$ \\
\hline $\begin{array}{l}\text { nmb38-15-20ccb } \\
\text { nmb38-15-20ccb } \\
\text { nmb38-15-20ccb } \\
\text { nmb38-15-20ccb } \\
\text { nmb38-15-20ccb }\end{array}$ & $\begin{array}{l}7,433.0 \\
7,434.0 \\
7,435.0 \\
7,436.0 \\
7,437.0\end{array}$ & $\begin{array}{l}7,434.0 \\
7,435.0 \\
7,436.0 \\
7,437.0 \\
7,438.0\end{array}$ & $\begin{array}{l}\text { 337LDVL } \\
337 \mathrm{LDVL} \\
337 \mathrm{LDVL} \\
\text { 337LDVL } \\
\text { 337LDVL }\end{array}$ & $\begin{array}{l}4.7 \\
3.3 \\
2.0 \\
2.1 \\
2.8\end{array}$ & $\begin{array}{l}1.500000 \\
0.700000 \\
1.000000 \\
1.600000 \\
1.200000\end{array}$ & $\begin{array}{l}\text { Coquina limestone } \\
\text { Coquina limestone } \\
\text { Coquina limestone } \\
\text { Coquina limestone } \\
\text { Coquina limestone }\end{array}$ \\
\hline $\begin{array}{l}\text { nmb38-15-20ccb } \\
\text { nmb38-15-20ccb } \\
\text { nmb38-15-20ccb } \\
\text { nmb38-15-20ccb } \\
\text { nmb38-15-20ccb }\end{array}$ & $\begin{array}{l}7,438.0 \\
7,439.0 \\
7,440.0 \\
7,441.0 \\
7,442.0\end{array}$ & $\begin{array}{l}7,439.0 \\
7,440.0 \\
7,441.0 \\
7,442.0 \\
7,443.0\end{array}$ & $\begin{array}{l}\text { 337LDVL } \\
337 \mathrm{LDVL} \\
337 \mathrm{LDVL} \\
337 \mathrm{LDVL} \\
337 \mathrm{LDVL}\end{array}$ & $\begin{array}{l}3.5 \\
3.6 \\
4.5 \\
3.1 \\
3.8\end{array}$ & $\begin{array}{l}0.500000 \\
1.400000 \\
1.900000 \\
2.400000 \\
3.100000\end{array}$ & $\begin{array}{l}\text { Coquina limestone } \\
\text { Coquina limestone } \\
\text { Coquina limestone } \\
\text { Coquina limestone } \\
\text { Coquina limestone }\end{array}$ \\
\hline $\begin{array}{l}\text { nmb } 38-15-20 c c b \\
\text { nmb38-15-20ccb } \\
\text { nmb 38-15-20ccb } \\
\text { nmb 38-15-20ccb } \\
\text { nmb38-15-20ccb }\end{array}$ & $\begin{array}{l}7,443.0 \\
7,444.0 \\
7,445.0 \\
7,446.0 \\
7,447.0\end{array}$ & $\begin{array}{l}7,444.0 \\
7,445.0 \\
7,446.0 \\
7,447.0 \\
7,448.0\end{array}$ & $\begin{array}{l}\text { 337LDVL } \\
\text { 337LDVL } \\
\text { 337LDVL } \\
\text { 337LDVL } \\
\text { 337LDVL }\end{array}$ & $\begin{array}{l}2.9 \\
3.1 \\
5.1 \\
2.8 \\
2.7\end{array}$ & $\begin{array}{l}1.600000 \\
1.700000 \\
1.100000 \\
1.100000 \\
2.700000\end{array}$ & $\begin{array}{l}\text { Coquina limestone } \\
\text { Coquina limestone } \\
\text { Coquina limestone } \\
\text { Coquina limestone } \\
\text { Coquina limestone }\end{array}$ \\
\hline $\begin{array}{l}\text { nmb 38-15-20ccb } \\
\text { nmb38-15-20ccb } \\
\text { nmb38-15-20ccb } \\
\text { nmb38-15-20ccb } \\
\text { nmb38-15-20ccb }\end{array}$ & $\begin{array}{l}7,448.0 \\
7,449.0 \\
7,450.0 \\
7,451.0 \\
7,452.0\end{array}$ & $\begin{array}{l}7,449.0 \\
7,450.0 \\
7,451.0 \\
7,452.0 \\
7,453.0\end{array}$ & $\begin{array}{l}\text { 337LDVL } \\
337 L D V L \\
337 L D V L \\
337 L D V L \\
337 L D V L\end{array}$ & $\begin{array}{l}3.5 \\
2.3 \\
2.7 \\
1.7 \\
2.3\end{array}$ & $\begin{array}{l}1.000000 \\
1.300000 \\
0.000000 \\
0.900000 \\
0.400000\end{array}$ & $\begin{array}{l}\text { Coquina limestone } \\
\text { Coquina limestone } \\
\text { Coquina limestone } \\
\text { Coquina limestone } \\
\text { Coquina limestone }\end{array}$ \\
\hline $\begin{array}{l}\text { nmb } 38-15-20 c c b \\
n m b 38-15-20 c c b \\
n m b 38-15-20 c c b \\
\text { nmb38-15-20ccb } \\
\text { nmb38-15-20ccb }\end{array}$ & $\begin{array}{l}7,453.0 \\
7,454.0 \\
7,455.0 \\
7,456.0 \\
7,457.0\end{array}$ & $\begin{array}{l}7,454.0 \\
7,455.0 \\
7,456.0 \\
7,457.0 \\
7,458.0\end{array}$ & $\begin{array}{l}\text { 337LDVL } \\
\text { 337LDVL } \\
\text { 337LDVL } \\
\text { 337LDVL } \\
\text { 337LDVL }\end{array}$ & $\begin{array}{l}1.7 \\
3.1 \\
4.9 \\
3.0 \\
3.1\end{array}$ & $\begin{array}{l}0.000000 \\
0.000000 \\
0.000000 \\
0.000000 \\
0.000000\end{array}$ & $\begin{array}{l}\text { Coquina limestone } \\
\text { Coquina limestone } \\
\text { Coquina limestone } \\
\text { Coquina limestone } \\
\text { Coquina limestone }\end{array}$ \\
\hline $\begin{array}{l}\text { nmb38-15-20ccb } \\
\text { nmb38-15-20ccb } \\
\text { nmb 38-15-20ccb } \\
\text { nmb38-15-20ccb } \\
\text { nmb38-15-20ccb }\end{array}$ & $\begin{array}{l}7,458.0 \\
7,459.0 \\
7,460.0 \\
7,461.0 \\
7,462.0\end{array}$ & $\begin{array}{l}7,459.0 \\
7,460.0 \\
7,461.0 \\
7,462.0 \\
7,463.0\end{array}$ & $\begin{array}{l}\text { 337LDVL } \\
337 L D V L \\
337 L D V L \\
337 L D V L \\
337 L D V L\end{array}$ & $\begin{array}{l}3.6 \\
2.0 \\
2.5 \\
3.1 \\
1.2\end{array}$ & $\begin{array}{l}0.000000 \\
0.000000 \\
0.000000 \\
0.000000 \\
0.000000\end{array}$ & $\begin{array}{l}\text { Coquina limestone } \\
\text { Coquina limestone } \\
\text { Coquina limestone } \\
\text { Coquina limestone } \\
\text { Coquina limestone }\end{array}$ \\
\hline $\begin{array}{l}\text { nmb } 38-15-20 c c b \\
n m b 38-15-20 c c b \\
n m b 38-15-20 c c b \\
n m b 38-15-20 c c b \\
n m b 38-15-20 c c b\end{array}$ & $\begin{array}{l}7,463.0 \\
7,464.0 \\
7,465.0 \\
7,466.0 \\
7,467.0\end{array}$ & $\begin{array}{l}7,464.0 \\
7,465.0 \\
7,466.0 \\
7,467.0 \\
7,468.0\end{array}$ & $\begin{array}{l}\text { 337LDVL } \\
\text { 337LDVL } \\
\text { 337LDVL } \\
\text { 337LDVL } \\
\text { 337LDVL }\end{array}$ & $\begin{array}{l}2.2 \\
3.1 \\
2.6 \\
1.6 \\
3.3\end{array}$ & $\begin{array}{l}0.000000 \\
0.000000 \\
0.000000 \\
0.000000 \\
0.700000\end{array}$ & $\begin{array}{l}\text { Coquina limestone } \\
\text { Coquina limestone } \\
\text { Coquina limestone } \\
\text { Coquina limestone } \\
\text { Coquina limestone }\end{array}$ \\
\hline $\begin{array}{l}\text { nmb38-15-20ccb } \\
\text { nmb38-15-20ccb } \\
\text { nmb38-15-20ccb } \\
\text { nmb 38-15-20ccb } \\
\text { nmb38-15-20ccb }\end{array}$ & $\begin{array}{l}7,468.0 \\
7,469.0 \\
7,470.0 \\
7,471.0 \\
7,472.0\end{array}$ & $\begin{array}{l}7,469.0 \\
7,470.0 \\
7,471.0 \\
7,472.0 \\
7,473.0\end{array}$ & $\begin{array}{l}\text { 337LDVL } \\
\text { 337LDVL } \\
\text { 337LDVL } \\
\text { 337LDVL } \\
\text { 337LDVL }\end{array}$ & $\begin{array}{l}2.9 \\
1.7 \\
3.9 \\
3.1 \\
2.8\end{array}$ & $\begin{array}{l}0.000000 \\
0.400000 \\
0.000000 \\
0.000000 \\
0.000000\end{array}$ & $\begin{array}{l}\text { Coquina limestone } \\
\text { Coquina limestone } \\
\text { Coquina limestone } \\
\text { Coquina limestone } \\
\text { Coquina limestone }\end{array}$ \\
\hline
\end{tabular}


Table 4.--Laboratory-determined porosity and permeability--Continued

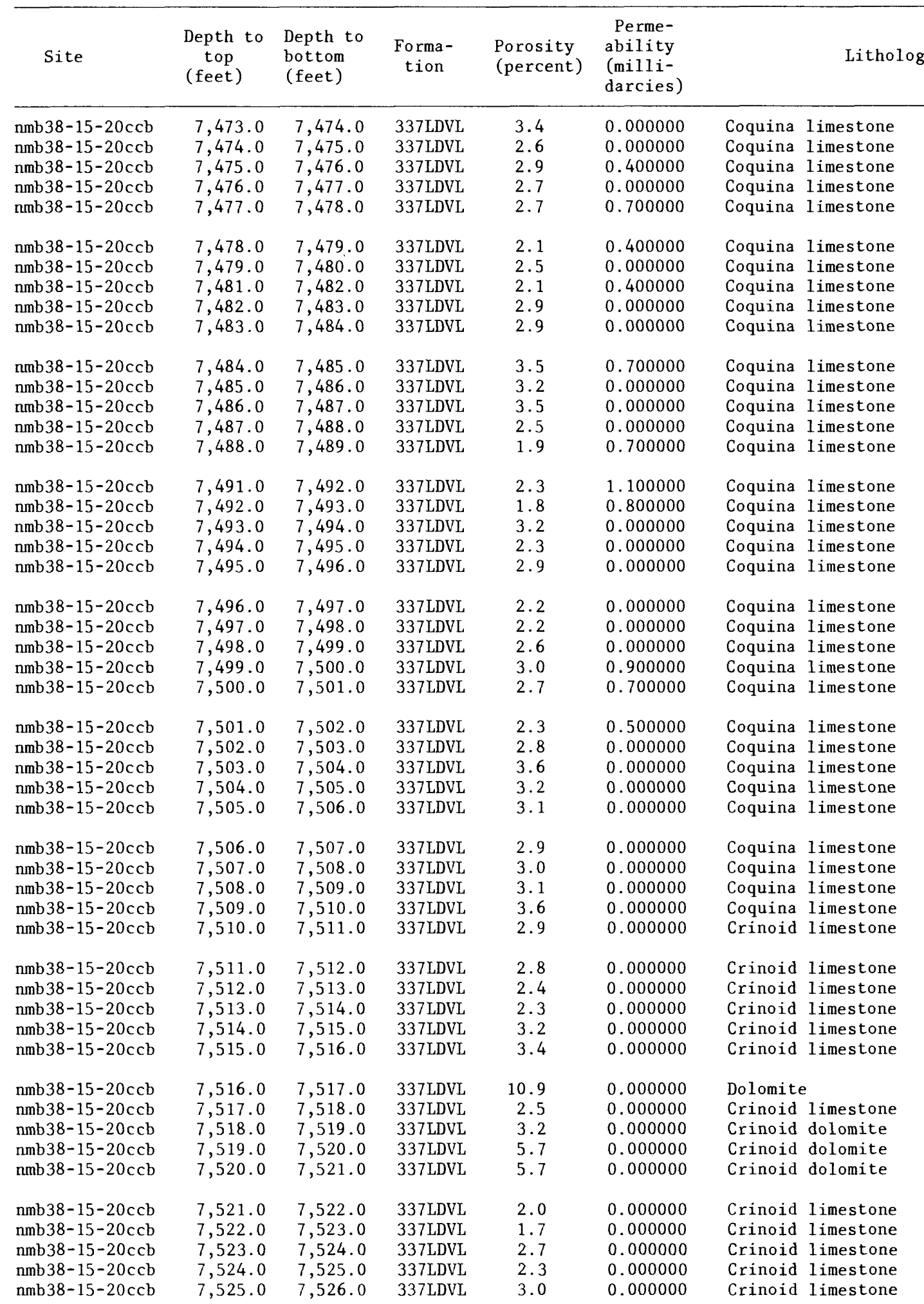


Table 4.--Laboratory-determined porosity and permeability--Continued

\begin{tabular}{|c|c|c|c|c|c|c|}
\hline Site & $\begin{array}{l}\text { Depth to } \\
\text { top } \\
\text { (feet) }\end{array}$ & $\begin{array}{l}\text { Depth to } \\
\text { bottom } \\
\text { (feet) }\end{array}$ & $\begin{array}{c}\text { Forma- } \\
\text { tion }\end{array}$ & $\begin{array}{l}\text { Porosity } \\
\text { (percent) }\end{array}$ & $\begin{array}{l}\text { Perme- } \\
\text { ability } \\
\text { (milli- } \\
\text { darcies) }\end{array}$ & Lithology \\
\hline nmb 38-15-20ccb & $7,526.0$ & $7,527.0$ & 337LDVL & 3.7 & 0.000000 & Crinoid dolomite \\
\hline $\mathrm{nmb} 38-15-20 \mathrm{ccb}$ & $7,527.0$ & $7,528.0$ & 337LDVL & 3.6 & 0.000000 & Crinoid dolomite \\
\hline $\mathrm{nmb} 38-15-20 \mathrm{ccb}$ & $7,528.0$ & $7,529.0$ & 337LDVL & 2.7 & 0.000000 & Coquina limestone \\
\hline nmb 38-15-20ccb & $7,529.0$ & $7,530.0$ & 337LDVL & 3.5 & 0.000000 & Coquina limestone \\
\hline$n m b 38-15-20 c c b$ & $7,530.0$ & $7,531.0$ & 337LDVL & 3.5 & 0.000000 & Coquina limestone \\
\hline $\mathrm{nmb} 38-15-20 \mathrm{ccb}$ & $7,531.0$ & $7,532.0$ & 337LDVL & 4.6 & 0.000000 & Coquina limestone \\
\hline$n m b 38-15-20 c c b$ & $7,532.0$ & $7,533.0$ & 337LDVL & 4.0 & 0.000000 & Coquina limestone \\
\hline $\mathrm{nmb} 38-15-20 \mathrm{ccb}$ & $7,533.0$ & $7,534.0$ & 337LDVL & 3.6 & 0.000000 & Coquina limestone \\
\hline $\mathrm{nmb} 38-15-20 \mathrm{ccb}$ & $7,534.0$ & $7,535.0$ & 337LDVL & 3.7 & 0.000000 & Coquina limestone \\
\hline $\mathrm{nmb} 38-15-20 \mathrm{ccb}$ & $7,535.0$ & $7,536.0$ & 337LDVL & 3.3 & 0.000000 & Coquina limestone \\
\hline nmb 38-15-20ccb & $7,536.0$ & $7,537.0$ & 337LDVL & 2.8 & 0.000000 & Coquina limestone \\
\hline$n m b 38-15-20 c c b$ & $7,537.0$ & $7,538.0$ & 337LDVL & 3.2 & 0.000000 & Coquina limestone \\
\hline $\mathrm{nmb} 38-15-20 \mathrm{ccb}$ & $7,538.0$ & $7,539.0$ & 337LDVL & 2.9 & 0.000000 & Coquina limestone \\
\hline nmb38-15-20ccb & $7,539.0$ & $7,540.0$ & 337LDVL & 2.7 & 0.000000 & Coquina limestone \\
\hline nmb38-15-20ccb & $7,540.0$ & $7,541.0$ & 337LDVL & 2.5 & 0.000000 & Coquina 1 imestone \\
\hline nmb38-15-20ccb & $7,541.0$ & $7,542.0$ & 337LDVL & 3.8 & 0.000000 & Coquina limestone \\
\hline nmb38-15-20ccb & $7,542.0$ & $7,543.0$ & 337LDVL & 3.5 & 0.000000 & Cherty limestone \\
\hline $\mathrm{nmb} 38-15-20 \mathrm{ccb}$ & $7,543.0$ & $7,544.0$ & 337LDVL & 1.9 & 0.000000 & Oolitic limestone \\
\hline nmb38-15-20ccb & $7,544.0$ & $7,545.0$ & 337LDVL & 3.1 & 0.000000 & Oolitic limestone \\
\hline $\mathrm{nmb} 38-15-20 \mathrm{ccb}$ & $7,545.0$ & $7,546.0$ & 337LDVL & 2.5 & 0.000000 & Oolitic limestone \\
\hline nmb38-15-20ccb & $7,546.0$ & $7,547.0$ & 337LDVL & 2.8 & 0.000000 & Oolitic limestone \\
\hline nmb38-15-20ccb & $7,547.0$ & $7,548.0$ & 337LDVL & 2.5 & 0.000000 & Oolitic limestone \\
\hline $\mathrm{nmb} 38-15-20 \mathrm{ccb}$ & $7,548.0$ & $7,549.0$ & 337LDVL & 2.9 & 0.000000 & Oolitic limestone \\
\hline nmb38-15-20ccb & $7,549.0$ & $7,550.0$ & 337LDVL & 1.7 & 0.000000 & Oolitic limestone \\
\hline nmb38-15-20ccb & $7,550.0$ & $7,551.0$ & 337LDVL & 1.7 & 0.000000 & Oolitic limestone \\
\hline nmb38-15-20ccb & $7,551.0$ & $7,552.0$ & 337LDVL & 1.2 & 0.000000 & Dolitic limestone \\
\hline nmb38-15-20ccb & $7,552.0$ & $7,553.0$ & 337LDVL & 2.2 & 0.000000 & Oolitic limestone \\
\hline nmb38-15-20ccb & $7,553.0$ & $7,554.0$ & 337LDVL & 2.2 & 0.000000 & Cherty limestone \\
\hline nmb38-15-20ccb & $7,554.0$ & $7,555.0$ & 337LDVL & 3.1 & 0.000000 & Cherty limestone \\
\hline nmb38-15-20ccb & $7,555.0$ & $7,556.0$ & 337LDVL & 7.0 & 0.000000 & Fine-grained limestone \\
\hline nmb38-15-20ccb & $7,556.0$ & $7,557.0$ & 337LDVL & 2.8 & 0.000000 & Fine-grained limestone \\
\hline nmb38-15-20ccb & $7,557.0$ & $7,558.0$ & 337LDVL & 3.8 & 0.000000 & Anhydritic dolomite \\
\hline nmb38-15-20ccb & $7,558.0$ & $7,559.0$ & 337LDVL & 2.6 & 0.000000 & Anhydritic dolomite \\
\hline nmb38-15-20ccb & $7,559.0$ & $7,560.0$ & 337LDVL & 5.5 & 0.000000 & Anhydritic dolomite \\
\hline nmb38-15-20ccb & $7,560.0$ & $7,561.0$ & 337LDVL & 7.8 & 0.000000 & Anhydritic dolomite \\
\hline nmb 38-15-20ccb & $7,561.0$ & $7,562.0$ & 337LDVL & 7.9 & 0.000000 & Anhydritic dolomite \\
\hline nmb38-15-20ccb & $7,562.0$ & $7,563.0$ & 337LDVL & 8.1 & 0.000000 & Anhydritic dolomite \\
\hline nmb 38-15-20ccb & $7,563.0$ & $7,564.0$ & 337LDVL & 9.0 & 0.000000 & Anhydritic dolomite \\
\hline nmb38-15-20ccb & $7,564.0$ & $7,565.0$ & 337LDVL & 11.7 & 1.300000 & Anhydritic dolomite \\
\hline nmb38-15-20ccb & $7,565.0$ & $7,566.0$ & 337LDVL & 11.1 & 0.000000 & Anhydritic dolomite \\
\hline nmb38-15-20ccb & $7,566.0$ & $7,567.0$ & 337LDVL & 6.0 & 0.000000 & Anhydritic dolomite \\
\hline nmb38-15-20ccb & $7,567.0$ & $7,568.0$ & 337LDVL & 4.1 & 0.000000 & Anhydritic dolomite \\
\hline nmb38-15-20ccb & $7,568.0$ & $7,569.0$ & 337LDVL & 11.2 & 1.100000 & Anhydritic dolomite \\
\hline nmb38-15-20ccb & $7,569.0$ & $7,570.0$ & $337 \mathrm{LDVL}$ & 10.5 & 0.000000 & Anhydritic dolomite \\
\hline $\mathrm{nmb} 38-15-20 \mathrm{ccb}$ & $7,571.0$ & $7,572.0$ & 337LDVL & 9.9 & 0.700000 & Anhydritic dolomite \\
\hline nmb38-15-20ccb & $7,572.0$ & $7,573.0$ & 337LDVL & 7.8 & 0.000000 & Anhydritic dolomite \\
\hline nmb38-15-20ccb & $7,573.0$ & $7,574.0$ & 337LDVL & 6.0 & 0.000000 & Anhydritic dolomite \\
\hline nmb38-15-20ccb & $7,574.0$ & $7,575.0$ & 337LDVL & 7.4 & 0.000000 & Anhydritic dolomite \\
\hline nmb38-15-20ccb & $7,575.0$ & $7,576.0$ & 337LDVL & 5.8 & 0.000000 & Anhydritic dolomite \\
\hline nmb38-15-20ccb & $7,576.0$ & $7,577.0$ & 337LDVL & 10.9 & 0.000000 & Anhydritic dolomite \\
\hline
\end{tabular}


Table 4.--Laboratory-determined porosity and permeability--Continued

\begin{tabular}{|c|c|c|c|c|c|c|}
\hline Site & $\begin{array}{l}\text { Depth to } \\
\text { top } \\
\text { (feet) }\end{array}$ & $\begin{array}{l}\text { Depth to } \\
\text { bottom } \\
\text { (feet) }\end{array}$ & $\begin{array}{c}\text { Forma- } \\
\text { tion }\end{array}$ & $\begin{array}{l}\text { Porosity } \\
\text { (percent) }\end{array}$ & $\begin{array}{l}\text { Perme- } \\
\text { ability } \\
\text { (milli- } \\
\text { darcies) }\end{array}$ & Litholog \\
\hline$n m b 38-15-20 c c b$ & $7,577.0$ & $7,578.0$ & 337LDVL & 7.0 & 0.000000 & Anhydritic dolomite \\
\hline nmb38-15-20ccb & $7,578.0$ & $7,579.0$ & $337 \mathrm{LDVL}$ & 4.2 & 0.000000 & Anhydritic dolomite \\
\hline nmb $38-15-20 \mathrm{ccb}$ & $7,579.0$ & $7,580.0$ & $337 \mathrm{LDVL}$ & 4.5 & 0.000000 & Anhydritic dolomite \\
\hline$n m b 38-15-20 c c b$ & $7,580.0$ & $7,581.0$ & 337LDVL & 5.5 & 0.000000 & Oolitic dolomite \\
\hline$n m b 38-15-20 c c b$ & $7,581.0$ & $7,582.0$ & 337LDVL & 5.4 & 0.000000 & Oolitic dolomite \\
\hline $\mathrm{nmb} 38-15-20 \mathrm{ccb}$ & $7,582.0$ & $7,583.0$ & 337LDVL & 2.6 & 0.000000 & Oolitic dolomite \\
\hline $\mathrm{nmb} 38-15-20 \mathrm{ccb}$ & $7,583.0$ & $7,584.0$ & $337 \mathrm{LDVL}$ & 1.5 & 0.000000 & Limestone breccia \\
\hline $\mathrm{nmb} 38-15-20 \mathrm{ccb}$ & $7,584.0$ & $7,585.0$ & 337LDVL & 1.0 & 0.000000 & Limestone breccia \\
\hline $\mathrm{nmb} 38-15-20 \mathrm{ccb}$ & $7,585.0$ & $7,586.0$ & $337 \mathrm{LDVL}$ & 3.8 & 0.000000 & Anhydritic dolomite \\
\hline $\mathrm{nmb} 38-15-20 \mathrm{ccb}$ & $7,586.0$ & $7,587.0$ & 337LDVL & 9.1 & 0.000000 & Anhydritic dolomite \\
\hline nmb38-15-20ccb & $7,587.0$ & $7,588.0$ & 337LDVL & 7.0 & 0.100000 & Anhydritic dolomite \\
\hline $\mathrm{nmb} 38-15-20 \mathrm{ccb}$ & $7,588.0$ & $7,589.0$ & 337LDVL & 6.0 & 0.000000 & Anhydritic dolomite \\
\hline $\mathrm{nmb} 38-15-20 \mathrm{ccb}$ & $7,589.0$ & $7,590.0$ & 337LDVL & 2.0 & 0.000000 & Anhydritic dolomite \\
\hline $\mathrm{nmb} 38-15-20 \mathrm{ccb}$ & $7,590.0$ & $7,591.0$ & 337LDVL & 4.9 & 0.700000 & Anhydritic dolomite \\
\hline nmb $38-15-20 c c b$ & $7,591.0$ & $7,592.0$ & $337 \mathrm{LDVL}$ & 17.0 & 0.600000 & Anhydritic dolomite \\
\hline $\mathrm{nmb} 38-15-20 \mathrm{ccb}$ & $7,592.0$ & $7,593.0$ & 337LDVL & 14.7 & 0.000000 & Anhydritic dolomite \\
\hline$n m b 38-15-20 c c b$ & $7,593.0$ & $7,594.0$ & 337LDVL & 4.8 & 0.000000 & Anhydritic dolomite \\
\hline nmb $38-15-20 c c b$ & $7,594.0$ & $7,595.0$ & 337LDVL & 9.0 & 0.000000 & Anhydritic dolomite \\
\hline$n m b 38-15-20 c c b$ & $7,595.0$ & $7,596.0$ & 337LDVL & 2.2 & 0.000000 & Anhydritic dolomite \\
\hline $\mathrm{nmb} 38-15-20 \mathrm{ccb}$ & $7,596.0$ & $7,597.0$ & 337LDVL & 8.2 & 0.000000 & Anhydritic dolomite \\
\hline$n m b 38-15-20 c c b$ & $7,597.0$ & $7,598.0$ & 337LDVL & 12.1 & 0.000000 & Anhydritic dolomite \\
\hline $\mathrm{nmb} 38-15-20 \mathrm{ccb}$ & $7,598.0$ & $7,599.0$ & 337LDVL & 14.4 & 0.010000 & Anhydritic dolomite \\
\hline $\mathrm{nmb} 38-15-20 \mathrm{ccb}$ & $7,599.0$ & $7,600.0$ & 337 LDVL & 10.4 & 0.500000 & Anhydritic dolomite \\
\hline$n m b 38-15-20 c c b$ & $7,600.0$ & $7,601.0$ & 337LDVL & 6.1 & 0.000000 & Anhydritic dolomite \\
\hline$n m b 38-15-20 c c b$ & $7,601.0$ & $7,602.0$ & 337LDVL & 1.4 & 0.000000 & Sandy limestone \\
\hline$n m b 38-15-20 c c b$ & $7,602.0$ & $7,603.0$ & 337LDVL & 1.6 & 0.000000 & Sandy limestone \\
\hline$n m b 38-15-20 c c b$ & $7,603.0$ & $7,604.0$ & 337LDVL & 1.8 & 0.000000 & Sandy limestone \\
\hline$n m b 38-15-20 c c b$ & $7,604.0$ & $7,605.0$ & 337LDVL & 3.2 & 0.000000 & Sandy limestone \\
\hline$n m b 38-15-20 c c b$ & $7,605.0$ & $7,606.0$ & 337LDVL & 1.8 & 0.000000 & Sandy limestone \\
\hline$n m b 38-15-20 c c b$ & $7,606.0$ & $7,607.0$ & $337 \mathrm{LDVL}$ & 1.8 & 0.000000 & Sandy limestone \\
\hline \multirow[t]{2}{*}{$\mathrm{nmb} 38-15-20 \mathrm{ccb}$} & $7,607.0$ & $7,608.0$ & 337LDVL & 2.4 & 0.000000 & Sandy limestone \\
\hline & & & AVERAGE- - & 3.8 & 0.278622 & \\
\hline$n m b 38-15-20 c c b$ & $7,614.0$ & $7,615.0$ & 3410URY & 2.5 & 0.000000 & Shaly limestone \\
\hline nmb $38-15-20 c c b$ & $7,615.0$ & $7,616.0$ & $3410 U R Y$ & 3.1 & 0.000000 & Shaly limestone \\
\hline nmb $38-15-20 c c b$ & $7,616.0$ & $7,617.0$ & $3410 U R Y$ & 2.5 & 0.000000 & Shaly limestone \\
\hline$n m b 38-15-20 c c b$ & $7,617.0$ & $7,618.0$ & 3410URY & 4.1 & 0.000000 & Shaly limestone \\
\hline$n m b 38-15-20 c c b$ & $7,618.0$ & $7,619.0$ & $3410 U R Y$ & 2.7 & 0.000000 & Shaly limestone \\
\hline$n m b 38-15-20 c c b$ & $7,619.0$ & $7,620.0$ & 3410URY & 1.8 & 0.000000 & Shaly limestone \\
\hline$n m b 38-15-20 c c b$ & $7,620.0$ & $7,621.0$ & $3410 U R Y$ & 3.2 & 0.000000 & Shaly limestone \\
\hline$n m b 38-15-20 c c b$ & $7,621.0$ & $7,622.0$ & $3410 U R Y$ & 2.9 & 0.000000 & Shaly limestone \\
\hline nmb38-15-20ccb & $7,622.0$ & $7,623.0$ & 3410URY & 2.0 & 0.000000 & Coquina limestone \\
\hline$n m b 38-15-20 c c b$ & $7,623.0$ & $7,624.0$ & $3410 U R Y$ & 2.2 & 0.000000 & Coquina limestone \\
\hline$n m b 38-15-20 c c b$ & $7,624.0$ & $7,625.0$ & $3410 U R Y$ & 2.4 & 0.000000 & Coquina limestone \\
\hline$n m b 38-15-20 c c b$ & $7,625.0$ & $7,626.0$ & $3410 U R Y$ & 2.5 & 0.000000 & Coquina limestone \\
\hline$n m b 38-15-20 c c b$ & $7,626.0$ & $7,627.0$ & $3410 \mathrm{URY}$ & 1.9 & 0.000000 & Coquina limestone \\
\hline$n m b 38-15-20 c c b$ & $7,627.0$ & $7,628.0$ & 3410 URY & 2.8 & 0.000000 & Limy dolomite \\
\hline$n m b 38-15-20 c c b$ & $7,628.0$ & $7,629.0$ & 3410URY & 3.2 & 0.000000 & Anhydritic dolomite \\
\hline
\end{tabular}


Table 4.--Laboratory-determined porosity and permeability--Continued

\begin{tabular}{|c|c|c|c|c|c|c|}
\hline Site & $\begin{array}{l}\text { Depth to } \\
\text { top } \\
\text { (feet) }\end{array}$ & $\begin{array}{l}\text { Depth to } \\
\text { bottom } \\
\text { (feet) }\end{array}$ & $\begin{array}{l}\text { Forma- } \\
\text { tion }\end{array}$ & $\begin{array}{l}\text { Porosity } \\
\text { (percent) }\end{array}$ & $\begin{array}{l}\text { Perme- } \\
\text { ability } \\
\text { (milli- } \\
\text { darcies) }\end{array}$ & Lithology \\
\hline $\begin{array}{l}\text { nmb38-15-20ccb } \\
\text { nmb38-15-20ccb } \\
\text { nmb38-15-20ccb } \\
\text { nmb38-15-20ccb } \\
\text { nmb38-15-20ccb }\end{array}$ & $\begin{array}{l}7,629.0 \\
7,630.0 \\
7,631.0 \\
7,632.0 \\
7,633.0\end{array}$ & $\begin{array}{l}7,630.0 \\
7,631.0 \\
7,632.0 \\
7,633.0 \\
7,634.0\end{array}$ & $\begin{array}{l}3410 \text { URY } \\
3410 \text { URY } \\
3410 U R Y \\
3410 U R Y \\
3410 U R Y\end{array}$ & $\begin{array}{l}3.8 \\
5.5 \\
3.9 \\
3.6 \\
4.7\end{array}$ & $\begin{array}{l}0.000000 \\
0.000000 \\
0.000000 \\
0.000000 \\
0.000000\end{array}$ & $\begin{array}{l}\text { Anhydritic dolomite } \\
\text { Shaly dolomite } \\
\text { Shaly dolomite } \\
\text { Shaly dolomite } \\
\text { Shaly dolomite }\end{array}$ \\
\hline $\begin{array}{l}n m b 38-15-20 c c b \\
n m b 38-15-20 c c b \\
n m b 38-15-20 c c b \\
n m b 38-15-20 c c b \\
n m b 38-15-20 c c b\end{array}$ & $\begin{array}{l}7,634.0 \\
7,635.0 \\
7,636.0 \\
7,637.0 \\
7,638.0\end{array}$ & $\begin{array}{l}7,635.0 \\
7,636.0 \\
7,637.0 \\
7,638.0 \\
7,639.0\end{array}$ & $\begin{array}{l}3410 \text { URY } \\
3410 U R Y \\
3410 U R Y \\
3410 U R Y \\
3410 U R Y\end{array}$ & $\begin{array}{l}4.6 \\
2.6 \\
3.2 \\
3.5 \\
2.8\end{array}$ & $\begin{array}{l}0.000000 \\
0.000000 \\
0.000000 \\
0.000000 \\
0.000000\end{array}$ & $\begin{array}{l}\text { Shaly dolomite } \\
\text { Shaly dolomite } \\
\text { Shaly dolomite } \\
\text { Shaly dolomite } \\
\text { Shaly dolomite }\end{array}$ \\
\hline $\begin{array}{l}\text { nmb38-15-20ccb } \\
\text { nmb38-15-20ccb } \\
\text { nmb38-15-20ccb } \\
\text { nmb38-15-20ccb } \\
\text { nmb38-15-20ccb }\end{array}$ & $\begin{array}{l}7,639.0 \\
7,640.0 \\
7,641.0 \\
7,642.0 \\
7,643.0\end{array}$ & $\begin{array}{l}7,640.0 \\
7,641.0 \\
7,642.0 \\
7,643.0 \\
7,644.0\end{array}$ & $\begin{array}{l}3410 U R Y \\
3410 U R Y \\
3410 U R Y \\
3410 U R Y \\
3410 U R Y\end{array}$ & $\begin{array}{l}4.3 \\
4.7 \\
3.8 \\
3.6 \\
4.9\end{array}$ & $\begin{array}{l}0.000000 \\
0.000000 \\
0.000000 \\
0.000000 \\
0.000000\end{array}$ & $\begin{array}{l}\text { Shaly dolomite } \\
\text { Shaly dolomite } \\
\text { Shaly dolomite } \\
\text { Shaly dolomite } \\
\text { Shaly dolomite }\end{array}$ \\
\hline $\begin{array}{l}\text { nmb38-15-20ccb } \\
\text { nmb38-15-20ccb } \\
\text { nmb38-15-20ccb } \\
\text { nmb38-15-20ccb } \\
\text { nmb38-15-20ccb }\end{array}$ & $\begin{array}{l}7,644.0 \\
7,645.0 \\
7,646.0 \\
7,647.0 \\
7,648.0\end{array}$ & $\begin{array}{l}7,645.0 \\
7,646.0 \\
7,647.0 \\
7,648.0 \\
7,649.0\end{array}$ & $\begin{array}{l}3410 U R Y \\
3410 U R Y \\
3410 U R Y \\
3410 U R Y \\
3410 U R Y\end{array}$ & $\begin{array}{l}2.8 \\
1.9 \\
3.1 \\
2.9 \\
2.8\end{array}$ & $\begin{array}{l}0.000000 \\
0.000000 \\
0.000000 \\
0.000000 \\
0.000000\end{array}$ & $\begin{array}{l}\text { Shaly dolomite } \\
\text { Shaly dolomite } \\
\text { Shaly dolomite } \\
\text { Shaly dolomite } \\
\text { Shaly dolomite }\end{array}$ \\
\hline $\begin{array}{l}\mathrm{nmb} 38-15-20 \mathrm{ccb} \\
\mathrm{nmb} 38-15-20 \mathrm{ccb} \\
\mathrm{nmb} 38-15-20 \mathrm{ccb} \\
\mathrm{nmb} 38-15-20 \mathrm{ccb} \\
\mathrm{nmb} 38-15-20 \mathrm{ccb}\end{array}$ & $\begin{array}{l}7,649.0 \\
7,650.0 \\
7,651.0 \\
7,652.0 \\
7,653.0\end{array}$ & $\begin{array}{l}7,650.0 \\
7,651.0 \\
7,652.0 \\
7,653.0 \\
7,654.0\end{array}$ & $\begin{array}{l}3410 \text { URY } \\
3410 U R Y \\
3410 U R Y \\
3410 U R Y \\
3410 U R Y\end{array}$ & $\begin{array}{l}3.2 \\
3.3 \\
2.2 \\
3.2 \\
3.2\end{array}$ & $\begin{array}{l}0.000000 \\
0.000000 \\
0.000000 \\
0.000000 \\
0.000000\end{array}$ & $\begin{array}{l}\text { Shaly dolomite } \\
\text { Shaly dolomite } \\
\text { Shaly dolomite } \\
\text { Shaly dolomite } \\
\text { Shaly dolomite }\end{array}$ \\
\hline $\begin{array}{l}\text { nmb38-15-20ccb } \\
\text { nmb38-15-20ccb } \\
\text { nmb38-15-20ccb } \\
\text { nmb38-15-20ccb } \\
\text { nmb38-15-20ccb }\end{array}$ & $\begin{array}{l}7,654.0 \\
7,655.0 \\
7,656.0 \\
7,657.0 \\
7,658.0\end{array}$ & $\begin{array}{l}7,655.0 \\
7,656.0 \\
7,657.0 \\
7,658.0 \\
7,659.0\end{array}$ & $\begin{array}{l}3410 \text { URY } \\
3410 U R Y \\
3410 U R Y \\
3410 U R Y \\
3410 U R Y\end{array}$ & $\begin{array}{l}2.9 \\
3.0 \\
2.8 \\
3.7 \\
2.5\end{array}$ & $\begin{array}{l}0.000000 \\
0.000000 \\
0.000000 \\
0.000000 \\
0.000000\end{array}$ & $\begin{array}{l}\text { Shaly dolomite } \\
\text { Shaly dolomite } \\
\text { Shaly dolomite } \\
\text { Shaly dolomite } \\
\text { Shaly dolomite }\end{array}$ \\
\hline $\begin{array}{l}\text { nmb38-15-20ccb } \\
\text { nmb38-15-20ccb } \\
\text { nmb38-15-20ccb } \\
\text { nmb38-15-20ccb } \\
\text { nmb38-15-20ccb }\end{array}$ & $\begin{array}{l}7,659.0 \\
7,660.0 \\
7,661.0 \\
7,662.0 \\
7,663.0\end{array}$ & $\begin{array}{l}7,660.0 \\
7,661.0 \\
7,662.0 \\
7,663.0 \\
7,664.0\end{array}$ & $\begin{array}{l}3410 U R Y \\
3410 U R Y \\
3410 U R Y \\
3410 U R Y \\
3410 U R Y\end{array}$ & $\begin{array}{l}2.9 \\
2.9 \\
3.0 \\
3.8 \\
4.1\end{array}$ & $\begin{array}{l}0.000000 \\
0.000000 \\
0.000000 \\
0.000000 \\
0.000000\end{array}$ & $\begin{array}{l}\text { Shaly dolomite } \\
\text { Shaly dolomite } \\
\text { Shaly dolomite } \\
\text { Shaly dolomite } \\
\text { Shaly dolomite }\end{array}$ \\
\hline $\begin{array}{l}\mathrm{nmb} 38-15-20 c \mathrm{cb} \\
\mathrm{nmb} 38-15-20 \mathrm{ccb} \\
\mathrm{nmb} 38-15-20 \mathrm{ccb} \\
\mathrm{nmb} 38-15-20 \mathrm{ccb} \\
\mathrm{nmb} 38-15-20 \mathrm{ccb}\end{array}$ & $\begin{array}{l}7,664.0 \\
7,665.0 \\
7,666.0 \\
7,667.0 \\
7,668.0\end{array}$ & $\begin{array}{l}7,665.0 \\
7,666.0 \\
7,667.0 \\
7,668.0 \\
7,669.0\end{array}$ & $\begin{array}{l}3410 U R Y \\
3410 U R Y \\
3410 U R Y \\
3410 U R Y \\
3410 U R Y\end{array}$ & $\begin{array}{l}3.6 \\
3.0 \\
4.2 \\
2.1 \\
3.5\end{array}$ & $\begin{array}{l}0.000000 \\
0.000000 \\
0.000000 \\
0.000000 \\
0.000000\end{array}$ & $\begin{array}{l}\text { Shaly dolomite } \\
\text { Shaly dolomite } \\
\text { Shaly dolomite } \\
\text { Shaly dolomite } \\
\text { Shaly dolomite }\end{array}$ \\
\hline $\begin{array}{l}\mathrm{nmb} 38-15-20 c \mathrm{cb} \\
\mathrm{nmb} 38-15-20 \mathrm{ccb} \\
\mathrm{nmb} 38-15-20 \mathrm{ccb} \\
\mathrm{nmb} 38-15-20 \mathrm{ccb} \\
\mathrm{nmb} 38-15-20 \mathrm{ccb}\end{array}$ & $\begin{array}{l}7,669.0 \\
7,670.0 \\
7,671.0 \\
7,672.0 \\
7,673.0\end{array}$ & $\begin{array}{l}7,670.0 \\
7,671.0 \\
7,672.0 \\
7,673.0 \\
7,674.0\end{array}$ & $\begin{array}{l}3410 \text { URY } \\
3410 U R Y \\
3410 U R Y \\
3410 U R Y \\
3410 U R Y\end{array}$ & $\begin{array}{l}3.4 \\
3.8 \\
3.3 \\
3.4 \\
2.8\end{array}$ & $\begin{array}{l}0.000000 \\
0.000000 \\
0.000000 \\
0.000000 \\
0.000000\end{array}$ & $\begin{array}{l}\text { Shaly dolomite } \\
\text { Shaly dolomite } \\
\text { Shaly dolomite } \\
\text { Shaly dolomite } \\
\text { Shaly dolomite }\end{array}$ \\
\hline $\begin{array}{l}\text { nmb38-15-20ccb } \\
\text { nmb38-15-20ccb } \\
\text { nmb38-15-20ccb } \\
\text { nmb38-15-20ccb } \\
\text { nmb38-15-20ccb }\end{array}$ & $\begin{array}{l}7,674.0 \\
7,675.0 \\
7,676.0 \\
7,677.0 \\
7,678.0\end{array}$ & $\begin{array}{l}7,675.0 \\
7,676.0 \\
7,677.0 \\
7,678.0 \\
7,679.0\end{array}$ & $\begin{array}{l}3410 U R Y \\
3410 U R Y \\
3410 U R Y \\
3410 U R Y \\
3410 U R Y\end{array}$ & $\begin{array}{l}2.7 \\
2.6 \\
4.2 \\
4.9 \\
1.6\end{array}$ & $\begin{array}{l}0.000000 \\
0.000000 \\
0.000000 \\
0.000000 \\
0.000000\end{array}$ & $\begin{array}{l}\text { Oolitic dolomite } \\
\text { Oolitic dolomite } \\
\text { Fine-grained dolomite } \\
\text { Fine-grained dolomite } \\
\text { Fine-grained dolomite }\end{array}$ \\
\hline
\end{tabular}


Table 4.--Laboratory-determined porosity and permeability--Continued

\begin{tabular}{|c|c|c|c|c|c|c|}
\hline Site & $\begin{array}{l}\text { Depth to } \\
\text { top } \\
\text { (feet) }\end{array}$ & $\begin{array}{l}\text { Depth to } \\
\text { bottom } \\
\text { (feet) }\end{array}$ & $\begin{array}{c}\text { Forma- } \\
\text { tion }\end{array}$ & $\begin{array}{l}\text { Porosity } \\
\text { (percent) }\end{array}$ & $\begin{array}{l}\text { Perme- } \\
\text { ability } \\
\text { (milli- } \\
\text { darcies) }\end{array}$ & Lithology \\
\hline $\begin{array}{l}\text { nmb38-15-20ccb } \\
\text { nmb38-15-20ccb } \\
\text { nmb38-15-20ccb } \\
\text { nmb38-15-20ccb } \\
\text { nmb38-15-20ccb }\end{array}$ & $\begin{array}{l}7,679.0 \\
7,680.0 \\
7,681.0 \\
7,683.0 \\
7,684.0\end{array}$ & $\begin{array}{l}7,680.0 \\
7,681.0 \\
7,682.0 \\
7,684.0 \\
7,685.0\end{array}$ & $\begin{array}{l}\text { 3410URY } \\
3410 U R Y \\
3410 U R Y \\
3410 U R Y \\
3410 U R Y\end{array}$ & $\begin{array}{l}4.7 \\
0.7 \\
2.0 \\
1.5 \\
1.8 \\
\end{array}$ & $\begin{array}{l}0.000000 \\
0.000000 \\
0.000000 \\
0.000000 \\
0.000000 \\
\end{array}$ & $\begin{array}{l}\text { Fine-grained dolomite } \\
\text { Fine-grained dolomite } \\
\text { Fine-grained dolomite } \\
\text { Fine-grained dolomite } \\
\text { Fine-grained dolomite }\end{array}$ \\
\hline & & & AVERAGE-- & 3.1 & 0.000000 & \\
\hline $\begin{array}{l}\mathrm{nmb} 38-15-20 \mathrm{ccb} \\
\mathrm{nmb} 38-15-20 \mathrm{ccb} \\
\mathrm{nmb} 38-15-20 \mathrm{ccb} \\
\mathrm{nmb} 38-15-20 \mathrm{ccb} \\
\mathrm{nmb} 38-15-20 \mathrm{ccb}\end{array}$ & $\begin{array}{l}7,690.0 \\
7,691.0 \\
7,692.0 \\
7,693.0 \\
7,694.0\end{array}$ & $\begin{array}{l}7,691.0 \\
7,692.0 \\
7,693.0 \\
7,694.0 \\
7,695.0\end{array}$ & $\begin{array}{l}341 \mathrm{ELBR} \\
341 \mathrm{ELBR} \\
341 \mathrm{ELBR} \\
341 \mathrm{ELBR} \\
341 \mathrm{ELBR}\end{array}$ & $\begin{array}{l}2.5 \\
2.4 \\
1.8 \\
2.0 \\
2.9\end{array}$ & $\begin{array}{l}0.000000 \\
0.000000 \\
0.000000 \\
0.000000 \\
0.000000\end{array}$ & $\begin{array}{l}\text { Shaly, sandy dolomite } \\
\text { Shaly, sandy dolomite } \\
\text { Shaly, sandy dolomite } \\
\text { Shaly, sandy dolomite } \\
\text { Shaly, sandy dolomite }\end{array}$ \\
\hline $\begin{array}{l}\mathrm{nmb} 38-15-20 \mathrm{ccb} \\
\mathrm{nmb} 38-15-20 \mathrm{ccb} \\
\mathrm{nmb} 38-15-20 \mathrm{ccb} \\
\mathrm{nmb} 38-15-20 \mathrm{ccb} \\
\mathrm{nmb} 38-15-20 \mathrm{ccb}\end{array}$ & $\begin{array}{l}7,695.0 \\
7,696.0 \\
7,697.0 \\
7,698.0 \\
7,699.0\end{array}$ & $\begin{array}{l}7,696.0 \\
7,697.0 \\
7,698.0 \\
7,699.0 \\
7,700.0\end{array}$ & $\begin{array}{l}341 \mathrm{ELBR} \\
341 \mathrm{ELBR} \\
341 \mathrm{ELBR} \\
341 \mathrm{ELBR} \\
341 \mathrm{ELBR}\end{array}$ & $\begin{array}{l}2.6 \\
2.3 \\
3.1 \\
2.0 \\
1.7\end{array}$ & $\begin{array}{l}0.000000 \\
0.000000 \\
0.000000 \\
0.000000 \\
0.000000\end{array}$ & $\begin{array}{l}\text { Shaly, sandy dolomite } \\
\text { Shaly, sandy dolomite } \\
\text { Shaly, sandy dolomite } \\
\text { Shaly, sandy dolomite } \\
\text { Shaly, sandy dolomite }\end{array}$ \\
\hline $\begin{array}{l}\mathrm{nmb} 38-15-20 c \mathrm{cb} \\
\mathrm{nmb} 38-15-20 \mathrm{ccb} \\
\mathrm{nmb} 38-15-20 \mathrm{ccb} \\
\mathrm{nmb} 38-15-20 \mathrm{ccb} \\
\mathrm{nmb} 38-15-20 \mathrm{ccb}\end{array}$ & $\begin{array}{l}7,700.0 \\
7,701.0 \\
7,702.0 \\
7,703.0 \\
7,704.0\end{array}$ & $\begin{array}{l}7,701.0 \\
7,702.0 \\
7,703.0 \\
7,704.0 \\
7,705.0\end{array}$ & $\begin{array}{l}341 \mathrm{ELBR} \\
341 \mathrm{ELBR} \\
341 \mathrm{ELBR} \\
341 \mathrm{ELBR} \\
341 \mathrm{ELBR}\end{array}$ & $\begin{array}{l}2.3 \\
1.8 \\
1.7 \\
2.6 \\
1.7\end{array}$ & $\begin{array}{l}0.000000 \\
0.000000 \\
0.000000 \\
0.000000 \\
0.000000\end{array}$ & $\begin{array}{l}\text { Shaly, sandy dolomite } \\
\text { Shaly, sandy dolomite } \\
\text { Shaly, sandy dolomite } \\
\text { Shaly, sandy dolomite } \\
\text { Shaly, sandy dolomite }\end{array}$ \\
\hline $\begin{array}{l}\text { nmb38-15-20ccb } \\
\text { nmb38-15-20ccb } \\
\text { nmb38-15-20ccb } \\
\text { nmb38-15-20ccb } \\
\text { nmb38-15-20ccb }\end{array}$ & $\begin{array}{l}7,705.0 \\
7,706.0 \\
7,707.0 \\
7,708.0 \\
7,709.0\end{array}$ & $\begin{array}{l}7,706.0 \\
7,707.0 \\
7,708.0 \\
7,709.0 \\
7,710.0\end{array}$ & $\begin{array}{l}341 \mathrm{ELBR} \\
341 \mathrm{ELBR} \\
341 \mathrm{ELBR} \\
341 \mathrm{ELBR} \\
341 \mathrm{ELBR}\end{array}$ & $\begin{array}{l}2.1 \\
1.7 \\
2.1 \\
1.9 \\
1.5\end{array}$ & $\begin{array}{l}0.000000 \\
0.000000 \\
0.000000 \\
0.000000 \\
0.000000\end{array}$ & $\begin{array}{l}\text { Shaly, sandy dolomite } \\
\text { Shaly, sandy dolomite } \\
\text { Shaly, sandy dolomite } \\
\text { Shaly, sandy dolomite } \\
\text { Shaly, sandy dolomite }\end{array}$ \\
\hline $\begin{array}{l}\mathrm{nmb} 38-15-20 c \mathrm{cb} \\
\mathrm{nmb} 38-15-20 \mathrm{ccb} \\
\mathrm{nmb} 38-15-20 \mathrm{ccb} \\
\mathrm{nmb} 38-15-20 \mathrm{ccb} \\
\mathrm{nmb} 38-15-20 \mathrm{ccb}\end{array}$ & $\begin{array}{l}7,710.0 \\
7,711.0 \\
7,712.0 \\
7,713.0 \\
7,714.0\end{array}$ & $\begin{array}{l}7,711.0 \\
7,712.0 \\
7,713.0 \\
7,714.0 \\
7,715.0\end{array}$ & $\begin{array}{l}341 \mathrm{ELBR} \\
341 \mathrm{ELBR} \\
341 \mathrm{ELBR} \\
341 \mathrm{ELBR} \\
341 \mathrm{ELBR}\end{array}$ & $\begin{array}{l}1.5 \\
2.5 \\
1.6 \\
2.2 \\
0.4\end{array}$ & $\begin{array}{l}0.000000 \\
0.000000 \\
0.000000 \\
0.000000 \\
0.000000\end{array}$ & $\begin{array}{l}\text { Shaly, sandy dolomite } \\
\text { Shaly, sandy dolomite } \\
\text { Shaly, sandy dolomite } \\
\text { Shaly, sandy dolomite } \\
\text { Shaly, sandy dolomite }\end{array}$ \\
\hline $\begin{array}{l}\text { nmb38-15-20ccb } \\
\text { nmb38-15-20ccb } \\
\text { nmb38-15-20ccb } \\
\text { nmb38-15-20ccb } \\
\text { nmb38-15-20ccb }\end{array}$ & $\begin{array}{l}7,715.0 \\
7,716.0 \\
7,717.0 \\
7,718.0 \\
7,719.0\end{array}$ & $\begin{array}{l}7,716.0 \\
7,717.0 \\
7,718.0 \\
7,719.0 \\
7,720.0\end{array}$ & $\begin{array}{l}341 \mathrm{ELBR} \\
341 \mathrm{ELBR} \\
341 \mathrm{ELBR} \\
341 \mathrm{ELBR} \\
341 \mathrm{ELBR}\end{array}$ & $\begin{array}{l}3.0 \\
3.7 \\
1.9 \\
3.2 \\
3.0\end{array}$ & $\begin{array}{l}0.000000 \\
0.000000 \\
0.000000 \\
0.000000 \\
0.000000\end{array}$ & $\begin{array}{l}\text { Shaly, sandy dolomite } \\
\text { Shaly, sandy dolomite } \\
\text { Shaly, sandy dolomite } \\
\text { Shaly, sandy dolomite } \\
\text { Shaly, sandy dolomite }\end{array}$ \\
\hline $\begin{array}{l}\mathrm{nmb} 38-15-20 \mathrm{ccb} \\
\mathrm{nmb} 38-15-20 \mathrm{ccb} \\
\mathrm{nmb} 38-15-20 \mathrm{ccb} \\
\mathrm{nmb} 38-15-20 \mathrm{ccb} \\
\mathrm{nmb} 38-15-20 \mathrm{ccb}\end{array}$ & $\begin{array}{l}7,720.0 \\
7,721.0 \\
7,722.0 \\
7,723.0 \\
7,724.0\end{array}$ & $\begin{array}{l}7,721.0 \\
7,722.0 \\
7,723.0 \\
7,724.0 \\
7,725.0\end{array}$ & $\begin{array}{l}341 \mathrm{ELBR} \\
341 \mathrm{ELBR} \\
341 \mathrm{ELBR} \\
341 \mathrm{ELBR} \\
341 \mathrm{ELBR}\end{array}$ & $\begin{array}{l}2.3 \\
2.3 \\
2.4 \\
3.1 \\
1.6 \\
\end{array}$ & $\begin{array}{l}0.000000 \\
0.000000 \\
0.000000 \\
0.000000 \\
0.000000 \\
\end{array}$ & $\begin{array}{l}\text { Shaly, sandy dolomite } \\
\text { Shaly, sandy dolomite } \\
\text { Shaly, sandy dolomite } \\
\text { Shaly, sandy dolomite } \\
\text { Shaly, sandy dolomite }\end{array}$ \\
\hline SITE AVERAGE- & 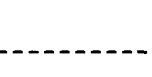 & 20 & AVERAGE-- & $\frac{2.2}{4.2}$ & $\frac{0.000000}{1.156667}$ & \\
\hline $\begin{array}{l}\text { nmb38-15-21bcb } \\
\text { nmb38-15-21bcb } \\
\text { nmb38-15-21bcb } \\
\text { nmb38-15-21bcb } \\
\text { nmb38-15-21bcb }\end{array}$ & $\begin{array}{l}3,270.0 \\
3,271.0 \\
3,272.0 \\
3,273.0 \\
3,274.0\end{array}$ & $\begin{array}{l}3,271.0 \\
3,272.0 \\
3,273.0 \\
3,274.0 \\
3,275.0\end{array}$ & $\begin{array}{l}317 \text { CTLR } \\
317 \text { CTLR } \\
\text { 317CTLR } \\
\text { 317CTLR } \\
\text { 317CTLR }\end{array}$ & $\begin{array}{r}6.3 \\
8.4 \\
3.2 \\
11.2 \\
14.4\end{array}$ & $\begin{array}{r}4.500000 \\
0.830000 \\
0.000000 \\
27.400000 \\
23.600000\end{array}$ & $\begin{array}{l}\text { Arkosic sandstone } \\
\text { Arkosic sandstone } \\
\text { Arkosic sandstone } \\
\text { Arkosic sandstone } \\
\text { Arkosic sandstone }\end{array}$ \\
\hline
\end{tabular}


Table 4.--Laboratory-determined porosity and permeability--Continued

\begin{tabular}{|c|c|c|c|c|c|c|}
\hline Site & $\begin{array}{l}\text { Depth to } \\
\text { top } \\
\text { (feet) }\end{array}$ & $\begin{array}{l}\text { Depth to } \\
\text { bottom } \\
\text { (feet) }\end{array}$ & $\begin{array}{c}\text { Forma- } \\
\text { tion }\end{array}$ & $\begin{array}{l}\text { Porosity } \\
\text { (percent) }\end{array}$ & $\begin{array}{l}\text { Perme- } \\
\text { ability } \\
\text { (milli- } \\
\text { darcies) }\end{array}$ & Lithology \\
\hline $\begin{array}{l}\text { nmb38-15-21bcb } \\
\text { nmb38-15-21bcb } \\
\text { nmb38-15-21bcb } \\
\text { nmb38-15-21bcb } \\
\text { nmb38-15-21bcb }\end{array}$ & $\begin{array}{l}3,275.0 \\
3,276.0 \\
3,277.0 \\
3,278.0 \\
3,279.0\end{array}$ & $\begin{array}{l}3,276.0 \\
3,277.0 \\
3,278.0 \\
3,279.0 \\
3,280.0\end{array}$ & $\begin{array}{l}317 \text { CTLR } \\
\text { 317CTLR } \\
\text { 317CTLR } \\
\text { 317CTLR } \\
\text { 317CTLR }\end{array}$ & $\begin{array}{r}10.8 \\
9.2 \\
11.9 \\
6.5 \\
10.2\end{array}$ & $\begin{array}{r}16.600000 \\
6.900000 \\
11.900000 \\
55.000000 \\
16.500000\end{array}$ & $\begin{array}{l}\text { Arkosic sandstone } \\
\text { Arkosic sandstone } \\
\text { Arkosic sandstone } \\
\text { Arkosic sandstone } \\
\text { Arkosic sandstone }\end{array}$ \\
\hline $\begin{array}{l}\text { nmb38-15-21bcb } \\
\text { nmb38-15-21bcb } \\
\text { nmb38-15-21bcb } \\
\text { nmb38-15-21bcb } \\
\text { nmb38-15-21bcb }\end{array}$ & $\begin{array}{l}3,280.0 \\
3,281.0 \\
3,282.0 \\
3,283.0 \\
3,284.0\end{array}$ & $\begin{array}{l}3,281.0 \\
3,282.0 \\
3,283.0 \\
3,284.0 \\
3,285.0\end{array}$ & $\begin{array}{l}\text { 317CTLR } \\
\text { 317CTLR } \\
\text { 317CTLR } \\
\text { 317CTLR } \\
\text { 317CTLR }\end{array}$ & $\begin{array}{r}10.6 \\
12.2 \\
11.4 \\
12.9 \\
5.7\end{array}$ & $\begin{array}{r}20.700000 \\
20.700000 \\
13.900000 \\
16.400000 \\
8.100000\end{array}$ & $\begin{array}{l}\text { Arkosic sandstone } \\
\text { Arkosic sandstone } \\
\text { Arkosic sandstone } \\
\text { Arkosic sandstone } \\
\text { Arkosic sandstone }\end{array}$ \\
\hline $\begin{array}{l}\text { nmb 38-15-21bcb } \\
\text { nmb 38-15-21bcb } \\
\text { nmb 38-15-21bcb } \\
\text { nmb 38-15-21bcb } \\
\text { nmb38-15-21bcb }\end{array}$ & $\begin{array}{l}3,285.0 \\
3,286.0 \\
3,287.0 \\
3,288.0 \\
3,289.0\end{array}$ & $\begin{array}{l}3,286.0 \\
3,287.0 \\
3,288.0 \\
3,289.0 \\
3,290.0\end{array}$ & $\begin{array}{l}\text { 317CTLR } \\
\text { 317CTLR } \\
\text { 317CTLR } \\
\text { 317CTLR } \\
\text { 317CTLR }\end{array}$ & $\begin{array}{r}10.6 \\
6.2 \\
8.8 \\
11.4 \\
12.0\end{array}$ & $\begin{array}{r}16.500000 \\
13.500000 \\
5.200000 \\
20.700000 \\
23.800000\end{array}$ & $\begin{array}{l}\text { Arkosic sandstone } \\
\text { Arkosic sandstone } \\
\text { Arkosic sandstone } \\
\text { Arkosic sandstone } \\
\text { Arkosic sandstone }\end{array}$ \\
\hline $\begin{array}{l}\text { nmb 38-15-21bcb } \\
\text { nmb38-15-21bcb } \\
\text { nmb38-15-21bcb } \\
\text { nmb38-15-21bcb } \\
\text { nmb38-15-21bcb }\end{array}$ & $\begin{array}{l}3,290.0 \\
3,291.0 \\
3,292.0 \\
3,293.0 \\
3,629.0\end{array}$ & $\begin{array}{l}3,291.0 \\
3,292.0 \\
3,293.0 \\
3,294.0 \\
3,630.0\end{array}$ & $\begin{array}{l}\text { 317CTLR } \\
\text { 317CTLR } \\
\text { 317CTLR } \\
\text { 317CTLR } \\
\text { 317CTLR }\end{array}$ & $\begin{array}{l}8.2 \\
8.5 \\
1.4 \\
7.3 \\
9.0\end{array}$ & $\begin{array}{r}9.500000 \\
13.900000 \\
0.000000 \\
2.400000 \\
3.800000\end{array}$ & $\begin{array}{l}\text { Arkosic sandstone } \\
\text { Arkosic sandstone } \\
\text { Arkosic sandstone } \\
\text { Arkosic sandstone } \\
\text { Arkosic sandstone }\end{array}$ \\
\hline $\begin{array}{l}\text { nmb38-15-21bcb } \\
\text { nmb38-15-21bcb } \\
\text { nmb38-15-21bcb } \\
\text { nmb38-15-21bcb } \\
\text { nmb38-15-21bcb }\end{array}$ & $\begin{array}{l}3,630.0 \\
3,631.0 \\
3,632.0 \\
3,633.0 \\
3,634.0\end{array}$ & $\begin{array}{l}3,631.0 \\
3,632.0 \\
3,633.0 \\
3,634.0 \\
3,635.0\end{array}$ & $\begin{array}{l}317 \text { CTLR } \\
\text { 317CTLR } \\
\text { 317CTLR } \\
\text { 317CTLR } \\
\text { 317CTLR }\end{array}$ & $\begin{array}{r}10.4 \\
16.1 \\
4.7 \\
7.8 \\
8.1\end{array}$ & $\begin{array}{r}12.500000 \\
3.200000 \\
0.400000 \\
4.200000 \\
40.200000\end{array}$ & $\begin{array}{l}\text { Arkosic sandstone } \\
\text { Arkosic sandstone } \\
\text { Arkosic sandstone } \\
\text { Arkosic sandstone } \\
\text { Arkosic sandstone }\end{array}$ \\
\hline $\begin{array}{l}\text { nmb 38-15-21bcb } \\
\text { nmb38-15-21bcb } \\
\text { nmb38-15-21bcb } \\
\text { nmb38-15-21bcb } \\
\text { nmb38-15-21bcb }\end{array}$ & $\begin{array}{l}3,635.0 \\
3,636.0 \\
3,637.0 \\
3,638.0 \\
3,639.0\end{array}$ & $\begin{array}{l}3,636.0 \\
3,637.0 \\
3,638.0 \\
3,639.0 \\
3,640.0\end{array}$ & $\begin{array}{l}\text { 317CTLR } \\
\text { 317CTLR } \\
\text { 317CTLR } \\
\text { 317CTLR } \\
\text { 317CTLR }\end{array}$ & $\begin{array}{l}8.7 \\
6.5 \\
3.5 \\
4.5 \\
7.5\end{array}$ & $\begin{array}{l}3.800000 \\
1.300000 \\
0.800000 \\
0.500000 \\
5.400000\end{array}$ & $\begin{array}{l}\text { Arkosic sandstone } \\
\text { Arkosic sandstone } \\
\text { Arkosic sandstone } \\
\text { Arkosic sandstone } \\
\text { Arkosic sandstone }\end{array}$ \\
\hline $\begin{array}{l}\text { nmb38-15-21bcb } \\
\text { nmb38-15-21bcb } \\
\text { nmb38-15-21bcb } \\
\text { nmb38-15-21bcb } \\
\text { nmb38-15-21bcb }\end{array}$ & $\begin{array}{l}3,640.0 \\
3,641.0 \\
3,642.0 \\
3,643.0 \\
3,644.0\end{array}$ & $\begin{array}{l}3,641.0 \\
3,642.0 \\
3,643.0 \\
3,644.0 \\
3,645.0\end{array}$ & $\begin{array}{l}\text { 317CTLR } \\
\text { 317CTLR } \\
\text { 317CTLR } \\
\text { 317CTLR } \\
\text { 317CTLR }\end{array}$ & $\begin{array}{l}6.9 \\
9.5 \\
9.3 \\
7.5 \\
9.3\end{array}$ & $\begin{array}{r}7.300000 \\
32.200000 \\
13.500000 \\
1.300000 \\
2.000000\end{array}$ & $\begin{array}{l}\text { Arkosic sandstone } \\
\text { Arkosic sandstone } \\
\text { Arkosic sandstone } \\
\text { Arkosic sandstone } \\
\text { Arkosic sandstone }\end{array}$ \\
\hline $\begin{array}{l}\text { nmb38-15-21bcb } \\
\text { nmb38-15-21bcb } \\
\text { nmb38-15-21bcb } \\
\text { nmb38-15-21bcb } \\
\text { nmb38-15-21bcb }\end{array}$ & $\begin{array}{l}3,645.0 \\
3,646.0 \\
3,647.0 \\
3,648.0 \\
3,649.0\end{array}$ & $\begin{array}{l}3,646.0 \\
3,647.0 \\
3,648.0 \\
3,649.0 \\
3,650.0\end{array}$ & $\begin{array}{l}\text { 317CTLR } \\
317 \text { CTLR } \\
317 \text { CTLR } \\
\text { 317CTLR } \\
\text { 317CTLR }\end{array}$ & $\begin{array}{l}6.3 \\
6.3 \\
8.1 \\
4.2 \\
6.3\end{array}$ & $\begin{array}{l}0.800000 \\
0.700000 \\
5.700000 \\
3.800000 \\
6.800000\end{array}$ & $\begin{array}{l}\text { Arkosic sandstone } \\
\text { Arkosic sandstone } \\
\text { Arkosic sandstone } \\
\text { Arkosic sandstone } \\
\text { Arkosic sandstone }\end{array}$ \\
\hline $\begin{array}{l}\text { nmb38-15-21bcb } \\
\text { SITE AVERAGE--- }\end{array}$ & $3,650.0$ & $3,651.0$ & $\begin{array}{l}317 \text { CTLR } \\
\text { AVERAGE-- }\end{array}$ & $\frac{7.1}{\frac{8.4}{8.4}}$ & $\frac{1.700000}{\frac{10.878913}{10.878913}}$ & Arkosic sandstone \\
\hline $\begin{array}{l}\text { nmb39-14-08dcc } \\
\text { nmb39-14-08dcc } \\
\text { nmb39-14-08dcc } \\
\text { nmb39-14-08dcc } \\
\text { nmb39-14-08dcc }\end{array}$ & $\begin{array}{l}5,130.0 \\
5,131.0 \\
5,132.0 \\
5,133.0 \\
5,134.0\end{array}$ & $\begin{array}{l}5,131.0 \\
5,132.0 \\
5,133.0 \\
5,134.0 \\
5,135.0\end{array}$ & $\begin{array}{l}321 \mathrm{HKTL} \\
321 \mathrm{HKTL} \\
321 \mathrm{HKTL} \\
321 \mathrm{HKTL} \\
321 \mathrm{HKTL}\end{array}$ & $\begin{array}{l}3.2 \\
3.6 \\
3.9 \\
3.7 \\
3.6\end{array}$ & $\begin{array}{l}0.000000 \\
0.000000 \\
0.000000 \\
0.000000 \\
0.000000\end{array}$ & $\begin{array}{l}\text { Finely crystalline limestone } \\
\text { Finely crystalline limestone } \\
\text { Finely crystalline limestone } \\
\text { Finely crystalline limestone } \\
\text { Finely crystalline limestone }\end{array}$ \\
\hline
\end{tabular}


Table 4.--Laboratory-determined porosity and permeability--Continued

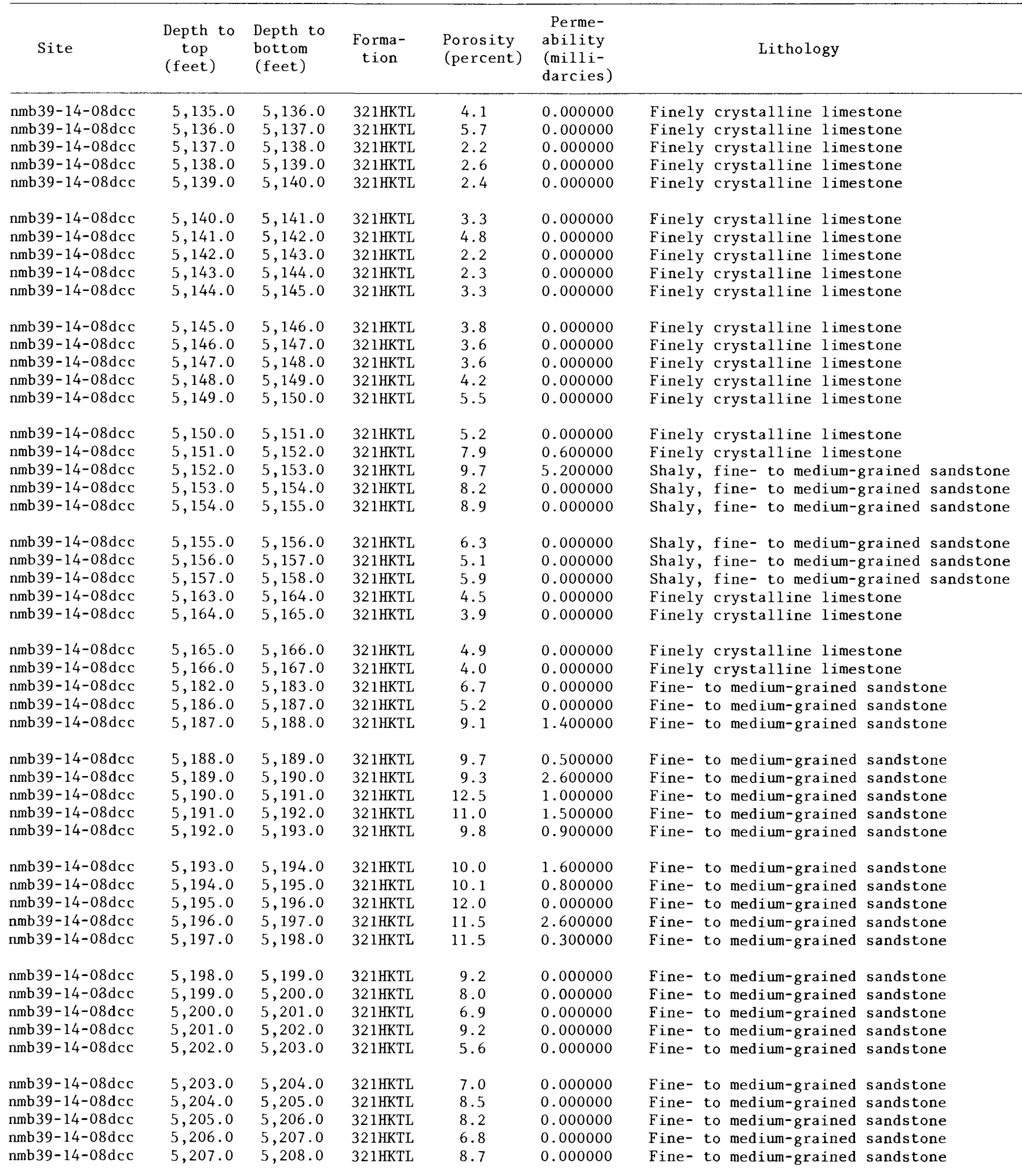


Table 4.--Laboratory-determined porosity and permeability--Continued

\begin{tabular}{|c|c|c|c|c|c|c|}
\hline Site & $\begin{array}{l}\text { Depth to } \\
\text { top } \\
\text { (feet) }\end{array}$ & $\begin{array}{l}\text { Depth to } \\
\text { bottom } \\
\text { (feet) }\end{array}$ & $\begin{array}{c}\text { Forma- } \\
\text { tion }\end{array}$ & $\begin{array}{l}\text { Porosity } \\
\text { (percent) }\end{array}$ & $\begin{array}{l}\text { Perme- } \\
\text { ability } \\
\text { (milli- } \\
\text { darcies) }\end{array}$ & Lithology \\
\hline$n m b 39-14-08 d c c$ & $5,224.0$ & $5,225.0$ & 321HKTL & 5.3 & 0.000000 & Fine-grained sandstone \\
\hline$n m b 39-14-08 d c c$ & $5,225.0$ & $5,226.0$ & 321HKTL & 6.3 & 0.000000 & Fine-grained sandstone \\
\hline nmb39-14-08dcc & $5,226.0$ & $5,227.0$ & 321 HKTL & 7.2 & 0.000000 & Fine-grained sandstone \\
\hline$n m b 39-14-08 d c c$ & $5,227.0$ & $5,228.0$ & 321HKTL & 6.4 & 0.000000 & Fine-grained sandstone \\
\hline nmb39-14-08dcc & $5,228.0$ & $5,229.0$ & $321 \mathrm{HKTL}$ & 6.9 & 0.000000 & Fine-grained sandstone \\
\hline $\mathrm{nmb} 39-14-08 \mathrm{dcc}$ & $5,229.0$ & $5,230.0$ & 321HKTL & 6.2 & 0.000000 & Fine-grained sandstone \\
\hline nmb39-14-08dcc & $5,230.0$ & $5,231.0$ & 321HKTL & 4.5 & 0.000000 & Fine-grained sandstone \\
\hline $\mathrm{nmb} 39-14-08 \mathrm{dcc}$ & $5,231.0$ & $5,232.0$ & $321 \mathrm{HKTL}$ & 7.0 & 0.000000 & Fine-grained sandstone \\
\hline nmb39-14-08dcc & $5,232.0$ & $5,233.0$ & $321 \mathrm{HKTL}$ & 8.1 & 0.000000 & Fine-grained sandstone \\
\hline nmb39-14-08dcc & $5,233.0$ & $5,234.0$ & $321 \mathrm{HKTL}$ & 7.1 & 0.000000 & Fine-grained sandstone \\
\hline$n m b 39-14-08 d c c$ & $5,234.0$ & $5,235.0$ & $321 \mathrm{HKTL}$ & 8.1 & 0.000000 & Fine-grained sandstone \\
\hline nmb39-14-08dcc & $5,235.0$ & $5,236.0$ & 321 HKTL & 8.5 & 0.000000 & Fine-grained sandstone \\
\hline$n m b 39-14-08 d c c$ & $5,236.0$ & $5,237.0$ & $321 \mathrm{HKTL}$ & 9.2 & 0.000000 & Fine-grained sandstone \\
\hline$n m b 39-14-08 d c c$ & $5,237.0$ & $5,238.0$ & $321 \mathrm{HKTL}$ & 8.6 & 0.000000 & Fine-grained sandstone \\
\hline nmb39-14-08dcc & $5,238.0$ & $5,239.0$ & $321 \mathrm{HKTL}$ & 6.9 & 0.000000 & Fine-grained sandstone \\
\hline nmb39-14-08dcc & $5,239.0$ & $5,240.0$ & $321 \mathrm{HKTL}$ & 7.0 & 0.000000 & Fine-grained sandstone \\
\hline nmb39-14-08dcc & $5,240.0$ & $5,241.0$ & $321 \mathrm{HKTL}$ & 8.4 & 0.000000 & Fine-grained sandstone \\
\hline $\mathrm{nmb} 39-14-08 \mathrm{dcc}$ & $5,241.0$ & $5,242.0$ & $321 \mathrm{HKTL}$ & 7.3 & 0.000000 & Fine-grained sandstone \\
\hline$n m b 39-14-08 d c c$ & $5,242.0$ & $5,243.0$ & $321 \mathrm{HKTL}$ & 6.0 & 0.000000 & Fine-grained sandstone \\
\hline nmb39-14-08dcc & $5,243.0$ & $5,244.0$ & $321 \mathrm{HKTL}$ & 4.7 & 0.000000 & Fine-grained sandstone \\
\hline nmb39-14-08dcc & $5,244.0$ & $5,245.0$ & 321HKTL & 6.2 & 0.000000 & Fine-grained sandstone \\
\hline nmb39-14-08dcc & $5,254.0$ & $5,255.0$ & $321 \mathrm{HKTL}$ & 4.0 & 0.000000 & Limestone and shale \\
\hline $\mathrm{nmb} 39-14-08 \mathrm{dcc}$ & $5,256.0$ & $5,257.0$ & $321 \mathrm{HKTL}$ & 2.1 & 0.000000 & Limestone and shale \\
\hline$n m b 39-14-08 d c c$ & $5,258.0$ & $5,259.0$ & $321 \mathrm{HKTL}$ & 4.3 & 0.000000 & Limestone and shale \\
\hline nmb39-14-08dcc & $5,262.0$ & $5,263.0$ & $321 \mathrm{HKTL}$ & 3.2 & 0.000000 & Limestone and shale \\
\hline nmb39-14-08dcc & $5,264.0$ & $5,265.0$ & 321HKTL & 6.0 & 0.000000 & Limestone and shale \\
\hline$n m b 39-14-08 d c c$ & $5,266.0$ & $5,267.0$ & $321 \mathrm{HKTL}$ & 3.5 & 0.000000 & Limestone and shale \\
\hline nmb39-14-08dcc & $5,268.0$ & $5,269.0$ & $321 \mathrm{HKTL}$ & 2.9 & 0.000000 & Limestone and shale \\
\hline nmb39-14-08dcc & $5,270.0$ & $5,271.0$ & $321 \mathrm{HKTL}$ & 3.0 & 0.000000 & Limestone and shale \\
\hline nmb39-14-08dcc & $5,272.0$ & $5,273.0$ & $321 \mathrm{HKTL}$ & 4.2 & 0.000000 & Limestone and shale \\
\hline nmb39-14-08dcc & $5,274.0$ & $5,275.0$ & $321 \mathrm{HKTL}$ & 2.6 & 0.000000 & Limestone and shale \\
\hline nmb39-14-08dcc & $5,276.0$ & $5,277.0$ & $321 \mathrm{HKTL}$ & 5.2 & 0.000000 & Limestone and shale \\
\hline $\mathrm{nmb} 39-14-08 \mathrm{dcc}$ & $5,282.0$ & $5,283.0$ & $321 \mathrm{HKTL}$ & 4.8 & 0.000000 & Finely crystalline limestone \\
\hline nmb39-14-08dcc & $5,284.0$ & $5,285.0$ & $321 \mathrm{HKTL}$ & 7.2 & 0.000000 & Finely crystalline limestone \\
\hline nmb39-14-08dcc & $5,286.0$ & $5,287.0$ & 321HKTL & 3.9 & 0.000000 & Shaly, fine-grained sandstone \\
\hline $\mathrm{nmb} 39-14-08 \mathrm{dcc}$ & $5,287.0$ & $5,288.0$ & $321 \mathrm{HKTL}$ & 3.3 & 0.000000 & Shaly, fine-grained sandstone \\
\hline $\mathrm{nmb} 39-14-08 \mathrm{dcc}$ & $5,288.0$ & $5,289.0$ & $321 \mathrm{HKTL}$ & 6.4 & 0.000000 & Shaly, fine-grained sandstone \\
\hline nmb39-14-08dcc & $5,289.0$ & $5,290.0$ & $321 \mathrm{HKTL}$ & 6.7 & 0.000000 & Shaly, fine-grained sandstone \\
\hline $\mathrm{nmb} 39-14-08 \mathrm{dcc}$ & $5,290.0$ & $5,291.0$ & $321 \mathrm{HKTL}$ & 8.8 & 0.000000 & Shaly, fine-grained sandstone \\
\hline nmb39-14-08dcc & $5,291.0$ & $5,292.0$ & $321 \mathrm{HKTL}$ & 5.1 & 0.000000 & Shaly, fine-grained sandstone \\
\hline nmb39-14-08dcc & $5,292.0$ & $5,293.0$ & $321 \mathrm{HKTL}$ & 8.5 & 0.000000 & Shaly, fine-grained sandstone \\
\hline nmb39-14-08dcc & $5,293.0$ & $5,294.0$ & 321HKTL & 8.8 & 0.000000 & Shaly, fine-grained sandstone \\
\hline nmb39-14-08dcc & $5,294.0$ & $5,295.0$ & $321 \mathrm{HKTL}$ & 6.6 & 0.000000 & Shaly, fine-grained sandstone \\
\hline nmb39-14-08dcc & $5,295.0$ & $5,296.0$ & $321 \mathrm{HKTL}$ & 6.7 & 0.000000 & Finely crystalline limestone \\
\hline nmb39-14-08dcc & $5,296.0$ & $5,297.0$ & $321 \mathrm{HKTL}$ & 7.7 & 0.000000 & Shaly, finely crystalline limestone \\
\hline$n m b 39-14-08 d c c$ & $5,297.0$ & $5,298.0$ & $321 \mathrm{HKTL}$ & 3.3 & 0.000000 & Shaly, finely crystalline limestone \\
\hline nmb39-14-08dcc & $5,299.0$ & $5,300.0$ & $321 \mathrm{HKTL}$ & 3.6 & 0.000000 & Shaly, finely crystalline limestone \\
\hline nmb39-14-08dcc & $5,301.0$ & $5,302.0$ & $321 \mathrm{HKTL}$ & 6.7 & 0.000000 & Shaly, fine-grained, limy sandstone \\
\hline nmb39-14-08dcc & $5,302.0$ & $5,303.0$ & $321 \mathrm{HKTL}$ & 4.5 & 0.000000 & Shaly, fine-grained, limy sandstone \\
\hline $\mathrm{nmb} 39-14-08 \mathrm{dcc}$ & $5,303.0$ & $5,304.0$ & $321 \mathrm{HKTL}$ & 3.8 & 0.000000 & Shaly, fine-grained, limy sandstone \\
\hline
\end{tabular}


Table 4.--Laboratory-determined porosity and permeability--Continued

\begin{tabular}{|c|c|c|c|c|c|c|}
\hline Site & $\begin{array}{l}\text { Depth to } \\
\text { top } \\
\text { (feet) }\end{array}$ & $\begin{array}{l}\text { Depth to } \\
\text { bottom } \\
\text { (feet) }\end{array}$ & $\begin{array}{c}\text { Forma- } \\
\text { tion }\end{array}$ & $\begin{array}{l}\text { Porosity } \\
\text { (percent) }\end{array}$ & $\begin{array}{l}\text { Perme- } \\
\text { ability } \\
\text { (milli- } \\
\text { darcies) }\end{array}$ & Lithology \\
\hline $\begin{array}{l}\mathrm{nmb} 39-14-08 d c c \\
\mathrm{nmb} 39-14-08 d c c \\
\mathrm{nmb} 39-14-08 d c c \\
\mathrm{nmb} 39-14-08 d c c \\
\mathrm{nmb} 39-14-08 d c c\end{array}$ & $\begin{array}{l}5,304 \cdot 0 \\
5,305 \cdot 0 \\
5,306 \cdot 0 \\
5,307.0 \\
5,309.0\end{array}$ & $\begin{array}{l}5,305.0 \\
5,306.0 \\
5,307.0 \\
5,308.0 \\
5,310.0\end{array}$ & $\begin{array}{l}\text { 321HKTL } \\
321 \text { HKTL } \\
321 \text { HKTL } \\
\text { 321HKTL } \\
\text { 321HKTL }\end{array}$ & $\begin{array}{l}1.2 \\
5.6 \\
5.5 \\
5.6 \\
3.9\end{array}$ & $\begin{array}{l}0.000000 \\
0.000000 \\
0.000000 \\
0.000000 \\
0.000000\end{array}$ & $\begin{array}{l}\text { Shaly, fine-grained, limy sandstone } \\
\text { Shaly, fine-grained, limy sandstone } \\
\text { Shaly, fine-grained, limy sandstone } \\
\text { Shaly, fine-grained, limy sandstone } \\
\text { Shaly, fine-grained, limy sandstone }\end{array}$ \\
\hline $\begin{array}{l}\mathrm{nmb} 39-14-08 d c c \\
\mathrm{nmb} 39-14-08 d c c \\
\mathrm{nmb} 39-14-08 d c c \\
\mathrm{nmb} 39-14-08 d c c \\
\mathrm{nmb} 39-14-08 d c c\end{array}$ & $\begin{array}{l}5,310.0 \\
5,312.0 \\
5,313.0 \\
5,314.0 \\
5,315.0\end{array}$ & $\begin{array}{l}5,311.0 \\
5,313.0 \\
5,314.0 \\
5,315.0 \\
5,316.0\end{array}$ & $\begin{array}{l}\text { 321HKTL } \\
\text { 321HKTL } \\
\text { 321HKTL } \\
\text { 321HKTL } \\
\text { 321HKTL }\end{array}$ & $\begin{array}{l}5.0 \\
4.7 \\
8.1 \\
8.9 \\
7.5\end{array}$ & $\begin{array}{l}0.000000 \\
0.000000 \\
0.000000 \\
0.000000 \\
0.000000\end{array}$ & $\begin{array}{l}\text { Shaly sandstone } \\
\text { Shaly sandstone } \\
\text { Shaly sandstone } \\
\text { Shaly sandstone } \\
\text { Shaly sandstone }\end{array}$ \\
\hline $\begin{array}{l}n m b 39-14-08 d c c \\
n m b 39-14-08 d c c \\
n m b 39-14-08 d c c \\
n m b 39-14-08 d c c \\
n m b 39-14-08 d c c\end{array}$ & $\begin{array}{l}5,323.0 \\
5,324.0 \\
5,325.0 \\
5,326.0 \\
5,334.0\end{array}$ & $\begin{array}{l}5,324.0 \\
5,325.0 \\
5,326.0 \\
5,327.0 \\
5,335.0\end{array}$ & $\begin{array}{l}\text { 321HKTL } \\
321 \text { HKTL } \\
321 \text { HKTL } \\
321 \text { HKTL } \\
321 \text { HKTL }\end{array}$ & $\begin{array}{l}7.6 \\
8.0 \\
7.1 \\
7.7 \\
4.2\end{array}$ & $\begin{array}{l}0.000000 \\
0.000000 \\
0.000000 \\
0.000000 \\
0.000000\end{array}$ & $\begin{array}{l}\text { Shaly sandstone } \\
\text { Shaly sandstone } \\
\text { Shaly sandstone } \\
\text { Limestone } \\
\text { Limestone }\end{array}$ \\
\hline $\begin{array}{l}n m b 39-14-08 d c c \\
n m b 39-14-08 d c c \\
n m b 39-14-08 d c c \\
n m b 39-14-08 d c c \\
n m b 39-14-08 d c c\end{array}$ & $\begin{array}{l}5,335 \cdot 0 \\
5,344.0 \\
5,345.0 \\
5,355.0 \\
5,357.2\end{array}$ & $\begin{array}{l}5,336.0 \\
5,345.0 \\
5,346.0 \\
5,357.2 \\
5,359.0\end{array}$ & $\begin{array}{l}\text { 321HKTL } \\
321 \text { HKTL } \\
321 \text { HKTL } \\
321 \text { HKTL } \\
321 \text { HKTL }\end{array}$ & $\begin{array}{l}4.6 \\
4.6 \\
5.5 \\
1.1 \\
1.3\end{array}$ & $\begin{array}{l}0.000000 \\
0.000000 \\
0.000000 \\
0.000000 \\
0.000000\end{array}$ & $\begin{array}{l}\text { Limestone } \\
\text { Shaly, fine-grained limestone } \\
\text { Shaly, fine-grained limestone } \\
\text { Limestone } \\
\text { Limestone }\end{array}$ \\
\hline $\begin{array}{l}\mathrm{nmb} 39-14-08 d c c \\
\mathrm{nmb} 39-14-08 d c c \\
\mathrm{nmb} 39-14-08 d c c \\
\mathrm{nmb} 39-14-08 d c c \\
\mathrm{nmb} 39-14-08 d c c\end{array}$ & $\begin{array}{l}5,359 \cdot 0 \\
5,360 \cdot 5 \\
5,362 \cdot 5 \\
5,370.0 \\
5,372.8\end{array}$ & $\begin{array}{l}5,360.5 \\
5,362.5 \\
5,364.0 \\
5,372.8 \\
5,373.7\end{array}$ & $\begin{array}{l}\text { 321HKTL } \\
321 \text { HKTL } \\
\text { 321HKTL } \\
\text { 321HKTL } \\
\text { 321HKTL }\end{array}$ & $\begin{array}{l}0.6 \\
1.1 \\
1.1 \\
1.5 \\
2.1\end{array}$ & $\begin{array}{l}0.000000 \\
1.600000 \\
1.200000 \\
0.000000 \\
0.200000\end{array}$ & $\begin{array}{l}\text { Limestone } \\
\text { Limestone } \\
\text { Limestone } \\
\text { Limestone } \\
\text { Limestone }\end{array}$ \\
\hline $\begin{array}{l}n m b 39-14-08 d c c \\
n m b 39-14-08 d c c \\
n m b 39-14-08 d c c \\
n m b 39-14-08 d c c \\
n m b 39-14-08 d c c\end{array}$ & $\begin{array}{l}5,373 \cdot 7 \\
5,375 \cdot 4 \\
5,377 \cdot 4 \\
5,379.2 \\
5,380.7\end{array}$ & $\begin{array}{l}5,375 \cdot 4 \\
5,377 \cdot 4 \\
5,379.2 \\
5,380.7 \\
5,382.7\end{array}$ & $\begin{array}{l}\text { 321HKTL } \\
321 \text { HKTL } \\
321 \text { HKTL } \\
321 \text { HKTL } \\
321 \text { HKTL }\end{array}$ & $\begin{array}{l}2.4 \\
2.4 \\
2.3 \\
2.1 \\
0.7\end{array}$ & $\begin{array}{l}0.020000 \\
0.000000 \\
0.010000 \\
0.000000 \\
0.080000\end{array}$ & $\begin{array}{l}\text { Limestone } \\
\text { Limestone } \\
\text { Limestone } \\
\text { Limestone } \\
\text { Limestone }\end{array}$ \\
\hline $\begin{array}{l}\mathrm{nmb} 39-14-08 d c c \\
\mathrm{nmb} 39-14-08 d c c \\
\mathrm{nmb} 39-14-08 d c c \\
\mathrm{nmb} 39-14-08 d c c \\
\mathrm{nmb} 39-14-08 d c c\end{array}$ & $\begin{array}{l}5,382.7 \\
5,384.5 \\
5,386.4 \\
5,388.0 \\
5,389.4\end{array}$ & $\begin{array}{l}5,384.5 \\
5,386.4 \\
5,388.0 \\
5,389.4 \\
5,391.5\end{array}$ & $\begin{array}{l}\text { 321HKTL } \\
321 \text { HKTL } \\
\text { 321HKTL } \\
321 \text { HKTL } \\
\text { 321HKTL }\end{array}$ & $\begin{array}{l}2.1 \\
1.2 \\
1.9 \\
2.9 \\
3.8\end{array}$ & $\begin{array}{l}0.000000 \\
0.100000 \\
0.700000 \\
0.000000 \\
1.400000\end{array}$ & $\begin{array}{l}\text { Limestone } \\
\text { Limestone } \\
\text { Limestone } \\
\text { Limestone } \\
\text { Limestone }\end{array}$ \\
\hline $\begin{array}{l}n m b 39-14-08 d c c \\
n m b 39-14-08 d c c \\
n m b 39-14-08 d c c \\
n m b 39-14-08 d c c \\
n m b 39-14-08 d c c\end{array}$ & $\begin{array}{l}5,391.5 \\
5,393.0 \\
5,394.8 \\
5,396.5 \\
5,419.0\end{array}$ & $\begin{array}{l}5,393.0 \\
5,394.8 \\
5,396.5 \\
5,398.0 \\
5,420.0\end{array}$ & $\begin{array}{l}\text { 321HKTL } \\
321 \text { HKTL } \\
321 \text { HKTL } \\
321 \text { HKTL } \\
321 \text { HKTL }\end{array}$ & $\begin{array}{l}1.9 \\
4.0 \\
3.0 \\
1.3 \\
2.6\end{array}$ & $\begin{array}{l}0.100000 \\
0.200000 \\
0.100000 \\
0.100000 \\
0.000000\end{array}$ & $\begin{array}{l}\text { Limestone } \\
\text { Limestone } \\
\text { Limestone } \\
\text { Limestone } \\
\text { Shaly, finely crystalline limestone }\end{array}$ \\
\hline $\begin{array}{l}n m b 39-14-08 d c c \\
n m b 39-14-08 d c c \\
n m b 39-14-08 d c c \\
n m b 39-14-08 d c c \\
n m b 39-14-08 d c c\end{array}$ & $\begin{array}{l}5,420.0 \\
5,427.0 \\
5,428.0 \\
5,429.0 \\
5,430.0\end{array}$ & $\begin{array}{l}5,421.0 \\
5,428.0 \\
5,429.0 \\
5,430.0 \\
5,431.0\end{array}$ & $\begin{array}{l}\text { 321HKTL } \\
321 \text { HKTL } \\
321 \text { HKTL } \\
321 \text { HKTL } \\
\text { 321HKTL }\end{array}$ & $\begin{array}{l}2.9 \\
4.8 \\
4.8 \\
5.8 \\
3.2\end{array}$ & $\begin{array}{l}0.000000 \\
0.000000 \\
0.000000 \\
0.000000 \\
0.000000\end{array}$ & $\begin{array}{l}\text { Shaly, finely crystalline limestone } \\
\text { Fine-grained sandstone } \\
\text { Fine-grained sandstone } \\
\text { Fine-grained sandstone } \\
\text { Fine-grained sandstone }\end{array}$ \\
\hline $\begin{array}{l}\mathrm{nmb} 39-14-08 d c c \\
\mathrm{nmb} 39-14-08 d c c \\
\mathrm{nmb} 39-14-08 d c c \\
\mathrm{nmb} 39-14-08 d c c \\
\mathrm{nmb} 39-14-08 d c c\end{array}$ & $\begin{array}{l}5,431.0 \\
5,432.0 \\
5,434.0 \\
5,435.0 \\
5,436.0\end{array}$ & $\begin{array}{l}5,432.0 \\
5,433.0 \\
5,435.0 \\
5,436.0 \\
5,437.0\end{array}$ & $\begin{array}{l}\text { 321HKTL } \\
321 \text { HKTL } \\
321 \text { HKTL } \\
321 \text { HKTL } \\
\text { 321HKTL }\end{array}$ & $\begin{array}{l}5.7 \\
9.4 \\
2.7 \\
2.7 \\
3.5\end{array}$ & $\begin{array}{l}0.000000 \\
0.000000 \\
0.000000 \\
0.000000 \\
0.000000\end{array}$ & $\begin{array}{l}\text { Fine-grained sandstone } \\
\text { Fine-grained sandstone } \\
\text { Finely crystalline limestone } \\
\text { Finely crystalline limestone } \\
\text { Finely crystalline limestone }\end{array}$ \\
\hline
\end{tabular}


Table 4.--Laboratory-determined porosity and permeability--Continued

\begin{tabular}{|c|c|c|c|c|c|c|}
\hline Site & $\begin{array}{l}\text { Depth to } \\
\text { top } \\
\text { (feet) }\end{array}$ & $\begin{array}{l}\text { Depth to } \\
\text { bottom } \\
\text { (feet) }\end{array}$ & $\begin{array}{l}\text { Forma- } \\
\text { tion }\end{array}$ & $\begin{array}{l}\text { Porosity } \\
\text { (percent) }\end{array}$ & $\begin{array}{l}\text { Perme- } \\
\text { ability } \\
\text { (milli- } \\
\text { darcies) }\end{array}$ & Lithology \\
\hline nmb39-14-08dcc & $5,437.0$ & $5,438.0$ & 321HKTL & 2.8 & 0.000000 & Finely crystalline limestone \\
\hline$n m b 39-14-08 d c c$ & $5,438.0$ & $5,439.0$ & $321 \mathrm{HKTL}$ & 3.0 & 0.000000 & Finely crystalline limestone \\
\hline nmb39-14-08dcc & $5,439.0$ & $5,440.0$ & 321HKTL & 3.0 & 0.000000 & Finely crystalline limestone \\
\hline $\mathrm{nmb} 39-14-08 \mathrm{dcc}$ & $5,440.0$ & $5,441.0$ & $321 \mathrm{HKTL}$ & 2.8 & 0.000000 & Finely crystalline limestone \\
\hline nmb39-14-08dcc & $5,441.0$ & $5,442.0$ & $321 \mathrm{HKTL}$ & 2.6 & 0.000000 & Finely crystalline 1 imestone \\
\hline$n m b 39-14-08 d c c$ & $5,442.0$ & $5,443.0$ & $321 \mathrm{HKTL}$ & 5.4 & 0.000000 & Finely crystalline limestone \\
\hline nmb39-14-08dcc & $5,443.0$ & $5,445.2$ & $321 \mathrm{HKTL}$ & 1.1 & 0.200000 & Limestone \\
\hline nmb39-14-08dcc & $5,445.2$ & $5,447.0$ & $321 \mathrm{HKTL}$ & 1.3 & 6.700000 & Limestone \\
\hline nmb39-14-08dcc & $5,447.0$ & $5,449.0$ & $321 \mathrm{HKTL}$ & 1.3 & 0.060000 & Limestone \\
\hline$n m b 39-14-08 d c c$ & $5,449.0$ & $5,450.5$ & $321 \mathrm{HKTL}$ & 1.5 & 0.000000 & Limestone \\
\hline $\mathrm{nmb} 39-14-08 \mathrm{dcc}$ & $5,450.5$ & $5,452.1$ & $321 \mathrm{HKTL}$ & 1.0 & 1.600000 & Limestone \\
\hline nmb39-14-08dcc & $5,452.1$ & $5,453.8$ & $321 \mathrm{HKTL}$ & 1.3 & 2.100000 & Limestone \\
\hline nmb39-14-08dcc & $5,453.8$ & $5,455.5$ & $321 \mathrm{HKTL}$ & 1.4 & 0.010000 & Limestone \\
\hline nmb39-14-08dcc & $5,455.5$ & $5,457.2$ & $321 \mathrm{HKTL}$ & 1.1 & 1.300000 & Limestone \\
\hline nmb39-14-08dcc & $5,457.2$ & $5,458.8$ & $321 \mathrm{HKTL}$ & 1.0 & 0.000000 & Limestone \\
\hline$n m b 39-14-08 d c c$ & $5,458.8$ & $5,460.0$ & $321 \mathrm{HKTL}$ & 1.5 & 0.300000 & Limestone \\
\hline nmb39-14-08dcc & $5,460.0$ & $5,462.0$ & $321 \mathrm{HKTL}$ & 0.9 & 0.800000 & Limestone \\
\hline nmb39-14-08dcc & $5,462.0$ & $5,463.7$ & $321 \mathrm{HKTL}$ & 1.0 & 5.900000 & Limestone \\
\hline nmb39-14-08dcc & $5,463.7$ & $5,465.6$ & $321 \mathrm{HKTL}$ & 0.6 & 0.300000 & Limestone \\
\hline nmb39-14-08dcc & $5,465.6$ & $5,467.4$ & $321 \mathrm{HKTL}$ & 0.6 & 1.600000 & Limestone \\
\hline$n m b 39-14-08 d c c$ & $5,467.4$ & $5,469.4$ & $321 \mathrm{HKTL}$ & 1.6 & 0.000000 & Limestone \\
\hline nmb39-14-08dcc & $5,469.4$ & $5,471.1$ & $321 \mathrm{HKTL}$ & 0.6 & 0.000000 & Limestone \\
\hline nmb39-14-08dcc & $5,471.1$ & $5,472.9$ & $321 \mathrm{HKTL}$ & 2.7 & 0.000000 & Limestone \\
\hline nmb39-14-08dcc & $5,472.9$ & $5,474.9$ & $321 \mathrm{HKTL}$ & 1.8 & 0.000000 & Limestone \\
\hline nmb39-14-08dcc & $5,474.9$ & $5,476.8$ & $321 \mathrm{HKTL}$ & 2.1 & 0.000000 & Limestone \\
\hline$n m b 39-14-08 d c c$ & $5,476.8$ & $5,478.6$ & $321 \mathrm{HKTL}$ & 2.3 & 0.010000 & Limestone \\
\hline nmb39-14-08dcc & $5,478.6$ & $5,480.3$ & $321 \mathrm{HKTL}$ & 2.0 & 0.000000 & Limestone \\
\hline $\mathrm{nmb} 39-14-08 \mathrm{dcc}$ & $5,480.3$ & $5,482.2$ & $321 \mathrm{HKTL}$ & 1.8 & 0.170000 & Limestone \\
\hline$n m b 39-14-08 d c c$ & $5,482.2$ & $5,484.2$ & $321 \mathrm{HKTL}$ & 1.6 & 0.010000 & Limestone \\
\hline nmb39-14-08dcc & $5,484.2$ & $5,486.1$ & $321 \mathrm{HKTL}$ & 2.8 & 0.000000 & Limestone \\
\hline nmb39-14-08dcc & $5,486.1$ & $5,488.0$ & $321 \mathrm{HKTL}$ & 2.8 & 0.020000 & Limestone \\
\hline $\mathrm{nmb} 39-14-08 \mathrm{dcc}$ & $5,488.0$ & $5,489.6$ & $321 \mathrm{HKTL}$ & 1.9 & 0.010000 & Limestone \\
\hline nmb39-14-08dcc & $5,489.6$ & $5,491.6$ & $321 \mathrm{HKTL}$ & 1.9 & 0.000000 & Limestone \\
\hline nmb39-14-08dcc & $5,491.6$ & $5,493.6$ & $321 \mathrm{HKTL}$ & 1.9 & 0.000000 & Limestone \\
\hline$n m b 39-14-08 d c c$ & $5,493.6$ & $5,495.3$ & $321 \mathrm{HKTL}$ & 2.2 & 0.010000 & Limestone \\
\hline$n m b 39-14-08 d c c$ & $5,495.3$ & $5,497.0$ & $321 \mathrm{HKTL}$ & 3.0 & 0.000000 & Limestone \\
\hline nmb39-14-08dcc & $5,497.0$ & $5,498.8$ & $321 \mathrm{HKTL}$ & 2.6 & 0.000000 & Limestone \\
\hline nmb39-14-08dcc & $5,498.8$ & $5,501.0$ & $321 \mathrm{HKTL}$ & 3.7 & 0.000000 & Limestone \\
\hline$n m b 39-14-08 d c c$ & $5,501.0$ & $5,503.0$ & $321 \mathrm{HKTL}$ & 3.4 & 0.050000 & Limestone \\
\hline & & & AVERAGE - - & 4.7 & 0.236907 & \\
\hline SITE AVERAGE--- & $\ldots$ & $-0-1$ & $-\cdots-n-1$ & $\overline{4.7}$ & $\overline{0.236907}$ & \\
\hline$n m b 39-16-16 a c a$ & $5,617.0$ & $5,618.0$ & 324PRDX & 1.3 & 0.170000 & Anhydritic Iimestone \\
\hline nmb39-16-16aca & $5,620.0$ & $5,621.0$ & $324 \mathrm{PRDX}$ & 1.2 & 0.040000 & Anhydritic limestone \\
\hline nmb39-16-16aca & $5,621.0$ & $5,622.0$ & 324PRDX & 0.3 & 0.200000 & Anhydritic Iimestone \\
\hline nmb39-16-16aca & $5,622.0$ & $5,623.0$ & $324 \mathrm{PRDX}$ & 0.5 & 0.370000 & Anhydritic limestone \\
\hline nmb39-16-16aca & $5,623.0$ & $5,624.0$ & $324 \mathrm{PRDX}$ & 0.5 & 0.050000 & Anhydritic Iimestone \\
\hline nmb39-16-16aca & $5,624.0$ & $5,625.0$ & 324PRDX & 0.7 & 0.030000 & Anhydritic Iimestone \\
\hline nmb39-16-16aca & $5,625.0$ & $5,626.0$ & $324 \mathrm{PRDX}$ & 1.0 & 0.050000 & Anhydritic limestone \\
\hline$n m b 39-16-16$ aca & $5,626.0$ & $5,627.0$ & $324 \mathrm{PRDX}$ & 0.7 & 0.040000 & Anhydritic Iimestone \\
\hline nmb39-16-16aca & $5,627.0$ & $5,628.0$ & $324 \mathrm{PRDX}$ & 0.6 & 0.020000 & Anhydritic limestone \\
\hline nmb39-16-16aca & $5,628.0$ & $5,629.0$ & 324PRDX & 0.8 & 0.050000 & Anhydritic Iimestone \\
\hline
\end{tabular}


Table 4.--Laboratory-determined porosity and permeability--Continued

\begin{tabular}{|c|c|c|c|c|c|c|}
\hline Site & $\begin{array}{l}\text { Depth to } \\
\text { top } \\
\text { (feet) }\end{array}$ & $\begin{array}{l}\text { Depth to } \\
\text { bottom } \\
\text { (feet) }\end{array}$ & $\begin{array}{c}\text { Forma- } \\
\text { tion }\end{array}$ & $\begin{array}{l}\text { Porosity } \\
\text { (percent) }\end{array}$ & $\begin{array}{l}\text { Perme- } \\
\text { ability } \\
\text { (milli- } \\
\text { darcies) }\end{array}$ & Lithology \\
\hline $\mathrm{nmb} 41-18-33 \mathrm{ab}$ & $4,287.0$ & $4,288.0$ & $321 \mathrm{HKTL}$ & 1.6 & 0.010000 & Silty sandstone \\
\hline $\mathrm{nmb} 41-18-33 \mathrm{ab}$ & $4,288.0$ & $4,289.0$ & 321HKTL & 3.0 & 0.010000 & Silty sandstone \\
\hline $\mathrm{nmb} 41-18-33 \mathrm{ab}$ & $4,293.0$ & $4,294.0$ & $321 \mathrm{HKTL}$ & 1.8 & 0.010000 & Finely crystalline limestone \\
\hline $\mathrm{nmb} 41-18-33 \mathrm{ab}$ & $4,294.0$ & $4,295.0$ & $321 \mathrm{HKTL}$ & 1.1 & 0.010000 & Finely crystalline limestone \\
\hline$n m b 41-18-33 a b$ & $4,295.0$ & $4,296.0$ & $321 \mathrm{HKTL}$ & 1.8 & 0.010000 & Finely crystalline limestone \\
\hline $\mathrm{nmb} 41-18-33 \mathrm{ab}$ & $4,298.0$ & $4,299.0$ & 321HKTL & 1.4 & 0.010000 & Fossiliferous, finely crystalline limestone \\
\hline$n m b 41-18-33 a b$ & $4,299.0$ & $4,300.0$ & $321 \mathrm{HKTL}$ & 1.1 & 0.020000 & Shaly, finely crystalline limestone \\
\hline$n m b 41-18-33 a b$ & $4,302.0$ & $4,303.0$ & 321HKTL & 1.7 & 0.040000 & Finely crystalline limestone \\
\hline$n m b 41-18-33 a b$ & $4,303.0$ & $4,304.0$ & $321 \mathrm{HKTL}$ & 1.2 & 0.010000 & Finely crystalline limestone \\
\hline $\mathrm{nmb} 41-18-33 \mathrm{ab}$ & $4,304.0$ & $4,305.0$ & $321 \mathrm{HKTL}$ & 1.1 & 0.010000 & Finely crystalline limestone \\
\hline $\mathrm{nmb} 41-18-33 \mathrm{ab}$ & $4,305.0$ & $4,306.0$ & $321 \mathrm{HKTL}$ & 1.4 & 0.010000 & Finely crystalline limestone \\
\hline $\mathrm{nmb} 41-18-33 \mathrm{ab}$ & $4,306.0$ & $4,307.0$ & $321 \mathrm{HKTL}$ & 1.1 & 0.010000 & Fossiliferous, finely crystalline limestone \\
\hline$n m b 41-18-33 a b$ & $4,307.0$ & $4,308.0$ & $321 \mathrm{HKTL}$ & 0.7 & 0.010000 & Finely crystalline limestone \\
\hline $\mathrm{nmb} 41-18-33 \mathrm{ab}$ & $4,308.0$ & $4,309.0$ & $321 \mathrm{HKTL}$ & 1.0 & 0.010000 & Finely crystalline limestone \\
\hline$n m b 41-18-33 a b$ & $4,309.0$ & $4,310.0$ & $321 \mathrm{HKTL}$ & 0.7 & 0.010000 & Finely crystalline limestone \\
\hline$n m b 41-18-33 a b$ & $4,310.0$ & $4,311.0$ & $321 \mathrm{HKTL}$ & 0.6 & 0.010000 & Finely crystalline limestone \\
\hline$n m b 41-18-33 a b$ & $4,311.0$ & $4,312.0$ & $321 \mathrm{HKTL}$ & 1.1 & 0.010000 & Finely crystalline limestone \\
\hline$n m b 41-18-33 a b$ & $4,312.0$ & $4,313.0$ & $321 \mathrm{HKTL}$ & 2.3 & 0.010000 & Shaly, finely crystalline limestone \\
\hline & & & AVERAGE-- & 3.0 & 0.029474 & \\
\hline SITE AVERAG & $-\pi-a-\alpha$ & 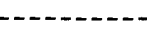 & $-1-n-1-2$ & 3.0 & 0.029474 & \\
\hline $\mathrm{nmb} 44-19-16 \mathrm{bcc}$ & $8,906.0$ & $8,907.8$ & 337LDVL & 1.6 & 0.100000 & Dolomite \\
\hline nmb44-19-16bcc & $8,907.8$ & $8,909.8$ & 337LDVL & 2.5 & 0.000000 & Dolomite \\
\hline nmb44-19-16bcc & $8,909.8$ & $8,911.5$ & $337 \mathrm{LDVL}$ & 4.0 & 0.000000 & Shale \\
\hline nmb44-19-16bcc & $8,911.5$ & $8,913.4$ & 337LDVL & 5.4 & 0.100000 & Dolomite and dolomitic shale \\
\hline nmb44-19-16bcc & $8,913.4$ & $8,915.4$ & 337LDVL & 4.5 & 9.500000 & Fine- to medium-crystalline dolomite \\
\hline nmb44-19-16bcc & $8,915.4$ & $8,917.0$ & $337 \mathrm{LDVL}$ & 1.6 & 0.000000 & Limestone and dolomite \\
\hline nmb44-19-16bcc & $8,917.0$ & $8,918.9$ & 337LDVL & 2.8 & 0.000000 & Fine-grained dolomite and limestone \\
\hline $\mathrm{nmb} 44-19-16 \mathrm{bcc}$ & $8,918.9$ & $8,920.9$ & 337LDVL & 1.5 & 0.000000 & Finely crystalline- to sucrose-limestone \\
\hline nmb44-19-16bcc & $8,920.9$ & $8,922.8$ & 337LDVL & 2.2 & 0.000000 & Finely crystalline- to sucrose-limestone \\
\hline nmb44-19-16bcc & $8,922.8$ & $8,925.0$ & 337LDVL & 2.8 & 0.000000 & Shaly, sucrose limestone \\
\hline nmb44-19-16bcc & $8,925.0$ & $8,926.8$ & 337LDVL & 1.3 & 0.000000 & Shaly, finely crystalline limestone \\
\hline nmb44-19-16bcc & $8,926.8$ & $8,929.0$ & $337 \mathrm{LDVL}$ & 1.0 & 0.000000 & Shaly, finely crystalline limestone \\
\hline nmb44-19-16bcc & $8,929.0$ & $8,931.0$ & 337LDVL & 3.8 & 0.000000 & Dolomitic shale and shaly limestone \\
\hline nmb44-19-16bcc & $8,931.0$ & $8,933.0$ & $337 \mathrm{LDVL}$ & 3.2 & 0.000000 & Shale and finely crystalline limestone \\
\hline nmb44-19-16bcc & $8,933.0$ & $8,934.8$ & 337LDVL & 1.3 & 0.000000 & Shaly, finely crystalline limestone \\
\hline nmb44-19-16bcc & $8,934.8$ & $8,936.3$ & 337LDVL & 0.7 & 20.000000 & Shaly, finely crystalline limestone \\
\hline nmb44-19-16bcc & $8,936.3$ & $8,938.3$ & $337 \mathrm{LDVL}$ & 0.5 & 0.000000 & Shaly, finely crystalline limestone \\
\hline nmb44-19-16bcc & $8,938.3$ & $8,940.0$ & 337LDVL & 1.8 & 0.000000 & Shaly, finely crystalline limestone \\
\hline nmb44-19-16bcc & $8,940.0$ & $8,941.9$ & 337LDVL & 1.0 & 0.000000 & Finely crystalline limestone \\
\hline nmb44-19-16bcc & $8,941.9$ & $8,943.0$ & 337LDVL & 1.7 & 0.000000 & Finely crystalline limestone \\
\hline nmb44-19-16bcc & $8,943.0$ & $8,945.0$ & 337LDVL & 0.9 & 1.100000 & Limestone \\
\hline nmb44-19-16bcc & $8,945.0$ & $8,946.7$ & 337LDVL & 0.9 & 0.100000 & Limes tone \\
\hline nmb44-19-16bcc & $8,946.7$ & $8,948.2$ & 337LDVL & 1.1 & 0.000000 & Limestone \\
\hline nmb44-19-16bcc & $8,948.2$ & $8,950.0$ & 337LDVL & 1.7 & 0.000000 & Limestone and shale \\
\hline nmb44-19-16bcc & $8,950.0$ & $8,951.5$ & 337LDVL & 1.1 & 0.200000 & Finely crystalline limestone \\
\hline nmb44-19-16bcc & $8,951.5$ & $8,953.0$ & 337LDVL & 1.7 & 0.000000 & Finely crystalline limestone \\
\hline $\mathrm{nmb} 44-19-16 \mathrm{bcc}$ & $8,953.0$ & $8,955.0$ & 337LDVL & 1.3 & 0.000000 & Finely crystalline limestone \\
\hline $\mathrm{nmb} 44-19-16 \mathrm{bcc}$ & $8,955.0$ & $8,956.5$ & 337LDVL & 1.5 & 0.000000 & Fossiliferous limestone \\
\hline nmb44-19-16bcc & $8,956.5$ & $8,958.2$ & 337LDVL & 1.2 & 0.000000 & Finely to coarsely crystalline limestone \\
\hline nmb44-19-16bcc & $8,958.2$ & $8,960.0$ & 337LDVL & 1.4 & 0.000000 & Finely to coarsely crystalline limestone \\
\hline
\end{tabular}


Table 4.--Laboratory-determined porosity and permeability--Continued

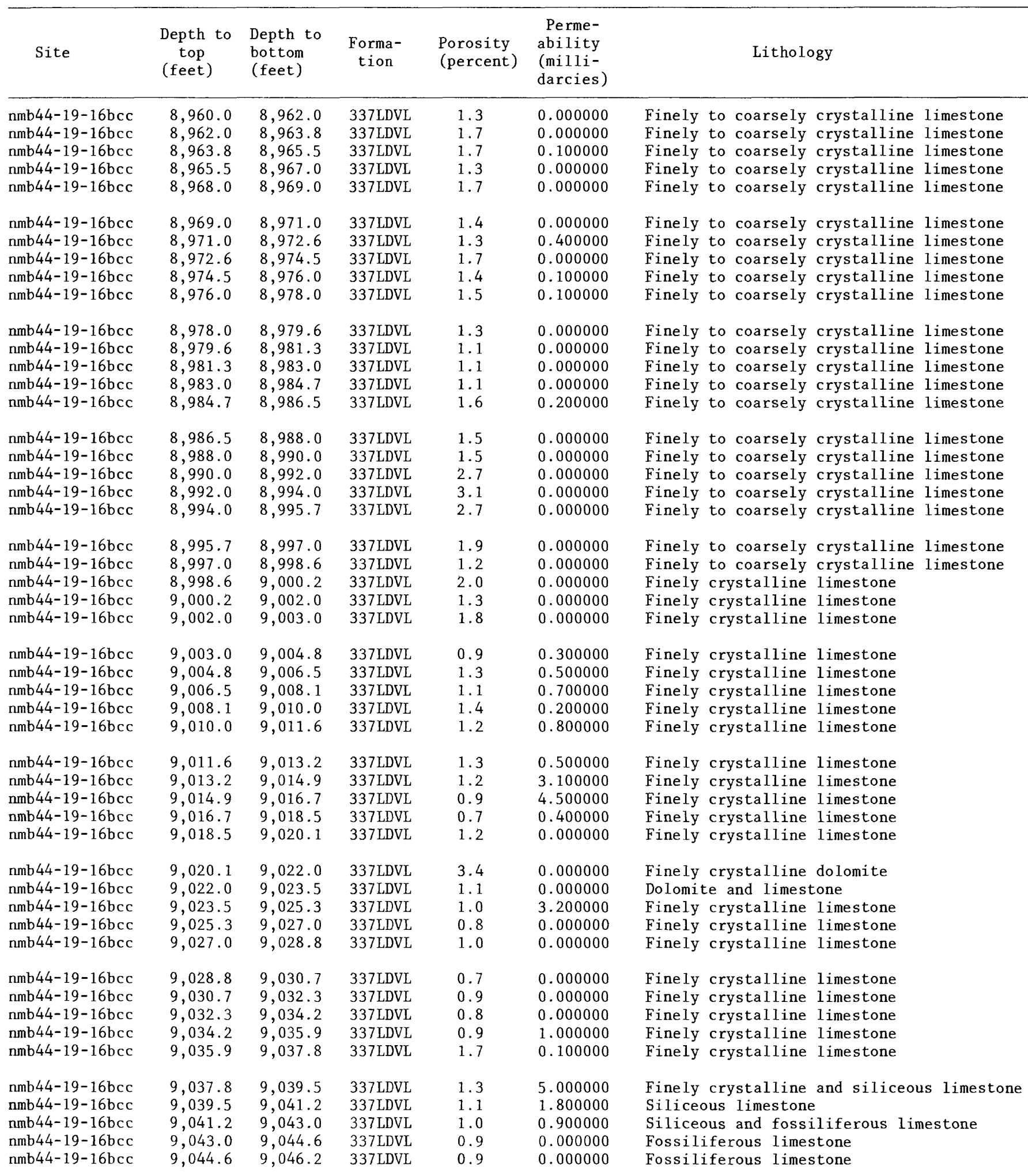


Table 4.--Laboratory-determined porosity and permeability--Continued

\begin{tabular}{|c|c|c|c|c|c|c|}
\hline Site & $\begin{array}{l}\text { Depth to } \\
\text { top } \\
\text { (feet) }\end{array}$ & $\begin{array}{l}\text { Depth to } \\
\text { bottom } \\
\text { (feet) }\end{array}$ & $\begin{array}{c}\text { Forma- } \\
\text { tion }\end{array}$ & $\begin{array}{l}\text { Porosity } \\
\text { (percent) }\end{array}$ & $\begin{array}{c}\text { Perme- } \\
\text { ability } \\
\text { (milli- } \\
\text { darcies) }\end{array}$ & Lithology \\
\hline$n m b 44-19-16 b c c$ & $9,046.2$ & $9,048.0$ & 337LDVL & 1.0 & 0.000000 & Fossiliferous limestone \\
\hline nmb44-19-16bcc & $9,048.0$ & $9,049.5$ & 337LDVL & 1.2 & 0.000000 & Fossiliferous limestone \\
\hline $\mathrm{nmb} 44-19-16 \mathrm{bcc}$ & $9,049.5$ & $9,051.2$ & $337 \mathrm{LDVL}$ & 0.9 & 0.000000 & Fossiliferous limestone \\
\hline $\mathrm{nmb} 44-19-16 \mathrm{bcc}$ & $9,051.2$ & $9,053.0$ & 337LDVL & 1.0 & 0.000000 & Fossiliferous limestone \\
\hline$n m b 44-19-16 b c c$ & $9,053.0$ & $9,056.5$ & 337LDVL & 1.0 & 0.000000 & Fossiliferous limestone \\
\hline$n m b 44-19-16 b c c$ & $9,056.5$ & $9,058.0$ & 337LDVL & 1.4 & 0.000000 & Fossiliferous limestone \\
\hline nmb44-19-16bcc & $9,058.0$ & $9,060.0$ & 337LDVL & 0.9 & 0.000000 & Fossiliferous limestone \\
\hline nmb44-19-16bcc & $9,060.0$ & $9,062.0$ & 337LDVL & 0.8 & 0.000000 & Fossiliferous limestone \\
\hline $\mathrm{nmb} 44-19-16 \mathrm{bcc}$ & $9,062.0$ & $9,064.9$ & 337LDVL & 2.2 & 0.000000 & Limestone and dolomitic limestone \\
\hline$n m b 44-19-16 b c c$ & $9,064.9$ & $9,066.5$ & $337 \mathrm{LDVL}$ & 10.9 & 21.000000 & Dolomite \\
\hline$n m b 44-19-16 b c c$ & $9,066.5$ & $9,068.3$ & 337LDVL & 13.7 & 3.200000 & Dolomite \\
\hline$n m b 44-19-16 b c c$ & $9,068.3$ & $9,070.2$ & 337LDVL & 18.9 & 2.400000 & Dolomite \\
\hline nmb44-19-16bcc & $9,070.2$ & $9,071.5$ & 337LDVL & 18.6 & 11.000000 & Dolomite \\
\hline nmb44-19-16bcc & $9,071.5$ & $9,073.2$ & 337LDVL & 14.8 & 1.500000 & Dolomite \\
\hline nmb44-19-16bcc & $9,073.2$ & $9,074.8$ & 337LDVL & 14.6 & 5.500000 & Dolomite \\
\hline nmb44-19-16bcc & $9,074.8$ & $9,076.6$ & 337LDVL & 9.9 & 78.000000 & Dolomite \\
\hline nmb44-19-16bcc & $9,076.6$ & $9,078.3$ & 337LDVL & 12.9 & 6.800000 & Dolomite \\
\hline nmb44-19-16bcc & $9,078.3$ & $9,080.0$ & $337 \mathrm{LDVL}$ & 2.4 & 0.200000 & Dolomite and limestone \\
\hline nmb44-19-16bcc & $9,080.0$ & $9,081.5$ & 337LDVL & 0.4 & 0.000000 & Fossiliferous, finely crystalline limestone \\
\hline$n m b 44-19-16 b c c$ & $9,081.5$ & $9,083.5$ & $337 \mathrm{LDVL}$ & 0.7 & 0.000000 & Fossiliferous, finely crystalline limestone \\
\hline$n m b 44-19-16 b c c$ & $9,083.5$ & $9,085.0$ & 337LDVL & 0.4 & 0.300000 & Fossiliferous, finely crystalline limestone \\
\hline nmb44-19-16bcc & $9,085.0$ & $9,086.8$ & 337LDVL & 0.5 & 0.000000 & Fossiliferous, finely crystalline limestone \\
\hline nmb44-19-16bcc & $9,086.8$ & $9,087.9$ & $337 \mathrm{LDVL}$ & 1.5 & 0.300000 & Fossiliferous, finely crystalline limestone \\
\hline nmb44-19-16bcc & $9,087.9$ & $9,089.2$ & 337LDVL & 2.1 & 0.000000 & Finely crystalline limestone \\
\hline$n m b 44-19-16 b c c$ & $9,089.2$ & $9,091.0$ & $337 \mathrm{LDVL}$ & 1.9 & 0.000000 & Finely crystalline limestone \\
\hline $\mathrm{nmb} 44-19-16 \mathrm{bcc}$ & $9,091.0$ & $9,093.0$ & 337LDVL & 4.1 & 0.000000 & Dolomite \\
\hline$n m b 44-19-16 b c c$ & $9,093.0$ & $9,095.0$ & 337LDVL & 2.8 & 2.800000 & Dolomite \\
\hline nmb44-19-16bcc & $9,095.0$ & $9,096.5$ & $337 \mathrm{LDVL}$ & 5.0 & 673.000000 & Dolomite \\
\hline$n m b 44-19-16 b c c$ & $9,096.5$ & $9,098.0$ & 337LDVL & 7.8 & 0.100000 & Dolomite \\
\hline$n m b 44-19-16 b c c$ & $9,098.0$ & $9,100.0$ & $337 \mathrm{LDVL}$ & 7.1 & 0.220000 & Dolomite \\
\hline nmb44-19-16bcc & $9,100.0$ & $9,102.5$ & 337LDVL & 7.9 & 5.100000 & Dolomite \\
\hline $\mathrm{nmb} 44-19-16 \mathrm{bcc}$ & $9,102.5$ & $9,104.5$ & 337LDVL & 6.3 & 0.100000 & Dolomite \\
\hline nmb44-19-16bcc & $9,104.5$ & $9,106.1$ & 337 LDVL & 6.0 & 0.000000 & Dolomite \\
\hline nmb44-19-16bcc & $9,106.1$ & $9,108.0$ & $337 \mathrm{LDVL}$ & 9.9 & 0.290000 & Dolomite \\
\hline$n m b 44-19-16 b c c$ & $9,108.0$ & $9,110.0$ & $337 \mathrm{LDVL}$ & 10.4 & 1.500000 & Dolomite \\
\hline $\mathrm{nmb} 44-19-16 \mathrm{bcc}$ & $9,110.0$ & $9,111.9$ & 337LDVL & 7.1 & 0.100000 & Dolomite \\
\hline nmb44-19-16bcc & $9,111.9$ & $9,113.6$ & 337LDVL & 11.6 & 22.000000 & Finely crystalline dolomite \\
\hline nmb44-19-16bcc & $9,113.6$ & $9,115.5$ & 337LDVL & 9.2 & 0.500000 & Finely crystalline dolomite \\
\hline nmb44-19-16bcc & $9,115.5$ & $9,117.2$ & $337 \mathrm{LDVL}$ & 2.9 & 53.000000 & Finely crystalline dolomite \\
\hline nmb44-19-16bcc & $9,117.2$ & $9,119.0$ & $337 \mathrm{LDVL}$ & 3.9 & 9.500000 & Finely crystalline dolomite \\
\hline nmb44-19-16bcc & $9,119.0$ & $9,121.0$ & 337LDVL & 2.8 & 0.600000 & Finely crystalline dolomite \\
\hline nmb44-19-16bcc & $9,121.0$ & $9,123.0$ & 337LDVL & 3.4 & 0.000000 & Finely crystalline dolomite \\
\hline nmb44-19-16bcc & $9,123.0$ & $9,124.8$ & 337LDVL & 5.1 & 0.660000 & Finely crystalline dolomite \\
\hline nmb44-19-16bcc & $9,124.8$ & $9,126.4$ & 337LDVL & 4.8 & 0.000000 & Finely crystalline, dolomitic limestone \\
\hline $\mathrm{nmb} 44-19-16 \mathrm{bcc}$ & $9,126.4$ & $9,128.3$ & $337 \mathrm{LDVL}$ & 2.5 & 0.000000 & Dolomitic limestone and dolomite \\
\hline nmb44-19-16bcc & $9,128.3$ & $9,130.1$ & 337LDVL & 5.1 & 0.000000 & Finely crystalline dolomite \\
\hline $\mathrm{nmb} 44-19-16 \mathrm{bcc}$ & $9,130.1$ & $9,132.8$ & 337LDVL & 5.5 & 1.300000 & Finely crystalline dolomite \\
\hline $\mathrm{nmb} 44-19-16 \mathrm{bcc}$ & $9,132.8$ & $9,134.9$ & 337LDVL & 4.9 & 1.600000 & Finely crystalline dolomite \\
\hline nmb44-19-16bcc & $9,134.9$ & $9,136.8$ & 337LDVL & 5.6 & 39.000000 & Finely crystalline dolomite \\
\hline$n m b 44-19-16 b c c$ & $9,136.8$ & $9,138.2$ & 337LDVL & 1.8 & 0.800000 & Finely crystalline limestone and dolomite \\
\hline
\end{tabular}


Table 4.--Laboratory-determined porosity and permeability--Continued

\begin{tabular}{|c|c|c|c|c|c|c|}
\hline Site & $\begin{array}{l}\text { Depth to } \\
\text { top } \\
\text { (feet) }\end{array}$ & $\begin{array}{l}\text { Depth to } \\
\text { bottom } \\
\text { (feet) }\end{array}$ & $\begin{array}{c}\text { Forma- } \\
\text { tion }\end{array}$ & $\begin{array}{l}\text { Porosity } \\
\text { (percent) }\end{array}$ & $\begin{array}{l}\text { Perme- } \\
\text { ability } \\
\text { (milli- } \\
\text { darcies) }\end{array}$ & Lithology \\
\hline \multirow[t]{2}{*}{$\mathrm{nmb} 44-19-16 \mathrm{bcc}$} & \multirow[t]{2}{*}{$9,138.2$} & \multirow[t]{2}{*}{$9,140.0$} & \multirow{2}{*}{$\begin{array}{l}\text { 337LDVL } \\
\text { AVERAGE-- }\end{array}$} & 1.6 & 0.300000 & \multirow[t]{2}{*}{ Shaly, finely crystalline dolomite } \\
\hline & & & & 3.1 & 7.615802 & \\
\hline $\mathrm{nmb} 44-19-16 \mathrm{bcc}$ & $9,203.0$ & $9,204.0$ & 3410URY & 0.5 & 0.070000 & Shaly, finely crystalline limestone \\
\hline$n m b 44-19-16 b c c$ & $9,205.0$ & $9,206.0$ & 3410URY & 1.0 & 0.010000 & Shaly, finely crystalline limestone \\
\hline nmb44-19-16bcc & $9,207.0$ & $9,208.0$ & $3410 U R Y$ & 1.5 & 0.010000 & Shaly, finely crystalline limestone \\
\hline $\mathrm{nmb} 44-19-16 \mathrm{bcc}$ & $9,209.0$ & $9,210.0$ & 3410URY & 0.7 & 0.000000 & Shaly, finely crystalline limestone \\
\hline $\mathrm{nmb} 44-19-16 \mathrm{bcc}$ & $9,211.0$ & $9,212.0$ & $3410 U R Y$ & 0.6 & 0.000000 & Shaly, finely crystalline limestone \\
\hline$n m b 44-19-16 b c c$ & $9,213.0$ & $9,214.0$ & 34 10URY & 0.5 & 0.000000 & Shaly, finely crystalline limestone \\
\hline$n m b 44-19-16 b c c$ & $9,215.0$ & $9,216.0$ & $3410 U R Y$ & 1.2 & 0.000000 & Shaly, finely crystalline limestone \\
\hline $\mathrm{nmb} 44-19-16 \mathrm{bcc}$ & $9,217.0$ & $9,218.0$ & 3410URY & 1.0 & 0.000000 & Shaly, finely crystalline limestone \\
\hline $\mathrm{nmb} 44-19-16 \mathrm{bcc}$ & $9,219.0$ & $9,220.0$ & 3410URY & 0.6 & 0.000000 & Shaly, finely crystalline limestone \\
\hline$n m b 44-19-16 b c c$ & $9,221.0$ & $9,222.0$ & 34 10URY & 3.3 & 0.000000 & Shaly, finely crystalline limestone \\
\hline $\mathrm{nmb} 44-19-16 \mathrm{bcc}$ & $9,223.0$ & $9,224.0$ & 3410URY & 0.3 & 0.020000 & Shaly, finely crystalline limestone \\
\hline $\mathrm{nmb} 44-19-16 \mathrm{bcc}$ & $9,225.0$ & $9,226.0$ & $3410 U R Y$ & 0.7 & 0.000000 & Shaly, finely crystalline limestone \\
\hline nmb44-19-16bcc & $9,227.0$ & $9,228.0$ & $3410 U R Y$ & 0.3 & 0.000000 & Shaly limestone \\
\hline nmb44-19-16bcc & $9,229.0$ & $9,230.0$ & 3410URY & 0.5 & 0.000000 & Shaly limestone \\
\hline$n m b 44-19-16 b c c$ & $9,231.0$ & $9,232.0$ & 34 10URY & 0.3 & 0.000000 & Shaly Iimestone \\
\hline$n m b 44-19-16 b c c$ & $9,233.0$ & $9,234.0$ & $3410 U R Y$ & 0.3 & 0.000000 & Shaly limestone \\
\hline $\mathrm{nmb} 44-19-16 \mathrm{bcc}$ & $9,235.0$ & $9,236.0$ & 3410URY & 0.5 & 0.000000 & Shaly limestone \\
\hline $\mathrm{nmb} 44-19-16 \mathrm{bcc}$ & $9,237.0$ & $9,238.0$ & 3410URY & 0.7 & 0.000000 & Shaly limestone \\
\hline $\mathrm{nmb} 44-19-16 \mathrm{bcc}$ & $9,239.0$ & $9,240.0$ & 3410URY & 0.7 & 0.000000 & Shaly limestone \\
\hline $\mathrm{nmb} 44-19-16 \mathrm{bcc}$ & $9,241.0$ & $9,242.0$ & 3410 URY & 0.7 & 0.000000 & Shaly Iimestone \\
\hline$n m b 44-19-16 b c c$ & $9,243.0$ & $9,244.0$ & 3410URY & 0.8 & 0.000000 & Shaly limestone \\
\hline $\mathrm{nmb} 44-19-16 \mathrm{bcc}$ & $9,245.0$ & $9,246.0$ & 3410URY & 0.5 & 0.000000 & Shaly limestone \\
\hline nmb44-19-16bcc & $9,247.0$ & $9,248.0$ & 3410URY & 0.5 & 0.010000 & Shaly limestone \\
\hline $\mathrm{nmb} 44-19-16 \mathrm{bcc}$ & $9,251.0$ & $9,252.0$ & 3410URY & 0.5 & 0.000000 & Shaly limestone \\
\hline nmb44-19-16bcc & $9,253.0$ & $9,254.0$ & 3410URY & 0.7 & 0.000000 & Shaly limestone \\
\hline nmb44-19-16bcc & $9,255.0$ & $9,256.0$ & 3410URY & & 00 & Shaly, medium-crystalline limestone \\
\hline $\mathrm{nmb} 44-19-16 \mathrm{bcc}$ & $9,257.0$ & $9,258.0$ & 34 10URY & 0.5 & 0.000000 & Shaly, medium-crystalline limestone \\
\hline$n m b 44-19-16 b c c$ & $9,259.0$ & $9,260.0$ & 34 1OURY & 0.5 & 0.000000 & Shaly, medium-crystalline limestone \\
\hline $\mathrm{nmb} 44-19-16 \mathrm{bcc}$ & $9,261.0$ & $9,262.0$ & 3410 URY & 1.0 & 0.000000 & Shaly, medium-crystalline limestone \\
\hline \multirow[t]{2}{*}{$\mathrm{nmb} 44-19-16 \mathrm{bcc}$} & \multirow[t]{2}{*}{$9,263.0$} & \multirow[t]{2}{*}{$9,264.0$} & $3410 U R Y$ & 1.8 & 0.000000 & \multirow[t]{2}{*}{ Finely crystalline limestone } \\
\hline & & & AVERAGE-- & 0.8 & 0.004000 & \\
\hline $\mathrm{nmb} 44-19-16 \mathrm{bcc}$ & $9,268.0$ & 9 , & $341 \mathrm{ELBR}$ & 1 . & 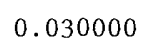 & Dol \\
\hline$m b 44-19-16 b c c$ & $9,273.0$ & $9,274.0$ & $341 \mathrm{ELBR}$ & 2.0 & 0.000000 & Finely crystalline dolomite \\
\hline nmb44-19-16bcc & $9,278.0$ & $9,279.0$ & $341 E L B R$ & 1.5 & 0.000000 & Finely crystalline dolomite \\
\hline$n m b 44-19-16 b c c$ & $9,283.0$ & $9,284.0$ & $341 \mathrm{ELBR}$ & 5.9 & 0.030000 & Finely crystalline dolomite \\
\hline $\mathrm{nmb} 44-19-16 \mathrm{bcc}$ & $9,288.0$ & $9,289.0$ & 341ELBR & 1.1 & 0.020000 & Finely crystalline dolomite \\
\hline $\mathrm{nmb} 44-19-16 \mathrm{bcc}$ & $9,293.0$ & $9,294.0$ & 341ELBR & 0.9 & 00 & Shaly, finely crystalline dolomite \\
\hline$n m b 44-19-16 b c c$ & $9,298.0$ & $9,299.0$ & 341ELBR & 1.1 & 0.020000 & Shaly, finely crystalline dolomite \\
\hline $\mathrm{nmb} 44-19-16 \mathrm{bcc}$ & $9,303.0$ & $9,304.0$ & $341 \mathrm{ELBR}$ & 0.5 & 0.000000 & Finely crystalline dolomite \\
\hline$n m b 44-19-16 b c c$ & $9,308.0$ & $9,309.0$ & $341 E L B R$ & 1.0 & 0.010000 & Finely crystalline dolomite \\
\hline$n m b 44-19-16 b c c$ & $9,311.0$ & $9,312.0$ & $341 E L B R$ & 1.2 & 0.000000 & Finely crystalline dolomite \\
\hline $\mathrm{nmb} 44-19-16 \mathrm{bcc}$ & $9,312.0$ & $9,313.0$ & 341ELBR & 0.3 & 0.000000 & Shale \\
\hline nmb44-19-16bcc & $9,324.0$ & $9,325.0$ & $341 E L B R$ & 0.5 & 0.000000 & Finely crystalline dolomite \\
\hline nmb44-19-16bcc & $9,344.0$ & $9,345.0$ & $341 \mathrm{ELBR}$ & 1.2 & 0.000000 & Shaly, finely crystalline dolomite \\
\hline$n m b 44-19-16 b c c$ & $9,345.0$ & $9,346.0$ & 341ELBR & 1.2 & 0.130000 & Shaly, finely crystalline dolomite \\
\hline nmb44-19-16bcc & $9,346.0$ & $9,347.0$ & 341ELBR & 0.8 & 0.020000 & Shaly, finely crystalline dolomite \\
\hline
\end{tabular}


Table 4.--Laboratory-determined porosity and permeability--Continued

\begin{tabular}{|c|c|c|c|c|c|c|}
\hline Site & $\begin{array}{l}\text { Depth to } \\
\text { top } \\
\text { (feet) }\end{array}$ & $\begin{array}{l}\text { Depth to } \\
\text { bottom } \\
\text { (feet) }\end{array}$ & $\begin{array}{c}\text { Forma- } \\
\text { tion }\end{array}$ & $\begin{array}{l}\text { Porosity } \\
\text { (percent) }\end{array}$ & $\begin{array}{l}\text { Perme- } \\
\text { ability } \\
\text { (milli- } \\
\text { darcies) }\end{array}$ & Lithology \\
\hline $\begin{array}{l}\mathrm{nmb} 44-19-16 \mathrm{bcc} \\
\mathrm{nmb} 44-19-16 \mathrm{bcc} \\
\mathrm{nmb} 44-19-16 \mathrm{bcc} \\
\mathrm{nmb} 44-19-16 \mathrm{bcc} \\
\mathrm{nmb} 44-19-16 \mathrm{bcc}\end{array}$ & $\begin{array}{l}9,347.0 \\
9,348.0 \\
9,349.0 \\
9,350.0 \\
9,351.0\end{array}$ & $\begin{array}{l}9,348.0 \\
9,349.0 \\
9,350.0 \\
9,351.0 \\
9,352.0\end{array}$ & $\begin{array}{l}341 E L B R \\
341 E L B R \\
341 E L B R \\
341 E L B R \\
341 E L B R\end{array}$ & $\begin{array}{l}1.9 \\
4.3 \\
7.4 \\
6.6 \\
5.5\end{array}$ & $\begin{array}{l}0.080000 \\
4.400000 \\
7.000000 \\
0.210000 \\
0.400000\end{array}$ & $\begin{array}{l}\text { Shaly, finely crystalline dolomite } \\
\text { Crystalline dolomite } \\
\text { Crystalline dolomite } \\
\text { Crystalline dolomite } \\
\text { Crystalline dolomite }\end{array}$ \\
\hline $\begin{array}{l}\mathrm{nmb} 44-19-16 \mathrm{bcc} \\
\mathrm{nmb} 44-19-16 \mathrm{bcc} \\
\mathrm{nmb} 44-19-16 \mathrm{bcc} \\
\mathrm{nmb} 44-19-16 \mathrm{bcc} \\
\mathrm{nmb} 44-19-16 \mathrm{bcc}\end{array}$ & $\begin{array}{l}9,352.0 \\
9,353.0 \\
9,354.0 \\
9,356.0 \\
9,358.0\end{array}$ & $\begin{array}{l}9,353.0 \\
9,354.0 \\
9,355.0 \\
9,357.0 \\
9,359.0\end{array}$ & $\begin{array}{l}341 \mathrm{ELBR} \\
341 \mathrm{ELBR} \\
341 \mathrm{ELBR} \\
341 \mathrm{ELBR} \\
341 \mathrm{ELBR}\end{array}$ & $\begin{array}{l}2.2 \\
3.2 \\
4.1 \\
1.2 \\
3.0\end{array}$ & $\begin{array}{l}0.020000 \\
1.000000 \\
0.230000 \\
0.010000 \\
0.210000\end{array}$ & $\begin{array}{l}\text { Crystalline dolomite } \\
\text { Crystalline dolomite } \\
\text { Crystalline dolomite } \\
\text { Shaly, finely crystalline dolomite } \\
\text { Crystalline dolomite }\end{array}$ \\
\hline $\begin{array}{l}\mathrm{nmb} 44-19-16 \mathrm{bcc} \\
\mathrm{nmb} 44-19-16 \mathrm{bcc} \\
\mathrm{nmb} 44-19-16 \mathrm{bcc} \\
\mathrm{nmb} 44-19-16 \mathrm{bcc} \\
\mathrm{nmb} 44-19-16 \mathrm{bcc}\end{array}$ & $\begin{array}{l}9,360.0 \\
9,362.0 \\
9,364.0 \\
9,366.0 \\
9,368.0\end{array}$ & $\begin{array}{l}9,361.0 \\
9,363.0 \\
9,365.0 \\
9,367.0 \\
9,369.0\end{array}$ & $\begin{array}{l}341 E L B R \\
341 E L B R \\
341 E L B R \\
341 E L B R \\
341 E L B R\end{array}$ & $\begin{array}{l}6.0 \\
2.8 \\
0.5 \\
2.3 \\
4.9\end{array}$ & $\begin{array}{l}0.030000 \\
0.380000 \\
0.000000 \\
0.030000 \\
0.290000\end{array}$ & $\begin{array}{l}\text { Crystalline dolomite } \\
\text { Crystalline dolomite } \\
\text { Crystalline dolomite } \\
\text { Crystalline dolomite } \\
\text { Crystalline dolomite }\end{array}$ \\
\hline $\begin{array}{l}\mathrm{nmb} 44-19-16 \mathrm{bcc} \\
\mathrm{nmb} 44-19-16 \mathrm{bcc} \\
\mathrm{nmb} 44-19-16 \mathrm{bcc} \\
\mathrm{nmb} 44-19-16 \mathrm{bcc} \\
\mathrm{nmb} 44-19-16 \mathrm{bcc}\end{array}$ & $\begin{array}{l}9,370.0 \\
9,372.0 \\
9,374.0 \\
9,376.0 \\
9,378.0\end{array}$ & $\begin{array}{l}9,371.0 \\
9,373.0 \\
9,375.0 \\
9,377.0 \\
9,379.0\end{array}$ & $\begin{array}{l}341 E L B R \\
341 E L B R \\
341 E L B R \\
341 E L B R \\
341 E L B R\end{array}$ & $\begin{array}{l}1.0 \\
2.3 \\
1.2 \\
0.6 \\
1.4\end{array}$ & $\begin{array}{l}0.070000 \\
0.030000 \\
0.000000 \\
0.010000 \\
0.010000\end{array}$ & $\begin{array}{l}\text { Crystalline dolomite } \\
\text { Crystalline dolomite } \\
\text { Crystalline dolomite } \\
\text { Crystalline dolomite } \\
\text { Crystalline dolomite }\end{array}$ \\
\hline $\begin{array}{l}\mathrm{nmb} 44-19-16 \mathrm{bcc} \\
\mathrm{nmb} 44-19-16 \mathrm{bcc} \\
\mathrm{nmb} 44-19-16 \mathrm{bcc} \\
\mathrm{nmb} 44-19-16 \mathrm{bcc} \\
\mathrm{nmb} 44-19-16 \mathrm{bcc}\end{array}$ & $\begin{array}{l}9,380.0 \\
9,382.0 \\
9,384.0 \\
9,386.0 \\
9,388.0\end{array}$ & $\begin{array}{l}9,381.0 \\
9,383.0 \\
9,385.0 \\
9,387.0 \\
9,389.0\end{array}$ & $\begin{array}{l}341 E L B R \\
341 E L B R \\
341 E L B R \\
341 E L B R \\
341 E L B R\end{array}$ & $\begin{array}{l}0.5 \\
0.8 \\
0.8 \\
2.4 \\
0.6\end{array}$ & $\begin{array}{l}0.000000 \\
0.000000 \\
0.070000 \\
0.010000 \\
0.010000\end{array}$ & $\begin{array}{l}\text { Crystalline dolomite } \\
\text { Crystalline dolomite } \\
\text { Crystalline dolomite } \\
\text { Crystalline dolomite } \\
\text { Crystalline dolomite }\end{array}$ \\
\hline $\begin{array}{l}\mathrm{nmb} 44-19-16 \mathrm{bcc} \\
\mathrm{nmb} 44-19-16 \mathrm{bcc} \\
\mathrm{nmb} 44-19-16 \mathrm{bcc} \\
\mathrm{nmb} 44-19-16 \mathrm{bcc} \\
\mathrm{nmb} 44-19-16 \mathrm{bcc}\end{array}$ & $\begin{array}{l}9,390.0 \\
9,392.0 \\
9,394.0 \\
9,396.0 \\
9,398.0\end{array}$ & $\begin{array}{l}9,391.0 \\
9,393.0 \\
9,395.0 \\
9,397.0 \\
9,399.0\end{array}$ & $\begin{array}{l}341 \mathrm{ELBR} \\
341 \mathrm{ELBR} \\
341 \mathrm{ELBR} \\
341 \mathrm{ELBR} \\
341 \mathrm{ELBR}\end{array}$ & $\begin{array}{l}0.8 \\
0.8 \\
3.4 \\
1.1 \\
1.3\end{array}$ & $\begin{array}{l}0.000000 \\
0.010000 \\
1.100000 \\
0.000000 \\
0.020000\end{array}$ & $\begin{array}{l}\text { Crystalline dolomite } \\
\text { Crystalline dolomite } \\
\text { Crystalline dolomite } \\
\text { Fine- to medium-grained dolomitic sandstone } \\
\text { Fine- to medium-grained dolomitic sandstone }\end{array}$ \\
\hline $\begin{array}{l}\mathrm{nmb} 44-19-16 \mathrm{bcc} \\
\mathrm{nmb} 44-19-16 \mathrm{bcc} \\
\mathrm{nmb} 44-19-16 \mathrm{bcc} \\
\mathrm{nmb} 44-19-16 \mathrm{bcc} \\
\mathrm{nmb} 44-19-16 \mathrm{bcc}\end{array}$ & $\begin{array}{l}9,400.0 \\
9,402.0 \\
9,404.0 \\
9,406.0 \\
9,408.0\end{array}$ & $\begin{array}{l}9,401.0 \\
9,403.0 \\
9,405.0 \\
9,407.0 \\
9,409.0\end{array}$ & $\begin{array}{l}341 E L B R \\
341 E L B R \\
341 E L B R \\
341 E L B R \\
341 E L B R\end{array}$ & $\begin{array}{l}2.1 \\
6.0 \\
1.2 \\
4.0 \\
3.6\end{array}$ & $\begin{array}{l}0.020000 \\
0.320000 \\
0.020000 \\
0.010000 \\
0.020000\end{array}$ & $\begin{array}{l}\text { Fine- to medium-grained dolomitic sandstone } \\
\text { Fine- to medium-grained dolomitic sandstone } \\
\text { Fine- to medium-grained quartz sandstone } \\
\text { Finely crystalline dolomite } \\
\text { Shaly, medium-crystalline dolomite }\end{array}$ \\
\hline $\begin{array}{l}\mathrm{nmb} 44-19-16 \mathrm{bcc} \\
\mathrm{nmb} 44-19-16 \mathrm{bcc} \\
\mathrm{nmb} 44-19-16 \mathrm{bcc} \\
\mathrm{nmb} 44-19-16 \mathrm{bcc} \\
\mathrm{nmb} 44-19-16 \mathrm{bcc}\end{array}$ & $\begin{array}{l}9,410.0 \\
9,412.0 \\
9,414.0 \\
9,416.0 \\
9,418.0\end{array}$ & $\begin{array}{l}9,411.0 \\
9,413.0 \\
9,415.0 \\
9,417.0 \\
9,419.0\end{array}$ & $\begin{array}{l}341 \mathrm{ELBR} \\
341 \mathrm{ELBR} \\
341 \mathrm{ELBR} \\
341 \mathrm{ELBR} \\
341 \mathrm{ELBR}\end{array}$ & $\begin{array}{l}1.7 \\
2.0 \\
1.4 \\
3.9 \\
1.0\end{array}$ & $\begin{array}{l}0.000000 \\
0.000000 \\
0.000000 \\
0.070000 \\
0.100000\end{array}$ & $\begin{array}{l}\text { Shaly, medium-crystalline dolomite } \\
\text { Fine-grained, glauconitic and dolomitic sandstone } \\
\text { Shale } \\
\text { Shaly, fine- to medium-grained sandstone } \\
\text { Shaly quartzite }\end{array}$ \\
\hline $\begin{array}{l}\mathrm{nmb} 44-19-16 \mathrm{bcc} \\
\mathrm{nmb} 44-19-16 \mathrm{bcc} \\
\mathrm{nmb} 44-19-16 \mathrm{bcc} \\
\mathrm{nmb} 44-19-16 \mathrm{bcc} \\
\mathrm{nmb} 44-19-16 \mathrm{bcc}\end{array}$ & $\begin{array}{l}9,420.0 \\
9,422.0 \\
9,424.0 \\
9,426.0 \\
9,428.0\end{array}$ & $\begin{array}{l}9,421.0 \\
9,423.0 \\
9,425.0 \\
9,427.0 \\
9,429.0\end{array}$ & $\begin{array}{l}341 \mathrm{ELBR} \\
341 \mathrm{ELBR} \\
341 \mathrm{ELBR} \\
341 \mathrm{ELBR} \\
341 \mathrm{ELBR}\end{array}$ & $\begin{array}{l}1.1 \\
1.9 \\
2.2 \\
2.2 \\
1.2\end{array}$ & $\begin{array}{l}0.110000 \\
0.000000 \\
0.000000 \\
0.000000 \\
0.000000\end{array}$ & $\begin{array}{l}\text { Shaly quartzite } \\
\text { Shaly quartzite } \\
\text { Medium-crystalline dolomite } \\
\text { Medium-crystalline dolomite } \\
\text { Quartzite }\end{array}$ \\
\hline $\begin{array}{l}\mathrm{nmb} 44-19-16 \mathrm{bcc} \\
\mathrm{nmb} 44-19-16 \mathrm{bcc} \\
\mathrm{nmb} 44-19-16 \mathrm{bcc} \\
\mathrm{nmb} 44-19-16 \mathrm{bcc} \\
\mathrm{nmb} 44-19-16 \mathrm{bcc}\end{array}$ & $\begin{array}{l}9,430.0 \\
9,432.0 \\
9,434.0 \\
9,436.0 \\
9,438.0\end{array}$ & $\begin{array}{l}9,431.0 \\
9,433.0 \\
9,435.0 \\
9,437.0 \\
9,439.0\end{array}$ & $\begin{array}{l}341 \mathrm{ELBR} \\
341 \mathrm{ELBR} \\
341 \mathrm{ELBR} \\
341 \mathrm{ELBR} \\
341 \mathrm{ELBR}\end{array}$ & $\begin{array}{l}2.5 \\
3.1 \\
1.6 \\
1.2 \\
2.4\end{array}$ & $\begin{array}{l}0.010000 \\
0.000000 \\
0.000000 \\
0.000000 \\
0.000000\end{array}$ & $\begin{array}{l}\text { Sandy shale } \\
\text { Shaly, finely crystalline dolomite } \\
\text { Shaly, finely crystalline dolomite } \\
\text { Shaly, finely crystalline dolomite } \\
\text { Shaly, finely crystalline dolomite }\end{array}$ \\
\hline
\end{tabular}


Table 4.--Laboratory-determined porosity and permeability--Continued

\begin{tabular}{|c|c|c|c|c|c|c|}
\hline Site & $\begin{array}{l}\text { Depth to } \\
\text { top } \\
\text { (feet) }\end{array}$ & $\begin{array}{l}\text { Depth to } \\
\text { bottom } \\
\text { (feet) }\end{array}$ & $\begin{array}{c}\text { Forma- } \\
\text { tion }\end{array}$ & $\begin{array}{l}\text { Porosity } \\
\text { (percent) }\end{array}$ & $\begin{array}{l}\text { Perme- } \\
\text { ability } \\
\text { (milli- } \\
\text { darcies) }\end{array}$ & Lithology \\
\hline$n m b 44-19-16 b c c$ & $9,440.0$ & $9,441.0$ & $341 E L B R$ & 3.1 & 0.000000 & Shaly, finely crystalline dolomite \\
\hline nmb44-19-16bcc & $9,442.0$ & $9,443.0$ & $341 \mathrm{ELBR}$ & 1.3 & 0.000000 & Shaly, finely crystalline dolomite \\
\hline nmb44-19-16bcc & $9,444.0$ & $9,445.0$ & 341ELBR & 1.9 & 0.000000 & Fine-grained quartz sandstone \\
\hline$n m b 44-19-16 b c c$ & $9,446.0$ & $9,447.0$ & $341 E L B R$ & 1.0 & 0.000000 & Shaly, fine- to medium-crystalline dolomite \\
\hline nmb44-19-16bcc & $9,448.0$ & $9,449.0$ & $341 \mathrm{ELBR}$ & 2.5 & 0.360000 & Shaly, fine- to medium-crystalline dolomite \\
\hline $\mathrm{nmb} 44-19-16 \mathrm{bcc}$ & $9,450.0$ & $9,451.0$ & $341 \mathrm{ELBR}$ & 6.5 & 0.040000 & Fine-grained glauconitic sandstone \\
\hline $\mathrm{nmb} 44-19-16 \mathrm{bcc}$ & $9,452.0$ & $9,453.0$ & $341 \mathrm{ELBR}$ & 5.0 & 0.000000 & Fine-grained glauconitic sandstone \\
\hline$n m b 44-19-16 b c c$ & $9,454.0$ & $9,455.0$ & $341 \mathrm{ELBR}$ & 4.1 & 0.020000 & Fine- to medium-grained glauconitic quartzit \\
\hline $\mathrm{nmb} 44-19-16 \mathrm{bcc}$ & $9,456.0$ & $9,457.0$ & $341 \mathrm{ELBR}$ & 8.5 & 0.710000 & Fine- to medium-grained dolomitic sandstone \\
\hline nmb44-19-16bcc & $9,458.0$ & $9,459.0$ & 341ELBR & 8.1 & 3.700000 & Fine- to medium-grained dolomitic sandstone \\
\hline $\mathrm{nmb} 44-19-16 \mathrm{bcc}$ & $9,460.0$ & $9,461.0$ & $341 E L B R$ & 5.9 & 0.380000 & Fine- to medium-grained dolomitic sandstone \\
\hline $\mathrm{nmb} 44-19-16 \mathrm{bcc}$ & $9,462.0$ & $9,463.0$ & $341 \mathrm{ELBR}$ & 5.7 & 0.310000 & Fine- to medium-grained dolomitic sandstone \\
\hline nmb44-19-16bcc & $9,464.0$ & $9,465.0$ & 341ELBR & 3.2 & 0.000000 & Fine- to medium-grained quartz sandstone \\
\hline $\mathrm{nmb} 44-19-16 \mathrm{bcc}$ & $9,466.0$ & $9,467.0$ & $341 E L B R$ & 5.1 & 0.120000 & Fine- to medium-grained quartz sandstone \\
\hline nmb44-19-16bcc & $9,468.0$ & $9,469.0$ & $341 \mathrm{ELBR}$ & 4.6 & 0.000000 & Shaly, finely crystalline dolomite \\
\hline$n m b 44-19-16 b c c$ & $9,470.0$ & $9,471.0$ & $341 \mathrm{ELBR}$ & 5.2 & 0.000000 & Shaly, finely crystalline dolomite \\
\hline nmb44-19-16bcc & $9,472.0$ & $9,473.0$ & 341ELBR & 3.4 & 0.010000 & Coarse-grained sandstone \\
\hline nmb44-19-16bcc & $9,474.0$ & $9,475.0$ & $341 \mathrm{ELBR}$ & 2.7 & 0.040000 & Coarse-grained sandstone \\
\hline nmb44-19-16bcc & $9,476.0$ & $9,477.0$ & 341ELBR & 3.8 & 0.000000 & Coarse-grained sandstone \\
\hline & & & AVERAGE - - & 2.7 & 0.265000 & \\
\hline SITE AVERAGE- & 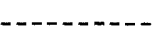 & & 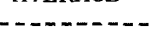 & 2.7 & 4.163469 & \\
\hline $\mathrm{nmb} 47-18-10 \mathrm{cbb}$ & 67.0 & 67.1 & 317CTLR & 14.8 & 1.400000 & Very fine-grained sandstone \\
\hline $\mathrm{nmb} 47-18-10 \mathrm{cbb}$ & 68.0 & 68.1 & 317 CTLR & 15.4 & 3.800000 & Very fine-grained sandstone \\
\hline $\mathrm{nmb} 47-18-10 \mathrm{cbb}$ & 69.0 & 69.1 & 317CTLR & 17.1 & 2.100000 & Very fine-grained sandstone \\
\hline $\mathrm{nmb} 47-18-10 \mathrm{cbb}$ & 70.0 & 70.1 & 317CTLR & 17.9 & 1.300000 & Very fine-grained sandstone \\
\hline $\mathrm{nmb} 47-18-10 \mathrm{cbb}$ & 71.2 & 71.3 & 317CTLR & 9.4 & 0.020000 & Very fine-grained sandstone \\
\hline$n m b 47-18-10 c b b$ & 72.0 & 72.1 & 317CTLR & 11.9 & 2.400000 & Very fine-grained sandstone \\
\hline nmb47-18-10cbb & 73.1 & 73.2 & 317CTLR & 13.0 & 0.350000 & Very fine-grained sandstone \\
\hline$n m b 47-18-10 c b b$ & 74.1 & 74.2 & 317CTLR & 13.4 & 0.400000 & Very fine-grained sandstone \\
\hline$n m b 47-18-10 c b b$ & 95.1 & 95.2 & 317CTLR & 6.3 & 0.020000 & Medium- to coarse-grained sandstone \\
\hline$n m b 47-18-10 \mathrm{cbb}$ & 96.0 & 96.1 & 317CTLR & 15.3 & 40.000000 & Medium- to coarse-grained sandstone \\
\hline $\mathrm{nmb} 47-18-10 \mathrm{cbb}$ & 97.3 & 97.4 & 317CTLR & 18.2 & 34.000000 & Friable, medium- to coarse-grained sandstone \\
\hline $\mathrm{nmb} 47-18-10 \mathrm{cbb}$ & 133.2 & 133.3 & 317CTLR & 10.1 & 0.200000 & Very fine-grained sandstone \\
\hline $\mathrm{nmb} 47-18-10 \mathrm{cbb}$ & 134.0 & 134.1 & 317CTLR & 11.0 & 0.070000 & Very fine-grained sandstone \\
\hline $\mathrm{nmb} 47-18-10 \mathrm{cbb}$ & 135.0 & 135.1 & 317CTLR & 7.9 & 0.040000 & Very fine-grained sandstone \\
\hline $\mathrm{nmb} 47-18-10 \mathrm{cbb}$ & 154.9 & 155.0 & 317CTLR & 18.6 & 3.300000 & Very fine- to coarse-grained sandstone \\
\hline$n m b 47-18-10 c b b$ & 156.1 & 156.2 & 317CTLR & 17.3 & 27.000000 & Coarse-grained sandstone \\
\hline$n m b 47-18-10 \mathrm{cbb}$ & 157.4 & 157.5 & 317CTLR & 13.1 & 0.300000 & Very fine- to coarse-grained sandstone \\
\hline $\mathrm{nmb} 47-18-10 \mathrm{cbb}$ & 223.3 & 223.4 & 317CTLR & 9.6 & 0.110000 & Very fine-grained sandstone \\
\hline $\mathrm{nmb} 47-18-10 \mathrm{cbb}$ & 224.3 & 224.4 & 317 CTLR & 11.6 & 8.900000 & Very fine-grained sandstone \\
\hline $\mathrm{nmb} 47-18-10 \mathrm{cbb}$ & 236.3 & 236.4 & 317CTLR & 12.5 & 0.200000 & Very fine-grained sandstone \\
\hline $\mathrm{nmb} 47-18-10 \mathrm{cbb}$ & 237.3 & 237.4 & 317CTLR & 13.1 & 0.250000 & Very fine-grained sandstone \\
\hline $\mathrm{nmb} 47-18-10 \mathrm{cbb}$ & 238.3 & 238.4 & 317CTLR & 16.9 & 0.600000 & Fine-grained sandstone \\
\hline$n m b 47-18-10 c b b$ & 239.3 & 239.4 & 317 CTLR & 13.3 & 0.250000 & Fine- to coarse-grained sandstone \\
\hline $\mathrm{nmb} 47-18-10 \mathrm{cbb}$ & 240.3 & 240.4 & 317 CTLR & 14.3 & 0.600000 & Fine- to coarse-grained sandstone \\
\hline nmb47-18-10cbb & 241.3 & 241.4 & 317CTLR & 16.3 & 6.000000 & Fine- to coarse-grained sandstone \\
\hline
\end{tabular}


Table 4.--Laboratory-determined porosity and permeability--Continued

\begin{tabular}{|c|c|c|c|c|c|c|}
\hline Site & $\begin{array}{l}\text { Depth to } \\
\text { top } \\
\text { (feet) }\end{array}$ & $\begin{array}{l}\text { Depth to } \\
\text { bottom } \\
\text { (feet) }\end{array}$ & $\begin{array}{l}\text { Forma- } \\
\text { tion }\end{array}$ & $\begin{array}{l}\text { Porosity } \\
\text { (percent) }\end{array}$ & $\begin{array}{l}\text { Perme- } \\
\text { ability } \\
\text { (milli- } \\
\text { darcies) }\end{array}$ & Lithology \\
\hline $\mathrm{nmb} 47-18-10 \mathrm{cbb}$ & 242.3 & 242.4 & 317CTLR & 16.3 & 0.500000 & Fine- to coarse-grained sandstone \\
\hline nmb47-18-10cbb & 243.5 & 243.6 & 317CTLR & 15.7 & 1.000000 & Fine- to coarse-grained sandstone \\
\hline$n m b 47-18-10 c b b$ & 244.5 & 244.6 & 317CTLR & 15.0 & 1.400000 & Fine- to coarse-grained sandstone \\
\hline $\mathrm{nmb} 47-18-10 \mathrm{cbb}$ & 245.6 & 245.7 & 317CTLR & 15.3 & 0.600000 & Fine- to coarse-grained sandstone \\
\hline nmb47-18-10cbb & 246.6 & 246.7 & 317CTLR & 15.4 & 0.700000 & Fine- to coarse- grained sandstone \\
\hline $\mathrm{nmb} 47-18-10 \mathrm{cbb}$ & 247.3 & 247.4 & 317CTLR & 16.0 & 18.000000 & Sandstone with coarse streak \\
\hline$n m b 47-18-10 c b b$ & 248.0 & 248.1 & 317CTLR & 14.1 & 2.500000 & Fine- to coarse-grained sandstone \\
\hline $\mathrm{nmb} 47-18-10 \mathrm{cbb}$ & 249.0 & 249.1 & 317CTLR & 11.9 & 0.300000 & Siltstone \\
\hline nmb47-18-10cbb & 254.9 & 255.0 & 317CTLR & 14.1 & 13.000000 & Sandstone with coarse streak \\
\hline nmb47-18-10cbb & 255.9 & 256.0 & 317 CTLR & 17.1 & 26.000000 & Sandstone with coarse streak \\
\hline $\mathrm{nmb} 47-18-10 \mathrm{cbb}$ & 256.9 & 257.0 & 317CTLR & 14.2 & 36.000000 & Fine- to coarse-grained sandstone \\
\hline $\mathrm{nmb} 47-18-10 \mathrm{cbb}$ & 262.8 & 262.9 & 317CTLR & 14.9 & 0.500000 & Fine- to medium-grained sandstone \\
\hline$n m b 47-18-10 c b b$ & 263.8 & 263.9 & 317CTLR & 13.7 & 0.350000 & Fine- to medium-grained sandstone \\
\hline nmb47-18-10cbb & 264.7 & 264.8 & 317CTLR & 14.2 & 1.800000 & Fine- to medium-grained sandstone \\
\hline $\mathrm{nmb} 47-18-10 \mathrm{cbb}$ & 265.9 & 266.0 & 317CTLR & 6.6 & 0.460000 & Fine- to coarse-grained sandstone \\
\hline SITE AVERAGE--- & 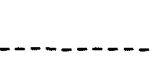 & $------\infty$ & AVERAGE-- & $\frac{13.8}{13.8}$ & $\frac{5.918000}{5.918000}$ & \\
\hline sb02-101-31dab & $6,160.0$ & $6,161.0$ & $321 \mathrm{WEBR}$ & 1.8 & 0.010000 & Silty sandstone \\
\hline sb02-101-31dab & $6,162.0$ & $6,163.0$ & $321 \mathrm{WEBR}$ & 3.1 & 0.020000 & Silty sandstone \\
\hline sb02-101-31dab & $6,164.0$ & $6,165.0$ & $321 \mathrm{WEBR}$ & 4.5 & 0.080000 & Silty sandstone \\
\hline sb02-101-31dab & $6,166.0$ & $6,167.0$ & $321 \mathrm{WEBR}$ & 7.2 & 0.450000 & Silty sandstone \\
\hline sb02-101-31dab & $6,168.0$ & $6,169.0$ & $321 \mathrm{WEBR}$ & 6.3 & 0.090000 & Silty sandstone \\
\hline sb02-101-31dab & $6,170.0$ & $6,171.0$ & 321WEBR & 7.1 & 0.090000 & Silty sandstone \\
\hline sb02-101-31dab & $6,172.0$ & $6,173.0$ & 321WEBR & 9.4 & 0.010000 & Silty sandstone \\
\hline sb02-101-31dab & $6,174.0$ & $6,175.0$ & 321WEBR & 7.7 & 0.120000 & Silty sandstone \\
\hline sb02-101-31dab & $6,176.0$ & $6,177.0$ & 321WEBR & 3.2 & 0.020000 & Silty sandstone \\
\hline sb02-101-31dab & $6,178.0$ & $6,179.0$ & $321 \mathrm{WEBR}$ & 4.5 & 0.010000 & Silty sandstone \\
\hline sb02-101-31dab & $6,181.0$ & $6,182.0$ & $321 \mathrm{WEBR}$ & 4.5 & 0.020000 & Silty sandstone \\
\hline sb02-101-31dab & $6,183.0$ & $6,184.0$ & 321WEBR & 7.1 & 0.320000 & Silty sandstone \\
\hline sb02-101-31dab & $6,185.0$ & $6,186.0$ & $321 \mathrm{WEBR}$ & 7.7 & 0.460000 & Silty sandstone \\
\hline sb02-101-31dab & $6,187.0$ & $6,188.0$ & $321 \mathrm{WEBR}$ & 8.4 & 0.120000 & Silty sandstone \\
\hline sb02-101-31dab & $6,189.0$ & $6,190.0$ & $321 \mathrm{WEBR}$ & 9.4 & 3.600000 & Silty sandstone \\
\hline sb02-101-31dab & $6,191.0$ & $6,192.0$ & $321 \mathrm{WEBR}$ & 9.5 & 0.040000 & Silty sandstone \\
\hline sb02-101-31dab & $6,192.0$ & $6,193.0$ & $321 \mathrm{WEBR}$ & 8.3 & 0.380000 & Silty sandstone \\
\hline sb02-101-31dab & $6,193.0$ & $6,194.0$ & 321WEBR & 5.4 & 0.050000 & Silty sandstone \\
\hline sb02-101-31dab & $6,194.0$ & $6,195.0$ & $321 \mathrm{WEBR}$ & 7.4 & 0.190000 & Silty sandstone \\
\hline sb02-101-31dab & $6,195.0$ & $6,196.0$ & $321 \mathrm{WEBR}$ & 3.2 & 0.040000 & Silty sandstone \\
\hline sb02-101-31dab & $6,202.0$ & $6,203.0$ & 321WEBR & 3.9 & 0.040000 & Silty sandstone \\
\hline sb02-101-31dab & $6,204.0$ & $6,205.0$ & 321WEBR & 3.3 & 0.030000 & Silty sandstone \\
\hline sb02-101-31dab & $6,206.0$ & $6,207.0$ & 321WEBR & 4.7 & 0.060000 & Silty sandstone \\
\hline sb02-101-31dab & $6,208.0$ & $6,209.0$ & $321 \mathrm{WEBR}$ & 6.1 & 0.140000 & Silty sandstone \\
\hline $\mathrm{sb} 02-101-31 \mathrm{dab}$ & $6,210.0$ & $6,211.0$ & $321 \mathrm{WEBR}$ & 6.7 & 0.070000 & Silty sandstone \\
\hline sb02-101-31dab & $6,212.0$ & $6,213.0$ & 321WEBR & 9.6 & 0.100000 & Silty sandstone \\
\hline sb02-101-31dab & $6,214.0$ & $6,215.0$ & 321WEBR & 7.0 & 0.190000 & Silty sandstone \\
\hline sb02-101-31dab & $6,216.0$ & $6,217.0$ & $321 \mathrm{WEBR}$ & 4.1 & 0.030000 & Silty sandstone \\
\hline $\mathrm{sb02-101-31dab}$ & $6,217.0$ & $6,218.0$ & $321 \mathrm{WEBR}$ & 3.7 & 0.040000 & Silty sandstone \\
\hline sb02-101-31dab & $6,222.0$ & $6,223.0$ & 321WEBR & 1.8 & 0.020000 & Silty sandstone \\
\hline
\end{tabular}


Table 4.--Laboratory-determined porosity and permeability--Continued

\begin{tabular}{|c|c|c|c|c|c|c|}
\hline Site & $\begin{array}{l}\text { Depth to } \\
\text { top } \\
\text { (feet) }\end{array}$ & $\begin{array}{l}\text { Depth to } \\
\text { bottom } \\
\text { (feet) }\end{array}$ & $\begin{array}{c}\text { Forma- } \\
\text { tion }\end{array}$ & $\begin{array}{l}\text { Porosity } \\
\text { (percent) }\end{array}$ & $\begin{array}{l}\text { Perme- } \\
\text { ability } \\
\text { (milli- } \\
\text { darcies) }\end{array}$ & Lithology \\
\hline sb02-101-31dab & $6,224.0$ & $6,225.0$ & 321WEBR & 13.3 & 0.060000 & Silty sandstone \\
\hline sb02-101-31dab & $6,225.0$ & $6,226.0$ & $321 \mathrm{WEBR}$ & 9.4 & 4.600000 & Silty sandstone \\
\hline sb02-101-31dab & $6,227.0$ & $6,228.0$ & 321WEBR & 5.6 & 0.170000 & Silty sandstone \\
\hline sb02-101-31dab & $6,228.0$ & $6,229.0$ & $321 \mathrm{WEBR}$ & 10.7 & 0.170000 & Silty sandstone \\
\hline sb02-101-31dab & $6,233.0$ & $6,234.0$ & 321WEBR & 6.3 & 0.050000 & Silty sandstone \\
\hline sb02-101-31dab & $6,234.0$ & $6,235.0$ & $321 \mathrm{WEBR}$ & 3.1 & 0.030000 & Silty sandstone \\
\hline sb02-101-31dab & $6,236.0$ & $6,237.0$ & 321WEBR & 5.4 & 0.070000 & Silty sandstone \\
\hline sb02-101-31dab & $6,238.0$ & $6,239.0$ & $321 \mathrm{WEBR}$ & 4.6 & 0.040000 & Silty sandstone \\
\hline sb02-101-31dab & $6,240.0$ & $6,241.0$ & $321 \mathrm{WEBR}$ & 4.6 & 0.050000 & Silty sandstone \\
\hline sb02-101-31dab & $6,242.0$ & $6,243.0$ & $321 \mathrm{WEBR}$ & 6.8 & 0.130000 & Silty sandstone \\
\hline sb02-101-31dab & $6,244.0$ & $6,245.0$ & $321 \mathrm{WEBR}$ & 9.3 & 0.430000 & Silty sandstone \\
\hline sb02-101-31dab & $6,246.0$ & $6,247.0$ & $321 \mathrm{WEBR}$ & 11.0 & 0.070000 & Silty sandstone \\
\hline $\mathrm{sb} 02-101-31 \mathrm{dab}$ & $6,248.0$ & $6,249.0$ & $321 \mathrm{WEBR}$ & 4.9 & 0.060000 & Silty sandstone \\
\hline sb02-101-31dab & $6,249.0$ & $6,250.0$ & $321 \mathrm{WEBR}$ & 2.9 & 0.040000 & Silty sandstone \\
\hline sb02-101-31dab & $6,252.0$ & $6,253.0$ & 321WEBR & 2.2 & 0.030000 & Silty sandstone \\
\hline sb02-101-31dab & $6,254.0$ & $6,255.0$ & $321 \mathrm{WEBR}$ & 3.8 & 0.250000 & Silty sandstone \\
\hline sb02-101-31dab & $6,256.0$ & $6,257.0$ & $321 \mathrm{WEBR}$ & 4.3 & 0.260000 & Silty sandstone \\
\hline $\mathrm{sb} 02-101-31 \mathrm{dab}$ & $6,258.0$ & $6,259.0$ & $321 \mathrm{WEBR}$ & 9.8 & 0.270000 & Silty sandstone \\
\hline sb02-101-31dab & $6,260.0$ & $6,261.0$ & $321 \mathrm{WEBR}$ & 7.1 & 0.070000 & Silty sandstone \\
\hline $\mathrm{sb} 02-101-31 \mathrm{dab}$ & $6,262.0$ & $6,263.0$ & $321 \mathrm{WEBR}$ & 6.8 & 0.160000 & Silty sandstone \\
\hline sb02-101-31dab & $6,264.0$ & $6,265.0$ & $321 \mathrm{WEBR}$ & 8.0 & 0.090000 & Silty sandstone \\
\hline sb02-101-31dab & $6,265.0$ & $6,266.0$ & $321 \mathrm{WEBR}$ & 5.8 & 0.050000 & Silty sandstone \\
\hline $\mathrm{sb} 02-101-31 \mathrm{dab}$ & $6,267.0$ & $6,268.0$ & $321 \mathrm{WEBR}$ & 6.8 & 0.160000 & Silty sandstone \\
\hline sb02-101-31dab & $6,269.0$ & $6,270.0$ & 321WEBR & 7.1 & 0.190000 & Silty sandstone \\
\hline sb02-101-31dab & $6,271.0$ & $6,272.0$ & $321 \mathrm{WEBR}$ & 4.0 & 0.030000 & Silty sandstone \\
\hline sb02-101-31dab & $6,273.0$ & $6,274.0$ & $321 \mathrm{WEBR}$ & 2.9 & 0.030000 & Silty sandstone \\
\hline $\mathrm{sb} 02-101-31 \mathrm{dab}$ & $6,275.0$ & $6,276.0$ & $321 \mathrm{WEBR}$ & 2.9 & 0.020000 & Silty sandstone \\
\hline $\mathrm{sb} 02-101-31 \mathrm{dab}$ & $6,277.0$ & $6,278.0$ & $321 \mathrm{WEBR}$ & 3.2 & 0.040000 & Silty sandstone \\
\hline sb02-101-31dab & $6,279.0$ & $6,280.0$ & $321 \mathrm{WEBR}$ & 10.1 & 0.070000 & Silty sandstone \\
\hline $\mathrm{sb} 02-101-31 \mathrm{dab}$ & $6,281.0$ & $6,282.0$ & $321 \mathrm{WEBR}$ & 12.7 & 0.620000 & Silty sandstone \\
\hline $\mathrm{sb} 02-101-31 \mathrm{dab}$ & $6,283.0$ & $6,284.0$ & $321 \mathrm{WEBR}$ & 11.0 & 0.680000 & Silty sandstone \\
\hline sb02-101-31dab & $6,285.0$ & $6,286.0$ & $321 \mathrm{WEBR}$ & 9.3 & 0.110000 & Silty sandstone \\
\hline $\mathrm{sb02}-101-31 \mathrm{dab}$ & $6,287.0$ & $6,288.0$ & $321 \mathrm{WEBR}$ & 14.5 & 1.900000 & Silty sandstone \\
\hline $\mathrm{sb} 02-101-31 \mathrm{dab}$ & $6,289.0$ & $6,290.0$ & $321 \mathrm{WEBR}$ & 8.1 & 0.780000 & Silty sandstone \\
\hline sb02-101-31dab & $6,291.0$ & $6,292.0$ & 321WEBR & 9.3 & 0.130000 & Silty sandstone \\
\hline sb02-101-31dab & $6,293.0$ & $6,294.0$ & 321WEBR & 6.8 & 0.060000 & Silty sandstone \\
\hline $\mathrm{sb} 02-101-31 \mathrm{dab}$ & $6,295.0$ & $6,296.0$ & $321 \mathrm{WEBR}$ & 2.7 & 0.060000 & Silty sandstone \\
\hline sb02-101-31dab & $6,297.0$ & $6,298.0$ & $321 \mathrm{WEBR}$ & 3.2 & 0.030000 & Silty sandstone \\
\hline sb02-101-31dab & $6,299.0$ & $6,300.0$ & $321 \mathrm{WEBR}$ & 2.9 & 0.020000 & Silty sandstone \\
\hline sb02-101-31dab & $6,301.0$ & $6,302.0$ & 321WEBR & 4.0 & 0.030000 & Silty sandstone \\
\hline sb02-101-31dab & $6,303.0$ & $6,304.0$ & 321WEBR & 6.9 & 0.070000 & Silty sandstone \\
\hline sb02-101-31dab & $6,305.0$ & $6,306.0$ & $321 \mathrm{WEBR}$ & 5.4 & 0.070000 & Silty sandstone \\
\hline sb02-101-31dab & $6,307.0$ & $6,308.0$ & $321 \mathrm{WEBR}$ & 5.3 & 0.170000 & Silty sandstone \\
\hline sb02-101-31dab & $6,309.0$ & $6,310.0$ & $321 \mathrm{WEBR}$ & 7.4 & 0.030000 & Silty sandstone \\
\hline sb02-101-31dab & $6,311.0$ & $6,312.0$ & $321 \mathrm{WEBR}$ & 6.5 & 0.120000 & Silty sandstone \\
\hline $\mathrm{sb} 02-101-31 \mathrm{dab}$ & $6,313.0$ & $6,314.0$ & 321WEBR & 5.6 & 0.130000 & Silty sandstone \\
\hline sb02-101-31dab & $6,315.0$ & $6,316.0$ & $321 \mathrm{WEBR}$ & 3.9 & 0.030000 & Silty sandstone \\
\hline sb02-101-31dab & $6,317.0$ & $6,318.0$ & $321 \mathrm{WEBR}$ & 4.6 & 0.090000 & Silty sandstone \\
\hline sb02-101-31dab & $6,319.0$ & $6,320.0$ & $321 \mathrm{WEBR}$ & 2.3 & 0.050000 & Silty sandstone \\
\hline sb02-101-31dab & $6,321.0$ & $6,322.0$ & $321 \mathrm{WEBR}$ & 7.4 & 0.110000 & Silty sandstone \\
\hline
\end{tabular}


Table 4.--Laboratory-determined porosity and permeability--Continued

\begin{tabular}{|c|c|c|c|c|c|c|}
\hline Site & $\begin{array}{l}\text { Depth to } \\
\text { top } \\
\text { (feet) }\end{array}$ & $\begin{array}{l}\text { Depth to } \\
\text { bottom } \\
\text { (feet) }\end{array}$ & $\begin{array}{c}\text { Forma- } \\
\text { tion }\end{array}$ & $\begin{array}{l}\text { Porosity } \\
\text { (percent) }\end{array}$ & $\begin{array}{l}\text { Perme- } \\
\text { ability } \\
\text { (milli- } \\
\text { darcies) }\end{array}$ & Lithology \\
\hline sb02-101-31dab & $6,322.0$ & $6,323.0$ & $321 \mathrm{WEBR}$ & 8.2 & 0.120000 & Silty sandstone \\
\hline sb02-101-31dab & $6,324.0$ & $6,325.0$ & $321 \mathrm{WEBR}$ & 6.6 & 0.440000 & Silty sandstone \\
\hline sb02-101-31dab & $6,326.0$ & $6,327.0$ & $321 \mathrm{WEBR}$ & 2.8 & 0.090000 & Silty sandstone \\
\hline sb02-101-31dab & $6,328.0$ & $6,329.0$ & $321 \mathrm{WEBR}$ & 3.7 & 0.100000 & Silty sandstone \\
\hline sb02-101-31dab & $6,330.0$ & $6,331.0$ & $321 \mathrm{WEBR}$ & 2.8 & 0.040000 & Silty sandstone \\
\hline sb02-101-31dab & $6,334.0$ & $6,335.0$ & $321 \mathrm{WEBR}$ & 8.0 & 0.560000 & Silty sandstone \\
\hline sb02-101-31dab & $6,336.0$ & $6,337.0$ & $321 \mathrm{WEBR}$ & 10.7 & 0.570000 & Silty sandstone \\
\hline sb02-101-31dab & $6,338.0$ & $6,339.0$ & $321 \mathrm{WEBR}$ & 9.8 & 0.310000 & Silty sandstone \\
\hline sb02-101-31dab & $6,340.0$ & $6,341.0$ & $321 \mathrm{WEBR}$ & 10.7 & 0.120000 & Silty sandstone \\
\hline sb02-101-31dab & $6,342.0$ & $6,343.0$ & $321 \mathrm{WEBR}$ & 3.4 & 0.030000 & Silty sandstone \\
\hline sb02-101-31dab & $6,344.0$ & $6,345.0$ & $321 \mathrm{WEBR}$ & 10.2 & 2.900000 & Silty sandstone \\
\hline sb02-101-31dab & $6,346.0$ & $6,347.0$ & $321 \mathrm{WEBR}$ & 6.9 & 0.120000 & Silty sandstone \\
\hline sb02-101-31dab & $6,348.0$ & $6,349.0$ & $321 \mathrm{WEBR}$ & 4.8 & 0.070000 & Silty sandstone \\
\hline sb02-101-31dab & $6,350.0$ & $6,351.0$ & $321 \mathrm{WEBR}$ & 2.5 & 0.040000 & Silty sandstone \\
\hline sb02-101-31dab & $6,351.0$ & $6,352.0$ & $321 \mathrm{WEBR}$ & 2.0 & 0.030000 & Silty sandstone \\
\hline sb02-101-31dab & $6,358.0$ & $6,359.0$ & $321 \mathrm{WEBR}$ & 2.3 & 2.300000 & Silty sandstone \\
\hline sb02-101-31dab & $6,360.0$ & $6,361.0$ & $321 \mathrm{WEBR}$ & 12.0 & 0.380000 & Silty sandstone \\
\hline $\mathrm{sb} 02-101-31 \mathrm{dab}$ & $6,362.0$ & $6,363.0$ & $321 \mathrm{WE} B R$ & 4.4 & 0.250000 & Silty sandstone \\
\hline sb02-101-31dab & $6,364.0$ & $6,365.0$ & $321 \mathrm{WEBR}$ & 3.7 & 0.070000 & Silty sandstone \\
\hline sb02-101-31dab & $6,366.0$ & $6,367.0$ & $321 \mathrm{WEBR}$ & 13.7 & 3.400000 & Silty sandstone \\
\hline $\mathrm{sb} 02-101-31 \mathrm{dab}$ & $6,368.0$ & $6,369.0$ & $321 \mathrm{WEBR}$ & 13.7 & 0.470000 & Silty sandstone \\
\hline sb02-101-31dab & $6,370.0$ & $6,371.0$ & $321 \mathrm{WEBR}$ & 3.6 & 0.070000 & Silty sandstone \\
\hline $\mathrm{sb} 02-101-31 \mathrm{dab}$ & $6,372.0$ & $6,373.0$ & $321 \mathrm{WEBR}$ & 8.1 & 0.750000 & Silty sandstone \\
\hline sb02-101-31dab & $6,373.0$ & $6,374.0$ & $321 \mathrm{WEBR}$ & 6.7 & 0.100000 & Silty sandstone \\
\hline sb02-101-31dab & $6,375.0$ & $6,376.0$ & $321 \mathrm{WEBR}$ & 3.7 & 0.160000 & Silty sandstone \\
\hline sb02-101-31dab & $6,378.0$ & $6,379.0$ & $321 \mathrm{WEBR}$ & 3.9 & 0.090000 & Silty sandstone \\
\hline sb02-101-31dab & $6,379.0$ & $6,380.0$ & $321 \mathrm{WEBR}$ & 3.6 & 0.080000 & Silty sandstone \\
\hline sb02-101-31dab & $6,381.0$ & $6,382.0$ & $321 \mathrm{WEBR}$ & 3.2 & 0.020000 & Silty sandstone \\
\hline$s b 02-101-31 \mathrm{dab}$ & $6,383.0$ & $6,384.0$ & $321 \mathrm{WEBR}$ & 3.6 & 10.000000 & Shaly, silty sandstone \\
\hline sb02-101-31dab & $6,385.0$ & $6,386.0$ & $321 \mathrm{WEBR}$ & 3.1 & 0.040000 & Silty sandstone \\
\hline sb02-101-31dab & $6,387.0$ & $6,388.0$ & $321 \mathrm{WEBR}$ & 3.1 & 0.020000 & Shaly, silty sandstone \\
\hline $\mathrm{sb02}-101-31 \mathrm{dab}$ & $6,389.0$ & $6,390.0$ & $321 \mathrm{WEBR}$ & 3.8 & 0.060000 & Shaly, silty sandstone \\
\hline sb02-101-31dab & $6,391.0$ & $6,392.0$ & $321 \mathrm{WEBR}$ & 3.3 & 0.070000 & Shaly, silty sandstone \\
\hline $\mathrm{sb} 02-101-31 \mathrm{dab}$ & $6,393.0$ & $6,394.0$ & $321 \mathrm{WEBR}$ & 2.7 & 0.020000 & Shaly, silty sandstone \\
\hline sb02-101-31dab & $6,396.0$ & $6,397.0$ & $321 \mathrm{WEBR}$ & 3.9 & 0.070000 & Shaly, silty sandstone \\
\hline sb02-101-31dab & $6,397.0$ & $6,398.0$ & $321 \mathrm{WEBR}$ & 9.7 & 0.090000 & Silty sandstone \\
\hline sb02-101-31dab & $6,398.0$ & $6,399.0$ & $321 \mathrm{WEBR}$ & 10.9 & 0.200000 & Silty sandstone \\
\hline$s b 02-101-31 d a b$ & $6,399.0$ & $6,400.0$ & $321 \mathrm{WEBR}$ & 14.6 & 0.540000 & Silty sandstone \\
\hline sb02-101-31dab & $6,400.0$ & $6,401.0$ & $321 \mathrm{WEBR}$ & 12.5 & 3.100000 & Silty sandstone \\
\hline sb02-101-31dab & $6,401.0$ & $6,402.0$ & $321 \mathrm{WEBR}$ & 10.3 & 0.500000 & Silty sandstone \\
\hline sb02-101-31dab & $6,402.0$ & $6,403.0$ & $321 \mathrm{WEBR}$ & 13.0 & 1.500000 & Silty sandstone \\
\hline sb02-101-31dab & $6,403.0$ & $6,404.0$ & $321 \mathrm{WEBR}$ & 13.6 & 7.100000 & Silty sandstone \\
\hline sb02-101-31dab & $6,404.0$ & $6,405.0$ & $321 \mathrm{WEBR}$ & 13.8 & 2.800000 & Silty sandstone \\
\hline sb02-101-31dab & $6,408.0$ & $6,409.0$ & $321 \mathrm{WEBR}$ & 11.1 & 0.740000 & Silty sandstone \\
\hline sb02-101-31dab & $6,411.0$ & $6,412.0$ & $321 \mathrm{WEBR}$ & 16.2 & 6.300000 & Silty sandstone \\
\hline $\mathrm{sb} 02-101-31 \mathrm{dab}$ & $6,412.0$ & $6,413.0$ & $321 \mathrm{WEBR}$ & 12.5 & 2.000000 & Silty sandstone \\
\hline $\mathrm{sb} 02-101-31 \mathrm{dab}$ & $6,413.0$ & $6,414.0$ & $321 \mathrm{WEBR}$ & 15.8 & 1.200000 & Silty sandstone \\
\hline sb02-101-31dab & $6,414.0$ & $6,415.0$ & $321 \mathrm{WEBR}$ & 12.5 & 2.600000 & Silty sandstone \\
\hline $\mathrm{sb} 02-101-31 \mathrm{dab}$ & $6,415.0$ & $6,416.0$ & 321WEBR & 12.5 & 0.870000 & Silty sandstone \\
\hline sb02-101-31dab & $6,416.0$ & $6,417.0$ & $321 \mathrm{WEBR}$ & 15.5 & 16.000000 & Silty sandstone \\
\hline
\end{tabular}


Table 4.--Laboratory-determined porosity and permeability--Continued

\begin{tabular}{|c|c|c|c|c|c|c|}
\hline Site & $\begin{array}{l}\text { Depth to } \\
\text { top } \\
\text { (feet) }\end{array}$ & $\begin{array}{l}\text { Depth to } \\
\text { bottom } \\
\text { (feet) }\end{array}$ & $\begin{array}{c}\text { Forma- } \\
\text { tion }\end{array}$ & $\begin{array}{l}\text { Porosity } \\
\text { (percent) }\end{array}$ & $\begin{array}{l}\text { Perme- } \\
\text { ability } \\
\text { (milli- } \\
\text { darcies) }\end{array}$ & Lithology \\
\hline $\begin{array}{l}\text { sb02-101-31dab } \\
\text { sb02-101-31dab } \\
\text { sb02-101-31dab } \\
\text { sb02-101-31dab } \\
\text { sb02-101-31dab }\end{array}$ & $\begin{array}{l}6,417.0 \\
6,418.0 \\
6,420.0 \\
6,423.0 \\
6,424.0\end{array}$ & $\begin{array}{l}6,418.0 \\
6,419.0 \\
6,421.0 \\
6,424.0 \\
6,425.0\end{array}$ & $\begin{array}{l}321 \mathrm{WEBR} \\
321 \mathrm{WEBR} \\
321 \mathrm{WEBR} \\
321 \mathrm{WEBR} \\
321 \mathrm{WEBR}\end{array}$ & $\begin{array}{l}15.3 \\
14.8 \\
13.5 \\
16.8 \\
15.1\end{array}$ & $\begin{array}{r}13.000000 \\
6.100000 \\
18.000000 \\
10.000000 \\
14.000000\end{array}$ & $\begin{array}{l}\text { Silty sandstone } \\
\text { Silty sandstone } \\
\text { Silty sandstone } \\
\text { Silty sandstone } \\
\text { Silty sandstone }\end{array}$ \\
\hline $\begin{array}{l}\text { sb02-101-31dab } \\
\text { sb02-101-31dab } \\
\text { sb02-101-31dab } \\
\text { sb02-101-31dab } \\
\text { sb02-101-31dab }\end{array}$ & $\begin{array}{l}6,425.0 \\
6,426.0 \\
6,427.0 \\
6,428.0 \\
6,429.0\end{array}$ & $\begin{array}{l}6,426.0 \\
6,427.0 \\
6,428.0 \\
6,429.0 \\
6,430.0\end{array}$ & $\begin{array}{l}321 \mathrm{WEBR} \\
321 \mathrm{WEBR} \\
321 \mathrm{WEBR} \\
321 \mathrm{WEBR} \\
321 \mathrm{WEBR}\end{array}$ & $\begin{array}{r}13.7 \\
13.1 \\
10.5 \\
3.8 \\
9.5\end{array}$ & $\begin{array}{l}6.300000 \\
3.800000 \\
1.900000 \\
0.100000 \\
0.110000\end{array}$ & $\begin{array}{l}\text { Silty sandstone } \\
\text { Silty sandstone } \\
\text { Silty sandstone } \\
\text { Silty sandstone } \\
\text { Silty sandstone }\end{array}$ \\
\hline $\begin{array}{l}\text { sb02-101-31dab } \\
\text { sb02-101-31dab } \\
\text { sb02-101-31dab } \\
\text { sb02-101-31dab } \\
\text { sb02-101-31dab }\end{array}$ & $\begin{array}{l}6,430.0 \\
6,431.0 \\
6,432.0 \\
6,433.0 \\
6,434.0\end{array}$ & $\begin{array}{l}6,431.0 \\
6,432.0 \\
6,433.0 \\
6,434.0 \\
6,435.0\end{array}$ & $\begin{array}{l}321 \mathrm{WEBR} \\
321 \mathrm{WEBR} \\
321 \mathrm{WEBR} \\
321 \mathrm{WEBR} \\
321 \mathrm{WEBR}\end{array}$ & $\begin{array}{r}4.9 \\
14.1 \\
11.2 \\
11.6 \\
11.4\end{array}$ & $\begin{array}{r}0.070000 \\
20.000000 \\
0.130000 \\
1.700000 \\
9.200000\end{array}$ & $\begin{array}{l}\text { Silty sandstone } \\
\text { Silty sandstone } \\
\text { Silty sandstone } \\
\text { Silty sandstone } \\
\text { Silty sandstone }\end{array}$ \\
\hline $\begin{array}{l}\text { sb02-101-31dab } \\
\text { sb02-101-31dab } \\
\text { sb02-101-31dab } \\
\text { sb02-101-31dab }\end{array}$ & $\begin{array}{l}6,435.0 \\
6,436.0 \\
6,437.0 \\
6,438.0\end{array}$ & $\begin{array}{l}6,436.0 \\
6,437.0 \\
6,438.0 \\
6,439.0\end{array}$ & $\begin{array}{l}\text { 321WEBR } \\
\text { 321WEBR } \\
\text { 321WEBR } \\
\text { 321WEBR }\end{array}$ & $\begin{array}{l}15.1 \\
11.3 \\
19.9 \\
13.2 \\
\end{array}$ & $\begin{array}{r}26.000000 \\
8.400000 \\
35.000000 \\
24.000000 \\
\end{array}$ & $\begin{array}{l}\text { Silty sandstone } \\
\text { Silty sandstone } \\
\text { Silty sandstone } \\
\text { Silty sandstone }\end{array}$ \\
\hline SITE AVERAGE--- & $-\cdots-1$ & $----1--$ & AVERAGE-- & $\frac{7.6}{7.6}$ & $\frac{1.927047}{1.927047}$ & \\
\hline $\begin{array}{l}\text { sb02-102-36bbb } \\
\text { sb02-102-36bbb } \\
\text { sb02-102-36bbb } \\
\text { sb02-102-36bbb } \\
\text { sb02-102-36bbb }\end{array}$ & $\begin{array}{l}5,930.0 \\
5,931.0 \\
5,932.0 \\
5,933.0 \\
5,934.0\end{array}$ & $\begin{array}{l}5,931.0 \\
5,932.0 \\
5,933.0 \\
5,934.0 \\
5,935.0\end{array}$ & $\begin{array}{l}321 \mathrm{WEBR} \\
321 \mathrm{WEBR} \\
321 \mathrm{WEBR} \\
321 \mathrm{WEBR} \\
321 \mathrm{WEBR}\end{array}$ & $\begin{array}{r}11.8 \\
12.6 \\
9.2 \\
3.2 \\
4.9\end{array}$ & $\begin{array}{l}2.600000 \\
1.700000 \\
0.850000 \\
0.000000 \\
0.000000\end{array}$ & $\begin{array}{l}\text { Sands tone } \\
\text { Sands tone } \\
\text { Sandstone } \\
\text { Sandstone } \\
\text { Sands tone }\end{array}$ \\
\hline $\begin{array}{l}\text { sb02-102-36bbb } \\
\text { sb02-102-36bbb } \\
\text { sb02-102-36bbb } \\
\text { sb02-102-36bbb } \\
\text { sb02-102-36bbb }\end{array}$ & $\begin{array}{l}5,935.0 \\
5,940.0 \\
5,941.0 \\
5,942.0 \\
5,943.0\end{array}$ & $\begin{array}{l}5,936.0 \\
5,941.0 \\
5,942.0 \\
5,943.0 \\
5,944.0\end{array}$ & $\begin{array}{l}321 \mathrm{WEBR} \\
321 \mathrm{WEBR} \\
321 \mathrm{WEBR} \\
321 \mathrm{WEBR} \\
321 \mathrm{WEBR}\end{array}$ & $\begin{array}{l}6.4 \\
6.3 \\
6.7 \\
3.8 \\
3.3\end{array}$ & $\begin{array}{l}0.000000 \\
0.000000 \\
0.000000 \\
0.000000 \\
0.000000\end{array}$ & $\begin{array}{l}\text { Sands tone } \\
\text { Sands tone } \\
\text { Sands tone } \\
\text { Sands tone } \\
\text { Sands tone }\end{array}$ \\
\hline $\begin{array}{l}\text { sb02-102-36bbb } \\
\text { sb02-102-36bbb } \\
\text { sb02-102-36bbb } \\
\text { sb02-102-36bbb } \\
\text { sb02-102-36bbb }\end{array}$ & $\begin{array}{l}5,944.0 \\
5,950.0 \\
5,951.0 \\
5,953.0 \\
5,954.0\end{array}$ & $\begin{array}{l}5,945.0 \\
5,951.0 \\
5,952.0 \\
5,954.0 \\
5,955.0\end{array}$ & $\begin{array}{l}321 \mathrm{WEBR} \\
321 \mathrm{WEBR} \\
321 \mathrm{WEBR} \\
321 \mathrm{WEBR} \\
321 \mathrm{WEBR}\end{array}$ & $\begin{array}{r}3.2 \\
13.5 \\
13.0 \\
12.5 \\
11.2\end{array}$ & $\begin{array}{l}0.000000 \\
5.500000 \\
1.350000 \\
1.600000 \\
1.000000\end{array}$ & $\begin{array}{l}\text { Sandstone } \\
\text { Sandstone } \\
\text { Sands tone } \\
\text { Sands tone } \\
\text { Sands tone }\end{array}$ \\
\hline $\begin{array}{l}\text { sb02-102-36bbb } \\
\text { sb02-102-36bbb } \\
\text { sb02-102-36bbb } \\
\text { sb02-102-36bbb } \\
\text { sb02-102-36bbb }\end{array}$ & $\begin{array}{l}5,955.0 \\
5,962.0 \\
5,963.0 \\
5,974.0 \\
5,978.0\end{array}$ & $\begin{array}{l}5,956.0 \\
5,963.0 \\
5,964.0 \\
5,975.0 \\
5,979.0\end{array}$ & $\begin{array}{l}321 \mathrm{WEBR} \\
321 \mathrm{WEBR} \\
321 \mathrm{WEBR} \\
321 \mathrm{WEBR} \\
321 \mathrm{WEBR}\end{array}$ & $\begin{array}{r}5.2 \\
3.3 \\
2.9 \\
7.6 \\
11.0\end{array}$ & $\begin{array}{l}0.000000 \\
0.000000 \\
0.000000 \\
0.000000 \\
1.000000\end{array}$ & $\begin{array}{l}\text { Sands tone } \\
\text { Sands tone } \\
\text { Sands tone } \\
\text { Sands tone } \\
\text { Sandstone }\end{array}$ \\
\hline $\begin{array}{l}\text { sb02-102-36bbb } \\
\text { sb02-102-36bbb } \\
\text { sb02-102-36bbb } \\
\text { sb02-102-36bbb } \\
\text { sb02-102-36bbb }\end{array}$ & $\begin{array}{l}5,979.0 \\
5,980.0 \\
6,005.0 \\
6,006.0 \\
6,007.0\end{array}$ & $\begin{array}{l}5,980.0 \\
5,981.0 \\
6,006.0 \\
6,007.0 \\
6,008.0\end{array}$ & $\begin{array}{l}321 \mathrm{WEBR} \\
321 \mathrm{WEBR} \\
321 \mathrm{WEBR} \\
321 \mathrm{WEBR} \\
321 \mathrm{WEBR}\end{array}$ & $\begin{array}{r}11.0 \\
9.5 \\
8.7 \\
9.7 \\
7.7\end{array}$ & $\begin{array}{r}3.800000 \\
11.500000 \\
1.000000 \\
1.750000 \\
0.200000\end{array}$ & $\begin{array}{l}\text { Sands tone } \\
\text { Sands tone } \\
\text { Sands tone } \\
\text { Sands tone } \\
\text { Sands tone }\end{array}$ \\
\hline $\begin{array}{l}\text { sb02-102-36bbb } \\
\text { sb02-102-36bbb } \\
\text { sb02-102-36bbb } \\
\text { sb02-102-36bbb } \\
\text { sb02-102-36bbb }\end{array}$ & $\begin{array}{l}6,008.0 \\
6,009.0 \\
6,012.0 \\
6,013.0 \\
6,014.0\end{array}$ & $\begin{array}{l}6,009.0 \\
6,010.0 \\
6,013.0 \\
6,014.0 \\
6,015.0\end{array}$ & $\begin{array}{l}321 \mathrm{WEBR} \\
321 \mathrm{WEBR} \\
321 \mathrm{WEBR} \\
321 \mathrm{WEBR} \\
321 \mathrm{WEBR}\end{array}$ & $\begin{array}{l}5.6 \\
7.7 \\
3.6 \\
4.8 \\
5.8\end{array}$ & $\begin{array}{l}0.000000 \\
0.000000 \\
0.000000 \\
0.000000 \\
0.000000\end{array}$ & $\begin{array}{l}\text { Sands tone } \\
\text { Sands tone } \\
\text { Sands tone } \\
\text { Sands tone } \\
\text { Sands tone }\end{array}$ \\
\hline
\end{tabular}


Table 4.--Laboratory-determined porosity and permeability--Continued

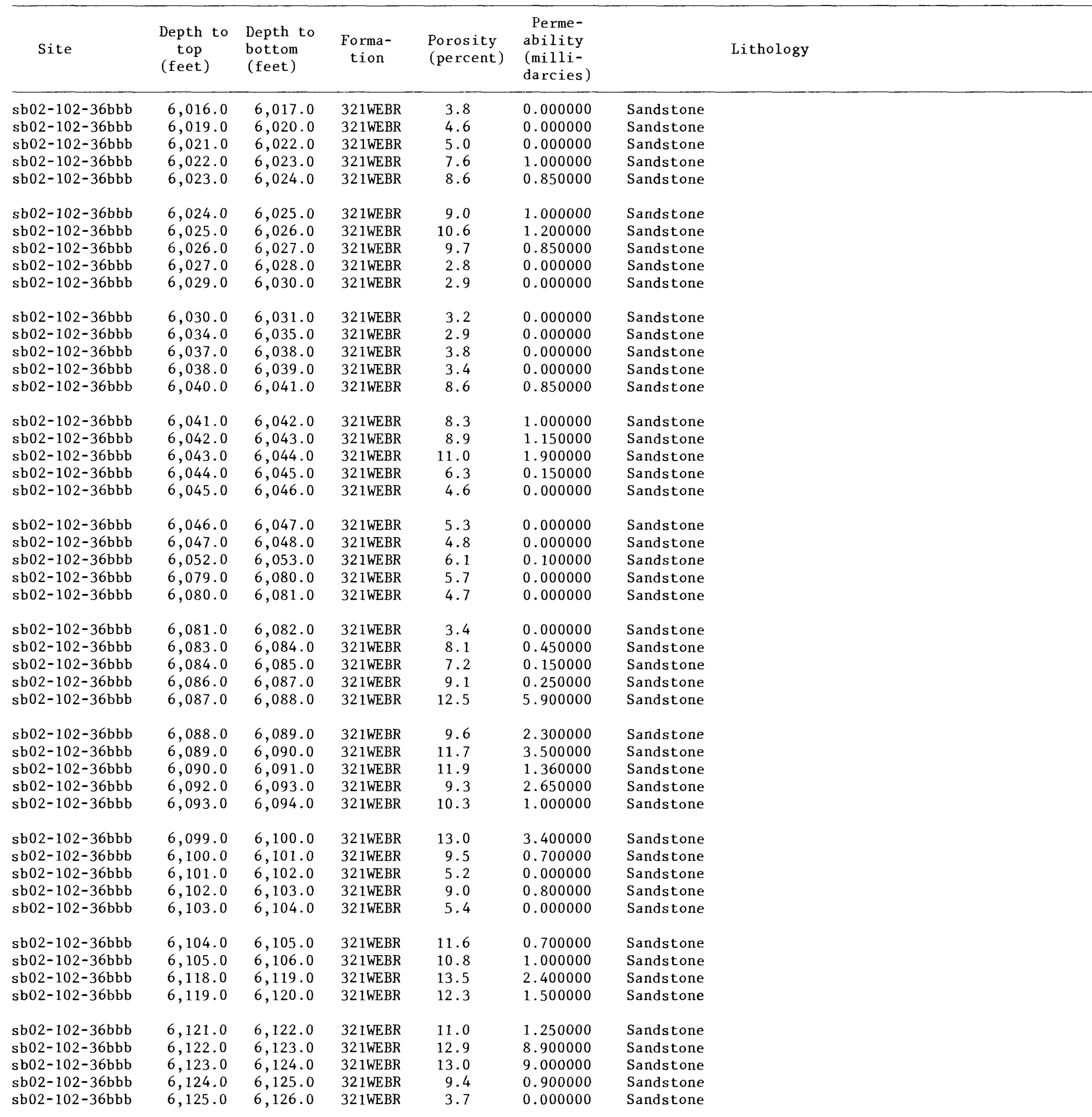


Table 4.--Laboratory-determined porosity and permeability--Continued

\begin{tabular}{|c|c|c|c|c|c|c|}
\hline Site & $\begin{array}{l}\text { Depth to } \\
\text { top } \\
\text { (feet) }\end{array}$ & $\begin{array}{l}\text { Depth to } \\
\text { bottom } \\
\text { (feet) }\end{array}$ & $\begin{array}{c}\text { Forma- } \\
\text { tion }\end{array}$ & $\begin{array}{l}\text { Porosity } \\
\text { (percent) }\end{array}$ & $\begin{array}{l}\text { Perme- } \\
\text { ability } \\
\text { (milli- } \\
\text { darcies) }\end{array}$ & Lithology \\
\hline $\begin{array}{l}\text { sb02-102-36bbb } \\
\text { sb02-102-36bbb } \\
\text { sb02-102-36bbb } \\
\text { sb02-102-36bbb } \\
\text { sb02-102-36bbb }\end{array}$ & $\begin{array}{l}6,126.0 \\
6,128.0 \\
6,132.0 \\
6,133.0 \\
6,154.0\end{array}$ & $\begin{array}{l}6,127.0 \\
6,129.0 \\
6,133.0 \\
6,134.0 \\
6,155.0\end{array}$ & $\begin{array}{l}\text { 321WEBR } \\
321 \mathrm{WEBR} \\
321 \mathrm{WEBR} \\
321 \mathrm{WEBR} \\
321 \mathrm{WEBR}\end{array}$ & $\begin{array}{r}3.4 \\
14.6 \\
8.8 \\
9.8 \\
11.5\end{array}$ & $\begin{array}{l}0.000000 \\
3.400000 \\
0.200000 \\
0.250000 \\
1.000000\end{array}$ & $\begin{array}{l}\text { Sands tone } \\
\text { Sands tone } \\
\text { Sands tone } \\
\text { Sands tone } \\
\text { Sands tone }\end{array}$ \\
\hline $\begin{array}{l}\text { sb02-102-36bbb } \\
\text { sb02-102-36bbb } \\
\text { sb02-102-36bbb } \\
\text { sb02-102-36bbb } \\
\text { sb02-102-36bbb }\end{array}$ & $\begin{array}{l}6,159.0 \\
6,160.0 \\
6,162.0 \\
6,163.0 \\
6,164.0\end{array}$ & $\begin{array}{l}6,160.0 \\
6,161.0 \\
6,163.0 \\
6,164.0 \\
6,165.0\end{array}$ & $\begin{array}{l}\text { 321WEBR } \\
\text { 321WEBR } \\
321 \mathrm{WEBR} \\
\text { 321WEBR } \\
\text { 321WEBR }\end{array}$ & $\begin{array}{r}4.5 \\
10.5 \\
9.3 \\
10.5 \\
11.8\end{array}$ & $\begin{array}{l}0.000000 \\
1.300000 \\
1.100000 \\
1.600000 \\
1.600000\end{array}$ & $\begin{array}{l}\text { Sandstone } \\
\text { Sandstone } \\
\text { Sands tone } \\
\text { Sands tone } \\
\text { Sands tone }\end{array}$ \\
\hline $\begin{array}{l}\text { sb02-102-36bbb } \\
\text { sb02-102-36bbb } \\
\text { sb02-102-36bbb } \\
\text { sb02-102-36bbb } \\
\text { sb02-102-36bbb }\end{array}$ & $\begin{array}{l}6,167.0 \\
6,169.0 \\
6,170.0 \\
6,171.0 \\
6,172.0\end{array}$ & $\begin{array}{l}6,168.0 \\
6,170.0 \\
6,171.0 \\
6,172.0 \\
6,173.0\end{array}$ & $\begin{array}{l}321 \mathrm{WEBR} \\
321 \mathrm{WEBR} \\
321 \mathrm{WEBR} \\
321 \mathrm{WEBR} \\
321 \mathrm{WEBR}\end{array}$ & $\begin{array}{r}13.8 \\
10.9 \\
10.2 \\
7.3 \\
10.0\end{array}$ & $\begin{array}{l}2.600000 \\
2.700000 \\
2.400000 \\
0.850000 \\
2.100000\end{array}$ & $\begin{array}{l}\text { Sands tone } \\
\text { Sands tone } \\
\text { Sands tone } \\
\text { Sands tone } \\
\text { Sands tone }\end{array}$ \\
\hline $\begin{array}{l}\text { sb02-102-36bbb } \\
\text { sb02-102-36bbb } \\
\text { sb02-102-36bbb } \\
\text { sb02-102-36bbb } \\
\text { sb02-102-36bbb }\end{array}$ & $\begin{array}{l}6,173.0 \\
6,174.0 \\
6,175.0 \\
6,220.0 \\
6,221.0\end{array}$ & $\begin{array}{l}6,174.0 \\
6,175.0 \\
6,176.0 \\
6,221.0 \\
6,222.0\end{array}$ & $\begin{array}{l}321 \text { WEBR } \\
321 \text { WEBR } \\
321 \text { WEBR } \\
321 \text { WEBR } \\
321 \text { WEBR }\end{array}$ & $\begin{array}{r}8.7 \\
8.5 \\
10.2 \\
8.6 \\
5.9\end{array}$ & $\begin{array}{l}1.200000 \\
0.600000 \\
1.000000 \\
0.850000 \\
0.100000\end{array}$ & $\begin{array}{l}\text { Sands tone } \\
\text { Sands tone } \\
\text { Sands tone } \\
\text { Sands tone } \\
\text { Sands tone }\end{array}$ \\
\hline $\begin{array}{l}\text { sb02-102-36bbb } \\
\text { sb02-102-36bbb } \\
\text { sb02-102-36bbb } \\
\text { sb02-102-36bbb } \\
\text { sb02-102-36bbb }\end{array}$ & $\begin{array}{l}6,222.0 \\
6,223.0 \\
6,224.0 \\
6,225.0 \\
6,226.0\end{array}$ & $\begin{array}{l}6,223.0 \\
6,224.0 \\
6,225.0 \\
6,226.0 \\
6,227.0\end{array}$ & $\begin{array}{l}\text { 321WEBR } \\
\text { 321WEBR } \\
\text { 321WEBR } \\
\text { 321WEBR } \\
\text { 321WEBR }\end{array}$ & $\begin{array}{r}8.7 \\
10.8 \\
6.3 \\
6.9 \\
13.0\end{array}$ & $\begin{array}{r}0.750000 \\
1.700000 \\
0.200000 \\
0.150000 \\
150.000000\end{array}$ & $\begin{array}{l}\text { Sands tone } \\
\text { Sands tone } \\
\text { Sands tone } \\
\text { Sands tone } \\
\text { Sands tone }\end{array}$ \\
\hline $\begin{array}{l}\text { sb02-102-36bbb } \\
\text { sb02-102-36bbb } \\
\text { sb02-102-36bbb } \\
\text { sb02-102-36bbb } \\
\text { sb02-102-36bbb }\end{array}$ & $\begin{array}{l}6,229.0 \\
6,229.0 \\
6,230.0 \\
6,231.0 \\
6,232.0\end{array}$ & $\begin{array}{l}6,230.0 \\
6,230.0 \\
6,231.0 \\
6,232.0 \\
6,233.0\end{array}$ & $\begin{array}{l}\text { 321WEBR } \\
\text { 321WEBR } \\
\text { 321WEBR } \\
\text { 321WEBR } \\
\text { 321WEBR }\end{array}$ & $\begin{array}{r}11.6 \\
11.6 \\
5.5 \\
15.0 \\
12.9\end{array}$ & $\begin{array}{r}20.000000 \\
20.000000 \\
0.000000 \\
29.000000 \\
6.300000\end{array}$ & $\begin{array}{l}\text { Sands tone } \\
\text { Sands tone } \\
\text { Sands tone } \\
\text { Sands tone } \\
\text { Sands tone }\end{array}$ \\
\hline $\begin{array}{l}\text { sb02-102-36bbb } \\
\text { sb02-102-36bbb } \\
\text { sb02-102-36bbb } \\
\text { sb02-102-36bbb } \\
\text { sb02-102-36bbb }\end{array}$ & $\begin{array}{l}6,237.0 \\
6,238.0 \\
6,239.0 \\
6,240.0 \\
6,241.0\end{array}$ & $\begin{array}{l}6,238.0 \\
6,239.0 \\
6,240.0 \\
6,241.0 \\
6,242.0\end{array}$ & $\begin{array}{l}\text { 321WEBR } \\
\text { 321WEBR } \\
\text { 321WEBR } \\
\text { 321WEBR } \\
\text { 321WEBR }\end{array}$ & $\begin{array}{r}9.7 \\
7.4 \\
12.0 \\
12.4 \\
7.9\end{array}$ & $\begin{array}{l}2.100000 \\
0.850000 \\
6.800000 \\
7.400000 \\
0.950000\end{array}$ & $\begin{array}{l}\text { Sands tone } \\
\text { Sands tone } \\
\text { Sands tone } \\
\text { Sands tone } \\
\text { Sands tone }\end{array}$ \\
\hline $\begin{array}{l}\text { sb02-102-36bbb } \\
\text { sb02-102-36bbb } \\
\text { sb02-102-36bbb } \\
\text { sb02-102-36bbb } \\
\text { sb02-102-36bbb }\end{array}$ & $\begin{array}{l}6,242.0 \\
6,243.0 \\
6,244.0 \\
6,245.0 \\
6,245.0\end{array}$ & $\begin{array}{l}6,243.0 \\
6,244.0 \\
6,245.0 \\
6,246.0 \\
6,246.0\end{array}$ & $\begin{array}{l}\text { 321WEBR } \\
\text { 32 IWEBR } \\
321 \mathrm{WEBR} \\
\text { 321WEBR } \\
\text { 321WEBR }\end{array}$ & $\begin{array}{r}13.1 \\
8.7 \\
13.3 \\
10.7 \\
10.9\end{array}$ & $\begin{array}{r}10.000000 \\
1.300000 \\
22.000000 \\
5.300000 \\
5.300000\end{array}$ & $\begin{array}{l}\text { Sands tone } \\
\text { Sands tone } \\
\text { Sands tone } \\
\text { Sands tone } \\
\text { Sandstone }\end{array}$ \\
\hline $\begin{array}{l}\text { sb02-102-36bbb } \\
\text { sb02-102-36bbb } \\
\text { sb02-102-36bbb } \\
\text { sb02-102-36bbb } \\
\text { sb02-102-36bbb }\end{array}$ & $\begin{array}{l}6,246.0 \\
6,247.0 \\
6,248.0 \\
6,249.0 \\
6,252.0\end{array}$ & $\begin{array}{l}6,247.0 \\
6,248.0 \\
6,249.0 \\
6,250.0 \\
6,253.0\end{array}$ & $\begin{array}{l}\text { 32 IWEBR } \\
321 \mathrm{WEBR} \\
321 \mathrm{WEBR} \\
321 \mathrm{WEBR} \\
\text { 321WEBR }\end{array}$ & $\begin{array}{l}12.3 \\
14.0 \\
12.0 \\
13.6 \\
15.2\end{array}$ & $\begin{array}{r}19.000000 \\
20.000000 \\
5.900000 \\
10.000000 \\
42.000000\end{array}$ & $\begin{array}{l}\text { Sands tone } \\
\text { Sands tone } \\
\text { Sands tone } \\
\text { Sands tone } \\
\text { Sands tone }\end{array}$ \\
\hline $\begin{array}{l}\text { sb02-102-36bbb } \\
\text { sb02-102-36bbb } \\
\text { sb02-102-36bbb } \\
\text { sb02-102-36bbb } \\
\text { sb02-102-36bbb }\end{array}$ & $\begin{array}{l}6,253.0 \\
6,254.0 \\
6,255.0 \\
6,257.0 \\
6,258.0\end{array}$ & $\begin{array}{l}6,254.0 \\
6,255.0 \\
6,256.0 \\
6,258.0 \\
6,259.0\end{array}$ & $\begin{array}{l}\text { 321WEBR } \\
321 \mathrm{WEBR} \\
321 \mathrm{WEBR} \\
321 \mathrm{WEBR} \\
321 \mathrm{WEBR}\end{array}$ & $\begin{array}{l}13.9 \\
15.3 \\
12.8 \\
14.7 \\
15.5\end{array}$ & $\begin{array}{r}135.000000 \\
35.000000 \\
11.000000 \\
16.000000 \\
47.000000\end{array}$ & $\begin{array}{l}\text { Sandstone } \\
\text { Sandstone } \\
\text { Sands tone } \\
\text { Sands tone } \\
\text { Sands tone }\end{array}$ \\
\hline
\end{tabular}


Table 4.--Laboratory-determined porosity and permeability--Continued

\begin{tabular}{|c|c|c|c|c|c|c|}
\hline Site & $\begin{array}{l}\text { Depth to } \\
\text { top } \\
\text { (feet) }\end{array}$ & $\begin{array}{l}\text { Depth to } \\
\text { bottom } \\
\text { (feet) }\end{array}$ & $\begin{array}{c}\text { Forma- } \\
\text { tion }\end{array}$ & $\begin{array}{l}\text { Porosity } \\
\text { (percent) }\end{array}$ & $\begin{array}{l}\text { Perme- } \\
\text { ability } \\
\text { (milli- } \\
\text { darcies) }\end{array}$ & Lithology \\
\hline sb02-102-36bbb & $6,260.0$ & $6,261.0$ & 321WEBR & 14.7 & 22.000000 & Sandstone \\
\hline sb02-102-36bbb & $6,261.0$ & $6,262.0$ & $321 \mathrm{WEBR}$ & 14.7 & 31.000000 & Sandstone \\
\hline sb02-102-36bbb & $6,262.0$ & $6,263.0$ & 321 WEBR & 16.5 & 54.000000 & Sandstone \\
\hline sb02-102-36bbb & $6,263.0$ & $6,264.0$ & $321 \mathrm{WEBR}$ & 19.2 & 75.000000 & Sandstone \\
\hline sb02-102-36bbb & $6,264.0$ & $6,265.0$ & $321 \mathrm{WEBR}$ & 16.6 & 62.000000 & Sandstone \\
\hline sb02-102-36bbb & $6,265.0$ & $6,266.0$ & 321WEBR & 14.9 & 27.000000 & Sandstone \\
\hline sb02-102-36bbb & $6,266.0$ & $6,267.0$ & $321 \mathrm{WEBR}$ & 15.5 & 27.000000 & Sandstone \\
\hline sb02-102-36bbb & $6,267.0$ & $6,268.0$ & $321 \mathrm{WEBR}$ & 15.6 & 26.000000 & Sandstone \\
\hline sb02-102-36bbb & $6,270.0$ & $6,271.0$ & $321 \mathrm{WEBR}$ & 13.4 & 13.500000 & Sandstone \\
\hline sb02-102-36bbb & $6,271.0$ & $6,272.0$ & $321 \mathrm{WEBR}$ & 14.5 & 19.000000 & Sandstone \\
\hline sb02-102-36bbb & $6,276.0$ & $6,277.0$ & $321 \mathrm{WEBR}$ & 11.9 & 14.000000 & Sandstone \\
\hline sb02-102-36bbb & $6,277.0$ & $6,278.0$ & $321 \mathrm{WEBR}$ & 15.4 & 54.000000 & Sandstone \\
\hline sb02-102-36bbb & $6,278.0$ & $6,279.0$ & $321 \mathrm{WEBR}$ & 11.9 & 5.500000 & Sandstone \\
\hline sb02-102-36bbb & $6,279.0$ & $6,280.0$ & $321 \mathrm{WEBR}$ & 11.0 & 3.500000 & Sandstone \\
\hline sb02-102-36bbb & $6,280.0$ & $6,281.0$ & $321 \mathrm{WEBR}$ & 10.2 & 0.850000 & Sandstone \\
\hline sb02-102-36bbb & $6,281.0$ & $6,282.0$ & $321 \mathrm{WEBR}$ & 15.4 & 29.000000 & Sandstone \\
\hline sb02-102-36bbb & $6,282.0$ & $6,283.0$ & $321 \mathrm{WEBR}$ & 16.3 & 52.000000 & Sandstone \\
\hline sb02-102-36bbb & $6,283.0$ & $6,284.0$ & $321 \mathrm{WEBR}$ & 13.4 & 11.500000 & Sandstone \\
\hline sb02-102-36bbb & $6,284.0$ & $6,285.0$ & $321 \mathrm{WEBR}$ & 9.7 & 1.300000 & Sandstone \\
\hline sb02-102-36bbb & $6,285.0$ & $6,286.0$ & $321 \mathrm{WEBR}$ & 13.3 & 125.000000 & Sandstone \\
\hline sb02-102-36bbb & $6,286.0$ & $6,287.0$ & 321WEBR & 12.5 & 5.900000 & Sandstone \\
\hline sb02-102-36bbb & $6,290.0$ & $6,291.0$ & $321 \mathrm{WEBR}$ & 6.2 & 0.000000 & Sandstone \\
\hline sb02-102-36bbb & $6,292.0$ & $6,293.0$ & $321 \mathrm{WEBR}$ & 6.2 & 0.000000 & Sandstone \\
\hline sb02-102-36bbb & $6,294.0$ & $6,295.0$ & $321 \mathrm{WEBR}$ & 11.2 & 3.200000 & Sandstone \\
\hline sb02-102-36bbb & $6,296.0$ & $6,297.0$ & $321 \mathrm{WEBR}$ & 12.6 & 6.300000 & Sandstone \\
\hline sb02-102-36bbb & $6,298.0$ & $6,299.0$ & $321 \mathrm{WEBR}$ & 11.5 & 6.800000 & Sandstone \\
\hline sb02-102-36bbb & $6,300.0$ & $6,301.0$ & 321 WEBR & 12.3 & 6.800000 & Sandstone \\
\hline $\mathrm{sb02}-102-36 \mathrm{bbb}$ & $6,302.0$ & $6,303.0$ & $321 \mathrm{WEBR}$ & 14.0 & 8.000000 & Sandstone \\
\hline sb02-102-36bbb & $6,306.0$ & $6,307.0$ & $321 \mathrm{WEBR}$ & 9.1 & 1.050000 & Sandstone \\
\hline sb02-102-36bbb & $6,308.0$ & $6,309.0$ & $321 \mathrm{WEBR}$ & 6.6 & 0.000000 & Sandstone \\
\hline $\mathrm{sb} 02-102-36 \mathrm{bbb}$ & $6,310.0$ & $6,311.0$ & $321 \mathrm{WEBR}$ & 11.3 & 3.800000 & Sandstone \\
\hline sb02-102-36bbb & $6,311.0$ & $6,312.0$ & $321 \mathrm{WEBR}$ & 12.4 & 10.000000 & Sandstone \\
\hline sb02-102-36bbb & $6,312.0$ & $6,313.0$ & $321 \mathrm{WEBR}$ & 11.1 & 3.800000 & Sands tone \\
\hline sb02-102-36bbb & $6,316.0$ & $6,317.0$ & $321 \mathrm{WEBR}$ & 9.0 & 1.150000 & Sandstone \\
\hline sb02-102-36bbb & $6,319.0$ & $6,320.0$ & $321 \mathrm{WEBR}$ & 14.2 & 22.000000 & Sandstone \\
\hline sb02-102-36bbb & $6,320.0$ & $6,321.0$ & $321 \mathrm{WEBR}$ & 12.6 & 43.000000 & Sandstone \\
\hline sb02-102-36bbb & $6,321.0$ & $6,322.0$ & $321 \mathrm{WEBR}$ & 13.5 & 10.000000 & Sandstone \\
\hline sb02-102-36bbb & $6,341.0$ & $6,342.0$ & $321 \mathrm{WEBR}$ & 11.3 & 5.100000 & Sandstone \\
\hline sb02-102-36bbb & $6,342.0$ & $6,343.0$ & $321 \mathrm{WEBR}$ & 12.7 & 6.300000 & Sandstone \\
\hline sb02-102-36bbb & $6,344.0$ & $6,345.0$ & 321 WEBR & 11.7 & 16.000000 & Sandstone \\
\hline sb02-102-36bbb & $6,345.0$ & $6,346.0$ & $321 \mathrm{WEBR}$ & 13.3 & 13.500000 & Sandstone \\
\hline sb02-102-36bbb & $6,346.0$ & $6,347.0$ & $321 \mathrm{WEBR}$ & 13.7 & 16.000000 & Sands tone \\
\hline sb02-102-36bbb & $6,348.0$ & $6,349.0$ & $321 \mathrm{WEBR}$ & 10.6 & 2.400000 & Sandstone \\
\hline sb02-102-36bbb & $6,349.0$ & $6,350.0$ & $321 \mathrm{WEBR}$ & 10.2 & 1.400000 & Sandstone \\
\hline sb02-102-36bbb & $6,350.0$ & $6,351.0$ & $321 \mathrm{WEBR}$ & 9.1 & 0.250000 & Sandstone \\
\hline sb02-102-36bbb & $6,363.0$ & $6,364.0$ & $321 \mathrm{WEBR}$ & 10.6 & 0.550000 & Sandstone \\
\hline sb02-102-36bbb & $6,364.0$ & $6,365.0$ & $321 \mathrm{WEBR}$ & 8.8 & 0.850000 & Sands tone \\
\hline sb02-102-36bbb & $6,368.0$ & $6,369.0$ & $321 \mathrm{WEBR}$ & 14.3 & 22.000000 & Sandstone \\
\hline sb02-102-36bbb & $6,369.0$ & $6,370.0$ & $321 \mathrm{WEBR}$ & 12.5 & 9.500000 & Sandstone \\
\hline sb02-102-36bbb & $6,370.0$ & $6,371.0$ & $321 \mathrm{WEBR}$ & 7.4 & 1.750000 & Sandstone \\
\hline
\end{tabular}


Table 4.--Laboratory-determined porosity and permeability--Continued

\begin{tabular}{|c|c|c|c|c|c|c|}
\hline Site & $\begin{array}{l}\text { Depth to } \\
\text { top } \\
\text { (feet) }\end{array}$ & $\begin{array}{l}\text { Depth to } \\
\text { bottom } \\
\text { (feet) }\end{array}$ & $\begin{array}{c}\text { Forma- } \\
\text { tion }\end{array}$ & $\begin{array}{l}\text { Porosity } \\
\text { (percent) }\end{array}$ & $\begin{array}{c}\text { Perme- } \\
\text { ability } \\
\text { (milli- } \\
\text { darcies) }\end{array}$ & Lithology \\
\hline sb02-102-36bbb & $6,371.0$ & $6,372.0$ & 321WEBR & 13.4 & 15.000000 & Sands tone \\
\hline $\mathrm{sb02}-102-36 \mathrm{bbb}$ & $6,373.0$ & $6,374.0$ & $321 \mathrm{WEBR}$ & 11.4 & 6.500000 & Sands tone \\
\hline sb02-102-36bbb & $6,375.0$ & $6,376.0$ & 321WEBR & 10.1 & 3.000000 & Sands tone \\
\hline sb02-102-36bbb & $6,376.0$ & $6,377.0$ & $321 \mathrm{WEBR}$ & 9.2 & 2.000000 & Sands tone \\
\hline SITE AVERAGE--- & $\ldots-\ldots$ & $\cdots-$ & AVERAGE-- & $\frac{9.9}{9.9}$ & $\frac{9.043224}{9.043224}$ & \\
\hline sb02-103-11ddc & $6,569.0$ & $6,570.0$ & 321WEBR & 16.4 & 35.000000 & Sandstone \\
\hline sb02-103-11ddc & $6,570.0$ & $6,571.0$ & $321 \mathrm{WEBR}$ & 15.8 & 21.000000 & Sandstone \\
\hline sb02-103-11ddc & $6,571.0$ & $6,572.0$ & $321 \mathrm{WEBR}$ & 11.5 & 43.000000 & Sandstone \\
\hline sb02-103-11ddc & $6,572.0$ & $6,573.0$ & $321 \mathrm{WEBR}$ & 17.1 & 26.000000 & Sands tone \\
\hline sb02-103-11ddc & $6,573.0$ & $6,574.0$ & 321WEBR & 15.3 & 10.000000 & Sands tone \\
\hline sb02-103-11ddc & $6,574.0$ & $6,575.0$ & 321WEBR & 14.9 & 15.000000 & Sands tone \\
\hline sb02-103-11ddc & $6,575.0$ & $6,576.0$ & 321WEBR & 14.3 & 2.200000 & Sands tone \\
\hline sb02-103-11ddc & $6,576.0$ & $6,577.0$ & $321 \mathrm{WEBR}$ & 14.2 & 26.000000 & Sands tone \\
\hline sb02-103-11ddc & $6,577.0$ & $6,578.0$ & $321 \mathrm{WEBR}$ & 16.1 & 5.700000 & Sandstone \\
\hline sb02-103-11ddc & $6,578.0$ & $6,579.0$ & 321WEBR & 18.4 & 6.400000 & Sands tone \\
\hline sb02-103-11ddc & $6,579.0$ & $6,580.0$ & $321 \mathrm{WEBR}$ & 15.8 & 35.000000 & Sands tone \\
\hline sb02-103-11ddc & $6,580.0$ & $6,581.0$ & $321 \mathrm{WEBR}$ & 19.9 & 67.000000 & Sands tone \\
\hline sb02-103-11ddc & $6,581.0$ & $6,582.0$ & $321 \mathrm{WEBR}$ & 15.3 & 15.000000 & Sands tone \\
\hline sb02-103-11ddc & $6,582.0$ & $6,583.0$ & $321 \mathrm{WEBR}$ & 14.8 & 5.600000 & Sands tone \\
\hline sb02-103-11ddc & $6,583.0$ & $6,584.0$ & $321 \mathrm{WEBR}$ & 14.6 & 21.000000 & Sands tone \\
\hline sb02-103-11ddc & $6,584.0$ & $6,585.0$ & 321WEBR & 13.3 & 3.500000 & Sandstone \\
\hline sb02-103-11ddc & $6,585.0$ & $6,586.0$ & $321 \mathrm{WEBR}$ & 14.6 & 10.000000 & Sands tone \\
\hline sb02-103-11ddc & $6,586.0$ & $6,587.0$ & 321WEBR & 14.3 & 12.000000 & Sands tone \\
\hline sb02-103-11ddc & $6,587.0$ & $6,588.0$ & $321 \mathrm{WEBR}$ & 11.8 & 1.400000 & Sands tone \\
\hline sb02-103-11ddc & $6,588.0$ & $6,589.0$ & 321WEBR & 11.0 & 6.700000 & Sandstone \\
\hline sb02-103-11ddc & $6,589.0$ & $6,590.0$ & 321WEBR & 17.4 & 28.000000 & Sands tone \\
\hline sb02-103-11ddc & $6,592.0$ & $6,593.0$ & 321WEBR & 15.4 & 1.700000 & Sands tone \\
\hline sb02-103-11ddc & $6,593.0$ & $6,594.0$ & $321 \mathrm{WEBR}$ & 16.5 & 0.900000 & Sandstone \\
\hline sb02-103-11ddc & $6,594.0$ & $6,595.0$ & $321 \mathrm{WEBR}$ & 14.9 & 18.000000 & Sandstone \\
\hline sb02-103-11ddc & $6,595.0$ & $6,596.0$ & 321WEBR & 23.0 & 2.700000 & Sandstone \\
\hline sb02-103-11ddc & $6,597.0$ & $6,598.0$ & 321 WEBR & 12.6 & 1.800000 & Sandstone \\
\hline sb02-103-11ddc & $6,598.0$ & $6,599.0$ & $321 \mathrm{WEBR}$ & 15.0 & 7.800000 & Sands tone \\
\hline sb02-103-11ddc & $6,599.0$ & $6,600.0$ & 321WEBR & 12.1 & 7.800000 & Sands tone \\
\hline sb02-103-11ddc & $6,600.0$ & $6,601.0$ & $321 \mathrm{WEBR}$ & 13.3 & 2.400000 & Sandstone \\
\hline sb02-103-11ddc & $6,601.0$ & $6,602.0$ & 321WEBR & 21.4 & 19.000000 & Sandstone \\
\hline sb02-103-11ddc & $6,602.0$ & $6,603.0$ & $321 \mathrm{WEBR}$ & 17.0 & 3.900000 & Sands tone \\
\hline sb02-103-11ddc & $6,603.0$ & $6,604.0$ & 321WEBR & 19.8 & 91.000000 & Sands tone \\
\hline sb02-103-11ddc & $6,604.0$ & $6,605.0$ & 321WEBR & 16.8 & 97.000000 & Sands tone \\
\hline $\mathrm{sb} 02-103-11 \mathrm{ddc}$ & $6,605.0$ & $6,606.0$ & $321 \mathrm{WEBR}$ & 24.3 & 111.000000 & Sandstone \\
\hline sb02-103-11ddc & $6,606.0$ & $6,607.0$ & 321WEBR & 19.3 & 111.000000 & Sandstone \\
\hline sb02-103-11ddc & $6,607.0$ & $6,608.0$ & 321WEBR & 18.1 & 64.000000 & Sandstone \\
\hline sb02-103-11ddc & $6,608.0$ & $6,609.0$ & $321 \mathrm{WEBR}$ & 18.3 & 3.200000 & Sandstone \\
\hline sb02-103-11ddc & $6,609.0$ & $6,610.0$ & 321WEBR & 16.8 & 41.000000 & Sands tone \\
\hline sb02-103-11ddc & $6,610.0$ & $6,611.0$ & $321 \mathrm{WEBR}$ & 19.4 & 7.600000 & Sands tone \\
\hline SITE AVERAGE- & & & AVERAGE-- & $\frac{16.2}{16.2}$ & $\frac{25.315385}{25.315385}$ & \\
\hline
\end{tabular}


Table 4.--Laboratory-determined porosity and permeability--Continued

\begin{tabular}{|c|c|c|c|c|c|c|}
\hline Site & $\begin{array}{l}\text { Depth to } \\
\text { top } \\
\text { (feet) }\end{array}$ & $\begin{array}{l}\text { Depth to } \\
\text { bottom } \\
\text { (feet) }\end{array}$ & $\begin{array}{c}\text { Forma- } \\
\text { tion }\end{array}$ & $\begin{array}{l}\text { Porosity } \\
\text { (percent) }\end{array}$ & $\begin{array}{l}\text { Perme- } \\
\text { ability } \\
\text { (milli- } \\
\text { darcies) }\end{array}$ & Lithology \\
\hline $\mathrm{sb} 02-88-10 \mathrm{dbb}$ & $2,052.0$ & $2,053.0$ & 321WEBR & 2.9 & 0.000000 & Gravelly, fine-grained, \\
\hline $\mathrm{sb} 02-88-10 \mathrm{dbb}$ & $2,057.0$ & $2,058.0$ & $321 \mathrm{WEBR}$ & 4.5 & 0.000000 & Gravelly, silty, limy \\
\hline $\mathrm{sb} 02-88-10 \mathrm{dbb}$ & $2,061.0$ & $2,062.0$ & $321 \mathrm{WEBR}$ & 5.3 & 1.400000 & Silty, clay-mottled qua \\
\hline sb02-88-10dbb & $2,064.0$ & $2,065.0$ & $321 \mathrm{WEBR}$ & 6.3 & 1.300000 & Silty, clay-mottled qua \\
\hline $\mathrm{sb} 02-88-10 \mathrm{dbb}$ & $2,097.0$ & $2,098.0$ & $321 \mathrm{WEBR}$ & 15.0 & 20.000000 & Silty, clay-mottled qua \\
\hline $\mathrm{sb} 02-88-10 \mathrm{dbb}$ & $2,098.0$ & $2,099.0$ & $321 \mathrm{WEBR}$ & 15.7 & 2.300000 & Silty, clay-mottled qua \\
\hline $\mathrm{sb} 02-88-10 \mathrm{dbb}$ & $2,099.0$ & $2,100.0$ & $321 \mathrm{WEBR}$ & 13.5 & 1.200000 & Silty, clay-mottled qua \\
\hline $\mathrm{sb} 02-88-10 \mathrm{dbb}$ & $2,100.0$ & $2,101.0$ & $321 \mathrm{WEBR}$ & 10.0 & 0.400000 & Silty, clay-mottled qua \\
\hline $\mathrm{sb} 02-88-10 \mathrm{dbb}$ & $2,101.0$ & $2,102.0$ & $321 \mathrm{WEBR}$ & 11.9 & 0.900000 & Silty, clay-mottled qua \\
\hline sb02-88-10dbb & $2,102.0$ & $2,103.0$ & $321 \mathrm{WEBR}$ & 8.1 & 0.300000 & Silty, clay-mottled qua \\
\hline $\mathrm{sb} 02-88-10 \mathrm{dbb}$ & $2,103.0$ & $2,104.0$ & $321 \mathrm{WEBR}$ & 7.7 & 0.200000 & Gypsiferous, fin \\
\hline $\mathrm{sb} 02-88-10 \mathrm{dbb}$ & $2,104.0$ & $2,105.0$ & $321 \mathrm{WEBR}$ & 7.9 & 0.300000 & Gypsiferous, fine-gra \\
\hline $\mathrm{sb} 02-88-10 \mathrm{dbb}$ & $2,105.0$ & $2,106.0$ & $321 \mathrm{WEBR}$ & 12.1 & 0.200000 & Silty, clay-mottled qua \\
\hline $\mathrm{sb} 02-88-10 \mathrm{dbb}$ & $2,106.0$ & $2,107.0$ & $321 \mathrm{WEBR}$ & 15.5 & 2.400000 & Fractured, fine-grained \\
\hline $\mathrm{sb} 02-88-10 \mathrm{dbb}$ & $2,111.0$ & $2,112.0$ & $321 \mathrm{WEBR}$ & 11.3 & 1.200000 & Silty, clay-mottled qua \\
\hline sb02-88-10dbb & $2,113.0$ & $2,114.0$ & $321 \mathrm{WEBR}$ & 15.1 & 1.100000 & Fractured, fine-grained \\
\hline $\mathrm{sb} 02-88-10 \mathrm{dbb}$ & $2,114.0$ & $2,115.0$ & $321 \mathrm{WEBR}$ & 15.2 & 4.900000 & Fractured, fine-grained \\
\hline sb02-88-10dbb & $2,117.0$ & $2,118.0$ & 321WEBR & 12.0 & 0.600000 & Fine-grained, clay-mott \\
\hline & & & AVERAGE-- & 10.6 & 2.150000 & \\
\hline SITE & & & 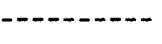 & $\overline{10.6}$ & 2.150000 & \\
\hline sb03-104-12bba & $9,303.0$ & $9,304.0$ & $321 \mathrm{WEBR}$ & 15.7 & 3.800000 & Fine-grained sandstone \\
\hline sb03-104-12bba & $9,304.0$ & $9,305.0$ & $321 \mathrm{WEBR}$ & 14.1 & 10.000000 & Fine-grained sandstone \\
\hline sb03-104-12bba & $9,305.0$ & $9,306.0$ & $321 \mathrm{WEBR}$ & 15.9 & 20.000000 & Fine-grained sandstone \\
\hline sb03-104-12bba & $9,306.0$ & $9,307.0$ & $321 \mathrm{WEBR}$ & 13.7 & 22.000000 & Fine-grained sandstone \\
\hline $\mathrm{sb} 03-104-12 \mathrm{bba}$ & $9,307.0$ & $9,308.0$ & $321 \mathrm{WEBR}$ & 9.8 & 0.380000 & Fine-grained sandstone \\
\hline sb03-104-12bba & $9,308.0$ & $9,309.0$ & $321 \mathrm{WEBR}$ & 11.9 & 0.55 & Fine-grained sandstone \\
\hline sb03-104-12bba & $9,309.0$ & $9,310.0$ & $321 \mathrm{WEBR}$ & 9.7 & 0.100000 & Fine-grained sandstone \\
\hline sb03-104-12bba & $9,310.0$ & $9,311.0$ & $321 \mathrm{WEBR}$ & 10.7 & 0.870000 & Fine-grained sandstone \\
\hline sb03-104-12bba & $9,311.0$ & $9,312.0$ & $321 \mathrm{WEBR}$ & 10.0 & 0.210000 & Fine-grained sandstone \\
\hline $\mathrm{sb03-104-12bba}$ & $9,312.0$ & $9,313.0$ & $321 \mathrm{WEBR}$ & 10.5 & 0.230000 & Fine-grained sandstone \\
\hline sb03-104-12bba & $9,313.0$ & $9,314.0$ & $321 \mathrm{WEBR}$ & 8.4 & 0.140000 & Fine-grained san \\
\hline sb03-104-12bba & $9,314.0$ & 15.0 & $321 \mathrm{WEBR}$ & 9.8 & 0.2 & Fine-grained sandstone \\
\hline sb03-104-12bba & $9,315.0$ & $9,316.0$ & $321 \mathrm{WEBR}$ & 6.7 & 0.240000 & Fine-grained sandstone \\
\hline $\mathrm{sb} 03-104-12 \mathrm{bba}$ & $9,316.0$ & $9,317.0$ & $321 \mathrm{WEBR}$ & 7.8 & 0.360000 & Fine-grained sandstone \\
\hline sb03-104-12bba & $9,317.0$ & $9,318.0$ & $321 \mathrm{WEBR}$ & 8.8 & 0.310000 & Fine-grained sandstone \\
\hline sb03-104-12bba & $9,318.0$ & $9,319.0$ & $321 \mathrm{WEBR}$ & 7.4 & 0.130000 & Fine-grained sandstone \\
\hline $\mathrm{sb} 03-104-12 \mathrm{bba}$ & $9,319.0$ & $9,320.0$ & $321 \mathrm{WEBR}$ & 6.5 & 0.060000 & Fine-grained sandstone \\
\hline sb03-104-12bba & $9,320.0$ & $9,321.0$ & $321 \mathrm{WEBR}$ & 7.0 & 0.080000 & Fine-grained sandstone \\
\hline sb03-104-12bba & $9,321.0$ & $9,322.0$ & $321 \mathrm{WEBR}$ & 7.7 & 0.250000 & Fine-grained sands tone \\
\hline sb03-104-12bba & $9,322.0$ & $9,323.0$ & $321 \mathrm{WEBR}$ & 6.4 & 0.110000 & Fine-grained sandstone \\
\hline sb03-104-12bba & $9,323.0$ & $9,324.0$ & $321 \mathrm{WEBR}$ & 6.0 & 0.060000 & Fine-grained sandstone \\
\hline sb03-104-12bba & $9,324.0$ & $9,325.0$ & $321 \mathrm{WEBR}$ & 7.2 & 0.130000 & Fine-grained sandstone \\
\hline sb03-104-12bba & $9,325.0$ & $9,326.0$ & $321 \mathrm{WEBR}$ & 5.0 & 0.050000 & Fine-grained sandstone \\
\hline $\mathrm{sb03}-104-12 \mathrm{bba}$ & $9,326.0$ & $9,327.0$ & $321 \mathrm{WEBR}$ & 5.5 & 0.060000 & Fine-grained sandstone \\
\hline sb03-104-12bba & $9,327.0$ & $9,328.0$ & $321 \mathrm{WEBR}$ & 6.0 & 0.070000 & Fine-grained sandstone \\
\hline sb03-104-12bba & $9,328.0$ & $9,329.0$ & 321WEBR & 7.4 & 0.150000 & Fine-grained san \\
\hline sb03-104-12bba & $9,329.0$ & $9,330.0$ & $321 \mathrm{WEBR}$ & 7.1 & 0.220000 & Fine-grained sands tone \\
\hline sb03-104-12bba & $9,438.0$ & $9,439.0$ & $321 \mathrm{WEBR}$ & 12.2 & 2.500000 & Fine-grained sandstone \\
\hline sb03-104-12bba & $9,442.0$ & $9,443.0$ & $321 \mathrm{WEBR}$ & 11.1 & 2.500000 & Fine-grained sandstone \\
\hline sb03-104-12bba & $9,445.0$ & $9,446.0$ & $321 \mathrm{WEBR}$ & 11.9 & 7.500000 & Fine-grained sandstone \\
\hline
\end{tabular}


Table 4.--Laboratory-determined porosity and permeability--Continued

\begin{tabular}{|c|c|c|c|c|c|c|}
\hline Site & $\begin{array}{l}\text { Depth to } \\
\text { top } \\
\text { (feet) }\end{array}$ & $\begin{array}{l}\text { Depth to } \\
\text { bottom } \\
\text { (feet) }\end{array}$ & $\begin{array}{c}\text { Forma- } \\
\text { tion }\end{array}$ & $\begin{array}{l}\text { Porosity } \\
\text { (percent) }\end{array}$ & $\begin{array}{l}\text { Perme- } \\
\text { ability } \\
\text { (milli- } \\
\text { darcies) }\end{array}$ & Lithology \\
\hline sb03-104-12bba & $9,448.0$ & $9,449.0$ & $321 \mathrm{WEBR}$ & $\underline{12.0}$ & 7.800000 & Fine-grained sandstone \\
\hline SITE AVERAGE- & & & AVERAGE-- & $\frac{9.4}{9.4}$ & $\frac{2.616774}{2.616774}$ & \\
\hline $\begin{array}{l}\text { sb04-99-12aaa } \\
\text { sb04-99-12aaa } \\
\text { sb04-99-12aaa } \\
\text { sb04-99-12aaa } \\
\text { sb04-99-12aaa }\end{array}$ & $\begin{array}{l}8,808.0 \\
8,809.0 \\
8,810.0 \\
8,813.0 \\
8,814.0\end{array}$ & $\begin{array}{l}8,809.0 \\
8,810.0 \\
8,811.0 \\
8,814.0 \\
8,815.0\end{array}$ & $\begin{array}{l}\text { 321WEBR } \\
321 \mathrm{WEBR} \\
321 \mathrm{WEBR} \\
321 \mathrm{WEBR} \\
\text { 321WEBR }\end{array}$ & $\begin{array}{l}4.5 \\
3.8 \\
4.1 \\
5.0 \\
6.2\end{array}$ & $\begin{array}{l}0.000000 \\
0.000000 \\
0.000000 \\
0.000000 \\
0.000000\end{array}$ & $\begin{array}{l}\text { Fine-grained, limy sandstone } \\
\text { Fine-grained, limy sandstone } \\
\text { Fine-grained, limy sandstone } \\
\text { Fine-grained, limy sandstone } \\
\text { Fine-grained, limy sandstone }\end{array}$ \\
\hline $\begin{array}{l}\text { sb04-99-12aaa } \\
\text { sb04-99-12aa a } \\
\text { sb04-99-12aaa } \\
\text { sb04-99-12aaa } \\
\text { sb04-99-12aaa }\end{array}$ & $\begin{array}{l}8,815.0 \\
8,816.0 \\
8,817.0 \\
8,818.0 \\
8,819.0\end{array}$ & $\begin{array}{l}8,816.0 \\
8,817.0 \\
8,818.0 \\
8,819.0 \\
8,820.0\end{array}$ & $\begin{array}{l}321 \mathrm{WEBR} \\
321 \mathrm{WEBR} \\
321 \mathrm{WEBR} \\
321 \mathrm{WEBR} \\
321 \mathrm{WEBR}\end{array}$ & $\begin{array}{r}6.7 \\
6.6 \\
5.7 \\
9.1 \\
11.2\end{array}$ & $\begin{array}{l}0.000000 \\
0.000000 \\
0.000000 \\
0.000000 \\
0.300000\end{array}$ & $\begin{array}{l}\text { Fine-grained, limy sandstone } \\
\text { Fine-grained, limy sandstone } \\
\text { Fine-grained, limy sandstone } \\
\text { Fine-grained sandstone } \\
\text { Medium-grained sandstone }\end{array}$ \\
\hline $\begin{array}{l}\text { sb04-99-12aaa } \\
\text { sb04-99-12aaa } \\
\text { sb04-99-12aaa } \\
\text { sb04-99-12aaa } \\
\text { sb04-99-12aaa }\end{array}$ & $\begin{array}{l}8,820.0 \\
8,821.0 \\
8,822.0 \\
8,823.0 \\
8,824.0\end{array}$ & $\begin{array}{l}8,821.0 \\
8,822.0 \\
8,823.0 \\
8,824.0 \\
8,825.0\end{array}$ & $\begin{array}{l}321 \mathrm{WEBR} \\
321 \mathrm{WEBR} \\
321 \mathrm{WEBR} \\
321 \mathrm{WEBR} \\
321 \mathrm{WEBR}\end{array}$ & $\begin{array}{l}10.3 \\
11.8 \\
10.9 \\
10.9 \\
11.7\end{array}$ & $\begin{array}{l}0.900000 \\
0.100000 \\
0.100000 \\
1.000000 \\
0.200000\end{array}$ & $\begin{array}{l}\text { Medium-grained sandstone } \\
\text { Medium-grained sandstone } \\
\text { Medium-grained sandstone } \\
\text { Medium-grained sandstone } \\
\text { Medium-grained sandstone }\end{array}$ \\
\hline $\begin{array}{l}\text { sb04-99-12aaa } \\
\text { sb04-99-12aaa } \\
\text { sb04-99-12aaa } \\
\text { sb04-99-12a a } \\
\text { sb04-99-12aaa }\end{array}$ & $\begin{array}{l}8,825.0 \\
8,826.0 \\
8,827.0 \\
8,828.0 \\
8,829.0\end{array}$ & $\begin{array}{l}8,826.0 \\
8,827.0 \\
8,828.0 \\
8,829.0 \\
8,830.0\end{array}$ & $\begin{array}{l}321 \mathrm{WEBR} \\
321 \mathrm{WEBR} \\
321 \mathrm{WEBR} \\
321 \mathrm{WEBR} \\
321 \mathrm{WEBR}\end{array}$ & $\begin{array}{r}10.3 \\
7.9 \\
5.5 \\
5.4 \\
3.5\end{array}$ & $\begin{array}{l}0.100000 \\
0.000000 \\
0.000000 \\
0.000000 \\
0.000000\end{array}$ & $\begin{array}{l}\text { Medium-grained sandstone } \\
\text { Fine-grained, limy sandstone } \\
\text { Fine-grained, limy sandstone } \\
\text { Fine-grained, limy sandstone } \\
\text { Fine-grained, limy sandstone }\end{array}$ \\
\hline $\begin{array}{l}\text { sb04-99-12aaa } \\
\text { sb04-99-12aaa } \\
\text { sb04-99-12aaa } \\
\text { sb04-99-12aaa } \\
\text { sb04-99-12aaa }\end{array}$ & $\begin{array}{l}8,830.0 \\
8,831.0 \\
8,832.0 \\
8,833.0 \\
8,834.0\end{array}$ & $\begin{array}{l}8,831.0 \\
8,832.0 \\
8,833.0 \\
8,834.0 \\
8,835.0\end{array}$ & $\begin{array}{l}\text { 321WEBR } \\
321 \mathrm{WEBR} \\
321 \mathrm{WEBR} \\
321 \mathrm{WEBR} \\
321 \mathrm{WEBR}\end{array}$ & $\begin{array}{r}6.1 \\
9.3 \\
7.6 \\
6.7 \\
10.7\end{array}$ & $\begin{array}{l}0.000000 \\
0.000000 \\
0.000000 \\
0.000000 \\
0.600000\end{array}$ & $\begin{array}{l}\text { Fine-grained, limy sandstone } \\
\text { Fine-grained sandstone } \\
\text { Medium-grained sandstone } \\
\text { Medium-grained sandstone } \\
\text { Medium-grained sandstone }\end{array}$ \\
\hline $\begin{array}{l}\text { sb04-99-12aaa } \\
\text { sb04-99-12aaa } \\
\text { sb04-99-12aaa } \\
\text { sb04-99-12aaa } \\
\text { sb04-99-12aaa }\end{array}$ & $\begin{array}{l}8,835.0 \\
8,836.0 \\
8,837.0 \\
8,838.0 \\
8,839.0\end{array}$ & $\begin{array}{l}8,836.0 \\
8,837.0 \\
8,838.0 \\
8,839.0 \\
8,840.0\end{array}$ & $\begin{array}{l}321 \mathrm{WEBR} \\
321 \mathrm{WEBR} \\
321 \mathrm{WEBR} \\
321 \mathrm{WEBR} \\
321 \mathrm{WEBR}\end{array}$ & $\begin{array}{r}11.1 \\
11.9 \\
11.8 \\
9.5 \\
13.5\end{array}$ & $\begin{array}{r}0.100000 \\
1.200000 \\
1.700000 \\
0.300000 \\
10.000000\end{array}$ & $\begin{array}{l}\text { Medium-grained sandstone } \\
\text { Medium-grained sandstone } \\
\text { Medium-grained sandstone } \\
\text { Medium-grained sandstone } \\
\text { Medium-grained sandstone }\end{array}$ \\
\hline $\begin{array}{l}\text { sb04-99-12aaa } \\
\text { sb04-99-12aaa } \\
\text { sb04-99-12aaa } \\
\text { sb04-99-12aaa } \\
\text { sb04-99-12aaa }\end{array}$ & $\begin{array}{l}8,840.0 \\
8,841.0 \\
8,842.0 \\
8,843.0 \\
8,844.0\end{array}$ & $\begin{array}{l}8,841.0 \\
8,842.0 \\
8,843.0 \\
8,844.0 \\
8,845.0\end{array}$ & $\begin{array}{l}321 \mathrm{WEBR} \\
321 \mathrm{WEBR} \\
321 \mathrm{WEBR} \\
321 \mathrm{WEBR} \\
321 \mathrm{WEBR}\end{array}$ & $\begin{array}{r}11.7 \\
9.6 \\
7.7 \\
9.4 \\
7.9\end{array}$ & $\begin{array}{l}4.300000 \\
1.000000 \\
0.100000 \\
1.000000 \\
0.000000\end{array}$ & $\begin{array}{l}\text { Medium-grained sandstone } \\
\text { Medium-grained sandstone } \\
\text { Medium-grained sandstone } \\
\text { Limy, medium-grained sandstone } \\
\text { Fine-grained, limy sandstone }\end{array}$ \\
\hline $\begin{array}{l}\text { sb04-99-12aaa } \\
\text { sb04-99-12aaa } \\
\text { sb04-99-12a a a } \\
\text { sb04-99-12a a } \\
\text { sb04-99-12aa a }\end{array}$ & $\begin{array}{l}8,845.0 \\
8,846.0 \\
8,856.0 \\
8,857.0 \\
8,858.0\end{array}$ & $\begin{array}{l}8,846.0 \\
8,847.0 \\
8,857.0 \\
8,858.0 \\
8,859.0\end{array}$ & $\begin{array}{l}\text { 321WEBR } \\
321 \mathrm{WEBR} \\
321 \mathrm{WEBR} \\
321 \mathrm{WEBR} \\
\text { 321WEBR }\end{array}$ & $\begin{array}{r}6.1 \\
7.0 \\
11.5 \\
8.4 \\
5.7\end{array}$ & $\begin{array}{r}0.000000 \\
0.000000 \\
20.000000 \\
6.900000 \\
0.000000\end{array}$ & $\begin{array}{l}\text { Fine-grained, limy sandstone } \\
\text { Fine-grained, limy sandstone } \\
\text { Medium-grained sandstone } \\
\text { Medium-grained sandstone } \\
\text { Fine-grained, limy sandstone }\end{array}$ \\
\hline $\begin{array}{l}\text { sb04-99-12aaa } \\
\text { sb04-99-12aaa } \\
\text { sb04-99-12aaa } \\
\text { sb04-99-12aaa } \\
\text { sb04-99-12aaa }\end{array}$ & $\begin{array}{l}8,859.0 \\
8,860.0 \\
8,861.0 \\
8,862.0 \\
8,863.0\end{array}$ & $\begin{array}{l}8,860.0 \\
8,861.0 \\
8,862.0 \\
8,863.0 \\
8,864.0\end{array}$ & $\begin{array}{l}321 \mathrm{WEBR} \\
321 \mathrm{WEBR} \\
321 \mathrm{WEBR} \\
321 \mathrm{WEBR} \\
321 \mathrm{WEBR}\end{array}$ & $\begin{array}{l}4.8 \\
4.4 \\
5.5 \\
4.4 \\
3.8\end{array}$ & $\begin{array}{l}0.000000 \\
0.000000 \\
0.000000 \\
0.000000 \\
0.000000\end{array}$ & $\begin{array}{l}\text { Fine-grained, limy sandstone } \\
\text { Fine-grained, limy sandstone } \\
\text { Fine-grained, limy sandstone } \\
\text { Fine-grained, limy sandstone } \\
\text { Fine-grained, limy sandstone }\end{array}$ \\
\hline
\end{tabular}


Table 4.--Laboratory-determined porosity and permeability--Continued

\begin{tabular}{|c|c|c|c|c|c|c|}
\hline Site & $\begin{array}{l}\text { Depth to } \\
\text { top } \\
\text { (feet) }\end{array}$ & $\begin{array}{l}\text { Depth to } \\
\text { bottom } \\
\text { (feet) }\end{array}$ & $\begin{array}{c}\text { Forma- } \\
\text { tion }\end{array}$ & $\begin{array}{l}\text { Porosity } \\
\text { (percent) }\end{array}$ & $\begin{array}{l}\text { Perme- } \\
\text { ability } \\
\text { (milli- } \\
\text { darcies) }\end{array}$ & Lithology \\
\hline $\begin{array}{l}\text { sb04-99-12a aa } \\
\text { sb04-99-12a a a } \\
\text { sb04-99-12a aa } \\
\text { sb04-99-12a aa } \\
\text { sb04-99-12a aa }\end{array}$ & $\begin{array}{l}8,864.0 \\
8,865.0 \\
8,866.0 \\
8,867.0 \\
8,868.0\end{array}$ & $\begin{array}{l}8,865.0 \\
8,866.0 \\
8,867.0 \\
8,868.0 \\
8,869.0\end{array}$ & $\begin{array}{l}321 \mathrm{WEBR} \\
321 \mathrm{WEBR} \\
321 \mathrm{WEBR} \\
321 \mathrm{WEBR} \\
321 \mathrm{WEBR}\end{array}$ & $\begin{array}{l}4.4 \\
4.0 \\
4.2 \\
4.2 \\
2.8\end{array}$ & $\begin{array}{l}1.200000 \\
0.000000 \\
0.000000 \\
0.000000 \\
0.000000\end{array}$ & $\begin{array}{l}\text { Fine-grained, limy sandstone } \\
\text { Fine-grained, limy sandstone } \\
\text { Fine-grained, limy sandstone } \\
\text { Fine-grained, limy sandstone } \\
\text { Fine-grained, limy sandstone }\end{array}$ \\
\hline $\begin{array}{l}\text { sb04-99-12a aa } \\
\text { sb04-99-12a aa } \\
\text { sb04-99-12a aa }\end{array}$ & $\begin{array}{l}8,869.0 \\
8,876.0 \\
8,885.0\end{array}$ & $\begin{array}{l}8,870.0 \\
8,877.0 \\
8,886.0\end{array}$ & $\begin{array}{l}\text { 321WEBR } \\
321 \mathrm{WEBR} \\
\text { 321WEBR }\end{array}$ & $\begin{array}{l}4.8 \\
5.4 \\
5.5 \\
\end{array}$ & $\begin{array}{l}0.000000 \\
0.000000 \\
0.000000 \\
\end{array}$ & $\begin{array}{l}\text { Fine-grained, limy sandstone } \\
\text { Fine-grained, limy sandstone } \\
\text { Fine-grained, limy sandstone }\end{array}$ \\
\hline SITE AVERAGE- & 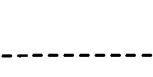 & ----- & AVERAGE-- & $\frac{7.4}{7.4}$ & $\frac{0.964151}{0.964151}$ & \\
\hline $\begin{array}{l}\text { sb05-95-02bab } \\
\text { sb05-95-02bab } \\
\text { sb05-95-02bab } \\
\text { sb05-95-02bab } \\
\text { sb05-95-02bab }\end{array}$ & $\begin{array}{l}2,199.0 \\
2,200.0 \\
2,201.0 \\
2,202.0 \\
2,208.0\end{array}$ & $\begin{array}{l}2,200.0 \\
2,201.0 \\
2,202.0 \\
2,203.0 \\
2,209.0\end{array}$ & $\begin{array}{l}321 \mathrm{WEBR} \\
321 \mathrm{WEBR} \\
321 \mathrm{WEBR} \\
321 \mathrm{WEBR} \\
321 \mathrm{WEBR}\end{array}$ & $\begin{array}{r}14.3 \\
13.2 \\
4.3 \\
12.7 \\
11.0\end{array}$ & $\begin{array}{r}41.000000 \\
19.000000 \\
0.000000 \\
13.000000 \\
3.200000\end{array}$ & $\begin{array}{l}\text { Fine-grained sandstone } \\
\text { Fine-grained sandstone } \\
\text { Fine-grained sandstone } \\
\text { Fine-grained sandstone } \\
\text { Fine-grained sandstone and siltstone }\end{array}$ \\
\hline $\begin{array}{l}\text { sb05-95-02bab } \\
\text { sb05-95-02bab } \\
\text { sb05-95-02bab } \\
\text { sb05-95-02bab } \\
\text { sb05-95-02bab }\end{array}$ & $\begin{array}{l}2,212.0 \\
2,225.0 \\
2,227.0 \\
2,230.0 \\
2,236.0\end{array}$ & $\begin{array}{l}2,213.0 \\
2,226.0 \\
2,228.0 \\
2,231.0 \\
2,237.0\end{array}$ & $\begin{array}{l}\text { 321WEBR } \\
321 \mathrm{WEBR} \\
321 \mathrm{WEBR} \\
321 \mathrm{WEBR} \\
321 \mathrm{WEBR}\end{array}$ & $\begin{array}{l}9.6 \\
5.4 \\
7.0 \\
6.5 \\
8.4\end{array}$ & $\begin{array}{l}0.600000 \\
0.000000 \\
0.000000 \\
0.000000 \\
0.300000\end{array}$ & $\begin{array}{l}\text { Fine-grained sandstone and siltstone } \\
\text { Fine-grained sandstone and siltstone } \\
\text { Fine-grained sandstone and siltstone } \\
\text { Siltstone } \\
\text { Fine-grained sandstone }\end{array}$ \\
\hline $\begin{array}{l}\text { sb05-95-02bab } \\
\text { sb05-95-02bab } \\
\text { sb05-95-02bab } \\
\text { sb05-95-02bab } \\
\text { sb05-95-02bab }\end{array}$ & $\begin{array}{l}2,237.0 \\
2,238.0 \\
2,239.0 \\
2,240.0 \\
2,243.0\end{array}$ & $\begin{array}{l}2,238.0 \\
2,239.0 \\
2,240.0 \\
2,241.0 \\
2,244.0\end{array}$ & $\begin{array}{l}321 \mathrm{WEBR} \\
321 \mathrm{WEBR} \\
321 \mathrm{WEBR} \\
321 \mathrm{WEBR} \\
321 \mathrm{WEBR}\end{array}$ & $\begin{array}{l}7.3 \\
4.3 \\
5.1 \\
5.3 \\
5.3\end{array}$ & $\begin{array}{l}0.000000 \\
0.000000 \\
0.000000 \\
0.000000 \\
0.000000\end{array}$ & $\begin{array}{l}\text { Fine-grained sandstone } \\
\text { Fine-grained sandstone } \\
\text { Fine-grained sandstone } \\
\text { Fine-grained sandstone } \\
\text { Fine-grained sandstone and siltstone }\end{array}$ \\
\hline $\begin{array}{l}\text { sb05-95-02bab } \\
\text { sb05-95-02bab } \\
\text { sb05-95-02bab } \\
\text { sb05-95-02bab } \\
\text { sb05-95-02bab }\end{array}$ & $\begin{array}{l}2,351.0 \\
2,352.0 \\
2,353.0 \\
2,354.0 \\
2,355.0\end{array}$ & $\begin{array}{l}2,352.0 \\
2,353.0 \\
2,354.0 \\
2,355.0 \\
2,356.0\end{array}$ & $\begin{array}{l}\text { 321WEBR } \\
321 \mathrm{WEBR} \\
321 \mathrm{WEBR} \\
321 \mathrm{WEBR} \\
\text { 321WEBR }\end{array}$ & $\begin{array}{l}8.5 \\
9.8 \\
8.0 \\
7.8 \\
6.3\end{array}$ & $\begin{array}{l}0.030000 \\
0.050000 \\
0.030000 \\
0.020000 \\
0.000000\end{array}$ & $\begin{array}{l}\text { Fine- to medium-grained quartz sandstone } \\
\text { Fine- to medium-grained quartz sandstone } \\
\text { Fine- to medium-grained quartz sandstone } \\
\text { Fine- to medium-grained quartz sandstone } \\
\text { Fine- to medium-grained quartz sandstone }\end{array}$ \\
\hline $\begin{array}{l}\text { sb05-95-02bab } \\
\text { sb05-95-02bab } \\
\text { sb05-95-02bab } \\
\text { sb05-95-02bab } \\
\text { sb05-95-02bab }\end{array}$ & $\begin{array}{l}2,356.0 \\
2,357.0 \\
2,358.0 \\
2,359.0 \\
2,360.0\end{array}$ & $\begin{array}{l}2,357.0 \\
2,358.0 \\
2,359.0 \\
2,360.0 \\
2,361.0\end{array}$ & $\begin{array}{l}321 \mathrm{WEBR} \\
321 \mathrm{WEBR} \\
321 \mathrm{WEBR} \\
321 \mathrm{WEBR} \\
\text { 321WEBR }\end{array}$ & $\begin{array}{r}8.6 \\
9.3 \\
12.1 \\
11.0 \\
8.5\end{array}$ & $\begin{array}{l}0.030000 \\
0.050000 \\
1.100000 \\
0.370000 \\
0.030000\end{array}$ & $\begin{array}{l}\text { Fine- to medium-grained quartz sandstone } \\
\text { Fine- to medium-grained quartz sandstone } \\
\text { Fine- to medium-grained quartz sandstone } \\
\text { Fine- to medium-grained quartz sandstone } \\
\text { Fine- to medium-grained quartz sandstone }\end{array}$ \\
\hline $\begin{array}{l}\text { sb05-95-02bab } \\
\text { sb05-95-02bab } \\
\text { sb05-95-02bab } \\
\text { sb05-95-02bab } \\
\text { sb05-95-02bab }\end{array}$ & $\begin{array}{l}2,362.0 \\
2,365.0 \\
2,368.0 \\
2,371.0 \\
2,375.0\end{array}$ & $\begin{array}{l}2,363.0 \\
2,366.0 \\
2,369.0 \\
2,372.0 \\
2,376.0\end{array}$ & $\begin{array}{l}321 \mathrm{WEBR} \\
321 \mathrm{WEBR} \\
321 \mathrm{WEBR} \\
321 \mathrm{WEBR} \\
321 \mathrm{WEBR}\end{array}$ & $\begin{array}{r}9.1 \\
12.0 \\
9.9 \\
9.8 \\
10.1\end{array}$ & $\begin{array}{l}0.170000 \\
0.250000 \\
0.050000 \\
0.030000 \\
0.050000\end{array}$ & $\begin{array}{l}\text { Fine- to medium-grained quartz sandstone } \\
\text { Fine- to medium-grained quartz sandstone } \\
\text { Fine- to medium-grained quartz sandstone } \\
\text { Fine- to medium-grained quartz sandstone } \\
\text { Fine- to medium-grained quartz sandstone }\end{array}$ \\
\hline
\end{tabular}


Table 4.--Laboratory-determined porosity and permeability--Continued

\begin{tabular}{|c|c|c|c|c|c|c|}
\hline Site & $\begin{array}{l}\text { Depth to } \\
\text { top } \\
\text { (feet) }\end{array}$ & $\begin{array}{l}\text { Depth to } \\
\text { bottom } \\
\text { (feet) }\end{array}$ & $\begin{array}{c}\text { Forma- } \\
\text { tion }\end{array}$ & $\begin{array}{l}\text { Porosity } \\
\text { (percent) }\end{array}$ & $\begin{array}{l}\text { Perme- } \\
\text { ability } \\
\text { (milli- } \\
\text { darcies) }\end{array}$ & Lithology \\
\hline $\begin{array}{l}\text { sb05-95-02bab } \\
\text { sb05-95-02bab } \\
\text { sb05-95-02bab }\end{array}$ & $\begin{array}{l}2,377.0 \\
2,381.0 \\
2,384.0\end{array}$ & $\begin{array}{l}2,378.0 \\
2,382.0 \\
2,385.0\end{array}$ & $\begin{array}{l}321 \mathrm{WEBR} \\
321 \mathrm{WEBR} \\
321 \mathrm{WEBR}\end{array}$ & $\begin{array}{r}7.3 \\
10.9 \\
11.3 \\
\end{array}$ & $\begin{array}{l}0.020000 \\
0.330000 \\
0.050000 \\
\end{array}$ & $\begin{array}{l}\text { Fine- to medium-grained quartz sandstone } \\
\text { Shaly, gravelly, dolomitic sandstone } \\
\text { Shaly, gravelly, dolomitic sandstone }\end{array}$ \\
\hline SITE AVERAGE-- & & & AVERAGE-- & $\frac{8.0}{8.0}$ & $\frac{1.865349}{1.865349}$ & \\
\hline $\begin{array}{l}\text { sb05-95-19bcb } \\
\text { sb05-95-19bcb }\end{array}$ & $\begin{array}{l}6,976.5 \\
6,984.0\end{array}$ & $\begin{array}{l}6,978: 0 \\
6,985: 0\end{array}$ & $\begin{array}{l}\text { 317PRKC } \\
\text { 317PRKC }\end{array}$ & $\begin{array}{l}6.5 \\
1.8 \\
\end{array}$ & $\begin{array}{l}0.030000 \\
0.010000 \\
\end{array}$ & $\begin{array}{l}\text { Shaly dolomite } \\
\text { Shaly dolomite }\end{array}$ \\
\hline & & & AVERAGE-- & 4.2 & 0.020000 & \\
\hline $\begin{array}{l}\text { sb05-95-19bcb } \\
\text { sb05-95-19bcb } \\
\text { sb05-95-19bcb } \\
\text { sb05-95-19bcb } \\
\text { sb05-95-19bcb }\end{array}$ & $\begin{array}{l}7,109.0 \\
7,112.0 \\
7,115.0 \\
7,116.0 \\
7,118.0\end{array}$ & $\begin{array}{l}7,110.0 \\
7,113.0 \\
7,116.0 \\
7,117.0 \\
7,119.0\end{array}$ & $\begin{array}{l}321 \text { WEBR } \\
321 \text { WEBR } \\
321 \text { WEBR } \\
321 \text { WEBR } \\
321 \text { WEBR }\end{array}$ & $\begin{array}{l}3.9 \\
3.1 \\
3.7 \\
2.3 \\
4.5 \\
\end{array}$ & $\begin{array}{l}0.010000 \\
0.010000 \\
0.030000 \\
0.010000 \\
0.020000 \\
\end{array}$ & $\begin{array}{l}\text { Silty, quartz sandstone } \\
\text { Silty, quartz sandstone } \\
\text { Fine-grained sandstone } \\
\text { Fine-grained sandstone } \\
\text { Fine-grained sandstone }\end{array}$ \\
\hline SITE AVERAGE- & & & AVERAGE-- & $\frac{3.5}{3.7}$ & $\frac{0.016000}{0.017143}$ & \\
\hline $\begin{array}{l}\text { sb13-105-35adb } \\
\text { sb13-105-35adb } \\
\text { sb13-105-35adb } \\
\text { sb13-105-35adb } \\
\text { sb13-105-35adb }\end{array}$ & $\begin{array}{l}14,699.0 \\
14,701.0 \\
14,702.0 \\
14,703.0 \\
14,704.0\end{array}$ & $\begin{array}{l}14,700.0 \\
14,702.0 \\
14,703.0 \\
14,704.0 \\
14,705.0\end{array}$ & $\begin{array}{l}\text { 317PRKC } \\
317 \text { PRKC } \\
317 \text { PRKC } \\
317 \text { PRKC } \\
317 \text { PRKC }\end{array}$ & $\begin{array}{l}2.9 \\
1.9 \\
1.7 \\
2.1 \\
2.0\end{array}$ & $\begin{array}{l}0.000000 \\
0.500000 \\
0.000000 \\
0.200000 \\
0.100000\end{array}$ & $\begin{array}{l}\text { Finely crystalline, limy dolomite } \\
\text { Finely crystalline, limy dolomite } \\
\text { Finely crystalline, limy dolomite } \\
\text { Finely crystalline, limy dolomite } \\
\text { Shaly, finely crystalline, limy dolomite }\end{array}$ \\
\hline $\begin{array}{l}\text { sb13-105-35adb } \\
\text { sb13-105-35adb } \\
\text { sb13-105-35adb } \\
\text { sb13-105-35adb } \\
\text { sb13-105-35adb }\end{array}$ & $\begin{array}{l}14,705.0 \\
14,706.0 \\
14,707.0 \\
14,708.0 \\
14,709.0\end{array}$ & $\begin{array}{l}14,706.0 \\
14,707.0 \\
14,708.0 \\
14,709.0 \\
14,710.0\end{array}$ & $\begin{array}{l}317 \text { PRKC } \\
317 \text { PRKC } \\
317 \text { PRKC } \\
317 \text { PRKC } \\
\text { 317PRKC }\end{array}$ & $\begin{array}{l}1.8 \\
2.9 \\
2.5 \\
3.7 \\
4.7\end{array}$ & $\begin{array}{l}0.000000 \\
0.000000 \\
0.000000 \\
0.000000 \\
0.000000\end{array}$ & $\begin{array}{l}\text { Finely crystalline, limy dolomite } \\
\text { Finely crystalline, limy dolomite } \\
\text { Finely crystalline, limy dolomite } \\
\text { Shaly, finely crystalline, limy dolomite } \\
\text { Finely crystalline, limy dolomite }\end{array}$ \\
\hline $\begin{array}{l}\text { sb } 13-105-35 a d b \\
\text { sb13-105-35adb } \\
\text { sb13-105-35adb } \\
\text { sb13-105-35adb } \\
\text { sb13-105-35adb }\end{array}$ & $\begin{array}{l}14,712.0 \\
14,713.0 \\
14,714.0 \\
14,715.0 \\
14,727.0\end{array}$ & $\begin{array}{l}14,713.0 \\
14,714.0 \\
14,715.0 \\
14,716.0 \\
14,728.0\end{array}$ & $\begin{array}{l}317 \text { PRKC } \\
317 \text { PRKC } \\
317 \text { PRKC } \\
317 \text { PRKC } \\
\text { 317PRKC }\end{array}$ & $\begin{array}{l}2.2 \\
2.2 \\
1.9 \\
4.5 \\
3.5\end{array}$ & $\begin{array}{l}0.000000 \\
0.000000 \\
0.000000 \\
0.000000 \\
0.000000\end{array}$ & $\begin{array}{l}\text { Finely crystalline, limy dolomite } \\
\text { Anhydritic, finely crystalline dolomite } \\
\text { Finely crystalline, limy dolomite } \\
\text { Finely crystalline, limy dolomite } \\
\text { Finely crystalline, limy dolomite }\end{array}$ \\
\hline $\begin{array}{l}\text { sb } 13-105-35 a d b \\
\text { sb13-105-35adb } \\
\text { sb13-105-35adb } \\
\text { sb13-105-35adb } \\
\text { sb13-105-35adb }\end{array}$ & $\begin{array}{l}14,728.0 \\
14,729.0 \\
14,730.0 \\
14,731.0 \\
14,732.0\end{array}$ & $\begin{array}{l}14,729.0 \\
14,730.0 \\
14,731.0 \\
14,732.0 \\
14,733.0\end{array}$ & $\begin{array}{l}317 \text { PRKC } \\
317 \text { PRKC } \\
317 \text { PRKC } \\
317 \text { PRKC } \\
317 \text { PRKC }\end{array}$ & $\begin{array}{l}3.6 \\
5.3 \\
2.7 \\
2.2 \\
4.2\end{array}$ & $\begin{array}{l}0.000000 \\
0.000000 \\
0.000000 \\
0.000000 \\
0.000000\end{array}$ & $\begin{array}{l}\text { Finely crystalline, limy dolomite } \\
\text { Finely crystalline, limy dolomite } \\
\text { Finely crystalline, limy dolomite } \\
\text { Finely crystalline, limy dolomite } \\
\text { Finely crystalline, limy dolomite }\end{array}$ \\
\hline $\begin{array}{l}\text { sb13-105-35adb } \\
\text { sb13-105-35adb } \\
\text { sb13-105-35adb } \\
\text { sb13-105-35adb } \\
\text { sb13-105-35adb }\end{array}$ & $\begin{array}{l}14,733.0 \\
14,734.0 \\
14,735.0 \\
14,736.0 \\
14,737.0\end{array}$ & $\begin{array}{l}14,734.0 \\
14,735.0 \\
14,736.0 \\
14,737.0 \\
14,738.0\end{array}$ & $\begin{array}{l}317 \text { PRKC } \\
317 \text { PRKC } \\
317 \text { PRKC } \\
317 \text { PRKC } \\
317 \text { PRKC }\end{array}$ & $\begin{array}{l}2.5 \\
3.8 \\
3.9 \\
2.6 \\
3.6\end{array}$ & $\begin{array}{l}0.000000 \\
0.000000 \\
0.000000 \\
0.000000 \\
0.000000\end{array}$ & $\begin{array}{l}\text { Finely crystalline, limy dolomite } \\
\text { Finely crystalline, limy dolomite } \\
\text { Finely crystalline, limy dolomite } \\
\text { Finely crystalline, limy dolomite } \\
\text { Finely crystalline, limy dolomite }\end{array}$ \\
\hline $\begin{array}{l}\text { sb } 13-105-35 a d b \\
\text { sb13-105-35adb } \\
\text { sb13-105-35adb } \\
\text { sb13-105-35adb } \\
\text { sb13-105-35adb }\end{array}$ & $\begin{array}{l}14,738.0 \\
14,739.0 \\
14,740.0 \\
14,741.0 \\
14,742.0\end{array}$ & $\begin{array}{l}14,739.0 \\
14,740.0 \\
14,741.0 \\
14,742.0 \\
14,743.0\end{array}$ & $\begin{array}{l}317 \text { PRKC } \\
317 \text { PRKC } \\
317 \text { PRKC } \\
317 \text { PRKC } \\
\text { 317PRKC }\end{array}$ & $\begin{array}{l}2.7 \\
3.8 \\
4.3 \\
4.2 \\
2.7\end{array}$ & $\begin{array}{l}0.000000 \\
0.000000 \\
0.000000 \\
0.000000 \\
0.000000\end{array}$ & $\begin{array}{l}\text { Finely crystalline, limy dolomite } \\
\text { Finely crystalline, limy dolomite } \\
\text { Finely crystalline, limy dolomite } \\
\text { Finely crystalline, limy dolomite } \\
\text { Finely crystalline, limy dolomite }\end{array}$ \\
\hline $\begin{array}{l}\text { sb13-105-35adb } \\
\text { sb13-105-35adb } \\
\text { sb } 13-105-35 a d b \\
\text { sb13-105-35adb } \\
\text { sb13-105-35adb }\end{array}$ & $\begin{array}{l}14,778.0 \\
14,779.0 \\
14,781.0 \\
14,782.0 \\
14,783.0\end{array}$ & $\begin{array}{l}14,779.0 \\
14,780.0 \\
14,782.0 \\
14,783.0 \\
14,784.0\end{array}$ & $\begin{array}{l}\text { 317PRKC } \\
317 \mathrm{PRKC} \\
317 \mathrm{PRKC} \\
317 \mathrm{PRKC} \\
317 \mathrm{PRKC}\end{array}$ & $\begin{array}{l}2.0 \\
1.3 \\
0.8 \\
1.0 \\
0.8\end{array}$ & $\begin{array}{l}0.000000 \\
0.000000 \\
0.000000 \\
0.000000 \\
0.000000\end{array}$ & $\begin{array}{l}\text { Finely crystalline, limy dolomite } \\
\text { Anhydritic, finely crystalline, limy dolomite } \\
\text { Anhydritic, finely crystalline, limy dolomite } \\
\text { Anhydritic, finely crystalline, limy dolomite } \\
\text { Anhydritic, finely crystalline, limy dolomite }\end{array}$ \\
\hline
\end{tabular}


Table 4.--Laboratory-determined porosity and permeability--Continued

\begin{tabular}{|c|c|c|c|c|c|c|}
\hline Site & $\begin{array}{l}\text { Depth to } \\
\text { top } \\
\text { (feet) }\end{array}$ & $\begin{array}{l}\text { Depth to } \\
\text { bottom } \\
\text { (feet) }\end{array}$ & $\begin{array}{l}\text { Forma- } \\
\text { tion }\end{array}$ & $\begin{array}{l}\text { Porosity } \\
\text { (percent) }\end{array}$ & $\begin{array}{l}\text { Perme- } \\
\text { ability } \\
\text { (milli- } \\
\text { darcies) }\end{array}$ & Lithology \\
\hline $\begin{array}{l}\text { sb13-105-35adb } \\
\text { sb13-105-35adb } \\
\text { sb13-105-35adb } \\
\text { sb13-105-35adb } \\
\text { sb13-105-35adb }\end{array}$ & $\begin{array}{l}14,784.0 \\
14,785.0 \\
14,786.0 \\
14,787.0 \\
14,788.0\end{array}$ & $\begin{array}{l}14,785.0 \\
14,786.0 \\
14,787.0 \\
14,788.0 \\
14,789.0\end{array}$ & $\begin{array}{l}317 \text { PRKC } \\
317 \text { PRKC } \\
317 \text { PRKC } \\
317 \text { PRKC } \\
317 \text { PRKC }\end{array}$ & $\begin{array}{l}1.3 \\
1.7 \\
2.2 \\
2.9 \\
1.9\end{array}$ & $\begin{array}{l}0.000000 \\
0.000000 \\
0.000000 \\
0.000000 \\
0.000000\end{array}$ & $\begin{array}{l}\text { Anhydritic, finely crystalline, limy dolomite } \\
\text { Finely crystalline, limy dolomite } \\
\text { Finely crystalline, limy dolomite } \\
\text { Finely crystalline, limy dolomite } \\
\text { Finely crystalline, limy dolomite }\end{array}$ \\
\hline $\begin{array}{l}\text { sb13-105-35adb } \\
\text { sb13-105-35adb } \\
\text { sb13-105-35adb } \\
\text { sb13-105-35adb } \\
\text { sb13-105-35adb }\end{array}$ & $\begin{array}{l}14,789.0 \\
14,790.0 \\
14,791.0 \\
14,807.0 \\
14,809.0\end{array}$ & $\begin{array}{r}14,790.0 \\
4,791.0 \\
14,792.0 \\
14,808.0 \\
14,810.0\end{array}$ & $\begin{array}{l}317 \mathrm{PRKC} \\
317 \mathrm{PRKC} \\
317 \mathrm{PRKC} \\
317 \mathrm{PRKC} \\
317 \mathrm{PRKC}\end{array}$ & $\begin{array}{l}2.1 \\
1.0 \\
1.3 \\
1.2 \\
1.6\end{array}$ & $\begin{array}{l}0.000000 \\
0.000000 \\
0.000000 \\
0.000000 \\
0.000000\end{array}$ & $\begin{array}{l}\text { Anhydritic, finely crystalline, limy dolomite } \\
\text { Anhydritic, finely crystalline, limy dolomite } \\
\text { Anhydritic, finely crystalline, limy dolomite } \\
\text { Anhydritic, finely crystalline, limy dolomite } \\
\text { Chalky, carbonaceous, finely crystalline dolomite }\end{array}$ \\
\hline \multirow[t]{2}{*}{$\begin{array}{l}\text { sb } 13-105-35 a d b \\
\text { sb13-105-35adb } \\
\text { sb13-105-35adb } \\
\text { sb13-105-35adb }\end{array}$} & $\begin{array}{l}14,811.0 \\
14,813.0 \\
14,815.0 \\
14,817.0\end{array}$ & $\begin{array}{l}14,812 \cdot 0 \\
14,814.0 \\
14,816.0 \\
14,818.0\end{array}$ & $\begin{array}{l}\text { 317PRKC } \\
\text { 317PRKC } \\
\text { 317PRKC } \\
\text { 317PRKC }\end{array}$ & $\begin{array}{l}1.5 \\
1.0 \\
1.2 \\
2.2 \\
\end{array}$ & $\begin{array}{l}0.000000 \\
0.000000 \\
0.000000 \\
0.000000 \\
\end{array}$ & $\begin{array}{l}\text { Finely crystalline, limy dolomite } \\
\text { Finely crystalline, limy dolomite } \\
\text { Finely crystalline, limy dolomite } \\
\text { Chalky, finely crystalline dolomite }\end{array}$ \\
\hline & & & AVERAGE-- & 2.5 & 0.016327 & \\
\hline $\begin{array}{l}\text { sb13-105-35adb } \\
\text { sb13-105-35adb } \\
\text { sb13-105-35adb } \\
\text { sb13-105-35adb } \\
\text { sb13-105-35adb }\end{array}$ & $\begin{array}{l}14,855 \cdot 0 \\
14,856.0 \\
14,857.0 \\
14,858.0 \\
14,859.0\end{array}$ & $\begin{array}{l}14,856.0 \\
14,857.0 \\
14,858 \cdot 0 \\
14,859.0 \\
14,860.0\end{array}$ & $\begin{array}{l}\text { 321WEBR } \\
\text { 321WEBR } \\
\text { 321WEBR } \\
\text { 321WEBR } \\
\text { 321WEBR }\end{array}$ & $\begin{array}{l}2.7 \\
2.0 \\
1.3 \\
1.6 \\
1.8\end{array}$ & $\begin{array}{l}0.000000 \\
0.000000 \\
0.000000 \\
0.000000 \\
0.000000\end{array}$ & $\begin{array}{l}\text { Fine-grained, limy sandstone } \\
\text { Fine-grained, limy sandstone } \\
\text { Fine-grained, limy sandstone } \\
\text { Fine-grained, limy sandstone } \\
\text { Fine-grained, limy sandstone }\end{array}$ \\
\hline $\begin{array}{l}\text { sb13-105-35adb } \\
\text { sb13-105-35adb } \\
\text { sb13-105-35adb } \\
\text { sb13-105-35adb } \\
\text { sb13-105-35adb }\end{array}$ & $\begin{array}{l}14,860.0 \\
14,861.0 \\
14,862.0 \\
14,863.0 \\
14,864.0\end{array}$ & $\begin{array}{l}14,861.0 \\
14,862.0 \\
14,863.0 \\
14,864.0 \\
14,865.0\end{array}$ & $\begin{array}{l}\text { 321WEBR } \\
321 \mathrm{WEBR} \\
\text { 321WEBR } \\
\text { 321WEBR } \\
\text { 321WEBR }\end{array}$ & $\begin{array}{l}1.9 \\
2.0 \\
1.4 \\
2.0 \\
3.4\end{array}$ & $\begin{array}{l}0.000000 \\
0.000000 \\
0.000000 \\
0.000000 \\
0.100000\end{array}$ & $\begin{array}{l}\text { Fine-grained, limy sandstone } \\
\text { Fine-grained, limy sandstone } \\
\text { Fine-grained, limy sandstone } \\
\text { Fine-grained, limy sandstone } \\
\text { Fine-grained, limy sandstone }\end{array}$ \\
\hline $\begin{array}{l}\text { sb13-105-35adb } \\
\text { sb13-105-35adb } \\
\text { sb13-105-35adb } \\
\text { sb13-105-35adb } \\
\text { sb13-105-35adb }\end{array}$ & $\begin{array}{l}14,865 \cdot 0 \\
14,866 \cdot 0 \\
14,867 \cdot 0 \\
14,868 \cdot 0 \\
14,869.0\end{array}$ & $\begin{array}{l}14,866.0 \\
14,867.0 \\
14,868.0 \\
14,869.0 \\
14,870.0\end{array}$ & $\begin{array}{l}\text { 321WEBR } \\
\text { 321WEBR } \\
\text { 321WEBR } \\
\text { 321WEBR } \\
\text { 321WEBR }\end{array}$ & $\begin{array}{l}3.5 \\
4.6 \\
3.1 \\
3.2 \\
2.7\end{array}$ & $\begin{array}{l}0.100000 \\
0.100000 \\
0.100000 \\
0.100000 \\
0.100000\end{array}$ & $\begin{array}{l}\text { Fine-grained, limy sandstone } \\
\text { Fine-grained, limy sandstone } \\
\text { Fine-grained, limy sandstone } \\
\text { Fine-grained, limy sandstone } \\
\text { Fine-grained, limy sandstone }\end{array}$ \\
\hline $\begin{array}{l}\text { sb13-105-35adb } \\
\text { sb13-105-35adb } \\
\text { sb13-105-35adb } \\
\text { sb13-105-35adb } \\
\text { sb13-105-35adb }\end{array}$ & $\begin{array}{l}14,870.0 \\
14,871.0 \\
14,872.0 \\
14,873.0 \\
14,874.0\end{array}$ & $\begin{array}{l}14,871.0 \\
14,872.0 \\
14,873.0 \\
14,874.0 \\
14,875.0\end{array}$ & $\begin{array}{l}321 \mathrm{WEBR} \\
321 \mathrm{WEBR} \\
321 \mathrm{WEBR} \\
321 \mathrm{WEBR} \\
\text { 321WEBR }\end{array}$ & $\begin{array}{l}1.8 \\
2.0 \\
2.2 \\
1.6 \\
1.5\end{array}$ & $\begin{array}{l}0.100000 \\
0.100000 \\
0.100000 \\
0.100000 \\
0.000000\end{array}$ & $\begin{array}{l}\text { Fine-grained, limy sandstone } \\
\text { Fine-grained, limy sandstone } \\
\text { Fine-grained, limy sandstone } \\
\text { Fine-grained sandstone } \\
\text { Fine-grained sandstone }\end{array}$ \\
\hline $\begin{array}{l}\text { sb13-105-35adb } \\
\text { sb13-105-35adb } \\
\text { sb13-105-35adb } \\
\text { sb13-105-35adb } \\
\text { sb13-105-35adb }\end{array}$ & $\begin{array}{l}14,875.0 \\
15,002.0 \\
15,003 \cdot 0 \\
15,004.0 \\
15,005.0\end{array}$ & $\begin{array}{l}14,876.0 \\
15,003.0 \\
15,004.0 \\
15,005.0 \\
15,006.0\end{array}$ & $\begin{array}{l}\text { 321WEBR } \\
321 \mathrm{WEBR} \\
\text { 321WEBR } \\
\text { 321WEBR } \\
\text { 321WEBR }\end{array}$ & $\begin{array}{l}1.0 \\
1.5 \\
1.7 \\
1.0 \\
1.6\end{array}$ & $\begin{array}{l}0.000000 \\
0.000000 \\
0.000000 \\
0.000000 \\
0.000000\end{array}$ & $\begin{array}{l}\text { Fine-grained sandstone } \\
\text { Fine-grained, quartzitic sandstone } \\
\text { Fine-grained, quartzitic sandstone } \\
\text { Fine-grained, quartzitic sandstone } \\
\text { Fine-grained, quartzitic sandstone }\end{array}$ \\
\hline $\begin{array}{l}\text { sb13-105-35adb } \\
\text { sb13-105-35adb } \\
\text { sb13-105-35adb } \\
\text { sb } 13-105-35 a d b\end{array}$ & $\begin{array}{l}15,006.0 \\
15,007.0 \\
15,008.0 \\
15,009.0\end{array}$ & $\begin{array}{l}15,007.0 \\
15,008.0 \\
15,009.0 \\
15,010.0\end{array}$ & $\begin{array}{l}\text { 321WEBR } \\
\text { 321WEBR } \\
\text { 321WEBR } \\
\text { 321WEBR }\end{array}$ & $\begin{array}{l}1.6 \\
2.1 \\
3.4 \\
3.9 \\
\end{array}$ & $\begin{array}{l}0.000000 \\
0.000000 \\
0.100000 \\
0.000000 \\
\end{array}$ & $\begin{array}{l}\text { Fine-grained, quartzitic sandstone } \\
\text { Fine-grained, quartzitic sandstone } \\
\text { Fine-grained, quartzitic sandstone } \\
\text { Fine-grained, quartzitic sandstone }\end{array}$ \\
\hline SITE AVERA & & & AVERAGE-- & $\frac{2.2}{2.4}$ & $\frac{0.037931}{0.024359}$ & \\
\hline $\begin{array}{l}\text { sb14-112-06bab } \\
\text { sb14-112-06bab } \\
\text { sb14-112-06bab }\end{array}$ & $\begin{array}{l}18,358.0 \\
18,359.0 \\
18,360.0\end{array}$ & $\begin{array}{l}18,359.0 \\
18,360.0 \\
18,361.0\end{array}$ & $\begin{array}{l}\text { 324MRGN } \\
324 M R G N \\
324 M R G N\end{array}$ & $\begin{array}{l}1.6 \\
2.3 \\
1.7 \\
\end{array}$ & $\begin{array}{l}0.010000 \\
0.030000 \\
0.010000 \\
\end{array}$ & $\begin{array}{l}\text { Shaly, crystalline, limy dolomite } \\
\text { Shaly, crystalline, limy dolomite } \\
\text { Shaly, crystalline, limy dolomite }\end{array}$ \\
\hline & & & AVERAGE-- & 1.9 & 0.016667 & \\
\hline
\end{tabular}


Table 4.--Laboratory-determined porosity and permeability--Continued

\begin{tabular}{|c|c|c|c|c|c|c|}
\hline Site & $\begin{array}{l}\text { Depth to } \\
\text { top } \\
\text { (feet) }\end{array}$ & $\begin{array}{l}\text { Depth to } \\
\text { bottom } \\
\text { (feet) }\end{array}$ & $\begin{array}{c}\text { Forma- } \\
\text { tion }\end{array}$ & $\begin{array}{l}\text { Porosity } \\
\text { (percent) }\end{array}$ & $\begin{array}{l}\text { Perme- } \\
\text { ability } \\
\text { (milli- } \\
\text { darcies) }\end{array}$ & Lithology \\
\hline $\begin{array}{l}\text { sb14-112-06bab } \\
\text { sb14-112-06bab } \\
\text { sb14-112-06bab } \\
\text { sb14-112-06bab } \\
\text { sb14-112-06bab }\end{array}$ & $\begin{array}{l}18,383.0 \\
18,384.0 \\
18,385.0 \\
18,386.0 \\
18,387.0\end{array}$ & $\begin{array}{l}18,384.0 \\
18,385.0 \\
18,386.0 \\
18,387.0 \\
18,388.0\end{array}$ & $\begin{array}{l}\text { 327RDVL } \\
\text { 327RDVL } \\
\text { 327RDVL } \\
\text { 327RDVL } \\
\text { 327RDVL }\end{array}$ & $\begin{array}{r}2.4 \\
14.3 \\
23.2 \\
22.2 \\
22.0\end{array}$ & $\begin{array}{l}0.010000 \\
7.600000 \\
2.700000 \\
8.600000 \\
6.200000\end{array}$ & $\begin{array}{l}\text { Crystalline dolomite } \\
\text { Sucrose dolomite } \\
\text { Vuggy, sucrose dolomite } \\
\text { Vuggy, sucrose dolomite } \\
\text { Sucrose dolomite }\end{array}$ \\
\hline $\begin{array}{l}\text { sb14-112-06bab } \\
\text { sb14-112-06bab } \\
\text { sb14-112-06bab } \\
\text { sb14-112-06bab } \\
\text { sb14-112-06bab }\end{array}$ & $\begin{array}{l}18,388.0 \\
18,389.0 \\
18,390.0 \\
18,392.0 \\
18,393.0\end{array}$ & $\begin{array}{l}18,389.0 \\
18,390.0 \\
18,391.0 \\
18,393.0 \\
18,394.0\end{array}$ & $\begin{array}{l}\text { 327RDVL } \\
\text { 327RDVL } \\
\text { 327RDVL } \\
\text { 327RDVL } \\
\text { 327RDVL }\end{array}$ & $\begin{array}{r}14.8 \\
11.3 \\
8.4 \\
3.1 \\
2.7\end{array}$ & $\begin{array}{l}6.700000 \\
7.800000 \\
0.070000 \\
0.040000 \\
0.010000\end{array}$ & $\begin{array}{l}\text { Crystalline dolomite } \\
\text { Crystalline dolomite } \\
\text { Crystalline dolomite } \\
\text { Crystalline limestone } \\
\text { Crystalline, dolomitic limestone }\end{array}$ \\
\hline $\begin{array}{l}\text { sb14-112-06bab } \\
\text { sb14-112-06bab } \\
\text { sb14-112-06bab } \\
\text { sb14-112-06bab } \\
\text { sb14-112-06bab }\end{array}$ & $\begin{array}{l}18,394.0 \\
18,395.0 \\
18,396.0 \\
18,397.0 \\
18,398.0\end{array}$ & $\begin{array}{l}18,395.0 \\
18,396.0 \\
18,397.0 \\
18,398.0 \\
18,399.0\end{array}$ & $\begin{array}{l}\text { 327RDVL } \\
\text { 327RDVL } \\
\text { 327RDVL } \\
\text { 327RDVL } \\
\text { 327RDVL }\end{array}$ & $\begin{array}{l}2.9 \\
1.1 \\
2.3 \\
2.6 \\
3.9\end{array}$ & $\begin{array}{l}2.900000 \\
0.220000 \\
0.020000 \\
0.020000 \\
0.070000\end{array}$ & $\begin{array}{l}\text { Crystalline, dolomitic limestone } \\
\text { Crystalline dolomite } \\
\text { Crystalline dolomite } \\
\text { Crystalline dolomite } \\
\text { Crystalline dolomite }\end{array}$ \\
\hline $\begin{array}{l}\text { sb14-112-06bab } \\
\text { sb14-112-06bab } \\
\text { sb14-112-06bab } \\
\text { sb14-112-06bab } \\
\text { sb14-112-06bab }\end{array}$ & $\begin{array}{l}18,399.0 \\
18,400.0 \\
18,401.0 \\
18,402.0 \\
18,403.0\end{array}$ & $\begin{array}{l}18,400.0 \\
18,401.0 \\
18,402.0 \\
18,403.0 \\
18,404.0\end{array}$ & $\begin{array}{l}\text { 327RDVL } \\
\text { 327RDVL } \\
\text { 327RDVL } \\
\text { 327RDVL } \\
\text { 327RDVL }\end{array}$ & $\begin{array}{r}2.6 \\
3.4 \\
10.6 \\
3.8 \\
2.5\end{array}$ & $\begin{array}{l}0.150000 \\
0.010000 \\
0.010000 \\
0.010000 \\
0.010000\end{array}$ & $\begin{array}{l}\text { Crystalline dolomite } \\
\text { Crystalline dolomite } \\
\text { Crystalline dolomite } \\
\text { Crystalline, dolomitic limestone } \\
\text { Crystalline, dolomitic limestone }\end{array}$ \\
\hline $\begin{array}{l}\text { sb14-112-06bab } \\
\text { sb14-112-06bab } \\
\text { sb14-112-06bab } \\
\text { sb14-112-06bab } \\
\text { sb14-112-06bab }\end{array}$ & $\begin{array}{l}18,404.0 \\
18,405.0 \\
18,406.0 \\
18,407.0 \\
18,408.0\end{array}$ & $\begin{array}{l}18,405.0 \\
18,406.0 \\
18,407.0 \\
18,408.0 \\
18,409.0\end{array}$ & $\begin{array}{l}\text { 327RDVL } \\
\text { 327RDVL } \\
\text { 327RDVL } \\
\text { 327RDVL } \\
\text { 327RDVL }\end{array}$ & $\begin{array}{l}2.8 \\
3.1 \\
2.5 \\
2.4 \\
2.5\end{array}$ & $\begin{array}{l}0.010000 \\
0.010000 \\
0.040000 \\
0.070000 \\
0.010000\end{array}$ & $\begin{array}{l}\text { Crystalline, dolomitic limestone } \\
\text { Crystalline, dolomitic limestone } \\
\text { Crystalline, dolomitic limestone } \\
\text { Crystalline dolomite } \\
\text { Crystalline, dolomitic limestone }\end{array}$ \\
\hline $\begin{array}{l}\text { sb14-112-06bab } \\
\text { sb14-112-06bab } \\
\text { sb14-112-06bab } \\
\text { sb14-112-06bab } \\
\text { sb14-112-06bab }\end{array}$ & $\begin{array}{l}18,409.0 \\
18,410.0 \\
18,411.0 \\
18,412.0 \\
18,413.0\end{array}$ & $\begin{array}{l}18,410.0 \\
18,411.0 \\
18,412.0 \\
18,413.0 \\
18,414.0\end{array}$ & $\begin{array}{l}\text { 327RDVL } \\
\text { 327RDVL } \\
\text { 327RDVL } \\
\text { 327RDVL } \\
\text { 327RDVL }\end{array}$ & $\begin{array}{l}2.5 \\
2.1 \\
4.6 \\
2.0 \\
2.6\end{array}$ & $\begin{array}{l}0.020000 \\
0.020000 \\
9.100000 \\
0.010000 \\
0.010000\end{array}$ & $\begin{array}{l}\text { Crystalline limestone } \\
\text { Crystalline dolomite } \\
\text { Crystalline dolomite } \\
\text { Shaly, crystalline dolomite } \\
\text { Shaly, crystalline dolomite }\end{array}$ \\
\hline $\begin{array}{l}\text { sb14-112-06bab } \\
\text { sb14-112-06bab } \\
\text { sb14-112-06bab } \\
\text { sb14-112-06bab } \\
\text { sb14-112-06bab }\end{array}$ & $\begin{array}{l}18,414.0 \\
18,415.0 \\
18,416.0 \\
18,417.0 \\
18,418.0\end{array}$ & $\begin{array}{l}18,415.0 \\
18,416.0 \\
18,417.0 \\
18,418.0 \\
18,419.0\end{array}$ & $\begin{array}{l}\text { 327RDVL } \\
\text { 327RDVL } \\
\text { 327RDVL } \\
\text { 327RDVL } \\
\text { 327RDVL }\end{array}$ & $\begin{array}{l}4.4 \\
3.1 \\
1.9 \\
1.2 \\
3.7\end{array}$ & $\begin{array}{l}0.010000 \\
0.040000 \\
0.040000 \\
0.010000 \\
0.010000\end{array}$ & $\begin{array}{l}\text { Crystalline dolomite } \\
\text { Crystalline, dolomitic limestone } \\
\text { Crystalline limestone } \\
\text { Crystalline dolomite } \\
\text { Crystalline dolomite }\end{array}$ \\
\hline $\begin{array}{l}\text { sb14-112-06bab } \\
\text { sb14-112-06bab } \\
\text { sb14-112-06bab } \\
\text { sb14-112-06bab } \\
\text { sb14-112-06bab }\end{array}$ & $\begin{array}{l}18,419.0 \\
18,420.0 \\
18,421.0 \\
18,422.0 \\
18,423.0\end{array}$ & $\begin{array}{l}18,420.0 \\
18,421.0 \\
18,422.0 \\
18,423.0 \\
18,424.0\end{array}$ & $\begin{array}{l}\text { 327RDVL } \\
\text { 327RDVL } \\
\text { 327RDVL } \\
\text { 327RDVL } \\
\text { 327RDVL }\end{array}$ & $\begin{array}{l}3.1 \\
2.0 \\
1.9 \\
2.0 \\
5.3\end{array}$ & $\begin{array}{l}0.010000 \\
0.010000 \\
0.040000 \\
0.460000 \\
0.010000\end{array}$ & $\begin{array}{l}\text { Crystalline limestone } \\
\text { Crystalline limestone } \\
\text { Crystalline limestone } \\
\text { Fractured, crystalline limestone } \\
\text { Crystalline limestone }\end{array}$ \\
\hline $\begin{array}{l}\text { sb14-112-06bab } \\
\text { sb14-112-06bab } \\
\text { sb14-112-06bab } \\
\text { sb14-112-06bab } \\
\text { sb14-112-06bab }\end{array}$ & $\begin{array}{l}18,424.0 \\
18,425.0 \\
18,426.0 \\
18,427.0 \\
18,428.0\end{array}$ & $\begin{array}{l}18,425.0 \\
18,426.0 \\
18,427.0 \\
18,428.0 \\
18,429.0\end{array}$ & $\begin{array}{l}\text { 327RDVL } \\
\text { 327RDVL } \\
327 R D V L \\
327 R D V L \\
327 R D V L\end{array}$ & $\begin{array}{l}1.3 \\
5.8 \\
5.2 \\
7.0 \\
5.4\end{array}$ & $\begin{array}{l}0.010000 \\
0.200000 \\
0.550000 \\
0.030000 \\
0.020000\end{array}$ & $\begin{array}{l}\text { Crystalline limestone } \\
\text { Crystalline dolomite } \\
\text { Crystalline dolomite } \\
\text { Crystalline dolomite } \\
\text { Crystalline dolomite }\end{array}$ \\
\hline $\begin{array}{l}\text { sb14-112-06bab } \\
\text { sb14-112-06bab } \\
\text { sb14-112-06bab } \\
\text { sb14-112-06bab } \\
\text { sb14-112-06bab }\end{array}$ & $\begin{array}{l}18,429.0 \\
18,430.0 \\
18,431.0 \\
18,432.0 \\
18,433.0\end{array}$ & $\begin{array}{l}18,430.0 \\
18,431.0 \\
18,432.0 \\
18,433.0 \\
18,434.0\end{array}$ & $\begin{array}{l}\text { 327RDVL } \\
\text { 327RDVL } \\
\text { 327RDVL } \\
\text { 327RDVL } \\
\text { 327RDVL }\end{array}$ & $\begin{array}{r}6.6 \\
3.0 \\
8.1 \\
8.5 \\
10.9\end{array}$ & $\begin{array}{r}0.090000 \\
0.070000 \\
15.000000 \\
0.390000 \\
0.010000\end{array}$ & $\begin{array}{l}\text { Crystalline dolomite } \\
\text { Crystalline dolomite } \\
\text { Fractured, crystalline dolomite } \\
\text { Crystalline dolomite } \\
\text { Crystalline dolomite }\end{array}$ \\
\hline
\end{tabular}


Table 4.--Laboratory-determined porosity and permeability--Continued

\begin{tabular}{|c|c|c|c|c|c|c|}
\hline Site & $\begin{array}{l}\text { Depth to } \\
\text { top } \\
\text { (feet) }\end{array}$ & $\begin{array}{l}\text { Depth to } \\
\text { bottom } \\
\text { (feet) }\end{array}$ & $\begin{array}{c}\text { Forma- } \\
\text { tion }\end{array}$ & $\begin{array}{l}\text { Porosity } \\
\text { (percent) }\end{array}$ & $\begin{array}{l}\text { Perme- } \\
\text { ability } \\
\text { (milli- } \\
\text { darcies) }\end{array}$ & Lithology \\
\hline $\begin{array}{l}\text { sb14-112-06bab } \\
\text { sb14-112-06bab } \\
\text { sb14-112-06bab } \\
\text { sb14-112-06bab } \\
\text { sb14-112-06bab }\end{array}$ & $\begin{array}{l}18,489.0 \\
18,490.0 \\
18,491.0 \\
18,492.0 \\
18,493.0\end{array}$ & $\begin{array}{l}18,490.0 \\
18,491.0 \\
18,492.0 \\
18,493.0 \\
18,494.0\end{array}$ & $\begin{array}{l}\text { 327RDVL } \\
\text { 327RDVL } \\
\text { 327RDVL } \\
\text { 327RDVL } \\
\text { 327RDVL }\end{array}$ & $\begin{array}{l}4.7 \\
2.2 \\
4.0 \\
3.4 \\
3.2\end{array}$ & $\begin{array}{l}0.590000 \\
0.000000 \\
0.280000 \\
0.070000 \\
0.010000\end{array}$ & $\begin{array}{l}\text { Crystalline dolomite } \\
\text { Crystalline limestone } \\
\text { Crystalline limestone } \\
\text { Crystalline limestone } \\
\text { Crystalline limestone }\end{array}$ \\
\hline $\begin{array}{l}\text { sb14-112-06bab } \\
\text { sb14-112-06bab } \\
\text { sb14-112-06bab } \\
\text { sb14-112-06bab } \\
\text { sb14-112-06bab }\end{array}$ & $\begin{array}{l}18,494.0 \\
18,495.0 \\
18,496.0 \\
18,497.0 \\
18,498.0\end{array}$ & $\begin{array}{l}18,495.0 \\
18,496.0 \\
18,497.0 \\
18,498.0 \\
18,499.0\end{array}$ & $\begin{array}{l}\text { 327RDVL } \\
\text { 327RDVL } \\
\text { 327RDVL } \\
\text { 327RDVL } \\
\text { 327RDVL }\end{array}$ & $\begin{array}{l}6.9 \\
2.4 \\
2.2 \\
2.9 \\
3.8\end{array}$ & $\begin{array}{l}1.500000 \\
0.010000 \\
0.000000 \\
0.010000 \\
0.080000\end{array}$ & $\begin{array}{l}\text { Crystalline limestone } \\
\text { Crystalline dolomite } \\
\text { Crystalline dolomite } \\
\text { Crystalline dolomite } \\
\text { Crystalline dolomite }\end{array}$ \\
\hline $\begin{array}{l}\text { sb14-112-06bab } \\
\text { sb14-112-06bab } \\
\text { sb14-112-06bab } \\
\text { sb14-112-06bab } \\
\text { sb14-112-06bab }\end{array}$ & $\begin{array}{l}18,499.0 \\
18,500.0 \\
18,501.0 \\
18,507.0 \\
18,508.0\end{array}$ & $\begin{array}{l}18,500.0 \\
18,501.0 \\
18,502.0 \\
18,508.0 \\
18,509.0\end{array}$ & $\begin{array}{l}\text { 327RDVL } \\
\text { 327RDVL } \\
\text { 327RDVL } \\
\text { 327RDVL } \\
\text { 327RDVL }\end{array}$ & $\begin{array}{l}2.2 \\
5.6 \\
3.9 \\
5.1 \\
8.3\end{array}$ & $\begin{array}{l}0.000000 \\
0.230000 \\
0.000000 \\
0.000000 \\
0.000000\end{array}$ & $\begin{array}{l}\text { Crystalline limestone } \\
\text { Crystalline limestone } \\
\text { Crystalline dolomite } \\
\text { Crystalline dolomite } \\
\text { Crystalline dolomite }\end{array}$ \\
\hline $\begin{array}{l}\text { sb14-112-06bab } \\
\text { sb14-112-06bab } \\
\text { sb14-112-06bab } \\
\text { sb14-112-06bab } \\
\text { sb14-112-06bab }\end{array}$ & $\begin{array}{l}18,509.0 \\
18,510.0 \\
18,511.0 \\
18,512.0 \\
18,513.0\end{array}$ & $\begin{array}{r}18,510.0 \\
18,511.0 \\
18,512.0 \\
18,513.0 \\
8,514.0\end{array}$ & $\begin{array}{l}\text { 327RDVL } \\
\text { 327RDVL } \\
\text { 327RDVL } \\
\text { 327RDVL } \\
\text { 327RDVL }\end{array}$ & $\begin{array}{l}3.4 \\
2.4 \\
8.4 \\
4.0 \\
3.6\end{array}$ & $\begin{array}{l}0.590000 \\
0.010000 \\
0.040000 \\
0.110000 \\
0.090000\end{array}$ & $\begin{array}{l}\text { Crystalline, dolomitic limestone } \\
\text { Crystalline dolomite } \\
\text { Crystalline dolomite } \\
\text { Crystalline dolomite } \\
\text { Crystalline dolomite }\end{array}$ \\
\hline $\begin{array}{l}\text { sb14-112-06bab } \\
\text { sb14-112-06bab } \\
\text { sb14-112-06bab } \\
\text { sb14-112-06bab } \\
\text { sb14-112-06bab }\end{array}$ & $\begin{array}{l}18,514.0 \\
18,515.0 \\
18,516.0 \\
18,517.0 \\
18,518.0\end{array}$ & $\begin{array}{l}18,515.0 \\
18,516.0 \\
18,517.0 \\
18,518.0 \\
18,519.0\end{array}$ & $\begin{array}{l}\text { 327RDVL } \\
\text { 327RDVL } \\
\text { 327RDVL } \\
\text { 327RDVL } \\
\text { 327RDVL }\end{array}$ & $\begin{array}{l}2.9 \\
2.9 \\
3.2 \\
3.0 \\
2.4\end{array}$ & $\begin{array}{l}0.000000 \\
0.000000 \\
0.000000 \\
0.000000 \\
0.770000\end{array}$ & $\begin{array}{l}\text { Crystalline dolomite } \\
\text { Crystalline dolomite } \\
\text { Crystalline dolomite } \\
\text { Crystalline dolomite } \\
\text { Crystalline dolomite }\end{array}$ \\
\hline $\begin{array}{l}\text { sb14-112-06bab } \\
\text { sb14-112-06bab } \\
\text { sb14-112-06bab } \\
\text { sb14-112-06bab } \\
\text { sb14-112-06bab }\end{array}$ & $\begin{array}{l}18,519.0 \\
18,520.0 \\
18,521.0 \\
18,524.0 \\
18,525.0\end{array}$ & $\begin{array}{l}18,520.0 \\
18,521.0 \\
18,522.0 \\
18,525.0 \\
18,526.0\end{array}$ & $\begin{array}{l}\text { 327RDVL } \\
\text { 327RDVL } \\
\text { 327RDVL } \\
\text { 327RDVL } \\
\text { 327RDVL }\end{array}$ & $\begin{array}{l}2.8 \\
2.9 \\
5.0 \\
2.9 \\
2.8\end{array}$ & $\begin{array}{r}0.010000 \\
0.160000 \\
0.020000 \\
449.000000 \\
0.020000\end{array}$ & $\begin{array}{l}\text { Crystalline limestone } \\
\text { Shaly, crystalline dolomite } \\
\text { Crystalline dolomite } \\
\text { Crystalline dolomite } \\
\text { Crystalline dolomite }\end{array}$ \\
\hline $\begin{array}{l}\text { sb14-112-06bab } \\
\text { sb14-112-06bab } \\
\text { sb14-112-06bab } \\
\text { sb14-112-06bab }\end{array}$ & $\begin{array}{l}18,528.0 \\
18,539.0 \\
18,540.0 \\
18,542.0\end{array}$ & $\begin{array}{l}18,529.0 \\
18,540.0 \\
18,541.0 \\
18,543.0\end{array}$ & $\begin{array}{l}\text { 327RDVL } \\
\text { 327RDVL } \\
\text { 327RDVL } \\
\text { 327RDVL } \\
\text { AVERAGE-- }\end{array}$ & $\begin{array}{l}3.0 \\
3.1 \\
2.1 \\
1.9 \\
4.6\end{array}$ & $\begin{array}{l}2.200000 \\
0.000000 \\
0.210000 \\
0.000000 \\
4.454394\end{array}$ & $\begin{array}{l}\text { Crystalline limestone } \\
\text { Shaly, anhydritic, crystalline limestone } \\
\text { Shaly, anhydritic, crystalline limestone } \\
\text { Shaly, crystalline limestone }\end{array}$ \\
\hline SITE AVERAGE-- & - & $\ldots$ & $\ldots \ldots$ & 4.5 & 4.355778 & \\
\hline $\begin{array}{l}\text { sb14-91-13bab } \\
\text { sb14-91-13bab } \\
\text { sb14-91-13bab } \\
\text { sb14-91-13bab } \\
\text { sb14-91-13bab }\end{array}$ & $\begin{array}{l}11,048.0 \\
11,049.0 \\
11,050.0 \\
11,051.0 \\
11,052.0\end{array}$ & $\begin{array}{l}11,049 \cdot 0 \\
11,050.0 \\
11,051.0 \\
11,052.0 \\
11,053.0\end{array}$ & $\begin{array}{l}321 \text { TSLP } \\
321 \text { TSLP } \\
321 \text { TSLP } \\
321 \text { TSLP } \\
\text { 321TSLP }\end{array}$ & $\begin{array}{l}0.6 \\
0.7 \\
0.8 \\
0.6 \\
0.5\end{array}$ & $\begin{array}{l}0.100000 \\
0.030000 \\
0.030000 \\
0.080000 \\
0.020000\end{array}$ & $\begin{array}{l}\text { Fine-grained, quartzitic sandstone } \\
\text { Fine-grained, quartzitic sandstone } \\
\text { Fine-grained, quartzitic sandstone } \\
\text { Fine-grained, quartzitic sandstone } \\
\text { Fine-grained, quartzitic sandstone }\end{array}$ \\
\hline $\begin{array}{l}\text { sb14-91-13bab } \\
\text { sb14-91-13bab } \\
\text { sb14-91-13bab } \\
\text { sb14-91-13bab } \\
\text { sb14-91-13bab }\end{array}$ & $\begin{array}{l}11,053 \cdot 0 \\
11,054.0 \\
11,059.0 \\
11,060.0 \\
11,061.0\end{array}$ & $\begin{array}{l}11,054 \cdot 0 \\
11,055 \cdot 0 \\
11,060.0 \\
11,061.0 \\
11,062.0\end{array}$ & $\begin{array}{l}\text { 321TSLP } \\
\text { 321TSLP } \\
\text { 321TSLP } \\
\text { 321TSLP } \\
\text { 321TSLP }\end{array}$ & $\begin{array}{l}0.6 \\
0.7 \\
0.5 \\
0.2 \\
0.2\end{array}$ & $\begin{array}{l}0.020000 \\
0.060000 \\
0.060000 \\
2.800000 \\
0.030000\end{array}$ & $\begin{array}{l}\text { Fine-grained, quartzitic sandstone } \\
\text { Fine-grained, quartzitic sandstone } \\
\text { Fine-grained, quartzitic sandstone } \\
\text { Fine-grained, quartzitic sandstone } \\
\text { Fine-grained, quartzitic sandstone }\end{array}$ \\
\hline
\end{tabular}


Table 4.--Laboratory-determined porosity and permeability--Continued

\begin{tabular}{|c|c|c|c|c|c|c|}
\hline Site & $\begin{array}{l}\text { Depth to } \\
\text { top } \\
\text { (feet) }\end{array}$ & $\begin{array}{l}\text { Depth to } \\
\text { bottom } \\
\text { (feet) }\end{array}$ & $\begin{array}{l}\text { Forma- } \\
\text { tion }\end{array}$ & $\begin{array}{l}\text { Porosity } \\
\text { (percent) }\end{array}$ & $\begin{array}{l}\text { Perme- } \\
\text { ability } \\
\text { (milli- } \\
\text { darcies) }\end{array}$ & Lithology \\
\hline $\begin{array}{l}\text { sb14-91-13bab } \\
\text { sb14-91-13bab } \\
\text { sb14-91-13bab } \\
\text { sb14-91-13bab } \\
\text { sb14-91-13bab }\end{array}$ & $\begin{array}{l}11,062 \cdot 0 \\
11,063 \cdot 0 \\
11,067 \cdot 0 \\
11,068.0 \\
11,069.0\end{array}$ & $\begin{array}{l}11,063 \cdot 0 \\
11,064.0 \\
11,068.0 \\
11,069.0 \\
11,070.0\end{array}$ & $\begin{array}{l}321 \mathrm{TSLP} \\
321 \mathrm{TSLP} \\
321 \mathrm{TSLP} \\
321 \mathrm{TSLP} \\
\text { 321TSLP }\end{array}$ & $\begin{array}{l}0.6 \\
0.2 \\
1.1 \\
0.9 \\
0.7\end{array}$ & $\begin{array}{l}0.030000 \\
0.040000 \\
0.090000 \\
0.550000 \\
0.040000\end{array}$ & $\begin{array}{l}\text { Fine-grained, quartzitic sandstone } \\
\text { Fine-grained, quartzitic sandstone } \\
\text { Fine-grained, quartzitic sandstone } \\
\text { Fine-grained, quartzitic sandstone } \\
\text { Fine-grained, quartzitic sandstone }\end{array}$ \\
\hline $\begin{array}{l}\text { sb14-91-13bab } \\
\text { sb14-91-13bab } \\
\text { sb14-91-13bab } \\
\text { sb14-91-13bab } \\
\text { sb14-91-13bab }\end{array}$ & $\begin{array}{l}11,070.0 \\
11,071.0 \\
11,072.0 \\
11,073.0 \\
11,074.0\end{array}$ & $\begin{array}{l}11,071.0 \\
11,072.0 \\
11,073.0 \\
11,074.0 \\
11,075.0\end{array}$ & $\begin{array}{l}321 T S L P \\
321 T S L P \\
321 T S L P \\
321 T S L P \\
321 T S L P\end{array}$ & $\begin{array}{l}0.6 \\
1.4 \\
0.9 \\
1.0 \\
0.7\end{array}$ & $\begin{array}{r}81.000000 \\
450.000000 \\
0.040000 \\
0.020000 \\
2.100000\end{array}$ & $\begin{array}{l}\text { Fine-grained, quartzitic sandstone } \\
\text { Fine-grained, quartzitic sandstone } \\
\text { Fine-grained, quartzitic sandstone } \\
\text { Fine-grained, quartzitic sandstone } \\
\text { Fine-grained, quartzitic sandstone }\end{array}$ \\
\hline $\begin{array}{l}\text { sb14-91-13bab } \\
\text { sb14-91-13bab } \\
\text { sb14-91-13bab } \\
\text { sb14-91-13bab } \\
\text { sb14-91-13bab }\end{array}$ & $\begin{array}{l}11,075.0 \\
11,076.0 \\
11,077.0 \\
11,078.0 \\
11,079.0\end{array}$ & $\begin{array}{l}11,076.0 \\
11,077.0 \\
11,078.0 \\
11,079.0 \\
11,080.0\end{array}$ & $\begin{array}{l}321 \text { TSLP } \\
321 \text { TSLP } \\
321 \text { TSLP } \\
321 \text { TSLP } \\
321 T S L P\end{array}$ & $\begin{array}{l}2.0 \\
1.0 \\
0.3 \\
7.3 \\
1.0\end{array}$ & $\begin{array}{r}87.000000 \\
0.020000 \\
0.030000 \\
94.000000 \\
0.010000\end{array}$ & $\begin{array}{l}\text { Fine-grained, quartzitic sandstone } \\
\text { Fine-grained, quartzitic sandstone } \\
\text { Fine-grained, quartzitic sandstone } \\
\text { Fine-grained, quartzitic sandstone } \\
\text { Fine-grained, quartzitic sandstone }\end{array}$ \\
\hline $\begin{array}{l}\text { sb14-91-13bab } \\
\text { sb14-91-13bab } \\
\text { sb14-91-13bab } \\
\text { sb14-91-13bab } \\
\text { sb14-91-13bab }\end{array}$ & $\begin{array}{l}11,081 \cdot 0 \\
11,082 \cdot 0 \\
11,083 \cdot 0 \\
11,084 \cdot 0 \\
11,085 \cdot 0\end{array}$ & $\begin{array}{l}11,082.0 \\
11,083.0 \\
11,084.0 \\
11,085.0 \\
11,086.0\end{array}$ & $\begin{array}{l}321 T S L P \\
321 T S L P \\
321 T S L P \\
321 T S L P \\
321 T S L P\end{array}$ & $\begin{array}{l}1.4 \\
1.6 \\
1.4 \\
1.8 \\
1.3 \\
\end{array}$ & $\begin{array}{l}0.050000 \\
0.030000 \\
0.040000 \\
0.030000 \\
0.020000 \\
\end{array}$ & $\begin{array}{l}\text { Fine-grained, quartzitic sandstone } \\
\text { Fine-grained, quartzitic sandstone } \\
\text { Fine-grained, quartzitic sandstone } \\
\text { Fine-grained, quartzitic sandstone } \\
\text { Fine-grained, quartzitic sandstone }\end{array}$ \\
\hline SITE AVERAGE-- & 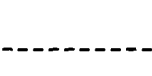 & $\cos ^{-1}$ & AVERAGE-- & $\frac{1.1}{1.1}$ & $\frac{23.945667}{23.945667}$ & \\
\hline $\begin{array}{l}\text { sb16-101-11ddc } \\
\text { sb16-101-11ddc } \\
\text { sb16-101-11ddc } \\
\text { sb16-101-11ddc } \\
\text { sb16-101-11ddc }\end{array}$ & $\begin{array}{l}14,070.0 \\
14,072.0 \\
14,073.0 \\
14,074.0 \\
14,078.0\end{array}$ & $\begin{array}{l}14,071.0 \\
14,073.0 \\
14,074.0 \\
14,075.0 \\
14,079.0\end{array}$ & $\begin{array}{l}317 \mathrm{PRKC} \\
317 \mathrm{PRKC} \\
317 \mathrm{PRKC} \\
317 \mathrm{PRKC} \\
317 \mathrm{PRKC}\end{array}$ & $\begin{array}{l}0.7 \\
1.4 \\
2.3 \\
1.3 \\
2.6\end{array}$ & $\begin{array}{l}0.020000 \\
2.600000 \\
0.190000 \\
0.090000 \\
0.030000\end{array}$ & $\begin{array}{l}\text { Fine-grained dolomite } \\
\text { Crystalline dolomite } \\
\text { Crystalline dolomite } \\
\text { Finely crystalline dolomite } \\
\text { Crystalline dolomite }\end{array}$ \\
\hline $\begin{array}{l}\text { sb16-101-11ddc } \\
\text { sb16-101-11ddc } \\
\text { sb } 16-101-11 d d c \\
\text { sb } 16-101-11 d d c \\
\text { sb } 16-101-11 d d c\end{array}$ & $\begin{array}{l}14,079.0 \\
14,081.0 \\
14,082.0 \\
14,083.0 \\
14,085.0\end{array}$ & $\begin{array}{l}14,080.0 \\
14,082.0 \\
14,083.0 \\
14,084.0 \\
14,086.0\end{array}$ & $\begin{array}{l}317 \mathrm{PRKC} \\
317 \mathrm{PRKC} \\
317 \mathrm{PRKC} \\
317 \mathrm{PRKC} \\
317 \mathrm{PRKC}\end{array}$ & $\begin{array}{l}0.9 \\
0.8 \\
1.1 \\
0.8 \\
2.3\end{array}$ & $\begin{array}{r}0.890000 \\
0.030000 \\
0.120000 \\
10.300000 \\
0.060000\end{array}$ & $\begin{array}{l}\text { Crystalline dolomite } \\
\text { Fine-grained dolomite } \\
\text { Crystalline dolomite } \\
\text { Vuggy, crystalline dolomite } \\
\text { Crystalline dolomite }\end{array}$ \\
\hline $\begin{array}{l}\text { sb16-101-11ddc } \\
\text { sb16-101-11ddc } \\
\text { sb16-101-11ddc } \\
\text { sb16-101-11ddc } \\
\text { sb16-101-11ddc }\end{array}$ & $\begin{array}{l}14,086.0 \\
14,087.0 \\
14,088.0 \\
14,089.0 \\
14,090.0\end{array}$ & $\begin{array}{l}14,087.0 \\
14,088.0 \\
14,089.0 \\
14,090.0 \\
14,091.0\end{array}$ & $\begin{array}{l}317 \mathrm{PRKC} \\
317 \mathrm{PRKC} \\
317 \mathrm{PRKC} \\
317 \mathrm{PRKC} \\
317 \mathrm{PRKC}\end{array}$ & $\begin{array}{r}0.7 \\
6.8 \\
1.0 \\
1.3 \\
11.4\end{array}$ & $\begin{array}{r}4.500000 \\
0.100000 \\
23.600000 \\
0.070000 \\
0.100000\end{array}$ & $\begin{array}{l}\text { Fine-grained dolomite } \\
\text { Crystalline dolomite } \\
\text { Fine-grained dolomite } \\
\text { Fine-grained limy dolomite } \\
\text { Sucrose dolomite }\end{array}$ \\
\hline $\begin{array}{l}\text { sb16-101-11ddc } \\
\text { sb16-101-11ddc } \\
\text { sb16-101-11ddc }\end{array}$ & $\begin{array}{l}14,091.0 \\
14,092.0 \\
14,093.0\end{array}$ & $\begin{array}{l}14,092.0 \\
14,093.0 \\
14,094.0\end{array}$ & $\begin{array}{l}317 P R K C \\
317 P R K C \\
317 P R K C\end{array}$ & $\begin{array}{r}10.2 \\
1.8 \\
3.4\end{array}$ & $\begin{array}{l}0.040000 \\
0.030000 \\
1.600000\end{array}$ & $\begin{array}{l}\text { Sucrose dolomite } \\
\text { Finely crystalline dolomite } \\
\text { Crystalline dolomite with } \\
\text { calcite-filled vugs }\end{array}$ \\
\hline $\begin{array}{l}\text { sb16-101-11ddc } \\
\text { sb16-101-11ddc }\end{array}$ & $\begin{array}{l}14,094.0 \\
14,095.0\end{array}$ & $\begin{array}{l}14,095.0 \\
14,096.0\end{array}$ & $\begin{array}{l}\text { 317PRKC } \\
\text { 317PRKC }\end{array}$ & $\begin{array}{l}2.8 \\
6.9\end{array}$ & $\begin{array}{l}3.400000 \\
0.890000\end{array}$ & $\begin{array}{l}\text { Vuggy, sucrose dolomite } \\
\text { Sucrose dolomite }\end{array}$ \\
\hline $\begin{array}{l}\text { sb16-101-11ddc } \\
\text { sb16-101-11ddc } \\
\text { sb16-101-11ddc } \\
\text { sb16-101-11ddc } \\
\text { sb16-101-11ddc }\end{array}$ & $\begin{array}{l}14,096.0 \\
14,097 \cdot 0 \\
14,098 \cdot 0 \\
14,099.0 \\
14,100.0\end{array}$ & $\begin{array}{l}14,097.0 \\
14,098.0 \\
14,099.0 \\
14,100.0 \\
14,101.0\end{array}$ & $\begin{array}{l}317 \mathrm{PRKC} \\
317 \mathrm{PRKC} \\
317 \mathrm{PRKC} \\
317 \mathrm{PRKC} \\
317 \mathrm{PRKC}\end{array}$ & $\begin{array}{l}1.5 \\
0.8 \\
1.2 \\
1.6 \\
3.9\end{array}$ & $\begin{array}{r}88.000000 \\
0.300000 \\
3.900000 \\
15.000000 \\
1.600000\end{array}$ & $\begin{array}{l}\text { Fractured, vuggy, crystalline dolomite } \\
\text { Fine-grained dolomite } \\
\text { Fine-grained dolomite } \\
\text { Fine-grained dolomite } \\
\text { Sucrose dolomite }\end{array}$ \\
\hline
\end{tabular}


Table 4.--Laboratory-determined porosity and permeability--Continued

\begin{tabular}{|c|c|c|c|c|c|c|}
\hline Site & $\begin{array}{l}\text { Depth to } \\
\text { top } \\
\text { (feet) }\end{array}$ & $\begin{array}{l}\text { Depth to } \\
\text { bottom } \\
\text { (feet) }\end{array}$ & $\begin{array}{c}\text { Forma- } \\
\text { tion }\end{array}$ & $\begin{array}{l}\text { Porosity } \\
\text { (percent) }\end{array}$ & $\begin{array}{l}\text { Perme- } \\
\text { ability } \\
\text { (milli- } \\
\text { darcies) }\end{array}$ & Lithology \\
\hline sb16-101-11ddc & $14,101.0$ & $14,102.0$ & 317PRKC & 0.7 & 0.100000 & Crystalline dolomite \\
\hline sb16-101-11ddc & $14,102.0$ & $14,103.0$ & 317 PRKC & 7.7 & 0.070000 & Sucrose dolomite \\
\hline sb16-101-11ddc & $14,104.0$ & $14,105.0$ & 317 PRKC & 3.7 & 1.500000 & Crystalline dolomite \\
\hline sb16-101-11ddc & $14,106.0$ & $14,107.0$ & 317PRKC & 1.9 & 0.860000 & Dolomite with calcite-filled vugs \\
\hline sb16-101-11ddc & $14,108.0$ & $14,109.0$ & 317 PRKC & 1.8 & 0.100000 & Finely crystalline dolomite \\
\hline sb16-101-11ddc & $14,110.0$ & $14,111.0$ & 317 PRKC & 13.8 & 0.420000 & Sucrose dolomite \\
\hline sb $16-101-11 d d c$ & $14,111.0$ & $14,112.0$ & 317PRKC & 6.8 & 36.000000 & Vuggy, sucrose dolomite \\
\hline sb16-101-11ddc & $14,112.0$ & $14,113.0$ & 317 PRKC & 2.7 & 0.090000 & Fine-grained dolomite \\
\hline $\mathrm{sb} 16-101-11 \mathrm{ddc}$ & $14,113.0$ & $14,114.0$ & 317 PRKC & 15.7 & 0.160000 & Sucrose dolomite \\
\hline sb16-101-11ddc & $14,114.0$ & $14,115.0$ & 317PRKC & 10.0 & 0.160000 & Sucrose dolomite \\
\hline sb16-101-11ddc & $14,119.0$ & $14,120.0$ & 317PRKC & 3.1 & 0.030000 & Crystalline dolomite with calcite inclusions \\
\hline sb16-101-11ddc & $14,120.0$ & $14,121.0$ & 317 PRKC & 2.8 & 0.030000 & Crystalline dolomite \\
\hline sb16-101-11ddc & $14,121.0$ & $14,122.0$ & 317 PRKC & 4.7 & 7.800000 & Crystalline dolomite \\
\hline sb16-101-11ddc & $14,122.0$ & $14,123.0$ & 317 PRKC & 4.3 & 1.300000 & Crystalline dolomite with calcite inclusions \\
\hline sb16-101-11ddc & $14,124.0$ & $14,125.0$ & 317PRKC & 8.0 & 0.110000 & Sucrose dolomite \\
\hline sb16-101-11ddc & $14,126.0$ & $14,127.0$ & 317 PRKC & 0.7 & 0.030000 & Crystalline dolomite \\
\hline sb16-101-11ddc & $14,128.0$ & $14,129.0$ & 317 PRKC & 4.5 & 0.910000 & Crystalline dolomite \\
\hline$s b 16-101-11 d d c$ & $14,130.0$ & $14,131.0$ & 317PRKC & 3.2 & 4.300000 & Crystalline dolomite \\
\hline sb16-101-11ddc & $14,132.0$ & $14,133.0$ & 317 PRKC & 3.0 & 0.040000 & Crystalline dolomite \\
\hline sb16-101-11ddc & $14,134.0$ & $14,135.0$ & 317PRKC & 3.1 & 0.030000 & Crystalline dolomite \\
\hline sb16-101-11ddc & $14,136.0$ & $14,137.0$ & 317 PRKC & 1.2 & 0.120000 & Crystalline limestone \\
\hline $\mathrm{sb} 16-101-11 \mathrm{ddc}$ & $14,138.0$ & $14,139.0$ & 317 PRKC & 2.2 & 0.980000 & Fine-grained dolomite \\
\hline sb16-101-11ddc & $14,140.0$ & $14,141.0$ & 317 PRKC & 1.6 & 0.030000 & Fine-grained dolomite \\
\hline sb16-101-11ddc & $14,142.0$ & $14,143.0$ & 317 PRKC & 0.5 & 0.040000 & Crystalline dolomite with calcite inclusions \\
\hline sb16-101-11ddc & $14,144.0$ & $14,145.0$ & 317PRKC & 1.2 & 0.020000 & Crystalline dolomite \\
\hline sb16-101-11ddc & $14,145.0$ & $14,146.0$ & 317PRKC & 1.8 & 0.120000 & Crystalline dolomite \\
\hline sb16-101-11ddc & $14,146.0$ & $14,147.0$ & 317 PRKC & 1.4 & 0.020000 & Crystalline dolomite \\
\hline sb16-101-11ddc & $14,147.0$ & $14,148.0$ & 317 PRKC & 1.7 & 0.590000 & Fine-grained dolomite \\
\hline sb16-101-11ddc & $14,149.0$ & $14,150.0$ & 317 PRKC & 1.7 & 0.020000 & Fine-grained dolomite \\
\hline sb16-101-11ddc & $14,150.0$ & $14,151.0$ & 317 PRKC & 11.2 & 0.510000 & Crystalline dolomite \\
\hline sb16-101-11ddc & $14,151.0$ & $14,152.0$ & 317 PRKC & 1.9 & 0.270000 & Crystalline dolomite \\
\hline $\mathrm{sb} 16-101-11 \mathrm{ddc}$ & $14,152.0$ & $14,153.0$ & 317PRKC & 1.0 & 0.080000 & Crystalline dolomite \\
\hline sb16-101-11ddc & $14,153.0$ & $14,154.0$ & 317 PRKC & 1.3 & 0.360000 & Crystalline dolomite \\
\hline sb16-101-11ddc & $14,154.0$ & $14,155.0$ & 317PRKC & 1.2 & 0.130000 & Crystalline dolomite \\
\hline sb16-101-11ddc & $14,155.0$ & $14,156.0$ & 317 PRKC & 2.0 & 0.520000 & Crystalline dolomite \\
\hline sb16-101-11ddc & $14,157.0$ & $14,158.0$ & 317 PRKC & 1.7 & 0.030000 & Crystalline dolomite \\
\hline sb16-101-11ddc & $14,158.0$ & $14,159.0$ & 317 PRKC & 1.6 & 0.110000 & Crystalline dolomite \\
\hline sb16-101-11ddc & $14,159.0$ & $14,160.0$ & 317 PRKC & 1.9 & 0.020000 & Crystalline dolomite \\
\hline sb16-101-11ddc & $14,206.0$ & $14,207.0$ & 317 PRKC & 1.6 & 0.140000 & Crystalline dolomite \\
\hline sb16-101-11ddc & $14,230.0$ & $14,231.0$ & 317PRKC & 0.6 & 0.090000 & Crystalline dolomite with calcite inclusions \\
\hline sb16-101-11ddc & $14,231.0$ & $14,232.0$ & 317PRKC & 2.6 & 0.260000 & Crystalline dolomite with calcite inclusions \\
\hline sb16-101-11ddc & $14,232.0$ & $14,233.0$ & 317PRKC & 2.0 & 0.290000 & Crystalline dolomite with calcite inclusions \\
\hline sb16-101-11ddc & $14,233.0$ & $14,234.0$ & 317PRKC & 3.4 & 7.800000 & Crystalline dolomite with calcite inclusions \\
\hline sb16-101-11ddc & $14,234.0$ & $14,235.0$ & 317 PRKC & 0.9 & 0.450000 & Sucrose dolomite \\
\hline sb16-101-11ddc & $14,235.0$ & $14,236.0$ & 317 PRKC & 8.7 & 0.030000 & Vuggy, crystalline dolomite \\
\hline sb16-101-11ddc & $14,236.0$ & $14,237.0$ & 317 PRKC & 12.6 & 4.500000 & Sucrose dolomite \\
\hline sb16-101-11ddc & $14,237.0$ & $14,238.0$ & 317 PRKC & 12.3 & 0.030000 & Vuggy, sucrose dolomite \\
\hline sb16-101-11ddc & $14,238.0$ & $14,239.0$ & 317PRKC & 10.0 & 0.040000 & Vuggy, sucrose dolomite \\
\hline sb16-101-11ddc & $14,239.0$ & $14,240.0$ & $317 \mathrm{PRKC}$ & 9.9 & 3.200000 & Sucrose dolomite \\
\hline \multirow[t]{2}{*}{ sb16-101-11ddc } & $14,242.0$ & $14,243.0$ & 317PRKC & 1.5 & 3.400000 & Crystalline dolomite \\
\hline & & & AVERAGE - - & 3.7 & 3.142667 & \\
\hline
\end{tabular}


Table 4.--Laboratory-determined porosity and permeability--Continued

\begin{tabular}{|c|c|c|c|c|c|c|}
\hline Site & $\begin{array}{l}\text { Depth to } \\
\text { top } \\
\text { (feet) }\end{array}$ & $\begin{array}{l}\text { Depth to } \\
\text { bottom } \\
\text { (feet) }\end{array}$ & $\begin{array}{c}\text { Forma- } \\
\text { tion }\end{array}$ & $\begin{array}{l}\text { Porosity } \\
\text { (percent) }\end{array}$ & $\begin{array}{l}\text { Perme- } \\
\text { ability } \\
\text { (milli- } \\
\text { darcies) }\end{array}$ & Lithology \\
\hline sb16-101-11ddc & $14,282.0$ & $14,283.0$ & 321TSLP & 1.2 & 0.260000 & Fine- to coarse-grained, limy sandstone \\
\hline $\mathrm{sb} 16-101-11 \mathrm{ddc}$ & $14,283.0$ & $14,284.0$ & $321 \mathrm{TSLP}$ & 1.9 & 0.240000 & Fine-grained dolomite \\
\hline sb16-101-11ddc & $14,284.0$ & $14,285.0$ & 321TSLP & 0.7 & 0.050000 & Medium- to coarse-grained, limy sandstone \\
\hline $\mathrm{sb} 16-101-11 \mathrm{ddc}$ & $14,285.0$ & $14,286.0$ & $321 \mathrm{TSLP}$ & 1.0 & 0.150000 & Medium- to coarse-grained, limy sandstone \\
\hline $\mathrm{sb} 16-101-11 \mathrm{ddc}$ & $14,287.0$ & $14,288.0$ & 321TSLP & 1.2 & 0.060000 & Fine- to medium-grained, limy sandstone \\
\hline $\mathrm{sb} 16-101-11 \mathrm{ddc}$ & $14,288.0$ & $14,289.0$ & 321TSLP & 6.5 & 22.000000 & Fine to medium-grained, limy sandstone \\
\hline$s b 16-101-11 d d c$ & $14,289.0$ & $14,290.0$ & $321 \mathrm{TSLP}$ & 2.0 & 1.100000 & Fine- to medium-grained, limy sandstone \\
\hline sb16-101-11ddc & $14,291.0$ & $14,292.0$ & $321 \mathrm{TSLP}$ & 0.9 & 0.030000 & Fine- to medium-grained, limy sandstone \\
\hline sb16-101-11ddc & $14,293.0$ & $14,294.0$ & $321 T S L P$ & 1.9 & 0.030000 & Fine- to medium-grained, limy sandstone \\
\hline sb16-101-11ddc & $14,295.0$ & $14,296.0$ & 321 TSLP & 1.1 & 0.030000 & Fine- to medium-grained, limy sandstone \\
\hline $\mathrm{sb16}-101-11 \mathrm{ddc}$ & $14,297.0$ & $14,298.0$ & $321 \mathrm{TSLP}$ & 1.1 & 23.000000 & Fine-grained sandstone \\
\hline sb16-101-11ddc & $14,298.0$ & $14,299.0$ & $321 T S L P$ & 1.0 & 0.040000 & Fine-grained, limy sandstone \\
\hline sb16-101-11ddc & $14,299.0$ & $14,300.0$ & $321 \mathrm{TSLP}$ & 0.8 & 0.030000 & Fine- to medium-grained, limy sandstone \\
\hline sb16-101-11ddc & $14,300.0$ & $14,301.0$ & 321 TSLP & 1.1 & 0.170000 & Fine- to medium-grained, limy sandstone \\
\hline $\mathrm{sb} 16-101-11 \mathrm{ddc}$ & $14,301.0$ & $14,302.0$ & $321 \mathrm{TSLP}$ & 0.8 & 0.050000 & Fine- to medium-grained, limy sandstone \\
\hline $\mathrm{sb16}-101-11 \mathrm{ddc}$ & $14,302.0$ & $14,303.0$ & $321 \mathrm{TSLP}$ & 2.4 & 3.100000 & Fractured, fine- to medium-grained, limy sandstone \\
\hline sb16-101-11ddc & $14,303.0$ & $14,304.0$ & $321 \mathrm{TSLP}$ & 4.3 & 0.030000 & Fine- to medium-grained, limy sandstone \\
\hline sb16-101-11ddc & $14,304.0$ & $14,305.0$ & $321 T S L P$ & 5.2 & 0.130000 & Fine- to medium-grained sandstone \\
\hline $\mathrm{sb} 16-101-11 \mathrm{ddc}$ & $14,305.0$ & $14,306.0$ & 321TSLP & 5.7 & 0.070000 & Fine- to medium-grained, limy sandstone \\
\hline sb16-101-11ddc & $14,306.0$ & $14,307.0$ & $321 \mathrm{TSLP}$ & 9.0 & 0.800000 & Fine-grained sandstone \\
\hline sb16-101-11ddc & $14,308.0$ & $14,309.0$ & 321TSLP & 9.3 & 1.800000 & Fine-grained sandstone \\
\hline $\mathrm{sb} 16-101-11 \mathrm{ddc}$ & $14,309.0$ & $14,310.0$ & 321 TSLP & 8.9 & 2.400000 & Fine-grained sandstone \\
\hline $\mathrm{sb} 16-101-11 \mathrm{ddc}$ & $14,313.0$ & $14,314.0$ & 321TSLP & 4.0 & 0.030000 & Fine-grained sandstone \\
\hline sb16-101-11ddc & $14,315.0$ & $14,316.0$ & $321 \mathrm{TSLP}$ & 6.7 & 0.030000 & Fine-grained sandstone \\
\hline $\mathrm{sb} 16-101-11 \mathrm{ddc}$ & $14,317.0$ & $14,318.0$ & 321TSLP & 6.0 & 0.160000 & Fine-grained sandstone \\
\hline $\mathrm{sb} 16-101-11 \mathrm{ddc}$ & $14,319.0$ & $14,320.0$ & $321 \mathrm{TSLP}$ & 6.8 & 0.710000 & Fine-grained sandstone \\
\hline sb16-101-11ddc & $14,321.0$ & $14,322.0$ & 321TSLP & 7.2 & 0.470000 & Fine- to medium-grained sandstone \\
\hline sb16-101-11ddc & $14,323.0$ & $14,324.0$ & $321 \mathrm{TSLP}$ & 10.3 & 0.760000 & Fine- to medium-grained sandstone \\
\hline sb16-101-11ddc & $14,325.0$ & $14,326.0$ & $321 \mathrm{TSLP}$ & 12.0 & 1.700000 & Fine-grained sandstone \\
\hline $\mathrm{sb} 16-101-11 \mathrm{ddc}$ & $14,327.0$ & $14,328.0$ & 321TSLP & 11.2 & 2.400000 & Fine-grained sandstone \\
\hline sb16-101-11ddc & $14,329.0$ & $14,330.0$ & 321TSLP & 8.6 & 2.700000 & Fine-grained, limy sandstone \\
\hline $\mathrm{sb} 16-101-11 \mathrm{ddc}$ & $14,331.0$ & $14,332.0$ & $321 \mathrm{TSLP}$ & 5.9 & 0.080000 & Fine-grained sandstone \\
\hline sb16-101-11ddc & $14,333.0$ & $14,334.0$ & 321TSLP & 8.5 & 1.400000 & Fine-grained sandstone \\
\hline sb16-101-11ddc & $14,335.0$ & $14,336.0$ & 321 TSLP & 7.0 & 0.660000 & Fine- to medium-grained sandstone \\
\hline sb $16-101-11 d d c$ & $14,337.0$ & $14,338.0$ & $321 \mathrm{TSLP}$ & 9.2 & 15.000000 & Fine- to medium-grained sandstone \\
\hline sb16-101-11ddc & $14,339.0$ & $14,340.0$ & $321 T S L P$ & 9.8 & 13.000000 & Fine- to medium-grained sandstone \\
\hline $\mathrm{sb} 16-101-11 \mathrm{ddc}$ & $14,340.0$ & $14,341.0$ & 321TSLP & 8.8 & 14.000000 & Fine- to medium-grained sandstone \\
\hline $\mathrm{sb} 16-101-11 \mathrm{ddc}$ & $14,341.0$ & $14,342.0$ & $321 \mathrm{TSLP}$ & 7.3 & 2.500000 & Fine- to coarse-grained sandstone \\
\hline sb16-101-11ddc & $14,342.0$ & $14,343.0$ & 321TSLP & 9.2 & 24.000000 & Fine- to medium-grained sandstone \\
\hline $\mathrm{sb} 16-101-11 \mathrm{ddc}$ & $14,343.0$ & $14,344.0$ & $321 \mathrm{TSLP}$ & 2.4 & 0.040000 & Fine- to medium-grained sandstone \\
\hline $\mathrm{sb} 16-101-11 \mathrm{ddc}$ & $14,345.0$ & $14,346.0$ & $321 \mathrm{TSLP}$ & 5.2 & 0.040000 & Fine- to medium-grained sandstone \\
\hline sb16-101-11ddc & $14,347.0$ & $14,348.0$ & $321 \mathrm{TSLP}$ & 4.9 & 0.030000 & Fine- to medium-grained sandstone \\
\hline $\operatorname{sb} 16-101-11 d d c$ & $14,349.0$ & $14,350.0$ & 321TSLP & 3.7 & 0.030000 & Fine- to medium-grained, limy sandstone \\
\hline $\mathrm{sb} 16-101-11 \mathrm{ddc}$ & $14,351.0$ & $14,352.0$ & 321TSLP & 4.1 & 0.720000 & Fine- to medium-grained sandstone \\
\hline sb16-101-11ddc & $14,353.0$ & $14,354.0$ & 321TSLP & 5.8 & 0.060000 & Fine- to medium-grained sandstone \\
\hline $\operatorname{sb} 16-101-11 d d c$ & $14,355.0$ & $14,356.0$ & 321TSLP & 3.7 & 0.060000 & Fine- to medium-grained sandstone \\
\hline sb16-101-11ddc & $14,357.0$ & $14,358.0$ & $321 \mathrm{TSLP}$ & 3.5 & 0.100000 & Fine- to medium-grained sandstone \\
\hline $\mathrm{sb} 16-101-11 \mathrm{ddc}$ & $14,359.0$ & $14,360.0$ & $321 T S L P$ & 2.3 & 0.030000 & Fine- to medium-grained, limy sandstone \\
\hline sb16-101-11ddc & $14,361.0$ & $14,362.0$ & $321 \mathrm{TSLP}$ & 1.3 & 0.080000 & Fine- to medium-grained sandstone \\
\hline sb16-101-11ddc & $14,363.0$ & $14,364.0$ & $321 \mathrm{TSLP}$ & 3.4 & 0.030000 & Fine- to medium-grained sandstone \\
\hline
\end{tabular}


Table 4.--Laboratory-determined porosity and permeability--Continued

\begin{tabular}{|c|c|c|c|c|c|c|}
\hline Site & $\begin{array}{l}\text { Depth to } \\
\text { top } \\
\text { (feet) }\end{array}$ & $\begin{array}{l}\text { Depth to } \\
\text { bottom } \\
\text { (feet) }\end{array}$ & $\begin{array}{c}\text { Forma- } \\
\text { tion }\end{array}$ & $\begin{array}{l}\text { Porosity } \\
\text { (percent) }\end{array}$ & $\begin{array}{c}\text { Perme- } \\
\text { ability } \\
\text { (milli- } \\
\text { darcies) }\end{array}$ & Lithology \\
\hline $\mathrm{sb} 16-101-11 \mathrm{ddc}$ & $14,365.0$ & $14,366.0$ & $321 \mathrm{TSLP}$ & 6.0 & 0.140000 & Fine- to medium-grained sandstone \\
\hline sb16-101-11ddc & $14,367.0$ & $14,368.0$ & $321 \mathrm{TSLP}$ & 6.8 & 0.740000 & Fine- to medium-grained sandstone \\
\hline sb16-101-11ddc & $14,369.0$ & $14,370.0$ & 321TSLP & 6.7 & 0.600000 & Fine- to medium-grained sandstone \\
\hline $\mathrm{sb} 16-101-11 \mathrm{ddc}$ & $14,371.0$ & $14,372.0$ & 321TSLP & 3.6 & 0.530000 & Fine- to medium-grained sandstone \\
\hline sb16-101-11ddc & $14,372.0$ & $14,373.0$ & $321 \mathrm{TSLP}$ & 6.4 & 1.200000 & Fine- to medium-grained sandstone \\
\hline sb16-101-11ddc & $14,374.0$ & $14,375.0$ & 321TSLP & 5.4 & 0.660000 & Fine- to medium-grained sandstone \\
\hline sb16-101-11ddc & $14,376.0$ & $14,377.0$ & $321 \mathrm{TSLP}$ & 2.8 & 0.030000 & Fine- to medium-grained sandstone \\
\hline sb16-101-11ddc & $14,378.0$ & $14,379.0$ & 321TSLP & 5.2 & 0.470000 & Fine- to medium-grained sandstone \\
\hline sb $16-101-11 \mathrm{ddc}$ & $14,380.0$ & $14,381.0$ & 321TSLP & 1.7 & 0.010000 & Fine- to medium-grained sandstone \\
\hline sb $16-101-11 d d c$ & $14,382.0$ & $14,383.0$ & $321 \mathrm{TSLP}$ & 2.0 & 0.040000 & Fine- to medium-grained sandstone \\
\hline sb16-101-11ddc & $14,384.0$ & $14,385.0$ & $321 \mathrm{TSLP}$ & 1.2 & 0.040000 & Fine- to medium-grained sandstone \\
\hline sb16-101-11ddc & $14,386.0$ & $14,387.0$ & 321TSLP & 3.2 & 0.040000 & Fine- to medium-grained sandstone \\
\hline sb16-101-11ddc & $14,388.0$ & $14,389.0$ & $321 \mathrm{TSLP}$ & 5.6 & 0.040000 & Fine- to medium-grained sandstone \\
\hline sb16-101-11ddc & $14,390.0$ & $14,391.0$ & 321TSLP & 5.8 & 0.040000 & Fine- to medium-grained sandstone \\
\hline sb $16-101-11 d d c$ & $14,392.0$ & $14,393.0$ & 321TSLP & 6.0 & 0.230000 & Fine- to medium-grained sandstone \\
\hline sb16-101-11ddc & $14,394.0$ & $14,395.0$ & $321 \mathrm{TSLP}$ & 5.6 & 0.070000 & Fine- to medium-grained sandstone \\
\hline sb16-101-11ddc & $14,396.0$ & $14,397.0$ & 321TSLP & 5.8 & 0.700000 & Fine- to medium-grained sandstone \\
\hline sb16-101-11ddc & $14,398.0$ & $14,399.0$ & 321TSLP & 7.3 & 0.980000 & Fine- to medium-grained sandstone \\
\hline sb16-101-11ddc & $14,400.0$ & $14,401.0$ & 321TSLP & 6.6 & 6.500000 & Medium-grained sandstone \\
\hline sb16-101-11ddc & $14,402.0$ & $14,403.0$ & 321TSLP & 7.2 & 0.300000 & Fine- to medium-grained sandstone \\
\hline sb16-101-11ddc & $14,404.0$ & $14,405.0$ & $321 \mathrm{TSLP}$ & 5.8 & 0.440000 & Fine-grained sandstone \\
\hline sb16-101-11ddc & $14,405.0$ & $14,406.0$ & $321 \mathrm{TSLP}$ & 6.2 & 0.580000 & Fine- to medium-grained sandstone \\
\hline sb16-101-11ddc & $14,406.0$ & $14,407.0$ & 321TSLP & 5.4 & 0.030000 & Fine- to medium-grained sandstone \\
\hline sb16-101-11ddc & $14,407.0$ & $14,408.0$ & 321TSLP & 10.6 & 9.200000 & Fine- to medium-grained sandstone \\
\hline sb16-101-11ddc & $14,408.0$ & $14,409.0$ & $321 \mathrm{TSLP}$ & 8.2 & 3.300000 & Fine-grained sandstone \\
\hline sb16-101-11ddc & $14,409.0$ & $14,410.0$ & 321TSLP & 9.9 & 8.600000 & Fine-grained sandstone \\
\hline sb16-101-11ddc & $14,410.0$ & $14,411.0$ & $321 T S L P$ & 6.0 & 0.700000 & Fine- to medium-grained sandstone \\
\hline sb16-101-11ddc & $14,411.0$ & $4,412.0$ & $321 \mathrm{TSLP}$ & 7.8 & 2.100000 & Fine-grained sandstone \\
\hline sb16-101-11ddc & $14,412.0$ & $14,413.0$ & $321 \mathrm{TSLP}$ & 10.3 & 8.300000 & Fine-grained sandstone \\
\hline sb16-101-11ddc & $14,414.0$ & $14,415.0$ & $321 \mathrm{TSLP}$ & 9.2 & 2.900000 & Fine-grained sandstone \\
\hline sb $16-101-11 d d c$ & $14,416.0$ & $14,417.0$ & $321 \mathrm{TSLP}$ & 8.1 & 1.500000 & Fine-grained sandstone \\
\hline $\mathrm{sb} 16-101-11 \mathrm{ddc}$ & $14,418.0$ & $14,419.0$ & $321 \mathrm{TSLP}$ & 7.3 & 2.000000 & Fine-grained sandstone \\
\hline sb16-101-11ddc & $14,420.0$ & $14,421.0$ & $321 \mathrm{TSLP}$ & 9.6 & 3.500000 & Fine-grained sandstone \\
\hline $\mathrm{sb} 16-101-11 \mathrm{ddc}$ & $14,422.0$ & $14,423.0$ & $321 \mathrm{TSLP}$ & 5.7 & 0.140000 & Fine- to medium-grained sandstone \\
\hline sb16-101-11ddc & $14,424.0$ & $14,425.0$ & 321TSLP & 6.2 & 0.190000 & Fine-grained sandstone \\
\hline sb $16-101-11 d d c$ & $14,426.0$ & $14,427.0$ & 321TSLP & 2.2 & 0.610000 & Fine- to medium-grained sandstone \\
\hline sb16-101-11ddc & $14,428.0$ & $14,429.0$ & $321 \mathrm{TSLP}$ & 3.3 & 0.010000 & Fine- to medium-grained sandstone \\
\hline sb16-101-11ddc & $14,430.0$ & $14,431.0$ & $321 \mathrm{TSLP}$ & 3.0 & 7.700000 & Fine- to medium-grained sandstone \\
\hline sb16-101-11ddc & $14,431.0$ & $14,432.0$ & $321 \mathrm{TSLP}$ & 4.0 & 0.030000 & Fine- to medium-grained sandstone \\
\hline sb $16-101-11 d d c$ & $14,432.0$ & $14,433.0$ & $321 \mathrm{TSLP}$ & 2.2 & 2.700000 & Fine- to medium-grained sandstone \\
\hline sb $16-101-11 d d c$ & $14,433.0$ & $14,434.0$ & 321TSLP & 3.6 & 0.010000 & Fine- to medium-grained sandstone \\
\hline sb $16-101-11 d d c$ & $14,435.0$ & $14,436.0$ & $321 \mathrm{TSLP}$ & 3.0 & 0.030000 & Fine- to medium-grained sandstone \\
\hline sb $16-101-11 d d c$ & $14,437.0$ & $14,438.0$ & $321 \mathrm{TSLP}$ & 3.4 & 0.990000 & Fine- to medium-grained sandstone \\
\hline sb $16-101-11 d d c$ & $14,439.0$ & $14,440.0$ & $321 \mathrm{TSLP}$ & 2.8 & 0.050000 & Fine- to medium-grained sandstone \\
\hline sb $16-101-11 d d c$ & $14,441.0$ & $14,442.0$ & $321 \mathrm{TSLP}$ & 3.8 & 0.520000 & Fine- to medium-grained sandstone \\
\hline sb $16-101-11 d d c$ & $14,443.0$ & $14,444.0$ & $321 \mathrm{TSLP}$ & 2.7 & 132.000000 & Fine- to medium-grained sandstone \\
\hline sb $16-101-11 d d c$ & $14,444.0$ & $14,445.0$ & $321 \mathrm{TSLP}$ & 4.3 & 0.040000 & Fine- to medium-grained sandstone \\
\hline sb $16-101-11 d d c$ & $14,445.0$ & $14,446.0$ & $321 \mathrm{TSLP}$ & 6.4 & 0.150000 & Fine- to medium-grained sandstone \\
\hline sb16-101-11ddc & $14,446.0$ & $14,447.0$ & $321 \mathrm{TSLP}$ & 7.0 & 1.200000 & Fine- to medium-grained sandstone \\
\hline $\mathrm{sb} 16-101-11 \mathrm{ddc}$ & $14,447.0$ & $14,448.0$ & 321 TSLP & 10.6 & 7.000000 & Fine- to medium-grained sandstone \\
\hline
\end{tabular}


Table 4.--Laboratory-determined porosity and permeability--Continued

\begin{tabular}{|c|c|c|c|c|c|c|}
\hline Site & $\begin{array}{l}\text { Depth to } \\
\text { top } \\
\text { (feet) }\end{array}$ & $\begin{array}{l}\text { Depth to } \\
\text { bottom } \\
\text { (feet) }\end{array}$ & $\begin{array}{c}\text { Forma- } \\
\text { tion }\end{array}$ & $\begin{array}{l}\text { Porosity } \\
\text { (percent) }\end{array}$ & $\begin{array}{l}\text { Perme- } \\
\text { ability } \\
\text { (milli- } \\
\text { darcies) }\end{array}$ & Lithology \\
\hline sb16-101-11ddc & $14,448.0$ & $14,449.0$ & 321TSLP & 7.7 & 1.000000 & Fine- to medium-grained sandstone \\
\hline sb16-101-11ddc & $14,449.0$ & $14,450.0$ & $321 \mathrm{TSLP}$ & 5.9 & 1.600000 & Fine- to medium-grained sandstone \\
\hline sb16-101-11ddc & $14,450.0$ & $14,451.0$ & $321 T S L P$ & 5.3 & 0.270000 & Fine- to medium-grained sandstone \\
\hline sb16-101-11ddc & $14,451.0$ & $14,452.0$ & $321 \mathrm{TSLP}$ & 6.1 & 6.300000 & Fine- to medium-grained sandstone \\
\hline sb16-101-11ddc & $14,452.0$ & $14,453.0$ & $321 \mathrm{TSLP}$ & 5.7 & 0.970000 & Fine- to medium-grained sandstone \\
\hline sb16-101-11ddc & $14,453.0$ & $14,454.0$ & $321 \mathrm{TSLP}$ & 3.0 & 0.090000 & Fine- to medium-grained sandstone \\
\hline sb16-101-11ddc & $14,455.0$ & $14,456.0$ & $321 \mathrm{TSLP}$ & 3.0 & 0.010000 & Fine- to medium-grained sandstone \\
\hline sb16-101-11ddc & $14,457.0$ & $14,458.0$ & $321 \mathrm{TSLP}$ & 3.6 & 0.010000 & Fine- to medium-grained sandstone \\
\hline sb $16-101-11 d d c$ & $14,459.0$ & $14,460.0$ & 321TSLP & 4.2 & 0.010000 & Fine- to medium-grained sandstone \\
\hline sb16-101-11ddc & $14,461.0$ & $14,462.0$ & $321 \mathrm{TSLP}$ & 3.1 & 0.420000 & Fine-grained sandstone \\
\hline sb16-101-11ddc & $14,462.0$ & $14,463.0$ & 321TSLP & 3.4 & 0.030000 & Fine-grained sandstone \\
\hline sb16-101-11ddc & $14,464.0$ & $14,465.0$ & $321 \mathrm{TSLP}$ & 1.9 & 0.030000 & Fine- to medium-grained sandstone \\
\hline sb $16-101-11 d d c$ & $14,466.0$ & $14,467.0$ & $321 \mathrm{TSLP}$ & 2.4 & 0.010000 & Fine- to medium-grained sandstone \\
\hline sb16-101-11ddc & $14,468.0$ & $14,469.0$ & $321 \mathrm{TSLP}$ & 2.5 & 2.300000 & Fine- to medium-grained sandstone \\
\hline sb16-101-11ddc & $14,469.0$ & $14,470.0$ & 321 TSLP & 0.9 & 0.030000 & Fine- to medium-grained sandstone \\
\hline sb16-101-11ddc & $14,470.0$ & $14,471.0$ & 321TSLP & 3.7 & 0.030000 & Fine- to medium-grained sandstone \\
\hline sb16-101-11ddc & $14,472.0$ & $14,473.0$ & $321 \mathrm{TSLP}$ & 2.4 & 0.010000 & Fine- to medium-grained sandstone \\
\hline sb $16-101-11 d d c$ & $14,474.0$ & $14,475.0$ & $321 \mathrm{TSLP}$ & 3.2 & 0.010000 & Fine- to medium-grained sandstone \\
\hline sb16-101-11ddc & $14,476.0$ & $14,477.0$ & $321 \mathrm{TSLP}$ & 1.8 & 0.030000 & Fine- to medium-grained sandstone \\
\hline sb16-101-11ddc & $14,478.0$ & $14,479.0$ & $321 \mathrm{TSLP}$ & 2.7 & 0.040000 & Fine- to medium-grained sandstone \\
\hline sb16-101-11ddc & $14,480.0$ & $14,481.0$ & $321 \mathrm{TSLP}$ & 4.3 & 0.050000 & Fine- to medium-grained sandstone \\
\hline sb16-101-11ddc & $14,481.0$ & $14,482.0$ & $321 \mathrm{TSLP}$ & 5.6 & 0.120000 & Fine- to medium-grained sandstone \\
\hline sb16-101-11ddc & $14,482.0$ & $14,483.0$ & $321 \mathrm{TSLP}$ & 3.8 & 0.030000 & Fine-grained sandstone \\
\hline sb $16-101-11 d d c$ & $14,484.0$ & $14,485.0$ & $321 \mathrm{TSLP}$ & 1.6 & 0.060000 & Fine- to medium-grained sandstone \\
\hline SITE AVERAGE-- & & & AVERAGE-- & $\frac{5.0}{4.5}$ & $\frac{2.901048}{2.992111}$ & \\
\hline sb16-101-15aaa & $13,781.0$ & $13,782.0$ & 317 PRKC & 0.5 & 0.020000 & Shaly, crystalline dolomite \\
\hline sb16-101-15aaa & $13,782.0$ & $13,783.0$ & $317 \mathrm{PRKC}$ & 0.5 & 0.020000 & Fine-grained dolomite with calcite inclusions \\
\hline sb16-101-15aaa & $13,783.0$ & $13,784.0$ & 317 PRKC & 0.4 & 0.020000 & Fine-grained dolomite \\
\hline sb16-101-15aaa & $13,784.0$ & $13,785.0$ & 317 PRKC & 0.5 & 0.010000 & Fine-grained dolomite \\
\hline sb16-101-15aaa & $13,785.0$ & $13,786.0$ & 317PRKC & 0.3 & 0.000000 & Fine-grained dolomite \\
\hline sb16-101-15aaa & $13,786.0$ & $13,787.0$ & 317PRKC & 0.3 & 0.500000 & Fine-grained dolomite \\
\hline sb16-101-15aaa & $13,787.0$ & $13,788.0$ & 317 PRKC & 0.3 & 0.000000 & Fine-grained dolomite \\
\hline sb16-101-15aa a & $13,788.0$ & $13,789.0$ & 317 PRKC & 0.3 & 0.010000 & Fine-grained dolomite \\
\hline sb16-101-15aaa & $13,789.0$ & $13,790.0$ & $317 \mathrm{PRKC}$ & 0.3 & 0.020000 & Fine-grained, carbonaceous dolomite \\
\hline sb16-101-15aaa & $13,790.0$ & $13,791.0$ & 317PRKC & 0.5 & 0.010000 & Fine-grained dolomite \\
\hline sb16-101-15aaa & $13,791.0$ & $13,792.0$ & 317PRKC & 1.2 & 0.010000 & Fine-grained dolomite \\
\hline sb16-101-15aaa & $13,792.0$ & $13,793.0$ & 317 PRKC & 0.8 & 0.040000 & Fine-grained dolomite \\
\hline sb16-101-15aaa & $13,793.0$ & $13,794.0$ & 317PRKC & 0.3 & 0.010000 & Fine-grained, carbonaceous dolomite \\
\hline sb16-101-15aaa & $13,794.0$ & $13,795.0$ & 317 PRKC & 0.3 & 0.010000 & Fine-grained dolomite \\
\hline sb16-101-15aaa & $13,795.0$ & $13,796.0$ & 317PRKC & 0.4 & 0.010000 & Fine-grained, carbonaceous dolomite \\
\hline sb16-101-15aaa & $13,796.0$ & $13,797.0$ & 317PRKC & 1.0 & 0.020000 & Fine-grained, carbonaceous dolomite \\
\hline sb16-101-15aaa & $13,797.0$ & $13,798.0$ & 317 PRKC & 0.6 & 0.010000 & Fine-grained, carbonaceous dolomite \\
\hline sb16-101-15aaa & $13,798.0$ & $13,799.0$ & $317 \mathrm{PRKC}$ & 1.5 & 0.040000 & Fine-grained, carbonaceous dolomite \\
\hline sb16-101-15aaa & $13,799.0$ & $13,800.0$ & 317 PRKC & 1.5 & 0.290000 & Fine-grained, carbonaceous dolomite \\
\hline sb16-101-15aa a & $13,800.0$ & $13,801.0$ & 317 PRKC & 1.3 & 0.040000 & Fine-grained, carbonaceous dolomite \\
\hline sb16-101-15aaa & $13,801.0$ & $13,802.0$ & $317 \mathrm{PRKC}$ & 0.6 & 0.010000 & Fine-grained dolomite \\
\hline sb16-101-15aaa & $13,802.0$ & $13,803.0$ & 317 PRKC & 1.2 & 0.500000 & Fine-grained dolomite \\
\hline sb16-101-15aaa & $13,803.0$ & $13,804.0$ & 317 PRKC & 1.5 & 0.110000 & Fine-grained dolomite \\
\hline sb16-101-15aaa & $13,804.0$ & $13,805.0$ & 317 PRKC & 1.1 & 0.030000 & Fine-grained dolomite \\
\hline sb16-101-15aaa & $13,805.0$ & $13,806.0$ & 317 PRKC & 0.9 & 0.750000 & Fine-grained dolomite with calcite crystals \\
\hline
\end{tabular}


Table 4.--Laboratory-determined porosity and permeability--Continued

\begin{tabular}{|c|c|c|c|c|c|c|}
\hline Site & $\begin{array}{l}\text { Depth to } \\
\text { top } \\
\text { (feet) }\end{array}$ & $\begin{array}{l}\text { Depth to } \\
\text { bottom } \\
\text { (feet) }\end{array}$ & $\begin{array}{c}\text { Forma- } \\
\text { tion }\end{array}$ & $\begin{array}{l}\text { Porosity } \\
\text { (percent) }\end{array}$ & $\begin{array}{l}\text { Perme- } \\
\text { ability } \\
\text { (milli- } \\
\text { darcies) }\end{array}$ & Lithology \\
\hline sb16-101-15aaa & $13,806.0$ & $13,807.0$ & 317PRKC & 0.6 & 0.800000 & Crystalline dolomite \\
\hline sb16-101-15aaa & $13,807.0$ & $13,808.0$ & 317PRKC & 0.7 & 0.010000 & Crystalline dolomite \\
\hline sb16-101-15aaa & $13,808.0$ & $13,809.0$ & 317PRKC & 0.9 & 0.040000 & Crystalline dolomite with calcite crystals \\
\hline sb16-101-15aaa & $13,809.0$ & $13,810.0$ & 317PRKC & 0.4 & 0.000000 & Crystalline dolomite with calcite crystals \\
\hline sb16-101-15aaa & $13,810.0$ & $13,811.0$ & 317 PRKC & 3.5 & 0.020000 & Fine-grained dolomite \\
\hline sb16-101-15aaa & $13,811.0$ & $13,812.0$ & 317 PRKC & 2.5 & 0.010000 & Fine-grained dolomite \\
\hline sb16-101-15aaa & $13,812.0$ & $13,813.0$ & 317PRKC & 12.6 & 0.850000 & Sucrose dolomite \\
\hline sb16-101-15aaa & $13,813.0$ & $13,814.0$ & 317PRKC & 10.8 & 0.430000 & Sucrose dolomite \\
\hline \multirow[t]{2}{*}{ sb16-101-15aaa } & $13,814.0$ & $13,815.0$ & 317PRKC & 9.1 & 0.100000 & Sucrose dolomite \\
\hline & & & AVERAGE-- & 1.7 & 0.139706 & \\
\hline sb16-101-15aaa & $13,816.0$ & $13,817.0$ & 321TSLP & 0.7 & 0.030000 & Fine-grained sandstone \\
\hline sb16-101-15aaa & $13,817.0$ & $13,818.0$ & 321TSLP & 0.9 & 0.030000 & Fine-grained sandstone \\
\hline sb16-101-15aaa & $13,818.0$ & $13,819.0$ & $321 \mathrm{TSLP}$ & 1.3 & 0.040000 & Fine-grained sandstone \\
\hline sb16-101-15aaa & $13,819.0$ & $13,820.0$ & 321TSLP & 1.9 & 0.030000 & Fine-grained sandstone \\
\hline sb16-101-15aaa & $13,820.0$ & $13,821.0$ & $321 \mathrm{TSLP}$ & 0.8 & 0.030000 & Fine-grained sandstone \\
\hline sb16-101-15aaa & $13,821.0$ & $13,822.0$ & $321 \mathrm{TSLP}$ & 1.3 & 0.040000 & Fine-grained sandstone \\
\hline sb16-101-15aaa & $13,822.0$ & $13,823.0$ & 321TSLP & 1.6 & 0.040000 & Fine-grained sandstone \\
\hline sb16-101-15aaa & $13,823.0$ & $13,824.0$ & $321 \mathrm{TSLP}$ & 1.3 & 0.030000 & Fine-grained sandstone with calcite inclusions \\
\hline sb16-101-15aaa & $13,824.0$ & $13,825.0$ & $321 \mathrm{TSLP}$ & 1.8 & 0.030000 & Fine-grained sandstone with calcite inclusions \\
\hline sb16-101-15aaa & $13,825.0$ & $13,826.0$ & $321 \mathrm{TSLP}$ & 1.9 & 0.040000 & Fine-grained sandstone with calcite inclusions \\
\hline sb16-101-15aaa & $13,826.0$ & $13,827.0$ & $321 \mathrm{TSLP}$ & 2.7 & 0.150000 & Fine-grained sandstone with calcite inclusions \\
\hline sb16-101-15aaa & $13,827.0$ & $13,828.0$ & 321TSLP & 1.3 & 0.030000 & Fine-grained sandstone with calcite inclusions \\
\hline sb16-101-15aaa & $13,828.0$ & $13,829.0$ & 321TSLP & 2.7 & 0.050000 & Fine-grained sandstone with calcite inclusions \\
\hline sb16-101-15aaa & $13,829.0$ & $13,830.0$ & $321 \mathrm{TSLP}$ & 1.1 & 0.030000 & Fine-grained sandstone with calcite inclusions \\
\hline sb16-101-15aaa & $13,830.0$ & $13,831.0$ & $321 \mathrm{TSLP}$ & 2.5 & 0.040000 & Fine-grained sandstone with calcite inclusions \\
\hline sb16-101-15aaa & $13,831.0$ & $13,832.0$ & 321TSLP & 1.3 & 0.060000 & Fine-grained sandstone with calcite inclusions \\
\hline sb16-101-15aaa & $13,832.0$ & $13,833.0$ & 321TSLP & 1.5 & 0.050000 & Fine-grained sandstone with calcite inclusions \\
\hline sb16-101-15aaa & $13,833.0$ & $13,834.0$ & 321TSLP & 1.7 & 0.050000 & Fine-grained sandstone with calcite inclusions \\
\hline sb16-101-15aaa & $13,834.0$ & $13,835.0$ & $321 \mathrm{TSLP}$ & 2.4 & 0.030000 & Fine-grained sandstone with calcite inclusions \\
\hline sb16-101-15aaa & $13,835.0$ & $13,836.0$ & $321 \mathrm{TSLP}$ & 5.6 & 0.030000 & Fine-grained sandstone with calcite inclusions \\
\hline sb16-101-15aaa & $13,836.0$ & $13,837.0$ & $321 \mathrm{TSLP}$ & 2.2 & 0.040000 & Fine-grained sandstone with calcite inclusions \\
\hline sb16-101-15aaa & $13,837.0$ & $13,838.0$ & $321 \mathrm{TSLP}$ & 1.3 & 0.030000 & Fine-grained sandstone with calcite inclusions \\
\hline sb16-101-15aaa & $13,838.0$ & $13,839.0$ & $321 \mathrm{TSLP}$ & 1.8 & 0.060000 & Fine-grained sandstone with calcite inclusions \\
\hline sb16-101-15aaa & $13,839.0$ & $13,840.0$ & 321TSLP & 1.4 & 0.030000 & Fine-grained sandstone with calcite inclusions \\
\hline sb16-101-15aaa & $13,840.0$ & $13,841.0$ & $321 \mathrm{TSLP}$ & 2.2 & 0.040000 & Fine-grained sandstone \\
\hline sb16-101-15aaa & $13,841.0$ & $13,842.0$ & $321 \mathrm{TSLP}$ & 2.8 & 0.030000 & Fine-grained sandstone \\
\hline sb16-101-15aaa & $13,842.0$ & $13,843.0$ & $321 \mathrm{TSLP}$ & 2.0 & 0.030000 & Fine-grained sandstone \\
\hline sb16-101-15aaa & $13,843.0$ & $13,844.0$ & $321 \mathrm{TSLP}$ & 3.4 & 0.030000 & Fine-grained sandstone \\
\hline sb16-101-15aaa & $13,844.0$ & $13,845.0$ & $321 \mathrm{TSLP}$ & 3.9 & 0.020000 & Fine-grained sandstone \\
\hline sb $16-101-15$ a a a & $13,845.0$ & $13,846.0$ & $321 \mathrm{TSLP}$ & 2.2 & 0.030000 & Fine-grained sandstone \\
\hline sb $16-101-15$ aaa & $13,846.0$ & $13,847.0$ & 321TSLP & 1.8 & 0.020000 & Fine-grained sandstone \\
\hline sb16-101-15aaa & $13,847.0$ & $13,848.0$ & $321 \mathrm{TSLP}$ & 3.0 & 0.050000 & Fine-grained sandstone with calcite inclusions \\
\hline sb16-101-15aaa & $13,848.0$ & $13,849.0$ & $321 \mathrm{TSLP}$ & 2.2 & 0.050000 & Fine-grained sandstone with calcite inclusions \\
\hline sb16-101-15aaa & $13,849.0$ & $13,850.0$ & 321TSLP & 2.7 & 0.060000 & Fine-grained sandstone with calcite inclusions \\
\hline sb $16-101-15$ aaa & $13,850.0$ & $13,851.0$ & $321 \mathrm{TSLP}$ & 4.2 & 0.040000 & Fine-grained sandstone with calcite inclusions \\
\hline sb16-101-15aaa & $13,851.0$ & $13,852.0$ & $321 \mathrm{TSLP}$ & 0.9 & 0.030000 & Fine-grained, quartzitic sandstone with calcite \\
\hline sb16-101-15aaa & $13,852.0$ & $13,853.0$ & $321 \mathrm{TSLP}$ & 1.4 & 0.030000 & Fine-grained, quartzitic sandstone with calcite \\
\hline sb16-101-15aaa & $13,853.0$ & $13,854.0$ & $321 \mathrm{TSLP}$ & 0.5 & 0.020000 & Fine-grained, quartzitic sandstone with calcite \\
\hline sb $16-101-15$ aaa & $13,854.0$ & $13,855.0$ & $321 \mathrm{TSLP}$ & 0.7 & 0.030000 & Fine-grained, quartzitic sandstone with calcite \\
\hline sb16-101-15aaa & $13,855.0$ & $13,856.0$ & $321 \mathrm{TSLP}$ & 3.5 & 0.470000 & Fine-grained sandstone with calcite inclusions \\
\hline
\end{tabular}


Table 4.--Laboratory-determined porosity and permeability--Continued

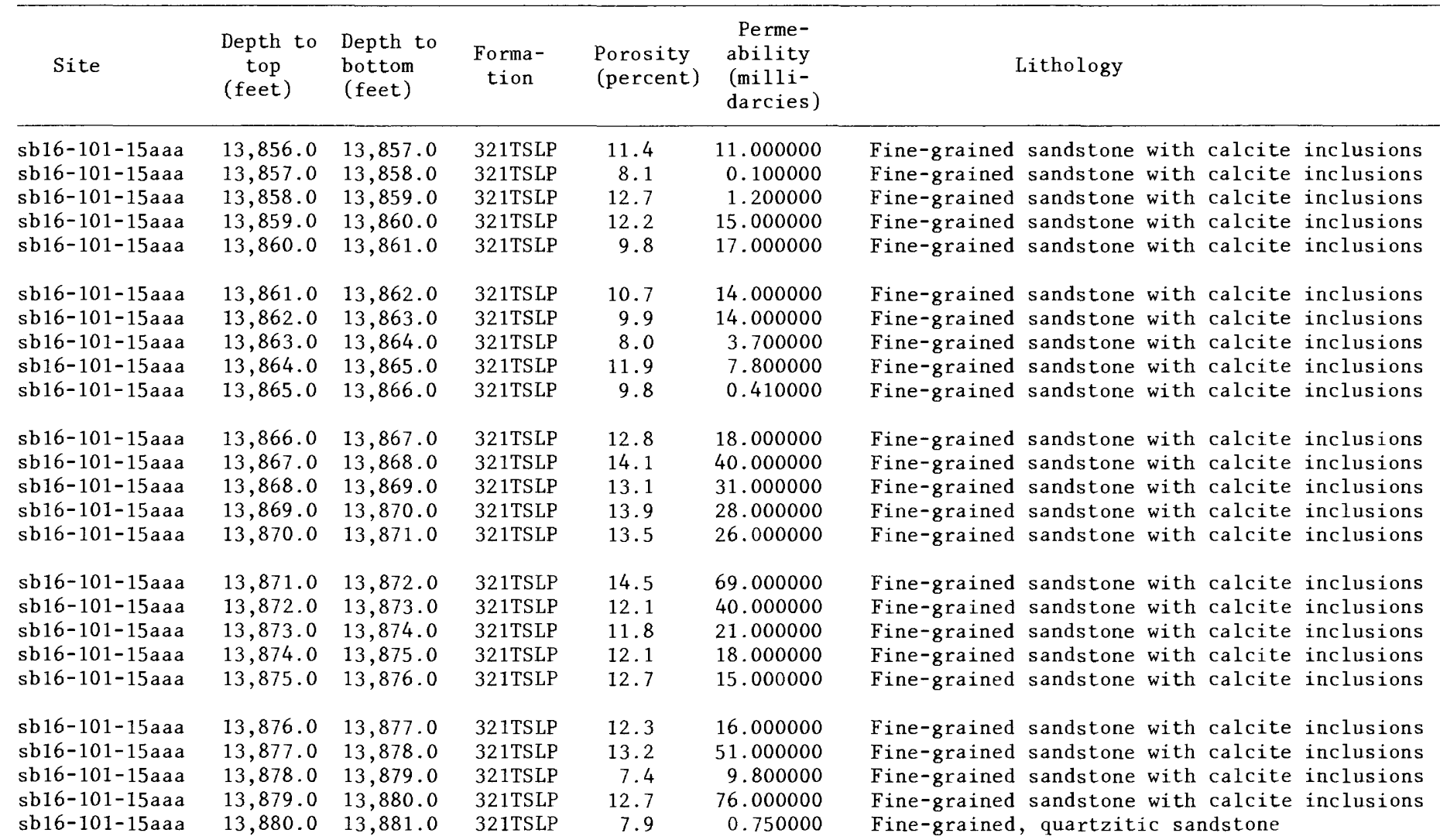

$\mathrm{sb} 16-101-15 \mathrm{aaa}$ sb16-101-15aaa sb16-101-15aaa sb16-101-15aaa sb16-101-15aaa

sb16-101-15aa a sb16-101-15aaa sb16-101-15aaa sb16-101-15aaa sb16-101-15aaa

sb16-101-15aaa sb16-101-15aaa sb16-101-15aaa sb16-101-15aaa sb16-101-15aa

$\mathrm{sb} 16-101-15 \mathrm{aaa}$ sb16-101-15aaa sb16-101-15aaa sb16-101-15aaa sb16-101-15aaa

sb16-101-15aaa sb16-101-15aaa sb16-101-15aaa sb16-101-15aaa sb16-101-15aaa

$\begin{array}{rrrrr}13,881.0 & 13,882.0 & 321 \mathrm{TSLP} & 9.9 & 1.200000 \\ 13,882.0 & 13,883.0 & 321 \mathrm{TSLP} & 7.1 & 0.030000 \\ 13,883.0 & 13,884.0 & 321 \mathrm{TSLP} & 1.8 & 0.200000 \\ 13,884.0 & 13,885.0 & 321 \mathrm{TSLP} & 2.8 & 2.100000 \\ 13,885.0 & 13,886.0 & 321 \mathrm{TSLP} & 2.7 & 0.030000 \\ & & & & \\ 13,886.0 & 13,887.0 & 321 \mathrm{TSLP} & 1.8 & 0.030000 \\ 13,887.0 & 13,887.5 & 321 \mathrm{TSLP} & 1.3 & 0.020000 \\ 13,887.5 & 13,888.0 & 321 \mathrm{TSLP} & 7.2 & 0.610000 \\ 13,888.0 & 13,889.0 & 321 \mathrm{TSLP} & 15.0 & 32.000000 \\ 13,889.0 & 13,890.0 & 321 \mathrm{TSLP} & 9.4 & 2.100000 \\ & & & & \\ 13,890.0 & 13,891.0 & 321 \mathrm{TSLP} & 8.5 & 0.610000 \\ 13,891.0 & 13,892.0 & 321 \mathrm{TSLP} & 8.1 & 0.130000 \\ 13,892.0 & 13,893.0 & 321 \mathrm{TSLP} & 7.7 & 0.460000 \\ 13,893.0 & 13,894.0 & 321 \mathrm{TSLP} & 5.8 & 2.400000 \\ 13,894.0 & 13,895.0 & 321 \mathrm{TSLP} & 5.7 & 0.750000 \\ & & & & \\ 13,895.0 & 13,896.0 & 321 \mathrm{TSLP} & 7.2 & 0.230000 \\ 13,896.0 & 13,897.0 & 321 \mathrm{TSLP} & 6.2 & 0.060000 \\ 13,897.0 & 13,898.0 & 321 \mathrm{TSLP} & 7.6 & 0.070000 \\ 13,898.0 & 13,899.0 & \text { 321TSLP } & 5.2 & 0.040000 \\ 13,899.0 & 13,900.0 & 321 \mathrm{TSLP} & 1.4 & 0.030000 \\ & & & & \\ 13,900.0 & 13,901.0 & \text { 321TSLP } & 4.7 & 0.030000 \\ 13,901.0 & 13,902.0 & \text { 321TSLP } & 3.0 & 0.060000 \\ 13,902.0 & 13,903.0 & 321 \mathrm{TSLP} & 3.4 & 0.030000 \\ 13,903.0 & 13,904.0 & 321 \mathrm{TSLP} & 3.9 & 0.050000 \\ 13,904.0 & 13,905.0 & 321 \mathrm{TSLP} & 3.5 & 0.030000\end{array}$

Fine-grained, quartzitic sandstone Fine-grained, quartzitic sandstone Fine-grained, quartzitic sandstone Fine-grained, quartzitic sandstone Fine-grained, quartzitic sandstone

Fine-grained, quartzitic sandstone Fine-grained, quartzitic sandstone Fine-grained, quartzitic sandstone Fine-grained sandstone Fine-grained sandstone

Fine-grained sandstone Fine-grained sandstone Fine-grained sandstone Fine-grained sandstone Fine-grained sandstone

Fine-grained sandstone Fine-grained sandstone Fine-grained sandstone Fine-grained sandstone Fine-grained sandstone

Fine-grained sandstone Fine-grained sandstone

Fine-grained sandstone with calcite inclusions Fine-grained sandstone with calcite inclusions Fine-grained sandstone with calcite inclusions 
Table 4.--Laboratory-determined porosity and permeability--Continued

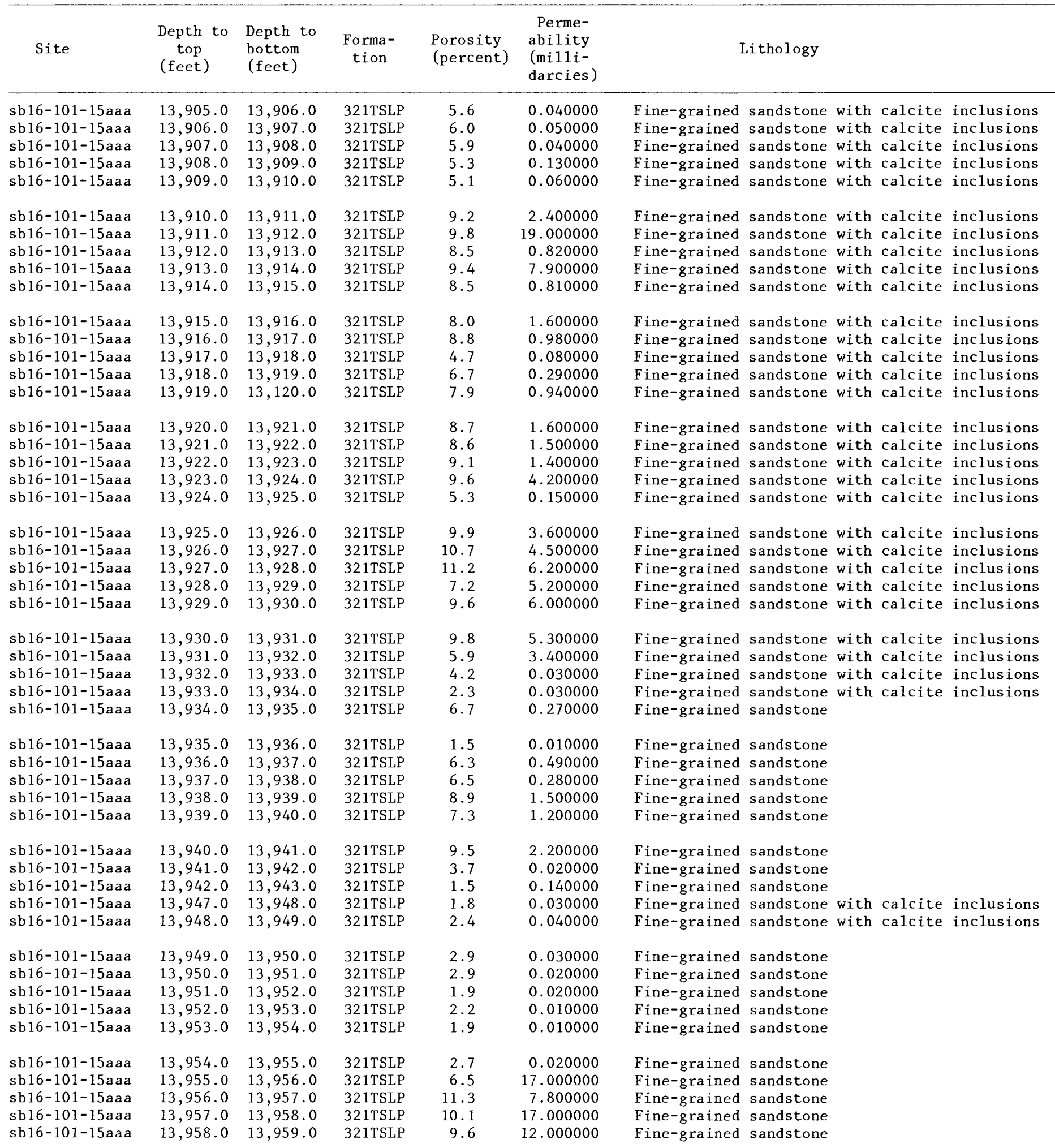


Table 4.--Laboratory-determined porosity and permeability--Continued

\begin{tabular}{|c|c|c|c|c|c|c|}
\hline Site & $\begin{array}{l}\text { Depth to } \\
\text { top } \\
\text { (feet) }\end{array}$ & $\begin{array}{l}\text { Depth to } \\
\text { bottom } \\
\text { (feet) }\end{array}$ & $\begin{array}{c}\text { Forma- } \\
\text { tion }\end{array}$ & $\begin{array}{l}\text { Porosity } \\
\text { (percent) }\end{array}$ & $\begin{array}{l}\text { Perme- } \\
\text { ability } \\
\text { (milli- } \\
\text { darcies) }\end{array}$ & Lithology \\
\hline sb16-101-15aаa & $13,959.0$ & $13,960.0$ & $321 \mathrm{TSLP}$ & 1.0 & 0.020000 & Fine-grained sandstone \\
\hline sb16-101-15aaa & $13,960.0$ & $13,961.0$ & $321 \mathrm{TSLP}$ & 8.1 & 0.030000 & Fine-grained sandstone \\
\hline sb16-101-15aaa & $13,961.0$ & $13,962.0$ & $321 \mathrm{TSLP}$ & 9.4 & 5.200000 & Fine-grained sandstone \\
\hline sb16-101-15aaa & $13,962.0$ & $13,963.0$ & 321TSLP & 7.7 & 0.030000 & Fine-grained sandstone \\
\hline sb16-101-15aaa & $13,963.0$ & $13,964.0$ & $321 \mathrm{TSLP}$ & 11.1 & 3.300000 & Fine-grained sandstone \\
\hline sb16-101-15aaa & $13,964.0$ & $13,965.0$ & $321 \mathrm{TSLP}$ & 10.3 & 5.600000 & Fine-grained sandstone \\
\hline sb16-101-15aaa & $13,965.0$ & $13,966.0$ & $321 \mathrm{TSLP}$ & 7.7 & 4.600000 & Fine-grained sandstone \\
\hline sb16-101-15aaa & $13,966.0$ & $13,967.0$ & $321 \mathrm{TSLP}$ & 11.6 & 12.000000 & Fine-grained sandstone \\
\hline sb16-101-15aaa & $13,967.0$ & $13,968.0$ & $321 \mathrm{TSLP}$ & 9.6 & 16.000000 & Fine-grained sandstone \\
\hline sb16-101-15aaa & $13,968.0$ & $13,969.0$ & $321 \mathrm{TSLP}$ & 6.0 & 0.070000 & Fine-grained sandstone \\
\hline sb16-101-15aaa & $13,969.0$ & $13,970.0$ & 321TSLP & 3.0 & 0.050000 & Fine-grained san \\
\hline sb16-101-15aaa & $13,970.0$ & $13,971.0$ & 321TSLP & 2.6 & 0.020000 & Fine-grained sandstone \\
\hline sb16-101-15aaa & $13,971.0$ & $13,972.0$ & 321TSLP & 10.5 & 0.760000 & Fine-grained sandstone \\
\hline sb16-101-15aaa & $13,972.0$ & $13,973.0$ & $321 \mathrm{TSLP}$ & 10.6 & 2.200000 & Fine-grained sandstone \\
\hline sb16-101-15aaa & $13,973.0$ & $13,974.0$ & 321TSLP & 12.1 & 5.200000 & Fine-grained sandstone \\
\hline sb16-101-15aaa & $13,974.0$ & $13,975.0$ & $321 \mathrm{TSLP}$ & 7.3 & 3.700000 & Fine-grained sandstone \\
\hline sb16-101-15aaa & $13,975.0$ & $13,976.0$ & 321TSLP & 9.5 & 0.460000 & Fine-grained sandstone \\
\hline sb16-101-15aaa & $13,976.0$ & $13,977.0$ & 321 TSLP & 9.2 & 0.730000 & Fine-grained sandstone \\
\hline sb16-101-15aaa & $13,977.0$ & $13,978.0$ & 321TSLP & 7.4 & 2.800000 & Fine-grained sandstone \\
\hline sb16-101-15aaa & $13,983.0$ & $13,984.0$ & 321TSLP & 7.2 & 0.280000 & Fine-grained sandstone \\
\hline sb16-101-15aaa & $13,984.0$ & $13,985.0$ & $321 \mathrm{TSLP}$ & 12.0 & 1.000000 & Fine-grained sandstone \\
\hline sb16-101-15aaa & $13,985.0$ & $13,986.0$ & 321TSLP & 13.8 & 11.000000 & Very fractured, fine-grained sandstone \\
\hline sb16-101-15aaa & $13,986.0$ & $13,987.0$ & 321TSLP & 13.2 & 8.500000 & Very fractured, fine-grained sandstone \\
\hline sb16-101-15aaa & $13,987.0$ & $13,988.0$ & 321TSLP & 12.7 & 3.000000 & Very fractured, fine-grained sandstone \\
\hline sb16-101-15aaa & $13,988.0$ & $13,989.0$ & 321TSLP & 13.4 & 1.600000 & Very fractured, fine-grained sandstone \\
\hline sb16-101-15aаa & $13,989.0$ & $13,990.0$ & $321 T S L P$ & 9.7 & 4.000000 & Very fractured, fine-grained sandstone \\
\hline sb16-101-15aaa & $13,990.0$ & $13,991.0$ & $321 \mathrm{TSLP}$ & 9.8 & 2.400000 & Very fractured, fine-grained sandstone \\
\hline sb16-101-15aaa & $13,991.0$ & $13,992.0$ & $321 \mathrm{TSLP}$ & 10.2 & 1.100000 & Fine-grained sandstone \\
\hline
\end{tabular}

sb16-101-15aaa

$13,991.0 \quad 13,992.0 \quad 321$ TSLP

1.100000

AVERAGE-- $\quad 6.5 \quad 4.899107$

4.098020

SITE AVERAGE - -

5.7

$\begin{array}{lllll}13,457.0 & 13,458.0 & 317 \mathrm{PRKC} & 5.5 & 0.010000 \\ 13,458.0 & 13,459.0 & \text { 317PRKC } & 7.1 & 0.010000\end{array}$

sb17-100-04ccc

$13,458.0 \quad 13,459.0 \quad 317 \mathrm{PRKC}$

$13,459.0 \quad 13,460.0 \quad 317 \mathrm{PRKC}$

$\mathrm{sb} 17-100-04 \mathrm{ccc}$

$13,460.0 \quad 13,461.0 \quad 317 \mathrm{PRKC}$

$8.7 \quad 0.010000$

$1.9 \quad 0.010000$

sb17-100-04ccc

$13,461.0 \quad 13,462.0 \quad 317$ PRKC

0.010000

$\mathrm{sb} 17-100-04 \mathrm{ccc}$

$13,462.0 \quad 13,463.0 \quad 317 \mathrm{PRKC}$

9.5

0.010000

7.0

$13,463.0 \quad 13,464.0$

317PRKC

sb17-100-04ccc

sb17-100-04ccc

$13,464.013,465.0$

317PRKC

$13,465.0 \quad 13,466.0 \quad 317 \mathrm{PRKC}$

$10.7 \quad 0.010000$

$9.5 \quad 0.010000$

$11.1 \quad 0.010000$

sb17-100-04ccc

sb17-100-04ccc

$13,466.0 \quad 13,467.0 \quad 317 \mathrm{PRKC}$

0.010000

$13,467.0 \quad 13,468.0 \quad 317 \mathrm{PRKC}$

$13,468.0 \quad 13,469.0 \quad 317$ PRKC

sb17-100-04ccc

sb17-100-04ccc

sb17-100-04ccc

sb17-100-04ccc

$\mathrm{sb} 17-100-04 \mathrm{ccc}$

sb17-100-04ccc

sb17-100-04ccc

sb17-100-04ccc

sb $17-100-04 \mathrm{ccc}$

$13,469.0 \quad 13,470.0 \quad 317 \mathrm{PRKC}$

$13,470.0 \quad 13,471.0 \quad 317$ PRKC

5.7

0.250000

10.9

7.3

0.010000

0.010000

0.020000

$\begin{array}{lllll}13,471.0 & 13,472.0 & 317 \text { PRKC } & 9.5 & 0.010000\end{array}$

Shaly, cherty, crystalline limestone Shaly, cherty, crystalline limestone Shaly, cherty, crystalline limestone Shaly, cherty, crystalline limestone Shaly, cherty, crystalline limestone

Shaly, cherty, crystalline limestone Dolomite with calcite crystals Dolomite with calcite crystals Dolomite with calcite crystals Dolomite with calcite crystals

Dolomite with calcite crystals Dolomite with calcite crystals Dolomite with calcite crystals Dolomite with calcite crystals Dolomite with calcite crystals

$13,472.0 \quad 13,473.0 \quad 317 \mathrm{PRKC}$

$13,473.0 \quad 13,474.0 \quad 317$ PRKC

$13,474.0 \quad 13,475.0 \quad 317$ PRKC

$13,475.0 \quad 13,476.0 \quad 317$ PRKC

$0.9 \quad 0.020000$

Dolomite with calcite crystals

$1.0 \quad 0.010000$

0.010000

0.010000

Dolomite with calcite-filled vugs

Dolomite with calcite-filled vugs

Dolomite with calcite-filled vugs

$\begin{array}{lllll}13,476.0 & 13,477.0 & 317 \text { PRKC } & 0.7 & 0.010000\end{array}$

Dolomite with calcite-filled vugs 
Table 4.--Laboratory-determined porosity and permeability--Continued

\begin{tabular}{|c|c|c|c|c|c|c|}
\hline Site & $\begin{array}{l}\text { Depth to } \\
\text { top } \\
\text { (feet) }\end{array}$ & $\begin{array}{l}\text { Depth to } \\
\text { bottom } \\
\text { (feet) }\end{array}$ & $\begin{array}{c}\text { Forma- } \\
\text { tion }\end{array}$ & $\begin{array}{l}\text { Porosity } \\
\text { (percent) }\end{array}$ & $\begin{array}{l}\text { Perme- } \\
\text { ability } \\
\text { (milli- } \\
\text { darcies) }\end{array}$ & Lithology \\
\hline $\mathrm{sb} 17-100-04 \mathrm{ccc}$ & $13,477.0$ & $13,478.0$ & $317 \mathrm{PRKC}$ & 1.0 & 0.010000 & Dolomite with calcite-filled vugs \\
\hline $\mathrm{sb} 17-100-04 \mathrm{ccc}$ & $13,478.0$ & $13,479.0$ & 317PRKC & 1.4 & 0.010000 & Dolomite with calcite-filled vugs \\
\hline $\mathrm{sb} 17-100-04 \mathrm{ccc}$ & $13,479.0$ & $13,480.0$ & 317 PRKC & 1.3 & 0.010000 & Dolomite with calcite-filled vugs \\
\hline $\mathrm{sb} 17-100-04 \mathrm{ccc}$ & $13,480.0$ & $13,481.0$ & 317PRKC & 9.3 & 0.310000 & Dolomite with calcite-filled vugs \\
\hline $\mathrm{sb17}-100-04 \mathrm{ccc}$ & $13,481.0$ & $13,482.0$ & 317PRKC & 2.1 & 0.040000 & Dolomite with calcite-filled vugs \\
\hline $\mathrm{sb} 17-100-04 \mathrm{ccc}$ & $13,482.0$ & $13,483.0$ & 317PRKC & 2.0 & 0.060000 & Dolomite with calcite-filled vugs \\
\hline $\mathrm{sb} 17-100-04 \mathrm{ccc}$ & $13,483.0$ & $13,484.0$ & 317PRKC & 11.7 & 0.190000 & Dolomite with calcite-filled vugs \\
\hline $\mathrm{sb} 17-100-04 \mathrm{ccc}$ & $13,484.0$ & $13,485.0$ & 317PRKC & 5.0 & 9.200000 & Dolomite with calcite-filled vugs \\
\hline sb17-100-04ccc & $13,485.0$ & $13,486.0$ & 317PRKC & 12.8 & 0.050000 & Dolomite with calcite-filled vugs \\
\hline $\mathrm{sb} 17-100-04 \mathrm{ccc}$ & $13,486.0$ & $13,487.0$ & 317PRKC & 9.7 & 0.200000 & Dolomite with calcite-filled vugs \\
\hline $\mathrm{sb17}-100-04 \mathrm{ccc}$ & $13,487.0$ & $13,488.0$ & 317PRKC & 7.0 & 0.070000 & Dolomite with calcite-filled vugs \\
\hline $\mathrm{sb} 17-100-04 \mathrm{ccc}$ & $13,488.0$ & $13,489.0$ & 317PRKC & 5.9 & 0.010000 & Dolomite with calcite, chert, and phosphate nodules \\
\hline $\mathrm{sb} 17-100-04 \mathrm{ccc}$ & $13,489.0$ & $13,490.0$ & 317PRKC & 2.8 & 0.010000 & Dolomite with calcite, chert, and phosphate nodules \\
\hline sb $17-100-04 \mathrm{ccc}$ & $13,490.0$ & $13,491.0$ & 317PRKC & 3.8 & 0.020000 & Dolomite with calcite, chert, and phosphate nodules \\
\hline $\mathrm{sb} 17-100-04 \mathrm{ccc}$ & $13,491.0$ & $13,492.0$ & 317PRKC & 3.6 & 0.010000 & Dolomite with calcite, chert, and phosphate nodules \\
\hline sb $17-100-04 \mathrm{ccc}$ & $13,492.0$ & $13,493.0$ & 317PRKC & 3.7 & 0.010000 & Dolomite with calcite, chert, and phosphate nodules \\
\hline sb17-100-04ccc & $13,493.0$ & $13,494.0$ & 317PRKC & 5.5 & 0.160000 & Dolomite with calcite, chert, and phosphate nodules \\
\hline $\mathrm{sb17}-100-04 \mathrm{ccc}$ & $13,494.0$ & $13,495.0$ & 317PRKC & 4.7 & 0.010000 & Dolomite with calcite, chert, and phosphate nodules \\
\hline sb $17-100-04 \mathrm{ccc}$ & $13,495.0$ & $13,496.0$ & 317PRKC & 10.5 & 0.030000 & Dolomite with calcite, chert, and phosphate nodules \\
\hline sb17-100-04ccc & $13,496.0$ & $13,497.0$ & 317PRKC & 6.0 & 0.040000 & Dolomite with calcite, chert, and phosphate nodules \\
\hline $\mathrm{sb} 17-100-04 \mathrm{ccc}$ & $13,497.0$ & $13,498.0$ & 317PRKC & 7.4 & 4.400000 & Dolomite with calcite crystals \\
\hline sb17-100-04ccc & $13,498.0$ & $13,499.0$ & 317PRKC & 9.4 & 0.010000 & Dolomite with calcite crystals \\
\hline sb17-100-04ccc & $13,499.0$ & $13,500.0$ & 317PRKC & 2.8 & 0.010000 & Dolomite with calcite crystals \\
\hline sb $17-100-04 \mathrm{ccc}$ & $13,500.0$ & $13,501.0$ & 317PRKC & 8.8 & 0.190000 & Dolomite with calcite crystals \\
\hline sb17-100-04ccc & $13,501.0$ & $13,502.0$ & 317PRKC & 3.9 & 3.100000 & Dolomite with calcite crystals \\
\hline sb17-100-04ccc & $13,502.0$ & $13,503.0$ & 317PRKC & 0.0 & 3.100000 & Dolomite with calcite crystals \\
\hline sb17-100-04ccc & $13,503.0$ & $13,504.0$ & 317PRKC & 2.3 & 0.010000 & Dolomite with calcite crystals \\
\hline sb17-100-04ccc & $13,504.0$ & $13,505.0$ & 317PRKC & 4.5 & 0.010000 & Dolomite with calcite-filled vugs \\
\hline sb17-100-04ccc & $13,505.0$ & $13,506.0$ & 317PRKC & 10.2 & 0.320000 & Dolomite with calcite-filled vugs \\
\hline sb17-100-04ccc & $13,506.0$ & $13,507.0$ & 317PRKC & 12.0 & 0.890000 & Dolomite with calcite-filled vugs \\
\hline sb17-100-04ccc & $13,507.0$ & $13,508.0$ & 317PRKC & 10.8 & 0.890000 & Dolomite with calcite-filled vugs \\
\hline sb17-100-04ccc & $13,508.0$ & $13,509.0$ & 317PRKC & 5.5 & 2.900000 & Dolomite with calcite-filled vugs \\
\hline sb $17-100-04 \mathrm{ccc}$ & $13,509.0$ & $13,510.0$ & 317PRKC & 3.9 & 0.090000 & Dolomite with calcite-filled vugs \\
\hline sb $17-100-04 \mathrm{ccc}$ & $13,510.0$ & $13,511.0$ & 317PRKC & 2.2 & 0.010000 & Dolomite with calcite-filled vugs \\
\hline sb17-100-04ccc & $13,511.0$ & $13,512.0$ & 317PRKC & 0.9 & 0.010000 & Dolomite with calcite-filled vugs \\
\hline sb $17-100-04 \mathrm{ccc}$ & $13,512.0$ & $13,513.0$ & 317PRKC & 1.3 & 0.060000 & Finely crystalline dolomite \\
\hline sb17-100-04ccc & $13,513.0$ & $13,514.0$ & 317PRKC & 1.0 & 0.040000 & Finely crystalline dolomite \\
\hline $\mathrm{sb} 17-100-04 \mathrm{ccc}$ & $13,514.0$ & $13,515.0$ & 317PRKC & 0.3 & 0.060000 & Finely crystalline dolomite \\
\hline $\mathrm{sb} 17-100-04 \mathrm{ccc}$ & $13,515.0$ & $13,516.0$ & 317PRKC & 1.2 & 0.420000 & Finely crystalline dolomite \\
\hline $\mathrm{sb} 17-100-04 \mathrm{ccc}$ & $13,516.0$ & $13,517.0$ & 317PRKC & 0.8 & 0.120000 & Finely crystalline dolomite \\
\hline $\mathrm{sb} 17-100-04 \mathrm{ccc}$ & $13,517.0$ & $13,518.0$ & 317PRKC & 2.4 & 0.030000 & Finely crystalline dolomite \\
\hline sb17-100-04ccc & $13,518.0$ & $13,519.0$ & 317PRKC & 2.0 & 0.020000 & Finely crystalline dolomite with phosphate nodules \\
\hline sb17-100-04ccc & $13,519.0$ & $13,520.0$ & 317PRKC & 1.9 & 0.020000 & Finely crystalline dolomite with phosphate nodules \\
\hline sb17-100-04ccc & $13,520.0$ & $13,521.0$ & 317PRKC & 3.8 & 0.030000 & Finely crystalline dolomite with phosphate nodules \\
\hline sb17-100-04ccc & $13,521.0$ & $13,522.0$ & 317PRKC & 3.0 & 0.030000 & Finely crystalline dolomite with phosphate nodules \\
\hline $\mathrm{sb} 17-100-04 \mathrm{ccc}$ & $13,522.0$ & $13,523.0$ & 317PRKC & 1.9 & 0.030000 & Fossiliferous, finely crystalline dolomite \\
\hline $\mathrm{sb} 17-100-04 \mathrm{ccc}$ & $13,523.0$ & $13,524.0$ & 317PRKC & 1.1 & 0.030000 & Fossiliferous, finely crystalline dolomite \\
\hline $\mathrm{sb} 17-100-04 \mathrm{ccc}$ & $13,524.0$ & $13,525.0$ & 317PRKC & 2.0 & 0.030000 & Fossiliferous, finely crystalline dolomite \\
\hline $\mathrm{sb} 17-100-04 \mathrm{ccc}$ & $13,525.0$ & $13,526.0$ & 317PRKC & 1.3 & 0.020000 & Fossiliferous, finely crystalline dolomite \\
\hline $\mathrm{sb} 17-100-04 \mathrm{ccc}$ & $13,526.0$ & $13,527.0$ & 317PRKC & 0.7 & 0.010000 & Fossiliferous, finely crystalline dolomite \\
\hline
\end{tabular}


Table 4.--Laboratory-determined porosity and permeability--Continued

\begin{tabular}{|c|c|c|c|c|c|c|}
\hline Site & $\begin{array}{l}\text { Depth to } \\
\text { top } \\
\text { (feet) }\end{array}$ & $\begin{array}{l}\text { Depth to } \\
\text { bottom } \\
\text { (feet) }\end{array}$ & $\begin{array}{c}\text { Forma- } \\
\text { tion }\end{array}$ & $\begin{array}{l}\text { Porosity } \\
\text { (percent) }\end{array}$ & $\begin{array}{l}\text { Perme- } \\
\text { ability } \\
\text { (milli- } \\
\text { darcies) }\end{array}$ & Lithology \\
\hline $\mathrm{sb} 17-100-04 \mathrm{ccc}$ & $13,527.0$ & $13,528.0$ & 317PRKC & 2.0 & 0.020000 & Fossiliferous, finely crystalline dolomite \\
\hline $\mathrm{sb} 17-100-04 \mathrm{ccc}$ & $13,528.0$ & $13,529.0$ & 317PRKC & 0.7 & 0.020000 & Fossiliferous, finely crystalline dolomite \\
\hline $\mathrm{sb} 17-100-04 \mathrm{ccc}$ & $13,529.0$ & $13,530.0$ & 317PRKC & 1.7 & 0.030000 & Fossiliferous, finely crystalline dolomite \\
\hline $\mathrm{sb} 17-100-04 \mathrm{ccc}$ & $13,530.0$ & $13,531.0$ & 317PRKC & 1.4 & 0.010000 & Fossiliferous, finely crystalline dolomite \\
\hline $\mathrm{sb} 17-100-04 \mathrm{ccc}$ & $13,531.0$ & $13,532.0$ & 317 PRKC & 1.8 & 0.010000 & Fossiliferous, finely crystalline dolomite \\
\hline $\mathrm{sb} 17-100-04 \mathrm{ccc}$ & $13,532.0$ & $13,533.0$ & 317PRKC & 2.2 & 0.020000 & Fossiliferous, finely crystalline dolomite \\
\hline $\mathrm{sb} 17-100-04 \mathrm{ccc}$ & $13,533.0$ & $13,534.0$ & 317PRKC & 2.1 & 0.030000 & Fossiliferous, finely crystalline dolomite \\
\hline $\mathrm{sb} 17-100-04 \mathrm{ccc}$ & $13,534.0$ & $13,534.6$ & 317PRKC & 1.9 & 0.020000 & Fossiliferous, finely crystalline dolomite \\
\hline $\mathrm{sb} 17-100-04 \mathrm{ccc}$ & $13,534.6$ & $13,535.0$ & 317PRKC & 1.0 & 0.170000 & Finely crystalline dolomite \\
\hline $\mathrm{sb} 17-100-04 \mathrm{ccc}$ & $13,535.0$ & $13,536.0$ & 317PRKC & 1.1 & 4.800000 & Finely crystalline dolomite \\
\hline sb $17-100-04 c c c$ & $13,536.0$ & $13,537.0$ & 317PRKC & 0.5 & 0.130000 & Finely crystalline dolomite \\
\hline $\mathrm{sb} 17-100-04 \mathrm{ccc}$ & $13,537.0$ & $13,538.0$ & 317PRKC & 1.0 & 0.180000 & Finely crystalline dolomite \\
\hline $\mathrm{sb} 17-100-04 \mathrm{ccc}$ & $13,538.0$ & $13,539.0$ & 317PRKC & 0.6 & 0.400000 & Finely crystalline dolomite \\
\hline $\mathrm{sb} 17-100-04 \mathrm{ccc}$ & $13,539.0$ & $13,540.0$ & $317 \mathrm{PRKC}$ & 1.1 & 0.420000 & Finely crystalline dolomite \\
\hline $\mathrm{sb} 17-100-04 \mathrm{ccc}$ & $13,540.0$ & $13,541.0$ & $317 \mathrm{PRKC}$ & 0.8 & 1.100000 & Finely crystalline dolomite \\
\hline sb $17-100-04 \mathrm{ccc}$ & $13,541.0$ & $13,542.0$ & 317PRKC & 1.0 & 0.010000 & Finely crystalline dolomite \\
\hline $\mathrm{sb} 17-100-04 \mathrm{ccc}$ & $13,542.0$ & $13,543.0$ & 317PRKC & 0.9 & 0.010000 & Finely crystalline dolomite \\
\hline sb17-100-04ccc & $13,543.0$ & $13,544.0$ & 317PRKC & 0.6 & 0.310000 & Finely crystalline dolomite \\
\hline $\mathrm{sb} 17-100-04 \mathrm{ccc}$ & $13,544.0$ & $13,545.0$ & 317PRKC & 1.0 & 0.180000 & Finely crystalline dolomite \\
\hline $\mathrm{sb} 17-100-04 \mathrm{ccc}$ & $13,545.0$ & $13,546.0$ & 317 PRKC & 1.0 & 0.250000 & Finely crystalline dolomite \\
\hline $\mathrm{sb} 17-100-04 \mathrm{ccc}$ & $13,546.0$ & $13,547.0$ & 317PRKC & 1.0 & 0.190000 & Finely crystalline dolomite \\
\hline $\mathrm{sb} 17-100-04 \mathrm{ccc}$ & $13,547.0$ & $13,548.0$ & 317PRKC & 1.2 & 0.090000 & Finely crystalline dolomite \\
\hline $\mathrm{sb} 17-100-04 \mathrm{ccc}$ & $13,548.0$ & $13,549.0$ & 317 PRKC & 1.1 & 0.230000 & Finely crystalline dolomite \\
\hline sb17-100-04ccc & $13,549.0$ & $13,550.0$ & 317PRKC & 1.5 & 0.690000 & Finely crystalline dolomite \\
\hline $\mathrm{sb} 17-100-04 \mathrm{ccc}$ & $13,550.0$ & $13,551.0$ & $317 \mathrm{PRKC}$ & 1.8 & 0.200000 & Finely crystalline dolomite \\
\hline $\mathrm{sb} 17-100-04 \mathrm{ccc}$ & $13,551.0$ & $13,552.0$ & 317PRKC & 0.6 & 0.090000 & Finely crystalline dolomite \\
\hline sb $17-100-04 c c c$ & $13,552.0$ & $13,553.0$ & 317PRKC & 1.2 & 0.160000 & Finely crystalline dolomite \\
\hline $\mathrm{sb} 17-100-04 \mathrm{ccc}$ & $13,553.0$ & $13,554.0$ & 317PRKC & 1.3 & 0.010000 & Finely crystalline dolomite \\
\hline sb 17-100-04ccc & $13,554.0$ & $13,555.0$ & 317PRKC & 1.4 & 0.190000 & Finely crystalline dolomite \\
\hline $\mathrm{sb} 17-100-04 \mathrm{ccc}$ & $13,555.0$ & $13,556.0$ & 317PRKC & 1.1 & 0.180000 & Finely crystalline dolomite \\
\hline $\mathrm{sb} 17-100-04 \mathrm{ccc}$ & $13,556.0$ & $13,557.0$ & 317PRKC & 1.4 & 0.430000 & Finely crystalline dolomite \\
\hline sb17-100-04ccc & $13,557.0$ & $13,558.0$ & 317PRKC & 1.3 & 0.160000 & Finely crystalline dolomite \\
\hline $\mathrm{sb} 17-100-04 \mathrm{ccc}$ & $13,558.0$ & $13,559.0$ & 317PRKC & 1.0 & 0.150000 & Finely crystalline dolomite \\
\hline sb17-100-04ccc & $13,559.0$ & $13,560.0$ & 317PRKC & 1.4 & 0.010000 & Finely crystalline dolomite \\
\hline $\mathrm{sb} 17-100-04 \mathrm{ccc}$ & $13,560.0$ & $13,561.0$ & 317PRKC & 1.4 & 0.010000 & Finely crystalline dolomite \\
\hline $\mathrm{sb} 17-100-04 \mathrm{ccc}$ & $13,561.0$ & $13,562.0$ & 317PRKC & 1.5 & 0.100000 & Finely crystalline dolomite \\
\hline sb17-100-04ccc & $13,562.0$ & $13,563.0$ & 317PRKC & 1.5 & 0.010000 & Finely crystalline dolomite \\
\hline $\mathrm{sb} 17-100-04 \mathrm{ccc}$ & $13,563.0$ & $13,564.0$ & 317PRKC & 1.9 & 0.010000 & Finely crystalline dolomite \\
\hline $\mathrm{sb} 17-100-04 \mathrm{ccc}$ & $13,564.0$ & $13,565.0$ & 317PRKC & 0.9 & 0.190000 & Finely crystalline dolomite \\
\hline $\mathrm{sb} 17-100-04 \mathrm{ccc}$ & $13,565.0$ & $13,566.0$ & 317 PRKC & 0.5 & 0.010000 & Finely crystalline dolomite \\
\hline $\mathrm{sb} 17-100-04 \mathrm{ccc}$ & $13,566.0$ & $13,567.0$ & 317PRKC & 0.3 & 0.200000 & Finely crystalline dolomite \\
\hline $\mathrm{sb} 17-100-04 \mathrm{ccc}$ & $13,567.0$ & $13,568.0$ & 317PRKC & 0.6 & 0.350000 & Finely crystalline dolomite \\
\hline $\mathrm{sb} 17-100-04 \mathrm{ccc}$ & $13,568.0$ & $13,569.0$ & 317PRKC & 1.3 & 0.030000 & Finely crystalline dolomite \\
\hline sb $17-100-04 \mathrm{ccc}$ & $13,569.0$ & $13,570.0$ & 317PRKC & 1.2 & 0.010000 & Finely crystalline dolomite \\
\hline $\mathrm{sb} 17-100-04 \mathrm{ccc}$ & $13,570.0$ & $13,571.0$ & 317PRKC & 1.7 & 0.010000 & Finely crystalline dolomite \\
\hline $\mathrm{sb} 17-100-04 \mathrm{ccc}$ & $13,571.0$ & $13,572.0$ & 317PRKC & 0.6 & 1.800000 & Finely crystalline dolomite \\
\hline $\mathrm{sb} 17-100-04 \mathrm{ccc}$ & $13,572.0$ & $13,573.0$ & 317PRKC & 1.4 & 0.260000 & Finely crystalline dolomite \\
\hline $\mathrm{sb} 17-100-04 \mathrm{ccc}$ & $13,573.0$ & $13,574.0$ & 317PRKC & 1.9 & 0.030000 & Finely crystalline dolomite \\
\hline sb $17-100-04 \mathrm{ccc}$ & $13,574.0$ & $13,575.0$ & 317PRKC & 1.2 & 0.010000 & Finely crystalline dolomite \\
\hline $\mathrm{sb} 17-100-04 \mathrm{ccc}$ & $13,575.0$ & $13,576.0$ & 317PRKC & 2.0 & 0.010000 & Finely crystalline dolomite \\
\hline
\end{tabular}


Table 4.--Laboratory-determined porosity and permeability--Continued

\begin{tabular}{|c|c|c|c|c|c|c|}
\hline Site & $\begin{array}{l}\text { Depth to } \\
\text { top } \\
\text { (feet) }\end{array}$ & $\begin{array}{l}\text { Depth to } \\
\text { bottom } \\
\text { (feet) }\end{array}$ & $\begin{array}{c}\text { Forma- } \\
\text { tion }\end{array}$ & $\begin{array}{l}\text { Porosity } \\
\text { (percent) }\end{array}$ & $\begin{array}{l}\text { Perme- } \\
\text { ability } \\
\text { (milli- } \\
\text { darcies) }\end{array}$ & Lithology \\
\hline $\mathrm{sb} 17-100-04 \mathrm{ccc}$ & $13,576.0$ & $13,577.0$ & 317PRKC & 1.0 & 0.120000 & Finely crystalline dolomite \\
\hline $\mathrm{sb} 17-100-04 \mathrm{ccc}$ & $13,577.0$ & $13,578.0$ & 317 PRKC & 0.7 & 0.150000 & Finely crystalline dolomite \\
\hline $\mathrm{sb} 17-100-04 \mathrm{ccc}$ & $13,578.0$ & $13,579.0$ & $317 \mathrm{PRKC}$ & 0.8 & 0.380000 & Finely crystalline dolomite \\
\hline sb17-100-04ccc & $13,579.0$ & $13,580.0$ & 317PRKC & 1.7 & 0.280000 & Finely crystalline dolomite \\
\hline $\mathrm{sb} 17-100-04 \mathrm{ccc}$ & $13,580.0$ & $13,581.0$ & $317 \mathrm{PRKC}$ & 1.8 & 0.010000 & Finely crystalline dolomite \\
\hline $\mathrm{sb} 17-100-04 \mathrm{ccc}$ & $13,581.0$ & $13,582.0$ & $317 \mathrm{PRKC}$ & 0.5 & 3.800000 & Finely crystalline dolomite \\
\hline sb17-100-04ccc & $13,582.0$ & $13,583.0$ & $317 \mathrm{PRKC}$ & 0.7 & 0.440000 & Finely crystalline dolomite \\
\hline $\mathrm{sb} 17-100-04 \mathrm{ccc}$ & $13,583.0$ & $13,584.0$ & 317PRKC & 1.0 & 0.220000 & Finely crystalline dolomite \\
\hline $\mathrm{sb} 17-100-04 \mathrm{ccc}$ & $13,584.0$ & $13,585.0$ & 317PRKC & 0.5 & 0.010000 & Finely crystalline dolomite \\
\hline $\mathrm{sb} 17-100-04 \mathrm{ccc}$ & $13,585.0$ & $13,586.0$ & $317 \mathrm{PRKC}$ & 0.4 & 11.000000 & Finely crystalline dolomite \\
\hline sb17-100-04ccc & $13,586.0$ & $13,587.0$ & 317PRKC & 0.4 & 0.390000 & Anhydrite \\
\hline $\mathrm{sb} 17-100-04 \mathrm{ccc}$ & $13,587.0$ & $13,588.0$ & 317PRKC & 0.4 & 0.010000 & Anhydrite \\
\hline $\mathrm{sb} 17-100-04 \mathrm{ccc}$ & $13,588.0$ & $13,589.0$ & 317PRKC & 0.4 & 0.170000 & Finely crystalline dolomite \\
\hline $\mathrm{sb} 17-100-04 \mathrm{ccc}$ & $13,589.0$ & $13,590.0$ & 317PRKC & 0.6 & 0.140000 & Finely crystalline dolomite \\
\hline $\mathrm{sb} 17-100-04 \mathrm{ccc}$ & $13,590.0$ & $13,591.0$ & $317 \mathrm{PRKC}$ & 0.4 & 0.330000 & Finely crystalline dolomite \\
\hline $\mathrm{sb} 17-100-04 \mathrm{ccc}$ & $13,591.0$ & $13,592.0$ & 317PRKC & 0.3 & 0.030000 & Finely crystalline dolomite \\
\hline sb $17-100-04 \mathrm{ccc}$ & $13,592.0$ & $13,593.0$ & 317PRKC & 0.3 & 0.690000 & Finely crystalline dolomite \\
\hline $\mathrm{sb} 17-100-04 \mathrm{ccc}$ & $13,593.0$ & $13,594.0$ & 317PRKC & 0.7 & 0.090000 & Finely crystalline dolomite \\
\hline $\mathrm{sb} 17-100-04 \mathrm{ccc}$ & $13,594.0$ & $13,595.0$ & 317PRKC & 0.8 & 0.020000 & Finely crystalline dolomite \\
\hline $\mathrm{sb} 17-100-04 \mathrm{ccc}$ & $13,595.0$ & $13,596.0$ & $317 \mathrm{PRKC}$ & 0.6 & 0.020000 & Finely crystalline dolomite \\
\hline sb $17-100-04 \mathrm{ccc}$ & $13,596.0$ & $13,597.0$ & 317 PRKC & 1.0 & 0.270000 & Finely crystalline dolomite \\
\hline $\mathrm{sb} 17-100-04 \mathrm{ccc}$ & $13,597.0$ & $13,598.0$ & 317PRKC & 0.7 & 0.090000 & Finely crystalline dolomite \\
\hline $\mathrm{sb} 17-100-04 \mathrm{ccc}$ & $13,598.0$ & $13,599.0$ & 317PRKC & 1.0 & 0.310000 & Finely crystalline dolomite \\
\hline sb17-100-04ccc & $13,599.0$ & $13,600.0$ & 317PRKC & 0.7 & 0.260000 & Finely crystalline dolomite \\
\hline $\mathrm{sb} 17-100-04 \mathrm{ccc}$ & $13,600.0$ & $13,601.0$ & $317 \mathrm{PRKC}$ & 0.6 & 12.000000 & Finely crystalline dolomite \\
\hline $\mathrm{sb} 17-100-04 \mathrm{ccc}$ & $13,601.0$ & $13,602.0$ & 317PRKC & 0.7 & 0.080000 & Finely crystalline dolomite \\
\hline $\operatorname{sb} 17-100-04 \mathrm{ccc}$ & $13,602.0$ & $13,603.0$ & 317 PRKC & 0.9 & 0.020000 & Finely crystalline dolomite \\
\hline sb17-100-04ccc & $13,603.0$ & $13,604.0$ & 317PRKC & 0.2 & 0.120000 & Finely crystalline dolomite with phosphate nodules \\
\hline $\mathrm{sb} 17-100-04 \mathrm{ccc}$ & $13,604.0$ & $13,605.0$ & 317 PRKC & 0.4 & 0.010000 & Finely crystalline dolomite with phosphate nodules \\
\hline $\mathrm{sb} 17-100-04 \mathrm{ccc}$ & $13,605.0$ & $13,606.0$ & 317PRKC & 0.3 & 0.020000 & Finely crystalline dolomite with phosphate nodules \\
\hline sb $17-100-04 \mathrm{ccc}$ & $13,606.0$ & $13,607.0$ & 317PRKC & 0.3 & 0.370000 & Finely crystalline dolomite with calcite crystals \\
\hline sb $17-100-04 \mathrm{ccc}$ & $13,607.0$ & $13,608.0$ & 317PRKC & 1.5 & 0.050000 & Finely crystalline dolomite with calcite crystals \\
\hline sb $17-100-04 \mathrm{ccc}$ & $13,608.0$ & $13,609.0$ & $317 \mathrm{PRKC}$ & 2.0 & 0.050000 & Finely crystalline dolomite with calcite crystals \\
\hline sb17-100-04ccc & $13,609.0$ & $13,610.0$ & 317PRKC & 1.1 & 0.050000 & Finely crystalline dolomite with phosphate nodules \\
\hline $\mathrm{sb} 17-100-04 \mathrm{ccc}$ & $13,610.0$ & $13,611.0$ & $317 \mathrm{PRKC}$ & 1.8 & 0.480000 & Finely crystalline dolomite with phosphate nodules \\
\hline $\mathrm{sb} 17-100-04 \mathrm{ccc}$ & $13,611.0$ & $13,612.0$ & 317 PRKC & 0.5 & 0.030000 & Finely crystalline dolomite with phosphate nodules \\
\hline $\mathrm{sb} 17-100-04 \mathrm{ccc}$ & $13,612.0$ & $13,613.0$ & 317PRKC & 6.6 & 46.000000 & Finely crystalline dolomite with phosphate nodules \\
\hline sb $17-100-04 \mathrm{ccc}$ & $13,613.0$ & $13,614.0$ & 317PRKC & 6.0 & 0.670000 & Finely crystalline dolomite with phosphate nodules \\
\hline $\mathrm{sb} 17-100-04 \mathrm{ccc}$ & $13,614.0$ & $13,615.0$ & 317PRKC & 0.7 & 0.010000 & Finely crystalline dolomite with phosphate nodules \\
\hline $\mathrm{sb} 17-100-04 \mathrm{ccc}$ & $13,615.0$ & $13,616.0$ & $317 \mathrm{PRKC}$ & 1.7 & 0.240000 & Finely crystalline dolomite with phosphate nodules \\
\hline sb17-100-04ccc & $13,616.0$ & $13,617.0$ & 317PRKC & 5.6 & 0.050000 & Finely crystalline dolomite \\
\hline $\mathrm{sb} 17-100-04 \mathrm{ccc}$ & $13,617.0$ & $13,618.0$ & 317PRKC & 1.1 & 0.330000 & Finely crystalline dolomite \\
\hline sb17-100-04ccc & $13,618.0$ & $13,619.0$ & $317 \mathrm{PRKC}$ & 3.2 & 0.050000 & Finely crystalline dolomite \\
\hline sb17-100-04ccc & $13,619.0$ & $13,620.0$ & 317PRKC & 1.3 & 0.140000 & Finely crystalline dolomite \\
\hline $\mathrm{sb} 17-100-04 \mathrm{ccc}$ & $13,620.0$ & $13,621.0$ & 317 PRKC & 1.1 & 0.080000 & Dolomite with calcite crystals \\
\hline sb $17-100-04 c c c$ & $13,621.0$ & $13,622.0$ & 317PRKC & 0.6 & 0.880000 & Dolomite with calcite crystals \\
\hline $\mathrm{sb} 17-100-04 \mathrm{ccc}$ & $13,622.0$ & $13,623.0$ & 317PRKC & 1.5 & 0.920000 & Dolomite with calcite crystals \\
\hline sb $17-100-04 \mathrm{ccc}$ & $13,623.0$ & $13,624.0$ & 317PRKC & 0.9 & 0.040000 & Coarsely crystalline dolomite with phosphate nodules \\
\hline sb $17-100-04 \mathrm{ccc}$ & $13,624.0$ & $13,625.0$ & 317 PRKC & 1.0 & 0.010000 & Coarsely crystalline dolomite with phosphate nodules \\
\hline $\mathrm{sb} 17-100-04 \mathrm{ccc}$ & $13,625.0$ & $13,626.0$ & 317PRKC & 0.7 & 0.420000 & Coarsely crystalline dolomite with phosphate nodules \\
\hline
\end{tabular}


Table 4.--Laboratory-determined porosity and permeability--Continued

\begin{tabular}{|c|c|c|c|c|c|c|}
\hline Site & $\begin{array}{l}\text { Depth to } \\
\text { top } \\
\text { (feet) }\end{array}$ & $\begin{array}{l}\text { Depth to } \\
\text { bottom } \\
\text { (feet) }\end{array}$ & $\begin{array}{c}\text { Forma- } \\
\text { tion }\end{array}$ & $\begin{array}{l}\text { Porosity } \\
\text { (percent) }\end{array}$ & $\begin{array}{l}\text { Perme- } \\
\text { ability } \\
\text { (milli- } \\
\text { darcies) }\end{array}$ & Lithology \\
\hline sb17-100-04ccc & $13,626.0$ & $13,627.0$ & 317PRKC & 0.7 & 0.400000 & Coarsely crystalline dolomite with phosphate nodules \\
\hline $\mathrm{sb} 17-100-04 \mathrm{ccc}$ & $13,627.0$ & $13,628.0$ & 317PRKC & 0.5 & 0.330000 & Coarsely crystalline dolomite with phosphate nodules \\
\hline sb $17-100-04 \mathrm{ccc}$ & $13,628.0$ & $13,629.0$ & 317 PRKC & 0.6 & 0.030000 & Coarsely crystalline dolomite with phosphate nodules \\
\hline $\mathrm{sb} 17-100-04 \mathrm{ccc}$ & $13,629.0$ & $13,630.0$ & 317PRKC & 0.5 & 0.010000 & Coarsely crystalline dolomite with phosphate nodules \\
\hline $\mathrm{sb} 17-100-04 \mathrm{ccc}$ & $13,630.0$ & $13,631.0$ & 317 PRKC & 0.4 & 0.010000 & Coarsely crystalline dolomite with phosphate nodules \\
\hline sb17-100-04ccc & $13,631.0$ & $13,632.0$ & 317 PRKC & 0.2 & 0.010000 & Coarsely crystalline dolomite with phosphate nodules \\
\hline sb17-100-04ccc & $13,632.0$ & $13,633.0$ & $317 \mathrm{PRKC}$ & 3.2 & 0.180000 & Coarsely crystalline dolomite with phosphate nodules \\
\hline sb $17-100-04 \mathrm{ccc}$ & $13,633.0$ & $13,634.0$ & $317 \mathrm{PRKC}$ & 0.3 & 0.020000 & Coarsely crystalline dolomite with phosphate nodules \\
\hline sb17-100-04ccc & $13,634.0$ & $13,635.0$ & 317 PRKC & 0.9 & 0.610000 & Coarsely crystalline dolomite with phosphate nodules \\
\hline $\mathrm{sb} 17-100-04 \mathrm{ccc}$ & $13,635.0$ & $13,636.0$ & 317 PRKC & 0.4 & 0.500000 & Finely crystalline dolomite \\
\hline sb17-100-04ccc & $13,636.0$ & $13,637.0$ & $317 \mathrm{PRKC}$ & 1.2 & 0.020000 & Finely crystalline dolomite \\
\hline sb17-100-04ccc & $13,637.0$ & $13,638.0$ & 317PRKC & 0.4 & 0.010000 & Finely crystalline dolomite \\
\hline sb $17-100-04 \mathrm{ccc}$ & $13,638.0$ & $13,639.0$ & $317 \mathrm{PRKC}$ & 1.5 & 3.400000 & Finely crystalline dolomite \\
\hline sb $17-100-04 \mathrm{ccc}$ & $13,639.0$ & $13,640.0$ & $317 \mathrm{PRKC}$ & 1.4 & 0.030000 & Finely crystalline dolomite \\
\hline sb $17-100-04 \mathrm{ccc}$ & $13,640.0$ & $13,641.0$ & $317 \mathrm{PRKC}$ & 0.4 & 0.320000 & Finely crystalline dolomite \\
\hline sb 17-100-04ccc & $13,641.0$ & $13,642.0$ & 317PRKC & 0.4 & 0.560000 & Finely crystalline dolomite \\
\hline sb17-100-04ccc & $13,642.0$ & $13,643.0$ & 317 PRKC & 0.5 & 0.010000 & Finely crystalline dolomite with phosphate nodules \\
\hline sb17-100-04ccc & $13,643.0$ & $13,644.0$ & 317 PRKC & 1.0 & 0.480000 & Finely crystalline dolomite with phosphate nodules \\
\hline $\mathrm{sb} 17-100-04 \mathrm{ccc}$ & $13,644.0$ & $13,645.0$ & 317PRKC & 2.3 & 0.030000 & Finely crystalline dolomite with phosphate nodules \\
\hline sb $17-100-04 \mathrm{ccc}$ & $13,645.0$ & $13,646.0$ & $317 \mathrm{PRKC}$ & 2.4 & 0.010000 & Finely crystalline dolomite with phosphate nodules \\
\hline $\mathrm{sb} 17-100-04 \mathrm{ccc}$ & $13,646.0$ & $13,647.0$ & 317 PRKC & 0.2 & 1.600000 & Finely crystalline dolomite with phosphate nodules \\
\hline sb $17-100-04 \mathrm{ccc}$ & $13,647.0$ & $13,648.0$ & 317PRKC & 0.6 & 0.010000 & Finely crystalline dolomite with phosphate nodules \\
\hline \multirow[t]{2}{*}{$\mathrm{sb} 17-100-04 \mathrm{ccc}$} & $13,648.0$ & $13,649.0$ & 317 PRKC & 1.8 & 0.010000 & Finely crystalline dolomite with phosphate nodules \\
\hline & & & AVERAGE-- & 2.5 & 0.687461 & \\
\hline sb17-100-04ccc & $13,683.0$ & $13,684.0$ & $321 \mathrm{TSLP}$ & 3.7 & 0.030000 & Fine-grained sandstone \\
\hline $\mathrm{sb} 17-100-04 \mathrm{ccc}$ & $13,684.0$ & $13,685.0$ & 321TSLP & 9.1 & 0.090000 & Fine-grained sandstone \\
\hline sb $17-100-04 \mathrm{ccc}$ & $13,685.0$ & $13,686.0$ & $321 \mathrm{TSLP}$ & 8.5 & 1.800000 & Fine-grained sandstone \\
\hline sb $17-100-04 \mathrm{ccc}$ & $13,686.0$ & $13,687.0$ & 321TSLP & 6.8 & 0.120000 & Fine-grained sandstone \\
\hline $\mathrm{sb} 17-100-04 \mathrm{ccc}$ & $13,687.0$ & $13,688.0$ & $321 \mathrm{TSLP}$ & 4.5 & 0.330000 & Fine-grained, quartzitic sandstone \\
\hline $\mathrm{sb} 17-100-04 \mathrm{ccc}$ & $13,688.0$ & $13,689.0$ & 321TSLP & 12.1 & 8.900000 & Fine-grained, quartzitic sandstone \\
\hline sb17-100-04ccc & $13,689.0$ & $13,690.0$ & $321 \mathrm{TSLP}$ & 12.5 & 26.000000 & Fine-grained, quartzitic sandstone \\
\hline $\mathrm{sb} 17-100-04 \mathrm{ccc}$ & $13,690.0$ & $13,691.0$ & 321TSLP & 13.6 & 38.000000 & Fine-grained, quartzitic sandstone \\
\hline sb17-100-04ccc & $13,691.0$ & $13,692.0$ & 321TSLP & 11.8 & 10.000000 & Fine-grained, quartzitic sandstone \\
\hline sb $17-100-04 \mathrm{ccc}$ & $13,692.0$ & $13,693.0$ & 321TSLP & 12.8 & 74.000000 & Fine-grained, quartzitic sandstone \\
\hline sb17-100-04ccc & $13,693.0$ & $13,694.0$ & 321TSLP & 12.5 & 53.000000 & Fine-grained, quartzitic sandstone \\
\hline $\mathrm{sb} 17-100-04 \mathrm{ccc}$ & $13,694.0$ & $13,695.0$ & 321TSLP & 12.3 & 30.000000 & Fine-grained, quartzitic sandstone \\
\hline sb17-100-04ccc & $13,695.0$ & $13,696.0$ & $321 \mathrm{TSLP}$ & 12.8 & 15.000000 & Fine-grained, quartzitic sandstone \\
\hline $\mathrm{sb} 17-100-04 \mathrm{ccc}$ & $13,696.0$ & $13,697.0$ & $321 \mathrm{TSLP}$ & 7.0 & 6.900000 & Fine-grained, quartzitic sandstone \\
\hline sb $17-100-04 c c c$ & $13,697.0$ & $13,698.0$ & $321 \mathrm{TSLP}$ & 10.8 & 2.500000 & Fine-grained sandstone \\
\hline sb 17-100-04ccc & $13,698.0$ & $13,699.0$ & $321 T S L P$ & 6.7 & 1.000000 & Fine-grained sandstone \\
\hline $\mathrm{sb} 17-100-04 \mathrm{ccc}$ & $13,699.0$ & $13,700.0$ & 321TSLP & 3.4 & 0.030000 & Fine-grained sandstone \\
\hline sb $17-100-04 \mathrm{ccc}$ & $13,700.0$ & $13,701.0$ & 321TSLP & 7.3 & 2.800000 & Fine-grained sandstone \\
\hline $\mathrm{sb} 17-100-04 \mathrm{ccc}$ & $13,701.0$ & $13,702.0$ & 321TSLP & 11.9 & 19.000000 & Fine-grained sandstone \\
\hline $\mathrm{sb} 17-100-04 \mathrm{ccc}$ & $13,702.0$ & $13,703.0$ & $321 \mathrm{TSLP}$ & 7.1 & 0.130000 & Fine-grained sandstone \\
\hline $\mathrm{sb} 17-100-04 \mathrm{ccc}$ & $13,703.0$ & $13,704.0$ & 321TSLP & 4.4 & 31.000000 & Fine-grained sandstone \\
\hline sb $17-100-04 c c c$ & $13,704.0$ & $13,705.0$ & 321TSLP & 5.8 & 0.350000 & Fine-grained sandstone \\
\hline $\mathrm{sb} 17-100-04 \mathrm{ccc}$ & $13,705.0$ & $13,706.0$ & 321TSLP & 3.5 & 0.110000 & Fine-to medium-grained, limy sandstone \\
\hline $\mathrm{sb} 17-100-04 \mathrm{ccc}$ & $13,706.0$ & $13,707.0$ & 321TSLP & 2.9 & 0.090000 & Fine-to medium-grained, limy sandstone \\
\hline $\mathrm{sb} 17-100-04 \mathrm{ccc}$ & $13,707.0$ & $13,708.0$ & 321TSLP & 2.6 & 0.200000 & Fine-to medium-grained, limy sandstone \\
\hline
\end{tabular}


Table 4.--Laboratory-determined porosity and permeability--Continued

\begin{tabular}{|c|c|c|c|c|c|}
\hline Site & $\begin{array}{l}\text { Depth to } \\
\text { top } \\
\text { (feet) }\end{array}$ & $\begin{array}{l}\text { Depth to } \\
\text { bottom } \\
\text { (feet) }\end{array}$ & $\begin{array}{c}\text { Forma- } \\
\text { tion }\end{array}$ & $\begin{array}{l}\text { Porosity } \\
\text { (percent) }\end{array}$ & $\begin{array}{l}\text { Perme- } \\
\text { ability } \\
\text { (milli- } \\
\text { darcies) }\end{array}$ \\
\hline sb17-100-04ccc & $13,708.0$ & $13,709.0$ & $321 \mathrm{TSLP}$ & 6.9 & 1.400000 \\
\hline $\mathrm{sb} 17-100-04 \mathrm{ccc}$ & $13,709.0$ & $13,710.0$ & $321 \mathrm{TSLP}$ & 7.9 & 5.700000 \\
\hline $\mathrm{sb} 17-100-04 \mathrm{ccc}$ & $13,710.0$ & $13,711.0$ & $321 \mathrm{TSLP}$ & 7.2 & 0.300000 \\
\hline $\mathrm{sb} 17-100-04 \mathrm{ccc}$ & $13,711.0$ & $13,712.0$ & $321 T S L P$ & 3.7 & 0.060000 \\
\hline $\mathrm{sb} 17-100-04 \mathrm{ccc}$ & $13,712.0$ & $13,713.0$ & $321 \mathrm{TSLP}$ & 2.5 & 0.060000 \\
\hline $\mathrm{sb} 17-100-04 \mathrm{ccc}$ & $13,713.0$ & $13,714.0$ & $321 \mathrm{TSLP}$ & 2.2 & 0.050000 \\
\hline $\mathrm{sb} 17-100-04 \mathrm{ccc}$ & $13,714.0$ & $13,715.0$ & $321 \mathrm{TSLP}$ & 7.5 & 0.520000 \\
\hline $\mathrm{sb} 17-100-04 \mathrm{ccc}$ & $13,715.0$ & $13,716.0$ & $321 \mathrm{TSLP}$ & 11.8 & 0.240000 \\
\hline $\mathrm{sb} 17-100-04 \mathrm{ccc}$ & $13,716.0$ & $13,717.0$ & $321 \mathrm{TSLP}$ & 8.8 & 0.680000 \\
\hline $\mathrm{sb} 17-100-04 \mathrm{ccc}$ & $13,717.0$ & $13,718.0$ & $321 \mathrm{TSLP}$ & 8.8 & 0.300000 \\
\hline $\mathrm{sb} 17-100-04 \mathrm{ccc}$ & $13,718.0$ & $13,719.0$ & $321 \mathrm{TSLP}$ & 10.3 & 0.770000 \\
\hline $\mathrm{sb} 17-100-04 \mathrm{ccc}$ & $13,719.0$ & $13,720.0$ & $321 \mathrm{TSLP}$ & 10.3 & 0.270000 \\
\hline $\mathrm{sb} 17-100-04 \mathrm{ccc}$ & $13,720.0$ & $13,721.0$ & $321 \mathrm{TSLP}$ & 6.8 & 0.390000 \\
\hline $\mathrm{sb} 17-100-04 \mathrm{ccc}$ & $13,721.0$ & $13,722.0$ & $321 \mathrm{TSLP}$ & 8.9 & 0.100000 \\
\hline $\mathrm{sb} 17-100-04 \mathrm{ccc}$ & $13,722.0$ & $13,723.0$ & $321 \mathrm{TSLP}$ & 8.7 & 21.000000 \\
\hline $\mathrm{sb} 17-100-04 \mathrm{ccc}$ & $13,723.0$ & $13,724.0$ & $321 \mathrm{TSLP}$ & 7.5 & 0.330000 \\
\hline $\mathrm{sb} 17-100-04 \mathrm{ccc}$ & $13,724.0$ & $13,725.0$ & $321 \mathrm{TSLP}$ & 7.7 & 3.100000 \\
\hline sb $17-100-04 c c c$ & $13,725.0$ & $13,726.0$ & $321 \mathrm{TSLP}$ & 7.3 & 3.800000 \\
\hline $\mathrm{sb} 17-100-04 \mathrm{ccc}$ & $13,726.0$ & $13,727.0$ & $321 \mathrm{TSLP}$ & 6.4 & 1.100000 \\
\hline $\mathrm{sb} 17-100-04 \mathrm{ccc}$ & $13,727.0$ & $13,728.0$ & $321 \mathrm{TSLP}$ & 8.1 & 14.000000 \\
\hline $\mathrm{sb} 17-100-04 \mathrm{ccc}$ & $13,728.0$ & $13,729.0$ & $321 \mathrm{TSLP}$ & 9.2 & 0.500000 \\
\hline $\mathrm{sb} 17-100-04 \mathrm{ccc}$ & $13,729.0$ & 13,7 & $321^{\prime}$ & 8. & 000 \\
\hline sb17-100-04ccc & $13,730.0$ & $13,731.0$ & 321TSLP & 9.3 & 4.300000 \\
\hline $\mathrm{sb} 17-100-04 \mathrm{ccc}$ & $13,731.0$ & $13,732.0$ & $321 T S L P$ & 10.4 & 0.620000 \\
\hline $\mathrm{sb} 17-100-04 \mathrm{ccc}$ & $13,732.0$ & $13,733.0$ & $321 \mathrm{TSLP}$ & 10.3 & 3.800000 \\
\hline $\mathrm{sb} 17-100-04 \mathrm{ccc}$ & $13,733.0$ & $13,734.0$ & $321 \mathrm{TSLP}$ & 10.9 & 5.400000 \\
\hline $\mathrm{sb} 17-100-04 \mathrm{ccc}$ & $13,734.0$ & $13,735.0$ & $321 \mathrm{TSLP}$ & 10.8 & 5.700000 \\
\hline $\mathrm{sb} 17-100-04 \mathrm{ccc}$ & $13,735.0$ & $13,736.0$ & $321 \mathrm{TSLP}$ & 9.4 & 5.700000 \\
\hline $\mathrm{sb} 17-100-04 \mathrm{ccc}$ & $13,736.0$ & $13,737.0$ & $321 \mathrm{TSLP}$ & 10.0 & 4.700000 \\
\hline $\mathrm{sb} 17-100-04 \mathrm{ccc}$ & $13,737.0$ & $13,738.0$ & $321 \mathrm{TSLP}$ & 9.3 & 4.100000 \\
\hline $\mathrm{sb} 17-100$ & 13,7 & 13,7 & $321 \mathrm{TSLP}$ & 8. & 3.100000 \\
\hline $\mathrm{sb} 17-1$ & $13,739.0$ & $13,740.0$ & $321 \mathrm{TSLP}$ & 9.3 & 0.920000 \\
\hline & & & AVERAGE- & 8.3 & 7.281930 \\
\hline SITE AVERAGE- & & & & 3.8 & 2.191000 \\
\hline sb18-98-28bca & $17,626.0$ & $17,627.0$ & $321 \mathrm{TSLP}$ & 0.4 & 0.010000 \\
\hline sb18-98-28bca & $17,627.0$ & $17,628.0$ & $321 \mathrm{TSLP}$ & 0.3 & 0.010000 \\
\hline sb $18-98-28 \mathrm{bca}$ & $17,628.0$ & $17,629.0$ & $321 \mathrm{TSLP}$ & 0.4 & 0.010000 \\
\hline sb18-98-28bca & $17,629.0$ & $17,630.0$ & $321 \mathrm{TSLP}$ & 0.4 & 0.010000 \\
\hline sb18-98-28bca & $17,630.0$ & $17,631.0$ & $321 \mathrm{TSLP}$ & 0.5 & 0.010000 \\
\hline sb $18-98-28$ bca & $17,631.0$ & $17,632.0$ & $321 \mathrm{TSLP}$ & 0.8 & 0.010000 \\
\hline $\mathrm{sb} 18-98-28 \mathrm{bca}$ & $17,632.0$ & $17,633.0$ & $321 \mathrm{TSLP}$ & 0.7 & 0.010000 \\
\hline sb18-98-28bca & $17,633.0$ & $17,634.0$ & $321 \mathrm{TSLP}$ & 0.9 & 0.010000 \\
\hline sb18-98-28bca & $17,634.0$ & $17,635.0$ & $321 \mathrm{TSLP}$ & 0.3 & 0.010000 \\
\hline sb18-98-28bca & $17,635.0$ & $17,636.0$ & $321 \mathrm{TSLP}$ & 0.7 & 0.010000 \\
\hline sb18-98-28bca & $17,636.0$ & $17,637.0$ & $321 \mathrm{TSLP}$ & 0.4 & 0.010000 \\
\hline sb18-98-28bca & $17,637.0$ & $17,638.0$ & $321 \mathrm{TSLP}$ & 0.7 & 0.010000 \\
\hline sb18-98-28bca & $17,638.0$ & $17,639.0$ & $321 \mathrm{TSLP}$ & 0.5 & 0.010000 \\
\hline sb18-98-28bca & $17,639.0$ & $17,640.0$ & 3211 SLP & 0.4 & 0.010000 \\
\hline sb18-98-28bca & $17,641.0$ & $17,642.0$ & $321 \mathrm{TSLP}$ & 0.6 & 0.020000 \\
\hline
\end{tabular}

Lithology

Fine- to medium-grained, limy sandstone Fine- to medium-grained, limy sandstone Fine- to medium-grained, limy sandstone Fine- to medium-grained, limy sandstone Fine- to medium-grained, limy sandstone

Fine- to medium-grained, limy sandstone Fine- to medium-grained, limy sandstone Fine- to medium-grained, limy sandstone Fine-grained, limy and quartzitic sandstone Fine-grained, limy and quartzitic sandstone

Fine-grained, limy and quartzitic sandstone Fine-grained, limy and quartzitic sandstone Fine-grained, limy and quartzitic sandstone Fine-grained, limy and quartzitic sandstone Fine-grained, limy and quartzitic sandstone

Fine-grained, limy and quartzitic sandstone Fine-grained, limy sandstone

Fine-grained, limy sandstone

Fine-grained, limy sandstone

Fine-grained sandstone

Fine-grained sands tone

Fine-grained sandstone

Fine-grained sandstone

Fine-grained sandstone

Fine-grained sandstone

Fine-grained sandstone

Fine-grained sandstone

Fine-grained sandstone

Fine-grained sandstone

Fine-grained sandstone

Fine-grained sandstone

Fine-grained sandstone

Fine- to medium-grained, quartzitic sandstone Fine- to medium-grained, quartzitic sandstone Fine- to medium-grained, quartzitic sandstone Fine- to medium-grained, quartzitic sandstone Fine- to medium-grained, quartzitic sandstone

Fine- to medium-grained, quartzitic sandstone Fine-grained, quartzitic sandstone Fine-grained, quartzitic sandstone Fine-grained, quartzitic sandstone Fine-grained, quartzitic sandstone

Fine- to medium-grained, quartzitic sandstone Fine- to medium-grained, quartzitic sandstone Fine- to medium-grained, quartzitic sandstone Fine- to medium-grained, quartzitic sandstone Fine-grained, quartzitic sandstone 
Table 4.--Laboratory-determined porosity and permeability--Continued

\begin{tabular}{|c|c|c|c|c|c|c|}
\hline Site & $\begin{array}{l}\text { Depth to } \\
\text { top } \\
\text { (feet) }\end{array}$ & $\begin{array}{l}\text { Depth to } \\
\text { bottom } \\
\text { (feet) }\end{array}$ & $\begin{array}{c}\text { Forma- } \\
\text { tion }\end{array}$ & $\begin{array}{l}\text { Porosity } \\
\text { (percent) }\end{array}$ & $\begin{array}{l}\text { Perme- } \\
\text { ability } \\
\text { (milli- } \\
\text { darcies) }\end{array}$ & Lithology \\
\hline sb $18-98-28$ bca & $17,642.0$ & $17,643.0$ & $321 \mathrm{TSLP}$ & 0.5 & 0.070000 & Fine-grained, quartzitic sandstone \\
\hline $\mathrm{sb} 18-98-28 \mathrm{bca}$ & $17,643.0$ & $17,644.0$ & $321 \mathrm{TSLP}$ & 0.3 & 0.010000 & Fine-grained, quartzitic sandstone \\
\hline sb $18-98-28 \mathrm{bca}$ & $17,644.0$ & $17,645.0$ & $321 \mathrm{TSLP}$ & 0.3 & 0.030000 & Fine-grained, quartzitic sandstone \\
\hline sb $18-98-28$ bca & $17,645.0$ & $17,646.0$ & $321 \mathrm{TSLP}$ & 0.3 & 0.010000 & Fine-grained, quartzitic sandstone \\
\hline $\mathrm{sb} 18-98-28 \mathrm{bca}$ & $17,646.0$ & $17,647.0$ & $321 \mathrm{TSLP}$ & 0.3 & 0.010000 & Fine-grained, quartzitic sandstone \\
\hline sb $18-98-28 b c a$ & $17,647.0$ & $17,648.0$ & $321 \mathrm{TSLP}$ & 0.3 & 0.010000 & Fine-grained, quartzitic sandstone \\
\hline sb $18-98-28$ bca & $17,648.0$ & $17,649.0$ & 321TSLP & 0.3 & 0.020000 & Fine-grained, quartzitic sandstone \\
\hline sb18-98-28bca & $17,649.0$ & $17,650.0$ & $321 \mathrm{TSLP}$ & 0.3 & 0.030000 & Fine-grained, quartzitic sandstone \\
\hline sb $18-98-28 b c a$ & $17,650.0$ & $17,651.0$ & $321 \mathrm{TSLP}$ & 0.4 & 0.010000 & Fine-grained, quartzitic sandstone \\
\hline $\mathrm{sb} 18-98-28 \mathrm{bca}$ & $17,651.0$ & $17,652.0$ & $321 \mathrm{TSLP}$ & 0.5 & 0.010000 & Fine-grained, quartzitic sandstone \\
\hline sb $18-98-28 b c a$ & $17,652.0$ & $17,653.0$ & $321 \mathrm{TSLP}$ & 0.5 & 0.010000 & Fine-grained, quartzitic sandstone \\
\hline sb $18-98-28$ bca & $17,653.0$ & $17,654.0$ & $321 \mathrm{TSLP}$ & 0.4 & 0.010000 & Fine-grained, quartzitic sandstone \\
\hline sb $18-98-28 b c a$ & $17,654.0$ & $17,655.0$ & $321 \mathrm{TSLP}$ & 0.6 & 0.010000 & Fine-grained, quartzitic sandstone \\
\hline sb18-98-28bca & $17,655.0$ & $17,656.0$ & $321 \mathrm{TSLP}$ & 0.6 & 0.020000 & Fine-grained, quartzitic sandstone \\
\hline sb $18-98-28$ bca & $17,656.0$ & $17,656.5$ & $321 \mathrm{TSLP}$ & 0.3 & 0.010000 & Fine-grained, quartzitic sandstone \\
\hline sb $18-98-28 b c a$ & $17,656.5$ & $17,657.0$ & $321 T S L P$ & 0.6 & 0.010000 & Fine-grained, quartzitic sandstone \\
\hline sb $18-98-28$ bca & $17,657.0$ & $17,658.0$ & $321 \mathrm{TSLP}$ & 0.5 & 0.030000 & Fine-grained, quartzitic sandstone \\
\hline sb $18-98-28$ bca & $17,658.0$ & $17,659.0$ & $321 \mathrm{TSLP}$ & 0.5 & 0.040000 & Fine-grained, quartzitic sandstone \\
\hline sb $18-98-28 b c a$ & $17,659.0$ & $17,660.0$ & $321 \mathrm{TSLP}$ & 0.9 & 0.030000 & Fine-grained, quartzitic sandstone \\
\hline sb $18-98-28$ bca & $17,660.0$ & $17,661.0$ & $321 \mathrm{TSLP}$ & 0.5 & 0.010000 & Fine-grained, quartzitic sandstone \\
\hline sb18-98-28bca & $17,661.0$ & $17,662.0$ & $321 \mathrm{TSLP}$ & 0.5 & 0.020000 & Fine-grained, quartzitic sandstone \\
\hline sb $18-98-28$ bca & $17,662.0$ & $17,663.0$ & $321 \mathrm{TSLP}$ & 0.5 & 0.030000 & Fine-grained, quartzitic sandstone \\
\hline $\mathrm{sb} 18-98-28 \mathrm{bca}$ & $17,663.0$ & $17,664.0$ & $321 \mathrm{TSLP}$ & 0.5 & 0.020000 & Fine-grained, quartzitic sandstone \\
\hline sb $18-98-28$ bca & $17,664.0$ & $17,665.0$ & $321 \mathrm{TSLP}$ & 0.4 & 0.090000 & Fine-grained, quartzitic sandstone \\
\hline sb $18-98-28$ bca & $17,665.0$ & $17,665.5$ & $321 \mathrm{TSLP}$ & 0.5 & 0.060000 & Fine-grained, quartzitic sandstone \\
\hline $\mathrm{sb} 18-98-28 \mathrm{bca}$ & $17,665.5$ & $17,666.0$ & $321 \mathrm{TSLP}$ & 3.0 & 0.150000 & Fine-grained, quartzitic sandstone \\
\hline sb $18-98-28 b c a$ & $17,666.0$ & $17,667.0$ & $321 \mathrm{TSLP}$ & 4.0 & 0.360000 & Fine-grained, quartzitic sandstone \\
\hline sb $18-98-28$ bca & $17,667.0$ & $17,668.0$ & $321 \mathrm{TSLP}$ & 2.0 & 0.070000 & Fine-grained, quartzitic sandstone \\
\hline sb $18-98-28 b c a$ & $17,668.0$ & $17,669.0$ & $321 \mathrm{TSLP}$ & 1.1 & 0.040000 & Fine-grained, quartzitic sandstone \\
\hline sb18-98-28bca & $17,669.0$ & $17,670.0$ & $321 \mathrm{TSLP}$ & 1.4 & 0.050000 & Fine-grained, quartzitic sandstone \\
\hline sb18-98-28bca & $17,670.0$ & $17,671.0$ & $321 \mathrm{TSLP}$ & 1.2 & 0.040000 & Fine-grained, quartzitic sandstone \\
\hline sb $18-98-28$ bca & $17,671.0$ & $17,672.0$ & $321 \mathrm{TSLP}$ & 1.1 & 0.460000 & Fine-grained, quartzitic sandstone \\
\hline $\mathrm{sb} 18-98-28 \mathrm{bca}$ & $17,672.0$ & $17,673.0$ & $321 \mathrm{TSLP}$ & 0.9 & 0.050000 & Fine-grained, quartzitic sandstone \\
\hline sb18-98-28bca & $17,673.0$ & $17,674.0$ & $321 \mathrm{TSLP}$ & 2.0 & 0.020000 & Fine-grained, quartzitic sandstone \\
\hline sb $18-98-28 b c a$ & $17,674.0$ & $17,675.0$ & $321 \mathrm{TSLP}$ & 1.7 & 0.030000 & Fine-grained, quartzitic sandstone \\
\hline sb $18-98-28$ bca & $17,675.0$ & $17,676.0$ & $321 \mathrm{TSLP}$ & 1.2 & 0.020000 & Fine-grained, quartzitic sandstone \\
\hline sb18-98-28bca & $17,676.0$ & $17,677.0$ & 321TSLP & 1.3 & 0.020000 & Fine-grained, quartzitic sandstone \\
\hline sb $18-98-28$ bca & $17,677.0$ & $17,678.0$ & $321 \mathrm{TSLP}$ & 1.3 & 0.020000 & Fine-grained, quartzitic sandstone \\
\hline sb $18-98-28$ bca & $17,680.0$ & $17,681.0$ & $321 \mathrm{TSLP}$ & 0.8 & 0.010000 & Fine-grained, quartzitic sandstone \\
\hline sb18-98-28bca & $17,681.0$ & $17,682.0$ & $321 \mathrm{TSLP}$ & 1.3 & 0.010000 & Fine-grained, quartzitic sandstone \\
\hline sb $18-98-28$ bca & $17,772.0$ & $17,773.0$ & $321 \mathrm{TSLP}$ & 1.6 & 0.020000 & Fine-grained sandstone \\
\hline sb $18-98-28$ bca & $17,773.0$ & $17,774.0$ & $321 \mathrm{TSLP}$ & 1.8 & 0.010000 & Fine-grained sandstone \\
\hline sb18-98-28bca & $17,774.0$ & $17,775.0$ & 321TSLP & 2.0 & 0.010000 & Fine-grained sandstone \\
\hline sb $18-98-28$ bca & $17,775.0$ & $17,776.0$ & $321 \mathrm{TSLP}$ & 1.5 & 0.010000 & Fine-grained sandstone \\
\hline sb $18-98-28 b c a$ & $17,776.0$ & $17,777.0$ & $321 \mathrm{TSLP}$ & 1.2 & 0.010000 & Fine-grained sandstone \\
\hline sb18-98-28bca & $17,777.0$ & $17,778.0$ & $321 \mathrm{TSLP}$ & 1.7 & 0.010000 & Fine-grained sandstone \\
\hline sb $18-98-28$ bca & $17,778.0$ & $17,779.0$ & $321 \mathrm{TSLP}$ & 1.7 & 0.010000 & Fine-grained sandstone \\
\hline sb18-98-28bca & $17,779.0$ & $17,780.0$ & $321 \mathrm{TSLP}$ & 2.0 & 0.010000 & Fine-grained sandstone \\
\hline sb18-98-28bca & $17,780.0$ & $17,781.0$ & $321 \mathrm{TSLP}$ & 1.3 & 0.010000 & Fine-grained sandstone \\
\hline sb $18-98-28 b c a$ & $17,781.0$ & $17,782.0$ & $321 \mathrm{TSLP}$ & 2.1 & 0.010000 & Fine-grained sandstone \\
\hline
\end{tabular}


Table 4.--Laboratory-determined porosity and permeability--Continued

\begin{tabular}{|c|c|c|c|c|c|c|}
\hline Site & $\begin{array}{l}\text { Depth to } \\
\text { top } \\
\text { (feet) }\end{array}$ & $\begin{array}{l}\text { Depth to } \\
\text { bottom } \\
\text { (feet) }\end{array}$ & $\begin{array}{c}\text { Forma- } \\
\text { tion }\end{array}$ & $\begin{array}{l}\text { Porosity } \\
\text { (percent) }\end{array}$ & $\begin{array}{l}\text { Perme- } \\
\text { ability } \\
\text { (milli- } \\
\text { darcies) }\end{array}$ & Lithology \\
\hline sb18-98-28bca & $17,782.0$ & $17,783.0$ & $321 \mathrm{TSLP}$ & 1.2 & 0.010000 & Fine-grained sandstone \\
\hline sb $18-98-28$ bca & $17,783.0$ & $17,784.0$ & $321 \mathrm{TSLP}$ & 1.3 & 0.010000 & Fine-grained sandstone \\
\hline sb18-98-28bca & $17,784.0$ & $17,785.0$ & 321TSLP & 1.2 & 0.030000 & Fine-grained sandstone \\
\hline sb18-98-28bca & $17,785.0$ & $17,786.0$ & 321 TSLP & 1.0 & 0.010000 & Fine-grained sandstone \\
\hline sb $18-98-28 \mathrm{bca}$ & $17,786.0$ & $17,787.0$ & $321 \mathrm{TSLP}$ & 1.3 & 0.010000 & Fine-grained sandstone \\
\hline sb18-98-28bca & $17,787.0$ & $17,788.0$ & 321TSLP & 1.0 & 0.010000 & Fine-grained sandstone \\
\hline sb $18-98-28$ bca & $17,788.0$ & $17,789.0$ & $321 \mathrm{TSLP}$ & 1.3 & 0.010000 & Fine-grained sandstone \\
\hline sb $18-98-28$ bca & $17,789.0$ & $17,790.0$ & $321 \mathrm{TSLP}$ & 2.7 & 0.010000 & Fine-grained sandstone \\
\hline sb $18-98-28$ bca & $17,790.0$ & $17,791.0$ & $321 \mathrm{TSLP}$ & 2.4 & 0.030000 & Fine-grained sandstone \\
\hline sb $18-98-28$ bca & $17,791.0$ & $17,792.0$ & $321 \mathrm{TSLP}$ & 0.7 & 0.010000 & Fine-grained sandstone \\
\hline sb18-98-28bca & $17,792.0$ & $17,793.0$ & $321 \mathrm{TSLP}$ & 1.4 & 0.010000 & Fine-grained sandstone \\
\hline sb $18-98-28$ bca & $17,793.0$ & $17,794.0$ & $321 \mathrm{TSLP}$ & 1.1 & 0.010000 & Fine-grained sandstone \\
\hline sb $18-98-28$ bca & $17,794.0$ & $17,795.0$ & $321 \mathrm{TSLP}$ & 1.0 & 0.130000 & Fine- to medium-grained sandstone \\
\hline sb $18-98-28$ bca & $17,795.0$ & $17,796.0$ & 321TSLP & 0.7 & 0.020000 & Fine- to medium-grained sandstone \\
\hline sb $18-98-28 b c a$ & $17,796.0$ & $17,797.0$ & 321TSLP & 0.9 & 0.010000 & Fine- to medium-grained sandstone \\
\hline $\mathrm{sb} 18-98-28 \mathrm{bca}$ & $17,797.0$ & $17,798.0$ & 321TSLP & 0.7 & 0.020000 & Fine- to medium-grained sandstone \\
\hline sb $18-98-28$ bca & $17,798.0$ & $17,799.0$ & $321 \mathrm{TSLP}$ & 0.6 & 0.010000 & Fine- to medium-grained sandstone \\
\hline sb $18-98-28$ bca & $17,799.0$ & $17,800.0$ & $321 \mathrm{TSLP}$ & 0.6 & 0.010000 & Fine- to medium-grained sandstone \\
\hline sb $18-98-28$ bca & $17,800.0$ & $17,801.0$ & $321 \mathrm{TSLP}$ & 0.7 & 0.020000 & Fine- to medium-grained sandstone \\
\hline sb $18-98-28$ bca & $17,801.0$ & $17,802.0$ & 321TSLP & 0.5 & 0.010000 & Fine- to medium-grained sandstone \\
\hline sb18-98-28bca & $17,802.0$ & $17,803.0$ & 321TSLP & 0.4 & 0.010000 & Fine- to medium-grained sandstone \\
\hline sb $18-98-28$ bca & $17,803.0$ & $17,804.0$ & $321 \mathrm{TSLP}$ & 1.7 & 0.010000 & Fine- to medium-grained sandstone \\
\hline sb $18-98-28$ bca & $17,804.0$ & $17,805.0$ & $321 \mathrm{TSLP}$ & 0.8 & 0.010000 & Fine-grained sandstone \\
\hline $\mathrm{sb} 18-98-28 \mathrm{bca}$ & $17,805.0$ & $17,806.0$ & $321 \mathrm{TSLP}$ & 1.0 & 0.020000 & Fine- to coarse-grained sandstone \\
\hline $\mathrm{sb} 18-98-28 \mathrm{bca}$ & $17,806.0$ & $17,807.0$ & $321 \mathrm{TSLP}$ & 0.4 & 0.010000 & Fine- to medium-grained sandstone \\
\hline sb18-98-28bca & $17,807.0$ & $17,808.0$ & $321 \mathrm{TSLP}$ & 2.4 & 0.050000 & Fine- to medium-grained sandstone \\
\hline sb18-98-28bca & $17,808.0$ & $17,809.0$ & $321 \mathrm{TSLP}$ & 2.5 & 0.030000 & Fine- to coarse-grained sandstone \\
\hline sb $18-98-28$ bca & $17,809.0$ & $17,810.0$ & $321 \mathrm{TSLP}$ & 2.1 & 0.040000 & Fine-grained sandstone \\
\hline sb $18-98-28$ bca & $17,810.0$ & $17,811.0$ & $321 \mathrm{TSLP}$ & 2.1 & 0.140000 & Fine-grained sandstone \\
\hline $\mathrm{sb} 18-98-28 \mathrm{bca}$ & $17,811.0$ & $17,812.0$ & $321 \mathrm{TSLP}$ & 2.0 & 0.080000 & Fine- to medium-grained sandstone \\
\hline sb18-98-28bca & $17,812.0$ & $17,813.0$ & 321TSLP & 0.7 & 0.030000 & Fine- to medium-grained sandstone \\
\hline sb $18-98-28$ bca & $17,813.0$ & $17,814.0$ & $321 \mathrm{TSLP}$ & 2.6 & 0.020000 & Fine- to medium-grained sandstone \\
\hline sb $18-98-28$ bca & $17,814.0$ & $17,815.0$ & $321 \mathrm{TSLP}$ & 0.8 & 0.010000 & Fine- to coarse-grained, quartzitic sandstone \\
\hline sb $18-98-28$ bca & $17,815.0$ & $17,816.0$ & $321 \mathrm{TSLP}$ & 0.8 & 0.010000 & Fine- to medium-grained, quartzitic sandstone \\
\hline sb18-98-28bca & $17,816.0$ & $17,817.0$ & 321TSLP & 1.3 & 0.010000 & Fine- to medium-grained, quartzitic sandstone \\
\hline $\mathrm{sb} 18-98-28 \mathrm{bca}$ & $17,817.0$ & $17,818.0$ & 321TSLP & 0.7 & 0.020000 & Fine- to medium-grained, quartzitic sandstone \\
\hline sb18-98-28bca & $17,818.0$ & $17,819.0$ & $321 \mathrm{TSLP}$ & 1.2 & 0.010000 & Fine-grained sandstone \\
\hline sb18-98-28bca & $17,819.0$ & $17,820.0$ & $321 \mathrm{TSLP}$ & 1.4 & 0.010000 & Fine-grained sandstone \\
\hline sb $18-98-28$ bca & $17,820.0$ & $17,821.0$ & 321 TSLP & 1.8 & 0.020000 & Fine- to medium-grained sandstone \\
\hline sb18-98-28bca & $17,822.0$ & $17,823.0$ & $321 \mathrm{TSLP}$ & 2.8 & 0.030000 & Fine-grained, slightly limy sandstone \\
\hline $\mathrm{sb} 18-98-28 \mathrm{bca}$ & $17,823.0$ & $17,824.0$ & $321 \mathrm{TSLP}$ & 1.6 & 0.020000 & Fine-grained, slightly limy sandstone \\
\hline sb $18-98-28$ bca & $17,824.0$ & $17,825.0$ & $321 \mathrm{TSLP}$ & 1.0 & 0.020000 & Fine-grained, slightly limy sandstone \\
\hline sb $18-98-28$ bca & $17,825.0$ & $17,826.0$ & $321 T S L P$ & 1.3 & 0.020000 & Fine-grained, slightly limy sandstone \\
\hline sb18-98-28bca & $17,826.0$ & $17,827.0$ & $321 \mathrm{TSLP}$ & 0.8 & 0.030000 & Fine-grained, slightly limy sandstone \\
\hline sb18-98-28bca & $17,827.0$ & $17,828.0$ & $321 \mathrm{TSLP}$ & 1.0 & 0.020000 & Fine-grained, slightly limy sandstone \\
\hline sb18-98-28bca & $17,828.0$ & $17,829.0$ & $321 \mathrm{TSLP}$ & 1.3 & 0.020000 & Fine-grained, slightly limy sandstone \\
\hline sb $18-98-28$ bca & $17,829.0$ & $17,830.0$ & 321TSLP & 0.9 & 0.030000 & Fine-grained, slightly limy sandstone \\
\hline sb $18-98-28$ bca & $17,830.0$ & $17,831.0$ & $321 \mathrm{TSLP}$ & 0.9 & 0.020000 & Fine-grained, slightly limy sandstone \\
\hline $\mathrm{sb} 18-98-28 \mathrm{bca}$ & $17,831.0$ & $17,832.0$ & $321 \mathrm{TSLP}$ & 0.9 & 0.020000 & Fine-grained, slightly limy sandstone \\
\hline $\mathrm{sb} 18-98-28 \mathrm{bca}$ & $17,832.0$ & $17,833.0$ & $321 \mathrm{TSLP}$ & 0.8 & 0.020000 & Fine-grained, slightly limy sandstone \\
\hline
\end{tabular}


Table 4.--Laboratory-determined porosity and permeability--Continued

\begin{tabular}{|c|c|c|c|c|c|c|}
\hline Site & $\begin{array}{l}\text { Depth to } \\
\text { top } \\
\text { (feet) }\end{array}$ & $\begin{array}{l}\text { Depth to } \\
\text { bottom } \\
\text { (feet) }\end{array}$ & $\begin{array}{c}\text { Forma- } \\
\text { tion }\end{array}$ & $\begin{array}{l}\text { Porosity } \\
\text { (percent) }\end{array}$ & $\begin{array}{l}\text { Perme- } \\
\text { ability } \\
\text { (milli- } \\
\text { darcies) }\end{array}$ & Lithology \\
\hline $\begin{array}{l}\text { sb18-98-28bca } \\
\text { sb18-98-28bca } \\
\text { sb18-98-28bca } \\
\text { sb } 18-98-28 \text { bca } \\
\text { sb } 18-98-28 \text { bca }\end{array}$ & $\begin{array}{l}17,834.0 \\
17,836.0 \\
17,838.0 \\
17,840.0 \\
17,842.0\end{array}$ & $\begin{array}{l}17,835.0 \\
17,837.0 \\
17,839.0 \\
17,841.0 \\
17,843.0\end{array}$ & $\begin{array}{l}321 \mathrm{TSLP} \\
321 \mathrm{TSLP} \\
321 \mathrm{TSLP} \\
321 \mathrm{TSLP} \\
321 \mathrm{TSLP}\end{array}$ & $\begin{array}{l}0.7 \\
1.3 \\
0.8 \\
1.2 \\
1.1\end{array}$ & $\begin{array}{l}0.030000 \\
0.020000 \\
0.010000 \\
0.100000 \\
0.010000\end{array}$ & $\begin{array}{l}\text { Fine-grained, slightly limy sandstone } \\
\text { Fine-grained, slightly limy sandstone } \\
\text { Fine-grained, slightly limy sandstone } \\
\text { Fine-grained, slightly limy sandstone } \\
\text { Fine-grained, slightly limy sandstone }\end{array}$ \\
\hline $\begin{array}{l}\text { sb } 18-98-28 \text { bca } \\
\text { sb18-98-28bca } \\
\text { sb18-98-28bca } \\
\text { sb18-98-28bca } \\
\text { sb18-98-28bca }\end{array}$ & $\begin{array}{l}17,844.0 \\
17,846.0 \\
17,848.0 \\
17,851.0 \\
17,853.0\end{array}$ & $\begin{array}{l}17,845.0 \\
17,847.0 \\
17,849.0 \\
17,852.0 \\
17,854.0\end{array}$ & $\begin{array}{l}321 \mathrm{TSLP} \\
321 \mathrm{TSLP} \\
321 \mathrm{TSLP} \\
321 \mathrm{TSLP} \\
\text { 321TSLP }\end{array}$ & $\begin{array}{l}0.7 \\
0.9 \\
1.7 \\
0.7 \\
0.7\end{array}$ & $\begin{array}{l}0.010000 \\
0.010000 \\
0.010000 \\
0.010000 \\
0.010000\end{array}$ & $\begin{array}{l}\text { Fine-grained, slightly limy sandstone } \\
\text { Fine-grained, slightly limy sandstone } \\
\text { Fine-grained, slightly limy sandstone } \\
\text { Fine-grained, slightly limy sandstone } \\
\text { Fine-grained, slightly limy sandstone }\end{array}$ \\
\hline $\begin{array}{l}\text { sb18-98-28bca } \\
\text { sb18-98-28bca } \\
\text { sb18-98-28bca } \\
\text { sb18-98-28bca } \\
\text { sb18-98-28bca }\end{array}$ & $\begin{array}{l}17,855.0 \\
17,857.0 \\
17,859.0 \\
17,861.0 \\
17,863.0\end{array}$ & $\begin{array}{l}17,856.0 \\
17,858 \cdot 0 \\
17,860.0 \\
17,862.0 \\
17,864.0\end{array}$ & $\begin{array}{l}321 \mathrm{TSLP} \\
321 \mathrm{TSLP} \\
321 \mathrm{TSLP} \\
321 \mathrm{TSLP} \\
321 \mathrm{TSLP}\end{array}$ & $\begin{array}{l}0.8 \\
1.4 \\
4.6 \\
1.2 \\
1.0\end{array}$ & $\begin{array}{l}0.010000 \\
0.010000 \\
0.010000 \\
0.010000 \\
0.010000\end{array}$ & $\begin{array}{l}\text { Fine-grained, slightly limy sandstone } \\
\text { Fine- to medium-grained limy sandstone } \\
\text { Fine-grained, slightly limy sandstone } \\
\text { Fine-grained, slightly limy sandstone } \\
\text { Fine-grained, slightly limy sandstone }\end{array}$ \\
\hline $\begin{array}{l}\text { sb } 18-98-28 \text { bca } \\
\text { sb } 18-98-28 \text { bca } \\
\text { sb } 18-98-28 \text { bca } \\
\text { sb } 18-98-28 \text { bca } \\
\text { sb18-98-28bca }\end{array}$ & $\begin{array}{l}17,865.0 \\
17,867.0 \\
17,869.0 \\
17,871.0 \\
17,873.0\end{array}$ & $\begin{array}{l}17,866.0 \\
17,868 \cdot 0 \\
17,870.0 \\
17,872.0 \\
17,874.0\end{array}$ & $\begin{array}{l}321 \mathrm{TSLP} \\
321 \mathrm{TSLP} \\
321 \mathrm{TSLP} \\
321 \mathrm{TSLP} \\
321 \mathrm{TSLP}\end{array}$ & $\begin{array}{l}1.9 \\
2.6 \\
2.6 \\
1.1 \\
1.3\end{array}$ & $\begin{array}{l}0.010000 \\
0.010000 \\
0.010000 \\
0.010000 \\
0.010000\end{array}$ & $\begin{array}{l}\text { Fine-grained, slightly limy sandstone } \\
\text { Fine- to medium-grained, slightly limy sandstone } \\
\text { Fine-grained, slightly limy sandstone } \\
\text { Fine-grained, slightly limy sandstone } \\
\text { Fine-grained, slightly limy sandstone }\end{array}$ \\
\hline $\begin{array}{l}\text { sb18-98-28bca } \\
\text { sb18-98-28bca } \\
\text { sb18-98-28bca } \\
\text { sb18-98-28bca } \\
\text { sb18-98-28bca }\end{array}$ & $\begin{array}{l}17,875.0 \\
17,877.0 \\
17,879.0 \\
17,881.0 \\
17,883.0\end{array}$ & $\begin{array}{l}17,876.0 \\
17,878.0 \\
17,880.0 \\
17,882.0 \\
17,884.0\end{array}$ & $\begin{array}{l}321 \mathrm{TSLP} \\
321 \mathrm{TSLP} \\
321 \mathrm{TSLP} \\
321 \mathrm{TSLP} \\
321 \mathrm{TSLP}\end{array}$ & $\begin{array}{l}2.1 \\
1.6 \\
1.1 \\
1.8 \\
1.8\end{array}$ & $\begin{array}{l}0.010000 \\
0.010000 \\
0.010000 \\
0.010000 \\
0.030000\end{array}$ & $\begin{array}{l}\text { Fine-grained, slightly limy sandstone } \\
\text { Fine- to medium-grained, slightly limy sandstone } \\
\text { Fine- to medium-grained, slightly limy sandstone } \\
\text { Fine- to medium-grained sandstone } \\
\text { Fine- to medium-grained, slightly limy sandstone }\end{array}$ \\
\hline $\begin{array}{l}\text { sb } 18-98-28 \text { bca } \\
\text { sb } 18-98-28 \text { bca } \\
\text { sb } 18-98-28 \text { bca } \\
\text { sb } 18-98-28 \text { bca } \\
\text { sb } 18-98-28 \text { bca }\end{array}$ & $\begin{array}{l}17,885.0 \\
17,887.0 \\
17,889.0 \\
17,891.0 \\
17,892.0\end{array}$ & $\begin{array}{l}17,886.0 \\
17,888.0 \\
17,890.0 \\
17,892.0 \\
17,893.0\end{array}$ & $\begin{array}{l}321 \mathrm{TSLP} \\
321 \mathrm{TSLP} \\
321 \mathrm{TSLP} \\
321 \mathrm{TSLP} \\
321 \mathrm{TSLP}\end{array}$ & $\begin{array}{l}1.7 \\
1.4 \\
1.0 \\
1.4 \\
1.6\end{array}$ & $\begin{array}{l}0.030000 \\
0.020000 \\
0.010000 \\
0.040000 \\
0.040000\end{array}$ & $\begin{array}{l}\text { Fine- to medium-grained sandstone } \\
\text { Fine- to medium-grained sandstone } \\
\text { Fine- to medium-grained sandstone } \\
\text { Fine- to medium-grained sandstone } \\
\text { Fine- to medium-grained, slightly limy sandstone }\end{array}$ \\
\hline SITE AVERAGE- & - & 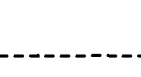 & AVERAGE-- & $\frac{1.1}{1.1}$ & $\frac{0.027172}{0.027172}$ & \\
\hline $\begin{array}{l}\text { sb20-87-30ddd } \\
\text { sb20-87-30ddd } \\
\text { sb20-87-30ddd } \\
\text { sb20-87-30ddd } \\
\text { sb20-87-30ddd }\end{array}$ & $\begin{array}{l}8,183.0 \\
8,184.0 \\
8,185.0 \\
8,186.0 \\
8,187.0\end{array}$ & $\begin{array}{l}8,184.0 \\
8,185.0 \\
8,186.0 \\
8,187.0 \\
8,188.0\end{array}$ & $\begin{array}{l}321 \mathrm{TSLP} \\
321 \mathrm{TSLP} \\
321 \mathrm{TSLP} \\
321 \mathrm{TSLP} \\
321 \mathrm{TSLP}\end{array}$ & $\begin{array}{l}4.0 \\
1.7 \\
1.5 \\
1.5 \\
4.2\end{array}$ & $\begin{array}{l}0.100000 \\
0.100000 \\
0.100000 \\
0.100000 \\
0.100000\end{array}$ & $\begin{array}{l}\text { Fine-grained, limy and quartzitic sandstone } \\
\text { Fine-grained, quartzitic sandstone } \\
\text { Fine-grained, quartzitic sandstone } \\
\text { Fine-grained, quartzitic sandstone } \\
\text { Fine-grained, quartzitic sandstone }\end{array}$ \\
\hline $\begin{array}{l}\text { sb20-87-30ddd } \\
\text { sb20-87-30ddd } \\
\text { sb20-87-30ddd } \\
\text { sb20-87-30ddd } \\
\text { sb20-87-30ddd }\end{array}$ & $\begin{array}{l}8,188.0 \\
8,189.0 \\
8,190.0 \\
8,191.0 \\
8,192.0\end{array}$ & $\begin{array}{l}8,189.0 \\
8,190.0 \\
8,191.0 \\
8,192.0 \\
8,193.0\end{array}$ & $\begin{array}{l}321 T S L P \\
321 T S L P \\
321 T S L P \\
321 T S L P \\
321 T S L P\end{array}$ & $\begin{array}{l}3.1 \\
2.9 \\
2.6 \\
1.5 \\
2.1\end{array}$ & $\begin{array}{l}0.400000 \\
0.000000 \\
0.100000 \\
0.400000 \\
0.000000\end{array}$ & $\begin{array}{l}\text { Fine-grained, quartzitic sandstone } \\
\text { Fine-grained, quartzitic sandstone } \\
\text { Fine-grained, quartzitic sandstone } \\
\text { Fine-grained, quartzitic sandstone } \\
\text { Fine-grained, quartzitic sandstone }\end{array}$ \\
\hline $\begin{array}{l}\text { sb20-87-30ddd } \\
\text { sb20-87-30ddd } \\
\text { sb20-87-30ddd } \\
\text { sb20-87-30ddd } \\
\text { sb20-87-30ddd }\end{array}$ & $\begin{array}{l}8,193.0 \\
8,194.0 \\
8,195.0 \\
8,196.0 \\
8,197.0\end{array}$ & $\begin{array}{l}8,194.0 \\
8,195.0 \\
8,196.0 \\
8,197.0 \\
8,198.0\end{array}$ & $\begin{array}{l}321 \mathrm{TSLP} \\
321 \mathrm{TSLP} \\
321 \mathrm{TSLP} \\
321 \mathrm{TSLP} \\
321 \mathrm{TSLP}\end{array}$ & $\begin{array}{l}2.8 \\
1.8 \\
2.1 \\
1.4 \\
1.6\end{array}$ & $\begin{array}{l}0.100000 \\
0.000000 \\
0.000000 \\
0.000000 \\
0.000000\end{array}$ & $\begin{array}{l}\text { Fine-grained, quartzitic sandstone } \\
\text { Fine-grained, quartzitic sandstone } \\
\text { Fine-grained, quartzitic sandstone } \\
\text { Fine-grained, quartzitic sandstone } \\
\text { Fine-grained, quartzitic sandstone }\end{array}$ \\
\hline
\end{tabular}


Table 4.--Laboratory-determined porosity and permeability--Continued

\begin{tabular}{|c|c|c|c|c|c|c|}
\hline Site & $\begin{array}{l}\text { Depth to } \\
\text { top } \\
\text { (feet) }\end{array}$ & $\begin{array}{l}\text { Depth to } \\
\text { bottom } \\
\text { (feet) }\end{array}$ & $\begin{array}{c}\text { Forma- } \\
\text { tion }\end{array}$ & $\begin{array}{l}\text { Porosity } \\
\text { (percent) }\end{array}$ & $\begin{array}{l}\text { Perme- } \\
\text { ability } \\
\text { (milli- } \\
\text { darcies) }\end{array}$ & Lithology \\
\hline $\begin{array}{l}\text { sb20-87-30ddd } \\
\text { sb20-87-30ddd } \\
\text { sb20-87-30ddd } \\
\text { sb20-87-30ddd } \\
\text { sb20-87-30ddd }\end{array}$ & $\begin{array}{l}8,198.0 \\
8,199.0 \\
8,200.0 \\
8,201.0 \\
8,202.0\end{array}$ & $\begin{array}{l}8,199.0 \\
8,200.0 \\
8,201.0 \\
8,202.0 \\
8,203.0\end{array}$ & $\begin{array}{l}321 T S L P \\
321 T S L P \\
321 T S L P \\
321 T S L P \\
321 T S L P\end{array}$ & $\begin{array}{l}1.0 \\
1.7 \\
1.3 \\
2.6 \\
2.0\end{array}$ & $\begin{array}{l}0.000000 \\
0.000000 \\
0.100000 \\
0.000000 \\
0.000000\end{array}$ & $\begin{array}{l}\text { Shaly, fine-grained, quartzitic sandstone } \\
\text { Shaly, fine-grained, quartzitic sandstone } \\
\text { Shaly, fine-grained, quartzitic sandstone } \\
\text { Shaly, fine-grained, quartzitic sandstone } \\
\text { Shaly, fine-grained, quartzitic sandstone }\end{array}$ \\
\hline $\begin{array}{l}\text { sb20-87-30ddd } \\
\text { sb20-87-30ddd } \\
\text { sb20-87-30ddd } \\
\text { sb20-87-30ddd } \\
\text { sb20-87-30ddd }\end{array}$ & $\begin{array}{l}8,203.0 \\
8,204.0 \\
8,205.0 \\
8,206.0 \\
8,207.0\end{array}$ & $\begin{array}{l}8,204.0 \\
8,205.0 \\
8,206.0 \\
8,207.0 \\
8,208.0\end{array}$ & $\begin{array}{l}321 \text { TSLP } \\
321 \text { TSLP } \\
321 \text { TSLP } \\
321 \text { TSLP } \\
321 \text { TSLP }\end{array}$ & $\begin{array}{l}1.2 \\
0.9 \\
0.9 \\
1.2 \\
1.1\end{array}$ & $\begin{array}{l}0.000000 \\
0.000000 \\
0.000000 \\
0.000000 \\
0.000000\end{array}$ & $\begin{array}{l}\text { Shaly, fine-grained, quartzitic sandstone } \\
\text { Fine-grained, quartzitic sandstone } \\
\text { Fine-grained, quartzitic sandstone } \\
\text { Fine-grained, quartzitic sandstone } \\
\text { Fine-grained, quartzitic sandstone }\end{array}$ \\
\hline $\begin{array}{l}\text { sb20-87-30ddd } \\
\text { sb20-87-30ddd } \\
\text { sb20-87-30ddd } \\
\text { sb20-87-30ddd } \\
\text { sb20-87-30ddd }\end{array}$ & $\begin{array}{l}8,208.0 \\
8,209.0 \\
8,210.0 \\
8,211.0 \\
8,212.0\end{array}$ & $\begin{array}{l}8,209.0 \\
8,210.0 \\
8,211.0 \\
8,212.0 \\
8,213.0\end{array}$ & $\begin{array}{l}321 \text { TSLP } \\
321 \text { TSLP } \\
321 T S L P \\
321 T S L P \\
321 T S L P\end{array}$ & $\begin{array}{l}2.0 \\
2.2 \\
2.0\end{array}$ & $\begin{array}{r}0.100000 \\
0.100000 \\
21.000000 \\
0.100000 \\
0.100000\end{array}$ & $\begin{array}{l}\text { Fine-grained, limy and quartzitic sandstone } \\
\text { Fine-grained, limy and quartzitic sandstone } \\
\text { Fine-grained, limy and quartzitic sandstone } \\
\text { Fine-grained, limy and quartzitic sandstone } \\
\text { Fine-grained, limy and quartzitic sandstone }\end{array}$ \\
\hline $\begin{array}{l}\text { sb20-87-30ddd } \\
\text { sb20-87-30ddd } \\
\text { sb20-87-30ddd } \\
\text { sb20-87-30ddd } \\
\text { sb20-87-30ddd }\end{array}$ & $\begin{array}{l}8,213.0 \\
8,214.0 \\
8,215.0 \\
8,216.0 \\
8,217.0\end{array}$ & $\begin{array}{l}8,214.0 \\
8,215.0 \\
8,216.0 \\
8,217.0 \\
8,218.0\end{array}$ & & $\begin{array}{l}2.3 \\
2.0 \\
2.5\end{array}$ & & $\begin{array}{l}\text { Fine-grained, limy and quartzitic sandstone } \\
\text { Fine-grained, limy and quartzitic sandstone } \\
\text { Fine-grained, limy and quartzitic sandstone } \\
\text { Fine-grained, limy and quartzitic sandstone } \\
\text { Fine-grained, limy and quartzitic sandstone }\end{array}$ \\
\hline $\begin{array}{l}\text { sb20-87-30ddd } \\
\text { sb20-87-30ddd } \\
\text { sb20-87-30ddd } \\
\text { sb20-87-30ddd } \\
\text { sb20-87-30ddd }\end{array}$ & $\begin{array}{l}8,218.0 \\
8,219.0 \\
8,220.0 \\
8,221.0 \\
8,222.0\end{array}$ & $\begin{array}{l}8,219.0 \\
8,220.0 \\
8,221.0 \\
8,222.0 \\
8,223.0\end{array}$ & $\begin{array}{l}321 \text { TSLP } \\
321 T S L P \\
321 \text { TSLP } \\
321 T S L P \\
321 T S L P\end{array}$ & $\begin{array}{l}2.2 \\
1.9 \\
2.4 \\
2.2 \\
2.2\end{array}$ & $\begin{array}{l}0.100000 \\
0.100000 \\
0.100000 \\
0.100000 \\
0.100000\end{array}$ & $\begin{array}{l}\text { Fine-grained, limy and quartzitic sandstone } \\
\text { Fine-grained, limy and quartzitic sandstone } \\
\text { Fine-grained, limy and quartzitic sandstone } \\
\text { Fine-grained, limy and quartzitic sandstone } \\
\text { Fine-grained, limy and quartzitic sandstone }\end{array}$ \\
\hline $\begin{array}{l}\text { sb20-87-30ddd } \\
\text { sb20-87-30ddd } \\
\text { sb20-87-30ddd } \\
\text { sb20-87-30ddd } \\
\text { sb20-87-30ddd }\end{array}$ & $\begin{array}{l}8,223.0 \\
8,224.0 \\
8,225.0 \\
8,226.0 \\
8,227.0\end{array}$ & $\begin{array}{l}8,224.0 \\
8,225.0 \\
8,226.0 \\
8,227.0 \\
8,228.0\end{array}$ & $\begin{array}{l}321 T S L P \\
321 T S L P \\
321 T S L P \\
321 T S L P \\
321 T S L P\end{array}$ & $\begin{array}{l}2.2 \\
1.9 \\
2.0 \\
2.0 \\
2.7\end{array}$ & & $\begin{array}{l}\text { Fine-grained, limy and quartzitic sandstone } \\
\text { Fine-grained, limy and quartzitic sandstone } \\
\text { Fine-grained, limy and quartzitic sandstone } \\
\text { Fine-grained, limy and quartzitic sandstone } \\
\text { Fine-grained, limy and quartzitic sandstone }\end{array}$ \\
\hline $\begin{array}{l}\text { sb20-87-30ddd } \\
\text { sb20-87-30ddd } \\
\text { sb20-87-30ddd } \\
\text { sb20-87-30ddd } \\
\text { sb20-87-30ddd }\end{array}$ & $\begin{array}{l}8,228.0 \\
8,229.0 \\
8,230.0 \\
8,231.0 \\
8,232.0\end{array}$ & $\begin{array}{l}8,229.0 \\
8,230.0 \\
8,231.0 \\
8,232.0 \\
8,233.0\end{array}$ & $\begin{array}{l}321 \text { TSLP } \\
321 \text { TSLP } \\
321 \text { TSLP } \\
321 \text { TSLP }\end{array}$ & $\begin{array}{l}1.8 \\
2.2 \\
2.2 \\
2.2 \\
1.3\end{array}$ & $\begin{array}{l}0.100000 \\
0.100000 \\
0.100000 \\
0.100000 \\
0.100000\end{array}$ & $\begin{array}{l}\text { Fine-grained, limy and quartzitic sandstone } \\
\text { Fine-grained, limy and quartzitic sandstone } \\
\text { Fine-grained, limy and quartzitic sandstone } \\
\text { Fine-grained, limy and quartzitic sandstone } \\
\text { Fine-grained, limy and quartzitic sandstone }\end{array}$ \\
\hline $\begin{array}{l}\text { sb20-87-30ddd } \\
\text { sb20-87-30ddd } \\
\text { sb20-87-30ddd } \\
\text { sb20-87-30ddd } \\
\text { sb20-87-30ddd }\end{array}$ & $\begin{array}{l}8,233.0 \\
8,234.0 \\
8,235.0 \\
8,236.0 \\
8,237.0\end{array}$ & $\begin{array}{l}8,234.0 \\
8,235.0 \\
8,236.0 \\
8,237.0 \\
8,238.0\end{array}$ & $\begin{array}{l}321 \text { TSLP } \\
321 \text { TSLP } \\
321 \text { TSLP } \\
321 \text { TSLP } \\
321 \text { TSLP }\end{array}$ & $\begin{array}{l}2.1 \\
1.9 \\
2.8 \\
3.4 \\
2.1\end{array}$ & $\begin{array}{l}0.100000 \\
0.100000 \\
0.100000 \\
0.100000 \\
0.100000\end{array}$ & $\begin{array}{l}\text { Medium-grained limy sandstone } \\
\text { Fine-grained, limy and quartzitic sandstone } \\
\text { Fine-grained, limy and quartzitic sandstone } \\
\text { Fine-grained, limy and quartzitic sandstone } \\
\text { Fine-grained, limy and quartzitic sandstone }\end{array}$ \\
\hline $\begin{array}{l}\text { sb20-87-30ddd } \\
\text { sb20-87-30ddd } \\
\text { sb20-87-30ddd } \\
\text { sb20-87-30ddd } \\
\text { sb20-87-30ddd }\end{array}$ & $\begin{array}{l}8,238.0 \\
8,239.0 \\
8,240.0 \\
8,241.0 \\
8,242.0\end{array}$ & $\begin{array}{l}8,239.0 \\
8,240.0 \\
8,241.0 \\
8,242.0 \\
8,243.0\end{array}$ & $\begin{array}{l}321 \text { TSLP } \\
321 \text { TSLP } \\
321 \text { TSLP } \\
321 \text { TSLP } \\
321 \text { TSLP }\end{array}$ & $\begin{array}{l}1.9 \\
2.0 \\
0.8 \\
1.9 \\
3.3\end{array}$ & $\begin{array}{l}2.200000 \\
0.100000 \\
0.100000 \\
0.100000 \\
0.100000\end{array}$ & $\begin{array}{l}\text { Fine-grained, limy and quartzitic sandstone } \\
\text { Fine-grained, limy and quartzitic sandstone } \\
\text { Fine-grained, limy and quartzitic sandstone } \\
\text { Fine-grained, limy and quartzitic sandstone } \\
\text { Fine-grained, limy and quartzitic sandstone }\end{array}$ \\
\hline $\begin{array}{l}\text { sb20-87-30ddd } \\
\text { sb20-87-30ddd } \\
\text { sb20-87-30ddd } \\
\text { sb20-87-30ddd } \\
\text { sb20-87-30ddd }\end{array}$ & $\begin{array}{l}8,243.0 \\
8,244.0 \\
8,245.0 \\
8,246.0 \\
8,247.0\end{array}$ & $\begin{array}{l}8,244.0 \\
8,245.0 \\
8,246.0 \\
8,247.0 \\
8,248.0\end{array}$ & $\begin{array}{l}321 T S L P \\
321 T S L P \\
321 T S L P \\
321 T S L P \\
321 T S L P\end{array}$ & $\begin{array}{l}2.4 \\
1.8 \\
2.6 \\
1.9 \\
1.8\end{array}$ & $\begin{array}{l}0.100000 \\
0.100000 \\
0.100000 \\
0.100000 \\
0.100000\end{array}$ & $\begin{array}{l}\text { Fine-grained, limy and quartzitic sandstone } \\
\text { Fine-grained, limy and quartzitic sandstone } \\
\text { Fine-grained, limy and quartzitic sandstone } \\
\text { Fine-grained, limy and quartzitic sandstone } \\
\text { Fine-grained, limy and quartzitic sandstone }\end{array}$ \\
\hline
\end{tabular}


Table 4.--Laboratory-determined porosity and permeability--Continued

\begin{tabular}{|c|c|c|c|c|c|c|}
\hline Site & $\begin{array}{l}\text { Depth to } \\
\text { top } \\
\text { (feet) }\end{array}$ & $\begin{array}{l}\text { Depth to } \\
\text { bottom } \\
\text { (feet) }\end{array}$ & $\begin{array}{c}\text { Forma- } \\
\text { tion }\end{array}$ & $\begin{array}{l}\text { Porosity } \\
\text { (percent) }\end{array}$ & $\begin{array}{l}\text { Perme- } \\
\text { ability } \\
\text { (milli- } \\
\text { darcies) }\end{array}$ & Lithology \\
\hline $\begin{array}{l}\text { sb20-87-30ddd } \\
\text { sb20-87-30ddd } \\
\text { sb20-87-30ddd } \\
\text { sb20-87-30ddd } \\
\text { sb20-87-30ddd }\end{array}$ & $\begin{array}{l}8,248.0 \\
8,249.0 \\
8,250.0 \\
8,251.0 \\
8,252.0\end{array}$ & $\begin{array}{l}8,249.0 \\
8,250.0 \\
8,251.0 \\
8,252.0 \\
8,253.0\end{array}$ & $\begin{array}{l}321 \mathrm{TSLP} \\
321 \mathrm{TSLP} \\
321 \mathrm{TSLP} \\
321 \mathrm{TSLP} \\
321 \mathrm{TSLP}\end{array}$ & $\begin{array}{l}2.7 \\
1.9 \\
2.1 \\
3.0 \\
1.9\end{array}$ & $\begin{array}{l}0.100000 \\
0.100000 \\
0.100000 \\
0.400000 \\
0.100000\end{array}$ & $\begin{array}{l}\text { Fine-grained, limy and quartzitic sandstone } \\
\text { Fine-grained, limy and quartzitic sandstone } \\
\text { Fine-grained, limy and quartzitic sandstone } \\
\text { Fine-grained, limy and quartzitic sandstone } \\
\text { Fine-grained, limy and quartzitic sandstone }\end{array}$ \\
\hline $\begin{array}{l}\text { sb20-87-30ddd } \\
\text { sb20-87-30ddd } \\
\text { sb20-87-30ddd } \\
\text { sb20-87-30ddd } \\
\text { sb20-87-30ddd }\end{array}$ & $\begin{array}{l}8,253.0 \\
8,254.0 \\
8,255.0 \\
8,256.0 \\
8,257.0\end{array}$ & $\begin{array}{l}8,254.0 \\
8,255.0 \\
8,256.0 \\
8,257.0 \\
8,258.0\end{array}$ & $\begin{array}{l}321 \mathrm{TSLP} \\
321 \mathrm{TSLP} \\
321 \mathrm{TSLP} \\
321 \mathrm{TSLP} \\
321 \mathrm{TSLP}\end{array}$ & $\begin{array}{l}2.5 \\
2.3 \\
1.4 \\
1.3 \\
0.9\end{array}$ & $\begin{array}{l}0.100000 \\
0.100000 \\
0.100000 \\
0.100000 \\
0.100000\end{array}$ & $\begin{array}{l}\text { Fine-grained, limy and quartzitic sandstone } \\
\text { Fine-grained, limy and quartzitic sandstone } \\
\text { Fine-grained, limy and quartzitic sandstone } \\
\text { Fine-grained, limy and quartzitic sandstone } \\
\text { Fine-grained, limy and quartzitic sandstone }\end{array}$ \\
\hline $\begin{array}{l}\text { sb20-87-30ddd } \\
\text { sb20-87-30ddd } \\
\text { sb20-87-30ddd } \\
\text { sb20-87-30ddd } \\
\text { sb20-87-30ddd }\end{array}$ & $\begin{array}{l}8,258.0 \\
8,259.0 \\
8,260.0 \\
8,261.0 \\
8,262.0\end{array}$ & $\begin{array}{l}8,259.0 \\
8,260.0 \\
8,261.0 \\
8,262.0 \\
8,263.0\end{array}$ & $\begin{array}{l}321 \mathrm{TSLP} \\
321 \mathrm{TSLP} \\
321 \mathrm{TSLP} \\
\text { 321TSLP } \\
\text { 321TSLP }\end{array}$ & $\begin{array}{l}1.1 \\
0.8 \\
1.6 \\
1.7 \\
1.9\end{array}$ & $\begin{array}{l}0.100000 \\
0.100000 \\
0.000000 \\
0.000000 \\
0.400000\end{array}$ & $\begin{array}{l}\text { Fine-grained, limy and quartzitic sandstone } \\
\text { Fine-grained, limy and quartzitic sandstone } \\
\text { Cross-bedded, shaly, fine-grained, limy sandstone } \\
\text { Cross-bedded, shaly, fine-grained, limy sandstone } \\
\text { Cross-bedded, shaly, fine-grained, limy sandstone }\end{array}$ \\
\hline $\begin{array}{l}\text { sb20-87-30ddd } \\
\text { sb20-87-30ddd } \\
\text { sb20-87-30ddd } \\
\text { sb20-87-30ddd } \\
\text { sb20-87-30ddd }\end{array}$ & $\begin{array}{l}8,263.0 \\
8,264.0 \\
8,265.0 \\
8,266.0 \\
8,267.0\end{array}$ & $\begin{array}{l}8,264.0 \\
8,265.0 \\
8,266.0 \\
8,267.0 \\
8,268.0\end{array}$ & $\begin{array}{l}321 \mathrm{TSLP} \\
321 \mathrm{TSLP} \\
321 \mathrm{TSLP} \\
\text { 321TSLP } \\
\text { 321TSLP }\end{array}$ & $\begin{array}{l}2.6 \\
1.8 \\
1.9 \\
2.0 \\
2.0\end{array}$ & $\begin{array}{l}0.000000 \\
0.000000 \\
0.000000 \\
0.100000 \\
0.000000\end{array}$ & $\begin{array}{l}\text { Cross-bedded, shaly, fine-grained, limy sandstone } \\
\text { Cross-bedded, shaly, fine-grained, limy sandstone } \\
\text { Cross-bedded, shaly, fine-grained, limy sandstone } \\
\text { Cross-bedded, shaly, fine-grained, limy sandstone } \\
\text { Cross-bedded, shaly, fine-grained, limy sandstone }\end{array}$ \\
\hline $\begin{array}{l}\text { sb } 20-87-30 d d d \\
\text { sb20-87-30ddd } \\
\text { sb20-87-30ddd } \\
\text { sb20-87-30ddd } \\
\text { sb20-87-30ddd }\end{array}$ & $\begin{array}{l}8,268.0 \\
8,269.0 \\
8,270.0 \\
8,271.0 \\
8,272.0\end{array}$ & $\begin{array}{l}8,269.0 \\
8,270.0 \\
8,271.0 \\
8,272.0 \\
8,273.0\end{array}$ & $\begin{array}{l}321 \mathrm{TSLP} \\
321 \mathrm{TSLP} \\
321 \mathrm{TSLP} \\
321 \mathrm{TSLP} \\
321 \mathrm{TSLP}\end{array}$ & $\begin{array}{l}2.1 \\
2.0 \\
1.4 \\
2.0 \\
1.6\end{array}$ & $\begin{array}{l}0.000000 \\
0.000000 \\
0.000000 \\
0.000000 \\
0.000000\end{array}$ & $\begin{array}{l}\text { Cross-bedded, shaly, fine-grained, limy sandstone } \\
\text { Cross-bedded, shaly, fine-grained, limy sandstone } \\
\text { Cross-bedded, shaly, fine-grained, limy sandstone } \\
\text { Cross-bedded, shaly, fine-grained, limy sandstone } \\
\text { Cross-bedded, shaly, fine-grained, limy sandstone }\end{array}$ \\
\hline $\begin{array}{l}\text { sb20-87-30ddd } \\
\text { sb20-87-30ddd } \\
\text { sb20-87-30ddd } \\
\text { sb20-87-30ddd } \\
\text { sb20-87-30ddd }\end{array}$ & $\begin{array}{l}8,273.0 \\
8,274.0 \\
8,275.0 \\
8,276.0 \\
8,277.0\end{array}$ & $\begin{array}{l}8,274.0 \\
8,275.0 \\
8,276.0 \\
8,277.0 \\
8,278.0\end{array}$ & $\begin{array}{l}321 \mathrm{TSLP} \\
321 \mathrm{TSLP} \\
321 \mathrm{TSLP} \\
321 \mathrm{TSLP} \\
\text { 321TSLP }\end{array}$ & $\begin{array}{l}2.6 \\
2.1 \\
1.6 \\
2.0 \\
1.3\end{array}$ & $\begin{array}{l}0.000000 \\
0.000000 \\
0.100000 \\
0.000000 \\
0.000000\end{array}$ & $\begin{array}{l}\text { Cross-bedded, shaly, fine-grained, limy sandstone } \\
\text { Cross-bedded, shaly, fine-grained, limy sandstone } \\
\text { Cross-bedded, shaly, fine-grained, limy sandstone } \\
\text { Cross-bedded, shaly, fine-grained, limy sandstone } \\
\text { Cross-bedded, shaly, fine-grained, limy sandstone }\end{array}$ \\
\hline $\begin{array}{l}\text { sb20-87-30ddd } \\
\text { sb20-87-30ddd } \\
\text { sb20-87-30ddd } \\
\text { sb20-87-30ddd } \\
\text { sb20-87-30ddd }\end{array}$ & $\begin{array}{l}8,278.0 \\
8,279.0 \\
8,280.0 \\
8,281.0 \\
8,282.0\end{array}$ & $\begin{array}{l}8,279.0 \\
8,280.0 \\
8,281.0 \\
8,282.0 \\
8,283.0\end{array}$ & $\begin{array}{l}321 \mathrm{TSLP} \\
321 \mathrm{TSLP} \\
321 \mathrm{TSLP} \\
\text { 321TSLP } \\
\text { 321TSLP }\end{array}$ & $\begin{array}{l}1.7 \\
1.8 \\
1.7 \\
2.1 \\
1.8\end{array}$ & $\begin{array}{l}0.100000 \\
0.100000 \\
0.100000 \\
0.000000 \\
0.100000\end{array}$ & $\begin{array}{l}\text { Cross-bedded, shaly, fine-grained, limy sandstone } \\
\text { Cross-bedded, shaly, fine-grained, limy sandstone } \\
\text { Cross-bedded, shaly, fine-grained, limy sandstone } \\
\text { Cross-bedded, shaly, fine-grained, limy sandstone } \\
\text { Cross-bedded, shaly, fine-grained, limy sandstone }\end{array}$ \\
\hline $\begin{array}{l}\text { sb20-87-30ddd } \\
\text { sb20-87-30ddd } \\
\text { sb20-87-30ddd } \\
\text { sb20-87-30ddd } \\
\text { sb20-87-30ddd }\end{array}$ & $\begin{array}{l}8,283.0 \\
8,284.0 \\
8,285.0 \\
8,286.0 \\
8,287.0\end{array}$ & $\begin{array}{l}8,284.0 \\
8,285.0 \\
8,286.0 \\
8,287.0 \\
8,288.0\end{array}$ & $\begin{array}{l}321 T S L P \\
321 T S L P \\
321 T S L P \\
321 T S L P \\
321 T S L P\end{array}$ & $\begin{array}{l}2.3 \\
1.4 \\
1.2 \\
1.7 \\
1.7\end{array}$ & $\begin{array}{l}0.100000 \\
0.400000 \\
0.400000 \\
0.100000 \\
0.400000\end{array}$ & $\begin{array}{l}\text { Cross-bedded, shaly, fine-grained, limy sandstone } \\
\text { Cross-bedded, shaly, fine-grained, limy sandstone } \\
\text { Cross-bedded, shaly, fine-grained, limy sandstone } \\
\text { Cross-bedded, shaly, fine-grained, limy sandstone } \\
\text { Cross-bedded, shaly, fine-grained, limy sandstone }\end{array}$ \\
\hline $\begin{array}{l}\text { sb20-87-30ddd } \\
\text { sb20-87-30ddd } \\
\text { sb20-87-30ddd } \\
\text { sb20-87-30ddd } \\
\text { sb20-87-30ddd }\end{array}$ & $\begin{array}{l}8,288.0 \\
8,289.0 \\
8,290.0 \\
8,291.0 \\
8,292.0\end{array}$ & $\begin{array}{l}8,289.0 \\
8,290.0 \\
8,291.0 \\
8,292.0 \\
8,293.0\end{array}$ & $\begin{array}{l}321 T S L P \\
321 T S L P \\
321 T S L P \\
321 T S L P \\
\text { 321TSLP }\end{array}$ & $\begin{array}{l}2.1 \\
2.2 \\
2.0 \\
2.4 \\
2.4\end{array}$ & $\begin{array}{l}0.100000 \\
0.400000 \\
0.400000 \\
0.400000 \\
0.400000\end{array}$ & $\begin{array}{l}\text { Cross-bedded, shaly, fine-grained, limy sandstone } \\
\text { Cross-bedded, shaly, fine-grained, limy sandstone } \\
\text { Cross-bedded, shaly, fine-grained, limy sandstone } \\
\text { Cross-bedded, shaly, fine-grained, limy sandstone } \\
\text { Cross-bedded, shaly, fine-grained, limy sandstone }\end{array}$ \\
\hline $\begin{array}{l}\text { sb20-87-30ddd } \\
\text { sb20-87-30ddd } \\
\text { sb20-87-30ddd } \\
\text { sb20-87-30ddd } \\
\text { sb20-87-30ddd }\end{array}$ & $\begin{array}{l}8,293.0 \\
8,294.0 \\
8,295.0 \\
8,296.0 \\
8,297.0\end{array}$ & $\begin{array}{l}8,294.0 \\
8,295.0 \\
8,296.0 \\
8,297.0 \\
8,298.0\end{array}$ & $\begin{array}{l}\text { 321TSLP } \\
\text { 321TSLP } \\
\text { 321TSLP } \\
\text { 321TSLP } \\
\text { 321TSLP }\end{array}$ & $\begin{array}{l}1.0 \\
2.1 \\
1.9 \\
1.2 \\
1.4\end{array}$ & $\begin{array}{l}0.100000 \\
0.100000 \\
0.100000 \\
0.400000 \\
0.400000\end{array}$ & $\begin{array}{l}\text { Fine-grained, limy sandstone } \\
\text { Fine-grained, limy sandstone } \\
\text { Fine-grained, limy sandstone } \\
\text { Fine-grained, limy sandstone } \\
\text { Fine-grained, limy sandstone }\end{array}$ \\
\hline
\end{tabular}


Table 4.--Laboratory-determined porosity and permeability--Continued

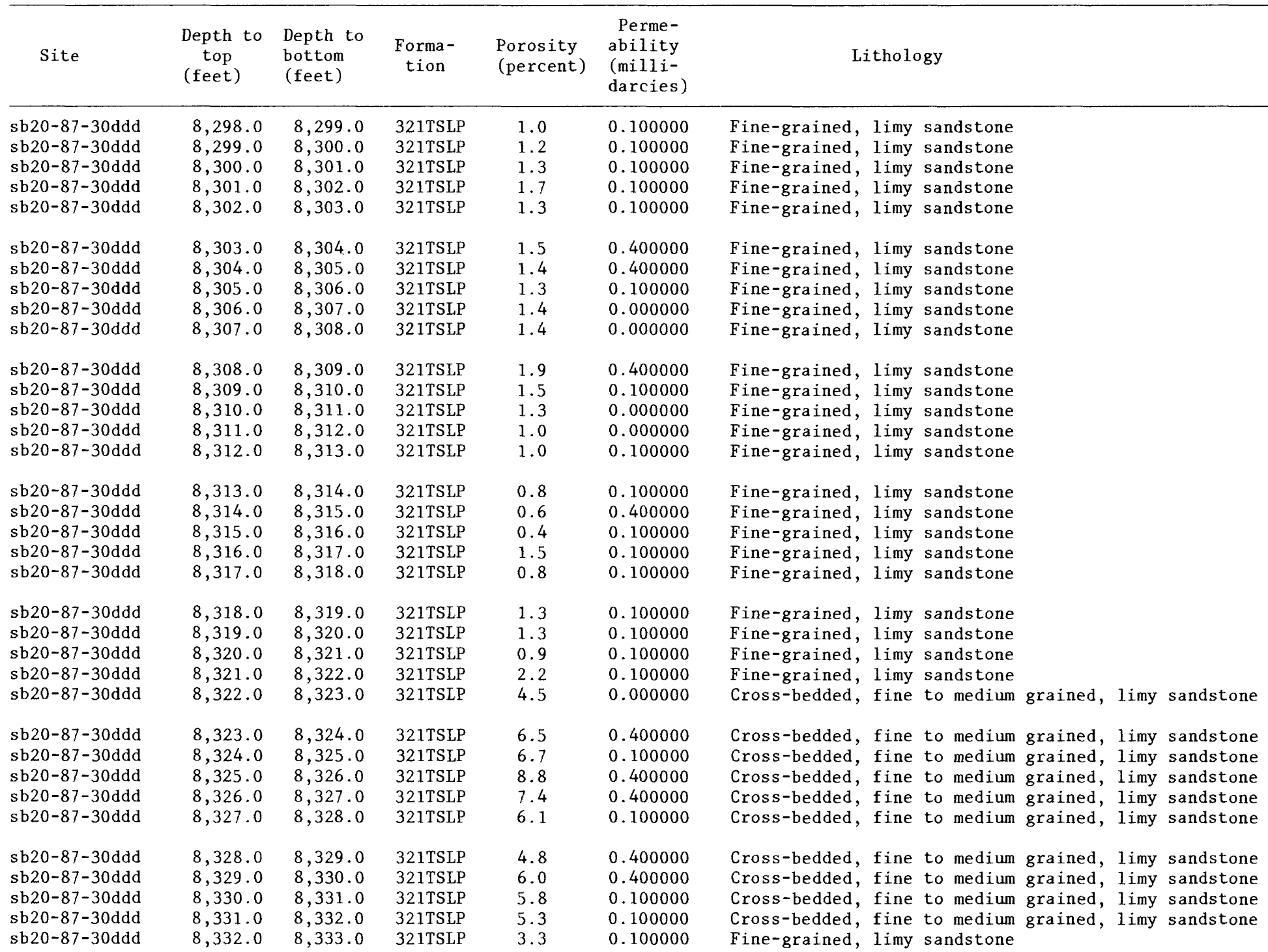

sb20-87-30ddd

sb20-87-30ddd

sb20-87-30ddd

sb20-87-30ddd

sb20-87-30ddd

sb20-87-30ddd

sb20-87-30ddd

sb20-87-30ddd

sb20-87-30ddd

sb20-87-30ddd

sb20-87-30ddd

sb20-87-30ddd

sb $20-87-30$ ddd

sb20-87-30ddd

sb20-87-30ddd

$\begin{array}{lllll}8,333.0 & 8,334.0 & \text { 321TSLP } & 2.3 & 0.000000 \\ 8,334.0 & 8,335.0 & \text { 321TSLP } & 2.6 & 0.100000 \\ 8,335.0 & 8,336.0 & 321 \mathrm{TSLP} & 1.8 & 0.000000 \\ 8,336.0 & 8,337.0 & 321 \mathrm{TSLP} & 2.3 & 0.100000 \\ 8,337.0 & 8,338.0 & 321 \mathrm{TSLP} & 1.6 & 0.000000 \\ & & & & \\ 8,338.0 & 8,339.0 & 321 \mathrm{TSLP} & 2.3 & 0.000000 \\ 8,339.0 & 8,340.0 & 321 \mathrm{TSLP} & 2.0 & 0.000000 \\ 8,340.0 & 8,341.0 & 321 \mathrm{TSLP} & 1.7 & 0.000000 \\ 8,341.0 & 8,342.0 & 321 \mathrm{TSLP} & 1.2 & 0.000000 \\ 8,342.0 & 8,343.0 & 321 \mathrm{TSLP} & 1.2 & 0.000000 \\ & & & & \\ 8,343.0 & 8,344.0 & 321 \mathrm{TSLP} & 1.5 & 0.100000 \\ 8,344.0 & 8,345.0 & 321 \mathrm{TSLP} & 1.4 & 0.000000 \\ 8,345.0 & 8,346.0 & 321 \mathrm{TSLP} & 1.4 & 0.000000 \\ 8,346.0 & 8,347.0 & 321 \mathrm{TSLP} & 1.4 & 0.000000 \\ 8,347.0 & 8,348.0 & 321 \mathrm{TSLP} & 1.7 & 0.100000\end{array}$

Fine-grained, limy sandstone

Shaly, fine-grained, limy sandstone

Fine-grained, limy sandstone

Fine to medium grained sandstone

Fine-grained, limy sandstone

Fine-grained, limy sandstone

Fine-grained, limy sandstone

Fine-grained, limy sandstone

Fine-grained, limy sandstone

Fine-grained, limy sandstone

Fine-grained, limy sandstone

Fine-grained, limy sandstone

Fine-grained, limy sandstone

Fine-grained, limy sandstone

Fine-grained, limy sandstone 
Table 4.--Laboratory-determined porosity and permeability--Continued

\begin{tabular}{|c|c|c|c|c|c|c|}
\hline Site & $\begin{array}{l}\text { Depth to } \\
\text { top } \\
\text { (feet) }\end{array}$ & $\begin{array}{l}\text { Depth to } \\
\text { bottom } \\
\text { (feet) }\end{array}$ & $\begin{array}{c}\text { Forma- } \\
\text { tion }\end{array}$ & $\begin{array}{l}\text { Porosity } \\
\text { (percent) }\end{array}$ & $\begin{array}{l}\text { Perme- } \\
\text { ability } \\
\text { (milli- } \\
\text { darcies) }\end{array}$ & Lithology \\
\hline sb20-87-30ddd & $8,348.0$ & $8,349.0$ & 321TSLP & 1.4 & 0.000000 & Fine-grained, limy sandstone \\
\hline sb20-87-30ddd & $8,349.0$ & $8,350.0$ & $321 \mathrm{TSLP}$ & 1.3 & 0.000000 & Fine-grained, limy sandstone \\
\hline sb20-87-30ddd & $8,350.0$ & $8,351.0$ & 321TSLP & 1.5 & 0.400000 & Fine-grained, limy sandstone \\
\hline sb20-87-30ddd & $8,351.0$ & $8,352.0$ & 321TSLP & 1.6 & 0.100000 & Fine-grained, limy sandstone \\
\hline sb20-87-30ddd & $8,352.0$ & $8,353.0$ & $321 \mathrm{TSLP}$ & 1.0 & 0.100000 & Fine-grained, limy sandstone \\
\hline sb20-87-30ddd & $8,353.0$ & $8,354.0$ & $321 \mathrm{TSLP}$ & 1.3 & 0.000000 & Fine-grained, limy sandstone \\
\hline sb20-87-30ddd & $8,354.0$ & $8,355.0$ & $321 \mathrm{TSLP}$ & 1.3 & 0.000000 & Fine-grained, limy sandstone \\
\hline sb20-87-30ddd & $8,355.0$ & $8,356.0$ & 321TSLP & 1.7 & 0.000000 & Fine-grained, limy sandstone \\
\hline sb20-87-30ddd & $8,356.0$ & $8,357.0$ & $321 \mathrm{TSLP}$ & 1.8 & 0.100000 & Fine-grained, limy sandstone \\
\hline sb20-87-30ddd & $8,357.0$ & $8,358.0$ & 321TSLP & 1.8 & 0.000000 & Fine-grained, limy sandstone \\
\hline sb20-87-30ddd & $8,358.0$ & $8,359.0$ & 321TSLP & 1.4 & 0.000000 & Fine-grained, limy sandstone \\
\hline sb20-87-30ddd & $8,359.0$ & $8,360.0$ & 321 TSLP & 1.8 & 0.100000 & Fine-grained, limy sandstone \\
\hline $\mathrm{sb} 20-87-30 \mathrm{ddd}$ & $8,360.0$ & $8,361.0$ & $321 \mathrm{TSLP}$ & 2.3 & 0.000000 & Fine-grained, limy sandstone \\
\hline sb20-87-30ddd & $8,361.0$ & $8,362.0$ & $321 \mathrm{TSLP}$ & 2.4 & 0.000000 & Fine-grained, limy sandstone \\
\hline sb20-87-30ddd & $8,362.0$ & $8,363.0$ & $321 \mathrm{TSLP}$ & 2.3 & 0.000000 & Fine-grained, limy sandstone \\
\hline sb20-87-30ddd & $8,363.0$ & $8,364.0$ & $321 \mathrm{TSLP}$ & 2.2 & 0.000000 & Fine-grained, limy sandstone \\
\hline $\mathrm{sb} 20-87-30 \mathrm{ddd}$ & $8,364.0$ & $8,365.0$ & $321 \mathrm{TSLP}$ & 2.3 & 0.000000 & Fine-grained, limy sandstone \\
\hline sb20-87-30ddd & $8,367.0$ & $8,368.0$ & $321 \mathrm{TSLP}$ & 2.2 & 0.000000 & Fine-grained, limy sandstone \\
\hline $\operatorname{sb} 20-87-30 \mathrm{ddd}$ & $8,368.0$ & $8,369.0$ & 321 TSLP & 2.0 & 0.000000 & Fine-grained, limy sandstone \\
\hline sb20-87-30ddd & $8,369.0$ & $8,370.0$ & $321 \mathrm{TSLP}$ & 1.8 & 0.000000 & Fine-grained, limy sandstone \\
\hline $\mathrm{sb} 20-87-30 \mathrm{ddd}$ & $8,370.0$ & $8,371.0$ & 321 TSLP & 1.5 & 0.000000 & Fine-grained, limy sandstone \\
\hline sb20-87-30ddd & $8,371.0$ & $8,372.0$ & $321 T S L P$ & 1.7 & 0.000000 & Fine-grained, limy sandstone \\
\hline $\operatorname{sb} 20-87-30 \mathrm{ddd}$ & $8,372.0$ & $8,373.0$ & $321 \mathrm{TSLP}$ & 1.4 & 0.000000 & Fine-grained, limy sandstone \\
\hline sb20-87-30ddd & $8,373.0$ & $8,374.0$ & 321TSLP & 2.0 & 0.100000 & Fine-grained, limy sandstone \\
\hline sb20-87-30ddd & $8,374.0$ & $8,375.0$ & $321 T S L P$ & 2.3 & 0.100000 & Fine-grained, limy sandstone \\
\hline $\mathrm{sb} 20-87-30 \mathrm{ddd}$ & $8,375.0$ & $8,376.0$ & $321 \mathrm{TSLP}$ & 1.7 & 0.000000 & Fine-grained, limy sandstone \\
\hline sb20-87-30ddd & $8,376.0$ & $8,377.0$ & $321 \mathrm{TSLP}$ & 2.6 & 0.400000 & Fine-grained, limy sandstone \\
\hline sb20-87-30ddd & $8,377.0$ & $8,378.0$ & $321 \mathrm{TSLP}$ & 3.1 & 0.100000 & Fine-grained, limy sandstone \\
\hline $\operatorname{sb} 20-87-30 \mathrm{ddd}$ & $8,378.0$ & $8,379.0$ & $321 \mathrm{TSLP}$ & 2.9 & 0.000000 & Fine-grained, limy sandstone \\
\hline sb20-87-30ddd & $8,379.0$ & $8,380.0$ & $321 T S L P$ & 2.2 & 0.000000 & Fine-grained, limy sandstone \\
\hline sb20-87-30ddd & $8,380.0$ & $8,381.0$ & 321TSLP & 1.2 & 0.000000 & Fine-grained, limy sandstone \\
\hline sb20-87-30ddd & $8,381.0$ & $8,382.0$ & 321TSLP & 1.3 & 0.000000 & Fine-grained, limy sandstone \\
\hline sb20-87-30ddd & $8,382.0$ & $8,383.0$ & $321 \mathrm{TSLP}$ & 1.0 & 0.100000 & Fine-grained, limy sandstone \\
\hline sb20-87-30ddd & $8,383.0$ & $8,384.0$ & $321 T S L P$ & 1.0 & 0.000000 & Fine-grained, limy sandstone \\
\hline sb20-87-30ddd & $8,384.0$ & $8,385.0$ & $321 \mathrm{TSLP}$ & 1.4 & 0.100000 & Fine-grained, limy sandstone \\
\hline sb20-87-30ddd & $8,385.0$ & $8,386.0$ & $321 \mathrm{TSLP}$ & 1.2 & 0.000000 & Fine-grained, limy sandstone \\
\hline sb20-87-30ddd & $8,386.0$ & $8,387.0$ & 321 TSLP & 1.3 & 0.000000 & Fine-grained, limy sandstone \\
\hline $\mathrm{sb} 20-87-30 \mathrm{ddd}$ & $8,387.0$ & $8,388.0$ & $321 T S L P$ & 0.7 & 0.000000 & Fine-grained, limy sandstone \\
\hline sb20-87-30ddd & $8,388.0$ & $8,389.0$ & $321 \mathrm{TSLP}$ & 1.2 & 0.100000 & Fine-grained, limy sandstone \\
\hline sb20-87-30ddd & $8,389.0$ & $8,390.0$ & $321 \mathrm{TSLP}$ & 0.9 & 0.000000 & Fine-grained, limy sandstone \\
\hline sb20-87-30ddd & $8,390.0$ & $8,391.0$ & $321 \mathrm{TSLP}$ & 1.1 & 0.000000 & Fine-grained, limy sandstone \\
\hline sb20-87-30ddd & $8,391.0$ & $8,392.0$ & $321 \mathrm{TSLP}$ & 0.6 & 0.000000 & Fine-grained, limy sandstone \\
\hline $\mathrm{sb} 20-87-30 \mathrm{ddd}$ & $8,392.0$ & $8,393.0$ & 321 TSLP & 0.9 & 0.000000 & Fine-grained, limy sandstone \\
\hline $\mathrm{sb} 20-87-30 \mathrm{ddd}$ & $8,393.0$ & $8,394.0$ & $321 T S L P$ & 0.8 & 0.000000 & Fine-grained, limy sandstone \\
\hline sb20-87-30ddd & $8,394.0$ & $8,395.0$ & 321TSLP & 0.7 & 0.000000 & Fine-grained, limy sandstone \\
\hline sb20-87-30ddd & $8,395.0$ & $8,396.0$ & 321TSLP & 1.2 & 0.000000 & Fine-grained, limy sandstone \\
\hline sb20-87-30ddd & $8,396.0$ & $8,397.0$ & $321 \mathrm{TSLP}$ & 1.3 & 0.000000 & Fine-grained, limy sandstone \\
\hline sb20-87-30ddd & $8,397.0$ & $8,398.0$ & 321TSLP & 0.8 & 0.100000 & Fine-grained, limy sandstone \\
\hline sb20-87-30ddd & $8,398.0$ & $8,399.0$ & $321 \mathrm{TSLP}$ & 0.3 & 0.000000 & Fine-grained, limy sandstone \\
\hline sb20-87-30ddd & $8,399.0$ & $8,400.0$ & $321 \mathrm{TSLP}$ & 0.6 & 0.000000 & Fine-grained, limy sandstone \\
\hline
\end{tabular}


Table 4.--Laboratory-determined porosity and permeability--Continued

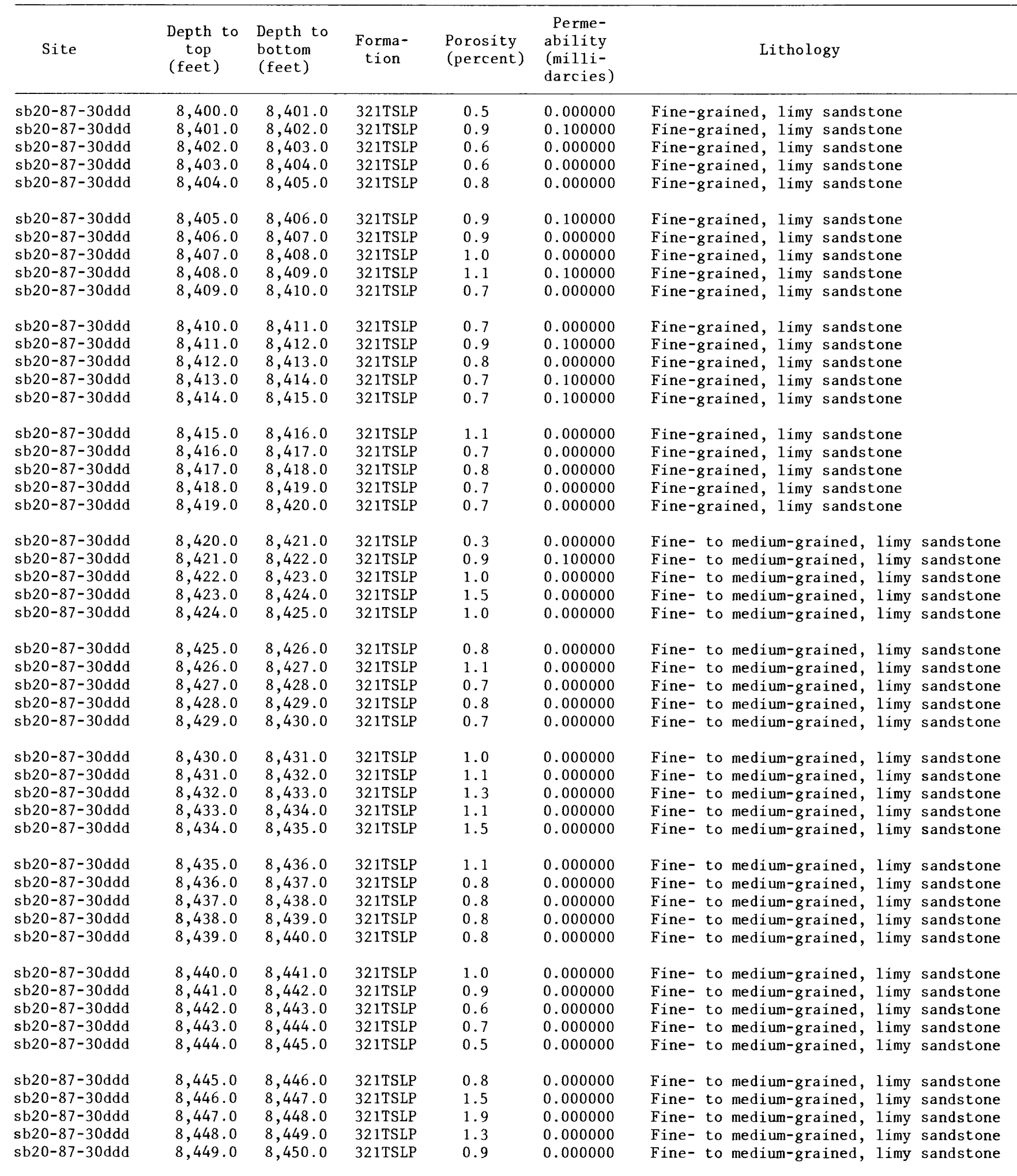


Table 4.--Laboratory-determined porosity and permeability--Continued

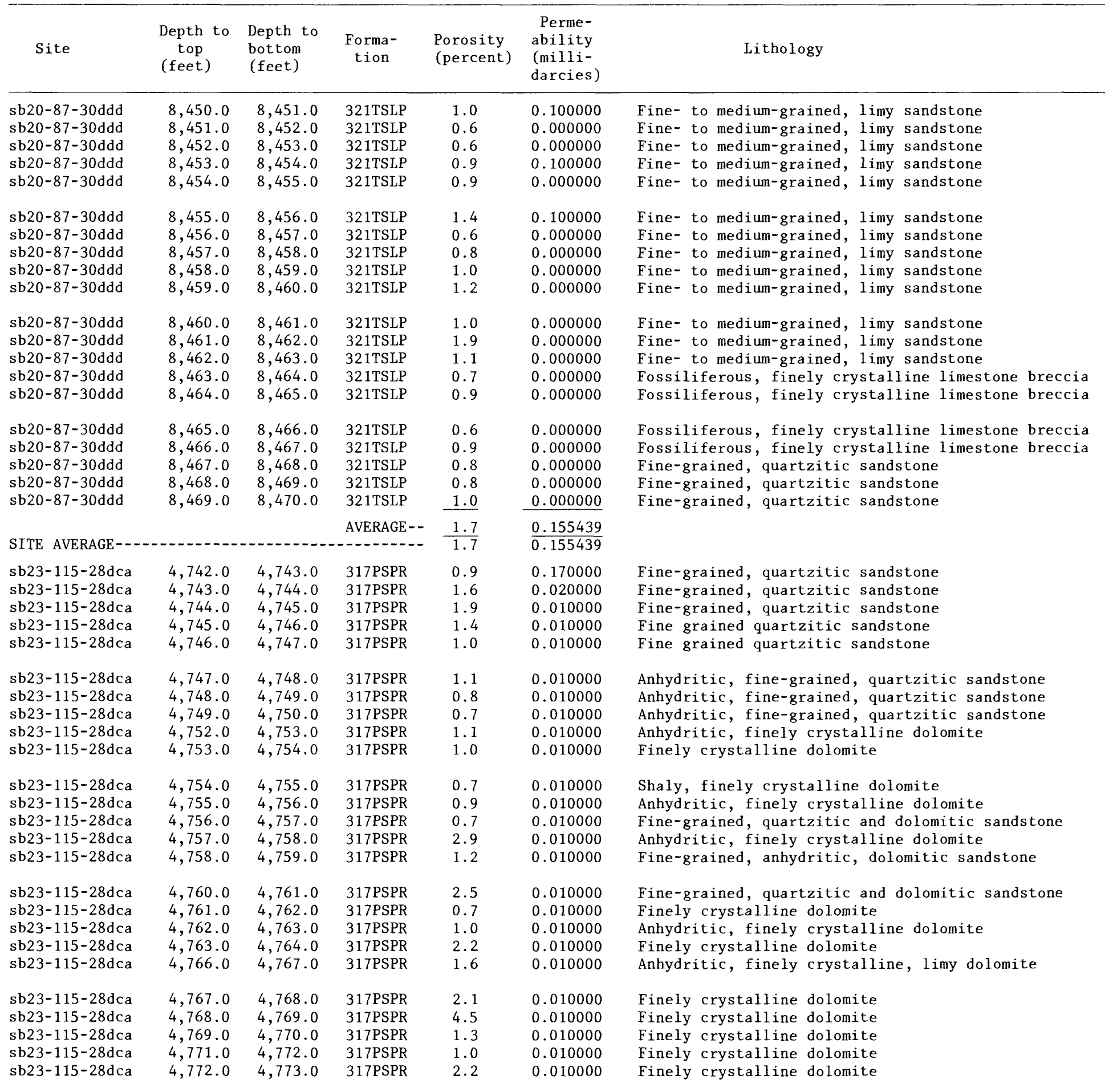


Table 4.--Laboratory-determined porosity and permeability--Continued

\begin{tabular}{|c|c|c|c|c|c|}
\hline Site & $\begin{array}{l}\text { Depth to } \\
\text { top } \\
\text { (feet) }\end{array}$ & $\begin{array}{l}\text { Depth to } \\
\text { bottom } \\
\text { (feet) }\end{array}$ & $\begin{array}{c}\text { Forma- } \\
\text { tion }\end{array}$ & $\begin{array}{l}\text { Porosity } \\
\text { (percent) }\end{array}$ & $\begin{array}{l}\text { Perme- } \\
\text { ability } \\
\text { (milli- } \\
\text { darcies) }\end{array}$ \\
\hline sb23-115-28dca & $4,774.0$ & $4,775.0$ & 317PSPR & 1.8 & 0.010000 \\
\hline sb23-115-28dca & $4,780.0$ & $4,781.0$ & 317PSPR & 1.4 & 0.010000 \\
\hline sb23-115-28dca & $4,781.0$ & $4,782.0$ & 317 PSPR & 1.8 & 0.010000 \\
\hline sb23-115-28dca & $4,783.0$ & $4,784.0$ & 317PSPR & 3.8 & 0.010000 \\
\hline \multirow[t]{2}{*}{$\mathrm{sb} 23-115-28 \mathrm{dca}$} & $4,784.0$ & $4,785.0$ & 317PSPR & 5.2 & 0.010000 \\
\hline & & & AVERAGE-- & 1.7 & 0.015667 \\
\hline ITE AVERAGE- & & & - & 1.7 & 0.015667 \\
\hline $\mathrm{sb} 24-87-01 \mathrm{bcd}$ & $6,290.0$ & $6,291.0$ & 321TSLP & 1.1 & 0.000000 \\
\hline sb24-87-01bcd & $6,291.0$ & $6,292.0$ & $321 \mathrm{TSLP}$ & 3.1 & .000000 \\
\hline sb24-87-01bcd & $6,292.0$ & $6,293.0$ & $321 \mathrm{TSLP}$ & 4.4 & 0.000000 \\
\hline sb24-87-01bcd & $6,293.0$ & $6,294.0$ & $321 \mathrm{TSLP}$ & 5.1 & 0.000000 \\
\hline sb24-87-01bcd & $6,294.0$ & $6,295.0$ & 321TSLP & 3.6 & 0.000000 \\
\hline sb24-87-01bcd & $6,295.0$ & $6,296.0$ & $321 \mathrm{TSLP}$ & 2.3 & 0.000000 \\
\hline sb24-87-01bcd & $6,296.0$ & $6,297.0$ & $321 \mathrm{TSLP}$ & 1.4 & 0.000000 \\
\hline sb24-87-01bcd & $6,297.0$ & $6,298.0$ & $321 \mathrm{TSLP}$ & 2.6 & 0.000000 \\
\hline sb24-87-01bcd & $6,298.0$ & $6,299.0$ & $321 \mathrm{TSLP}$ & 2.8 & 0.000000 \\
\hline sb24-87-01bcd & $6,299.0$ & $6,300.0$ & $321 \mathrm{TSLP}$ & 1.4 & 0.000000 \\
\hline sb24-87-01bcd & $6,300.0$ & $6,301.0$ & 321TSLP & 4.9 & 0.000000 \\
\hline sb24-87-01bcd & $6,301.0$ & $6,302.0$ & $321 \mathrm{TSLP}$ & 4.4 & 0.000000 \\
\hline sb24-87-01bcd & $6,302.0$ & $6,303.0$ & $321 \mathrm{TSLP}$ & 2.6 & 0.000000 \\
\hline sb24-87-01bcd & $6,303.0$ & $6,304.0$ & $321 \mathrm{TSLP}$ & 2.8 & 0.000000 \\
\hline sb24-87-01bcd & $6,304.0$ & $6,305.0$ & $321 T S L P$ & 4.6 & 0.000000 \\
\hline sb24-87-01bcd & $6,305.0$ & $6,306.0$ & $\mathrm{P}$ & & 000 \\
\hline sb24-87-01bcd & $6,306.0$ & $6,307.0$ & $321 \mathrm{TSLP}$ & 3.6 & 0.000000 \\
\hline sb24-87-01bcd & $6,307.0$ & $6,308.0$ & $321 \mathrm{TSLP}$ & 3.0 & 0.000000 \\
\hline sb24-87-01bcd & $6,308.0$ & $6,309.0$ & $321 \mathrm{TSLP}$ & 3.9 & 0.000000 \\
\hline sb24-87-01bcd & $6,309.0$ & $6,310.0$ & $321 \mathrm{TSLP}$ & 3.6 & 0.000000 \\
\hline sb24-87-01bcd & $6,310.0$ & $6,311.0$ & $321 \mathrm{TSLP}$ & 2.2 & 0.000000 \\
\hline $\operatorname{sb} 24-87-01 \mathrm{bcd}$ & $6,311.0$ & $6,312.0$ & $321 \mathrm{TSLP}$ & 1.2 & 0.000000 \\
\hline sb24-87-01bcd & $6,312.0$ & $6,313.0$ & $321 \mathrm{TSLP}$ & 3.4 & 0.000000 \\
\hline sb24-87-01bcd & $6,324.0$ & $6,325.0$ & $321 \mathrm{TSLP}$ & 4.9 & 0.170000 \\
\hline sb24-87-01bcd & $6,326.0$ & $6,327.0$ & $321 T S L P$ & 4.0 & 0.000000 \\
\hline sb24-87-01bcd & $6,327.0$ & $6,328.0$ & $321 \mathrm{TSLP}$ & $3 . J$ & 0.000000 \\
\hline sb24-87-01bcd & $6,332.0$ & $6,333.0$ & $321 \mathrm{TSLP}$ & 3.6 & 0.000000 \\
\hline & & & AVERAGE-- & 3. & 0.006296 \\
\hline SITE AVERAGE- & & & - & 3.3 & 0.006296 \\
\hline $\mathrm{sb} 25-88-31 d c d$ & $5,065.0$ & $5,066.0$ & $321 \mathrm{TSLP}$ & 0,1 & 000 \\
\hline $8-31 d c d$ & $5,066.0$ & 5,0 & 32 & & 000 \\
\hline sb25-88-31dcd & 5,067 & 5,0 & & & 0.010000 \\
\hline sb25-88-31dcd & $5,068.0$ & $5,069.0$ & $321 \mathrm{TSLP}$ & 5.9 & 0.430000 \\
\hline sb25-88-31dcd & $5,069.0$ & $5,070.0$ & $321 \mathrm{TSLP}$ & 4.9 & 0.110000 \\
\hline $\mathrm{sb} 25-88-31 \mathrm{dcd}$ & $5,070.0$ & $5,071.0$ & 32 & 6.4 & 0.300000 \\
\hline sb25-88-31dcd & $5,071.0$ & $5,072.0$ & $321 \mathrm{TSLP}$ & 4.3 & 0.110000 \\
\hline sb25-88-31dcd & $5,072.0$ & $5,073.0$ & $321 \mathrm{TSLP}$ & 3.8 & 0.040000 \\
\hline sb25-88-31dcd & $5,073.0$ & $5,074.0$ & 321TSLP & 4.1 & 0.020000 \\
\hline $\mathrm{sb} 25-88-31 \mathrm{dcd}$ & $5,074.0$ & $5,075.0$ & $321 \mathrm{TSLP}$ & 7.5 & 0.840000 \\
\hline sb25-88-31dcd & $5,075.0$ & $5,076.0$ & 321 & 5.3 & 0.080000 \\
\hline $\mathrm{sb} 25-88-31 \mathrm{dcd}$ & $5,076.0$ & $5,077.0$ & $321 \mathrm{TSLP}$ & 3.3 & 0.000000 \\
\hline sb25-88-31dcd & $5,077.0$ & $5,078.0$ & $321 \mathrm{TSLP}$ & 2.3 & 0.000000 \\
\hline sb25-88-31dcd & $5,078.0$ & $5,079.0$ & 321TSLP & 2.0 & 0.000000 \\
\hline $\mathrm{sb} 25-88-31 \mathrm{dcd}$ & $5,079.0$ & $5,080.0$ & 321TSLP & 1.9 & 0.000000 \\
\hline
\end{tabular}

Lithology

Finely crystalline, limy dolomite

Finely crystalline dolomite

Finely crystalline dolomite

Finely crystalline dolomite

Finely crystalline dolomite

Fine-grained, quartzitic and dolomitic sandstone Fine-grained, quartzitic and dolomitic sandstone Fine-grained, quartzitic and dolomitic sandstone Fine-grained, quartzitic and dolomitic sandstone Fine-grained, quartzitic and dolomitic sandstone

Fine-grained, quartzitic and dolomitic sandstone Fine-grained, quartzitic and dolomitic sandstone Fine-grained, quartzitic and dolomitic sandstone Fine-grained, quartzitic and dolomitic sandstone Fine-grained, quartzitic and dolomitic sandstone

Fine-grained, quartzitic and dolomitic sandstone Fine-grained, quartzitic and dolomitic sandstone Fine-grained, quartzitic and dolomitic sandstone Fine-grained, quartzitic and dolomitic sandstone Fine-grained, quartzitic and dolomitic sandstone

Fine-grained, quartzitic and dolomitic sandstone Fine-grained, quartzitic and dolomitic sandstone Fine-grained, quartzitic and dolomitic sandstone Fine-grained, quartzitic and dolomitic sandstone Fine-grained, quartzitic and dolomitic sandstone

Fine-grained, quartzitic and dolomitic sandstone Fine-grained, quartzitic and dolomitic sandstone Fine-grained, quartzitic and dolomitic sandstone Vuggy, fine- to medium-crystalline dolomite

Fine-grained, quartzitic and dolomitic sandstone

Fine-grained, quartzitic and dolomitic sandstone Fine-grained, quartzitic and dolomitic sandstone

Fine grained, limy sandstone Fine grained, limy sandstone Fine grained, limy sandstone Fine grained, limy sandstone Fine grained, limy sandstone

Fine grained, limy sandstone Fine grained, limy sandstone Fine-grained, limy sandstone Fine-grained, limy sandstone Fine-grained, limy sandstone

Fine-grained, limy sandstone Fine-grained, limy sandstone Fine-grained, limy sandstone Fine-grained, limy sandstone Fine-grained, quartzitic sandstone 
Table 4.--Laboratory-determined porosity and permeability--Continued

\begin{tabular}{|c|c|c|c|c|c|c|}
\hline Site & $\begin{array}{l}\text { Depth to } \\
\text { top } \\
\text { (feet) }\end{array}$ & $\begin{array}{l}\text { Depth to } \\
\text { bottom } \\
\text { (feet) }\end{array}$ & $\begin{array}{c}\text { Forma- } \\
\text { tion }\end{array}$ & $\begin{array}{l}\text { Porosity } \\
\text { (percent) }\end{array}$ & $\begin{array}{l}\text { Perme- } \\
\text { ability } \\
\text { (milli- } \\
\text { darcies) }\end{array}$ & Lithology \\
\hline $\mathrm{sb} 25-88-31 \mathrm{dcd}$ & $5,080.0$ & $5,081.0$ & $321 \mathrm{TSLP}$ & 2.1 & 0.000000 & Fine-grained, quartzitic sandstone \\
\hline $\mathrm{sb} 25-88-31 \mathrm{dcd}$ & $5,081.0$ & $5,082.0$ & $321 \mathrm{TSLP}$ & 1.9 & 0.010000 & Fine-grained, quartzitic sandstone \\
\hline $\mathrm{sb} 25-88-31 \mathrm{dcd}$ & $5,082.0$ & $5,083.0$ & 321TSLP & 2.2 & 0.010000 & Fine-grained, quartzitic sandstone \\
\hline $\mathrm{sb} 25-88-31 \mathrm{dcd}$ & $5,083.0$ & $5,084.0$ & $321 \mathrm{TSLP}$ & 0.9 & 0.000000 & Fine-grained, quartzitic sandstone \\
\hline $\mathrm{sb} 25-88-31 \mathrm{dcd}$ & $5,084.0$ & $5,085.0$ & $321 \mathrm{TSLP}$ & 0.2 & 0.000000 & Fine-grained, quartzitic sandstone \\
\hline $\mathrm{sb} 25-88-31 \mathrm{dcd}$ & $5,085.0$ & $5,086.0$ & $321 \mathrm{TSLP}$ & 3.0 & 0.000000 & Fine-grained, quartzitic sandstone \\
\hline $\mathrm{sb} 25-88-31 \mathrm{dcd}$ & $5,086.0$ & $5,087.0$ & $321 \mathrm{TSLP}$ & 4.0 & 0.020000 & Fine-grained, quartzitic sandstone \\
\hline sb25-88-31dcd & $5,087.0$ & $5,088.0$ & $321 \mathrm{TSLP}$ & 4.4 & 0.020000 & Fine-grained, quartzitic sandstone \\
\hline $\mathrm{sb} 25-88-31 \mathrm{dcd}$ & $5,088.0$ & $5,089.0$ & $321 \mathrm{TSLP}$ & 3.2 & 0.000000 & Fine-grained, quartzitic sandstone \\
\hline $\mathrm{sb} 25-88-31 \mathrm{dcd}$ & $5,089.0$ & $5,090.0$ & $321 \mathrm{TSLP}$ & 1.4 & 0.000000 & Fine-grained, quartzitic sandstone \\
\hline $\mathrm{sb} 25-88-31 \mathrm{dcd}$ & $5,090.0$ & $5,091.0$ & $321 \mathrm{TSLP}$ & 2.9 & 0.000000 & Fine-grained, quartzitic sandstone \\
\hline $\mathrm{sb} 25-88-31 \mathrm{dcd}$ & $5,091.0$ & $5,092.0$ & $321 \mathrm{TSLP}$ & 2.3 & 0.000000 & Fine-grained, quartzitic sandstone \\
\hline $\mathrm{sb} 25-88-31 \mathrm{dcd}$ & $5,092.0$ & $5,093.0$ & $321 \mathrm{TSLP}$ & 3.3 & 0.010000 & Fine-grained, quartzitic sandstone \\
\hline $\mathrm{sb} 25-88-31 \mathrm{dcd}$ & $5,093.0$ & $5,094.0$ & $321 \mathrm{TSLP}$ & 2.8 & 0.000000 & Fine-grained, quartzitic sandstone \\
\hline $\mathrm{sb} 25-88-31 \mathrm{dcd}$ & $5,094.0$ & $5,095.0$ & $321 \mathrm{TSLP}$ & 3.9 & 0.000000 & Fine-grained, quartzitic sandstone \\
\hline $\mathrm{sb} 25-88-31 \mathrm{dcd}$ & $5,095.0$ & $5,096.0$ & $321 \mathrm{TSLP}$ & 2.6 & 0.000000 & Fine-grained, quartzitic sandstone \\
\hline $\mathrm{sb} 25-88-31 \mathrm{dcd}$ & $5,096.0$ & $5,097.0$ & $321 \mathrm{TSLP}$ & 2.8 & 0.010000 & Fine-grained, quartzitic sandstone \\
\hline $\mathrm{sb} 25-88-31 \mathrm{dcd}$ & $5,097.0$ & $5,098.0$ & $321 \mathrm{TSLP}$ & 1.4 & 0.000000 & Fractured, fine-grained, quartzitic sandstone \\
\hline $\mathrm{sb} 25-88-31 \mathrm{dcd}$ & $5,098.0$ & $5,099.0$ & $321 \mathrm{TSLP}$ & 3.0 & 0.000000 & Fractured, fine-grained, quartzitic sandstone \\
\hline $\mathrm{sb} 25-88-31 \mathrm{dcd}$ & $5,099.0$ & $5,100.0$ & $321 \mathrm{TSLP}$ & 2.5 & 0.000000 & Fractured, fine-grained, quartzitic sandstone \\
\hline $\mathrm{sb} 25-88-31 \mathrm{dcd}$ & $5,100.0$ & $5,101.0$ & $321 \mathrm{TSLP}$ & 1.7 & 0.000000 & Fractured, fine-grained, quartzitic sandstone \\
\hline $\mathrm{sb} 25-88-31 \mathrm{dcd}$ & $5,101.0$ & $5,102.0$ & $321 \mathrm{TSLP}$ & 1.8 & 0.000000 & Fine-grained, quartzitic sandstone \\
\hline $\mathrm{sb} 25-88-31 \mathrm{dcd}$ & $5,102.0$ & $5,103.0$ & $321 \mathrm{TSLP}$ & 2.1 & 0.000000 & Fractured, fine-grained, quartzitic sandstone \\
\hline
\end{tabular}

SITE AVERAGE-

sb26-87-30ddc sb26-87-30ddc sb26-87-30ddc sb26-87-30ddc sb26-87-30ddc

sb26-87-30ddc sb26-87-30ddc sb26-87-30ddc sb26-87-30ddc sb26-87-30ddc

sb26-87-30ddc sb26-87-30ddc sb26-87-30ddc sb26-87-30ddc sb26-87-30ddc

sb26-87-30ddc sb26-87-30ddc sb26-87-30ddc sb26-87-30ddc sb26-87-30ddc

sb26-87-30ddc sb26-87-30ddc sb26-87-30ddc sb26-87-30ddc sb26-87-30ddc

\begin{tabular}{|c|c|c|c|c|}
\hline $4,353.0$ & $4,354.0$ & $321 \mathrm{TSLP}$ & 2.7 & 0.000000 \\
\hline $4,354.0$ & $4,355.0$ & $321 \mathrm{TSLP}$ & 3.9 & 0.000000 \\
\hline $4,355.0$ & $4,356.0$ & $321 \mathrm{TSLP}$ & 7.3 & 0.000000 \\
\hline $4,356.0$ & $4,357.0$ & $321 \mathrm{TSLP}$ & 8.5 & 0.600000 \\
\hline $4,357.0$ & $4,358.0$ & $321 \mathrm{TSLP}$ & 4.8 & 9.900000 \\
\hline $4,358.0$ & $4,359.0$ & $321 \mathrm{TSLP}$ & 9.2 & 2.000000 \\
\hline $4,359.0$ & $4,360.0$ & $321 \mathrm{TSLP}$ & 11.0 & 16.000000 \\
\hline $4,360.0$ & $4,361.0$ & $321 \mathrm{TSLP}$ & 8.4 & 2.000000 \\
\hline $4,361.0$ & $4,362.0$ & $321 \mathrm{TSLP}$ & 7.2 & 1.200000 \\
\hline $4,362.0$ & $4,363.0$ & $321 \mathrm{TSLP}$ & 8.7 & 1.200000 \\
\hline $4,363.0$ & $4,364.0$ & $321 \mathrm{TSLP}$ & 8.8 & 1.500000 \\
\hline $4,364.0$ & $4,365.0$ & $321 \mathrm{TSLP}$ & 9.3 & 1.200000 \\
\hline $4,365.0$ & $4,366.0$ & $321 \mathrm{TSLP}$ & 9.2 & 0.200000 \\
\hline $4,366.0$ & $4,367.0$ & $321 \mathrm{TSLP}$ & 9.2 & 0.000000 \\
\hline $4,367.0$ & $4,368.0$ & $321 \mathrm{TSLP}$ & 8.7 & 0.200000 \\
\hline $4,368.0$ & $4,369.0$ & $321 \mathrm{TSLP}$ & 8.5 & 0.000000 \\
\hline $4,369.0$ & $4,370.0$ & 321TSLP & 8.1 & 0.000000 \\
\hline $4,370.0$ & $4,371.0$ & $321 \mathrm{TSLP}$ & 7.2 & 0.000000 \\
\hline $4,371.0$ & $4,372.0$ & $321 \mathrm{TSLP}$ & 5.7 & 0.000000 \\
\hline $4,372.0$ & $4,373.0$ & $321 \mathrm{TSLP}$ & 4.5 & 0.000000 \\
\hline $4,373.0$ & $4,374.0$ & $321 \mathrm{TSLP}$ & 4.9 & 0.000000 \\
\hline $4,374.0$ & $4,375.0$ & 321TSLP & 3.8 & 0.000000 \\
\hline $4,385.0$ & $4,386.0$ & 321TSLP & 8.0 & 0.200000 \\
\hline $4,386.0$ & $4,387.0$ & $321 \mathrm{TSLP}$ & 9.6 & 0.600000 \\
\hline 4,38 & $4,388.0$ & $321 \mathrm{TSLP}$ & 4.2 & 0.000000 \\
\hline
\end{tabular}

Anhydrite

Sandstone and anhydrite

Sandstone

Sandstone

Sandstone

Sandstone

Sands tone

Sandstone

Sandstone

Sandstone

Sandstone

Sandstone

Sandstone

Sandstone

Sandstone

Fine-grained sandstone

Fine-grained sandstone

Anhydritic, fine-grained sandstone

Anhydritic, fine-grained sandstone

Anhydritic, fine-grained sandstone

Anhydritic, fine-grained sandstone

Anhydritic, fine-grained sandstone

Fine-grained sandstone

Fine-grained sandstone

Fine-grained sandstone 
Table 4.--Laboratory-determined porosity and permeability--Continued

\begin{tabular}{|c|c|c|c|c|c|c|}
\hline Site & $\begin{array}{l}\text { Depth to } \\
\text { top } \\
\text { (feet) }\end{array}$ & $\begin{array}{l}\text { Depth to } \\
\text { bottom } \\
\text { (feet) }\end{array}$ & $\begin{array}{c}\text { Forma- } \\
\text { tion }\end{array}$ & $\begin{array}{l}\text { Porosity } \\
\text { (percent) }\end{array}$ & $\begin{array}{l}\text { Perme- } \\
\text { ability } \\
\text { (milli- } \\
\text { darcies) }\end{array}$ & Lithology \\
\hline $\mathrm{sb} 26-87-30 \mathrm{ddc}$ & $4,388.0$ & $4,389.0$ & 321TSLP & 8.6 & 0.000000 & Fine-grained sandstone \\
\hline sb26-87-30ddc & $4,389.0$ & $4,390.0$ & $321 \mathrm{TSLP}$ & 7.2 & 0.000000 & Fine-grained sandstone \\
\hline sb26-87-30ddc & $4,390.0$ & $4,391.0$ & $321 \mathrm{TSLP}$ & 7.1 & 0.000000 & Fine-grained sandstone \\
\hline sb26-87-30ddc & $4,391.0$ & $4,392.0$ & $321 \mathrm{TSLP}$ & 8.7 & 0.000000 & Fine-grained sandstone \\
\hline $\mathrm{sb} 26-87-30 \mathrm{ddc}$ & $4,392.0$ & $4,393.0$ & $321 \mathrm{TSLP}$ & 8.8 & 0.000000 & Fine-grained sandstone \\
\hline sb26-87-30ddc & $4,393.0$ & $4,394.0$ & 321TSLP & 5.8 & 0.000000 & Fine-grained sandstone \\
\hline $\mathrm{sb} 26-87-30 \mathrm{ddc}$ & $4,394.0$ & $4,395.0$ & $321 T S L P$ & 4.0 & 0.000000 & Fine-grained sandstone \\
\hline sb $26-87-30 d d c$ & $4,395.0$ & $4,396.0$ & $321 \mathrm{TSLP}$ & 3.7 & 0.000000 & Fine-grained sandstone \\
\hline $\mathrm{sb} 26-87-30 \mathrm{ddc}$ & $4,396.0$ & $4,397.0$ & $321 \mathrm{TSLP}$ & 4.0 & 0.000000 & Fine-grained sandstone \\
\hline sb26-87-30ddc & $4,397.0$ & $4,398.0$ & $321 \mathrm{TSLP}$ & 4.0 & 0.000000 & Fine-grained sandstone \\
\hline $\mathrm{sb} 26-87-30 \mathrm{ddc}$ & $4,398.0$ & $4,399.0$ & $321 \mathrm{TSLP}$ & 3.5 & 0.000000 & Fine-grained sandstone \\
\hline sb26-87-30ddc & $4,399.0$ & $4,400.0$ & $321 \mathrm{TSLP}$ & 5.5 & 0.000000 & Fine-grained sandstone \\
\hline sb26-87-30ddc & $4,400.0$ & $4,401.0$ & $321 \mathrm{TSLP}$ & 6.1 & 0.000000 & Fine-grained sandstone \\
\hline sb26-87-30ddc & $4,401.0$ & $4,402.0$ & $321 \mathrm{TSLP}$ & 3.7 & 0.000000 & Fine-grained sandstone \\
\hline sb26-87-30ddc & $4,402.0$ & $4,403.0$ & $321 \mathrm{TSLP}$ & 3.1 & 0.000000 & Fine-grained sandstone \\
\hline $\mathrm{sb} 26-87-30 \mathrm{ddc}$ & $4,403.0$ & $4,404.0$ & $321 \mathrm{TSLP}$ & 2.7 & 0.000000 & Friable, fine- to medium-grained sandstone \\
\hline $\mathrm{sb} 26-87-30 \mathrm{ddc}$ & $4,404.0$ & $4,405.0$ & $321 \mathrm{TSLP}$ & 6.0 & 0.000000 & Friable, fine- to medium-grained sandstone \\
\hline $\mathrm{sb} 26-87-30 \mathrm{ddc}$ & $4,405.0$ & $4,406.0$ & $321 \mathrm{TSLP}$ & 5.3 & 0.000000 & Friable, fine- to medium-grained sandstone \\
\hline $\mathrm{sb} 26-87-30 \mathrm{ddc}$ & $4,406.0$ & $4,407.0$ & $321 \mathrm{TSLP}$ & 4.0 & 0.000000 & Friable, fine- to medium-grained sandstone \\
\hline sb26-87-30ddc & $4,407.0$ & $4,408.0$ & $321 \mathrm{TSLP}$ & 6.0 & 0.000000 & Fine-grained sandstone \\
\hline $\mathrm{sb} 26-87-30 \mathrm{ddc}$ & $4,408.0$ & $4,409.0$ & 321TSLP & 7.8 & 0.000000 & Fine-grained sandstone \\
\hline $\mathrm{sb} 26-87-30 \mathrm{ddc}$ & $4,409.0$ & $4,410.0$ & $321 \mathrm{TSLP}$ & 9.5 & 0.300000 & Fine-grained sandstone \\
\hline $\mathrm{sb} 26-87-30 \mathrm{ddc}$ & $4,410.0$ & $4,411.0$ & $321 \mathrm{TSLP}$ & 8.9 & 1.500000 & Fine-grained sandstone \\
\hline sb26-87-30ddc & $4,411.0$ & $4,412.0$ & $321 \mathrm{TSLP}$ & 6.0 & 1.400000 & Fine-grained sandstone \\
\hline $\mathrm{sb} 26-87-30 \mathrm{ddc}$ & $4,412.0$ & $4,413.0$ & $321 \mathrm{TSLP}$ & 6.4 & 0.100000 & Fine-grained sandstone \\
\hline $\mathrm{sb} 26-87-30 \mathrm{ddc}$ & $4,413.0$ & $4,414.0$ & 321TSLP & 6.7 & 0.000000 & Sandstone \\
\hline sb26-87-30ddc & $4,414.0$ & $4,415.0$ & $321 \mathrm{TSLP}$ & 6.9 & 1.100000 & Sandstone \\
\hline $\mathrm{sb} 26-87-30 \mathrm{ddc}$ & $4,416.0$ & $4,417.0$ & $321 \mathrm{TSLP}$ & 10.7 & 3.000000 & Fine-grained sandstone \\
\hline sb26-87-30ddc & $4,417.0$ & $4,418.0$ & $321 \mathrm{TSLP}$ & 10.4 & 3.300000 & Fine-grained sandstone \\
\hline sb26-87-30ddc & $4,418.0$ & $4,419.0$ & $321 \mathrm{TSLP}$ & 10.4 & 2.500000 & Fine-grained sandstone \\
\hline $\mathrm{sb} 26-87-30 \mathrm{ddc}$ & $4,419.0$ & $4,420.0$ & $321 \mathrm{TSLP}$ & 8.9 & 0.900000 & Fine-grained sandstone \\
\hline sb26-87-30ddc & $4,420.0$ & $4,421.0$ & $321 \mathrm{TSLP}$ & 10.1 & 1.500000 & Fine-grained sandstone \\
\hline sb26-87-30ddc & $4,421.0$ & $4,422.0$ & $321 \mathrm{TSLP}$ & 9.3 & 1.100000 & Fine-grained sandstone \\
\hline sb26-87-30ddc & $4,422.0$ & $4,423.0$ & $321 \mathrm{TSLP}$ & 10.0 & 1.400000 & Fine-grained sandstone \\
\hline sb26-87-30ddc & $4,423.0$ & $4,424.0$ & 321TSLP & 7.0 & 0.000000 & Fine-grained sandstone \\
\hline $\mathrm{sb} 26-87-30 \mathrm{ddc}$ & $4,424.0$ & $4,425.0$ & 321TSLP & 5.9 & 0.000000 & Fine-grained sandstone \\
\hline sb26-87-30ddc & $4,425.0$ & $4,426.0$ & $321 \mathrm{TSLP}$ & 7.1 & 0.000000 & Fine-grained sandstone \\
\hline$s b 26-87-30 d d c$ & $4,426.0$ & $4,427.0$ & $321 \mathrm{TSLP}$ & 10.4 & 2.500000 & Fine-grained sandstone \\
\hline sb26-87-30ddc & $4,427.0$ & $4,428.0$ & $321 \mathrm{TSLP}$ & 8.4 & 1.200000 & Fine-grained sandstone \\
\hline $\mathrm{sb} 26-87-30 \mathrm{ddc}$ & $4,428.0$ & $4,429.0$ & $321 \mathrm{TSLP}$ & 9.0 & 4.100000 & Shaly, fine-grained sandstone \\
\hline $\mathrm{sb} 26-87-30 \mathrm{ddc}$ & $4,429.0$ & $4,430.0$ & 321TSLP & 8.2 & 3.000000 & Shaly, fine-grained sandstone \\
\hline $\mathrm{sb} 26-87-30 \mathrm{ddc}$ & $4,430.0$ & $4,431.0$ & 321TSLP & 9.8 & 6.400000 & Shaly, fine-grained sandstone \\
\hline $\mathrm{sb} 26-87-30 \mathrm{ddc}$ & $4,431.0$ & $4,432.0$ & $321 \mathrm{TSLP}$ & 9.8 & 6.100000 & Shaly, fine- to medium-grained sandstone \\
\hline sb26-87-30ddc & $4,432.0$ & $4,433.0$ & $321 T S L P$ & 7.8 & 1.800000 & Shaly, fine- to medium-grained sandstone \\
\hline $\mathrm{sb} 26-87-30 \mathrm{ddc}$ & $4,433.0$ & $4,434.0$ & 321TSLP & 6.0 & 0.000000 & Shaly, fine- to medium-grained sandstone \\
\hline sb26-87-30ddc & $4,434.0$ & $4,435.0$ & 321TSLP & 5.1 & 0.000000 & Shaly, fine-grained sandstone \\
\hline $\mathrm{sb} 26-87-30 \mathrm{ddc}$ & $4,435.0$ & $4,436.0$ & $321 \mathrm{TSLP}$ & 6.0 & 0.000000 & Shaly, fine-grained sandstone \\
\hline $\mathrm{sb} 26-87-30 \mathrm{ddc}$ & $4,436.0$ & $4,437.0$ & $321 \mathrm{TSLP}$ & 5.9 & 0.000000 & Fine-grained sandstone \\
\hline $\mathrm{sb} 26-87-30 \mathrm{ddc}$ & $4,437.0$ & $4,438.0$ & 321TSLP & 5.9 & 0.000000 & Fine-grained sandstone \\
\hline sb26-87-30ddc & $4,438.0$ & $4,439.0$ & $321 \mathrm{TSLP}$ & 7.0 & 0.000000 & Fine-grained sandstone \\
\hline
\end{tabular}


Table 4.--Laboratory-determined porosity and permeability--Continued

\begin{tabular}{|c|c|c|c|c|c|c|}
\hline Site & $\begin{array}{l}\text { Depth to } \\
\text { top } \\
\text { (feet) }\end{array}$ & $\begin{array}{l}\text { Depth to } \\
\text { bottom } \\
\text { (feet) }\end{array}$ & $\begin{array}{c}\text { Forma- } \\
\text { tion }\end{array}$ & $\begin{array}{l}\text { Porosity } \\
\text { (percent) }\end{array}$ & $\begin{array}{l}\text { Perme- } \\
\text { ability } \\
\text { (milli- } \\
\text { darcies) }\end{array}$ & Lithology \\
\hline $\begin{array}{l}\text { sb26-87-30ddc } \\
\text { sb26-87-30ddc } \\
\text { sb26-87-30ddc } \\
\text { sb26-87-30ddc } \\
\text { sb26-87-30ddc }\end{array}$ & $\begin{array}{l}4,439.0 \\
4,440.0 \\
4,441.0 \\
4,442.0 \\
4,443.0\end{array}$ & $\begin{array}{l}4,440.0 \\
4,441.0 \\
4,442.0 \\
4,443.0 \\
4,444.0\end{array}$ & $\begin{array}{l}321 \mathrm{TSLP} \\
321 \mathrm{TSLP} \\
321 \mathrm{TSLP} \\
321 \mathrm{TSLP} \\
321 \mathrm{TSLP}\end{array}$ & $\begin{array}{l}7.2 \\
7.6 \\
7.5 \\
7.1 \\
8.9\end{array}$ & $\begin{array}{l}0.000000 \\
0.000000 \\
0.000000 \\
0.000000 \\
0.000000\end{array}$ & $\begin{array}{l}\text { Fine-grained sandstone } \\
\text { Fine-grained sandstone } \\
\text { Fine-grained sandstone } \\
\text { Fine-grained sandstone } \\
\text { Fine-grained sandstone }\end{array}$ \\
\hline $\begin{array}{l}\text { sb26-87-30ddc } \\
\text { sb26-87-30ddc } \\
\text { sb26-87-30ddc } \\
\text { sb26-87-30ddc } \\
\text { sb26-87-30ddc }\end{array}$ & $\begin{array}{l}4,444.0 \\
4,445.0 \\
4,446.0 \\
4,447.0 \\
4,448.0\end{array}$ & $\begin{array}{l}4,445.0 \\
4,446.0 \\
4,447.0 \\
4,448.0 \\
4,449.0\end{array}$ & $\begin{array}{l}321 \text { TSLP } \\
321 \text { TSLP } \\
321 \text { TSLP } \\
321 \text { TSLP } \\
\text { 321TSLP }\end{array}$ & $\begin{array}{r}7.3 \\
8.3 \\
10.5 \\
11.1 \\
11.1\end{array}$ & $\begin{array}{l}0.000000 \\
0.000000 \\
1.300000 \\
2.900000 \\
3.500000\end{array}$ & $\begin{array}{l}\text { Fine-grained sandstone } \\
\text { Fractured, fine-grained sandstone } \\
\text { Friable, fine-grained sandstone } \\
\text { Friable, fine-grained sandstone } \\
\text { Friable, fine-grained sandstone }\end{array}$ \\
\hline $\begin{array}{l}\text { sb26-87-30ddc } \\
\text { sb26-87-30ddc } \\
\text { sb26-87-30ddc } \\
\text { sb26-87-30ddc } \\
\text { sb26-87-30ddc }\end{array}$ & $\begin{array}{l}4,449.0 \\
4,450.0 \\
4,451.0 \\
4,452.0 \\
4,453.0\end{array}$ & $\begin{array}{l}4,450.0 \\
4,451.0 \\
4,452.0 \\
4,453.0 \\
4,454.0\end{array}$ & $\begin{array}{l}321 \text { TSLP } \\
321 \text { TSLP } \\
321 \text { TSLP } \\
321 \text { TSLP } \\
\text { 321TSLP }\end{array}$ & $\begin{array}{r}12.1 \\
14.0 \\
14.6 \\
13.7 \\
6.7\end{array}$ & $\begin{array}{r}12.000000 \\
30.000000 \\
17.000000 \\
28.000000 \\
0.000000\end{array}$ & $\begin{array}{l}\text { Friable, fine-grained sandstone } \\
\text { Friable, fine-grained sandstone } \\
\text { Friable, fine-grained sandstone } \\
\text { Friable, fine-grained sandstone } \\
\text { Friable, fine-grained sandstone }\end{array}$ \\
\hline $\begin{array}{l}\text { sb26-87-30ddc } \\
\text { sb26-87-30ddc } \\
\text { sb26-87-30ddc } \\
\text { sb26-87-30ddc } \\
\text { sb26-87-30ddc }\end{array}$ & $\begin{array}{l}4,454.0 \\
4,455.0 \\
4,456.0 \\
4,457.0 \\
4,458.0\end{array}$ & $\begin{array}{l}4,455.0 \\
4,456.0 \\
4,457.0 \\
4,458.0 \\
4,459.0\end{array}$ & $\begin{array}{l}321 \text { TSLP } \\
321 \text { TSLP } \\
321 \text { TSLP } \\
321 \text { TSLP } \\
321 \text { TSLP }\end{array}$ & $\begin{array}{r}11.9 \\
7.9 \\
9.2 \\
9.5 \\
10.2\end{array}$ & $\begin{array}{r}11.000000 \\
1.000000 \\
0.700000 \\
0.900000 \\
6.200000\end{array}$ & $\begin{array}{l}\text { Friable, fine-grained sandstone } \\
\text { Friable, fine-grained sandstone } \\
\text { Friable, fine-grained sandstone } \\
\text { Friable, fine-grained sandstone } \\
\text { Fine- to medium-grained sandstone }\end{array}$ \\
\hline $\begin{array}{l}\text { sb26-87-30ddc } \\
\text { sb26-87-30ddc } \\
\text { sb26-87-30ddc } \\
\text { sb26-87-30ddc } \\
\text { sb26-87-30ddc }\end{array}$ & $\begin{array}{l}4,459.0 \\
4,460.0 \\
4,461.0 \\
4,462.0 \\
4,463.0\end{array}$ & $\begin{array}{l}4,460.0 \\
4,461.0 \\
4,462.0 \\
4,463.0 \\
4,464.0\end{array}$ & $\begin{array}{l}321 \text { TSLP } \\
321 \text { TSLP } \\
321 \text { TSLP } \\
321 \text { TSLP } \\
\text { 321TSLP }\end{array}$ & $\begin{array}{r}9.9 \\
11.8 \\
10.5 \\
12.4 \\
12.1\end{array}$ & $\begin{array}{r}2.500000 \\
9.100000 \\
4.900000 \\
14.000000 \\
10.000000\end{array}$ & $\begin{array}{l}\text { Fine- to medium-grained sandstone } \\
\text { Sandstone } \\
\text { Sandstone } \\
\text { Fine-grained sandstone } \\
\text { Fine-grained sandstone }\end{array}$ \\
\hline $\begin{array}{l}\text { sb26-87-30ddc } \\
\text { sb26-87-30ddc } \\
\text { sb26-87-30ddc } \\
\text { sb26-87-30ddc } \\
\text { sb26-87-30ddc }\end{array}$ & $\begin{array}{l}4,477.0 \\
4,478.0 \\
4,479.0 \\
4,480.0 \\
4,481.0\end{array}$ & $\begin{array}{l}4,478.0 \\
4,479.0 \\
4,480.0 \\
4,481.0 \\
4,482.0\end{array}$ & $\begin{array}{l}321 \text { TSLP } \\
321 \text { TSLP } \\
321 \text { TSLP } \\
321 \text { TSLP } \\
\text { 321TSLP }\end{array}$ & $\begin{array}{l}4.1 \\
6.0 \\
5.8 \\
4.3 \\
6.8\end{array}$ & $\begin{array}{l}0.000000 \\
0.000000 \\
0.000000 \\
0.000000 \\
0.400000\end{array}$ & $\begin{array}{l}\text { Fine-grained sandstone } \\
\text { Fine-grained sandstone } \\
\text { Fine-grained sandstone } \\
\text { Fine-grained sandstone } \\
\text { Fine-grained sandstone }\end{array}$ \\
\hline $\begin{array}{l}\text { sb26-87-30ddc } \\
\text { sb26-87-30ddc } \\
\text { sb26-87-30ddc } \\
\text { sb26-87-30ddc } \\
\text { sb26-87-30ddc }\end{array}$ & $\begin{array}{l}4,482.0 \\
4,483.0 \\
4,484.0 \\
4,485.0 \\
4,486.0\end{array}$ & $\begin{array}{l}4,483.0 \\
4,484.0 \\
4,485.0 \\
4,486.0 \\
4,487.0\end{array}$ & $\begin{array}{l}321 T S L P \\
321 T S L P \\
321 T S L P \\
321 T S L P \\
321 T S L P\end{array}$ & $\begin{array}{l}7.1 \\
8.1 \\
7.0 \\
8.5 \\
9.2\end{array}$ & $\begin{array}{l}0.400000 \\
1.200000 \\
0.000000 \\
2.400000 \\
4.100000\end{array}$ & $\begin{array}{l}\text { Shaly, fine-grained sandstone } \\
\text { Fine-grained sandstone } \\
\text { Fine-grained sandstone } \\
\text { Fine-grained sandstone } \\
\text { Friable, fine-grained sandstone }\end{array}$ \\
\hline $\begin{array}{l}\text { sb26-87-30ddc } \\
\text { sb26-87-30ddc } \\
\text { sb26-87-30ddc } \\
\text { sb26-87-30ddc } \\
\text { sb26-87-30ddc }\end{array}$ & $\begin{array}{l}4,487.0 \\
4,488.0 \\
4,489.0 \\
4,490.0 \\
4,491.0\end{array}$ & $\begin{array}{l}4,488.0 \\
4,489.0 \\
4,490.0 \\
4,491.0 \\
4,492.0\end{array}$ & $\begin{array}{l}321 \text { TSLP } \\
321 \text { TSLP } \\
321 \text { TSLP } \\
\text { 321TSLP } \\
\text { 321TSLP }\end{array}$ & $\begin{array}{r}11.0 \\
10.7 \\
8.0 \\
8.7 \\
10.1\end{array}$ & $\begin{array}{r}14.000000 \\
25.000000 \\
1.600000 \\
2.700000 \\
12.000000\end{array}$ & $\begin{array}{l}\text { Friable, fine-grained sandstone } \\
\text { Fine-grained sandstone } \\
\text { Fine-grained sandstone } \\
\text { Fine-grained sandstone } \\
\text { Fine-grained sandstone }\end{array}$ \\
\hline $\begin{array}{l}\text { sb26-87-30ddc } \\
\text { sb26-87-30ddc } \\
\text { sb26-87-30ddc } \\
\text { sb26-87-30ddc } \\
\text { sb26-87-30ddc }\end{array}$ & $\begin{array}{l}4,498 \cdot 0 \\
4,499.0 \\
4,500 \cdot 0 \\
4,501.0 \\
4,502.0\end{array}$ & $\begin{array}{l}4,499.0 \\
4,500.0 \\
4,501.0 \\
4,502.0 \\
4,503.0\end{array}$ & $\begin{array}{l}321 \text { TSLP } \\
321 \text { TSLP } \\
321 \text { TSLP } \\
321 \text { TSLP } \\
\text { 321TSLP }\end{array}$ & $\begin{array}{l}8.3 \\
7.7 \\
7.4 \\
6.0 \\
8.7\end{array}$ & $\begin{array}{l}2.900000 \\
1.300000 \\
0.900000 \\
0.000000 \\
5.200000\end{array}$ & $\begin{array}{l}\text { Fine-grained sandstone } \\
\text { Fine-grained sandstone } \\
\text { Fine-grained sandstone } \\
\text { Fine-grained sandstone } \\
\text { Fine-grained sandstone }\end{array}$ \\
\hline $\begin{array}{l}\text { sb26-87-30ddc } \\
\text { sb26-87-30ddc } \\
\text { sb26-87-30ddc } \\
\text { sb26-87-30ddc } \\
\text { sb26-87-30ddc }\end{array}$ & $\begin{array}{l}4,503 \cdot 0 \\
4,504.0 \\
4,505.0 \\
4,506.0 \\
4,507.0\end{array}$ & $\begin{array}{l}4,504.0 \\
4,505.0 \\
4,506.0 \\
4,507.0 \\
4,508.0\end{array}$ & $\begin{array}{l}321 \mathrm{TSLP} \\
321 \mathrm{TSLP} \\
321 \mathrm{TSLP} \\
321 \mathrm{TSLP} \\
\text { 321TSLP }\end{array}$ & $\begin{array}{r}8.9 \\
5.7 \\
7.4 \\
3.8 \\
10.5\end{array}$ & $\begin{array}{l}3.600000 \\
0.000000 \\
1.700000 \\
0.000000 \\
4.400000\end{array}$ & $\begin{array}{l}\text { Fine-grained sandstone } \\
\text { Fine-grained sandstone } \\
\text { Fine-grained sandstone } \\
\text { Fine-grained sandstone } \\
\text { Fine-grained sandstone }\end{array}$ \\
\hline
\end{tabular}


Table 4.--Laboratory-determined porosity and permeability--Continued

\begin{tabular}{|c|c|c|c|c|c|c|}
\hline Site & $\begin{array}{l}\text { Depth to } \\
\text { top } \\
\text { (feet) }\end{array}$ & $\begin{array}{l}\text { Depth to } \\
\text { bottom } \\
\text { (feet) }\end{array}$ & $\begin{array}{c}\text { Forma- } \\
\text { tion }\end{array}$ & $\begin{array}{l}\text { Porosity } \\
\text { (percent) }\end{array}$ & $\begin{array}{l}\text { Perme- } \\
\text { ability } \\
\text { (milli- } \\
\text { darcies) }\end{array}$ & Lithology \\
\hline $\begin{array}{l}\text { sb26-87-30ddc } \\
\text { sb26-87-30ddc } \\
\text { sb26-87-30ddc } \\
\text { sb26-87-30ddc } \\
\text { sb26-87-30ddc }\end{array}$ & $\begin{array}{l}4,508 \cdot 0 \\
4,509.0 \\
4,510.0 \\
4,518.0 \\
4,519.0\end{array}$ & $\begin{array}{l}4,509.0 \\
4,510.0 \\
4,511.0 \\
4,519.0 \\
4,520.0\end{array}$ & $\begin{array}{l}\text { 321TSLP } \\
321 T S L P \\
\text { 321TSLP } \\
\text { 321TSLP } \\
\text { 321TSLP }\end{array}$ & $\begin{array}{l}8.6 \\
7.5 \\
5.5 \\
6.8 \\
5.9\end{array}$ & $\begin{array}{l}0.700000 \\
0.700000 \\
0.000000 \\
0.000000 \\
0.000000\end{array}$ & $\begin{array}{l}\text { Fine-grained sandstone } \\
\text { Fine-grained sandstone } \\
\text { Fine-grained sandstone } \\
\text { Fine-grained sandstone } \\
\text { Fine-grained sandstone }\end{array}$ \\
\hline $\begin{array}{l}\text { sb26-87-30ddc } \\
\text { sb26-87-30ddc } \\
\text { sb26-87-30ddc } \\
\text { sb26-87-30ddc } \\
\text { sb26-87-30ddc }\end{array}$ & $\begin{array}{l}4,520.0 \\
4,521.0 \\
4,522.0 \\
4,523.0 \\
4,524.0\end{array}$ & $\begin{array}{l}4,521.0 \\
4,522.0 \\
4,523.0 \\
4,524.0 \\
4,525.0\end{array}$ & $\begin{array}{l}\text { 321TSLP } \\
321 \mathrm{TSLP} \\
\text { 321TSLP } \\
\text { 321TSLP } \\
\text { 321TSLP }\end{array}$ & $\begin{array}{l}6.1 \\
6.0 \\
5.8 \\
6.3 \\
4.5\end{array}$ & $\begin{array}{l}0.000000 \\
0.000000 \\
0.000000 \\
0.000000 \\
0.000000\end{array}$ & $\begin{array}{l}\text { Fine-grained sandstone } \\
\text { Fine-grained sandstone } \\
\text { Fine-grained sandstone } \\
\text { Fine-grained sandstone } \\
\text { Fine-grained sandstone }\end{array}$ \\
\hline $\begin{array}{l}\text { sb26-87-30ddc } \\
\text { sb26-87-30ddc } \\
\text { sb26-87-30ddc } \\
\text { sb26-87-30ddc } \\
\text { sb26-87-30ddc }\end{array}$ & $\begin{array}{l}4,525 \cdot 0 \\
4,526.0 \\
4,527.0 \\
4,528.0 \\
4,529.0\end{array}$ & $\begin{array}{l}4,526.0 \\
4,527.0 \\
4,528.0 \\
4,529.0 \\
4,530.0\end{array}$ & $\begin{array}{l}\text { 321TSLP } \\
\text { 321TSLP } \\
\text { 321TSLP } \\
\text { 321TSLP } \\
\text { 321TSLP }\end{array}$ & $\begin{array}{l}5.2 \\
3.2 \\
5.8 \\
3.4 \\
1.4\end{array}$ & $\begin{array}{l}0.000000 \\
0.000000 \\
0.000000 \\
0.000000 \\
0.000000\end{array}$ & $\begin{array}{l}\text { Fine-grained sandstone } \\
\text { Fine-grained sandstone } \\
\text { Fine-grained sandstone } \\
\text { Fine-grained sandstone } \\
\text { Fine-grained sandstone }\end{array}$ \\
\hline $\begin{array}{l}\text { sb26-87-30ddc } \\
\text { sb26-87-30ddc } \\
\text { sb26-87-30ddc } \\
\text { sb26-87-30ddc } \\
\text { sb26-87-30ddc }\end{array}$ & $\begin{array}{l}4,530.0 \\
4,531.0 \\
4,532.0 \\
4,533.0 \\
4,534.0\end{array}$ & $\begin{array}{l}4,531.0 \\
4,532.0 \\
4,533.0 \\
4,534.0 \\
4,535.0\end{array}$ & $\begin{array}{l}\text { 321TSLP } \\
\text { 321TSLP } \\
\text { 321TSLP } \\
\text { 321TSLP } \\
\text { 321TSLP }\end{array}$ & $\begin{array}{l}3.9 \\
3.5 \\
4.4 \\
3.7 \\
4.8\end{array}$ & $\begin{array}{l}0.000000 \\
0.000000 \\
0.000000 \\
0.000000 \\
0.000000\end{array}$ & $\begin{array}{l}\text { Fine-grained sandstone } \\
\text { Fine-grained sandstone } \\
\text { Fine-grained sandstone } \\
\text { Fine-grained sandstone } \\
\text { Fine-grained sandstone }\end{array}$ \\
\hline $\begin{array}{l}\text { sb26-87-30ddc } \\
\text { sb26-87-30ddc } \\
\text { sb26-87-30ddc } \\
\text { sb26-87-30ddc } \\
\text { sb26-87-30ddc }\end{array}$ & $\begin{array}{l}4,535.0 \\
4,536.0 \\
4,537.0 \\
4,538 \cdot 0 \\
4,539.0\end{array}$ & $\begin{array}{l}4,536.0 \\
4,537.0 \\
4,538.0 \\
4,539.0 \\
4,540.0\end{array}$ & $\begin{array}{l}321 \mathrm{TSLP} \\
\text { 321TSLP } \\
\text { 321TSLP } \\
\text { 321TSLP } \\
\text { 321TSLP }\end{array}$ & $\begin{array}{l}4.1 \\
4.1 \\
6.2 \\
4.9 \\
6.2\end{array}$ & $\begin{array}{l}0.000000 \\
0.000000 \\
0.000000 \\
0.000000 \\
0.000000\end{array}$ & $\begin{array}{l}\text { Shaly, fine-grained sandstone } \\
\text { Shaly, fine-grained sandstone } \\
\text { Shaly, fine-grained sandstone } \\
\text { Shaly, fine-grained sandstone } \\
\text { Shaly, fine-grained sandstone }\end{array}$ \\
\hline $\begin{array}{l}\text { sb26-87-30ddc } \\
\text { sb26-87-30ddc } \\
\text { sb26-87-30ddc } \\
\text { sb26-87-30ddc } \\
\text { sb26-87-30ddc }\end{array}$ & $\begin{array}{l}4,540.0 \\
4,541.0 \\
4,542.0 \\
4,543.0 \\
4,544.0\end{array}$ & $\begin{array}{l}4,541.0 \\
4,542.0 \\
4,543.0 \\
4,544.0 \\
4,545.0\end{array}$ & $\begin{array}{l}\text { 321TSLP } \\
\text { 321TSLP } \\
\text { 321TSLP } \\
\text { 321TSLP } \\
\text { 321TSLP }\end{array}$ & $\begin{array}{l}5.2 \\
4.4 \\
5.2 \\
6.3 \\
3.7\end{array}$ & $\begin{array}{l}0.000000 \\
0.000000 \\
0.000000 \\
0.000000 \\
0.000000\end{array}$ & $\begin{array}{l}\text { Shaly, fine-grained sandstone } \\
\text { Fine-grained sandstone } \\
\text { Fine-grained sandstone } \\
\text { Shaly, fine-grained sandstone } \\
\text { Shaly, fine-grained sandstone }\end{array}$ \\
\hline $\mathrm{sb} 26-87-30 \mathrm{ddc}$ & $4,546.0$ & $4,547.0$ & $321 \mathrm{TSLP}$ & 4.0 & 0.000000 & Shaly, fine-grained sandstone \\
\hline SITE AVERAGE-- & - & & AVERAGE-- & $\frac{7.2}{7.2}$ & $\frac{2.052564}{2.052564}$ & \\
\hline $\begin{array}{l}\text { sb26-87-32bbb } \\
\text { sb26-87-32bbb } \\
\text { sb26-87-32bbb } \\
\text { sb26-87-32bbb } \\
\text { sb26-87-32bbb }\end{array}$ & $\begin{array}{l}4,432.0 \\
4,433.0 \\
4,434.0 \\
4,435.0 \\
4,436.0\end{array}$ & $\begin{array}{l}4,433.0 \\
4,434.0 \\
4,435.0 \\
4,436.0 \\
4,437.0\end{array}$ & $\begin{array}{l}\text { 321TSLP } \\
\text { 321TSLP } \\
\text { 321TSLP } \\
\text { 321TSLP } \\
\text { 321TSLP }\end{array}$ & $\begin{array}{l}2.8 \\
7.3 \\
4.9 \\
4.9 \\
6.0\end{array}$ & $\begin{array}{l}0.000000 \\
1.700000 \\
0.000000 \\
0.000000 \\
0.000000\end{array}$ & $\begin{array}{l}\text { Friable, fine-grained sandstone } \\
\text { Friable, fine-grained sandstone } \\
\text { Friable, fine-grained sandstone } \\
\text { Fine-grained sandstone } \\
\text { Fine-grained sandstone }\end{array}$ \\
\hline $\begin{array}{l}\mathrm{sb} 26-87-32 \mathrm{bbb} \\
\mathrm{sb} 26-87-32 \mathrm{bbb} \\
\mathrm{sb} 26-87-32 \mathrm{bbb} \\
\mathrm{sb} 26-87-32 \mathrm{bbb} \\
\mathrm{sb} 26-87-32 \mathrm{bbb}\end{array}$ & $\begin{array}{l}4,437.0 \\
4,438.0 \\
4,439.0 \\
4,440.0 \\
4,441.0\end{array}$ & $\begin{array}{l}4,438.0 \\
4,439.0 \\
4,440.0 \\
4,441.0 \\
4,442.0\end{array}$ & $\begin{array}{l}\text { 321TSLP } \\
\text { 321TSLP } \\
\text { 321TSLP } \\
\text { 321TSLP } \\
\text { 321TSLP }\end{array}$ & $\begin{array}{l}4.3 \\
4.5 \\
9.2 \\
8.1 \\
9.4\end{array}$ & $\begin{array}{l}0.000000 \\
0.000000 \\
1.300000 \\
3.300000 \\
1.100000\end{array}$ & $\begin{array}{l}\text { Fine-grained sandstone } \\
\text { Fine-grained sandstone } \\
\text { Fine-grained sandstone } \\
\text { Friable, fine-grained sandstone } \\
\text { Friable, fine-grained sandstone }\end{array}$ \\
\hline $\begin{array}{l}\text { sb26-87-32bbb } \\
\text { sb26-87-32bbb } \\
\text { sb26-87-32bbb } \\
\text { sb26-87-32bbb } \\
\text { sb26-87-32bbb }\end{array}$ & $\begin{array}{l}4,442.0 \\
4,443.0 \\
4,444.0 \\
4,464.0 \\
4,465.0\end{array}$ & $\begin{array}{l}4,443.0 \\
4,444.0 \\
4,445.0 \\
4,465.0 \\
4,466.0\end{array}$ & $\begin{array}{l}\text { 321TSLP } \\
\text { 321TSLP } \\
\text { 321TSLP } \\
\text { 321TSLP } \\
\text { 321TSLP }\end{array}$ & $\begin{array}{r}10.3 \\
10.5 \\
8.9 \\
9.4 \\
4.4\end{array}$ & $\begin{array}{l}4.000000 \\
3.100000 \\
2.400000 \\
1.100000 \\
0.000000\end{array}$ & $\begin{array}{l}\text { Friable, fine-grained sandstone } \\
\text { Friable, fine-grained sandstone } \\
\text { Friable, fine-grained sandstone } \\
\text { Friable, fine-grained sandstone } \\
\text { Fine-grained, quartzitic sandstone }\end{array}$ \\
\hline
\end{tabular}


Table 4.--Laboratory-determined porosity and permeability--Continued

\begin{tabular}{|c|c|c|c|c|c|c|}
\hline Site & $\begin{array}{l}\text { Depth to } \\
\text { top } \\
\text { (feet) }\end{array}$ & $\begin{array}{l}\text { Depth to } \\
\text { bottom } \\
\text { (feet) }\end{array}$ & $\begin{array}{c}\text { Forma- } \\
\text { tion }\end{array}$ & $\begin{array}{l}\text { Porosity } \\
\text { (percent) }\end{array}$ & $\begin{array}{l}\text { Perme- } \\
\text { ability } \\
\text { (milli- } \\
\text { darcies) }\end{array}$ & Lithology \\
\hline $\begin{array}{l}\text { sb26-87-32bbb } \\
\text { sb26-87-32bbb } \\
\text { sb26-87-32bbb } \\
\text { sb26-87-32bbb } \\
\text { sb26-87-32bbb }\end{array}$ & $\begin{array}{l}4,466.0 \\
4,467.0 \\
4,468.0 \\
4,469.0 \\
4,470.0\end{array}$ & $\begin{array}{l}4,467.0 \\
4,468.0 \\
4,469.0 \\
4,470.0 \\
4,471.0\end{array}$ & $\begin{array}{l}321 \mathrm{TSLP} \\
\text { 321TSLP } \\
321 \mathrm{TSLP} \\
\text { 321TSLP } \\
\text { 321TSLP }\end{array}$ & $\begin{array}{r}6.9 \\
9.4 \\
11.4 \\
6.3 \\
10.1\end{array}$ & $\begin{array}{l}0.000000 \\
0.800000 \\
7.400000 \\
0.000000 \\
0.300000\end{array}$ & $\begin{array}{l}\text { Friable, fine-grained sandstone } \\
\text { Friable, fine-grained sandstone } \\
\text { Friable, fine-grained sandstone } \\
\text { Friable- to quartzitic, fine-grained sandstone } \\
\text { Fine-grained, quartzitic sandstone }\end{array}$ \\
\hline $\begin{array}{l}\text { sb26-87-32bbb } \\
\text { sb26-87-32bbb } \\
\text { sb26-87-32bbb } \\
\text { sb26-87-32bbb } \\
\text { sb26-87-32bbb }\end{array}$ & $\begin{array}{l}4,471.0 \\
4,472.0 \\
4,473.0 \\
4,474.0 \\
4,475.0\end{array}$ & $\begin{array}{l}4,472.0 \\
4,473.0 \\
4,474.0 \\
4,475.0 \\
4,476.0\end{array}$ & $\begin{array}{l}321 \mathrm{TSLP} \\
321 \mathrm{TSLP} \\
321 \mathrm{TSLP} \\
321 \mathrm{TSLP} \\
\text { 321TSLP }\end{array}$ & $\begin{array}{l}6.0 \\
5.8 \\
3.6 \\
8.3 \\
8.3\end{array}$ & $\begin{array}{l}0.000000 \\
0.000000 \\
0.000000 \\
1.100000 \\
0.400000\end{array}$ & $\begin{array}{l}\text { Fine-grained, quartzitic sandstone } \\
\text { Fine-grained, quartzitic sandstone } \\
\text { Fine-grained, quartzitic sandstone } \\
\text { Fine-grained, quartzitic sandstone } \\
\text { Fine-grained, quartzitic sandstone }\end{array}$ \\
\hline $\begin{array}{l}\text { sb26-87-32bbb } \\
\text { sb26-87-32bbb } \\
\text { sb26-87-32bbb } \\
\text { sb26-87-32bbb } \\
\text { sb26-87-32bbb }\end{array}$ & $\begin{array}{l}4,476.0 \\
4,477.0 \\
4,478.0 \\
4,479.0 \\
4,480.0\end{array}$ & $\begin{array}{l}4,477.0 \\
4,478.0 \\
4,479.0 \\
4,480.0 \\
4,481.0\end{array}$ & $\begin{array}{l}321 \mathrm{TSLP} \\
321 \mathrm{TSLP} \\
321 \mathrm{TSLP} \\
321 \mathrm{TSLP} \\
321 \mathrm{TSLP}\end{array}$ & $\begin{array}{r}8.6 \\
12.1 \\
7.8 \\
11.1 \\
10.2\end{array}$ & $\begin{array}{r}0.800000 \\
5.200000 \\
0.200000 \\
12.000000 \\
3.500000\end{array}$ & $\begin{array}{l}\text { Fine-grained, quartzitic sandstone } \\
\text { Fine-grained, quartzitic sandstone } \\
\text { Fine-grained, quartzitic sandstone } \\
\text { Friable, fine-grained sandstone } \\
\text { Friable- to quartzitic, fine-grained sandstone }\end{array}$ \\
\hline $\begin{array}{l}\text { sb26-87-32bbb } \\
\text { sb26-87-32bbb } \\
\text { sb26-87-32bbb } \\
\text { sb26-87-32bbb } \\
\text { sb26-87-32bbb }\end{array}$ & $\begin{array}{l}4,481.0 \\
4,482.0 \\
4,483.0 \\
4,484.0 \\
4,485.0\end{array}$ & $\begin{array}{l}4,482.0 \\
4,483.0 \\
4,484.0 \\
4,485.0 \\
4,486.0\end{array}$ & $\begin{array}{l}\text { 321TSLP } \\
\text { 321TSLP } \\
\text { 321TSLP } \\
\text { 321TSLP } \\
\text { 321TSLP }\end{array}$ & $\begin{array}{r}9.6 \\
9.2 \\
7.6 \\
10.5 \\
10.1\end{array}$ & $\begin{array}{l}3.000000 \\
2.300000 \\
1.000000 \\
6.300000 \\
4.300000\end{array}$ & $\begin{array}{l}\text { Friable- to quartzitic, fine-grained sandstone } \\
\text { Fine-grained, quartzitic sandstone } \\
\text { Friable- to quartzitic, fine-grained sandstone } \\
\text { Fine-grained sandstone } \\
\text { Fine-grained sandstone }\end{array}$ \\
\hline $\begin{array}{l}\text { sb26-87-32bbb } \\
\text { sb26-87-32bbb } \\
\text { sb26-87-32bbb } \\
\text { sb26-87-32bbb } \\
\text { sb26-87-32bbb }\end{array}$ & $\begin{array}{l}4,486.0 \\
4,487.0 \\
4,488.0 \\
4,489.0 \\
4,490.0\end{array}$ & $\begin{array}{l}4,487.0 \\
4,488.0 \\
4,489.0 \\
4,490.0 \\
4,491.0\end{array}$ & $\begin{array}{l}321 \mathrm{TSLP} \\
\text { 321TSLP } \\
\text { 321TSLP } \\
\text { 321TSLP } \\
\text { 321TSLP }\end{array}$ & $\begin{array}{r}7.6 \\
10.5 \\
9.4 \\
9.9 \\
9.4\end{array}$ & $\begin{array}{l}0.000000 \\
5.600000 \\
2.700000 \\
5.000000 \\
1.800000\end{array}$ & $\begin{array}{l}\text { Fine-grained sandstone } \\
\text { Fine-grained sandstone } \\
\text { Fine-grained sandstone } \\
\text { Shaly, fine-grained sandstone } \\
\text { Fine-grained sandstone }\end{array}$ \\
\hline $\begin{array}{l}\text { sb26-87-32bbb } \\
\text { sb26-87-32bbb } \\
\text { sb26-87-32bbb } \\
\text { sb26-87-32bbb } \\
\text { sb26-87-32bbb }\end{array}$ & $\begin{array}{l}4,491.0 \\
4,492.0 \\
4,493.0 \\
4,494.0 \\
4,495.0\end{array}$ & $\begin{array}{l}4,492.0 \\
4,493.0 \\
4,494.0 \\
4,495.0 \\
4,496.0\end{array}$ & $\begin{array}{l}321 \text { TSLP } \\
\text { 321TSLP } \\
\text { 321TSLP } \\
\text { 321TSLP } \\
\text { 321TSLP }\end{array}$ & $\begin{array}{r}11.4 \\
4.2 \\
5.5 \\
6.4 \\
8.5\end{array}$ & $\begin{array}{r}10.000000 \\
0.000000 \\
0.000000 \\
0.000000 \\
0.600000\end{array}$ & $\begin{array}{l}\text { Shaly, fine- to medium-grained sandstone } \\
\text { Fine-grained, quartzitic sandstone } \\
\text { Fine-grained, quartzitic sandstone } \\
\text { Fine-grained, quartzitic sandstone } \\
\text { Fine-grained, quartzitic sandstone }\end{array}$ \\
\hline $\begin{array}{l}\text { sb26-87-32bbb } \\
\text { sb26-87-32bbb } \\
\text { sb26-87-32bbb } \\
\text { sb26-87-32bbb } \\
\text { sb26-87-32bbb }\end{array}$ & $\begin{array}{l}4,496.0 \\
4,497.0 \\
4,498.0 \\
4,499.0 \\
4,500.0\end{array}$ & $\begin{array}{l}4,497.0 \\
4,498.0 \\
4,499.0 \\
4,500.0 \\
4,501.0\end{array}$ & $\begin{array}{l}321 \mathrm{TSLP} \\
\text { 321TSLP } \\
\text { 321TSLP } \\
\text { 321TSLP } \\
\text { 321TSLP }\end{array}$ & $\begin{array}{l}10.7 \\
11.7 \\
11.8 \\
11.2 \\
10.1\end{array}$ & $\begin{array}{r}5.400000 \\
14.000000 \\
14.000000 \\
8.200000 \\
3.000000\end{array}$ & $\begin{array}{l}\text { Fine-grained, quartzitic sandstone } \\
\text { Fine-grained sandstone } \\
\text { Fine-grained sandstone } \\
\text { Fine-grained sandstone } \\
\text { Fine-grained sandstone }\end{array}$ \\
\hline $\begin{array}{l}\text { sb26-87-32bbb } \\
\text { sb26-87-32bbb } \\
\text { sb26-87-32bbb } \\
\text { sb26-87-32bbb } \\
\text { sb26-87-32bbb }\end{array}$ & $\begin{array}{l}4,501 \cdot 0 \\
4,502.0 \\
4,503.0 \\
4,504.0 \\
4,505.0\end{array}$ & $\begin{array}{l}4,502.0 \\
4,503.0 \\
4,504.0 \\
4,505.0 \\
4,506.0\end{array}$ & $\begin{array}{l}\text { 321TSLP } \\
\text { 321TSLP } \\
\text { 321TSLP } \\
\text { 321TSLP } \\
\text { 321TSLP }\end{array}$ & $\begin{array}{l}9.5 \\
9.2 \\
8.5 \\
8.3 \\
8.6\end{array}$ & $\begin{array}{l}1.900000 \\
1.900000 \\
1.700000 \\
0.600000 \\
1.100000\end{array}$ & $\begin{array}{l}\text { Friable, fine-grained sandstone } \\
\text { Friable, fine-grained sandstone } \\
\text { Fine-grained, quartzitic sandstone } \\
\text { Fine-grained, quartzitic sandstone } \\
\text { Fine-grained, quartzitic sandstone }\end{array}$ \\
\hline $\begin{array}{l}\text { sb26-87-32bbb } \\
\text { sb26-87-32bbb } \\
\text { sb26-87-32bbb } \\
\text { sb26-87-32bbb } \\
\text { sb26-87-32bbb }\end{array}$ & $\begin{array}{l}4,506 \cdot 0 \\
4,507.0 \\
4,508.0 \\
4,514.0 \\
4,520.0\end{array}$ & $\begin{array}{l}4,507 \cdot 0 \\
4,508 \cdot 0 \\
4,509 \cdot 0 \\
4,515 \cdot 0 \\
4,521 \cdot 0\end{array}$ & $\begin{array}{l}321 \mathrm{TSLP} \\
\text { 321TSLP } \\
\text { 321TSLP } \\
\text { 321TSLP } \\
\text { 321TSLP }\end{array}$ & $\begin{array}{r}9.4 \\
8.6 \\
8.2 \\
10.9 \\
10.9\end{array}$ & $\begin{array}{l}1.400000 \\
1.000000 \\
0.000000 \\
4.300000 \\
4.200000\end{array}$ & $\begin{array}{l}\text { Fine-grained, quartzitic sandstone } \\
\text { Fine-grained, quartzitic sandstone } \\
\text { Fine-grained sandstone } \\
\text { Friable, fine-grained sandstone } \\
\text { Fine-grained sandstone }\end{array}$ \\
\hline $\begin{array}{l}\text { sb26-87-32bbb } \\
\text { sb26-87-32bbb } \\
\text { sb26-87-32bbb } \\
\text { sb26-87-32bbb } \\
\text { sb26-87-32bbb }\end{array}$ & $\begin{array}{l}4,525 \cdot 0 \\
4,530.0 \\
4,535.0 \\
4,540.0 \\
4,545.0\end{array}$ & $\begin{array}{l}4,526.0 \\
4,531.0 \\
4,536.0 \\
4,541.0 \\
4,546.0\end{array}$ & $\begin{array}{l}\text { 321TSLP } \\
\text { 321TSLP } \\
\text { 321TSLP } \\
\text { 321TSLP } \\
\text { 321TSLP }\end{array}$ & $\begin{array}{r}9.2 \\
11.2 \\
10.6 \\
9.6 \\
7.3\end{array}$ & $\begin{array}{l}2.200000 \\
7.800000 \\
5.900000 \\
3.300000 \\
0.300000\end{array}$ & $\begin{array}{l}\text { Fine-grained sandstone } \\
\text { Fine-grained sandstone } \\
\text { Fine-grained sandstone } \\
\text { Fine-grained sandstone } \\
\text { Fine-grained sandstone }\end{array}$ \\
\hline
\end{tabular}


Table 4.--Laboratory-determined porosity and permeability--Continued

\begin{tabular}{|c|c|c|c|c|c|c|}
\hline Site & $\begin{array}{l}\text { Depth to } \\
\text { top } \\
\text { (feet) }\end{array}$ & $\begin{array}{l}\text { Depth to } \\
\text { bottom } \\
\text { (feet) }\end{array}$ & $\begin{array}{c}\text { Forma- } \\
\text { tion }\end{array}$ & $\begin{array}{l}\text { Porosity } \\
\text { (percent) }\end{array}$ & $\begin{array}{l}\text { Perme- } \\
\text { ability } \\
\text { (milli- } \\
\text { darcies) }\end{array}$ & Lithology \\
\hline $\mathrm{sb} 26-87-32 \mathrm{bbb}$ & $4,550.0$ & $4,551.0$ & 321TSLP & 5.3 & 1.400000 & Fine-grained sandstone \\
\hline $\mathrm{sb} 26-87-32 \mathrm{bbb}$ & $4,555.0$ & $4,556.0$ & $321 \mathrm{TSLP}$ & 8.5 & 1.000000 & Fine- to medium-grained sandstone \\
\hline $\mathrm{sb} 26-87-32 \mathrm{bbb}$ & $4,560.0$ & $4,561.0$ & 321TSLP & 6.0 & 0.300000 & Friable, fine-grained sandstone \\
\hline $\mathrm{sb} 26-87-32 \mathrm{bbb}$ & $4,563.0$ & $4,564.0$ & $321 \mathrm{TSLP}$ & 6.2 & 2.100000 & Fine-grained sandstone \\
\hline $\mathrm{sb} 26-87-32 \mathrm{bbb}$ & $4,565.0$ & $4,566.0$ & 321TSLP & 2.1 & 0.100000 & Fine- to medium-grained, quartzitic sandstone \\
\hline & & & AVERAGE-- & 8.3 & 2.562857 & \\
\hline SITE AVERAGE-- & $-\cdots-n$ & $-n$ & $-\cdots-n-n-n$ & 8.3 & 2.562857 & \\
\hline $\mathrm{sb} 26-89-17 \mathrm{dba}$ & $7,317.0$ & $7,318.0$ & 321TSLP & 0.5 & 0.010000 & Limestone and sandstone \\
\hline $\mathrm{sb} 26-89-17 \mathrm{dba}$ & $7,320.0$ & $7,321.0$ & $321 \mathrm{TSLP}$ & 0.4 & 0.010000 & Limestone and sandstone \\
\hline sb26-89-17dba & $7,321.0$ & $7,322.0$ & $321 \mathrm{TSLP}$ & 0.3 & 0.010000 & Limestone and sandstone \\
\hline $\mathrm{sb} 26-89-17 \mathrm{dba}$ & $7,328.0$ & $7,329.0$ & $321 \mathrm{TSLP}$ & 0.3 & 0.000000 & Limestone and sandstone \\
\hline $\mathrm{sb} 26-89-17 \mathrm{dba}$ & $7,329.0$ & $7,330.0$ & 321TSLP & 1.1 & 0.000000 & Sandstone \\
\hline $\mathrm{sb} 26-89-17 \mathrm{dba}$ & $7,330.0$ & $7,331.0$ & 321TSLP & 1.1 & 0.000000 & Sandstone \\
\hline $\mathrm{sb} 26-89-17 \mathrm{dba}$ & $7,331.0$ & $7,332.0$ & $321 \mathrm{TSLP}$ & 1.0 & 0.000000 & Sandstone \\
\hline $\mathrm{sb} 26-89-17 \mathrm{dba}$ & $7,332.0$ & $7,333.0$ & 321TSLP & 1.0 & 0.000000 & Sandstone \\
\hline $\mathrm{sb} 26-89-17 \mathrm{dba}$ & $7,333.0$ & $7,334.0$ & $321 \mathrm{TSLP}$ & 1.1 & 0.000000 & Sands tone \\
\hline $\mathrm{sb} 26-89-17 \mathrm{dba}$ & $7,336.0$ & $7,337.0$ & 321TSLP & 1.1 & 0.000000 & Sands tone \\
\hline $\mathrm{sb} 26-89-17 \mathrm{dba}$ & $7,337.0$ & $7,338.0$ & $321 \mathrm{TSLP}$ & 1.0 & 0.000000 & Sandstone \\
\hline $\mathrm{sb} 26-89-17 \mathrm{dba}$ & $7,339.0$ & $7,340.0$ & $321 \mathrm{TSLP}$ & 4.0 & 0.000000 & Sandstone \\
\hline $\mathrm{sb} 26-89-17 \mathrm{dba}$ & $7,340.0$ & $7,341.0$ & $321 \mathrm{TSLP}$ & 1.2 & 0.000000 & Sandstone \\
\hline $\mathrm{sb} 26-89-17 \mathrm{dba}$ & $7,341.0$ & $7,342.0$ & $321 \mathrm{TSLP}$ & 1.0 & 0.000000 & Sands tone \\
\hline sb26-89-17dba & $7,342.0$ & $7,343.0$ & $321 \mathrm{TSLP}$ & 1.0 & 0.000000 & Sands tone \\
\hline $\mathrm{sb} 26-89-17 \mathrm{dba}$ & $7,343.0$ & $7,344.0$ & 321TSLP & 1.9 & 0.020000 & Sandstone \\
\hline$s b 26-89-17 d b a$ & $7,344.0$ & $7,345.0$ & 321TSLP & 2.1 & 0.010000 & Sandstone \\
\hline $\mathrm{sb} 26-89-17 \mathrm{dba}$ & $7,345.0$ & $7,346.0$ & $321 \mathrm{TSLP}$ & 2.4 & 0.010000 & Sands tone \\
\hline $\mathrm{sb} 26-89-17 \mathrm{dba}$ & $7,346.0$ & $7,347.0$ & 321TSLP & 2.1 & 0.000000 & Sandstone \\
\hline $\mathrm{sb} 26-89-17 \mathrm{dba}$ & $7,348.0$ & $7,349.0$ & 321TSLP & 2.5 & 0.000000 & Sands tone \\
\hline sb26-89-17dba & $7,349.0$ & $7,350.0$ & 321TSLP & 2.4 & 0.000000 & Sandstone \\
\hline $\mathrm{sb} 26-89-17 \mathrm{dba}$ & $7,350.0$ & $7,351.0$ & $321 \mathrm{TSLP}$ & 2.2 & 0.000000 & Sandstone \\
\hline sb26-89-17dba & $7,351.0$ & $7,352.0$ & 321TSLP & 1.7 & 0.000000 & Sandstone \\
\hline & & & AVERAGE - - & 1.5 & 0.003043 & \\
\hline SITE AVERAGE- & 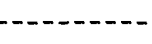 & $=-------$ & 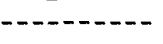 & 1.5 & 0.003043 & \\
\hline sb26-90-01cda & $6,338.0$ & $6,339.0$ & 321TSLP & 10.9 & 9.400000 & Fine-grained sandstone \\
\hline sb26-90-01cda & $6,339.0$ & $6,340.0$ & $321 T S L P$ & 10.4 & 8.200000 & Fine-grained sandstone \\
\hline sb26-90-01cda & $6,340.0$ & $6,341.0$ & 321TSLP & 8.9 & 0.100000 & Fine-grained sandstone \\
\hline sb26-90-01cda & $6,341.0$ & $6,342.0$ & 321TSLP & 7.2 & 1.500000 & Fine-grained sandstone \\
\hline sb26-90-01cda & $6,342.0$ & $6,343.0$ & 321TSLP & 8.3 & 0.900000 & Fine-grained sandstone \\
\hline sb26-90-01cda & $6,343.0$ & $6,344.0$ & $321 \mathrm{TSLP}$ & 5.7 & 0.450000 & Silty, fine-grained sandstone \\
\hline sb26-90-01cda & $6,344.0$ & $6,345.0$ & 321TSLP & 3.0 & 0.020000 & Fine-grained sandstone \\
\hline sb26-90-01cda & $6,345.0$ & $6,346.0$ & $321 \mathrm{TSLP}$ & 1.8 & 0.040000 & Fine-grained sandstone with siltstone laminae \\
\hline sb26-90-01cda & $6,346.0$ & $6,347.0$ & 321 TSLP & 3.3 & 0.050000 & Fine-grained, cemented sandstone \\
\hline $\mathrm{sb} 26-90-01 \mathrm{cda}$ & $6,347.0$ & $6,348.0$ & 321TSLP & 3.4 & 0.080000 & Fine-grained sandstone \\
\hline $\mathrm{sb} 26-90-01 \mathrm{cda}$ & $6,348.0$ & $6,349.0$ & 321TSLP & 4.3 & 0.050000 & Fine-grained sandstone \\
\hline $\mathrm{sb} 26-90-01 \mathrm{cda}$ & $6,349.0$ & $6,350.0$ & $321 \mathrm{TSLP}$ & 6.4 & 0.120000 & Fine-grained sandstone \\
\hline $\mathrm{sb} 26-90-01 \mathrm{cda}$ & $6,350.0$ & $6,351.0$ & $321 \mathrm{TSLP}$ & 8.0 & 0.870000 & Fine-grained sandstone \\
\hline sb26-90-01cda & $6,351.0$ & $6,352.0$ & $321 \mathrm{TSLP}$ & 8.4 & 0.160000 & Fine-grained sandstone \\
\hline $\mathrm{sb} 26-90-01 \mathrm{cda}$ & $6,352.0$ & $6,353.0$ & 321TSLP & 8.2 & 0.740000 & Fine-grained sandstone \\
\hline
\end{tabular}


Table 4.--Laboratory-determined porosity and permeability--Continued

\begin{tabular}{|c|c|c|c|c|c|c|}
\hline Site & $\begin{array}{l}\text { Depth to } \\
\text { top } \\
\text { (feet) }\end{array}$ & $\begin{array}{l}\text { Depth to } \\
\text { bottom } \\
\text { (feet) }\end{array}$ & $\begin{array}{c}\text { Forma- } \\
\text { tion }\end{array}$ & $\begin{array}{l}\text { Porosity } \\
\text { (percent) }\end{array}$ & $\begin{array}{c}\text { Perme- } \\
\text { ability } \\
\text { (milli- } \\
\text { darcies) }\end{array}$ & Lithology \\
\hline sb26-90-01cda & $6,353.0$ & $6,354.0$ & $321 \mathrm{TSLP}$ & 9.9 & 1.800000 & Fine-grained sandstone \\
\hline sb26-90-01cda & $6,354.0$ & $6,355.0$ & $321 \mathrm{TSLP}$ & 9.8 & 0.650000 & Fine-grained sandstone \\
\hline sb26-90-01cda & $6,355.0$ & $6,356.0$ & $321 T S L P$ & 8.1 & 1.600000 & Fine-grained, cemented sandstone \\
\hline sb26-90-01cda & $6,356.0$ & $6,357.0$ & $321 \mathrm{TSLP}$ & 10.5 & 26.000000 & Fine-grained sandstone \\
\hline sb26-90-01cda & $6,357.0$ & $6,358.0$ & $321 \mathrm{TSLP}$ & 9.9 & 1.500000 & Friable, fine-grained sandstone \\
\hline sb26-90-01cda & $6,358.0$ & $6,359.0$ & 321TSLP & 4.2 & 2.400000 & Fine-grained, cemented sandstone \\
\hline sb26-90-01cda & $6,359.0$ & $6,360.0$ & $321 \mathrm{TSLP}$ & 1.8 & 0.040000 & Fine-grained sandstone \\
\hline sb26-90-01cda & $6,360.0$ & $6,361.0$ & 321TSLP & 2.3 & 0.060000 & Fine-grained sandstone with siltstone laminae \\
\hline sb26-90-01cda & $6,361.0$ & $6,362.0$ & $321 \mathrm{TSLP}$ & 2.3 & 0.080000 & Fine-grained sandstone \\
\hline sb26-90-01cda & $6,362.0$ & $6,363.0$ & $321 \mathrm{TSLP}$ & 2.9 & 0.050000 & Fine-grained sandstone \\
\hline sb26-90-01cda & $6,363.0$ & $6,364.0$ & 321TSLP & 4.9 & 0.040000 & Fine-grained sandstone \\
\hline sb26-90-01cda & $6,364.0$ & $6,365.0$ & $321 \mathrm{TSLP}$ & 6.8 & 0.900000 & Fine-grained sandstone \\
\hline sb26-90-01cda & $6,365.0$ & $6,366.0$ & $321 \mathrm{TSLP}$ & 6.2 & 0.200000 & Fine-grained sandstone \\
\hline sb26-90-01cda & $6,366.0$ & $6,367.0$ & $321 \mathrm{TSLP}$ & 4.4 & 0.090000 & Fine-grained sandstone \\
\hline sb26-90-01cda & $6,367.0$ & $6,368.0$ & $321 \mathrm{TSLP}$ & 9.4 & 3.600000 & Fine-grained sandstone \\
\hline sb26-90-01cda & $6,368.0$ & $6,369.0$ & $321 \mathrm{TSLP}$ & 5.6 & 0.030000 & Fine-grained sandstone \\
\hline sb26-90-01cda & $6,369.0$ & $6,370.0$ & $321 \mathrm{TSLP}$ & 6.0 & 0.040000 & Dolomite \\
\hline sb26-90-01cda & $6,370.0$ & $6,371.0$ & 321TSLP & 5.3 & 0.030000 & Dolomite \\
\hline sb26-90-01cda & $6,371.0$ & $6,372.0$ & $321 \mathrm{TSLP}$ & 4.3 & 0.040000 & Fine-grained sandstone \\
\hline sb26-90-01cda & $6,372.0$ & $6,373.0$ & 321TSLP & 8.4 & 1.600000 & Fine-grained sandstone \\
\hline sb26-90-01cda & $6,373.0$ & $6,374.0$ & $321 \mathrm{TSLP}$ & 4.7 & 0.250000 & Fine-grained sandstone \\
\hline sb26-90-01cda & $6,374.0$ & $6,375.0$ & 321TSLP & 10.5 & 0.120000 & Fine-grained sandstone \\
\hline sb26-90-01cda & $6,375.0$ & $6,376.0$ & 321TSLP & 14.4 & 13.000000 & Fine-grained sandstone \\
\hline sb26-90-01cda & $6,376.0$ & $6,377.0$ & $321 \mathrm{TSLP}$ & 13.1 & 3.600000 & Fine-grained sandstone \\
\hline sb26-90-01cda & $6,377.0$ & $6,378.0$ & $321 \mathrm{TSLP}$ & 8.0 & 0.050000 & Fine-grained sandstone \\
\hline sb26-90-01cda & $6,378.0$ & $6,379.0$ & $321 \mathrm{TSLP}$ & 10.9 & 0.650000 & Fine-grained sandstone \\
\hline sb26-90-01cda & $6,379.0$ & $6,380.0$ & 321TSLP & 10.3 & 3.700000 & Fine-grained sandstone \\
\hline sb26-90-01cda & $6,380.0$ & $6,381.0$ & $321 \mathrm{TSLP}$ & 4.9 & 0.060000 & Fine-grained sandstone \\
\hline sb26-90-01cda & $6,381.0$ & $6,382.0$ & $321 \mathrm{TSLP}$ & 8.8 & 1.800000 & Fine-grained sandstone \\
\hline sb26-90-01cda & $6,382.0$ & $6,383.0$ & $321 \mathrm{TSLP}$ & 12.0 & 5.100000 & Fine-grained sandstone \\
\hline sb26-90-01cda & $6,383.0$ & $6,384.0$ & $321 \mathrm{TSLP}$ & 17.1 & 36.000000 & Fine-grained sandstone \\
\hline sb26-90-01cda & $6,384.0$ & $6,385.0$ & 321TSLP & 17.4 & 51.000000 & Fine-grained sandstone \\
\hline sb26-90-01cda & $6,385.0$ & $6,386.0$ & $321 \mathrm{TSLP}$ & 17.3 & 50.000000 & Fine-grained sandstone \\
\hline sb26-90-01cda & $6,386.0$ & $6,387.0$ & $321 \mathrm{TSLP}$ & 16.7 & 0.830000 & Fine-grained sandstone \\
\hline sb26-90-01cda & $6,387.0$ & $6,388.0$ & 321TSLP & 12.9 & 0.240000 & Fine-grained sandstone \\
\hline sb26-90-01cda & $6,388.0$ & $6,389.0$ & 321TSLP & 9.4 & 0.100000 & Fine-grained sandstone \\
\hline sb26-90-01cda & $6,389.0$ & $6,390.0$ & $321 \mathrm{TSLP}$ & 15.8 & 8.900000 & Fine-grained sandstone \\
\hline sb26-90-01cda & $6,390.0$ & $6,391.0$ & 321TSLP & 13.6 & 8.400000 & Fine-grained sandstone \\
\hline sb26-90-01cda & $6,391.0$ & $6,392.0$ & $321 \mathrm{TSLP}$ & 22.5 & 327.000000 & Fine-grained sandstone \\
\hline sb26-90-01cda & $6,392.0$ & $6,393.0$ & $321 \mathrm{TSLP}$ & 24.0 & 330.000000 & Fine-grained sandstone \\
\hline sb26-90-01cda & $6,393.0$ & $6,394.0$ & 321TSLP & 21.6 & 305.000000 & Fine-grained sandstone \\
\hline sb26-90-01cda & $6,394.0$ & $6,395.0$ & $321 \mathrm{TSLP}$ & 19.2 & 107.000000 & Fine-grained sandstone \\
\hline sb26-90-01cda & $6,395.0$ & $6,396.0$ & $321 \mathrm{TSLP}$ & 19.7 & 177.000000 & Fine-grained sandstone \\
\hline sb26-90-01cda & $6,396.0$ & $6,397.0$ & $321 T S L P$ & 17.7 & 161.000000 & Fine-grained sandstone \\
\hline sb26-90-01cda & $6,397.0$ & $6,398.0$ & $321 \mathrm{TSLP}$ & 17.3 & 96.000000 & Fine-grained sandstone \\
\hline sb26-90-01cda & $6,398.0$ & $6,399.0$ & $321 \mathrm{TSLP}$ & 17.5 & 67.000000 & Fine-grained sandstone \\
\hline sb26-90-01cda & $6,399.0$ & $6,400.0$ & $321 T S L P$ & 16.7 & 174.000000 & Fine-grained sandstone \\
\hline sb26-90-01cda & $6,400.0$ & $6,401.0$ & 321TSLP & 18.4 & 133.000000 & Fine-grained sandstone \\
\hline sb26-90-01cda & $6,401.0$ & $6,402.0$ & $321 \mathrm{TSLP}$ & 17.8 & 130.000000 & Fine-grained sandstone \\
\hline sb26-90-01cda & $6,402.0$ & $6,403.0$ & $321 \mathrm{TSLP}$ & 17.6 & 116.000000 & Fine-grained sandstone \\
\hline
\end{tabular}


Table 4.--Laboratory-determined porosity and permeability--Continued

\begin{tabular}{|c|c|c|c|c|c|c|c|}
\hline Site & $\begin{array}{l}\text { Depth to } \\
\text { top } \\
\text { (feet) }\end{array}$ & $\begin{array}{l}\text { Depth to } \\
\text { bottom } \\
\text { (feet) }\end{array}$ & $\begin{array}{c}\text { Forma- } \\
\text { tion }\end{array}$ & $\begin{array}{l}\text { Porosity } \\
\text { (percent) }\end{array}$ & $\begin{array}{c}\text { Perme- } \\
\text { ability } \\
\text { (milli- } \\
\text { darcies) }\end{array}$ & & Lithology \\
\hline sb26-90-01cda & $6,403.0$ & $6,404.0$ & $321 \mathrm{TSLP}$ & 21.1 & 249.000000 & Fine-grained & sandstone \\
\hline sb26-90-01cda & $6,404.0$ & $6,405.0$ & 321TSLP & 19.6 & 204.000000 & Fine-grained & sandstone \\
\hline sb26-90-01cda & $6,405.0$ & $6,406.0$ & $321 \mathrm{TSLP}$ & 19.6 & 264.000000 & Fine-grained & sandstone \\
\hline sb26-90-01cda & $6,406.0$ & $6,407.0$ & $321 \mathrm{TSLP}$ & 16.2 & 127.000000 & Fine-grained & sandstone \\
\hline sb26-90-01cda & $6,407.0$ & $6,408.0$ & $321 \mathrm{TSLP}$ & 12.7 & 46.000000 & Fine-grained & sandstone \\
\hline $\mathrm{sb} 26-90-01 \mathrm{cda}$ & $6,408.0$ & $6,409.0$ & 321TSLP & 10.1 & 7.500000 & Fine-grained & sandstone \\
\hline sb26-90-01cda & $6,409.0$ & $6,410.0$ & $321 \mathrm{TSLP}$ & 10.0 & 20.000000 & Fine-grained & sandstone \\
\hline sb26-90-01cda & $6,410.0$ & $6,411.0$ & 321TSLP & 17.2 & 179.000000 & Fine-grained & sandstone \\
\hline sb26-90-01cda & $6,411.0$ & $6,412.0$ & 321TSLP & 17.2 & 156.000000 & Fine-grained & sandstone \\
\hline sb26-90-01cda & $6,412.0$ & $6,413.0$ & 321TSLP & 18.4 & 266.000000 & Fine-grained & sandstone \\
\hline sb26-90-01cda & $6,412.0$ & $6,413.0$ & $321 \mathrm{TSLP}$ & 18.4 & 266.000000 & Fine-grained & sandstone \\
\hline sb26-90-01cda & $6,413.0$ & $6,414.0$ & $321 \mathrm{TSLP}$ & 18.9 & 0.000000 & Fine-grained & sandstone \\
\hline sb26-90-01cda & $6,414.0$ & $6,415.0$ & 321TSLP & 13.1 & 12.000000 & Fine-grained & sandstone \\
\hline sb26-90-01cda & $6,415.0$ & $6,516.0$ & $321 \mathrm{TSLP}$ & 13.0 & 33.000000 & Fine-grained & sandstone \\
\hline sb26-90-01cda & $6,416.0$ & $6,417.0$ & $321 \mathrm{TSLP}$ & 14.9 & 124.000000 & Fine-grained & sandstone \\
\hline sb26-90-01cda & $6,417.0$ & $6,418.0$ & $321 \mathrm{TSLP}$ & 14.5 & 69.000000 & Fine-grained & sandstone \\
\hline sb26-90-01cda & $6,420.0$ & $6,421.0$ & $321 \mathrm{TSLP}$ & 14.4 & 54.000000 & Fine-grained & 1 sandstone \\
\hline sb26-90-01cda & $6,421.0$ & $6,422.0$ & $321 \mathrm{TSLP}$ & 14.1 & 95.000000 & Fine-grained & sandstone \\
\hline sb26-90-01cda & $6,422.0$ & $6,423.0$ & 321TSLP & 14.4 & 57.000000 & Fine-grained & sandstone \\
\hline sb26-90-01cda & $6,423.0$ & $6,424.0$ & $321 \mathrm{TSLP}$ & 16.1 & 111.000000 & Fine-grained & sandstone \\
\hline sb26-90-01cda & $6,424.0$ & $6,425.0$ & $321 \mathrm{TSLP}$ & 14.0 & 77.000000 & Fine-grained & sandstone \\
\hline sb26-90-01cda & $6,425.0$ & $6,426.0$ & $321 \mathrm{TSLP}$ & 14.4 & 89.000000 & Fine-grained & sandstone \\
\hline sb26-90-01cda & $6,426.0$ & $6,427.0$ & 321TSLP & 14.0 & 105.000000 & Fine-grained & sandstone \\
\hline sb26-90-01cda & $6,427.0$ & $6,428.0$ & 321TSIP & 14.8 & 84.000000 & Fine-grained & sandstone \\
\hline sb26-90-01cda & $6,428.0$ & $6,429.0$ & $321 \mathrm{TSLP}$ & 14.0 & 70.000000 & Fine-grained & sandstone \\
\hline sb26-90-01cda & $6,429.0$ & $6,430.0$ & 321TSLP & 14.3 & 43.0 & Fine-grained & san \\
\hline sb26-90-01cda & $6,430.0$ & $6,431.0$ & 321TSLP & 13.3 & 38.000000 & Fine-grained & stone \\
\hline sb26-90-01cda & $6,431.0$ & $6,432.0$ & $321 \mathrm{TSLP}$ & 13.1 & 52.000000 & Fine-grained & sandstone \\
\hline sb26-90-01cda & $6,432.0$ & $6,433.0$ & $321 \mathrm{TSLP}$ & 13.3 & 46.000000 & Fine-grained & sandstone \\
\hline sb26-90-01cda & $6,433.0$ & $6,434.0$ & 321TSLP & 14.4 & 98.000000 & Fine-grained & sandstone \\
\hline $\mathrm{sb} 26-90-01 \mathrm{cda}$ & $6,434.0$ & $6,435.0$ & $321 \mathrm{TSLP}$ & 13.7 & 64.000000 & Fine-grained & sandstone \\
\hline sb26-90-01cda & $6,435.0$ & $6,436.0$ & 321TSLP & 16.0 & 33.000000 & Fine-grained & sandstone \\
\hline sb26-90-01cda & $6,436.0$ & $6,437.0$ & 321TSLP & 13.4 & 154.000000 & Fine-grained & sandstone \\
\hline sb26-90-01cda & $6,437.0$ & $6,438.0$ & 321TSLP & 3.7 & 67.000000 & Fine-grained & s andstone \\
\hline sb26-90-01cda & $6,438.0$ & $6,439.0$ & 321TSLP & 12.9 & 8.500000 & Fine-grained & sandstone \\
\hline $\mathrm{sb} 26-90-01 \mathrm{cda}$ & 6,439 & $6,440.0$ & $321 \mathrm{TSLP}$ & 12.8 & 0000 & Fine-grained & tone \\
\hline sb26-90-01cda & $6,440.0$ & $6,441.0$ & $321 \mathrm{TSLP}$ & 12.2 & 58.000000 & Fine-grained & sands tone \\
\hline sb26-90-01cda & $6,441.0$ & $6,442.0$ & $321 \mathrm{TSLP}$ & 14.3 & 80.000000 & Fine-grained & sandstone \\
\hline sb26-90-01cda & $6,442.0$ & $6,443.0$ & $321 \mathrm{TSLP}$ & 14.9 & 41.000000 & Fine-grained & sandstone \\
\hline sb26-90-01cda & $6,443.0$ & $6,444.0$ & $321 \mathrm{TSLP}$ & 14.2 & 73.000000 & Fine-grained & sands tone \\
\hline sb26-90-01cda & $6,444.0$ & $6,445.0$ & 321TSLP & 17.3 & 54.000000 & Fine-grained & sandstone \\
\hline sb26-90-01cda & $6,444.0$ & $6,445.0$ & 321TSLP & 17.3 & 54.000000 & Fine-grained & sandstone \\
\hline sb26-90-01cda & $6,445.0$ & $6,446.0$ & 321TSLP & 14.4 & 71.000000 & Fine-grained & sandstone \\
\hline sb26-90-01cda & $6,446.0$ & $6,447.0$ & 321TSLP & 5.6 & 65.000000 & Fine-grained & 1 sandstone \\
\hline sb26-90-01cda & $6,447.0$ & $6,448.0$ & 321TSLP & 14.9 & 46.000000 & Fine-grained & sandstone \\
\hline sb26-90-01cda & $6,448.0$ & $6,449.0$ & $321 \mathrm{TSLP}$ & 14.2 & 57.000000 & Fine-grained & sandstone \\
\hline sb26-90-01cda & $6,449.0$ & $6,450.0$ & 321TSLP & 13.5 & 31.000000 & Fine-grained & 1 sandstone \\
\hline sb26-90-01cda & $6,450.0$ & $6,451.0$ & $321 \mathrm{TSLP}$ & 14.7 & 64.000000 & Fine-grained & sandstone \\
\hline sb26-90-01cda & $6,451.0$ & $6,452.0$ & $321 \mathrm{TSLP}$ & 14.2 & 39.000000 & Fine-grained & sandstone \\
\hline sb26-90-01cda & $6,452.0$ & $6,453.0$ & $321 \mathrm{TSLP}$ & 13.7 & 38.000000 & Fine-grained & s andstone \\
\hline
\end{tabular}


Table 4.--Laboratory-determined porosity and permeability--Continued

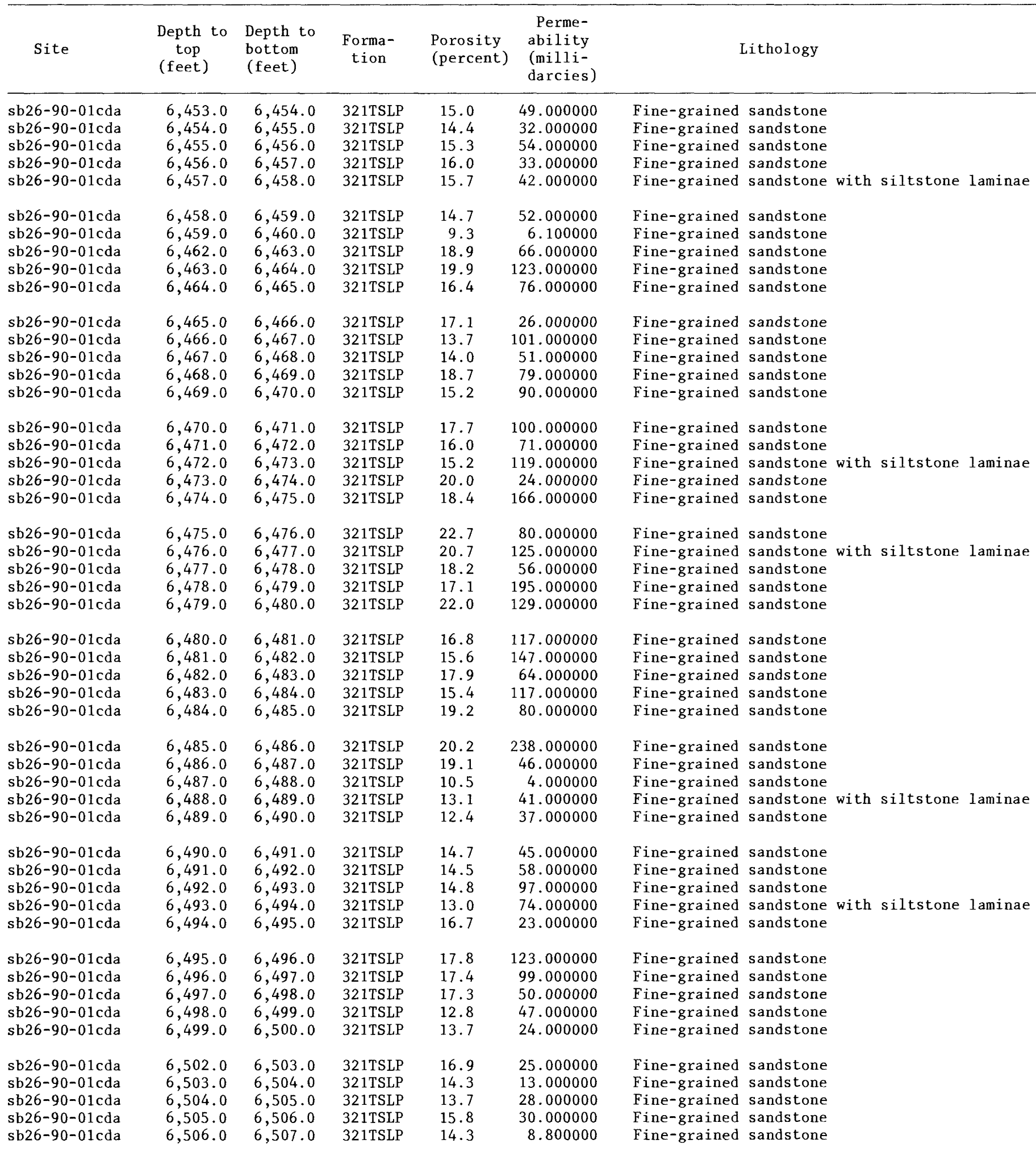


Table 4.--Laboratory-determined porosity and permeability--Continued

\begin{tabular}{|c|c|c|c|c|c|c|}
\hline Site & $\begin{array}{l}\text { Depth to } \\
\text { top } \\
\text { (feet) }\end{array}$ & $\begin{array}{l}\text { Depth to } \\
\text { bottom } \\
\text { (feet) }\end{array}$ & $\begin{array}{c}\text { Forma- } \\
\text { tion }\end{array}$ & $\begin{array}{l}\text { Porosity } \\
\text { (percent) }\end{array}$ & $\begin{array}{c}\text { Perme- } \\
\text { ability } \\
\text { (milli- } \\
\text { darcies) }\end{array}$ & Lithology \\
\hline $\begin{array}{l}\text { sb26-90-01cda } \\
\text { sb26-90-01cda } \\
\text { sb26-90-01cda } \\
\text { sb26-90-01cda } \\
\text { sb26-90-01cda }\end{array}$ & $\begin{array}{l}6,507.0 \\
6,508 \cdot 0 \\
6,509 \cdot 0 \\
6,510 \cdot 0 \\
6,511.0\end{array}$ & $\begin{array}{l}6,508.0 \\
6,509.0 \\
6,510.0 \\
6,511.0 \\
6,512.0\end{array}$ & $\begin{array}{l}321 \mathrm{TSLP} \\
321 \mathrm{TSLP} \\
321 \mathrm{TSLP} \\
321 \mathrm{TSLP} \\
321 \mathrm{TSLP}\end{array}$ & $\begin{array}{l}13.3 \\
14.0 \\
10.0 \\
16.9 \\
16.1\end{array}$ & $\begin{array}{r}11.000000 \\
7.800000 \\
76.000000 \\
155.000000 \\
57.000000\end{array}$ & $\begin{array}{l}\text { Fine-grained sandstone } \\
\text { Fine-grained sandstone } \\
\text { Fine-grained sandstone } \\
\text { Fine-grained sandstone } \\
\text { Fine-grained sandstone }\end{array}$ \\
\hline $\begin{array}{l}\text { sb26-90-01cda } \\
\text { sb26-90-01cda } \\
\text { sb26-90-01cda } \\
\text { sb26-90-01cda } \\
\text { sb26-90-01cda }\end{array}$ & $\begin{array}{l}6,512.0 \\
6,513.0 \\
6,514.0 \\
6,515.0 \\
6,516.0\end{array}$ & $\begin{array}{l}6,513.0 \\
6,514.0 \\
6,515.0 \\
6,516.0 \\
6,517.0\end{array}$ & $\begin{array}{l}321 \mathrm{TSLP} \\
321 \mathrm{TSLP} \\
321 \mathrm{TSLP} \\
321 \mathrm{TSLP} \\
321 \mathrm{TSLP}\end{array}$ & $\begin{array}{r}12.1 \\
20.9 \\
20.1 \\
9.8 \\
8.8\end{array}$ & $\begin{array}{r}13.000000 \\
17.000000 \\
3.100000 \\
0.600000 \\
0.450000\end{array}$ & $\begin{array}{l}\text { Fine-grained sandstone } \\
\text { Fine-grained sandstone } \\
\text { Fine-grained sandstone } \\
\text { Fine-grained sandstone } \\
\text { Fine-grained sandstone }\end{array}$ \\
\hline $\begin{array}{l}\text { sb26-90-01cda } \\
\text { sb26-90-01cda } \\
\text { sb26-90-01cda } \\
\text { sb26-90-01cda } \\
\text { sb26-90-01cda }\end{array}$ & $\begin{array}{l}6,517.0 \\
6,518.0 \\
6,519.0 \\
6,520.0 \\
6,521.0\end{array}$ & $\begin{array}{l}6,518.0 \\
6,519.0 \\
6,520.0 \\
6,521.0 \\
6,522.0\end{array}$ & $\begin{array}{l}321 \mathrm{TSLP} \\
321 \mathrm{TSLP} \\
321 \mathrm{TSLP} \\
321 \mathrm{TSLP} \\
321 \mathrm{TSLP}\end{array}$ & $\begin{array}{r}7.8 \\
10.1 \\
10.5 \\
8.0 \\
10.6\end{array}$ & $\begin{array}{r}1.100000 \\
2.100000 \\
14.000000 \\
1.500000 \\
6.100000\end{array}$ & $\begin{array}{l}\text { Fine-grained sandstone } \\
\text { Fine-grained sandstone } \\
\text { Fine-grained sandstone } \\
\text { Fine-grained sandstone } \\
\text { Fine-grained sandstone }\end{array}$ \\
\hline $\begin{array}{l}\text { sb26-90-01cda } \\
\text { sb26-90-01cda } \\
\text { sb26-90-01cda } \\
\text { sb26-90-01cda } \\
\text { sb26-90-01cda }\end{array}$ & $\begin{array}{l}6,522 \cdot 0 \\
6,523 \cdot 0 \\
6,524.0 \\
6,525 \cdot 0 \\
6,526 \cdot 0\end{array}$ & $\begin{array}{l}6,523.0 \\
6,524.0 \\
6,525.0 \\
6,526.0 \\
6,527.0\end{array}$ & $\begin{array}{l}321 \mathrm{TSLP} \\
321 \mathrm{TSLP} \\
321 \mathrm{TSLP} \\
321 \mathrm{TSLP} \\
321 \mathrm{TSLP}\end{array}$ & $\begin{array}{r}10.5 \\
8.4 \\
10.5 \\
10.1 \\
7.5\end{array}$ & $\begin{array}{l}2.100000 \\
1.800000 \\
2.700000 \\
6.200000 \\
3.100000\end{array}$ & $\begin{array}{l}\text { Fine-grained sandstone } \\
\text { Fine-grained sandstone } \\
\text { Fine-grained sandstone } \\
\text { Fine-grained sandstone } \\
\text { Fine-grained sandstone }\end{array}$ \\
\hline $\begin{array}{l}\text { sb26-90-01cda } \\
\text { sb26-90-01cda } \\
\text { sb26-90-01cda } \\
\text { sb26-90-01cda } \\
\text { sb26-90-01cda }\end{array}$ & $\begin{array}{l}6,527 \cdot 0 \\
6,528 \cdot 0 \\
6,529 \cdot 0 \\
6,530.0 \\
6,531 \cdot 0\end{array}$ & $\begin{array}{l}6,528.0 \\
6,529.0 \\
6,530.0 \\
6,531.0 \\
6,532.0\end{array}$ & $\begin{array}{l}321 \mathrm{TSLP} \\
321 \mathrm{TSLP} \\
321 \mathrm{TSLP} \\
321 \mathrm{TSLP} \\
321 \mathrm{TSLP}\end{array}$ & $\begin{array}{l}11.2 \\
10.1 \\
14.0 \\
13.3 \\
12.2\end{array}$ & $\begin{array}{r}8.700000 \\
8.700000 \\
9.900000 \\
25.000000 \\
51.000000\end{array}$ & $\begin{array}{l}\text { Fine-grained sandstone } \\
\text { Fine-grained sandstone } \\
\text { Fine-grained sandstone } \\
\text { Fine-grained sandstone } \\
\text { Fine-grained sandstone }\end{array}$ \\
\hline $\begin{array}{l}\text { sb26-90-01cda } \\
\text { sb26-90-01cda } \\
\text { sb26-90-01cda } \\
\text { sb26-90-01cda } \\
\text { sb26-90-01cda }\end{array}$ & $\begin{array}{l}6,532.0 \\
6,533 \cdot 0 \\
6,534.0 \\
6,535.0 \\
6,536.0\end{array}$ & $\begin{array}{l}6,533.0 \\
6,534.0 \\
6,535.0 \\
6,536.0 \\
6,537.0\end{array}$ & $\begin{array}{l}321 \text { TSLP } \\
321 \text { TSLP } \\
321 T S L P \\
321 \text { TSLP } \\
\text { 321TSLP }\end{array}$ & $\begin{array}{r}11.0 \\
12.7 \\
14.2 \\
8.6 \\
6.7\end{array}$ & $\begin{array}{r}23.000000 \\
44.000000 \\
58.000000 \\
6.800000 \\
3.300000\end{array}$ & $\begin{array}{l}\text { Fine-grained sandstone } \\
\text { Fine-grained sandstone } \\
\text { Fine-grained sandstone } \\
\text { Silty, fine-grained sandstone } \\
\text { Fine-grained sandstone }\end{array}$ \\
\hline $\begin{array}{l}\text { sb26-90-01cda } \\
\text { sb26-90-01cda } \\
\text { sb26-90-01cda } \\
\text { sb26-90-01cda } \\
\text { sb26-90-01cda }\end{array}$ & $\begin{array}{l}6,537 \cdot 0 \\
6,538 \cdot 0 \\
6,539 \cdot 0 \\
6,540.0 \\
6,543.0\end{array}$ & $\begin{array}{l}6,538.0 \\
6,539.0 \\
6,540.0 \\
6,541.0 \\
6,544.0\end{array}$ & $\begin{array}{l}321 \mathrm{TSLP} \\
321 \mathrm{TSLP} \\
321 \mathrm{TSLP} \\
321 \mathrm{TSLP} \\
321 \mathrm{TSLP}\end{array}$ & $\begin{array}{r}11.7 \\
10.6 \\
11.3 \\
10.1 \\
0.0\end{array}$ & $\begin{array}{r}27.000000 \\
38.000000 \\
17.000000 \\
7.800000 \\
0.020000\end{array}$ & $\begin{array}{l}\text { Fine-grained sandstone } \\
\text { Fine-grained sandstone } \\
\text { Fine-grained sandstone } \\
\text { Fine-grained sandstone } \\
\text { Fine-grained, cemented sandstone }\end{array}$ \\
\hline $\begin{array}{l}\text { sb26-90-01cda } \\
\text { sb26-90-01cda } \\
\text { sb26-90-01cda } \\
\text { sb26-90-01cda } \\
\text { sb26-90-01cda }\end{array}$ & $\begin{array}{l}6,544.0 \\
6,545.0 \\
6,546.0 \\
6,547.0 \\
6,548.0\end{array}$ & $\begin{array}{l}6,545.0 \\
6,546.0 \\
6,547.0 \\
6,548.0 \\
6,549.0\end{array}$ & $\begin{array}{l}321 \mathrm{TSLP} \\
321 \mathrm{TSLP} \\
321 \mathrm{TSLP} \\
321 \mathrm{TSLP} \\
321 \mathrm{TSLP}\end{array}$ & $\begin{array}{l}1.1 \\
2.4 \\
1.4 \\
1.8 \\
2.3\end{array}$ & $\begin{array}{l}0.020000 \\
0.040000 \\
0.040000 \\
0.030000 \\
0.050000\end{array}$ & $\begin{array}{l}\text { Fine-grained sandstone } \\
\text { Fine-grained sandstone } \\
\text { Fine-grained sandstone } \\
\text { Fine-grained sandstone } \\
\text { Fine-grained sandstone }\end{array}$ \\
\hline $\begin{array}{l}\text { sb26-90-01cda } \\
\text { sb26-90-01cda } \\
\text { sb26-90-01cda } \\
\text { sb26-90-01cda } \\
\text { sb26-90-01cda }\end{array}$ & $\begin{array}{l}6,549.0 \\
6,550 \cdot 0 \\
6,551.0 \\
6,552.0 \\
6,553.0\end{array}$ & $\begin{array}{l}6,550.0 \\
6,551.0 \\
6,552.0 \\
6,553.0 \\
6,554.0\end{array}$ & $\begin{array}{l}321 \mathrm{TSLP} \\
321 \mathrm{TSLP} \\
321 \mathrm{TSLP} \\
321 \mathrm{TSLP} \\
321 \mathrm{TSLP}\end{array}$ & $\begin{array}{l}2.6 \\
2.3 \\
2.9 \\
2.4 \\
4.4\end{array}$ & $\begin{array}{l}0.040000 \\
0.050000 \\
0.100000 \\
0.050000 \\
0.170000\end{array}$ & $\begin{array}{l}\text { Fine-grained sandstone } \\
\text { Fine-grained sandstone } \\
\text { Fine-grained sandstone } \\
\text { Fine-grained sandstone } \\
\text { Fine-grained sandstone }\end{array}$ \\
\hline $\begin{array}{l}\text { sb26-90-01cda } \\
\text { sb26-90-01cda } \\
\text { sb26-90-01cda } \\
\text { sb26-90-01cda } \\
\text { sb26-90-01cda }\end{array}$ & $\begin{array}{l}6,554 \cdot 0 \\
6,555 \cdot 0 \\
6,556.0 \\
6,557.0 \\
6,558.0\end{array}$ & $\begin{array}{l}6,555.0 \\
6,556.0 \\
6,557.0 \\
6,558.0 \\
6,559.0\end{array}$ & $\begin{array}{l}321 T S L P \\
321 T S L P \\
321 T S L P \\
321 T S L P \\
321 T S L P\end{array}$ & $\begin{array}{r}4.2 \\
7.9 \\
4.2 \\
8.9 \\
11.2\end{array}$ & $\begin{array}{l}0.080000 \\
3.600000 \\
0.100000 \\
1.800000 \\
0.270000\end{array}$ & $\begin{array}{l}\text { Fine-grained sandstone } \\
\text { Fine-grained sandstone } \\
\text { Fine-grained sandstone } \\
\text { Fine-grained sandstone } \\
\text { Fine-grained sandstone }\end{array}$ \\
\hline
\end{tabular}


Table 4.--Laboratory-determined porosity and permeability--Continued

\begin{tabular}{|c|c|c|c|c|c|c|}
\hline Site & $\begin{array}{l}\text { Depth to } \\
\text { top } \\
\text { (feet) }\end{array}$ & $\begin{array}{l}\text { Depth to } \\
\text { bottom } \\
\text { (feet) }\end{array}$ & $\begin{array}{c}\text { Forma- } \\
\text { tion }\end{array}$ & $\begin{array}{l}\text { Porosity } \\
\text { (percent) }\end{array}$ & $\begin{array}{l}\text { Perme- } \\
\text { ability } \\
\text { (milli- } \\
\text { darcies) }\end{array}$ & Lithology \\
\hline $\begin{array}{l}\text { sb26-90-01cda } \\
\text { sb26-90-01cda } \\
\text { sb26-90-01cda } \\
\text { sb26-90-01cda } \\
\text { sb26-90-01cda }\end{array}$ & $\begin{array}{l}6,559 \cdot 0 \\
6,560.0 \\
6,561.0 \\
6,562.0 \\
6,563.0\end{array}$ & $\begin{array}{l}6,560 \cdot 0 \\
6,561.0 \\
6,562.0 \\
6,563.0 \\
6,564.0\end{array}$ & $\begin{array}{l}321 T S L P \\
321 T S L P \\
321 T S L P \\
321 T S L P \\
321 T S L P\end{array}$ & $\begin{array}{l}9.3 \\
8.3 \\
9.1 \\
9.5 \\
9.7\end{array}$ & $\begin{array}{l}8.100000 \\
1.600000 \\
6.500000 \\
2.000000 \\
9.100000\end{array}$ & $\begin{array}{l}\text { Fine-grained sandstone } \\
\text { Fine-grained sandstone } \\
\text { Fine-grained sandstone } \\
\text { Fine-grained sandstone } \\
\text { Fine-grained sandstone }\end{array}$ \\
\hline $\begin{array}{l}\text { sb26-90-01cda } \\
\text { sb26-90-01cda } \\
\text { sb26-90-01cda } \\
\text { sb26-90-01cda } \\
\text { sb26-90-01cda }\end{array}$ & $\begin{array}{l}6,564.0 \\
6,565.0 \\
6,566.0 \\
6,567.0 \\
6,568.0\end{array}$ & $\begin{array}{l}6,565 \cdot 0 \\
6,566.0 \\
6,567.0 \\
6,568.0 \\
6,569.0\end{array}$ & $\begin{array}{l}321 T S L P \\
321 T S L P \\
321 T S L P \\
321 T S L P \\
321 T S L P\end{array}$ & $\begin{array}{r}11.1 \\
10.6 \\
9.3 \\
10.0 \\
11.6\end{array}$ & $\begin{array}{l}0.870000 \\
2.900000 \\
5.500000 \\
3.600000 \\
3.600000\end{array}$ & $\begin{array}{l}\text { Fine-grained sandstone } \\
\text { Silty, fine-grained sandstone } \\
\text { Fine-grained sandstone } \\
\text { Fine-grained sandstone } \\
\text { Fine-grained sandstone }\end{array}$ \\
\hline $\begin{array}{l}\text { sb26-90-01cda } \\
\text { sb26-90-01cda } \\
\text { sb26-90-01cda } \\
\text { sb26-90-01cda } \\
\text { sb26-90-01cda }\end{array}$ & $\begin{array}{l}6,569.0 \\
6,570.0 \\
6,571.0 \\
6,572.0 \\
6,573.0\end{array}$ & $\begin{array}{l}6,570.0 \\
6,571.0 \\
6,572.0 \\
6,573.0 \\
6,574.0\end{array}$ & $\begin{array}{l}321 \mathrm{TSLP} \\
321 \mathrm{TSLP} \\
321 \mathrm{TSLP} \\
321 \mathrm{TSLP} \\
321 \mathrm{TSLP}\end{array}$ & $\begin{array}{r}11.9 \\
9.5 \\
4.9 \\
6.8 \\
7.8\end{array}$ & $\begin{array}{l}1.700000 \\
5.600000 \\
0.280000 \\
0.230000 \\
0.570000\end{array}$ & $\begin{array}{l}\text { Fine-grained sandstone } \\
\text { Fine-grained sandstone } \\
\text { Fine-grained sandstone } \\
\text { Fine-grained sandstone } \\
\text { Fine-grained sandstone }\end{array}$ \\
\hline $\begin{array}{l}\text { sb26-90-01cda } \\
\text { sb26-90-01cda } \\
\text { sb26-90-01cda } \\
\text { sb26-90-01cda } \\
\text { sb26-90-01cda }\end{array}$ & $\begin{array}{l}6,574.0 \\
6,575.0 \\
6,576.0 \\
6,577.0 \\
6,578.0\end{array}$ & $\begin{array}{l}6,575.0 \\
6,576.0 \\
6,577.0 \\
6,578.0 \\
6,579.0\end{array}$ & $\begin{array}{l}321 T S L P \\
321 T S L P \\
321 T S L P \\
321 T S L P \\
321 T S L P\end{array}$ & $\begin{array}{l}4.6 \\
6.8 \\
6.5 \\
6.3 \\
6.3\end{array}$ & $\begin{array}{l}0.160000 \\
0.240000 \\
0.520000 \\
0.760000 \\
0.220000\end{array}$ & $\begin{array}{l}\text { Fine-grained sandstone } \\
\text { Fine-grained sandstone } \\
\text { Fine-grained sandstone } \\
\text { Fine-grained sandstone } \\
\text { Fine-grained sandstone }\end{array}$ \\
\hline $\begin{array}{l}\text { sb26-90-01cda } \\
\text { sb26-90-01cda } \\
\text { sb26-90-01cda } \\
\text { sb26-90-01cda } \\
\text { sb26-90-01cda }\end{array}$ & $\begin{array}{l}6,579.0 \\
6,580.0 \\
6,584.0 \\
6,585.0 \\
6,586.0\end{array}$ & $\begin{array}{l}6,580 \cdot 0 \\
6,581 \cdot 0 \\
6,585 \cdot 0 \\
6,586.0 \\
6,587 \cdot 0\end{array}$ & $\begin{array}{l}321 T S L P \\
321 T S L P \\
321 T S L P \\
321 T S L P \\
321 T S L P\end{array}$ & $\begin{array}{r}9.4 \\
12.3 \\
11.9 \\
11.3 \\
9.7\end{array}$ & $\begin{array}{r}3.600000 \\
27.000000 \\
12.000000 \\
5.300000 \\
4.000000\end{array}$ & $\begin{array}{l}\text { Fine-grained sandstone } \\
\text { Fine-grained sandstone } \\
\text { Fine-grained sandstone } \\
\text { Fine-grained sandstone } \\
\text { Fine-grained sandstone }\end{array}$ \\
\hline $\begin{array}{l}\text { sb26-90-01cda } \\
\text { sb26-90-01cda } \\
\text { sb26-90-01cda } \\
\text { sb26-90-01cda } \\
\text { sb26-90-01cda }\end{array}$ & $\begin{array}{l}6,587.0 \\
6,588.0 \\
6,589.0 \\
6,590.0 \\
6,591.0\end{array}$ & $\begin{array}{l}6,588 \cdot 0 \\
6,589.0 \\
6,590.0 \\
6,591.0 \\
6,592.0\end{array}$ & $\begin{array}{l}321 T S L P \\
321 T S L P \\
321 T S L P \\
321 T S L P \\
\text { 321TSLP }\end{array}$ & $\begin{array}{l}11.8 \\
12.4 \\
10.1 \\
12.0 \\
12.4\end{array}$ & $\begin{array}{r}9.300000 \\
72.000000 \\
21.000000 \\
26.000000 \\
15.000000\end{array}$ & $\begin{array}{l}\text { Fine-grained sandstone } \\
\text { Fine-grained sandstone } \\
\text { Fine-grained sandstone } \\
\text { Fine-grained sandstone } \\
\text { Fine-grained sandstone }\end{array}$ \\
\hline $\begin{array}{l}\text { sb26-90-01cda } \\
\text { sb26-90-01cda } \\
\text { sb26-90-01cda } \\
\text { sb26-90-01cda } \\
\text { sb26-90-01cda }\end{array}$ & $\begin{array}{l}6,592.0 \\
6,593.0 \\
6,594.0 \\
6,595.0 \\
6,596.0\end{array}$ & $\begin{array}{l}6,593.0 \\
6,594.0 \\
6,595.0 \\
6,596.0 \\
6,597.0\end{array}$ & $\begin{array}{l}\text { 321TSLP } \\
\text { 321TSLP } \\
\text { 321TSLP } \\
\text { 321TSLP } \\
\text { 321TSLP }\end{array}$ & $\begin{array}{r}10.4 \\
6.5 \\
10.8 \\
7.6 \\
10.5\end{array}$ & $\begin{array}{r}0.220000 \\
14.000000 \\
85.000000 \\
6.100000 \\
17.000000\end{array}$ & $\begin{array}{l}\text { Silty, fine-grained sandstone } \\
\text { Silty, fine-grained sandstone } \\
\text { Fine-grained sandstone } \\
\text { Fine-grained sandstone } \\
\text { Fine-grained sandstone }\end{array}$ \\
\hline $\begin{array}{l}\text { sb26-90-01cda } \\
\text { sb26-90-01cda } \\
\text { sb26-90-01cda } \\
\text { sb26-90-01cda } \\
\text { sb26-90-01cda }\end{array}$ & $\begin{array}{l}6,597.0 \\
6,598.0 \\
6,599.0 \\
6,600.0 \\
6,601.0\end{array}$ & $\begin{array}{l}6,598 \cdot 0 \\
6,599.0 \\
6,600 \cdot 0 \\
6,601.0 \\
6,602 \cdot 0\end{array}$ & $\begin{array}{l}\text { 321TSLP } \\
\text { 321TSLP } \\
\text { 321TSLP } \\
\text { 321TSLP } \\
\text { 321TSLP }\end{array}$ & $\begin{array}{l}8.9 \\
9.5 \\
9.1 \\
9.6 \\
9.2\end{array}$ & $\begin{array}{r}9.100000 \\
7.000000 \\
12.000000 \\
0.100000 \\
15.000000\end{array}$ & $\begin{array}{l}\text { Fine-grained sandstone } \\
\text { Fine-grained sandstone } \\
\text { Fine-grained sandstone } \\
\text { Fine-grained sandstone } \\
\text { Fine-grained sandstone }\end{array}$ \\
\hline $\begin{array}{l}\text { sb26-90-01cda } \\
\text { sb26-90-01cda } \\
\text { sb26-90-01cda } \\
\text { sb26-90-01cda } \\
\text { sb26-90-01cda }\end{array}$ & $\begin{array}{l}6,602 \cdot 0 \\
6,603 \cdot 0 \\
6,604.0 \\
6,605 \cdot 0 \\
6,606.0\end{array}$ & $\begin{array}{l}6,603 \cdot 0 \\
6,604.0 \\
6,605.0 \\
6,606.0 \\
6,607.0\end{array}$ & $\begin{array}{l}321 T S L P \\
\text { 321TSLP } \\
\text { 321TSLP } \\
\text { 321TSLP } \\
\text { 321TSLP }\end{array}$ & $\begin{array}{r}10.3 \\
10.0 \\
5.9 \\
5.8 \\
2.1\end{array}$ & $\begin{array}{r}12.000000 \\
3.700000 \\
0.200000 \\
0.200000 \\
0.080000\end{array}$ & $\begin{array}{l}\text { Fine-grained sandstone } \\
\text { Fine-grained sandstone } \\
\text { Fine-grained sandstone } \\
\text { Fine-grained sandstone } \\
\text { Fine-grained sandstone with siltstone laminae }\end{array}$ \\
\hline $\begin{array}{l}\text { sb26-90-01cda } \\
\text { sb26-90-01cda } \\
\text { sb26-90-01cda } \\
\text { sb26-90-01cda } \\
\text { sb26-90-01cda }\end{array}$ & $\begin{array}{l}6,607.0 \\
6,608 \cdot 0 \\
6,609.0 \\
6,610.0 \\
6,611.0\end{array}$ & $\begin{array}{l}6,608 \cdot 0 \\
6,609 \cdot 0 \\
6,610.0 \\
6,611.0 \\
6,612.0\end{array}$ & $\begin{array}{l}\text { 321TSLP } \\
\text { 321TSLP } \\
\text { 321TSLP } \\
\text { 321TSLP } \\
\text { 321TSLP }\end{array}$ & $\begin{array}{r}11.2 \\
15.3 \\
13.6 \\
14.8 \\
4.9\end{array}$ & $\begin{array}{r}80.000000 \\
82.000000 \\
31.000000 \\
68.000000 \\
1.600000\end{array}$ & $\begin{array}{l}\text { Fine-grained sandstone } \\
\text { Fine-grained sandstone } \\
\text { Fine-grained sandstone } \\
\text { Fine-grained sandstone } \\
\text { Silty, fine-grained sandstone }\end{array}$ \\
\hline
\end{tabular}


Table 4.--Laboratory-determined porosity and permeability--Continued

\begin{tabular}{|c|c|c|c|c|c|c|}
\hline Site & $\begin{array}{l}\text { Depth to } \\
\text { top } \\
\text { (feet) }\end{array}$ & $\begin{array}{l}\text { Depth to } \\
\text { bottom } \\
\text { (feet) }\end{array}$ & $\begin{array}{c}\text { Forma- } \\
\text { tion }\end{array}$ & $\begin{array}{l}\text { Porosity } \\
\text { (percent) }\end{array}$ & $\begin{array}{l}\text { Perme- } \\
\text { ability } \\
\text { (milli- } \\
\text { darcies) }\end{array}$ & Lithology \\
\hline $\begin{array}{l}\text { sb26-90-01cda } \\
\text { sb26-90-01cda } \\
\text { sb26-90-01cda }\end{array}$ & $\begin{array}{l}6,612.0 \\
6,613.0 \\
6,614.0\end{array}$ & $\begin{array}{l}6,613.0 \\
6,614.0 \\
6,615.0\end{array}$ & $\begin{array}{l}321 T S L P \\
321 T S L P \\
321 T S L P\end{array}$ & $\begin{array}{l}9.1 \\
4.1 \\
4.0 \\
\end{array}$ & $\begin{array}{l}0.600000 \\
0.040000 \\
0.020000 \\
\end{array}$ & $\begin{array}{l}\text { Fine-grained sandstone } \\
\text { Silty, fine-grained sandstone } \\
\text { Fine-grained sandstone }\end{array}$ \\
\hline sb26-90-01cda & & & AVERAGE - - & 11.7 & 43.043470 & \\
\hline $\begin{array}{l}\text { sb26-90-01cda } \\
\text { sb26-90-01cda } \\
\text { sb26-90-01cda } \\
\text { sb26-90-01cda } \\
\text { sb26-90-01cda }\end{array}$ & $\begin{array}{l}6,615 \cdot 0 \\
6,616.0 \\
6,617 \cdot 0 \\
6,618 \cdot 0 \\
6,619.0\end{array}$ & $\begin{array}{l}6,616.0 \\
6,617.0 \\
6,618.0 \\
6,619.0 \\
6,620.0\end{array}$ & $\begin{array}{l}\text { 324AMSD } \\
\text { 324AMSD } \\
\text { 324AMSD } \\
\text { 324AMSD } \\
\text { 324AMSD }\end{array}$ & $\begin{array}{r}10.0 \\
4.8 \\
5.6 \\
4.1 \\
2.5\end{array}$ & $\begin{array}{l}0.040000 \\
0.050000 \\
0.060000 \\
0.030000 \\
0.980000\end{array}$ & $\begin{array}{l}\text { Vuggy dolomite } \\
\text { Vuggy dolomite } \\
\text { Dolomite } \\
\text { Fine-grained dolomite } \\
\text { Fine-grained dolomite }\end{array}$ \\
\hline $\begin{array}{l}\text { sb26-90-01cda } \\
\text { sb26-90-01cda } \\
\text { sb26-90-01cda } \\
\text { sb26-90-01cda } \\
\text { sb26-90-01cda }\end{array}$ & $\begin{array}{l}6,620 \cdot 0 \\
6,621 \cdot 0 \\
6,624 \cdot 0 \\
6,625 \cdot 0 \\
6,626 \cdot 0\end{array}$ & $\begin{array}{l}6,621 \cdot 0 \\
6,622 \cdot 0 \\
6,625 \cdot 0 \\
6,626.0 \\
6,627.0\end{array}$ & $\begin{array}{l}\text { 324AMSD } \\
\text { 324AMSD } \\
\text { 324AMSD } \\
\text { 324AMSD } \\
\text { 324AMSD }\end{array}$ & $\begin{array}{l}1.3 \\
0.7 \\
1.3 \\
1.6 \\
7.4\end{array}$ & $\begin{array}{l}0.030000 \\
0.010000 \\
0.480000 \\
0.630000 \\
0.190000\end{array}$ & $\begin{array}{l}\text { Fine-grained dolomite } \\
\text { Fine-grained dolomite } \\
\text { Fine-grained dolomite } \\
\text { Fine-grained dolomite } \\
\text { Fine-grained sandstone }\end{array}$ \\
\hline $\begin{array}{l}\text { sb26-90-01cda } \\
\text { sb26-90-01cda } \\
\text { sb26-90-01cda } \\
\text { sb26-90-01cda }\end{array}$ & $\begin{array}{l}6,627 \cdot 0 \\
6,628 \cdot 0 \\
6,629 \cdot 0 \\
6,630 \cdot 0\end{array}$ & $\begin{array}{l}6,628 \cdot 0 \\
6,629 \cdot 0 \\
6,630 \cdot 0 \\
6,631 \cdot 0\end{array}$ & $\begin{array}{l}\text { 324AMSD } \\
\text { 324AMSD } \\
\text { 324AMSD } \\
\text { 324AMSD }\end{array}$ & $\begin{array}{l}1.4 \\
5.2 \\
5.3 \\
8.5 \\
\end{array}$ & $\begin{array}{r}0.060000 \\
0.130000 \\
1.800000 \\
27.000000 \\
\end{array}$ & $\begin{array}{l}\text { Fine-grained sandstone } \\
\text { Fine-grained sandstone } \\
\text { Fine-grained sandstone } \\
\text { Fine-grained sandstone }\end{array}$ \\
\hline sb26-90-01cda & & & AVERAGE-- & 4.3 & 2.249286 & \\
\hline SITE AVERAGE-- & $-\cdots-1$ & $----n$ & $--n-1---$ & 11.3 & 41.018227 & \\
\hline $\begin{array}{l}\text { sc05-92-03cba } \\
\text { sc05-92-03cba } \\
\text { sc05-92-03cba } \\
\text { sc05-92-03cba } \\
\text { sc05-92-03cba }\end{array}$ & $\begin{array}{l}1,486.0 \\
1,489.0 \\
1,491.0 \\
1,493.0 \\
1,496.0\end{array}$ & $\begin{array}{l}1,488.0 \\
1,491.0 \\
1,493.0 \\
1,495.0 \\
1,498.0\end{array}$ & $\begin{array}{l}\text { 321WEBR } \\
321 \mathrm{WEBR} \\
\text { 321WEBR } \\
\text { 321WEBR } \\
\text { 321WEBR }\end{array}$ & $\begin{array}{r}9.4 \\
11.2 \\
9.8 \\
9.8 \\
10.3\end{array}$ & $\begin{array}{l}0.000000 \\
0.000000 \\
0.000000 \\
0.000000 \\
0.000000\end{array}$ & $\begin{array}{l}\text { Micaceous sandstone } \\
\text { Shale } \\
\text { Micaceous sandstone } \\
\text { Micaceous sandstone } \\
\text { Micaceous sandstone }\end{array}$ \\
\hline $\begin{array}{l}\text { sc05-92-03cba } \\
\text { sc05-92-03cba } \\
\text { sc05-92-03cba } \\
\text { sc05-92-03cba } \\
\text { sc05-92-03cba }\end{array}$ & $\begin{array}{l}1,499.0 \\
1,502.0 \\
1,505.0 \\
1,521.0 \\
1,523.0\end{array}$ & $\begin{array}{l}1,501.0 \\
1,504.0 \\
1,507.0 \\
1,523.0 \\
1,525.0\end{array}$ & $\begin{array}{l}\text { 321WEBR } \\
\text { 321WEBR } \\
\text { 321WEBR } \\
\text { 321WEBR } \\
\text { 321WEBR }\end{array}$ & $\begin{array}{l}11.8 \\
16.2 \\
12.3 \\
12.3 \\
11.0\end{array}$ & $\begin{array}{l}0.190000 \\
2.320000 \\
0.000000 \\
1.860000 \\
1.390000\end{array}$ & $\begin{array}{l}\text { Micaceous sandstone } \\
\text { Micaceous sandstone } \\
\text { Micaceous sandstone } \\
\text { Micaceous sandstone } \\
\text { Micaceous sandstone }\end{array}$ \\
\hline $\begin{array}{l}\text { sc05-92-03cba } \\
\text { sc05-92-03cba } \\
\text { sc05-92-03cba } \\
\text { sc05-92-03cba } \\
\text { sc05-92-03cba }\end{array}$ & $\begin{array}{l}1,525.0 \\
1,527.0 \\
1,531.0 \\
1,537.0 \\
1,540.0\end{array}$ & $\begin{array}{l}1,527.0 \\
1,529.0 \\
1,533.0 \\
1,539.0 \\
1,542.0\end{array}$ & $\begin{array}{l}\text { 321WEBR } \\
\text { 321WEBR } \\
\text { 321WEBR } \\
\text { 321WEBR } \\
\text { 321WEBR }\end{array}$ & $\begin{array}{r}8.0 \\
8.3 \\
12.3 \\
7.1 \\
7.7\end{array}$ & $\begin{array}{l}0.000000 \\
0.000000 \\
0.000000 \\
0.000000 \\
0.000000\end{array}$ & $\begin{array}{l}\text { Micaceous sandstone } \\
\text { Micaceous sandstone } \\
\text { Micaceous sandstone } \\
\text { Micaceous sandstone } \\
\text { Micaceous sandstone }\end{array}$ \\
\hline $\begin{array}{l}\text { sc05-92-03cba } \\
\text { sc05-92-03cba } \\
\text { sc05-92-03cba } \\
\text { sc05-92-03cba } \\
\text { sc05-92-03cba }\end{array}$ & $\begin{array}{l}1,542.0 \\
1,544.0 \\
1,546.0 \\
1,548.0 \\
1,550.0\end{array}$ & $\begin{array}{l}1,544.0 \\
1,546.0 \\
1,548.0 \\
1,550.0 \\
1,552.0\end{array}$ & $\begin{array}{l}\text { 321WEBR } \\
\text { 321WEBR } \\
\text { 321WEBR } \\
\text { 321WEBR } \\
\text { 321WEBR }\end{array}$ & $\begin{array}{l}6.5 \\
5.9 \\
6.8 \\
6.2 \\
4.9\end{array}$ & $\begin{array}{l}0.000000 \\
0.000000 \\
0.000000 \\
0.000000 \\
1.860000\end{array}$ & $\begin{array}{l}\text { Micaceous sandstone } \\
\text { Micaceous sandstone } \\
\text { Micaceous sandstone } \\
\text { Micaceous sandstone } \\
\text { Micaceous sandstone }\end{array}$ \\
\hline $\begin{array}{l}\text { sc05-92-03cba } \\
\text { sc05-92-03cba } \\
\text { sc05-92-03cba } \\
\text { sc05-92-03cba } \\
\text { sc05-92-03cba }\end{array}$ & $\begin{array}{l}1,552.0 \\
1,554.0 \\
1,556.0 \\
1,558.0 \\
1,664.5\end{array}$ & $\begin{array}{l}1,554.0 \\
1,556.0 \\
1,558.0 \\
1,560.0 \\
1,666.5\end{array}$ & $\begin{array}{l}\text { 321WEBR } \\
\text { 321WEBR } \\
\text { 321WEBR } \\
\text { 321WEBR } \\
\text { 321WEBR }\end{array}$ & $\begin{array}{r}4.9 \\
5.9 \\
11.8 \\
10.1 \\
9.9\end{array}$ & $\begin{array}{l}0.000000 \\
0.000000 \\
0.930000 \\
0.000000 \\
0.000000\end{array}$ & $\begin{array}{l}\text { Micaceous sandstone } \\
\text { Micaceous sandstone } \\
\text { Micaceous sandstone } \\
\text { Micaceous sandstone } \\
\text { Micaceous sandstone and claystone }\end{array}$ \\
\hline $\begin{array}{l}\mathrm{sc0}-92-03 \mathrm{cba} \\
\mathrm{sc0}-92-03 \mathrm{cba} \\
\mathrm{sc0}-92-03 \mathrm{cba} \\
\mathrm{sc0}-92-03 \mathrm{cba} \\
\mathrm{sc05}-92-03 \mathrm{cba}\end{array}$ & $\begin{array}{l}1,666.5 \\
1,668.5 \\
1,670.5 \\
1,672.5 \\
1,674.5\end{array}$ & $\begin{array}{l}1,668.5 \\
1,670.5 \\
1,672.5 \\
1,674.5 \\
1,676.5\end{array}$ & $\begin{array}{l}\text { 321WEBR } \\
\text { 321WEBR } \\
\text { 321WEBR } \\
\text { 321WEBR } \\
\text { 321WEBR }\end{array}$ & $\begin{array}{r}10.9 \\
8.0 \\
7.4 \\
7.4 \\
8.3\end{array}$ & $\begin{array}{l}1.390000 \\
0.320000 \\
0.000000 \\
3.250000 \\
0.000000\end{array}$ & $\begin{array}{l}\text { Micaceous sandstone } \\
\text { Micaceous sandstone } \\
\text { Micaceous sandstone and claystone } \\
\text { Micaceous sandstone } \\
\text { Micaceous sandstone }\end{array}$ \\
\hline
\end{tabular}


Table 4.--Laboratory-determined porosity and permeability--Continued

\begin{tabular}{|c|c|c|c|c|c|c|}
\hline Site & $\begin{array}{l}\text { Depth to } \\
\text { top } \\
\text { (feet) }\end{array}$ & $\begin{array}{l}\text { Depth to } \\
\text { bottom } \\
\text { (feet) }\end{array}$ & $\begin{array}{c}\text { Forma- } \\
\text { tion }\end{array}$ & $\begin{array}{l}\text { Porosity } \\
\text { (percent) }\end{array}$ & $\begin{array}{l}\text { Perme- } \\
\text { ability } \\
\text { (milli- } \\
\text { darcies) }\end{array}$ & Lithology \\
\hline sc05-92-03cba & $1,676.5$ & $1,678.5$ & $321 \mathrm{WEBR}$ & 6.5 & 0.000000 & Micaceous sandstone and claystone \\
\hline SITE AVERAGE-- & -..... & -- & AVERAGE-- & $\frac{9.0}{9.0}$ & $\frac{0.435806}{0.435806}$ & \\
\hline $\begin{array}{l}\text { sla03-24-22bda } \\
\text { sla03-24-22bda } \\
\text { sla03-24-22bda } \\
\text { sla03-24-22bda } \\
\text { sla03-24-22bda }\end{array}$ & $\begin{array}{l}8,753.0 \\
8,756.0 \\
8,801.0 \\
8,886.0 \\
8,890.0\end{array}$ & $\begin{array}{l}8,756.0 \\
8,759.0 \\
8,804.0 \\
8,890.0 \\
8,894.0\end{array}$ & $\begin{array}{l}317 \text { PRKC } \\
317 \text { PRKC } \\
317 \text { PRKC } \\
317 \text { PRKC } \\
317 \text { PRKC }\end{array}$ & $\begin{array}{l}7.9 \\
6.4 \\
2.8 \\
6.5 \\
6.1\end{array}$ & $\begin{array}{l}0.000000 \\
0.000000 \\
0.000000 \\
0.040000 \\
0.030000\end{array}$ & $\begin{array}{l}\text { Phosphatic, fine-grained sandstone } \\
\text { Phosphatic, fine-grained sandstone } \\
\text { Sandy, dolomitic limestone } \\
\text { Siliceous dolomite } \\
\text { Siliceous dolomite }\end{array}$ \\
\hline $\begin{array}{l}\text { sla03-24-22bda } \\
\text { sla03-24-22bda } \\
\text { sla03-24-22bda }\end{array}$ & $\begin{array}{l}8,906.0 \\
8,963.0 \\
8,967.0\end{array}$ & $\begin{array}{l}8,907.0 \\
8,967.0 \\
8,968.0\end{array}$ & $\begin{array}{l}317 \mathrm{PRKC} \\
317 \mathrm{PRKC} \\
317 \mathrm{PRKC}\end{array}$ & $\begin{array}{l}10.1 \\
16.3 \\
10.9 \\
\end{array}$ & $\begin{array}{l}0.030000 \\
0.050000 \\
0.060000 \\
\end{array}$ & $\begin{array}{l}\text { Fine-grained, limy, cherty sandstone } \\
\text { Phosphatic, fine-grained sandstone } \\
\text { Phosphatic, fine-grained sandstone }\end{array}$ \\
\hline & & & AVERAGE-- & 8.4 & 0.026250 & \\
\hline $\begin{array}{l}\text { sla03-24-22bda } \\
\text { sla03-24-22bda } \\
\text { sla03-24-22bda } \\
\text { sla03-24-22bda } \\
\text { sla03-24-22bda }\end{array}$ & $\begin{array}{l}9,040.5 \\
9,044 \cdot 5 \\
9,046.5 \\
9,048.5 \\
9,050.5\end{array}$ & $\begin{array}{l}9,041 \cdot 5 \\
9,045 \cdot 5 \\
9,047 \cdot 5 \\
9,049 \cdot 5 \\
9,051 \cdot 5\end{array}$ & $\begin{array}{l}321 \text { WEBR } \\
321 \text { WEBR } \\
321 \text { WEBR } \\
321 \text { WEBR } \\
321 \text { WEBR }\end{array}$ & $\begin{array}{l}6.5 \\
5.1 \\
4.7 \\
4.6 \\
4.5\end{array}$ & $\begin{array}{l}0.000000 \\
0.030000 \\
0.040000 \\
0.050000 \\
0.040000\end{array}$ & $\begin{array}{l}\text { Fine- to medium-grained sandstone } \\
\text { Fine- to medium-grained sandstone } \\
\text { Fine- to medium-grained sandstone } \\
\text { Fine- to medium-grained sandstone } \\
\text { Fine- to medium-grained sandstone }\end{array}$ \\
\hline $\begin{array}{l}\text { sla03-24-22bda } \\
\text { sla03-24-22bda } \\
\text { sla03-24-22bda } \\
\text { sla03-24-22bda } \\
\text { sla03-24-22bda }\end{array}$ & $\begin{array}{l}9,052.5 \\
9,054.5 \\
9,056.5 \\
9,058.5 \\
9,060.5\end{array}$ & $\begin{array}{l}9,053 \cdot 5 \\
9,055 \cdot 5 \\
9,057 \cdot 5 \\
9,059 \cdot 5 \\
9,061 \cdot 5\end{array}$ & $\begin{array}{l}321 \text { WEBR } \\
321 \text { WEBR } \\
321 \text { WEBR } \\
321 \text { WEBR } \\
321 \text { WEBR }\end{array}$ & $\begin{array}{l}3.7 \\
3.9 \\
4.7 \\
4.6 \\
4.9\end{array}$ & $\begin{array}{l}0.020000 \\
0.020000 \\
0.030000 \\
0.030000 \\
0.030000\end{array}$ & $\begin{array}{l}\text { Fine- to medium-grained sandstone } \\
\text { Fine- to medium-grained sandstone } \\
\text { Fine- to medium-grained sandstone } \\
\text { Fine- to medium-grained sandstone } \\
\text { Fine- to medium-grained sandstone }\end{array}$ \\
\hline $\begin{array}{l}\text { sla03-24-22bda } \\
\text { sla03-24-22bda } \\
\text { sla03-24-22bda } \\
\text { sla03-24-22bda } \\
\text { sla03-24-22bda }\end{array}$ & $\begin{array}{l}9,062 \cdot 5 \\
9,064 \cdot 5 \\
9,066.5 \\
9,068.5 \\
9,070.5\end{array}$ & $\begin{array}{l}9,063 \cdot 5 \\
9,065 \cdot 5 \\
9,067 \cdot 5 \\
9,069 \cdot 5 \\
9,071 \cdot 5\end{array}$ & $\begin{array}{l}321 \mathrm{WEBR} \\
321 \mathrm{WEBR} \\
321 \mathrm{WEBR} \\
321 \mathrm{WEBR} \\
321 \mathrm{WEBR}\end{array}$ & $\begin{array}{l}5.3 \\
3.7 \\
4.9 \\
6.2 \\
8.1\end{array}$ & $\begin{array}{l}0.040000 \\
0.040000 \\
0.020000 \\
0.050000 \\
0.130000\end{array}$ & $\begin{array}{l}\text { Fine- to medium-grained sandstone } \\
\text { Fine- to medium-grained sandstone } \\
\text { Cross-bedded, fine- to medium-grained sandstone } \\
\text { Cross-bedded, fine- to medium-grained sandstone } \\
\text { Cross-bedded, fine- to medium-grained sandstone }\end{array}$ \\
\hline $\begin{array}{l}\text { sla03-24-22bda } \\
\text { sla03-24-22bda } \\
\text { sla03-24-22bda } \\
\text { sla03-24-22bda } \\
\text { sla03-24-22bda }\end{array}$ & $\begin{array}{l}9,072.5 \\
9,074.5 \\
9,076.5 \\
9,078.5 \\
9,080.5\end{array}$ & $\begin{array}{l}9,073 \cdot 5 \\
9,075 \cdot 5 \\
9,077 \cdot 5 \\
9,079.5 \\
9,081 \cdot 5\end{array}$ & $\begin{array}{l}321 \text { WEBR } \\
321 \text { WEBR } \\
321 \text { WEBR } \\
321 \text { WEBR } \\
321 \text { WEBR }\end{array}$ & $\begin{array}{l}7.8 \\
7.5 \\
5.0 \\
6.8 \\
6.8\end{array}$ & $\begin{array}{l}0.150000 \\
0.100000 \\
0.020000 \\
0.070000 \\
0.110000\end{array}$ & $\begin{array}{l}\text { Cross-bedded, fine- to medium-grained sandstone } \\
\text { Cross-bedded, fine- to medium-grained sandstone } \\
\text { Cross-bedded, fine- to medium-grained sandstone } \\
\text { Cross-bedded, fine- to medium-grained sandstone } \\
\text { Cross-bedded, fine- to medium-grained sandstone }\end{array}$ \\
\hline $\begin{array}{l}\text { sla03-24-22bda } \\
\text { sla03-24-22bda } \\
\text { sla03-24-22bda } \\
\text { sla03-24-22bda } \\
\text { sla03-24-22bda }\end{array}$ & $\begin{array}{l}9,082.5 \\
9,084 \cdot 5 \\
9,086.5 \\
9,088.5 \\
9,090.5\end{array}$ & $\begin{array}{l}9,083 \cdot 5 \\
9,085 \cdot 5 \\
9,087 \cdot 5 \\
9,089 \cdot 5 \\
9,091 \cdot 5\end{array}$ & $\begin{array}{l}321 \mathrm{WEBR} \\
321 \mathrm{WEBR} \\
321 \mathrm{WEBR} \\
321 \mathrm{WEBR} \\
321 \mathrm{WEBR}\end{array}$ & $\begin{array}{l}6.0 \\
5.9 \\
6.1 \\
4.6 \\
5.2\end{array}$ & $\begin{array}{l}0.040000 \\
0.030000 \\
0.030000 \\
0.020000 \\
0.040000\end{array}$ & $\begin{array}{l}\text { Cross-bedded, fine- to medium-grained sandstone } \\
\text { Cross-bedded, fine- to medium-grained sandstone } \\
\text { Cross-bedded, fine- to medium-grained sandstone } \\
\text { Cross-bedded, fine- to medium-grained sandstone } \\
\text { Cross-bedded, fine- to medium-grained sandstone }\end{array}$ \\
\hline $\begin{array}{l}\text { sla03-24-22bda } \\
\text { sla03-24-22bda } \\
\text { sla03-24-22bda } \\
\text { sla03-24-22bda } \\
\text { sla03-24-22bda }\end{array}$ & $\begin{array}{l}9,092.5 \\
9,094.5 \\
9,096.5 \\
9,098.5 \\
9,100.5\end{array}$ & $\begin{array}{l}9,093 \cdot 5 \\
9,095 \cdot 5 \\
9,097.5 \\
9,099.5 \\
9,101.5\end{array}$ & $\begin{array}{l}321 \mathrm{WEBR} \\
321 \mathrm{WEBR} \\
321 \mathrm{WEBR} \\
321 \mathrm{WEBR} \\
321 \mathrm{WEBR}\end{array}$ & $\begin{array}{l}6.5 \\
5.5 \\
6.8 \\
4.9 \\
7.6\end{array}$ & $\begin{array}{l}0.350000 \\
0.030000 \\
0.090000 \\
0.040000 \\
0.300000\end{array}$ & $\begin{array}{l}\text { Cross-bedded, fine- to medium-grained sandstone } \\
\text { Cross-bedded, fine- to medium-grained sandstone } \\
\text { Cross-bedded, fine- to medium-grained sandstone } \\
\text { Cross-bedded, fine- to medium-grained sandstone } \\
\text { Cross-bedded, fine- to medium-grained sandstone }\end{array}$ \\
\hline $\begin{array}{l}\text { sla03-24-22bda } \\
\text { sla03-24-22bda } \\
\text { sla03-24-22bda } \\
\text { sla03-24-22bda } \\
\text { sla03-24-22bda }\end{array}$ & $\begin{array}{l}9,102.5 \\
9,104.5 \\
9,192.5 \\
9,194.5 \\
9,196.5\end{array}$ & $\begin{array}{l}9,103 \cdot 5 \\
9,105.5 \\
9,193.5 \\
9,195.5 \\
9,197.5\end{array}$ & $\begin{array}{l}321 \mathrm{WEBR} \\
321 \mathrm{WEBR} \\
321 \mathrm{WEBR} \\
321 \mathrm{WEBR} \\
321 \mathrm{WEBR}\end{array}$ & $\begin{array}{r}9.9 \\
9.0 \\
13.7 \\
15.2 \\
12.7\end{array}$ & $\begin{array}{l}1.040000 \\
0.600000 \\
6.630000 \\
9.020000 \\
6.940000\end{array}$ & $\begin{array}{l}\text { Cross-bedded, fine- to medium-grained sandstone } \\
\text { Cross-bedded, fine- to medium-grained sandstone } \\
\text { Cross-bedded, fine- to medium-grained sandstone } \\
\text { Cross-bedded, fine- to medium-grained sandstone } \\
\text { Cross-bedded, fine- to medium-grained sandstone }\end{array}$ \\
\hline
\end{tabular}


Table 4.--Laboratory-determined porosity and permeability--Continued

\begin{tabular}{|c|c|c|c|c|c|}
\hline Site & $\begin{array}{l}\text { Depth to } \\
\text { top } \\
\text { (feet) }\end{array}$ & $\begin{array}{l}\text { Depth to } \\
\text { bottom } \\
\text { (feet) }\end{array}$ & $\begin{array}{c}\text { Forma- } \\
\text { tion }\end{array}$ & $\begin{array}{l}\text { Porosity } \\
\text { (percent) }\end{array}$ & $\begin{array}{l}\text { Perme- } \\
\text { ability } \\
\text { (milli- } \\
\text { darcies) }\end{array}$ \\
\hline
\end{tabular}

\begin{tabular}{l}
\hline sla03-24-22bda \\
sla03-24-22bda \\
sla03-24-22bda \\
sla03-24-22bda \\
sla03-24-22bda \\
sla03-24-22bda
\end{tabular}

sla03-24-22bda sla03-24-22bda s la $03-24-22 \mathrm{bda}$ sla03-24-22bda sla03-24-22bda

sla03-24-22bda sla03-24-22bda sla03-24-22bda sla03-24-22bda sla03-24-22bda

s la 03-24-22bda sla 03-24-22bda sla03-24-22bda s la03-24-22bda sla03-24-22bda

s la03-24-22bda s la03-24-22bda sla03-24-22bda s la03-24-22bda sla03-24-22bda

sla03-24-22bda s la 03-24-22bda sla 03-24-22bda s la 03-24-22bda sla03-24-22bda

sla03-24-22bda sla03-24-22bda sla 03-24-22bda sla03-24-22bda sla03-24-22bda

sla03-24-22bda sla03-24-22bda sla03-24-22bda s la 03-24-22bda s la 03-24-22bda

sla03-24-22bda sla 03-24-22bda s la 03-24-22bda s la 03-24-22bda s la03-24-22bda

sla03-24-22bda s la03-24-22bda s la 03-24-22bda s la 03-24-22bda s la03-24-22bda

\begin{tabular}{|c|c|c|c|c|}
\hline $9,198.5$ & $9,199.5$ & $321 \mathrm{WEBR}$ & 4.9 & 0.000000 \\
\hline $9,200.5$ & $9,201.5$ & $321 \mathrm{WEBR}$ & 7.3 & 0.130000 \\
\hline $9,202.5$ & $9,203.5$ & $321 \mathrm{WEBR}$ & 7.2 & 0.130000 \\
\hline $9,204.5$ & $9,205.5$ & 321WEBR & 4.3 & 0.010000 \\
\hline $9,206.5$ & $9,207.5$ & $321 \mathrm{WEBR}$ & 6.1 & 0.180000 \\
\hline $9,209.5$ & $9,210.5$ & 321WEBR & 3.7 & 0.020000 \\
\hline $9,212.5$ & $9,213.5$ & 321WEBR & 3.2 & 0.000000 \\
\hline $9,214.5$ & $9,215.5$ & $321 \mathrm{WEBR}$ & 4.7 & 0.000000 \\
\hline $9,216.5$ & $9,217.5$ & $321 \mathrm{WEBR}$ & 4.5 & 0.000000 \\
\hline $9,218.5$ & $9,219.5$ & 321WEBR & 4.9 & 0.000000 \\
\hline $9,220.5$ & $9,221.5$ & 321WEBR & 6.0 & 0.000000 \\
\hline $9,222.5$ & $9,223.5$ & $321 \mathrm{WEBR}$ & 4.7 & 0.000000 \\
\hline $9,224.5$ & $9,225.5$ & 321WEBR & 3.1 & 0.000000 \\
\hline $9,226.5$ & $9,227.5$ & $321 \mathrm{WEBR}$ & 5.0 & 0.020000 \\
\hline $9,228.5$ & $9,229.5$ & $321 \mathrm{WEBR}$ & 4.7 & 0.000000 \\
\hline $9,230.5$ & $9,231.5$ & $321 \mathrm{WEBR}$ & 4.6 & 0.020000 \\
\hline $9,232.5$ & $9,233.5$ & $321 \mathrm{WEBR}$ & 4.8 & 0.030000 \\
\hline $9,234.5$ & $9,235.5$ & $321 \mathrm{WEBR}$ & 5.1 & 0.020000 \\
\hline $9,236.5$ & $9,237.5$ & 321WEBR & 5.2 & 0.020000 \\
\hline $9,238.5$ & $9,239.5$ & $321 \mathrm{WEBR}$ & 4.3 & 0.000000 \\
\hline $9,240.5$ & $9,241.5$ & $321 \mathrm{WEBR}$ & 4.6 & 0.000000 \\
\hline $9,242.5$ & $9,243.5$ & $321 \mathrm{WEBR}$ & 4.3 & 0.000000 \\
\hline $9,244.5$ & $9,245.5$ & $321 \mathrm{WEBR}$ & 4.2 & 0.000000 \\
\hline $9,246.5$ & $9,247.5$ & $321 \mathrm{WEBR}$ & 4.7 & 0.120000 \\
\hline $9,248.5$ & $9,249.5$ & $321 \mathrm{WEBR}$ & 4.2 & 0.000000 \\
\hline $9,250.5$ & $9,251.5$ & $321 \mathrm{WEBR}$ & 4.9 & 0.000000 \\
\hline $9,252.5$ & $9,253.5$ & $321 \mathrm{WEBR}$ & 7.5 & 0.110000 \\
\hline $9,254.5$ & $9,255.5$ & $321 \mathrm{WEBR}$ & 3.9 & 0.000000 \\
\hline $9,256.5$ & $9,257.5$ & $321 \mathrm{WEBR}$ & 5.7 & 0.010000 \\
\hline $9,258.5$ & $9,259.5$ & 321WEBR & 2.7 & 0.000000 \\
\hline $9,260.5$ & $9,261.5$ & $321 \mathrm{WEBR}$ & 3.2 & 0.000000 \\
\hline $9,262.5$ & $9,263.5$ & $321 \mathrm{WEBR}$ & 7.9 & 0.790000 \\
\hline $9,269.5$ & $9,270.5$ & $321 \mathrm{WEBR}$ & 3.7 & 0.020000 \\
\hline $9,280.5$ & $9,281.5$ & 321WEBR & 9.2 & 0.150000 \\
\hline $9,282.5$ & $9,283.5$ & $321 \mathrm{WEBR}$ & 10.0 & 3.480000 \\
\hline $9,284.5$ & $9,285.5$ & $321 \mathrm{WEBR}$ & 4.8 & 0.020000 \\
\hline $9,286.5$ & $9,287.5$ & $321 \mathrm{WEBR}$ & 4.6 & 0.010000 \\
\hline $9,288.5$ & $9,289.5$ & 321WEBR & 3.0 & 0.000000 \\
\hline $9,290.5$ & $9,291.5$ & $321 \mathrm{WEBR}$ & 4.7 & 0.130000 \\
\hline $9,292.5$ & $9,293.5$ & $321 \mathrm{WEBR}$ & 11.1 & 14.000000 \\
\hline $9,294.5$ & $9,295.5$ & $321 \mathrm{WEBR}$ & 11.8 & 16.000000 \\
\hline $9,296.5$ & $9,297.5$ & $321 \mathrm{WEBR}$ & 8.6 & 0.850000 \\
\hline $9,298.5$ & $9,299.5$ & $321 \mathrm{WEBR}$ & 10.6 & 2.600000 \\
\hline $9,300.5$ & $9,301.5$ & $321 \mathrm{WEBR}$ & 9.6 & 0.700000 \\
\hline $9,302.5$ & $9,303.5$ & $321 \mathrm{WEBR}$ & 9.8 & 0.860000 \\
\hline $9,304.5$ & $9,305.5$ & $321 \mathrm{WEBR}$ & 9.9 & 1.230000 \\
\hline $9,306.5$ & $9,307.5$ & $321 \mathrm{WEBR}$ & 9.5 & 0.910000 \\
\hline $9,308.5$ & $9,309.5$ & $321 \mathrm{WEBR}$ & 10.0 & 1.530000 \\
\hline $9,310.5$ & $9,311.5$ & $321 \mathrm{WEBR}$ & 9.5 & 0.670000 \\
\hline $9,312.5$ & $9,313.5$ & $321 \mathrm{WEBR}$ & 10.6 & 0.950000 \\
\hline
\end{tabular}

Cross-bedded, fine- to medium-grained sandstone Cross-bedded, fine- to medium-grained sandstone Cross-bedded, fine- to medium-grained sandstone Cross-bedded, fine- to medium-grained sandstone Cross-bedded, fine- to medium-grained sandstone

Cross-bedded, fine- to medium-grained sandstone Cross-bedded, fine- to medium-grained sandstone Cross-bedded, fine- to medium-grained sandstone Cross-bedded, fine- to medium-grained sandstone Cross-bedded, fine- to medium-grained sandstone

Cross-bedded, fine- to medium-grained sandstone Cross-bedded, fine- to medium-grained sandstone Cross-bedded, fine- to medium-grained sandstone Cross-bedded, fine- to medium-grained sandstone Cross-bedded, fine- to medium-grained sandstone

Cross-bedded, fine- to medium-grained sandstone Cross-bedded, fine- to medium-grained sandstone Cross-bedded, fine- to medium-grained sandstone Cross-bedded, fine- to medium-grained sandstone Cross-bedded, fine- to medium-grained sandstone

Cross-bedded, fine- to medium-grained sandstone Cross-bedded, fine- to medium-grained sandstone Cross-bedded, fine- to medium-grained sandstone Cross-bedded, fine- to medium-grained sandstone Cross-bedded, fine- to medium-grained sandstone

Cross-bedded, fine- to medium-grained sandstone Cross-bedded, fine- to medium-grained sandstone Cross-bedded, fine- to medium-grained sandstone Cross-bedded, fine- to medium-grained sandstone Cross-bedded, fine- to medium-grained sandstone

Cross-bedded, fine- to medium-grained sandstone Cross-bedded, fine- to medium-grained sandstone Cross-bedded, fine- to medium-grained sandstone Cross-bedded, fine- to medium-grained sandstone Cross-bedded, fine- to medium-grained sandstone

Cross-bedded, fine- to medium-grained sandstone Cross-bedded, fine- to medium-grained sandstone Cross-bedded, fine- to medium-grained sandstone Cross-bedded, fine- to medium-grained sandstone Cross-bedded, fine- to medium-grained sandstone

Cross-bedded, fine- to medium-grained sandstone Cross-bedded, fine- to medium-grained sandstone Cross-bedded, fine- to medium-grained sandstone Cross-bedded, fine- to medium-grained sandstone Cross-bedded, fine- to medium-grained sandstone

Cross-bedded, fine- to medium-grained sandstone Cross-bedded, fine- to medium-grained sandstone Cross-bedded, fine- to medium-grained sandstone Cross-bedded, fine- to medium-grained sandstone Cross-bedded, fine- to medium-grained sandstone 
Table 4.--Laboratory-determined porosity and permeability--Continued

\begin{tabular}{|c|c|c|c|c|c|c|}
\hline Site & $\begin{array}{l}\text { Depth to } \\
\text { top } \\
\text { (feet) }\end{array}$ & $\begin{array}{l}\text { Depth to } \\
\text { bottom } \\
\text { (feet) }\end{array}$ & $\begin{array}{c}\text { Forma- } \\
\text { tion }\end{array}$ & $\begin{array}{l}\text { Porosity } \\
\text { (percent) }\end{array}$ & $\begin{array}{l}\text { Perme- } \\
\text { ability } \\
\text { (milli- } \\
\text { darcies) }\end{array}$ & Lithology \\
\hline sla03-24-22bda & $9,314.5$ & $9,315.5$ & $321 W E B R$ & 9.3 & 0.400000 & Cross-bedded, fine- to medium-grained sandstone \\
\hline sla03-24-22bda & $9,316.5$ & $9,317.5$ & $321 \mathrm{WEBR}$ & 7.6 & 0.030000 & Cross-bedded, fine- to medium-grained sandstone \\
\hline sla03-24-22bda & $9,318.5$ & $9,319.5$ & $321 \mathrm{WEBR}$ & 6.7 & 0.050000 & Cross-bedded, fine- to medium-grained sandstone \\
\hline s $1 \mathrm{a} 03-24-22 \mathrm{bda}$ & $9,320.5$ & $9,321.5$ & $321 \mathrm{WEBR}$ & 4.6 & 0.020000 & Cross-bedded, fine- to medium-grained sandstone \\
\hline sla03-24-22bda & $9,322.5$ & $9,323.5$ & $321 \mathrm{WEBR}$ & 3.4 & 0.020000 & Cross-bedded, fine- to medium-grained sandstone \\
\hline sla03-24-22bda & $9,324.5$ & $9,325.5$ & $321 \mathrm{WEBR}$ & 2.8 & 0.000000 & Cross-bedded, fine- to medium-grained sandstone \\
\hline $\mathrm{s} 1 \mathrm{a} 03-24-22 \mathrm{bda}$ & $9,326.5$ & $9,327.5$ & $321 \mathrm{WEBR}$ & 4.5 & 0.020000 & Cross-bedded, fine- to medium-grained sandstone \\
\hline sla03-24-22bda & $9,328.5$ & $9,329.5$ & $321 \mathrm{WEBR}$ & 5.6 & 0.050000 & Cross-bedded, fine- to medium-grained sandstone \\
\hline $\mathrm{s} 1 \mathrm{a} 03-24-22 \mathrm{bda}$ & $9,330.5$ & $9,331.5$ & $321 \mathrm{WEBR}$ & 3.3 & 0.030000 & Cross-bedded, fine- to medium-grained sandstone \\
\hline s la $03-24-22 b d a$ & $9,332.5$ & $9,333.5$ & $321 \mathrm{WEBR}$ & 3.3 & 0.040000 & Cross-bedded, fine- to medium-grained sandstone \\
\hline sla03-24-22bda & $9,334.5$ & $9,335.5$ & $321 \mathrm{WEBR}$ & 5.0 & 0.060000 & Cross-bedded, fine- to medium-grained sandstone \\
\hline sla03-24-22bda & $9,336.5$ & $9,337.5$ & 321WEBR & 5.0 & 0.050000 & Cross-bedded, fine- to medium-grained sandstone \\
\hline $\mathrm{sla03-24-22bda}$ & $9,338.5$ & $9,339.5$ & 321WEBR & 4.6 & 0.050000 & Cross-bedded, fine- to medium-grained sandstone \\
\hline sla03-24-22bda & $9,340.5$ & $9,341.5$ & 321 WEBR & 5.5 & 0.060000 & Cross-bedded, fine- to medium-grained sandstone \\
\hline s $1 \mathrm{a} 03-24-22 \mathrm{bda}$ & $9,342.5$ & $9,343.5$ & $321 \mathrm{WEBR}$ & 7.3 & 0.300000 & Cross-bedded, fine- to medium-grained sandstone \\
\hline s la03-24-22bda & $9,344.5$ & $9,345.5$ & $321 \mathrm{WEBR}$ & 6.8 & 0.100000 & Cross-bedded, fine- to medium-grained sandstone \\
\hline sla03-24-22bda & $9,346.5$ & $9,347.5$ & $321 \mathrm{WEBR}$ & 3.3 & 0.000000 & Cross-bedded, fine- to medium-grained sandstone \\
\hline s1a03-24-22bda & $9,348.5$ & $9,349.5$ & $321 \mathrm{WEBR}$ & 4.2 & 0.000000 & Cross-bedded, fine- to medium-grained sandstone \\
\hline sla03-24-22bda & $9,350.5$ & $9,351.5$ & $321 \mathrm{WEBR}$ & 2.9 & 0.000000 & Cross-bedded, fine- to medium-grained sandstone \\
\hline sla03-24-22bda & $9,352.5$ & $9,353.5$ & 321 WEBR & 4.3 & 0.020000 & Cross-bedded, fine- to medium-grained sandstone \\
\hline sla03-24-22bda & $9,354.5$ & $9,355.5$ & $321 \mathrm{WEBR}$ & 3.7 & 0.030000 & Cross-bedded, fine- to medium-grained sandstone \\
\hline & & & AVERAGE-- & 6.0 & 0.691226 & \\
\hline SITE AVERAGE-- & $=-$ & & ------1 & 6.2 & 0.644561 & \\
\hline sld $03-20-05 c c b$ & 0.0 & 0.4 & $321 \mathrm{WEBR}$ & 12.8 & 70.000000 & Sandstone \\
\hline & & & AVERAGE-- & 12.8 & 70.000000 & \\
\hline SITE AVERAGE- & 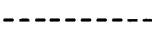 & 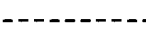 & $---1---1$ & 12.8 & 70.000000 & \\
\hline s $1 \mathrm{~d} 04-20-04 \mathrm{ddc}$ & $4,477.0$ & $4,478.0$ & 317 PRKC & 4.1 & 0.270000 & Finely crystalline dolomite \\
\hline sld04-20-04ddc & $4,478.0$ & $4,479.0$ & 317PRKC & 7.2 & 0.170000 & Finely crystalline dolomite \\
\hline s $1 d 04-20-04 d d c$ & $4,479.0$ & $4,480.0$ & 317 PRKC & 4.5 & 0.020000 & Finely crystalline dolomite \\
\hline s $1 \mathrm{~d} 04-20-04 \mathrm{ddc}$ & $4,480.0$ & $4,481.0$ & 317PRKC & 7.0 & 4.100000 & Finely crystalline dolomite \\
\hline s1d04-20-04ddc & $4,481.0$ & $4,482.0$ & 317 PRKC & 13.2 & 7.600000 & Cherty, finely crystalline dolomite \\
\hline s1d04-20-04ddc & $4,482.0$ & $4,483.0$ & 317 PRKC & 1.6 & 6.600000 & Finely crystalline dolomite \\
\hline s $1 \mathrm{~d} 04-20-04 \mathrm{ddc}$ & $4,483.0$ & $4,484.0$ & 317PRKC & 13.6 & 0.000000 & Finely crystalline dolomite \\
\hline s1d04-20-04ddc & $4,484.0$ & $4,485.0$ & 317 PRKC & 9.1 & 20.000000 & Anhydritic, finely crystalline dolomite \\
\hline s1d04-20-04ddc & $4,485.0$ & $4,486.0$ & 317 PRKC & 10.1 & 0.070000 & Finely crystalline dolomite \\
\hline s1d04-20-04ddc & $4,486.0$ & $4,487.0$ & 317 PRKC & 7.3 & 0.050000 & Finely crystalline dolomite \\
\hline s $1 \mathrm{~d} 04-20-04 \mathrm{ddc}$ & $4,487.0$ & $4,488.0$ & 317 PRKC & 9.9 & 3.100000 & Finely crystalline dolomite \\
\hline s $1 \mathrm{~d} 04-20-04 \mathrm{ddc}$ & $4,488.0$ & $4,489.0$ & 317 PRKC & 11.4 & 0.040000 & Finely crystalline dolomite \\
\hline s $1 \mathrm{~d} 04-20-04 \mathrm{ddc}$ & $4,489.0$ & $4,490.0$ & 317 PRKC & 11.1 & 52.000000 & Finely crystalline dolomite \\
\hline s $1 \mathrm{~d} 04-20-04 \mathrm{ddc}$ & $4,490.0$ & $4,491.0$ & 317 PRKC & 21.8 & 3.200000 & Finely crystalline dolomite \\
\hline s $1 \mathrm{~d} 04-20-04 \mathrm{ddc}$ & $4,491.0$ & $4,492.0$ & 317 PRKC & 16.6 & 1.500000 & Finely crystalline dolomite \\
\hline s1d04-20-04ddc & $4,492.0$ & $4,493.0$ & 317 PRKC & 14.0 & 0.330000 & Anhydritic, finely crystalline dolomite \\
\hline sld $04-20-04 d d c$ & $4,493.0$ & $4,494.0$ & 317PRKC & 12.3 & 0.350000 & Finely crystalline dolomite \\
\hline sld04-20-04ddc & $4,494.0$ & $4,495.0$ & $317 \mathrm{PRKC}$ & 16.0 & 15.000000 & Finely crystalline dolomite \\
\hline s $1 \mathrm{~d} 04-20-04 \mathrm{ddc}$ & $4,495.0$ & $4,496.0$ & 317 PRKC & 7.4 & 12.000000 & Finely crystalline dolomite \\
\hline s $1 \mathrm{~d} 04-20-04 \mathrm{ddc}$ & $4,496.0$ & $4,497.0$ & 317 PRKC & 14.7 & 0.070000 & Anhydritic, finely crystalline dolomite \\
\hline
\end{tabular}


Table 4.--Laboratory-determined porosity and permeability--Continued

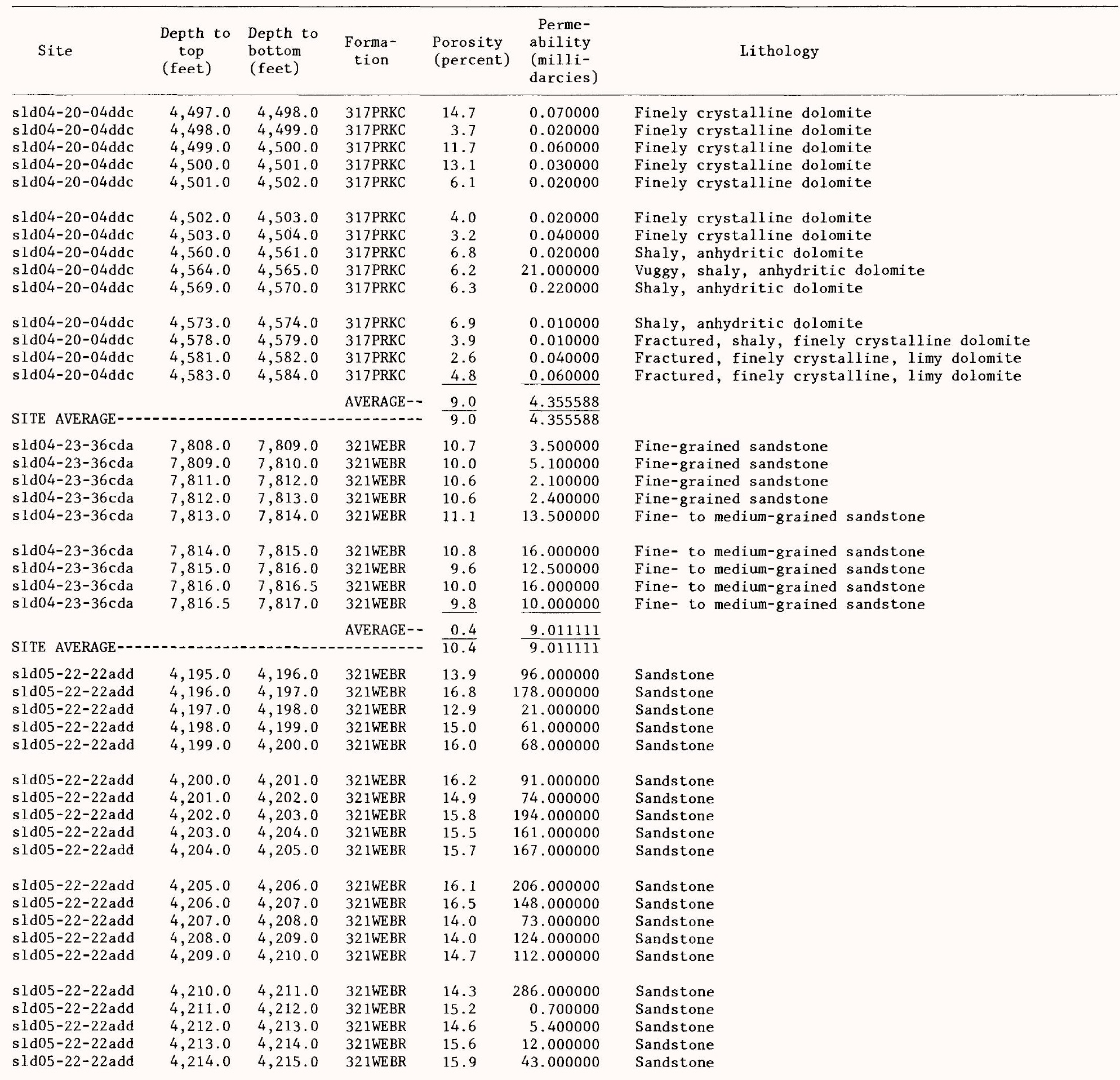


Table 4.--Laboratory-determined porosity and permeability--Continued

\begin{tabular}{|c|c|c|c|c|c|c|}
\hline Site & $\begin{array}{l}\text { Depth to } \\
\text { top } \\
\text { (feet) }\end{array}$ & $\begin{array}{l}\text { Depth to } \\
\text { bottom } \\
\text { (feet) }\end{array}$ & $\begin{array}{c}\text { Forma- } \\
\text { tion }\end{array}$ & $\begin{array}{l}\text { Porosity } \\
\text { (percent) }\end{array}$ & $\begin{array}{l}\text { Perme- } \\
\text { ability } \\
\text { (milli- } \\
\text { darcies) }\end{array}$ & Lithology \\
\hline sld05-22-22add & $4,215.0$ & $4,216.0$ & $321 \mathrm{WEBR}$ & 13.2 & 15.000000 & Sandstone \\
\hline s1d05-22-22add & $4,216.0$ & $4,217.0$ & 321WEBR & 15.2 & 12.000000 & Sandstone \\
\hline sld05-22-22add & $4,217.0$ & $4,218.0$ & 321WEBR & 12.3 & 8.300000 & Sandstone \\
\hline s1d05-22-22add & $4,218.0$ & $4,219.0$ & 321WEBR & 13.0 & 5.500000 & Sandstone \\
\hline s $1 d 05-22-22 a d d$ & $4,219.0$ & $4,220.0$ & $321 \mathrm{WEBR}$ & 14.7 & 112.000000 & Sandstone \\
\hline s $1 d 05-22-22 a d d$ & $4,220.0$ & $4,221.0$ & 321WEBR & 14.7 & 28.000000 & Sandstone \\
\hline s 1 do5-22-22add & $4,221.0$ & $4,222.0$ & 321WEBR & 12.5 & 14.000000 & Sands tone \\
\hline s $1 d 05-22-22 a d d$ & $4,222.0$ & $4,223.0$ & 321WEBR & 10.9 & 0.900000 & Sandstone \\
\hline s1d05-22-22add & $4,223.0$ & $4,224.0$ & $321 \mathrm{WEBR}$ & 13.7 & 74.000000 & Sandstone \\
\hline sldo5-22-22add & $4,224.0$ & $4,225.0$ & $321 \mathrm{WEBR}$ & 13.7 & 13.000000 & Sandstone \\
\hline s $1 d 05-22-22 a d d$ & $4,225.0$ & $4,226.0$ & 321WEBR & 12.3 & 15.000000 & Sandstone \\
\hline s $1 d 05-22-22 a d d$ & $4,226.0$ & $4,227.0$ & 321WEBR & 14.9 & 20.000000 & Sands tone \\
\hline s 1 do5-22-22add & $4,227.0$ & $4,228.0$ & 321WEBR & 14.3 & 25.000000 & Sandst one \\
\hline s $1 d 05-22-22 a d d$ & $4,228.0$ & $4,229.0$ & 321WEBR & 15.3 & 45.000000 & Sandstone \\
\hline s 1 d05-22-22add & $4,233.0$ & $4,234.0$ & $321 \mathrm{WEBR}$ & 14.1 & 43.000000 & Sandstone \\
\hline sld05-22-22add & $4,234.0$ & $4,235.0$ & 321WEBR & 14.3 & 36.000000 & Sandstone \\
\hline sld05-22-22add & $4,235.0$ & $4,236.0$ & 321WEBR & 13.7 & 30.000000 & Sandstone \\
\hline sld05-22-22add & $4,236.0$ & $4,237.0$ & $321 \mathrm{WEBR}$ & 13.5 & 20.000000 & Sandstone \\
\hline s $1 d 05-22-22 a d d$ & $4,237.0$ & $4,238.0$ & 321WEBR & 14.8 & 55.000000 & Sandstone \\
\hline sldo5-22-22add & $4,238.0$ & $4,239.0$ & $321 \mathrm{WEBR}$ & 12.6 & 41.000000 & Sandstone \\
\hline sldo5-22-22add & $4,239.0$ & $4,240.0$ & 321WEBR & 13.8 & 43.000000 & Sandstone \\
\hline sldo5-22-22add & $4,240.0$ & $4,241.0$ & 321WEBR & 14.1 & 36.000000 & Sandstone \\
\hline sld05-22-22add & $4,241.0$ & $4,242.0$ & $321 \mathrm{WEBR}$ & 13.2 & 41.000000 & Sands tone \\
\hline s1d05-22-22add & $4,242.0$ & $4,243.0$ & 321WEBR & 11.4 & 15.000000 & Limy sandstone \\
\hline s1d05-22-22add & $4,243.0$ & $4,244.0$ & $321 \mathrm{WEBR}$ & 9.2 & 2.400000 & Limy sandstone \\
\hline s $1 d 05-22-22 a d d$ & $4,244.0$ & $4,245.0$ & 321WEBR & 11.4 & 16.000000 & Limy sandstone \\
\hline s $1 d 05-22-22 a d d$ & $4,245.0$ & $4,246.0$ & 321WEBR & 11.1 & 15.000000 & Limy sandstone \\
\hline s $1 d 05-22-22 a d d$ & $4,246.0$ & $4,247.0$ & 321WEBR & 10.7 & 56.000000 & Limy sandstone \\
\hline s $1 d 05-22-22 a d d$ & $4,247.0$ & $4,248.0$ & $321 \mathrm{WEBR}$ & 12.7 & 101.000000 & Limy sandstone \\
\hline sldo5-22-22add & $4,248.0$ & $4,249.0$ & $321 \mathrm{WEBR}$ & 12.8 & 39.000000 & Limy sandstone \\
\hline s1d05-22-22add & $4,249.0$ & $4,250.0$ & 321WEBR & 13.1 & 64.000000 & Limy sandstone \\
\hline sld05-22-22add & $4,250.0$ & $4,251.0$ & $321 \mathrm{WEBR}$ & 12.8 & 36.000000 & Limy sandstone \\
\hline s1d05-22-22add & $4,251.0$ & $4,252.0$ & 321WEBR & 9.8 & 13.000000 & Sandstone \\
\hline sld05-22-22add & $4,252.0$ & $4,253.0$ & $321 \mathrm{WEBR}$ & 4.0 & 0.500000 & Limy sandstone \\
\hline s1d05-22-22add & $4,253.0$ & $4,254.0$ & 321WEBR & 2.7 & 0.000000 & Limy sandstone \\
\hline sld05-22-22add & $4,254.0$ & $4,255.0$ & $321 \mathrm{WEBR}$ & 3.5 & 0.000000 & Limy sandstone \\
\hline s1d05-22-22add & $4,255.0$ & $4,256.0$ & $321 \mathrm{WEBR}$ & 3.6 & 0.000000 & Limy sandstone \\
\hline s 1 d05-22-22add & $4,256.0$ & $4,257.0$ & 321WEBR & 4.6 & 0.000000 & Limy sandstone \\
\hline sld05-22-22add & $4,257.0$ & $4,258.0$ & $321 \mathrm{WEBR}$ & 11.3 & 0.100000 & Limy sandstone \\
\hline s $1 d 05-22-22 a d d$ & $4,258.0$ & $4,259.0$ & 321WEBR & 12.4 & 0.000000 & Limy sandstone \\
\hline sld05-22-22add & $4,259.0$ & $4,260.0$ & $321 \mathrm{WEBR}$ & 14.8 & 0.400000 & Limy sandstone \\
\hline sld05-22-22add & $4,260.0$ & $4,261.0$ & $321 \mathrm{WEBR}$ & 12.6 & 0.100000 & Limy sandstone \\
\hline s $1 d 05-22-22$ add & $4,261.0$ & $4,262.0$ & $321 \mathrm{WEBR}$ & 12.7 & 6.200000 & Sands tone \\
\hline s $1 d 05-22-22$ add & $4,262.0$ & $4,263.0$ & 321WEBR & 14.0 & 14.000000 & Sands tone \\
\hline sld05-22-22add & $4,263.0$ & $4,264.0$ & $321 \mathrm{WEBR}$ & 13.1 & 17.000000 & Sands tone \\
\hline sld $05-22-22$ add & $4,264.0$ & $4,265.0$ & 321WEBR & 12.5 & 32.000000 & Sands tone \\
\hline sldo5-22-22add & $4,265.0$ & $4,266.0$ & $321 \mathrm{WEBR}$ & 13.7 & 63.000000 & Sandstone \\
\hline s $1 d 05-22-22 a d d$ & $4,266.0$ & $4,267.0$ & 321WEBR & 12.2 & 31.000000 & Sandstone \\
\hline sldo5-22-22add & $4,267.0$ & $4,268.0$ & 321WEBR & 5.5 & 0.000000 & Limy sandstone \\
\hline s $1 d 05-22-22 a d d$ & $4,268.0$ & $4,269.0$ & $321 \mathrm{WEBR}$ & 11.3 & 11.000000 & Sands tone \\
\hline
\end{tabular}


Table 4.--Laboratory-determined porosity and permeability--Continued

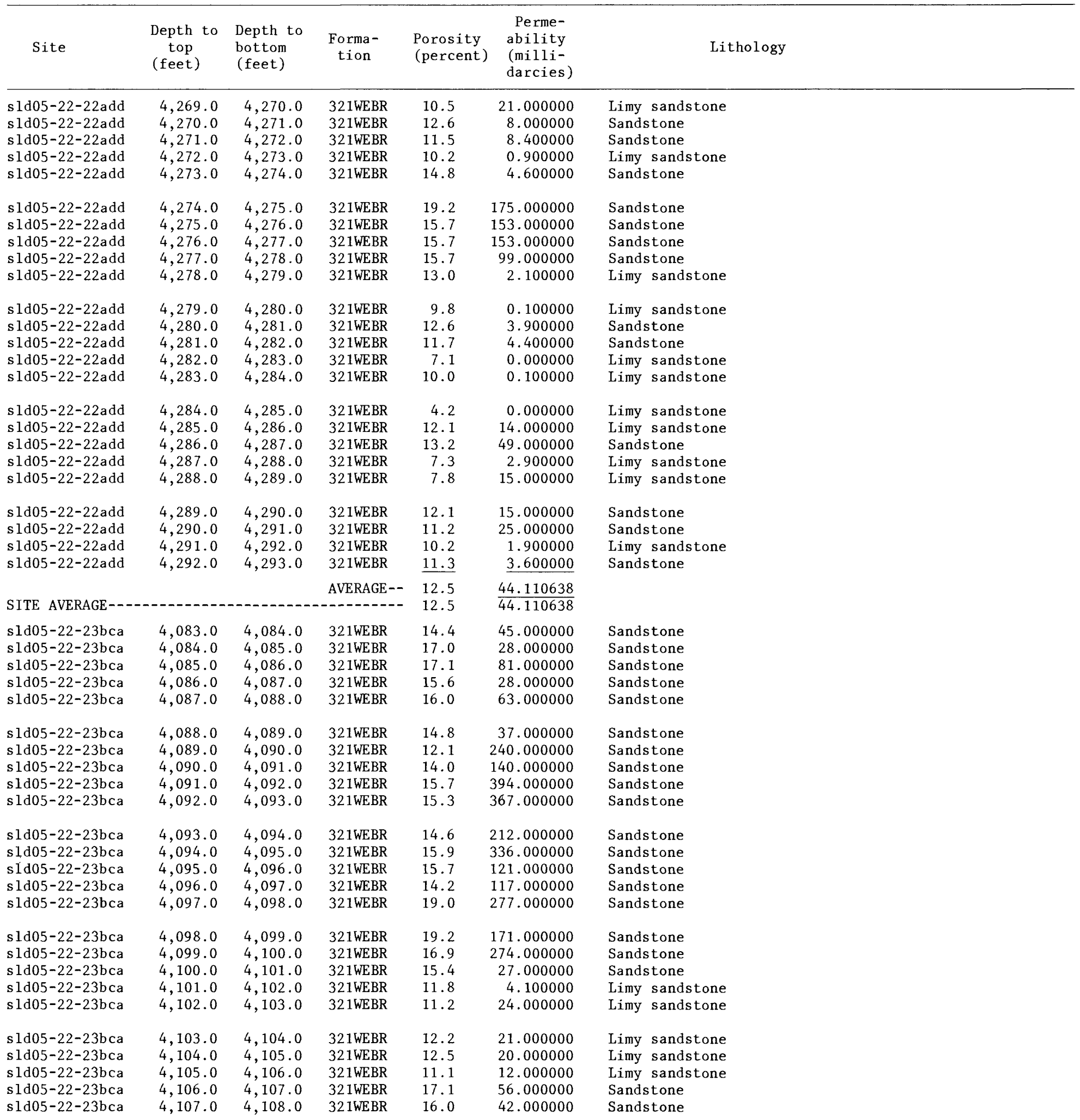


Table 4.--Laboratory-determined porosity and permeability--Continued

\begin{tabular}{|c|c|c|c|c|c|c|}
\hline Site & $\begin{array}{l}\text { Depth to } \\
\text { top } \\
\text { (feet) }\end{array}$ & $\begin{array}{l}\text { Depth to } \\
\text { bottom } \\
\text { (feet) }\end{array}$ & $\begin{array}{c}\text { Forma- } \\
\text { tion }\end{array}$ & $\begin{array}{l}\text { Porosity } \\
\text { (percent) }\end{array}$ & $\begin{array}{l}\text { Perme- } \\
\text { ability } \\
\text { (milli- } \\
\text { darcies) }\end{array}$ & Lithology \\
\hline sld05-22-23bca & $4,108.0$ & $4,109.0$ & $321 \mathrm{WEBR}$ & 15.9 & 34.000000 & Sands tone \\
\hline sld05-22-23bca & $4,109.0$ & $4,110.0$ & 321WEBR & 17.2 & 27.000000 & Sands tone \\
\hline sldo5-22-23bca & $4,110.0$ & $4,111.0$ & $321 \mathrm{WEBR}$ & 17.0 & 47.000000 & Sands tone \\
\hline sld $05-22-23 b c a$ & $4,111.0$ & $4,112.0$ & $321 \mathrm{WEBR}$ & 16.5 & 51.000000 & Sands tone \\
\hline sld $05-22-23 b c a$ & $4,112.0$ & $4,113.0$ & $321 \mathrm{WEBR}$ & 17.2 & 37.000000 & Sands tone \\
\hline sld05-22-23bca & $4,113.0$ & $4,114.0$ & 321WEBR & 16.0 & 26.000000 & Sands tone \\
\hline sld05-22-23bca & $4,114.0$ & $4,115.0$ & $321 \mathrm{WEBR}$ & 14.7 & 27.000000 & Sandstone \\
\hline sld05-22-23bca & $4,115.0$ & $4,116.0$ & $321 \mathrm{WEBR}$ & 12.3 & 26.000000 & Sands tone \\
\hline sld05-22-23bca & $4,116.0$ & $4,117.0$ & $321 \mathrm{WEBR}$ & 2.9 & 0.000000 & Limy sandstone \\
\hline s $1 d 05-22-23 b c a$ & $4,117.0$ & $4,118.0$ & $321 \mathrm{WEBR}$ & 2.0 & 0.000000 & Limy sandstone \\
\hline sld05-22-23bca & $4,118.0$ & $4,119.0$ & 321WEBR & 4.1 & 0.000000 & Limy sandstone \\
\hline sld05-22-23bca & $4,135.0$ & $4,136.0$ & $321 \mathrm{WEBR}$ & 9.4 & 23.000000 & Limy sandstone \\
\hline sld05-22-23bca & $4,136.0$ & $4,137.0$ & $321 \mathrm{WEBR}$ & 7.3 & 4.500000 & Limy sandstone \\
\hline sld05-22-23bca & $4,137.0$ & $4,138.0$ & $321 \mathrm{WEBR}$ & 3.4 & 0.000000 & Limy sandstone \\
\hline sld $05-22-23 b c a$ & $4,138.0$ & $4,139.0$ & $321 \mathrm{WEBR}$ & 11.8 & 59.000000 & Sandstone \\
\hline s1d05-22-23bca & $4,139.0$ & $4,140.0$ & 321WEBR & 11.2 & 9.300000 & Sands tone \\
\hline sld05-22-23bca & $4,140.0$ & $4,141.0$ & $321 \mathrm{WEBR}$ & 9.6 & 6.300000 & Sandstone \\
\hline sld05-22-23bca & $4,141.0$ & $4,142.0$ & $321 \mathrm{WEBR}$ & 11.0 & 2.700000 & Sands tone \\
\hline sld05-22-23bca & $4,142.0$ & $4,143.0$ & $321 \mathrm{WEBR}$ & 12.9 & 26.000000 & Sands tone \\
\hline s1d05-22-23bca & $4,143.0$ & $4,144.0$ & $321 \mathrm{WEBR}$ & 13.5 & 26.000000 & Sands tone \\
\hline sld05-22-23bca & $4,144.0$ & $4,145.0$ & $321 \mathrm{WEBR}$ & 11.3 & 21.000000 & Sandstone \\
\hline sld05-22-23bca & $4,145.0$ & $4,146.0$ & $321 \mathrm{WEBR}$ & 12.1 & 26.000000 & Sands tone \\
\hline s $1 d 05-22-23 b c a$ & $4,146.0$ & $4,147.0$ & 321WEBR & 10.7 & 11.000000 & Sandstone \\
\hline sldo5-22-23bca & $4,147.0$ & $4,148.0$ & $321 \mathrm{WEBR}$ & 5.3 & 0.000000 & Limy sandstone \\
\hline s $1 \mathrm{~d} 05-22-23 \mathrm{bca}$ & $4,148.0$ & $4,149.0$ & $321 \mathrm{WEBR}$ & 0.4 & 0.000000 & Limy sandstone \\
\hline sld05-22-23bca & $4,149.0$ & $4,150.0$ & $321 \mathrm{WEBR}$ & 3.7 & 0.000000 & Limy sandstone \\
\hline sld05-22-23bca & $4,150.0$ & $4,151.0$ & $321 \mathrm{WEBR}$ & 14.5 & 1.900000 & Limy sandstone \\
\hline sld05-22-23bca & $4,151.0$ & $4,152.0$ & $321 \mathrm{WEBR}$ & 9.5 & 0.100000 & Limy sandstone \\
\hline sld05-22-23bca & $4,152.0$ & $4,153.0$ & $321 \mathrm{WEBR}$ & 10.8 & 21.000000 & Limy sandstone \\
\hline sld05-22-23bca & $4,181.0$ & $4,182.0$ & 321WEBR & 10.4 & 2.000000 & Sandstone \\
\hline s $1 d 05-22-23 b c a$ & $4,182.0$ & $4,183.0$ & $321 \mathrm{WEBR}$ & 11.0 & 11.300000 & Sands tone \\
\hline sld05-22-23bca & $4,183.0$ & $4,184.0$ & 321WEBR & 10.7 & 4.400000 & Sands tone \\
\hline s $1 d 05-22-23 b c a$ & $4,184.0$ & $4,185.0$ & $321 \mathrm{WEBR}$ & 11.4 & 3.600000 & Sandstone \\
\hline sldo5-22-23bca & $4,185.0$ & $4,186.0$ & $321 \mathrm{WEBR}$ & 11.9 & 15.000000 & Sandstone \\
\hline s1d05-22-23bca & $4,186.0$ & $4,187.0$ & $321 \mathrm{WEBR}$ & 6.9 & 5.000000 & Limy sandstone \\
\hline sld05-22-23bca & $4,187.0$ & $4,188.0$ & $321 \mathrm{WEBR}$ & 10.3 & 1.000000 & Limy sandstone \\
\hline sld05-22-23bca & $4,188.0$ & $4,189.0$ & 321WEBR & 7.7 & 0.000000 & Limy sandstone \\
\hline s $1 d 05-22-23 b c a$ & $4,189.0$ & $4,190.0$ & $321 \mathrm{WEBR}$ & 11.8 & 0.900000 & Limy sandstone \\
\hline sld05-22-23bca & $4,190.0$ & $4,191.0$ & $321 \mathrm{WEBR}$ & 11.6 & 1.100000 & Limy sandstone \\
\hline sld05-22-23bca & $4,191.0$ & $4,192.0$ & $321 \mathrm{WEBR}$ & 10.8 & 0.900000 & Limy sandstone \\
\hline sld05-22-23bca & $4,192.0$ & $4,193.0$ & $321 \mathrm{WEBR}$ & 11.5 & 0.700000 & Limy sandstone \\
\hline sld05-22-23bca & $4,193.0$ & $4,194.0$ & $321 \mathrm{WEBR}$ & 11.9 & 0.600000 & Limy sandstone \\
\hline sld05-22-23bca & $4,194.0$ & $4,195.0$ & $321 \mathrm{WEBR}$ & 7.9 & 0.000000 & Sands tone \\
\hline sldo5-22-23bca & $4,195.0$ & $4,196.0$ & $321 \mathrm{WEBR}$ & 10.2 & 0.400000 & Sands tone \\
\hline sld05-22-23bca & $4,196.0$ & $4,197.0$ & $321 \mathrm{WE} B R$ & 12.8 & 6.600000 & Sandstone \\
\hline sld05-22-23bca & $4,197.0$ & $4,198.0$ & 321WEBR & 10.2 & 0.700000 & Sands tone \\
\hline s $1 d 05-22-23 b c a$ & $4,198.0$ & $4,199.0$ & $321 \mathrm{WEBR}$ & 12.0 & 9.100000 & Sands tone \\
\hline sld05-22-23bca & $4,199.0$ & $4,200.0$ & $321 \mathrm{WEBR}$ & 12.7 & 4.100000 & Sands tone \\
\hline s $1 d 05-22-23 b c a$ & $4,200.0$ & $4,201.0$ & 321WEBR & 11.4 & 1.000000 & Sandstone \\
\hline sld05-22-23bca & $4,201.0$ & $4,202.0$ & $321 \mathrm{WEBR}$ & 11.2 & 0.300000 & Sandstone \\
\hline
\end{tabular}


Table 4.--Laboratory-determined porosity and permeability--Continued

\begin{tabular}{|c|c|c|c|c|c|c|}
\hline Site & $\begin{array}{l}\text { Depth to } \\
\text { top } \\
\text { (feet) }\end{array}$ & $\begin{array}{l}\text { Depth to } \\
\text { bottom } \\
\text { (feet) }\end{array}$ & $\begin{array}{c}\text { Forma- } \\
\text { tion }\end{array}$ & $\begin{array}{l}\text { Porosity } \\
\text { (percent) }\end{array}$ & $\begin{array}{l}\text { Perme- } \\
\text { ability } \\
\text { (milli- } \\
\text { darcies) }\end{array}$ & Lithology \\
\hline sld $05-22-23 b c a$ & $4,202.0$ & $4,203.0$ & 321WEBR & 10.2 & 0.100000 & Limy sandstone \\
\hline sld05-22-23bca & $4,203.0$ & $4,204.0$ & 321WEBR & 10.2 & 0.100000 & Limy sandstone \\
\hline s $1 d 05-22-23 b c a$ & $4,204.0$ & $4,205.0$ & 321WEBR & 9.6 & 3.100000 & Limy sandstone \\
\hline s $1 d 05-22-23 b c a$ & $4,205.0$ & $4,206.0$ & $321 \mathrm{WEBR}$ & 7.6 & 2.000000 & Limy sandstone \\
\hline s $1 d 05-22-23 b c a$ & $4,206.0$ & $4,207.0$ & $321 \mathrm{WEBR}$ & 5.6 & 0.000000 & Limy sandstone \\
\hline sld05-22-23bca & $4,207.0$ & $4,208.0$ & 321WEBR & 4.9 & 0.000000 & Limy sandstone \\
\hline s1d05-22-23bca & $4,208.0$ & $4,209.0$ & $321 \mathrm{WEBR}$ & 4.3 & 0.000000 & Limy sandstone \\
\hline s $1 d 05-22-23 b c a$ & $4,209.0$ & $4,210.0$ & 321 WEBR & 8.2 & 0.000000 & Limy sandstone \\
\hline s 1 d $05-22-23 b c a$ & $4,210.0$ & $4,211.0$ & 321WEBR & 6.9 & 0.000000 & Limy sandstone \\
\hline s $1 d 05-22-23 b c a$ & $4,211.0$ & $4,212.0$ & $321 \mathrm{WEBR}$ & 8.4 & 0.000000 & Limy sandstone \\
\hline s $1 d 05-22-23 b c a$ & $4,212.0$ & $4,213.0$ & 321WEBR & 8.8 & 0.000000 & Limy sandstone \\
\hline sld $05-22-23 \mathrm{bca}$ & $4,213.0$ & $4,214.0$ & $321 \mathrm{WEBR}$ & 6.2 & 0.000000 & Limy sandstone \\
\hline s 1 d05-22-23bca & $4,214.0$ & $4,215.0$ & 321WEBR & 8.3 & 0.000000 & Limy sandstone \\
\hline sld $05-22-23 b c a$ & $4,215.0$ & $4,216.0$ & $321 \mathrm{WEBR}$ & 8.3 & 0.000000 & Limy sandstone \\
\hline sld $05-22-23 b c a$ & $4,216.0$ & $4,217.0$ & 321WEBR & 12.1 & 0.300000 & Limy sandstone \\
\hline s $1 d 05-22-23 b c a$ & $4,217.0$ & $4,218.0$ & $321 \mathrm{WEBR}$ & 11.8 & 1.400000 & Sandstone \\
\hline sld05-22-23bca & $4,218.0$ & $4,219.0$ & 321WEBR & 6.9 & 0.000000 & Limy sandstone \\
\hline sldo5-22-23bca & $4,219.0$ & $4,220.0$ & $321 \mathrm{WEBR}$ & 10.3 & 0.100000 & Limy sandstone \\
\hline s $1 d 05-22-23 b c a$ & $4,220.0$ & $4,221.0$ & $321 \mathrm{WEBR}$ & 8.0 & 0.400000 & Limy sandstone \\
\hline sldo5-22-23bca & $4,221.0$ & $4,222.0$ & $321 \mathrm{WEBR}$ & 11.0 & 12.000000 & Limy sandstone \\
\hline s1d05-22-23bca & $4,222.0$ & $4,223.0$ & 321WEBR & 10.2 & 1.800000 & Limy sandstone \\
\hline s 1 do $05-22-23$ bca & $4,223.0$ & $4,224.0$ & $321 \mathrm{WEBR}$ & 9.0 & 0.500000 & Limy sandstone \\
\hline sld05-22-23bca & $4,224.0$ & $4,225.0$ & $321 \mathrm{WEBR}$ & 9.8 & 0.700000 & Limy sandstone \\
\hline s $1 d 05-22-23 b c a$ & $4,225.0$ & $4,226.0$ & $321 \mathrm{WEBR}$ & 12.7 & 0.500000 & Limy sandstone \\
\hline s1d05-22-23bca & $4,235.0$ & $4,236.0$ & $321 \mathrm{WEBR}$ & 14.3 & 9.200000 & Sandstone \\
\hline sld05-22-23bca & $4,236.0$ & $4,237.0$ & 321WEBR & 14.8 & 28.000000 & Sandstone \\
\hline s1d05-22-23bca & $4,237.0$ & $4,238.0$ & $321 \mathrm{WEBR}$ & 14.2 & 11.000000 & Sandstone \\
\hline sld $05-22-23 b c a$ & $4,238.0$ & $4,239.0$ & 321WEBR & 16.3 & 49.000000 & Sandstone \\
\hline s 1 do5-22-23bca & $4,239.0$ & $4,240.0$ & $321 \mathrm{WEBR}$ & 13.0 & 5.500000 & Limy sandstone \\
\hline s1d05-22-23bca & $4,240.0$ & $4,241.0$ & $321 \mathrm{WEBR}$ & 11.0 & 1.700000 & Sandstone \\
\hline sldo5-22-23bca & $4,241.0$ & $4,242.0$ & 321WEBR & 12.3 & 3.300000 & Limy sandstone \\
\hline s 1 d $05-22-23$ bca & $4,242.0$ & $4,243.0$ & $321 \mathrm{WEBR}$ & 12.1 & 3.500000 & Sandstone \\
\hline sldo5-22-23bca & $4,243.0$ & $4,244.0$ & $321 \mathrm{WEBR}$ & 11.7 & 3.900000 & Limy sandstone \\
\hline sldo5-22-23bca & $4,244.0$ & $4,245.0$ & $321 \mathrm{WEBR}$ & 13.4 & 12.000000 & Sandstone \\
\hline s $1 d 05-22-23 b c a$ & $4,245.0$ & $4,246.0$ & $321 \mathrm{WEBR}$ & 12.2 & 7.600000 & Sandstone \\
\hline sldo5-22-23bca & $4,246.0$ & $4,247.0$ & $321 \mathrm{WEBR}$ & 14.1 & 7.000000 & Sandstone \\
\hline s 1 d05-22-23bca & $4,247.0$ & $4,248.0$ & $321 \mathrm{WEBR}$ & 12.6 & 2.100000 & Sandstone \\
\hline s 1 d05-22-23bca & $4,248.0$ & $4,249.0$ & $321 \mathrm{WEBR}$ & 12.8 & 17.000000 & Sandstone \\
\hline s 1 d05-22-23bca & $4,249.0$ & $4,250.0$ & $321 \mathrm{WEBR}$ & 14.1 & 63.000000 & Sandstone \\
\hline sldo5-22-23bca & $4,250.0$ & $4,251.0$ & $321 \mathrm{WEBR}$ & 15.3 & 10.000000 & Sandstone \\
\hline sldo5-22-23bca & $4,251.0$ & $4,252.0$ & $321 \mathrm{WEBR}$ & 12.7 & 111.000000 & Sandstone \\
\hline s1d05-22-23bca & $4,252.0$ & $4,253.0$ & $321 \mathrm{WEBR}$ & 15.0 & 28.000000 & Sandstone \\
\hline sldo5-22-23bca & $4,253.0$ & $4,254.0$ & $321 \mathrm{WEBR}$ & 18.3 & 38.000000 & Sandstone \\
\hline sldo5-22-23bca & $4,254.0$ & $4,255.0$ & $321 \mathrm{WEBR}$ & 15.8 & 37.000000 & Sands tone \\
\hline s1d05-22-23bca & $4,255.0$ & $4,256.0$ & $321 \mathrm{WEBR}$ & 13.6 & 7.800000 & Sandstone \\
\hline sldo5-22-23bca & $4,256.0$ & $4,257.0$ & $321 \mathrm{WEBR}$ & 15.9 & 23.000000 & Sandstone \\
\hline s1d05-22-23bca & $4,257.0$ & $4,258.0$ & $321 \mathrm{WEBR}$ & 8.8 & 0.100000 & Limestone and sandstone \\
\hline s 1 d05-22-23bca & $4,258.0$ & $4,259.0$ & $321 \mathrm{WE} B R$ & 12.6 & 17.000000 & Limestone and sandstone \\
\hline s1d05-22-23bca & $4,259.0$ & $4,260.0$ & $321 \mathrm{WEBR}$ & 12.6 & 3.200000 & Limestone and sandstone \\
\hline s1d05-22-23bca & $4,260.0$ & $4,261.0$ & $321 \mathrm{WEBR}$ & 19.0 & 40.000000 & Limestone and sandstone \\
\hline
\end{tabular}


Table 4.--Laboratory-determined porosity and permeability--Continued

\begin{tabular}{|c|c|c|c|c|c|c|}
\hline Site & $\begin{array}{l}\text { Depth to } \\
\text { top } \\
\text { (feet) }\end{array}$ & $\begin{array}{l}\text { Depth to } \\
\text { bottom } \\
\text { (feet) }\end{array}$ & $\begin{array}{c}\text { Forma- } \\
\text { tion }\end{array}$ & $\begin{array}{l}\text { Porosity } \\
\text { (percent) }\end{array}$ & $\begin{array}{c}\text { Perme- } \\
\text { ability } \\
(\text { milli- } \\
\text { darcies) }\end{array}$ & Lithology \\
\hline $\begin{array}{l}\text { sld05-22-23bca } \\
\text { sld05-22-23bca } \\
\text { sld05-22-23bca } \\
\text { sld05-22-23bca } \\
\text { sld05-22-23bca }\end{array}$ & $\begin{array}{l}4,261.0 \\
4,262.0 \\
4,263.0 \\
4,264.0 \\
4,265.0\end{array}$ & $\begin{array}{l}4,262.0 \\
4,263.0 \\
4,264.0 \\
4,265.0 \\
4,266.0\end{array}$ & $\begin{array}{l}321 \mathrm{WEBR} \\
321 \mathrm{WEBR} \\
321 \mathrm{WEBR} \\
321 \mathrm{WEBR} \\
\text { 321WEBR }\end{array}$ & $\begin{array}{r}11.2 \\
8.7 \\
13.0 \\
14.6 \\
14.1\end{array}$ & $\begin{array}{r}2.900000 \\
3.600000 \\
45.000000 \\
26.000000 \\
28.000000\end{array}$ & $\begin{array}{l}\text { Sands tone } \\
\text { Limy sands tone } \\
\text { Sands tone } \\
\text { Sands tone } \\
\text { Sands tone }\end{array}$ \\
\hline $\begin{array}{l}\text { sld05-22-23bca } \\
\text { sld05-22-23bca } \\
\text { sld05-22-23bca }\end{array}$ & $\begin{array}{l}4,266.0 \\
4,267.0 \\
4,268.0\end{array}$ & $\begin{array}{l}4,267.0 \\
4,268.0 \\
4,269.0\end{array}$ & $\begin{array}{l}\text { 321WEBR } \\
\text { 321WEBR } \\
\text { 321WEBR }\end{array}$ & $\begin{array}{l}12.8 \\
10.0 \\
20.3 \\
\end{array}$ & $\begin{array}{l}55.000000 \\
13.000000 \\
51.000000 \\
\end{array}$ & $\begin{array}{l}\text { Sands tone } \\
\text { Sands tone } \\
\text { Sands tone }\end{array}$ \\
\hline SITE AVERAGE--- & 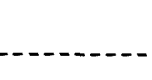 & $--1-n$ & AVERAGE-- & $\begin{array}{l}11.9 \\
11.9\end{array}$ & $\begin{array}{l}33.872180 \\
33.872180\end{array}$ & \\
\hline $\begin{array}{l}\text { sld05-22-23cba } \\
\text { sld05-22-23cba } \\
\text { sld05-22-23cba } \\
\text { sld05-22-23cba } \\
\text { sld05-22-23cba }\end{array}$ & $\begin{array}{l}4,053.0 \\
4,054.0 \\
4,055.0 \\
4,056.0 \\
4,057.0\end{array}$ & $\begin{array}{l}4,054.0 \\
4,055.0 \\
4,056.0 \\
4,057.0 \\
4,058.0\end{array}$ & $\begin{array}{l}\text { 317PRKC } \\
317 \mathrm{PRKC} \\
317 \mathrm{PRKC} \\
317 \mathrm{PRKC} \\
317 \mathrm{PRKC}\end{array}$ & $\begin{array}{r}7.2 \\
10.9 \\
8.4 \\
10.3 \\
10.2\end{array}$ & $\begin{array}{l}1.760000 \\
3.800000 \\
5.100000 \\
8.400000 \\
0.350000\end{array}$ & $\begin{array}{l}\text { Sandy dolomite } \\
\text { Sandy dolomite } \\
\text { Sandy dolomite } \\
\text { Sandy dolomite } \\
\text { Sandy dolomite }\end{array}$ \\
\hline $\begin{array}{l}\text { sld05-22-23cba } \\
\text { sld05-22-23cba } \\
\text { sld05-22-23cba } \\
\text { sld05-22-23cba } \\
\text { sld05-22-23cba }\end{array}$ & $\begin{array}{l}4,058.0 \\
4,059.0 \\
4,060.0 \\
4,061.0 \\
4,062.0\end{array}$ & $\begin{array}{l}4,059.0 \\
4,060.0 \\
4,061.0 \\
4,062.0 \\
4,063.0\end{array}$ & $\begin{array}{l}\text { 317PRKC } \\
\text { 317PRKC } \\
\text { 317PRKC } \\
\text { 317PRKC } \\
\text { 317PRKC } \\
\text { AVERAGE-- }\end{array}$ & $\begin{array}{l}7.3 \\
7.1 \\
6.7 \\
5.3 \\
7.2 \\
8.1\end{array}$ & $\begin{array}{l}0.250000 \\
0.300000 \\
0.120000 \\
0.000000 \\
0.350000 \\
2.043000\end{array}$ & $\begin{array}{l}\text { Sandy dolomite } \\
\text { Sandy dolomite } \\
\text { Sandy dolomite } \\
\text { Dolomite } \\
\text { Sandy dolomite }\end{array}$ \\
\hline $\begin{array}{l}\text { sld } 05-22-23 \mathrm{cba} \\
\text { sld05-22-23cba } \\
\text { sld } 05-22-23 \mathrm{cba} \\
\text { sld05-22-23cba } \\
\text { sld05-22-23cba }\end{array}$ & $\begin{array}{l}4,097.0 \\
4,098.0 \\
4,099.0 \\
4,100.0 \\
4,101.0\end{array}$ & $\begin{array}{l}4,098.0 \\
4,099.0 \\
4,100.0 \\
4,101.0 \\
4,102.0\end{array}$ & $\begin{array}{l}\text { 321WEBR } \\
321 \mathrm{WEBR} \\
321 \mathrm{WEBR} \\
\text { 321WEBR } \\
\text { 321WEBR }\end{array}$ & $\begin{array}{l}15.8 \\
13.0 \\
14.9 \\
15.8 \\
11.4\end{array}$ & $\begin{array}{r}48.000000 \\
21.000000 \\
44.000000 \\
68.000000 \\
9.500000\end{array}$ & $\begin{array}{l}\text { Sands tone } \\
\text { Sands tone } \\
\text { Sands tone } \\
\text { Sands tone } \\
\text { Sands tone }\end{array}$ \\
\hline $\begin{array}{l}\text { sld05-22-23cba } \\
\text { sld05-22-23cba } \\
\text { sld05-22-23cba } \\
\text { sld05-22-23cba } \\
\text { sld05-22-23cba }\end{array}$ & $\begin{array}{l}4,102.0 \\
4,103.0 \\
4,104.0 \\
4,105.0 \\
4,106.0\end{array}$ & $\begin{array}{l}4,103.0 \\
4,104.0 \\
4,105.0 \\
4,106.0 \\
4,107.0\end{array}$ & $\begin{array}{l}\text { 321WEBR } \\
321 \mathrm{WEBR} \\
321 \mathrm{WEBR} \\
321 \mathrm{WEBR} \\
\text { 321WEBR }\end{array}$ & $\begin{array}{l}14.5 \\
14.2 \\
14.5 \\
14.6 \\
13.2\end{array}$ & $\begin{array}{l}18.000000 \\
18.000000 \\
54.000000 \\
58.000000 \\
41.000000\end{array}$ & $\begin{array}{l}\text { Sands tone } \\
\text { Sands tone } \\
\text { Sands tone } \\
\text { Sands tone } \\
\text { Sands tone }\end{array}$ \\
\hline $\begin{array}{l}\text { sld } 05-22-23 \mathrm{cba} \\
\text { sld05-22-23cba } \\
\text { sld05-22-23cba } \\
\text { sld05-22-23cba } \\
\text { sld05-22-23cba }\end{array}$ & $\begin{array}{l}4,112.0 \\
4,113.0 \\
4,114.0 \\
4,115.0 \\
4,116.0\end{array}$ & $\begin{array}{l}4,113.0 \\
4,114.0 \\
4,115.0 \\
4,116.0 \\
4,117.0\end{array}$ & $\begin{array}{l}\text { 321WEBR } \\
\text { 321WEBR } \\
\text { 321WEBR } \\
\text { 321WEBR } \\
\text { 321WEBR }\end{array}$ & $\begin{array}{l}13.2 \\
13.4 \\
14.7 \\
15.0 \\
14.9\end{array}$ & $\begin{array}{r}136.000000 \\
380.000000 \\
94.000000 \\
94.000000 \\
94.000000\end{array}$ & $\begin{array}{l}\text { Sands tone } \\
\text { Sands tone } \\
\text { Sands tone } \\
\text { Sands tone } \\
\text { Sands tone }\end{array}$ \\
\hline $\begin{array}{l}\text { sld } 05-22-23 \mathrm{cba} \\
\text { sld05-22-23cba } \\
\text { sld } 05-22-23 \mathrm{cba} \\
\text { sld } 05-22-23 \mathrm{cba} \\
\text { sld05-22-23cba }\end{array}$ & $\begin{array}{l}4,117.0 \\
4,118.0 \\
4,119.0 \\
4,120.0 \\
4,121.0\end{array}$ & $\begin{array}{l}4,118.0 \\
4,119.0 \\
4,120.0 \\
4,121.0 \\
4,122.0\end{array}$ & $\begin{array}{l}\text { 321WEBR } \\
321 \mathrm{WEBR} \\
321 \mathrm{WEBR} \\
321 \mathrm{WEBR} \\
\text { 321WEBR }\end{array}$ & $\begin{array}{l}15.8 \\
15.3 \\
16.2 \\
15.0 \\
13.8\end{array}$ & $\begin{array}{l}220.000000 \\
105.000000 \\
300.000000 \\
105.000000 \\
170.000000\end{array}$ & $\begin{array}{l}\text { Sands tone } \\
\text { Sands tone } \\
\text { Sands tone } \\
\text { Sands tone } \\
\text { Sands tone }\end{array}$ \\
\hline $\begin{array}{l}\text { sld } 05-22-23 \mathrm{cba} \\
\text { sld05-22-23cba } \\
\text { sld05-22-23cba } \\
\text { sld } 05-22-23 \mathrm{cba} \\
\text { sld05-22-23cba }\end{array}$ & $\begin{array}{l}4,123.0 \\
4,124.0 \\
4,125.0 \\
4,126.0 \\
4,127.0\end{array}$ & $\begin{array}{l}4,124.0 \\
4,125.0 \\
4,126.0 \\
4,127.0 \\
4,128.0\end{array}$ & $\begin{array}{l}\text { 321WEBR } \\
321 \mathrm{WEBR} \\
321 \mathrm{WEBR} \\
321 \mathrm{WEBR} \\
\text { 321WEBR }\end{array}$ & $\begin{array}{l}14.3 \\
13.5 \\
16.7 \\
14.6 \\
16.0\end{array}$ & $\begin{array}{r}136.000000 \\
44.000000 \\
75.000000 \\
62.000000 \\
220.000000\end{array}$ & $\begin{array}{l}\text { Sands tone } \\
\text { Sands tone } \\
\text { Sands tone } \\
\text { Sands tone } \\
\text { Sands tone }\end{array}$ \\
\hline $\begin{array}{l}\text { sld } 05-22-23 \mathrm{cba} \\
\text { sld05-22-23cba } \\
\text { sld } 05-22-23 \mathrm{cba} \\
\text { sld } 05-22-23 \mathrm{cba} \\
\text { sld05-22-23cba }\end{array}$ & $\begin{array}{l}4,128.0 \\
4,129.0 \\
4,130.0 \\
4,131.0 \\
4,132.0\end{array}$ & $\begin{array}{l}4,129.0 \\
4,130.0 \\
4,131.0 \\
4,132.0 \\
4,133.0\end{array}$ & $\begin{array}{l}\text { 321WEBR } \\
321 \mathrm{WEBR} \\
321 \mathrm{WEBR} \\
321 \mathrm{WEBR} \\
321 \mathrm{WEBR}\end{array}$ & $\begin{array}{l}12.5 \\
13.4 \\
11.5 \\
14.2 \\
13.9\end{array}$ & $\begin{array}{l}29.000000 \\
33.000000 \\
44.000000 \\
38.000000 \\
58.000000\end{array}$ & $\begin{array}{l}\text { Sands tone } \\
\text { Sands tone } \\
\text { Sands tone } \\
\text { Sands tone } \\
\text { Sands tone }\end{array}$ \\
\hline
\end{tabular}


Table 4.--Laboratory-determined porosity and permeability--Continued

\begin{tabular}{|c|c|c|c|c|c|c|}
\hline Site & $\begin{array}{l}\text { Depth to } \\
\text { top } \\
\text { (feet) }\end{array}$ & $\begin{array}{l}\text { Depth to } \\
\text { bottom } \\
\text { (feet) }\end{array}$ & $\begin{array}{c}\text { Forma- } \\
\text { tion }\end{array}$ & $\begin{array}{l}\text { Porosity } \\
\text { (percent) }\end{array}$ & $\begin{array}{c}\text { Perme- } \\
\text { ability } \\
\text { (milli- } \\
\text { darcies) }\end{array}$ & Lithology \\
\hline $\mathrm{s} 1 \mathrm{~d} 05-22-23 \mathrm{cba}$ & $4,133.0$ & $4,134.0$ & $321 \mathrm{WEBR}$ & 14.7 & 38.000000 & Sandstone \\
\hline sld $05-22-23 \mathrm{cba}$ & $4,134.0$ & $4,135.0$ & $321 \mathrm{WEBR}$ & 13.7 & 62.000000 & Sands tone \\
\hline sld $05-22-23 \mathrm{cba}$ & $4,135.0$ & $4,136.0$ & $321 \mathrm{WEBR}$ & 16.2 & 83.000000 & Sandstone \\
\hline sld $05-22-23 \mathrm{cba}$ & $4,136.0$ & $4,137.0$ & 321WEBR & 16.0 & 125.000000 & Sandstone \\
\hline s $1 d 05-22-23 c b a$ & $4,137.0$ & $4,138.0$ & $321 \mathrm{WEBR}$ & 16.0 & 72.000000 & Sandstone \\
\hline s $1 \mathrm{~d} 05-22-23 \mathrm{cba}$ & $4,138.0$ & $4,139.0$ & $321 \mathrm{WEBR}$ & 15.8 & 105.000000 & Sandstone \\
\hline s $1 d 05-22-23 \mathrm{cba}$ & $4,139.0$ & $4,140.0$ & 321WEBR & 15.8 & 83.000000 & Sandstone \\
\hline $\mathrm{s} 1 \mathrm{~d} 05-22-23 \mathrm{cba}$ & $4,140.0$ & $4,141.0$ & $321 \mathrm{WEBR}$ & 14.7 & 75.000000 & Sandstone \\
\hline s 1 d $05-22-23 \mathrm{cba}$ & $4,141.0$ & $4,142.0$ & $321 \mathrm{WEBR}$ & 15.3 & 79.000000 & Sandstone \\
\hline s $1 d 05-22-23 c b a$ & $4,142.0$ & $4,143.0$ & $321 \mathrm{WEBR}$ & 16.5 & 100.000000 & Sandstone \\
\hline $\mathrm{s} 1 \mathrm{~d} 05-22-23 \mathrm{cba}$ & $4,143.0$ & $4,144.0$ & 321WEBR & 14.0 & 72.000000 & Sandstone \\
\hline s $1 d 05-22-23 \mathrm{cba}$ & $4,144.0$ & $4,145.0$ & 321WEBR & 12.6 & 42.000000 & Sandstone \\
\hline sld05-22-23cba & $4,145.0$ & $4,146.0$ & $321 \mathrm{WEBR}$ & 12.8 & 27.000000 & Sandstone \\
\hline sld05-22-23cba & $4,146.0$ & $4,147.0$ & 321WEBR & 12.2 & 22.000000 & Sandstone \\
\hline s $1 d 05-22-23 c b a$ & $4,147.0$ & $4,148.0$ & $321 \mathrm{WEBR}$ & 14.5 & 66.000000 & Sandstone \\
\hline s 1 d05-22-23cba & $4,148.0$ & $4,149.0$ & 321 WEBR & 11.8 & 8.000000 & Sandstone \\
\hline s $1 \mathrm{~d} 05-22-23 \mathrm{cba}$ & $4,149.0$ & $4,150.0$ & 321WEBR & 13.3 & 24.000000 & Sands tone \\
\hline s $1 \mathrm{~d} 05-22-23 \mathrm{cba}$ & $4,150.0$ & $4,151.0$ & $321 \mathrm{WEBR}$ & 12.1 & 33.000000 & Sands tone \\
\hline sId $05-22-23 \mathrm{cba}$ & $4,151.0$ & $4,152.0$ & 32 IWEBR & 14.7 & 66.000000 & Sands tone \\
\hline s1d05-22-23cba & $4,152.0$ & $4,153.0$ & $321 \mathrm{WEBR}$ & 14.7 & 83.000000 & Sandstone \\
\hline $\mathrm{s} 1 \mathrm{~d} 05-22-23 \mathrm{cba}$ & $4,153.0$ & $4,154.0$ & $321 \mathrm{WEBR}$ & 14.9 & 50.000000 & Sandstone \\
\hline s1d05-22-23cba & $4,154.0$ & $4,155.0$ & $321 \mathrm{WEBR}$ & 14.7 & 72.000000 & Sandstone \\
\hline s1d05-22-23cba & $4,155.0$ & $4,156.0$ & 321WEBR & 16.1 & 50.000000 & Sandstone \\
\hline s $1 d 05-22-23 \mathrm{cba}$ & $4,156.0$ & $4,157.0$ & 321WEBR & 13.5 & 47.000000 & Sandstone \\
\hline sld $05-22-23 \mathrm{cba}$ & $4,157.0$ & $4,158.0$ & $321 \mathrm{WEBR}$ & 15.5 & 94.000000 & Sandstone \\
\hline s1d05-22-23cba & $4,158.0$ & $4,159.0$ & $321 \mathrm{WEBR}$ & 15.0 & 88.000000 & Sandstone \\
\hline s $1 d 05-22-23 c b a$ & $4,159.0$ & $4,160.0$ & $321 \mathrm{WEBR}$ & 15.7 & 83.000000 & Sands tone \\
\hline s $1 d 05-22-23 \mathrm{cba}$ & $4,160.0$ & $4,161.0$ & 321WEBR & 16.3 & 88.000000 & Sandstone \\
\hline s1d05-22-23cba & $4,161.0$ & $4,162.0$ & $321 \mathrm{WEBR}$ & 11.8 & 79.000000 & Sandstone \\
\hline s1d05-22-23cba & $4,162.0$ & $4,163.0$ & $321 \mathrm{WEBR}$ & 12.8 & 75.000000 & Sandstone \\
\hline s1d05-22-23cba & $4,163.0$ & $4,164.0$ & $321 \mathrm{WEBR}$ & 14.0 & 68.000000 & Sandstone \\
\hline s $1 d 05-22-23 c b a$ & $4,164.0$ & $4,165.0$ & $321 \mathrm{WEBR}$ & 14.3 & 47.000000 & Sands tone \\
\hline s $1 d 05-22-23 c b a$ & $4,165.0$ & $4,166.0$ & $321 W E B R$ & 10.8 & 33.000000 & Sandstone \\
\hline SITE AVERAGE-- & 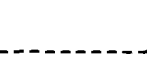 & - -2 & AVERAGE-- & $\frac{14.3}{13.5}$ & $\frac{78.658730}{68.163425}$ & \\
\hline sld $05-23-18 \mathrm{cca}$ & $4,117.0$ & $4,118.0$ & 317 PRKC & 2.9 & 0.000000 & Sucrose limestone \\
\hline s1d05-23-18cca & $4,118.0$ & $4,119.0$ & 317PRKC & 2.8 & 0.000000 & Sucrose limestone \\
\hline s 1 d0 $05-23-18$ cca & $4,119.0$ & $4,120.0$ & 317PRKC & 2.6 & 0.000000 & Sucrose limestone \\
\hline sld $05-23-18 \mathrm{cca}$ & $4,120.0$ & $4,121.0$ & 317 PRKC & 5.8 & 0.000000 & Sucrose limestone \\
\hline s1d05-23-18cca & $4,121.0$ & $4,122.0$ & $317 \mathrm{PRKC}$ & 3.1 & 0.000000 & Fine-grained, dolomitic limestone \\
\hline s $1 \mathrm{~d} 05-23-18 \mathrm{cca}$ & $4,122.0$ & $4,123.0$ & 317PRKC & 2.4 & 0.000000 & Fine-grained, dolomitic limestone \\
\hline s $1 \mathrm{~d} 05-23-18 \mathrm{cca}$ & $4,123.0$ & $4,124.0$ & $317 \mathrm{PRKC}$ & 3.1 & 0.000000 & Fine-grained, dolomitic limestone \\
\hline sld $05-23-18 \mathrm{cca}$ & $4,124.0$ & $4,125.0$ & $317 \mathrm{PRKC}$ & 2.1 & 0.000000 & Fine-grained, dolomitic limestone \\
\hline sld05-23-18cca & $4,125.0$ & $4,126.0$ & 317PRKC & 2.4 & 0.000000 & Fine-grained, dolomitic limestone \\
\hline sld05-23-18cca & $4,126.0$ & $4,127.0$ & 317PRKC & 2.3 & 0.000000 & Fine-grained, dolomitic limestone \\
\hline sld $05-23-18 c c a$ & $4,127.0$ & $4,128.0$ & $317 \mathrm{PRKC}$ & 3.3 & 0.000000 & Fine-grained to sandy limestone \\
\hline s $1 \mathrm{~d} 05-23-18 \mathrm{cca}$ & $4,128.0$ & $4,129.0$ & 317 PRKC & 3.9 & 0.000000 & Fine-grained to sandy limestone \\
\hline s 1 d $05-23-18$ cca & $4,129.0$ & $4,130.0$ & 317 PRKC & 2.7 & 0.000000 & Fine-grained to sandy limestone \\
\hline s 1 d $05-23-18 \mathrm{cca}$ & $4,156.0$ & $4,157.0$ & 317 PRKC & 3.5 & 0.000000 & Finely crystalline to sandy limestone \\
\hline sld $05-23-18$ cca & $4,157.0$ & $4,158.0$ & $317 \mathrm{PRKC}$ & 2.4 & 0.000000 & Finely crystalline to sandy limestone \\
\hline
\end{tabular}


Table 4.--Laboratory-determined porosity and permeability--Continued

\begin{tabular}{|c|c|c|c|c|c|c|}
\hline Site & $\begin{array}{l}\text { Depth to } \\
\text { top } \\
\text { (feet) }\end{array}$ & $\begin{array}{l}\text { Depth to } \\
\text { bottom } \\
\text { (feet) }\end{array}$ & $\begin{array}{c}\text { Forma- } \\
\text { tion }\end{array}$ & $\begin{array}{l}\text { Porosity } \\
\text { (percent) }\end{array}$ & $\begin{array}{l}\text { Perme- } \\
\text { ability } \\
\text { (milli- } \\
\text { darcies) }\end{array}$ & Lithology \\
\hline $\begin{array}{l}\text { sld05-23-18cca } \\
\text { sld05-23-18cca } \\
\text { sld05-23-18cca } \\
\text { sld05-23-18cca } \\
\text { sld05-23-18cca }\end{array}$ & $\begin{array}{l}4,158 \cdot 0 \\
4,159 \cdot 0 \\
4,160 \cdot 0 \\
4,161 \cdot 0 \\
4,162 \cdot 0\end{array}$ & $\begin{array}{l}4,179.0 \\
4,160.0 \\
4,161.0 \\
4,162.0 \\
4,163.0\end{array}$ & $\begin{array}{l}317 \text { PRKC } \\
317 \text { PRKC } \\
317 \text { PRKC } \\
317 \text { PRKC } \\
317 \text { PRKC }\end{array}$ & $\begin{array}{l}2.5 \\
2.1 \\
2.3 \\
2.1 \\
2.2\end{array}$ & $\begin{array}{l}0.000000 \\
0.000000 \\
0.000000 \\
0.000000 \\
0.000000\end{array}$ & $\begin{array}{l}\text { Finely crystalline to sandy limestone } \\
\text { Finely crystalline to sandy limestone } \\
\text { Finely crystalline to sandy limestone } \\
\text { Finely crystalline to sandy limestone } \\
\text { Finely crystalline to sandy limestone }\end{array}$ \\
\hline $\begin{array}{l}\text { sld05-23-18cca } \\
\text { sld05-23-18cca } \\
\text { sld05-23-18cca } \\
\text { sld05-23-18cca } \\
\text { sld05-23-18cca }\end{array}$ & $\begin{array}{l}4,163 \cdot 0 \\
4,164 \cdot 0 \\
4,165.0 \\
4,166.0 \\
4,167.0\end{array}$ & $\begin{array}{l}4,164 \cdot 0 \\
4,165 \cdot 0 \\
4,166.0 \\
4,167.0 \\
4,168.0\end{array}$ & $\begin{array}{l}317 \text { PRKC } \\
317 \text { PRKC } \\
317 \text { PRKC } \\
317 \text { PRKC } \\
317 \text { PRKC }\end{array}$ & $\begin{array}{l}3.4 \\
2.9 \\
4.1 \\
2.8 \\
2.8\end{array}$ & $\begin{array}{r}0.000000 \\
0.000000 \\
17.000000 \\
0.160000 \\
0.110000\end{array}$ & $\begin{array}{l}\text { Finely crystalline to sandy limestone } \\
\text { Finely crystalline to sandy limestone } \\
\text { Finely crystalline to sandy limestone } \\
\text { Fractured, finely crystalline limestone } \\
\text { Limestone breccia and shale }\end{array}$ \\
\hline $\begin{array}{l}\text { s } 1 d 05-23-18 c c a \\
\text { sld05-23-18cca } \\
\text { sld05-23-18cca } \\
\text { sld05-23-18cca } \\
\text { sld05-23-18cca }\end{array}$ & $\begin{array}{l}4,168 \cdot 0 \\
4,169.0 \\
4,170.0 \\
4,171.0 \\
4,172.0\end{array}$ & $\begin{array}{l}4,169.0 \\
4,170.0 \\
4,171.0 \\
4,172.0 \\
4,173.0\end{array}$ & $\begin{array}{l}317 \mathrm{PRKC} \\
317 \mathrm{PRKC} \\
317 \mathrm{PRKC} \\
317 \mathrm{PRKC} \\
317 \mathrm{PRKC}\end{array}$ & $\begin{array}{l}2.7 \\
5.4 \\
3.1 \\
5.0 \\
2.1\end{array}$ & $\begin{array}{l}0.160000 \\
0.010000 \\
0.000000 \\
0.000000 \\
0.000000\end{array}$ & $\begin{array}{l}\text { Fractured, sucrose limestone } \\
\text { Vuggy limestone } \\
\text { Vuggy limestone } \\
\text { Vuggy limestone } \\
\text { Crystalline limestone }\end{array}$ \\
\hline $\begin{array}{l}\text { sld } 05-23-18 c c a \\
\text { sld05-23-18cca } \\
\text { sld05-23-18cca } \\
\text { s } 1 \text { d05-23-18cca } \\
\text { s } 1 d 05-23-18 c c a\end{array}$ & $\begin{array}{l}4,173 \cdot 0 \\
4,174 \cdot 0 \\
4,175 \cdot 0 \\
4,176.0 \\
4,187.0\end{array}$ & $\begin{array}{l}4,174.0 \\
4,175.0 \\
4,176.0 \\
4,177.0 \\
4,188.0\end{array}$ & $\begin{array}{l}317 \mathrm{PRKC} \\
317 \mathrm{PRKC} \\
317 \mathrm{PRKC} \\
317 \mathrm{PRKC} \\
317 \mathrm{PRKC}\end{array}$ & $\begin{array}{l}4.5 \\
4.5 \\
2.0 \\
9.7 \\
6.2\end{array}$ & $\begin{array}{l}0.070000 \\
0.000000 \\
0.010000 \\
0.010000 \\
0.010000\end{array}$ & $\begin{array}{l}\text { Fractured, shaly and crystalline limestone } \\
\text { Shale } \\
\text { Fractured, shaly and crystalline limestone } \\
\text { Fractured, shaly and crystalline limestone } \\
\text { Shaly, sucrose limestone }\end{array}$ \\
\hline $\begin{array}{l}\text { sld05-23-18cca } \\
\text { sld05-23-18cca } \\
\text { sld05-23-18cca } \\
\text { sld05-23-18cca } \\
\text { sld05-23-18cca }\end{array}$ & $\begin{array}{l}4,196.0 \\
4,197.0 \\
4,198.0 \\
4,199.0 \\
4,200.0\end{array}$ & $\begin{array}{l}4,197.0 \\
4,198.0 \\
4,199.0 \\
4,200.0 \\
4,201.0\end{array}$ & $\begin{array}{l}\text { 317PRKC } \\
\text { 317PRKC } \\
\text { 317PRKC } \\
\text { 317PRKC } \\
\text { 317PRKC }\end{array}$ & $\begin{array}{l}6.2 \\
1.3 \\
1.9 \\
2.1 \\
1.8\end{array}$ & $\begin{array}{l}0.000000 \\
0.000000 \\
0.000000 \\
0.000000 \\
0.000000\end{array}$ & $\begin{array}{l}\text { Shaly limestone } \\
\text { Shaly limestone } \\
\text { Shaly limestone } \\
\text { Shaly limestone } \\
\text { Shaly limestone }\end{array}$ \\
\hline $\begin{array}{l}\text { sld } 05-23-18 c c a \\
\text { sld05-23-18cca } \\
\text { sld05-23-18cca } \\
\text { sld05-23-18cca } \\
\text { sld05-23-18cca }\end{array}$ & $\begin{array}{l}4,201 \cdot 0 \\
4,202 \cdot 0 \\
4,203 \cdot 0 \\
4,204 \cdot 0 \\
4,205 \cdot 0\end{array}$ & $\begin{array}{l}4,202 \cdot 0 \\
4,203 \cdot 0 \\
4,204.0 \\
4,205.0 \\
4,206.0\end{array}$ & $\begin{array}{l}\text { 317PRKC } \\
317 \text { PRKC } \\
317 \text { PRKC } \\
\text { 317PRKC } \\
\text { 317PRKC }\end{array}$ & $\begin{array}{r}1.6 \\
2.1 \\
16.8 \\
17.9 \\
15.2\end{array}$ & $\begin{array}{r}0.000000 \\
0.010000 \\
28.000000 \\
19.000000 \\
2.300000\end{array}$ & $\begin{array}{l}\text { Fine-grained, limy sandstone } \\
\text { Sandy limestone } \\
\text { Fine-grained, limy sandstone } \\
\text { Fine-grained, limy sandstone } \\
\text { Fine-grained sandstone and limestone }\end{array}$ \\
\hline $\begin{array}{l}\text { sld } 05-23-18 c c a \\
\text { sld05-23-18cca } \\
\text { sld05-23-18cca } \\
\text { sld05-23-18cca } \\
\text { sld } 05-23-18 c c a\end{array}$ & $\begin{array}{l}4,206.0 \\
4,207.0 \\
4,208.0 \\
4,209.0 \\
4,210.0\end{array}$ & $\begin{array}{l}4,207.0 \\
4,208 \cdot 0 \\
4,209.0 \\
4,210.0 \\
4,211.0\end{array}$ & $\begin{array}{l}317 \text { PRKC } \\
317 \text { PRKC } \\
317 \text { PRKC } \\
317 \text { PRKC } \\
317 \text { PRKC }\end{array}$ & $\begin{array}{l}13.7 \\
12.8 \\
14.6 \\
13.9 \\
15.9\end{array}$ & $\begin{array}{r}187.000000 \\
36.000000 \\
83.000000 \\
10.000000 \\
30.000000\end{array}$ & $\begin{array}{l}\text { Fractured, fine to coarse grained, limy sandstone } \\
\text { Sandy dolomite } \\
\text { Fine to medium grained, limy sandstone } \\
\text { Fine to medium grained limy sandstone } \\
\text { Shaly sandstone }\end{array}$ \\
\hline $\begin{array}{l}\text { sld05-23-18cca } \\
\text { sld05-23-18cca } \\
\text { sld05-23-18cca } \\
\text { sld05-23-18cca }\end{array}$ & $\begin{array}{l}4,211.0 \\
4,212.0 \\
4,213.0 \\
4,214.0\end{array}$ & $\begin{array}{l}4,212.0 \\
4,213 \cdot 0 \\
4,214.0 \\
4,215.0\end{array}$ & $\begin{array}{l}\text { 317PRKC } \\
\text { 317PRKC } \\
\text { 317PRKC } \\
\text { 317PRKC } \\
\text { AVERAGE-- }\end{array}$ & $\begin{array}{r}9.9 \\
14.5 \\
13.6 \\
13.8 \\
5.7\end{array}$ & $\begin{array}{r}2.500000 \\
31.000000 \\
60.000000 \\
13.000000 \\
9.617593\end{array}$ & $\begin{array}{l}\text { Fine-grained, quartzitic sandstone } \\
\text { Fine-grained, quartzitic sandstone } \\
\text { Fine-grained, quartzitic sandstone } \\
\text { Fine-grained, quartzitic sandstone }\end{array}$ \\
\hline $\begin{array}{l}\text { sld } 05-23-18 c c a \\
\text { sld05-23-18cca } \\
\text { sld05-23-18cca } \\
\text { s } 1 d 05-23-18 c c a \\
\text { sld05-23-18cca }\end{array}$ & $\begin{array}{l}6,076.0 \\
6,077.0 \\
6,078.0 \\
6,079.0 \\
6,080.0\end{array}$ & $\begin{array}{l}6,077.0 \\
6,078.0 \\
6,079.0 \\
6,080.0 \\
6,081.0\end{array}$ & $\begin{array}{l}331 \mathrm{HMBG} \\
331 \mathrm{HMBG} \\
331 \mathrm{HMBG} \\
331 \mathrm{HMBG} \\
331 \mathrm{HMBG}\end{array}$ & $\begin{array}{r}7.7 \\
15.7 \\
10.4 \\
8.6 \\
13.2\end{array}$ & $\begin{array}{r}56.000000 \\
60.000000 \\
1.000000 \\
19.000000 \\
31.000000\end{array}$ & $\begin{array}{l}\text { Fine-grained sandstone } \\
\text { Fine-grained sandstone } \\
\text { Fine-grained sandstone } \\
\text { Fine-grained sandstone } \\
\text { Fine-grained sandstone }\end{array}$ \\
\hline $\begin{array}{l}\text { sld05-23-18cca } \\
\text { s } 1 d 05-23-18 c c a \\
\text { sld05-23-18cca } \\
\text { sld05-23-18cca } \\
\text { sld05-23-18cca }\end{array}$ & $\begin{array}{l}6,081 \cdot 0 \\
6,082 \cdot 0 \\
6,083 \cdot 0 \\
6,084 \cdot 0 \\
6,085 \cdot 0\end{array}$ & $\begin{array}{l}6,082.0 \\
6,083.0 \\
6,084.0 \\
6,085.0 \\
6,086.0\end{array}$ & $\begin{array}{l}\text { 331HMBG } \\
331 \mathrm{HMBG} \\
331 \mathrm{HMBG} \\
331 \mathrm{HMBG} \\
331 \mathrm{HMBG}\end{array}$ & $\begin{array}{r}10.1 \\
6.3 \\
10.0 \\
6.8 \\
5.2\end{array}$ & $\begin{array}{r}0.250000 \\
2.100000 \\
28.000000 \\
7.300000 \\
0.080000\end{array}$ & $\begin{array}{l}\text { Fine-grained sandstone } \\
\text { Fine-grained sandstone } \\
\text { Fine-grained sandstone } \\
\text { Fine-grained sandstone } \\
\text { Fine-grained sandstone }\end{array}$ \\
\hline
\end{tabular}


Table 4.--Laboratory-determined porosity and permeability--Continued

\begin{tabular}{|c|c|c|c|c|c|c|}
\hline Site & $\begin{array}{l}\text { Depth to } \\
\text { top } \\
\text { (feet) }\end{array}$ & $\begin{array}{l}\text { Depth to } \\
\text { bottom } \\
\text { (feet) }\end{array}$ & $\begin{array}{l}\text { Forma- } \\
\text { tion }\end{array}$ & $\begin{array}{l}\text { Porosity } \\
\text { (percent) }\end{array}$ & $\begin{array}{l}\text { Perme- } \\
\text { ability } \\
\text { (milli- } \\
\text { darcies) }\end{array}$ & Lithology \\
\hline $\begin{array}{l}\text { s } 1 d 05-23-18 \text { cca } \\
\text { s } 1 d 05-23-18 \text { cca } \\
\text { s } 1 d 05-23-18 c c a\end{array}$ & $\begin{array}{l}6,086.0 \\
6,087.0 \\
6,088.0\end{array}$ & $\begin{array}{l}6,087.0 \\
6,088.0 \\
6,089.0\end{array}$ & $\begin{array}{l}\text { 331HMBG } \\
331 \mathrm{HMBG} \\
331 \mathrm{HMBG}\end{array}$ & $\begin{array}{l}9.1 \\
4.3 \\
1.4 \\
\end{array}$ & $\begin{array}{r}7.500000 \\
21.000000 \\
2.800000 \\
\end{array}$ & $\begin{array}{l}\text { Fine-grained sandstone } \\
\text { Fine-grained sandstone } \\
\text { Fine-grained sandstone }\end{array}$ \\
\hline SITE AVERAGE--- & - & 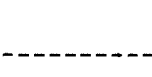 & AVERAGE-- & $\frac{8.4}{6.2}$ & $\frac{18.156154}{11.274328}$ & \\
\hline $\begin{array}{l}\text { s1d05-23-21 aaa } \\
\text { sld05-23-21aaa } \\
\text { sld05-23-21 aaa } \\
\text { s } 1 \text { d } 05-23-21 \text { aaa } \\
\text { s1d05-23-21 aaa }\end{array}$ & $\begin{array}{l}3,334.0 \\
3,335.0 \\
3,336.0 \\
3,337.0 \\
3,338.0\end{array}$ & $\begin{array}{l}3,335.0 \\
3,336.0 \\
3,337.0 \\
3,338.0 \\
3,339.0\end{array}$ & $\begin{array}{l}321 \mathrm{WEBR} \\
321 \mathrm{WEBR} \\
321 \mathrm{WEBR} \\
321 \mathrm{WEBR} \\
321 \mathrm{WEBR}\end{array}$ & $\begin{array}{r}13.8 \\
8.1 \\
12.7 \\
11.4 \\
13.0\end{array}$ & $\begin{array}{r}68.000000 \\
0.150000 \\
15.000000 \\
40.000000 \\
23.000000\end{array}$ & $\begin{array}{l}\text { Fine-grained, limy sandstone } \\
\text { Fine-grained, limy sandstone } \\
\text { Fine-grained, limy sandstone } \\
\text { Fine- to coarse-grained, limy sandstone } \\
\text { Fine-grained, limy sandstone }\end{array}$ \\
\hline $\begin{array}{l}\text { s1d05-23-21aaa } \\
\text { s1d05-23-21 aaa } \\
\text { s1d05-23-21aaa } \\
\text { sld05-23-21 aaa } \\
\text { s1d05-23-21 aaa }\end{array}$ & $\begin{array}{l}3,339.0 \\
3,340.0 \\
3,341.0 \\
3,342.0 \\
3,343.0\end{array}$ & $\begin{array}{l}3,340.0 \\
3,341.0 \\
3,342.0 \\
3,343.0 \\
3,344.0\end{array}$ & $\begin{array}{l}321 \mathrm{WEBR} \\
321 \mathrm{WEBR} \\
321 \mathrm{WEBR} \\
321 \mathrm{WEBR} \\
321 \mathrm{WEBR}\end{array}$ & $\begin{array}{l}12.5 \\
13.2 \\
12.9 \\
11.8 \\
12.1\end{array}$ & $\begin{array}{l}40.000000 \\
33.000000 \\
98.000000 \\
37.000000 \\
17.000000\end{array}$ & $\begin{array}{l}\text { Fine-grained, limy sandstone } \\
\text { Fine-grained, limy sandstone } \\
\text { Fine- to coarse-grained, limy sandstone } \\
\text { Fine-grained, limy sandstone } \\
\text { Fine- to medium-grained, limy sandstone }\end{array}$ \\
\hline s1d05-23-21aaa & $3,344.0$ & $3,345.0$ & $321 \mathrm{WEBR}$ & 10.4 & 31.000000 & Shaly, fine to medium-grained sandstone \\
\hline SITE AVERAGE--- & $-2--2-0-1$ & & AVERAGE-- & $\frac{12.0}{12.0}$ & $\frac{36.559091}{36.559091}$ & \\
\hline $\begin{array}{l}\text { sld19-09-08bdb } \\
\text { sld19-09-08bdb } \\
\text { sld19-09-08bdb } \\
\text { sld19-09-08bdb } \\
\text { sld19-09-08bdb }\end{array}$ & $\begin{array}{l}5,148.0 \\
5,151.0 \\
5,154.0 \\
5,157.0 \\
5,160.0\end{array}$ & $\begin{array}{l}5,149.0 \\
5,152.0 \\
5,155.0 \\
5,158.0 \\
5,161.0\end{array}$ & $\begin{array}{l}317 \text { PRKC } \\
317 \text { PRKC } \\
317 \text { PRKC } \\
317 \text { PRKC } \\
317 \text { PRKC }\end{array}$ & $\begin{array}{l}4.3 \\
3.1 \\
4.6 \\
4.5 \\
3.1\end{array}$ & $\begin{array}{l}0.000000 \\
0.000000 \\
0.100000 \\
0.100000 \\
0.000000\end{array}$ & $\begin{array}{l}\text { Shaly, finely crystalline dolomite } \\
\text { Finely crystalline limestone } \\
\text { Shaly, finely crystalline dolomite } \\
\text { Finely crystalline dolomite } \\
\text { Finely crystalline limy dolomite }\end{array}$ \\
\hline $\begin{array}{l}\text { s } 1 d 19-09-08 b d b \\
\text { sld19-09-08bdb } \\
\text { sld19-09-08bdb } \\
\text { sld19-09-08bdb } \\
\text { sld19-09-08bdb }\end{array}$ & $\begin{array}{l}5,163.0 \\
5,166.0 \\
5,169.0 \\
5,172.0 \\
5,175.0\end{array}$ & $\begin{array}{l}5,164.0 \\
5,167.0 \\
5,170.0 \\
5,173.0 \\
5,176.0\end{array}$ & $\begin{array}{l}317 \text { PRKC } \\
317 \text { PRKC } \\
317 \text { PRKC } \\
317 \text { PRKC } \\
317 \text { PRKC }\end{array}$ & $\begin{array}{l}3.8 \\
1.3 \\
5.2 \\
3.0 \\
3.0\end{array}$ & $\begin{array}{l}0.100000 \\
0.000000 \\
0.000000 \\
0.000000 \\
0.100000\end{array}$ & $\begin{array}{l}\text { Finely crystalline dolomite } \\
\text { Shaly, finely crystalline dolomite } \\
\text { Shaly, finely crystalline dolomite } \\
\text { Finely crystalline dolomite } \\
\text { Finely crystalline dolomite }\end{array}$ \\
\hline SITE AVERAGE--- & - - & $=-$ & AVERAGE-- & $\frac{3.6}{3.6}$ & $\frac{0.040000}{0.040000}$ & \\
\hline $\begin{array}{l}\text { s } 1 \text { d } 21-11-25 d d d \\
\text { sld21-11-25ddd }\end{array}$ & $\begin{array}{l}0.2 \\
1.4\end{array}$ & $\begin{array}{l}0.5 \\
1.8\end{array}$ & $\begin{array}{l}\text { 317WTRM } \\
\text { 317WTRM }\end{array}$ & $\begin{array}{l}15.1 \\
18.8 \\
\end{array}$ & $\begin{array}{l}375.000000 \\
970.000000 \\
\end{array}$ & $\begin{array}{l}\text { Medium-grained quartzitic sandstone } \\
\text { Medium-grained quartzitic sandstone }\end{array}$ \\
\hline SITE AVERAGE-- & 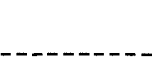 & & AVERAGE-- & $\frac{17.0}{17.0}$ & $\frac{672.500000}{672.500000}$ & \\
\hline $\begin{array}{l}\text { sld } 23-11-27 b c a \\
\text { sld23-11-27bca }\end{array}$ & $\begin{array}{l}1.4 \\
2.5\end{array}$ & $\begin{array}{l}2.1 \\
2.8\end{array}$ & $\begin{array}{l}\text { 317WTRM } \\
\text { 317WTRM }\end{array}$ & $\begin{array}{l}21.7 \quad 1, \\
24.6 \quad 3,\end{array}$ & $\begin{array}{l}, 390.000000 \\
, 080.000000 \\
\end{array}$ & $\begin{array}{l}\text { Quartz sandstone } \\
\text { Quartz sandstone }\end{array}$ \\
\hline & & & AVERAGE-- & $23.2 \quad 2$, &, 235.000000 & \\
\hline SITE AVERAGE--- & $-\cdots-1--$ & 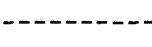 & ------- & 23.22 , &, 235.000000 & \\
\hline $\begin{array}{l}\text { sld23-17-17ada } \\
\text { s } 1 d 23-17-17 \text { ada } \\
\text { s1d23-17-17ada } \\
\text { sld23-17-17ada } \\
\text { s } 1 d 23-17-17 \text { ada }\end{array}$ & $\begin{array}{l}8,693.0 \\
8,697.0 \\
8,699.0 \\
8,706.0 \\
8,709.0\end{array}$ & $\begin{array}{l}8,694.0 \\
8,698.0 \\
8,700.0 \\
8,707.0 \\
8,710.0\end{array}$ & $\begin{array}{l}\text { 337RDLL } \\
\text { 337RDLL } \\
\text { 337RDLL } \\
\text { 337RDLL } \\
\text { 337RDLL }\end{array}$ & $\begin{array}{l}8.2 \\
5.7 \\
8.1 \\
5.0 \\
4.0\end{array}$ & $\begin{array}{l}2.400000 \\
0.210000 \\
2.600000 \\
2.800000 \\
0.160000\end{array}$ & $\begin{array}{l}\text { Dolomite } \\
\text { Dolomite } \\
\text { Dolomite } \\
\text { Dolomite } \\
\text { Dolomite }\end{array}$ \\
\hline $\begin{array}{l}\text { sld23-17-17ada } \\
\text { sld23-17-17ada } \\
\text { sld23-17-17ada } \\
\text { sld23-17-17ada } \\
\text { sld23-17-17ada }\end{array}$ & $\begin{array}{l}8,713.0 \\
8,719.0 \\
8,723.0 \\
8,729.0 \\
8,731.0\end{array}$ & $\begin{array}{l}8,714.0 \\
8,720.0 \\
8,724.0 \\
8,730.0 \\
8,732.0\end{array}$ & $\begin{array}{l}\text { 337RDLL } \\
\text { 337RDLL } \\
\text { 337RDLL } \\
\text { 337RDLL } \\
\text { 337RDLL }\end{array}$ & $\begin{array}{l}6.2 \\
7.0 \\
3.4 \\
3.7 \\
3.6\end{array}$ & $\begin{array}{r}6.700000 \\
0.100000 \\
11.800000 \\
2.300000 \\
2.400000\end{array}$ & $\begin{array}{l}\text { Dolomite } \\
\text { Dolomite } \\
\text { Dolomite } \\
\text { Dolomite } \\
\text { Dolomite }\end{array}$ \\
\hline
\end{tabular}


Table 4.--Laboratory-determined porosity and permeability--Continued

\begin{tabular}{|c|c|c|c|c|c|c|}
\hline Site & $\begin{array}{l}\text { Depth to } \\
\text { top } \\
\text { (feet) }\end{array}$ & $\begin{array}{l}\text { Depth to } \\
\text { bottom } \\
\text { (feet) }\end{array}$ & $\begin{array}{c}\text { Forma- } \\
\text { tion }\end{array}$ & $\begin{array}{l}\text { Porosity } \\
\text { (percent) }\end{array}$ & $\begin{array}{l}\text { Perme- } \\
\text { ability } \\
\text { (milli- } \\
\text { darcies) }\end{array}$ & Lithology \\
\hline sld23-17-17ada & $8,733.0$ & $8,734.0$ & 337RDLL & 4.7 & 15.000000 & Dolomite \\
\hline sld23-17-17ada & $8,734.0$ & $8,735.0$ & 337RDLL & 4.0 & 23.000000 & Dolomite \\
\hline s $1 d 23-17-17 \mathrm{ada}$ & $8,736.0$ & $8,737.0$ & 337RDLL & 3.8 & 2.700000 & Dolomite \\
\hline sld23-17-17ada & $8,738.0$ & $8,739.0$ & 337RDLL & 6.4 & 1.300000 & Dolomite \\
\hline sld23-17-17ada & $8,740.0$ & $8,741.0$ & 337RDLL & 6.7 & 7.000000 & Dolomite \\
\hline sld23-17-17ada & $8,745.0$ & $8,746.0$ & 337RDLL & 6.8 & 5.500000 & Dolomite \\
\hline sld23-17-17ada & $8,746.0$ & $8,747.0$ & 337RDLL & 6.8 & 73.000000 & Dolomite \\
\hline sld23-17-17ada & $8,748.0$ & $8,749.0$ & 337RDLL & 10.4 & 2.600000 & Dolomite \\
\hline s1d23-17-17ada & $8,751.0$ & $8,752.0$ & 337RDLL & 4.4 & 24.000000 & Dolomite \\
\hline sld23-17-17ada & $8,753.0$ & $8,754.0$ & 337RDLL & 4.6 & 2.200000 & Dolomite \\
\hline sld23-17-17ada & $8,755.0$ & $8,756.0$ & 337RDLL & 4.8 & 0.300000 & Dolomite \\
\hline sld23-17-17ada & $8,757.0$ & $8,758.0$ & 337RDLL & 7.3 & 0.670000 & Dolomite \\
\hline sld23-17-17ada & $8,760.0$ & $8,761.0$ & 337RDLL & 5.9 & 2.100000 & Dolomite \\
\hline sld23-17-17ada & $8,761.0$ & $8,762.0$ & 337RDLL & 20.9 & 28.000000 & Dolomite \\
\hline sld23-17-17ada & $8,764.0$ & $8,765.0$ & 337RDLL & 7.1 & 2.100000 & Limestone \\
\hline sld23-17-17ada & $8,766.0$ & $8,767.0$ & 337RDLL & 12.9 & 0.000000 & Dolomite \\
\hline sld23-17-17ada & $8,767.0$ & $8,768.0$ & 337RDLL & 5.2 & 0.000000 & Dolomite \\
\hline sld23-17-17ada & $8,768.0$ & $8,769.0$ & 337RDLL & 5.9 & 1.000000 & Dolomite \\
\hline sld23-17-17ada & $8,770.0$ & $8,771.0$ & 337RDLL & 6.3 & 0.670000 & Dolomite \\
\hline sld $23-17-17$ a da & $8,773.0$ & $8,774.0$ & 337RDLL & .10 .8 & 1.000000 & Dolomite \\
\hline sld23-17-17ada & $8,775.0$ & $8,776.0$ & 337RDLL & 4.6 & 0.170000 & Dolomite \\
\hline s1d23-17-17ada & $8,781.0$ & $8,782.0$ & 337RDLL & 8.0 & 0.560000 & Dolomite \\
\hline sld23-17-17ada & $8,782.0$ & $8,783.0$ & 337RDLL & 12.9 & 1.100000 & Dolomite \\
\hline s $1 \mathrm{~d} 23-17-17$ a da & $8,785.0$ & $8,786.0$ & 337RDLL & 6.6 & 0.200000 & Dolomite \\
\hline sld $23-17-17$ ada & $8,786.0$ & $8,787.0$ & 337RDLL & 8.9 & 0.400000 & Dolomite \\
\hline sld23-17-17ada & $8,788.0$ & $8,789.0$ & 337RDLL & 7.6 & 50.000000 & Dolomite \\
\hline sld $23-17-17 a d a$ & $8,791.0$ & $8,792.0$ & 337RDLL & 8.2 & 0.200000 & Dolomite \\
\hline sld23-17-17ada & $8,793.0$ & $8,794.0$ & 337RDLL & 8.9 & 0.600000 & Dolomite \\
\hline sld23-17-17ada & $8,795.0$ & $8,796.0$ & 337RDLL & 15.3 & 8.200000 & Dolomite \\
\hline sld $23-17-17$ ada & $8,797.0$ & $8,798.0$ & 337RDLL & 7.4 & 0.000000 & Dolomite \\
\hline sld23-17-17ada & $8,799.0$ & $8,800.0$ & 337RDLL & 4.0 & 0.500000 & Dolomite \\
\hline sld23-17-17ada & $8,802.0$ & $8,803.0$ & 337RDLL & 7.5 & 0.200000 & Dolomite \\
\hline sld23-17-17ada & $8,803.0$ & $8,804.0$ & 337RDLL & 7.2 & 0.100000 & Dolomite \\
\hline $\operatorname{sld} 23-17-17 \mathrm{ada}$ & $8,805.0$ & $8,806.0$ & 337RDLL & 6.9 & 0.200000 & Dolomite \\
\hline sld $23-17-17$ ada & $8,807.0$ & $8,808.0$ & 337RDLL & 10.2 & 12.000000 & Dolomite \\
\hline sld23-17-17ada & $8,808.0$ & $8,809.0$ & 337RDLL & 7.4 & 0.500000 & Dolomite \\
\hline sld $23-17-17 \mathrm{ada}$ & $8,810.0$ & $8,811.0$ & 337RDLL & 13.0 & 0.400000 & Dolomite \\
\hline sld23-17-17ada & $8,812.0$ & $8,813.0$ & 337RDLL & 8.2 & 0.400000 & Dolomite \\
\hline s $1 \mathrm{~d} 23-17-17 \mathrm{ada}$ & $8,816.0$ & $8,817.0$ & 337RDLL & 6.9 & 0.010000 & Dolomite \\
\hline s $1 d 23-17-17$ ada & $8,817.0$ & $8,818.0$ & 337RDLL & 7.6 & 0.300000 & Dolomite \\
\hline sld $23-17-17$ a da & $8,821.0$ & $8,822.0$ & 337RDLL & 7.5 & 7.400000 & Dolomite \\
\hline sld $23-17-17$ ada & $8,823.0$ & $8,824.0$ & 337RDLL & 8.8 & 0.020000 & Dolomite \\
\hline & & & AVERAGE-- & 7.4 & 5.905192 & \\
\hline SITE AVERAGE-- & 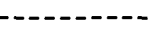 & & 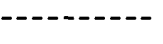 & 7.4 & 5.905192 & \\
\hline sld $24-13-23 c d d$ & $2,200.0$ & $2,201.0$ & $317 \mathrm{KIBB}$ & 0.8 & 0.040000 & Finely crystalline dolomite \\
\hline s ld $24-13-23$ cdd & $2,201.0$ & $2,202.0$ & $317 \mathrm{KIBB}$ & 0.7 & 0.030000 & Finely crystalline dolomite \\
\hline sld24-13-23cdd & $2,202.0$ & $2,203.0$ & $317 \mathrm{KIBB}$ & 0.9 & 0.040000 & Finely crystalline dolomite \\
\hline sld $24-13-23 c d d$ & $2,203.0$ & $2,204.0$ & $317 \mathrm{KIBB}$ & 0.7 & 0.040000 & Finely crystalline dolomite \\
\hline s $1 d 24-13-23 c d d$ & $2,204.0$ & $2,205.0$ & $317 \mathrm{KIBB}$ & 0.4 & 0.040000 & Finely crystalline dolomite \\
\hline
\end{tabular}


Table 4.--Laboratory-determined porosity and permeability--Continued

\begin{tabular}{|c|c|c|c|c|c|c|}
\hline Site & $\begin{array}{l}\text { Depth to } \\
\text { top } \\
\text { (feet) }\end{array}$ & $\begin{array}{l}\text { Depth to } \\
\text { bottom } \\
\text { (feet) }\end{array}$ & $\begin{array}{c}\text { Forma- } \\
\text { tion }\end{array}$ & $\begin{array}{l}\text { Porosity } \\
\text { (percent) }\end{array}$ & $\begin{array}{l}\text { Perme- } \\
\text { ability } \\
\text { (milli- } \\
\text { darcies) }\end{array}$ & Lithology \\
\hline sld24-13-23cdd & $2,205.0$ & $2,206.0$ & $317 \mathrm{KIBB}$ & 2.0 & 0.050000 & Finely crystalline dolomite \\
\hline s $1 d 24-13-23 c d d$ & $2,206.0$ & $2,207.0$ & $317 \mathrm{KIBB}$ & 1.5 & 0.040000 & Finely crystalline dolomite \\
\hline s $1 d 24-13-23 c d d$ & $2,207.0$ & $2,208.0$ & $317 \mathrm{KIBB}$ & 1.4 & 0.040000 & Anhydritic, crystalline dolomite conglomerate \\
\hline $\mathrm{s} 1 \mathrm{~d} 24-13-23 \mathrm{cdd}$ & $2,208.0$ & $2,209.0$ & $317 \mathrm{KIBB}$ & 1.0 & 0.030000 & Finely crystalline dolomite \\
\hline s $1 d 24-13-23 c d d$ & $2,209.0$ & $2,210.0$ & $317 \mathrm{KIBB}$ & 0.9 & 0.040000 & Anhydritic, crystalline dolomite conglomerate \\
\hline $\mathrm{s} 1 \mathrm{~d} 24-13-23 \mathrm{cdd}$ & $2,210.0$ & $2,211.0$ & $317 \mathrm{KIBB}$ & 1.4 & 0.040000 & Anhydritic, crystalline dolomite conglomerate \\
\hline s1d24-13-23cdd & $2,211.0$ & $2,212.0$ & $317 \mathrm{KIBB}$ & 1.7 & 0.030000 & Anhydritic, crystalline dolomite conglomerate \\
\hline sld24-13-23cdd & $2,212.0$ & $2,213.0$ & $317 \mathrm{KIBB}$ & 1.7 & 0.030000 & Anhydritic, crystalline dolomite conglomerate \\
\hline s $1 d 24-13-23 c d d$ & $2,213.0$ & $2,214.0$ & $317 \mathrm{KIBB}$ & 1.5 & 0.030000 & Anhydritic, crystalline dolomite conglomerate \\
\hline s $1 d 24-13-23 c d d$ & $2,214.0$ & $2,215.0$ & $317 \mathrm{KIBB}$ & 1.2 & 0.020000 & Anhydritic, crystalline dolomite conglomerate \\
\hline $\mathrm{s} 1 \mathrm{~d} 24-13-23 \mathrm{cdd}$ & $2,215.0$ & $2,216.0$ & $317 \mathrm{KIBB}$ & 0.8 & 0.020000 & Anhydritic, crystalline dolomite conglomerate \\
\hline s 1 d24-13-23cdd & $2,216.0$ & $2,217.0$ & $317 \mathrm{KIBB}$ & 0.7 & 0.030000 & Anhydritic, crystalline dolomite conglomerate \\
\hline s $1 d 24-13-23 c d d$ & $2,217.0$ & $2,218.0$ & $317 \mathrm{KIBB}$ & 2.0 & 0.060000 & Anhydritic, crystalline dolomite conglomerate \\
\hline s $1 d 24-13-23 c d d$ & $2,218.0$ & $2,219.0$ & $317 \mathrm{KIBB}$ & 2.4 & 0.040000 & Anhydritic, crystalline dolomite conglomerate \\
\hline s $1 d 24-13-23 c d d$ & $2,219.0$ & $2,220.0$ & $317 \mathrm{KIBB}$ & 2.2 & 0.030000 & Anhydritic, crystalline dolomite conglomerate \\
\hline s $1 d 24-13-23 c d d$ & $2,220.0$ & $2,221.0$ & $317 \mathrm{KIBB}$ & 1.8 & 0.030000 & Anhydritic, crystalline dolomite conglomerate \\
\hline s $1 d 24-13-23 c d d$ & $2,221.0$ & $2,222.0$ & $317 \mathrm{KIBB}$ & 2.4 & 0.010000 & Anhydritic, crystalline dolomite conglomerate \\
\hline $\mathrm{s} 1 \mathrm{~d} 24-13-23 \mathrm{cdd}$ & $2,222.0$ & $2,223.0$ & $317 \mathrm{KIBB}$ & 13.5 & 2.700000 & Anhydritic, crystalline dolomite conglomerate \\
\hline s $1 d 24-13-23 c d d$ & $2,223.0$ & $2,224.0$ & $317 \mathrm{KIBB}$ & 8.1 & 0.150000 & Anhydritic, crystalline dolomite conglomerate \\
\hline $\operatorname{s} 1 d 24-13-23 c d d$ & $2,224.0$ & $2,225.0$ & $317 \mathrm{KIBB}$ & 4.9 & 0.070000 & Anhydritic, crystalline dolomite conglomerate \\
\hline $\mathrm{s} 1 \mathrm{~d} 24-13-23 \mathrm{cdd}$ & $2,225.0$ & $2,226.0$ & $317 \mathrm{KIBB}$ & 8.8 & 0.100000 & Anhydritic, crystalline dolomite conglomerate \\
\hline s $1 d 24-13-23 c d d$ & $2,226.0$ & $2,227.0$ & $317 \mathrm{KIBB}$ & 8.1 & 0.090000 & Anhydritic, crystalline dolomite conglomerate \\
\hline $\mathrm{s} 1 \mathrm{~d} 24-13-23 \mathrm{cdd}$ & $2,227.0$ & $2,228.0$ & $317 \mathrm{KIBB}$ & 7.3 & 0.070000 & Anhydritic, crystalline dolomite conglomerate \\
\hline $\operatorname{s} 1 d 24-13-23 c d d$ & $2,228.0$ & $2,229.0$ & $317 \mathrm{KIBB}$ & 4.6 & 0.050000 & Anhydritic, crystalline dolomite conglomerate \\
\hline s $1 d 24-13-23 c d d$ & $2,229.0$ & $2,230.0$ & $317 \mathrm{KIBB}$ & 6.0 & 0.050000 & Anhydritic, crystalline dolomite conglomerate \\
\hline s $1 d 24-13-23 c d d$ & $2,230.0$ & $2,231.0$ & $317 \mathrm{KIBB}$ & 12.0 & 2.800000 & Anhydritic, crystalline dolomite conglomerate \\
\hline $\mathrm{s} 1 \mathrm{~d} 24-13-23 \mathrm{cdd}$ & $2,231.0$ & $2,232.0$ & $317 \mathrm{KIBB}$ & 10.5 & 0.780000 & Anhydritic, crystalline dolomite conglomerate \\
\hline s $1 d 24-13-23 c d d$ & $2,232.0$ & $2,233.0$ & $317 \mathrm{KIBB}$ & 7.4 & 0.170000 & Anhydritic, crystalline dolomite conglomerate \\
\hline s $1 d 24-13-23 c d d$ & $2,233.0$ & $2,234.0$ & $317 \mathrm{KIBB}$ & 8.8 & 0.190000 & Anhydritic, crystalline dolomite conglomerate \\
\hline s $1 d 24-13-23 c d d$ & $2,234.0$ & $2,235.0$ & $317 \mathrm{KIBB}$ & 9.1 & 1.900000 & Anhydritic, crystalline dolomite conglomerate \\
\hline $\mathrm{s} 1 \mathrm{~d} 24-13-23 \mathrm{cdd}$ & $2,235.0$ & $2,236.0$ & $317 \mathrm{KIBB}$ & 8.8 & 0.060000 & Anhydritic, crystalline dolomite conglomerate \\
\hline & & & AVERAGE-- & 4.1 & 0.276111 & \\
\hline SITE AVERAGE- & & & & 4.1 & 0.276111 & \\
\hline $\mathrm{sld} 25-13-12 \mathrm{bac}$ & $3,823.0$ & $3,824.0$ & 337RDLL & 11.2 & 0.360000 & Finely crystalline dolomite \\
\hline s1d $25-13-12 b a c$ & $3,824.0$ & $3,825.0$ & 337RDLL & 12.0 & 0.700000 & Finely crystalline dolomite \\
\hline $\mathrm{s} 1 \mathrm{~d} 25-13-12 \mathrm{bac}$ & $3,825.0$ & $3,826.0$ & 337RDLL & 7.2 & 0.390000 & Finely crystalline dolomite \\
\hline $\operatorname{sld} 25-13-12 \mathrm{bac}$ & $3,826.0$ & $3,827.0$ & 337RDLL & 8.0 & 0.170000 & Finely crystalline dolomite \\
\hline sld $25-13-12 \mathrm{bac}$ & $3,827.0$ & $3,828.0$ & 337RDLL & 12.2 & 0.700000 & Finely crystalline dolomite \\
\hline $\mathrm{s} 1 \mathrm{~d} 25-13-12 \mathrm{bac}$ & $3,828.0$ & $3,829.0$ & 337RDLL & 20.2 & 2.600000 & Finely crystalline dolomite \\
\hline s $1 d 25-13-12 \mathrm{bac}$ & $3,829.0$ & $3,830.0$ & 337RDLL & 19.6 & 1.100000 & Finely crystalline dolomite \\
\hline $\mathrm{s} 1 \mathrm{~d} 25-13-12 \mathrm{bac}$ & $3,830.0$ & $3,831.0$ & 337RDLL & 19.7 & 1.000000 & Finely crystalline dolomite \\
\hline sld $25-13-12 b a c$ & $3,831.0$ & $3,832.0$ & 337RDLL & 18.6 & 0.910000 & Finely crystalline dolomite \\
\hline s $1 d 25-13-12 b a c$ & $3,832.0$ & $3,833.0$ & 337RDLL & 21.3 & 1.500000 & Finely crystalline dolomite \\
\hline $\mathrm{s} 1 \mathrm{~d} 25-13-12 \mathrm{bac}$ & $3,833.0$ & $3,834.0$ & 337RDLL & 17.7 & 1.500000 & Finely crystalline dolomite \\
\hline s $1 d 25-13-12$ bac & $3,834.0$ & $3,835.0$ & 337RDLL & 21.6 & 6.000000 & Chalky, finely crystalline dolomite \\
\hline s $1 d 25-13-12$ bac & $3,835.0$ & $3,836.0$ & 337RDLL & 15.8 & 7.000000 & Chalky, finely crystalline dolomite \\
\hline s $1 d 25-13-12 b a c$ & $3,836.0$ & $3,837.0$ & 337RDLL & 20.1 & 5.100000 & Chalky, finely crystalline dolomite \\
\hline $\mathrm{s} 1 \mathrm{~d} 25-13-12 \mathrm{bac}$ & $3,837.0$ & $3,838.0$ & 337RDLL & 11.4 & 0.280000 & Chalky, finely crystalline dolomite \\
\hline
\end{tabular}


Table 4.--Laboratory-determined porosity and permeability--Continued

\begin{tabular}{|c|c|c|c|c|c|c|}
\hline Site & $\begin{array}{l}\text { Depth to } \\
\text { top } \\
\text { (feet) }\end{array}$ & $\begin{array}{l}\text { Depth to } \\
\text { bottom } \\
\text { (feet) }\end{array}$ & $\begin{array}{c}\text { Forma- } \\
\text { tion }\end{array}$ & $\begin{array}{l}\text { Porosity } \\
\text { (percent) }\end{array}$ & $\begin{array}{l}\text { Perme- } \\
\text { ability } \\
\text { (milli- } \\
\text { darcies) }\end{array}$ & Lithology \\
\hline $\begin{array}{l}\text { s1d } 25-13-12 \text { bac } \\
\text { s1d } 25-13-12 \text { bac } \\
\text { sld25-13-12bac } \\
\text { sld } 25-13-12 \text { bac } \\
\text { s } 1 d 25-13-12 \text { bac }\end{array}$ & $\begin{array}{l}3,838.0 \\
3,839.0 \\
3,941.0 \\
3,942.0 \\
3,943.0\end{array}$ & $\begin{array}{l}3,839.0 \\
3,840.0 \\
3,942.0 \\
3,943.0 \\
3,944.0\end{array}$ & $\begin{array}{l}\text { 337RDLL } \\
\text { 337RDLL } \\
\text { 337RDLL } \\
\text { 337RDLL } \\
\text { 337RDLL }\end{array}$ & $\begin{array}{r}11.3 \\
13.2 \\
1.5 \\
4.3 \\
2.1\end{array}$ & $\begin{array}{l}0.230000 \\
0.430000 \\
0.080000 \\
0.060000 \\
0.020000\end{array}$ & $\begin{array}{l}\text { Chalky, finely crystalline dolomite } \\
\text { Chalky, finely crystalline dolomite } \\
\text { Anhydritic, finely crystalline dolomite } \\
\text { Anhydritic, finely crystalline dolomite } \\
\text { Finely crystalline dolomite }\end{array}$ \\
\hline $\begin{array}{l}\text { s } 1 d 25-13-12 \text { bac } \\
\text { sld } 25-13-12 \text { bac } \\
\text { sld25-13-12bac } \\
\text { sld25-13-12bac } \\
\text { sld } 25-13-12 \text { bac }\end{array}$ & $\begin{array}{l}3,944.0 \\
3,945.0 \\
3,946.0 \\
3,947.0 \\
3,948.0\end{array}$ & $\begin{array}{l}3,945.0 \\
3,946.0 \\
3,947.0 \\
3,948.0 \\
3,949.0\end{array}$ & $\begin{array}{l}\text { 337RDLL } \\
\text { 337RDLL } \\
\text { 337RDLL } \\
\text { 337RDLL } \\
\text { 337RDLL }\end{array}$ & $\begin{array}{r}6.5 \\
10.0 \\
6.6 \\
8.3 \\
10.3\end{array}$ & $\begin{array}{l}0.150000 \\
0.210000 \\
0.040000 \\
0.050000 \\
0.110000\end{array}$ & $\begin{array}{l}\text { Finely crystalline dolomite } \\
\text { Finely crystalline dolomite } \\
\text { Anhydritic, finely crystalline dolomite } \\
\text { Finely crystalline dolomite } \\
\text { Finely crystalline dolomite }\end{array}$ \\
\hline $\begin{array}{l}\text { s } 1 d 25-13-12 \text { bac } \\
\text { sld } 25-13-12 \text { bac } \\
\text { sld25-13-12bac } \\
\text { sld25-13-12bac } \\
\text { sld25-13-12bac }\end{array}$ & $\begin{array}{l}3,949.0 \\
3,950.0 \\
3,951.0 \\
3,952.0 \\
3,953.0\end{array}$ & $\begin{array}{l}3,950.0 \\
3,951.0 \\
3,952.0 \\
3,953.0 \\
3,954.0\end{array}$ & $\begin{array}{l}\text { 337RDLL } \\
\text { 337RDLL } \\
\text { 337RDLL } \\
\text { 337RDLL } \\
\text { 337RDLL }\end{array}$ & $\begin{array}{l}9.1 \\
8.3 \\
7.7 \\
5.9 \\
6.0\end{array}$ & $\begin{array}{l}0.180000 \\
0.070000 \\
0.030000 \\
0.020000 \\
0.020000\end{array}$ & $\begin{array}{l}\text { Finely crystalline dolomite } \\
\text { Finely crystalline dolomite } \\
\text { Finely crystalline dolomite } \\
\text { Finely crystalline dolomite } \\
\text { Finely crystalline dolomite }\end{array}$ \\
\hline $\begin{array}{l}\text { sld25-13-12bac } \\
\text { sld } 25-13-12 \text { bac } \\
\text { sld25-13-12bac } \\
\text { sld25-13-12bac } \\
\text { sld25-13-12bac }\end{array}$ & $\begin{array}{l}3,954.0 \\
3,955.0 \\
3,956.0 \\
3,957.0 \\
3,958.0\end{array}$ & $\begin{array}{l}3,955.0 \\
3,956.0 \\
3,957.0 \\
3,958.0 \\
3,959.0\end{array}$ & $\begin{array}{l}\text { 337RDLL } \\
\text { 337RDLL } \\
\text { 337RDLL } \\
\text { 337RDLL } \\
\text { 337RDLL }\end{array}$ & $\begin{array}{l}7.1 \\
6.2 \\
6.1 \\
5.2 \\
5.8\end{array}$ & $\begin{array}{l}0.030000 \\
8.200000 \\
0.020000 \\
0.020000 \\
0.030000\end{array}$ & $\begin{array}{l}\text { Finely crystalline dolomite } \\
\text { Finely crystalline dolomite } \\
\text { Finely crystalline dolomite } \\
\text { Finely crystalline dolomite } \\
\text { Finely crystalline dolomite }\end{array}$ \\
\hline sld $25-13-12 b a c$ & $3,959.0$ & $3,960.0$ & $\begin{array}{l}337 \text { RDLL } \\
\text { AVERAGE-- }\end{array}$ & $\frac{4.6}{\frac{10.6}{10.6}}$ & $\frac{0.020000}{\frac{1.092500}{1.092500}}$ & Finely crystalline dolomite \\
\hline $\begin{array}{l}\text { sld26-20-29ada } \\
\text { sld26-20-29ada } \\
\text { sld26-20-29ada } \\
\text { sld26-20-29ada } \\
\text { sld26-20-29ada }\end{array}$ & $\begin{array}{l}7,638.0 \\
7,643.0 \\
7,648.0 \\
7,664.0 \\
7,734.0\end{array}$ & $\begin{array}{l}7,639.0 \\
7,644.0 \\
7,649.0 \\
7,665.0 \\
7,735.0\end{array}$ & $\begin{array}{l}\text { 337RDLL } \\
\text { 337RDLL } \\
\text { 337RDLL } \\
\text { 337RDLL } \\
\text { 337RDLL }\end{array}$ & $\begin{array}{l}1.8 \\
1.9 \\
6.8 \\
2.8 \\
8.8\end{array}$ & $\begin{array}{r}0.400000 \\
0.400000 \\
1.400000 \\
68.000000 \\
2.500000\end{array}$ & $\begin{array}{l}\text { Crinoid limestone } \\
\text { Crinoid limestone } \\
\text { Anhydritic dolomite } \\
\text { Algal limestone } \\
\text { Crinoid dolomite }\end{array}$ \\
\hline $\begin{array}{l}\text { sld26-20-29ada } \\
\text { sld26-20-29ada } \\
\text { sld26-20-29ada } \\
\text { sld26-20-29ada } \\
\text { sld26-20-29ada }\end{array}$ & $\begin{array}{l}8,295.0 \\
8,296.0 \\
8,297.0 \\
8,298.0 \\
8,299.0\end{array}$ & $\begin{array}{l}8,296.0 \\
8,297.0 \\
8,298.0 \\
8,299.0 \\
8,300.0\end{array}$ & $\begin{array}{l}341 \mathrm{ELBR} \\
341 \mathrm{ELBR} \\
341 \mathrm{ELBR} \\
341 \mathrm{ELBR} \\
341 \mathrm{ELBR}\end{array}$ & $\begin{array}{r}3.2 \\
7.0 \\
8.4 \\
11.9 \\
10.0\end{array}$ & $\begin{array}{l}0.040000 \\
0.100000 \\
0.130000 \\
0.680000 \\
1.000000\end{array}$ & $\begin{array}{l}\text { Quartzitic sandstone } \\
\text { Quartzitic sandstone } \\
\text { Quartzitic sandstone } \\
\text { Quartzitic sandstone } \\
\text { Quartzitic sandstone }\end{array}$ \\
\hline $\begin{array}{l}\text { sld26-20-29ada } \\
\text { sld26-20-29ada } \\
\text { sld26-20-29ada }\end{array}$ & $\begin{array}{l}8,301.0 \\
8,302.0 \\
8,303.0\end{array}$ & $\begin{array}{l}8,302.0 \\
8,303.0 \\
8,304.0\end{array}$ & $\begin{array}{l}\text { 341ELBR } \\
\text { 341ELBR } \\
\text { 341ELBR }\end{array}$ & $\begin{array}{l}6.9 \\
6.2 \\
6.9 \\
\end{array}$ & $\begin{array}{l}0.060000 \\
5.500000 \\
1.600000 \\
\end{array}$ & $\begin{array}{l}\text { Quartzitic sandstone } \\
\text { Quartzitic sandstone } \\
\text { Quartzitic sandstone }\end{array}$ \\
\hline SITE AVERAGE-- & 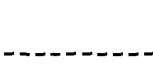 & (-e-ces & AVERAGE-- & $\frac{7.6}{7.5}$ & $\frac{1.138750}{7.788125}$ & \\
\hline $\begin{array}{l}\text { sld27-12-09aca } \\
\text { sld27-12-09aca } \\
\text { sld27-12-09aca } \\
\text { sld27-12-09aca } \\
\text { sld27-12-09aca }\end{array}$ & $\begin{array}{l}2,670.0 \\
2,671.0 \\
2,672.0 \\
2,673.0 \\
2,674.0\end{array}$ & $\begin{array}{l}2,671.0 \\
2,672.0 \\
2,673.0 \\
2,674.0 \\
2,675.0\end{array}$ & $\begin{array}{l}317 \mathrm{KIBB} \\
317 \mathrm{KIBB} \\
317 \mathrm{KIBB} \\
317 \mathrm{KIBB} \\
317 \mathrm{KIBB}\end{array}$ & $\begin{array}{r}6.5 \\
6.4 \\
9.2 \\
7.7 \\
10.6\end{array}$ & $\begin{array}{r}2.100000 \\
1.700000 \\
1.500000 \\
0.000000 \\
18.100000\end{array}$ & $\begin{array}{l}\text { Dolomite } \\
\text { Dolomite } \\
\text { Dolomite } \\
\text { Dolomite } \\
\text { Dolomite }\end{array}$ \\
\hline
\end{tabular}


Table 4.--Laboratory-determined porosity and permeability--Continued

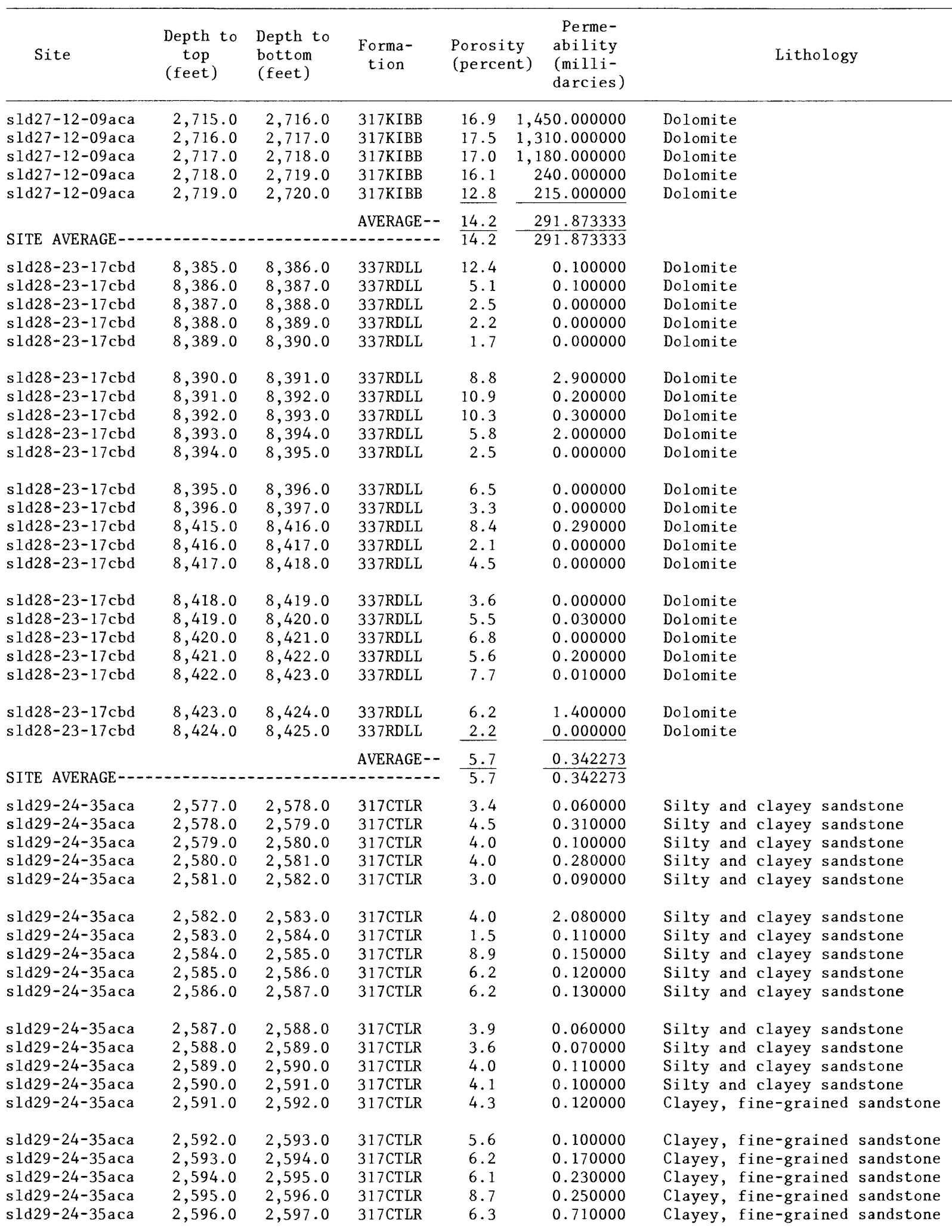


Table 4.--Laboratory-determined porosity and permeability--Continued

\begin{tabular}{|c|c|c|c|c|c|c|}
\hline Site & $\begin{array}{l}\text { Depth to } \\
\text { top } \\
\text { (feet) }\end{array}$ & $\begin{array}{l}\text { Depth to } \\
\text { bottom } \\
\text { (feet) }\end{array}$ & $\begin{array}{c}\text { Forma- } \\
\text { tion }\end{array}$ & $\begin{array}{l}\text { Porosity } \\
\text { (percent) }\end{array}$ & $\begin{array}{c}\text { Perme- } \\
\text { ability } \\
\text { (milli- } \\
\text { darcies) }\end{array}$ & Lithology \\
\hline $\begin{array}{l}\text { sld29-24-35aca } \\
\text { sld29-24-35aca } \\
\text { sld29-24-35aca } \\
\text { sld29-24-35aca } \\
\text { sld29-24-35aca }\end{array}$ & $\begin{array}{l}2,597.0 \\
2,598.0 \\
2,599.0 \\
2,600.0 \\
2,601.0\end{array}$ & $\begin{array}{l}2,598.0 \\
2,599.0 \\
2,600.0 \\
2,601.0 \\
2,602.0\end{array}$ & $\begin{array}{l}\text { 317CTLR } \\
\text { 317CTLR } \\
\text { 317CTLR } \\
\text { 317CTLR } \\
\text { 317CTLR }\end{array}$ & $\begin{array}{l}14.5 \\
20.4 \\
17.2 \\
15.2 \\
14.6\end{array}$ & $\begin{array}{l}0.600000 \\
0.570000 \\
1.500000 \\
0.230000 \\
1.400000\end{array}$ & $\begin{array}{l}\text { Clayey, fine-grained sandstone } \\
\text { Clayey, fine-grained sandstone } \\
\text { Clayey, fine-grained sandstone } \\
\text { Clayey, fine-grained sandstone } \\
\text { Clayey, fine-grained sandstone }\end{array}$ \\
\hline $\begin{array}{l}\text { sld29-24-35aca } \\
\text { SITE AVERAGE-- }\end{array}$ & $2,602.0$ & $2,603.0$ & $\begin{array}{l}317 \text { CTLR } \\
\text { AVERAGE-- }\end{array}$ & $\frac{13.7}{\frac{7.5}{7.5}}$ & $\frac{10.200000}{\frac{0.763462}{0.763462}}$ & Fine-grained sandstone \\
\hline $\begin{array}{l}\text { sld30-16-16dcd } \\
\text { s1d30-16-16dcd } \\
\text { s1d30-16-16dcd } \\
\text { s1d30-16-16dcd } \\
\text { s1d30-16-16dcd }\end{array}$ & $\begin{array}{l}1,474.0 \\
1,475.0 \\
1,476.0 \\
1,477.0 \\
1,478.0\end{array}$ & $\begin{array}{l}1,475.0 \\
1,476.0 \\
1,477.0 \\
1,478.0 \\
1,479.0\end{array}$ & $\begin{array}{l}317 \text { WTRM } \\
317 \text { WTRM } \\
317 \text { WTRM } \\
\text { 317WTRM } \\
\text { 317WTRM }\end{array}$ & $\begin{array}{r}13.2 \\
9.7 \\
14.2 \\
12.0 \\
11.8\end{array}$ & $\begin{array}{r}16.000000 \\
0.290000 \\
1.200000 \\
3.100000 \\
17.000000\end{array}$ & $\begin{array}{l}\text { Fine-grained sandstone } \\
\text { Fine- to medium-grained sandstone } \\
\text { Fine-grained sandstone } \\
\text { Fine-grained sandstone } \\
\text { Fine-grained sandstone }\end{array}$ \\
\hline $\begin{array}{l}\text { sld30-16-16dcd } \\
\text { sld30-16-16dcd } \\
\text { sld30-16-16dcd } \\
\text { sld30-16-16dcd } \\
\text { sld30-16-16dcd }\end{array}$ & $\begin{array}{l}1,479.0 \\
1,480.0 \\
1,481.0 \\
1,482.0 \\
1,483.0\end{array}$ & $\begin{array}{l}1,480.0 \\
1,481.0 \\
1,482.0 \\
1,483.0 \\
1,484.0\end{array}$ & $\begin{array}{l}\text { 317WTRM } \\
317 \text { WTRM } \\
317 \text { WTRM } \\
\text { 317WTRM } \\
\text { 317WTRM }\end{array}$ & $\begin{array}{l}13.0 \\
13.2 \\
13.6 \\
17.8 \\
12.3\end{array}$ & $\begin{array}{r}2.000000 \\
2.000000 \\
0.260000 \\
73.000000 \\
1.300000\end{array}$ & $\begin{array}{l}\text { Fine- to medium-grained sandstone } \\
\text { Fine- to medium-grained sandstone } \\
\text { Fine-grained sandstone } \\
\text { Fine-grained sandstone } \\
\text { Fine-grained sandstone }\end{array}$ \\
\hline $\begin{array}{l}\text { sld30-16-16dcd } \\
\text { sld30-16-16dcd } \\
\text { sld30-16-16dcd } \\
\text { sld30-16-16dcd } \\
\text { sld30-16-16dcd }\end{array}$ & $\begin{array}{l}1,484.0 \\
1,485.0 \\
1,486.0 \\
1,487.0 \\
1,488.0\end{array}$ & $\begin{array}{l}1,485.0 \\
1,486.0 \\
1,487.0 \\
1,488.0 \\
1,489.0\end{array}$ & $\begin{array}{l}317 \text { WTRM } \\
317 \text { WTRM } \\
317 \text { WTRM } \\
\text { 317WTRM } \\
\text { 317WTRM }\end{array}$ & $\begin{array}{r}8.6 \\
10.4 \\
17.0 \\
11.2 \\
21.9\end{array}$ & $\begin{array}{r}0.530000 \\
2.200000 \\
72.000000 \\
2.700000 \\
168.000000\end{array}$ & $\begin{array}{l}\text { Clayey, fine-grained sandstone } \\
\text { Clayey, fine-grained sandstone } \\
\text { Fine-grained sandstone } \\
\text { Clayey, fine-grained sandstone } \\
\text { Fine-grained sandstone }\end{array}$ \\
\hline $\begin{array}{l}\text { sld30-16-16dcd } \\
\text { sld30-16-16dcd } \\
\text { sld30-16-16dcd } \\
\text { sld30-16-16dcd } \\
\text { sld30-16-16dcd }\end{array}$ & $\begin{array}{l}1,489.0 \\
1,490.0 \\
1,491.0 \\
1,492.0 \\
1,493.0\end{array}$ & $\begin{array}{l}1,490.0 \\
1,491.0 \\
1,492.0 \\
1,493.0 \\
1,494.0\end{array}$ & $\begin{array}{l}317 \text { WTRM } \\
317 \text { WTRM } \\
317 \text { WTRM } \\
317 \text { WTRM } \\
\text { 317WTRM }\end{array}$ & $\begin{array}{l}23.3 \\
24.7 \\
25.0 \\
20.7 \\
17.8\end{array}$ & $\begin{array}{l}290.000000 \\
459.000000 \\
667.000000 \\
349.000000 \\
135.000000\end{array}$ & $\begin{array}{l}\text { Fine-grained sandstone } \\
\text { Fine-grained sandstone } \\
\text { Fine-grained sandstone } \\
\text { Fine-grained sandstone } \\
\text { Fine- to medium-grained sandstone }\end{array}$ \\
\hline $\begin{array}{l}\text { sld30-16-16dcd } \\
\text { sld30-16-16dcd } \\
\text { sld30-16-16dcd } \\
\text { sld30-16-16dcd } \\
\text { sld30-16-16dcd }\end{array}$ & $\begin{array}{l}1,494.0 \\
1,495.0 \\
1,496.0 \\
1,497.0 \\
1,498.0\end{array}$ & $\begin{array}{l}1,495.0 \\
1,496.0 \\
1,497.0 \\
1,498.0 \\
1,499.0\end{array}$ & $\begin{array}{l}317 \text { WTRM } \\
317 \text { WTRM } \\
317 \text { WTRM } \\
\text { 317WTRM } \\
\text { 317WTRM }\end{array}$ & $\begin{array}{l}21.2 \\
19.4 \\
22.9 \\
15.9 \\
17.1\end{array}$ & $\begin{array}{r}354.000000 \\
643.000000 \\
472.000000 \\
4.500000 \\
149.000000\end{array}$ & $\begin{array}{l}\text { Fine- to medium-grained sandstone } \\
\text { Clayey, fine- to medium-grained sandstone } \\
\text { Clayey, fine- to medium-grained sandstone } \\
\text { Fine- to medium-grained, quartzitic sandstone } \\
\text { Fine-grained, quartzitic sandstone }\end{array}$ \\
\hline $\begin{array}{l}\text { sld30-16-16dcd } \\
\text { sld30-16-16dcd } \\
\text { sld30-16-16dcd } \\
\text { sld30-16-16dcd } \\
\text { sld30-16-16dcd }\end{array}$ & $\begin{array}{l}1,499.0 \\
1,500.0 \\
1,501.0 \\
1,502.0 \\
1,503.0\end{array}$ & $\begin{array}{l}1,500.0 \\
1,501.0 \\
1,502.0 \\
1,503.0 \\
1,504.0\end{array}$ & $\begin{array}{l}317 \text { WTRM } \\
317 \text { WTRM } \\
317 \text { WTRM } \\
\text { 317WTRM } \\
\text { 317WTRM }\end{array}$ & $\begin{array}{l}17.1 \\
16.2 \\
16.5 \\
20.3 \\
24.1 \\
1,1\end{array}$ & $\begin{array}{r}244.000000 \\
17.000000 \\
135.000000 \\
269.000000 \\
320.000000\end{array}$ & $\begin{array}{l}\text { Fine-grained sandstone } \\
\text { Fine-grained sandstone } \\
\text { Fine-grained sandstone } \\
\text { Fine-grained sandstone } \\
\text { Fine-grained sandstone }\end{array}$ \\
\hline $\begin{array}{l}\text { sld30-16-16dcd } \\
\text { sld30-16-16dcd } \\
\text { s1d30-16-16dcd } \\
\text { sld30-16-16dcd } \\
\text { sld30-16-16dcd }\end{array}$ & $\begin{array}{l}1,504.0 \\
1,505.0 \\
1,506.0 \\
1,507.0 \\
1,508.0\end{array}$ & $\begin{array}{l}1,505.0 \\
1,506.0 \\
1,507.0 \\
1,508.0 \\
1,509.0\end{array}$ & $\begin{array}{l}317 \text { WTRM } \\
317 \text { WTRM } \\
317 \text { WTRM } \\
317 \text { WTRM } \\
\text { 317WTRM }\end{array}$ & $\begin{array}{l}20.4 \\
24.4 \\
25.0 \\
22.2 \\
22.4\end{array}$ & $\begin{array}{l}115.000000 \\
516.000000 \\
603.000000 \\
430.000000 \\
437.000000\end{array}$ & $\begin{array}{l}\text { Fine-grained sandstone } \\
\text { Fine-grained sandstone } \\
\text { Fine-grained sandstone } \\
\text { Fine-grained sandstone } \\
\text { Fine-grained sandstone }\end{array}$ \\
\hline $\begin{array}{l}\text { sld30-16-16dcd } \\
\text { sld30-16-16dcd } \\
\text { sld30-16-16dcd } \\
\text { sld30-16-16dcd } \\
\text { sld30-16-16dcd }\end{array}$ & $\begin{array}{l}1,509.0 \\
1,510.0 \\
1,511.0 \\
1,512.0 \\
1,513.0\end{array}$ & $\begin{array}{l}1,510.0 \\
1,511.0 \\
1,512.0 \\
1,513.0 \\
1,514.0\end{array}$ & $\begin{array}{l}\text { 317WTRM } \\
317 \text { WTRM } \\
317 \text { WTRM } \\
\text { 317WTRM } \\
\text { 317WTRM }\end{array}$ & $\begin{array}{l}20.8 \\
20.9 \\
26.1 \\
26.6 \\
26.6\end{array}$ & $\begin{array}{l}232.000000 \\
506.000000 \\
445.000000 \\
475.000000 \\
713.000000\end{array}$ & $\begin{array}{l}\text { Fine-grained sandstone } \\
\text { Fine-grained sandstone } \\
\text { Fine-grained sandstone } \\
\text { Fine- to medium-grained sandstone } \\
\text { Fine- to medium-grained sandstone }\end{array}$ \\
\hline
\end{tabular}


Table 4.--Laboratory-determined porosity and permeability--Continued

\begin{tabular}{|c|c|c|c|c|c|c|}
\hline Site & $\begin{array}{l}\text { Depth to } \\
\text { top } \\
\text { (feet) }\end{array}$ & $\begin{array}{l}\text { Depth to } \\
\text { bottom } \\
\text { (feet) }\end{array}$ & $\begin{array}{c}\text { Forma- } \\
\text { tion }\end{array}$ & $\begin{array}{l}\text { Porosit } \\
\text { (percen }\end{array}$ & $\begin{array}{ll} & \text { Perme- } \\
\text { ty } & \text { ability } \\
\text { (milli- } & \text { darcies })\end{array}$ & Lithology \\
\hline s $1 d 30-16-16 d c d$ & $1,514.0$ & $1,515.0$ & 317WTRM & 25.5 & 549.000000 & Fine- to medium-grained sandstone \\
\hline $\mathrm{s} 1 \mathrm{~d} 30-16-16 \mathrm{dcd}$ & $1,515.0$ & $1,516.0$ & 317 WTRM & 27.5 & $1,380.000000$ & Fine- to medium-grained sandstone \\
\hline s $1 d 30-16-16 d c d$ & $1,516.0$ & $1,517.0$ & 317WTRM & 26.3 & $1,450.000000$ & Fine- to medium-grained sandstone \\
\hline s 1 d $30-16-16 \mathrm{dcd}$ & $1,517.0$ & $1,518.0$ & 317WTRM & 26.8 & 792.000000 & Fine- to medium-grained sandstone \\
\hline $\mathrm{s} 1 \mathrm{~d} 30-16-16 \mathrm{dcd}$ & $1,518.0$ & $1,519.0$ & 317 WTRM & 26.0 & 648.000000 & Fine- to medium-grained sandstone \\
\hline $\mathrm{s} 1 \mathrm{~d} 30-16-16 \mathrm{dcd}$ & $1,519.0$ & $1,520.0$ & 317WTRM & 25.9 & 971.000000 & Fine- to medium-grained sandstone \\
\hline sld $30-16-16 d c d$ & $1,520.0$ & $1,521.0$ & 317 WTRM & 26.4 & $1,780.000000$ & Medium grained sandstone \\
\hline $\mathrm{s} 1 \mathrm{~d} 30-16-16 \mathrm{dcd}$ & $1,521.0$ & $1,522.0$ & 317 WTRM & 22.5 & 486.000000 & Fine-grained sandstone \\
\hline $\mathrm{s} 1 \mathrm{~d} 30-16-16 \mathrm{dcd}$ & $1,522.0$ & $1,523.0$ & 317WTRM & 26.2 & $1,055.000000$ & Fine-grained sandstone \\
\hline $\mathrm{s} 1 \mathrm{~d} 30-16-16 \mathrm{dcd}$ & $1,523.0$ & $1,524.0$ & 317WTRM & 25.4 & 620.000000 & Fine- to medium-grained sandstone \\
\hline $\operatorname{s} 1 d 30-16-16 d c d$ & $1,524.0$ & $1,525.0$ & 317WTRM & 22.7 & 806.000000 & Fine-grained sandstone \\
\hline $\operatorname{sid} 30-16-16 \mathrm{dcd}$ & $1,525.0$ & $1,526.0$ & 317WTRM & 26.4 & $1,330.000000$ & Fine- to medium-grained sandstone \\
\hline $\mathrm{s} 1 \mathrm{~d} 30-16-16 \mathrm{dcd}$ & $1,526.0$ & $1,527.0$ & 317 WTRM & 26.1 & 405.000000 & Fine-grained sandstone \\
\hline sld30-16-16dcd & $1,527.0$ & $1,528.0$ & 317WTRM & 27.7 & 9.000000 & Fine- to medium-grained sandstone \\
\hline $\operatorname{s} 1 \mathrm{~d} 30-16-16 \mathrm{dcd}$ & $1,528.0$ & $1,529.0$ & 317 WTRM & 27.6 & 0.240000 & Fine-grained sandstone \\
\hline s $1 d 30-16-16 d c d$ & $1,529.0$ & $1,530.0$ & 317WTRM & 27.6 & 0.210000 & Fine- to medium-grained sandstone \\
\hline s1d30-16-16dcd & $1,530.0$ & $1,531.0$ & 317WTRM & 20.9 & 0.620000 & Fine-grained, quartzitic sandstone \\
\hline $\mathrm{s} 1 \mathrm{~d} 30-16-16 \mathrm{dcd}$ & $1,531.0$ & $1,532.0$ & 317WTRM & 21.8 & 0.160000 & Medium-grained, quartzitic sandstone \\
\hline $\mathrm{s} 1 \mathrm{~d} 30-16-16 \mathrm{dcd}$ & $1,532.0$ & $1,533.0$ & 317 WTRM & 18.2 & 0.760000 & Fine-grained, quartzitic sandstone \\
\hline $\mathrm{s} 1 \mathrm{~d} 30-16-16 \mathrm{dcd}$ & $1,533.0$ & $1,534.0$ & 317WTRM & 17.9 & 103.000000 & Fine-grained sandstone \\
\hline $\mathrm{s} 1 \mathrm{~d} 30-16-16 \mathrm{dcd}$ & $1,534.0$ & $1,535.0$ & 317WTRM & 21.4 & 332.000000 & Fine-grained sandstone \\
\hline s $1 d 30-16-16 d c d$ & $1,535.0$ & $1,536.0$ & 317WTRM & 23.5 & 622.000000 & Fine-grained sandstone \\
\hline $\mathrm{s} 1 \mathrm{~d} 30-16-16 \mathrm{dcd}$ & $1,536.0$ & $1,537.0$ & 317WTRM & 26.2 & 421.000000 & Fine-grained sandstone \\
\hline s1d $30-16-16 \mathrm{dcd}$ & $1,537.0$ & $1,538.0$ & 317WTRM & 23.0 & 911.000000 & Fine-grained sandstone \\
\hline $\operatorname{s} 1 \mathrm{~d} 30-16-16 \mathrm{dcd}$ & $1,538.0$ & $1,539.0$ & 317WTRM & 20.7 & 112.000000 & Fine- to medium-grained sandstone \\
\hline s $1 \mathrm{~d} 30-16-16 \mathrm{dcd}$ & $1,539.0$ & $1,540.0$ & 317WTRM & 17.7 & 89.000000 & Clayey, fine-grained, quartzitic sandstone \\
\hline $\mathrm{s} 1 \mathrm{~d} 30-16-16 \mathrm{dcd}$ & $1,540.0$ & $1,541.0$ & 317WTRM & 22.0 & 292.000000 & Fine- to medium-grained sandstone \\
\hline $\mathrm{s} 1 \mathrm{~d} 30-16-16 \mathrm{dcd}$ & $1,541.5$ & $1,542.0$ & 317 WTRM & 20.4 & 933.000000 & Clayey, fine- to medium-grained sandstone \\
\hline $\mathrm{s} 1 \mathrm{~d} 30-16-16 \mathrm{dcd}$ & $1,542.0$ & $1,543.0$ & 317WTRM & 24.8 & $1,310.000000$ & Fine- to medium-grained sandstone \\
\hline $\mathrm{s} 1 \mathrm{~d} 30-16-16 \mathrm{dcd}$ & $1,543.0$ & $1,544.0$ & 317WTRM & 24.9 & 990.000000 & Fine- to medium-grained sandstone \\
\hline $\mathrm{s} 1 \mathrm{~d} 30-16-16 \mathrm{dcd}$ & $1,544.0$ & $1,545.0$ & 317WTRM & 25.4 & $1,075.000000$ & Fine- to medium-grained sandstone \\
\hline sld $30-16-16 \mathrm{dcd}$ & $1,545.0$ & $1,546.0$ & 317WTRM & 24.4 & 777.000000 & Fine- to medium-grained sandstone \\
\hline $\mathrm{s} 1 \mathrm{~d} 30-16-16 \mathrm{dcd}$ & $1,546.0$ & $1,547.0$ & 317WTRM & 13.2 & 25.000000 & Clayey, fine-grained sandstone \\
\hline $\mathrm{s} 1 \mathrm{~d} 30-16-16 \mathrm{dcd}$ & $1,547.0$ & $1,548.0$ & 317WTRM & 13.2 & 12.000000 & Clayey, fine-grained sandstone \\
\hline s $1 \mathrm{~d} 30-16-16 \mathrm{dcd}$ & $1,548.0$ & $1,549.0$ & 317WTRM & 14.9 & 11.000000 & Clayey, fine-grained sandstone \\
\hline $\mathrm{s} 1 \mathrm{~d} 30-16-16 \mathrm{dcd}$ & $1,549.0$ & $1,550.0$ & 317WTRM & 18.8 & 190.000000 & Clayey, fine- to medium-grained sandstone \\
\hline $\mathrm{s} 1 \mathrm{~d} 30-16-16 \mathrm{dcd}$ & $1,550.0$ & $1,551.0$ & 317WTRM & 15.5 & 26.000000 & Clayey, fine- to medium-grained sandstone \\
\hline sld $30-16-16 \mathrm{dcd}$ & $1,551.0$ & $1,552.0$ & 317WTRM & 17.2 & 22.000000 & Clayey, fine-grained sandstone \\
\hline $\mathrm{s} 1 \mathrm{~d} 30-16-16 \mathrm{dcd}$ & $1,552.0$ & $1,553.0$ & 317WTRM & 16.7 & 71.000000 & Clayey, fine-grained sandstone \\
\hline $\mathrm{s} 1 \mathrm{~d} 30-16-16 \mathrm{dcd}$ & $1,553.0$ & $1,554.0$ & 317 WTRM & 20.4 & 133.000000 & Clayey, fine-grained sandstone \\
\hline $\mathrm{s} 1 \mathrm{~d} 30-16-16 \mathrm{dcd}$ & $1,554.0$ & $1,555.0$ & 317WTRM & 21.6 & 253.000000 & Clayey, fine-grained sandstone \\
\hline s $1 d 30-16-16 d c d$ & $1,555.0$ & $1,556.0$ & 317WTRM & 19.0 & 355.000000 & Clayey, fine-grained sandstone \\
\hline $\mathrm{s} 1 \mathrm{~d} 30-16-16 \mathrm{dcd}$ & $1,556.0$ & $1,557.0$ & 317WTRM & 16.2 & 183.000000 & Clayey, fine-grained sandstone \\
\hline s $1 \mathrm{~d} 30-16-16 \mathrm{dcd}$ & $1,557.0$ & $1,558.0$ & 317WTRM & 25.0 & 830.000000 & Clayey, fine- to medium-grained sandstone \\
\hline $\operatorname{s} 1 d 30-16-16 \mathrm{dcd}$ & $1,558.0$ & $1,559.0$ & 317WTRM & 23.8 & 835.000000 & Clayey, fine- to medium-grained sandstone \\
\hline $\mathrm{s} 1 \mathrm{~d} 30-16-16 \mathrm{dcd}$ & $1,559.0$ & $1,560.0$ & 317 WTRM & 21.1 & 180.000000 & Clayey, fine- to medium-grained sandstone \\
\hline $\mathrm{s} 1 \mathrm{~d} 30-16-16 \mathrm{dcd}$ & $1,562.0$ & $1,563.0$ & 317WTRM & 23.4 & 337.000000 & Clayey, fine- to medium-grained sandstone \\
\hline s $1 \mathrm{~d} 30-16-16 \mathrm{dcd}$ & $1,564.0$ & $1,565.0$ & 317WTRM & 14.0 & 3.300000 & Clayey, fine-grained, quartzitic sandstone \\
\hline $\mathrm{s} 1 \mathrm{~d} 30-16-16 \mathrm{dcd}$ & $1,566.0$ & $1,567.0$ & 317WTRM & 13.7 & 7.800000 & Clayey, fine-grained sandstone \\
\hline $\mathrm{s} 1 \mathrm{~d} 30-16-16 \mathrm{dcd}$ & $1,568.0$ & $1,569.0$ & 317WTRM & 11.8 & 3.700000 & Clayey, fine-grained sandstone \\
\hline
\end{tabular}


Table 4.--Laboratory-determined porosity and permeability--Continued

\begin{tabular}{|c|c|c|c|c|c|c|}
\hline Site & $\begin{array}{l}\text { Depth to } \\
\text { top } \\
\text { (feet) }\end{array}$ & $\begin{array}{l}\text { Depth to } \\
\text { bottom } \\
\text { (feet) }\end{array}$ & $\begin{array}{c}\text { Forma- } \\
\text { tion }\end{array}$ & $\begin{array}{l}\text { Porosity } \\
\text { (percent) }\end{array}$ & $\begin{array}{l}\text { Perme- } \\
\text { ability } \\
\text { (milli- } \\
\text { darcies) }\end{array}$ & Lithology \\
\hline $\operatorname{sld} 30-16-16 \mathrm{dcd}$ & $1,570.0$ & $1,571.0$ & 317WTRM & 18.2 & 18.000000 & Clayey, fine-grained sandstone \\
\hline sld30-16-16dcd & $1,572.0$ & $1,573.0$ & 317WTRM & 16.7 & 50.000000 & Clayey, fine-grained sandstone \\
\hline $\operatorname{sld} 30-16-16 \mathrm{dcd}$ & $1,574.0$ & $1,575.0$ & 317WTRM & 16.4 & 60.000000 & Clayey, fine-grained sandstone \\
\hline $\operatorname{sld} 30-16-16 \mathrm{dcd}$ & $1,576.0$ & $1,577.0$ & 317WTRM & 18.8 & 54.000000 & Clayey, fine-grained sandstone \\
\hline $\operatorname{sld} 30-16-16 \mathrm{dcd}$ & $1,578.0$ & $1,579.0$ & 317 WTRM & 22.4 & 95.000000 & Clayey, fine-grained sandstone \\
\hline sld30-16-16dcd & $1,580.0$ & $1,581.0$ & 317WTRM & 23.8 & 90.000000 & Clayey, fine-grained sandstone \\
\hline sld $30-16-16 \mathrm{dcd}$ & $1,582.0$ & $1,583.0$ & 317WTRM & 17.0 & 95.000000 & Clayey, fine-grained sandstone \\
\hline $\operatorname{sld} 30-16-16 \mathrm{dcd}$ & $1,584.0$ & $1,585.0$ & 317WTRM & 20.0 & 58.000000 & Clayey, fine-grained sandstone \\
\hline $\operatorname{s} 1 d 30-16-16 \mathrm{dcd}$ & $1,586.0$ & $1,587.0$ & 317WTRM & 18.8 & 63.000000 & Clayey, fine-grained sandstone \\
\hline $\operatorname{sld} 30-16-16 \mathrm{dcd}$ & $1,588.0$ & $1,589.0$ & $317 W T R M$ & 14.7 & 83.000000 & Clayey, fine-grained sandstone \\
\hline $\operatorname{sid} 30-16-16 \mathrm{dcd}$ & $1,590.0$ & $1,591.0$ & 317WTRM & 18.7 & 83.000000 & Clayey, fine-grained sandstone \\
\hline $\operatorname{sid} 30-16-16 \mathrm{dcd}$ & $1,592.0$ & $1,593.0$ & 317WTRM & 18.7 & 41.000000 & Clayey, fine-grained sandstone \\
\hline SITE AVERAGE-- & 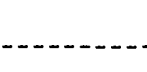 & 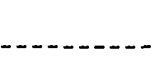 & AVERAGE-- & $\frac{20.1}{20.1}$ & $\frac{351.557549}{351.557549}$ & \\
\hline $\begin{array}{l}\text { sld30-16-19daa } \\
\text { sld30-16-19daa } \\
\text { sld30-16-19daa } \\
\text { sld30-16-19daa } \\
\text { sld30-16-19daa }\end{array}$ & $\begin{array}{l}1,540.0 \\
1,543.0 \\
1,546.0 \\
1,548.0 \\
1,550.0\end{array}$ & $\begin{array}{l}1,541.0 \\
1,544.0 \\
1,547.0 \\
1,549.0 \\
1,501.0\end{array}$ & $\begin{array}{l}317 \text { WTRM } \\
317 \text { WTRM } \\
317 \text { WTRM } \\
317 \text { WTRM } \\
\text { 317WTRM }\end{array}$ & $\begin{array}{r}8.1 \\
8.7 \\
8.7 \\
9.1 \\
12.0\end{array}$ & $\begin{array}{l}0.040000 \\
0.020000 \\
0.010000 \\
0.010000 \\
0.390000\end{array}$ & $\begin{array}{l}\text { Clayey, fine-grained, dolomitic sandstone } \\
\text { Clayey, fine-grained, dolomitic sandstone } \\
\text { Clayey, fine-grained, dolomitic sandstone } \\
\text { Clayey, fine-grained sandstone } \\
\text { Clayey, fine-grained sandstone }\end{array}$ \\
\hline $\begin{array}{l}\text { sld30-16-19daa } \\
\text { sld30-16-19daa } \\
\text { sld30-16-19daa } \\
\text { sld30-16-19daa } \\
\text { sld30-16-19daa }\end{array}$ & $\begin{array}{l}1,552.0 \\
1,553.0 \\
1,554.0 \\
1,555.0 \\
1,556.0\end{array}$ & $\begin{array}{l}1,553.0 \\
1,554.0 \\
1,555.0 \\
1,556.0 \\
1,557.0\end{array}$ & $\begin{array}{l}317 \text { WTRM } \\
317 \text { WTRM } \\
317 \text { WTRM } \\
\text { 317WTRM } \\
\text { 317WTRM }\end{array}$ & $\begin{array}{r}11.8 \\
9.4 \\
10.8 \\
15.6 \\
12.7\end{array}$ & $\begin{array}{l}0.290000 \\
0.030000 \\
0.170000 \\
1.500000 \\
0.780000\end{array}$ & $\begin{array}{l}\text { Clayey, fine-grained sandstone } \\
\text { Clayey, fine-grained sandstone } \\
\text { Fine-grained sandstone } \\
\text { Fine- to medium-grained sandstone } \\
\text { Fine- to medium-grained sandstone }\end{array}$ \\
\hline $\begin{array}{l}\text { sld30-16-19daa } \\
\text { sld30-16-19daa } \\
\text { sld30-16-19daa } \\
\text { sld30-16-19daa } \\
\text { sld30-16-19daa }\end{array}$ & $\begin{array}{l}1,557.0 \\
1,558.0 \\
1,559.0 \\
1,560.0 \\
1,561.0\end{array}$ & $\begin{array}{l}1,558.0 \\
1,559.0 \\
1,560.0 \\
1,561.0 \\
1,562.0\end{array}$ & $\begin{array}{l}\text { 317WTRM } \\
\text { 317WTRM } \\
\text { 317WTRM } \\
\text { 317WTRM } \\
\text { 317WTRM }\end{array}$ & $\begin{array}{l}15.1 \\
13.2 \\
15.1 \\
11.6 \\
10.2\end{array}$ & $\begin{array}{l}4.200000 \\
1.800000 \\
1.700000 \\
0.210000 \\
0.090000\end{array}$ & $\begin{array}{l}\text { Fine-grained sandstone } \\
\text { Fine- to medium-grained sandstone } \\
\text { Fine- to medium-grained sandstone } \\
\text { Fine- to medium-grained sandstone } \\
\text { Fine- to medium-grained sandstone }\end{array}$ \\
\hline $\begin{array}{l}\text { sld30-16-19daa } \\
\text { sld30-16-19daa } \\
\text { sld30-16-19daa } \\
\text { sld30-16-19daa } \\
\text { sld30-16-19daa }\end{array}$ & $\begin{array}{l}1,562.0 \\
1,563.0 \\
1,564.0 \\
1,565.0 \\
1,566.0\end{array}$ & $\begin{array}{l}1,563.0 \\
1,564.0 \\
1,565.0 \\
1,566.0 \\
1,567.0\end{array}$ & $\begin{array}{l}317 \text { WTRM } \\
317 \text { WTRM } \\
317 \text { WTRM } \\
317 \text { WTRM } \\
317 \text { WTRM }\end{array}$ & $\begin{array}{l}10.0 \\
12.2 \\
21.2 \\
20.0 \\
16.5\end{array}$ & $\begin{array}{r}0.080000 \\
0.180000 \\
769.000000 \\
801.000000 \\
66.000000\end{array}$ & $\begin{array}{l}\text { Fine- to medium-grained sandstone } \\
\text { Fine-grained sandstone } \\
\text { Fine- to medium-grained sandstone } \\
\text { Fine- to medium-grained sandstone } \\
\text { Fine- to medium-grained sandstone }\end{array}$ \\
\hline $\begin{array}{l}\text { sld30-16-19daa } \\
\text { sld30-16-19daa } \\
\text { sld30-16-19daa } \\
\text { sld30-16-19daa } \\
\text { sld30-16-19daa }\end{array}$ & $\begin{array}{l}1,567.0 \\
1,572.0 \\
1,573.0 \\
1,574.0 \\
1,575.0\end{array}$ & $\begin{array}{l}1,568.0 \\
1,573.0 \\
1,574.0 \\
1,575.0 \\
1,576.0\end{array}$ & $\begin{array}{l}\text { 317WTRM } \\
317 \text { WTRM } \\
317 \text { WTRM } \\
317 \text { WTRM } \\
317 \text { WTRM }\end{array}$ & $\begin{array}{l}17.9 \\
14.4 \\
15.4 \\
14.1 \\
15.7\end{array}$ & $\begin{array}{r}209.000000 \\
1.400000 \\
0.780000 \\
1.600000 \\
1.300000\end{array}$ & $\begin{array}{l}\text { Fine- to medium-grained sandstone } \\
\text { Clayey, fine-grained sandstone } \\
\text { Clayey, fine- to medium-grained sandstone } \\
\text { Clayey, fine- to medium-grained sandstone } \\
\text { Clayey, fine- to medium-grained sandstone }\end{array}$ \\
\hline $\begin{array}{l}\text { sld30-16-19daa } \\
\text { sld30-16-19daa } \\
\text { sld30-16-19daa } \\
\text { sld30-16-19daa } \\
\text { s } 1 d 30-16-19 \text { daa }\end{array}$ & $\begin{array}{l}1,576.0 \\
1,577.0 \\
1,580.0 \\
1,581.0 \\
1,582.0\end{array}$ & $\begin{array}{l}1,577.0 \\
1,578.0 \\
1,581.0 \\
1,582.0 \\
1,583.0\end{array}$ & $\begin{array}{l}\text { 317WTRM } \\
\text { 317WTRM } \\
\text { 317WTRM } \\
\text { 317WTRM } \\
\text { 317WTRM }\end{array}$ & $\begin{array}{r}11.8 \\
9.9 \\
11.3 \\
5.8 \\
19.2\end{array}$ & $\begin{array}{r}0.070000 \\
0.050000 \\
0.280000 \\
0.060000 \\
134.0000000\end{array}$ & $\begin{array}{l}\text { Clayey, fine-grained sandstone } \\
\text { Fine-grained sandstone } \\
\text { Fine- to medium-grained sandstone } \\
\text { Fine-grained, quartzitic sandstone } \\
\text { Fine-grained sandstone }\end{array}$ \\
\hline $\begin{array}{l}\text { sld30-16-19daa } \\
\text { sld30-16-19daa } \\
\text { sld30-16-19daa } \\
\text { sld30-16-19daa } \\
\text { sld30-16-19daa }\end{array}$ & $\begin{array}{l}1,583.0 \\
1,584.0 \\
1,585.0 \\
1,586.0 \\
1,587.0\end{array}$ & $\begin{array}{l}1,584.0 \\
1,585.0 \\
1,586.0 \\
1,587.0 \\
1,588.0\end{array}$ & $\begin{array}{l}\text { 317WTRM } \\
\text { 317WTRM } \\
\text { 317WTRM } \\
\text { 317WTRM } \\
\text { 317WTRM }\end{array}$ & $\begin{array}{l}19.6 \\
20.8 \\
17.9 \\
17.0 \\
16.8\end{array}$ & $\begin{array}{r}137.000000 \\
125.000000 \\
108.000000 \\
85.000000 \\
82.000000\end{array}$ & $\begin{array}{l}\text { Fine-grained sandstone } \\
\text { Fine-grained sandstone } \\
\text { Fine-grained sandstone } \\
\text { Fine-grained sandstone } \\
\text { Fine-grained sandstone }\end{array}$ \\
\hline
\end{tabular}


Table 4.--Laboratory-determined porosity and permeability--Continued

\begin{tabular}{|c|c|c|c|c|c|c|}
\hline Site & $\begin{array}{l}\text { Depth to } \\
\text { top } \\
\text { (feet) }\end{array}$ & $\begin{array}{l}\text { Depth to } \\
\text { bottom } \\
\text { (feet) }\end{array}$ & $\begin{array}{c}\text { Forma- } \\
\text { tion }\end{array}$ & $\begin{array}{l}\text { Porosity } \\
\text { (percent) }\end{array}$ & $\begin{array}{c}\text { Perme- } \\
\text { ability } \\
\text { (milli- } \\
\text { darcies) }\end{array}$ & Lithology \\
\hline $\begin{array}{l}\text { sld30-16-19daa } \\
\text { sld30-16-19daa } \\
\text { sld30-16-19daa } \\
\text { sld30-16-19daa } \\
\text { sld30-16-19daa }\end{array}$ & $\begin{array}{l}1,588.0 \\
1,589.0 \\
1,590.0 \\
1,591.0 \\
1,592.0\end{array}$ & $\begin{array}{l}1,589.0 \\
1,590.0 \\
1,591.0 \\
1,592.0 \\
1,593.0\end{array}$ & $\begin{array}{l}\text { 317WTRM } \\
\text { 317WTRM } \\
\text { 317WTRM } \\
\text { 317WTRM } \\
\text { 317WTRM }\end{array}$ & $\begin{array}{l}16.6 \\
18.8 \\
18.8 \\
17.3 \\
18.6\end{array}$ & $\begin{array}{r}102.000000 \\
83.000000 \\
160.000000 \\
84.000000 \\
122.000000\end{array}$ & $\begin{array}{l}\text { Fine-grained sandstone } \\
\text { Fine-grained sandstone } \\
\text { Fine-grained sandstone } \\
\text { Fine-grained sandstone } \\
\text { Fine-grained sandstone }\end{array}$ \\
\hline $\begin{array}{l}\text { sld30-16-19daa } \\
\text { sld30-16-19daa } \\
\text { sld30-16-19daa } \\
\text { sld30-16-19daa } \\
\text { sld30-16-19daa }\end{array}$ & $\begin{array}{l}1,593.0 \\
1,594.0 \\
1,595.0 \\
1,596.0 \\
1,597.0\end{array}$ & $\begin{array}{l}1,594.0 \\
1,595.0 \\
1,596.0 \\
1,597.0 \\
1,598.0\end{array}$ & $\begin{array}{l}\text { 317WTRM } \\
\text { 317WTRM } \\
\text { 317WTRM } \\
\text { 317WTRM } \\
\text { 317WTRM }\end{array}$ & $\begin{array}{l}17.7 \\
17.2 \\
17.3 \\
19.3 \\
15.3\end{array}$ & $\begin{array}{r}90.000000 \\
32.000000 \\
22.000000 \\
48.000000 \\
8.600000\end{array}$ & $\begin{array}{l}\text { Fine-grained sandstone } \\
\text { Fine-grained sandstone } \\
\text { Fine-grained sandstone } \\
\text { Fine-grained sandstone } \\
\text { Fine-grained sandstone }\end{array}$ \\
\hline $\begin{array}{l}\text { sld30-16-19daa } \\
\text { sld30-16-19daa } \\
\text { sld30-16-19daa } \\
\text { sld30-16-19daa } \\
\text { sld30-16-19daa }\end{array}$ & $\begin{array}{l}1,598.0 \\
1,599.0 \\
1,600.0 \\
1,601.0 \\
1,602.0\end{array}$ & $\begin{array}{l}1,599.0 \\
1,600.0 \\
1,601.0 \\
1,602.0 \\
1,603.0\end{array}$ & $\begin{array}{l}\text { 317WTRM } \\
\text { 317WTRM } \\
\text { 317WTRM } \\
\text { 317WTRM } \\
\text { 317WTRM }\end{array}$ & $\begin{array}{l}15.5 \\
20.9 \\
21.3 \\
21.5 \\
22.4\end{array}$ & $\begin{array}{r}13.000000 \\
110.000000 \\
138.000000 \\
161.000000 \\
333.000000\end{array}$ & $\begin{array}{l}\text { Fine-grained sandstone } \\
\text { Fine-grained sandstone } \\
\text { Fine-grained sandstone } \\
\text { Fine-grained sandstone } \\
\text { Fine-grained sandstone }\end{array}$ \\
\hline $\begin{array}{l}\text { sld30-16-19daa } \\
\text { sld30-16-19daa } \\
\text { sld30-16-19daa } \\
\text { sld30-16-19daa } \\
\text { sld30-16-19daa }\end{array}$ & $\begin{array}{l}1,603.0 \\
1,627.0 \\
1,628.0 \\
1,629.0 \\
1,630.0\end{array}$ & $\begin{array}{l}1,604.0 \\
1,628.0 \\
1,629.0 \\
1,630.0 \\
1,631.0\end{array}$ & $\begin{array}{l}\text { 317WTRM } \\
\text { 317WTRM } \\
\text { 317WTRM } \\
\text { 317WTRM } \\
\text { 317WTRM }\end{array}$ & $\begin{array}{l}20.1 \\
17.0 \\
17.3 \\
17.1 \\
16.2\end{array}$ & $\begin{array}{r}133.000000 \\
74.000000 \\
130.000000 \\
74.000000 \\
34.000000\end{array}$ & $\begin{array}{l}\text { Fine-grained sandstone } \\
\text { Fine-grained sandstone } \\
\text { Fine-grained sandstone } \\
\text { Fine-grained sandstone } \\
\text { Fine-grained sandstone }\end{array}$ \\
\hline $\begin{array}{l}\text { sld30-16-19daa } \\
\text { sld30-16-19daa } \\
\text { sld30-16-19daa } \\
\text { sld30-16-19daa } \\
\text { sld30-16-19daa }\end{array}$ & $\begin{array}{l}1,631.0 \\
1,632.0 \\
1,633.0 \\
1,634.0 \\
1,635.0\end{array}$ & $\begin{array}{r}1,632.0 \\
1,633.0 \\
1,634.0 \\
1,635.0 \\
11,636.0\end{array}$ & $\begin{array}{l}\text { 317WTRM } \\
\text { 317WTRM } \\
\text { 317WTRM } \\
\text { 317WTRM } \\
\text { 317WTRM }\end{array}$ & $\begin{array}{l}19.0 \\
18.7 \\
19.0 \\
18.8 \\
15.5\end{array}$ & $\begin{array}{r}149.000000 \\
196.000000 \\
107.000000 \\
183.000000 \\
51.000000\end{array}$ & $\begin{array}{l}\text { Fine-grained sandstone } \\
\text { Fine- to medium-grained sandstone } \\
\text { Fine-grained sandstone } \\
\text { Fine- to medium-grained sandstone } \\
\text { Fine-grained sandstone }\end{array}$ \\
\hline $\begin{array}{l}\text { sld30-16-19daa } \\
\text { sld30-16-19daa } \\
\text { sld30-16-19daa } \\
\text { sld30-16-19daa } \\
\text { sld30-16-19daa }\end{array}$ & $\begin{array}{l}1,636.0 \\
1,637.0 \\
1,638.0 \\
1,639.0 \\
1,640.0\end{array}$ & $\begin{array}{l}1,637.0 \\
1,638.0 \\
1,639.0 \\
1,640.0 \\
1,641.0\end{array}$ & $\begin{array}{l}\text { 317WTRM } \\
\text { 317WTRM } \\
\text { 317WTRM } \\
\text { 317WTRM } \\
\text { 317WTRM }\end{array}$ & $\begin{array}{l}15.2 \\
15.6 \\
22.9 \\
22.2 \\
14.8\end{array}$ & $\begin{array}{r}65.000000 \\
179.000000 \\
753.000000 \\
566.000000 \\
46.000000\end{array}$ & $\begin{array}{l}\text { Fine- to medium-grained sandstone } \\
\text { Fine- to medium-grained sandstone } \\
\text { Fine- to medium-grained sandstone } \\
\text { Fine- to medium-grained sandstone } \\
\text { Fine-grained sandstone }\end{array}$ \\
\hline $\begin{array}{l}\text { sld30-16-19daa } \\
\text { sld30-16-19daa } \\
\text { sld30-16-19daa } \\
\text { sld30-16-19daa } \\
\text { sld30-16-19daa }\end{array}$ & $\begin{array}{l}1,641.0 \\
1,642.0 \\
1,643.0 \\
1,644.0 \\
1,645.0\end{array}$ & $\begin{array}{l}1,642.0 \\
1,643.0 \\
1,644.0 \\
1,645.0 \\
1,646.0\end{array}$ & $\begin{array}{l}\text { 317WTRM } \\
\text { 317WTRM } \\
\text { 317WTRM } \\
\text { 317WTRM } \\
\text { 317WTRM }\end{array}$ & $\begin{array}{l}17.7 \\
20.2 \\
19.1 \\
22.2 \\
17.9\end{array}$ & $\begin{array}{r}90.000000 \\
236.000000 \\
373.000000 \\
468.000000 \\
519.000000\end{array}$ & $\begin{array}{l}\text { Fine-grained sandstone } \\
\text { Fine-grained sandstone } \\
\text { Fine- to medium-grained sandstone } \\
\text { Fine- to medium-grained sandstone } \\
\text { Fine- to medium-grained sandstone }\end{array}$ \\
\hline $\begin{array}{l}\text { s } 1 d 30-16-19 \text { daa } \\
\text { sld30-16-19daa } \\
\text { sld30-16-19daa } \\
\text { sld30-16-19daa } \\
\text { sld30-16-19daa }\end{array}$ & $\begin{array}{l}1,646.0 \\
1,647.0 \\
1,648.0 \\
1,649.0 \\
1,650.0\end{array}$ & $\begin{array}{l}1,647.0 \\
1,648.0 \\
1,649.0 \\
1,650.0 \\
1,651.0\end{array}$ & $\begin{array}{l}317 \text { WTRM } \\
\text { 317WTRM } \\
\text { 317WTRM } \\
\text { 317WTRM } \\
\text { 317WTRM }\end{array}$ & $\begin{array}{l}17.8 \\
11.1 \\
13.2 \\
12.0 \\
14.1\end{array}$ & $\begin{array}{l}2.100000 \\
1.100000 \\
0.590000 \\
2.900000 \\
0.900000\end{array}$ & $\begin{array}{l}\text { Fine- to medium-grained sandstone } \\
\text { Fine- to medium-grained sandstone } \\
\text { Fine-grained sandstone } \\
\text { Fine- to medium-grained sandstone } \\
\text { Fine- to medium-grained sandstone }\end{array}$ \\
\hline $\begin{array}{l}\text { sld30-16-19daa } \\
\text { sld30-16-19daa } \\
\text { sld30-16-19daa } \\
\text { sld30-16-19daa } \\
\text { sld30-16-19daa }\end{array}$ & $\begin{array}{l}1,657.0 \\
1,658.0 \\
1,659.0 \\
1,660.0 \\
1,661.0\end{array}$ & $\begin{array}{l}1,658.0 \\
1,659.0 \\
1,660.0 \\
1,661.0 \\
1,662.0\end{array}$ & $\begin{array}{l}\text { 317WTRM } \\
\text { 317WTRM } \\
\text { 317WTRM } \\
\text { 317WTRM } \\
\text { 317WTRM }\end{array}$ & $\begin{array}{l}19.8 \\
14.5 \\
20.2 \\
18.8 \\
20.9\end{array}$ & $\begin{array}{r}232.000000 \\
38.000000 \\
129.000000 \\
68.000000 \\
190.000000\end{array}$ & $\begin{array}{l}\text { Fine-grained sandstone } \\
\text { Clayey, fine- to medium-grained sandstone } \\
\text { Clayey, fine- to medium-grained sandstone } \\
\text { Clayey, fine- to medium-grained sandstone } \\
\text { Clayey, fine- to medium-grained sandstone }\end{array}$ \\
\hline $\begin{array}{l}\text { sld30-16-19daa } \\
\text { sld30-16-19daa } \\
\text { sld30-16-19daa } \\
\text { sld30-16-19daa } \\
\text { sld30-16-19daa }\end{array}$ & $\begin{array}{l}1,662.0 \\
1,663.0 \\
1,664.0 \\
1,665.0 \\
1,666.0\end{array}$ & $\begin{array}{l}1,663.0 \\
1,664.0 \\
1,665.0 \\
1,666.0 \\
1,667.0\end{array}$ & $\begin{array}{l}\text { 317WTRM } \\
\text { 317WTRM } \\
\text { 317WTRM } \\
\text { 317WTRM } \\
\text { 317WTRM }\end{array}$ & $\begin{array}{l}17.0 \\
19.7 \\
15.0 \\
20.8 \\
14.5\end{array}$ & $\begin{array}{r}39.000000 \\
207.000000 \\
37.000000 \\
154.000000 \\
29.000000\end{array}$ & $\begin{array}{l}\text { Clayey, fine-grained sandstone } \\
\text { Clayey, fine- to medium-grained sandstone } \\
\text { Clayey, medium- to coarse-grained sandstone } \\
\text { Clayey, fine- to medium-grained sandstone } \\
\text { Clayey, fine- to medium-grained sandstone }\end{array}$ \\
\hline
\end{tabular}


Table 4.--Laboratory-determined porosity and permeability--Continued

\begin{tabular}{|c|c|c|c|c|c|c|}
\hline Site & $\begin{array}{l}\text { Depth to } \\
\text { top } \\
\text { (feet) }\end{array}$ & $\begin{array}{l}\text { Depth to } \\
\text { bottom } \\
\text { (feet) }\end{array}$ & $\begin{array}{c}\text { Forma- } \\
\text { tion }\end{array}$ & $\begin{array}{l}\text { Porosity } \\
\text { (percent) }\end{array}$ & $\begin{array}{l}\text { Perme- } \\
\text { ability } \\
\text { (milli- } \\
\text { darcies) }\end{array}$ & Lithology \\
\hline $\begin{array}{l}\text { sld30-16-19daa } \\
\text { sld30-16-19daa } \\
\text { sld30-16-19daa } \\
\text { sld30-16-19daa } \\
\text { sld30-16-19daa }\end{array}$ & $\begin{array}{l}1,667.0 \\
1,668.0 \\
1,669.0 \\
1,670.0 \\
1,672.0\end{array}$ & $\begin{array}{l}1,668.0 \\
1,669.0 \\
1,670.0 \\
1,671.0 \\
1,673.0\end{array}$ & $\begin{array}{l}\text { 317WTRM } \\
\text { 317WTRM } \\
\text { 317WTRM } \\
\text { 317WTRM } \\
\text { 317WTRM }\end{array}$ & $\begin{array}{l}18.3 \\
17.0 \\
14.5 \\
17.1 \\
17.2\end{array}$ & $\begin{array}{r}57.000000 \\
32.000000 \\
3.400000 \\
38.000000 \\
62.000000\end{array}$ & $\begin{array}{l}\text { Clayey, fine- to medium-grained sandstone } \\
\text { Clayey, fine-grained sandstone } \\
\text { Clayey, fine-grained sandstone } \\
\text { Clayey, fine-grained sandstone } \\
\text { Clayey, fine-grained sandstone }\end{array}$ \\
\hline $\begin{array}{l}\text { sld30-16-19daa } \\
\text { sld30-16-19daa } \\
\text { sld30-16-19daa } \\
\text { sld30-16-19daa } \\
\text { sid30-16-19daa }\end{array}$ & $\begin{array}{l}1,674.0 \\
1,676.0 \\
1,688.0 \\
1,690.0 \\
1,692.0\end{array}$ & $\begin{array}{l}1,675.0 \\
1,677.0 \\
1,689.0 \\
1,691.0 \\
1,693.0\end{array}$ & $\begin{array}{l}317 \text { WTRM } \\
\text { 317WTRM } \\
\text { 317WTRM } \\
\text { 317WTRM } \\
\text { 317WTRM }\end{array}$ & $\begin{array}{l}14.1 \\
12.1 \\
17.4 \\
16.0 \\
14.2\end{array}$ & $\begin{array}{r}26.000000 \\
2.600000 \\
30.000000 \\
14.000000 \\
18.000000\end{array}$ & $\begin{array}{l}\text { Clayey, fine- to medium-grained, quartzitic sandstone } \\
\text { Clayey, quartzitic, medium- to coarse-sandstone } \\
\text { Clayey, fine-grained, quartzitic sandstone } \\
\text { Clayey, fine-grained sandstone } \\
\text { Clayey, quartzitic, medium- to coarse-sandstone }\end{array}$ \\
\hline $\begin{array}{l}\text { sld30-16-19daa } \\
\text { sld30-16-19daa } \\
\text { s } 1 \text { d30-16-19daa }\end{array}$ & $\begin{array}{l}1,694.0 \\
1,696.0 \\
1,698.0\end{array}$ & $\begin{array}{l}1,695.0 \\
1,697.0 \\
1,699.0\end{array}$ & $\begin{array}{l}\text { 317WTRM } \\
\text { 317WTRM } \\
\text { 317WTRM }\end{array}$ & $\begin{array}{l}14.3 \\
13.0 \\
14.9 \\
\end{array}$ & $\begin{array}{l}0.420000 \\
0.390000 \\
0.880000 \\
\end{array}$ & $\begin{array}{l}\text { Clayey, fine-grained sandstone } \\
\text { Clayey, fine-grained sandstone } \\
\text { Clayey, fine-grained sandstone }\end{array}$ \\
\hline SITE AVERAGE--- & ------ & 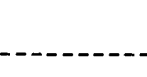 & AVERAGE-- & $\frac{16.1}{16.1}$ & $\frac{100.825714}{100.825714}$ & \\
\hline $\begin{array}{l}\text { s } 1 d 30-21-21 d d d \\
\text { s } 1 d 30-21-21 d d d \\
\text { s } 1 d 30-21-21 d d d \\
\text { s } 1 d 30-21-21 d d d\end{array}$ & $\begin{array}{r}92.0 \\
02.0 \\
99.0 \\
1,222.0\end{array}$ & $\begin{array}{r}92.1 \\
02.1 \\
99.1 \\
1,222.1\end{array}$ & $\begin{array}{l}317 \text { CDRM } \\
317 \text { CDRM } \\
317 \text { CDRM } \\
\text { 317CDRM } \\
\text { AVERAGE-- }\end{array}$ & $\begin{array}{r}9.4 \\
7.9 \\
20.0 \\
12.5 \\
12.5\end{array}$ & $\begin{array}{l}0.150000 \\
0.016000 \\
0.640000 \\
0.000710 \\
0.201678\end{array}$ & $\begin{array}{l}\text { Sandstone } \\
\text { Sandy siltstone } \\
\text { Sandstone } \\
\text { Sandstone }\end{array}$ \\
\hline $\begin{array}{l}\text { s } 1 d 30-21-21 d d d \\
\text { s } 1 \text { d } 30-21-21 d d d \\
\text { sld } 30-21-21 d d d \\
\text { s } 1 \text { d } 30-21-21 d d d \\
\text { s } 1 d 30-21-21 d d d\end{array}$ & $\begin{array}{l}1,631.0 \\
1,842.0 \\
2,024.0 \\
2,450.0 \\
2,559.0\end{array}$ & $\begin{array}{l}1,631 \cdot 1 \\
1,842 \cdot 1 \\
2,024.1 \\
2,450.1 \\
2,559.1\end{array}$ & $\begin{array}{l}\text { 321HKTL } \\
\text { 321HKTL } \\
\text { 321HKTL } \\
\text { 321HKTL } \\
\text { 321HKTL } \\
\text { AVERAGE-- }\end{array}$ & $\begin{array}{r}1.3 \\
15.5 \\
5.2 \\
5.4 \\
3.7 \\
6.2\end{array}$ & $\begin{array}{r}0.000000 \\
14.000000 \\
0.000000 \\
0.000000 \\
0.000000 \\
2.800000\end{array}$ & $\begin{array}{l}\text { Limestone } \\
\text { Sandstone } \\
\text { Limy siltstone } \\
\text { Silty limestone } \\
\text { Limestone }\end{array}$ \\
\hline $\begin{array}{l}\text { s } 1 \text { d } 30-21-21 d d d \\
\text { s } 1 \text { d } 30-21-21 d d d \\
\text { sld } 30-21-21 d d d \\
\text { s } 1 d 30-21-21 d d d \\
\text { s } 1 d 30-21-21 d d d\end{array}$ & $\begin{array}{l}2,912.0 \\
2,947.0 \\
3,037.0 \\
3,043.0 \\
3,129.0\end{array}$ & $\begin{array}{l}2,912.1 \\
2,947.1 \\
3,037.1 \\
3,043.1 \\
3,129.1\end{array}$ & $\begin{array}{l}\text { 324PRDX } \\
\text { 324PRDX் } \\
\text { 324PRDX } \\
\text { 324PRDX } \\
\text { 324PRDX }\end{array}$ & $\begin{array}{r}7.9 \\
3.2 \\
0.6 \\
11.4 \\
0.3\end{array}$ & $\begin{array}{l}0.012000 \\
0.000600 \\
0.000170 \\
0.001800 \\
0.000000\end{array}$ & $\begin{array}{l}\text { Dolomitic limestone } \\
\text { Silty claystone } \\
\text { Anhydrite } \\
\text { Mudstone } \\
\text { Halite }\end{array}$ \\
\hline $\begin{array}{l}\text { sld30-21-21ddd } \\
\text { s } 1 \text { d } 30-21-21 d d d \\
\text { s } 1 \text { d } 30-21-21 d d d \\
\text { s } 1 \text { d } 30-21-21 d d d \\
\text { s } 1 \text { d } 30-21-21 d d d\end{array}$ & $\begin{array}{l}3,344.0 \\
3,367.0 \\
3,380.0 \\
3,445.0 \\
3,526.0\end{array}$ & $\begin{array}{l}3,344.1 \\
3,367.1 \\
3,380.1 \\
3,445.1 \\
3,526.1\end{array}$ & $\begin{array}{l}\text { 324PRDX } \\
\text { 324PRDX } \\
\text { 324PRDX } \\
\text { 324PRDX } \\
\text { 324PRDX }\end{array}$ & $\begin{array}{r}0.2 \\
10.2 \\
0.5 \\
0.6 \\
2.5\end{array}$ & $\begin{array}{l}0.000000 \\
0.000270 \\
0.000220 \\
0.000440 \\
0.012000\end{array}$ & $\begin{array}{l}\text { Halite } \\
\text { Mudstone and anhydrite } \\
\text { Halite } \\
\text { Anhydrite } \\
\text { Anhydrite }\end{array}$ \\
\hline $\begin{array}{l}\text { s } 1 d 30-21-21 d d d \\
\text { s } 1 d 30-21-21 d d d \\
\text { s } 1 d 30-21-21 d d d \\
\text { s } 1 d 30-21-21 d d d \\
\text { s } 1 d 30-21-21 d d d\end{array}$ & $\begin{array}{l}3,541.0 \\
3,843.0 \\
4,206.0 \\
4,214.0 \\
5,265.0\end{array}$ & $\begin{array}{l}3,541.1 \\
3,843 \cdot 1 \\
4,206 \cdot 1 \\
4,214.1 \\
5,265.1\end{array}$ & $\begin{array}{l}\text { 324PRDX } \\
\text { 324PRDX } \\
\text { 324PRDX } \\
\text { 324PRDX } \\
\text { 324PRDX } \\
\text { AVERAGE-- }\end{array}$ & $\begin{array}{r}12.6 \\
0.4 \\
0.6 \\
0.8 \\
6.5 \\
3.9\end{array}$ & $\begin{array}{l}0.008000 \\
0.000690 \\
0.000210 \\
0.000400 \\
0.000390 \\
0.002479\end{array}$ & $\begin{array}{l}\text { Mudstone } \\
\text { Siltstone } \\
\text { Halite } \\
\text { Siltstone } \\
\text { Silty limestone }\end{array}$ \\
\hline $\begin{array}{l}\text { s1d } 30-21-21 d d d \\
\text { s1d } 30-21-21 d d d\end{array}$ & $\begin{array}{l}5,579.0 \\
5,596.0\end{array}$ & $\begin{array}{l}5,579.1 \\
5,596.1\end{array}$ & $\begin{array}{l}\text { 324PKTL } \\
\text { 324PKTL } \\
\text { AVERAGE-- }\end{array}$ & $\begin{array}{l}4.5 \\
2.9 \\
3.7\end{array}$ & $\begin{array}{l}0.000000 \\
0.000000 \\
0.000000\end{array}$ & $\begin{array}{l}\text { Limy siltstone } \\
\text { Limy siltstone }\end{array}$ \\
\hline s1d $30-21-21 d d d$ & $5,806.0$ & $5,806.1$ & $\begin{array}{l}\text { 327MOLS } \\
\text { AVERAGE-- }\end{array}$ & $\frac{2.4}{2.4}$ & $\frac{0.000190}{0.000190}$ & Limy sandstone \\
\hline
\end{tabular}


Table 4.--Laboratory-determined porosity and permeability--Continued

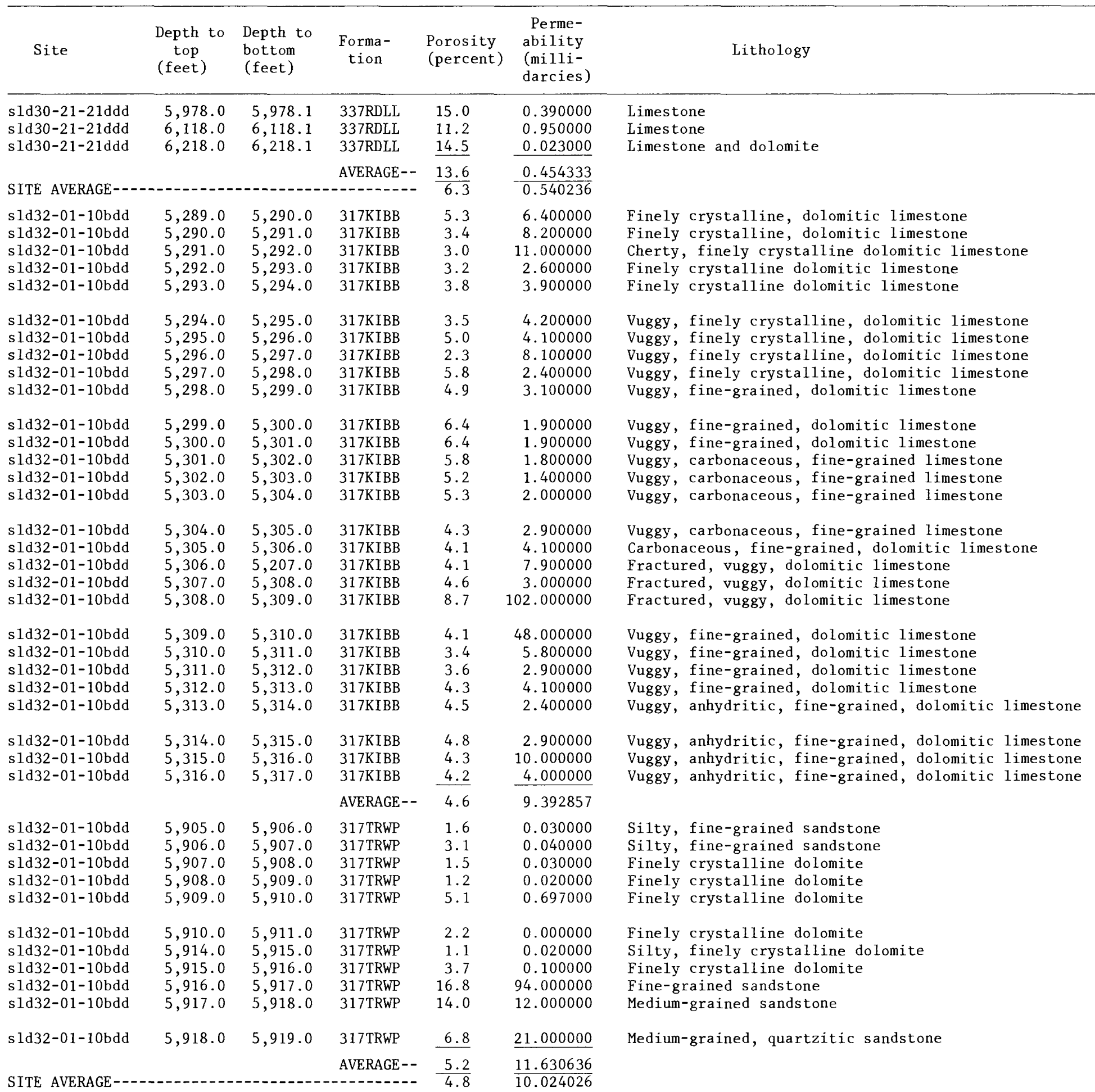


Table 4.--Laboratory-determined porosity and permeability--Continued

\begin{tabular}{|c|c|c|c|c|c|c|c|c|}
\hline Site & $\begin{array}{l}\text { Depth to } \\
\text { top } \\
\text { (feet) }\end{array}$ & $\begin{array}{l}\text { Depth to } \\
\text { bottom } \\
\text { (feet) }\end{array}$ & $\begin{array}{c}\text { Forma- } \\
\text { tion }\end{array}$ & $\begin{array}{l}\text { Porosity } \\
\text { (percent) }\end{array}$ & $\begin{array}{l}\text { Perme- } \\
\text { ability } \\
\text { (milli- } \\
\text { darcies) }\end{array}$ & \multicolumn{3}{|c|}{ Lithology } \\
\hline sld34-21-09bbc & $6,377.0$ & $6,378.0$ & 337RDLL & 0.6 & 0.000000 & Finely & crystalline & limestone \\
\hline s $1 \mathrm{~d} 34-21-09 \mathrm{bbc}$ & $6,378.0$ & $6,379.0$ & 337RDLL & 0.9 & 0.000000 & Finely & crystalline & limestone \\
\hline sld34-21-09bbc & $6,379.0$ & $6,380.0$ & 337RDLL & 0.7 & 0.000000 & Finely & crystalline & limestone \\
\hline sld34-21-09bbc & $6,380.0$ & $6,381.0$ & 337RDLL & 0.8 & 0.000000 & Finely & crystalline & limestone \\
\hline $\operatorname{sld} 34-21-09 b \mathrm{bc}$ & $6,381.0$ & $6,382.0$ & 337RDLL & 0.8 & 0.000000 & Finely & crystalline & limestone \\
\hline $\mathrm{sld} 34-21-09 \mathrm{bbc}$ & $6,382.0$ & $6,383.0$ & 337RDLL & 0.6 & 0.000000 & Finely & crystalline & limestone \\
\hline sld34-21-09bbc & 6.383 .0 & $6,384.0$ & 337RDLL & 0.8 & 0.000000 & Finely & crystalline & limestone \\
\hline sld34-21-09bbc & $6,384.0$ & $6,385.0$ & 337RDLL & 0.7 & 0.900000 & Finely & crystalline & limestone \\
\hline sld34-21-09bbc & $6,385.0$ & $6,386.0$ & 337RDLL & 0.8 & 0.000000 & Finely & crystalline & limestone \\
\hline sld34-21-09bbc & $6,386.0$ & $6,387.0$ & 337RDLL & 0.7 & 0.000000 & Finely & crystalline & limestone \\
\hline sld34-21-09bbc & $6,387.0$ & $6,388.0$ & 337RDLL & 1.2 & 0.000000 & Finely & crystalline & limestone \\
\hline $\mathrm{s} 1 \mathrm{~d} 34-21-09 \mathrm{bbc}$ & $6,388.0$ & $6,389.0$ & 337RDLL & 1.0 & 0.000000 & Finely & crystalline & limestone \\
\hline $\operatorname{sld} 34-21-09 b b c$ & $6,389.0$ & $6,390.0$ & 337RDLL & 0.5 & 0.000000 & Finely & crystalline & limestone \\
\hline sld34-21-09bbc & $6,390.0$ & $6,391.0$ & 337RDLL & 0.6 & 0.000000 & Finely & crystalline & limestone \\
\hline sld34-21-09bbc & $6,391.0$ & $6,392.0$ & 337RDLL & 0.7 & 0.000000 & Finely & crystalline & limestone \\
\hline sld34-21-09bbc & $6,392.0$ & $6,393.0$ & 337RDLL & 5.1 & 0.000000 & Finely & crystalline & limestone \\
\hline sld $34-21-09 b b c$ & $6,393.0$ & $6,394.0$ & 337RDLL & 10.0 & 0.000000 & Finely & crystalline & limestone \\
\hline s ld $34-21-09 b b c$ & $6,394.0$ & $6,395.0$ & 337RDLL & 9.1 & 0.000000 & Finely & crystalline & limestone \\
\hline s $1 d 34-21-09 b b c$ & $6,395.0$ & $6,396.0$ & 337RDLL & 4.5 & 0.000000 & Finely & crystalline & limestone \\
\hline sld34-21-09bbc & $6,396.0$ & $6,397.0$ & $337 \mathrm{RDLL}$ & 5.5 & 0.000000 & Finely & crystalline & limestone \\
\hline sld34-21-09bbc & $6,397.0$ & $6,398.0$ & 337 RDLL & 5.4 & 0.000000 & Finely & crystalline & limestone \\
\hline sld34-21-09bbc & $6,398.0$ & $6,399.0$ & 337RDLL & 6.9 & 0.000000 & Finely & crystalline & limestone \\
\hline sld34-21-09bbc & $6,399.0$ & $6,400.0$ & 337RDLL & 0.9 & 0.000000 & Finely & crystalline & limestone \\
\hline s $1 d 34-21-09 b b c$ & $6,400.0$ & $6,401.0$ & 337RDLL & 1.5 & 0.000000 & Finely & crystalline & limestone \\
\hline sld34-21-09bbc & $6,401.0$ & $6,402.0$ & 337RDLL & 0.9 & 0.000000 & Finely & crystalline & limestone \\
\hline sld34-21-09bbc & $6,402.0$ & $6,403.0$ & 337RDLL & 0.7 & 0.000000 & Finely & crystalline & limestone \\
\hline sld34-21-09bbc & $6,403.0$ & $6,404.0$ & 337RDLL & 0.8 & 0.000000 & Finely & crystalline & limestone \\
\hline sld34-21-09bbc & $6,404.0$ & $6,405.0$ & 337RDLL & 1.2 & 0.000000 & Finely & crystalline & limestone \\
\hline sld34-21-09bbc & $6,405.0$ & $6,406.0$ & 337RDLL & 1.2 & 0.000000 & Finely & crystalline & limestone \\
\hline sld34-21-09bbc & $6,406.0$ & $6,407.0$ & 337RDLL & 1.0 & 0.000000 & Finely & crystalline & limestone \\
\hline sld34-21-09bbc & $6,407.0$ & $6,408.0$ & 337RDLL & 1.4 & 0.000000 & Finely & crystalline & limestone \\
\hline s1d34-21-09bbc & $6,408.0$ & $6,409.0$ & 337RDLL & 1.0 & 0.000000 & Finely & crystalline & limestone \\
\hline sld34-21-09bbc & $6,409.0$ & $6,410.0$ & 337RDLL & 2.0 & 0.000000 & Finely & crystalline & limestone \\
\hline sld34-21-09bbc & $6,410.0$ & $6,411.0$ & 337RDLL & 1.1 & 0.000000 & Finely & crystalline & limestone \\
\hline sld34-21-09bbc & $6,411.0$ & $6,412.0$ & 337RDLL & 1.6 & 0.000000 & Finely & crystalline & limestone \\
\hline sld34-21-09bbc & $6,412.0$ & $6,413.0$ & 337RDLL & 1.6 & 0.000000 & Finely & crystalline & limestone \\
\hline $\mathrm{sld} 34-21-09 \mathrm{bbc}$ & $6,413.0$ & $6,414.0$ & 337RDLL & 1.1 & 0.000000 & Finely & crystalline & limestone \\
\hline sld34-21-09bbc & $6,414.0$ & $6,415.0$ & 337RDLL & 1.1 & 0.000000 & Finely & crystalline & limestone \\
\hline s1d34-21-09bbc & $6,415.0$ & $6,416.0$ & $337 \mathrm{RDLL}$ & 1.0 & 0.000000 & Finely & crystalline & limestone \\
\hline $\operatorname{sld} 34-21-09 b b c$ & $6,416.0$ & $6,417,0$ & 337RDLL & 0.8 & 0.000000 & Finely & crystalline & limestone \\
\hline sld34-21-09bbc & $6,417.0$ & $6,418.0$ & 337RDLL & 1.1 & 0.000000 & Finely & crystalline & limestone \\
\hline s $1 d 34-21-09 b b c$ & $6,418.0$ & $6,419.0$ & 337RDLL & 0.9 & 0.000000 & Finely & crystalline & limestone \\
\hline sld34-21-09bbc & $6,419.0$ & $6,420.0$ & 337RDLL & 1.0 & 0.000000 & Finely & crystalline & limestone \\
\hline sld34-21-09bbc & $6,420.0$ & $6,421.0$ & $337 R D L L$ & 1.3 & 0.000000 & Finely & crystalline & limestone \\
\hline sld34-21-09bbc & $6,421.0$ & $6,422.0$ & 337 RDLL & 0.9 & 0.000000 & Finely & crystalline & limestone \\
\hline $\mathrm{s} 1 \mathrm{~d} 34-21-09 \mathrm{bbc}$ & $6,422.0$ & $6,423.0$ & 337RDLL & 1.1 & 0.000000 & Finely & crystalline & limestone \\
\hline
\end{tabular}


Table 4.--Laboratory-determined porosity and permeability--Continued

\begin{tabular}{|c|c|c|c|c|c|c|}
\hline Site & $\begin{array}{l}\text { Depth to } \\
\text { top } \\
\text { (feet) }\end{array}$ & $\begin{array}{l}\text { Depth to } \\
\text { bottom } \\
\text { (feet) }\end{array}$ & $\begin{array}{c}\text { Forma- } \\
\text { tion }\end{array}$ & $\begin{array}{l}\text { Porosity } \\
\text { (percent) }\end{array}$ & $\begin{array}{l}\text { Perme- } \\
\text { ability } \\
\text { (milli- } \\
\text { darcies) }\end{array}$ & Lithology \\
\hline s ld 34-21-09bbc & $6,423.0$ & $6,424.0$ & 337RDLL & 1.3 & 0.000000 & Finely crystalline limestone \\
\hline sld34-21-09bbc & $6,424.0$ & $6,425.0$ & 337RDLL & 0.7 & 0.000000 & Finely crystalline limestone \\
\hline sld34-2l-09bbc & $6,425.0$ & $6,426.0$ & 337RDLL & 1.0 & 0.000000 & Finely crystalline limestone \\
\hline s1d34-21-09bbc & $6,426.0$ & $6,427.0$ & 337RDLL & 1.4 & 0.000000 & Finely crystalline limestone \\
\hline sld34-21-09bbc & $6,427.0$ & $6,428.0$ & 337RDLL & 1.0 & 0.000000 & Finely crystalline limestone \\
\hline s $1 d 34-21-09 b b c$ & $6,428.0$ & $6,429.0$ & 337RDLL & 1.0 & 0.000000 & Finely crystalline limestone \\
\hline s $1 d 34-21-09 b b c$ & $6,429.0$ & $6,430.0$ & 337RDLL & 0.8 & 0.000000 & Finely crystalline limestone \\
\hline s1d34-21-09bbc & $6,430.0$ & $6,431.0$ & 337RDLL & 0.9 & 0.000000 & Finely crystalline limestone \\
\hline s $1 d 34-21-09 b b c$ & $6,431.0$ & $6,432.0$ & 337RDLL & 1.0 & 0.000000 & Finely crystalline limestone \\
\hline sld34-21-09bbc & $6,432.0$ & $6,433.0$ & 337RDLL & 1.1 & 0.000000 & Finely crystalline limestone \\
\hline sld34-2l-09bbc & $6,433.0$ & $6,434.0$ & 337RDLL & 0.7 & 0.000000 & Finely crystalline limestone \\
\hline s1d34-21-09bbc & $6,434.0$ & $6,435.0$ & 337RDLL & 1.7 & 0.000000 & Finely crystalline limestone \\
\hline sld34-21-09bbc & $6,474.0$ & $6,475.0$ & 337RDLL & 15.5 & 0.140000 & Anhydritic, finely crystalline dolomite \\
\hline sld34-21-09bbc & $6,475.0$ & $6,476.0$ & 337RDLL & 10.7 & 0.020000 & Anhydritic, finely crystalline dolomite \\
\hline sld34-21-09bbc & $6,476.0$ & $6,477.0$ & 337RDLL & 12.6 & 0.040000 & Anhydritic, finely crystalline dolomite \\
\hline s $1 d 34-21-09 b b c$ & $6,477.0$ & $6,478.0$ & 337RDLL & 11.7 & 0.030000 & Anhydritic, finely crystalline dolomite \\
\hline s ld34-2l-09bbc & $6,478.0$ & $6,479.0$ & 337RDLL & 17.1 & 0.130000 & Anhydritic, finely crystalline dolomite \\
\hline sld34-21-09bbc & $6,479.0$ & $6,480.0$ & 337RDLL & 10.0 & 0.010000 & Anhydritic, finely crystalline dolomite \\
\hline sld34-21-09bbc & $6,480.0$ & $6,481.0$ & 337RDLL & 12.0 & 0.030000 & Anhydritic, finely crystalline dolomite \\
\hline sld34-21-09bbc & $6,481.0$ & $6,482.0$ & 337RDLL & 6.4 & 0.010000 & Anhydritic, finely crystalline dolomite \\
\hline sld34-21-09bbc & $6,482.0$ & $6,483.0$ & 337RDLL & 12.4 & 0.020000 & Anhydritic, finely crystalline dolomite \\
\hline s $1 \mathrm{~d} 34-21-09 \mathrm{bbc}$ & $6,483.0$ & $6,484.0$ & 337RDLL & 7.4 & 0.010000 & Anhydritic, finely crystalline dolomite \\
\hline $\operatorname{sld} 34-21-09 b b c$ & $6,484.0$ & $6,485.0$ & 337RDLL & 8.4 & 0.010000 & Anhydritic, finely crystalline dolomite \\
\hline s $1 d 34-21-09 b b c$ & $6,485.0$ & $6,486.0$ & 337RDLL & 8.1 & 0.010000 & Anhydritic, finely crystalline dolomite \\
\hline sld34-21-09bbc & $6,486.0$ & $6,487.0$ & 337RDLL & 7.0 & 0.010000 & Anhydritic, finely crystalline dolomite \\
\hline s $1 \mathrm{~d} 34-21-09 \mathrm{bbc}$ & $6,487.0$ & $6,488.0$ & 337RDLL & 9.2 & 0.010000 & Anhydritic, finely crystalline dolomite \\
\hline s $1 d 34-21-09 b b c$ & $6,488.0$ & $6,489.0$ & 337RDLL & 9.6 & 0.010000 & Anhydritic, finely crystalline dolomite \\
\hline s $1 \mathrm{~d} 34-21-09 \mathrm{bbc}$ & $6,489.0$ & $6,490.0$ & 337RDLL & 3.3 & 0.010000 & Anhydritic, finely crystalline dolomite \\
\hline s $1 d 34-21-09 b b c$ & $6,490.0$ & $6,491.0$ & 337RDLL & 2.1 & 0.010000 & Anhydritic, finely crystalline dolomite \\
\hline sld34-21-09bbc & $6,491.0$ & $6,492.0$ & 337RDLL & 1.7 & 0.010000 & Anhydritic, finely crystalline dolomite \\
\hline sld34-21-09bbc & $6,492.0$ & $6,493.0$ & 337RDLL & 2.2 & 0.010000 & Anhydritic, finely crystalline dolomite \\
\hline sld34-21-09bbc & $6,493.0$ & $6,494.0$ & 337RDLL & 2.5 & 0.010000 & Anhydritic, finely crystalline dolomite \\
\hline sld $34-21-09 b b c$ & $6,494.0$ & $6,495.0$ & 337RDLL & 5.3 & 0.010000 & Anhydritic, finely crystalline dolomite \\
\hline s $1 \mathrm{~d} 34-21-09 \mathrm{bbc}$ & $6,495.0$ & $6,496.0$ & 337RDLL & 10.3 & 0.010000 & Anhydritic, finely crystalline dolomite \\
\hline sld34-21-09bbc & $6,496.0$ & $6,497.0$ & 337RDLL & 11.5 & 0.050000 & Anhydritic, finely crystalline dolomite \\
\hline s $1 d 34-21-09 b b c$ & $6,497.0$ & $6,498.0$ & 337RDLL & 6.6 & 0.010000 & Anhydritic, finely crystalline dolomite \\
\hline s $1 d 34-21-09 b b c$ & $6,498.0$ & $6,499.0$ & 337RDLL & 11.4 & 0.010000 & Anhydritic, finely crystalline dolomite \\
\hline sld34-21-09bbc & $6,499.0$ & $6,500.0$ & 337RDLL & 10.0 & 0.020000 & Anhydritic, finely crystalline dolomite \\
\hline sld34-21-09bbc & $6,500.0$ & $6,501.0$ & 337RDLL & 7.9 & 0.020000 & Anhydritic, finely crystalline dolomite \\
\hline s $1 d 34-21-09 b b c$ & $6,501.0$ & $6,502.0$ & 337RDLL & 5.6 & 0.010000 & Anhydritic, finely crystalline dolomite \\
\hline s $1 \mathrm{~d} 34-21-09 \mathrm{bbc}$ & $6,502.0$ & $6,503.0$ & 337RDLL & 6.3 & 0.010000 & Anhydritic, finely crystalline dolomite \\
\hline s $1 \mathrm{~d} 34-21-09 \mathrm{bbc}$ & $6,503.0$ & $6,504.0$ & 337RDLL & 14.3 & 0.010000 & Anhydritic, finely crystalline dolomite \\
\hline sld34-21-09bbc & $6,504.0$ & $6,505.0$ & 337RDLL & 15.2 & 0.010000 & Anhydritic, finely crystalline dolomite \\
\hline s $1 d 34-21-09 b b c$ & $6,505.0$ & $6,506.0$ & 337RDLL & 12.5 & 0.010000 & Anhydritic, finely crystalline dolomite \\
\hline sld $34-21-09 b b c$ & $6,506.0$ & $6,507.0$ & 337RDLL & 13.9 & 0.030000 & Anhydritic, finely crystalline dolomite \\
\hline s $1 d 34-21-09 b b c$ & $6,507.0$ & $6,508.0$ & 337RDLL & 13.9 & 0.020000 & Anhydritic, finely crystalline dolomite \\
\hline s $1 d 34-21-09 b b c$ & $6,508.0$ & $6,509.0$ & 337RDLL & 12.4 & 0.010000 & Anhydritic, finely crystalline dolomite \\
\hline s $1 \mathrm{~d} 34-21-09 \mathrm{bbc}$ & $6,509.0$ & $6,510.0$ & 337RDLL & 14.5 & 0.010000 & Anhydritic, finely crystalline dolomite \\
\hline s $1 d 34-21-09 b b c$ & $6,510.0$ & $6,511.0$ & 337RDLL & 14.5 & 0.010000 & Anhydritic, finely crystalline dolomite \\
\hline s ld $34-21-09 b b c$ & $6,511.0$ & $6,512.0$ & 337RDLL & 14.7 & 0.010000 & Anhydritic, finely crystalline dolomite \\
\hline
\end{tabular}


Table 4.--Laboratory-determined porosity and permeability--Continued

\begin{tabular}{|c|c|c|c|c|c|c|}
\hline Site & $\begin{array}{l}\text { Depth to } \\
\text { top } \\
\text { (feet) }\end{array}$ & $\begin{array}{l}\text { Depth to } \\
\text { bottom } \\
\text { (feet) }\end{array}$ & $\begin{array}{c}\text { Forma- } \\
\text { tion }\end{array}$ & $\begin{array}{l}\text { Porosity } \\
\text { (percent) }\end{array}$ & $\begin{array}{l}\text { Perme- } \\
\text { ability } \\
\text { (milli- } \\
\text { darcies) }\end{array}$ & Lithology \\
\hline s $1 d 34-21-09 b b c$ & $6,512.0$ & $6,513.0$ & 337RDLL & 7.2 & 0.010000 & Anhydritic, finely crystalline do \\
\hline $\mathrm{s} 1 \mathrm{~d} 34-21-09 \mathrm{bbc}$ & $6,513.0$ & $6,514.0$ & 337RDLL & 8.8 & 0.200000 & Anhydritic, finely crystalline do \\
\hline sld34-21-09bbc & $6,514.0$ & $6,515.0$ & 337RDLL & 13.5 & 0.010000 & Anhydritic, finely crystalline do \\
\hline $\mathrm{s} 1 \mathrm{~d} 34-21-09 \mathrm{bbc}$ & $6,515.0$ & $6,516.0$ & 337RDLL & 11.7 & 0.010000 & Anhydritic, finely crystalline do \\
\hline sld34-21-09bbc & $6,516.0$ & $6,517.0$ & 337RDLL & 13.7 & 0.010000 & Anhydritic, finely crystalline do \\
\hline sld34-21-09bbc & $6,517.0$ & $6,518.0$ & 337RDLL & 11.5 & 0.010000 & Anhydritic, finely crystalline do \\
\hline $\mathrm{s} 1 \mathrm{~d} 34-21-09 \mathrm{bbc}$ & $6,518.0$ & $6,519.0$ & 337RDLL & 10.4 & 0.010000 & Anhydritic, finely crystalline do \\
\hline sld34-21-09bbc & $6,519.0$ & $6,520.0$ & 337RDLL & $\underline{12.9 i}$ & 0.010000 & Anhydritic, finely crystalline do \\
\hline & & & AVERAGE-- & 5.4 & 0.019038 & \\
\hline SITE AVERAGE-- & 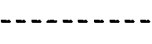 & 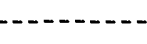 & & 5.4 & 0.019038 & \\
\hline $\mathrm{s} 1 \mathrm{~d} 36-10-21 \mathrm{bdb}$ & $2,972.0$ & $2,973.0$ & 317WTRM & 12.9 & 54.000000 & Fine-grained sandstone \\
\hline s $1 d 36-10-21 b d b$ & $2,973.0$ & $2,974.0$ & 317WTRM & 18.1 & 161.000000 & Fine-grained sandstone \\
\hline $\mathrm{s} 1 \mathrm{~d} 36-10-21 \mathrm{bdb}$ & $2,974.0$ & $2,975.0$ & 317WTRM & 18.1 & 259.000000 & Fine-grained sandstone \\
\hline s $1 d 36-10-21 b d b$ & $2,975.0$ & $2,976.0$ & 317WTRM & 19.3 & 14.000000 & Fine-grained, dolomitic sandstone \\
\hline $\operatorname{sid} 36-10-21 b d b$ & $2,976.0$ & $2,977.0$ & 317WTRM & 19.2 & 262.000000 & Fine-grained, dolomitic sandstone \\
\hline $\mathrm{s} 1 \mathrm{~d} 36-10-21 \mathrm{bdb}$ & $2,977.0$ & $2,978.0$ & 317WTRM & 18.0 & 51.000000 & Fine-grained, dolomitic sandst \\
\hline sld $36-10-21 b d b$ & $2,978.0$ & $2,979.0$ & 317WTRM & 23.2 & 981.000000 & Fine-grained sandstone \\
\hline $\mathrm{s} 1 \mathrm{~d} 36-10-21 \mathrm{bdb}$ & $2,979.0$ & $2,980.0$ & 317 WTRM & 26.0 & 346.000000 & Fine-grained sands tone \\
\hline $\mathrm{s} 1 \mathrm{~d} 36-10-21 \mathrm{bdb}$ & $2,980.0$ & $2,981.0$ & 317WTRM & 21.1 & 673.000000 & Fine-grained sands tone \\
\hline s $1 d 36-10-21 b d b$ & $2,981.0$ & $2,981.5$ & 317WTRM & 18.6 & 149.000000 & Fine- to coarse-grained sandstone \\
\hline $\mathrm{s} 1 \mathrm{~d} 36-10-21 \mathrm{bdb}$ & $2,982.0$ & $2,983.0$ & 317WTRM & 15.1 & 576.000000 & Fine- to coarse-grained sandstone \\
\hline $\operatorname{s} 1 \mathrm{~d} 36-10-21 \mathrm{bdb}$ & $2,983.0$ & $2,984.0$ & 317WTRM & 17.4 & 731.000000 & Fine- to coarse-grained sandstone \\
\hline $\operatorname{sld} 36-10-21 b d b$ & $2,984.0$ & $2,985.0$ & 317WTRM & 13.3 & 496.000000 & Fine- to coarse-grained sandstone \\
\hline $\operatorname{s} 1 d 36-10-21 b d b$ & $2,985.0$ & $2,986.0$ & 317WTRM & 15.1 & 28.000000 & Fine- to coarse-grained sandstone \\
\hline s $1 d 36-10-21 b d b$ & $2,986.0$ & $2,987.0$ & 317 WTRM & 20.7 & 981.000000 & Fine- to coarse-grained sandstone \\
\hline
\end{tabular}

$\operatorname{sld} 36-10-21 \mathrm{bdb}$ sld36-10-21bdb sld36-10-21bdb s $1 \mathrm{~d} 36-10-21 \mathrm{bdb}$ sld36-10-21bdb

s $1 \mathrm{~d} 36-10-21 \mathrm{bdb}$ s $1 \mathrm{~d} 36-10-21 \mathrm{bdb}$ s $1 \mathrm{~d} 36-10-21 \mathrm{bdb}$ sld $36-10-21 b d b$ s1d36-10-21bdb

sld $36-10-21 b d b$ sld36-10-21bdb s $1 d 36-10-21 b d b$ s1d36-10-21bdb sld36-10-21bdb

sld36-10-21bdb s 1 d $36-10-21 b d b$ sld36-10-21bdb $\operatorname{sid} 36-10-21 b d b$ sld36-10-21bdb

$2,987.0 \quad 2,988.0 \quad 317$ WTRM

$2,988.0 \quad 2,989.0 \quad 317$ WTRM

$2,989.0 \quad 2,990.0 \quad 317$ WTRM

$2,990.0 \quad 2,991.0 \quad 317$ WTRM

2,991.0 2,991.5 317WTRM

$20.6 \quad 1,115.000000$

$20.5 \quad 654.000000$

$18.3 \quad 673.000000$

$15.4 \quad 731.000000$

$18.82,115.000000$

$\begin{array}{lllll}2,992.0 & 2,993.0 & 317 \text { WTRM } & 18.1 & 542.000000\end{array}$

$\begin{array}{lllll}2,993.0 & 2,994.0 & 317 W T R M & 14.5 & 788.000000\end{array}$

$\begin{array}{lllll}2,994.0 & 2,995.0 & 317 \text { WTRM } & 16.0 & 167.000000\end{array}$

$\begin{array}{lllll}2,995.0 & 2,996.0 & 317 \text { WTRM } & 15.8 & 357.000000\end{array}$

$\begin{array}{lllll}2,996.0 & 2,997.0 & 317 \text { WTRM } & 17.2 & 1,192.000000\end{array}$

$\begin{array}{lllll}2,997.0 & 2,998.0 & 317 \text { WTRM } & 14.0 & 15.000000\end{array}$

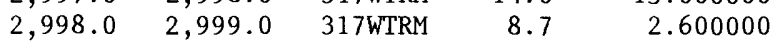

$\begin{array}{lllll}2,999.0 & 3,000.0 & 317 \text { WTRM } & 9.8 & 16.000000\end{array}$

$\begin{array}{lllll}3,000.0 & 3,001.0 & 317 \text { WTRM } & 11.4 & 21.000000\end{array}$

$\begin{array}{lllll}3,001.0 & 3,002.0 & 317 \text { WTRM } & 13.3 & 21.000000\end{array}$

$\begin{array}{lllll}3,002.0 & 3,003.0 & 317 \text { WTRM } & 14.4 & 44.000000\end{array}$

$3,003.0 \quad 3,004.0 \quad 317$ WTRM $\quad 14.6 \quad 57.000000$

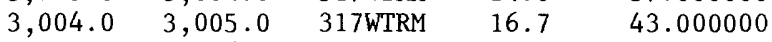

$\begin{array}{lllll}3,005.0 & 3,006.0 & 317 \text { WTRM } & 17.0 & 141.000000\end{array}$

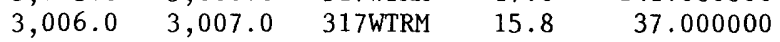

$\operatorname{sid} 36-10-21 b d b$ $\operatorname{sld} 36-10-21 b d b$ $\operatorname{sld} 36-10-21 b d b$ sld $36-10-21 b d b$ sld36-10-21bdb

$3,007.0 \quad 3,008.0 \quad 317$ WTRM $\quad 1 \% .4$

$3,008.0 \quad 3,009.0 \quad 317$ WTRM $\quad 14.7$

$3,009.0 \quad 3,010.0 \quad 317$ WTRM $\quad 14.9$

$3,010.0 \quad 3,011.0 \quad 317$ WTRM $\quad 16.9$

$\begin{array}{llll}3,011.0 & 3,012.0 \quad 317 \text { WTRM } & 16.2\end{array}$
72.000000 90.000000 49.000000 155.000000 101.000000
Fine-grained sandstone

Fine-grained sandstone

Fine-grained sandstone

Fine-grained sandstone

Fine-grained sandstone

Fine-grained sandstone

Fine-grained sandstone

Fine- to medium-grained sandstone Fine- to medium-grained sandstone Medium-grained sandstone

Fine-grained sandstone

Fine-grained sandstone

Fine-grained sandstone

Fine-grained sandstone

Fine-grained sandstone

Fine- to medium-grained sandstone Fine- to medium-grained sandstone Fine-grained sandstone

Fine-grained sandstone

Fine-grained sandstone

Fine-grained sandstone

Fine-grained sandstone

Fine-grained sandstone

Fine-grained sandstone

Fine-grained sandstone 
Table 4.--Laboratory-determined porosity and permeability--Continued

\begin{tabular}{|c|c|c|c|c|c|c|}
\hline Site & $\begin{array}{l}\text { Depth to } \\
\text { top } \\
\text { (feet) }\end{array}$ & $\begin{array}{l}\text { Depth to } \\
\text { bottom } \\
\text { (feet) }\end{array}$ & $\begin{array}{c}\text { Forma- } \\
\text { tion }\end{array}$ & $\begin{array}{l}\text { Porosity } \\
\text { (percent) }\end{array}$ & $\begin{array}{c}\text { Perme- } \\
\text { ability } \\
\text { (milli- } \\
\text { darcies) }\end{array}$ & Lithology \\
\hline s $1 d 36-25-27 b b a$ & $5,852.0$ & $5,853.0$ & $324 \mathrm{PRDX}$ & 10.6 & 5.000000 & Anhydritic, finely crystalline dolomite \\
\hline $\mathrm{s} 1 \mathrm{~d} 36-25-27 \mathrm{bba}$ & $5,853.0$ & $5,854.0$ & 324PRDX & 13.6 & 527.000000 & Anhydritic, finely crystalline dolomite \\
\hline $\mathrm{s} l \mathrm{~d} 36-25-27 \mathrm{bba}$ & $5,854.0$ & $5,855.0$ & 324PRDX & 12.4 & 17.000000 & Anhydritic, finely crystalline dolomite \\
\hline $\mathrm{s} 1 \mathrm{~d} 36-25-27 \mathrm{bba}$ & $5,855.0$ & $5,856.0$ & 324PRDX & 13.1 & 488.000000 & Anhydritic, finely crystalline dolomite \\
\hline sld $36-25-27 \mathrm{bba}$ & $5,856.0$ & $5,857.0$ & 324PRDX & 2.6 & 0.010000 & Anhydritic, finely crystalline dolomite \\
\hline $\mathrm{s} 1 \mathrm{~d} 36-25-27 \mathrm{bba}$ & $5,857.0$ & $5,858.0$ & 324PRDX & 14.3 & 0.370000 & Finely crystalline dolomite \\
\hline $\mathrm{s} 1 \mathrm{~d} 36-25-27 \mathrm{bba}$ & $5,858.0$ & $5,859.0$ & 324PRDX & 8.9 & 0.090000 & Finely crystalline dolomite \\
\hline sld $36-25-27 b b a$ & $5,859.0$ & $5,860.0$ & 324PRDX & 8.0 & 0.080000 & Finely crystalline dolomite \\
\hline \multicolumn{3}{|l|}{ SITE AVERAGE-- } & AVERAGE-- & $\frac{10.7}{10.7}$ & $\frac{174.114348}{174.114348}$ & \\
\hline sld36-26-16cac & $6,284.0$ & $6,285.0$ & 324PRDX & 6.9 & 2.500000 & Anhydritic, finely crystalline dolomite \\
\hline sld36-26-16cac & $6,285.0$ & $6,286.0$ & 324PRDX & 7.8 & 5.900000 & Vuggy, anhydritic, medium-crystalline dolomite \\
\hline s1d36-26-16cac & $6,286.0$ & $6,287.0$ & 324PRDX & 5.7 & 0.810000 & Vuggy, anhydritic, medium-crystalline dolomite \\
\hline s ld $36-26-16 \mathrm{cac}$ & $6,287.0$ & $6,288.0$ & 324PRDX & 12.5 & 86.000000 & Vuggy, anhydritic, medium-crystalline dolomite \\
\hline s $1 \mathrm{~d} 36-26-16 \mathrm{cac}$ & $6,288.0$ & $6,289.0$ & 324PRDX & 13.7 & 64.000000 & Vuggy, anhydritic, medium-crystalline dolomite \\
\hline sld36-26-16cac & $6,289.0$ & $6,290.0$ & 324PRDX & 14.5 & 92.000000 & Vuggy, anhydritic, medium-crystalline dolomite \\
\hline sld36-26-16 cac & $6,290.0$ & $6,291.0$ & 324PRDX & 14.4 & 39.000000 & Vuggy, anhydritic, medium-crystalline dolomite \\
\hline $\mathrm{s} 1 \mathrm{~d} 36-26-16 \mathrm{cac}$ & $6,291.0$ & $6,292.0$ & 324PRDX & 13.0 & 56.000000 & Vuggy, anhydritic, medium-crystalline dolomite \\
\hline sld $36-26-16 \mathrm{cac}$ & $6,292.0$ & $6,293.0$ & $324 \mathrm{PRDX}$ & 16.1 & 51.000000 & Vuggy, anhydritic, medium-crystalline dolomite \\
\hline $\operatorname{sld} 36-26-16 \mathrm{cac}$ & $6,293.0$ & $6,294.0$ & 324PRDX & 14.2 & 41.000000 & Vuggy, anhydritic, medium-crystalline dolomite \\
\hline s $1 d 36-26-16 \mathrm{cac}$ & $6,294.0$ & $6,295.0$ & $324 \mathrm{PRDX}$ & 13.5 & 87.000000 & Vuggy, anhydritic, medium-crystalline dolomite \\
\hline sld36-26-16cac & $6,295.0$ & $6,296.0$ & 324PRDX & 11.9 & 39.000000 & Vuggy, anhydritic, medium-crystalline dolomite \\
\hline sld $36-26-16 \mathrm{cac}$ & $6,296.0$ & $6,297.0$ & $324 \mathrm{PRDX}$ & 10.2 & 27.000000 & Vuggy, anhydritic, medium-crystalline dolomite \\
\hline sld36-26-16cac & $6,297.0$ & $6,298.0$ & $324 \mathrm{PRDX}$ & 10.5 & 18.000000 & Vuggy, anhydritic, medium-crystalline dolomite \\
\hline $\operatorname{sld} 36-26-16 \mathrm{cac}$ & $6,298.0$ & $6,299.0$ & $324 \mathrm{PRDX}$ & 8.2 & 7.200000 & Vuggy, anhydritic, medium-crystalline dolomite \\
\hline $\operatorname{sld} 36-26-16 \mathrm{cac}$ & $6,299.0$ & $6,300.0$ & $324 \mathrm{PRDX}$ & 8.4 & 7.800000 & Vuggy, anhydritic, medium-crystalline dolomite \\
\hline s1d36-26-16cac & $6,300.0$ & $6,301.0$ & $324 \mathrm{PRDX}$ & 5.2 & 1.300000 & Anhydritic, finely crystalline dolomite \\
\hline \multirow{2}{*}{\multicolumn{4}{|c|}{ SITE AVERAGE }} & 11.0 & 36.794706 & \\
\hline & & & & $\overline{11.0}$ & 36.794706 & \\
\hline s1d36-26-22baa & $6,319.0$ & $6,320.0$ & 324PRDX & 6.5 & 0.190000 & Shaly, finely crystalline dolomite \\
\hline sld $36-26-22$ baa & $6,320.0$ & $6,321.0$ & $324 \mathrm{PRDX}$ & 6.0 & 0.330000 & Finely crystalline dolomite \\
\hline sld36-26-22baa & $6,321.0$ & $6,322.0$ & $324 \mathrm{PRDX}$ & 3.4 & 0.070000 & Vuggy, anhydritic, medium-crystalline dolomite \\
\hline sld36-26-22baa & $6,322.0$ & $6,323.0$ & 324PRDX & 5.7 & 4.900000 & Vuggy, anhydritic, medium-crystalline dolomite \\
\hline $\operatorname{sld} 36-26-22 \mathrm{baa}$ & $6,323.0$ & $6,324.0$ & 324PRDX & 7.7 & 62.000000 & Vuggy, anhydritic, medium-crystalline dolomite \\
\hline s 1 d $36-26-22$ baa & $6,324.0$ & $6,325.0$ & $324 \mathrm{PRDX}$ & 8.5 & 7.000000 & Vuggy, anhydritic, medium-crystalline dolomite \\
\hline $\mathrm{s} 1 \mathrm{~d} 36-26-22 \mathrm{baa}$ & $6,325.0$ & $6,326.0$ & 324PRDX & 10.4 & 16.000000 & Vuggy, anhydritic, medium-crystalline dolomite \\
\hline $\operatorname{sld} 36-26-22 \mathrm{baa}$ & $6,326.0$ & $6,327.0$ & 324PRDX & 8.7 & 6.200000 & Vuggy, anhydritic, medium-crystalline dolomite \\
\hline $\operatorname{sld} 36-26-22 \mathrm{baa}$ & $6,327.0$ & $6,328.0$ & 324PRDX & 10.5 & 7.500000 & Vuggy, anhydritic, medium-crystalline dolomite \\
\hline sld $36-26-22 \mathrm{baa}$ & $6,328.0$ & $6,329.0$ & 324PRDX & 7.6 & 10.000000 & Vuggy, anhydritic, medium-crystalline dolomite \\
\hline s $1 d 36-26-22 b a a$ & $6,329.0$ & $6,330.0$ & 324PRDX & 5.0 & 0.400000 & Shaly, finely crystalline dolomite \\
\hline s $1 d 36-26-22$ baa & $6,330.0$ & $6,331.0$ & 324PRDX & 0.5 & 0.010000 & Shaly, finely crystalline dolomite \\
\hline s $1 \mathrm{~d} 36-26-22 \mathrm{baa}$ & $6,331.0$ & $6,332.0$ & 324PRDX & 3.6 & 0.010000 & Shaly, finely crystalline dolomite \\
\hline s $1 d 36-26-22$ baa & $6,332.0$ & $6,333.0$ & 324PRDX & 1.3 & 0.010000 & Shaly, finely crystalline dolomite \\
\hline sld $36-26-22 \mathrm{baa}$ & $6,333.0$ & $6,334.0$ & $324 \mathrm{PRDX}$ & 0.9 & 0.600000 & Shaly, finely crystalline dolomite \\
\hline sld36-26-22baa & $6,334.0$ & $6,335.0$ & $324 \mathrm{PRDX}$ & 0.8 & 0.010000 & Shaly, finely crystalline dolomite \\
\hline s 1 d $36-26-22$ baa & $6,335.0$ & $6,336.0$ & 324PRDX & 3.1 & 0.010000 & Shaly, finely crystalline dolomite \\
\hline sld $36-26-22 \mathrm{baa}$ & $6,336.0$ & $6,337.0$ & 324PRDX & 5.2 & 8.600000 & Shaly, finely crystalline dolomite \\
\hline $\mathrm{sld} 36-26-22 \mathrm{baa}$ & $6,337.0$ & $6,338.0$ & 324PRDX & 4.8 & 4.100000 & Shaly, finely crystalline dolomite \\
\hline sld $36-26-22 b a a$ & $6,338.0$ & $6,339.0$ & $324 \mathrm{PRDX}$ & 3.8 & 0.140000 & Shaly, finely crystalline dolomite \\
\hline
\end{tabular}


Table 4.--Laboratory-determined porosity and permeability--Continued

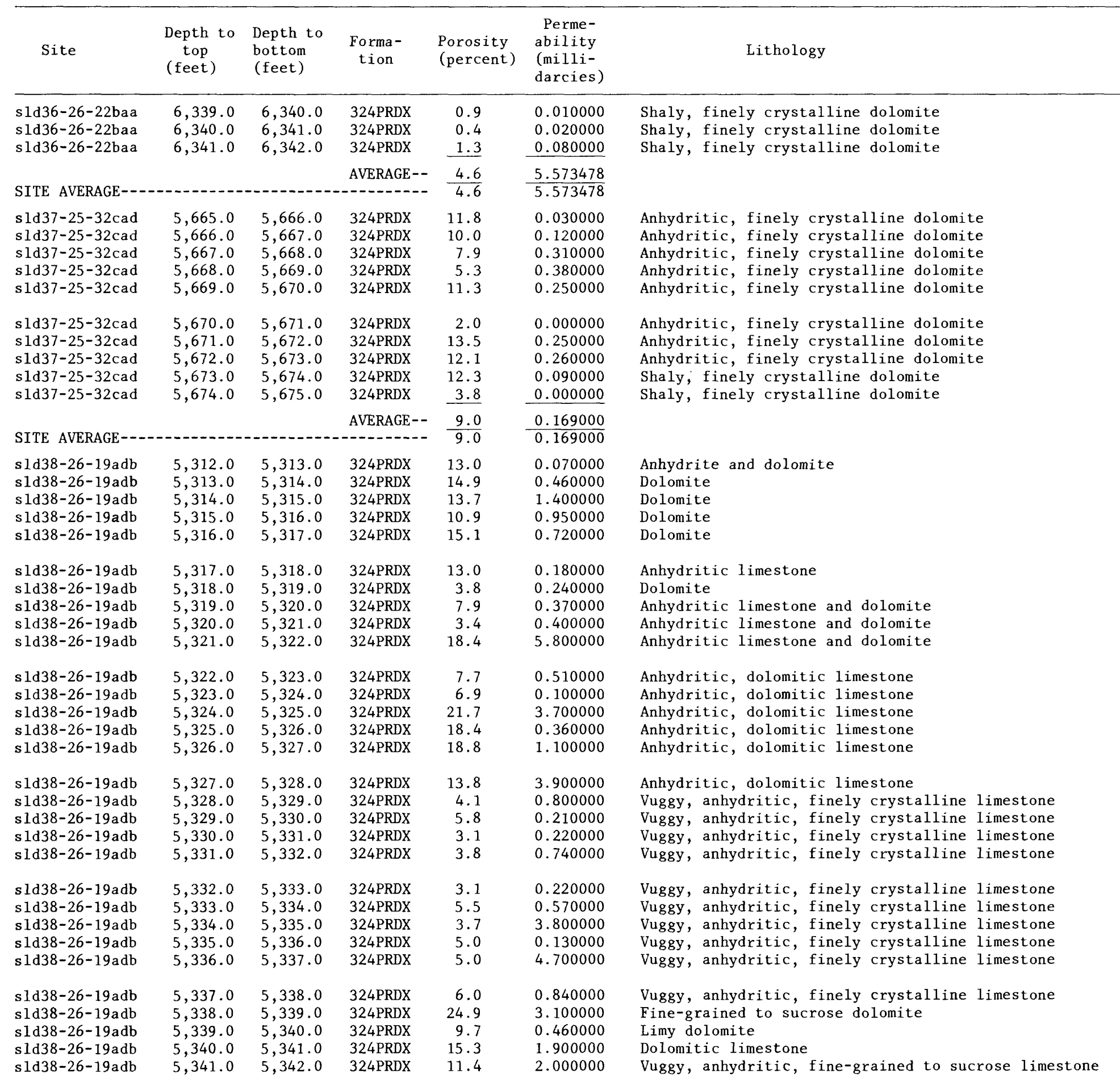


Table 4.--Laboratory-determined porosity and permeability--Continued

\begin{tabular}{|c|c|c|c|c|c|c|}
\hline Site & $\begin{array}{l}\text { Depth to } \\
\text { top } \\
\text { (feet) }\end{array}$ & $\begin{array}{l}\text { Depth to } \\
\text { bottom } \\
\text { (feet) }\end{array}$ & $\begin{array}{c}\text { Forma- } \\
\text { tion }\end{array}$ & $\begin{array}{l}\text { Porosity } \\
\text { (percent) }\end{array}$ & $\begin{array}{l}\text { Perme- } \\
\text { ability } \\
\text { (milli- } \\
\text { darcies) }\end{array}$ & Lithology \\
\hline $\mathrm{s} 1 \mathrm{~d} 38-26-19 \mathrm{adb}$ & $5,342.0$ & $5,343.0$ & 324PRDX & 8.0 & 8.600000 & Vuggy, anhydritic, fine-grained to sucrose limestone \\
\hline $\operatorname{sid} 38-26-19 a \mathrm{db}$ & $5,343.0$ & $5,344.0$ & 324PRDX & 10.8 & 7.700000 & Vuggy, anhydritic, fine-grained to sucrose limestone \\
\hline s1d38-26-19adb & $5,344.0$ & $5,345.0$ & $324 \mathrm{PRDX}$ & 4.6 & 0.410000 & Sparsely vuggy, anhydritic limestone \\
\hline s $1 \mathrm{~d} 38-26-19 \mathrm{adb}$ & $5,345.0$ & $5,346.0$ & $324 \mathrm{PRDX}$ & 4.2 & 0.110000 & Sparsely vuggy, anhydritic limestone \\
\hline $\operatorname{sld} 38-26-19 a d b$ & $5,346.0$ & $5,347.0$ & 324PRDX & 3.2 & 0.110000 & Sparsely vuggy, anhydritic limestone \\
\hline $\operatorname{sid} 38-26-19 a d b$ & $5,347.0$ & $5,348.0$ & $324 \mathrm{PRDX}$ & 2.6 & 0.100000 & Anhydritic limestone \\
\hline s1d38-26-19adb & $5,348.0$ & $5,349.0$ & $324 \mathrm{PRDX}$ & 3.7 & 1.100000 & Anhydritic limestone \\
\hline s $1 d 38-26-19 a d b$ & $5,349.0$ & $5,350.0$ & $324 \mathrm{PRDX}$ & 3.6 & 0.070000 & Anhydritic limestone \\
\hline s $1 d 38-26-19 a d b$ & $5,350.0$ & $5,351.0$ & $324 \mathrm{PRDX}$ & 6.0 & 0.110000 & Anhydritic limestone \\
\hline s $1 d 38-26-19 a d b$ & $5,351.0$ & $5,352.0$ & $324 \mathrm{PRDX}$ & 5.3 & 0.120000 & Anhydritic limestone \\
\hline sld $38-26-19 a d b$ & $5,352.0$ & $5,353.0$ & 324PRDX & 3.2 & 0.250000 & Anhydritic limestone \\
\hline s $1 d 38-26-19 a d b$ & $5,353.0$ & $5,354.0$ & $324 \mathrm{PRDX}$ & 4.0 & 0.110000 & Anhydritic limestone \\
\hline s $1 \mathrm{~d} 38-26-19 \mathrm{adb}$ & $5,354.0$ & $5,355.0$ & $324 \mathrm{PRDX}$ & 3.6 & 0.200000 & Anhydritic limestone \\
\hline s $1 d 38-26-19 a d b$ & $5,355.0$ & $5,356.0$ & 324PRDX & 4.2 & 0.110000 & Anhydritic limestone \\
\hline sld $38-26-19 a d b$ & $5,356.0$ & $5,357.0$ & 324PRDX & 2.8 & 0.120000 & Anhydritic limestone \\
\hline $\operatorname{sid} 38-26-19 a d b$ & $5,357.0$ & $5,358.0$ & $324 \mathrm{PRDX}$ & 2.5 & 0.160000 & Anhydritic limestone \\
\hline s $1 d 38-26-19 a d b$ & $5,358.0$ & $5,359.0$ & $324 \mathrm{PRDX}$ & 2.0 & 0.650000 & Anhydritic limestone \\
\hline s $1 d 38-26-19 a d b$ & $5,359.0$ & $5,360.0$ & 324PRDX & 1.4 & 0.330000 & Anhydritic limestone \\
\hline sld $38-26-19 a d b$ & $5,360.0$ & $5,361.0$ & 324PRDX & 2.1 & 0.100000 & Anhydritic limestone \\
\hline $\operatorname{sld} 38-26-19 a d b$ & $5,361.0$ & $5,362.0$ & 324PRDX & 1.3 & 0.090000 & Anhydritic limestone \\
\hline $\operatorname{sld} 38-26-19 a d b$ & $5,362.0$ & $5,363.0$ & 324PRDX & 0.9 & 0.040000 & Anhydritic limestone \\
\hline sld $38-26-19 a d b$ & $5,363.0$ & $5,364.0$ & $324 \mathrm{PRDX}$ & 2.0 & 0.130000 & Limy dolomite \\
\hline s $1 d 38-26-19 a d b$ & $5,364.0$ & $5,365.0$ & $324 \mathrm{PRDX}$ & 17.3 & 0.790000 & Shaly dolomite \\
\hline $\operatorname{sld} 38-26-19 a d b$ & $5,365.0$ & $5,366.0$ & $324 \mathrm{PRDX}$ & 17.1 & 0.890000 & Shaly dolomite \\
\hline $\operatorname{sld} 38-26-19 a d b$ & $5,366.0$ & $5,367.0$ & $324 \mathrm{PRDX}$ & 10.2 & 0.260000 & Shaly, anhydritic dolomite \\
\hline $\operatorname{sld} 38-26-19 a d b$ & $5,367.0$ & $5,368.0$ & 324PRDX & 20.3 & 1.590000 & Shaly, anhydritic dolomite \\
\hline s $1 d 38-26-19 a d b$ & $5,368.0$ & $5,369.0$ & $324 \mathrm{PRDX}$ & 8.2 & 0.080000 & Shaly dolomite \\
\hline sld38-26-19adb & $5,369.0$ & $5,370.0$ & $324 \mathrm{PRDX}$ & 2.2 & 0.040000 & Shaly, fossiliferous limestone \\
\hline sld38-26-19adb & $5,370.0$ & $5,371.0$ & 324PRDX & 2.0 & 0.050000 & Shaly, fossiliferous limestone \\
\hline s $1 d 38-26-19 a d b$ & $5,371.0$ & $5,372.0$ & $324 \mathrm{PRDX}$ & 1.4 & 0.050000 & Shaly, fossiliferous limestone \\
\hline sld $38-26-19 a d b$ & $5,372.0$ & $5,373.0$ & $324 \mathrm{PRDX}$ & 1.3 & 0.050000 & Shaly, fossiliferous limestone \\
\hline s $1 d 38-26-19 a d b$ & $5,373.0$ & $5,374.0$ & 324PRDX & 1.0 & 0.050000 & Shaly, fossiliferous limestone \\
\hline s $1 d 38-26-19 a d b$ & $5,374.0$ & $5,375.0$ & $324 \mathrm{PRDX}$ & 1.6 & 0.080000 & Shaly, fossiliferous limestone \\
\hline sld $38-26-19 a d b$ & $5,375.0$ & $5,376.0$ & 324PRDX & 1.7 & 0.090000 & Shaly, fossiliferous limestone \\
\hline sld $38-26-19 a d b$ & $5,376.0$ & $5,377.0$ & 324PRDX & 2.0 & 0.050000 & Shaly, fossiliferous limestone \\
\hline $\operatorname{sld} 38-26-19 a d b$ & $5,377.0$ & $5,378.0$ & 324PRDX & 1.7 & 0.060000 & Shaly, fossiliferous limestone \\
\hline & & & AVERAGE-- & 7.2 & 0.981818 & \\
\hline SITE AVERAGE-- & 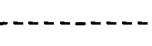 & 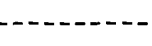 & क्ष & 7.2 & 0.981818 & \\
\hline sld $38-26-22$ add & $5,139.0$ & $5,140.0$ & $324 \mathrm{PRDX}$ & 1.8 & 1.300000 & Limestone and shale \\
\hline s ld $38-26-22$ add & $5,140.0$ & $5,141.0$ & 324 PRDX & 0.6 & 0.060000 & Limestone and shale \\
\hline s1d38-26-22add & $5,141.0$ & $5,142.0$ & $324 \mathrm{PRDX}$ & 0.5 & 0.050000 & Laminated limestone \\
\hline sld $38-26-22$ add & $5,142.0$ & $5,143.0$ & $324 \mathrm{PRDX}$ & 0.6 & 0.060000 & Limestone and shale \\
\hline sld38-26-22add & $5,143.0$ & $5,144.0$ & 324PRDX & 0.6 & 0.050000 & Limestone and shale \\
\hline sld38-26-22add & $5,144.0$ & $5,145.0$ & $324 \mathrm{PRDX}$ & 0.5 & 0.070000 & Limestone and shale \\
\hline s1d38-26-22add & $5,145.0$ & $5,146.0$ & 324PRDX & 4.9 & 0.230000 & Anhydritic limestone \\
\hline sld $38-26-22$ add & $5,146.0$ & $5,147.0$ & $324 \mathrm{PRDX}$ & 8.3 & 0.090000 & Anhydritic limestone \\
\hline s $1 d 38-26-22 a d d$ & $5,147.0$ & $5,148.0$ & $324 \mathrm{PRDX}$ & 9.0 & 0.070000 & Anhydritic limestone \\
\hline sld $38-26-22$ add & $5,148.0$ & $5,149.0$ & $324 \mathrm{PRDX}$ & 4.6 & 0.070000 & Anhydritic limestone \\
\hline
\end{tabular}


Table 4.--Laboratory-determined porosity and permeability--Continued

\begin{tabular}{|c|c|c|c|c|c|c|}
\hline Site & $\begin{array}{l}\text { Depth to } \\
\text { top } \\
\text { (feet) }\end{array}$ & $\begin{array}{l}\text { Depth to } \\
\text { bottom } \\
\text { (feet) }\end{array}$ & $\begin{array}{c}\text { Forma- } \\
\text { tion }\end{array}$ & $\begin{array}{l}\text { Porosity } \\
\text { (percent) }\end{array}$ & $\begin{array}{c}\text { Perme- } \\
\text { ability } \\
\text { (milli- } \\
\text { darcies) }\end{array}$ & Lithology \\
\hline $\begin{array}{l}\text { sld38-26-22add } \\
\text { sld } 38-26-22 a \text { dd } \\
\text { sld38-26-22add } \\
\text { sld38-26-22add } \\
\text { s } 1 \text { d } 38-26-22 a d d\end{array}$ & $\begin{array}{l}5,149.0 \\
5,150.0 \\
5,151.0 \\
5,152.0 \\
5,153.0\end{array}$ & $\begin{array}{l}5,150.0 \\
5,151.0 \\
5,152.0 \\
5,153.0 \\
5,154.0\end{array}$ & $\begin{array}{l}\text { 324PRDX } \\
324 \mathrm{PRDX} \\
324 \mathrm{PRDX} \\
\text { 324PRDX } \\
\text { 324PRDX }\end{array}$ & $\begin{array}{l}0.3 \\
0.3 \\
0.5 \\
1.6 \\
4.5\end{array}$ & $\begin{array}{l}0.530000 \\
0.040000 \\
0.050000 \\
0.110000 \\
0.180000\end{array}$ & $\begin{array}{l}\text { Fine-grained limestone } \\
\text { Fine-grained limestone } \\
\text { Fine-grained limestone } \\
\text { Silty, fossiliferous dolomite } \\
\text { Silty, fossiliferous dolomite }\end{array}$ \\
\hline $\begin{array}{l}\text { s ld38-26-22add } \\
\text { sld38-26-22add } \\
\text { sld38-26-22add } \\
\text { sld38-26-22add } \\
\text { s } 1 \text { d } 38-26-22 \text { add }\end{array}$ & $\begin{array}{l}5,154.0 \\
5,155.0 \\
5,156.0 \\
5,157.0 \\
5,158.0\end{array}$ & $\begin{array}{l}5,155.0 \\
5,156.0 \\
5,157.0 \\
5,158.0 \\
5,159.0\end{array}$ & $\begin{array}{l}324 \mathrm{PRDX} \\
324 \mathrm{PRDX} \\
324 \mathrm{PRDX} \\
324 \mathrm{PRDX} \\
324 \mathrm{PRDX}\end{array}$ & $\begin{array}{r}8.9 \\
8.9 \\
12.9 \\
0.6 \\
1.2\end{array}$ & $\begin{array}{l}0.080000 \\
0.110000 \\
0.250000 \\
0.040000 \\
0.030000\end{array}$ & $\begin{array}{l}\text { Silty, fossiliferous dolomite } \\
\text { Silty, fossiliferous dolomite } \\
\text { Silty, fossiliferous dolomite } \\
\text { Fine-grained limestone } \\
\text { Fine-grained limestone }\end{array}$ \\
\hline $\begin{array}{l}\text { s } 1 d 38-26-22 a \text { dd } \\
\text { sld38-26-22add } \\
\text { sld38-26-22add } \\
\text { sld38-26-22add } \\
\text { sld38-26-22add }\end{array}$ & $\begin{array}{l}5,159.0 \\
5,160.0 \\
5,161.0 \\
5,177.0 \\
5,178.0\end{array}$ & $\begin{array}{l}5,160.0 \\
5,161.0 \\
5,162.0 \\
5,178.0 \\
5,179.0\end{array}$ & $\begin{array}{l}\text { 324PRDX } \\
324 \mathrm{PRDX} \\
324 \mathrm{PRDX} \\
324 \mathrm{PRDX} \\
\text { 324PRDX }\end{array}$ & $\begin{array}{l}0.5 \\
0.5 \\
0.6 \\
0.7 \\
1.0\end{array}$ & $\begin{array}{l}0.030000 \\
0.030000 \\
0.030000 \\
0.030000 \\
0.040000\end{array}$ & $\begin{array}{l}\text { Fine-grained limestone } \\
\text { Fine-grained limestone } \\
\text { Fine-grained limestone } \\
\text { Limestone } \\
\text { Limestone }\end{array}$ \\
\hline $\begin{array}{l}\text { s } 1 d 38-26-22 a \text { dd } \\
\text { sld38-26-22add } \\
\text { sld38-26-22add } \\
\text { sld38-26-22add } \\
\text { sld38-26-22add }\end{array}$ & $\begin{array}{l}5,179.0 \\
5,180.0 \\
5,181.0 \\
5,182.0 \\
5,183.0\end{array}$ & $\begin{array}{l}5,180.0 \\
5,181.0 \\
5,182.0 \\
5,183.0 \\
5,184.0\end{array}$ & $\begin{array}{l}324 \mathrm{PRDX} \\
324 \mathrm{PRDX} \\
324 \mathrm{PRDX} \\
324 \mathrm{PRDX} \\
324 \mathrm{PRDX}\end{array}$ & $\begin{array}{r}0.9 \\
0.8 \\
0.5 \\
0.6 \\
10.4\end{array}$ & $\begin{array}{l}0.040000 \\
0.040000 \\
0.040000 \\
0.050000 \\
0.090000\end{array}$ & $\begin{array}{l}\text { Limes tone } \\
\text { Limestone } \\
\text { Limestone } \\
\text { Limestone } \\
\text { Chalky limestone }\end{array}$ \\
\hline $\begin{array}{l}\text { sld } 38-26-22 a \text { dd } \\
\text { sld38-26-22add } \\
\text { s1d38-26-22add } \\
\text { sld38-26-22add } \\
\text { s1d38-26-22add }\end{array}$ & $\begin{array}{l}5,184.0 \\
5,185.0 \\
5,186.0 \\
5,187.0 \\
5,188.0\end{array}$ & $\begin{array}{l}5,185.0 \\
5,186.0 \\
5,187.0 \\
5,188.0 \\
5,189.0\end{array}$ & $\begin{array}{l}324 \mathrm{PRDX} \\
324 \mathrm{PRDX} \\
324 \mathrm{PRDX} \\
324 \mathrm{PRDX} \\
324 \mathrm{PRDX}\end{array}$ & $\begin{array}{l}0.4 \\
3.5 \\
4.3 \\
4.2 \\
9.5\end{array}$ & $\begin{array}{r}0.030000 \\
1.300000 \\
1.200000 \\
3.700000 \\
80.000000\end{array}$ & $\begin{array}{l}\text { Chalky limestone } \\
\text { Vuggy limestone } \\
\text { Vuggy limestone } \\
\text { Vuggy limestone } \\
\text { Vuggy limestone }\end{array}$ \\
\hline $\begin{array}{l}\text { sld38-26-22a dd } \\
\text { sld38-26-22add } \\
\text { sld } 38-26-22 \text { add } \\
\text { sld38-26-22add } \\
\text { sld38-26-22add }\end{array}$ & $\begin{array}{l}5,189.0 \\
5,190.0 \\
5,191.0 \\
5,192.0 \\
5,193.0\end{array}$ & $\begin{array}{l}5,190.0 \\
5,191.0 \\
5,192.0 \\
5,193.0 \\
5,194.0\end{array}$ & $\begin{array}{l}324 \mathrm{PRDX} \\
324 \mathrm{PRDX} \\
324 \mathrm{PRDX} \\
324 \mathrm{PRDX} \\
324 \mathrm{PRDX}\end{array}$ & $\begin{array}{r}10.7 \\
11.6 \\
10.4 \\
9.1 \\
8.2\end{array}$ & $\begin{array}{r}51.000000 \\
16.000000 \\
10.000000 \\
7.500000 \\
0.340000\end{array}$ & $\begin{array}{l}\text { Vuggy limestone } \\
\text { Vuggy limestone } \\
\text { Vuggy limestone } \\
\text { Vuggy limestone } \\
\text { Vuggy limestone }\end{array}$ \\
\hline $\begin{array}{l}\text { sld } 38-26-22 a \text { dd } \\
\text { sld38-26-22add } \\
\text { sld } 38-26-22 a \text { dd } \\
\text { sld38-26-22add } \\
\text { sld38-26-22add }\end{array}$ & $\begin{array}{l}5,194.0 \\
5,195.0 \\
5,196.0 \\
5,197.0 \\
5,198.0\end{array}$ & $\begin{array}{l}5,195.0 \\
5,196.0 \\
5,197.0 \\
5,198.0 \\
5,199.0\end{array}$ & $\begin{array}{l}324 \mathrm{PRDX} \\
324 \mathrm{PRDX} \\
324 \mathrm{PRDX} \\
324 \mathrm{PRDX} \\
324 \mathrm{PRDX}\end{array}$ & $\begin{array}{r}10.1 \\
10.1 \\
10.9 \\
7.3 \\
8.6\end{array}$ & $\begin{array}{r}1.400000 \\
9.100000 \\
85.000000 \\
7.900000 \\
395.000000\end{array}$ & $\begin{array}{l}\text { Vuggy limestone } \\
\text { Vuggy limestone } \\
\text { Vuggy limestone } \\
\text { Vuggy limestone } \\
\text { Vuggy limestone }\end{array}$ \\
\hline $\begin{array}{l}\text { sld } 38-26-22 a \text { dd } \\
\text { sld38-26-22add } \\
\text { s ld } 38-26-22 \text { add } \\
\text { sld38-26-22add } \\
\text { sld38-26-22add }\end{array}$ & $\begin{array}{l}5,199.0 \\
5,200.0 \\
5,201.0 \\
5,202.0 \\
5,203.0\end{array}$ & $\begin{array}{l}5,200.0 \\
5,201.0 \\
5,202.0 \\
5,203.0 \\
5,204.0\end{array}$ & $\begin{array}{l}324 \mathrm{PRDX} \\
324 \mathrm{PRDX} \\
324 \mathrm{PRDX} \\
324 \mathrm{PRDX} \\
324 \mathrm{PRDX}\end{array}$ & $\begin{array}{r}10.0 \\
8.1 \\
8.2 \\
12.9 \\
15.7\end{array}$ & $\begin{array}{r}27.000000 \\
19.000000 \\
27.000000 \\
1.200000 \\
29.000000\end{array}$ & $\begin{array}{l}\text { Vuggy limestone } \\
\text { Vuggy limestone } \\
\text { Vuggy limestone } \\
\text { Limestone } \\
\text { Limestone }\end{array}$ \\
\hline $\begin{array}{l}\text { sld38-26-22add } \\
\text { sld38-26-22add } \\
\text { sld } 38-26-22 a d d \\
\text { sld38-26-22add } \\
\text { sld38-26-22add }\end{array}$ & $\begin{array}{l}5,204.0 \\
5,205.0 \\
5,206.0 \\
5,207.0 \\
5,208.0\end{array}$ & $\begin{array}{l}5,205.0 \\
5,206.0 \\
5,207.0 \\
5,208.0 \\
5,209.0\end{array}$ & $\begin{array}{l}324 \mathrm{PRDX} \\
324 \mathrm{PRDX} \\
324 \mathrm{PRDX} \\
324 \mathrm{PRDX} \\
324 \mathrm{PRDX}\end{array}$ & $\begin{array}{l}17.4 \\
16.5 \\
16.9 \\
17.3 \\
13.8\end{array}$ & $\begin{array}{l}14.000000 \\
18.000000 \\
57.000000 \\
25.000000 \\
21.000000\end{array}$ & $\begin{array}{l}\text { Limestone } \\
\text { Limestone } \\
\text { Limes tone } \\
\text { Limes tone } \\
\text { Limes tone }\end{array}$ \\
\hline $\begin{array}{l}\text { sld } 38-26-22 a \text { dd } \\
\text { sld38-26-22a dd } \\
\text { sld38-26-22add } \\
\text { sld } 38-26-22 a \text { dd } \\
\text { sld38-26-22add }\end{array}$ & $\begin{array}{l}5,209.0 \\
5,210.0 \\
5,211.0 \\
5,212.0 \\
5,213.0\end{array}$ & $\begin{array}{l}5,210.0 \\
5,211.0 \\
5,212.0 \\
5,213.0 \\
5,214.0\end{array}$ & $\begin{array}{l}324 \mathrm{PRDX} \\
324 \mathrm{PRDX} \\
324 \mathrm{PRDX} \\
\text { 324PRDX } \\
324 \mathrm{PRDX}\end{array}$ & $\begin{array}{l}8.5 \\
4.8 \\
4.8 \\
4.3 \\
4.5\end{array}$ & $\begin{array}{l}9.500000 \\
6.500000 \\
2.200000 \\
2.900000 \\
0.300000\end{array}$ & $\begin{array}{l}\text { Limestone } \\
\text { Limestone } \\
\text { Limestone } \\
\text { Limestone } \\
\text { Chalky limestone }\end{array}$ \\
\hline
\end{tabular}


Table 4.--Laboratory-determined porosity and permeability--Continued

\begin{tabular}{|c|c|c|c|c|c|c|}
\hline Site & $\begin{array}{l}\text { Depth to } \\
\text { top } \\
\text { (feet) }\end{array}$ & $\begin{array}{l}\text { Depth to } \\
\text { bottom } \\
\text { (feet) }\end{array}$ & $\begin{array}{c}\text { Forma- } \\
\text { tion }\end{array}$ & $\begin{array}{l}\text { Porosity } \\
\text { (percent) }\end{array}$ & $\begin{array}{l}\text { Perme- } \\
\text { ability } \\
\text { (milli- } \\
\text { darcies) }\end{array}$ & Lithology \\
\hline sld $38-26-22 a d d$ & $5,214.0$ & $5,215.0$ & $324 \mathrm{PRDX}$ & 10.1 & 0.290000 & Chalky limestone \\
\hline sld $38-26-22 a d d$ & $5,215.0$ & $5,216.0$ & 324PRDX & 0.6 & 0.410000 & Chalky limestone \\
\hline sld $38-26-22$ add & $5,216.0$ & $5,217.0$ & 324PRDX & 0.7 & 0.030000 & Limestone \\
\hline sld $38-26-22 a d d$ & $5,217.0$ & $5,218.0$ & 324PRDX & 0.9 & 0.040000 & Limestone \\
\hline sld $38-26-22 a d d$ & $5,218.0$ & $5,219.0$ & $324 \mathrm{PRDX}$ & 0.9 & 0.040000 & Limestone \\
\hline sld38-26-22add & $5,219.0$ & $5,220.0$ & $324 \mathrm{PRDX}$ & 1.4 & 0.030000 & Limestone \\
\hline sld $38-26-22 a d d$ & $5,220.0$ & $5,221.0$ & 324PRDX & 0.5 & 0.030000 & Limestone breccia \\
\hline $\operatorname{sld} 38-26-22 \mathrm{add}$ & $5,221.0$ & $5,222.0$ & 324PRDX & 1.1 & 0.030000 & Limestone breccia \\
\hline sld $38-26-22 a d d$ & $5,222.0$ & $5,223.0$ & $324 \mathrm{PRDX}$ & 2.0 & 0.050000 & Limestone \\
\hline $\operatorname{sld} 38-26-22 \mathrm{add}$ & $5,223.0$ & $5,224.0$ & $324 \mathrm{PRDX}$ & 4.5 & 0.380000 & Limestone \\
\hline sld $38-26-22 a d d$ & $5,224.0$ & $5,225.0$ & 324PRDX & 5.1 & 3.200000 & Limestone \\
\hline sld $38-26-22$ add & $5,225.0$ & $5,226.0$ & $324 \mathrm{PRDX}$ & 6.5 & 3.700000 & Limestone \\
\hline sld $38-26-22$ add & $5,226.0$ & $5,227.0$ & 324PRDX & 7.7 & 6.400000 & Limestone \\
\hline sld $38-26-22$ add & $5,227.0$ & $5,228.0$ & 324PRDX & 6.6 & 0.670000 & Limestone \\
\hline sld $38-26-22 a d d$ & $5,228.0$ & $5,229.0$ & 324PRDX & 7.5 & 7.700000 & Limestone \\
\hline sld38-26-22add & $5,229.0$ & $5,230.0$ & 324PRDX & 7.3 & 0.490000 & Limestone \\
\hline sld $38-26-22$ add & $5,230.0$ & $5,231.0$ & $324 \mathrm{PRDX}$ & 4.6 & 0.370000 & Limestone \\
\hline $\operatorname{sld} 38-26-22 \mathrm{add}$ & $5,231.0$ & $5,232.0$ & 324PRDX & 2.0 & 0.030000 & Fossiliferous limestone \\
\hline sld $38-26-22$ add & $5,232.0$ & $5,233.0$ & 324PRDX & 1.1 & 0.030000 & Fossiliferous limestone \\
\hline $\operatorname{sld} 38-26-22 \mathrm{add}$ & $5,233.0$ & $5,234.0$ & 324PRDX & 0.9 & 0.090000 & Fossiliferous limestone \\
\hline $\operatorname{sld} 38-26-22 \mathrm{add}$ & $5,234.0$ & $5,235.0$ & $324 \mathrm{PRDX}$ & 0.7 & 0.030000 & Fossiliferous limestone \\
\hline sld $38-26-22 a d d$ & $5,235.0$ & $5,236.0$ & $324 \mathrm{PRDX}$ & 1.2 & 0.040000 & Fossiliferous limestone \\
\hline sld $38-26-22$ add & $5,236.0$ & $5,237.0$ & $324 \mathrm{PRDX}$ & 0.8 & 0.040000 & Fossiliferous limestone \\
\hline sld $38-26-22 a d d$ & $5,392.0$ & $5,393.0$ & $324 \mathrm{PRDX}$ & 1.2 & 0.050000 & Silty, limy shale \\
\hline sld $38-26-22$ add & $5,393.0$ & $5,394.0$ & $324 \mathrm{PRDX}$ & 2.8 & 0.090000 & Silty, limy shale \\
\hline $\operatorname{sld} 38-26-22 \mathrm{add}$ & $5,394.0$ & $5,395.0$ & 324PRDX & 14.6 & 0.100000 & Shaly limestone \\
\hline s ld $38-26-22$ add & $5,395.0$ & $5,396.0$ & $324 \mathrm{PRDX}$ & 3.7 & 0.100000 & Anhydritic, silty limestone \\
\hline s 1 d $38-26-22$ add & $5,398.0$ & $5,399.0$ & $324 \mathrm{PRDX}$ & 2.4 & 0.090000 & Anhydritic, silty limestone \\
\hline s 1 d $38-26-22$ add & $5,415.0$ & $5,416.0$ & $324 \mathrm{PRDX}$ & 2.0 & 0.080000 & Anhydrite and dolomite \\
\hline $\operatorname{sld} 38-26-22 \mathrm{add}$ & $5,416.0$ & $5,417.0$ & $324 \mathrm{PRDX}$ & 7.7 & 0.060000 & Sucrose dolomite \\
\hline $\operatorname{sld} 38-26-22$ add & $5,417.0$ & $5,418.0$ & 324PRDX & 14.0 & 2.700000 & Sucrose dolomite \\
\hline sld $38-26-22$ add & $5,418.0$ & $5,419.0$ & $324 \mathrm{PRDX}$ & 9.7 & 0.060000 & Anhydritic, sucrose dolomite \\
\hline sld $38-26-22$ add & $5,419.0$ & $5,420.0$ & $324 \mathrm{PRDX}$ & 3.8 & 2.300000 & Anhydritic dolomite \\
\hline sld $38-26-22 a d d$ & $5,420.0$ & $5,421.0$ & 324PRDX & 5.1 & 1.800000 & Anhydritic dolomite \\
\hline sld $38-26-22 a d d$ & $5,421.0$ & $5,422.0$ & 324PRDX & 4.6 & 3.200000 & Anhydritic dolomite \\
\hline sld38-26-22add & $5,422.0$ & $5,423.0$ & $324 \mathrm{PRDX}$ & 7.9 & 0.700000 & Anhydritic dolomite \\
\hline s ld $38-26-22 a d d$ & $5,423.0$ & $5,424.0$ & $324 \mathrm{PRDX}$ & 6.2 & 1.600000 & Dolomite \\
\hline s 1 d $38-26-22 a d d$ & $5,424.0$ & $5,425.0$ & $324 \mathrm{PRDX}$ & 3.3 & 0.290000 & Dolomite \\
\hline s 1 d $38-26-22 a d d$ & $5,425.0$ & $5,426.0$ & 324PRDX & 3.1 & 4.500000 & Dolomite \\
\hline sld $38-26-22$ add & $5,426.0$ & $5,427.0$ & $324 \mathrm{PRDX}$ & 7.2 & 0.100000 & Dolomite \\
\hline $\operatorname{sld} 38-26-22 \mathrm{add}$ & $5,427.0$ & $5,428.0$ & $324 \mathrm{PRDX}$ & 11.9 & 1.500000 & Earthy dolomite \\
\hline s $1 \mathrm{~d} 38-26-22 \mathrm{add}$ & $5,428.0$ & $5,429.0$ & 324PRDX & 13.0 & 0.240000 & Earthy dolomite \\
\hline s 1 d $38-26-22$ add & $5,429.0$ & $5,430.0$ & $324 \mathrm{PRDX}$ & 12.8 & 0.240000 & Earthy dolomite \\
\hline sld $38-26-22 a d d$ & $5,430.0$ & $5,431.0$ & 324PRDX & 6.3 & 0.060000 & Earthy dolomite \\
\hline s 1 d $38-26-22 a d d$ & $5,431.0$ & $5,432.0$ & 324PRDX & 3.4 & 0.050000 & Shaly dolomite \\
\hline $\operatorname{sld} 38-26-22 \mathrm{add}$ & $5,432.0$ & $5,433.0$ & 324PRDX & 7.4 & 0.070000 & Earthy dolomite \\
\hline sld $38-26-22 a d d$ & $5,433.0$ & $5,434.0$ & 324PRDX & 7.7 & 0.080000 & Earthy dolomite \\
\hline s 1 d $38-26-22$ add & $5,434.0$ & $5,435.0$ & 324PRDX & 5.6 & 0.050000 & Earthy dolomite \\
\hline sld $38-26-22$ add & $5,435.0$ & $5,436.0$ & 324PRDX & 4.8 & 0.050000 & Earthy dolomite \\
\hline $\operatorname{sid} 38-26-22 \mathrm{add}$ & $5,436.0$ & $5,437.0$ & 324PRDX & 9.7 & 0.070000 & Earthy dolomite \\
\hline
\end{tabular}


Table 4.--Laboratory-determined porosity and permeability--Continued

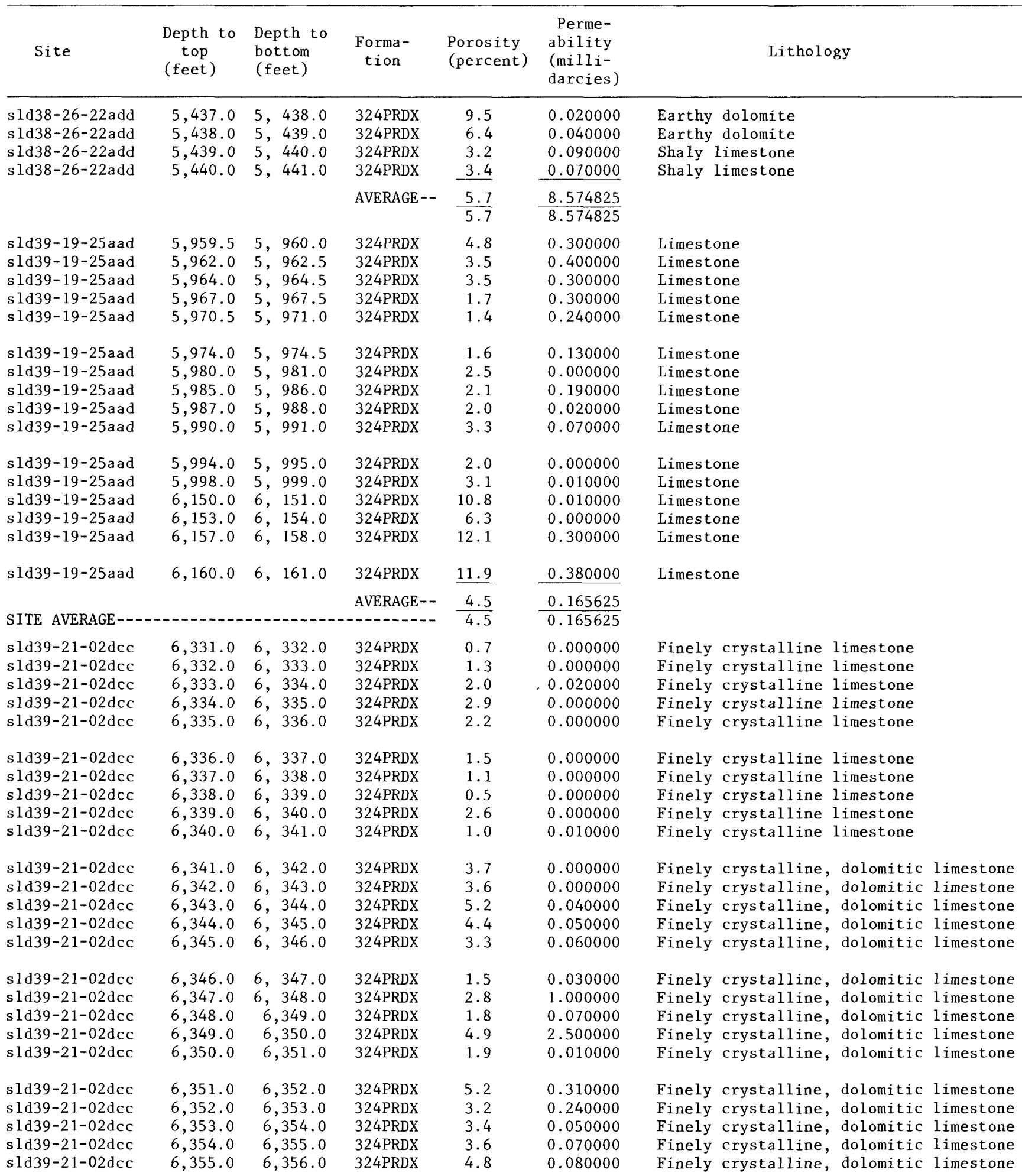


Table 4.--Laboratory-determined porosity and permeability--Continued

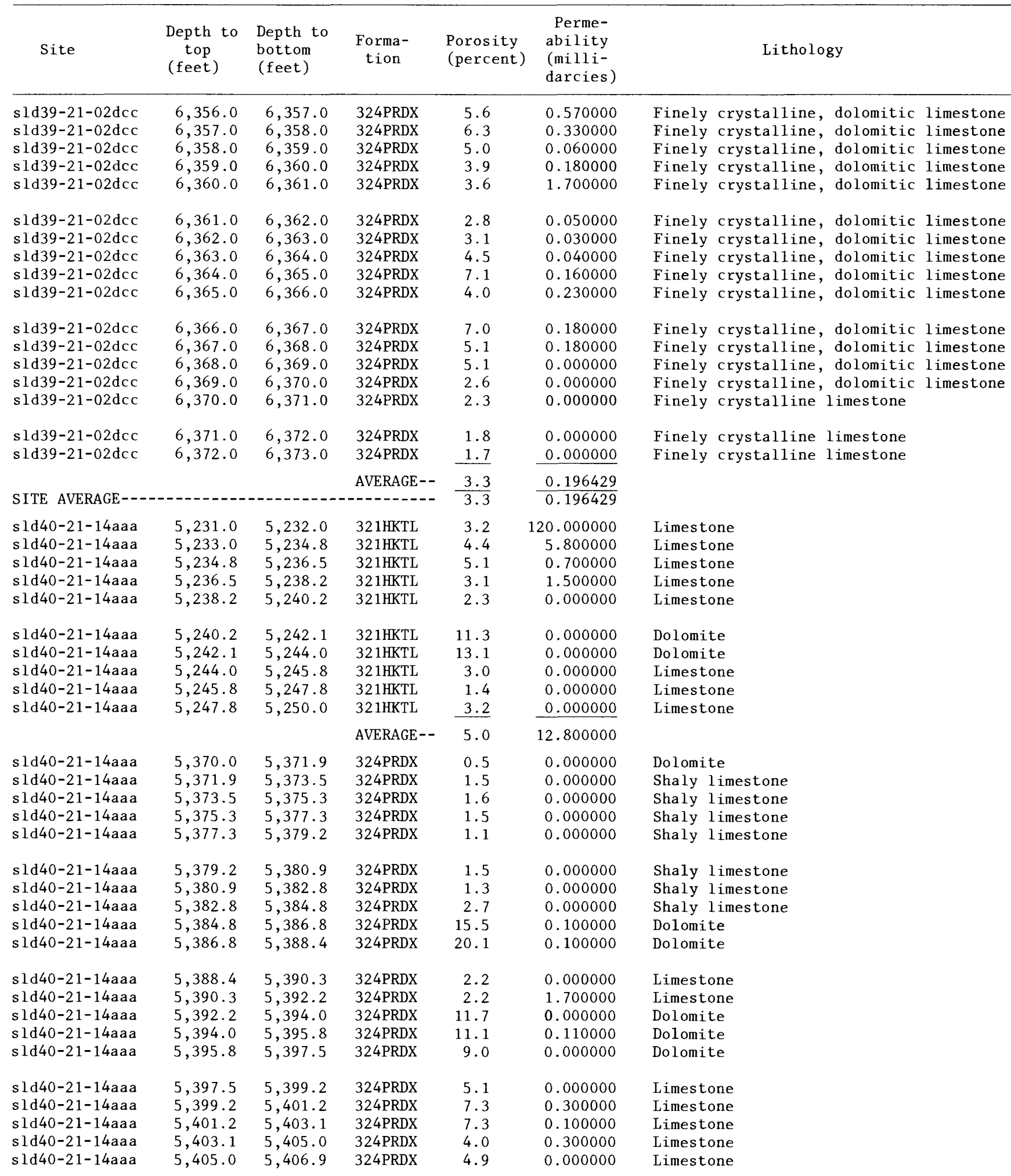


Table 4.--Laboratory-determined porosity and permeability--Continued

\begin{tabular}{|c|c|c|c|c|c|c|}
\hline Site & $\begin{array}{l}\text { Depth to } \\
\text { top } \\
\text { (feet) }\end{array}$ & $\begin{array}{l}\text { Depth to } \\
\text { bottom } \\
\text { (feet) }\end{array}$ & $\begin{array}{l}\text { Forma- } \\
\text { tion }\end{array}$ & $\begin{array}{l}\text { Porosity } \\
\text { (percent) }\end{array}$ & $\begin{array}{l}\text { Perme- } \\
\text { ability } \\
\text { (milli- } \\
\text { darcies) }\end{array}$ & Litho \\
\hline s1d40-21-14aaa & $5,406.9$ & $5,408.6$ & 324PRDX & 8.6 & 0.000000 & Limestone \\
\hline s1d40-21-14aaa & $5,408.6$ & $5,410.4$ & 324PRDX & 4.0 & 0.000000 & Dolomite \\
\hline s1d40-21-14aaa & $5,410.4$ & $5,412.1$ & $324 \mathrm{PRDX}$ & 3.2 & 0.100000 & Shaly limestone \\
\hline $\operatorname{sid} 40-21-14 \mathrm{aaa}$ & $5,412.1$ & $5,414.0$ & $324 \mathrm{PRDX}$ & 2.1 & 0.000000 & Shaly limestone \\
\hline & & & AVERAGE -- & 5.4 & 0.117083 & \\
\hline SITE AVERAGE--- &.-- & -- & $\ldots \ldots \ldots$ & 5.3 & 3.847353 & \\
\hline s1d40-23-13bdd & $5,696.0$ & $5,698.2$ & $324 \mathrm{PRDX}$ & 8.6 & 0.250000 & Algal limestone \\
\hline s1d40-23-13bdd & $5,698.2$ & $5,700.3$ & 324PRDX & 9.2 & 0.300000 & Algal limestone \\
\hline s $1 d 40-23-13 b d d$ & $5,700.3$ & $5,702.3$ & 324PRDX & 10.1 & 0.450000 & Algal limestone \\
\hline s1d40-23-13bdd & $5,702.5$ & $5,704.5$ & $324 \mathrm{PRDX}$ & 5.6 & 0.250000 & Algal limestone \\
\hline s1d40-23-13bdd & $5,704.8$ & $5,707.1$ & $324 \mathrm{PRDX}$ & 7.8 & 0.750000 & Algal limestone \\
\hline sld40-23-13bdd & $5,707.4$ & $5,709.1$ & 324PRDX & 21.0 & 14.500000 & Sandstone \\
\hline s1d40-23-13bdd & $5,709.4$ & $5,711.2$ & 324PRDX & 19.3 & 7.050000 & Sandstone \\
\hline sld40-23-13bdd & $5,711.2$ & $5,713.2$ & 324PRDX & 18.4 & 3.100000 & Sandstone \\
\hline s $1 d 40-23-13 b d d$ & $5,713.5$ & $5,715.2$ & 324PRDX & 13.8 & 0.800000 & Sandstone \\
\hline s1d40-23-13bdd & $5,715.5$ & $5,717.3$ & 324PRDX & 7.8 & 0.100000 & Sandy limestone \\
\hline s $1 d 40-23-13 b d d$ & $5,717.7$ & $5,719.2$ & 324PRDX & 7.8 & 0.200000 & Sandy limestone \\
\hline s1d40-23-13bdd & $5,719.5$ & $5,721.2$ & 324PRDX & 14.2 & 2.650000 & Algal limestone \\
\hline s1d40-23-13bdd & $5,721.5$ & $5,723.2$ & 324PRDX & 16.7 & 3.100000 & Algal limestone \\
\hline s1d40-23-13bdd & $5,723.5$ & $5,725.2$ & 324PRDX & 12.8 & 0.200000 & Algal limestone \\
\hline s1d40-23-13bdd & $5,725.2$ & $5,727.2$ & 324PRDX & 12.8 & 0.700000 & Algal limestone \\
\hline s $1 d 40-23-13 b d d$ & $5,727.5$ & $5,729.2$ & 324PRDX & 16.3 & 3.050000 & Algal limestone \\
\hline s1d40-23-13bdd & $5,729.5$ & $5,731.4$ & 324PRDX & 17.6 & 13.500000 & Algal limestone \\
\hline s $1 d 40-23-13 b d d$ & $5,731.7$ & $5,734.2$ & 324PRDX & 9.5 & 2.200000 & Shaly limestone \\
\hline sld40-23-13bdd & $5,734.5$ & $5,736.1$ & 324PRDX & 8.6 & 0.850000 & Shaly limestone \\
\hline s $1 d 40-23-13 b d d$ & $5,736.4$ & $5,738.1$ & $324 \mathrm{PRDX}$ & 7.4 & 0.800000 & Shaly limestone \\
\hline s $1 d 40-23-13 b d d$ & $5,738.4$ & $5,740.1$ & 324PRDX & 5.7 & 0.020000 & Shaly limestone \\
\hline sld40-23-13bdd & $5,740.1$ & $5,742.4$ & 324PRDX & 7.2 & 0.850000 & Shaly limestone \\
\hline s1d40-23-13bdd & $5,742.7$ & $5,745.0$ & 324PRDX & 7.0 & 1.600000 & Shaly limestone \\
\hline s1d40-23-13bdd & $5,746.0$ & $5,748.5$ & 324PRDX & 8.0 & 0.400000 & Shaly limestone \\
\hline s $1 d 40-23-13 b d d$ & $5,748.8$ & $5,750.6$ & 324PRDX & 8.2 & 8.900000 & Shaly limestone \\
\hline s1d40-23-13bdd & $5,750.9$ & $5,751.7$ & 324PRDX & 8.2 & 0.750000 & Shaly limestone \\
\hline sld40-23-13bdd & $5,752.6$ & $5,755.0$ & 324PRDX & 13.4 & 1.300000 & Cherty limestone \\
\hline sld40-23-13bdd & $5,755.0$ & $5,757.2$ & 324PRDX & 16.2 & 3.600000 & Cherty limestone \\
\hline s $1 d 40-23-13 b d d$ & $5,757.2$ & $5,759.4$ & 324PRDX & 17.2 & 2.900000 & Cherty limestone \\
\hline s1d40-23-13bdd & $5,759.4$ & $5,762.3$ & 324PRDX & 14.6 & 1.500000 & Cherty limestone \\
\hline s $1 d 40-23-13 b d d$ & $5,762.3$ & $5,768.4$ & 324PRDX & 12.7 & 4.950000 & Cherty limestone \\
\hline s $1 d 40-23-13 b d d$ & $5,768.4$ & $5,769.3$ & 324PRDX & 12.2 & 5.900000 & Cherty limestone \\
\hline s $1 d 40-23-13 b d d$ & $5,769.3$ & $5,771.2$ & $324 \mathrm{PRDX}$ & 10.7 & 4.980000 & Cherty limestone \\
\hline s $1 d 40-23-13 b d d$ & $5,771.2$ & $5,773.4$ & 324PRDX & 15.4 & 3.350000 & Cherty limestone \\
\hline sld40-23-13bdd & $5,773.6$ & $5,774.2$ & 324PRDX & 15.4 & 15.700000 & Cherty limestone \\
\hline sld40-23-13bdd & $5,774.5$ & $5,776.1$ & 324PRDX & 15.2 & 11.950000 & Cherty limestone \\
\hline s1d40-23-13bdd & $5,776.4$ & $5,778.1$ & 324PRDX & 14.3 & 26.850000 & Cherty limestone \\
\hline s $1 d 40-23-13 b d d$ & $5,778.4$ & $5,780.0$ & 324PRDX & 14.7 & 7.550000 & Cherty limestone \\
\hline s $1 d 40-23-13 b d d$ & $5,780.0$ & $5,782.1$ & 324PRDX & 2.9 & 0.000000 & Shale \\
\hline s1d40-23-13bdd & $5,782.4$ & $5,784.0$ & $324 \mathrm{PRDX}$ & 1.5 & 0.000000 & Shale \\
\hline
\end{tabular}


Table 4.--Laboratory-determined porosity and permeability--Continued

\begin{tabular}{|c|c|c|c|c|c|c|}
\hline Site & $\begin{array}{l}\text { Depth to } \\
\text { top } \\
\text { (feet) }\end{array}$ & $\begin{array}{l}\text { Depth to } \\
\text { bottom } \\
\text { (feet) }\end{array}$ & $\begin{array}{c}\text { Forma- } \\
\text { tion }\end{array}$ & $\begin{array}{l}\text { Porosity } \\
\text { (percent) }\end{array}$ & $\begin{array}{l}\text { Perme- } \\
\text { ability } \\
\text { (milli- } \\
\text { darcies) }\end{array}$ & Lithology \\
\hline $\begin{array}{l}\text { sld40-23-13bdd } \\
\text { s } 1 d 40-23-13 \text { bdd } \\
\text { sld40-23-13bdd } \\
\text { sld40-23-13bdd } \\
\text { s } 1 d 40-23-13 b d d\end{array}$ & $\begin{array}{l}5,784.3 \\
5,786.3 \\
5,787.2 \\
5,789.7 \\
5,793.1\end{array}$ & $\begin{array}{l}5,786.0 \\
5,787.2 \\
5,789.7 \\
5,793.1 \\
5,795.2\end{array}$ & $\begin{array}{l}324 \mathrm{PRDX} \\
324 \mathrm{PRDX} \\
324 \mathrm{PRDX} \\
324 \mathrm{PRDX} \\
324 \mathrm{PRDX}\end{array}$ & $\begin{array}{l}5.2 \\
3.8 \\
5.4 \\
3.5 \\
5.4\end{array}$ & $\begin{array}{l}0.100000 \\
0.000000 \\
1.500000 \\
1.050000 \\
0.250000\end{array}$ & $\begin{array}{l}\text { Shaly limestone } \\
\text { Shaly limestone } \\
\text { Shaly limestone } \\
\text { Shaly limestone } \\
\text { Shaly limestone }\end{array}$ \\
\hline $\begin{array}{l}\text { sld40-23-13bdd } \\
\text { sld40-23-13bdd } \\
\text { sld40-23-13bdd } \\
\text { sld40-23-13bdd } \\
\text { sld40-23-13bdd }\end{array}$ & $\begin{array}{l}5,795.5 \\
5,798.3 \\
5,800.6 \\
5,803.2 \\
5,805.7\end{array}$ & $\begin{array}{l}5,798.0 \\
5,700.3 \\
5,803.0 \\
5,805.4 \\
5,808.2\end{array}$ & $\begin{array}{l}324 \mathrm{PRDX} \\
324 \mathrm{PRDX} \\
324 \mathrm{PRDX} \\
324 \mathrm{PRDX} \\
324 \mathrm{PRDX}\end{array}$ & $\begin{array}{r}5.9 \\
14.8 \\
17.8 \\
6.2 \\
3.8\end{array}$ & $\begin{array}{r}0.100000 \\
8.000000 \\
17.450000 \\
0.000000 \\
0.150000\end{array}$ & $\begin{array}{l}\text { Shaly limestone } \\
\text { Cherty limestone } \\
\text { Cherty limestone } \\
\text { Shaly limestone } \\
\text { Shaly limestone }\end{array}$ \\
\hline $\begin{array}{l}\text { sld40-23-13bdd } \\
\text { sld40-23-13bdd } \\
\text { sld40-23-13bdd } \\
\text { sld40-23-13bdd } \\
\text { sld40-23-13bdd }\end{array}$ & $\begin{array}{l}5,808 \cdot 5 \\
5,810.4 \\
5,812.4 \\
5,814.4 \\
5,815.8\end{array}$ & $\begin{array}{l}5,810.1 \\
5,812.1 \\
5,814.1 \\
5,815.8 \\
5,817.0\end{array}$ & $\begin{array}{l}324 \mathrm{PRDX} \\
324 \mathrm{PRDX} \\
324 \mathrm{PRDX} \\
324 \mathrm{PRDX} \\
324 \mathrm{PRDX}\end{array}$ & $\begin{array}{l}1.7 \\
3.7 \\
4.6 \\
2.9 \\
3.9\end{array}$ & $\begin{array}{r}0.150000 \\
0.200000 \\
0.150000 \\
0.150000 \\
14.650000\end{array}$ & $\begin{array}{l}\text { Shaly limestone } \\
\text { Shaly limestone } \\
\text { Shaly limestone } \\
\text { Shaly limestone } \\
\text { Shaly limestone }\end{array}$ \\
\hline $\begin{array}{l}\text { sld40-23-13bdd } \\
\text { sld40-23-13bdd } \\
\text { sld40-23-13bdd } \\
\text { sld40-23-13bdd } \\
\text { sld40-23-13bdd }\end{array}$ & $\begin{array}{l}5,817 \cdot 0 \\
5,819 \cdot 4 \\
5,821 \cdot 3 \\
5,823 \cdot 3 \\
5,826.3\end{array}$ & $\begin{array}{l}5,819.1 \\
5,821.0 \\
5,823.3 \\
5,826.0 \\
5,828.1\end{array}$ & $\begin{array}{l}324 \mathrm{PRDX} \\
324 \mathrm{PRDX} \\
324 \mathrm{PRDX} \\
324 \mathrm{PRDX} \\
324 \mathrm{PRDX}\end{array}$ & $\begin{array}{l}4.8 \\
6.6 \\
6.9 \\
7.5 \\
7.4\end{array}$ & $\begin{array}{l}0.100000 \\
0.100000 \\
0.100000 \\
0.350000 \\
0.050000\end{array}$ & $\begin{array}{l}\text { Shaly limestone } \\
\text { Shaly limestone } \\
\text { Shaly limestone } \\
\text { Shaly limestone } \\
\text { Shaly limestone }\end{array}$ \\
\hline $\begin{array}{l}\text { sld40-23-13bdd } \\
\text { sld40-23-13bdd } \\
\text { sld40-23-13bdd } \\
\text { sld40-23-13bdd } \\
\text { sld40-23-13bdd }\end{array}$ & $\begin{array}{l}5,828.4 \\
5,830.4 \\
5,832.6 \\
5,834.6 \\
5,836.4\end{array}$ & $\begin{array}{l}5,830.1 \\
5,832.1 \\
5,834.2 \\
5,836.2 \\
5,838.5\end{array}$ & $\begin{array}{l}324 \mathrm{PRDX} \\
324 \mathrm{PRDX} \\
324 \mathrm{PRDX} \\
324 \mathrm{PRDX} \\
324 \mathrm{PRDX}\end{array}$ & $\begin{array}{r}15.8 \\
15.8 \\
11.2 \\
1.6 \\
1.5\end{array}$ & $\begin{array}{l}1.500000 \\
1.000000 \\
0.250000 \\
0.000000 \\
0.000000\end{array}$ & $\begin{array}{l}\text { Cherty limestone } \\
\text { Cherty limestone } \\
\text { Cherty limestone } \\
\text { Shaly limestone } \\
\text { Shaly limestone }\end{array}$ \\
\hline $\begin{array}{l}\text { sld40-23-13bdd } \\
\text { sld40-23-13bdd } \\
\text { sld40-23-13bdd } \\
\text { s ld40-23-13bdd } \\
\text { s ld40-23-13bdd }\end{array}$ & $\begin{array}{l}5,838.8 \\
5,841 \cdot 3 \\
5,843 \cdot 4 \\
5,845.4 \\
5,848.6\end{array}$ & $\begin{array}{l}5,841.0 \\
5,843.4 \\
5,845.4 \\
5,848.3 \\
5,851.0\end{array}$ & $\begin{array}{l}\text { 324PRDX } \\
324 \mathrm{PRDX} \\
324 \mathrm{PRDX} \\
324 \mathrm{PRDX} \\
324 \mathrm{PRDX}\end{array}$ & $\begin{array}{r}2.8 \\
11.1 \\
14.9 \\
4.4 \\
2.4 \\
\end{array}$ & $\begin{array}{l}0.000000 \\
0.500000 \\
3.100000 \\
0.000000 \\
0.050000\end{array}$ & $\begin{array}{l}\text { Shaly limestone } \\
\text { Cherty limestone } \\
\text { Cherty limestone } \\
\text { Shaly limestone } \\
\text { Shaly limestone }\end{array}$ \\
\hline SITE AVERAGE--- & 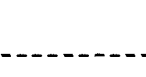 & - & AVERAGE-- & $\frac{9.6}{9.6}$ & $\frac{2.983571}{2.983571}$ & \\
\hline $\begin{array}{l}\text { sld40-24-22aba } \\
\text { sld40-24-22aba } \\
\text { sld40-24-22aba } \\
\text { sld40-24-22aba } \\
\text { s } 1 d 40-24-22 a b a\end{array}$ & $\begin{array}{l}5,771.0 \\
5,772.0 \\
5,773.0 \\
5,774.0 \\
5,775.0\end{array}$ & $\begin{array}{l}5,772.0 \\
5,773.0 \\
5,774.0 \\
5,775.0 \\
5,776.0\end{array}$ & $\begin{array}{l}\text { 324PRDX } \\
\text { 324PRDX } \\
\text { 324PRDX } \\
\text { 324PRDX } \\
\text { 324PRDX }\end{array}$ & $\begin{array}{r}4.3 \\
1.2 \\
9.6 \\
11.5 \\
12.7\end{array}$ & $\begin{array}{l}0.010000 \\
1.200000 \\
1.200000 \\
0.180000 \\
8.100000\end{array}$ & $\begin{array}{l}\text { Finely crystalline limestone } \\
\text { Finely crystalline limestone } \\
\text { Finely crystalline, dolomitic limestone } \\
\text { Finely crystalline, dolomitic limestone } \\
\text { Finely crystalline, dolomitic limestone }\end{array}$ \\
\hline $\begin{array}{l}\text { sld40-24-22aba } \\
\text { sld40-24-22aba } \\
\text { sld40-24-22aba } \\
\text { sld40-24-22aba } \\
\text { sld40-24-22aba }\end{array}$ & $\begin{array}{l}5,776.0 \\
5,777.0 \\
5,778.0 \\
5,779.0 \\
5,780.0\end{array}$ & $\begin{array}{l}5,777.0 \\
5,778.0 \\
5,779.0 \\
5,780.0 \\
5,781.0\end{array}$ & $\begin{array}{l}324 \mathrm{PRDX} \\
324 \mathrm{PRDX} \\
324 \mathrm{PRDX} \\
324 \mathrm{PRDX} \\
324 \mathrm{PRDX}\end{array}$ & $\begin{array}{l}9.1 \\
5.6 \\
5.7 \\
4.3 \\
1.5\end{array}$ & $\begin{array}{l}0.100000 \\
0.720000 \\
0.010000 \\
0.060000 \\
0.020000\end{array}$ & $\begin{array}{l}\text { Finely crystalline, dolomitic limestone } \\
\text { Finely crystalline limestone } \\
\text { Finely crystalline limestone } \\
\text { Finely crystalline limestone } \\
\text { Finely crystalline limestone }\end{array}$ \\
\hline $\begin{array}{l}\text { sld40-24-22aba } \\
\text { sld40-24-22aba } \\
\text { sld40-24-22aba } \\
\text { sld40-24-22aba } \\
\text { sld40-24-22aba }\end{array}$ & $\begin{array}{l}5,781.0 \\
5,782.0 \\
5,783.0 \\
5,784.0 \\
5,786.0\end{array}$ & $\begin{array}{l}5,782.0 \\
5,783.0 \\
5,784.0 \\
5,785.0 \\
5,787.0\end{array}$ & $\begin{array}{l}\text { 324PRDX } \\
324 \mathrm{PRDX} \\
324 \mathrm{PRDX} \\
324 \mathrm{PRDX} \\
324 \mathrm{PRDX}\end{array}$ & $\begin{array}{l}1.8 \\
0.4 \\
0.9 \\
0.5 \\
1.8\end{array}$ & $\begin{array}{l}1.800000 \\
0.000000 \\
0.010000 \\
0.000000 \\
0.000000\end{array}$ & $\begin{array}{l}\text { Finely crystalline limestone } \\
\text { Finely crystalline limestone } \\
\text { Finely crystalline limestone } \\
\text { Finely crystalline limestone } \\
\text { Finely crystalline limestone }\end{array}$ \\
\hline
\end{tabular}


Table 4.--Laboratory-determined porosity and permeability--Continued

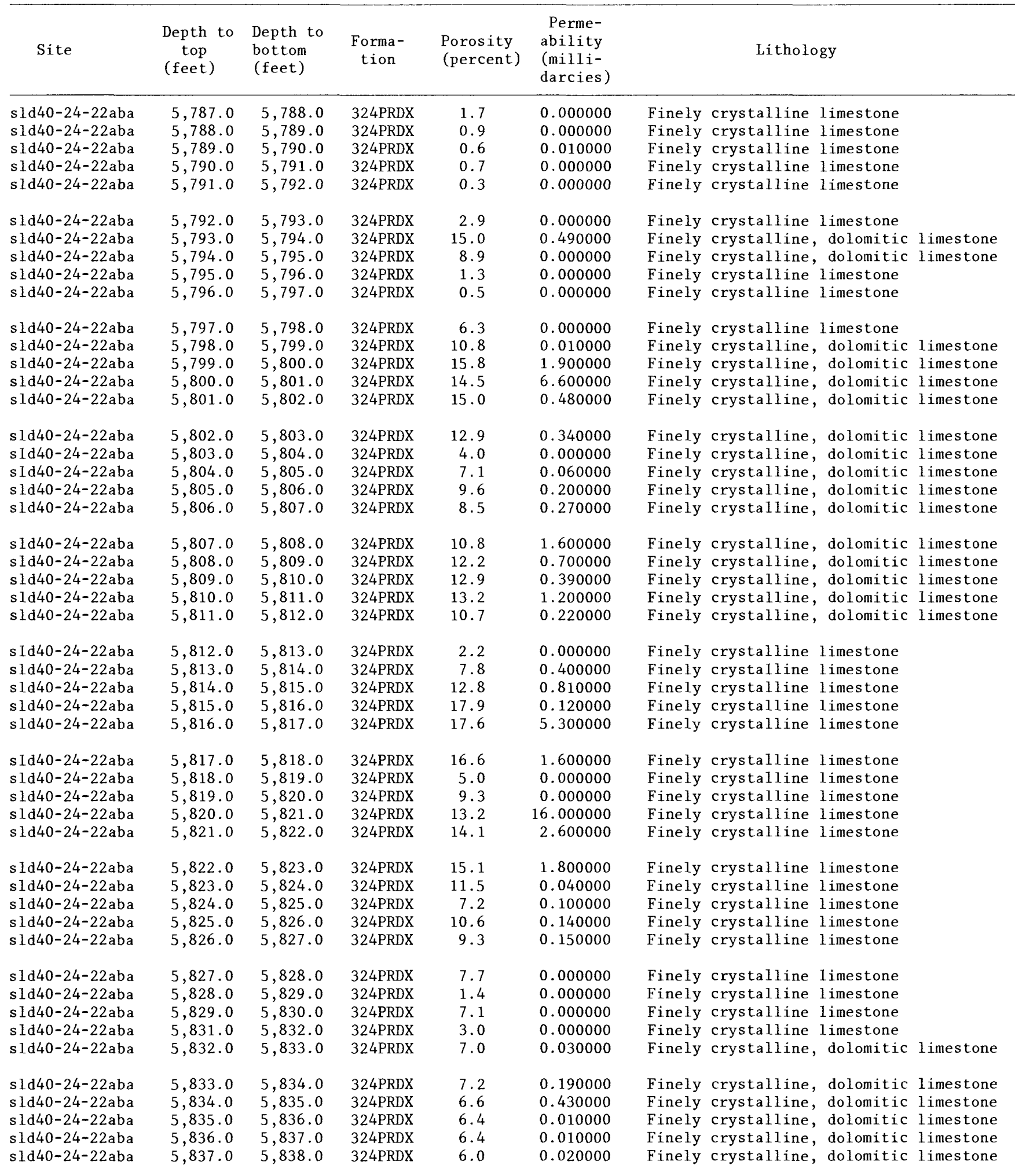


Table 4.--Laboratory-determined porosity and permeability--Continued

\begin{tabular}{|c|c|c|c|c|c|c|}
\hline Site & $\begin{array}{l}\text { Depth to } \\
\text { top } \\
\text { (feet) }\end{array}$ & $\begin{array}{l}\text { Depth to } \\
\text { bottom } \\
\text { (feet) }\end{array}$ & $\begin{array}{c}\text { Forma- } \\
\text { tion }\end{array}$ & $\begin{array}{l}\text { Porosity } \\
\text { (percent) }\end{array}$ & $\begin{array}{c}\text { Perme- } \\
\text { ability } \\
\text { (milli- } \\
\text { darcies) }\end{array}$ & Lithology \\
\hline sld40-24-22aba & $5,838.0$ & $5,839.0$ & 324PRDX & 5.5 & 0.030000 & Finely crystalline, dolomitic limestone \\
\hline sld40-24-22aba & $5,839.0$ & $5,840.0$ & 324PRDX & 4.7 & 0.080000 & Finely crystalline, dolomitic limestone \\
\hline sld40-24-22aba & $5,840.0$ & $5,841.0$ & 324PRDX & 7.1 & 0.040000 & Finely crystalline, dolomitic limestone \\
\hline sld40-24-22aba & $5,841.0$ & $5,842.0$ & $324 \mathrm{PRDX}$ & 1.4 & 0.010000 & Finely crystalline limestone \\
\hline sld40-24-22aba & $5,842.0$ & $5,843.0$ & $324 \mathrm{PRDX}$ & 0.8 & 0.010000 & Finely crystalline limestone \\
\hline sld40-24-22aba & $5,843.0$ & $5,844.0$ & 324PRDX & 0.8 & 0.010000 & Finely crystalline limestone \\
\hline s $1 d 40-24-22 a b a$ & $5,844.0$ & $5,845.0$ & 324PRDX & 0.0 & 0.500000 & Finely crystalline limestone \\
\hline sld40-24-22aba & $5,845.0$ & $5,846.0$ & 324PRDX & 0.8 & 0.000000 & Finely crystalline limestone \\
\hline sld40-24-22aba & $5,846.0$ & $5,847.0$ & 324PRDX & 1.1 & 0.000000 & Finely crystalline limestone \\
\hline sld40-24-22aba & $5,847.0$ & $5,848.0$ & 324PRDX & 1.3 & 0.000000 & Finely crystalline limestone \\
\hline s $1 d 40-24-22 a b a$ & $5,848.0$ & $5,849.0$ & $324 \mathrm{PRDX}$ & 0.6 & 0.000000 & Finely crystalline limestone \\
\hline s ld $40-24-22 a b a$ & $5,849.0$ & $5,850.0$ & $324 \mathrm{PRDX}$ & 0.8 & 0.020000 & Finely crystalline limestone \\
\hline s ld $40-24-22 a b a$ & $5,850.0$ & $5,851.0$ & $324 \mathrm{PRDX}$ & 0.8 & 0.000000 & Finely crystalline limestone \\
\hline sld40-24-22aba & $5,851.0$ & $5,852.0$ & $324 \mathrm{PRDX}$ & 0.7 & 0.000000 & Finely crystalline limestone \\
\hline sld40-24-22aba & $5,852.0$ & $5,853.0$ & 324PRDX & 0.9 & 0.010000 & Finely crystalline limestone \\
\hline s ld 40-24-22aba & $5,853.0$ & $5,854.0$ & $324 \mathrm{PRDX}$ & 1.1 & 0.000000 & Finely crystalline limestone \\
\hline $\operatorname{sld} 40-24-22 a b a$ & $5,854.0$ & $5,855.0$ & 324PRDX & 1.0 & 0.020000 & Finely crystalline limestone \\
\hline s ld $40-24-22 a b a$ & $5,855.0$ & $5,856.0$ & $324 \mathrm{PRDX}$ & 2.3 & 0.040000 & Finely crystalline limestone \\
\hline $\operatorname{sld} 40-24-22 a b a$ & $5,856.0$ & $5,857.0$ & 324PRDX & 1.9 & 0.000000 & Finely crystalline limestone \\
\hline sld40-24-22aba & $5,857.0$ & $5,858.0$ & $324 \mathrm{PRDX}$ & 1.1 & 0.510000 & Finely crystalline limestone \\
\hline sld40-24-22aba & $5,858.0$ & $5,859.0$ & $324 \mathrm{PRDX}$ & 4.8 & 0.000000 & Finely crystalline limestone \\
\hline $\operatorname{sld} 40-24-22 a b a$ & $5,859.0$ & $5,860.0$ & $324 \mathrm{PRDX}$ & 5.4 & 0.180000 & Finely crystalline limestone \\
\hline sld40-24-22aba & $5,860.0$ & $5,861.0$ & 324PRDX & 1.1 & 0.000000 & Finely crystalline limestone \\
\hline sld40-24-22aba & $5,861.0$ & $5,862.0$ & 324PRDX & 5.4 & 0.000000 & Finely crystalline limestone \\
\hline sld40-24-22aba & $5,862.0$ & $5,863.0$ & 324PRDX & 6.1 & 0.040000 & Vuggy, finely crystalline, dolomitic limestone \\
\hline sld40-24-22aba & $5,863.0$ & $5,864.0$ & 324PRDX & 10.3 & 10.000000 & Vuggy, finely crystaliine, dolomitic limestone \\
\hline $\operatorname{sld} 40-24-22 a b a$ & $5,864.0$ & $5,865.0$ & 324PRDX & 6.9 & 1.600000 & Vuggy, finely crystalline, dolomitic limestone \\
\hline s ld40-24-22aba & $5,865.0$ & $5,866.0$ & 324PRDX & 8.9 & 20.000000 & Vuggy, finely crystalline, dolomitic limestone \\
\hline sld40-24-22aba & $5,866.0$ & $5,867.0$ & 324PRDX & 5.7 & 1.000000 & Vuggy, finely crystalline, dolomitic limestone \\
\hline sld40-24-22aba & $5,867.0$ & $5,868.0$ & $324 \mathrm{PRDX}$ & 9.1 & 27.000000 & Vuggy, finely crystalline, dolomitic limestone \\
\hline sld40-24-22aba & $5,868.0$ & $5,869.0$ & $324 \mathrm{PRDX}$ & 3.7 & 0.040000 & Vuggy, finely crystalline, dolomitic limestone \\
\hline s ld40-24-22aba & $5,869.0$ & $5,870.0$ & 324PRDX & 3.5 & 0.010000 & Finely crystalline limestone \\
\hline s ld40-24-22aba & $5,870.0$ & $5,871.0$ & 324PRDX & 13.9 & 0.010000 & Finely crystalline limestone \\
\hline sld40-24-22aba & $5,871.0$ & $5,872.0$ & $324 \mathrm{PRDX}$ & 7.1 & 0.010000 & Finely crystalline limestone \\
\hline sld40-24-22aba & $5,872.0$ & $5,873.0$ & $324 \mathrm{PRDX}$ & 1.3 & 0.000000 & Finely crystalline limestone \\
\hline sld40-24-22aba & $5,873.0$ & $5,874.0$ & 324PRDX & 1.4 & 0.000000 & Finely crystalline limestone \\
\hline s $1 d 40-24-22 a b a$ & $5,874.0$ & $5,875.0$ & $324 \mathrm{PRDX}$ & 1.6 & 0.010000 & Finely crystalline limestone \\
\hline sld $40-24-22 a b a$ & $5,875.0$ & $5,876.0$ & 324PRDX & 9.2 & 0.000000 & Finely crystalline limestone \\
\hline sld40-24-22aba & $5,876.0$ & $5,877.0$ & 324PRDX & 2.0 & 0.000000 & Finely crystalline limestone \\
\hline sld40-24-22aba & $5,877.0$ & $5,878.0$ & 324PRDX & 2.2 & 0.010000 & Finely crystalline limestone \\
\hline sld $40-24-22 a b a$ & $5,878.0$ & $5,879.0$ & 324PRDX & 5.8 & 0.030000 & Finely crystalline limestone \\
\hline sld40-24-22aba & $5,879.0$ & $5,880.0$ & 324PRDX & 2.9 & 0.010000 & Finely crystalline limestone \\
\hline sld40-24-22aba & $5,880.0$ & $5,881.0$ & 324PRDX & 3.3 & 0.010000 & Finely crystalline limestone \\
\hline s $1 d 40-24-22 a b a$ & $5,881.0$ & $5,882.0$ & $324 \mathrm{PRDX}$ & 8.6 & 0.110000 & Finely crystalline limestone \\
\hline sld40-24-22aba & $5,882.0$ & $5,883.0$ & 324PRDX & 9.1 & 3.000000 & Vuggy, finely crystalline limestone \\
\hline s $1 d 40-24-22 a b a$ & $5,883.0$ & $5,884.0$ & 324PRDX & 12.0 & 97.000000 & Vuggy, finely crystalline limestone \\
\hline sld40-24-22aba & $5,884.0$ & $5,885.0$ & $324 \mathrm{PRDX}$ & 10.8 & 2.000000 & Vuggy, finely crystalline limestone \\
\hline sld40-24-22aba & $5,885.0$ & $5,886.0$ & $324 \mathrm{PRDX}$ & 7.3 & 13.000000 & Vuggy, finely crystalline limestone \\
\hline s ld40-24-22aba & $5,886.0$ & $5,887.0$ & 324PRDX & 6.7 & 121.000000 & Vuggy, finely crystalline limestone \\
\hline s ld 40-24-22aba & $5,887.0$ & $5,888.0$ & $324 \mathrm{PRDX}$ & 10.0 & 51.000000 & Vuggy, finely crystalline limestone \\
\hline
\end{tabular}


Table 4.--Laboratory-determined porosity and permeability--Continued

\begin{tabular}{|c|c|c|c|c|c|c|}
\hline Site & $\begin{array}{l}\text { Depth to } \\
\text { top } \\
\text { (feet) }\end{array}$ & $\begin{array}{l}\text { Depth to } \\
\text { bottom } \\
\text { (feet) }\end{array}$ & $\begin{array}{c}\text { Forma- } \\
\text { tion }\end{array}$ & $\begin{array}{l}\text { Porosity } \\
\text { (percent) }\end{array}$ & $\begin{array}{l}\text { Perme- } \\
\text { ability } \\
\text { (milli- } \\
\text { darcies) }\end{array}$ & Lithology \\
\hline $\begin{array}{l}\text { sld40-24-22aba } \\
\text { sld40-24-22aba } \\
\text { sld40-24-22aba } \\
\text { sld40-24-22aba } \\
\text { sld40-24-22aba }\end{array}$ & $\begin{array}{l}5,888.0 \\
5,889.0 \\
5,890.0 \\
5,891.0 \\
5,892.0\end{array}$ & $\begin{array}{l}5,889.0 \\
5,890.0 \\
5,891.0 \\
5,892.0 \\
5,893.0\end{array}$ & $\begin{array}{l}324 \mathrm{PRDX} \\
324 \mathrm{PRDX} \\
324 \mathrm{PRDX} \\
324 \mathrm{PRDX} \\
324 \mathrm{PRDX}\end{array}$ & $\begin{array}{r}11.0 \\
5.2 \\
8.7 \\
5.9 \\
9.8\end{array}$ & $\begin{array}{r}35.000000 \\
4.400000 \\
0.540000 \\
1.900000 \\
1.900000\end{array}$ & $\begin{array}{l}\text { Vuggy, finely crystalline limestone } \\
\text { Finely crystalline limestone } \\
\text { Finely crystalline limestone } \\
\text { Finely crystalline limestone } \\
\text { Finely crystalline limestone }\end{array}$ \\
\hline $\begin{array}{l}\text { sld40-24-22aba } \\
\text { sld40-24-22aba } \\
\text { sld40-24-22aba } \\
\text { s } 1 d 40-24-22 \text { aba } \\
\text { sld40-24-22aba }\end{array}$ & $\begin{array}{l}5,893.0 \\
5,894.0 \\
5,895.0 \\
5,896.0 \\
5,897.0\end{array}$ & $\begin{array}{l}5,894.0 \\
5,895.0 \\
5,896.0 \\
5,897.0 \\
5,898.0\end{array}$ & $\begin{array}{l}324 \mathrm{PRDX} \\
324 \mathrm{PRDX} \\
324 \mathrm{PRDX} \\
324 \mathrm{PRDX} \\
324 \mathrm{PRDX}\end{array}$ & $\begin{array}{l}5.2 \\
3.0 \\
4.1 \\
2.7 \\
3.2\end{array}$ & $\begin{array}{l}0.430000 \\
0.040000 \\
0.020000 \\
0.030000 \\
0.010000\end{array}$ & $\begin{array}{l}\text { Finely crystalline limestone } \\
\text { Finely crystalline limestone } \\
\text { Finely crystalline limestone } \\
\text { Finely crystalline limestone } \\
\text { Finely crystalline limestone }\end{array}$ \\
\hline $\begin{array}{l}\text { s } 1 d 40-24-22 a b a \\
\text { sld } 40-24-22 a b a \\
\text { s } 1 d 40-24-22 a b a \\
\text { sld } 40-24-22 a b a \\
\text { sld40-24-22aba }\end{array}$ & $\begin{array}{l}5,898.0 \\
5,899.0 \\
5,900.0 \\
5,901.0 \\
5,902.0\end{array}$ & $\begin{array}{l}5,899.0 \\
5,900.0 \\
5,901.0 \\
5,902.0 \\
5,903.0\end{array}$ & $\begin{array}{l}324 \mathrm{PRDX} \\
324 \mathrm{PRDX} \\
324 \mathrm{PRDX} \\
324 \mathrm{PRDX} \\
324 \mathrm{PRDX}\end{array}$ & $\begin{array}{l}3.2 \\
1.6 \\
1.9 \\
2.7 \\
2.0\end{array}$ & $\begin{array}{l}0.010000 \\
0.010000 \\
0.010000 \\
0.010000 \\
0.000000\end{array}$ & $\begin{array}{l}\text { Finely crystalline limestone } \\
\text { Finely crystalline limestone } \\
\text { Finely crystalline limestone } \\
\text { Finely crystalline limestone } \\
\text { Finely crystalline limestone }\end{array}$ \\
\hline $\begin{array}{l}\text { s } 1 d 40-24-22 a b a \\
\text { sld40-24-22aba } \\
\text { s } 1 d 40-24-22 a b a \\
\text { sld40-24-22aba } \\
\text { sld40-24-22aba }\end{array}$ & $\begin{array}{l}5,903.0 \\
5,904.0 \\
5,905.0 \\
5,906.0 \\
5,907.0\end{array}$ & $\begin{array}{l}5,904.0 \\
5,905.0 \\
5,906.0 \\
5,907.0 \\
5,908.0\end{array}$ & $\begin{array}{l}324 \mathrm{PRDX} \\
324 \mathrm{PRDX} \\
324 \mathrm{PRDX} \\
324 \mathrm{PRDX} \\
324 \mathrm{PRDX}\end{array}$ & $\begin{array}{l}1.2 \\
2.5 \\
2.1 \\
4.4 \\
4.0\end{array}$ & $\begin{array}{l}0.000000 \\
0.090000 \\
0.010000 \\
0.020000 \\
0.090000\end{array}$ & $\begin{array}{l}\text { Finely crystalline limestone } \\
\text { Finely crystalline limestone } \\
\text { Finely crystalline limestone } \\
\text { Finely crystalline limestone } \\
\text { Finely crystalline limestone }\end{array}$ \\
\hline $\begin{array}{l}\text { s } 1 d 40-24-22 a b a \\
\text { s } 1 d 40-24-22 a b a \\
\text { s } 1 d 40-24-22 a b a \\
\text { sld40-24-22aba } \\
\text { s } 1 d 40-24-22 a b a\end{array}$ & $\begin{array}{l}5,908.0 \\
5,909.0 \\
5,910.0 \\
5,911.0 \\
5,912.0\end{array}$ & $\begin{array}{l}5,909.0 \\
5,910.0 \\
5,911.0 \\
5,912.0 \\
5,913.0\end{array}$ & $\begin{array}{l}324 \mathrm{PRDX} \\
324 \mathrm{PRDX} \\
324 \mathrm{PRDX} \\
324 \mathrm{PRDX} \\
324 \mathrm{PRDX}\end{array}$ & $\begin{array}{l}3.9 \\
4.3 \\
2.5 \\
1.6 \\
1.2\end{array}$ & $\begin{array}{l}0.120000 \\
0.150000 \\
0.020000 \\
0.040000 \\
0.160000\end{array}$ & $\begin{array}{l}\text { Finely crystalline limestone } \\
\text { Finely crystalline limestone } \\
\text { Finely crystalline limestone } \\
\text { Finely crystalline limestone } \\
\text { Finely crystalline limestone }\end{array}$ \\
\hline $\begin{array}{l}\text { s } 1 d 40-25-26 b b b \\
\text { s } 1 d 40-25-26 b b b \\
\text { s } 1 d 40-25-26 b b b \\
\text { s } 1 d 40-25-26 b b b \\
\text { s } 1 d 40-25-26 b b b\end{array}$ & $\begin{array}{l}5,953 \cdot 0 \\
5,954 \cdot 5 \\
5,958 \cdot 0 \\
5,959.8 \\
5,961.7\end{array}$ & $\begin{array}{l}5,954.5 \\
5,956.0 \\
5,959.8 \\
5,961.7 \\
5,963.3\end{array}$ & $\begin{array}{l}321 \mathrm{HKTL} \\
321 \mathrm{HKTL} \\
321 \mathrm{HKTL} \\
321 \mathrm{HKTL} \\
321 \mathrm{HKTL}\end{array}$ & $\begin{array}{l}3.6 \\
2.6 \\
2.9 \\
3.1 \\
3.6\end{array}$ & $\begin{array}{l}0.200000 \\
2.400000 \\
0.300000 \\
0.400000 \\
0.300000\end{array}$ & $\begin{array}{l}\text { Limestone } \\
\text { Limestone } \\
\text { Limestone } \\
\text { Limestone } \\
\text { Limestone }\end{array}$ \\
\hline $\begin{array}{l}\text { s } 1 d 40-25-26 b b b \\
\text { s } 1 d 40-25-26 b b b \\
\text { sld } 40-25-26 b b b \\
\text { s } 1 d 40-25-26 b b b \\
\text { s } 1 d 40-25-26 b b b\end{array}$ & $\begin{array}{l}5,963 \cdot 3 \\
5,965 \cdot 2 \\
5,967 \cdot 2 \\
5,969 \cdot 2 \\
5,971 \cdot 0\end{array}$ & $\begin{array}{l}5,965.2 \\
5,967.2 \\
5,969.2 \\
5,971.0 \\
5,972.8\end{array}$ & $\begin{array}{l}321 \mathrm{HKTL} \\
321 \mathrm{HKTL} \\
321 \mathrm{HKTL} \\
321 \mathrm{HKTL} \\
321 \mathrm{HKTL}\end{array}$ & $\begin{array}{l}7.6 \\
5.8 \\
6.8 \\
6.7 \\
9.2\end{array}$ & $\begin{array}{l}0.600000 \\
0.100000 \\
0.500000 \\
4.700000 \\
2.100000\end{array}$ & $\begin{array}{l}\text { Limes tone } \\
\text { Limes tone } \\
\text { Limestone } \\
\text { Limestone } \\
\text { Limestone }\end{array}$ \\
\hline $\begin{array}{l}\text { sld } 40-25-26 b b b \\
\text { sld } 40-25-26 b b b \\
\text { sld } 40-25-26 b b b\end{array}$ & $\begin{array}{l}5,972.8 \\
5,974.7 \\
5,976.5\end{array}$ & $\begin{array}{l}5,974.7 \\
5,976.5 \\
5,979.0\end{array}$ & $\begin{array}{l}\text { 321HKTL } \\
321 \mathrm{HKTL} \\
321 \mathrm{HKTL}\end{array}$ & $\begin{array}{r}5.6 \\
6.2 \\
13.8 \\
\end{array}$ & $\begin{array}{r}6.400000 \\
60.000000 \\
43.000000 \\
\end{array}$ & $\begin{array}{l}\text { Limestone } \\
\text { Limestone } \\
\text { Limestone }\end{array}$ \\
\hline & & & AVERAGE- & 6.0 & 9.307692 & \\
\hline
\end{tabular}


Table 4.--Laboratory-determined porosity and permeability--Continued

\begin{tabular}{|c|c|c|c|c|c|c|}
\hline Site & $\begin{array}{l}\text { Depth to } \\
\text { top } \\
\text { (feet) }\end{array}$ & $\begin{array}{l}\text { Depth to } \\
\text { bottom } \\
\text { (feet) }\end{array}$ & $\begin{array}{c}\text { Forma- } \\
\text { tion }\end{array}$ & $\begin{array}{l}\text { Porosity } \\
\text { (percent) }\end{array}$ & $\begin{array}{l}\text { Perme- } \\
\text { ability } \\
\text { (milli- } \\
\text { darcies) }\end{array}$ & Lithology \\
\hline $\operatorname{sld} 40-25-26 b b b$ & $6,098.0$ & $6,100.5$ & 324PRDX & 8.8 & 1.400000 & Dolomite \\
\hline sld $40-25-26 b b b$ & $6,102.0$ & $6,104.0$ & $324 \mathrm{PRDX}$ & 9.0 & 1.600000 & Limestone and dolomite \\
\hline s $1 d 40-25-26 b b b$ & $6,104.0$ & $6,105.8$ & $324 \mathrm{PRDX}$ & 12.1 & 0.100000 & Limestone and dolomite \\
\hline s $1 d 40-25-26 b b b$ & $6,105.8$ & $6,108.0$ & $324 \mathrm{PRDX}$ & 10.4 & 0.200000 & Limestone and dolomite \\
\hline sld $40-25-26 \mathrm{bbb}$ & $6,109.0$ & $6,110.2$ & $324 \mathrm{PRDX}$ & 10.0 & 0.200000 & Dolomite \\
\hline SITE AVERAGE-- & - & & AVERAGE-- & $\frac{10.1}{7.1}$ & $\frac{0.700000}{6.916667}$ & \\
\hline $\operatorname{sld} 41-23-12 a a a$ & $5,478.0$ & $5,480.5$ & 324PRDX & 5.1 & 0.500000 & Algal limestone \\
\hline sld4 $1-23-12 \mathrm{aaa}$ & $5,480.5$ & $5,482.5$ & $324 \mathrm{PRDX}$ & 11.4 & 1.150000 & Algal limestone \\
\hline sld41-23-12aaa & $5,482.5$ & $5,485.0$ & $324 \mathrm{PRDX}$ & 1.7 & 0.050000 & Siltstone \\
\hline sld41-23-12aaa & $5,485.0$ & $5,487.0$ & $324 \mathrm{PRDX}$ & 1.2 & 0.100000 & Siltstone \\
\hline sld41-23-12aaa & $5,487.0$ & $5,490.0$ & $324 \mathrm{PRDX}$ & 1.2 & 0.050000 & Siltstone \\
\hline s $1 d 41-23-12 a a a$ & $5,490.0$ & $5,492.0$ & $324 \mathrm{PRDX}$ & 2.6 & 0.350000 & Siltstone \\
\hline sld41-23-12aa & $5,492.0$ & $5,495.0$ & $324 \mathrm{PRDX}$ & 11.2 & 22.500000 & Algal limestone \\
\hline sld41-23-12aa & $5,495.0$ & $5,497.0$ & $324 \mathrm{PRDX}$ & 13.3 & 0.950000 & Algal limestone \\
\hline sld41-23-12aa & $5,497.0$ & $5,499.0$ & $324 \mathrm{PRDX}$ & 13.0 & 2.600000 & Algal limestone \\
\hline s ld $41-23-12$ a a & $5,499.0$ & $5,501.0$ & $324 \mathrm{PRDX}$ & 8.5 & 0.950000 & Algal limestone \\
\hline $\operatorname{sld} 41-23-12 \mathrm{a} a \mathrm{a}$ & $5,501.0$ & $5,503.0$ & $324 \mathrm{PRDX}$ & 2.2 & 6.050000 & Algal limestone \\
\hline s ld41-23-12aa & $5,503.0$ & $5,505.5$ & 324PRDX & 2.5 & 0.150000 & Algal limestone \\
\hline s ld $41-23-12 \mathrm{aa}$ & $5,505.5$ & $5,507.5$ & $324 \mathrm{PRDX}$ & 5.8 & 0.750000 & Algal limestone \\
\hline $\operatorname{sld} 41-23-12$ aa & $5,507.5$ & $5,510.0$ & 324PRDX & 8.7 & 0.600000 & Algal limestone \\
\hline $\operatorname{sld} 41-23-12 \mathrm{a} a$ & $5,510.0$ & $5,512.0$ & $324 \mathrm{PRDX}$ & 6.6 & 1.750000 & Algal limestone \\
\hline sld41-23-12aaa & $5,512.0$ & $5,514.5$ & 324PRDX & 14.7 & 0.200000 & Cherty limestone \\
\hline sld41-23-12a a a & $5,514.5$ & $5,517.5$ & 324PRDX & 21.9 & 11.550000 & Cherty limestone \\
\hline sld4 $1-23-12 \mathrm{aaa}$ & $5,517.5$ & $5,519.3$ & $324 \mathrm{PRDX}$ & 5.1 & 3.800000 & Algal limestone \\
\hline sld41-23-12aaa & $5,519.3$ & $5,521.0$ & 324PRDX & 1.0 & 0.900000 & Algal limestone \\
\hline s ld41-23-12a a a & $5,521.0$ & $5,523.5$ & $324 \mathrm{PRDX}$ & 4.6 & 0.300000 & Algal limestone \\
\hline sld41-23-12a a a & $5,523.5$ & $5,526.0$ & 324PRDX & 4.0 & 0.000000 & Algal limestone \\
\hline sld41-23-12aa & $5,526.0$ & $5,528.0$ & $324 \mathrm{PRDX}$ & 1.8 & 0.000000 & Algal limestone \\
\hline sld41-23-12a a & $5,528.0$ & $5,529.7$ & 324PRDX & 1.2 & 0.100000 & Algal limestone \\
\hline sld41-23-12aa & $5,530.0$ & $5,531.2$ & 324PRDX & 0.8 & 2.250000 & Algal limestone \\
\hline sld41-23-12aaa & $5,531.5$ & $5,533.4$ & $324 \mathrm{PRDX}$ & 1.2 & 0.450000 & Algal limestone \\
\hline $\operatorname{sld} 41-23-12 \mathrm{a} a$ & $5,533.7$ & $5,535.3$ & 324PRDX & 2.2 & 1.700000 & Algal limestone \\
\hline s $1 d 41-23-12$ aa & $5,535.6$ & $5,537.5$ & 324PRDX & 2.9 & 0.000000 & Algal limestone \\
\hline $\operatorname{sld} 41-23-12 \mathrm{aaa}$ & $5,537.8$ & $5,539.4$ & 324PRDX & 2.4 & 0.050000 & Algal limestone \\
\hline sld41-23-12a a a & $5,539.7$ & $5,541.4$ & 324PRDX & 3.3 & 0.100000 & Algal limestone \\
\hline sld41-23-12aaa & $5,541.7$ & $5,543.3$ & $324 \mathrm{PRDX}$ & 2.0 & 0.100000 & Algal limestone \\
\hline $\operatorname{sld} 41-23-12 \mathrm{a} a \mathrm{a}$ & $5,543.6$ & $5,545.3$ & 324PRDX & 8.2 & 0.750000 & Cherty limestone \\
\hline sld41-23-12aa & $5,545.6$ & $5,547.3$ & $324 \mathrm{PRDX}$ & 9.2 & 0.550000 & Cherty limestone \\
\hline s $1 d 41-23-12$ a a & $5,547.6$ & $5,549.2$ & 324PRDX & 13.5 & 2.550000 & Cherty limestone \\
\hline sld41-23-12aa & $5,549.5$ & $5,551.3$ & $324 \mathrm{PRDX}$ & 20.0 & 5.800000 & Cherty limestone \\
\hline s1d41-23-12aaa & $5,551.6$ & $5,553.3$ & $324 \mathrm{PRDX}$ & 16.9 & 3.400000 & Cherty limestone \\
\hline sld41-23-12a a a & $5,553.6$ & $5,554.6$ & 324PRDX & 18.4 & 2.800000 & Cherty limestone \\
\hline s $1 d 41-23-12$ aaa & $5,554.9$ & $5,556.3$ & 324PRDX & 2.9 & 0.150000 & Shaly limestone \\
\hline sld41-23-12 aаa & $5,556.6$ & $5,558.4$ & 324PRDX & 1.4 & 0.100000 & Shaly limestone \\
\hline sld41-23-12aa & $5,558.7$ & $5,560.5$ & 324PRDX & 1.1 & 0.600000 & Shaly limestone \\
\hline s $1 d 41-23-12$ aa & $5,560.8$ & $5,562.3$ & $324 \mathrm{PRDX}$ & 0.9 & 0.050000 & Shaly limestone \\
\hline
\end{tabular}


Table 4.--Laboratory-determined porosity and permeability--Continued

\begin{tabular}{|c|c|c|c|c|c|c|}
\hline Site & $\begin{array}{l}\text { Depth to } \\
\text { top } \\
\text { (feet) }\end{array}$ & $\begin{array}{l}\text { Depth to } \\
\text { bottom } \\
\text { (feet) }\end{array}$ & $\begin{array}{l}\text { Forma- } \\
\text { tion }\end{array}$ & $\begin{array}{l}\text { Porosity } \\
\text { (percent) }\end{array}$ & $\begin{array}{c}\text { Perme- } \\
\text { ability } \\
\text { (milli- } \\
\text { darcies) }\end{array}$ & Lithology \\
\hline $\begin{array}{l}\text { sld41-23-12aaa } \\
\text { sld41-23-12aaa } \\
\text { sld41-23-12aaa } \\
\text { sld41-23-12aaa } \\
\text { sld41-23-12aaa }\end{array}$ & $\begin{array}{l}5,562.6 \\
5,564.6 \\
5,566.7 \\
5,569.6 \\
5,571.3\end{array}$ & $\begin{array}{l}5,564.3 \\
5,566.4 \\
5,569.3 \\
5,571.0 \\
5,573.5\end{array}$ & $\begin{array}{l}\text { 324PRDX } \\
324 \mathrm{PRDX} \\
324 \mathrm{PRDX} \\
324 \mathrm{PRDX} \\
324 \mathrm{PRDX}\end{array}$ & $\begin{array}{r}2.9 \\
8.6 \\
7.4 \\
8.6 \\
12.7\end{array}$ & $\begin{array}{r}0.500000 \\
2.400000 \\
36.600000 \\
89.500000 \\
585.000000\end{array}$ & $\begin{array}{l}\text { Shaly limestone } \\
\text { Cherty limestone } \\
\text { Cherty limestone } \\
\text { Cherty limestone } \\
\text { Cherty limestone }\end{array}$ \\
\hline $\begin{array}{l}\text { sld41-23-12aaa } \\
\text { sld41-23-12aaa } \\
\text { sld41-23-12aaa } \\
\text { sld41-23-12aaa } \\
\text { sld41-23-12aaa }\end{array}$ & $\begin{array}{l}5,573.8 \\
5,576.2 \\
5,577.7 \\
5,579.4 \\
5,581.5\end{array}$ & $\begin{array}{l}5,576.2 \\
5,577.7 \\
5,579.4 \\
5,581.5 \\
5,583.5\end{array}$ & $\begin{array}{l}\text { 324PRDX } \\
324 \mathrm{PRDX} \\
324 \mathrm{PRDX} \\
324 \mathrm{PRDX} \\
324 \mathrm{PRDX}\end{array}$ & $\begin{array}{r}11.8 \\
14.4 \\
14.0 \\
9.8 \\
14.2\end{array}$ & $\begin{array}{l}89.666666 \\
92.500000 \\
11.150000 \\
53.500000 \\
10.850000\end{array}$ & $\begin{array}{l}\text { Cherty limestone } \\
\text { Cherty limestone } \\
\text { Cherty limestone } \\
\text { Cherty limestone } \\
\text { Cherty limestone }\end{array}$ \\
\hline $\begin{array}{l}\text { sld41-23-12aaa } \\
\text { sld41-23-12aaa } \\
\text { sld41-23-12aaa } \\
\text { sld41-23-12aaa } \\
\text { sld41-23-12aaa }\end{array}$ & $\begin{array}{l}5,583 \cdot 5 \\
5,585 \cdot 3 \\
5,587 \cdot 2 \\
5,589 \cdot 3 \\
5,591 \cdot 3\end{array}$ & $\begin{array}{l}5,585 \cdot 3 \\
5,587 \cdot 2 \\
5,589 \cdot 3 \\
5,591 \cdot 3 \\
5,593 \cdot 6\end{array}$ & $\begin{array}{l}324 \mathrm{PRDX} \\
324 \mathrm{PRDX} \\
324 \mathrm{PRDX} \\
324 \mathrm{PRDX} \\
324 \mathrm{PRDX}\end{array}$ & $\begin{array}{r}13.6 \\
10.6 \\
13.5 \\
13.9 \\
9.3\end{array}$ & $\begin{array}{r}7.250000 \\
2.850000 \\
10.050000 \\
12.600000 \\
13.200000\end{array}$ & $\begin{array}{l}\text { Cherty limestone } \\
\text { Cherty limestone } \\
\text { Cherty limestone } \\
\text { Cherty limestone } \\
\text { Cherty limestone }\end{array}$ \\
\hline $\begin{array}{l}\text { sld41-23-12aaa } \\
\text { sld41-23-12aaa } \\
\text { sld41-23-12aaa } \\
\text { sld41-23-12aa } \\
\text { sld41-23-12aaa }\end{array}$ & $\begin{array}{l}5,593.6 \\
5,595 \cdot 1 \\
5,597.4 \\
5,598.6 \\
5,601.4\end{array}$ & $\begin{array}{l}5,595.1 \\
5,597.4 \\
5,598.6 \\
5,601.4 \\
5,603.8\end{array}$ & $\begin{array}{l}324 \mathrm{PRDX} \\
324 \mathrm{PRDX} \\
324 \mathrm{PRDX} \\
324 \mathrm{PRDX} \\
324 \mathrm{PRDX}\end{array}$ & $\begin{array}{l}8.8 \\
3.9 \\
5.6 \\
5.6 \\
6.7\end{array}$ & $\begin{array}{r}9.000000 \\
0.600000 \\
5.300000 \\
9.100000 \\
52.050000\end{array}$ & $\begin{array}{l}\text { Cherty limestone } \\
\text { Cherty limestone } \\
\text { Cherty limestone } \\
\text { Cherty limestone } \\
\text { Cherty limestone }\end{array}$ \\
\hline $\begin{array}{l}\text { sld41-23-12aaa } \\
\text { sld41-23-12aaa } \\
\text { sld41-23-12aaa } \\
\text { sld41-23-12aaa } \\
\text { sld41-23-12aaa }\end{array}$ & $\begin{array}{l}5,603 \cdot 8 \\
5,605 \cdot 5 \\
5,607 \cdot 6 \\
5,609 \cdot 6 \\
5,611 \cdot 3\end{array}$ & $\begin{array}{l}5,605 \cdot 8 \\
5,607 \cdot 6 \\
5,609 \cdot 6 \\
5,611.3 \\
5,613 \cdot 5\end{array}$ & $\begin{array}{l}\text { 324PRDX } \\
324 \mathrm{PRDX} \\
324 \mathrm{PRDX} \\
324 \mathrm{PRDX} \\
324 \mathrm{PRDX}\end{array}$ & $\begin{array}{l}7.2 \\
6.8 \\
7.2 \\
5.7 \\
4.4\end{array}$ & $\begin{array}{r}11.250000 \\
74.550000 \\
3.500000 \\
72.000000 \\
4.650000\end{array}$ & $\begin{array}{l}\text { Cherty limestone } \\
\text { Cherty limestone } \\
\text { Cherty limestone } \\
\text { Cherty limestone } \\
\text { Cherty limestone }\end{array}$ \\
\hline $\begin{array}{l}\text { sld41-23-12aaa } \\
\text { sld41-23-12aaa } \\
\text { sld41-23-12aaa } \\
\text { sld41-23-12aaa } \\
\text { sld41-23-12aaa }\end{array}$ & $\begin{array}{l}5,613 \cdot 5 \\
5,615 \cdot 2 \\
5,617 \cdot 6 \\
5,619 \cdot 5 \\
5,621 \cdot 6\end{array}$ & $\begin{array}{l}5,615 \cdot 2 \\
5,617.6 \\
5,619 \cdot 5 \\
5,621.6 \\
5,623.3\end{array}$ & $\begin{array}{l}\text { 324PRDX } \\
324 \mathrm{PRDX} \\
324 \mathrm{PRDX} \\
324 \mathrm{PRDX} \\
324 \mathrm{PRDX}\end{array}$ & $\begin{array}{l}5.3 \\
1.1 \\
0.8 \\
6.5 \\
5.3\end{array}$ & $\begin{array}{r}23.250000 \\
18.100000 \\
3.950000 \\
0.050000 \\
0.100000\end{array}$ & $\begin{array}{l}\text { Cherty limestone } \\
\text { Shale } \\
\text { Shale } \\
\text { Cherty limestone } \\
\text { Cherty limestone }\end{array}$ \\
\hline $\begin{array}{l}\text { sld41-23-12aaa } \\
\text { sld41-23-12aaa } \\
\text { sld41-23-12aaa } \\
\text { sld41-23-12aaa } \\
\text { sld41-23-12aaa }\end{array}$ & $\begin{array}{l}5,623 \cdot 3 \\
5,625 \cdot 2 \\
5,627 \cdot 0 \\
5,629 \cdot 2 \\
5,631 \cdot 3\end{array}$ & $\begin{array}{l}5,625 \cdot 2 \\
5,627 \cdot 0 \\
5,629 \cdot 2 \\
5,631 \cdot 3 \\
5,633 \cdot 6\end{array}$ & $\begin{array}{l}324 \mathrm{PRDX} \\
324 \mathrm{PRDX} \\
324 \mathrm{PRDX} \\
324 \mathrm{PRDX} \\
324 \mathrm{PRDX}\end{array}$ & $\begin{array}{r}7.9 \\
8.8 \\
1.9 \\
5.4 \\
11.7\end{array}$ & $\begin{array}{l}1.000000 \\
2.200000 \\
1.700000 \\
0.050000 \\
0.100000\end{array}$ & $\begin{array}{l}\text { Cherty limestone } \\
\text { Cherty limestone } \\
\text { Siltstone } \\
\text { Cherty limestone } \\
\text { Cherty limestone }\end{array}$ \\
\hline $\begin{array}{l}\text { sld41-23-12aaa } \\
\text { sld41-23-12aaa } \\
\text { sld41-23-12aaa } \\
\text { sld41-23-12aaa } \\
\text { sld41-23-12aaa }\end{array}$ & $\begin{array}{l}5,633 \cdot 6 \\
5,635 \cdot 5 \\
5,637 \cdot 4 \\
5,639 \cdot 4 \\
5,641 \cdot 5\end{array}$ & $\begin{array}{l}5,635 \cdot 5 \\
5,637 \cdot 4 \\
5,639 \cdot 4 \\
5,641 \cdot 5 \\
5,643 \cdot 5\end{array}$ & $\begin{array}{l}\text { 324PRDX } \\
324 \mathrm{PRDX} \\
\text { 324PRDX } \\
\text { 324PRDX } \\
\text { 324PRDX }\end{array}$ & $\begin{array}{r}14.0 \\
12.5 \\
1.5 \\
1.7 \\
1.9\end{array}$ & $\begin{array}{l}0.100000 \\
0.300000 \\
0.900000 \\
0.100000 \\
0.000000\end{array}$ & $\begin{array}{l}\text { Cherty limestone } \\
\text { Cherty limestone } \\
\text { Siltstone } \\
\text { Siltstone } \\
\text { Siltstone }\end{array}$ \\
\hline $\begin{array}{l}\text { sld41-23-12aaa } \\
\text { sld41-23-12aaa } \\
\text { sld41-23-12aaa } \\
\text { sld41-23-12a aa } \\
\text { s1d41-23-12aaa }\end{array}$ & $\begin{array}{l}5,643 \cdot 5 \\
5,645 \cdot 6 \\
5,647 \cdot 4 \\
5,649.7 \\
5,652 \cdot 1\end{array}$ & $\begin{array}{l}5,645 \cdot 6 \\
5,647 \cdot 4 \\
5,649 \cdot 7 \\
5,652 \cdot 1 \\
5,654.8\end{array}$ & $\begin{array}{l}324 \mathrm{PRDX} \\
324 \mathrm{PRDX} \\
324 \mathrm{PRDX} \\
324 \mathrm{PRDX} \\
324 \mathrm{PRDX}\end{array}$ & $\begin{array}{r}7.6 \\
12.7 \\
11.7 \\
10.6 \\
6.6 \\
\end{array}$ & $\begin{array}{l}2.300000 \\
2.550000 \\
3.000000 \\
0.366666 \\
0.250000 \\
\end{array}$ & $\begin{array}{l}\text { Cherty limestone } \\
\text { Cherty limestone } \\
\text { Cherty limestone } \\
\text { Cherty limestone } \\
\text { Cherty limestone }\end{array}$ \\
\hline SITE AVERAGE--- & $-\cdots$ & $--n-\infty-1$ & AVERAGE- - & $\frac{7.3}{7.3}$ & $\frac{16.432157}{16.432157}$ & \\
\hline
\end{tabular}


Table 4.--Laboratory-determined porosity and permeability--Continued

\begin{tabular}{|c|c|c|c|c|c|c|}
\hline Site & $\begin{array}{l}\text { Depth to } \\
\text { top } \\
\text { (feet) }\end{array}$ & $\begin{array}{l}\text { Depth to } \\
\text { bottom } \\
\text { (feet) }\end{array}$ & $\begin{array}{c}\text { Forma- } \\
\text { tion }\end{array}$ & $\begin{array}{l}\text { Porosity } \\
\text { (percent) }\end{array}$ & $\begin{array}{l}\text { Perme- } \\
\text { ability } \\
\text { (milli- } \\
\text { darcies) }\end{array}$ & Lithology \\
\hline s $1 d 41-24-18$ acd & $5,510.0$ & $5,511.0$ & 324PRDX & 0.9 & 0.020000 & Limestone \\
\hline s $1 d 41-24-18 a c d$ & $5,511.0$ & $5,512.0$ & 324PRDX & 1.2 & 0.050000 & Limestone \\
\hline sld41-24-18acd & $5,512.0$ & $5,513.0$ & 324PRDX & 0.6 & 0.010000 & Limy sandstone \\
\hline sld41-24-18acd & $5,513.0$ & $5,514.0$ & 324PRDX & 0.1 & 0.130000 & Sandstone \\
\hline s $1 d 41-24-18$ acd & $5,514.0$ & $5,515.0$ & $324 \mathrm{PRDX}$ & 0.7 & 0.020000 & Limestone \\
\hline s $1 d 41-24-18 a c d$ & $5,515.0$ & $5,516.0$ & 324PRDX & 0.1 & 0.040000 & Sandy limestone \\
\hline sld41-24-18acd & $5,516.0$ & $5,517.0$ & 324PRDX & 0.1 & 1.160000 & Sandstone \\
\hline s $1 d 41-24-18$ acd & $5,517.0$ & $5,518.0$ & $324 \mathrm{PRDX}$ & 0.1 & 1.400000 & Sands tone \\
\hline sld $41-24-18$ acd & $5,518.0$ & $5,519.0$ & 324PRDX & 0.3 & 0.070000 & Limestone \\
\hline s $1 d 41-24-18 a c d$ & $5,519.0$ & $5,520.0$ & 324PRDX & 0.4 & 0.040000 & Limestone \\
\hline s $1 d 41-24-18$ acd & $5,520.0$ & $5,520.5$ & 324PRDX & 0.6 & 0.020000 & Limestone \\
\hline s $1 d 41-24-18 a$ cd & $5,522.5$ & $5,524.0$ & $324 \mathrm{PRDX}$ & 1.9 & 0.010000 & Sandy limestone \\
\hline s $1 d 41-24-18 a c d$ & $5,525.0$ & $5,526.0$ & 324PRDX & 1.7 & 0.010000 & Sandstone \\
\hline s $1 d 41-24-18 a c d$ & $5,526.0$ & $5,527.0$ & 324PRDX & 1.3 & 0.010000 & Sands tone \\
\hline s $1 d 41-24-18 a c d$ & $5,527.0$ & $5,528.0$ & 324PRDX & 0.2 & 0.010000 & Sandstone \\
\hline s $1 d 41-24-18 a c d$ & $5,528.0$ & $5,529.0$ & 324PRDX & 0.2 & 0.020000 & Limy sandstone \\
\hline s $1 d 41-24-18 a c d$ & $5,529.0$ & $5,530.0$ & $324 \mathrm{PRDX}$ & 0.7 & 0.010000 & Limy sandstone \\
\hline s $1 d 41-24-18$ acd & $5,530.0$ & $5,531.0$ & $324 P R D X$ & 1.8 & 0.010000 & Limy sandstone \\
\hline s $1 d 41-24-18$ acd & $5,531.0$ & $5,532.0$ & 324PRDX & 2.0 & 0.010000 & Sandy limestone \\
\hline s $1 d 41-24-18 a c d$ & $5,535.0$ & $5,536.0$ & 324PRDX & 0.1 & 0.010000 & Fractured sandy limestone \\
\hline s $1 d 41-24-18 a c d$ & $5,536.0$ & $5,537.0$ & $324 \mathrm{PRDX}$ & 0.2 & 0.130000 & Sands tone \\
\hline $\operatorname{sld} 41-24-18 \mathrm{acd}$ & $5,537.0$ & $5,538.0$ & $324 \mathrm{PRDX}$ & 0.1 & 0.140000 & Sandstone \\
\hline s $1 d 41-24-18$ acd & $5,538.0$ & $5,539.0$ & 324PRDX & 0.2 & 0.120000 & Sandstone \\
\hline s $1 d 41-24-18 a c d$ & $5,539.0$ & $5,540.0$ & $324 \mathrm{PRDX}$ & 0.3 & 0.010000 & Limestone \\
\hline s $1 d 41-24-18$ acd & $5,540.0$ & $5,541.0$ & 324PRDX & 0.1 & 0.010000 & Limy dolomite \\
\hline s $1 d 41-24-18 a c d$ & $5,541.0$ & $5,542.0$ & $324 \mathrm{PRDX}$ & 0.1 & 0.010000 & Sands tone \\
\hline s $1 d 41-24-18 a c d$ & $5,542.0$ & $5,543.0$ & 324PRDX & 0.1 & 0.010000 & Sandstone \\
\hline s $1 d 41-24-18$ acd & $5,543.0$ & $5,544.0$ & 324PRDX & 0.1 & 0.030000 & Sands tone \\
\hline s1d41-24-18acd & $5,544.0$ & $5,545.0$ & $324 \mathrm{PRDX}$ & 0.1 & 0.010000 & Sands tone \\
\hline s $1 d 41-24-18 a c d$ & $5,545.0$ & $5,546.0$ & $324 \mathrm{PRDX}$ & 0.1 & 0.010000 & Sandstone \\
\hline s $1 d 41-24-18 a c d$ & $5,546.0$ & $5,547.0$ & 324PRDX & 0.1 & 0.010000 & Sandstone \\
\hline s $1 d 41-24-18$ a cd & $5,547.0$ & $5,548.0$ & $324 \mathrm{PRDX}$ & 0.1 & 0.600000 & Shaly sandstone \\
\hline s $1 d 41-24-18 a c d$ & $5,548.0$ & $5,549.0$ & 324PRDX & 0.2 & 2.880000 & Sandstone \\
\hline s $1 d 41-24-18 a c d$ & $5,549.0$ & $5,550.0$ & 324PRDX & 0.1 & 3.920000 & Sandstone \\
\hline s1d41-24-18acd & $5,550.0$ & $5,551.0$ & $324 \mathrm{PRDX}$ & 0.2 & 0.990000 & Fractured sandstone \\
\hline s $1 d 41-24-18$ acd & $5,551.0$ & $5,552.0$ & 324PRDX & 0.1 & 0.010000 & Sandstone \\
\hline s $1 d 41-24-18 a c d$ & $5,552.0$ & $5,553.0$ & 324PRDX & 0.1 & 0.010000 & Sandstone \\
\hline s $1 d 41-24-18 a c d$ & $5,553.0$ & $5,554.0$ & 324PRDX & 0.1 & 0.010000 & Sandstone \\
\hline s $1 d 41-24-18 a c d$ & $5,554.0$ & $5,555.0$ & 324PRDX & 0.1 & 0.010000 & Sands tone \\
\hline s $1 d 41-24-18$ acd & $5,555.0$ & $5,556.0$ & $324 \mathrm{PRDX}$ & 0.1 & 0.010000 & Sandstone \\
\hline s $1 d 41-24-18 a c d$ & $5,556.0$ & $5,557.0$ & 324PRDX & 0.2 & 0.040000 & Sandstone \\
\hline s $1 d 41-24-18 a c d$ & $5,557.0$ & $5,558.0$ & 324PRDX & 0.1 & 0.010000 & Shaly sandstone \\
\hline sld41-24-18acd & $5,558.0$ & $5,559.0$ & 324PRDX & 0.1 & 0.010000 & Shaly sandstone \\
\hline \multirow[t]{2}{*}{ s $1 d 41-24-18$ acd } & $5,559.0$ & $5,560.0$ & $324 \mathrm{PRDX}$ & 0.1 & 0.010000 & Shaly sandstone \\
\hline & & & AVERAGE-- & 0.4 & 0.274091 & \\
\hline SITE AVERAGE- & & & $-\ldots-\ldots$ & 0.4 & 0.274091 & \\
\hline
\end{tabular}


Table 4.--Laboratory-determined porosity and permeability--Continued

\begin{tabular}{|c|c|c|c|c|c|c|}
\hline Site & $\begin{array}{l}\text { Depth to } \\
\text { top } \\
\text { (feet) }\end{array}$ & $\begin{array}{l}\text { Depth to } \\
\text { bottom } \\
\text { (feet) }\end{array}$ & $\begin{array}{c}\text { Forma- } \\
\text { tion }\end{array}$ & $\begin{array}{l}\text { Porosity } \\
\text { (percent) }\end{array}$ & $\begin{array}{l}\text { Perme- } \\
\text { ability } \\
\text { (milli- } \\
\text { darcies) }\end{array}$ & Lithology \\
\hline $\operatorname{sid} 41-24-22 \mathrm{ccd}$ & $5,303.5$ & $5,304.7$ & $321 \mathrm{HKTL}$ & 0.9 & 0.000000 & Limestone \\
\hline $\operatorname{sid} 41-24-22 \mathrm{ccd}$ & $5,304.7$ & $5,306.0$ & $321 \mathrm{HKTL}$ & 1.8 & 0.000000 & Limestone \\
\hline $\operatorname{sid} 41-24-22 \operatorname{ccd}$ & $5,361.0$ & $5,363.0$ & $321 \mathrm{HKTL}$ & 2.0 & 0.100000 & Shaly limestone \\
\hline s $1 d 41-24-22 c c d$ & $5,363.0$ & $5,365.0$ & 321HKTL & 2.5 & 0.200000 & Shaly limestone \\
\hline $\operatorname{sid} 41-24-22 \mathrm{ccd}$ & $5,365.0$ & $5,367.0$ & $321 \mathrm{HKTL}$ & 1.3 & 3.000000 & Limestone \\
\hline s1d41-24-22ccd & $5,367.0$ & $5,369.0$ & 321HKTL & 0.4 & 1.900000 & Limestone \\
\hline $\operatorname{s} 1 d 41-24-22 \operatorname{ccd}$ & $5,369.0$ & $5,371.0$ & 321HKTL & 0.5 & 0.000000 & Limestone \\
\hline $\operatorname{sid} 41-24-22 \mathrm{ccd}$ & $5,371.0$ & $5,373.0$ & $321 \mathrm{HKTL}$ & 1.5 & 0.500000 & Limestone \\
\hline $\operatorname{s} 1 d 41-24-22 c c d$ & $5,373.0$ & $5,375.0$ & 321HKTL & 6.7 & 0.900000 & Limestone \\
\hline s $1 d 41-24-22 \mathrm{ccd}$ & $5,375.0$ & $5,377.0$ & $321 \mathrm{HKTL}$ & 13.0 & 3.500000 & Limestone \\
\hline s $1 d 41-24-22 c c d$ & $5,377.0$ & $5,379.0$ & 321HKTL & 7.6 & 2.000000 & Limestone \\
\hline s $1 d 41-24-22 c c d$ & $5,379.0$ & $5,381.0$ & $321 \mathrm{HKTL}$ & 4.4 & 0.100000 & Limestone \\
\hline s $1 d 41-24-22 \operatorname{ccd}$ & $5,381.0$ & $5,383.0$ & $321 \mathrm{HKTL}$ & 1.4 & 0.000000 & Limestone \\
\hline $\operatorname{s} 1 d 41-24-22 c c d$ & $5,407.0$ & $5,409.0$ & 321HKTL & 0.5 & 0.000000 & Shale \\
\hline s $1 d 41-24-22 c c d$ & $5,412.0$ & $5,414.0$ & 321HKTL & 1.0 & 0.000000 & Shale \\
\hline \multirow[t]{2}{*}{$\operatorname{sid} 41-24-22 c c d$} & \multirow[t]{2}{*}{$5,416.0$} & \multirow[t]{2}{*}{$5,418.0$} & 321HKTL & 0.8 & 0.000000 & \multirow[t]{2}{*}{ Shale } \\
\hline & & & AVERAGE-- & 2.9 & 0.762500 & \\
\hline sld41-24-22ccd & $5,443.0$ & $5,445.0$ & 324PRDX & 1.0 & 0.000000 & Shaly dolomite and limestone \\
\hline $\operatorname{sid} 41-24-22 \mathrm{ccd}$ & $5,445.0$ & $5,447.0$ & 324PRDX & 0.8 & 0.000000 & Shaly limestone \\
\hline s $1 d 41-24-22 \operatorname{ccd}$ & $5,447.0$ & $5,449.0$ & 324PRDX & 0.9 & 0.000000 & Shaly limestone \\
\hline $\operatorname{s} 1 d 41-24-22 c c d$ & $5,449.0$ & $5,451.0$ & $324 \mathrm{PRDX}$ & 1.1 & 0.000000 & Shaly limestone \\
\hline $\operatorname{sid} 41-24-22 \mathrm{ccd}$ & $5,451.0$ & $5,453.0$ & 324PRDX & 0.8 & 2.700000 & Shaly limestone \\
\hline $\operatorname{sid} 41-24-22 \mathrm{ccd}$ & $5,453.0$ & $5,455.0$ & 324PRDX & 0.7 & 0.100000 & Sha1y limestone \\
\hline s $1 d 41-24-22 c c d$ & $5,455.0$ & $5,457.0$ & $324 \mathrm{PRDX}$ & 0.7 & 0.800000 & Shaly limestone \\
\hline s $1 d 41-24-22 \mathrm{ccd}$ & $5,457.0$ & $5,459.0$ & $324 \mathrm{PRDX}$ & 0.6 & 6.600000 & Shaly limestone \\
\hline $\operatorname{sid} 41-24-22 \mathrm{ccd}$ & $5,459.0$ & $5,461.0$ & $324 \mathrm{PRDX}$ & 0.9 & 0.000000 & Shaly limestone \\
\hline s $1 d 41-24-22 c c d$ & $5,461.0$ & $5,463.0$ & 324PRDX & 0.6 & 0.000000 & Limestone \\
\hline $\mathrm{s} 1 \mathrm{~d} 41-24-22 \mathrm{ccd}$ & $5,463.0$ & $5,465.0$ & $324 \mathrm{PRDX}$ & 1.0 & 0.000000 & Limestone \\
\hline $\operatorname{sid} 41-24-22 \mathrm{ccd}$ & $5,465.0$ & $5,467.0$ & 324PRDX & 1.1 & 0.000000 & Shaly limestone \\
\hline s $1 d 41-24-22 c c d$ & $5,467.0$ & $5,469.0$ & 324PRDX & 1.7 & 0.000000 & Shaly limestone \\
\hline $\operatorname{sid} 41-24-22 \mathrm{ccd}$ & $5,469.0$ & $5,471.0$ & $324 \mathrm{PRDX}$ & 1.3 & 0.000000 & Shaly limestone \\
\hline $\operatorname{sid} 41-24-22 \mathrm{ccd}$ & $5,475.0$ & $5,476.8$ & 324PRDX & 0.9 & 0.400000 & Limestone \\
\hline s $1 d 41-24-22 \mathrm{ccd}$ & $5,476.8$ & $5,478.5$ & 324PRDX & 1.4 & 0.000000 & Limestone \\
\hline $\operatorname{sld} 41-24-22 \mathrm{ccd}$ & $5,478.5$ & $5,480.8$ & $324 \mathrm{PRDX}$ & 1.3 & 0.000000 & Limestone \\
\hline $\operatorname{s} 1 d 41-24-22 c c d$ & $5,480.8$ & $5,482.5$ & $324 \mathrm{PRDX}$ & 2.2 & 0.000000 & Limestone \\
\hline $\operatorname{s} 1 d 41-24-22 \mathrm{ccd}$ & $5,482.5$ & $5,484.5$ & 324PRDX & 3.7 & 0.000000 & Limestone \\
\hline $\operatorname{sid} 41-24-22 \operatorname{ccd}$ & $5,484.5$ & $5,486.4$ & 324PRDX & 2.5 & 0.000000 & Limestone \\
\hline s $1 d 41-24-22 \mathrm{ccd}$ & $5,486.4$ & $5,488.2$ & $324 \mathrm{PRDX}$ & 2.0 & 0.000000 & Limestone \\
\hline s $1 d 41-24-22 c c d$ & $5,488.2$ & $5,490.0$ & $324 \mathrm{PRDX}$ & 1.9 & 0.000000 & Limestone \\
\hline $\operatorname{s} 1 d 41-24-22 \mathrm{ccd}$ & $5,490.0$ & $5,492.0$ & 324PRDX & 1.4 & 0.000000 & Anhydrite \\
\hline s ld $41-24-22 \mathrm{ccd}$ & $5,492.0$ & $5,493.8$ & $324 \mathrm{PRDX}$ & 0.5 & 0.000000 & Anhydrite \\
\hline s $1 d 41-24-22 \mathrm{ccd}$ & $5,493.8$ & $5,495.5$ & 324PRDX & 0.2 & 0.000000 & Anhydrite \\
\hline $\operatorname{sid} 41-24-22 \mathrm{ccd}$ & $5,495.5$ & $5,497.2$ & $324 \mathrm{PRDX}$ & 0.2 & 0.000000 & Anhydrite \\
\hline $\operatorname{sid} 41-24-22 \operatorname{ccd}$ & $5,497.2$ & $5,499.1$ & 324PRDX & 0.5 & 0.000000 & Limestone \\
\hline $\operatorname{sid} 41-24-22 \mathrm{ccd}$ & $5,499.1$ & $5,501.0$ & $324 \mathrm{PRDX}$ & 0.8 & 0.000000 & Limestone \\
\hline sld41-24-22ccd & $5,501.0$ & $5,503.0$ & $324 \mathrm{PRDX}$ & 0.5 & 0.000000 & Limestone \\
\hline sld $41-24-22 \mathrm{ccd}$ & $5,503.0$ & $5,504.5$ & $324 \mathrm{PRDX}$ & 0.6 & 0.000000 & Anhydritic dolomite \\
\hline
\end{tabular}


Table 4.--Laboratory-determined porosity and permeability--Continued

\begin{tabular}{|c|c|c|c|c|c|c|}
\hline Site & $\begin{array}{l}\text { Depth to } \\
\text { top } \\
\text { (feet) }\end{array}$ & $\begin{array}{l}\text { Depth to } \\
\text { bottom } \\
\text { (feet) }\end{array}$ & $\begin{array}{c}\text { Forma- } \\
\text { tion }\end{array}$ & $\begin{array}{l}\text { Porosity } \\
\text { (percent) }\end{array}$ & $\begin{array}{l}\text { Perme- } \\
\text { ability } \\
\text { (milli- } \\
\text { darcies) }\end{array}$ & Lithology \\
\hline $\begin{array}{l}\text { sld41-24-22ccd } \\
\text { sld41-24-22ccd } \\
\text { sld41-24-22ccd } \\
\text { sld41-24-22ccd } \\
\text { sld41-24-22ccd }\end{array}$ & $\begin{array}{l}5,504 \cdot 5 \\
5,506.7 \\
5,508 \cdot 7 \\
5,510.3 \\
5,511 \cdot 3\end{array}$ & $\begin{array}{l}5,506.7 \\
5,508.7 \\
5,510.3 \\
5,511.3 \\
5,513.5\end{array}$ & $\begin{array}{l}324 \mathrm{PRDX} \\
324 \mathrm{PRDX} \\
324 \mathrm{PRDX} \\
324 \mathrm{PRDX} \\
324 \mathrm{PRDX}\end{array}$ & $\begin{array}{r}0.5 \\
0.6 \\
0.6 \\
1.2 \\
10.9\end{array}$ & $\begin{array}{l}0.000000 \\
0.000000 \\
0.000000 \\
0.000000 \\
0.400000\end{array}$ & $\begin{array}{l}\text { Shaly limestone } \\
\text { Shaly limestone } \\
\text { Shaly limestone } \\
\text { Shaly limestone } \\
\text { Fractured limestone }\end{array}$ \\
\hline $\begin{array}{l}\text { sld4 } 1-24-22 c c d \\
\text { sld41-24-22ccd } \\
\text { sld41-24-22ccd } \\
\text { sld4 } 1-24-22 c c d \\
\text { sld4 } 1-24-22 c c d\end{array}$ & $\begin{array}{l}5,513.5 \\
5,515.0 \\
5,516.8 \\
5,531.0 \\
5,533.0\end{array}$ & $\begin{array}{l}5,515.0 \\
5,516.8 \\
5,517.8 \\
5,533.0 \\
5,535.0\end{array}$ & $\begin{array}{l}\text { 324PRDX } \\
324 \mathrm{PRDX} \\
324 \mathrm{PRDX} \\
324 \mathrm{PRDX} \\
324 \mathrm{PRDX}\end{array}$ & $\begin{array}{l}2.5 \\
2.9 \\
3.3 \\
5.9 \\
1.1\end{array}$ & $\begin{array}{l}0.000000 \\
0.000000 \\
0.000000 \\
0.100000 \\
0.200000\end{array}$ & $\begin{array}{l}\text { Limes tone } \\
\text { Limestone } \\
\text { Limestone } \\
\text { Dolomite } \\
\text { Limestone }\end{array}$ \\
\hline $\begin{array}{l}\text { s1d41-24-22ccd } \\
\text { sld41-24-22ccd } \\
\text { sld41-24-22ccd } \\
\text { sld41-24-22ccd } \\
\text { sld41-24-22ccd }\end{array}$ & $\begin{array}{l}5,535.0 \\
5,537.0 \\
5,539.0 \\
5,540.5 \\
5,542.1\end{array}$ & $\begin{array}{l}5,537.0 \\
5,539.0 \\
5,540.5 \\
5,542.1 \\
5,544.0\end{array}$ & $\begin{array}{l}324 \mathrm{PRDX} \\
324 \mathrm{PRDX} \\
324 \mathrm{PRDX} \\
324 \mathrm{PRDX} \\
324 \mathrm{PRDX}\end{array}$ & $\begin{array}{l}2.7 \\
4.8 \\
4.0 \\
7.4 \\
5.2\end{array}$ & $\begin{array}{l}0.000000 \\
0.200000 \\
0.000000 \\
0.000000 \\
0.100000\end{array}$ & $\begin{array}{l}\text { Limestone } \\
\text { Dolomite } \\
\text { Dolomite } \\
\text { Dolomite } \\
\text { Dolomite }\end{array}$ \\
\hline $\begin{array}{l}\text { sld4 } 1-24-22 \operatorname{ccd} \\
\text { sld41-24-22ccd } \\
\text { sld4 } 1-24-22 c c d\end{array}$ & $\begin{array}{l}5,544.0 \\
5,545.0 \\
5,570.0\end{array}$ & $\begin{array}{l}5,545.0 \\
5,546.0 \\
5,572.0\end{array}$ & $\begin{array}{l}\text { 324PRDX } \\
324 \mathrm{PRDX} \\
324 \mathrm{PRDX}\end{array}$ & $\begin{array}{l}4.9 \\
2.0 \\
0.9 \\
\end{array}$ & $\begin{array}{l}0.300000 \\
0.200000 \\
0.000000 \\
\end{array}$ & $\begin{array}{l}\text { Dolomite } \\
\text { Shaly dolomite } \\
\text { Limestone }\end{array}$ \\
\hline SITE AVERAGE-- & $-0--0--$ & -----1 & AVERAGE-- & $\frac{2.0}{2.2}$ & $\frac{0.252083}{0.379687}$ & \\
\hline $\begin{array}{l}\text { sld41-24-34add } \\
\text { sld41-24-34add } \\
\text { sld41-24-34add } \\
\text { s1d41-24-34add } \\
\text { sld41-24-34add }\end{array}$ & $\begin{array}{l}5,634.0 \\
5,635.0 \\
5,636.0 \\
5,637.0 \\
5,638.0\end{array}$ & $\begin{array}{l}5,635.0 \\
5,636.0 \\
5,637.0 \\
5,638.0 \\
5,639.0\end{array}$ & $\begin{array}{l}\text { 324PRDX } \\
324 \mathrm{PRDX} \\
324 \mathrm{PRDX} \\
324 \mathrm{PRDX} \\
324 \mathrm{PRDX}\end{array}$ & $\begin{array}{r}24.2 \\
22.7 \\
9.5 \\
1.0 \\
0.7\end{array}$ & $\begin{array}{l}4.700000 \\
1.900000 \\
0.140000 \\
0.060000 \\
0.070000\end{array}$ & $\begin{array}{l}\text { Finely crystalline dolomite } \\
\text { Finely crystalline dolomite } \\
\text { Vuggy dolomite } \\
\text { Carbonaceous, limy dolomite } \\
\text { Carbonaceous, limy dolomite }\end{array}$ \\
\hline $\begin{array}{l}\text { sld41-24-34add } \\
\text { sld41-24-34add } \\
\text { sld41-24-34add } \\
\text { sld41-24-34add } \\
\text { sld4 } 1-24-34 \text { add }\end{array}$ & $\begin{array}{l}5,639.0 \\
5,640.0 \\
5,641.0 \\
5,642.0 \\
5,643.0\end{array}$ & $\begin{array}{l}5,640.0 \\
5,641.0 \\
5,642.0 \\
5,643.0 \\
5,644.0\end{array}$ & $\begin{array}{l}324 \mathrm{PRDX} \\
324 \mathrm{PRDX} \\
324 \mathrm{PRDX} \\
324 \mathrm{PRDX} \\
324 \mathrm{PRDX}\end{array}$ & $\begin{array}{r}1.7 \\
6.8 \\
12.9 \\
14.1 \\
14.3\end{array}$ & $\begin{array}{l}0.070000 \\
1.700000 \\
5.000000 \\
0.750000 \\
0.150000\end{array}$ & $\begin{array}{l}\text { Limy dolomite } \\
\text { Limy dolomite } \\
\text { Vuggy dolomite } \\
\text { Limy dolomite } \\
\text { Limy dolomite }\end{array}$ \\
\hline $\begin{array}{l}\text { sld41-24-34add } \\
\text { sld41-24-34add } \\
\text { sld41-24-34add } \\
\text { sld41-24-34add } \\
\text { sld41-24-34add }\end{array}$ & $\begin{array}{l}5,644.0 \\
5,645.0 \\
5,646.0 \\
5,647.0 \\
5,648.0\end{array}$ & $\begin{array}{l}5,645.0 \\
5,646.0 \\
5,647.0 \\
5,648.0 \\
5,649.0\end{array}$ & $\begin{array}{l}324 \mathrm{PRDX} \\
324 \mathrm{PRDX} \\
324 \mathrm{PRDX} \\
324 \mathrm{PRDX} \\
324 \mathrm{PRDX}\end{array}$ & $\begin{array}{l}12.2 \\
13.4 \\
14.7 \\
13.9 \\
13.9\end{array}$ & $\begin{array}{l}2.100000 \\
0.870000 \\
1.700000 \\
1.300000 \\
0.150000\end{array}$ & $\begin{array}{l}\text { Limy dolomite } \\
\text { Limy dolomite } \\
\text { Limy dolomite } \\
\text { Limy dolomite } \\
\text { Finely crystalline, limy dolomite }\end{array}$ \\
\hline $\begin{array}{l}\text { sld41-24-34add } \\
\text { sld41-24-34add } \\
\text { sld41-24-34add } \\
\text { sld41-24-34add } \\
\text { sld41-24-34add }\end{array}$ & $\begin{array}{l}5,649.0 \\
5,650.0 \\
5,651.0 \\
5,652.0 \\
5,653.0\end{array}$ & $\begin{array}{l}5,650.0 \\
5,651.0 \\
5,652.0 \\
5,653.0 \\
5,654.0\end{array}$ & $\begin{array}{l}\text { 324PRDX } \\
324 \mathrm{PRDX} \\
324 \mathrm{PRDX} \\
324 \mathrm{PRDX} \\
324 \mathrm{PRDX}\end{array}$ & $\begin{array}{r}0.5 \\
0.7 \\
3.4 \\
10.9 \\
8.0\end{array}$ & $\begin{array}{l}0.050000 \\
0.020000 \\
0.030000 \\
0.050000 \\
5.600000\end{array}$ & $\begin{array}{l}\text { Finely crystalline, limy dolomite } \\
\text { Finely crystalline, limy dolomite } \\
\text { Finely crystalline, limy dolomite } \\
\text { Finely crystalline, limy dolomite } \\
\text { Finely crystalline, limy dolomite }\end{array}$ \\
\hline $\begin{array}{l}\text { sld41-24-34add } \\
\text { sld41-24-34add } \\
\text { sld41-24-34add } \\
\text { sld41-24-34add } \\
\text { sld41-24-34add }\end{array}$ & $\begin{array}{l}5,654.0 \\
5,655.0 \\
5,656.0 \\
5,657.0 \\
5,658.0\end{array}$ & $\begin{array}{l}5,655.0 \\
5,656.0 \\
5,657.0 \\
5,658.0 \\
5,659.0\end{array}$ & $\begin{array}{l}\text { 324PRDX } \\
324 \mathrm{PRDX} \\
324 \mathrm{PRDX} \\
324 \mathrm{PRDX} \\
324 \mathrm{PRDX}\end{array}$ & $\begin{array}{r}6.0 \\
4.6 \\
1.6 \\
31.1 \\
23.8\end{array}$ & $\begin{array}{r}0.030000 \\
4.600000 \\
19.000000 \\
27.000000 \\
60.000000\end{array}$ & $\begin{array}{l}\text { Finely crystalline, limy dolomite } \\
\text { Fine-grained dolomite } \\
\text { Fine-grained dolomite } \\
\text { Vuggy, finely crystalline dolomite } \\
\text { Vuggy, finely crystalline dolomite }\end{array}$ \\
\hline $\begin{array}{l}\text { sld41-24-34add } \\
\text { sld41-24-34add } \\
\text { sld41-24-34add } \\
\text { s1d41-24-34add } \\
\text { sld41-24-34add }\end{array}$ & $\begin{array}{l}5,659.0 \\
5,660.0 \\
5,661.0 \\
5,662.0 \\
5,663.0\end{array}$ & $\begin{array}{l}5,660.0 \\
5,661.0 \\
5,662.0 \\
5,663.0 \\
5,664.0\end{array}$ & $\begin{array}{l}324 \mathrm{PRDX} \\
324 \mathrm{PRDX} \\
324 \mathrm{PRDX} \\
324 \mathrm{PRDX} \\
324 \mathrm{PRDX}\end{array}$ & $\begin{array}{r}15.8 \\
13.8 \\
22.3 \\
18.6 \\
7.0\end{array}$ & $\begin{array}{r}31.000000 \\
42.000000 \\
26.000000 \\
21.000000 \\
1.000000\end{array}$ & $\begin{array}{l}\text { Vuggy, finely crystalline dolomite } \\
\text { Vuggy, finely crystalline dolomite } \\
\text { Vuggy, finely crystalline dolomite } \\
\text { Vuggy, finely crystalline dolomite } \\
\text { Fine-grained, limy dolomite }\end{array}$ \\
\hline
\end{tabular}


Table 4.--Laboratory-determined porosity and permeability--Continued

\begin{tabular}{|c|c|c|c|c|c|c|}
\hline Site & $\begin{array}{l}\text { Depth to } \\
\text { top } \\
\text { (feet) }\end{array}$ & $\begin{array}{l}\text { Depth to } \\
\text { bottom } \\
\text { (feet) }\end{array}$ & $\begin{array}{c}\text { Forma- } \\
\text { tion }\end{array}$ & $\begin{array}{l}\text { Porosity } \\
\text { (percent) }\end{array}$ & $\begin{array}{l}\text { Perme- } \\
\text { ability } \\
\text { (milli- } \\
\text { darcies) }\end{array}$ & Lithology \\
\hline $\operatorname{sid} 41-24-34 a d d$ & $5,664.0$ & $5,665.0$ & $324 \mathrm{PRDX}$ & 1.2 & 0.130000 & Fine-grained, limy dolomite \\
\hline s1d41-24-34add & $5,665.0$ & $5,666.0$ & $324 \mathrm{PRDX}$ & 0.5 & 0.110000 & Carbonaceous, fine-grained dolomite \\
\hline s $1 d 41-24-34 a d d$ & $5,666.0$ & $5,667.0$ & $324 \mathrm{PRDX}$ & 1.0 & 0.860000 & Fine-grained dolomite \\
\hline s $1 d 41-24-34 a d d$ & $5,667.0$ & $5,668.0$ & $324 \mathrm{PRDX}$ & 0.3 & 0.130000 & Fine-grained dolomite \\
\hline s1d41-24-34add & $5,668.0$ & $5,669.0$ & $324 \mathrm{PRDX}$ & 0.4 & 0.100000 & Finely crystalline dolomite \\
\hline s $1 d 41-24-34 a d d$ & $5,669.0$ & $5,670.0$ & $324 \mathrm{PRDX}$ & 0.4 & 0.100000 & Finely crystalline dolomite \\
\hline s1d41-24-34add & $5,670.0$ & $5,671.0$ & 324PRDX & 0.5 & 0.030000 & Fine-grained dolomite \\
\hline s1d41-24-34add & $5,671.0$ & $5,672.0$ & $324 \mathrm{PRDX}$ & 0.3 & 0.050000 & Fine-grained dolomite \\
\hline s $1 d 41-24-34 a d d$ & $5,672.0$ & $5,673.0$ & 324PRDX & 0.3 & 0.080000 & Finely crystalline dolomite \\
\hline s $1 d 41-24-34 a d d$ & $5,673.0$ & $5,674.0$ & $324 \mathrm{PRDX}$ & 0.3 & 0.170000 & Finely crystalline dolomite \\
\hline s1d41-24-34add & $5,674.0$ & $5,675.0$ & 324PRDX & 0.3 & 0.090000 & Finely crystalline dolomite \\
\hline s1d41-24-34add & $5,675.0$ & $5,676.0$ & 324PRDX & 0.3 & 0.400000 & Finely crystalline dolomite \\
\hline s $1 d 41-24-34 a d d$ & $5,676.0$ & $5,677.0$ & 324PRDX & 0.3 & 0.030000 & Finely crystalline dolomite \\
\hline s1d41-24-34add & $5,677.0$ & $5,678.0$ & $324 \mathrm{PRDX}$ & 0.3 & 0.110000 & Finely crystalline dolomite \\
\hline s $1 d 41-24-34 a d d$ & $5,678.0$ & $5,679.0$ & 324PRDX & 0.3 & 0.080000 & Finely crystalline dolomite \\
\hline s $1 d 41-24-34$ add & $5,679.0$ & $5,680.0$ & $324 \mathrm{PRDX}$ & 0.7 & 0.050000 & Finely crystalline dolomite \\
\hline s $1 d 41-24-34 a d d$ & $5,680.0$ & $5,681.0$ & $324 \mathrm{PRDX}$ & 0.3 & 0.040000 & Finely crystalline dolomite \\
\hline s $1 d 41-24-34 a d d$ & $5,681.0$ & $5,682.0$ & $324 \mathrm{PRDX}$ & 0.3 & 0.040000 & Finely crystalline dolomite \\
\hline s1d41-24-34add & $5,682.0$ & $5,683.0$ & 324PRDX & 0.3 & 0.360000 & Finely crystalline dolomite \\
\hline s $1 d 41-24-34 a d d$ & $5,683.0$ & $5,684.0$ & $324 \mathrm{PRDX}$ & 1.6 & 0.090000 & Finely crystalline dolomite \\
\hline s1d41-24-34add & $5,684.0$ & $5,685.0$ & 324PRDX & 0.6 & 0.02 & Finely crystalline dolomite \\
\hline s $1 d 41-24-34 a d d$ & $5,685.0$ & $5,686.0$ & $324 \mathrm{PRDX}$ & 0.4 & 0.050000 & Finely crystalline dolomite \\
\hline s $1 d 41-24-34 a d d$ & $5,686.0$ & $5,687.0$ & 324PRDX & 0.3 & 0.230000 & Finely crystalline dolomite \\
\hline s $1 d 41-24-34 a d d$ & $5,687.0$ & $5,688.0$ & $324 \mathrm{PRDX}$ & 0.4 & 0.070000 & Finely crystalline dolomite \\
\hline s $1 d 41-24-34 a d d$ & $5,688.0$ & $5,689.0$ & 324PRDX & 1.3 & 0.120000 & Finely crystalline dolomite \\
\hline s $1 d 41-24-34$ add & $5,689.0$ & $5,690.0$ & 324PRDX & 0.7 & 0.050000 & Finely crystalline dolomite \\
\hline s $1 d 41-24-34 a d d$ & $5,690.0$ & $5,691.0$ & $324 \mathrm{PRDX}$ & 2.9 & 0.050000 & Finely crystalline dolomite \\
\hline s $1 d 41-24-34 a d d$ & $5,691.0$ & $5,692.0$ & $324 \mathrm{PRDX}$ & 3.5 & 0.050000 & Vuggy, finely crystalline dolomite \\
\hline s $1 d 41-24-34 a d d$ & $5,692.0$ & $5,693.0$ & 324PRDX & 2.9 & 0.040000 & Vuggy, finely crystalline dolomite \\
\hline s $1 d 41-24-34 a d d$ & $5,693.0$ & $5,694.0$ & $324 \mathrm{PRDX}$ & 2.1 & 5.500000 & Finely crystalline dolomite \\
\hline sld41-24-34add & $5,694.0$ & $5,695.0$ & $324 \mathrm{PRDX}$ & 5.7 & 0.520000 & Finely crystalline dolomite \\
\hline s $1 d 41-24-34 a d d$ & $5,695.0$ & $5,696.0$ & $324 \mathrm{PRDX}$ & 0.4 & 4.400000 & Finely crystalline dolomite \\
\hline s $1 d 41-24-34 a d d$ & $5,696.0$ & $5,697.0$ & 324PRDX & 0.3 & 0.130000 & Finely crystalline dolomite \\
\hline s $1 d 41-24-34 a d d$ & $5,697.0$ & $5,698.0$ & 324PRDX & 0.8 & 0.110000 & Finely crystalline dolomite \\
\hline sld41-24-34add & $5,698.0$ & $5,699.0$ & $324 \mathrm{PRDX}$ & 0.3 & 0.050000 & Finely crystalline dolomite \\
\hline s $1 d 41-24-34 a d d$ & $5,699.0$ & $5,700.0$ & 324PRDX & 0.4 & 0.020000 & Finely crystalline dolomite \\
\hline s 1d41-24-34add & $5,700.0$ & $5,701.0$ & 324PRDX & 1.3 & 3.300000 & Fine-grained dolomite \\
\hline sld41-24-34add & $5,701.0$ & $5,702.0$ & $324 \mathrm{PRDX}$ & 11.2 & 14.000000 & Finely crystalline dolomite \\
\hline sld $41-24-34$ add & $5,702.0$ & $5,703.0$ & $324 \mathrm{PRDX}$ & 8.4 & 16.000000 & Finely crystalline dolomite \\
\hline sld41-24-34add & $5,703.0$ & $5,704.0$ & $324 \mathrm{PRDX}$ & 5.0 & 0.040000 & Finely crystalline dolomite \\
\hline s1d41-24-34add & $5,704.0$ & $5,705.0$ & 324PRDX & 0.4 & 0.320000 & Crystalline, limy dolomite \\
\hline s $1 d 41-24-34$ add & $5,705.0$ & $5,706.0$ & $324 \mathrm{PRDX}$ & 0.6 & 0.130000 & Crystalline, limy dolomite \\
\hline s $1 d 41-24-34$ add & $5,706.0$ & $5,707.0$ & 324PRDX & 0.9 & 1.800000 & Crystalline limestone \\
\hline sld41-24-34add & $5,707.0$ & $5,708.0$ & $324 \mathrm{PRDX}$ & 2.1 & 0.110000 & Crystalline limestone \\
\hline s1d41-24-34add & $5,708.0$ & $5,709.0$ & 324PRDX & 0.8 & 0.110000 & Crystalline limestone \\
\hline sld41-24-34add & $5,709.0$ & $5,710.0$ & $324 \mathrm{PRDX}$ & 2.1 & 0.110000 & Crystalline limestone \\
\hline s $1 d 41-24-34$ add & $5,710.0$ & $5,711.0$ & $324 \mathrm{PRDX}$ & 2.0 & 0.140000 & Crystalline limestone \\
\hline sld41-24-34add & $5,711.0$ & $5,712.0$ & $324 \mathrm{PRDX}$ & 6.8 & 194.000000 & Vuggy, crystalline limestone \\
\hline sld41-24-34add & $5,712.0$ & $5,713.0$ & 324PRDX & 10.5 & 112.000000 & Vuggy, sucrose limestone \\
\hline s1d41-24-34add & $5,713.0$ & $5,714.0$ & $324 \mathrm{PRDX}$ & 10.0 & 63.000000 & Vuggy, sucrose limestone \\
\hline
\end{tabular}


Table 4.--Laboratory-determined porosity and permeability--Continued

\begin{tabular}{|c|c|c|c|c|c|c|}
\hline Site & $\begin{array}{l}\text { Depth to } \\
\text { top } \\
\text { (feet) }\end{array}$ & $\begin{array}{l}\text { Depth to } \\
\text { bottom } \\
\text { (feet) }\end{array}$ & $\begin{array}{c}\text { Forma- } \\
\text { tion }\end{array}$ & $\begin{array}{l}\text { Porosity } \\
\text { (percent) }\end{array}$ & $\begin{array}{c}\text { Perme- } \\
\text { ability } \\
(\text { milli- } \\
\text { darcies) }\end{array}$ & Lithology \\
\hline s $1 d 41-24-34$ add & $5,714.0$ & $5,715.0$ & $324 \mathrm{PRDX}$ & 10.7 & 487.000000 & Vuggy, sucrose limestone \\
\hline s $1 d 41-24-34 a d d$ & $5,715.0$ & $5,716.0$ & 324PRDX & 10.1 & 655.000000 & Vuggy, sucrose limestone \\
\hline s $1 d 41-24-34$ add & $5,716.0$ & $5,717.0$ & $324 \mathrm{PRDX}$ & 9.1 & 168.000000 & Vuggy, sucrose limestone \\
\hline sld41-24-34add & $5,717.0$ & $5,718.0$ & $324 \mathrm{PRDX}$ & 13.7 & 295.000000 & Vuggy, sucrose limestone \\
\hline s $1 d 41-24-34$ add & $5,718.0$ & $5,719.0$ & $324 \mathrm{PRDX}$ & 11.1 & 250.000000 & Vuggy, crystalline limestone \\
\hline $\operatorname{sid} 41-24-34 \mathrm{add}$ & $5,719.0$ & $5,720.0$ & 324PRDX & 9.6 & 613.000000 & Vuggy, crystalline limestone \\
\hline s $1 d 41-24-34 a d d$ & $5,720.0$ & $5,721.0$ & 324PRDX & 10.5 & 413.000000 & Vuggy, crystalline limestone \\
\hline $\operatorname{sid} 41-24-34 \mathrm{add}$ & $5,721.0$ & $5,722.0$ & $324 \mathrm{PRDX}$ & 10.7 & 216.000000 & Vuggy, crystalline limestone \\
\hline s $1 d 41-24-34$ add & $5,722.0$ & $5,723.0$ & $324 \mathrm{PRDX}$ & 8.81 , &, 291.000000 & Sucrose limestone \\
\hline s $1 d 41-24-34 a d d$ & $5,723.0$ & $5,724.0$ & $324 \mathrm{PRDX}$ & 8.4 & 260.000000 & Vuggy, sucrose limestone \\
\hline s $1 \mathrm{~d} 41-24-34 \mathrm{add}$ & $5,724.0$ & $5,725.0$ & 324PRDX & 13.32 , &, 435.000000 & Vuggy, crystalline limestone \\
\hline $\operatorname{s} 1 \mathrm{~d} 41-24-34 \mathrm{add}$ & $5,725.0$ & $5,726.0$ & 324PRDX & 9.9 & 475.000000 & Vuggy, sucrose limestone \\
\hline sld41-24-34add & $5,726.0$ & $5,727.0$ & 324PRDX & 8.4 & 36.000000 & Vuggy, sucrose limestone \\
\hline s $1 d 41-24-34 a d d$ & $5,731.0$ & $5,732.0$ & $324 \mathrm{PRDX}$ & 7.2 & 49.000000 & Vuggy, crystalline dolomite \\
\hline s ld $41-24-34 a d d$ & $5,732.0$ & $5,733.0$ & 324PRDX & 13.2 & 45.000000 & Vuggy, crystalline dolomite \\
\hline s $1 \mathrm{~d} 41-24-34 \mathrm{add}$ & $5,733.0$ & $5,734.0$ & $324 \mathrm{PRDX}$ & 13.6 & 29.000000 & Sucrose limestone \\
\hline $\operatorname{sid} 41-24-34 \mathrm{add}$ & $5,734.0$ & $5,735.0$ & $324 \mathrm{PRDX}$ & 11.8 & 31.000000 & Vuggy, crystalline dolomite \\
\hline sld41-24-34add & $5,735.0$ & $5,736.0$ & 324PRDX & 9.1 & 21.000000 & Vuggy, crystalline dolomite \\
\hline sld41-24-34add & $5,736.0$ & $5,737.0$ & $324 \mathrm{PRDX}$ & 9.9 & 8.400000 & Vuggy, crystalline dolomite \\
\hline s $1 d 41-24-34 a d d$ & $5,737.0$ & $5,738.0$ & $324 \mathrm{PRDX}$ & 8.5 & 26.000000 & Vuggy, crystalline dolomite \\
\hline s 1 d4 $1-24-34$ add & $5,738.0$ & $5,739.0$ & 324PRDX & 9.9 & 32.000000 & Vuggy, crystalline dolomite \\
\hline s $1 d 41-24-34$ add & $5,739.0$ & $5,740.0$ & $324 \mathrm{PRDX}$ & 8.3 & 19.000000 & Vuggy, crystalline dolomite \\
\hline s $1 \mathrm{~d} 41-24-34 \mathrm{add}$ & $5,740.0$ & $5,741.0$ & $324 \mathrm{PRDX}$ & 6.2 & 2.500000 & Vuggy, crystalline dolomite \\
\hline s $1 d 41-24-34$ add & $5,741.0$ & $5,742.0$ & $324 \mathrm{PRDX}$ & 3.8 & 0.140000 & Vuggy, crystalline dolomite \\
\hline sld41-24-34add & $5,742.0$ & $5,743.0$ & $324 \mathrm{PRDX}$ & 1.5 & 0.020000 & Fine-grained dolomite \\
\hline s $1 \mathrm{~d} 41-24-34 \mathrm{add}$ & $5,743.0$ & $5,744.0$ & 324PRDX & 2.0 & 9.400000 & Crystalline dolomite \\
\hline s 1 d4 $1-24-34 a d d$ & $5,744.0$ & $5,745.0$ & 324PRDX & 1.3 & 0.520000 & Crystalline dolomite \\
\hline s $1 d 41-24-34 a d d$ & $5,745.0$ & $5,746.0$ & $324 \mathrm{PRDX}$ & 8.6 & 0.010000 & Fine-grained dolomite \\
\hline sld41-24-34add & $5,746.0$ & $5,747.0$ & $324 \mathrm{PRDX}$ & 10.7 & 0.030000 & Crystalline dolomite \\
\hline s $1 d 41-24-34$ add & $5,747.0$ & $5,748.0$ & $324 \mathrm{PRDX}$ & 11.6 & 0.010000 & Crystalline dolomite \\
\hline sld41-24-34add & $5,748.0$ & $5,749.0$ & $324 \mathrm{PRDX}$ & 6.6 & 0.030000 & Crystalline dolomite \\
\hline $\operatorname{sld} 41-24-34 a d d$ & $5,749.0$ & $5,750.0$ & 324PRDX & 5.7 & 0.020000 & Crystalline dolomite \\
\hline $\operatorname{sid} 41-24-34 a d d$ & $5,750.0$ & $5,751.0$ & 324PRDX & 1.2 & 0.040000 & Fine-grained dolomite \\
\hline sld41-24-34add & $5,751.0$ & $5,752.0$ & $324 \mathrm{PRDX}$ & 0.8 & 0.040000 & Crystalline dolomite \\
\hline $\operatorname{sid} 41-24-34 a d d$ & $5,752.0$ & $5,753.0$ & $324 \mathrm{PRDX}$ & 16.3 & 0.090000 & Dolomite \\
\hline s $1 d 41-24-34$ add & $5,753.0$ & $5,754.0$ & $324 \mathrm{PRDX}$ & 3.1 & 0.020000 & Crystalline dolomite \\
\hline sld41-24-34add & $5,754.0$ & $5,755.0$ & 324PRDX & 8.9 & 2.100000 & Dolomite \\
\hline s $1 d 41-24-34 a d d$ & $5,755.0$ & $5,756.0$ & 324PRDX & 14.6 & 0.070000 & Dolomite \\
\hline sld41-24-34add & $5,756.0$ & $5,757.0$ & $324 \mathrm{PRDX}$ & 1.2 & 0.150000 & Limy dolomite \\
\hline $\operatorname{sld} 41-24-34 a d d$ & $5,757.0$ & $5,758.0$ & 324PRDX & 4.2 & 0.520000 & Vuggy, crystalline dolomite \\
\hline sld41-24-34add & $5,758.0$ & $5,759.0$ & $324 P R D X$ & 5.8 & 0.360000 & Vuggy, crystalline dolomite \\
\hline sld41-24-34add & $5,759.0$ & $5,760.0$ & $324 \mathrm{PRDX}$ & 2.6 & 0.130000 & Crystalline dolomite \\
\hline sld41-24-34add & $5,760.0$ & $5,761.0$ & $324 \mathrm{PRDX}$ & 4.5 & 0.360000 & Vuggy, crystalline dolomite \\
\hline sld41-24-34add & $5,761.0$ & $5,762.0$ & 324PRDX & 3.1 & 0.270000 & Crystalline dolomite \\
\hline sld41-24-34add & $5,762.0$ & $5,763.0$ & $324 \mathrm{PRDX}$ & 3.5 & 2.100000 & Crystalline dolomite \\
\hline sld41-24-34add & $5,763.0$ & $5,764.0$ & $324 \mathrm{PRDX}$ & 2.5 & 1.800000 & Crystalline dolomite \\
\hline s ld $41-24-34 \mathrm{add}$ & $5,764.0$ & $5,765.0$ & $324 \mathrm{PRDX}$ & 3.8 & 0.440000 & Crystalline dolomite \\
\hline sld $41-24-34$ add & $5,765.0$ & $5,766.0$ & $324 \mathrm{PRDX}$ & 3.3 & 0.680000 & Crystalline dolomite \\
\hline sld41-24-34add & $5,766.0$ & $5,767.0$ & $324 \mathrm{PRDX}$ & 1.9 & 0.080000 & Crystalline dolomite \\
\hline sld41-24-34add & $5,767.0$ & $5,768.0$ & 324PRDX & 7.0 & 6.300000 & Finely crystalline dolomite \\
\hline
\end{tabular}


Table 4.--Laboratory-determined porosity and permeability--Continued

\begin{tabular}{|c|c|c|c|c|c|c|}
\hline Site & $\begin{array}{l}\text { Depth to } \\
\text { top } \\
\text { (feet) }\end{array}$ & $\begin{array}{l}\text { Depth to } \\
\text { bottom } \\
\text { (feet) }\end{array}$ & $\begin{array}{c}\text { Forma- } \\
\text { tion }\end{array}$ & $\begin{array}{l}\text { Porosity } \\
\text { (percent) }\end{array}$ & $\begin{array}{l}\text { Perme- } \\
\text { ability } \\
\text { (milli- } \\
\text { darcies) }\end{array}$ & Lithology \\
\hline $\begin{array}{l}\text { sld41-24-34add } \\
\text { sld41-24-34add } \\
\text { sld41-24-34add } \\
\text { sld41-24-34add } \\
\text { sld41-24-34add }\end{array}$ & $\begin{array}{l}5,768.0 \\
5,769.0 \\
5,770.0 \\
5,771.0 \\
5,772.0\end{array}$ & $\begin{array}{l}5,769.0 \\
5,770.0 \\
5,771.0 \\
5,772.0 \\
5,773.0\end{array}$ & $\begin{array}{l}324 \mathrm{PRDX} \\
324 \mathrm{PRDX} \\
324 \mathrm{PRDX} \\
324 \mathrm{PRDX} \\
324 \mathrm{PRDX}\end{array}$ & $\begin{array}{l}1.0 \\
1.0 \\
1.2 \\
2.2 \\
4.7\end{array}$ & $\begin{array}{r}0.100000 \\
0.500000 \\
10.000000 \\
0.060000 \\
29.000000\end{array}$ & $\begin{array}{l}\text { Finely crystalline dolomite } \\
\text { Finely crystalline dolomite } \\
\text { Finely crystalline dolomite } \\
\text { Finely crystalline dolomite } \\
\text { Finely crystalline dolomite }\end{array}$ \\
\hline $\begin{array}{l}\text { s1d41-24-34add } \\
\text { sld41-24-34add } \\
\text { sld41-24-34add } \\
\text { s } 1 d 41-24-34 a d d \\
\text { sld41-24-34add }\end{array}$ & $\begin{array}{l}5,773.0 \\
5,774.0 \\
5,775.0 \\
5,776.0 \\
5,777.0\end{array}$ & $\begin{array}{l}5,774.0 \\
5,775.0 \\
5,776.0 \\
5,777.0 \\
5,778.0\end{array}$ & $\begin{array}{l}\text { 324PRDX } \\
324 \mathrm{PRDX} \\
324 \mathrm{PRDX} \\
324 \mathrm{PRDX} \\
\text { 324PRDX }\end{array}$ & $\begin{array}{l}8.3 \\
7.2 \\
7.7 \\
4.0 \\
3.0\end{array}$ & $\begin{array}{r}0.030000 \\
0.010000 \\
0.030000 \\
15.000000 \\
2.000000\end{array}$ & $\begin{array}{l}\text { Finely crystalline dolomite } \\
\text { Fine-grained dolomite } \\
\text { Finely crystalline dolomite } \\
\text { Finely crystalline dolomite } \\
\text { Finely crystalline dolomite }\end{array}$ \\
\hline $\begin{array}{l}\text { sld41-24-34add } \\
\text { sld41-24-34add } \\
\text { s } 1 d 41-24-34 a d d\end{array}$ & $\begin{array}{l}5,778.0 \\
5,779.0 \\
5,780.0\end{array}$ & $\begin{array}{l}5,779.0 \\
5,780.0 \\
5,781.0\end{array}$ & $\begin{array}{l}\text { 324PRDX } \\
\text { 324PRDX } \\
\text { 324PRDX }\end{array}$ & $\begin{array}{l}2.0 \\
3.2 \\
0.7 \\
\end{array}$ & $\begin{array}{l}3.100000 \\
0.600000 \\
1.100000 \\
\end{array}$ & $\begin{array}{l}\text { Finely crystalline dolomite } \\
\text { Finely crystalline dolomite } \\
\text { Finely crystalline dolomite }\end{array}$ \\
\hline SITE AVERAGE- & & & AVERAGE-- & $\frac{6.0}{6.0}$ & $\frac{60.291748}{60.291748}$ & \\
\hline $\begin{array}{l}\text { sld41-24-34cdd } \\
\text { sld41-24-34cdd } \\
\text { sld41-24-34cdd } \\
\text { s } 1 d 41-24-34 c d d \\
\text { s } 1 d 41-24-34 c d d\end{array}$ & $\begin{array}{l}5,657.0 \\
5,658.0 \\
5,659.0 \\
5,660.0 \\
5,661.0\end{array}$ & $\begin{array}{l}5,658.0 \\
5,659.0 \\
5,660.0 \\
5,661.0 \\
5,662.0\end{array}$ & $\begin{array}{l}\text { 324PRDX } \\
324 \mathrm{PRDX} \\
\text { 324PRDX } \\
\text { 324PRDX } \\
\text { 324PRDX }\end{array}$ & $\begin{array}{l}3.9 \\
7.4 \\
5.3 \\
3.2 \\
3.2\end{array}$ & $\begin{array}{l}0.020000 \\
0.310000 \\
0.980000 \\
0.790000 \\
0.790000\end{array}$ & $\begin{array}{l}\text { Sucrose limestone } \\
\text { Sucrose limestone } \\
\text { Sucrose limestone } \\
\text { Sucrose limestone } \\
\text { Sucrose limestone }\end{array}$ \\
\hline $\begin{array}{l}\text { s } 1 d 41-24-34 \text { cdd } \\
\text { s } 1441-24-34 c d d \\
\text { s } 1 d 41-24-34 c d d \\
\text { s } 1 d 41-24-34 c d d \\
\text { s } 1 d 41-24-34 c d d\end{array}$ & $\begin{array}{l}5,662.0 \\
5,663.0 \\
5,664.0 \\
5,665.0 \\
5,666.0\end{array}$ & $\begin{array}{l}5,663.0 \\
5,664.0 \\
5,665.0 \\
5,666.0 \\
5,667.0\end{array}$ & $\begin{array}{l}324 \mathrm{PRDX} \\
324 \mathrm{PRDX} \\
324 \mathrm{PRDX} \\
324 \mathrm{PRDX} \\
324 \mathrm{PRDX}\end{array}$ & $\begin{array}{l}7.5 \\
5.6 \\
5.5 \\
7.1 \\
2.3\end{array}$ & $\begin{array}{l}1.100000 \\
0.980000 \\
0.790000 \\
1.300000 \\
0.120000\end{array}$ & $\begin{array}{l}\text { Sucrose limestone } \\
\text { Sucrose limestone } \\
\text { Sucrose limestone } \\
\text { Sucrose limestone } \\
\text { Sucrose limestone }\end{array}$ \\
\hline $\begin{array}{l}\text { sld41-24-34cdd } \\
\text { sld41-24-34cdd } \\
\text { s } 1 d 41-24-34 c d d \\
\text { s } 1 d 41-24-34 c d d \\
\text { s } 1 d 41-24-34 c d d\end{array}$ & $\begin{array}{l}5,667.0 \\
5,668.0 \\
5,669.0 \\
5,670.0 \\
5,671.0\end{array}$ & $\begin{array}{l}5,668 \cdot 0 \\
5,669.0 \\
5,670.0 \\
5,671.0 \\
5,672.0\end{array}$ & $\begin{array}{l}\text { 324PRDX } \\
324 \mathrm{PRDX} \\
324 \mathrm{PRDX} \\
324 \mathrm{PRDX} \\
\text { 324PRDX }\end{array}$ & $\begin{array}{r}5.7 \\
16.9 \\
9.7 \\
16.0 \\
16.2\end{array}$ & $\begin{array}{r}0.050000 \\
2.600000 \\
2.800000 \\
19.000000 \\
1.200000\end{array}$ & $\begin{array}{l}\text { Sucrose limestone } \\
\text { Sucrose limestone } \\
\text { Sucrose limestone } \\
\text { Sucrose limestone } \\
\text { Sucrose limestone }\end{array}$ \\
\hline $\begin{array}{l}\text { s } 1 d 41-24-34 c d d \\
\text { sld41-24-34cdd } \\
\text { s1d41-24-34cdd } \\
\text { sld41-24-34cdd } \\
\text { sld41-24-34cdd }\end{array}$ & $\begin{array}{l}5,672.0 \\
5,673.0 \\
5,674.0 \\
5,675.0 \\
5,676.0\end{array}$ & $\begin{array}{l}5,673.0 \\
5,674.0 \\
5,675.0 \\
5,676.0 \\
5,677.0\end{array}$ & $\begin{array}{l}\text { 324PRDX } \\
324 \mathrm{PRDX} \\
324 \mathrm{PRDX} \\
324 \mathrm{PRDX} \\
324 \mathrm{PRDX}\end{array}$ & $\begin{array}{l}10.7 \\
12.3 \\
10.1 \\
12.5 \\
18.5\end{array}$ & $\begin{array}{l}2.100000 \\
2.300000 \\
1.500000 \\
0.540000 \\
4.100000\end{array}$ & $\begin{array}{l}\text { Sucrose limestone } \\
\text { Sucrose limestone } \\
\text { Sucrose limestone } \\
\text { Sucrose limestone } \\
\text { Sucrose limestone }\end{array}$ \\
\hline $\begin{array}{l}\text { sld41-24-34cdd } \\
\text { sld41-24-34cdd } \\
\text { sld41-24-34cdd } \\
\text { sld41-24-34cdd } \\
\text { sld41-24-34cdd }\end{array}$ & $\begin{array}{l}5,677.0 \\
5,678.0 \\
5,679.0 \\
5,680.0 \\
5,681.0\end{array}$ & $\begin{array}{l}5,678.0 \\
5,679.0 \\
5,680.0 \\
5,681.0 \\
5,682.0\end{array}$ & $\begin{array}{l}324 \mathrm{PRDX} \\
324 \mathrm{PRDX} \\
324 \mathrm{PRDX} \\
324 \mathrm{PRDX} \\
324 \mathrm{PRDX}\end{array}$ & $\begin{array}{l}13.6 \\
17.4 \\
16.2 \\
16.3 \\
11.2\end{array}$ & $\begin{array}{l}2.900000 \\
4.200000 \\
2.200000 \\
2.000000 \\
3.400000\end{array}$ & $\begin{array}{l}\text { Sucrose limestone } \\
\text { Sucrose limestone } \\
\text { Sucrose limestone } \\
\text { Sucrose limestone } \\
\text { Sucrose limestone }\end{array}$ \\
\hline $\begin{array}{l}\text { sld41-24-34cdd } \\
\text { s } 1 d 41-24-34 c d d \\
\text { sld41-24-34cdd } \\
\text { sld41-24-34cdd } \\
\text { s } 1 d 41-24-34 \text { cdd }\end{array}$ & $\begin{array}{l}5,682.0 \\
5,683.0 \\
5,684.0 \\
5,685.0 \\
5,686.0\end{array}$ & $\begin{array}{l}5,683.0 \\
5,684.0 \\
5,685.0 \\
5,686.0 \\
5,687.0\end{array}$ & $\begin{array}{l}324 \mathrm{PRDX} \\
324 \mathrm{PRDX} \\
324 \mathrm{PRDX} \\
324 \mathrm{PRDX} \\
\text { 324PRDX }\end{array}$ & $\begin{array}{r}10.0 \\
2.7 \\
7.6 \\
11.1 \\
11.8\end{array}$ & $\begin{array}{l}7.800000 \\
2.000000 \\
0.690000 \\
2.500000 \\
0.120000\end{array}$ & $\begin{array}{l}\text { Sucrose limestone } \\
\text { Sucrose dolomite } \\
\text { Sucrose dolomite } \\
\text { Sucrose dolomite } \\
\text { Sucrose, dolomitic limestone }\end{array}$ \\
\hline $\begin{array}{l}\text { sld41-24-34cdd } \\
\text { sld41-24-34cdd } \\
\text { sld41-24-34cdd } \\
\text { sld41-24-34cdd } \\
\text { sld41-24-34cdd }\end{array}$ & $\begin{array}{l}5,687.0 \\
5,688.0 \\
5,689.0 \\
5,690.0 \\
5,691.0\end{array}$ & $\begin{array}{l}5,688.0 \\
5,689.0 \\
5,690.0 \\
5,691.0 \\
5,692.0\end{array}$ & $\begin{array}{l}324 \mathrm{PRDX} \\
324 \mathrm{PRDX} \\
324 \mathrm{PRDX} \\
324 \mathrm{PRDX} \\
324 \mathrm{PRDX}\end{array}$ & $\begin{array}{r}7.7 \\
12.0 \\
13.5 \\
8.9 \\
16.3\end{array}$ & $\begin{array}{l}5.200000 \\
5.200000 \\
7.200000 \\
2.700000 \\
8.400000\end{array}$ & $\begin{array}{l}\text { Sucrose, dolomitic limestone } \\
\text { Sucrose, dolomitic limestone } \\
\text { Sucrose, dolomitic limestone } \\
\text { Sucrose, dolomitic limestone } \\
\text { Sucrose, dolomitic limestone }\end{array}$ \\
\hline
\end{tabular}


Table 4.--Laboratory-determined porosity and permeability--Continued

\begin{tabular}{|c|c|c|c|c|c|c|}
\hline Site & $\begin{array}{l}\text { Depth to } \\
\text { top } \\
\text { (feet) }\end{array}$ & $\begin{array}{l}\text { Depth to } \\
\text { bottom } \\
\text { (feet) }\end{array}$ & $\begin{array}{c}\text { Forma- } \\
\text { tion }\end{array}$ & $\begin{array}{l}\text { Porosity } \\
\text { (percent) }\end{array}$ & $\begin{array}{l}\text { Perme- } \\
\text { ability } \\
\text { (milli- } \\
\text { darcies) }\end{array}$ & Lithology \\
\hline $\begin{array}{l}\text { sld41-24-34cdd } \\
\text { sld41-24-34cdd } \\
\text { sld41-24-34cdd } \\
\text { sld41-24-34cdd } \\
\text { sld41-24-34cdd }\end{array}$ & $\begin{array}{l}5,692.0 \\
5,693.0 \\
5,694.0 \\
5,695.0 \\
5,696.0\end{array}$ & $\begin{array}{l}5,693.0 \\
5,694.0 \\
5,695.0 \\
5,696.0 \\
5,697.0\end{array}$ & $\begin{array}{l}\text { 324PRDX } \\
\text { 324PRDX } \\
\text { 324PRDX } \\
\text { 324PRDX } \\
\text { 324PRDX }\end{array}$ & $\begin{array}{r}18.4 \\
18.5 \\
18.3 \\
7.7 \\
21.6\end{array}$ & $\begin{array}{l}6.200000 \\
4.800000 \\
5.800000 \\
4.000000 \\
4.300000\end{array}$ & $\begin{array}{l}\text { Sucrose, dolomitic limestone } \\
\text { Sucrose, dolomitic limestone } \\
\text { Sucrose, limy dolomite } \\
\text { Sucrose, limy dolomite } \\
\text { Sucrose, limy dolomite }\end{array}$ \\
\hline $\begin{array}{l}\text { sld41-24-34cdd } \\
\text { sld41-24-34cdd } \\
\text { sld41-24-34cdd } \\
\text { sld } 41-24-34 c d d \\
\text { s } 1 d 41-24-34 c d d\end{array}$ & $\begin{array}{l}5,700.0 \\
5,701.0 \\
5,702.0 \\
5,703.0 \\
5,713.0\end{array}$ & $\begin{array}{l}5,701.0 \\
5,702.0 \\
5,703.0 \\
5,704.0 \\
5,714.0\end{array}$ & $\begin{array}{l}\text { 324PRDX } \\
\text { 324PRDX } \\
\text { 324PRDX } \\
\text { 324PRDX } \\
\text { 324PRDX }\end{array}$ & $\begin{array}{r}21.5 \\
18.6 \\
2.4 \\
4.9 \\
5.5\end{array}$ & $\begin{array}{l}4.600000 \\
3.400000 \\
1.200000 \\
1.100000 \\
0.740000\end{array}$ & $\begin{array}{l}\text { Sucrose, limy dolomite } \\
\text { Sucrose, limy dolomite } \\
\text { Sucrose, dolomitic limestone } \\
\text { Sucrose, dolomitic limestone } \\
\text { Sucrose, dolomitic limestone }\end{array}$ \\
\hline $\begin{array}{l}\text { sld41-24-34cdd } \\
\text { sld41-24-34cdd } \\
\text { s1d41-24-34cdd } \\
\text { sld } 41-24-34 c d d \\
\text { s } 1 d 41-24-34 c d d\end{array}$ & $\begin{array}{l}5,714.0 \\
5,715.0 \\
5,716.0 \\
5,719.0 \\
5,720.0\end{array}$ & $\begin{array}{l}5,715.0 \\
5,716.0 \\
5,717.0 \\
5,720.0 \\
5,721.0\end{array}$ & $\begin{array}{l}\text { 324PRDX } \\
\text { 324PRDX } \\
\text { 324PRDX } \\
\text { 324PRDX } \\
\text { 324PRDX }\end{array}$ & $\begin{array}{l}7.5 \\
5.0 \\
2.3 \\
4.6 \\
6.2\end{array}$ & $\begin{array}{l}3.000000 \\
0.020000 \\
0.510000 \\
0.320000 \\
3.200000\end{array}$ & $\begin{array}{l}\text { Sucrose, dolomitic limestone } \\
\text { Sucrose, dolomitic limestone } \\
\text { Sucrose, dolomitic limestone } \\
\text { Sucrose limestone } \\
\text { Sucrose limestone }\end{array}$ \\
\hline $\begin{array}{l}\text { sld41-24-34cdd } \\
\text { sld41-24-34cdd } \\
\text { sld41-24-34cdd } \\
\text { sld41-24-34cdd } \\
\text { sld } 41-24-34 c d d\end{array}$ & $\begin{array}{l}5,721.0 \\
5,722.0 \\
5,734.0 \\
5,735.0 \\
5,736.0\end{array}$ & $\begin{array}{l}5,722.0 \\
5,723.0 \\
5,735.0 \\
5,736.0 \\
5,737.0\end{array}$ & $\begin{array}{l}\text { 324PRDX } \\
\text { 324PRDX } \\
\text { 324PRDX } \\
\text { 324PRDX } \\
\text { 324PRDX }\end{array}$ & $\begin{array}{r}5.0 \\
3.3 \\
12.4 \\
6.7 \\
10.2\end{array}$ & $\begin{array}{l}0.140000 \\
0.040000 \\
0.200000 \\
0.140000 \\
0.100000\end{array}$ & $\begin{array}{l}\text { Sucrose limestone } \\
\text { Sucrose limestone } \\
\text { Sucrose, limy dolomite } \\
\text { Sucrose, limy dolomite } \\
\text { Sucrose, limy dolomite }\end{array}$ \\
\hline $\begin{array}{l}\text { sld41-24-34cdd } \\
\text { sld41-24-34cdd } \\
\text { sld41-24-34cdd } \\
\text { sld41-24-34cdd } \\
\text { sld41-24-34cdd }\end{array}$ & $\begin{array}{l}5,737.0 \\
5,745.0 \\
5,746.0 \\
5,747.0 \\
5,748.0\end{array}$ & $\begin{array}{l}5,738.0 \\
5,746.0 \\
5,747.0 \\
5,748.0 \\
5,749.0\end{array}$ & $\begin{array}{l}\text { 324PRDX } \\
\text { 324PRDX } \\
\text { 324PRDX } \\
\text { 324PRDX } \\
\text { 324PRDX }\end{array}$ & $\begin{array}{l}6.9 \\
3.6 \\
4.4 \\
8.6 \\
8.3\end{array}$ & $\begin{array}{l}0.070000 \\
0.200000 \\
5.100000 \\
0.620000 \\
0.140000\end{array}$ & $\begin{array}{l}\text { Sucrose, limy dolomite } \\
\text { Sucrose limestone } \\
\text { Sucrose limestone } \\
\text { Sucrose limestone } \\
\text { Sucrose limestone }\end{array}$ \\
\hline $\begin{array}{l}\text { sld41-24-34cdd } \\
\text { sld41-24-34cdd } \\
\text { sld41-24-34cdd } \\
\text { sld41-24-34cdd } \\
\text { sld41-24-34cdd }\end{array}$ & $\begin{array}{l}5,749.0 \\
5,750.0 \\
5,751.0 \\
5,752.0 \\
5,760.0\end{array}$ & $\begin{array}{l}5,750.0 \\
5,751.0 \\
5,752.0 \\
5,753.0 \\
5,761.0\end{array}$ & $\begin{array}{l}\text { 324PRDX } \\
324 \text { PRDX } \\
\text { 324PRDX } \\
\text { 324PRDX } \\
\text { 324PRDX }\end{array}$ & $\begin{array}{l}4.7 \\
6.2 \\
8.8 \\
8.6 \\
8.5\end{array}$ & $\begin{array}{r}0.350000 \\
0.400000 \\
0.690000 \\
0.970000 \\
115.000000\end{array}$ & $\begin{array}{l}\text { Sucrose limestone } \\
\text { Sucrose limestone } \\
\text { Sucrose limestone } \\
\text { Sucrose limestone } \\
\text { Sucrose limestone }\end{array}$ \\
\hline $\begin{array}{l}\text { sld41-24-34cdd } \\
\text { sld41-24-34cdd } \\
\text { s1d41-24-34cdd } \\
\text { s } 1 d 41-24-34 c d d \\
\text { s1d41-24-34cdd }\end{array}$ & $\begin{array}{l}5,761.0 \\
5,762.0 \\
5,763.0 \\
5,764.0 \\
5,765.0\end{array}$ & $\begin{array}{l}5,762.0 \\
5,763.0 \\
5,764.0 \\
5,765.0 \\
5,766.0\end{array}$ & $\begin{array}{l}\text { 324PRDX } \\
324 \mathrm{PRDX} \\
324 \mathrm{PRDX} \\
324 \mathrm{PRDX} \\
324 \mathrm{PRDX}\end{array}$ & $\begin{array}{l}7.4 \\
6.9 \\
5.7 \\
6.4 \\
5.1\end{array}$ & $\begin{array}{r}5.000000 \\
2.600000 \\
0.860000 \\
20.000000 \\
3.200000\end{array}$ & $\begin{array}{l}\text { Sucrose limestone } \\
\text { Sucrose limestone } \\
\text { Sucrose limestone } \\
\text { Sucrose limestone } \\
\text { Sucrose limestone }\end{array}$ \\
\hline $\begin{array}{l}\text { sld41-24-34cdd } \\
\text { s1d41-24-34cdd } \\
\text { s1d41-24-34cdd } \\
\text { s1d41-24-34cdd } \\
\text { s1d41-24-34cdd }\end{array}$ & $\begin{array}{l}5,766.0 \\
5,767.0 \\
5,768.0 \\
5,769.0 \\
5,774.0\end{array}$ & $\begin{array}{l}5,767.0 \\
5,768.0 \\
5,769.0 \\
5,770.0 \\
5,775.0\end{array}$ & $\begin{array}{l}\text { 324PRDX } \\
324 \mathrm{PRDX} \\
324 \mathrm{PRDX} \\
\text { 324PRDX } \\
324 \mathrm{PRDX}\end{array}$ & $\begin{array}{l}7.3 \\
6.1 \\
5.8 \\
4.3 \\
7.9\end{array}$ & $\begin{array}{r}5.000000 \\
0.590000 \\
16.000000 \\
0.170000 \\
0.100000\end{array}$ & $\begin{array}{l}\text { Sucrose limestone } \\
\text { Sucrose limestone } \\
\text { Sucrose limestone } \\
\text { Sucrose limestone } \\
\text { Fine-grained dolomite }\end{array}$ \\
\hline $\begin{array}{l}\text { sld41-24-34cdd } \\
\text { s } 1 d 41-24-34 c d d \\
\text { sld41-24-34cdd } \\
\text { s } 1 d 41-24-34 c d d \\
\text { s1d } 41-24-34 c d d\end{array}$ & $\begin{array}{l}5,775.0 \\
5,776.0 \\
5,777.0 \\
5,779.0 \\
5,780.0\end{array}$ & $\begin{array}{l}5,776.0 \\
5,777.0 \\
5,778.0 \\
5,780.0 \\
5,781.0\end{array}$ & $\begin{array}{l}\text { 324PRDX } \\
\text { 324PRDX } \\
\text { 324PRDX } \\
\text { 324PRDX } \\
\text { 324PRDX }\end{array}$ & $\begin{array}{r}12.5 \\
8.7 \\
7.1 \\
5.3 \\
3.4\end{array}$ & $\begin{array}{l}0.060000 \\
0.200000 \\
0.080000 \\
0.100000 \\
0.020000\end{array}$ & $\begin{array}{l}\text { Dolomite } \\
\text { Dolomite } \\
\text { Dolomite } \\
\text { Sucrose dolomite } \\
\text { Sucrose dolomite }\end{array}$ \\
\hline $\begin{array}{l}\text { sld41-24-34cdd } \\
\text { s } 1 d 41-24-34 c d d \\
\text { sld41-24-34cdd } \\
\text { s } 1 d 41-24-34 c d d \\
\text { sld41-24-34cdd }\end{array}$ & $\begin{array}{l}5,781.0 \\
5,782.0 \\
5,783.0 \\
5,784.0 \\
5,785.0\end{array}$ & $\begin{array}{l}5,782.0 \\
5,783.0 \\
5,784.0 \\
5,785.0 \\
5,786.0\end{array}$ & $\begin{array}{l}\text { 324PRDX } \\
\text { 324PRDX } \\
\text { 324PRDX } \\
\text { 324PRDX } \\
\text { 324PRDX }\end{array}$ & $\begin{array}{r}8.1 \\
8.3 \\
12.9 \\
11.4 \\
10.0\end{array}$ & $\begin{array}{r}0.020000 \\
0.040000 \\
0.040000 \\
1.100000 \\
145.000000\end{array}$ & $\begin{array}{l}\text { Sucrose dolomite } \\
\text { Sucrose dolomite } \\
\text { Sucrose dolomite } \\
\text { Sucrose dolomite } \\
\text { Sucrose dolomite }\end{array}$ \\
\hline
\end{tabular}


Table 4.--Laboratory-determined porosity and permeability--Continued

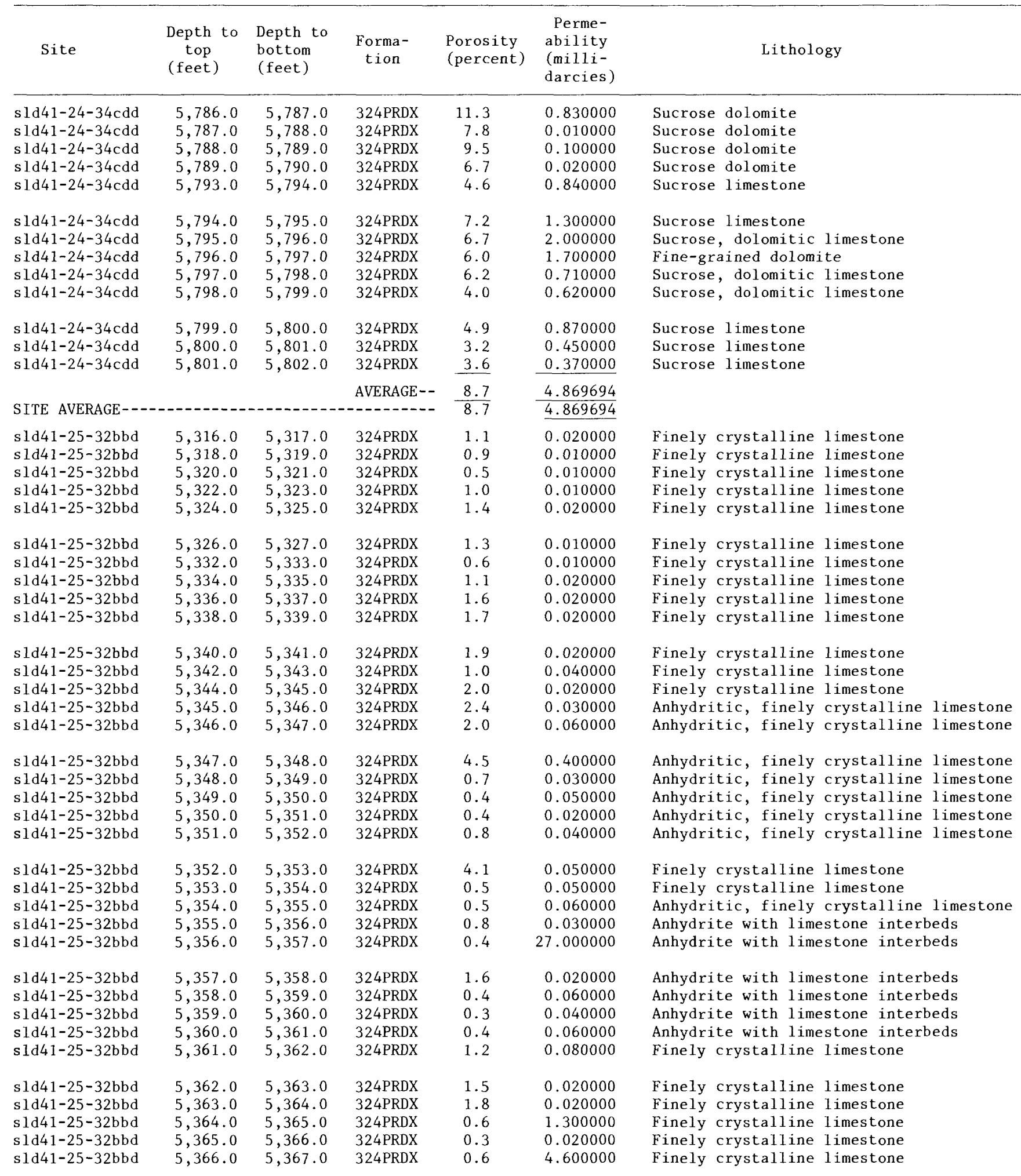


Table 4.--Laboratory-determined porosity and permeability--Continued

\begin{tabular}{|c|c|c|c|c|c|c|}
\hline Site & $\begin{array}{l}\text { Depth to } \\
\text { top } \\
\text { (feet) }\end{array}$ & $\begin{array}{l}\text { Depth to } \\
\text { bottom } \\
\text { (feet) }\end{array}$ & $\begin{array}{c}\text { Forma- } \\
\text { tion }\end{array}$ & $\begin{array}{l}\text { Porosity } \\
\text { (percent) }\end{array}$ & $\begin{array}{l}\text { Perme- } \\
\text { ability } \\
\text { (milli- } \\
\text { darcies) }\end{array}$ & Lithology \\
\hline s $1 d 41-25-32 b b d$ & $5,367.0$ & $5,368.0$ & 324PRDX & 1.1 & 0.010000 & Finely crystalline limestone \\
\hline s $1 d 41-25-32 b b d$ & $5,368.0$ & $5,369.0$ & 324PRDX & 1.6 & 0.040000 & Finely crystalline limestone \\
\hline sld $41-25-32 b b d$ & $5,369.0$ & $5,370.0$ & 324PRDX & 1.7 & 0.240000 & Finely crystalline limestone \\
\hline s $1 d 41-25-32 b b d$ & $5,370.0$ & $5,371.0$ & $324 \mathrm{PRDX}$ & 0.9 & 0.050000 & Finely crystalline limestone \\
\hline s $1 d 41-25-32 b b d$ & $5,371.0$ & $5,372.0$ & $324 \mathrm{PRDX}$ & 1.7 & 0.030000 & Finely crystalline limestone \\
\hline s $1 d 41-25-32 b b d$ & $5,372.0$ & $5,373.0$ & 324PRDX & 1.5 & 0.030000 & Finely crystalline limestone \\
\hline s $1 d 41-25-32 b b d$ & $5,373.0$ & $5,374.0$ & $324 \mathrm{PRDX}$ & 0.9 & 0.020000 & Finely crystalline limestone \\
\hline s $1 d 41-25-32 b b d$ & $5,374.0$ & $5,375.0$ & 324PRDX & 0.7 & 0.020000 & Finely crystalline limestone \\
\hline s $1 d 41-25-32 b b d$ & $5,375.0$ & $5,376.0$ & 324PRDX & 0.9 & 0.010000 & Shaly, finely crystalline, dolomitic limestone \\
\hline s $1 d 41-25-32 b b d$ & $5,376.0$ & $5,377.0$ & $324 \mathrm{PRDX}$ & 1.7 & 0.010000 & Finely crystalline limestone \\
\hline s $1 d 41-25-32 b b d$ & $5,415.0$ & $5,416.0$ & $324 \mathrm{PRDX}$ & 2.9 & 0.040000 & Shaly, finely crystalline, dolomitic limestone \\
\hline s $1 d 41-25-32 b b d$ & $5,416.0$ & $5,417.0$ & $324 \mathrm{PRDX}$ & 5.1 & 0.160000 & Shaly, finely crystalline, dolomitic limestone \\
\hline s $1 d 41-25-32 b b d$ & $5,417.0$ & $5,418.0$ & $324 \mathrm{PRDX}$ & 5.2 & 0.040000 & Shaly, finely crystalline, dolomitic limestone \\
\hline s $1 d 41-25-32 b b d$ & $5,418.0$ & $5,419.0$ & $324 \mathrm{PRDX}$ & 5.6 & 0.120000 & Shaly, finely crystalline, dolomitic limestone \\
\hline s $1 d 41-25-32 b b d$ & $5,419.0$ & $5,420.0$ & 324PRDX & 6.1 & 0.020000 & Shaly, finely crystalline, dolomitic limestone \\
\hline s $1 d 41-25-32 b b d$ & $5,420.0$ & $5,421.0$ & $324 \mathrm{PRDX}$ & 6.2 & 0.020000 & Shaly, finely crystalline, dolomitic limestone \\
\hline $\operatorname{sid} 41-25-32 b b d$ & $5,421.0$ & $5,422.0$ & 324PRDX & 7.8 & 0.020000 & Shaly, finely crystalline, dolomitic limestone \\
\hline s $1 d 41-25-32 b b d$ & $5,422.0$ & $5,423.0$ & $324 \mathrm{PRDX}$ & 0.7 & 0.070000 & Anhydritic, medium- to coarse-crystalline limestone \\
\hline sld $41-25-32 b b d$ & $5,423.0$ & $5,424.0$ & $324 \mathrm{PRDX}$ & 0.4 & 0.070000 & Anhydritic, medium- to coarse-crystalline limestone \\
\hline s $1 d 41-25-32 b b d$ & $5,424.0$ & $5,425.0$ & $324 \mathrm{PRDX}$ & 0.4 & 0.040000 & Anhydritic, medium- to coarse-crystalline limestone \\
\hline s $1 d 41-25-32 b b d$ & $5,425.0$ & $5,426.0$ & $324 \mathrm{PRDX}$ & 0.5 & 0.070000 & Anhydritic, medium- to coarse-crystalline limestone \\
\hline s $1 d 41-25-32 b b d$ & $5,435.0$ & $5,436.0$ & 324PRDX & 0.4 & 0.012000 & Anhydritic, medium- to coarse-crystalline limestone \\
\hline sld $41-25-32 b b d$ & $5,436.0$ & $5,437.0$ & 324PRDX & 0.4 & 0.010000 & Anhydritic, medium- to coarse-crystalline limestone \\
\hline s $1 d 41-25-32 b b d$ & $5,440.0$ & $5,441.0$ & 324PRDX & 2.6 & 0.020000 & Carbonaceous, crystalline, dolomitic limestone \\
\hline sld41-25-32bbd & $5,441.0$ & $5,442.0$ & $324 \mathrm{PRDX}$ & 2.4 & 0.020000 & Carbonaceous, crystalline, dolomitic limestone \\
\hline s $1 d 41-25-32 b b d$ & $5,442.0$ & $5,443.0$ & $324 \mathrm{PRDX}$ & 5.0 & 0.020000 & Finely crystalline, dolomitic limestone \\
\hline s $1 d 41-25-32 b b d$ & $5,443.0$ & $5,444.0$ & $324 \mathrm{PRDX}$ & 4.2 & 0.020000 & Finely crystalline, dolomitic limestone \\
\hline sld $41-25-32 b b d$ & $5,444.0$ & $5,445.0$ & $324 \mathrm{PRDX}$ & 3.2 & 0.030000 & Finely crystalline limestone \\
\hline s $1 d 41-25-32 b b d$ & $5,445.0$ & $5,446.0$ & $324 \mathrm{PRDX}$ & 6.2 & 0.050000 & Vuggy, finely crystalline limestone \\
\hline s $1 d 41-25-32 b b d$ & $5,446.0$ & $5,447.0$ & $324 \mathrm{PRDX}$ & 10.2 & 1.000000 & Vuggy, finely crystalline limestone \\
\hline s $1 d 41-25-32 b b d$ & $5,447.0$ & $5,448.0$ & $324 \mathrm{PRDX}$ & 18.1 & 3.100000 & Vuggy, finely crystalline, dolomitic limestone \\
\hline s $1 d 41-25-32 b b d$ & $5,448.0$ & $5,449.0$ & 324PRDX & 12.9 & 0.130000 & Vuggy, finely crystalline, dolomitic limestone \\
\hline s $1 d 41-25-32 b b d$ & $5,449.0$ & $5,450.0$ & $324 \mathrm{PRDX}$ & 6.9 & 0.210000 & Vuggy, finely crystalline, dolomitic limestone \\
\hline sld $41-25-32 b b d$ & $5,450.0$ & $5,451.0$ & $324 \mathrm{PRDX}$ & 2.3 & 0.060000 & Finely crystalline limestone \\
\hline s $1 d 41-25-32 b b d$ & $5,451.0$ & $5,452.0$ & $324 \mathrm{PRDX}$ & 1.0 & 0.030000 & Finely crystalline limestone \\
\hline s $1 d 41-25-32 b b d$ & $5,452.0$ & $5,453.0$ & $324 \mathrm{PRDX}$ & 0.7 & 0.010000 & Finely crystalline limestone \\
\hline s $1 d 41-25-32 b b d$ & $5,453.0$ & $5,454.0$ & $324 \mathrm{PRDX}$ & 0.6 & 0.020000 & Finely crystalline limestone \\
\hline sld41-25-32bbd & $5,454.0$ & $5,455.0$ & 324PRDX & 1.2 & 0.040000 & Fine- to medium-crystalline limestone \\
\hline s $1 d 41-25-32 b b d$ & $5,455.0$ & $5,456.0$ & 324PRDX & 0.8 & 0.040000 & Finely crystalline limestone \\
\hline s $1 d 41-25-32 b b d$ & $5,456.0$ & $5,457.0$ & $324 \mathrm{PRDX}$ & 0.7 & 0.050000 & Finely crystalline limestone \\
\hline s $1 d 41-25-32 b b d$ & $5,457.0$ & $5,458.0$ & $324 \mathrm{PRDX}$ & 0.7 & 0.040000 & Finely crystalline limestone \\
\hline sld $41-25-32 b b d$ & $5,458.0$ & $5,459.0$ & $324 \mathrm{PRDX}$ & 0.4 & 0.130000 & Finely crystalline limestone \\
\hline sld $41-25-32 b b d$ & $5,459.0$ & $5,460.0$ & 324PRDX & 0.5 & 0.050000 & Finely crystalline limestone \\
\hline s $1 d 41-25-32 b b d$ & $5,460.0$ & $5,461.0$ & $324 \mathrm{PRDX}$ & 0.7 & 0.040000 & Anhydritic, finely crystalline limestone \\
\hline s $1 d 41-25-32 b b d$ & $5,461.0$ & $5,462.0$ & 324PRDX & 0.7 & 0.050000 & Anhydritic, finely crystalline limestone \\
\hline s $1 d 41-25-32 b b d$ & $5,462.0$ & $5,463.0$ & 324PRDX & 0.8 & 0.060000 & Anhydritic, finely crystalline limestone \\
\hline s $1 d 41-25-32 b b d$ & $5,463.0$ & $5,464.0$ & $324 \mathrm{PRDX}$ & 1.5 & 0.330000 & Anhydritic, finely crystalline limestone \\
\hline s $1 d 41-25-32 b b d$ & $5,464.0$ & $5,465.0$ & $324 \mathrm{PRDX}$ & 0.6 & 0.040000 & Anhydritic, finely crystalline limestone \\
\hline $\operatorname{sld} 41-25-32 b b d$ & $5,465.0$ & $5,466.0$ & 324PRDX & 0.8 & 0.120000 & Anhydritic, finely crystalline limestone \\
\hline s $1 d 41-25-32 b b d$ & $5,466.0$ & $5,467.0$ & $324 \mathrm{PRDX}$ & 0.8 & 0.080000 & Anhydritic, finely crystalline limestone \\
\hline
\end{tabular}


Table 4.--Laboratory-determined porosity and permeability--Continued

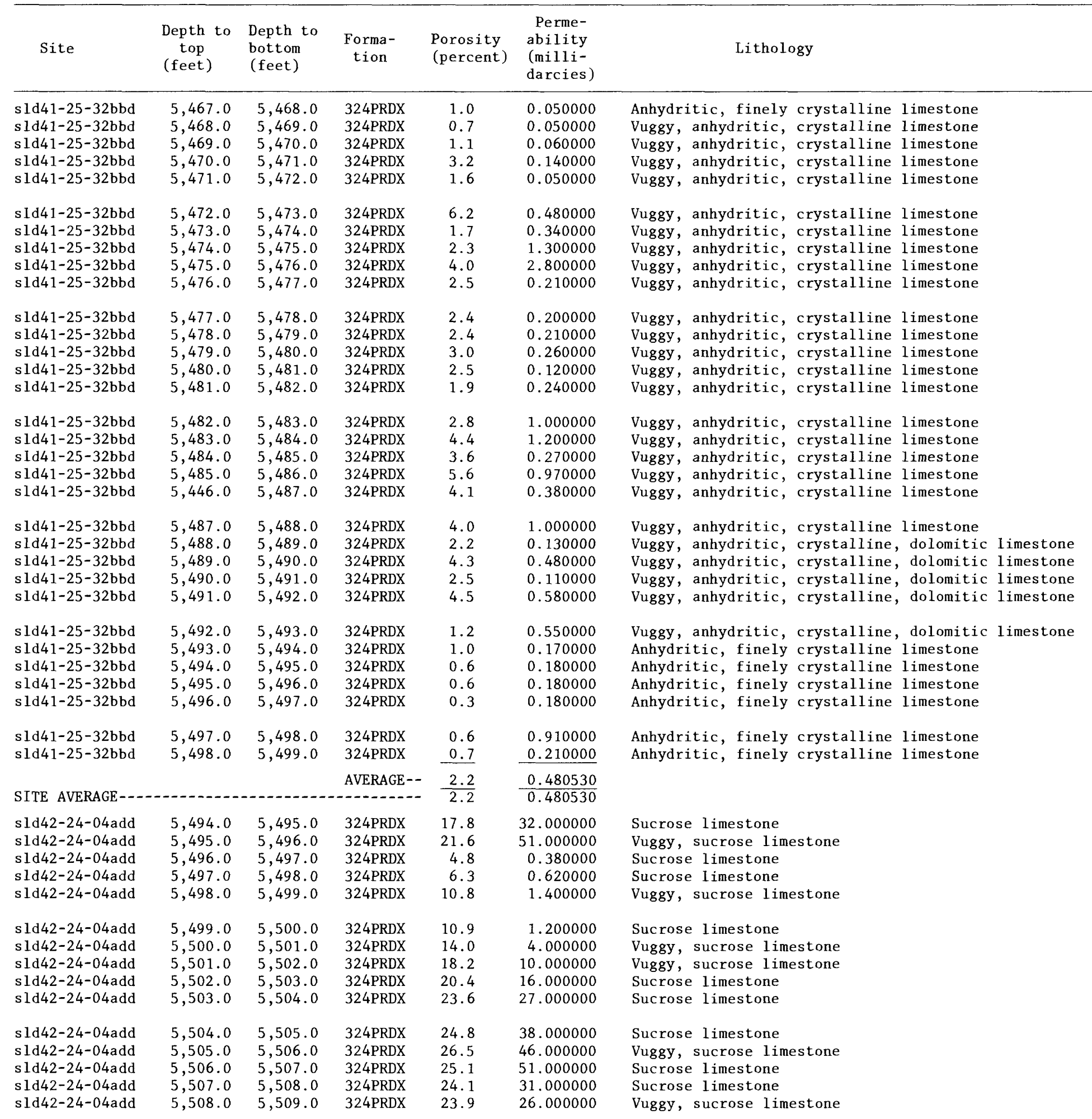


Table 4.--Laboratory-determined porosity and permeability--Continued

\begin{tabular}{|c|c|c|c|c|c|c|}
\hline Site & $\begin{array}{l}\text { Depth to } \\
\text { top } \\
\text { (feet) }\end{array}$ & $\begin{array}{l}\text { Depth to } \\
\text { bottom } \\
\text { (feet) }\end{array}$ & $\begin{array}{c}\text { Forma- } \\
\text { tion }\end{array}$ & $\begin{array}{l}\text { Porosity } \\
\text { (percent) }\end{array}$ & $\begin{array}{l}\text { Perme- } \\
\text { ability } \\
\text { (milli- } \\
\text { darcies) }\end{array}$ & Lithology \\
\hline $\begin{array}{l}\text { sld42-24-04add } \\
\text { sld42-24-04add } \\
\text { sld42-24-04add } \\
\text { sld42-24-04add } \\
\text { sld42-24-04add }\end{array}$ & $\begin{array}{l}5,509.0 \\
5,510.0 \\
5,511.0 \\
5,512.0 \\
5,513.0\end{array}$ & $\begin{array}{l}5,510.0 \\
5,511.0 \\
5,512.0 \\
5,513.0 \\
5,514.0\end{array}$ & $\begin{array}{l}324 \mathrm{PRDX} \\
324 \mathrm{PRDX} \\
324 \mathrm{PRDX} \\
324 \mathrm{PRDX} \\
324 \mathrm{PRDX}\end{array}$ & $\begin{array}{l}20.5 \\
13.9 \\
21.0 \\
23.9 \\
16.1\end{array}$ & $\begin{array}{r}9.200000 \\
9.000000 \\
21.000000 \\
37.000000 \\
8.900000\end{array}$ & $\begin{array}{l}\text { Sucrose limestone } \\
\text { Sucrose limestone } \\
\text { Sucrose limestone } \\
\text { Sucrose limestone } \\
\text { Vuggy, sucrose limestone }\end{array}$ \\
\hline $\begin{array}{l}\text { sld42-24-04add } \\
\text { sld42-24-04add } \\
\text { sld42-24-04add } \\
\text { sld42-24-04add } \\
\text { sld42-24-04add }\end{array}$ & $\begin{array}{l}5,514.0 \\
5,515.0 \\
5,516.0 \\
5,517.0 \\
5,518.0\end{array}$ & $\begin{array}{l}5,515.0 \\
5,516.0 \\
5,517.0 \\
5,518.0 \\
5,519.0\end{array}$ & $\begin{array}{l}324 \mathrm{PRDX} \\
324 \mathrm{PRDX} \\
324 \mathrm{PRDX} \\
324 \mathrm{PRDX} \\
324 \mathrm{PRDX}\end{array}$ & $\begin{array}{r}5.1 \\
5.2 \\
11.2 \\
10.0 \\
11.4\end{array}$ & $\begin{array}{r}0.370000 \\
0.100000 \\
0.490000 \\
36.000000 \\
1.400000\end{array}$ & $\begin{array}{l}\text { Vuggy, sucrose limestone } \\
\text { Vuggy, crystalline limestone } \\
\text { Vuggy, crystalline limestone } \\
\text { Crystalline limestone } \\
\text { Vuggy, crystalline limestone }\end{array}$ \\
\hline $\begin{array}{l}\text { sld42-24-04add } \\
\text { sld42-24-04add } \\
\text { sld42-24-04add } \\
\text { sld42-24-04add } \\
\text { sld42-24-04add }\end{array}$ & $\begin{array}{l}5,519.0 \\
5,520.0 \\
5,521.0 \\
5,522.0 \\
5,523.0\end{array}$ & $\begin{array}{l}5,520.0 \\
5,521.0 \\
5,522.0 \\
5,523.0 \\
5,524.0\end{array}$ & $\begin{array}{l}\text { 324PRDX } \\
324 \mathrm{PRDX} \\
324 \mathrm{PRDX} \\
324 \mathrm{PRDX} \\
324 \mathrm{PRDX}\end{array}$ & $\begin{array}{r}8.3 \\
15.2 \\
14.6 \\
15.2 \\
18.9\end{array}$ & $\begin{array}{r}7.500000 \\
61.000000 \\
0.290000 \\
0.700000 \\
3.900000\end{array}$ & $\begin{array}{l}\text { Vuggy, crystalline limestone } \\
\text { Vuggy, crystalline limestone } \\
\text { Vuggy, crystalline limestone } \\
\text { Vuggy, sucrose limestone } \\
\text { Vuggy, sucrose limestone }\end{array}$ \\
\hline $\begin{array}{l}\text { sld42-24-04add } \\
\text { sld42-24-04add } \\
\text { sld42-24-04add } \\
\text { sld42-24-04add } \\
\text { sld42-24-04add }\end{array}$ & $\begin{array}{l}5,524.0 \\
5,525.0 \\
5,526.0 \\
5,527.0 \\
5,528.0\end{array}$ & $\begin{array}{l}5,525.0 \\
5,526.0 \\
5,527.0 \\
5,528.0 \\
5,529.0\end{array}$ & $\begin{array}{l}324 \mathrm{PRDX} \\
324 \mathrm{PRDX} \\
324 \mathrm{PRDX} \\
324 \mathrm{PRDX} \\
324 \mathrm{PRDX}\end{array}$ & $\begin{array}{r}21.1 \\
10.3 \\
7.1 \\
0.8 \\
1.1\end{array}$ & $\begin{array}{l}2.300000 \\
0.710000 \\
2.800000 \\
0.310000 \\
0.310000\end{array}$ & $\begin{array}{l}\text { Vuggy, sucrose limestone } \\
\text { Vuggy, crystalline limestone } \\
\text { Finely crystalline limestone } \\
\text { Finely crystalline limestone } \\
\text { Finely crystalline limestone }\end{array}$ \\
\hline $\begin{array}{l}\text { sld42-24-04add } \\
\text { sld42-24-04add } \\
\text { sld42-24-04add } \\
\text { sld42-24-04add } \\
\text { sld42-24-04add }\end{array}$ & $\begin{array}{l}5,529.0 \\
5,543.0 \\
5,544.0 \\
5,545.0 \\
5,546.0\end{array}$ & $\begin{array}{l}5,530.0 \\
5,544.0 \\
5,545.0 \\
5,546.0 \\
5,547.0\end{array}$ & $\begin{array}{l}324 \mathrm{PRDX} \\
324 \mathrm{PRDX} \\
324 \mathrm{PRDX} \\
324 \mathrm{PRDX} \\
324 \mathrm{PRDX}\end{array}$ & $\begin{array}{l}0.7 \\
4.2 \\
2.5 \\
0.6 \\
1.1\end{array}$ & $\begin{array}{l}0.070000 \\
1.900000 \\
0.660000 \\
0.020000 \\
0.030000\end{array}$ & $\begin{array}{l}\text { Finely crystalline limestone } \\
\text { Carbonaceous, crystalline limestone } \\
\text { Crystalline limestone } \\
\text { Vuggy, crystalline limestone } \\
\text { Vuggy, crystalline limestone }\end{array}$ \\
\hline $\begin{array}{l}\text { sld42-24-04add } \\
\text { sld42-24-04add } \\
\text { sld42-24-04add } \\
\text { sld42-24-04add } \\
\text { sld42-24-04add }\end{array}$ & $\begin{array}{l}5,547.0 \\
5,548.0 \\
5,549.0 \\
5,550.0 \\
5,551.0\end{array}$ & $\begin{array}{l}5,548.0 \\
5,549.0 \\
5,550.0 \\
5,551.0 \\
5,552.0\end{array}$ & $\begin{array}{l}\text { 324PRDX } \\
324 \mathrm{PRDX} \\
324 \mathrm{PRDX} \\
324 \mathrm{PRDX} \\
\text { 324PRDX }\end{array}$ & $\begin{array}{l}1.2 \\
0.8 \\
0.6 \\
3.7 \\
6.9\end{array}$ & $\begin{array}{l}2.900000 \\
0.080000 \\
0.110000 \\
0.130000 \\
0.200000\end{array}$ & $\begin{array}{l}\text { Crystalline limestone } \\
\text { Crystalline limestone } \\
\text { Crystalline limestone } \\
\text { Crystalline limestone } \\
\text { Vuggy, crystalline limestone }\end{array}$ \\
\hline $\begin{array}{l}\text { sld42-24-04add } \\
\text { sld42-24-04add } \\
\text { sld42-24-04add } \\
\text { sld42-24-04add } \\
\text { sld42-24-04add }\end{array}$ & $\begin{array}{l}5,552.0 \\
5,553.0 \\
5,554.0 \\
5,555.0 \\
5,572.0\end{array}$ & $\begin{array}{l}5,553.0 \\
5,554.0 \\
5,555.0 \\
5,556.0 \\
5,573.0\end{array}$ & $\begin{array}{l}\text { 324PRDX } \\
324 \mathrm{PRDX} \\
324 \mathrm{PRDX} \\
324 \mathrm{PRDX} \\
\text { 324PRDX }\end{array}$ & $\begin{array}{l}4.2 \\
5.2 \\
5.1 \\
3.6 \\
1.7\end{array}$ & $\begin{array}{l}7.600000 \\
2.500000 \\
1.200000 \\
0.180000 \\
0.160000\end{array}$ & $\begin{array}{l}\text { Vuggy, crystalline limestone } \\
\text { Vuggy, crystalline limestone } \\
\text { Vuggy, crystalline limestone } \\
\text { Crystalline limestone } \\
\text { Crystalline limestone }\end{array}$ \\
\hline $\begin{array}{l}\text { s1d42-24-04add } \\
\text { sld42-24-04add } \\
\text { s } 1 d 42-24-04 \text { add } \\
\text { sld42-24-04add } \\
\text { sld42-24-04add }\end{array}$ & $\begin{array}{l}5,573.0 \\
5,574.0 \\
5,575.0 \\
5,576.0 \\
5,577.0\end{array}$ & $\begin{array}{l}5,574.0 \\
5,575.0 \\
5,576.0 \\
5,577.0 \\
5,578.0\end{array}$ & $\begin{array}{l}324 \mathrm{PRDX} \\
324 \mathrm{PRDX} \\
324 \mathrm{PRDX} \\
324 \mathrm{PRDX} \\
324 \mathrm{PRDX}\end{array}$ & $\begin{array}{l}3.2 \\
3.2 \\
3.9 \\
6.7 \\
4.1\end{array}$ & $\begin{array}{l}0.130000 \\
0.070000 \\
0.490000 \\
1.100000 \\
0.350000\end{array}$ & $\begin{array}{l}\text { Crystalline limestone } \\
\text { Vuggy, crystalline limestone } \\
\text { Vuggy, crystalline limestone } \\
\text { Vuggy, crystalline limestone } \\
\text { Finely crystalline limestone }\end{array}$ \\
\hline $\begin{array}{l}\text { sld42-24-04add } \\
\text { s1d42-24-04add } \\
\text { sld42-24-04add } \\
\text { sld } 42-24-04 \text { add } \\
\text { sld } 42-24-04 \text { add }\end{array}$ & $\begin{array}{l}5,578.0 \\
5,582.0 \\
5,583.0 \\
5,584.0 \\
5,585.0\end{array}$ & $\begin{array}{l}5,579.0 \\
5,583.0 \\
5,584.0 \\
5,585.0 \\
5,586.0\end{array}$ & $\begin{array}{l}\text { 324PRDX } \\
324 \mathrm{PRDX} \\
324 \mathrm{PRDX} \\
324 \mathrm{PRDX} \\
\text { 324PRDX }\end{array}$ & $\begin{array}{l}3.9 \\
0.8 \\
6.1 \\
8.2 \\
8.6\end{array}$ & $\begin{array}{l}2.500000 \\
0.090000 \\
0.770000 \\
0.440000 \\
2.500000\end{array}$ & $\begin{array}{l}\text { Crystalline limestone } \\
\text { Fine-grained limestone } \\
\text { Vuggy, crystalline limestone } \\
\text { Vuggy, crystalline limestone } \\
\text { Vuggy, crystalline limestone }\end{array}$ \\
\hline $\begin{array}{l}\text { sld42-24-04add } \\
\text { sld42-24-04add } \\
\text { sld42-24-04add } \\
\text { sld42-24-04add } \\
\text { sld42-24-04add }\end{array}$ & $\begin{array}{l}5,586.0 \\
5,587.0 \\
5,588.0 \\
5,589.0 \\
5,590.0\end{array}$ & $\begin{array}{l}5,587.0 \\
5,588.0 \\
5,589.0 \\
5,590.0 \\
5,591.0\end{array}$ & $\begin{array}{l}324 \mathrm{PRDX} \\
324 \mathrm{PRDX} \\
324 \mathrm{PRDX} \\
324 \mathrm{PRDX} \\
324 \mathrm{PRDX}\end{array}$ & $\begin{array}{l}1.0 \\
4.5 \\
5.6 \\
8.8 \\
6.1\end{array}$ & $\begin{array}{l}1.000000 \\
2.100000 \\
0.980000 \\
1.700000 \\
1.100000\end{array}$ & $\begin{array}{l}\text { Vuggy, crystalline limestone } \\
\text { Vuggy, crystalline limestone } \\
\text { Vuggy, crystalline limestone } \\
\text { Vuggy, crystalline limestone } \\
\text { Vuggy, crystalline limestone }\end{array}$ \\
\hline
\end{tabular}


Table 4.--Laboratory-determined porosity and permeability--Continued

\begin{tabular}{|c|c|c|c|c|c|c|}
\hline Site & $\begin{array}{l}\text { Depth to } \\
\text { top } \\
\text { (feet) }\end{array}$ & $\begin{array}{l}\text { Depth to } \\
\text { bottom } \\
\text { (feet) }\end{array}$ & $\begin{array}{l}\text { Forma- } \\
\text { tion }\end{array}$ & $\begin{array}{l}\text { Porosity } \\
\text { (percent) }\end{array}$ & $\begin{array}{l}\text { Perme- } \\
\text { ability } \\
\text { (milli- } \\
\text { darcies) }\end{array}$ & Lithology \\
\hline $\begin{array}{l}\text { sld42-24-04add } \\
\text { sld42-24-04add } \\
\text { sld42-24-04add } \\
\text { sld42-24-04add } \\
\text { sld42-24-04add }\end{array}$ & $\begin{array}{l}5,591.0 \\
5,592.0 \\
5,593.0 \\
5,594.0 \\
5,595.0\end{array}$ & $\begin{array}{l}5,592.0 \\
5,593.0 \\
5,594.0 \\
5,595.0 \\
5,596.0\end{array}$ & $\begin{array}{l}\text { 324PRDX } \\
324 \mathrm{PRDX} \\
324 \mathrm{PRDX} \\
324 \mathrm{PRDX} \\
324 \mathrm{PRDX}\end{array}$ & $\begin{array}{l}4.2 \\
8.0 \\
9.0 \\
7.2 \\
6.7\end{array}$ & $\begin{array}{l}0.200000 \\
4.900000 \\
9.500000 \\
4.800000 \\
1.900000\end{array}$ & $\begin{array}{l}\text { Vuggy, crystalline limestone } \\
\text { Vuggy, crystalline limestone } \\
\text { Vuggy, crystalline limestone } \\
\text { Vuggy, crystalline limestone } \\
\text { Vuggy, crystalline limestone }\end{array}$ \\
\hline $\begin{array}{l}\text { sld42-24-04add } \\
\text { sld42-24-04add } \\
\text { sld42-24-04add } \\
\text { sld42-24-04add } \\
\text { s1d42-24-04add }\end{array}$ & $\begin{array}{l}5,596.0 \\
5,597.0 \\
5,598.0 \\
5,599.0 \\
5,614.0\end{array}$ & $\begin{array}{l}5,597.0 \\
5,598.0 \\
5,599.0 \\
5,600.0 \\
5,615.0\end{array}$ & $\begin{array}{l}\text { 324PRDX } \\
324 \mathrm{PRDX} \\
324 \mathrm{PRDX} \\
324 \mathrm{PRDX} \\
\text { 324PRDX }\end{array}$ & $\begin{array}{r}7.3 \\
3.9 \\
3.3 \\
3.5 \\
10.8\end{array}$ & $\begin{array}{l}6.500000 \\
0.100000 \\
0.110000 \\
0.090000 \\
1.800000\end{array}$ & $\begin{array}{l}\text { Vuggy limestone } \\
\text { Carbonaceous, crystalline limestone } \\
\text { Crystalline limestone } \\
\text { Finely crystalline limestone } \\
\text { Sucrose limestone }\end{array}$ \\
\hline $\begin{array}{l}\text { sld42-24-04add } \\
\text { sld42-24-04add } \\
\text { sld42-24-04add } \\
\text { sld42-24-04add } \\
\text { sld42-24-04add }\end{array}$ & $\begin{array}{l}5,615.0 \\
5,616.0 \\
5,617.0 \\
5,622.0 \\
5,623.0\end{array}$ & $\begin{array}{l}5,616.0 \\
5,617.0 \\
5,618.0 \\
5,623.0 \\
5,624.0\end{array}$ & $\begin{array}{l}324 \mathrm{PRDX} \\
324 \mathrm{PRDX} \\
324 \mathrm{PRDX} \\
324 \mathrm{PRDX} \\
324 \mathrm{PRDX}\end{array}$ & $\begin{array}{r}17.1 \\
20.0 \\
13.9 \\
3.8 \\
5.1\end{array}$ & $\begin{array}{l}1.700000 \\
6.300000 \\
0.900000 \\
1.300000 \\
0.470000\end{array}$ & $\begin{array}{l}\text { Sucrose limestone } \\
\text { Fine-grained limestone } \\
\text { Fine-grained limestone } \\
\text { Fine-grained limestone } \\
\text { Crystalline limestone }\end{array}$ \\
\hline $\begin{array}{l}\text { sld42-24-04add } \\
\text { sld42-24-04add } \\
\text { sld42-24-04add } \\
\text { sld42-24-04add } \\
\text { sld42-24-04add }\end{array}$ & $\begin{array}{l}5,624.0 \\
5,625.0 \\
5,626.0 \\
5,627.0 \\
5,628.0\end{array}$ & $\begin{array}{l}5,625 \cdot 0 \\
5,626 \cdot 0 \\
5,627 \cdot 0 \\
5,628 \cdot 0 \\
5,629 \cdot 0\end{array}$ & $\begin{array}{l}324 \mathrm{PRDX} \\
324 \mathrm{PRDX} \\
324 \mathrm{PRDX} \\
324 \mathrm{PRDX} \\
324 \mathrm{PRDX}\end{array}$ & $\begin{array}{l}4.4 \\
4.5 \\
3.4 \\
4.4 \\
2.9\end{array}$ & $\begin{array}{l}6.400000 \\
0.560000 \\
0.360000 \\
0.400000 \\
0.130000\end{array}$ & $\begin{array}{l}\text { Vuggy, finely crystalline limestone } \\
\text { Crystalline limestone } \\
\text { Limestone } \\
\text { Crystalline limestone } \\
\text { Crystalline limestone }\end{array}$ \\
\hline $\begin{array}{l}\text { s1d42-24-04add } \\
\text { sld42-24-04add }\end{array}$ & $\begin{array}{l}5,629.0 \\
5,630.0\end{array}$ & $\begin{array}{l}5,630.0 \\
5,631.0\end{array}$ & $\begin{array}{l}\text { 324PRDX } \\
\text { 324PRDX }\end{array}$ & $\begin{array}{l}2.8 \\
2.0 \\
\end{array}$ & $\begin{array}{l}0.170000 \\
5.500000 \\
\end{array}$ & $\begin{array}{l}\text { Crystalline limestone } \\
\text { Finely crystalline limestone }\end{array}$ \\
\hline SITE AVERAGE--- & $---\infty$ & ------- & AVERAGE-- & $\frac{9.1}{9.1}$ & $\frac{7.195747}{7.195747}$ & \\
\hline $\begin{array}{l}\text { sld42-26-29dad } \\
\text { s } 1 \text { d } 42-26-29 \text { dad } \\
\text { sld42-26-29dad } \\
\text { s } 1 \text { d } 42-26-29 \text { dad } \\
\text { sld42-26-29dad }\end{array}$ & $\begin{array}{l}5,735.0 \\
5,736.0 \\
5,737.0 \\
5,738.0 \\
5,739.0\end{array}$ & $\begin{array}{l}5,736.0 \\
5,737.0 \\
5,738.0 \\
5,739.0 \\
5,740.0\end{array}$ & $\begin{array}{l}\text { 324PRDX } \\
324 \mathrm{PRDX} \\
324 \mathrm{PRDX} \\
324 \mathrm{PRDX} \\
324 \mathrm{PRDX}\end{array}$ & $\begin{array}{l}2.5 \\
6.7 \\
4.4 \\
5.9 \\
8.3\end{array}$ & $\begin{array}{r}0.100000 \\
0.100000 \\
0.010000 \\
0.060000 \\
142.000000\end{array}$ & $\begin{array}{l}\text { Shaly, fossiliferous limestone } \\
\text { Shaly, fossiliferous limestone } \\
\text { Shaly, fossiliferous limestone } \\
\text { Shaly, fossiliferous limestone } \\
\text { Shaly, fossiliferous limestone }\end{array}$ \\
\hline $\begin{array}{l}\text { s } 1 \text { d } 42-26-29 \text { dad } \\
\text { sld42-26-29dad } \\
\text { sld42-26-29dad } \\
\text { sld42-26-29dad } \\
\text { sld42-26-29dad }\end{array}$ & $\begin{array}{l}5,740.0 \\
5,741.0 \\
5,742.0 \\
5,743.0 \\
5,744.0\end{array}$ & $\begin{array}{l}5,741.0 \\
5,742.0 \\
5,743.0 \\
5,744.0 \\
5,745.0\end{array}$ & $\begin{array}{l}324 \mathrm{PRDX} \\
324 \mathrm{PRDX} \\
324 \mathrm{PRDX} \\
324 \mathrm{PRDX} \\
324 \mathrm{PRDX}\end{array}$ & $\begin{array}{r}11.2 \\
11.7 \\
13.3 \\
9.5 \\
9.4\end{array}$ & $\begin{array}{r}37.000000 \\
52.000000 \\
2.700000 \\
1.100000 \\
5.500000\end{array}$ & $\begin{array}{l}\text { Shaly, fossiliferous limestone } \\
\text { Shaly, fossiliferous limestone } \\
\text { Shaly, fossiliferous limestone } \\
\text { Shaly, fossiliferous limestone } \\
\text { Shaly, fossiliferous limestone }\end{array}$ \\
\hline $\begin{array}{l}\text { s } 1 d 42-26-29 \text { dad } \\
\text { s } 1 \text { d42-26-29dad } \\
\text { sld42-26-29dad }\end{array}$ & $\begin{array}{l}5,745.0 \\
5,746.0 \\
5,749.0\end{array}$ & $\begin{array}{l}5,746.0 \\
5,747.0 \\
5,750.0\end{array}$ & $\begin{array}{l}324 \mathrm{PRDX} \\
324 \mathrm{PRDX} \\
324 \mathrm{PRDX}\end{array}$ & $\begin{array}{l}7.5 \\
6.7 \\
1.4 \\
\end{array}$ & $\begin{array}{l}0.600000 \\
0.650000 \\
0.010000 \\
\end{array}$ & $\begin{array}{l}\text { Shaly, fossiliferous limestone } \\
\text { Shaly, fossiliferous limestone } \\
\text { Shaly, fine-grained limestone }\end{array}$ \\
\hline SITE AVERAGE--- & . & & AVERAGE-- & $\frac{7.6}{7.6}$ & $\frac{18.602308}{18.602308}$ & \\
\hline ua $02-01-18 a b a$ & 0.0 & 0.4 & 321 WEBR & 18.8 & 30.000000 & Cross-bedded sandstone \\
\hline SITE AVERAGE-- & ----1 & $=-1$ & AVERAGE-- & $\frac{18.8}{\frac{18.8}{6.5}}$ & $\frac{\frac{30.000000}{30.000000}}{21.733119}$ & \\
\hline
\end{tabular}


Table 5.--In-situ permeability and hydraulic conductivity

1. Formation codes are defined in table 1.

2. Depths to the top and bottom of tested intervals are measured from land surface, top of casing, the drilling platform, or the kelly bushing. The measuring point was unspecified or was not recorded in compiling the data in this table. An error of no more than 20 feet is introduced by assuming that the measuring point was the land surface. The number 99,999 in either of these columns means that the depth is unknown.

3. Test type--abbreviations used include: lab--estimated from average laboratory-determined permeability of core samples; lab:oc--estimated from average laboratory-determined permeability of outcrop samples; and geophys--estimated from average geophysically determined porosity in the interval. Other test types listed are largely self-explanatory and include slug (constant-rate) pumping, flow(ing well), bailing, (pressure) injection, drill-stem, and airlift (pumping) tests.

4. Permeability--All permeability values listed in this table either were determined directly by drill-stem tests or were converted to equivalent in-situ permeability from laboratory determined permeability, laboratory determined porosity, or geophysically determined porosity, by equations in table 2 and equations 5 and 6.

5. Hydraulic conductivity--Values in this column were measured directly by pressure-injection tests, calculated from transmissivity (usually by dividing by test-interval thickness), or calculated by equation 7 from in-situ permeability.

6. All values reported in this table are rounded to two significant figures (trailing zeroes are not significant).

7. Lithology--Lithologic descriptions are condensed from descriptions on geologic logs of test intervals and are considered subjective, rather than quantitative. The term shale in this column when used with siltstone can mean either mudstone, claystone, or marl.

8. Source--Sources used for information presented in this table are stated in condensed form to save space. Abbreviations used in this column include: CDWR--Colorado Division of Water Resources (Denver, Colo.); PI--Petroleum Information Corp. (Denver, Colo.); USBR--U.S. Bureau of Reclamation (Denver, Colo., and Salt Lake City, Utah); USGS--U.S. Geological Survey; Woodward-Clyde--Woodward-Clyde Consultants Corp. (Denver, Colo.). 


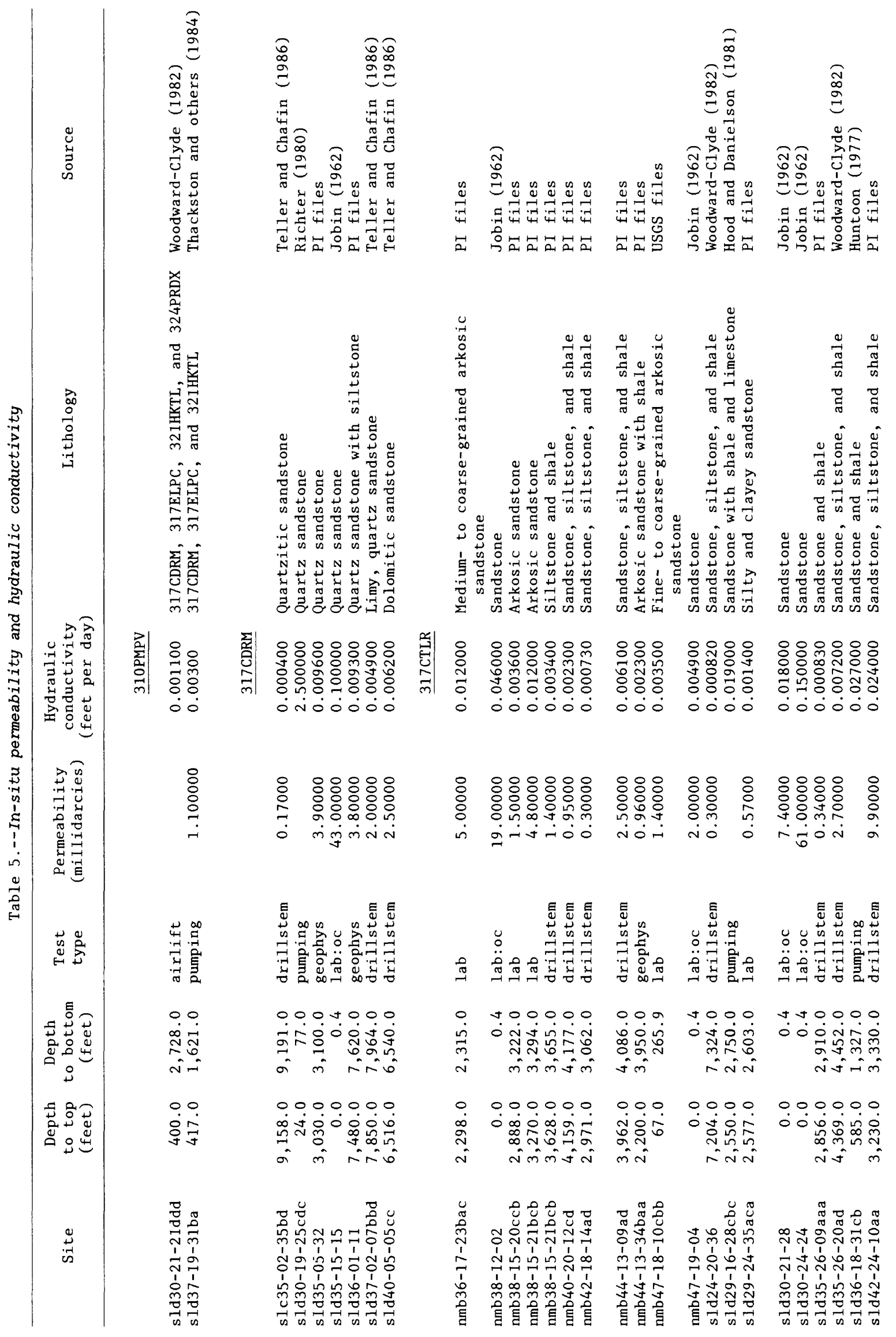




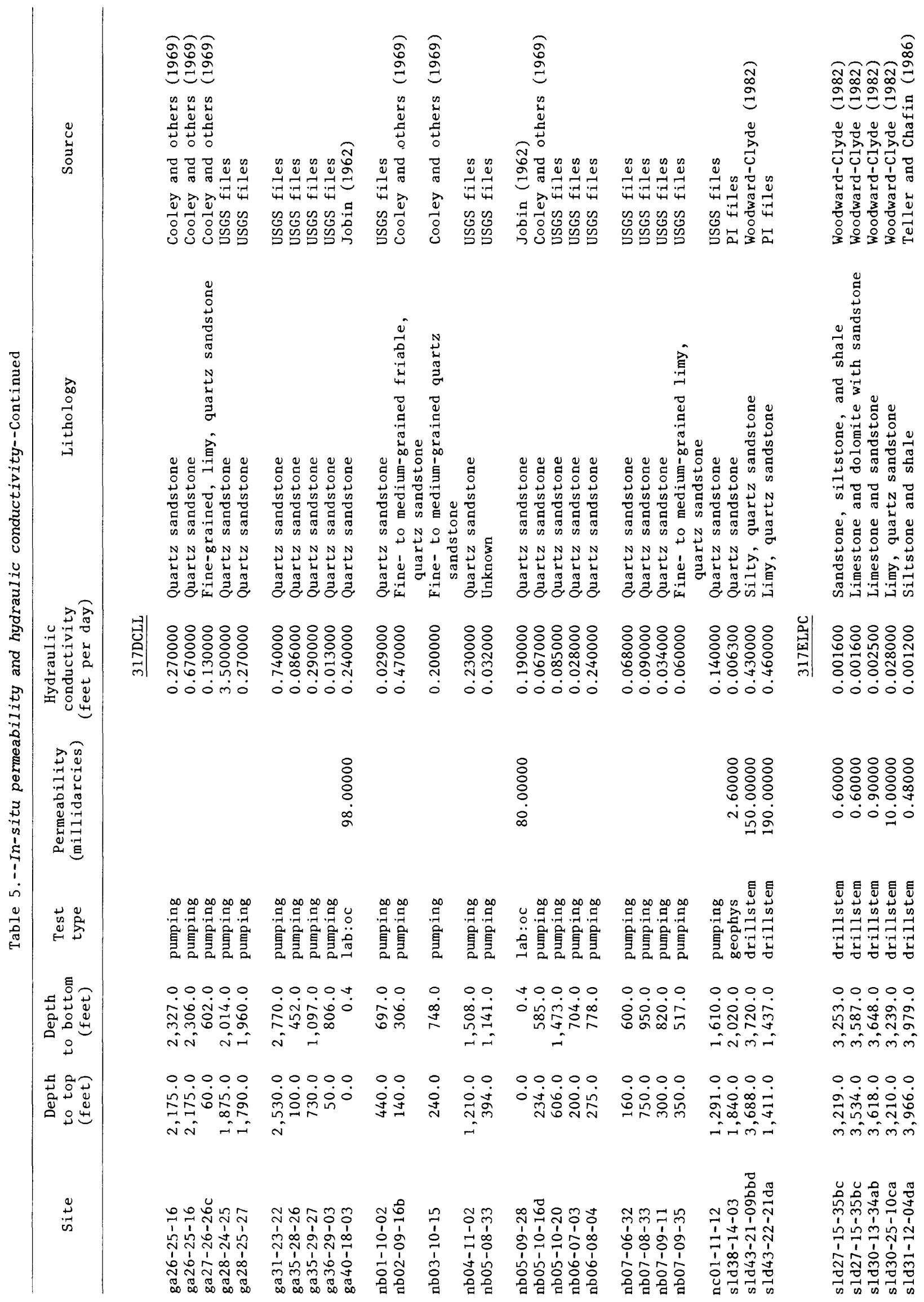




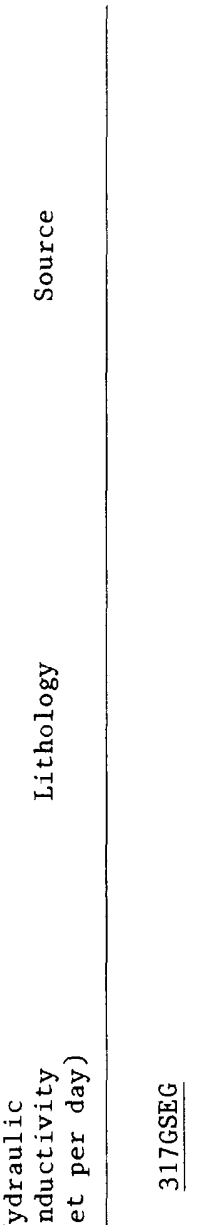

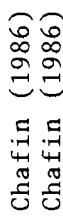

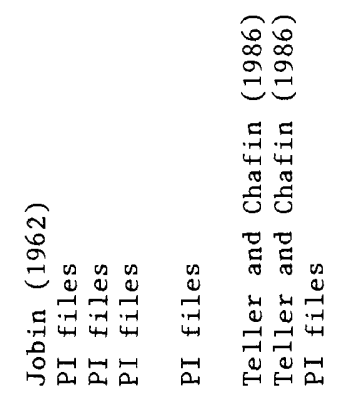

華蕠。

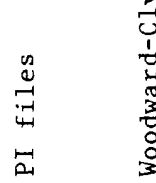

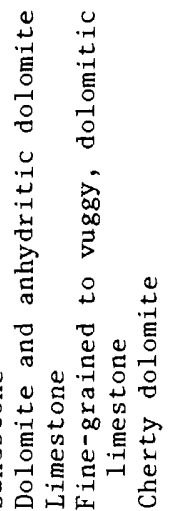

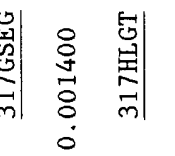

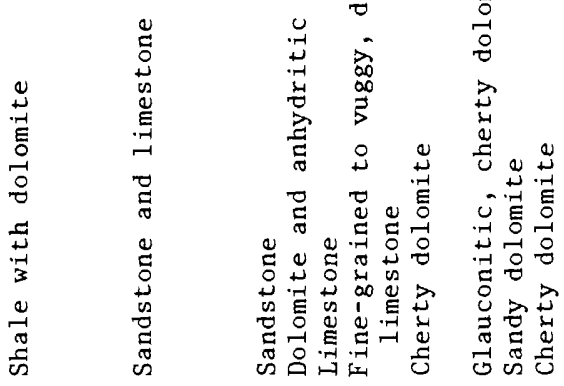

玄

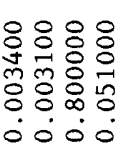

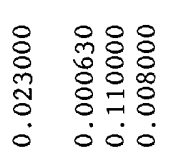

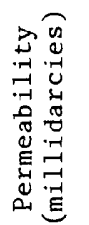

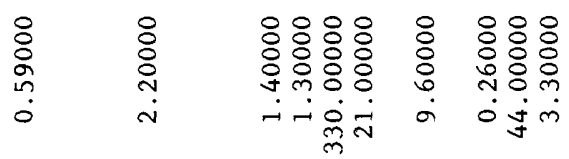

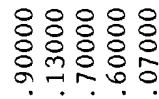

융유용요

त्रकं लं

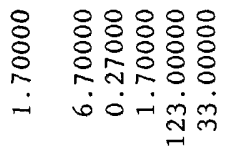

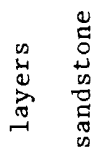

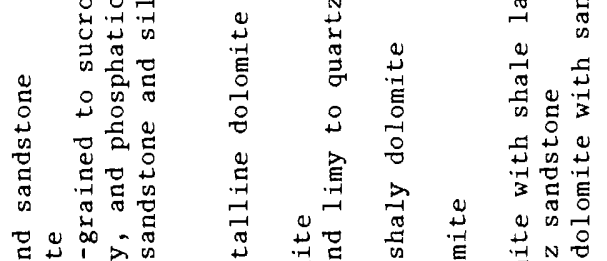

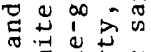

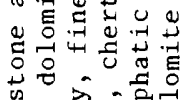

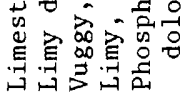

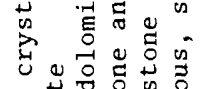

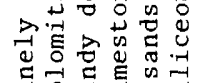

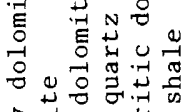

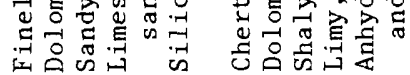

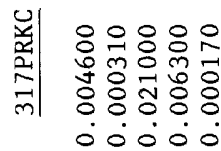

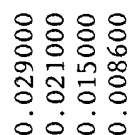

0
$\stackrel{0}{1}$
0
0
0

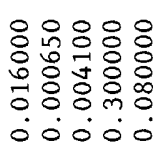

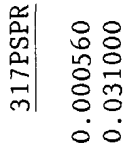

응유유



0000

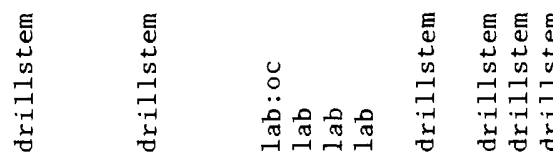

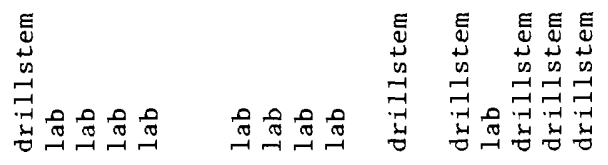

$\begin{array}{ll}0 & 0 \\ \stackrel{0}{\pi} & \dot{m} \\ 0 & \dot{m}\end{array}$

T000 0000

00000

0000

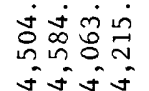

000000

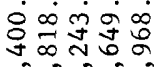

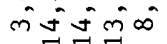

in mintiós

00000

0.00

$\circ$

00000

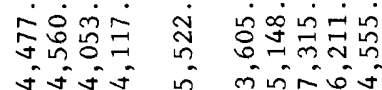

ivisio.

Nin

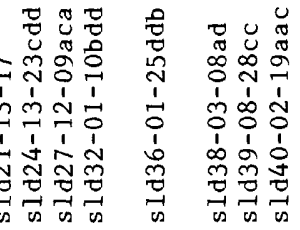

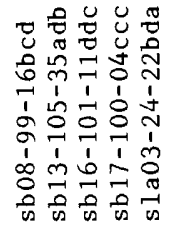

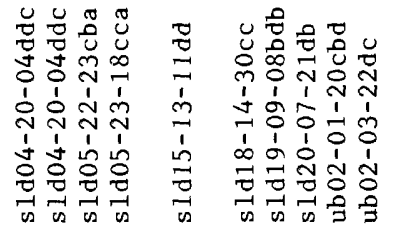

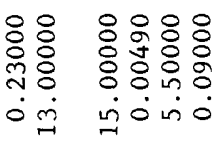

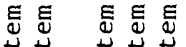

齐弯

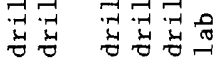

000000

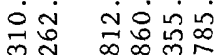

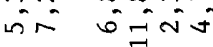

000000

ำ ำำง

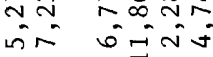

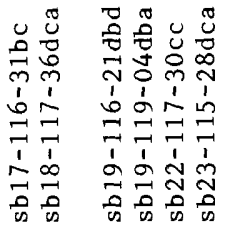




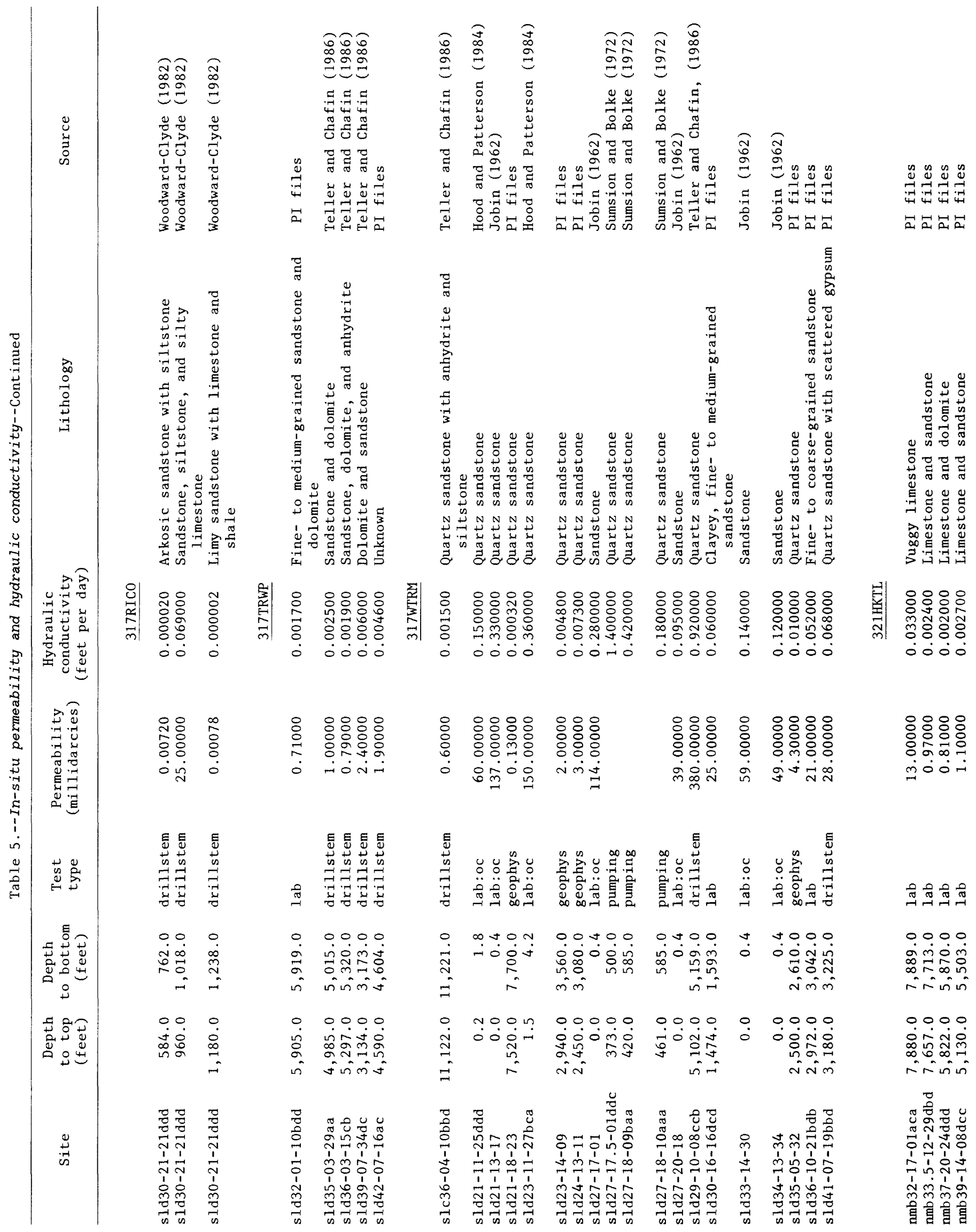




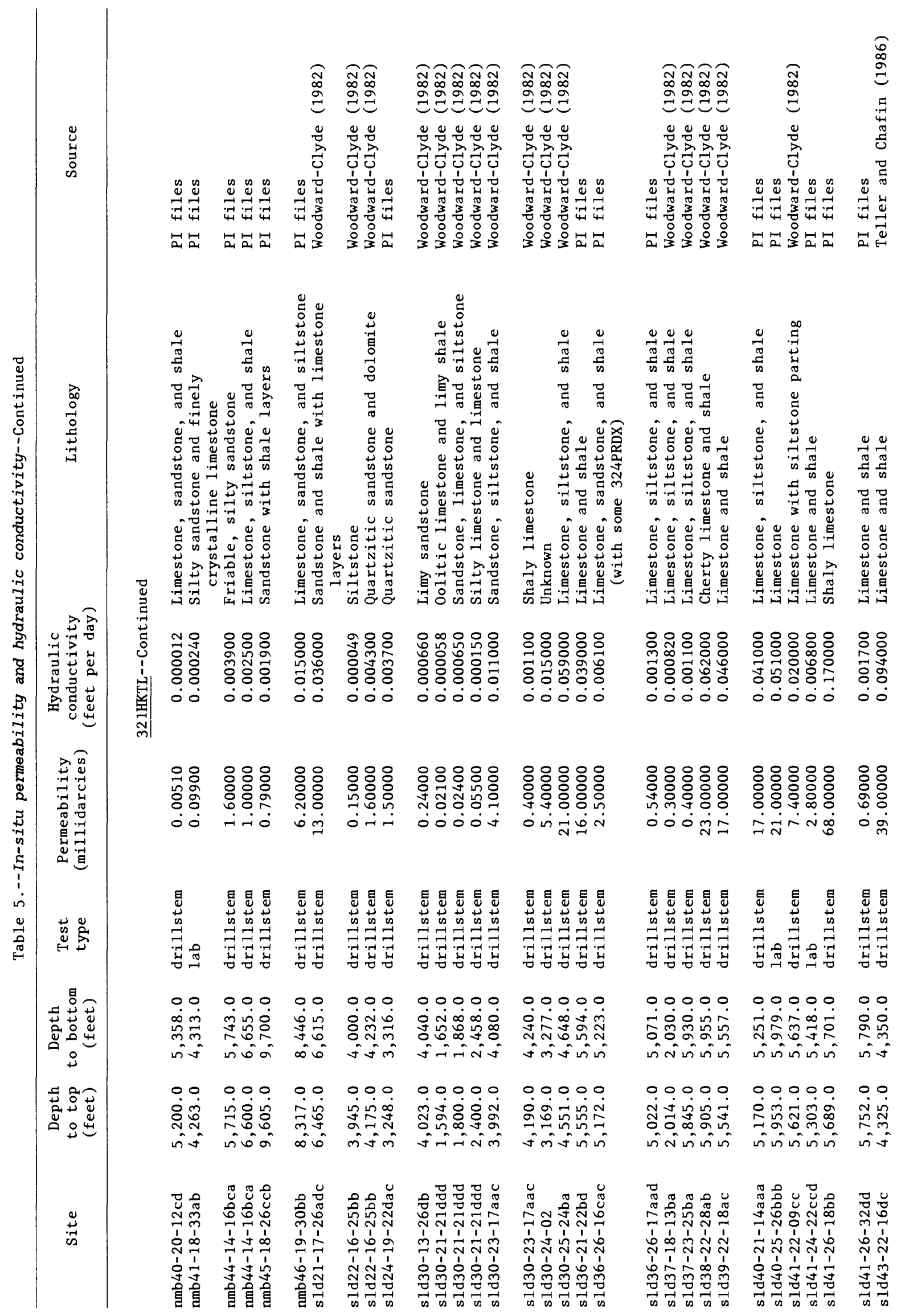




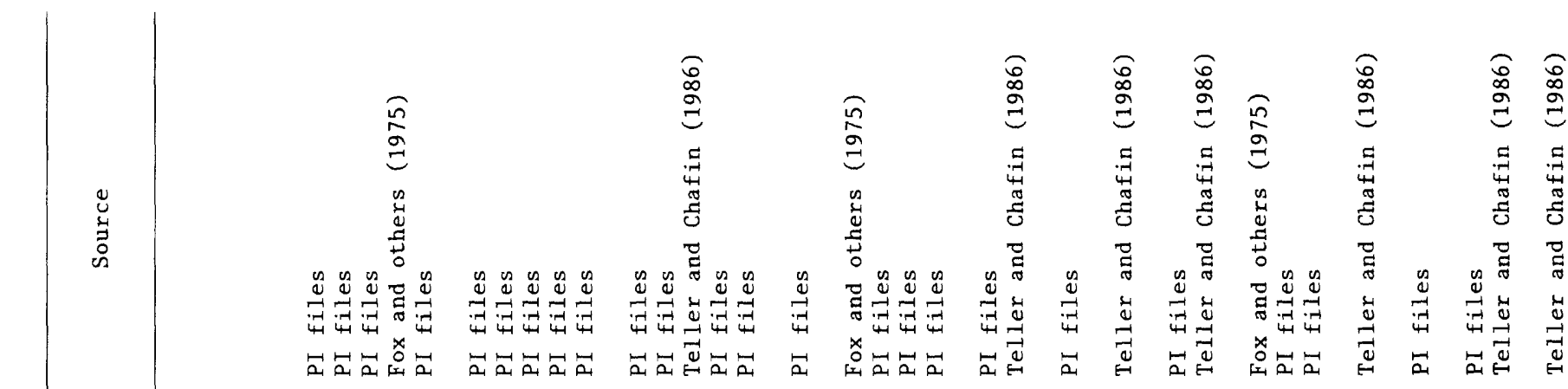

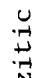

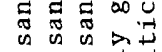

$N N N$

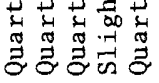

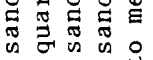

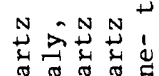

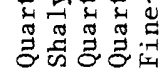

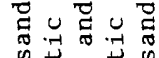

N N

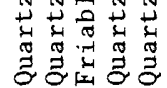

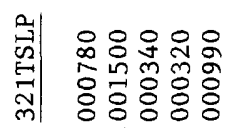

00000

운융융유유

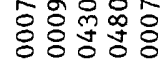

융ㅇㅇㅇㅇㅇㅇ

00000

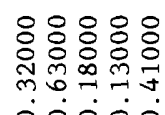

융: : :

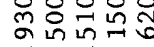

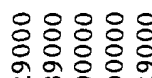

4⿳亠丷⿵冂丶

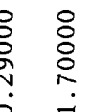

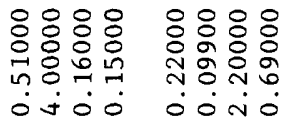

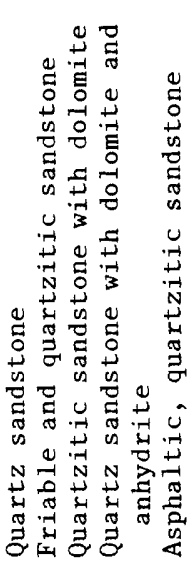

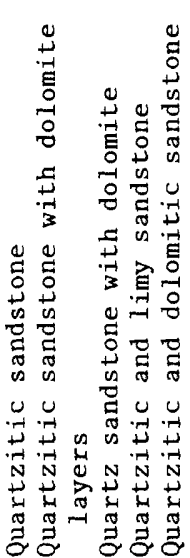

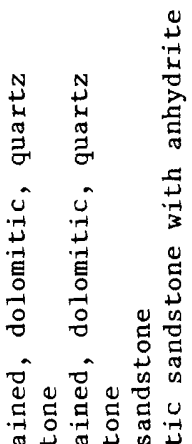

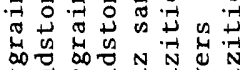

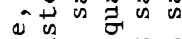

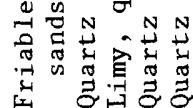

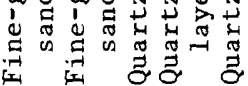

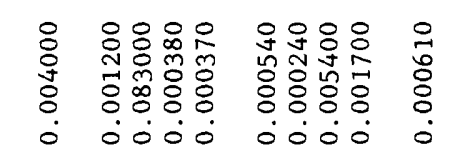

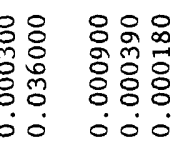

$\begin{array}{ll}\circ & 8 \\ 0 & 0 \\ \vdots & \vdots \\ 0 & 0 \\ 0 & 0\end{array}$

0
융
8
8
0
0
0

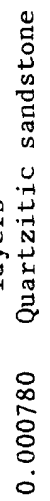

00000

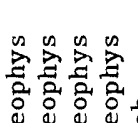

ह

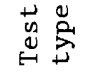

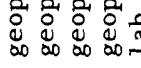

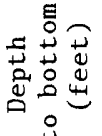

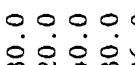

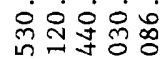

官完宫宫品

00 办

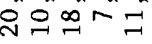

ज云望声

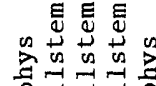

\section{西}

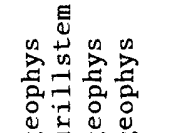

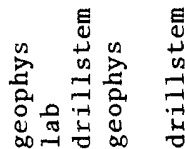

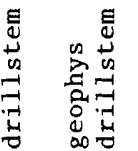

齐

离

융융

00000.

䒘守这岕客

00000

00000

00000

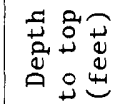

ลำラ

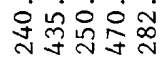

ज全至式

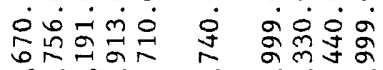

जn०0:

รํำ

ํํㅇํㅇㅇํㅇ

=50

i.

$\dot{0} \dot{0}$

000

$\stackrel{0}{\dot{5}}$

in

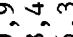

00000

- 0000

0000

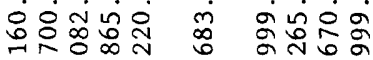

$\infty$ ำ

iุ

00

000

जि的全=

ร융ํํ

Iño

各点

ूे

0

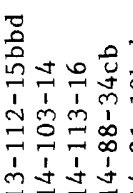

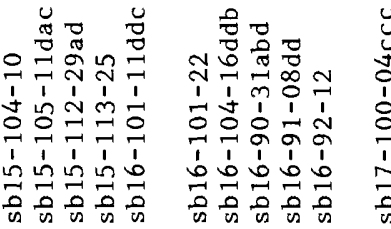

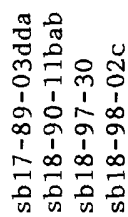

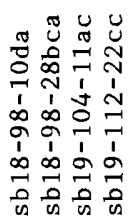

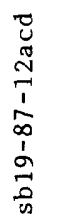

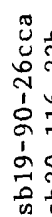

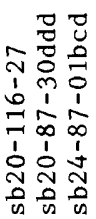

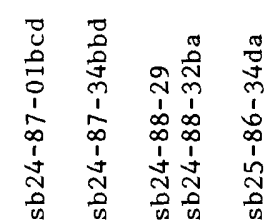




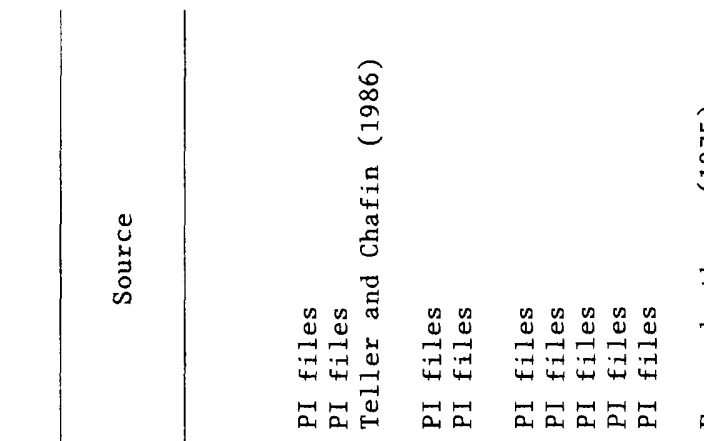

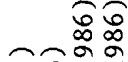

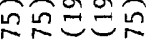

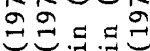

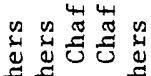

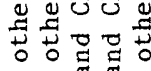

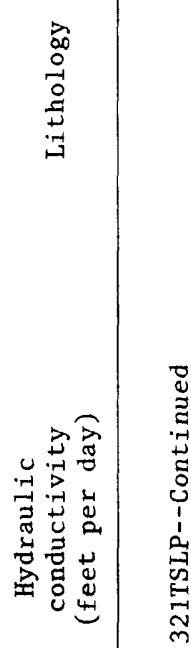

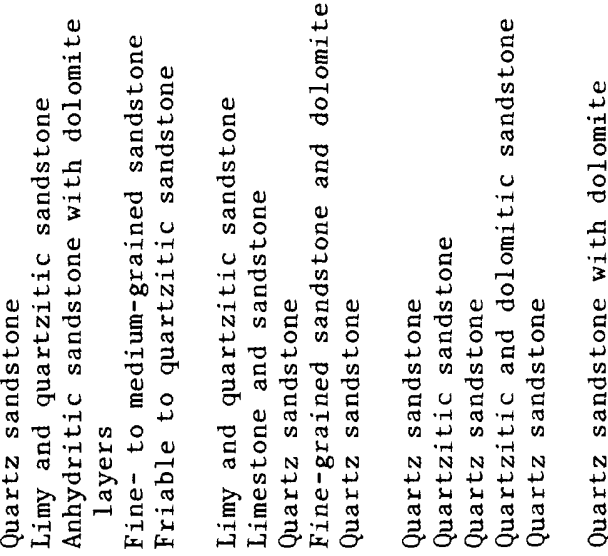

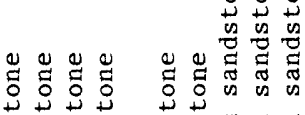

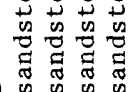

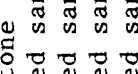

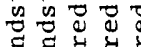

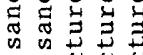

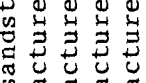

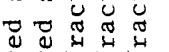

里哭

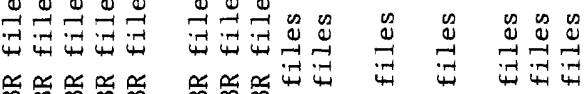

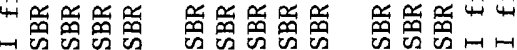

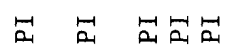
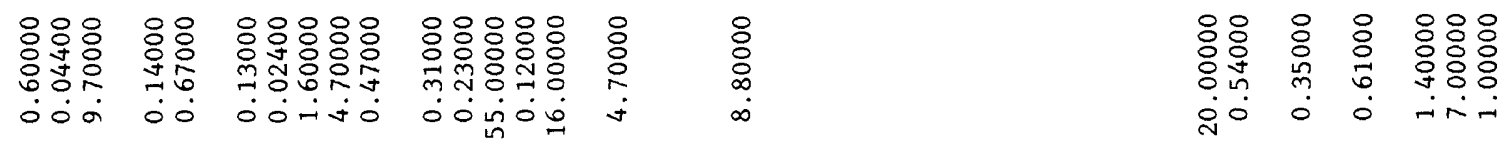

辰

岁苔

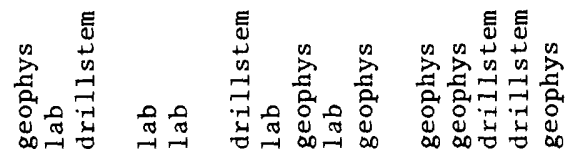

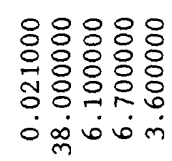

양융융융유

ivivi

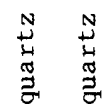

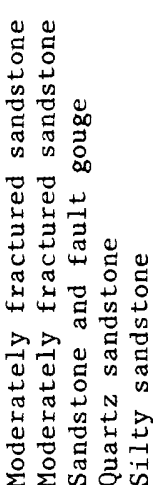

寒

$\Rightarrow$

क

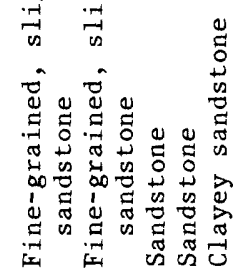

0000000000000

官官:

000000

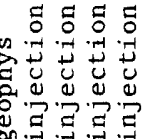

동형형

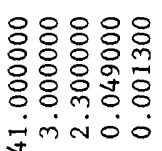

0
$\circ$
0
0
0

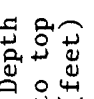

finin fin

于

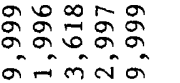

00000

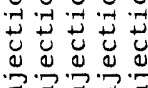

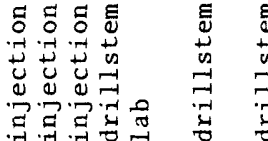

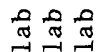

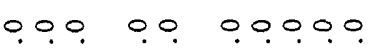

00000

ूंजु

कुष

$00+6$

$+N-0.0$

0.000

0 ఫิ ఫ)

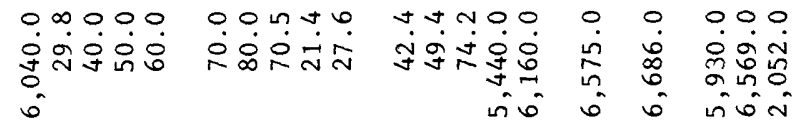

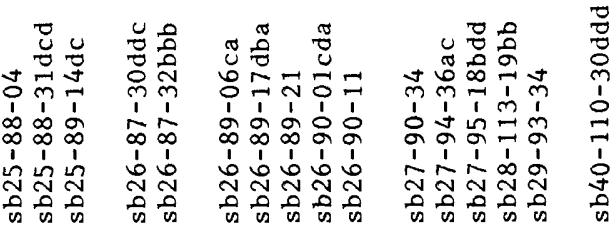

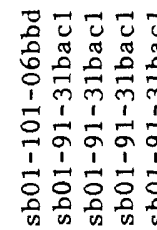

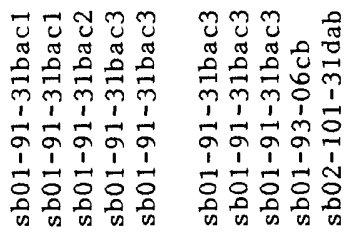

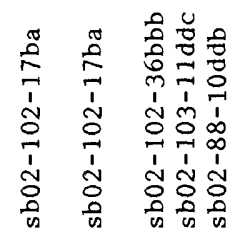




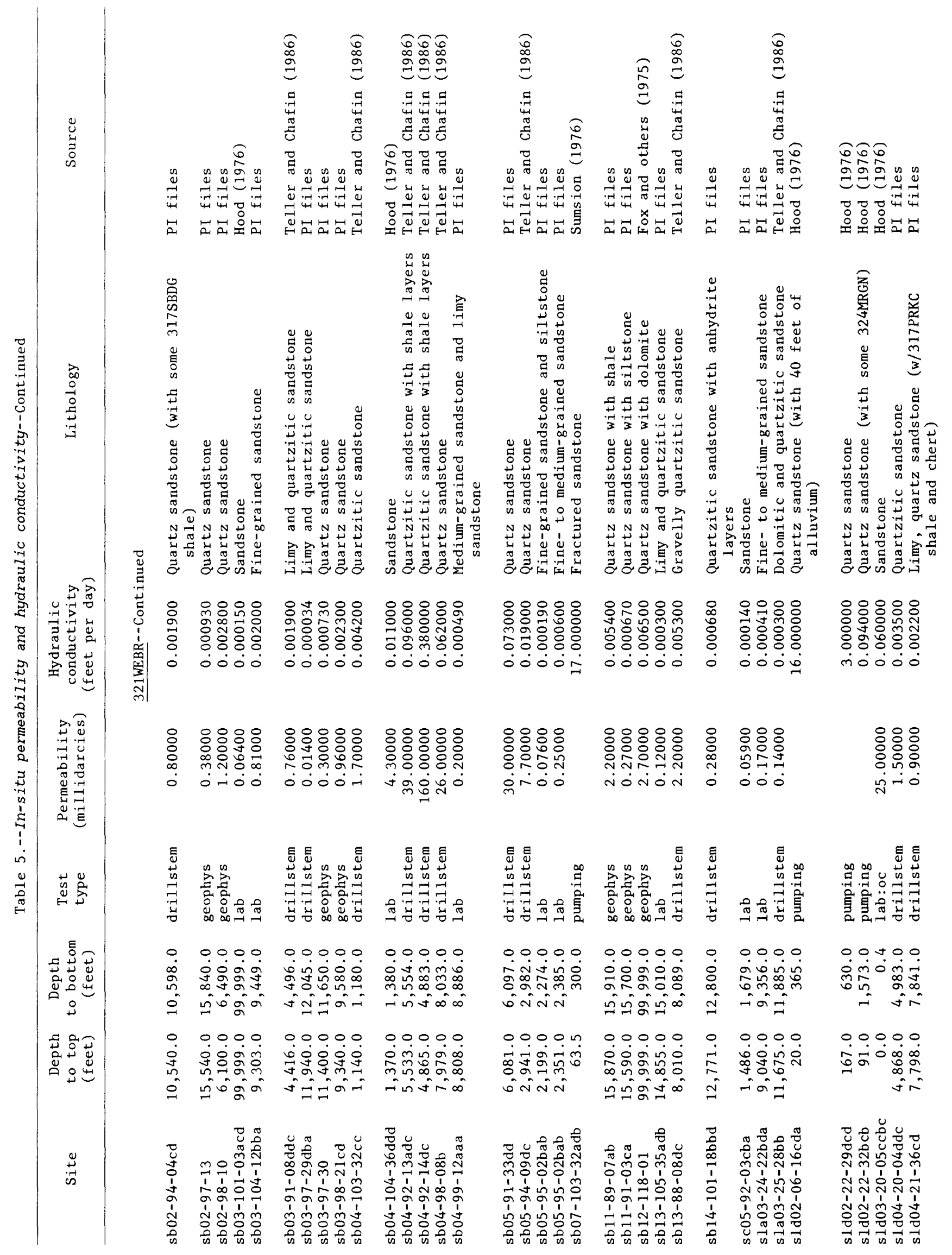




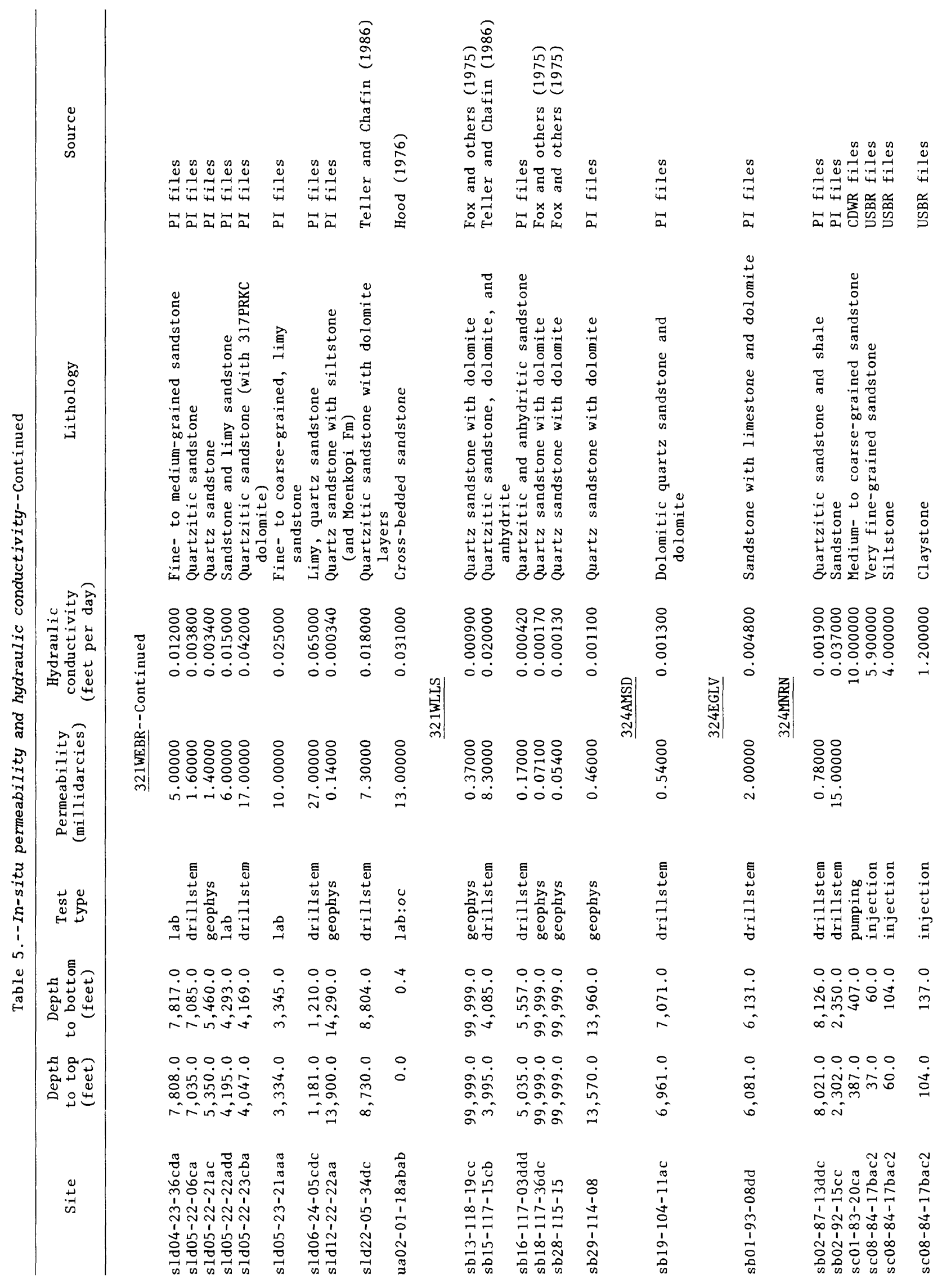




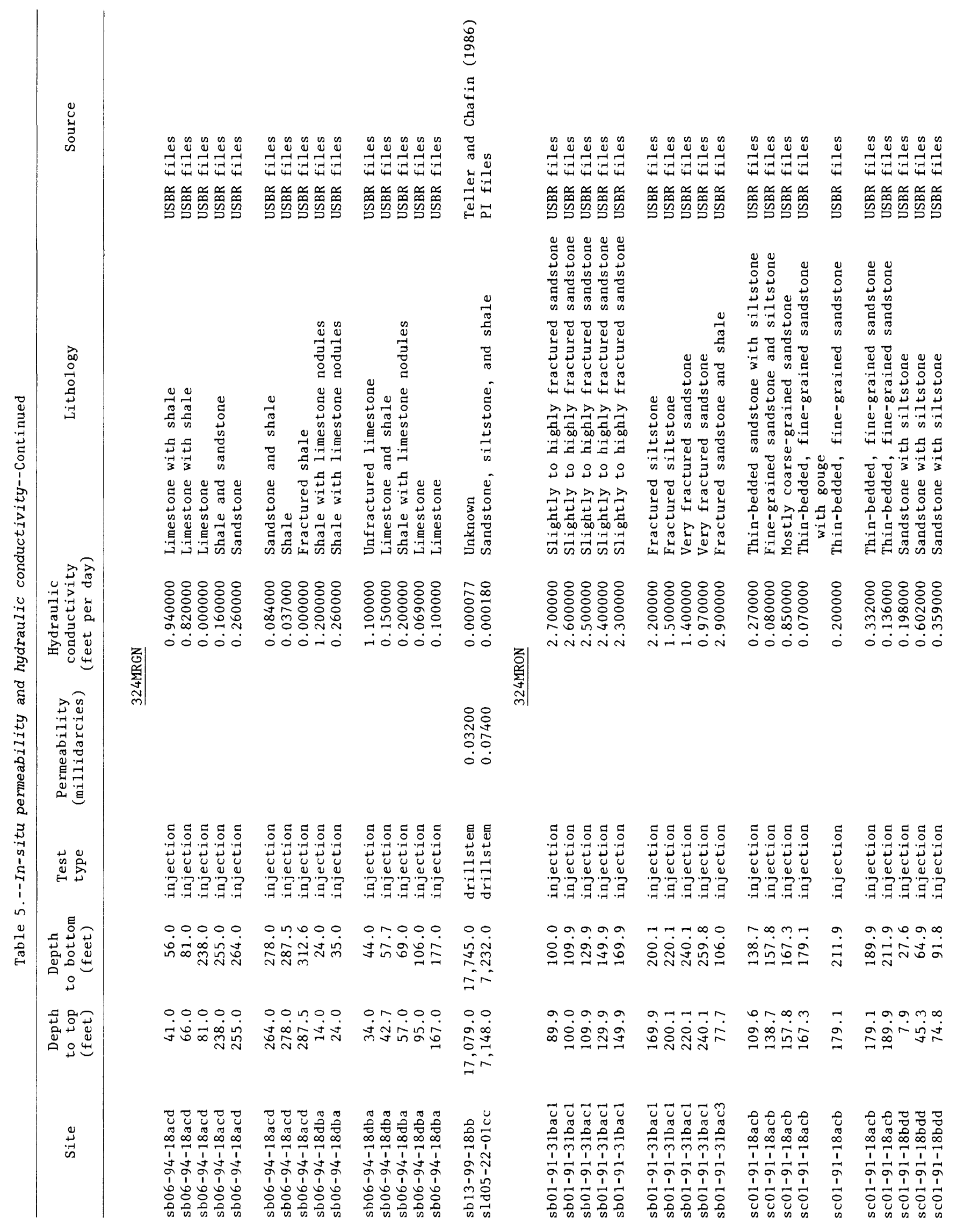




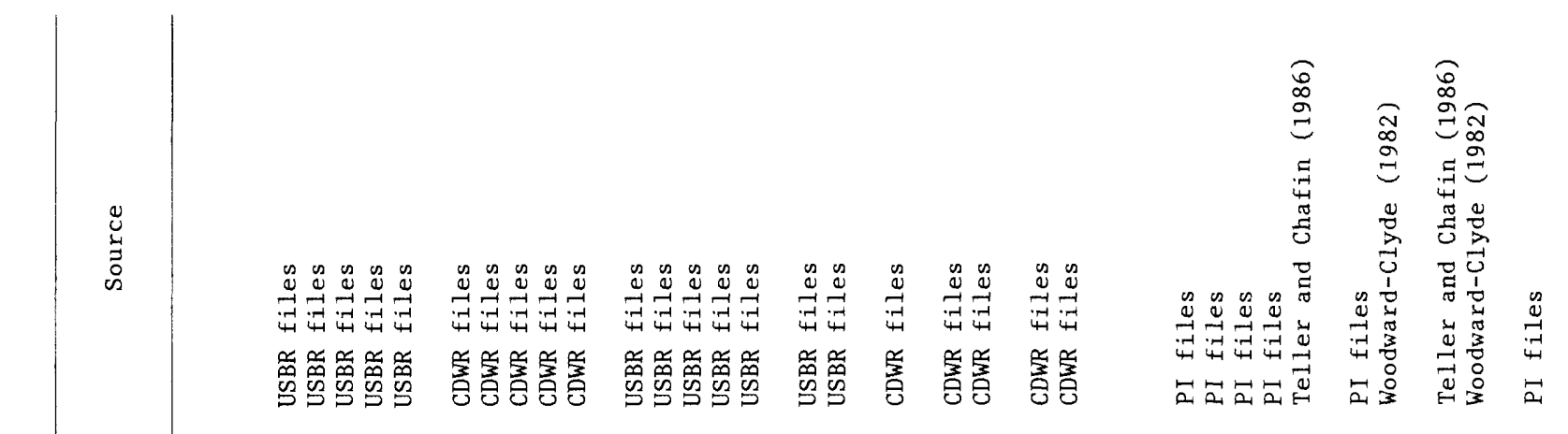

(1)
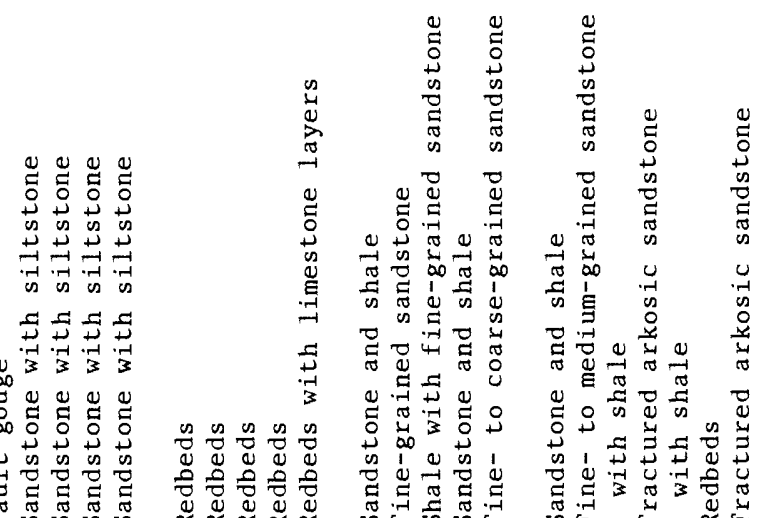

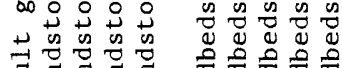

记

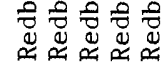

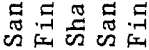

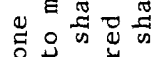

事递

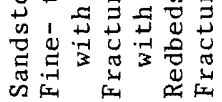

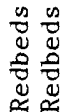

융ㅇㅇㅇㅇㅠ

กู่

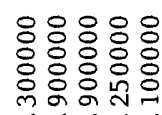

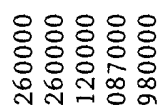

융

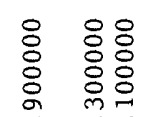

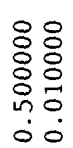

章

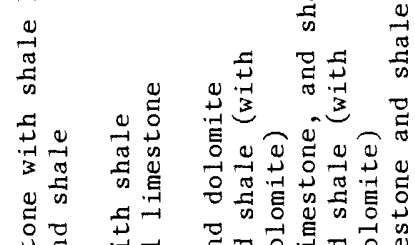

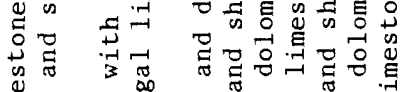

00000

نnivo

00000

ori

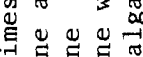

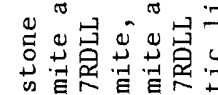

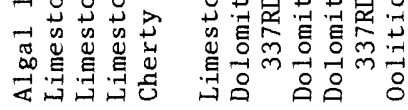

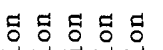

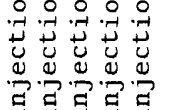

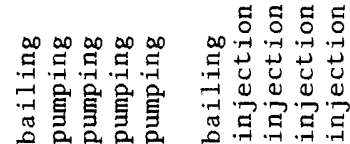

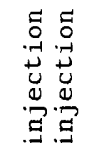

\section{:}

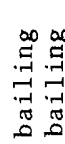

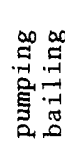

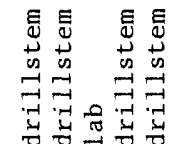

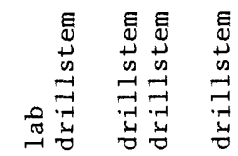

至

$\neg \rightarrow \infty 0$

00000

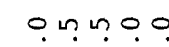

으

- 0000

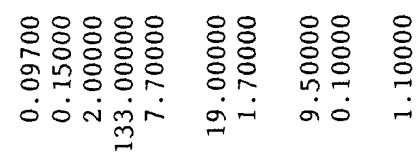

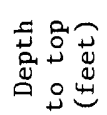

กิm

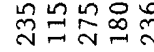

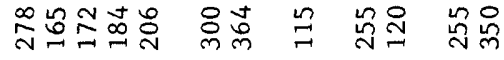

00000

00000

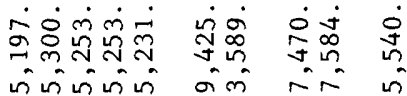

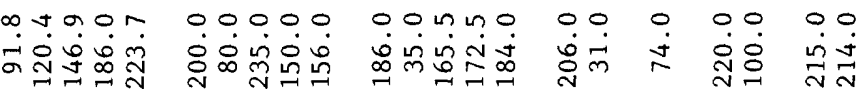

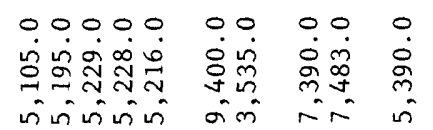

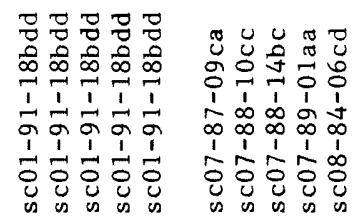

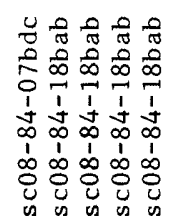
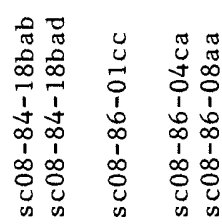

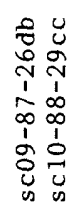

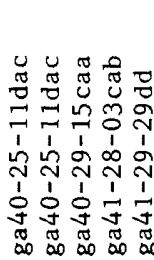

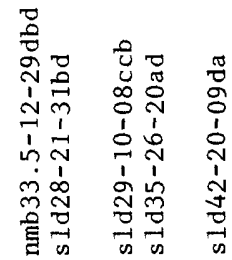




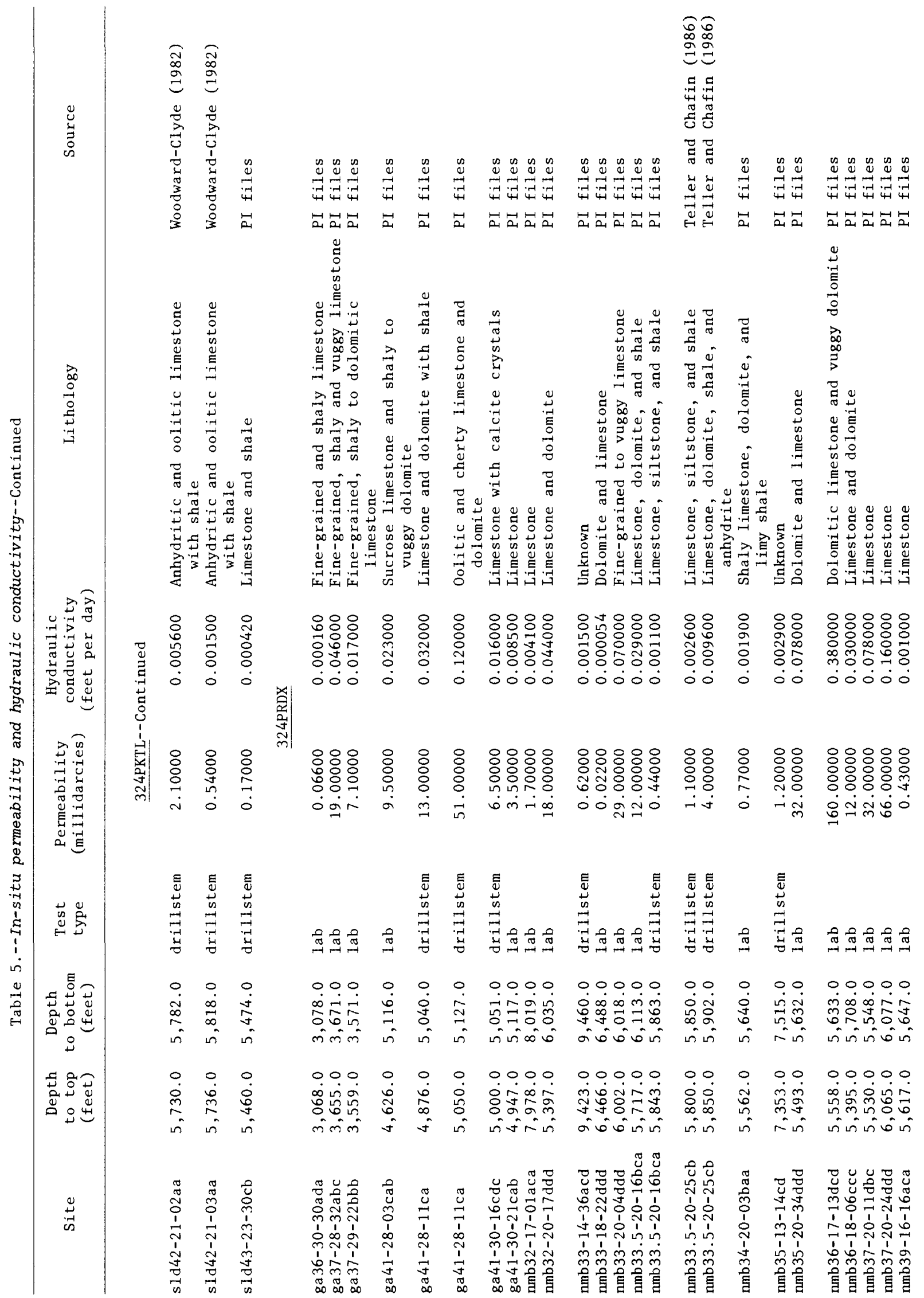




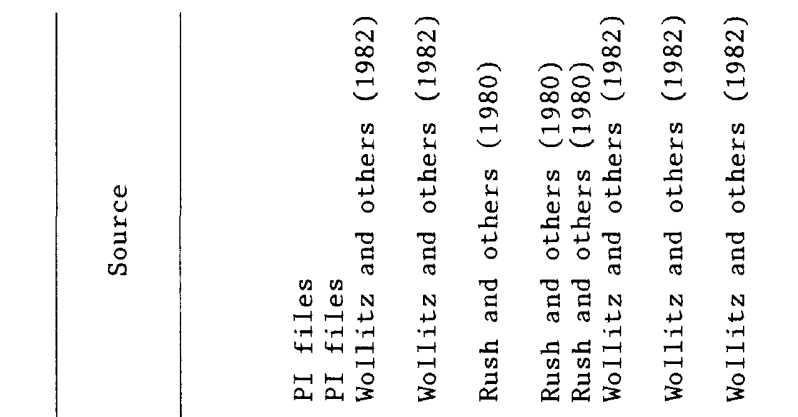

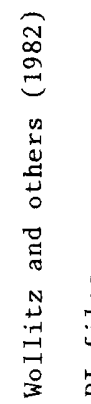
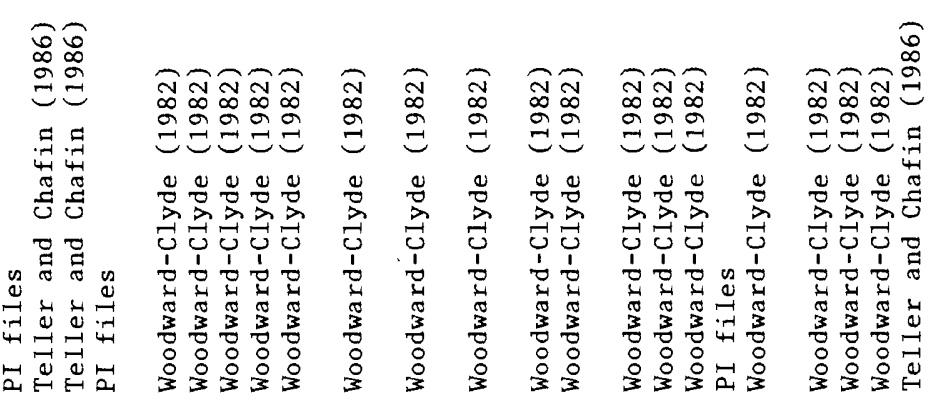

$\frac{2}{2}$

용요영

\begin{tabular}{l}
$808:$ \\
$88: 80$ \\
09080 \\
\hline
\end{tabular}

-ió -100

- 0 우

$0 \dot{0}+\dot{1}$

.

若总

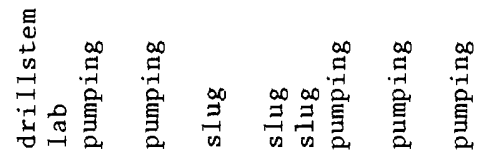

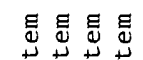

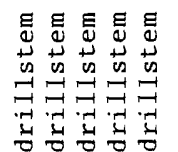

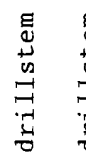

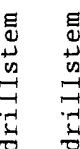

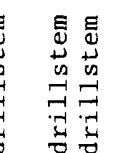

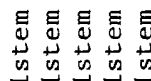

뿽혀

吾

000

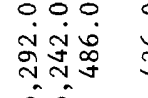

0
$\infty$
0
$\alpha$

우웅잉

吾

66

ฟีํํำ

号

0000.0000

\section{(}

- 0

00

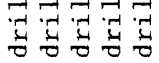

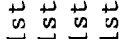

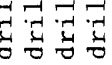

\section{$0.0 \%$ o $0.00 \%$ o.}

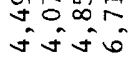

ingis

ते

웅

00000

0000

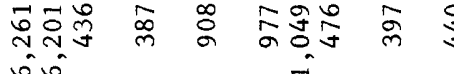

$\vec{n}$

0.00 .0 .000$$
\text { (n) }
$$

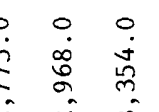

00

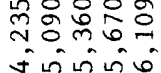

i

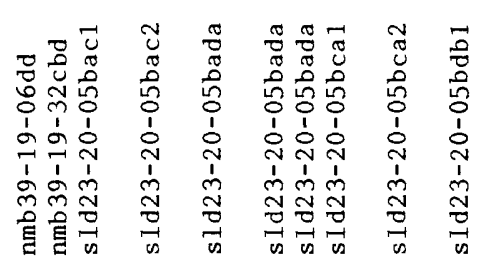

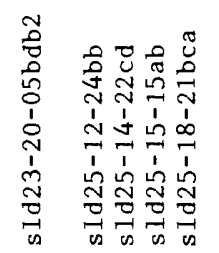

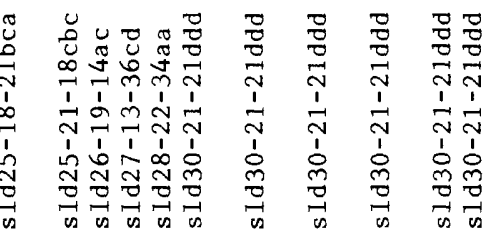
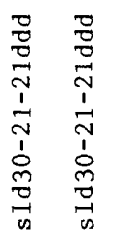

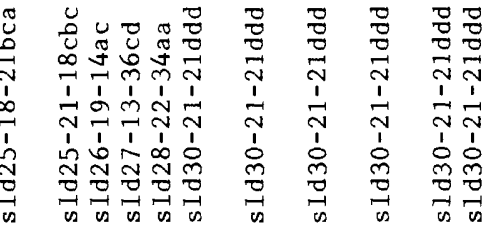

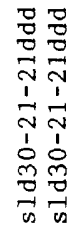




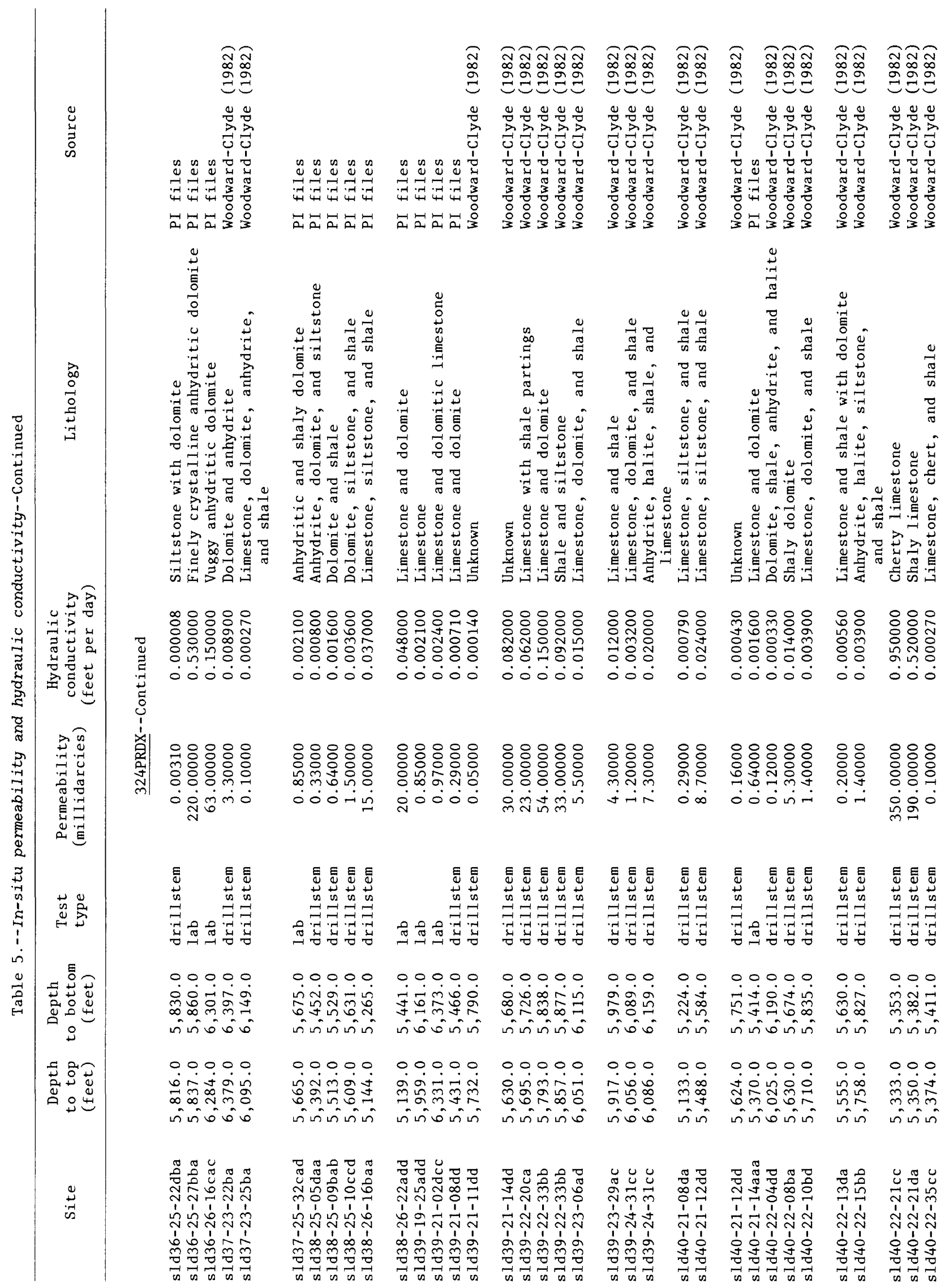




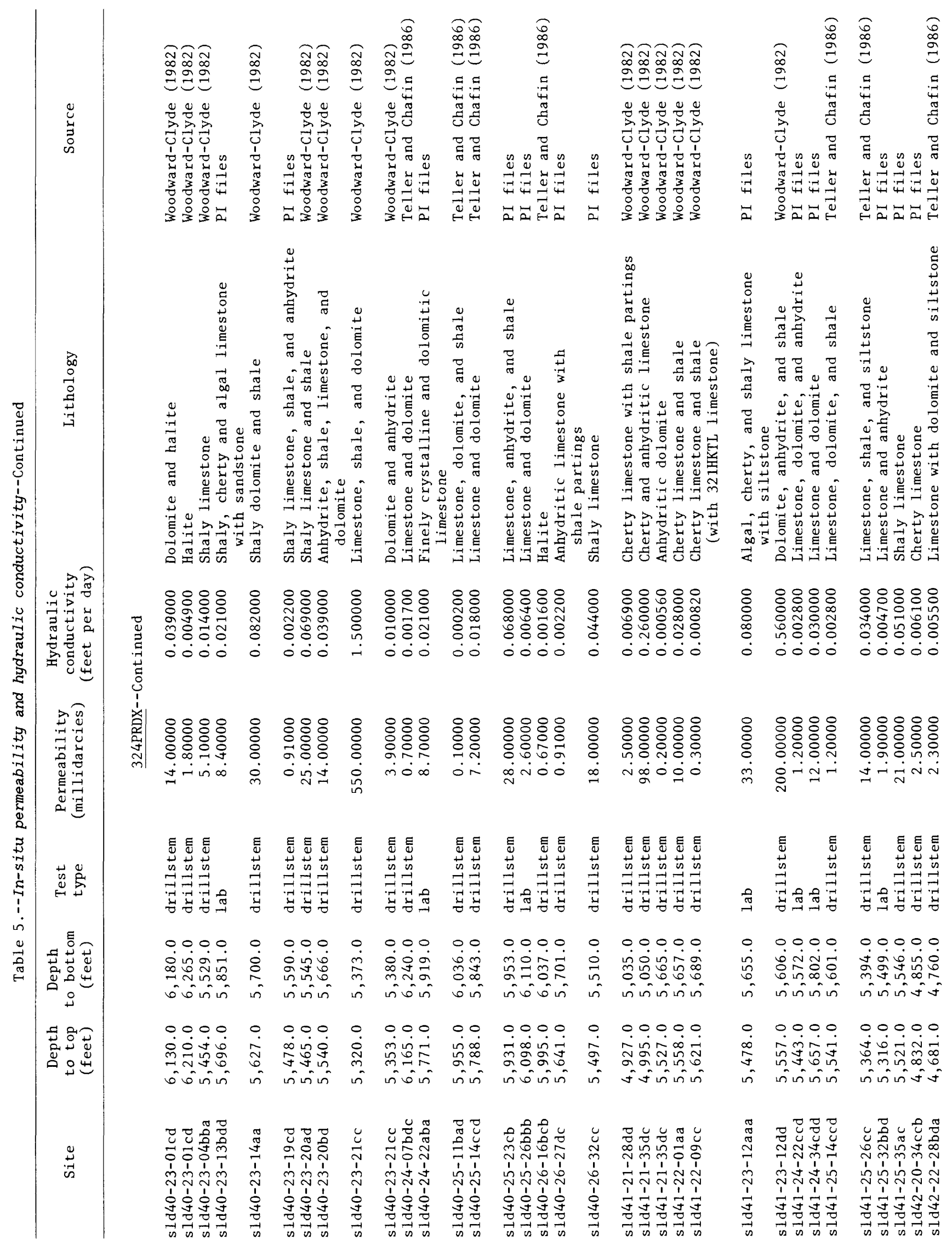




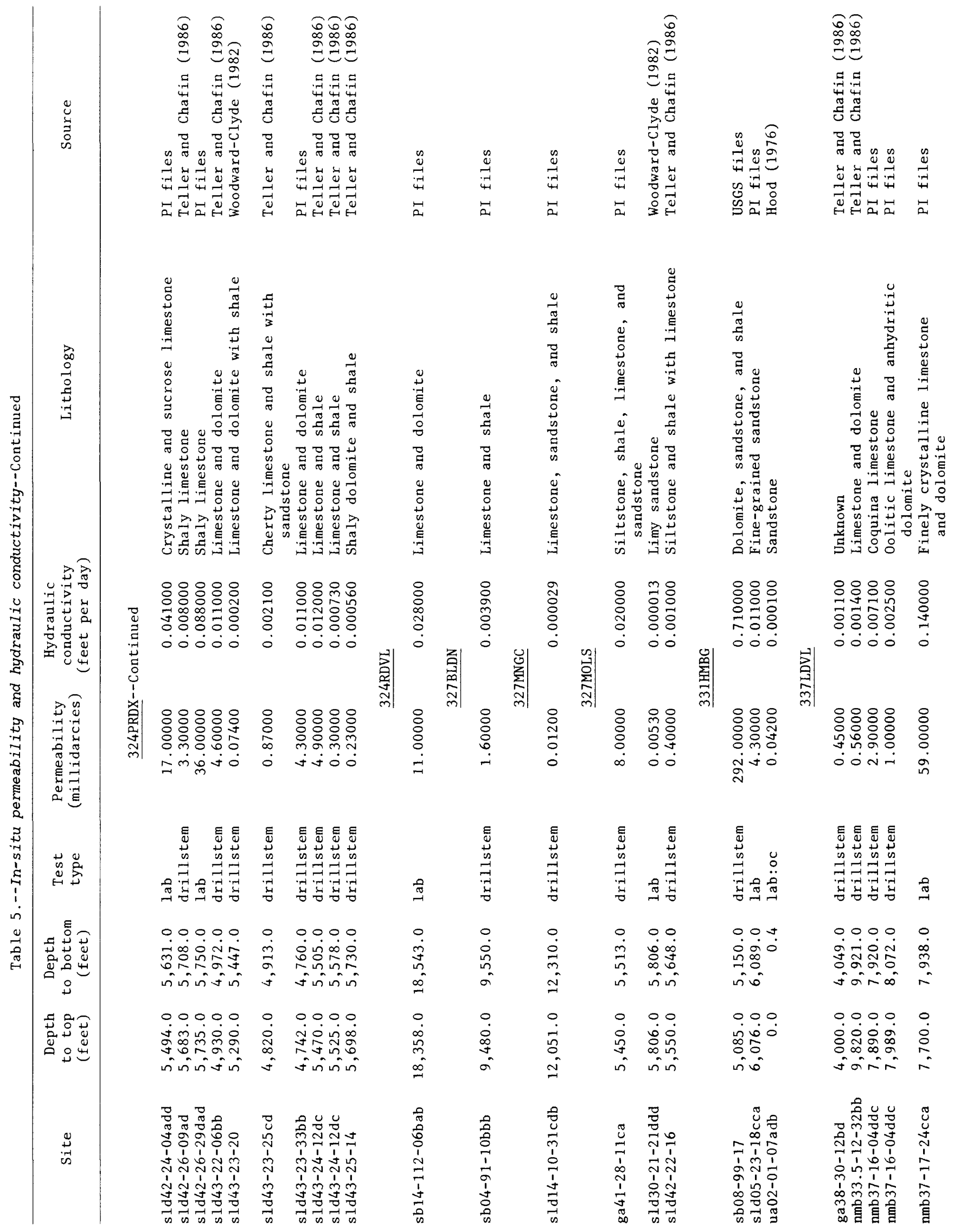




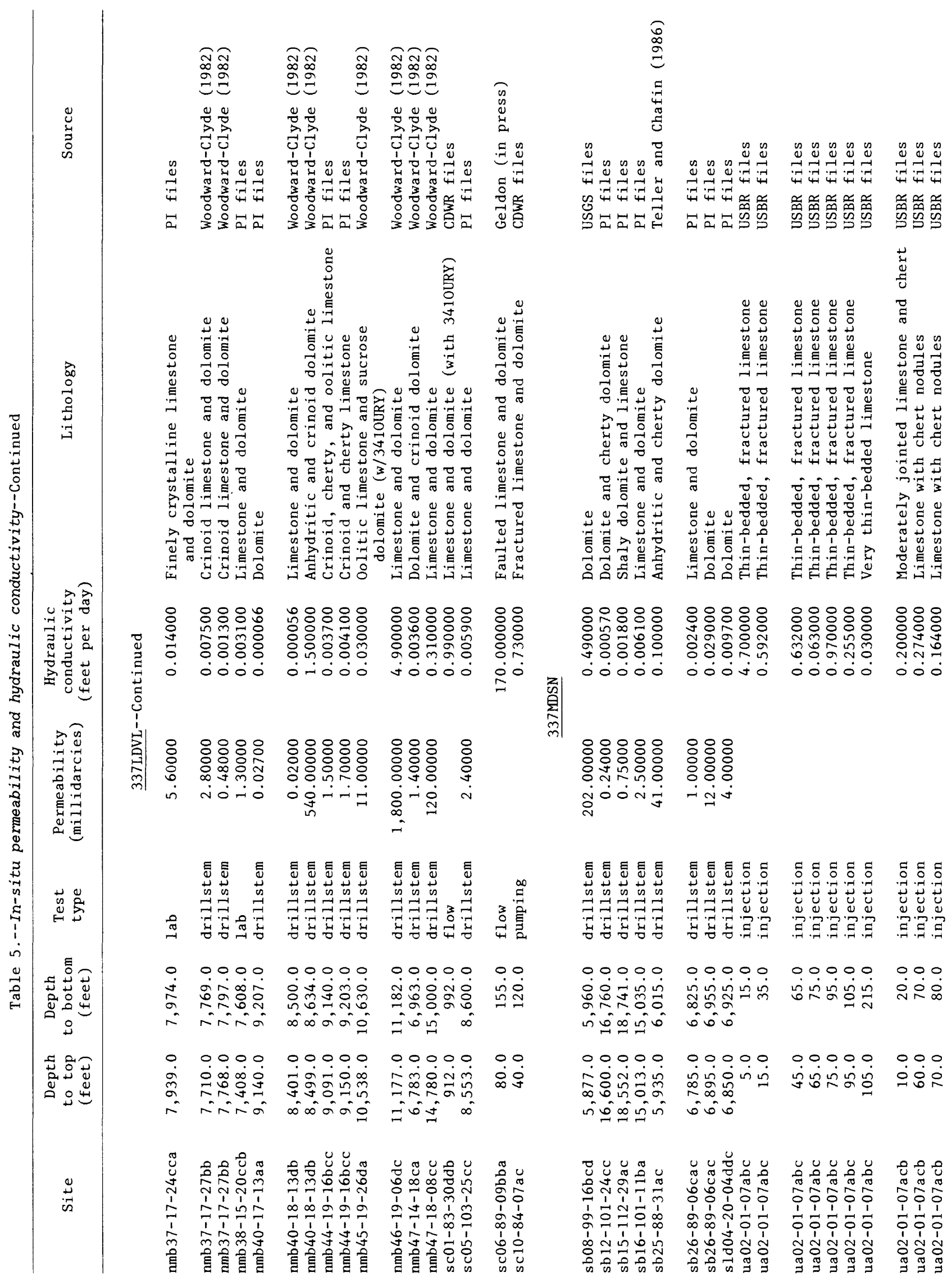




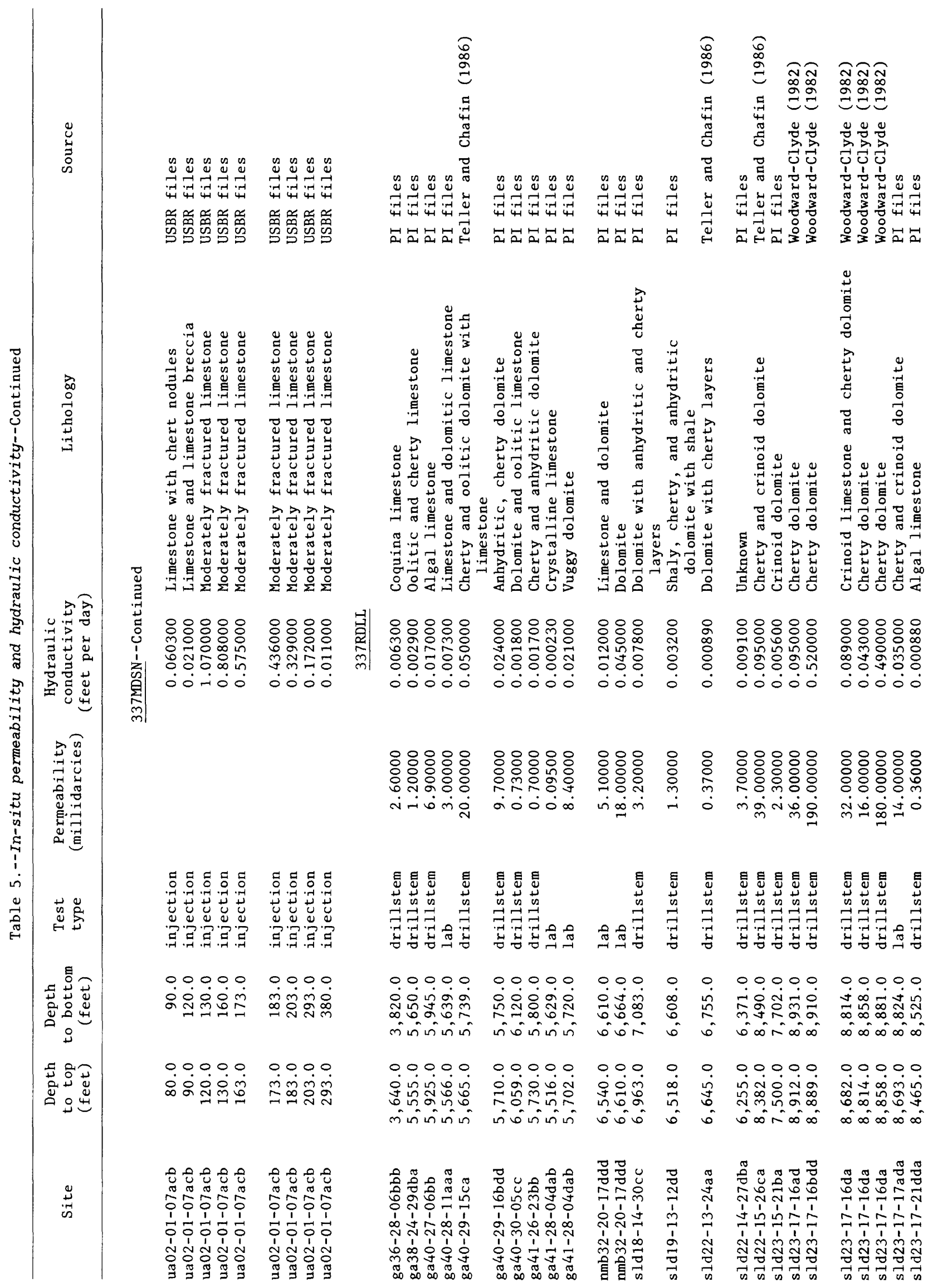




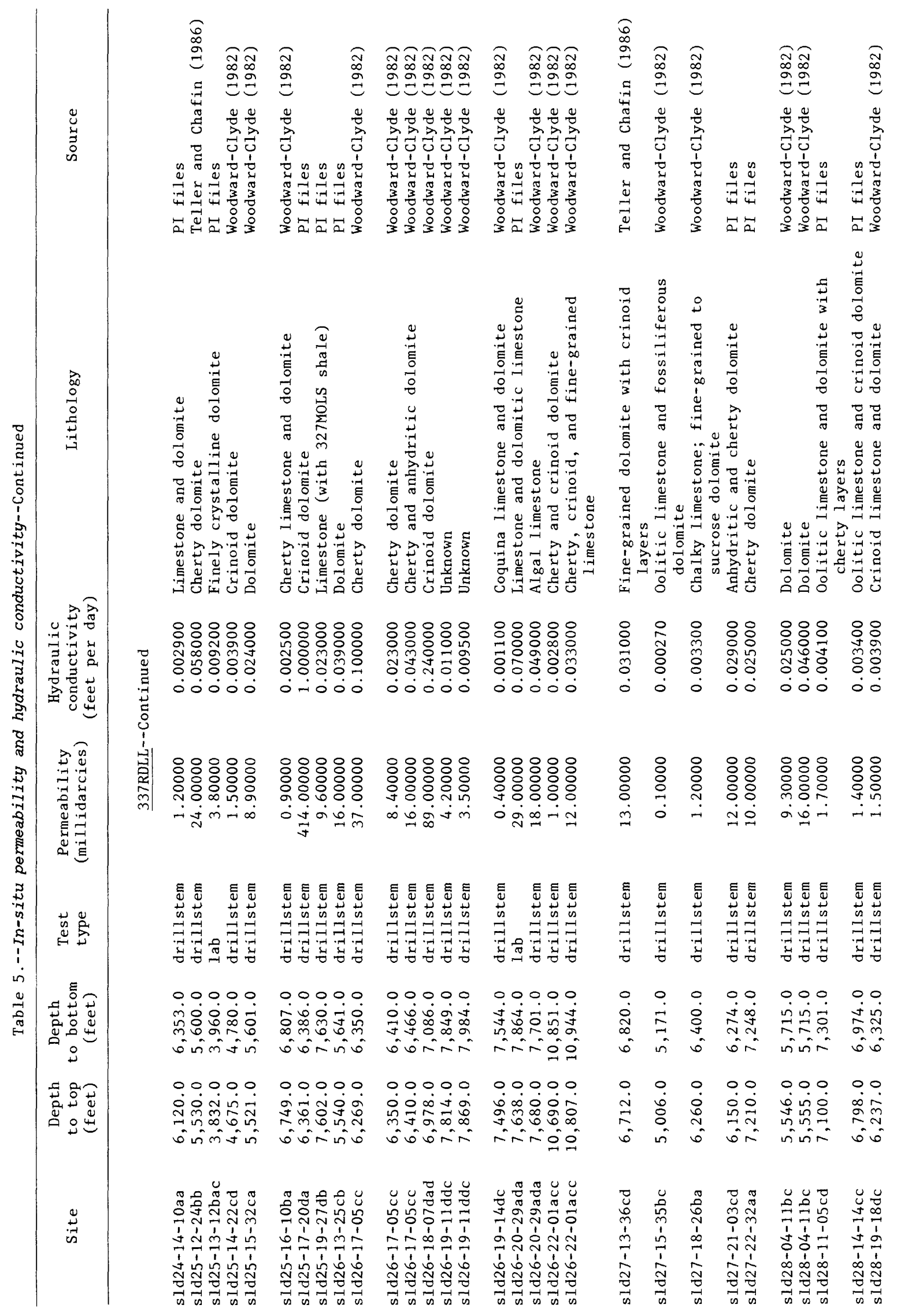




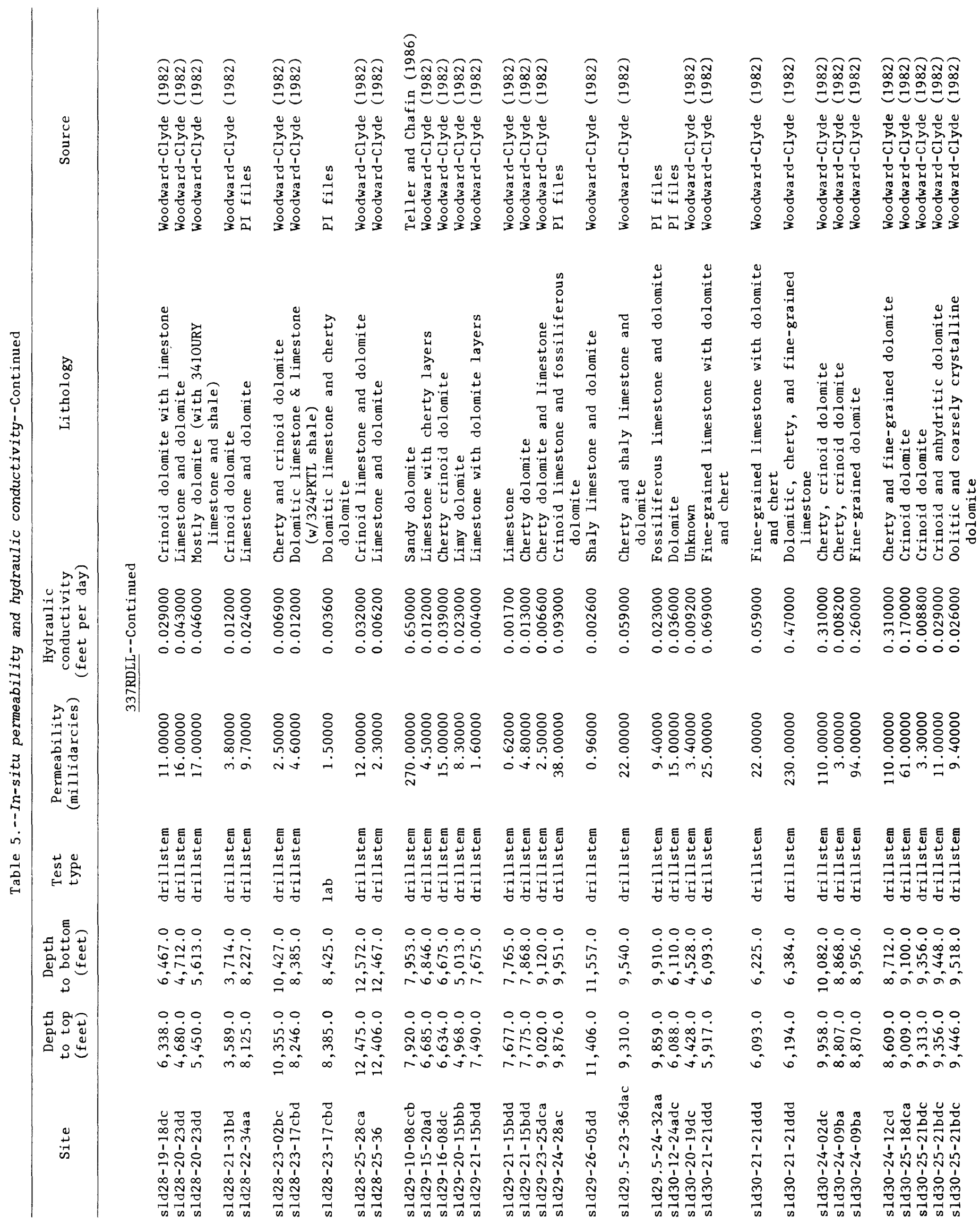




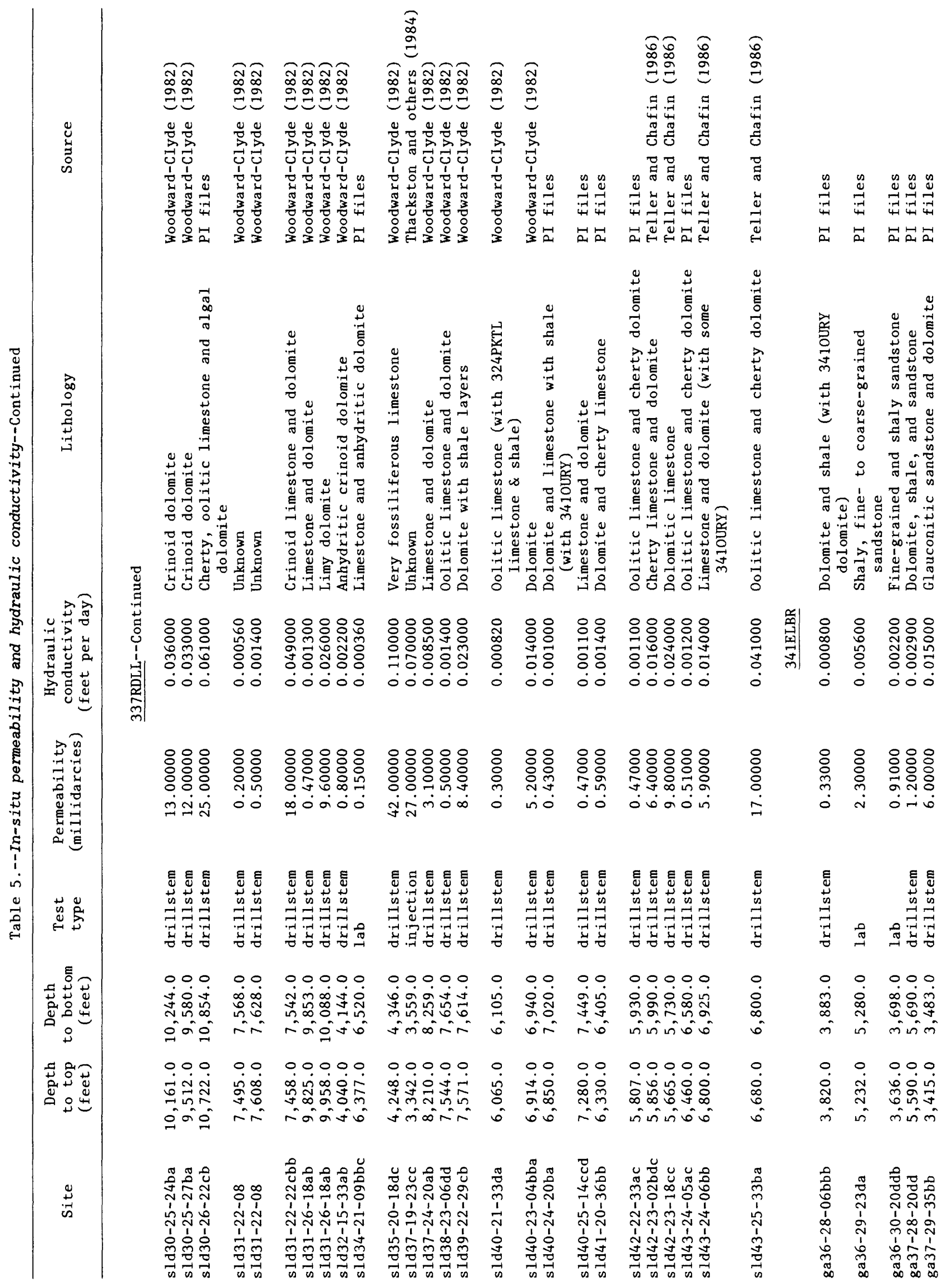




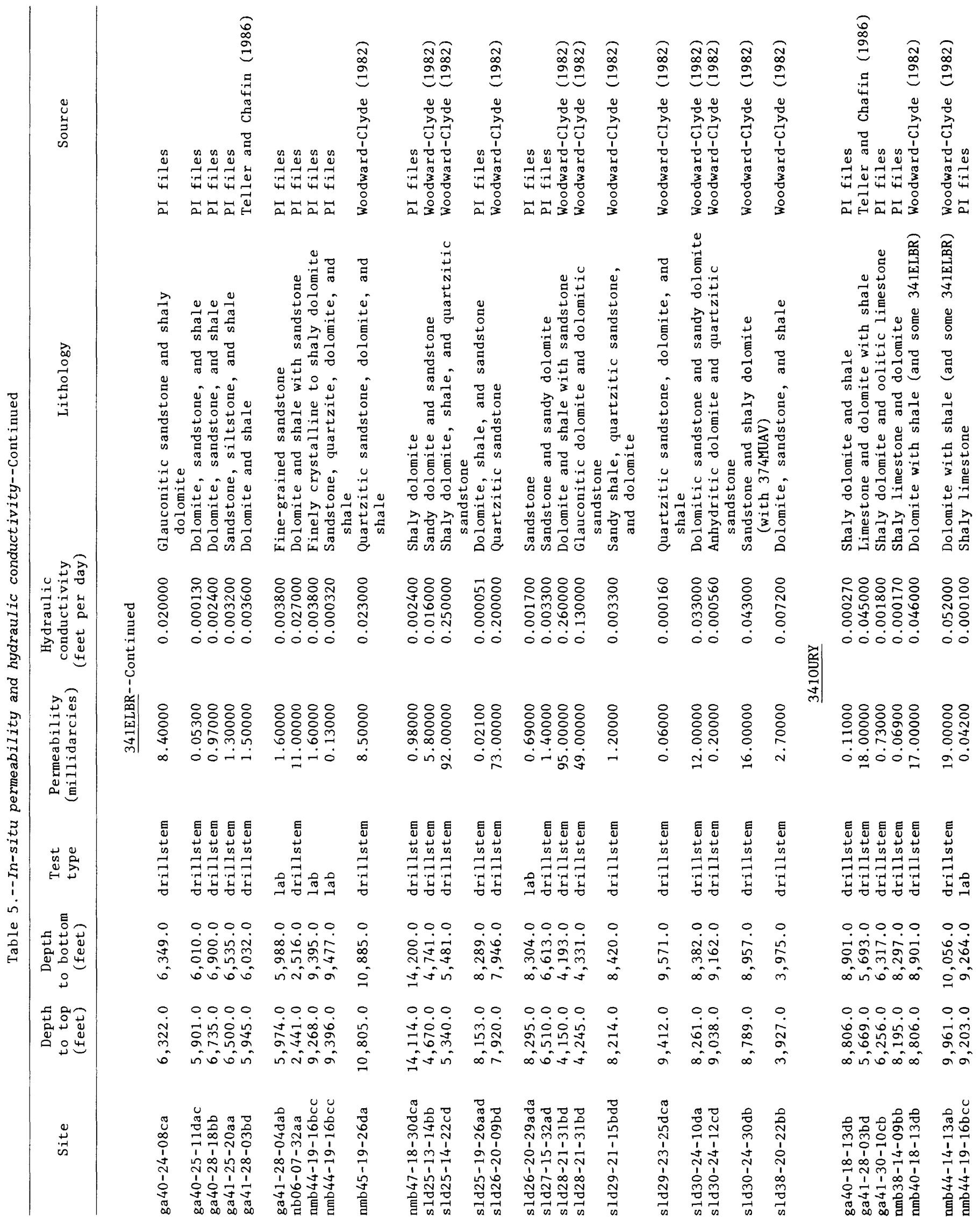




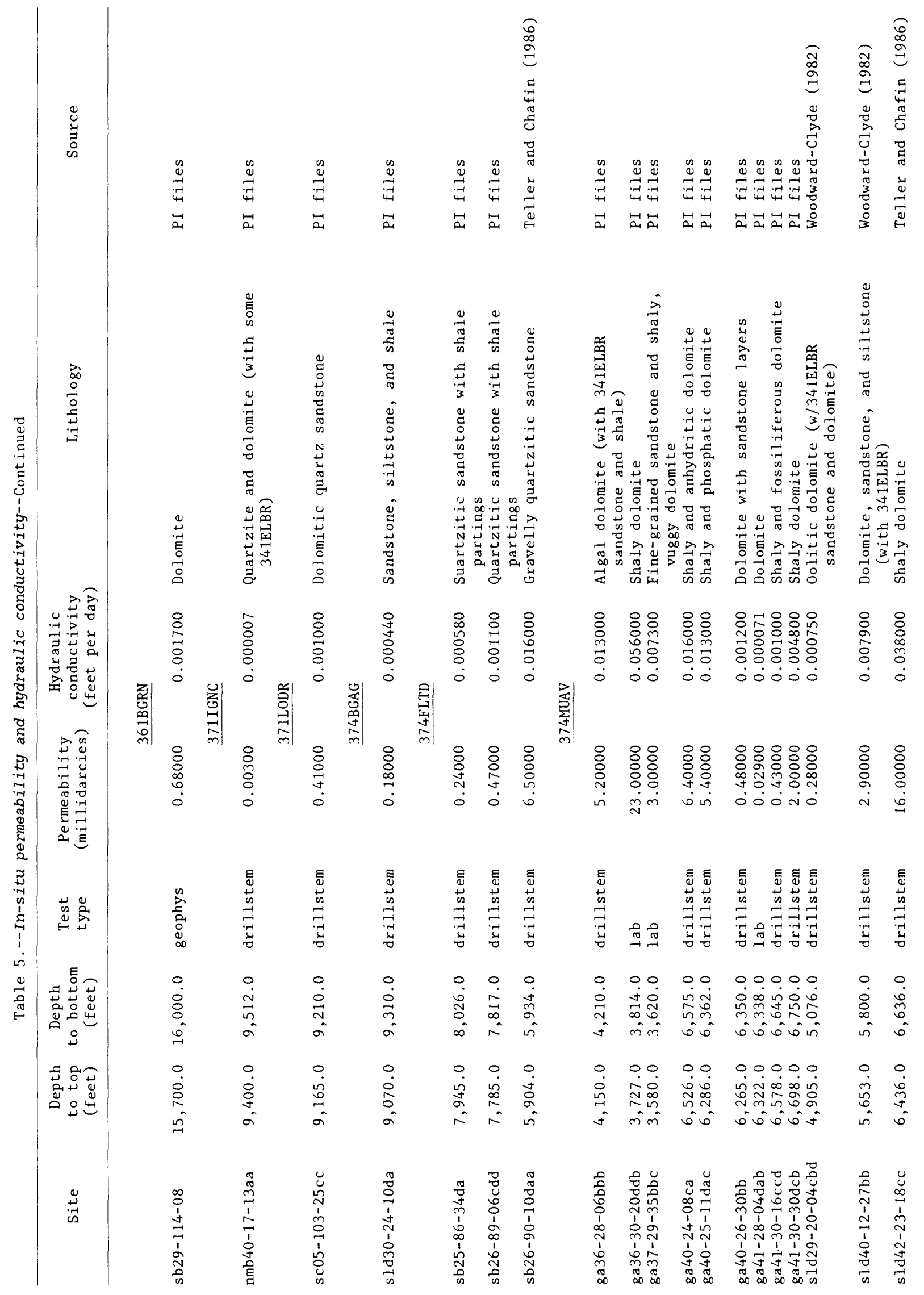




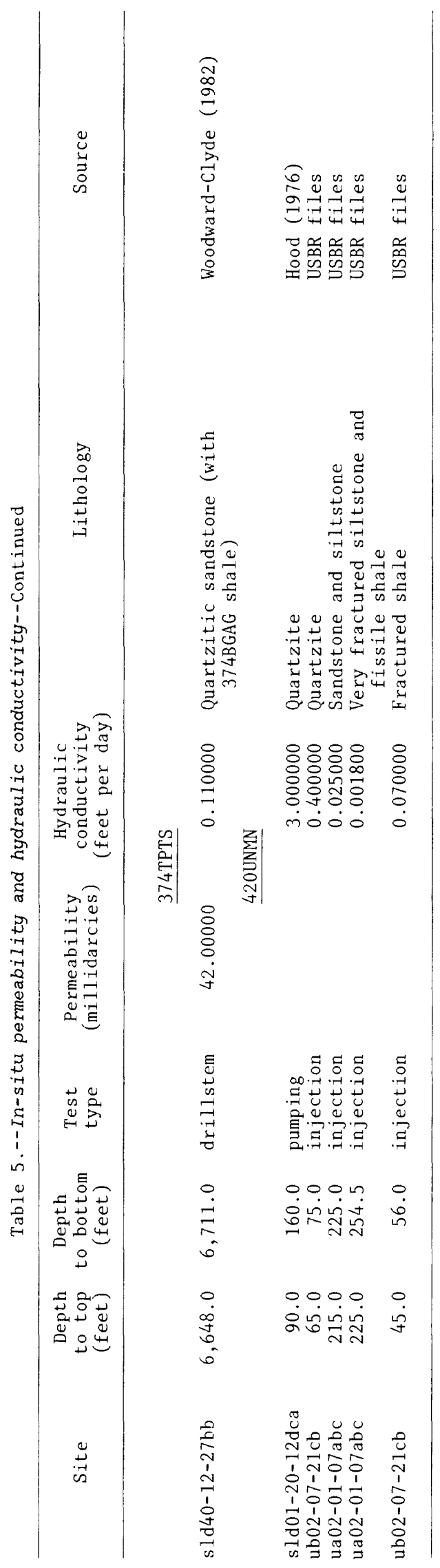

